\title{
LIQUID ACQUISITION DEVICES FOR ADVANCED IN-SPACE CRYOGENIC PROPULSION SYSTEMS
}

\author{
by \\ JASON WILLIAM HARTWIG
}

Submitted in partial fulfillment of the requirements

For the degree of Doctor of Philosophy

Dissertation adviser: Dr. Yasuhiro Kamotani

Department of Mechanical and Aerospace Engineering CASE WESTERN RESERVE UNIVERSITY

May, 2014 


\section{CASE WESTERN RESERVE UNIVERSITY \\ SCHOOL OF GRADUATE STUDIES}

We hereby approve the thesis/dissertation of
Jason William Hartwig
candidate for the Doctor of Philosophy degree*.

(signed) Yasuhiro Kamotani

(chair of the committee)

$\underline{\text { Jaikrishnan Kadambi }}$

$\underline{\text { Jay Adin Mann Jr. }}$

$\underline{\text { David Chato }}$

(date) $03 / 14 / 2014$

*We also certify that written approval has been obtained for any proprietary material contained therein 
Copyright @ 2014 by Jason William Hartwig

All rights reserved 


\section{Dedication}

I dedicate this dissertation to my teacher. Sir, your unwavering compassion, dedication, and guidance provided the backbone to which this work was made possible. Thank you for your constant daily inspiration and support. You inspired me to work with single pointed focus and determination throughout the course of this work. The character traits that you instill in me, I will always cherish in my heart. Sir thank you for believing in me, and inspiring me to work in a regulated and dedicated manner. Not a day passes without an overwhelming feeling of joy and gratitude for your presence in my life. 


\section{Table of Contents}

List of Tables $\quad$ xvii

List of Figures $\quad$ xxi

Acknowledgements $\quad$ xl

List of Abbreviations $\quad$ xlv

Glossary $\quad$ li

$\begin{array}{ll}\text { Abstract } & \text { lix }\end{array}$

\section{Chapter 1: Introduction}

1.1 The Flexible Path 1

1.2 Fundamental Cryogenic Fluids 4

1.3 Motivation for Cryogenic Propulsion Technology Development 6

1.4 Existing Challenges with Cryogenic Propellants 7

1.5 Cryogenic Fluid Management Subsystems 9

1.6 Future Cryogenic Fluid Management Applications 9

1.6.1 In-Space Cryogenic Engines 10

1.6.2 In-Space Cryogenic Fuel Depots 13

\section{Chapter 2: Background and Historical Review}

2.1 Propellant Management Device Purpose 27

2.2 Other Types of Propellant Management Devices 31

$\begin{array}{lll}2.3 & \text { Vanes } & 36\end{array}$

2.3.1 Design Concept, Basic Flow Physics, and Principle of Operation 36

2.3.2 Advantages and Disadvantages 39 
2.3.3 Storable Propellant Historical Examples 40

2.3.3.1 Space Experiments $\quad 40$

2.3.3.2 Vehicles and Missions $\quad 41$

2.4 Sponges 43

2.4.1 Design Concept, Basic Flow Physics, and Principle of Operation 43

2.4.2 Advantages and Disadvantages 45

2.4.3 Storable Propellant Historical Examples 46

2.4.3.1 Space Experiments $\quad 46$

2.4.3.2 Vehicles and Missions $\quad 47$

2.5 Screen Channel Liquid Acquisition Devices 53

2.5.1 Design Concept, Basic Flow Physics, and Principle of Operation 53

2.5.2 Mesh and Metal Type 56

2.5.3 Advantages and Disadvantages $\quad 59$

2.5.4 Storable Propellant Historical Examples 60

2.5.4.1 Space Experiments 61

2.5.4.2 Vehicles and Missions $\quad 62$

2.5.5 Cryogenic Propellant Historical Examples 65

2.6 Propellant Management Device Combinations 68

$\begin{array}{lll}2.7 & \text { NASA's Current Needs } & 70\end{array}$

\section{Chapter 3: Influential Factors and Physics- Based Modeling of Liquid Acquisition Devices}

$3.1 \quad$ 1-g One Dimensional Simplified Pressure Drop Model 73

3.2 The Room Temperature Bubble Point Pressure 76

$\begin{array}{lll}3.2 .1 & \text { Assumptions } & 77\end{array}$

3.2.2 Bubble Point Model Derivation 78

3.2.3 Types of Bubble Point Experiments 85 
3.2.4 Surface Tension Model 86

3.2.5 Specifying the Pore Diameter 89

3.2.6 Previously Reported Bubble Points 92

3.3 Hydrostatic Pressure Drop 96

$3.4 \quad$ Flow-through-Screen Pressure Drop 98

3.4.1 Model Derivation $\quad 98$

3.4.2 Model Parameters and Flow-through-Screen Experiment 101

3.4.3 Historical Data and Trends 103

3.5 Frictional and Dynamic Pressure Drop 108

$\begin{array}{lll}3.6 & \text { Wicking Rate } & 115\end{array}$

3.6.1 Model Derivation 116

3.6.2 Wicking Rate Experiment 120

$\begin{array}{lll}\text { 3.6.3 Historical Data and Trends } & 120\end{array}$

3.7 Screen Compliance 122

3.7.1 Model Derivation and Screen Compliance Experiment 122

3.7.2 Historical Data and Trends 123

$\begin{array}{lll}3.8 & \text { Material Compatibility } & 124\end{array}$

3.9 The Room Temperature Reseal Pressure Model 126

3.9.1 Model Derivation 127

3.9.2 Historical Data and Trends 130

3.9.3 Specifying the Reseal Diameter 131

3.10 Pressurant Gas Type 132

3.11 Concluding Remarks and Implications for Cryogenic Propulsion Systems 133

Chapter 4: Room Temperature Liquid Acquisition Device Performance Experiments

4.1 Pure Fluid Tests 
4.1.1 Scanning Electron Microscopy Analysis 139

4.1.2 Bubble Point Experimental Setup 142

4.1.3 Bubble Point Experimental Methodology and Data Reduction 145

$\begin{array}{lll}\text { 4.1.4 Contact Angle Measurements } & 148\end{array}$

4.1.5 Experimental Bubble Point Results 150

$\begin{array}{lll}4.2 & \text { Binary Mixture Tests } & 154\end{array}$

4.2.1 Experimental Setup, Methodology, and Data Reduction 154

4.2.2 Theoretical Predictions 155

4.2.2.1 Liquid/Vapor Surface Tension of the Methanol/Water Mixture

4.2.2.2 Contact Angle Measurements 157

4.2.3 Experimental Results 159

4.2.3.1 Bubble Point Pressure 159

4.2.3.2 Critical Zisman Surface Tension 166

$\begin{array}{lll}4.3 & \text { Reseal Pressure Tests } & 170\end{array}$

$\begin{array}{lll}4.4 & \text { Wicking Rate Tests } & 172\end{array}$

$\begin{array}{lll}\text { 4.5 Concluding Remarks } & 175\end{array}$

\section{Chapter 5: Parametric Analysis on the Liquid Hydrogen and Nitrogen Bubble Point Pressure}

$\begin{array}{lll}5.1 & \text { Test Purpose and Motivation } & 179\end{array}$

$\begin{array}{lll}5.2 & \text { Experimental Design } & 180\end{array}$

5.2.1 Test Article and Facility 180

5.2.2 Instrumentation and Data Acquisition 186

$\begin{array}{lll}\text { 5.2.3 Data Reduction } & 188\end{array}$

$\begin{array}{llr}\text { 5.2.4 Test Matrix } & 189\end{array}$ 
$\begin{array}{lll}5.3 & \text { Experimental Methodology } & 191\end{array}$

5.4 Experimental Results and Discussion 192

5.4.1 Screen Weave Dependence 197

5.4.2 Liquid Dependence 201

5.4.3 Liquid Temperature Dependence 204

5.4.4 Liquid Pressure Dependence 206

5.4.5 Pressurant Gas Dependence 210

5.5 Concluding Remarks 222

\section{Chapter 6: High Pressure Liquid Oxygen Bubble Point Experiments}

6.1 Test Purpose and Motivation 225

6.2 Experimental Design 228

6.2.1 Test Article and Facility 228

6.2.2 Instrumentation and Data Acquisition 232

6.2.3 Test Matrix 233

6.3 Experimental Methodology 234

6.4 Experimental Results and Discussion 236

6.4.1 Test Conditions 236

6.4.2 Elevated Temperature Dependence 239

6.4.3 Liquid Subcooling and Pressurant Gas Dependence 243

6.4.4 Heat Transfer Effects at Elevated Temperature 249

6.4.5 Analysis of Videos 257

$\begin{array}{lll}6.5 & \text { Concluding Remarks } & 258\end{array}$ 


\section{Chapter 7: High Pressure Liquid Methane Bubble Point Experiments}

7.1 Test Purpose and Motivation 263

$\begin{array}{lll}7.2 & \text { Experimental Design } & 264\end{array}$

7.2.1 Modifications to Facility, Test Article, and Instrumentation 264

$\begin{array}{lll}\text { 7.2.2 Test Matrix } & 270\end{array}$

7.3 Experimental Results and Discussion 271

$\begin{array}{lll}\text { 7.3.1 Test Conditions } & 271\end{array}$

7.3.2 Elevated Temperature Dependence 272

7.3.3 Liquid Subcooling and Pressurant Gas Dependence 275

$\begin{array}{lll}7.4 & \text { Thermal Analysis } & 280\end{array}$

7.4.1 Heat Transfer at Breakdown 282

7.4.2 Interfacial Temperature 290

7.4.3 Condensation and Evaporation Mass Flux 298

7.4.3.1 Temperature and Pressure Data-Based 300

7.4.3.2 Kinetic Theory 305

7.4.4 Screen Reynolds Number 307

7.4.5 Heat Conduction into Liquid 311

$\begin{array}{lll}7.5 & \text { Concluding Remarks } & 316\end{array}$

7.5.1 Pressurization with Gaseous Methane 318

7.5.2 Pressurization with Gaseous Helium 319

$\begin{array}{lll}\text { 7.5.3 Pressurization with Gaseous Nitrogen } & 321\end{array}$

\section{Chapter 8: Warm Pressurant Gas Effects on the Static Bubble Point Pressure for Cryogenic Liquid Acquisition Devices}


8.1 Test Purpose and Motivation $\quad 324$

8.2 Design Modifications 327

8.3 Experimental Methodology 329

$\begin{array}{lll}8.4 & \text { Test Matrix } & 330\end{array}$

8.5 Warm Pressurant Gas Liquid Hydrogen Experiments 330

8.6 Warm Pressurant Gas Liquid Nitrogen Experiments 337

8.7 Concluding Remarks 341

\section{Chapter 9: Full Scale Liquid Acquisition Device Outflow Tests in Liquid Hydrogen}

9.1 Test Purpose and Motivation 344

$\begin{array}{lll}9.2 & \text { Test Plan } & 346\end{array}$

9.3 Facility and Test Article 347

9.4 Horizontal Liquid Acquisition Device Tests 353

9.4.1 Test Description 353

9.4.2 Research Hardware 354

9.4.3 Instrumentation and Test Methodology 355

9.4.4 Experimental Results and Comparison to Model 356

9.5 Flow-through-Screen Tests 359

9.5.1 Test Description 359

9.5.2 Research Hardware 360

$\begin{array}{lll}\text { 9.5.3 Instrumentation } & 360\end{array}$

9.5.4 Test Methodology 363

9.5.5 Experimental Results and Comparison to Model 363

9.5.5.1 Screen Weave Dependence 363

9.5.5.2 Temperature Dependence 366 
9.6 1-g Inverted Vertical Liquid Acquisition Device Outflow Tests 371

$\begin{array}{lll}\text { 9.6.1 Test Description } & 371\end{array}$

9.6.2 Research Hardware 373

9.6.2.1 Standard 325x2300 Channel 373

9.6.2.2 Thermodynamic Vent System Cooled 325x2300 Channel 375

9.6.3 Thermodynamic Vent System Heat Exchanger Analysis 377

$\begin{array}{lll}\text { 9.6.4 Instrumentation } & 382\end{array}$

9.6.5 Test Methodology 385

9.6.6 Test Matrix 386

9.6.7 One Dimensional Steady State Pressure Drop Model General Trends

9.6.8 Experimental Results 391

9.6.8.1 Screen Channel Bubble Point Tests in Isopropyl Alcohol 391

9.6.8.2 Standard 325x2300 Channel Performance 394

9.6.8.3 Thermodynamic Vent System Cooled 325x2300 Channel Performance 397

9.6.8.4 Thermodynamic Vent System Efficiency 399

9.6.8.5 Subcooling Effect $\quad 402$

9.6.9 Comparison to One Dimensional Model 405

$\begin{array}{lll}9.7 & \text { Concluding Remarks } & 409\end{array}$

\section{Chapter 10: The Bubble Point Pressure Model for Cryogenic Propellants}

10.1 Current Model Limitations 414

10.2 Summary of Data 416

10.3 Room Temperature Pore Diameter Model 418

$\begin{array}{ll}\text { 10.3.1 Model } & 418\end{array}$

10.3.2 Maximum Bubble Point Pressure 423 
10.4 Temperature Dependent Pore Diameter and Pressurant Gas Model 427

10.5 Liquid Subcooling Model 440

10.6 Warm Pressurant Gas Model 448

10.7 Concluding Remarks 455

\section{Chapter 11: The Reseal Pressure Model for Cryogenic Propellants}

11.1 Current Model Limitations 460

$\begin{array}{lll}11.2 & \text { Summary of Data } & 461\end{array}$

11.3 Room Temperature Reseal Diameter Model 461

11.4 Temperature Dependent Reseal Diameter Model 464

11.5 Liquid Subcooling Model 472

11.6 Warm Pressurant Gas Model 480

11.7 Concluding Remarks 481

\section{Chapter 12: Analytical Model for Steady Flow through a Porous Liquid Acquisition Device Channel}

12.1 One Dimensional Pressure Drop Model Drawbacks 487

12.2 Literature Review 489

12.3 Analytical Model Formulation 493

$\begin{array}{lll}\text { 12.3.1 Assumptions } & 494\end{array}$

12.3.2 Governing Equations 495

12.3.3 Method of Solution 497

12.4 Model Results, Sensitivities, and Comparison to One Dimensional Model 504

12.4.1 Validation of Laminar Channel Flow Assumption 512 
12.4.2 Model Comparison to Liquid Oxygen Horizontal Liquid Acquisition Device Experiments

12.4.3 Model Comparison to Liquid Hydrogen 1-g Inverted Vertical Outflow Experiments

12.5 Dynamic Bubble Point Model 521

12.6 Convective Cooling of the Liquid Acquisition Device Screen 526

$\begin{array}{lll}12.7 & \text { Concluding Remarks } & 528\end{array}$

\section{Chapter 13: Optimal Liquid Acquisition Device Screen Weave for a Liquid Hydrogen Fuel Depot}

13.1 Background and Mission Requirements 533

13.2 Bubble Point Pressure and Flow-through-Screen Pressure Drop 536

13.3 Critical Mass Flux 542

13.4 Minimum Bubble Point 543

13.5 Minimum Screen Area 545

13.6 Other Considerations 549

13.7 Channel Number and Size 552

13.8 Concluding Remarks 553

\section{Chapter 14: Optimal Propellant Management Device for a Small Scale Liquid Hydrogen Propellant Tank}

14.1 Background and Mission Requirements 556

14.2 Analytical Screen Channel Flow Model in Microgravity 559

14.2.1 Extension of 1-g model to Microgravity 559

$\begin{array}{ll}\text { 14.2.2 Flow Model Derivation } & 561\end{array}$ 
$\begin{array}{ll}\text { 14.2.4 General Model Trends } & 570\end{array}$

14.3 Analytical Vane Model in Microgravity 577

14.4 Trade Study Variables $\quad 584$

14.5 Trade Study Results 587

$\begin{array}{lll}14.6 & \text { Concluding Remarks } & 597\end{array}$

\section{Chapter 15: Conclusions}

$\begin{array}{lll}15.1 & \text { Summary } & 600\end{array}$

$\begin{array}{lll}15.2 & \text { Future Work } & 606\end{array}$

\section{Appendices}

$\begin{array}{lll}\text { Appendix A Historical Depot Demonstration Missions } & 609\end{array}$

$\begin{array}{ll}\text { A.1 Project THERMO } & 610\end{array}$

A.2 Shuttle Derived Experiments 611

A.3 COLD-SAT 612

A.4 CONE 615

A.5 CRYOTE 616

$\begin{array}{ll}\text { A.6 CPST TDM } & 617\end{array}$

Appendix B Summary of Previously Reported Bubble Point Data 618

Appendix C Langmuir Isotherm for the Liquid/Vapor Case 623

Appendix D Langmuir Isotherms for the Solid/Liquid and Solid/Vapor Case 630

Appendix E Historical Heated Pressurant Gas Liquid Acquisition Device Tests 638

$\begin{array}{lll}\text { Appendix F Previously Reported Porous Channel Solutions } & 648\end{array}$

Appendix G Summary of Cryogenic Screen Channel LAD Design Tools 661

G.1 Bubble Point Pressure Model 661 
G.2 Reseal Pressure Model 663

G.3 Flow-through-Screen Pressure Drop $\quad 664$

G.4 Wicking Rate $\quad 664$

G.5 Total System Pressure Drop in 1-g or Microgravity 665

$\begin{array}{ll}\text { Bibliography } & 667\end{array}$ 


\section{List of Tables}

Chapter 1

1.1 - Relevant Thermophysical Properties for the Four Primary Cryogenic Liquids

1.2 Challenges and Implications for Future Liquid Oxygen/Liquid Hydrogen System Design

Chapter 3

3.1 - Fitting Parameters for the Surface Tension Model

3.2 - Measured and Calculated Screen Parameters

3.3 - Formulas for Calculating Screen Properties

105

3.4 - Fitting Parameters for Flow-through-Screen Pressure Drop Model

3.5 - Effective Wicking Diameters for Several Dutch Twill and Plain Dutch Weaves

3.6 - Screen Compliance Model Parameters for Three Different Screens

3.7 - Manufacturability of Liquid Acquisition Device Screen Meshes

3.8 - Room Temperature Reseal Diameters using Method 2

3.9 - Chronology of Recent Liquid Acquisition Device Technology

Development Program Conducted at NASA Glenn Research Center

Chapter 4

4.1 - Screen Parameters from Scanning Electron Microscopy Image Analysis

4.2 - Comparison of Three Methods for Determining the Effective Pore Diameter: 1. Fit to Equation 3.20 Using Pure Fluid Contact Angles, 2. Historical Data, 3. Scanning Electron Microscopy Image Analysis

4.3 - Updated Effective Wicking Diameters for Dutch Twill and Plain Dutch Weaves

Chapter 5

$5.1-450 \times 2750$ Test Matrix 


\section{Chapter 9}

9.1 - Summary of Reference Missions for Full Scale Outflow Experiment 346

9.2 - Horizontal Liquid Acquisition Device Instrumentation 356

9.3 - Flow-through-Screen Instrumentation 362

9.4 - Flow-through-Screen Fitting Parameters for Various Fluids for a $325 \times 2300$ Screen

9.5 - Instrumentation List for Inverted Vertical Outflow Tests

9.6 - Inverted Outflow Test Matrix

9.7 - Pressure Drop Contributions at Breakdown for Low and High Flows

9.8 - Thermodynamic Vent System Cooled 325x2300 Channel

Performance

9.9 - Gain in Bubble Point Pressure as a Function of the Level of Subcooling

Chapter 10

10.1 - Calculated Pore Diameters at Room Temperature

10.2 - Updated Set of Measured and Calculated Liquid Acquisition

Device Screen Parameters

10.3 - Gain or Loss in Bubble Point Pressure from Room Temperature Value at Liquid Hydrogen Temperatures

10.4 - Linear Thermal Contraction Coefficients for Screen Channel Liquid Acquisition Device Metals

10.5 - Temperature Dependent Pore Diameter Fitting Parameters

10.6 - Complete Coefficient Matrix for Subcooled Liquid Bubble Point Model

10.7 - Averaged Subcooled Liquid Gain Slopes as a Function of Gas/Liquid Pair 
10.9 - Comparison of Performance between Old Room Temperature and New Cryogenic Bubble Point Pressure Model

\section{Chapter 11}

11.1 - Comparison of Breakthrough Pore Diameters with Reseal

Diameters at Room Temperature

11.2 - Ratio of Reseal Pressure at 20.3K to Reseal Pressure at Room Temperature

11.3 - Fitting Parameters for Temperature Dependent Reseal Diameter Model

11.4 - Coefficient Matrix for Subcooled Liquid Reseal Pressure Model

11.5 - Coefficient Matrix for Heated Pressurant Gas Reseal Model

Chapter 12

12.1 - Flow-through-Screen Pressure Drop Coefficients for Different Screens

\section{Chapter 13}

13.1 - Depot Sizing for a Mars Exploration Mission for Two Types of Architectures

13.2 - Thermodynamic Properties of Liquid Hydrogen Which Bound Depot Operation

13.4 - Liquid Hydrogen Bubble Point Pressure Computed at the Normal Boiling Point for Candidate Screens for the Fuel Depot

13.5 - Computed Z Values for the Five Finest Dutch Twill Screens in Liquid Hydrogen

13.6 - Required Minimum Screen Area and Channel Width for Two Different Liquid Hydrogen Depot Concepts at a Demand Flow Rate of $2.75 \mathrm{~kg} / \mathrm{s}$ 


\section{Chapter 14}

14.1 - Comparison of Model Predicted Percent of Screen Exposed to Pressurant Gas at Breakdown in a) 1-g and b) Microgravity for a $325 \times 2300$ Screen and Channel in Liquid Hydrogen at a Demand Flow Rate of $0.01 \mathrm{~kg} / \mathrm{s}$

14.2 - Screen Channel Liquid Acquisition Device Expulsion Efficiencies for Variable Thermodynamic Conditions, Demand Flow Rates, and Screen Types

14.3 - Expulsion Efficiencies for Variable Sized Screen Channel Liquid Acquisition Devices in Terms of Height, Width, and Number of Arms

14.4 - Screen Channel Liquid Acquisition Device Sizing as a Function of Demand Flow Rate for Case A Ullage Bubble Growth

14.5 - Screen Channel Liquid Acquisition Device Sizing as a Function of Demand Flow Rate for Cases B and C Ullage Bubble Growth Rates

14.6 - Vane Propellant Management Device Sizing as a Function of Demand Flow Rate

Appendix A

A.1 - Shuttle Cargo Bay Derived Cryogenic Fluid Management Experiment Design Parameters

Appendix B

B.1 - Fine Mesh Dutch Twill Meshes

B.2 - Coarser Dutch Twill Meshes

B.3 - Plain Dutch Meshes

B.4 - Reverse Dutch Mesh

B.5 - Twilled Square Meshes

B.6 - Plain Square Meshes

Appendix D

D.1 - Langmuir Isotherm Fitting Parameters 


\section{List of Figures}

\section{Chapter 1}

1.1 - Triple Line Temperature, Normal Boiling Point, Critical

Temperature, and Surface Tension at the Normal Boiling Point for Several Fluids of Interest

1.2 - Operating Range of Temperatures and Pressures Over Which

Hydrogen, Nitrogen, Oxygen, and Methane Exist as Liquids

1.3 - Illustration of Cryogenic Fluid Management Components

1.4 - Lunar Mission Architecture

1.5 - Cryogenic Fuel Depot Concept

Chapter 2

2.1 - Illustration of Why Liquid Acquisition Devices are Required

2.2 - Generic Supply and Receiver System where the Downstream Customer is Either an Engine or Receiver Tank

2.3 - Schematic of a Spherical Bladder Lying Just Within the Tank Shell

2.4 - Schematic of a Diaphragm Assembly

2.5 - Example of a Trough

2.6 - A Baffle Welded to the Interior of a Propellant Tank

2.7 - Three Dimensional Image of a Vortex Suppressor

2.8 - Total Communication Vane with Center Post

2.9 - Schematic of Vane Flow Patterns in Low Gravity

2.10 - Fluid Acquisition and Resupply Experiment-II Vane and Sponge with $10 \%$ Liquid Remaining in the Tank

2.11 - Small Scale Total Communication Sponge

2.12 - Sponge Type Vane inside the Vented Tank Resupply Experiment 
2.14 - Schematic of the EUROSTAR Propellant Management Device and Tank Array Where Each Sponge is Positioned Away from the Spin Axis

2.15 - Sponge used in the Micro-satellite Technology Experiment

2.16 - Example of a Screen Channel Start Basket/Sump

2.17 - Example of a Total Communication Screen Channel Liquid Acquisition Device

2.18 - Scanning Electron Microscopy Image of a 200x1400 Dutch Twill Screen

2.19 - Three Dimensional Models of a) Twilled Square, b) Plain Dutch, and c) Dutch Twill Weave Styles

2.20 - Spherical Pleated Screen Liner used for Spacelab

2.21 - Fluid Acquisition and Resupply Experiment-I Gallery Arm

2.22 - Space Shuttle Screen Channel Liquid Acquisition Device Inside the

a) Reaction Control System and b) Orbital Maneuvering Propellant Tanks

2.23 - Buran Liquid Acquisition Device inside Liquid Oxygen Tank used for Orbital Maneuvering

2.24 - Combination Propellant Management Devices with a) Four Tall Vanes, a Small Sponge, and Baffles Positioned within the Sponge and b) Four Short Vanes, Center Post, Trap, and Trough

\section{Chapter 3}

3.1 - Vertically Oriented Screen Channel Liquid Acquisition Device in 1g Inverted Outflow at a) Full Tank Fill and b) Partial Tank Fill

3.2 - The Liquid/Vapor Interface of a Patch in the Dutch Twill Screen to Show the Geometry of the Curved Interface

3.3 - Approximating a Dutch Twill Pore as an Inverted Vertical Capillary Tube

3.4 - Progression of the Contact Angle with Time during Screen

Pressurization

3.5 - Model Generated Liquid Hydrogen and Nitrogen Surface Tension against Data from Stansfield (1958) and Jasper (1972) 
3.7 - Geometry of a Triangular Pore from Scanning Electron Microscopy Image

3.8 - Historical Bubble Point Data for a) 200x1400 and b) $325 \times 2300$ Mesh Screens

3.9 - Average Pore Diameter as a Function of the Fineness of the Mesh

3.10 - Hydrostatic Pressure Drop in a 1-g Inverted Vertical Outflow Configuration

3.11 - Friction Factor versus Screen Reynolds Number for Dutch Twill Weaves

3.12 - Friction Factor versus Screen Reynolds Number for All Other Weaves

3.13 - Frictional and Dynamic Pressure Losses down Liquid Acquisition Device Channel

3.14 - Illustration of the Wicking Phenomena

3.15 - Ideal Experimental Setup for a Screen Compliance Experiment

3.16 - Conceptual Image of a Liquid Acquisition Device Screen Pore Illustrating the Pore Mouth and Pore Throat

\section{Chapter 4}

4.1 - Scanning Electron Microscopy Image of a) 450x2750 Mesh and b) 510x3600 Mesh Dutch Twill Screen Samples

4.2 - Submerged Screen Sample Mounted within the Holding Cylinder

4.3 - Schematic of Room Temperature Bubble Point Experimental Setup

4.4 - Raw Differential Pressure Transducer Signal, Bubble Point, and Reseal Pressure as a Function of Time

4.5 - Water Droplets on a 510x3600 Screen Sample during Sessile Drop Measurements 
4.6 - Room Temperature Bubble Point Pressure as a Function of Surface Tension of Pure Fluids for the a) $325 \times 2300$, b) 450x2750, and c)

510x3600 Mesh Screen Samples

4.7 - Experimental Data and Langmuir Isotherm Fit to the $\mathrm{T}=20^{\circ} \mathrm{C}$

Methanol/Water Surface Tension Data from Vazquez et al. (1995)

4.8 - Contact Angle Measurements of Methanol/Water Mixtures on the $325 \times 2300$ Screen

4.9 - Binary Mixture Bubble Point Predictions as a Function of a) Mixture Surface Tension and b) Methanol Mass Fraction

4.10 - Bubble Point Pressure as a Function of Methanol/Water Mixtures as a Function of a) Surface Tension and b) Methanol Mass Fraction for the $325 \times 2300$ Screen

4.11 - Bubble Point Pressure as a Function of Methanol/Water Mixtures as a Function of a) Surface Tension and b) Methanol Mass Fraction for the $450 \times 2750$ Screen

4.12 - Bubble Point Pressure as a Function of Methanol/Water Mixtures as a Function of a) Surface Tension and b) Methanol Mass Fraction for the $510 \times 3600$ Screen

4.13 - Methanol Mole Fraction versus Mass Fraction

4.14 - Zisman Plot for Binary Methanol/Water and Stainless Steel 304 System

4.15 - Room Temperature Reseal Point Pressure as a Function of Surface Tension of Pure Fluids for the a) $325 \times 2300$, b) $450 \times 2750$, and c) 510x3600 Mesh Screen Samples

4.16 - Experimental Setup for Wicking Tests

4.17 - Wicking Distance as a Function of Time for a 325x2300, 450x2750, and 510x3600 Dutch Twill Screen in Isopropyl Alcohol

\section{Chapter 5}

5.1 - Screen Sample and Flange

5.2 - Low Pressure Liquid Acquisition Device Cup 
5.4 - Receiver Dewar for Low Pressure Liquid Hydrogen and Nitrogen Bubble Point Tests

5.5 - Cell 7 Liquid Hydrogen Flow System

5.6 - Test Matrix for the Low Pressure Cryogenic Bubble Point Tests

5.7 - Screenshot of an Ideal Test Run for a 450x2750 Screen with Gaseous Helium/Liquid Hydrogen Testing

5.8 - Thermodynamic State of the Liquid Hydrogen at Screen Breakdown for a) $325 \times 2300$, b) 450x2750, and c) 510x3600 Screen

5.9 - Thermodynamic State of the Liquid Nitrogen at Screen Breakdown for a) $325 \times 2300$, b) $450 \times 2750$, and c) $510 \times 3600$ Screen

5.10 - Bubble Point Pressure Dependence on Screen Mesh in a) Liquid Hydrogen and b) Liquid Nitrogen

5.11 - Bubble Point Pressure Dependence on Liquid for a) 325x2300, b) 450x2750, and c) 510x3600 Screen Mesh Samples

5.12 - a) Liquid Hydrogen and b) Liquid Nitrogen Bubble Point as a Function of Liquid Screen Side Temperature (SD1)

5.13 - Liquid Hydrogen Bubble Point Pressure Dependence on Liquid Temperature and Pressure using Helium as a Pressurant Gas for a) $325 \times 2300$, b) $450 \times 2750$, and c) $510 \times 3600$ Screens

5.14 - Liquid Nitrogen Bubble Point Pressure Dependence on Liquid Temperature and Pressure using Helium as a Pressurant Gas for a) $325 \times 2300$, b) $450 \times 2750$, and c) $510 \times 3600$ Screens

5.15 - Liquid Hydrogen Bubble Point Pressure Dependence on Pressurant Gas for a) $325 \times 2300$, b) $450 \times 2750$, and c) 510x3600 Screens

5.16 - Liquid Nitrogen Bubble Point Pressure Dependence on Pressurant Gas for a) $325 \times 2300$, b) 450x2750, and c) 510x3600 Screens

5.17 - 325x2300 Liquid Hydrogen Bubble Point Pressure as a Function of Liquid Temperature and Pressure using a) Gaseous Helium and b) Gaseous Hydrogen as a Pressurant

5.18 - 450x2750 Liquid Hydrogen Bubble Point Pressure as a Function of Liquid Temperature and Pressure using a) Gaseous Helium and b) Gaseous Hydrogen as a Pressurant 
5.19 - 510x3600 Liquid Hydrogen Bubble Point Pressure as a Function of Liquid Temperature and Pressure using a) Gaseous Helium and b)

Gaseous Hydrogen as a Pressurant

5.20 - 325x2300 Liquid Nitrogen Bubble Point Pressure as a Function of Liquid Temperature and Pressure using a) Gaseous Helium and b)

Gaseous Nitrogen as a Pressurant

5.21 - 450x2750 Liquid Nitrogen Bubble Point Pressure as a Function of Liquid Temperature and Pressure using a) Gaseous Helium and b)

Gaseous Nitrogen as a Pressurant

5.22 - 510x3600 Liquid Nitrogen Bubble Point Pressure as a Function of Liquid Temperature and Pressure using a) Gaseous Helium and b)

Gaseous Nitrogen as a Pressurant

Chapter 6

6.1 -Screen/Cup Assembly for High Pressure Bubble Point Experiments

6.2 - High Pressure Cryogenic Fluid Management Test Tank

6.3 -Screen and Cup Assembly, High Pressure Test Tank, and Dewar

6.4 - Summary of Test Conditions for Current High Pressure Liquid Oxygen Bubble Point Tests and Other Related Liquid Oxygen Technology Development Programs

6.5 - Liquid Oxygen Thermodynamic Conditions at Bubble Breakthrough as a Function of the Liquid Screen Side Pressure and a) Liquid Screen Side Temperature (SD1) and b) Bulk Liquid Temperature (SD4) for the 200x1400 Screen

6.6 - Liquid Oxygen Thermodynamic Conditions at Bubble Breakthrough as a Function of the Liquid Screen Side Pressure and a) Liquid Screen Side Temperature (SD1) and b) Bulk Liquid Temperature (SD4) for the $325 \times 2300$ Screen

6.7 - Liquid Oxygen Bubble Point Pressure as a Function of a) Temperature of the Liquid Side of the Screen and b) Bulk Liquid Temperature for the $200 \times 1400$ Screen

6.8 - Liquid Oxygen Bubble Point Pressure as a Function of a) Temperature of the Liquid Side of the Screen and b) Bulk Liquid Temperature for the $325 \times 2300$ Screen 
6.9 - Oxygen Saturation Temperature vs. a) Liquid Screen Side Temperature and b) Bulk Liquid Temperature at Bubble Breakthrough When Using Gaseous Oxygen for the 325x2300 Screen

6.10 - Liquid Oxygen Temperature Correlation for Gaseous Helium at Bubble Breakthrough for the $325 \times 2300$ Screen

6.11 - Liquid Oxygen 325x2300 Bubble Point as a Function of Liquid Screen Side Pressure

6.12 - Liquid Oxygen Bubble Point Pressure as a Function of the Thermodynamic State of the Liquid at the Screen for a) 200x1400 Using Gaseous Helium b) $325 \times 2300$ Using Gaseous Helium and c) 325x2300 Using Gaseous Oxygen

6.13 - Liquid Oxygen Bubble Point Pressure as a Function of the Temperature Difference a) Across the Screen and b) Between Liquid at Screen and Bulk Liquid in the Tank for the 200x1400 Screen

6.14 - Liquid Oxygen Bubble Point Pressure as a Function of the Temperature Difference a) Across the Screen and b) Between Liquid at Screen and Bulk Liquid in the Tank for the $325 \times 2300$ Screen

6.15 - Liquid Oxygen Bubble Point Pressure as a Function of Liquid Screen Side Temperature and a) Temperature Difference across the Screen and b) Temperature Difference between Liquid at Screen and Bulk Liquid in Tank at Breakdown for the 200x1400 Screen Using Gaseous Helium

6.16 - Liquid Oxygen Bubble Point Pressure as a Function of Liquid Screen Side Temperature and a) Temperature Difference across the Screen and b) Temperature Difference between Liquid at Screen and Bulk Liquid in Tank at Breakdown for the $325 \times 2300$ Screen Using Gaseous Helium

6.17 - Liquid Oxygen Bubble Point Pressure as a Function of Liquid Screen Side Temperature and a) Temperature Difference across the Screen and b) Temperature Difference between Liquid at Screen and Bulk Liquid in Tank at Breakdown for the 325x2300 Screen Using Gaseous Oxygen

6.18 - Time Trace of Temperature Sensors and Differential Pressure Transducer with Corresponding Images of Screen using a) Gaseous Helium and b) Gaseous Oxygen as Pressurants

\section{Chapter 7}

7.1 - Screen/Cup Assembly for High Pressure Methane Experiments 
7.2 - Updated Screen and Cup Assembly, High Pressure Test Tank, and Dewar

7.3 - Location of Temperature Measurements inside Cryogenic Fluid Management Test Tank

7.4 - Temperature and Liquid Level Measurement of the Bulk Liquid in the Cryogenic Fluid Management Test Tank as Viewed from the Bottom of the Tank

7.5 - Summary of the Primary Liquid Methane Analytical and Experimental Propellant Tank Test Conditions under NASA's Liquid Oxygen/Liquid Methane Technology Development Program

7.6 - Liquid Methane Thermodynamic Conditions at Bubble Breakthrough as a Function of the Liquid Screen Side Pressure and a) Liquid Screen Side Temperature (SD1) and b) Bulk Liquid Temperature (SD4)

7.7 - 325x2300 Liquid Methane Bubble Point Pressure as a Function of a) Temperature at the Liquid Side of the Screen and b) Bulk Liquid Temperature

7.8 - Methane Saturation Temperature versus Liquid Screen Side Temperature at Bubble Breakthrough

7.9 - Liquid Methane Temperature Correlation for a) Gaseous Helium and b) Gaseous Nitrogen at Breakthrough for a $325 \times 2300$ Screen

7.10 - Combined Liquid Temperature, Pressure, and Pressurant Gas Type Dependence on 325x2300 Liquid Methane Bubble Point Pressure using a) Helium, b) Nitrogen, and c) Methane as Pressurants

7.11 - Pressure Dependence on Liquid Methane Bubble Point Pressure for $325 \times 2300$ Screen

7.12 - Liquid Methane Bubble Point Pressure as a Function of the Temperature Difference a) Across the Screen and b) Between Liquid at Screen and Bulk Liquid in Tank for the 325x2300 Screen

7.13 - Liquid Methane Bubble Point Pressure as a Function of Liquid Screen Side Temperature and a) Temperature Difference across the Screen and b) Temperature Difference between Liquid at Screen and Bulk Liquid in Tank at Breakdown for the 325x2300 Screen using Gaseous Helium 
7.14 - Liquid Methane Bubble Point Pressure as a Function of Liquid Screen Side Temperature and a) Temperature Difference across the Screen and b) Temperature Difference between Liquid at Screen and Bulk Liquid in Tank at Breakdown for the 325x2300 Screen using Gaseous Nitrogen

7.15 - Liquid Methane Bubble Point Pressure as a Function of Liquid Screen Side Temperature and a) Temperature Difference across the Screen and b) Temperature Difference between Liquid at Screen and Bulk Liquid in Tank at Breakdown for the 325×2300 Screen using Gaseous Methane

7.16 - Ratio of the Computed Fritz Time to Inertial Time as a Function of the Test Number for Gaseous Helium, Nitrogen, and Methane

7.17 - Difference between the Calculated Interfacial Temperature and the Measured Bulk Liquid Temperature at Breakthrough for Pressurization with a) Gaseous Methane b) Gaseous Helium and c) Gaseous Nitrogen

7.18 - Difference between the Calculated Interfacial Temperature and the Bulk Liquid Temperature at Bubble Breakthrough as a Function of Liquid Subcooling

7.19 - Mass Flux across the Screen at Bubble Breakthrough as a Function of Liquid Methane Subcooling

7.20 - Mass Flux across the Screen at Bubble Breakthrough as a Function of the Bulk Liquid Temperature and Pressure using a) Gaseous Methane, b) Gaseous Helium, and c) Gaseous Nitrogen as a Pressurant

7.21 - Mass Flux across the Screen at Bubble Breakthrough using Gaseous Methane to Pressurize as a Function of the Liquid Temperature and Pressure using the Schrage Model from Kinetic Theory

7.22 - Screen Reynolds Number at Bubble Breakthrough as a Function of Liquid Subcooling

7.23 - Screen Reynolds Number at Bubble Breakthrough as a Function of Liquid Pressure and Temperature using a) Gaseous Methane, b) Gaseous Helium, and c) Gaseous Nitrogen to pressurize 
7.24 - Bubble Breakthroughs for: a) Gaseous Methane Pressurization at a Low Screen Reynolds Number $(\approx 400)$, b) Gaseous Methane

Pressurization at a High Screen Reynolds Number $(\approx 28,000)$, c) Gaseous Helium Pressurization at a Low Screen Reynolds Number $(\approx 20)$, d)

Gaseous Helium Pressurization at a High Screen Reynolds Number $(\approx 40)$, e) Gaseous Nitrogen Pressurization at a Low Screen Reynolds Number $(\approx$ 250), f) Gaseous Nitrogen Pressurization at a High Screen Reynolds Number $(\approx 2400)$

7.25 - Calculated Difference between Interface and Screen Liquid Side Temperature as a Function of Bulk Liquid Pressure and Temperature using Gaseous Methane to Pressurize

7.26 - Net Heat Flux into the Interface at Bubble Breakthrough as a Function of Liquid Pressure and Temperature using Gaseous Methane to Pressurize

7.27 - Qualitative Operating Regimes for Different Pressurant Gases with Liquid Methane

\section{Chapter 8}

8.1 - Sources of Heat Leak into a Cryogenic Propellant Tank

8.2 - Warm Pressurant Gas Heater Bank

8.3 - Cold Gas Liquid Hydrogen Bubble Point as a Function of the Liquid Screen Side Temperature

8.4 - Heated Pressurant Gas Liquid Hydrogen Bubble Point Pressure as a Function of the Temperature Difference between Pressurant Gas and Liquid

8.5 - Normalized Heated Pressurant Gas Liquid Hydrogen Bubble Point Pressure as a Function of the Temperature Difference between Pressurant Gas and Liquid at the Screen

8.6 - Cold Gas Liquid Nitrogen Bubble Point as a Function of the Liquid Screen Side Temperature

8.7 - Heated Pressurant Gas Liquid Nitrogen Bubble Point Pressure as a Function of the Temperature Difference between Pressurant Gas and Liquid 
8.8 - Normalized Heated Pressurant Gas Liquid Nitrogen Bubble Point Pressure as a Function of the Temperature Difference between Pressurant

Gas and Liquid at the Screen

Chapter 9

9.1 - Liquid Hydrogen Test Tank 348

9.2 - Test Tank Lid

9.3 - Test Tank Internal Hardware

9.4 - Vacuum Chamber Lid and Feedthroughs

350

9.5 - Integration of Test Tank into Facility

9.6 - Small Multipurpose Research Facility Liquid Hydrogen Flow Control System

9.7 - Computer Data Acquisition System

9.8 - Illustration of Horizontal Liquid Acquisition Device Testing

9.9 - Horizontal Liquid Acquisition Device Research Hardware

9.10 - Liquid Oxygen Frictional and Dynamic Pressure Loss Data versus Model Predictions in a) $90.6 \mathrm{~K}, 1.03 \mathrm{MPa}$ and b) $107 \mathrm{~K}, 1.65 \mathrm{MPa}$ Liquid

9.11 - Flow-through-Screen Research Hardware

9.12 - Screenshot of Flow-through-Screen Data Acquisition

9.13 - Typical Flow-through-Screen Test Run for the 325x2300 and 450x2750 Screen Samples in Liquid Hydrogen

9.14 - Typical Flow-through-Screen Test Run for the 325x2300 Screen Sample in Gaseous Helium

9.15 - Temperature Dependence of the Flow-through-Screen Pressure Drop

9.16 - Standard Deviation Plots of Room Temperature and Liquid Hydrogen Flow-through-Screen Model Curves 
9.17 - Percent Difference in Flow-through-Screen Pressure Drop Models between Liquid Hydrogen and Room Temperature as a Function of Mass Flux across the Screen

9.18 - Illustration of Inverted Liquid Acquisition Device Outflow Test

$9.19-325 \times 2300$ Standard Channel

9.20 - 325x2300 Thermodynamic Vent System Cooled Channel

9.21 - Isopropyl Alcohol Bubble Point Testing of the Completed $325 \times 2300$ Channel

9.22 - Completed 325x2300 Thermodynamic Vent System Cooled Channel

9.23 - Screenshot of Vertical Liquid Acquisition Device Outflow Data Acquisition

9.24 - General One Dimensional Steady State Pressure Drop Model Trends - Exposed Screen Length as a Function of Liquid Temperature and Mass Flow Rate through the Channel

9.25 - Relative Magnitude of Pressure Drop Contributions as a Function of Exposed Channel Length for a) Low Flow (0.005 kg/s) and b) High Flow $(0.025 \mathrm{~kg} / \mathrm{s})$

9.26 - Pre and Post Isopropyl Alcohol Bubble Point Testing of the a) $325 \times 2300$ Standard Channel and b) TVS Cooled Channel

9.27 - Exposed Screen Height as a Function of Tank Liquid Conditions and Mass Flow Rate through the Standard 325x2300 Channel at a) First Helium Bubble Ingestion and b) Total Breakdown

9.28 - Exposed Screen Height as a Function of Tank Liquid Conditions and Mass Flow Rate through the Thermodynamic Vent System Cooled $325 \times 2300$ Channel at a) First Helium Bubble Ingestion and b) Total Breakdown

9.29 - Exposed Screen Height as a Function of Mass Flow Rate through the Thermodynamic Vent System Cooled 325x2300 Channel with the Thermodynamic Vent System Engaged and Disengaged

9.30 - Thermodynamic Vent System Heat Exchanger Efficiency in Terms of the Temperature Difference between Bulk Liquid in the Tank and the Liquid inside the Channel 
9.31 - Gain in Dynamic Liquid Acquisition Device Performance Due to

Subcooling the Bulk Liquid Hydrogen in the Tank

9.32 - Gain in Static Liquid Acquisition Device Performance due to Subcooling the Bulk Liquid Hydrogen at the Screen Liquid/Vapor Interface

9.33 - One Dimensional Pressure Drop Model Simulations versus Data for Exposed Screen Height at Channel Breakdown Assuming 100\% Available Flow-through-Screen Flow Area

9.34 - One Dimensional Pressure Drop Model Simulations versus Data for Exposed Screen Height at Channel Breakdown Assuming Reduced Available Flow-through-Screen Flow Area

9.35 - Disparity between Data and One Dimensional Model Predicted Exposed Screen Height as a Function of Flow Rate through the Channel

Chapter 10

10.1 - Liquid Hydrogen Bubble Point Pressure Dependence on Pressurant Gas for the $325 \times 2300$ Screen

10.2 - Room Temperature Pore Diameter as a Function of Gap between Four Shute Wires

10.3 - Room Temperature Pore Diameter as a Function of the Fineness of the Mesh

10.4 - Room Temperature Pore Diameter as a Function of X

10.5 - Pore Diameter as a Function of Temperature for a) $325 \times 2300$, b) 450x2750, and c) 510x3600 Dutch Twill Screens

10.6 - Temperature Dependent Pore Diameter Fitting Coefficient versus the Fineness of the Mesh

10.7 - Updated Model Performance versus Bubble Point Data Taken in Normally Saturated Liquid States: a) 450x2750 Liquid Hydrogen Data using Gaseous Helium and Gaseous Hydrogen and b) 325x2300 Liquid Nitrogen Data using Gaseous Helium and Gaseous Nitrogen

10.8 - Model Generated 325x2300 Bubble Point Ratio as a Function of the Reduced Temperature for Normally Saturated Liquid Taken Over the Range of Conditions of the Data 
10.9 - Model Generated Subcooled Gain Curves for a 325x2300 Screen using a) Gaseous Helium and b) Autogenous Pressurization Schemes as a Function of Pressure Difference Above the Saturation Pressure at the Normal Boiling Point

10.10 - 325x2300 Liquid Nitrogen Bubble Point Data as a Function of Liquid Pressure and Temperature using a) Gaseous Helium and b) Gaseous Nitrogen as Pressurants

10.11 - Model Generated 325x2300 Liquid Nitrogen Bubble Point Pressure as a Function of the Liquid Pressure and Temperature using a) Gaseous Helium and b) Gaseous Nitrogen as Pressurants

10.12 - Comparison of 325x2300 Gaseous Helium/Liquid Methane Subcooled Gain as a Function of Liquid Pressure and Temperature at the Screen between the a) Data and b) Model

10.13 - Comparison of 325x2300 Gaseous Nitrogen/Liquid Methane Subcooled Gain as a Function of Liquid Pressure and Temperature at the Screen between the a) Data and b) Model

10.14 - Comparison of 325x2300 Gaseous Methane/Liquid Methane Subcooled Gain as a Function of Liquid Pressure and Temperature at the Screen between the a) Data and b) Model

10.15 - Model Generated Curves of the Ratio of the Heated Pressurant Gas Loss from Equation 10.17 for a) Liquid Hydrogen and b) Liquid Nitrogen

\section{Chapter 11}

11.1 - Ratio of Room Temperature Reseal Pressure to Bubble Point Pressure versus the Fineness of the Screen

11.3 - Reseal Diameter as a Function of Temperature for a) $325 \times 2300$, b) 450x2750, and c) 510x3600 Dutch Twill Screen

11.4 - Model Generated 325x2300 Reseal Pressure Ratio as a Function of the Reduced Temperature for Normally Saturated Liquid Taken Over the Range of Conditions of the Data 
11.5 - Model Generated Subcooled Gain Curves for a 325x2300 Screen using a) Gaseous Helium and b) Autogenous Pressurization Schemes as a Function of Pressure Difference Above the Saturation Pressure at the Normal Boiling Point

11.6 - a) Data and b) Model Generated Subcooled Gain as a Function of the Liquid Temperature and Pressure at the Screen for a 200x1400 Mesh in Liquid Oxygen using Gaseous Helium as a Pressurant

11.7 - a) Data and b) Model Generated Subcooled Gain as a Function of the Liquid Temperature and Pressure at the LAD Screen for a 325x2300 Mesh in Liquid Oxygen using Gaseous Helium as a Pressurant

11.8 - a) Data and b) Model Generated Bubble Point as a Function of the Liquid Temperature and Pressure at the LAD Screen for a 325x2300 Mesh in Liquid Nitrogen using Gaseous Helium as a Pressurant

11.9 - a) Data and b) Model Generated Bubble Point as a Function of the Liquid Temperature and Pressure at the LAD Screen for a 325x2300 Mesh in Liquid Nitrogen using Gaseous Nitrogen as a Pressurant

11.10 - Model Generated 450x2750 Liquid Hydrogen a) Bubble Point Pressure and b) Reseal Pressure using Gaseous Helium as a Pressurant

\section{Chapter 12}

12.1 - Illustration of Discrepancies between One Dimensional Pressure Drop Model and Data for the 1-g Inverted Outflow Configuration

12.2 - Liquid Acquisition Device Channel with Flow through Screen and Out of the Channel

12.4 - Velocity Vector Plots for a Range of Liquid Oxygen Mass Flow Rates and Channel Dimensions

12.5 - Normalized Flow-through-Screen Pressure Drop along the Channel for Four Different Dutch Twill Screens for a) $\operatorname{Re}_{\mathrm{e}}=10^{4}$, b) $\operatorname{Re}_{\mathrm{e}}=10^{5}$, c) $\operatorname{Re}_{\mathrm{e}}=10^{6}$, and d) $\operatorname{Re}_{\mathrm{e}}=10^{7}$

12.6 - Maximum Analytical Model Flow-through-Screen Pressure Drop Divided by the One Dimensional Model Flow-through-Screen Pressure Drop as a Function of Outlet Reynolds Number 
12.7 - Plot of the Worst-Case Turbulent Pressure Drop down the Channel as a Percentage of the Maximum Pressure Drop across the Screen

$12.8-61.0 \mathrm{~cm} \times 2.54 \mathrm{~cm} \times 5.08 \mathrm{~cm}$ (24 in $\times 1$ in $\times 2$ in) Wide Horizontal Liquid Acquisition Device Channel

12.9 - New Analytical Model Comparison with Data for Liquid Oxygen Flow inside a Horizontal Liquid Acquisition Device Channel at a) 1.034 $\mathrm{MPa}$ and $90.6 \mathrm{~K}$ and b) $1.655 \mathrm{MPa}$ and $107 \mathrm{~K}$

12.10 - Schematic of Liquid Acquisition Device Channel used to Model $1 \mathrm{~g}$ Inverted Outflow Tests

12.11 - Difference between Liquid Hydrogen Inverted Outflow Experimental Data from Chapter 9 and Analytical Model Predicted Exposed Screen Length at Breakdown as a Function of Mass Flow Rate using the New Model

12.12 - Illustration of Convective Heat Transfer across Screen for Two Liquid Acquisition Device Channel Sections with the Same Overall Mass Flow Rate with Velocity Vectors Shown by Blue Arrows for a) LAD Channel of Height $\mathrm{H}$ and b) LAD Channel of Height $2 \mathrm{H}$

12.13 - Residual Difference between Actual and Model Predicted Exposed Screen Length using Analytical Flow Model Coupled with Dynamic Bubble Point Model

12.14 - Illustration of the Internal Channel Velocity Profile Change at the Propellant Tank Liquid/Vapor Interface

12.15 - a) Heat Transfer Coefficient and b) Power Per Unit Length across the Exposed Portion of the Screen as a Function of Mass Flow Rate through the Channel

\section{Chapter 13}

13.1 - Model Predicted Liquid Hydrogen Bubble Point Pressure Over the Anticipated Operating Range of a Cryogenic Fuel Depot for a) Five Finest Dutch Twill, b) Coarse Dutch Twill and Plain Dutch, and c) Twilled and Plain Square Mesh Screens

13.2 - Model Predicted Liquid Hydrogen Flow-through-Screen Pressure Drop Over the Anticipated Operating Range of a Cryogenic Fuel Depot for a) Five Finest Dutch Twill, b) Coarse Dutch Twill and Plain Dutch, and c) Twilled and Plain Square Mesh Screens 
13.3 - A Screen Channel Liquid Acquisition Device under Steady Flow Conditions with Negligible Gravitational Force

13.4 - Minimum Cross Sectional Area versus Demand Mass Flow Rate for the Five Finest Dutch Twill Screens in Liquid Hydrogen

\section{Chapter 14}

14.1 - a) Screen Channel and b) Vane Propellant Management Devices Mounted Inside the Cylindrical Liquid Hydrogen Propellant Tank

14.2 - Comparison of Liquid/Vapor Interface in a) 1-g Environment and b) Microgravity Environment

14.3 - Liquid Flow through a Screen Channel Liquid Acquisition Device in Microgravity

14.4 - Comparison of Microgravity Liquid/Vapor Interface in a) Case B and b) Case $\mathrm{C}$

14.5 - Normalized Interface Velocity as a Function of Normalized Exposed Screen Length

14.6 - Velocity Vector Plots at Increasing Screen Exposure Percentages (Lower Tank Fill Levels) for a 325x2300 Liquid Acquisition Device Screen and Channel in Liquid Hydrogen at a Demand Flow Rate of 0.01 $\mathrm{kg} / \mathrm{s}$

14.7 - Flow-through-Screen Pressure Drop as a Function of Distance along the Liquid Acquisition Device Channel for Varying Demand Flow Rates

14.8 - Total Pressure Drop as a Function of Distance along Liquid Acquisition Device Channel for Demand Flow Rate of a) $0.001 \mathrm{~kg} / \mathrm{s}$ and $0.01 \mathrm{~kg} / \mathrm{s}$ at Breakdown

14.9 - Velocity Vector Plots inside a 325x2300 Liquid Acquisition Device Channel for Various Mass Flow Rates and Channel Dimensions

14.10 - Liquid Flow Path along Wall Mounted Vanes

14.11 - a) Side and b) Top-Down View of Vanes and Sump

14.12 - Upstream Radius of Curvature as a Function of Downstream Radius of Curvature for a Liquid Hydrogen Demand Flow Rate of $1 \times 10^{-4}$ $\mathrm{kg} / \mathrm{s}$ 
14.13 - Flow Chart Outlining Propellant Management Device Trade Study Model Inputs and Outputs

14.14 - Expulsion Efficiency as a Function of Demand Flow Rate for Vanes and Screen Channel Liquid Acquisition Devices

14.15 - Resultant Propellant Management Device System Mass as a Function of Demand Flow Rate for Vanes and Screen Channel Liquid Acquisition Devices

14.16 - Mass Flow Rate Delivered Per Vane Arm as a Function of Total Demand Mass Flow Rate

14.17 - Recommended Propellant Management Device for a Given Demand Flow Rate Range for a Small Scale Liquid Hydrogen Storage Tank in Microgravity

\section{Appendix D}

D.1 - Solid/Vapor Interfacial Tension as a Function of Liquid/Vapor Interfacial Tension for Binary Methanol/Water and Stainless Steel 304 System

D.2 - a) Solid/Vapor and b) Solid/Liquid Interfacial Tension as a Function of Methanol Mass Fraction

D.3 - a) Solid/Vapor and b) Solid/Liquid Spreading Pressure as a Function of "A"

Appendix E

E.1 - Heated Pressurant Gas Liquid Acquisition Device Performance Data for a $325 \times 2300$ Screen in Liquid Hydrogen

E.2 - Remaining Heated Pressurant Gas Liquid Acquisition Device Data in Liquid Hydrogen for Various Screens

E.3 - Inverted Liquid Hydrogen Outflow Hold Tests for a 325x2300

Screen from Meserole and Jones (1993) using Heated Pressure Gas

E.4 - Warm Pressurant Start Basket Liquid Acquisition Device Performance Tests in Liquid Hydrogen from Bennett (1987)

E.5 - Heated Non-inverted Bubble Point Data in Liquid Nitrogen from Castle (1972) 
E.6 - Warm Pressurant Gas Liquid Acquisition Device Performance Data for a 325x2300 Screen in Isopropyl Alcohol and Freon 


\section{Acknowledgments}

I would first like to acknowledge my dear friends Padmanabha and Gaurav. Padmanabha, without your constant support both professionally and emotionally, this work would not have been possible. I have been blessed to have you as a colleague, as a mentor, and most importantly as a dear friend for so many years. Your deep concern, compassion, and encouragement were felt throughout the course of the work. Thank you for inspiring me to work in a detached manner. Gaurav, I thank you for your support throughout this work and for keeping me on the straight and narrow. Our time together as colleagues and friends inside and out of Case are some of my fondest memories. Your maturity and level-headedness also inspired me to work in a regulated manner.

I acknowledge my Case Western Reserve University dissertation committee members. Dr. Kamotani, thank you for your many years of service as an instructor in the classroom, a mentor, and chair of the committee. Dr. Kadambi, thank you for your insightful comments and suggestions on improving the quality of this work. Dr. Mann, thank you for your years of service as an instructor, a consultant, and a personal friend. I cherish the time we spent together discussing both theoretical and experimental aspects of this dissertation. Our weekly thought provoking discussions inspired me to always work with determination and to continually improve in writing. Thank you for exhibiting patience, humility, and diligence. I would also like to recognize Dr. Alexis Abramson, Dr. Donald Feke, and Dr. Jackie Sung for their support, especially in the early stages. 
I acknowledge all of the NASA colleagues who I have had the privilege of working with over the course of this dissertation; in particular, I would like to thank Dr. Chato, Mike Doherty, Michael Meyer, and John Jurns for your years of service and support. Each one of you was especially vital in making this work a reality. Dave and Mike Meyer, thank you for allowing me pursue this field in near single pointed focus. I am grateful for your support professionally, and for our many technical talks. Somehow you were able to secure funding and testing for so long despite the constant uncertainty and changing complexion of our field. Thank you for having faith in me and for allowing me to grow professionally. Mike Doherty, I want to graciously thank you for your support throughout the years, both financially and professionally. Things were quite rough living semester by semester without any promise that I could juggle both full time employment and this degree. But because of your compassion and your faith in me, somehow we were able to have the shelter to do some very meaningful work. John, I thank you for your support and encouragement throughout the first few years of my tenure at NASA. Most of what I learned in cryogenics is derived from our daily technical conversations. Because you were so flexible and supportive in the beginning, I was able to juggle classes, research, and all of our tasks. I will never forget our time together or the sacrifices you made for me, John.

Throughout my brief time at NASA, I have always felt grateful to get to work with so many professional members of the scientific community. John McQuillen, thank you for our endless thought provoking discussions, for helping me to really polish professional writing skills, and for your help in conceiving and running experiments. You have always pushed me to think critically. Enrique Rame, I am grateful and happy for our 
many technical discussions and for your support in data analysis. Ben Stiegemeier, I appreciate all of our discussions at the board, in "hammering out" problems like the great scientists of old. Thanks for being such an amazing friend both professionally and personally.

I would like to acknowledge members of my Propellants and Propulsion branch for their years of technical service and assistance in reviewing technical papers, including Neil Van Dresar, Greg Zimmerli, and Dave Plachta. Neil, as an office mate, you have always been open and supportive in discussing so many technical matters. I feel fortunate to have the chance to work with all of you. I thank Mark Klem for his guidance and support during the final year of work. I am grateful to Jeff Moder and Steve Barsi for their many technical suggestions on modeling and analytical methods throughout the duration of this work. Jeff, thanks for your help with the thermal modeling. Joe Gaby, I feel so fortunate for our association throughout the years. You have really taught me well on the inner workings of test conception and on having a long term vision as a researcher.

I acknowledge the operations and technician staff at Cell-7 and SMiRF, including Joe Puskas, Jack Kowalewski, Helmut Bamberger, Craig Robinson, Marivel Baez, and Lori Arnet. It is only because of you all that we were able to run such successful experiments and collect so much meaningful data for NASA. It has been such a reward to work with such knowledgeable and eager engineers. Joe, I appreciate all the hard work in helping me design and run the liquid oxygen and methane tests, for helping us collect so much data in so little time. Jack, thanks for your support in both the room temperature and liquid hydrogen bubble point tests and for helping to run such a demanding hydrogen test. Helmut and Craig, thanks for your service in building and running the liquid 
hydrogen outflow experiment. As you said, this was the most complicated experiment, yet one that generated the most amount of relevant data in the history of SMiRF. I am grateful for your combined excellence and professionalism.

I acknowledge the design engineers who made the large scale outflow test a reality. Maureen Kudlac, thank you for your support in design, and for supporting and encouraging me to pursue this work. Frank Quinn and Jerry Vera I thank you for all the design support and our many wonderful technical discussions. I thank Wesley Johnson for his support, encouragement, and friendship since the beginning of my tenure at Glenn. I thank Leo Bolshinskiy for his thoughtful criticisms of every one of our data reviews. I am also grateful to Don Jaekle for our few, yet potent interactions. You truly strive for excellence, and your rigor and determination always supercharges me to pursue the highest quality work in that same manner of excellence.

I would like to acknowledge all of my interns throughout the course of this work. It is a blessing to work on challenging technical problems while having the chance to engage so many others in service. My heart lies in working with you all sincere students. I thank Rob Webb, Olivia Peachley, Ron Zeszut, and Michael Schubert for their assistance in the early stages, and Anthony Savas, Keaton Keefer, Jeremy Styborski, and Aalok Patel for their assistance in the later years. I thank my high school students, Dan Weis, Karan Lamba, and Ben Lew for their hard work. I am especially grateful to Samuel Darr for our association throughout the years. While pursuing a $\mathrm{PhD}$ has been a privilege, it has been an even greater privilege to witness you all grow both professionally and personally in your own respective ways. 
I acknowledge my family and close friends. Mom and Dad, thank you for always encouraging me to reach for the stars. Thank you for supporting me to pursue my dreams and desires, for the countless selfless sacrifices you have made, and for your emotional stability and well wishes throughout my entire life. I am grateful to my sister Lindsay. You have always given me so much loving support as both a sister and as a friend throughout my life. Judy, thank you for pushing me to pursue my dreams and for helping me to remain fearless in the face of uncertainty. I lastly acknowledge Tiffany. Thank you for being so enthusiastic, supportive, and dedicated in helping me to complete this work. It is because of your emotional strength and support that I was able to stay focused and steady in the final year.

While this work was externally difficult at times, it never once felt that way internally because of all of your love, support, and encouragement. I see this dissertation not as a compilation of my efforts, but rather as the sum total of all of your compassion, support, and service. Thank you!

The beginning portion of this work was funded through the Case Prime Fellowship. The middle portion of this work was funded by ASRC Aerospace Corporation. The remaining portion of this work was funded through NASA Grant Number NNX12AQ13G. 


\section{List of Abbreviations}

1D 2D 3D

Al

AME

APU

B2H6

BAC

$\mathrm{BC}$

BD

BP

$\mathrm{C}_{5} \mathrm{H}_{12}$

CCD

CCL-7

CEV

CFD

CFM

CFME

CFMF

COLD-SAT

CONE

COPV

CPST

CRYOTE

CSA

CV
One, Two, Three Dimensional

Aluminum

Ascent Main Engine

Auxiliary Power Unit

Diborane

Broad Area Cooling Shield

Boundary Condition

Burst Disc

Back Pressure

Pentane

Charge Coupled Device

Cryogenics Components Lab 7

Crew Exploration Vehicle

Computational Fluid Dynamics

Cryogenic Fluid Management

Cryogenic Fluid Management Experiment

Cryogenic Fluid Management Facility

Cryogenic Orbiting Liquid Depot-Storage, Acquisition, and Transfer

Cryogenic Orbital Nitrogen Experiment

Composite Overwrapped Pressure Vessel

Cryogenic Propellant Storage and Transfer Project

Cryogenic Orbital Testbed

Canadian Science Agency

Control Volume 


\begin{tabular}{|c|c|}
\hline $\mathrm{CZ}$ & Critical Zisman \\
\hline DAQ & Data Acquisition System \\
\hline DARPA & Defense Advanced Research Projects Agency \\
\hline DPT & Differential Pressure Transducer \\
\hline DVR & Digital Video Recorder \\
\hline EDAX & Energy Dispersive X-Ray Microanalysis \\
\hline EDS & Earth Departure Stage \\
\hline $\mathrm{EE}$ & Expulsion Efficiency \\
\hline EELV & Evolved Expendable Launch Vehicle \\
\hline EOL & End of Life \\
\hline ESA & European Space Agency \\
\hline FARE & Fluid Acquisition Resupply Experiment \\
\hline FM & Flow Meter \\
\hline FMU & Friction Multiplier \\
\hline FTS & Flow through Screen \\
\hline $\mathrm{GCH}_{4}$ & Gaseous Methane \\
\hline $\mathrm{GHe}$ & Gaseous Helium \\
\hline $\mathrm{GH}_{2}$ & Gaseous Hydrogen \\
\hline GOES & Geostationary Operational Environmental Satellite \\
\hline GOX & Gaseous Oxygen \\
\hline $\mathrm{GN}_{2}$ & Gaseous Nitrogen \\
\hline GRC & Glenn Research Center \\
\hline HEX & Heat Exchanger \\
\hline HLL & Heavy Launch Lift \\
\hline IBP & Inverted Bubble Point \\
\hline ICBM & Intercontinental Ballistic Missile \\
\hline IO & Inverted Outflow \\
\hline
\end{tabular}




\begin{tabular}{|c|c|}
\hline IPA & Isopropyl Alcohol \\
\hline IRIG-B & Inter-Range Instrumentation Group \\
\hline ISRU & In-space Resource Utilization \\
\hline ISS & International Space Station \\
\hline JSC & Johnson Space Center \\
\hline JT & Joule-Thompson \\
\hline JWST & James Webb Space Telescope \\
\hline $\mathrm{L} / \mathrm{V}$ & Liquid/Vapor \\
\hline LAD & Liquid Acquisition Device \\
\hline LAPS & Lunar Ascent Propulsion Study \\
\hline $\mathrm{LCH}_{4}$ & Liquid Methane \\
\hline LCT & Laser Communication Terminal \\
\hline LEO & Low Earth Orbit \\
\hline $\mathrm{LH}_{2}$ & Liquid Hydrogen \\
\hline LHe & Liquid Helium \\
\hline $\mathrm{Li}$ & Lithium \\
\hline LLO & Low Lunar Orbit \\
\hline $\mathrm{LN}_{2}$ & Liquid Nitrogen \\
\hline LOX & Liquid Oxygen \\
\hline LSAM & Lunar Surface Access Module \\
\hline MAWP & Maximum Allowable Working Pressure \\
\hline MDS & Molecular Dynamics Simulation \\
\hline MEOP & Maximum Expected Operating Pressure \\
\hline MG & Mass Gauging \\
\hline MGS & Mars Global Surveyor \\
\hline MHD & Magneto-hydrodynamic Flow \\
\hline MiTEx & Micro-satellite Technology Experiment \\
\hline
\end{tabular}




\begin{tabular}{|c|c|}
\hline MLI & Multi-layer Insulation \\
\hline MLSTC & Methane Lunar Surface Thermal Control \\
\hline МMH & Monomethyl Hydrazine \\
\hline MPS & Main Propulsion System \\
\hline $\mathrm{N}_{2} \mathrm{H}_{4}$ & Hydrazine \\
\hline $\mathrm{N}_{2} \mathrm{O}_{4}$ & Nitrous Tetraoxide \\
\hline NASA & National Aeronautics and Space Administration \\
\hline NBP & Normal Boiling Point \\
\hline NEAR & Near Earth Asteroid Rendezvous \\
\hline NEO & Near Earth Object \\
\hline NFIRE & Near Field Infrared Experiment \\
\hline NGO & Needs, Goals, and Objectives \\
\hline $\mathrm{Ni}$ & Nickel \\
\hline NIBP & Non-inverted Bubble Point \\
\hline NIST & National Institute of Standards and Technology \\
\hline NS & Navier Stokes \\
\hline NTO & Nitrous Tetraoxide \\
\hline NTU & Number of Heat Transfer Units \\
\hline NVF & No Vent Fill \\
\hline OD & Outer Diameter \\
\hline ODE & Ordinary Differential Equation \\
\hline OF & Oxidizer/Fuel Ratio \\
\hline $\mathrm{OF}_{2}$ & Oxygen Difluoride \\
\hline ORBCOMM & Orbital Communication Satellites \\
\hline OMS & Orbital Maneuvering System \\
\hline OTV & Orbital Transfer Vehicle \\
\hline PCA & Pressure Control Assembly \\
\hline
\end{tabular}




\begin{tabular}{|c|c|}
\hline PCAD & Propulsion and Cryogenics Advanced Development \\
\hline PDE & Partial Differential Equation \\
\hline PDR & Preliminary Design Review \\
\hline PID & Proportional-Integral-Derivative \\
\hline PIV & Particle Image Velocimetry \\
\hline PMD & Propellant Management Device \\
\hline POD & Point of Departure \\
\hline PT & Pressure Transducer \\
\hline RCA & Radio Corporation of America \\
\hline RCS & Reaction Control System \\
\hline $\mathrm{RD}$ & Receiver Dewar \\
\hline $\operatorname{Re}$ & Reynolds Number \\
\hline REFPROP & Reference Fluid Thermodynamic and Transport Properties \\
\hline $\mathrm{S} / \mathrm{L}$ & Solid/Liquid \\
\hline $\mathrm{S} / \mathrm{V}$ & Solid/Vapor \\
\hline SATCOM & Satellite - Communications \\
\hline $\mathrm{SD}$ & Silicon Diode \\
\hline SDO & Solar Dynamic Observatory \\
\hline SEM & Scanning Electron Microscope \\
\hline SF & Safety Factor \\
\hline SFHe & Superfluid Helium \\
\hline SHOOT & Superfluid Helium On-orbit Transfer \\
\hline SLPM & Standard Liters per Minute \\
\hline SMiRF & Small Multi-purpose Research Facility \\
\hline SNR & Signal to Noise Ratio \\
\hline SS & Stainless Steel \\
\hline ST & Supply Tank \\
\hline
\end{tabular}


STS

TDM

TDRS

THERMO

$\mathrm{Ti}$

TRL

TVS

ULA

$\mathrm{VC}$

VJ

VTRE

WSTF

YLE

ZBO
Shuttle Transportation System

Technology Demonstration Mission

Tracking and Data Relay Satellites

Thermo and Hydrodynamic Experiment Research Module in Orbit

Titanium

Technology Readiness Level

Thermodynamic Vent System

United Launch Alliance

Vacuum Chamber

Vacuum Jacketed

Vented Tank Resupply Experiment

White Sands Test Facility

Young-LaPlace Equation

Zero Boil Off 


\section{Glossary}

$A \quad$ Area, $\left[\mathrm{m}^{2}\right]$

$A_{C} \quad$ Cross sectional area, $\left[\mathrm{m}^{2}\right]$

$A_{m} \quad$ Mean cross sectional area, $\left[\mathrm{m}^{2}\right]$

a Surface area to volume ratio, $\left[\mathrm{m}^{-1}\right]$

$a_{C, i} \quad$ Activity, dimensionless

$a_{i} \quad$ Surface tension fitting parameters, dimensionless

$a_{\text {pore }} \quad$ Radius of screen pore tube, $[\mu \mathrm{m}]$

$B \quad$ Screen thickness, [m]

Bo $\quad$ Bond number, dimensionless

$b_{i} \quad$ Screen diameter fitting parameters, dimensionless

C Constant, dimensionless

$C_{D} \quad$ Chord length based on height of vane, [m]

$C_{h} \quad$ Heat capacity rate, $[\mathrm{W} / \mathrm{K}]$

$C_{\text {lam }} \quad$ Frictional flow-through-screen coefficient, $[1 / \mathrm{m}]$

$C_{\min } \quad$ Minimum heat capacity rate of the hot and cold fluids, $[\mathrm{W} / \mathrm{K}]$

$C_{S} \quad$ Chord length based on height of sump, [m]

$C_{\text {turb }} \quad$ Turbulent flow-through-screen coefficient, dimensionless

$c_{i} \quad$ Coefficient of thermal contraction fitting parameters, various dimensions

$c_{p, L A D} \quad$ Specific heat of liquid inside channel, $[\mathrm{J} / \mathrm{kgK}]$

$D_{C} \quad$ Capillary diameter, $[\mu \mathrm{m}]$

$D_{E} \quad$ Wicking diameter, $[\mu \mathrm{m}]$

$D_{H} \quad$ Hydraulic diameter, [m]

$D_{P} \quad$ Effective pore diameter, $[\mu \mathrm{m}]$

$D_{R} \quad$ Effective reseal diameter, $[\mu \mathrm{m}]$

$D_{S} \quad$ Diameter of screen sample, $[\mathrm{m}]$

$D_{V} \quad$ Distance between consecutive vanes, $[\mathrm{m}]$

$d_{s} \quad$ Diameter of shute wire, $[\mu \mathrm{m}]$

$d_{w} \quad$ Diameter of warp wire, $[\mu \mathrm{m}]$

EE Expulsion efficiency, dimensionless

$e \quad$ Pipe roughness, $[\mathrm{m}]$

$\vec{e}_{i} \quad$ Unit vector, dimensionless 
$F \quad$ Two phase multiplier, dimensionless

$F_{\sim B} \quad$ Body force due to gravity, $[\mathrm{N}]$

$F_{D} \quad$ Drag force on sphere, $[\mathrm{N}]$

$F_{T} \quad$ Drag force per area, $\left[\mathrm{N} / \mathrm{m}^{2}\right]$

$f \quad$ Friction factor, dimensionless

$g \quad$ Gravity, $\left[\mathrm{m} / \mathrm{s}^{2}\right]$

$H \quad$ Height of liquid acquisition device, [m]

$H_{M} \quad$ Mean radius of curvature, [m]

$H_{S} \quad$ Height of screend sump, [m]

$H_{V} \quad$ Height of the vane, [m]

$h_{\text {conv }} \quad$ Convective heat transfer coefficient, $\left[\mathrm{W} / \mathrm{m}^{2} \mathrm{~K}\right]$

$h_{f g} \quad$ Heat of vaporization, $[\mathrm{kJ} / \mathrm{kg}]$

$h_{i} \quad$ Heat transfer coefficient of cold fluid, $\left[\mathrm{W} / \mathrm{m}^{2} \mathrm{~K}\right]$

$h_{L} \quad$ Heat transfer coefficient assuming all liquid flow, $\left[\mathrm{W} / \mathrm{m}^{2} \mathrm{~K}\right]$

$h_{N B} \quad$ Heat transfer coefficient for nucleate boiling, $\left[\mathrm{W} / \mathrm{m}^{2} \mathrm{~K}\right]$

$h_{o} \quad$ Heat transfer coefficient of warm fluid, $\left[\mathrm{W} / \mathrm{m}^{2} \mathrm{~K}\right]$

$h_{p} \quad$ Height of triangular pore, $[\mu \mathrm{m}]$

ISP Specific impulse, $\left[\mathrm{s}^{-1}\right]$

$\vec{J}_{i} \quad$ Mass flux, $\left[\mathrm{kg} / \mathrm{m}^{2} \mathrm{~s}\right]$

$J_{C} \quad$ Condensation mass flux, $\left[\mathrm{kg} / \mathrm{m}^{2} \mathrm{~s}\right]$

$K \quad$ Surface tension fitting parameter, dimensionless

$K_{d} \quad$ Screen deflection constant, $\left[\mathrm{m}^{5 / 3} / \mathrm{N}^{1 / 3}\right]$

$k \quad$ Thermal conductivity, $[\mathrm{W} / \mathrm{m} \mathrm{K}]$

$k_{B} \quad$ Boltzmann constant, $[\mathrm{J} / \mathrm{K}]$

$k_{\text {eff }} \quad$ Effective screen thermal conductivity, [W/m K]

$k_{f} \quad$ Thermal conductivity of fluid, $[\mathrm{W} / \mathrm{m} \mathrm{K}]$

$k_{s} \quad$ Thermal conductivity of metal, $[\mathrm{W} / \mathrm{m} * \mathrm{~K}]$

$L \quad$ Length of liquid acquisition device, [m]

$L_{B} \quad$ Channel length at first exposure to vapor, [m]

$L_{b} \quad$ Channel length below liquid/vapor interface, [m]

$L_{C} \quad$ Characteristic length, $[\mathrm{m}]$

$L_{\text {Exposed }} \quad$ Length of channel exposed to vapor, [m] 


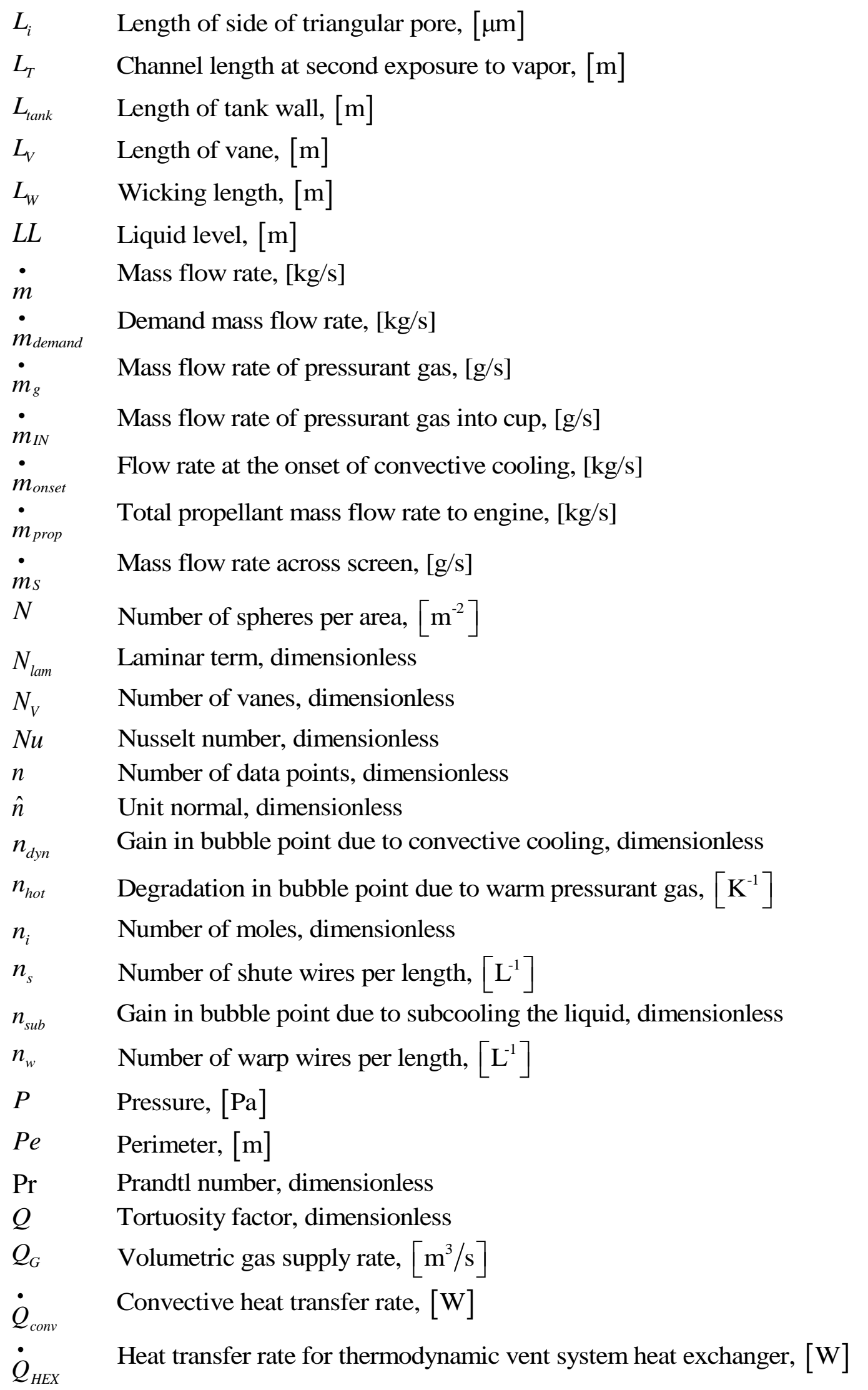




\begin{tabular}{|c|c|}
\hline$R_{i}$ & Radii of curvature, $[\mathrm{m}]$ \\
\hline$R_{G}$ & Gas constant, $[\mathrm{J} / \mathrm{kgK}]$ \\
\hline$R_{S}$ & Radius of screened sump, $[\mathrm{m}]$ \\
\hline$R_{T}$ & Thermal resistance, $[\mathrm{K} / \mathrm{W}]$ \\
\hline$R_{\text {tank }}$ & Radius of tank, [m] \\
\hline$R a_{F}$ & FRITZ radius, $[\mu \mathrm{m}]$ \\
\hline $\operatorname{Re}$ & Reynolds number, dimensionless \\
\hline$r$ & Radius of spherical particle, $[\mu \mathrm{m}]$ \\
\hline$r_{d}$ & Ratio of screen wire diameters, dimensionless \\
\hline$S$ & Entropy, $[\mathrm{J} / \mathrm{K}]$ \\
\hline $\bar{S}$ & Excess entropy per area, $\left[\mathrm{J} / \mathrm{m}^{2} \mathrm{~K}\right]$ \\
\hline$S_{C}$ & Characteristic screen length, $[\mathrm{m}]$ \\
\hline$S_{N}$ & Nucleate boiling supression factor, dimensionless \\
\hline$s$ & Wetted circumference, $[\mathrm{m}]$ \\
\hline$T$ & Temperature, $[\mathrm{K}]$ \\
\hline $\bar{T}$ & Average vehicle thrust, $[\mathrm{N}]$ \\
\hline$T_{c, i n}$ & Temperature of the cold liquid inside heat exchanger coil, $[\mathrm{K}]$ \\
\hline$T_{\text {Gas }}$ & Temperature of the pressurant gas, $[\mathrm{K}]$ \\
\hline$T_{h, i n}$ & Temperature of incoming liquid into channel, $[\mathrm{K}]$ \\
\hline$T_{h, \text { out }}$ & Temperature of the outgoing liquid out of the channel, $[\mathrm{K}]$ \\
\hline$T_{\text {Onset }}$ & Temperature at onset of degradation, $[\mathrm{K}]$ \\
\hline$t$ & Time, $[\mathrm{s}]$ \\
\hline$t_{C}$ & Thickness of thermodynamic vent system tube coil, [m] \\
\hline$U$ & Internal energy, $[\mathrm{J}]$ \\
\hline$U_{i}$ & Fluid velocity through screen, $[\mathrm{m} / \mathrm{s}]$ \\
\hline$U_{0}$ & Overall heat transfer coefficient, $\left[\mathrm{W} / \mathrm{m}^{2} \mathrm{~K}\right]$ \\
\hline$u_{e}$ & Channel exit velocity, $[\mathrm{m} / \mathrm{s}]$ \\
\hline$V$ & Volume, $\left[\mathrm{m}^{3}\right]$ \\
\hline$V_{\text {residuals }}$ & Remaining volume of liquid in tank, $\left[\mathrm{m}^{3}\right]$ \\
\hline$V_{\text {solid }}$ & Solid volume fraction of the screen, $[\mathrm{m}]$ \\
\hline$V_{\text {sphere }}$ & Volume of a spherical particle, $\left[\mathrm{m}^{3}\right]$ \\
\hline$V_{\text {tank }}$ & Internal volume of tank, $\left[\mathrm{m}^{3}\right]$ \\
\hline$\dot{V}$ & Volumetric flow rate, $\left[\mathrm{m}^{3} / \mathrm{s}\right]$ \\
\hline$\underset{\sim}{v}$ & Velocity vector, $[\mathrm{m} / \mathrm{s}]$ \\
\hline
\end{tabular}




$\begin{array}{ll}v_{i n j} & \text { Injection velocity, }[\mathrm{m} / \mathrm{s}] \\ v_{W} & \text { Wicking velocity, }[\mathrm{m} / \mathrm{s}] \\ W & \text { Width of the liquid acquisition device, }[\mathrm{m}] \\ W_{a} & \text { Work of adhesion, }\left[\mathrm{J} / \mathrm{m}^{2}\right] \\ W_{0} & \text { Screen deflection, }[\mathrm{m}] \\ X & \text { Pore diameter correlation factor, dimensionless } \\ X_{t t} & \text { Martinelli parameter, dimensionless } \\ X & \text { Quality, dimensionless } \\ Y & \text { Mass fraction, dimensionless } \\ Z & \text { Compressibility factor, dimensionless }\end{array}$

\section{Greek}

$\alpha$

$\alpha_{i}$

$\beta$

$\beta_{M}$

$\Gamma_{i}$

$\gamma_{L V}$

$\gamma_{S L}$

$\gamma_{S V}$

$\Delta G_{a}$

$\Delta G_{C}$

$\triangle P_{B P}$

$\triangle P_{B P, \text { dynamic }}$

$\triangle P_{B P, H o t}$

$\triangle P_{B P, N B P}$

$\triangle P_{B P, S a t}$

$\triangle P_{B P, \text { static }}$

$\triangle P_{B P, S u b}$

$\Delta P_{C}$

$\triangle P_{D P T}$
Viscous pressure drop coefficient, dimensionless

Activity coefficient, dimensionless

Inertial pressure drop coefficient, dimensionless

Work of adhesion fitting parameter, $\left[\mathrm{m}^{4} / \mathrm{J}^{2}\right]$

Excess composition per area, $\left[[\mathrm{C}] / \mathrm{m}^{2}\right]$

Liquid/vapor surface tension, $[\mathrm{mN} / \mathrm{m}]$

Solid/liquid surface tension, $[\mathrm{mN} / \mathrm{m}]$

Solid/vapor surface tension, $[\mathrm{mN} / \mathrm{m}]$

Work of adhesion, $\left[\mathrm{J} / \mathrm{m}^{2}\right]$

Work of cohesion, $\left[\mathrm{J} / \mathrm{m}^{2}\right]$

Bubble point pressure, $[\mathrm{Pa}]$

Dynamic bubble point pressure, $[\mathrm{Pa}]$

Bubble point pressure at an elevated pressurant gas temperature, $[\mathrm{Pa}]$

Bubble point pressure at the normal boiling point, $[\mathrm{Pa}]$

Bubble point pressure at a saturated liquid state, $[\mathrm{Pa}]$

Static bubble point pressure, $[\mathrm{Pa}]$

Bubble point pressure at subcooled liquid state, $[\mathrm{Pa}]$

Pressure difference in capillary tube, $[\mathrm{Pa}]$

Raw differential pressure reading, $[\mathrm{Pa}]$ 
$\Delta P_{d} \quad$ Pressure differential that causes a deflection, [Pa]

$\Delta P_{\text {drive }} \quad$ Vane driving pressure, $[\mathrm{Pa}]$

$\Delta P_{\text {dynamic }}$

Dynamic pressure drop down channel, $[\mathrm{Pa}]$

$\triangle P_{F T S}$

Flow-through-screen pressure drop, $[\mathrm{Pa}]$

$\Delta P_{\text {frictional }}$

Frictional pressure drop down channel, $[\mathrm{Pa}]$

$\Delta P_{\text {frictional,turbulent }}$

Turbulent portion of frictional pressure drop down channel, $[\mathrm{Pa}]$

$\Delta P_{\text {hydrostatic }}$

Hydrostatic pressure drop, $[\mathrm{Pa}]$

$\Delta P_{\text {inertial }}$

Inertial pressure loss, $[\mathrm{Pa}]$

$\Delta P_{\text {other }}$

Pressure drop due to sloshing, vibration, etc., $[\mathrm{Pa}]$

$\Delta P_{R S}$

Reseal pressure, $[\mathrm{Pa}]$

$\Delta P_{R S, H o t}$

$\triangle P_{R S, S a t}$

Reseal pressure at an elevated pressurant gas temperature, $[\mathrm{Pa}]$

$\Delta P_{R S, S u b}$

Reseal pressure for a saturated liquid state, $[\mathrm{Pa}]$

$\Delta P_{\text {sat }}$

Reseal pressure for a subcooled liquid state, $[\mathrm{Pa}]$

$\Delta P_{\text {total }}$

Difference between saturation pressure based on wall and fluid, $[\mathrm{Pa}]$

$\Delta P_{\text {viscous }}$

Total pressure drop, $[\mathrm{Pa}]$

$\Delta T_{e}$

Viscous pressure loss, $[\mathrm{Pa}]$

Difference between coil wall and saturation temperature, $[\mathrm{K}]$

$\varepsilon$

Void fraction, dimensionless

$\varepsilon_{H E X}$

$\theta_{C}$

Heat exchanger efficiency, dimensionless

$\theta_{R}$

Advancing contact angle, [degrees]

$\lambda_{n}$

Receding contact angle, [degrees]

$\mu$

Eigenvalue, $[1 / \mathrm{L}]$

Viscosity, $[\mathrm{kg} / \mathrm{m} \mathrm{s}]$

$\mu_{i} \quad$ Chemical potential, [J/particle $]$

$v \quad$ Kinematic viscosity, $\left[\mathrm{m}^{2} / \mathrm{s}\right]$

$\xi \quad$ Coefficient of thermal contraction, dimensionless

$\pi \quad$ Spreading pressure, $\left[\mathrm{J} / \mathrm{m}^{2}\right]$

$\rho \quad$ Density, $\left[\mathrm{kg} / \mathrm{m}^{3}\right]$

$\Sigma(t) \quad$ Time dependent interfacial surface, dimensionless

$\sigma_{i}$

Condensation and evaporation coefficient, dimensionless

$\tau$

Excess volume per unit area, [m]

$\Phi$

Heat flux, $\left[\mathrm{W} / \mathrm{m}^{2}\right]$ 


\section{Subscripts and Superscripts}

$\begin{array}{ll}+ & \text { Adsorbing } \\ - & \text { Desorbing } \\ 0,1,2 & \text { Fitting parameters } \\ 288 K & \text { Evaluated at } 288 \mathrm{~K} \\ 295 K & \text { Evaluated at } 295 \mathrm{~K} \\ b & \text { Below interface } \\ C & \text { Critical } \\ e & \text { Excess } \\ f & \text { Saturated liquid } \\ \text { interface } & \text { Interface } \\ L & \text { Liquid } \\ L V & \text { Liquid/vapor } \\ M A X & \text { Maximum } \\ M I N & \text { Minimum } \\ R & \text { Reduced } \\ r e f & \text { Reference state } \\ S A T & \text { Saturated } \\ S E M & \text { From scanning electron microscopy analysis } \\ \alpha & \\ \beta & \text { Phase 1 } \\ & \text { Phase 2 }\end{array}$

\section{Math}

cos Cosine of an angle

cosh Hyperbolic cosine

$\log \quad$ Logarithmic

tanh Hyperbolic tangent

$x, y, z \quad$ Standard Cartesian coordinate system

$\nabla \quad$ Del Operator, $[1 / \mathrm{m}]$

$d x \quad$ Differential, $[\mathrm{m}]$

$\frac{D}{D t} \quad$ Material derivative, $[1 / \mathrm{s}]$

$\frac{d}{d t} \quad$ Derivative with respect to time, $[1 / \mathrm{s}]$ 
$\frac{d}{d x} \quad$ Ordinary derivative with respect to space, $[1 / \mathrm{x}]$

$\frac{\partial}{\partial x} \quad$ Partial derivative with respect to space, $[1 / \mathrm{x}]$ 


\title{
LIQUID ACQUISITION DEVICES FOR ADVANCED IN-SPACE CRYOGENIC PROPULSION SYSTEMS
}

\author{
Abstract \\ by \\ JASON WILLIAM HARTWIG
}

The enabling of all future in-space cryogenic engines and cryogenic fuel depots for future manned and robotic space exploration missions begins with technology development of advanced cryogenic fluid management systems upstream in the propellant tank. Gravity affects many fluidic processes, such as the separation of the liquid and vapor phases within the propellant tank. By design, all in-space cryogenic engines and cryogenic fuel depots require vapor free liquid delivery. To meet these fluid transfer requirements over a wide range of mission flow rates, gravitational and thermal environments, propellant management devices will be required to favorably position liquid and vapor within the tank.

The purpose of this work is to develop such robust and flexible liquid acquisition devices (LAD), particularly for low surface tension cryogenic propellants operating in microgravity environments, through a battery of component level and full scale ground experiments, and development of analytical tools. Models are first developed from first principles for the influential factors which govern LAD performance, which include 
bubble point pressure, flow-through-screen pressure drop, wicking rate, and screen compliance. The literature is rigorously reviewed to gather data to validate the models. Then a series of parametric component level tests are conducted in room temperature liquids and cryogenic hydrogen, nitrogen, oxygen, and methane to determine the effect of varying screen type, liquid, liquid temperature and pressure, and pressurant gas type and temperature on the bubble point pressure. LAD channels are then constructed, and full scale LAD outflow tests are conducted in liquid hydrogen to simulate fluid transfer from a propellant tank in a variable thermal environment, to determine pressure drop contributions, and to assess reliability of the LADs at cryogenic temperatures. One of the channels is thermally flight representative with a custom designed internal heat exchanger. Experimental results are used to update and assess analytical predictive models in cryogenic liquids for bubble point, reseal pressure, and pressure drop in the porous channel, and the updated models are used to determine the optimal LAD screen for a liquid hydrogen fuel depot operating in Low Earth Orbit. Additionally, performance of vanes in cryogenic liquids is compared against screen channels. 


\section{Chapter 1}

\section{Introduction}

The purpose of this chapter is to present insight into the primary field of application for this dissertation, namely in-space cryogenic propulsion systems. First, the current path of cryogenic propulsion technology development is outlined. Next, the four primary cryogenic fluids of interest are briefly examined in terms of physical properties to familiarize the reader. Then, existing challenges when working with cryogenic fluids are discussed as well current mitigation strategies. Finally, cryogenic propellant engines and cryogenic propellant depots are briefly examined to show how cryogenic fluid management systems are required inside the propellant tank, particularly propellant management devices.

\subsection{The Flexible Path}

Manned space exploration remains at the forefront of mankind's relentless endeavor to search, explore, and thus understand the physical universe. Despite the recent de-emphasis on a single manned mission to the surface of the Moon, the National Aeronautics and Space Administration (NASA), as well as commercial space flight community still maintains strong interest in human and robotic missions beyond Low Earth Orbit (LEO) environments. A new strategy for human exploration was recently outlined in the Augustine Report, which shifts focus away from a single destination to 
that of several (Augustine 2010). Deemed the Flexible Path, travel to several locations of interest will follow a logical progression; NASA will continue to develop the technology necessary to orbit, probe, and eventually land on several celestial bodies of interest through an increase in research and funding to relevant technologies in the coming years (Crawley and Mindell 2010 and Korsmeyer et al. 2010). Inherent in this new focus will be the development, design, and optimization of the primary systems and subsystems of heavy launch lift (HLL) vehicles, LEO vehicles (such as in-space fuel storage and transfer vehicles, satellites, etc.), robotic probes and landers, and eventually, vehicles which could potentially deliver robots and humans to the surfaces of the Moon and Mars.

The logical progression of space exploration begins with developing LEO systems, followed by travel to Earth-Moon Lagrange points, then Near Earth Objects (NEOs) such as asteroids, followed by robotic satellites to probe the Moon, Mars, and beyond, robotic surface missions, and finally manned surface missions. Necessary for the exploration and study of these locations of interest will be the development of new and existing propulsion capabilities required to send both human and robot afar. Therefore advanced in-space propulsion systems, which are the backbone of any and all space flight programs, will be required, regardless of the mission, destination, or desire.

The three primary in-space propulsion systems include electric, chemical, and nuclear systems. The primary measure of the performance of a particular propulsion system is to determine the non-dimensional specific impulse (ISP):

$$
I S P=\frac{\bar{T}}{\dot{m}_{\text {prop }} g}
$$


where $\bar{T}$ is thrust, $\dot{m}_{\text {prop }}$ is the total mass flow rate of propellant, and $g$ is gravity. ISP provides a convenient method to compare efficiency of different propulsion systems. For example, electric propulsion systems generally have very high ISP and low thrust relative to chemical and nuclear systems, which makes them best suited for long duration robotic missions where human life support systems are not required. However, for long duration manned missions, electric systems may not be able to provide adequate thrust to propel humans to the final destination in a reasonable time. Comparatively, chemical and nuclear systems are generally characterized by lower ISP, but much higher thrust relative to electric systems. Emphasis in the current work is on general cryogenic propulsion technology development of cryogenic fluid management (CFM) components, which has applications for both chemical and nuclear systems because long term storage and transfer of cryogenic liquids is required for both.

According to NASA's long term space exploration vision, the development of cryogenic propulsion systems remains at the forefront of its research and technology development program (NASA’s Space Technology Roadmaps 2012). Cryogenic propellant technology can be used to enable future high performance in-space engines, fuel storage depots (defined in Section 1.6.2), life support systems, fuel cells, in-space resource utilization (ISRU) systems, cooling, refrigeration, liquefaction, and will thus enable every one of the aforementioned missions and destinations of interest. The challenge thus arises to develop technology that is flexible, broad based, and applicable to multiple missions under the Flexible Path, rather than applicable to a single mission. In addition, fundamental issues associated with the storage and transfer of cryogenic propellants must first be addressed before any of these missions become fully realizable. 


\subsection{Fundamental Cryogenic Fluids}

In physics, the field of cryogenics is loosely defined as the study of physical phenomena that occur at very low temperatures. A cryogenic liquid is therefore defined as a substance which exists as a liquid at extremely cold temperatures and a gas at room temperatures. However, "low" and "cold" are relative words since all substances can be evaporatively cooled into a liquid and then solid state, with the exception of helium. Liquid nitrogen $\left(\mathrm{LN}_{2}\right)$ is generally used as the standard reference cryogenic liquid, since nitrogen exists in abundance on Earth, and because the normal boiling point (NBP) of $77.4 \mathrm{~K}$ is very low relative to many substances which exist as liquids at room temperature.

This dissertation topic is focused on the general field of cryogenic liquid flow through porous liquid acquisition devices (LADs). Regardless of the temperature regime, the liquid state is always bound by the triple point, the point at which a liquid begins to solidify into a solid, and the critical point, the point at which liquid and gaseous phases become indistinguishable. Outlined in Figure 1.1 are the four primary fundamental cryogenic liquids, liquid hydrogen $\left(\mathrm{LH}_{2}\right), \mathrm{LN}_{2}$, liquid oxygen (LOX), and liquid methane $\left(\mathrm{LCH}_{4}\right)$, along with the triple line temperature, NBP temperature (saturation temperature at $1 \mathrm{~atm}$ of pressure), critical temperature $\left(\mathrm{T}_{\mathrm{C}}\right)$, and the liquid/vapor $(\mathrm{L} / \mathrm{V})$ surface tension at the NBP. Liquid helium (LHe) and water are listed for reference. As shown, even the warmest temperature at which methane exists as a liquid at the NBP is still only $111 \mathrm{~K}$. Surface tension decreases as the liquid saturation temperature decreases, with $\mathrm{LCH}_{4}$ and LOX having an order of magnitude higher surface tension over $\mathrm{LH}_{2}$. In general, as the saturation temperature decreases, the range over which a substance exists as a liquid 
becomes narrower; methane exists in the liquid phase over a $100 \mathrm{~K}$ range whereas hydrogen exists as a liquid over a $20 \mathrm{~K}$ range.

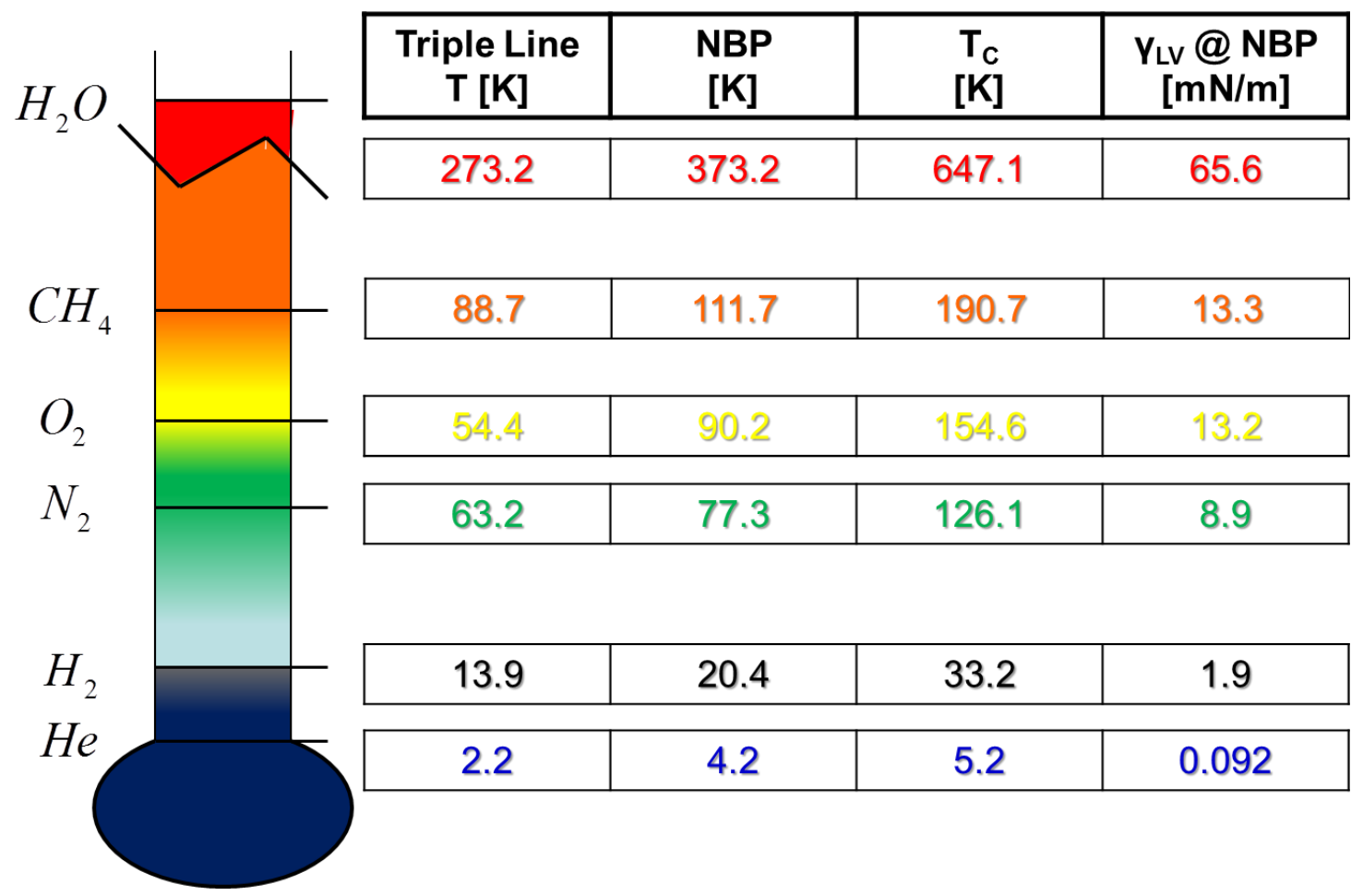

Figure 1.1 - Triple Line Temperature, Normal Boiling Point, Critical Temperature, and Surface Tension at the Normal Boiling Point for Several Fluids of Interest

For illustration, Figure 1.2 plots the temperature and pressure ranges over which the four fluids exist as liquids between the saturation curve and triple line. Temperature/Pressure (T/P) plots are used throughout the text to illustrate how the LAD performs as a function of the thermodynamic state of the liquid. For comparison, Table 1.1 lists the four primary cryogenic fluids along with thermophysical properties at the NBP saturation conditions relevant in the current work, such as the saturation temperature, heat of vaporization $h_{f g}$, liquid density $\rho_{f}$, kinematic viscosity $v$, and 
surface tension $\gamma_{L V}$. Clearly, advanced systems are required to store, maintain, and transfer such cold liquids.

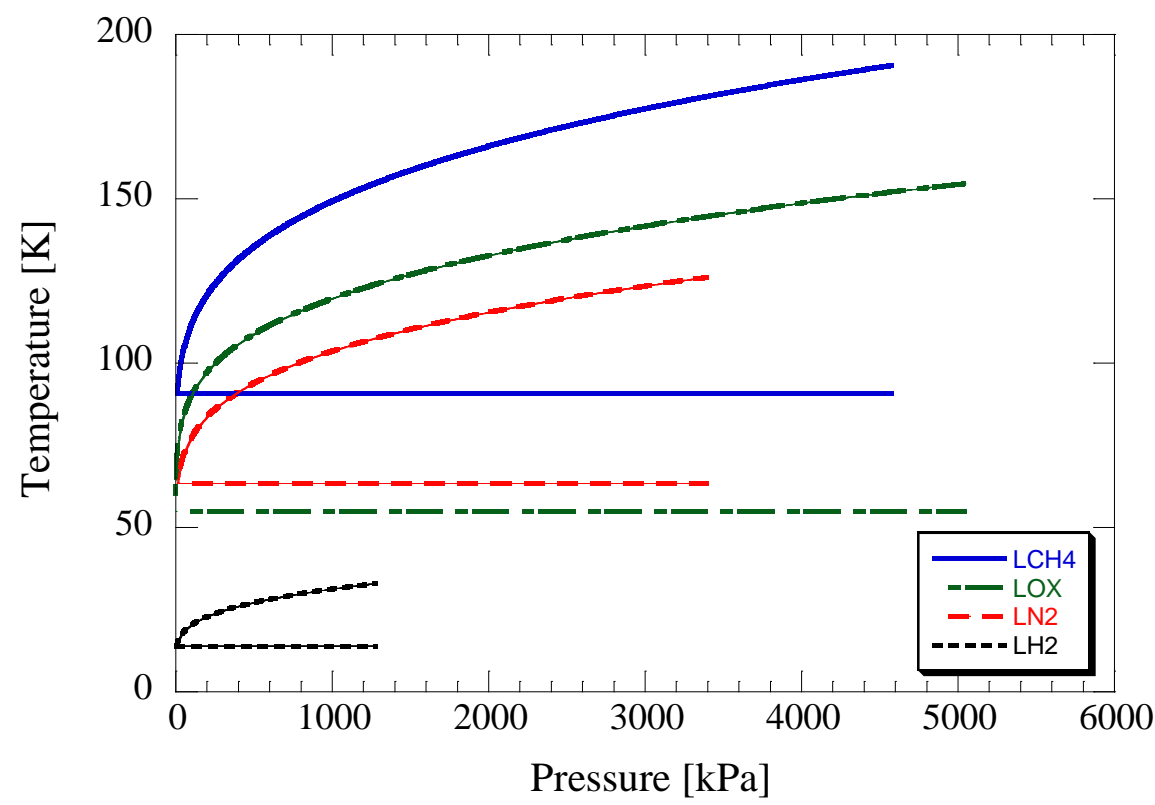

Figure 1.2 - Operating Range of Temperatures and Pressures Over Which Hydrogen, Nitrogen, Oxygen, and Methane Exist as Liquids. For each liquid, the saturation line and triple line are plotted up to the critical point.

\begin{tabular}{|c|c|c|c|c|c|}
\cline { 2 - 7 } \multicolumn{1}{c|}{} & Tsat [K] & hfg [kJ/kg] & of [kg/m^3] & $\mathbf{u}\left[\mathbf{m}^{\wedge} \mathbf{2} / \mathbf{s}\right]$ & $\mathbf{\gamma L V}[\mathbf{m N} / \mathbf{m}]$ \\
\hline Methane & 111.7 & 510.3 & 422.44 & $2.78 \mathrm{E}-07$ & 13.3 \\
\hline Oxygen & 90.2 & 213.2 & 1142 & $1.71 \mathrm{E}-07$ & 13.2 \\
\hline Nitrogen & 77.3 & 198.6 & 807 & $1.86 \mathrm{E}-07$ & 8.9 \\
\hline Hydrogen & 20.3 & 445.6 & 70.8 & $1.89 \mathrm{E}-07$ & 1.94 \\
\hline
\end{tabular}

Table 1.1 - Relevant Thermophysical Properties for the Four Primary Cryogenic Liquids

\subsection{Motivation for Cryogenic Propulsion Technology Development}

In recent years, there has been a decline in the proposed usage of the more toxic storable propellants, such as nitrous tetroxide $\left(\mathrm{N}_{2} \mathrm{O}_{4}\right)$ and monomethyl hydrazine $(\mathrm{MMH})$. 
Four reasons are cited to the proposed shift from storable propellants to cryogenic propellants. First, safety and environmental concerns over the use of storable propellants have led to the ongoing examination of non-toxic cryogenic propellants as the ideal fluid for on-orbit propulsion systems for future human and robotic exploration missions. There has also been a growing desire to research alternative fuel sources and propellant combinations which enable longer duration missions, such as $\mathrm{LOX} / \mathrm{LCH}_{4}$. Second, cryogenic propulsion systems like $\mathrm{LOX} / \mathrm{LH}_{2}$ also have proven flight heritage in Saturn $\mathrm{V}$ (S4, S4B, S2), upper stages (Centaur, Delta IV), and the Space Shuttle Transportation (STS) system. Third, $\mathrm{LOX} / \mathrm{LH}_{2}$ systems are attractive candidates for future in-space engines such as the $\mathrm{J}-2 \mathrm{X}$ and future LEO fuel depots.

But perhaps the most attractive and most often cited feature of cryogenic propulsion systems is the unmatched level of performance relative to storable propellant combinations. Aside from nuclear thermal propulsion systems (Robbins and Finger 1991), at optimal mixture ratios, no other known pure propellant combination can deliver a higher ISP than that of $\mathrm{LOX} / \mathrm{LH}_{2}$ (fluorine can be added to slightly increase performance). In the optimal design of any spacecraft however, high ISP is not the only consideration. From a systems engineering standpoint, long term human missions will require either reconsideration of propellant choice or a refinement of existing cryogenic propulsion technology.

\subsection{Existing Challenges with Cryogenic Propellants}

The importance and role of fuels such as hydrogen as a sustainable energy source have previously been cited (Momirlan and Veziroglu 2005 and Balat 2008). However, 
there are challenging aspects when working with cryogenic liquids such as $\mathrm{LH}_{2}$ due to the thermophysical properties as outlined in Table 1.1. So if high performance is the desire, then several key issues must first be addressed. Table 1.2 lists challenges and resultant implications on propellant tank system design for future $\mathrm{LOX} / \mathrm{LH}_{2}$ propulsion systems. For example, the low liquid density and low NBP makes $\mathrm{LH}_{2}$ difficult to package, requiring large and thick walled tanks. $\mathrm{LH}_{2}$ is also particularly susceptible to parasitic heat leak because it exists as a liquid only at extremely low temperatures, resulting in costly insulation systems and possible boil-off of precious propellant. Of particular interest in the current work is the difficulty associated with vapor free liquid transfer from the propellant tank due to low surface tension and low NBP.

\begin{tabular}{|l|l|l|}
\hline \multicolumn{1}{|c|}{ Challenge } & \multicolumn{1}{|c|}{ Reason } & \multicolumn{1}{c|}{ Implication } \\
\hline 1. Hard to "package" & Low liquid density and NBP & Requires larger and thicker walled storage tanks \\
\hline 2. Difficult to insulate & $\begin{array}{l}\text { Low NBP, sensitive to parasitic } \\
\text { heat leak }\end{array}$ & $\begin{array}{l}\text { Some critical amount of propellant will always be } \\
\text { sacrificed due to heat leak }\end{array}$ \\
\hline $\begin{array}{l}\text { 3. Limited engine design } \\
\text { start and run boxes }\end{array}$ & $\begin{array}{l}\text { Low critical temperature and } \\
\text { pressure }\end{array}$ & Limited operating range to avoid cavitation \\
\hline $\begin{array}{l}\text { 4. Added transfer system } \\
\text { complexity }\end{array}$ & $\begin{array}{l}\text { Vastly different NBP of LOX and } \\
\text { LH }_{2}\end{array}$ & $\begin{array}{l}\text { Requires additional thermal conditioning of } \\
\text { propellants prior to incidence at injector }\end{array}$ \\
\hline $\begin{array}{l}\text { 5. Difficult to transfer } \\
\text { single phase liquid }\end{array}$ & $\begin{array}{l}\text { Low resistance to flow, low } \\
\text { surface tension, low NBP }\end{array}$ & $\begin{array}{l}\text { High probability of vapor ingestion into a storage } \\
\text { tank or engine }\end{array}$ \\
\hline
\end{tabular}

Table 1.2 - Challenges and Implications for Future Liquid Oxygen/Liquid Hydrogen System

\section{Design}

All in-space cryogenic engines require single phase liquid up to the injectors. Cryogenic fuel depots will also require very high liquid volume fill fractions. Therefore without rigorous design of propellant management systems within the propellant tank, vapor ingestion in the transfer line, and thus to the engine or customer storage tank, is highly probable. 


\subsection{Cryogenic Fluid Management Subsystems}

To deal with the above mentioned challenges, a number of CFM subsystems can be implemented upstream within the propellant tank as illustrated in Figure 1.3 (Motil et al 2007). Passive multi-layer insulation (MLI) systems, lightweight, low thermal conductivity tank support struts, as well as optimized active cooling systems, such as broad area cooling (BAC) shields or direct tank applied cooling systems which use colder working fluids to circulate around the tank, can be used to intercept and reduce heat leak. Thermodynamic vent systems (TVS) can be used to control and maintain tank pressure due to heat leak into the cryogen. Advanced mass gauging (MG) systems can be used to determine the amount of liquid propellant inside the tank, irrespective of the g-level. Lighter weight Lithium (Li), Aluminum (Al), or Titanium (Ti) tanks may be used to reduce system mass (Tam et al. 2003 and 2006). Propellant management devices (PMDs), which are the primary focus of this work, can be used to maintain liquid flow out of the propellant tank to the transfer line by maintaining liquid over the tank outlet at all times of a mission. Many of these systems have flight heritage in storable propulsion systems, but only limited usage in ground experiments in cryogenic systems.

\subsection{Future Cryogenic Fluid Management Applications}

There are two primary customers or applications for CFM technology to provide in-space vapor free liquid transfer from the upstream propellant tank. In-space cryogenic engines will require vapor free propellant transfer over a wide range of flow rates in milli- and microgravity environments over a wide range of thermal environments. Future in-space cryogenic fuel depots, which are of particular interest in the current work, will 
also require efficient methods to store and transfer $\mathrm{LH}_{2}$ and $\mathrm{LOX}$ from a depot storage tank to a customer receiver tank.

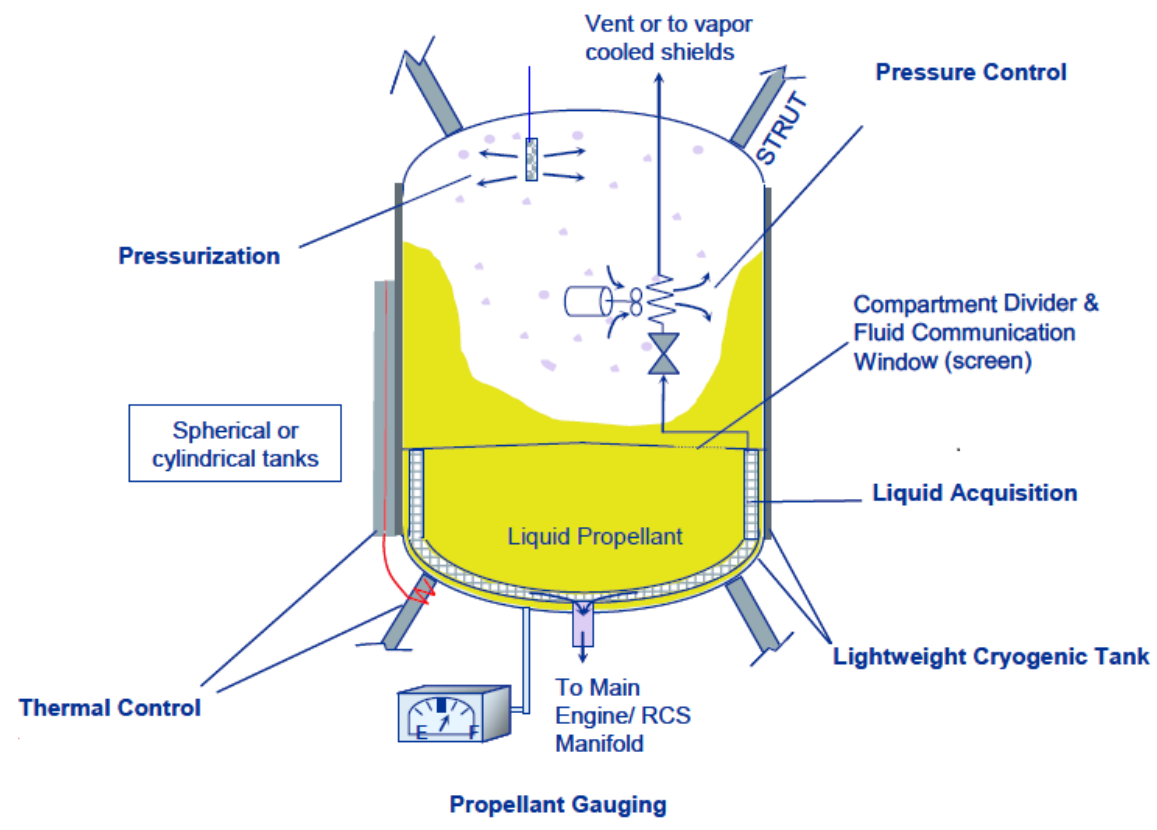

Figure 1.3 - Illustration of Cryogenic Fluid Management Components

\subsubsection{In-Space Cryogenic Engines}

To illustrate how LADs will be required at nearly all phases of a mission, Figure 1.4 outlines the mission architecture and timeline for a representative manned mission to the surface of the Moon for up to a six month stay, taken from Exploration Systems Architecture Study (Anonymous 2005). Two heavy lift $\mathrm{LOX} / \mathrm{LH}_{2}$ rockets would launch from Earth, one carrying the astronauts and crew exploration vehicle (CEV), and one carrying the Earth Departure Stage (EDS), Lunar Service Access Module (LSAM), LSAM ascent and descent stages, and equipment for the six month Lunar surface mission. Therefore, both systems carry onboard all the propellant required for the entire mission, which increases the size of the launch system dramatically. 


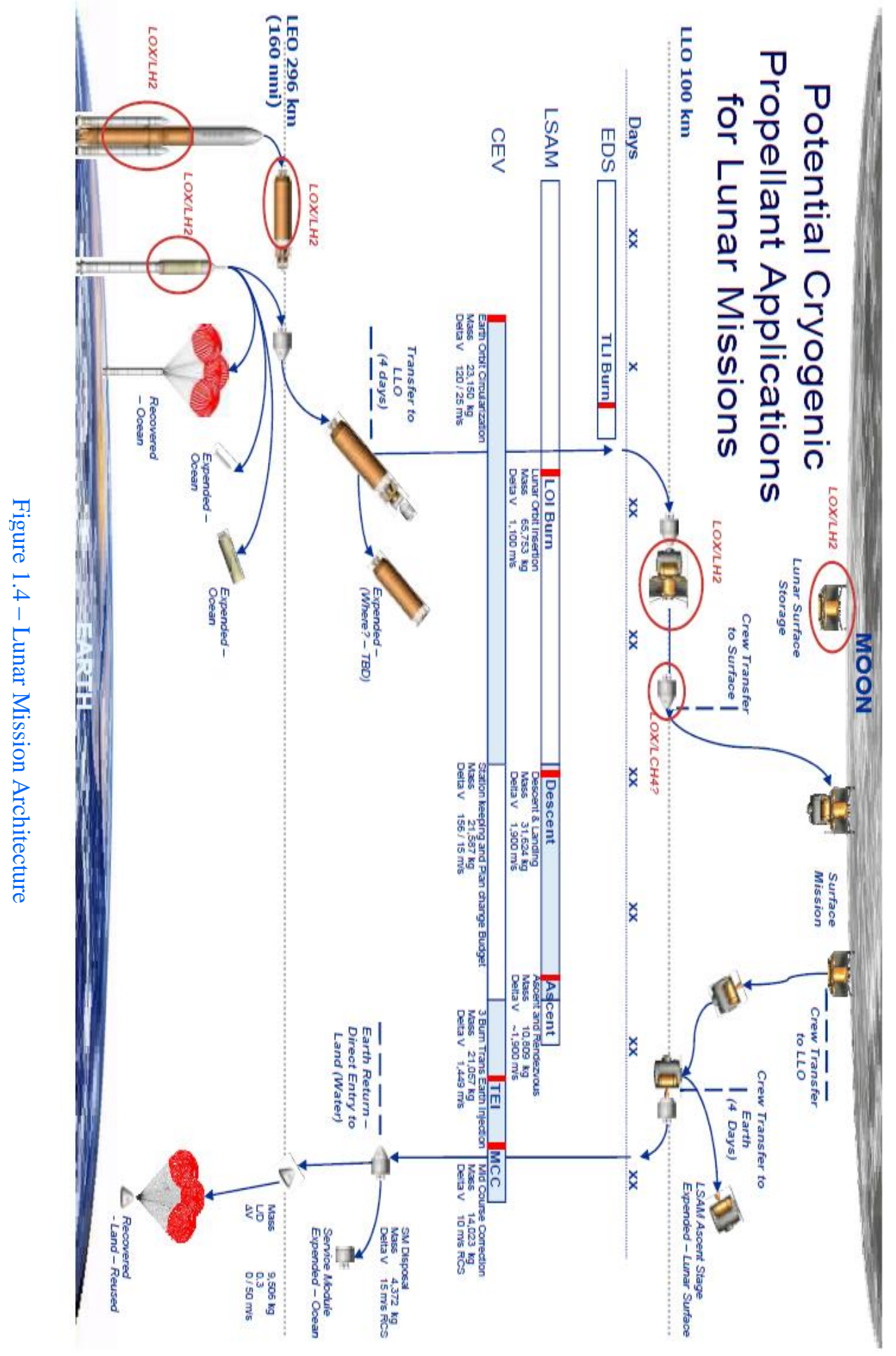


The simplified concept of operation is as follows: Both rockets launch from Earth within a week of each other. The CEV and EDS stages then rendezvous, dock, and join in LEO. After an approximate four day journey, the EDS engine stage is jettisoned as the crew enters Low Lunar Orbit (LLO). The CEV and LSAM then land on the surface of the Moon by using the descent engines. After the six month mission, the crew launches from the surface using the ascent main engine (AME) to return to LLO to rendezvous and dock with the CEV to return to Earth.

Examination of Figure 1.4 shows that cryogenic propulsion systems will be required for every phase of the mission. $\mathrm{LOX} / \mathrm{LH}_{2}$ systems are considered for the Earth launch, EDS, and LSAM descent stage while $\mathrm{LOX} / \mathrm{LCH}_{4}$ is considered for the LSAM ascent stage, making use of the ability of methane to absorb more parasitic heat leak than hydrogen over the course of the six month stay. PMDs are not required for the launch stage, since vehicle thrust and 1-g acceleration is sufficient to maintain liquid over the outlet. Advanced PMDs will however be required for the EDS stage to ensure liquid covers the outlet during minor docking thrust maneuvers when the two systems join in the reduced gravity of LEO, and to maintain liquid flow during the departure to LLO. Because LLO is also reduced gravity, advanced PMDs will be required for LLO loitering stage and the descent stage. Simple PMDs will also be required to ensure that LOX and $\mathrm{LCH}_{4}$ sufficiently cover the tank outlet at engine ignition in 1/6 gravity on the Lunar surface, as well for reaction control system (RCS) maneuvering through LLO, to LEO, and then back to Earth. Obviously, flexible and robust PMDs are required to deliver vapor free liquid to several different engines using several different cryogenic propellants 
over a range of gravitational and thermal environments in order to enable this long duration manned Lunar surface mission.

\subsubsection{In-Space Cryogenic Fuel Depots}

Perhaps the best future application for cryogenic LADs will be in cryogenic fuel depots. Large chemical or nuclear propulsion systems will likely be required to enable manned missions to destinations beyond LEO. For a Lunar or Martian surface return mission, the baseline method would be to launch from Earth carrying all the propellant required to reach the destination and return safely to Earth as depicted in Figure 1.4. This traditional method severely hinders the amount of payload that a single system can send into LEO or beyond. It also severely hinders the final achievable Earth departure velocity.

An alternative approach is to launch programmable robots and mining equipment months to years before humans reach the destination to extract or generate the required amount of propellant for the return portion of the mission on the surface. ISRU proposes the in-situ extraction of necessary chemical elements to temporarily sustain human life (ex. $\mathrm{O}_{2}$ ), but to also mine the surface for fuel (ex. $\mathrm{H}_{2}$ or $\left.\mathrm{CH}_{4}\right)$. Once adequate propellant has been generated, humans could then launch from Earth with empty or reusable tanks, land on the surface, conduct a mission, and then refill prior to departure. While this method eliminates the need to carry the additional payload, there is a great amount of speculation to its fundamental feasibility and cost. The ISRU concept is still in an infancy stage due to a lack of sufficient experimental data taken in-situ on the Moon or Mars. 
Fuel depots are a much more practical option for enabling long duration robotic and manned space missions. A fuel depot is defined as an Earth-orbiting propellant storage vessel that can house cryogenic propellant for an indefinite duration of time, and then transfer the propellant to any other orbiting vehicle. A spacecraft can launch with enough propellant to break the Earth gravity well, rendezvous and dock with the fuel depot, and then refill en route to the final destination. The depot would use LADs to supply liquid to the vehicle storage tank to first chill down and then refill the propellant tank. This process is analogous to refueling an automobile at a gas station on the ground. Large fuel depots can therefore contain a large percentage of the propellant required for a deep space mission, thus allowing more dry mass launched into orbit. Due to its size, a fuel depot will likely need to be launched in stages and assembled in LEO. The initial cost of assembly will quickly be outweighed by the long term, continuous usage of the fuel depot for routine commercial space flight. High performance in-space cryogenic engines coupled with large amounts of propellants equates to significantly higher Earth departure velocities and/or much longer duration missions beyond LEO. The lower overall cost, less risk, and fewer uncertainties in design and operation render the cryogenic fuel depot the primary candidate for enabling future long-duration manned missions.

Proposed full scale depots could potentially house storable or cryogenic propellants. The advantage of storing cryogenic fuels like $\mathrm{LH}_{2}$ is a significant boost in spacecraft performance as outlined in Section 1.3. However, Zero Boil Off (ZBO) active cooling systems for $\mathrm{LH}_{2}$ must first be demonstrated on the ground to prove that cold propellant can be stored indefinitely (Guernsey et al. 2005). In addition, the in-space 
storage and transfer of cryogenic propellants should also be demonstrated in a small depot demonstration mission, such as the mission previously proposed by the Cryogenic Propellant Storage and Transfer Project (CPST) at NASA. Other previously attempted, but never flown depot demonstration missions are discussed in Appendix A.

Figure 1.5 shows a typical cryogenic fuel depot concept which shows the $\mathrm{LH}_{2}$ and LOX tanks, sun shield for radiation shielding, as well as a docked Centaur upper stage (Kutter 2010). This particular depot is sized for a single mission; full scale depots supporting routine commercial space travel would obviously be much larger. Recent examples of proposed full scale fuel depot concepts are available in the literature for general (Kutter et al. 2005, Fikes et al. 2006, Street 2006, Kutter et al. 2008, Goff et al. 2009, Zegler and Kutter 2010, and McLean et al. 2011), Lunar (Young et al. 2006, Chandler et al. 2007, and Zegler et al. 2009), as well as Martian based architectures (Tanner et al. 2006). Compared to previous designs, the newer proposed full scale depots are much larger and flexible in that they could potentially service launch vehicles as a refueling station as well as facilitate life extension for existing satellites while taking advantage of new technological advances in cryogenic liquid storage (Kutter 2010).

Depots will remain in the microgravity of LEO for a period of several months up to an indefinite time. Therefore, in addition to implementing ZBO technology, LADs will also be required to control and maintain liquid flow out of the depot storage tank. The customer vehicle tanks will require very high final liquid volume fill fractions, necessitating the need for robust LADs upstream in the depot storage tank. While depot flow rate demands are likely smaller than in-space engine demands, depots may not have the ability to settle liquid over the tank outlet before propellant transfer in the same way 
that most engines operate; the need for unsettled vapor free, low surface tension cryogenic propellant in the microgravity of LEO makes LADs an excellent candidate to meet this requirement.

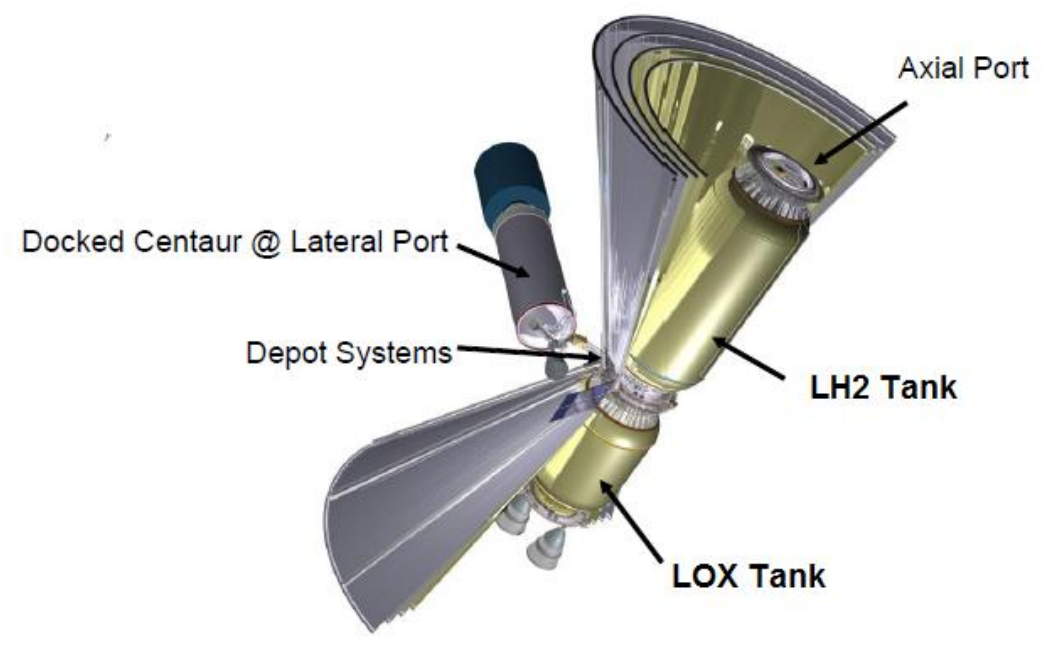

Figure 1.5 - Cryogenic Fuel Depot Concept

\subsection{Purpose of Dissertation and Overview by Chapter}

The enabling of all future in-space cryogenic engines and cryogenic propellant depots for future manned and robotic space exploration missions begins with technology development of LADs upstream in the propellant tank. Depending on the mission requirements, which include acceleration level, direction, and spin, mass flow rate, thermal environment, tank pressure, and desired final liquid fill level, LADs will be required to ensure that the tank outlet is sufficiently covered with liquid at all phases of the mission. By design, all in-space cryogenic engines and cryogenic fuel depots will require vapor free liquid delivery. LADs must first be rigorously qualified before they 
can be routinely used in flight cryogenic propellant tanks to ensure that both liquid and vapor are favorably positioned within the tank for any mission.

In addition, understanding the underlying fluid mechanics and heat transfer associated with the transfer of cryogens with LADs is not well understood in years past, because little data exists to characterize the system. While LADs have been studied for nearly five decades, and that they have flight heritage in storable propulsion systems, the combination of low surface tension, complications due to heat leak at low temperatures, a need for propellant transfer across a wide range of demand flow rates over a range of gravitational conditions, and uncertainty in the L/V interface within the propellant tank in microgravity makes LAD design in cryogenic propulsion systems quite challenging. Advancing the technology readiness level (TRL) by extending LADs from storable systems to operate in low gravity cryogenic systems is thus the primary focus of this work. The goal is to not only develop usable hardware for all future space vehicles, but to also understand fundamental cryogenic fluid behavior, provide relevant experimental data, and develop physics-based models and analytical design tools.

The purpose of this dissertation is therefore to develop robust and flexible liquid acquisition devices, particularly for low surface tension cryogenic propellants operating in microgravity, to meet the current and future demands of advanced in-space cryogenic propulsion systems. This purpose is pursued through development of fundamental models and analytical tools of the influential factors which govern LAD performance, and a comprehensive battery of component level and full scale LAD experiments in cryogenic hydrogen, nitrogen, oxygen, and methane. By providing the theoretical and experimental 
groundwork for LADs in cryogenic propellants, it is hoped that the tools developed in this work can be extended to all future cryogenic LAD design.

The overall outline of the dissertation is as follows: Chapters 1 and 2 provide motivation and background for conducting this work. Chapter 3 presents the influential factors that govern LAD performance along with preliminary models used to predict LAD performance. Chapters $4-9$ present all of the room temperature and cryogenic experiments conducted in this work. Chapters $10-14$ provide refined analytical and empirical models for cryogenic propulsion systems. Chapter 15 is the conclusion.

The detailed outline of the dissertation by chapter is as follows: Chapter 2 provides a background and historical review for PMDs. Specifically the three main types of capillary driven PMDs, vanes, sponges, and screen channel liquid acquisition devices are compared and contrasted, and screen channel LADs are down selected as the most robust option for cryogenic technology development. In Chapter 3, the set of influential factors that govern screen channel LAD performance are formulated and the basic physics and governing equations are derived and presented, which include static room temperature bubble point pressure, flow-through-screen (FTS) pressure drop, wicking rate, screen compliance, material compatibility, and static room temperature reseal pressure. Also presented is a simplified one dimensional (1D) pressure balance for flow through and down the LAD channel. The primary LAD screens of interest in the current work are subsequently identified. A comprehensive literature review is also conducted to gather all historical screen channel LAD ground test data to validate models. Chapter 4 presents the experimental methodology for ground testing LAD screens. Pure fluid as well as binary mixture room temperature bubble point data is presented, and several 
methods are compared for determining the effective screen pore diameter. Room temperature wicking tests are also presented. Chapter 5 then presents the low pressure cryogenic hydrogen and nitrogen experiments and data using three screens, two liquids and two different pressurization schemes. Tests are conducted in a parametric fashion to independently assess the effect of mesh type, liquid type, liquid temperature and pressure, and pressurant gas type on the cryogenic bubble point. Chapter 6 then presents higher pressure LOX bubble point tests and data for two screens and two different pressurization schemes. In addition to the trends examined in Chapter 5, the effect of subcooling the liquid on LAD performance is examined over a much wider range of test conditions. Heat transfer effects at LAD breakdown are also examined. Chapter 7 repeats higher pressure bubble point tests in $\mathrm{LCH}_{4}$ for a single screen using three different pressurant gases. Heat transfer effects are further examined by comparing features such as interfacial temperature, liquid subcooling, and condensation and evaporation mass flux rates between the three pressurant gases. Chapter 8 examines the effect of elevating the temperature of the pressurant gas temperature on $\mathrm{LH}_{2}$ and $\mathrm{LN}_{2}$ bubble point pressures. Chapter 9 presents the experimental methodology for testing full scale LAD channels. 1g vertical inverted outflow test results in $\mathrm{LH}_{2}$ are conducted to compare performance of two different full scale LAD channels. One of the channels is thermally flight representative due to the presence of a custom internal heat exchanger. Horizontal LAD and FTS $\mathrm{LH}_{2}$ test results are also presented to formulate the temperature dependent FTS model. Comparison is also given between the LAD outflow data and the simplified 1D pressure drop model from Chapter 3. Chapter 10 presents the revised static bubble point model for cryogenic liquids. Model dependencies are systematically presented to explain 
the trends in the data. A temperature dependent screen pore diameter is introduced, and subcooled gain and heated pressurant gas loss terms are also added to the new model. Chapter 11 echoes Chapter 10 by presenting a refined reseal pressure drop model, using cryogenic data to validate the model. Chapter 12 presents a new steady state analytical pressure drop model for flow through a channel with one porous wall with arbitrary variable suction or injection, which is directly applied to the LAD channel, by directly solving the Navier Stokes (NS) equations. The model is compared to the 1-g inverted outflow data to update the static bubble point model for convective cooling in the dynamic outflow case. Chapter 13 is the first of two chapters applying the revised set of cryogenic LAD performance models. Analysis and rationale are presented for choosing an optimal LAD screen weave for a large scale $\mathrm{LH}_{2}$ fuel depot orbiting in LEO. A critical mass flux and minimum bubble point are used along with the influential factors from Chapter 3 to down select the optimal screen. Chapter 14, the second model application chapter, uses the refined set of models to simulate outflow from a smaller scale $\mathrm{LH}_{2}$ propellant tank representative of a depot demonstration mission. Additionally, steady state analysis of vanes is presented to compare performance against screen channel LADs. Chapter 15 presents conclusions and future recommendations for cryogenic LAD technology development. Appendix A presents a historical summary of previously attempted depot demonstration missions. Appendix B presents the full list of screens types and meshes available for screen channel LADs as well as a summary of the data mining of all previously reported bubble point data. Appendices $\mathrm{C}$ and $\mathrm{D}$ present Langmuir isotherms for the data presented in Chapter 4 while Appendix E presents previously reported heated pressurant gas historical data relevant to Chapter 8. Appendix 
F presents the results of the exhaustive literature review into previous solutions for porous channel flow. Appendix G presents a summary of the updated suite of analytical models for cryogenic screen channel LADs.

Journal publications, conference papers, and conference presentations of relevance to this dissertation either published, under review, or in preparation at the time of submission are listed as follows:

\section{Chapter 3:}

Hartwig, J.W., Darr, S.R., McQuillen, J.B., Rame, E., and Chato, D.J. “A Steady State Pressure Drop Model for Screen Channel Liquid Acquisition Devices" $25^{\text {th }}$ Space Cryogenics Workshop, Anchorage, AK, June 23 - 25, 2013.

Hartwig, J.W. and Darr, S.R. "Influential Factors for Liquid Acquisition Device Screen Selection for Cryogenic Propulsion Systems" Applied Thermal Engineering 66, $548-562.2014$.

\section{Chapters 3 and 4:}

Hartwig, J.W. and Mann, J. "Room Temperature Bubble Point Tests on Porous Screens: Implications for Cryogenic Liquid Acquisition Devices” AIChE Annual Meeting, Pittsburgh, PA, October 29 - November 2, 2012.

Hartwig, J.W., Mann, J.A., Kamotani, Y., and Chato, D.J. “Liquid Acquisition Devices for Advanced In-space Cryogenic Propulsion Systems”, Research ShowCASE, April 12, 2013. 
Hartwig, J.W. and Mann, J.A. "Liquid Transport in Microgravity I: A Predictive Bubble Point Pressure Model for Porous LAD Screens” Journal of Porous Media (in press). 2014.

\section{Chapter 4:}

Hartwig, J.W. and Mann, J.A. "Bubble Point Pressures of Binary Mixtures Wetting Screens Against a Vapor: Implications for Low Gravity Cryogenic Liquid Acquisition” AIChE Annual Meeting, San Francisco, CA, November 3 - 8, 2013.

Hartwig, J.W. and Mann, J.A. "Liquid Transport in Microgravity II: Bubble Point Pressures of Binary Methanol/Water Mixtures in Fine-Mesh Screens" AIChE Journal 60, $730-739.2014$.

\section{Chapter 5:}

Hartwig, J.W., McQuillen, J.B., and Chato, D.J. "Performance Gains of Propellant Management Devices for Liquid Hydrogen Depots" AIAA-2013-0368, $51^{\text {st }}$ Annual Meeting of the Aerospace Sciences, Grapevine, TX, January 7 - 10, 2013.

Hartwig, J.W. and McQuillen, J., and Chato, D.J. "Screen Channel LAD Bubble Point Tests in Liquid Hydrogen" International Journal of Hydrogen Energy 39, 853 861. 2014.

Hartwig, J.W., Mann, J.A., Darr, S.R., and Chato, D.J. "Liquid Transport in Microgravity III: Parametric Analysis of the Liquid Hydrogen and Nitrogen Bubble Point Pressure for Cryogenic Liquid Acquisition Devices" Cryogenics (accepted for publication). 2014. 
Hartwig, J.W. and Chato, D.J. "Screen Channel LAD Bubble Point Tests in Liquid Nitrogen" (in prep.).

\section{Chapter 6:}

Jurns, J.M. and Hartwig, J.W. "Liquid Oxygen Liquid Acquisition Device Bubble Point Tests with High Pressure LOX at Elevated Temperature" $24^{\text {th }}$ Space Cryogenic Workshop, Coeur d'Alene, Idaho, June 8 - 10, 2011.

Hartwig, J.W. and McQuillen, J. “Analysis of Screen Channel LAD Bubble Point Tests in Liquid Oxygen at Elevated Temperature" AIAA-2011-3775, 42 ${ }^{\text {nd }}$ AIAA Thermophysics Conference, Honolulu, HI, June 27 - 30, 2011.

Jurns, J.M. and Hartwig, J.W. "Liquid Oxygen Liquid Acquisition Device Bubble Point Tests with High Pressure LOX at Elevated Temperature” Cryogenics, 52, 283 289. 2012.

Hartwig, J.W., McQuillen, J., and Jurns, J.M. "Screen Channel LAD Bubble Point Tests in Liquid Oxygen" Journal of Thermophysics and Heat Transfer (in press). 2014.

\section{Chapter 7:}

Hartwig, J.W. and McQuillen, J. “Analysis of Screen Channel LAD Bubble Point Tests in Liquid Methane at Elevated Temperature" AIAA-2012-759, 50 ${ }^{\text {th }}$ Annual Meeting of the Aerospace Sciences, Nashville, TN, January 9 - 12, 2012.

Hartwig, J.W. and McQuillen, J. "Screen Channel LAD Bubble Point Tests in Liquid Methane" Journal of Thermophysics and Heat Transfer (in press). 2014. 
Savas, A., Hartwig, J.W., and Moder, J.P. "Thermal Analysis of a Cryogenic Liquid Acquisition Device Barrier under Autogenous and Non-condensable Pressurization Schemes” International Journal of Heat and Mass Transfer (in press). 2014.

\section{Chapter 8:}

Hartwig, J.W., McQuillen, J.B., and Chato, D.J. "Warm Pressurant Gas Effects on the Liquid Hydrogen Bubble Point" AIAA-2012-3982, 49 ${ }^{\text {th }}$ Joint Propulsion Conference, San Jose, CA, July 15 - 17, 2013.

Hartwig, J.W., McQuillen, J.B., and Chato, D.J. "Warm Pressurant Gas Effects on the Static Bubble Point Pressure for Cryogenic LADs" Journal of Thermophysics and Heat Transfer (submitted). 2015.

\section{Chapter 9:}

Hartwig, J.W., Chato, D.J., McQuillen, J.B., Vera, J., Kudlac, M.T., and Quinn, F.D. "Liquid Acquisition Device Outflow Tests in Liquid Hydrogen" $25^{\text {th }}$ Space Cryogenics Workshop June 23 - 25, 2013.

Chato, D.J., Hartwig, J.W., Rame, E., and McQuillen, J.B. "Low Gravity Cryogenic Liquid Acquisition for Space Exploration" $29^{\text {th }}$ American Society for Gravitational and Space Research, Lake Buena Vista, FL, November 3 - 8, 2013.

Hartwig, J.W., Chato, D.J., McQuillen, J.B., Vera, J., Kudlac, M.T., and Quinn, F.D. "Liquid Acquisition Device Outflow Tests in Liquid Hydrogen” Cryogenics (in press). 2014. 
Hartwig, J.W., Darr, S.R., McQuillen, J.B., Rame, E., and Chato, D.J. “A Steady State Pressure Drop Model for Screen Channel Liquid Acquisition Devices" Cryogenics (in press). 2014.

\section{Chapter 10:}

Hartwig, J.W., Mann, J.A., and Kamotani, Y. "The Bubble Point Pressure Model for Screen Channel Liquid Acquisition Devices Operating in Cryogenic Propulsion Systems” Research ShowCASE, April 18, 2014.

Hartwig, J.W., McQuillen, J.B., and Chato, D.J. “The Static Bubble Point Pressure Model for Porous LAD Screens Operating in Cryogenic Propulsion Systems" $9^{\text {th }}$ International Conference on Two-Phase Systems for Ground and Space Applications, Baltimore, MD, September 22 - 26, 2014.

Hartwig, J.W. "The Bubble Point Pressure Model for Cryogenic Screen Channel Liquid Acquisition Devices" Physics of Fluids (in prep.).

\section{Chapter 11:}

Hartwig, J.W. and Chato, D.J. “The Reseal Pressure Model for Cryogenic Screen Channel Liquid Acquisition Devices" (in prep.).

\section{Chapter 12:}

Hartwig, J.W. and Darr, S.R. “Analytical Model for Steady Flow through a Channel with One Porous Wall with Variable Suction or Injection" Journal of Fluid Mechanics (in prep.). 


\section{Chapter 13:}

Darr, S.R. and Hartwig, J.W. "Optimal Liquid Acquisition Device Screen Weave for a Liquid Hydrogen Fuel Depot” International Journal of Hydrogen Energy 39, $4356-4366.2014$.

\section{Chapter 14:}

Patel, A. and Hartwig, J.W. "Analytical Model for Cryogenic Propellant Flow in a Porous Screen Channel LAD in Microgravity" 50 $0^{\text {th }}$ Joint Propulsion Conference, Cleveland, OH, July 28 - 30, 2014.

Hartwig, J.W. and Patel, A. "Optimal Propellant Management Device for a Small Scale Liquid Hydrogen Storage Tank in Microgravity" International Journal of Hydrogen Energy (in prep.). 


\section{Chapter 2}

\section{Background and Historical Review}

The purpose of this chapter is to present a comprehensive background and historical review of propellant management devices. The basic flow physics, design concept, and principle of operation are presented for each type of PMD. The three primary capillary driven PMD types of vanes, sponges, and screen channel LADs are then compared and contrasted. A detailed review of previous PMD applications is given for each type, which include space experiments as well as space missions and vehicles. Then the most robust PMD type is down selected as the most flexible and robust option for technology development for NASA's current needs as well as future cryogenic propulsion systems.

\subsection{Propellant Management Device Purpose}

Gravity affects many processes in space, such as the separation of the liquid and vapor phases within a propellant tank. In general, the lowest achievable potential energy state within a tank governs the location of the L/V interface. In the standard gravity field of Earth, fluid density dictates this location because the heavier liquid settles to the bottom and the lighter vapor rises to the top. In the microgravity conditions of space however, surface tension becomes the controlling mechanism for the phase separation because the liquid tends to wet the walls, leaving a gaseous core in the center. To meet 
vapor free transfer requirements for both in-space cryogenic engines and cryogenic fuel depots, any one of a number of PMDs may be required inside the tank.

Figure 2.1 illustrates why LADs are required for successful engine operation. On the ground or during launch, LADs are generally not required because vehicle thrust and high-g levels can maintain phase separation within the propellant tank. In microgravity however, in the absence of settling thrusting maneuvers to favorably position the liquid, there is no way to guarantee vapor free propellant flow out of the tank without using a LAD. After sufficient time, in an unsettled environment, liquid and gas phases will combine such that a two phase mixture may cover the outlet. At a bare minimum, a mixture of gas and liquid sent to the engine will cause combustion instabilities, and at worst, cause complete engine failure.

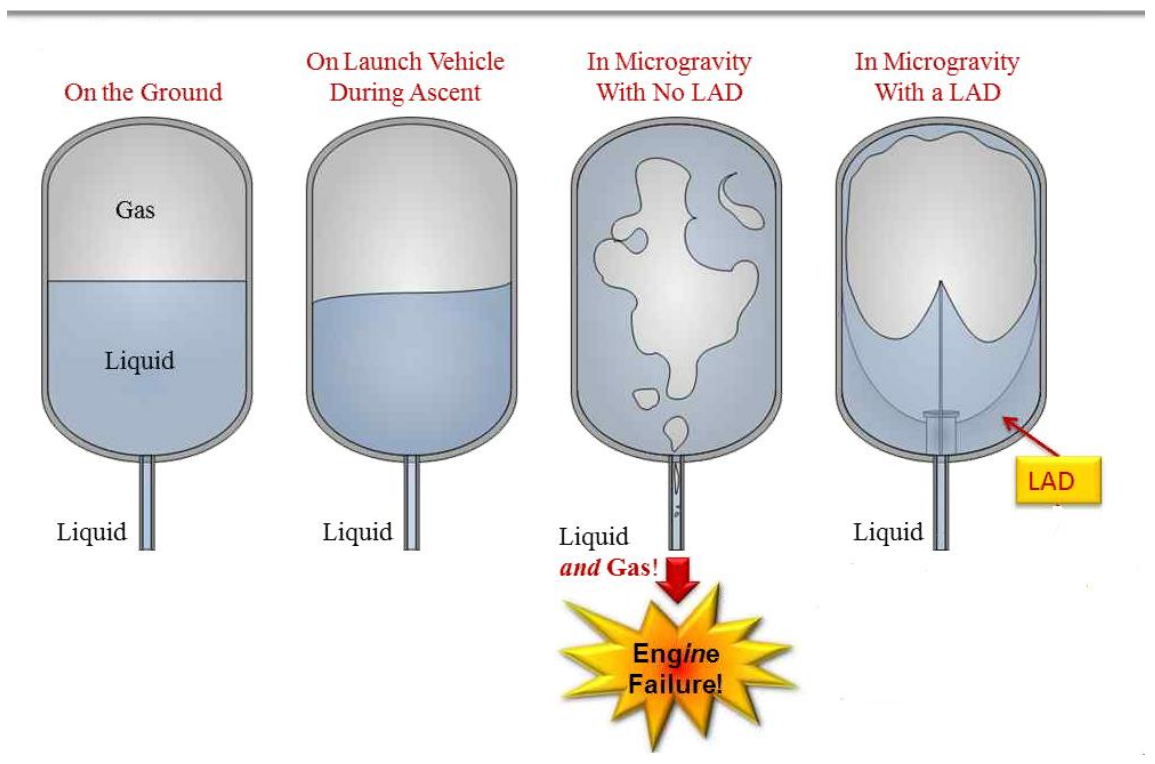

Figure 2.1 - Illustration of Why Liquid Acquisition Devices are Required

The purpose of a PMD is to separate liquid and gas phases within a propellant tank and to transfer vapor free propellant from a storage tank to a transfer line en route to 
one of two customers, an engine or receiver tank (depot application), in any gravitational or thermal environment. The generic system architecture for propellant transfer is shown in Figure 2.2. The process of propellant transfer from a storage tank to the customer is divided among the following four stages:

1) Vapor free liquid extraction from the supply tank

2) Chill-down of the transfer line

3) Chill-down of the receiver system

4) Fill of the receiver system

PMDs represent the first step in the propellant transfer process.

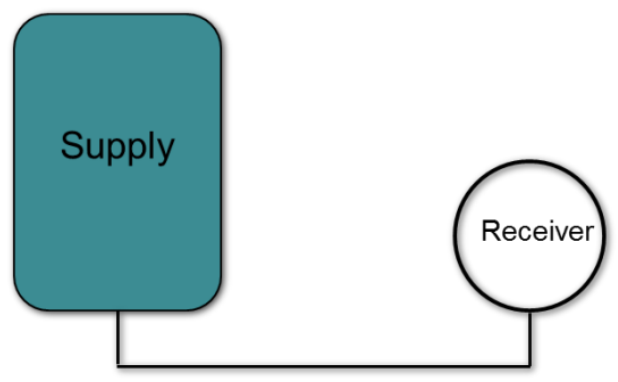

Figure 2.2 - Generic Supply and Receiver System where the Downstream Customer is Either an Engine or Receiver Tank

PMDs were born out of the desire to perform engine restarts in a low-g environment (Radcliffe and Transue 1961 and Behruzi et al. 2007). PMDs must be designed and implemented to ensure that there is always communication between the PMD and liquid anywhere within the tank, and to ensure that the tank outlet is sufficiently covered with liquid during any phase of the mission. In the 1-g field of Earth, 
transfer of liquid is easy because the L/V interface in the tank is always such that the heavier liquid resides at the bottom of the tank and the lighter vapor rises to the top; a simple hole in the bottom of the tank is sufficient. In reduced gravity environments $\left(10^{-2}\right.$ $-10^{-4} \mathrm{~g}$ ), at high liquid levels, settling thrusting maneuvers can be used to favorably position liquid over the tank outlet. At low liquid levels, simple bubble arrestors or sumps can be inserted over the tank outlet to prevent vapor ingestion into the transfer line in order to drain the remaining liquid residuals.

In the low Bond number microgravity environment of space however, where Bond number is defined as:

$B o=\frac{\rho g L_{C}^{2}}{\gamma_{L V}}$

where $\rho$ is the liquid density and $L_{C}$ is the characteristic length of the system, single phase liquid extraction becomes a challenge because surface tension forces generally become the driving force for phase separation and liquid flow. Liquid tends to wrap the outer walls, leaving a gaseous core in the center of the tank. Multiple PMDs may be required to sufficiently cover the outlet with liquid to counteract low g-levels. Full communication PMDs, or devices that maintain communication between liquid, PMD, and tank outlet at all times, are often required in microgravity systems so that propellant can be accessed from anywhere within the tank. When supplying cryogenic liquids to the outlet of the tank, low gravity fluid control and acquisition is further complicated over storable liquid due to the low surface tension and high susceptibility to parasitic heat leak associated with the low NBP of cryogenic propellants. 
PMDs come in numerous styles and designs, each with its own specific purpose. Multiple PMDs are often required to meet the demands of a particular mission, whether using storable or cryogenic propellants. PMDs have been used extensively in chemical storable propulsion systems and can even be implemented in electric propulsion systems (Polzin et al. 2007). PMD performance is determined by three primary characteristics: PMD system mass, demand mass flow rate, and expulsion efficiency $E E$, which is defined as

$$
E E=\frac{V_{\tan k}-V_{\text {residuals }}}{V_{\tan k}}
$$

where $V_{\text {residuals }}$ is the residual liquid propellant left in the tank when the PMD breaks down and admits vapor into the transfer line, and $V_{\tan k}$ is the internal volume of the tank. Therefore $E E$ is a measure of how much of the tank is drained through the LAD before the LAD breaks down. The emphasis of this dissertation is on full communication, flexible, and robust capillary driven PMDs, which are actually the most commonly used systems for flight (DeBrock 1968). The three most popular capillary driven PMDs are vanes, sponges, and screen channel LADs (Rollins et al. 1985, Jaekle 1991, 1993, 1997, and Purohit 1999), but there are many other non-capillary systems which have been used in previous years.

\subsection{Other Types of Propellant Management Devices}

The simplest PMD is simply a hole at the bottom of the tank. If acceleration levels are high enough, or if the propellant tank resides in reduced gravity $\left(10^{-2}-10^{-4} \mathrm{~g}\right)$, there may not be a need for a special PMD. If mission requirements will allow, there are 
numerous non-capillary driven PMD types which can be implemented for control and extraction of single phase liquid propellant. Over the years, many missions have incorporated tanks with positive expulsion devices, which include pistons, diaphragms, and bladders. Positive expulsion devices are used primarily to maintain the interface between pressurant gas and propellant through the presence of a barrier (Chu and Unterberg 1967). Pistons have been used as PMDs to divide the pressurant gas from the propellant, but leakage and low $E E$ led to the desire for better devices.

The bladder was one of the devices developed to replace the piston PMD on the Corporal (Ballinger et al. 1995). The bladder PMD resembles a balloon, where the propellant is located inside the membrane with a narrow opening leading to the tank outlet as shown in Figure 2.3 (Lark 1968). Because the bladder must encompass the entirety of the propellant, it is heavier than the diaphragm and so tank size is again limited. Also, the pressurant gas can potentially cause folding of the membrane, reducing $E E$ unless a support structure is added. However, the bladder maintains a smaller sealing area than the diaphragm, which needs to be welded to the entire circumference of the tank, allowing for easier installation and removal. Bladders were also used in the Mercury and Gemini missions (Biron 1990).

A diaphragm differs from the bladder in that it is composed of a flexible membrane to separate pressurant gas and liquid propellant (Coulbert et al. 1973 and Kreis et al. 1996). Figure 2.4 shows a diaphragm which uses an elastomeric barrier for phase separation (Ballinger et al. 1995). Like bladders, diaphragms are also advantageous in systems that require effective slosh control and elimination of reactions between pressurant gas and propellant. Because bladders and diaphragms span across the entire 
tank, the mass of the diaphragm may rival the mass of the tank walls, making these PMDs impractical in large scale applications. In addition, elastomeric material is not well suited for long life missions (De Brock et al. 1971).

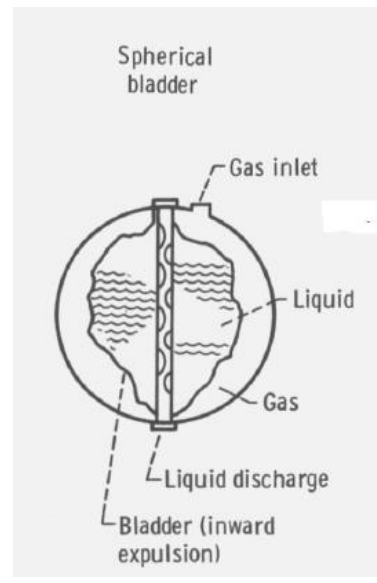

Figure 2.3 - Schematic of a Spherical Bladder Lying Just Within the Tank Shell
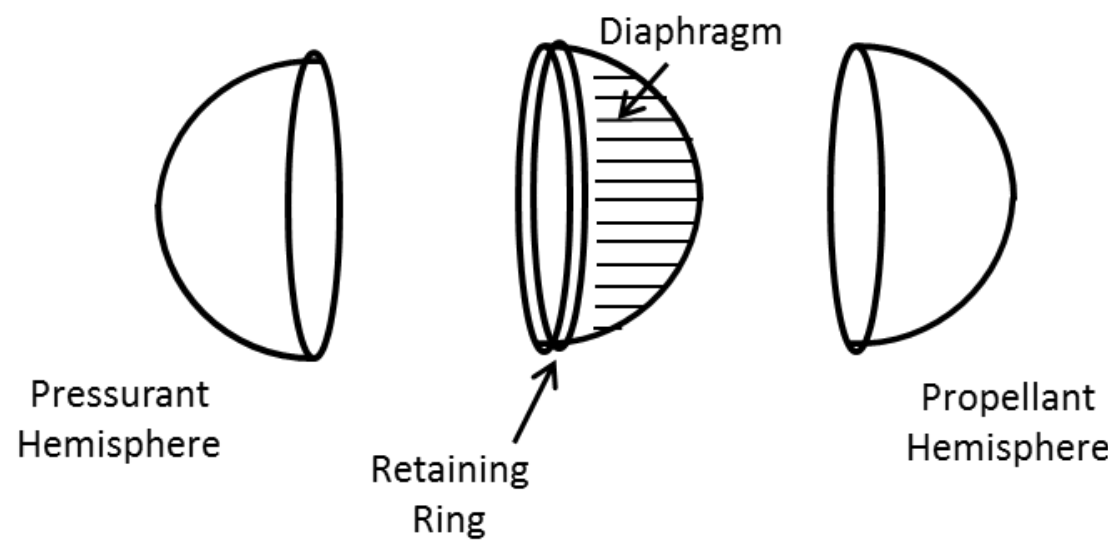

Hemisphere

Figure 2.4 - Schematic of a Diaphragm Assembly. The molded diaphragm is welded between the two hemispheres.

Newer missions tend to employ surface tension PMDs that can be built to be lighter and more reliable than positive expulsion devices. However, the diaphragm remains effective at maintaining gas free flow to the outlet and eliminating propellant 
slosh. For example, the Space Shuttle used three diaphragm tanks (Ballinger et al. 1995) for its Auxiliary Power Unit (APU), Cassini used a diaphragm tank for its RCS (Enright and Wong 1994), and recent computational analysis performed on diaphragms show they are good at dampening slosh (Lenahen et al. 2013).

Traps, troughs, baffles, and vortexes are considered capillary PMDs that are used as simple control devices, and not full communication devices. Traps use porous elements such as screens to trap gas outside of the structure while allowing liquid to flow through the trap and out of the tank (Giacalone 1993 and Jaekle 1995). Porous traps also allow the PMD to hold propellant at high accelerations. Traps are generally reliable and can be constructed out of lightweight materials. However, since most traps cannot passively reacquire propellant in low gravity environments, they are primarily used in systems which experience one-time maneuvers, such as launches or station keeping maneuvers. Traps have been given consideration inside the Arianne-5 upper stage tanks for restart (Behruzi and Netter 2003 and Behruzi and Michaelis 2006). A custom built trap PMD was used in the famous Apollo service module for liquid retention during adverse accelerations such as those caused by the RCS (Hines et al. 1967 and DeBrock et al. 1971). The capillary driven trap allowed the tank to hold liquid over the outlet while simultaneously preventing large gas bubbles from entering the engine feed line.

Troughs are highly reliable control PMDs that use hydrostatic forces to maintain control of liquid, although they can be designed to use surface tension to refill (Jaekle 1995 and Tam et al. 2001). An example of a trough is depicted in Figure 2.5. Troughs differ from traps in that they are passively refillable. They are effective at providing large quantities of propellant for high acceleration maneuvers beyond the capabilities of 
sponge PMDs. However, since they encompass the liquid that they hold and must be constructed of solid metal, they require more space and metal mass than sponges, and are thus less efficient at lower accelerations.

Baffles are control PMDs primarily used to reduce sloshing (Tam et al. 2002a). Baffles can include a wide variety of shapes, but all function to limit propellant movement. Shown in Figure 2.6 is an example of a baffle. The two baffles welded into the propellant tank resembled flattened rings that span the diameter of the tank with a hole in the center.

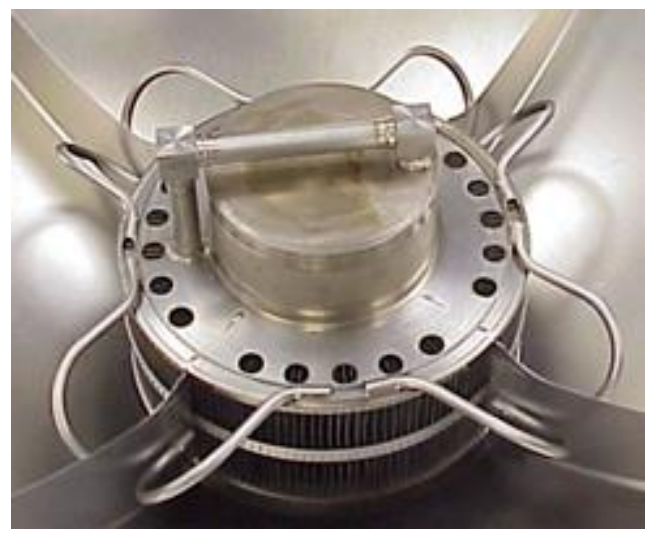

Figure 2.5 - Example of a Trough

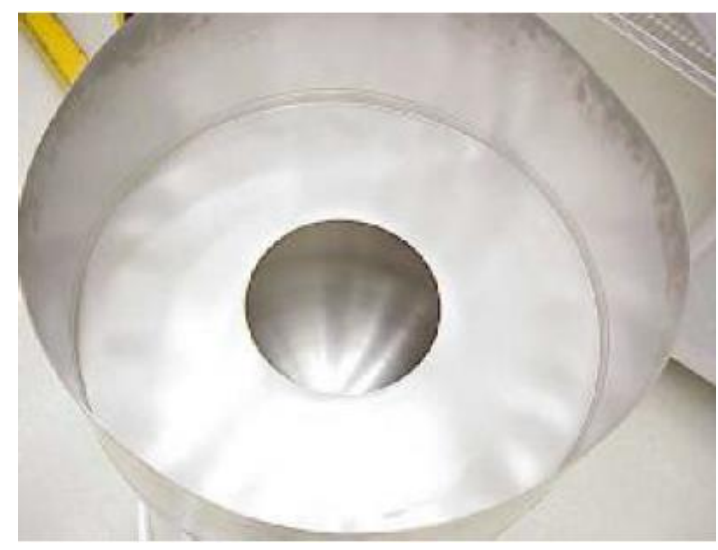

Figure 2.6 - A Baffle Welded to the Interior of a Propellant Tank 
Meanwhile a vortex suppressor is meant to reduce vortices at the tank outlet that appear during high mass flows. This allows the system to operate well under higher flow rates. The development of a vortex suppressor was needed for the Near Earth Asteroid Rendezvous (NEAR) oxidizer tank as shown in Figure 2.7 (Tam et al. 1995).

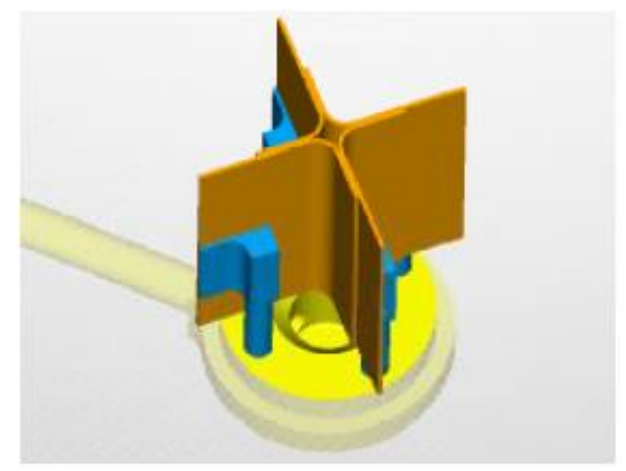

Figure 2.7 - Three Dimensional Image of a Vortex Suppressor. The yellow represents the tank outlet and outflow tube.

\subsection{Vanes}

The three primary total communication capillary driven PMDs include vanes, sponges, and screen channel LADs. Of the three, the simplest and most reliable PMD is the vane. Relative to screen channel LADs, vanes are open acquisition PMDs which allow for a much simpler design at the cost of not being able to sustain or supply higher flow rates. Vanes have rich flight heritage in storable propulsion systems but none in cryogenic systems.

\subsubsection{Design Concept, Basic Flow Physics, and Principle of Operation}

As shown in Figure 2.8, vanes are generally designed as thin metal plates that are mounted perpendicular to the tank walls so that distinct corners are formed between PMD 
and the wall (Jaekle 1991 and Tegart 1997). The metal plates can be tapered from "short" to "tall" from the center of the tank to the tank outlet as shown. This tapering allows the vane to utilize a weak capillary pumping force to move liquid from the center or aft end to the tank outlet in the absence of gravity. The size and number of vanes is determined by the flow rate requirements and $E E$.

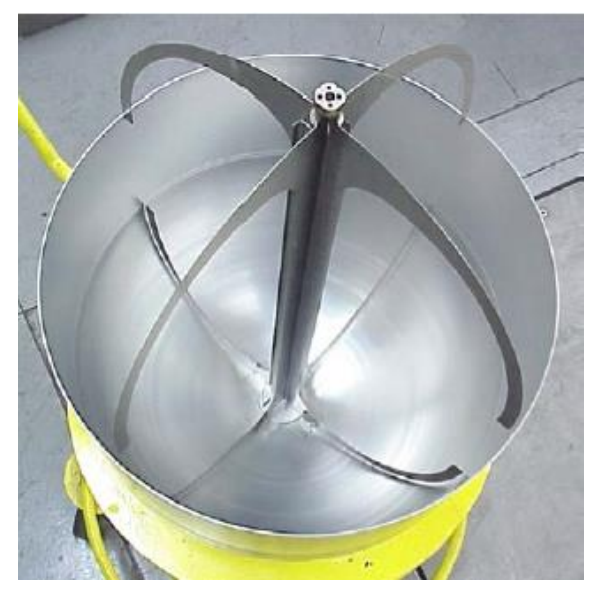

Figure 2.8 - Total Communication Vane with Center Post

Vanes are sized and numbered so that there is always communication between the propellant pool and vane. As shown in Figure 2.8, a center post can be used as an additional flow path for liquid to creep towards the exit. Vanes can be constructed out of the same metal as the tank wall, allowing for a very simple and lightweight design. For added robustness, a double vane or ribbon vane can be used to increase vane flow area, and thus total flow rate out of the tank.

Detailed steady state analysis of vanes is reserved for Chapter 14. The basic flow physics and principle of operation for vanes are as follows (Tam et al. 2000 and Griffin et al. 2003): In flight systems, vanes closely follow the contours of the tank walls. In low gravity, liquid naturally sticks to the vanes and walls in the absence of accelerations. The 
liquid propellant wets the plate surfaces, and surface tension causes the liquid to form a rounded fillet in the corners, thus enabling liquid to be transported along the fillet toward the outlet. Capillary forces then push liquid from one end of the vane to the other near the poles of the tank. Liquid from the pole opposite the tank outlet is carried across the tank along a center post (not shown) using similar weak capillary forces. These flow paths are depicted with blue arrows in Figure 2.9.

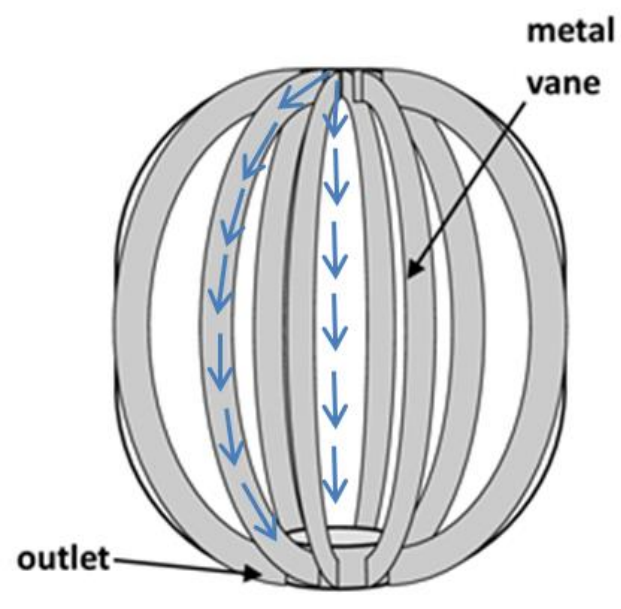

Figure 2.9 - Schematic of Vane Flow Patterns in Low Gravity

Because vanes are open PMDs, they cannot block gas ingestion into the outlet. As propellant is removed via the tank outlet, the weak capillary pumping force can only replace liquid over the tank outlet. This renders stand-alone vanes useless except for liquid resupply in very low acceleration environments with high surface tension propellants, since they are incapable of controlling or holding liquid over the tank outlet. To circumvent this problem, vanes are often used in conjunction with small control devices mounted over the tank outlet to provide a very robust PMD. There is a critical 
flow rate beyond which vanes cannot supply liquid to the outlet in a continuous outflow environment; this is quantified for a small scale $\mathrm{LH}_{2}$ tank in Chapter 14.

\subsubsection{Advantages and Disadvantages}

Perhaps the biggest advantage to choosing vanes over sponges or screen channel LADs is simplicity. Vanes are often constructed out of very thin sheet metal and are generally very easy to build, shape, and install into propellant tanks. The simplest design solution which meets experimental requirements is the best solution, so vanes are often the first choice. Second, vanes are also much lighter than sponges and gallery arms. For example, thin Ti sheet vanes can be installed into most storable propulsion systems that employ Ti tanks. Third, as a result of the simplicity in design, vanes are cheaper to manufacture over sponges and screen channel LADs. Finally, vanes are highly reliable. Because of the open flow path, vanes can generally achieve very high $E E$ before gas ingestion into the outlet.

The two disadvantages of a vane PMD are that it cannot supply nor sustain medium to large demand flow rates and it cannot sustain liquid only flow under medium to high adverse accelerations due to the weak capillary pumping force relative to sponges and galleries. This limits vanes to be implemented in systems that experience low glevels and require very low demand flow rates. For future cryogenic engines and cryogenic depot applications, it may be difficult to scale up the vane to meet the projected higher flow rate demands. 


\subsubsection{Storable Propellant Historical Examples}

Vanes have a rich flight heritage in storable propulsion systems in flight experiments as well as in numerous vehicles and missions. Vanes are particularly beneficial in satellite systems requiring periodic station keeping maneuvers because satellites only require occasional access to propellant over the course of a long duration mission. The lightweight vane is also ideal to reduce the size and system of the satellite. General examples of vane designs are available in the literature (Debrenceni et al. 1995b and 1998 and Netter et al. 1999).

\subsubsection{Space Experiments}

Historically, there are two space experiments which employed a vane type PMD. The Fluid Acquisition Resupply Experiment-II (FARE-II) tested a vane type LAD using a simulant fluid onboard of the Shuttle mission STS-57 as its primary PMD (Dominick and Tegart 1994 and Dominick et al. 2011). The secondary PMD resembled that of a sponge. The purpose of the experiment was to establish vane performance limits in terms of maximum achievable expulsion efficiencies under adverse acceleration levels. A snapshot of the FARE-II experiment is shown in Figure 2.10 (Dominick et al. 2011). This was a very successful mission which generated useful low-g data.

A vane type PMD was also used for the Vented Tank Resupply Experiment (VTRE) onboard the Shuttle mission STS-77 (Chato and Martin 2006). Twelve outer and twelve inner vanes were mounted inside a small scale see-through tank to conduct outflow tests using Refrigerant-113. A vane type PMD was also planned to be used in the Skylab mission (Tegart 1979). 


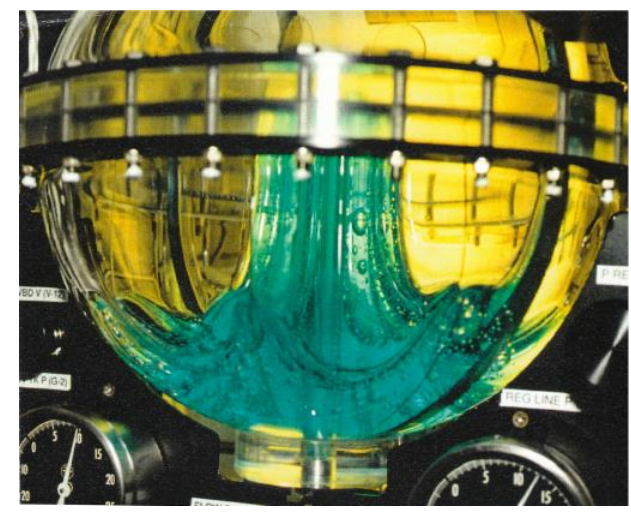

Figure 2.10 - Fluid Acquisition and Resupply Experiment-II Vane and Sponge with 10\% Liquid Remaining in the Tank

\subsubsection{Vehicles and Missions}

Many different variations of vanes have been used in numerous storable propulsion flight vehicles and missions. In 1975, the company Radio Corporation of America (RCA) launched several communications satellites (SATCOM) into orbit (Balzer et al. 1976). The mission objective was the provision of commercial satellite coverage to all fifty of the United States. A tank with a vane PMD was used to provide the propellant necessary for orbital insertion, regular station keeping, and to access propellant during coasting in low-g. Four vanes sprouted from the tank outlet and tapered all the way up to the other hemisphere of the tank, allowing the vanes to contact both tank ends.

The HS 601 Block I satellite was developed in 1987 as a commercial satellite (Tam et al. 1998). The tank assembly was comprised of several PMDs, but vanes were the primary system used to resupply propellant to a sponge and trap during low-g coasts (Tam et al. 1996). This satellite also only required small station keeping maneuvers, which were easily achievable with the vane. The HS 601 Block II satellite design 
completed in 1997 used a simple four vane arm PMD in its main propellant tank (Tam et al. 1998). This design was similar to its predecessor, except the longer cylindrical tank required longer vane arms and a slightly more complex trap assembly.

Vanes were used in the Orbital Communication (ORBCOMM) satellites (Jaekle 2013), which were responsible for handling low data transfer, limiting the communications to non-time sensitive information. These satellites allow two-way data communication, position determination, emergency alerting, and alphanumeric messaging (Deckett 1994). The design of the satellite was a simple disk with deployable solar panels and antenna (Stoltz et al. 1996). Vanes were used primarily for low thrust station keeping.

Many geosynchronous satellites also employ vanes. For example, a tank and vane PMD was developed in early 2000 for a commercial satellite (Tam et al. 2000). This particular system had hemispherical vanes which were not connected along the walls, but connected with a center post. Another example of a geosynchronous satellite was the Star-2 system which used a bi-propellant system with a single fuel tank and two oxidizer tanks (Rattenni 2001). Vanes were chosen because of the desire to achieve very high $E E$ and maintain very low residuals. The Boeing 601 was yet another example of a geosynchronous satellite employing a simple vane PMD (Narita and Yendler 2007).

Vanes were also used to supply propellant for the Near Field InfraRed Experiment (NFIRE) for station keeping (Jaekle 2013). The satellite was launched in 2007 (Ballweg and Wallrapp 2012) which carried two payloads: a Track Sensor Payload (NFIRE 2013) to detect and track missiles, and a Laser Communication Terminal (LCT) (Smutny and 
Lange 2006) to test laser communication with the German made TerraSar-X satellite. Vanes have also been used in the Iridium constellation (Garrison et al. 1997), the INSAT satellites (Kale et al. 1972, Menon 1972, and Netter and Prasad 1988), and the Arabsat television satellites (Rollins et al. 1984).

\subsection{Sponges}

The second total communication capillary driven PMD is the sponge. A sponge is defined as an open structure PMD that has the ability to maintain and refill propellant at the tank outlet, typically mounted directly over the outlet (Jaekle 1993). Of the three, sponges by far have been used in the widest range of storable propulsion liquid acquisition systems. Relative to vanes, the sponge is heavier and slightly more expensive; relative to screen channel LADs it is a much simpler design. Like vanes, sponges have no flight heritage in cryogenic propulsion systems.

\subsubsection{Design Concept, Basic Flow Physics, and Principle of Operation}

Similar to vanes, a sponge is composed of an array of fins or plates made from ultra-thin, lightweight metal. The distinguishing factor between vanes and sponges is that sponge fins or plates emanate from the center of the tank over the tank outlet while vanes are mounted alongside the tank wall. By this distinction, many of the vanes reported in the literature are actually sponges. Sponges also differ from vanes in that they can be designed to control the location of both the liquid and gaseous phases within the propellant tank; a wall mounted vane with center post can be used to position the both liquid and ullage but not nearly as efficiently as the sponge. Because the sponge is centrally located, and because it forces liquid to be centrally located, sponges are 
favorable for applications where tight center of mass control of the spacecraft is desired. Sponges are open PMDs and thus do not use porous elements or enclosures like traps or screen channel LADs.

Sponges can be designed in various ways, and generally consist of perforated, angled plates in contact with the tank outlet. Sponges are also designed to favorably position the ullage bubble; the plates can even be angled is such a way to drive bubbles away from the outlet and towards the aft end of the tank. Figure 2.11 shows a radial sponge where liquid is "absorbed" or drawn into the gaps between plates and then driven down toward the outlet by capillary forces (Tam et al. 2008a). Many of the basic flow principles that apply to vanes from Section 2.3.1 also apply to sponges.

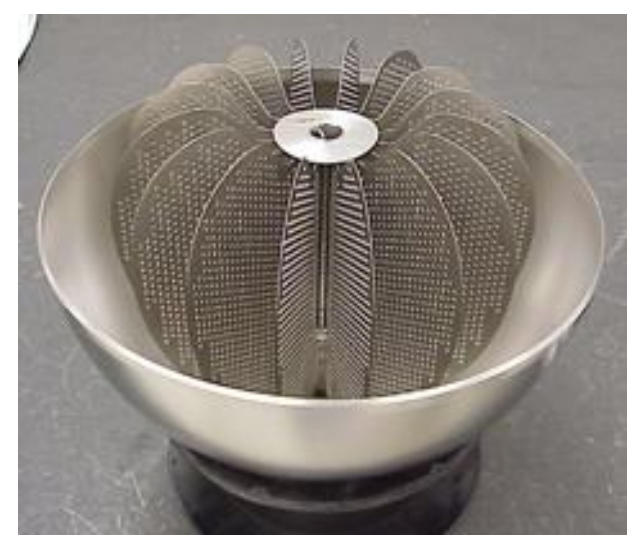

Figure 2.11 - Small Scale Total Communication Sponge

The size and number of plates is determined by the desired flow rate, $E E$, and whether or not access to ullage is desired. Plates are often perforated to reduce mass of the PMD, but this can also lead to a less efficient device. Depending on the size and number of holes, propellant acquisition can be greatly reduced and vapor ingestion can 
thus become an issue. Therefore, sponge mass is often traded with performance to determine the optimal design for a particular mission.

Sponges are most often employed for resupply for engine ignition, engine restart, or short duration maneuvers requiring a small quantity of propellant. For all of these applications, the sponge is sized to ensure there is sufficient propellant covering the outlet to carry out the restart or burn; afterwards, vehicle acceleration is sufficient to maintain liquid over the outlet. Sponges are also often used as control devices even though they are open PMDs. Sponges can easily be used as refill devices to maintain position of the liquid during minor slosh events or adverse accelerations in between engine burns to hold propellant for the next burn.

\subsubsection{Advantages and Disadvantages}

The primary advantage for choosing sponges over vanes is robustness. The sponge can handle the same low flow rates as vanes, but can also be used to control both ullage and liquid within the propellant tank. Second, sponges can be used to control the location of liquid under slightly higher adverse accelerations relative to the vane by increasing the number of sponge plates to decrease gap thickness. Relative to screen channel gallery arms, sponges are lighter weight, easier to fabricate, and more reliable. Higher reliability is achieved because of the simpler open PMD design. Sponges can be constructed from lightweight $\mathrm{Al}$ or Ti sheet metal, making them inherently less expensive.

The disadvantage to using a sponge over a vane is higher system mass. For the same desired $E E$, vanes are always the lighter design solution. The first and biggest 
disadvantage to using a sponge over a gallery arm is lower performance; sponges cannot supply medium to high flow rates and cannot control liquid position under medium to high adverse acceleration levels under either steady flow or restart conditions. Second, sponges simply do not scale with the projected size of larger propellant tanks because the size and mass of the sponge PMD rivals the size and mass of the propellant tank walls. Third, neither sponge nor vane performance is verifiable in ground tests prior to flight, making PMD design for both completely dependent on analysis.

\subsubsection{Storable Propellant Historical Examples}

Sponges have quite the rich flight heritage in storable propulsion systems in flight experiments as well as in numerous vehicles and missions. Sponges have particular success in missions that require refill, or for higher frequency station keeping maneuvers. General examples of sponge designs are available in the literature (Spencer et al. 1991, Tam et al. 2002b, and Tam et al. 2008a, b, and c).

\subsubsection{Space Experiments}

Sponges were employed as secondary PMDs on both the FARE-II and VTRE Shuttle experiments. Figure 2.12 shows the location of the sponge in the center of the VTRE tank. The sponge completed the mission objective of venting the tank in microgravity without losing precious liquid (Chato and Martin 2006).

In addition, sponges were also the PMD of choice for the recent Orbital Express mission in 2007 (Tam et al. 2008b). Orbital Express was a demonstration mission to test resupply of satellites with propellant in microgravity (Dipprey and Rotenberger 2003). The sponge consisted of 16 Ti plates that radiated from a central pickup assembly. 

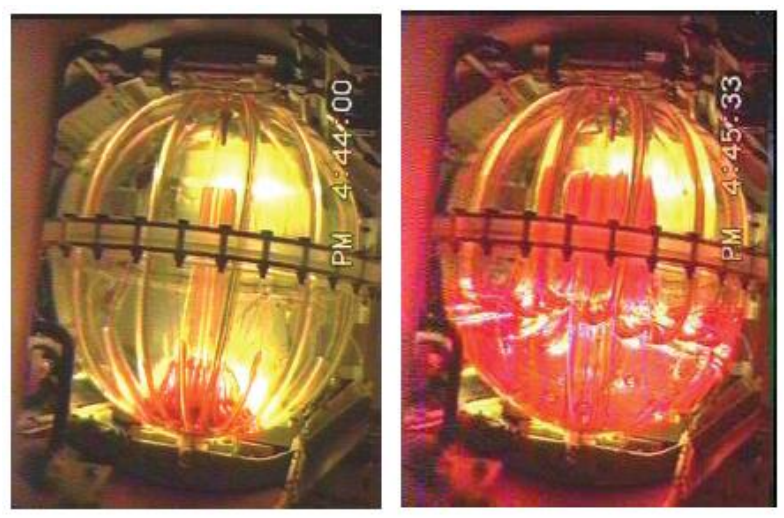

Figure 2.12 - Sponge Type Vane inside the Vented Tank Resupply Experiment

\subsubsection{Vehicles and Missions}

Sponges were the first ever PMD to obtain flight heritage in storable propellants. The Agena Upper Stage Rocket, first launched in 1959 (DiFrancesco and Booraday 1989), used a simple sponge composed of a hemispherical array of metal fins that fanned above a screened trap (De Brock et al. 1971, and DeBrock and Grove 1974 and 1975). The sponge also had a venting tube to allow any trapped vapor to be vented towards the aft end of the tank while liquid was moved toward the tank outlet. Agena flew on 361 successful launches, making it one of the most popular upper stage engines.

Sponges were used in an ion propulsion engine using liquid cesium propellant for an auxiliary station keeping thruster (De Brock et al. 1971). The propellant feed system required a surface tension PMD to transport the liquid from the reservoir to a vaporizing surface. A small storage tank incorporating a 120 fin compact sponge in the reservoir was used to acquire liquid, and then a porous rod transferred the cesium to the vaporizing surface. 
Sponges were also the first surface tension PMD to be incorporated in an interplanetary mission (Dowdy and De Brock 1973, Vote and Schatz 1973, Stultz 1977, Dowdy et al. 1977, and Morrisey 1992). Launched in 1975, Viking-1 and Viking-2 were a set of robotic orbiters and landers sent to explore the surface of Mars (Schmit et al. 1977). Because the Viking orbiters required controlled orbits around Mars, a sponge was chosen to ensure sufficient liquid to perform station keeping and coasting. It was also chosen to maintain a stable center of mass as the spacecraft orbited around the planet (Dowdy et al. 1977). As shown in Figure 2.13, this particular sponge was very large and tall so that liquid was always positioned near the center of the propellant tank (Dominick and Tegart 1981). Both orbiters outlived the expected mission lifespan of 510 days; both orbiters exceeded 1000 days, with Viking-1 lasting 1700 days (Schmit et al. 1980 and 1981).

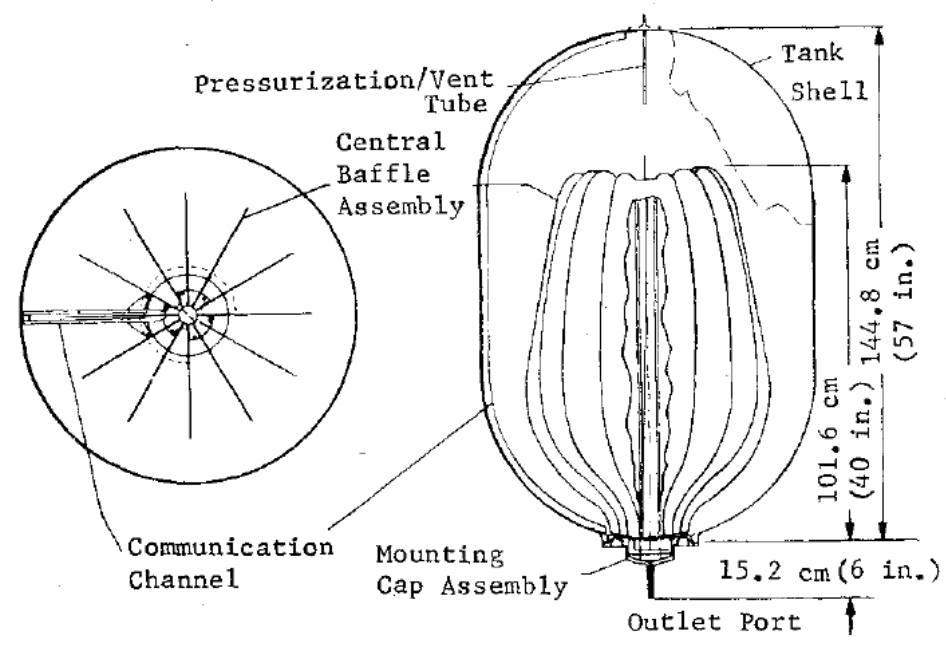

Figure 2.13 - Mars Viking Propellant Management Device

The British Aerospace EUROSTAR system featured a rather unique sponge PMD as shown in Figure 2.14 (Rollins et al. 1992 and Ducret et al. 1996). The system of 
communication satellites designed by Lockheed Martin employed a simple vane, sponge, baffle, and trap, with the sponge being the primary PMD. The vanes were used to refill the sponge during low-coast times until propellant was needed for another maneuver. The interesting feature of the EUROSTAR tank was that the sponge was placed off center of the vehicle axis, facing radially outward away from the spin axis (Rollins et al. 1986). During in-flight vehicle spin, the trap inlets were completely submerged in propellant, allowing for lower residual propellant delivery.

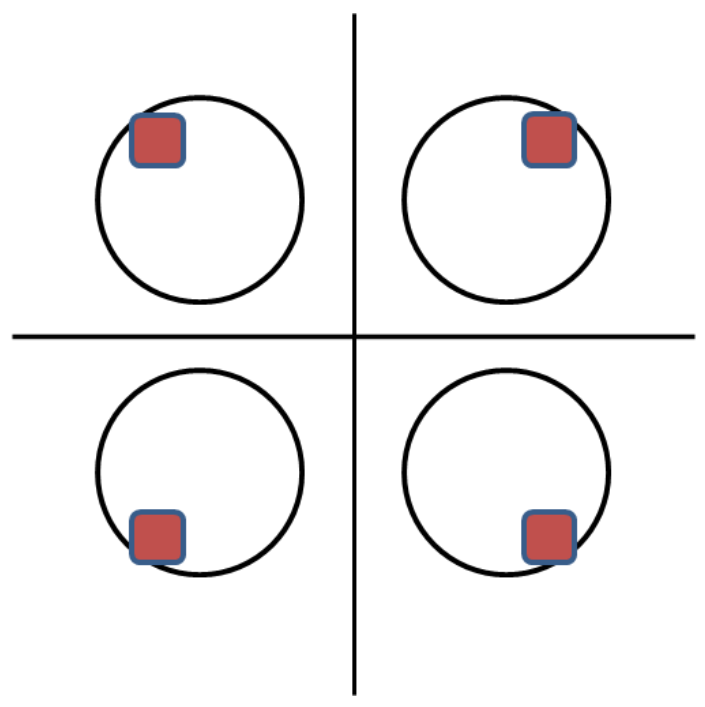

Figure 2.14 - Schematic of the EUROSTAR Propellant Management Device and Tank Array Where Each Sponge is Positioned Away from the Spin Axis. PMD is shown in red.

The Mars Global Surveyor (MGS) was launched in 1996 to continue the mission of the failed Mars Observer (Dominick 1999). Compared to its predecessor, MGS was smaller, lighter, and cheaper. Two identical propellant tanks contained PMD structures of a large sponge and an anti-slosh baffle. In order to control propellant slosh during spin, a ring baffle was installed around the inner circumference of the tank, at the midpoint. The 8 paneled sponge provided control of propellant for center of gravity purposes, and to 
keep propellant near the tank outlet, even under unfavorable conditions such as attitude control.

Sponges were the PMD of choice for the prestigious and successful Cassini Huygens mission to Saturn, which launched in 1997. The original purpose of Cassini was to analyze the rings of Saturn and probe the surface of the moon Titan (Wong and Breckenridge 1995, Lee and Hanover 2005, Mitchell 2005, Sarani 2005, Vandermey and Paczkowski 2006, Standley 2006, Burk and Bates 2008, Buffington et al. 2008, and Pilinski and Lee 2009). It has since provided surface and atmospheric data of numerous other bodies within the Saturn system. The main propellant tanks used large, 8 paneled sponge PMDs (Enright and Wong 1994). The main purpose of the PMD was to maintain the position of the propellant and ullage while in low-g environments as well as for basic thrust control (Chiang et al. 1996).

Sponges were employed in the 1999 Chandra X-ray telescope, where access to both liquid and gas was required. Chandra was launched into LEO and orbited Earth between $10,000 \mathrm{~km}$ and 140,000 $\mathrm{km}$ above the surface to provide unobstructed, deep space sight into the depths of the universe (Weisskopf et al. 2002 and Hefner and Davidson 2004). Although the Chandra PMD contained a center post, baffles, and trap along with the 8 paneled sponge, the primary PMD was the sponge, because mission requirements dictated the need to control both phases (Debreceni et al. 1997). Half of the triangular panels were used to dislodge trapped bubbles, and the other half extended outward into the baffles to reacquire liquid. 
Launched in 2010, the Solar Dynamic Observatory (SDO) was an Explorer-class mission which achieved geosynchronous orbit in order to observe the Sun (Mason and Starin 2011). SDO required a large amount of propellant, close to half of the overall mass of the vehicle (Willis 2012). The PMD used on this satellite was a sponge because maintaining a propellant center of mass and reducing liquid slosh were the two main objectives for PMD design.

Sponges were also used in the recent Messenger mission to Mercury in 2011 (Wiley and Dommer 2003 and Wilson et al. 2012 and 2013). A sponge will also be employed for the recently conceived James Webb Space Telescope (JWST), a collaborative effort by NASA, the European Science Agency (ESA), and Canadian Science Agency (CSA). The purpose of JWST mission is to study the evolution of galaxies and the birth of stars and planets from the Earth-Sun LaGrange-2 point (Hunter et al. 2004, Gardner et al. 2006).

In addition to basic science missions, sponges have rich heritage in geosynchronous satellites. A sponge PMD originally built for the oxidizer tank of a 1988 Mars exploration vehicle was reused for military satellites (Tam et al. 2008a). The Space Systems Loral 1300 bus, which employed simple sponges, was modified for use in the Intelsat-V, Geostationary Operational Environmental Satellites (GOES), and DIRECTV satellites (Hollingsworth et al. 2002, Kim and Wilson 2006, Kim et al. 2007) to manage the fuel in the bipropellant tanks (Jaekle 2013). The Boeing 601HP of 1995(Boeing 601 Fleet 2013) and Boeing 702HP of 2009 (Boeing 702HP Fleet 2013) were satellites designed to carry implements for DIRECTV (Narita and Yendler 2007). The later Boeing 702MP spacecraft used a hybrid of bipropellant and electric propulsion systems. The 
chemical propulsion system was used for boosting (Apfel 2006) while the Xenon fueled electric system was used to achieve geosynchronous orbit and maintain station keeping (Goebel et al. 2002). The chemical stages employed a sponge PMD.

Sponges were used in countless military applications as well. For example, in 2007, The Defense Advanced Research Projects Agency (DARPA) designed the Microsatellite Technology Experiment (MiTEx) as a test to demonstrate upper stage capabilities (Osborn et al. 2007 and Benton et al. 2007). The goal was to deliver two small satellites into geostationary orbit using an upper stage vehicle. For the MiTEx upper stage, a small sponge, a set of baffles, and a trap were used in the propellant tank. Two baffles, an axial baffle above the sponge, and a radial baffle around it, were installed to control propellant motion around the sponge. The sponge was small, with many panels leading to a center post as shown in Figure 2.15. Many of the other designs of military PMDs are classified and thus cannot be discussed in this work.

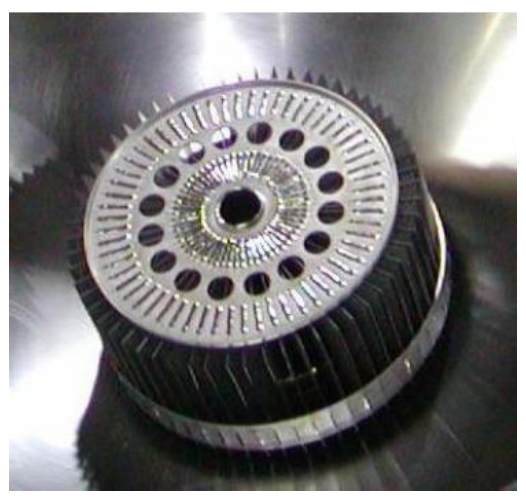

Figure 2.15 - Sponge used in the Micro-satellite Technology Experiment 


\subsection{Screen Channel Liquid Acquisition Devices}

The third total communication capillary driven PMD is the screen channel liquid acquisition device or gallery arm. A screen channel LAD is defined as a closed channel with three solid walls and one porous wall. Screen channel LADs use the same basic capillary pumping force as vanes and sponges, but offer a much more robust solution to liquid acquisition over a wider range of thermal and gravitational conditions. The primary difference between screen channels and vanes and sponges is that the channel creates an internal and closed flow path for liquid to flow from the bulk propellant in the tank to the outlet of the tank. The presence of the screen allows for relatively higher flow rates under more adverse accelerations and promotes higher resistance to gas ingestion, at the cost of a more complex and expensive design. Screen channel LADs have flight heritage in storable propulsion systems, and are the only PMD type to ever be used in a flight cryogenic system.

\subsubsection{Design Concept, Basic Flow Physics, and Principle of Operation}

For flight missions, screen channel LAD design is classified into two categories (Burge et al. 1972, Burge and Blackmon 1973a and 1973b, and Burge et al. 1973), namely start baskets and total communication devices. Start baskets, sumps, traps, start tanks, and pleated tubes (Boraas and LaBruna 1976) are considered small LADs that confine sufficient liquid over the tank outlet to start engines until relatively large vehicle accelerations can adequately settle the liquid for the large flow rates required for engine operation. Shown in Figure 2.16, start baskets are simply sized to ensure liquid covers the outlet, and are designed as the last line of defense against gas ingestion as a bubble 
arrestor. They allow liquid to flow across the screen but also act as a barrier to vapor ingestion if gas comes in contact with the screen, essentially trapping liquid inside the basket and preventing gas from entering. Start baskets are much simpler to design than full communication devices and are used in systems that experience large acceleration changes and high demand flow rates over short time scales. The particular sump shown in Figure 2.16 can also be used to feed the mixing pump located on top of the basket to recirculate liquid to destratify the tank.

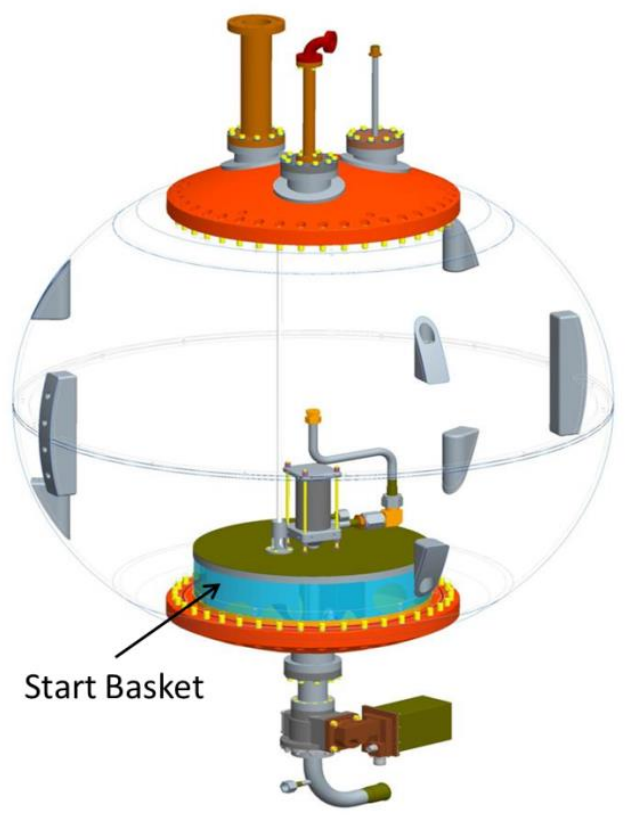

Figure 2.16 - Example of a Screen Channel Start Basket/Sump

Meanwhile, total communication devices are much more complex designs than start baskets, because they are required to ensure communication between propellant and outlet during all phases of a mission. As shown in Figure 2.17, total communication screen channel LADs, or gallery arms, run the full length of the propellant tank. These LADs are designed and manufactured in a variety of styles, sizes, and geometries. 
Typically they are rectangular shaped channels. Total communication devices, such as channels, distributors, and tank liners, are used in systems that experience small acceleration changes and demand lower flow rates over longer time scales.

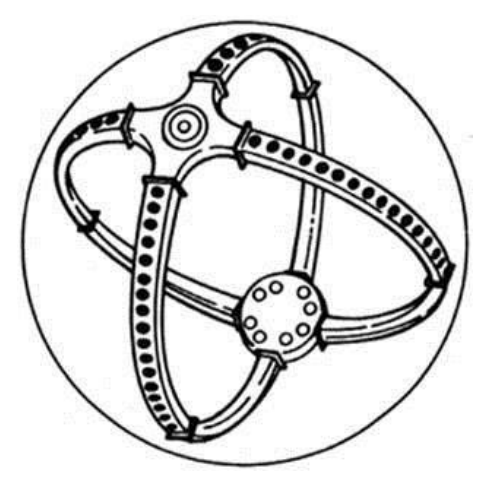

Figure 2.17 - Example of a Total Communication Screen Channel Liquid Acquisition Device

The basic flow physics and principle of operation for total communication screen channel LADs is as follows: In flight-like systems, these LADs tend to closely follow the contour of the propellant tank wall and can have different cross section geometries (typically a triangular or rectangular shape). The channel side that faces the wall has openings covered with a tightly woven fine mesh screen, which produces very small pores $(10-100 \mu \mathrm{m})$. The other three sides of the channel are solid metal. Because the propellant naturally tends toward the tank walls in low gravity environments, the screen side usually faces the wall. During either quiescent or transient flow environments, the screen serves three purposes:

1. To maintain communication between tank outlet and propellant during all phases of the mission. When liquid approaches the porous screen, the screen admits liquid into the channel. 
2. To separate and control phases. When pressurant gas or vapor approaches the screen, liquid surface tension forces within the screen pores block vapor admittance.

3. To rewet portions of the screen that dry out due to exposure to warm pressurant gas; the screen can wick liquid along the screen.

The channels all converge to a common location at the tank outlet in order to ensure that there is communication between propellant and tank outlet during the mission. As liquid is withdrawn from the tank and vapor approaches the screen, surface tension forces block vapor entrance into the channel, but allow the liquid to flow freely. Screen channel LADs succeed in preventing gas ingestion so long as the pressure differential across the screen does not exceed the bubble point pressure (defined in Section 3.2).

\subsubsection{Mesh and Metal Type}

The choice of screen for a particular mission is dictated by the mission requirements, which include gravitational and thermal environments, as well as desired demand flow rate. LAD screens are classified by the geometry, size, number of pores, and manufacturing style, which is compactly expressed as the screen weave. The screen weave refers to the number of wires per inch in each direction and the weave pattern used during manufacturing. Figure 2.18 displays a Scanning Electron Microscopy (SEM) image of a commonly used 200x1400 Dutch Twill screen mesh where there are 200 larger warp wires and 1400 smaller shute wires per square inch of screen material. The warp wires are not visible in Figure 2.18. 


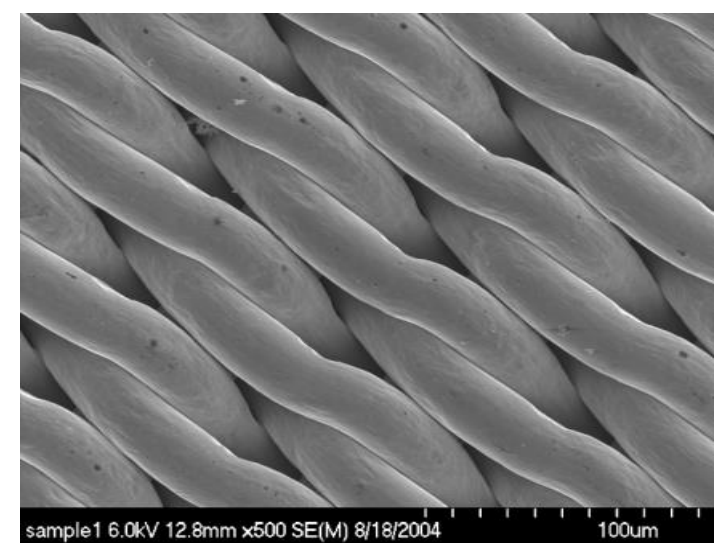

Figure 2.18 - Scanning Electron Microscopy Image of a 200x1400 Dutch Twill Screen

The screen weave is the most important parameter affecting the choice of screen channel LADs since certain weaves are capable of producing much finer pore sizes than other weaves. For example, finer screen meshes are desirable to ensure adequate resistance to vapor ingestion. However, they tend to generate large hydraulic pressure losses during propellant outflow. In addition, the smaller pore sizes also make finer screens more susceptible to potential clogging due to impurities that may exist within the propellant liquid.

In order of increasing complexity, the types of screen weaves available for screen channel LADs are Plain Square, Twilled Square, Plain Dutch, Reverse Dutch, and Dutch Twill. 3D models of the Twilled Square, Plain Dutch, and Dutch Twill weaves are shown in Figure 2.19, taken from bopp.com (2012). Each weave type has a different weave pattern of its larger warp (shown in red) and smaller shute wires (shown in gray), which run perpendicular to each other. The Plain Square weave is the simplest design because the warp and shute diameters are the same size, and the wires simply pass over and under each other in a square pattern. Pore sizes are generally large for this mesh. 


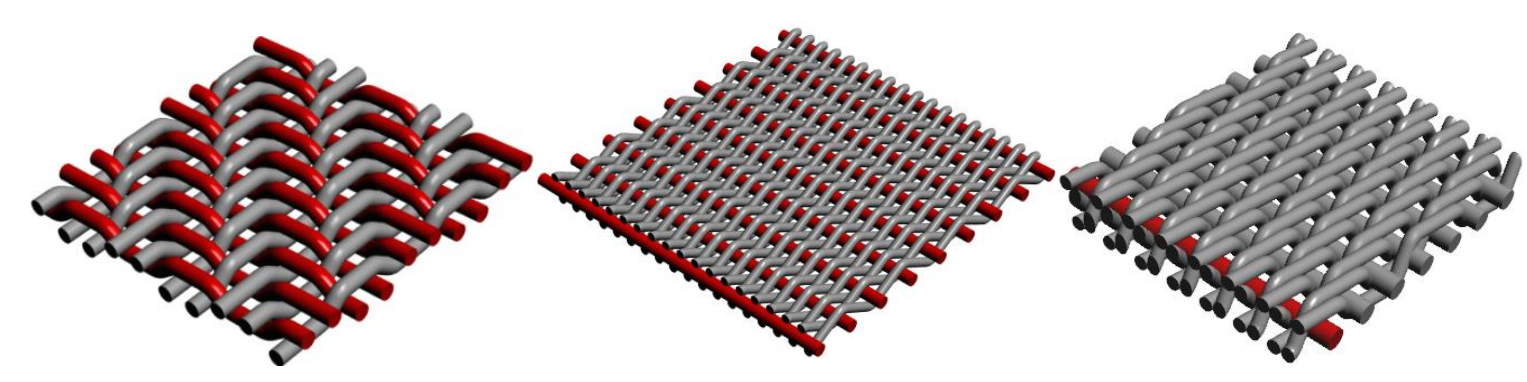

Figure 2.19 - Three Dimensional Models of a) Twilled Square, b) Plain Dutch, and c) Dutch

Twill Weave Styles. Warp wires are denoted in red and shute wires are denoted in gray.

The Twilled Square weave is the second most complex style; the warp and shute wires are also the same diameter, but each shute wires passes over two warp wires before going under the next two warp wires. The pattern then repeats. The Plain Dutch weave has the same pattern as the Plain Square, but the warp wires are larger in diameter than the shute wires, which create smaller pore sizes. The Reverse Dutch weave is the inverse of the Plain Dutch; the shute wires are larger than the warp wires. Lastly, the most complex screen weave is the Dutch Twill. This weave combines properties of both Plain Dutch and Twilled Square; it has the same weave pattern as the Twilled Square but has larger warp than shute wires like the Plain Dutch. Each shute wire again passes over one warp wire before passing under the next two warp wires. The Dutch Twill weave creates the smallest pore diameters and the most tortuous flow path for gas ingestion, thus making it an attractive candidate for low surface tension cryogenic liquid acquisition systems. A full list of all 40 available screen meshes is reserved in Appendix B.

The type of metal also affects screen selection, and thus LAD channel design and mass. Coarser meshes are available in many different metals, such as Ti and Al, while finer meshes are generally only available in heavier metals such as stainless steel (SS). 
As with vane and sponge PMDs, a screen channel LAD designer must often trade performance for system mass.

The ability to wick liquid along the screen makes woven screen superior to perforated plate. Pore sizes much smaller than $10 \mu \mathrm{m}$ are achievable using advanced laser drilling or machining techniques on a solid piece of metal. However, for flexible liquid acquisition systems, both the size and the number of holes affect performance. The number of pores in a woven wire screen is proportional to the product of the number of the warp and shute wires. Perforated plates are structurally more stable than woven screens at the cost of higher flow resistances due to fewer holes. However since perforated plates cannot wick liquid to areas that dry out due to evaporation, they are not recommended as a primary PMD in future cryogenic propulsion systems.

\subsubsection{Advantages and Disadvantages}

LAD screens are almost always in direct contact with liquid fuel, and can acquire propellant in almost any situation. This means that regardless of spin, direction, or acceleration, screen channel PMDs maintain contact with liquid and can continue to deliver that liquid to the outlet. This makes screen channels likely the most flexible PMD across a range of mission requirements (Jaekle 1997). Unlike other PMDs such as traps, sponges, or vanes, they can supply vapor free liquid under much higher accelerations, and then sustain high flow rates due to rapid reacquisition of liquid. Until the pressure drop across the screen exceeds the screen bubble point pressure, vapor free propellant will continue to the outlet. Because of these advantages, gallery arms are optimal PMDs for flights requiring high flow demands. Flexibility, robustness, the ability to maintain low, 
medium, or high flow rates, and the ability to acquire and supply liquid under very high adverse accelerations render the screen channel LAD advantageous over the vane and sponge type PMD.

However, there are some major disadvantages to screen channel LADs. As described above, there is a bubble point at which the surface tension of the wetted screens will fail and vapor ingestion will occur. Because of the delicacy of the fine mesh screens, reliability is reduced. Also, the sheer mass and size of the arms in relation to other PMDs, such as vanes and sponges, can be a disadvantage. For example, the finer screens can only be constructed from SS and are inherently heavier than coarser builds of Ti. Lastly, screen channel LADs are the most expensive and difficult to manufacture of the PMDs. They require a great deal of materials to construct, tests to analyze bubble points of different meshes, and can be difficult to manufacture. Therefore the high performance of screen channel LADs comes at the added cost, mass, complexity, and less overall reliability relative to vanes and sponges.

\subsubsection{Storable Propellant Historical Examples}

Screen channel LADs have a rich flight heritage with storable propellants. They have been used in both space experiments and flight vehicles. Design of these LADs is

well understood for storable systems (Schweickert 1981, Anglim 1981, and Rollins et al. 1988). Galleries have particular success in missions which require flexible demand systems, such as the STS. 


\subsubsection{Space Experiments}

Screen channel LADs were used in some of the earliest high altitude tests in the mid 1950's. The X-15 spacecraft, while never breaking LEO, was tested as a rocket powered space plane to determine the role of man as a future pilot (Walker and Weil 1963). Launched from modified B-52 airplanes at high altitude, the X-15 would continue to accelerate and increase in altitude to conduct high altitude entry maneuvers. A total communication gallery LAD was installed inside the propellant tank, which was a simple screen lining the entire interior tank wall, to access propellant throughout the altitude testing (DeBrock 1967 and DeBrock et al. 1971). A picture of this unique type of screen channel device is depicted in Figure 2.20, which was also to be used in the Spacelab experiment onboard the Shuttle (Cady 1976 and 1978 and Lord 1987). As shown, this gallery more closely resembles a group of closely packed vanes.

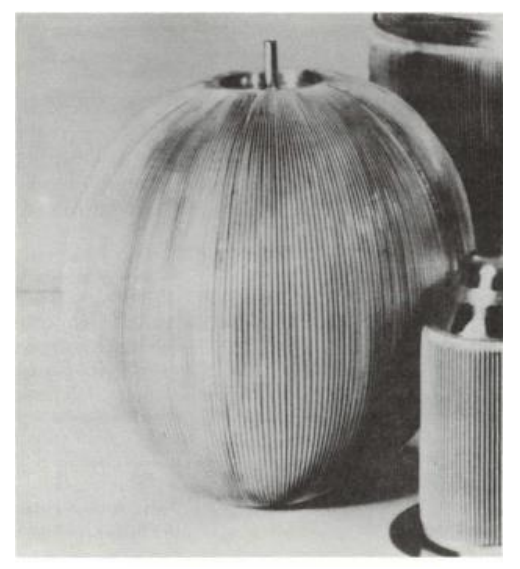

Figure 2.20 - Spherical Pleated Screen Liner used for Spacelab

Galleries were also used in the Boeing Peacekeeper missions. Originally built as an Intercontinental Ballistic Missile (ICBM) (Kumpel et al. 2002), the Boeing Peacekeeper was redesigned as an expendable resupply vehicle for space missions, 
specifically for the International Space Station (ISS). The Boeing Stage IV used a gallery arm to supply propellant for attitude control and also used ring baffles to prevent slosh (Gaines and Orton 1984). Testing was performed in 1-g and aboard a KC-135. 1-g testing was used to evaluate PMD refill and expulsion efficiency and to measure pressure drop across the screen to calculate bubble point pressures at engine startup; the low-g experiments tested slosh control.

The primary storable propellant space experiment employing screen channel LADs was the FARE-I mission onboard STS-53(Dominick and Driscoll 1993 and Dominick et al. 2011). FARE-I tested the fill and $E E$ of a four armed screen channel LAD assembly using the see-through tank shown in Figure 2.21. The galleries were able to achieve expulsion efficiencies near $98 \%$. Like FARE-II, FARE-I was a very successful mission towards understanding general low-g fluid behavior as well as PMD performance in microgravity.

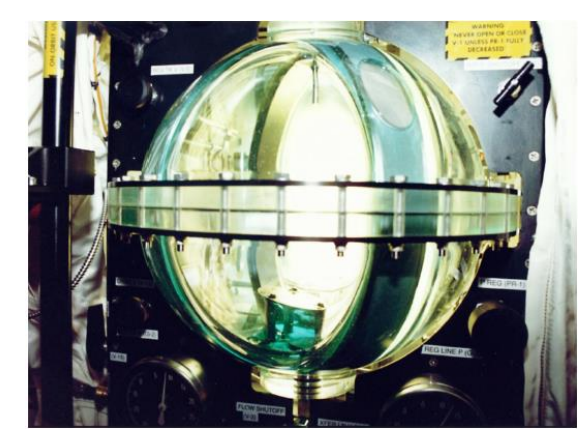

Figure 2.21 - Fluid Acquisition and Resupply Experiment-I Gallery Arm

\subsubsection{Vehicles and Missions}

The Agena Upper Stage Rocket launched in 1959 began using a screened start basket in 1964(DiFrancesco and Booraday 1989). The motivation for the incorporation of 
the basket was the desire to perform multiple restarts of the engine (De Brock et al. 1971). The Agena design featured a screened cone at the top of the sump that protruded into the bottom of the tank to allow for both refill of the sump and the preservation of gas free flow to the tank outlet.

Several GOES satellites employed a 200x1400 screen channel LAD. First launched in 1974 (Joselyn and Grubb 1985), GOES satellites were designed to observe weather on Earth, specifically for monitoring storms (Krummann 2000). Geosynchronous orbits required station keeping maneuvers several times every month using the gallery PMD (Joselyn and Grubb 1985 and Debreceni et al. 1995b).

Screen channel LADs were also employed in many communications satellites. For example, the bipropellant Intelsat VI, VIIA satellites used for improved phone and television coverage employed four screened gallery arms and a trap to maintain liquid over the tank outlet (Purohit and Prickett 1999). The fuel tank used a 200x1400 mesh while the oxidizer used a 165x800 mesh (Debreceni et al. 1995a and Jaekle 2013). Space Systems/Loral produced a series of Superbird Satellites for the Japanese owned Space Communications Corporation. Superbird-A was launched in 1992 (Murase et al. 1998) and provided consistent telecommunications services across a great part of Asia, including, China, Japan, and Taiwan. The Superbird satellite utilized a screen channel PMD to allow for liquid acquisition in low-g environments and to maximize $E E$ (Murase et al. 1998). The 702B, a recently evolved communications satellite based on the Boeing 702 MP platform, also used a screen channel PMD for its propellant tank (Jaekle 2013). The Milstar satellites, which provided worldwide communication for military personnel, employed a 30x160 mesh screen channel LAD for its PMD (Heubush and Pugmire 1988 
and Kwiatkowski et al. 1994). Galleries were also used in the only known NASA mission to have failed in the Mars Observer mission, due to "propulsion and pyrotechnic" problems (Saulsberry et al. 1999). However its descendant was the highly successful Mars Global Surveyor (Dominick 1999).

Perhaps the most well-known example of a screen channel LAD in a flight vehicle is in the STS. Shuttle is well known as NASA's workhorse vehicle from 1981 2011. The purpose of the Shuttle was to transport astronauts, cargo, and space experiments from ground to LEO. STS is the only reusable, winged manned vehicle to achieve orbit in LEO and land back on Earth. Some of the primary accomplishments included transport and assembly of the ISS, ferrying astronauts and supplies to the ISS, return, recovery, and/or repair of satellites, transportation of Spacelab (Lord 1987), Hubble Telescope (Zimmerman 2008), Chandra X-ray Observatory (Weisskopf et al. 2002), and numerous Tracking and Data Relay Satellites (TDRS). Since the destination was LEO, special thrusters were needed to make small adjustments to the vehicle position. STS was equipped with 14 RCS thrusters to change the attitude or direction. Meanwhile, to change orbits for rendezvous docking maneuvers, STS was also equipped with an Orbital Maneuvering System (OMS). Both systems were fueled by NTO and MMH. The Shuttle flew 135 missions in total, 133/133 successful operations with its PMD.

Both RCS and OMS tanks employed very complex total communication $325 \times 2300$ screen channel LADs. A schematic of the LAD inside the upper aft RCS pad is shown in Figure 2.22a. The RCS LAD was designed to supply propellant during all phases of the mission in LEO, as well as during re-entry. As shown, there were multiple 
sections of windowed screen material in total communication-like arms to access propellant at different acceleration levels, as well as a sump at the bottom of the tank (White 1980). Meanwhile OMS used both gas arrestors and gallery arms as shown in Figure 2.22b. Design details for both are well represented in the literature (Fester et al. 1974 and 1975, Tegart and Fester 1975, Regnier and Hess 1978, Hess and Regnier 1978, and Anglim 1979 and 1981). Although a non-toxic upgrade to cryogenic propellants was considered for the RCS/OMS systems (Lak et al. 1998), due to safety considerations it never came to fruition.
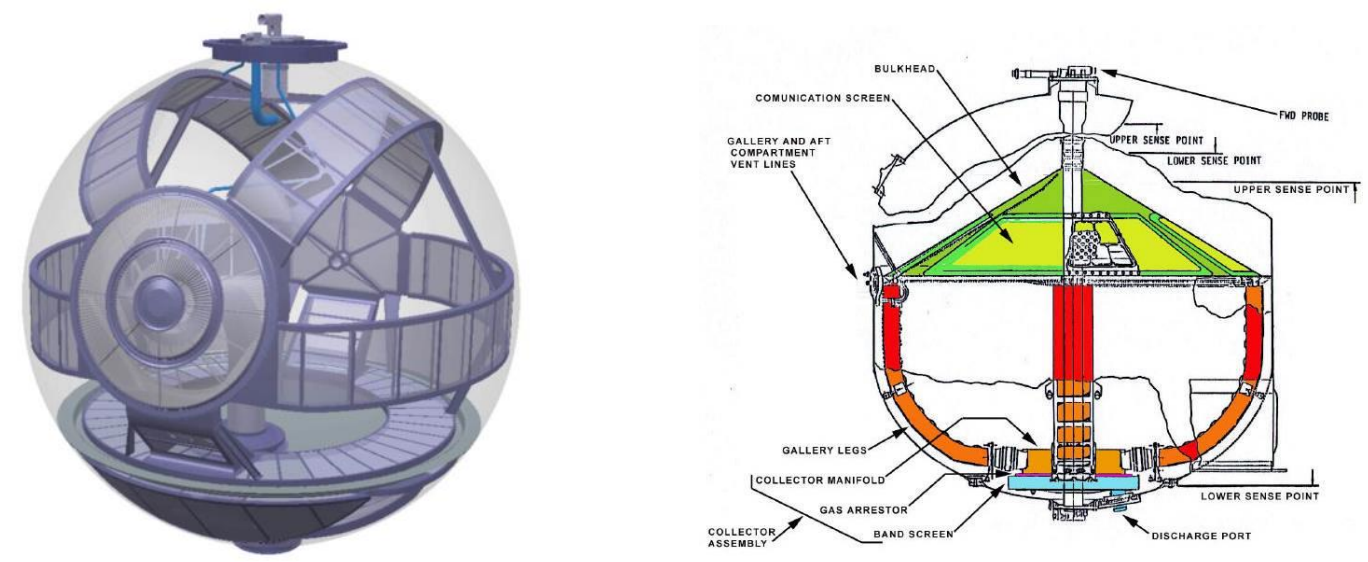

Figure 2.22 - Space Shuttle Screen Channel Liquid Acquisition Device Inside the a) Reaction Control System and b) Orbital Maneuvering Propellant Tanks

\subsubsection{Cryogenic Propellant Historical Examples}

Of all the PMDs, screen channel LADs are the only PMD type to actually have flight heritage with cryogenic liquids. The first attempt at using LADs inside a flight cryogenic propellant tank was in the popular Centaur upper stage. Short duration $(<6$ hours) upper stages typically use settling thrust maneuvers and thus vehicle acceleration to drive propellant to the tank outlet (Austad 2001). However, because of the sheer 
number of engine burns required to position propellant, and the possibility of vapor ingestion, the settling system was deemed undesirable for multi-burn mission (Blatt and Aydelott 1976 and Blatt et al. 1980). Therefore start baskets for both the $\mathrm{LOX}$ and $\mathrm{LH}_{2}$ tanks were designed and tested; the baskets even included passive subcooling systems to further mitigate the likelihood of vapor ingestion into the transfer line (Blatt 1970c, d, and e, Blatt 1971, Blatt and Walter 1975, Blatt et al. 1976, and Blatt and Ayedelott 1978). While the start basket weighed more than the previous settling system, faster engine preparation and less total number of required engine burns made the start basket the more long term viable option. While Centaur upper stages continue to be used for evolved expendable launch vehicles (EELV), start baskets were never installed into the propellant tanks. Several low-g CFM experiments were proposed (Chato et al. 2006) and modifications to convert the Centaur upper stage into a cryogenic test bed were conceived, but they never came to fruition (Kutter et al. 2006, Sakla et al. 2006, and Gravlee et al. 2010).

The second attempt at using screen channel LADs inside a flight cryogenic propellant tank was in the not well-known Russian Buran. Analogous to the United States Shuttle, and very similar in design, the purpose of the Buran was to ferry astronauts and cargo into LEO with a reusable vehicle. The Buran was also developed as a potential military application due to its very large payload capacity. The exact military capabilities are classified. The main difference between the two shuttles was that the Buran employed cryogenic $\mathrm{LOX} / \mathrm{LH}_{2}$ stages for its LEO maneuvering whereas the Shuttle used storable propellants for its RCS and OMS systems. Additionally, the Buran launch stage was four single LOX/kerosene rockets, whereas the Shuttle used a combination of solid rocket 
boosters and $\mathrm{LOX} / \mathrm{LH}_{2}$ stage. Buran only had a single launch in November, 1988 from the Baikonur Cosmodrome facility. Launched as an unmanned spacecraft on its 206 minute inaugural voyage, the Buran was sent into orbit, completed two full orbits in LEO around Earth, and then landed back in Russia. The Buran was unique because it was the first spacecraft of its size to perform fully automated launching, LEO maneuvers, re-entry and descent, and land back on Earth. The Buran LAD is shown in Figure 2.23 (Semenov et al. 1995) where \#3 and \#5 correspond to tube style screened LAD elements used to acquire liquid during orbital maneuvering, descent, and re-entry. The Buran LAD was never tested outside of this inaugural flight.

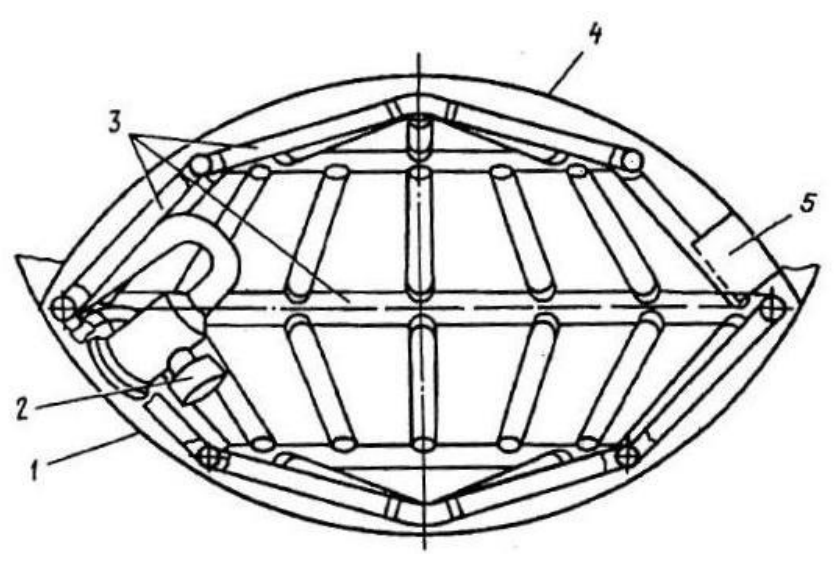

Figure 2.23 - Buran Liquid Acquisition Device inside Liquid Oxygen Tank used for Orbital Maneuvering

The third attempt at obtaining flight heritage with LADs in cryogenic liquids was in the successful Superfluid Helium On-Orbit Transfer (SHOOT) small scale experiment onboard STS-57 in June, 1991 (Lee et al. 1988, Gille et al. 1989, Castellano et al. 1989, DiPirro et al. 1990, Kashani et al. 1990, Hopkins and Mord 1990, and DiPirro et al. 1992). The purpose of SHOOT was to demonstrate autonomous transfer of Superfluid 
Helium (SFHe) between two storage tanks in low gravity, accurate mass gauging, successful operation of a screen channel LAD, as well as demonstrate accurate phase separation with SFHe and normal helium. SFHe, representing a unique fluid with zero entropy and zero viscosity, has applications in quantum solvents, spectroscopy, and cryocooling (Hendricks et al. 1988 and Frank 1997). SHOOT used a 325x2300 screen channel LAD to acquire the ultra-low surface tension SFHe, the details of which are well documented in the literature (Anderson et al. 1988, Anderson 1989, DiPirro 1989, Maddocks and Van Sciver 1989, DiPirro 1990, Nissen et al. 1990, and Nissen and Van Sciver 1991). Although helium is an inert, SHOOT represented a major step in the advancement of cryogenic propulsion system technology development through its simple demonstration mission. While basic experiments have been conducted to analyze liquid positioning, propellant slosh, chill down of hardware through the Saturn IV-B, Centaur, and Titan CFM flight tests, obtaining low-g performance data in cryogenic propellants like $\mathrm{LOX}$ and $\mathrm{LH}_{2}$ still remains to be one of the highest priority objectives for the space flight community (Chato 2008).

\subsection{Propellant Management Device Combinations}

It is instructive to note that many PMDs are actually combinations of several subsystem devices. Grouping several PMDs together in a single system, a designer can easily overcome the aforementioned disadvantages of each of the stand-alone systems. For example, sponges and baffles are often used in combination with simple vanes for resupply missions, as shown in Figure 2.24a (Debreceni et al. 2003). Vanes arms can continuously access propellant from the pool in the tank to refill the sponge, while the sponge can be used to store propellant for the next burn. Meanwhile a series of baffles 
can help prevent liquid movement within the sponge panels and increase the effectiveness of the sponge at maintaining propellant.

Another combination example is a vane, trap, and trough combination as shown in Figure 2.24b (Griffin et al. 2003). Reliable vanes and a center post aid in propellant acquisition from the tank walls, both on the outlet side and the pressurant gas side. Flow is forced from both the vanes and center post through a small hole at the top of a trough/trap combination. A double layer of perforated screen sits above the outlet to reduce vapor ingestion, while the hydrostatic forces used by the trough can keep large amounts of propellant near the outlet during tank spin. Many other examples of combination style PMDs exist in the literature (Debreceni et al. 2001, Tam et al. 2001, Griffin et al. 2003, Debreceni et al. 2004, and Tam and Jaekle 2005).
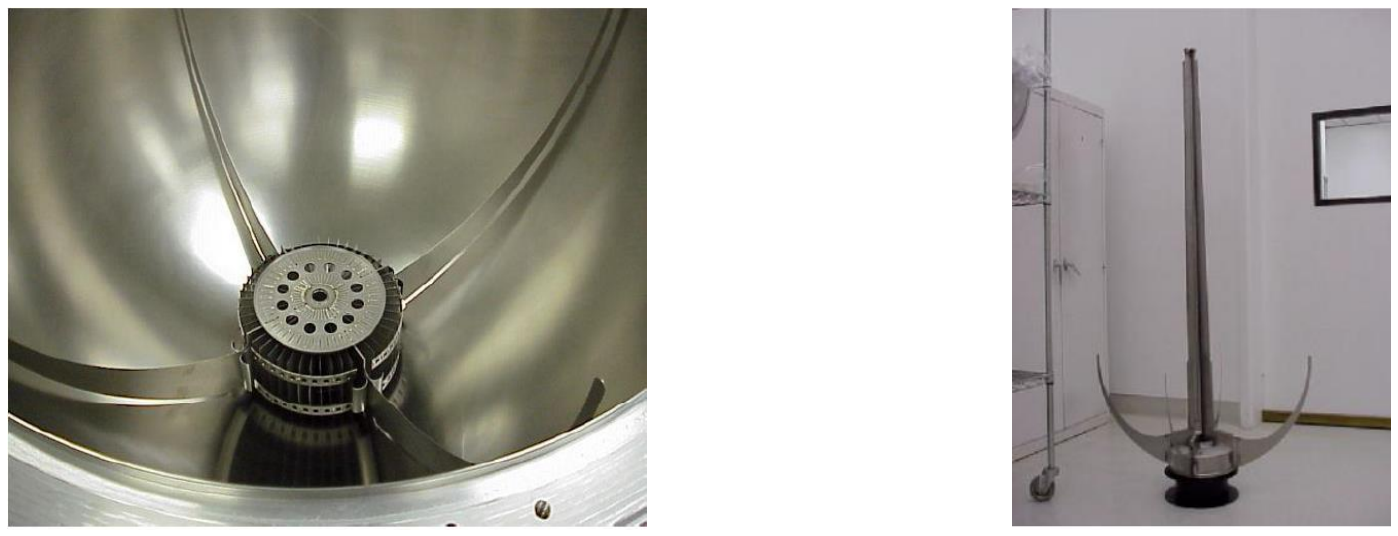

Figure 2.24 - Combination Propellant Management Device with a) Four Tall Vanes, a Small Sponge, and Baffles Positioned within the Sponge and b) Four Short Vanes, Center Post, Trap, and Trough 


\subsection{NASA's Current Needs}

In congruence with the current desire to develop technology for multiple cryogenic propulsion systems, a flexible, robust, and multi-purposeful LAD is required to meet NASA's current and future demands. Liquid acquisition devices need to be able to supply vapor free cryogenic propellant across a wide range of future vehicles, low surface tension cryogenic propellants, demand flow rates, and gravitational and thermal environments. For example, a LAD will be required for supplying higher pressure fed engines in the reduced gravity of Lunar or Martian ascent stages with up to $1.8 \mathrm{~kg} / \mathrm{s}$ (4 $\mathrm{lbm} / \mathrm{s})$ fuel and $5.45 \mathrm{~kg} / \mathrm{s}(12 \mathrm{lbm} / \mathrm{s})$ oxidizer flow rates. LADs will be required to supply fuel depot flow rates in excess of $0.11 \mathrm{~kg} / \mathrm{s}(0.25 \mathrm{lbm} / \mathrm{s})$ and $2.3 \mathrm{~kg} / \mathrm{s}(5 \mathrm{lbm} / \mathrm{s}) \mathrm{LH}_{2}$ and LOX, respectively in the microgravity of LEO. In addition, NASA maintains interest in developing payloads for small scale depot demonstration missions, of which the most recent attempt was entitled the CPST Technology Demonstration Mission (TDM). This mission required a flexible delivery system to demonstrate transfer of propellant at multiple $\mathrm{LH}_{2}$ fill levels.

Comparing the characteristics, strengths, and weaknesses of the three primary capillary driven vanes, sponges, and screen channel LADs, it is clear that vanes and sponges can supply vapor free propellant using a much simpler design and implementation than screen channel LADs. Both types are advantageous in systems which experience low-g levels and very low supply demands. However, sponges and vanes may not meet the high flow rate requirements of future propellant depots or inspace engines; sponges simply do not scale with the projected size of depot tanks, and vanes may not be able to supply large flow rates relative to screen channel LADs. Screen 
channel LADs are the most extensible and flexible cryogenic PMD for variable vehicle acceleration and direction, and can provide a wide range of flow rate regimes to supply single phase liquid for both engine restart and continuous engine operation, as well as fluid transfer for fuel cells, life support, and the proposed fuel depots. Therefore screen channel LADs are the recommended technology approach for future in-space cryogenic systems due to a rich ground technology development program, flight heritage in storable and cryogenic liquids, and higher performance, flexibility, and robustness relative to vanes and sponges.

The following four mission scenarios outline where a screen channel LAD would be required to extract cryogenic propellant from a storage tank in the microgravity conditions of space:

1. Main engine burn for an upper stage or engine in reduced gravity (ex. Lunar ascent stage).

2. Small thruster firing for a reaction control system.

3. Fluid circulation for a tank internal TVS or mixing pump (DiStefano et al. 1994 and Winters 1996).

4. Transfer from a cryogenic depot to another vehicle.

During a main engine burn, the thrust generated will cause a significant acceleration that will settle the propellant over the tank outlet. However, if the main engine burn occurs in a reduced gravity environment, a simplified PMD, such as a screen channel sump may be used to ensure liquid initially covers the tank outlet. Therefore, the 
LAD must be capable of delivering high flow rates under high acceleration levels without generating a large pressure drop or breaking down due to cavitation from dissolved pressurant gas or from vaporization within the liquid propellant.

During small thruster firing, thrust generated may again settle the propellant, but sump style LADs will still be required to ensure the outlet is sufficiently covered with liquid. In this scenario, smaller flow rates are anticipated under the same large acceleration changes. Therefore, a LAD must be capable of delivering smaller flow rates under adverse acceleration magnitudes and directions. The LAD screen must also be capable of withstanding loads that may occur during the transient restart of the engine.

During fluid circulation for a tank internal TVS system or mixing pump, the LAD must be capable of delivering smaller flow rates despite an unknown initial position of the $\mathrm{L} / \mathrm{V}$ interface within the tank. Therefore the LAD must have a high bubble point pressure, and be capable of delivering lower flows in low gravity conditions.

Finally, during fluid transfer from fuel depots in LEO, the storage tank would be subject to a wide range of acceleration levels, over a wide range of demand flow rates through the LAD. In this case, the LAD should provide flow with high resistance to vapor ingestion and incur low pressure losses across the screen. These four different mission scenarios hint at some of the desired features of the optimal screen channel LAD. 


\section{Chapter 3}

\section{Influential Factors and Physics-Based Modeling of Liquid Acquisition Devices}

The purpose of this chapter is to present a detailed list of the six factors which govern screen channel LAD behavior and determine screen selection for LAD design, and to derive and validate the physics-based models for these factors. A simplified 1D 1-

g pressure drop model is presented for the LAD. Bubble point (and reseal) pressure, flowthrough-screen pressure drop, wicking rate, screen compliance, material compatibility, and pressurant gas type are among the influential factors governing design. Governing equations and analytical models for these parameters are developed from first principles. A comprehensive literature review is then conducted to gather all previously reported data to validate the models. Basic experimental procedure is presented for each parameter. Finally the primary LAD screens are down selected for experiments for room temperature and cryogenic liquids. Additionally, analysis will be given on the impact of these influential factors for an $\mathrm{LH}_{2}$ fueled propulsion system.

\subsection{1-g One Dimensional Simplified Pressure Drop Model}

Either in 1-g or in microgravity, a LAD screen will separate liquid and vapor phases so long as the pressure differential across the screen does not exceed the bubble 
point pressure of that screen. Therefore the total pressure loss in the LAD system must be less than this pressure to prevent vapor ingestion into the channel:

$$
\Delta P_{\text {total }}<\Delta P_{B P}
$$

where $\Delta P_{B P}$ is the bubble point pressure, and the total pressure drop can be expressed as a sum of the constituent parts:

$$
\Delta P_{\text {total }}=\Delta P_{\text {hydrostatic }}+\Delta P_{F T S}+\Delta P_{\text {friction }}+\Delta P_{\text {dynamic }}+\Delta P_{\text {other }}
$$

where $\Delta P_{\text {hydrastatic }}$ is the hydrostatic pressure on the liquid within the channel, $\Delta P_{F T S}$ is the pressure drop due to liquid flow across the screen, $\Delta P_{\text {friction }}$ is the frictional loss down the LAD channel, $\Delta P_{\text {dynamic }}$ is the dynamic pressure drop due to inflow into the channel, and $\Delta P_{\text {other }}$ is the pressure loss contribution due to vibrations (Tegart and Aydelott 1978), propellant sloshing, and/or transients (Tegart 1976) at the start of flow demand (change in pore size due to screen bending or flexing). This is known as Jaekle's (1997) 1D pressure drop model.

Consider a vertically oriented rectangular shaped screen channel LAD of length $L$, depth $H$, and width $W$, mounted inside a propellant tank in 1-g as shown in Figure 3.1. The tank is initially full of liquid, with the LAD channel completely submerged as shown in Figure 3.1a. Then the tank is drained through the LAD, as liquid is routed through the screen, into the channel, and out of the top of the channel in an inverted outflow (IO) configuration as shown in Figure 3.1b. Points 1-4 depict the flow path. 
When a LAD is completely submerged in liquid, single phase liquid flow is inevitable. When the L/V interface inside the propellant tank drops below the top of the vertical LAD channel, assuming the channel pores are all completely wetted, the pressure drop inside the channel begins to rise. While the tank is drained and the LAD becomes exposed to gas, surface tension forces within the screen pores block vapor ingestion into the channel. Liquid will remain inside the part of the channel that is partially exposed to pressurant gas as shown in Figure 3.1b, so long as the sum total of pressure losses within the system do not exceed the bubble point pressure. Eventually, the LAD will break down at a certain liquid level in the tank.

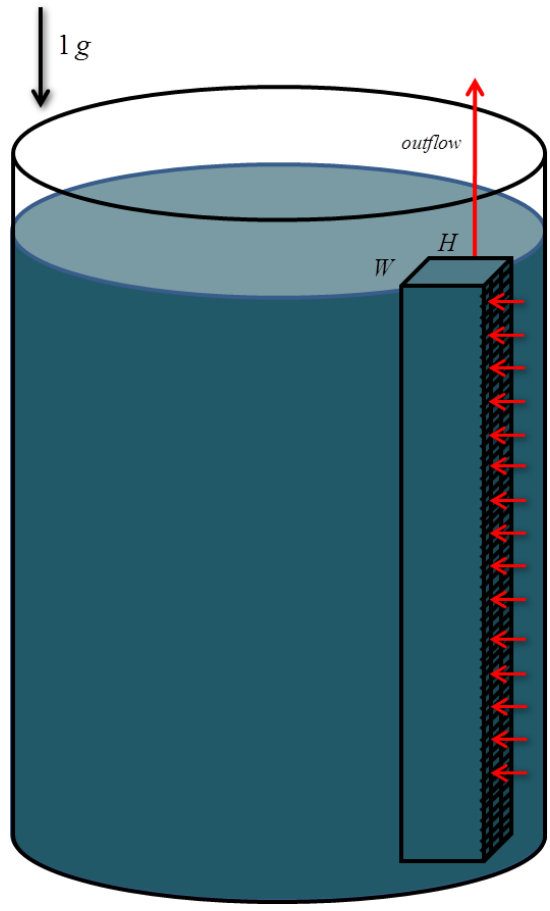

a)

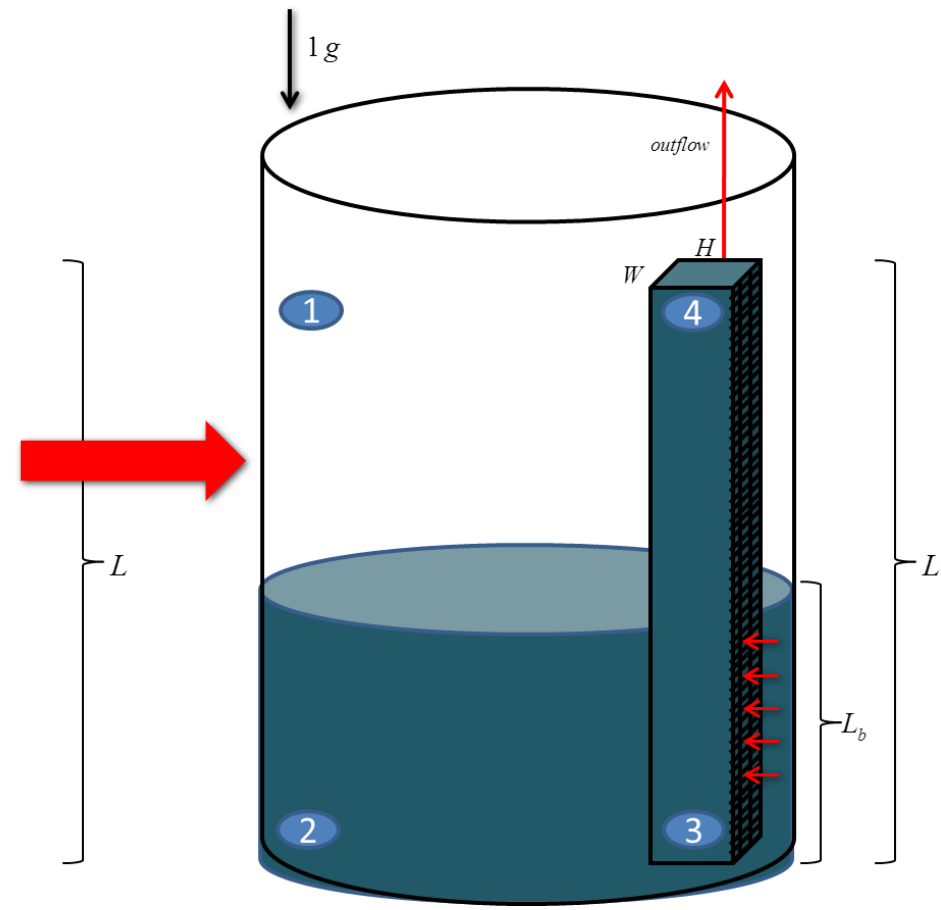

b)

Figure 3.1 - Vertically Oriented Screen Channel Liquid Acquisition Device in 1-g Inverted 
During a steady state outflow, all transient terms are negligible. For steady flow, Equation 3.2 therefore becomes:

$\Delta P_{\text {total }}=\Delta P_{\text {hydrostatic }}+\Delta P_{F T S}+\Delta P_{\text {friction }}+\Delta P_{\text {dynamic }}$

The maximum pressure drop across the screen in Figure $3.1 \mathrm{~b}$ occurs at the top of the channel, between points 1 and 4:

$P_{1}-P_{4}=\Delta P_{\text {hydrostatic }}+\Delta P_{F T S}+\Delta P_{\text {frictional }}+\Delta P_{\text {dynamic }}$

Models are now needed for the bubble point pressure and each of the steady state pressure drop terms.

\subsection{The Room Temperature Bubble Point Pressure}

The primary performance parameter for the screen channel LAD is the maximum bubble point pressure. For a given cryogenic propellant, screen mesh, and operating range of fluid conditions (pressure, temperature) for a given mission, the bubble point sets either the maximum allowable flow rate that a screen channel LAD can deliver out of a propellant tank or the maximum allowable adverse acceleration level against which liquid can be withdrawn from the tank. Following the method of Adamson and Gast (1997), a relation between the bubble point pressure, geometry of the screen pore, surface tension, and contact angle is constructed from a model based on the general YoungLaplace equation (YLE) for the pressure drop across a set of curved interfaces between two phases, which are embedded in the 3D space of the screen mesh (Fainerman et al. 1994). The theory which takes into account the complex local structure of the weaves in 
the fine mesh screens is isomorphic with a set of cylinder capillaries. This property leads to the simplified YLE that involves an effective pore diameter.

\subsubsection{Assumptions}

The following assumptions are required to derive the simplified bubble point model:

1. Reactions and diffusion at the $\mathrm{L} / \mathrm{V}$ interface and adsorption and desorption to and from the interface are negligible. During dormant times (i.e. no gas pressurization), gaseous helium (GHe) may diffuse into the liquid phase, but diffusion rates are negligible over testing time scales.

2. The L/V interface is treated as a zero thickness surface. In reality, higher liquid pressures may act to increase the interface thickness, but the effect is negligible for the LAD screen systems studied herein (Cahn 1978 and Turkevich and Mann 1990a).

3. The complex 3D pore structure can be modeled as the average of an ensemble of simple vertical capillary tubes. In reality, the Dutch Twill weave pattern creates a tortuous path and complex shaped pore.

4. The $\mathrm{L} / \mathrm{V}$ equilibrium interface formed between the warp and shute wires of the Dutch Twill screen is approximated as a 2D interface parallel to the screen face.

5. The continuum hypothesis is invoked; pressurization of the screen is done in an infinite number of quasi-static steps. 
6. The advancing contact angle between liquid and pore throat is approximately the same advancing contact angle between liquid and pore mouth.

7. The advancing contact angle that is pinned at the microscopic pore mouth is approximately the same advancing contact angle that is measured on the macroscopic screen surface.

\subsubsection{Bubble Point Model Derivation}

Consider the domain drawn in Figure 3.2 that represents the L/V interface patch in a Dutch twill screen. Figure 3.2 displays a general time dependent curved surface formed by two phases, a L/V interface, where the surface, $\Sigma(t)$ of area $A(t)$ may change in time. The normal $\hat{n}$ to the surface at $P$ points from the liquid phase to the vapor phase. The two vectors in the tangent plane at $P, \vec{e}_{1}, \vec{e}_{2}$ determine the normal vector, $\hat{n}=\vec{e}_{1} \times \vec{e}_{2} /\left|\vec{e}_{1} \times \vec{e}_{2}\right|$. The net flux of component $i$ for a multicomponent system, from the vapor phase is $-\hat{n} \bullet \vec{J}_{i}^{(+)}$, adsorbing (desorbing) onto the interface and there may be a net flux from the liquid phase, $+\hat{n} \bullet \vec{J}_{i}^{(-)}$, adsorbing (desorbing) into the interface. The pressure jump at $P$ is $P^{(+)}-P^{(-)}$. The surface excess of the $i^{t h}$ component is $\Gamma_{i}(\vec{x}, t)$. The principal radii of curvature are shown here as $R_{\max }, R_{\min }$. Neglecting surface reactions, diffusion, and any adsorption or desorption fluxes between the phases, but allowing a curved interface between two fluids within the screen, the general form of the YLE relates the pressure jump across that interface with the curvature of the surface:

$[P]=-\gamma_{L V} \nabla \cdot n$ 
where $\gamma_{L V}$ is the surface tension or surface free energy of the liquid, $n$ is the unit normal, and the pressure jump is expressed in the following manner:

$[P] \equiv P^{(+)}-P^{(-)}$

where $\mathrm{z}$ is aligned along the normal vector $n$. The divergence of the unit normal is related to the mean curvature $H_{M}$ of a surface through the following relation:

$2 H_{M} \stackrel{\text { def }}{=} \nabla \cdot n$

There is a sign convention that must be taken into account. Therefore, using assumption 2 for a smooth surface and smooth pressure jump,

$[P]=-2 \gamma H_{M}$

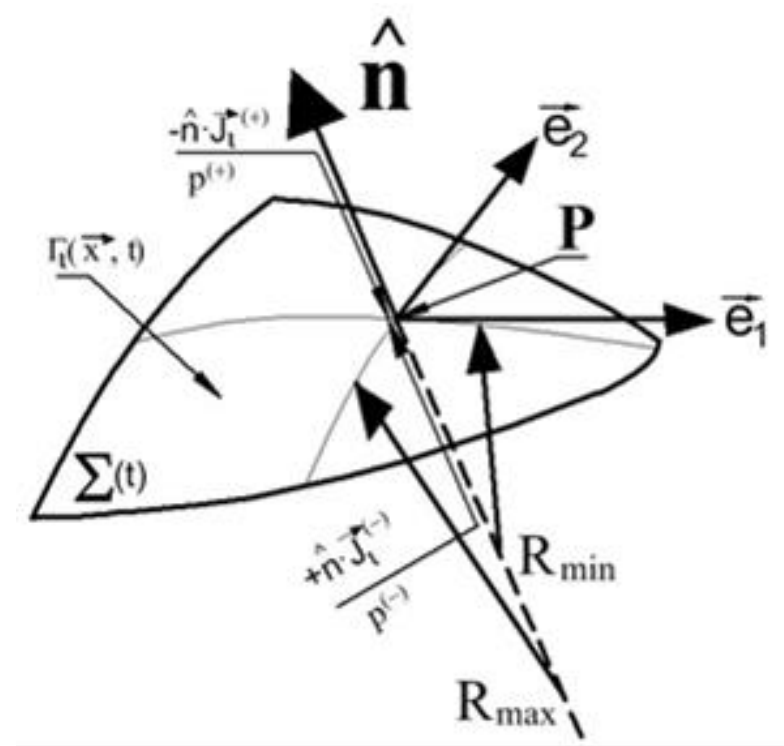

Figure 3.2 - The Liquid/Vapor Interface of a Patch in the Dutch Twill Screen to Show the Geometry of the Curved Interface 
The sign convention is illustrated by use of a specific, but general, representation of the patch shown in Figure 3.2 from which the tangent vectors are $\vec{e}_{1}=(1,0, \partial \zeta / \partial x)$, and $\vec{e}_{2}=(0,1, \partial \zeta / \partial y)$. The unit normal is computed to be:

$\Sigma(x, y, t)=\left(\begin{array}{c}x=x \\ y=y \\ z=\zeta(x, y, t)\end{array}\right)$

which points from the liquid to the vapor phase. These formulas make it clear that $\nabla \cdot n$ is a non-linear function (see Boruvka and Neumann 1977). In this representation, Equation 3.8 is a nonlinear differential equation, which requires boundary conditions invoked from the constraint requirements of the three-phase contact line. In principle, it is possible to formulate this boundary value problem on a "unit mesh" of the weave, and by symmetry seek a general solution to the bubble point pressure problem, but the weave is sufficiently complex.

Note that the case of axisymmetric geometry has been solved for drops spreading on flat surfaces (Boruvka and Neumann 1977). Also, the case of a bubble formed at the aperture of a capillary concerned with the maximum bubble pressure method is well discussed in the literature; see for example Berg (2010), page 72. Also, since the surface tension is known for various liquids it remains to manipulate the mean curvature of Equation 3.8 so as to provide an average pore diameter. There is an isomorphism between a spherical cap, Figure 3.3, and the actual surfaces formed in the screen as represented in Figure 3.2. That means that there exists a one-to-one mapping between a spherical cap and the patch of Figure 3.2. 
Figure 3.3 shows that two principle radii of curvature can be defined at any point along the surface, and that the mean curvature, $H_{M}$ is related to these radii through the simple relation:

$\Delta P=\gamma_{L V}\left(\frac{1}{R_{\max }}+\frac{1}{R_{\min }}\right)$

where the sign convention is used to compute $\Delta P$. Invoking assumption 3 , for the special case of a vertical capillary tube shown in Figure 3.3, the pressure jump across the interface is simplified:

$\Delta P=\gamma_{L V} \frac{2}{R}$

\section{Liquid}
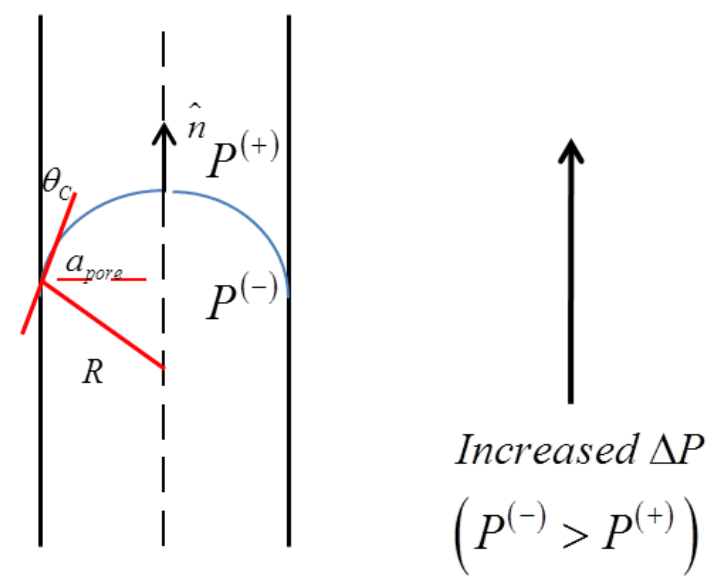

Gas

Figure 3.3 - Approximating a Dutch Twill Pore as an Inverted Vertical Capillary Tube. The normal is taken as pointing into the liquid phase. 
Note that the mean radius $R$ of the pore forms a perfect spherical meniscus and that this radius will depend on the radius of the tube, and contact angle between liquid and solid tube through the following relation:

$R \cos \theta_{C}=a_{\text {pore }}$

such that the pressure drop across the interface becomes:

$\Delta P=\frac{2 \gamma_{L V} \cos \theta_{C}}{a_{\text {pore }}}$

Note also the inverted configuration in Figure 3.3; for laboratory bubble point testing it is easier to deduce the bubble point when precisely controlling the liquid head pressure on top of the screen and pressurizing the pore from beneath.

It has been assumed in previous studies that cryogenic liquids such as $\mathrm{LH}_{2}, \mathrm{LN}_{2}$, LOX, and $\mathrm{LCH}_{4}$, as well as standard reference fluids like isopropyl alcohol (IPA), are close to perfect wetting with clean tank surfaces like SS and Al such that the contact angle $\theta_{c} \approx 0$. In general, the contact angle will depend on the properties of the fluid and the solid, and also the surface roughness. Therefore to validate this assumption, contact angles were measured for each screen and fluid pair and are reported in Chapter 4.

Assumption 4 is invoked to relate the pressure jump across the spherical interface with the actual bubble point pressure of the LAD screens. Therefore, the equivalent radius, $a_{\text {pore }}$, of the vertical capillary tube that is isomorphic with the mean curvature, $H_{M}$, can be approximated using a hydraulic diameter of a circle inscribed within the complex triangular pore shape such that Equation 3.13 becomes: 
$\Delta P=\frac{4 \gamma_{L V} \cos \theta_{C}}{D_{p}} \times f\left(\cos \theta_{C}, D_{p}\right)$

where $D_{p}$ is defined as the so called "effective" pore diameter:

$\frac{1}{D_{p}}=\frac{1}{R_{\max }}+\frac{1}{R_{\min }}$

The function $f\left(\cos \theta_{C}, D_{p}\right)$ corrects for the geometry of the weave and would necessarily be modeled if the geometry of the pore could be mapped in sufficient detail. It appears that taking $f\left(\cos \theta_{C}, D_{p}\right)=1$ is a reasonable approximation for the weaves investigated herein.

To achieve a bubble breakthrough, the pressure underneath the screen is gradually elevated such that the column of gas trapped within the screen slowly creeps up the pore. If the gas pressure is ramped in fixed, quasi-static steps as in assumption 5, then the contact angle between liquid and solid within the pore throat at time $=0$ will be the same advancing contact angle for all other times. Then the gas column will rise and the same contact angle within the pore throat will become "pinned" at the pore mouth through the use of assumption 6. After subsequent pressurization, a gas bubble will eventually detach and break away from the screen. From the definition of the bubble point, the differential pressure at this initial point of gas breakthrough is what defines the experimental bubble point. To permit relation of the microscopic contact angle at the pore mouth with the measurable macroscopic contact angle of the screen, assumption 7 is employed such that Equation 3.14 can be rewritten as: 
$\Delta P_{B P}=\frac{4 \gamma_{L V} \cos \theta_{C}}{D_{p}}$

Equation 3.16 is known as the simplified bubble point equation for a screen channel LAD, and by construction, is isomorphic to the interfacial governing equation based on the geometry of Figures 3.2 and 3.3 (Gauglitz and Radke 1990). If the relation is allowable, then macroscopic measurement of advancing contact angle via the Sessile Drop method is related to the advancing contact angle pinned at the pore mouth, which is related to the advancing contact angle at the pore throat. Figure 3.4 illustrates this concept and plots the contact angle with increased pressurization under the LAD screen. Thus detailed mathematical mappings are not required for the practical application of Equation 3.16 .

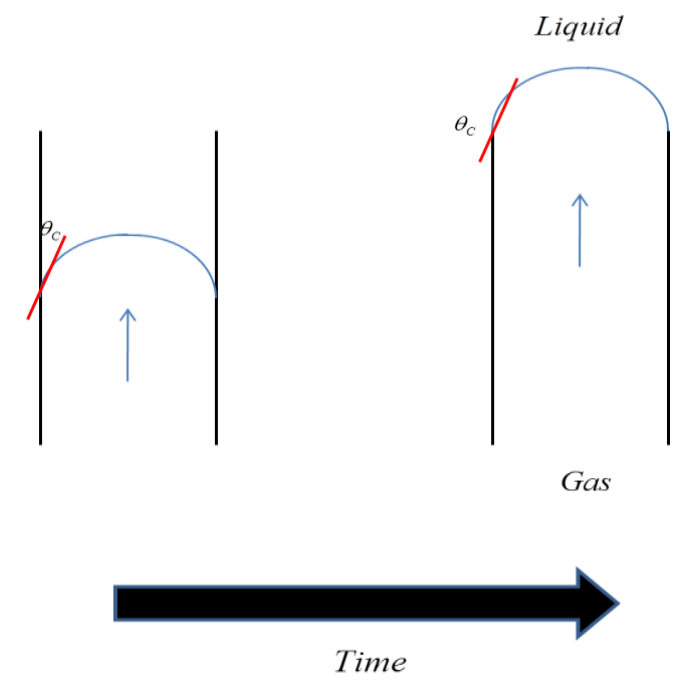

Figure 3.4 - Progression of the Contact Angle with Time during Screen Pressurization. Advancing and receding contact angles differ, and the hysteresis depends on surface roughness, "pre-wetting" at the monolayer level on the wire matrix, and on the rate of advancement or withdrawal. 
To be precise, since the gas bubble is expanding from within the pore throat to the pore mouth, the contact angle in Equation 3.16 is the advancing contact angle. If Equation 3.16 were applied to the pressurization process in reverse (i.e. if mass were pulled back down through the screen or if the top side of the screen was pressurized), then this angle would be the receding contact angle; one would anticipate hysteresis between the two. Therefore, the measured breakthrough pressure would be different from the measured reseal pressure on account of differences between advancing and receding contact angles, and also due to differences in the size of the pore throat and pore mouth.

\subsubsection{Types of Bubble Point Experiments}

Historically there are four different test configurations for measuring the bubble point pressure of a given screen sample. The ideal case is to use an inverted bubble point (IBP) configuration, which will be described in Chapter 4 where the liquid is on top of the screen and gas is beneath. The IBP configuration is preferred over the non-inverted bubble point (NIBP) configuration (wetted screen attached to a liquid filled section with pressurant gas blowing down on the screen) because it is much easier to control liquid head pressure, it is easier to control the uniformity of pressurization, and because data reduction is simplified because bubbles that break through the screen in the IBP configuration naturally rise away from the screen. An example of the NIBP configuration is in Castle (1972).

A third method for measuring the bubble point pressure of a LAD screen sample is to use a dynamic outflow configuration. Here, a screen sample is attached to a channel, mounted inside a tank, and the tank is drained by passing liquid through the channel as 
done in Meserole and Jones (1993). As the liquid level drains and the LAD becomes exposed to pressurant gas, the pressure differential across the screen slowly increases and eventually exceeds the bubble point pressure. While this configuration is more flight representative, it is inferior to the IBP configuration because it is even more difficult to control the location and direction of pressurization; even small changes in the uniformity of the pressurization can cause large differences in the breakdown height of the channel as shown in the data. A fourth method used by Bennett (1987) is to submerge a basket made of screen mesh into a liquid, lower the liquid level, and blow pressurant gas on the screen until breakdown. This method is best for start baskets, and not full communication devices. The majority of historical bubble point data collected, along with all new experimental data in the current work, is for the IBP configuration. Details of the IBP test configuration and methodology for room temperature and cryogenic fluids are reserved for Chapters 4 and 5, respectively.

\subsubsection{Surface Tension Model}

To compare predicted bubble points from Equation 3.16 to experimental data, knowledge of surface tension, contact angle, and effective pore diameter is required. In general, surface tension is known to be a function of temperature and is well represented by:

$\gamma_{L V}=\gamma_{0}\left(1-T_{R}\right)^{K}$

where $\gamma_{0}, K$ are constants and $T_{R}$ is the reduced temperature defined in the traditional sense: 
$T_{R}=\frac{T}{T_{C}}$

where $T$ is the temperature of the liquid at the LAD screen and $T_{C}$ is the critical temperature (Ferguson and Kennedy 1936). A modified form of Equation 3.17 is used to predict surface tension across a wide range of room temperature and cryogenic liquids for the current work:

$\gamma_{L V}=\gamma_{0}\left(1-\frac{T}{T_{C}}\right)^{a_{0}+a_{1} \frac{T}{T_{C}}+a_{2} \frac{T^{2}}{T_{C}}}$

where $\gamma_{0}, a_{0}, a_{1}, a_{2}$ are fitting parameters and all temperatures are evaluated in Kelvin. Tables B-1 - B-6 in Appendix B list the LAD screens, liquids, and pressurant gas types over which bubble point data exists in the literature, as well as data from the current work. As shown, there is bubble point data available using 20 different liquids, in room temperature liquids acetone, ammonia, ethanol, Freon-113, Freon-114, IPA, methanol, silicon oil, and water, in storable propellants diborane $\left(\mathrm{B}_{2} \mathrm{H}_{6}\right)$, hydrazine $\left(\mathrm{N}_{2} \mathrm{H}_{4}\right), \mathrm{MMH}$, nitrogen tetroxide (NTO), oxygen diflouride $\left(\mathrm{OF}_{2}\right)$, and pentane $\left(\mathrm{C}_{5} \mathrm{H}_{12}\right)$, and cryogenic liquids, $\mathrm{LCH}_{4}, \mathrm{LOX}, \mathrm{LN}_{2}, \mathrm{LH}_{2}$, and $\mathrm{SFHe}$. Surface tension and critical temperature data is readily available for all 9 of the storable fluids (Moore et al. 1968, Jasper 1972, Ambrose et al. 1974 and 1975, Sato et al. 1991, Gude and Teja 1995, and DDBSP 2011). Less extensive data is available for storable propellants (Laubengayer et al. 1941, Paynter 1970 and 1973b, Jasper 1972, McCarty 1986, and Arnold 1999) and for all cryogenic fluids (Stansfield 1958, Jasper 1972, Brooks and Donnelly 1977, Jensen et al. 1980, McCarty 1986, and Van Sciver 1986). 
Curves were fit to the data to minimize the least squares distance between model and data. To generate smooth curves as a function of liquid temperature, curves were fit across the range of available data using an anchor point of zero surface tension at the critical point. Figure 3.5 plots model generated $\mathrm{LH}_{2}$ and $\mathrm{LN}_{2}$ surface tensions as a function of the ratio of liquid temperature to critical temperature versus the sparse surface tension data available in the literature. Results for all fluids are compiled in Table 3.1, which lists the temperature range over which surface tension and bubble point data is available, critical temperature, fitting coefficients, and error between the model curve and data. As shown, an excellent fit to the data is achieved using three fitting parameters for all fluids except SFHe and water, where the full 4 parameter fit was required.

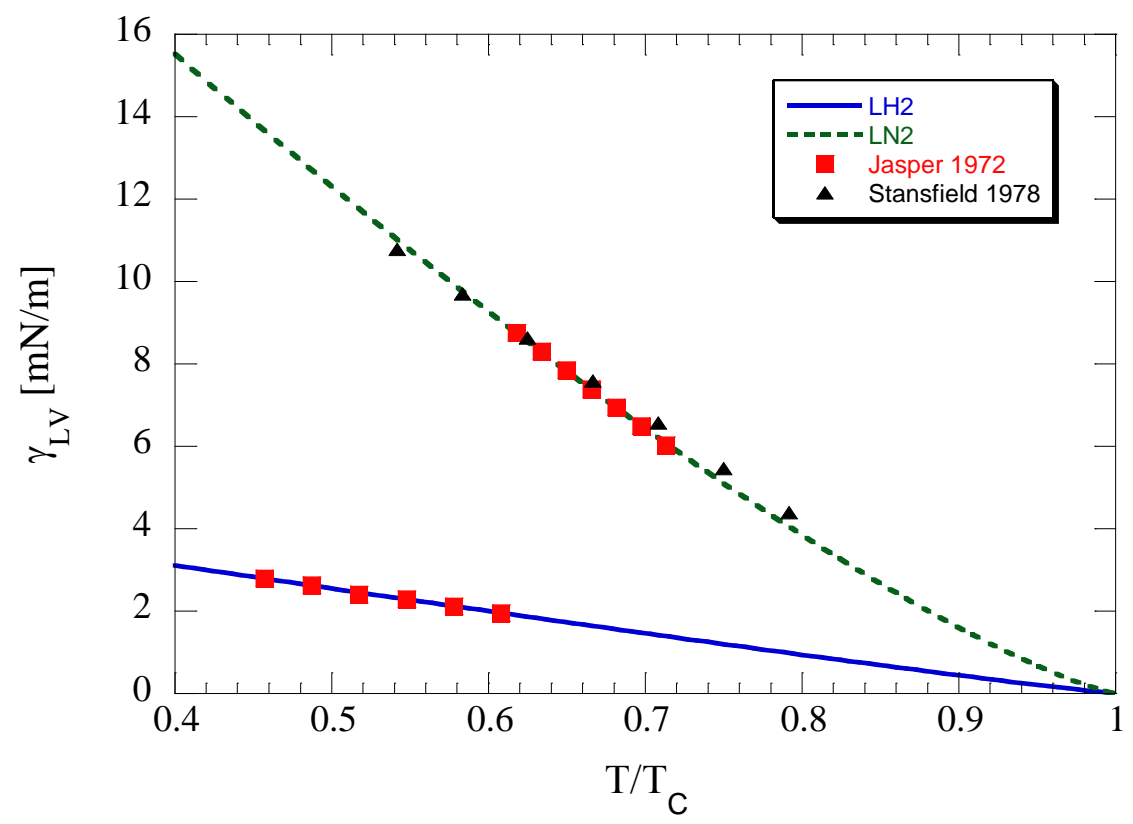

Figure 3.5 - Model Generated Liquid Hydrogen and Nitrogen Surface Tension against Data from Stansfield (1958) and Jasper (1972) 


\begin{tabular}{|c|c|c|c|c|c|c|c|c|c|}
\hline Fluid & $\operatorname{Tmin}[K]$ & $\operatorname{Tmax}[\mathrm{K}]$ & Tc [K] & $\gamma_{0}[\mathrm{mN} / \mathrm{m}]$ & $a_{0}$ & $a_{1}$ & $a_{2}$ & $r[\mathrm{mN} / \mathrm{m}]$ & Reference \\
\hline Acetone & 182 & 329 & 508.1 & 65.7 & 1.149 & 0 & 0 & $2.50 \mathrm{E}-05$ & Jasper 1972, Ambrose et al. 1974 \\
\hline B2H6 & 110 & 180 & 298.85 & 49.13 & 1.374 & 0 & 0 & $5.14 \mathrm{E}-05$ & Laubengayer et al. 1941, Jasper 1972 \\
\hline Ethanol & 220 & 350 & 513.9 & 46.2 & 0.525 & 0.545 & 0 & 3.11E-04 & Jasper 1972, Ambrose et al. 1975 \\
\hline Freon-113 & 200 & 375 & 487.21 & 52.72 & 1.095 & 0 & 0 & $1.81 \mathrm{E}-04$ & Jasper 1972 \\
\hline Freon-114 & 200 & 350 & 418.83 & 53.1 & 1.195 & 0 & 0 & $1.43 \mathrm{E}-06$ & Moore et al. 1968 \\
\hline LCH4 & 90 & 165 & 190.6 & 39.06 & 1.221 & 0 & 0 & $2.55 \mathrm{E}-10$ & McCarty 1986 \\
\hline LH2 & 14 & 24 & 32.94 & 5.431 & 1.090 & 0 & 0 & 4.74E-08 & Jasper 1972 \\
\hline LN2 & 65 & 120 & 126.2 & 29.71 & 1.271 & 0 & 0 & 4.00E-11 & Stansfield 1958, Jasper 1972 \\
\hline LOX & 70 & 135 & 154.6 & 38.46 & 1.222 & 0 & 0 & $8.00 \mathrm{E}-12$ & Jasper 1972 \\
\hline Methanol & 270 & 300 & 513.38 & 40.6 & 0.425 & 0.461 & 0 & $6.16 \mathrm{E}-05$ & Jasper 1972 \\
\hline NH & 200 & 330 & 405.4 & 90.9 & 1.096 & 0 & 0 & 1.57E-05 & Paynter 1970, Jasper 1972 \\
\hline OF2 & 110 & 170 & 213.45 & 51.8 & 1.280 & 0 & 0 & $2.02 \mathrm{E}-06$ & Paynter 1970 \\
\hline SFHe & 1 & 3 & 5.1953 & 0.39 & 0.115 & 0.811 & 0.428 & $1.92 \mathrm{E}-07$ & Jensen et al. 1980, Van Sciver 1986 \\
\hline Water & 220 & 350 & 647.096 & 0.086 & -0.012 & 0.371 & 0.803 & 3.93E-03 & Jasper 1972, Sato et al. 1991 \\
\hline
\end{tabular}

Table 3.1 - Fitting Parameters for the Surface Tension Model

\subsubsection{Specifying the Pore Diameter}

For popular mesh screens such as the Dutch Twill, as is evident in the SEM image in Figure 2.18, it is generally difficult to define a 2D pore diameter due to the complex 3D triangular shape of the $\mathrm{L} / \mathrm{V}$ interface within the screen. While previous studies have examined bubble points of single pores of various geometries to address this concern (Jurns and McQuillen 2008), there are three methods to estimate the effective pore diameter of the screen.

Method 1: The first and easiest way to approximate the pore diameter is to measure the bubble point using a "standard" reference fluid with known surface tension and use similitude to determine $D_{P}$ :

$$
D_{P}=\frac{4 \gamma_{r e f} \cos \theta_{c}}{\Delta P_{B P, r e f}}
$$


This requires knowledge of contact angle though. IPA is generally chosen as the reference fluid because its surface tension is much higher relative to cryogenic liquids, which permits a good calibration range for a given screen sample, and because the contact angle is expected to be small. This method is straightforward and is the most reliable.

Method 2: The second method to estimate the effective pore diameter is to calculate the diameter from the slope of a bubble point vs. surface tension (times the cosine of the contact angle) plot for each screen weave, summing over historical data available in the literature. This method is more attractive than the former single reference fluid measurement, since it is based on several different fluids across the surface tension range. However, there tends to be more variability in the final reported values due to sample to sample variation in pore diameters due to manufacturing defects, and due to the assumption of linearity between bubble point pressure and surface tension across the full temperature range.

Method 3: The third method to determine the effective pore diameter is to image the screens using an SEM and use geometry to estimate the 2D pore size. This is the only method than can be used if testing equipment or historical data is unavailable. Figure 3.6 shows an SEM image of the 325x2300 screen. Employing the previous methodology from Jurns and McQuillen (2008), a 2D triangular pore can be fit to the SEM image to approximate the pore diameter. The two dimensional triangular projection is broken up into two right triangles as shown in Figure 3.7, and the pore triangle is chosen to be the resultant hydraulic diameter of the planar surface: 
$D_{P, S E M} \approx \frac{4 A_{\mathrm{C}, S E M}}{P e}$

$A_{\mathrm{C}, S E M}$ is the cross sectional area normal to the fluid and $P e$ is the perimeter around the triangle:

$D_{P, S E M}=\frac{4\left(A_{I}+A_{I I}\right)}{\left(L_{1}+L_{2}+L_{3}\right)}$

where $A_{I}, A_{I I}$ and $L_{1}, L_{2}, L_{3}$ are the area and lengths of the triangle as shown in Figure 3.7, respectively. To determine accuracy of the SEM measurement against the simple room temperature bubble point method, these three methods for determining pore diameter will be compared in Chapter 4 for several different screens.

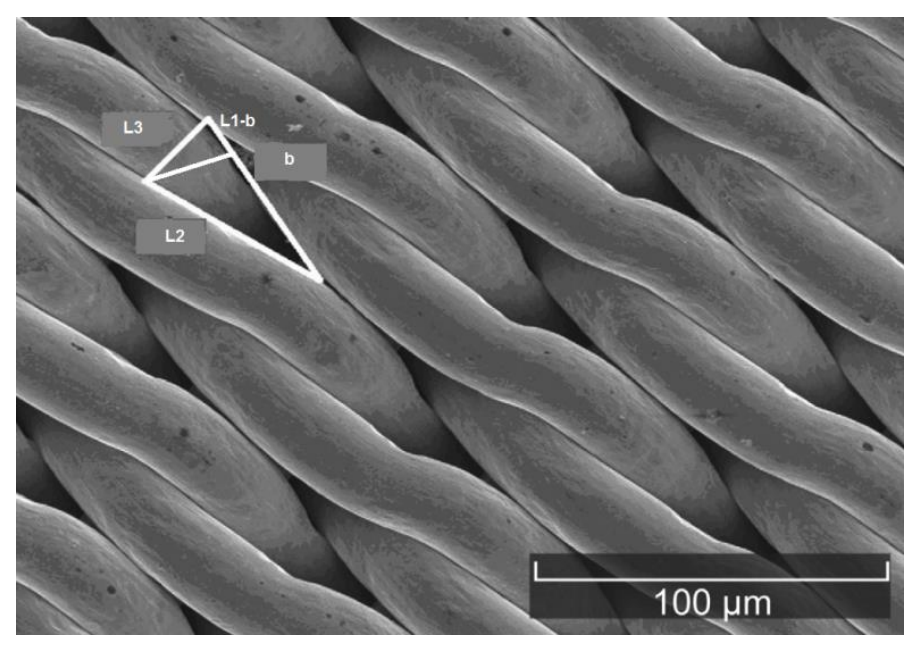

Figure 3.6 - Scanning Electron Microscopy Image of a 325x2300 Screen. Triangles are drawn to represent a simplified geometry of the complex screen pore. 


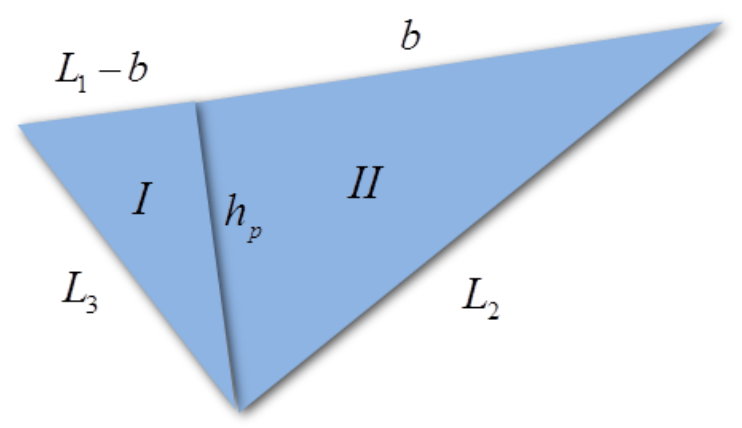

Figure 3.7 - Geometry of a Triangular Pore from Scanning Electron Microscopy Image

\subsubsection{Previously Reported Bubble Points}

Rigorous and comprehensive survey of the literature was performed to gather all previously reported bubble point data for all possible screens. Detailed results are reserved for Chapter 10, where the full cryogenic bubble point pressure model is constructed. Appendix B lists the 40 screens over which information is available in the literature. 24 of these 40 screens have actual historical bubble point data available; pore diameter data for two new screens are added for the current work for a total of 26 screens. Figures 3.8a and $\mathrm{b}$ plot historical bubble point data for the most popular two meshes, a 200x1400 and 325x2300 Dutch Twill mesh, respectively. Data is taken from Paynter (1973b), Burge and Blackmon (1973b), Cady (1973, 1975, and 1977), Chato and Kudlac (2002), Kudlac and Jurns (2006), and Jurns et al. (2007). Very few screens other than these two have data available in cryogenic liquids, and for screens where cryogenic bubble point data is available, the data is quite sparse. As shown, bubble point pressure scales with the surface tension of the liquid for both screens. Comparing Figures 3.8a to b, higher bubble points are obtained using the finer $325 \times 2300$ mesh over the $200 \times 1400$ mesh. 

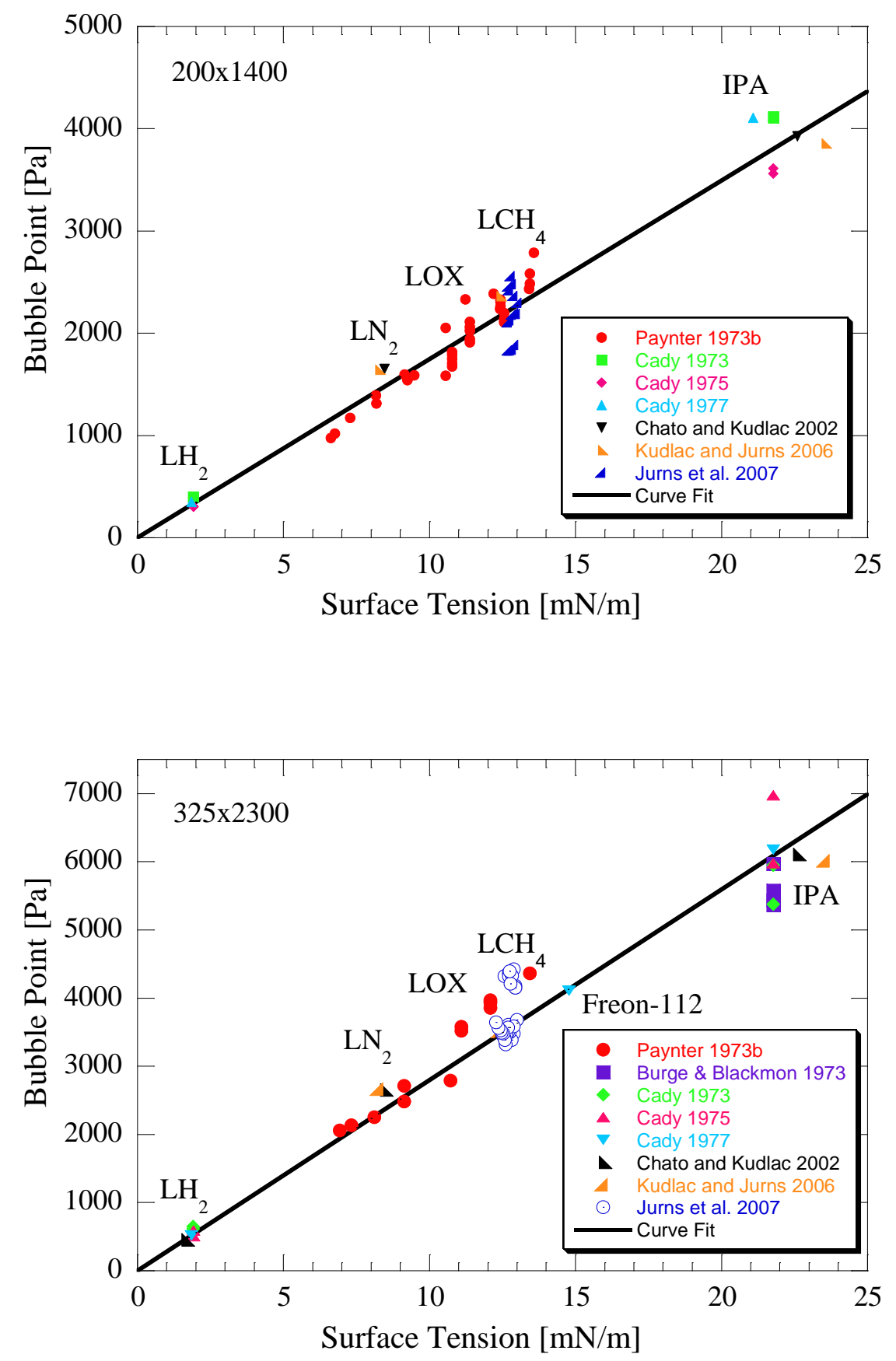

Figure 3.8 - Historical Bubble Point Data for a) 200x1400 and b) 325x2300 Mesh Screens

In addition to the above authors, in chronological order, historical bubble point data is also taken from Balzer et al. (1969), Alexander et al. (1970), Blatt (1970a and b), Heckman (1971), Castle (1972), Burge et al. 1973, Paynter (1973a), Stark et al. (1974), 
Simon 1979, Dodge and Bowles (1984), Bingham and Tegart (1977), Wilson and Meserole (1986), Meserole and Jones (1993), and Conrath and Dreyer (2009). Most historical references are associated with storable propulsion technology development programs such as the STS auxiliary propulsion system, and thus present data for high surface tension propellants with coarser mesh screens. Meanwhile, with the latest push towards advancing cryogenic propulsion technology, more recent references associated with Centaur upper stage CFM development, Orbital Transfer Vehicles (OTVs) (Heald and Merino 1979, Gilmore 1985, and Schuster and Brown 1987), and Space Station Freedom report data for finer mesh screens and lower surface tension cryogenic liquids. The CFM and CPST projects have funded most of the experiments from the current work.

Using Equation 3.20, a line can be fit to the bubble point versus surface tension data for a particular mesh to determine the average pore diameter across the range of liquid temperatures as exemplified by the black line in Figure 3.8. To obtain a rough order of magnitude of how the effective pore diameter scales with the fineness of the mesh, Table 3.2 lists the average effective pore diameter for the 24 historical screens where bubble point data is available. For each mesh, data was plotted, and a line was fit to the data to minimize the least squared distance between data and line to obtain a single value of $D_{P}$. Note that this assumes perfect linearity between bubble point pressure and surface tension, all the way down to cryogenic temperatures. 


\begin{tabular}{|c|c|c|c|c|c|c|c|c|}
\hline Mesh & Mesh Type & dwarp $[\mu \mathrm{m}]$ & dshute $[\mu \mathrm{m}]$ & Source, wire diameters & Avg. Dp [ $\mu \mathrm{m}]$ & $B[\mu \mathrm{m}]$ & $\mathrm{a}[\mu \mathrm{m}-1]$ & $\varepsilon$ \\
\hline $325 \times 2300$ & Dutch Twill & 38.1 & 25.4 & Industry & 14.1 & 91.4 & 0.1102 & 0.245 \\
\hline $325 \times 1900$ & Dutch Twill & 35.6 & 30.5 & Armour \& Cannon (1968) & - & 96.5 & 0.1005 & 0.215 \\
\hline $250 \times 1400$ & Dutch Twill & 55.9 & 38.1 & Industry & 19.1 & 132 & 0.0721 & 0.255 \\
\hline $250 \times 1370$ & Dutch Twill & 55.9 & 38.1 & Burge \& Blackmon (1973b) & 19.5 & 132 & 0.0708 & 0.267 \\
\hline $200 \times 1400$ & Dutch Twill & 71.1 & 40.6 & Industry & 23.1 & 152 & 0.0654 & 0.248 \\
\hline $165 \times 1400$ & Dutch Twill & 71.1 & 40.6 & Industry & - & 152 & 0.0611 & 0.306 \\
\hline $165 \times 800$ & Dutch Twill & 71.1 & 50.8 & Industry & 45.2 & 173 & 0.0415 & 0.43 \\
\hline $80 \times 700$ & Dutch Twill & 102 & 76.2 & Industry & 55.5 & 254 & 0.0318 & 0.369 \\
\hline $200 \times 600$ & Dutch Twill & 62.2 & 45.7 & Industry & 44.8 & 154 & 0.0356 & 0.552 \\
\hline $30 \times 500$ & Dutch Twill & 250 & 110 & Industry & - & 470 & 0.0171 & 0.461 \\
\hline $30 \times 160$ & Dutch Twill & 229 & 178 & Cady (1973) & - & 584 & 0.0078 & 0.635 \\
\hline $20 \times 250$ & Dutch Twill & 102 & 63.5 & Industry & - & 229 & 0.0097 & 0.835 \\
\hline $50 \times 250$ & Plain Dutch & 140 & 114 & Industry & 104 & 368 & 0.0131 & 0.611 \\
\hline $30 \times 250$ & Plain Dutch & 254 & 203 & Heckman (1971) & 155 & 660 & 0.0122 & 0.36 \\
\hline $24 \times 110$ & Plain Dutch & 381 & 267 & Industry & 200 & 914 & 0.0058 & 0.572 \\
\hline $720 \times 140$ & Reverse Dutch & 35.6 & 109 & Cady (1977) & 40 & 254 & 0.0439 & 0.03 \\
\hline $500 \times 500$ & Twill Square & 25.4 & 25.4 & Cady (1977) & 46 & 50.8 & 0.0655 & 0.584 \\
\hline $325 \times 325$ & Twill Square & 27.9 & 27.9 & Heckman (1971) & 65 & 55.9 & 0.0414 & 0.711 \\
\hline $180 \times 180$ & Twill Square & 53.3 & 53.3 & Paynter (1970) & 85.3 & 107 & 0.023 & 0.693 \\
\hline $120 \times 120$ & Twill Square & 50.8 & 50.8 & Heckman (1971) & 169 & 102 & 0.0257 & 0.674 \\
\hline $100 \times 100$ & Square & 114 & 114 & Industry & 118 & 228 & 0.0136 & 0.614 \\
\hline $230 \times 230$ & Square & 38.1 & 38.1 & Heckman (1971) & 94.5 & 76.2 & 0.0301 & 0.713 \\
\hline $200 \times 200$ & Square & 53.3 & 53.3 & Industry & 99.7 & 107 & 0.0268 & 0.643 \\
\hline $150 \times 150$ & Square & 66 & 66 & Heckman (1971) & 135 & 132 & 0.0199 & 0.671 \\
\hline $80 \times 80$ & Square & 140 & 140 & Heckman (1971) & 209 & 279 & 0.0108 & 0.622 \\
\hline $60 \times 60$ & Square & 191 & 191 & Heckman (1971) & 342 & 381 & 0.0081 & 0.612 \\
\hline $50 \times 50$ & Square & 229 & 229 & Industry & 307 & 458 & 0.0068 & 0.612 \\
\hline $40 \times 40$ & Square & 254 & 254 & Heckman (1971) & 544 & 508 & 0.0053 & 0.662 \\
\hline $30 \times 30$ & Square & 240 & 240 & Armour \& Cannon (1968) & 540 & 480 & 0.0039 & 0.769 \\
\hline
\end{tabular}

Table 3.2 - Measured and Calculated Screen Parameters

Figure 3.9 plots the average pore diameter based on Method 2, historical data as a function of the fineness of the mesh, in terms of the square root of the product of the number of warp wires, $n_{w}$ times shute wires, $n_{s}$. Results show that there is a fairly strong correlation between the effective pore diameter, which is not a physical parameter of the screen, and the fineness of the screen in terms of the square root of the number of pores per square inch, which is a physical parameter of the screen. Figure 3.9 implies that the finest mesh will have the highest bubble point pressure. 


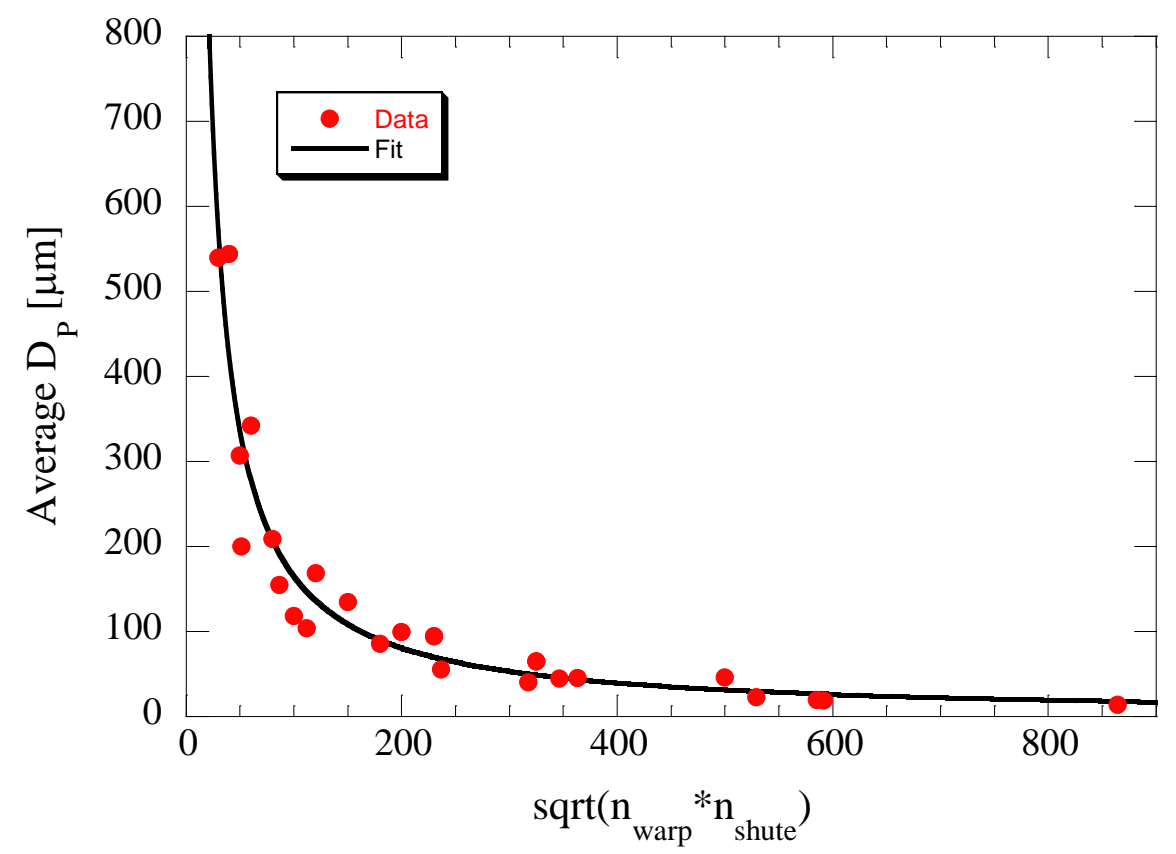

Figure 3.9 - Average Pore Diameter as a Function of the Fineness of the Mesh

\subsection{Hydrostatic Pressure Drop}

An important design consideration that influences LAD performance is the hydrostatic pressure of the liquid inside the channel, or more generally, the magnitude and direction of acceleration, be it from vehicle or from gravity, relative to the flow direction of the liquid inside the channel. Being able to supply vapor free liquid against high adverse accelerations is one of the primary reasons for choosing screen channels over other PMD types. For mission design, acceleration magnitude, level, and direction should be calculated first before selecting the appropriate screen. An example calculation is presented in Chapter 13 where a LAD is sized for a large scale $\mathrm{LH}_{2}$ fuel depot that experiences large adverse accelerations. 
The hydrostatic pressure drop is highlighted in red in Figure 3.10, which for a 1-g inverted vertical outflow configuration can be expressed simply as:

$\Delta P_{\text {hydrostatic }}=\rho g\left(L-L_{b}\right)$

where $\rho$ is the liquid density, $g$ is the gravitational constant, $L$ is the total length of the channel, and $L_{b}$ is the length of the channel below the tank L/V interface, which changes in time as the tank is drained. Therefore, as the liquid level in the tank drops, the liquid inside the channel must overcome a larger hydrostatic force. In LEO, calculation of hydrostatics is slightly more complex because propellant tank orbiting and spinning due to vehicle maneuvering turns the simple $1 \mathrm{D}$ problem into a $3 \mathrm{D}$ problem.

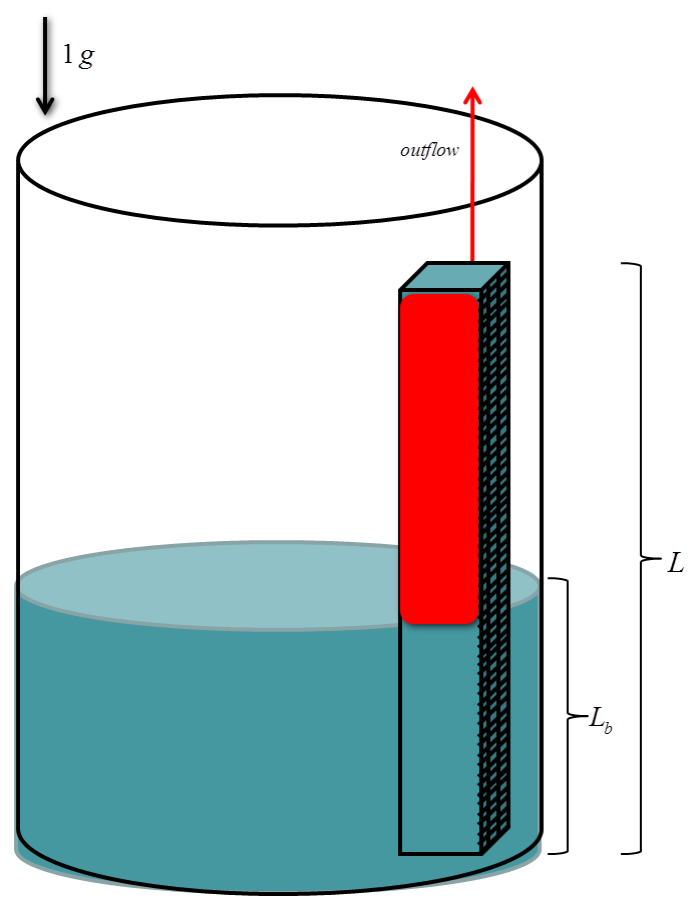

Figure 3.10 - Hydrostatic Pressure Drop in a 1-g Inverted Vertical Outflow Configuration 


\subsection{Flow-through-Screen Pressure Drop}

In 1-g or under significant vehicle acceleration, the dominant pressure drop in Equation 3.2 is hydrostatics. In the low Bond number conditions of space however, the dominant pressure loss will be the FTS pressure drop, since the hydrostatic pressure is generally negligible. For a given fluid, each screen has a specific FTS pressure drop associated with its specific geometry and manufacturing. Therefore the second primary influential factor governing LAD screen selection is the FTS pressure drop.

The FTS pressure drop is due to liquid flow through the wetted portion of the LAD screen in Figure 3.1. As the tank is drained, as the available area for liquid to flow is reduced, the FTS pressure drop increases proportionally. If the FTS pressure drop exceeds the bubble point value, bubbles will break through the screen. This parameter is also highly critical to LAD design, since finer meshes may produce higher bubble point pressures at the cost of higher FTS pressure drop. Therefore a balance between these two primary parameters is generally required for each mission.

\subsubsection{Model Derivation}

Due to the complex weave pattern for LAD screens, it is difficult to derive an exact solution for the flow through a LAD screen. An empirical solution from Armour and Cannon (1968) has been proposed as well as basic computational fluid dynamics simulations from Zhang et al. (2009) for the flow through a LAD screen. Using the logic from Armour and Cannon (1968), the approximate solution is formulated as the sum of the pressure drop due to viscous (laminar) and inertial (turbulent) resistance. 
For viscous resistance, the screen is approximated as a packed bed of spherical particles. In a screen of unit cross-sectional area, the number of particles is:

$$
N=\frac{V_{\text {solid }}}{V_{\text {sphere }}}
$$

where $N$ is the number of spheres per area, $V_{\text {solid }}$ is the solid volume fraction (units of length) and $V_{\text {sphere }}$ is the volume of a spherical particle with radius $r$. A simplified expression for $V_{\text {solid }}$ is obtained by rewriting it as $(1-\varepsilon) B$, where $\varepsilon$ is the dimensionless void fraction and $B$ is the thickness of the screen. Equation 3.24 can thus be rewritten as:

$$
N=\frac{(1-\varepsilon) B}{\frac{4}{3} \pi r^{3}}
$$

The total drag force on a unit cross-sectional area of the LAD screen is:

$$
F_{T}=N F_{D}
$$

where $F_{D}$ is the drag force around a single sphere. Because the viscous resistance term is fully laminar, Stokes solution for creeping flow around a sphere is used, and the total drag force becomes:

$$
F_{T}=\frac{3(1-\varepsilon) B}{4 \pi r^{3}} 6 \pi \mu r U
$$

where $U$ is the fluid velocity and $\mu$ is the viscosity. Since the screen wires are slightly crushed together during manufacturing, obviously Stokes solution is an idealization. An approximation which takes into account the error associated with the idealization can be 
made by multiplying the total drag force by a constant times the solid volume fraction, $C(1-\varepsilon)$ and replacing the velocity by the free stream velocity, $\frac{U}{\varepsilon}$. The constant must be determined through experiment. Equation 3.27 becomes:

$F_{T}=C(1-\varepsilon) \frac{3(1-\varepsilon) B}{4 \pi r^{3}} 6 \pi \mu r \frac{U}{\varepsilon}$

For a LAD screen, the drag force is equal to the viscous pressure drop times the void fraction. Simplifying, Equation 3.28 becomes:

$\Delta P_{v i s c} \varepsilon=C\left(\frac{3(1-\varepsilon)}{r}\right)^{2} \frac{B \mu U}{2 \varepsilon}$

The term in parenthesis is the surface area to volume ratio $a$, and it is this relationship between $a$ and $r$ that makes the expression applicable to non-spherical packings. The constant $C / 2$ is combined into a new constant $\alpha$, and the viscous pressure drop term becomes:

$\Delta P_{v i s c}=\alpha \frac{B a^{2} \mu}{\varepsilon^{2}} U$

Meanwhile, the inertial resistance term is dominated by turbulent flow. Pressure drop in turbulent pipe flow is proportional to a frictional coefficient times $\rho U^{2}$. Approximating the LAD screen as a bank of closely packed pipes, the relationship between turbulent pressure drop and velocity is simply:

$$
\Delta P_{\text {inertial }}=\beta \rho\left(\frac{U}{\varepsilon}\right)^{2} \frac{B}{D_{P}}
$$


where $D_{P}$ is the screen pore diameter and $\beta$ is the constant which accounts for non-ideal flow as in the case of the viscous pressure term. The total pressure drop for a LAD screen becomes:

$$
\Delta P_{F T S}=\alpha\left(\frac{B \mu a^{2}}{\varepsilon^{2}}\right) U+\beta\left(\frac{B}{\varepsilon^{2} D_{P}}\right) U^{2}
$$

Equation 3.32 holds for a standard Plain Square or Twilled Square screen, where there is a direct flow path through the screen. For the Dutch Twill screens used in the current work, the flow path is tortuous and so a tortuosity factor, $Q$ is incorporated into Equation 3.32. Finally, Equation 3.32 can be rewritten in terms of the mass flow rate and cross sectional area of the channel, and the final expression is obtained for the total FTS pressure drop:

$$
\Delta P_{F T S}=\alpha\left(\frac{Q B \mu a^{2}}{\rho \varepsilon^{2}}\right) \frac{\dot{m}}{A_{C}}+\beta\left(\frac{Q B}{\rho \varepsilon^{2} D_{P}}\right)\left(\frac{\dot{m}}{A_{C}}\right)^{2}
$$

where $A_{C}$ is the cross sectional area of the screen.

\subsubsection{Model Parameters and Flow-through-Screen Experiment}

Examination of Equation 3.33 indicates that the FTS pressure drop is a function of both fluid properties and screen parameters. All LAD screen properties can be estimated through simple knowledge of the number of warp and shute wires per square inch, $n_{w}, n_{s}$, respectively, and the diameters of the warp and shute wires, $d_{w}, d_{s}$, respectively. This simplicity makes pressure drop calculations facile. Table 3.3 lists the equations needed to calculate screen thickness $B$, surface area to volume ratio $a$, and void fraction (or 
porosity) $\varepsilon$ for Plain Square, Twilled Square, Plain Dutch, and Dutch Twill LAD screen types. While the formulas seem complex, they are all based on geometrical considerations. For example, the screen thickness $B$ of a Dutch Twill is simply twice the shute diameter thickness plus the warp diameter thickness. Values of $D_{P}$ can be taken from Table 3.2. Alternatively, one could approximate the effective pore diameter by interpolation using Figure 3.9.

Table 3.2 lists values for warp and shute wire diameters, along with calculated values for the screen thickness, surface area to volume ratio, and void fraction. Values of $d_{w}$ and $d_{s}$ are obtained from the literature (Armour and Cannon 1968, Paynter 1970, Heckman 1971, Burge and Blackmon 1973b, and Cady 1973) or from industry, where available. Tortuosity values are 1.0 for Plain Square and Twilled Square weaves and 1.3 for Reverse Twill, Plain Dutch, and Twilled Dutch weaves.

Details of the experimental procedure for conducting a FTS experiment are reserved for the LOX and $\mathrm{LH}_{2}$ FTS tests in Chapter 9; a brief description is presented here. A screen sample is fit into the cross-section of a pipe so that single phase (either gas or liquid) flow through the pipe must pass through the screen. This is especially difficult in cryogenic liquid flow. A differential pressure transducer (DPT) is installed so that it can measure the pressure drop across the screen. A flow meter is placed either downstream or upstream of the screen to measure the flow through the screen. Temperature and pressure measurements are taken near the screen to define the thermodynamic state of the fluid at the entrance to the screen. With these measurements 
taken over a range of flow rates, screens, and fluids, it is possible to obtain the fitting parameters $\alpha$ and $\beta$ in Equation 3.33.

\subsubsection{Historical Data and Trends}

To validate the FTS pressure drop model, data was compiled from 9 different historical studies where FTS data was available. Data was taken from Ingmanson (1961), Armour and Cannon (1968), Blatt (1970b), Castle (1972), Burge et al. (1973), Burge and Blackmon (1973b), Cady (1973 and 1977), and Ludewig et al. (1974). Data reported in Amenus (1965) was unusable because authors did not report mass flow rate. Data from Kressilk (1969), Hines (1971), and Wintec (1972) are included in the set from Cady (1973), for brevity.

To compare performance across all LAD screens, and to determine fitting parameters $\alpha$ and $\beta$, Equation 3.33 is cast into a non-dimensional form. The resulting equation is in terms of a modified Reynolds (Re) number based on characteristic length

of $\frac{1}{a^{2} D_{P}}$, friction factor $f$, and the equation relating the two, which are defined as:

$$
\begin{aligned}
& R e_{D_{P}}=\frac{1}{\mu a^{2} D_{P}}\left(\frac{\dot{m}}{A_{C}}\right) \\
& f=\frac{\Delta P_{F T S} \rho \varepsilon^{2} D_{P}}{Q B}\left(\frac{A_{C}}{\dot{m}}\right)^{2} \\
& f=\frac{\alpha}{\operatorname{Re}_{D_{P}}}+\beta
\end{aligned}
$$


The first term on the right hand side of Equation 3.36 is the contribution due to the laminar spherical particle model from Equation 3.30 while the second term is the contribution due to the turbulent flow model from Equation 3.31. Through substitution, friction factor and $R e_{D_{P}}$ are related through Equation 3.36. Figures 3.11 and Figures 3.12 plot $f$ against $R e_{D_{P}}$ on a $\log / \log$ plot for Dutch Twill, and all other weaves, respectively. At low $R e_{D_{P}}$ where laminar flow dominates, the data appears to be negatively linear on a $\log / \log$ scale. This confirms the inverse proportional relationship between $f$ and $R e_{D_{P}}$ from Equation 3.30, thus confirming the laminar spherical particle model. At high $R e_{D_{P}}$, the data asymptotically approaches a constant. This agrees with Equation 3.36, since the first term becomes very small for high $R e_{D_{P}}$, and $f$ is approximately equal to the constant $\beta$. Thus, the turbulent flow model is verified by the experimental data.

Table 3.4 lists the FTS pressure drop fitting parameters for Dutch Twill, and all other weaves, respectively. Dutch Twills yield smaller FTS pressure drops in both laminar and turbulent regimes, due to more available flow area, higher void fraction, etc. For most accurate results, a LAD designer should conduct a simple FTS test on a new LAD sample; data from Figures 3.11 and 3.12 are meant for preliminary design or where testing is unavailable. 


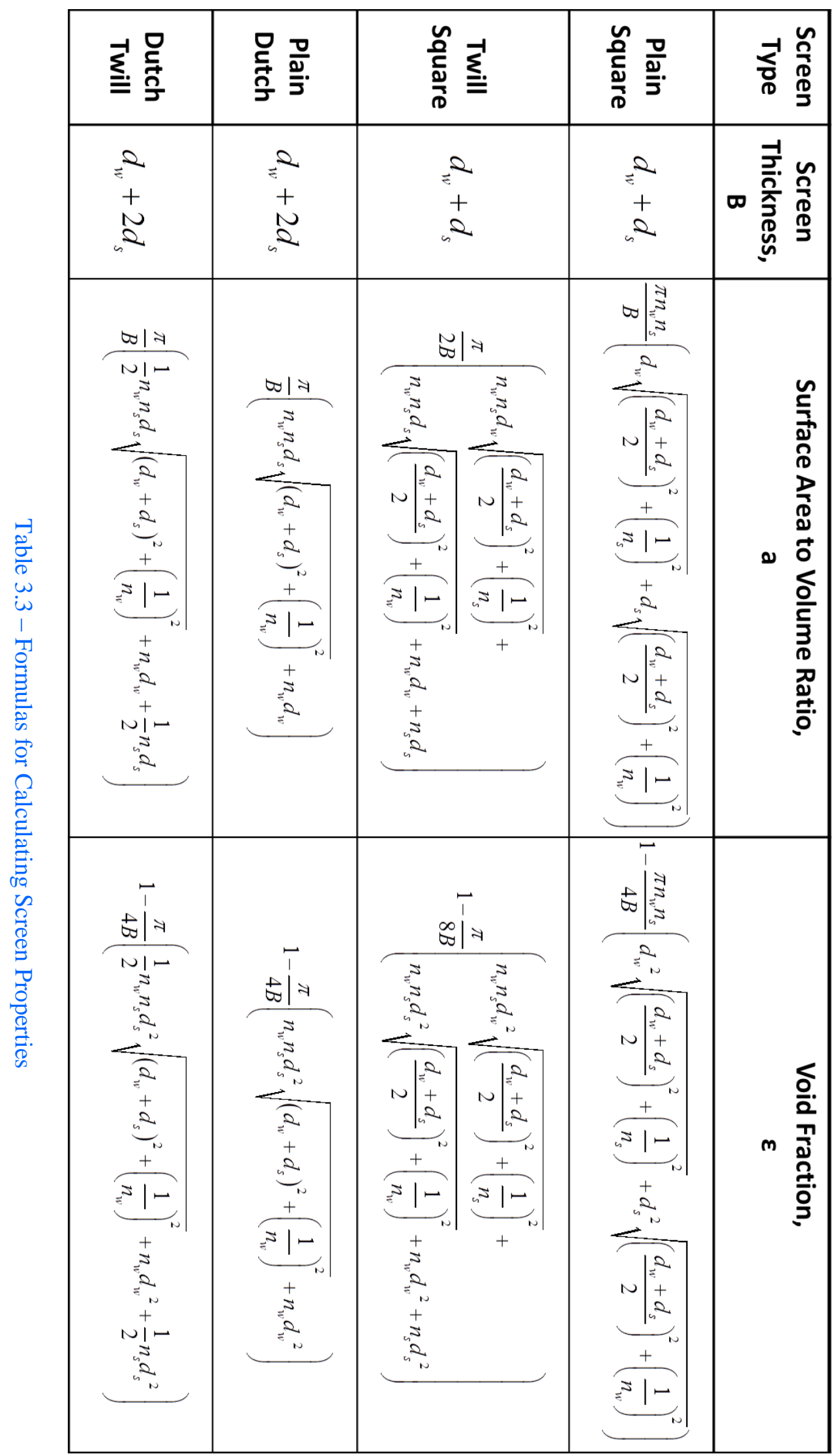




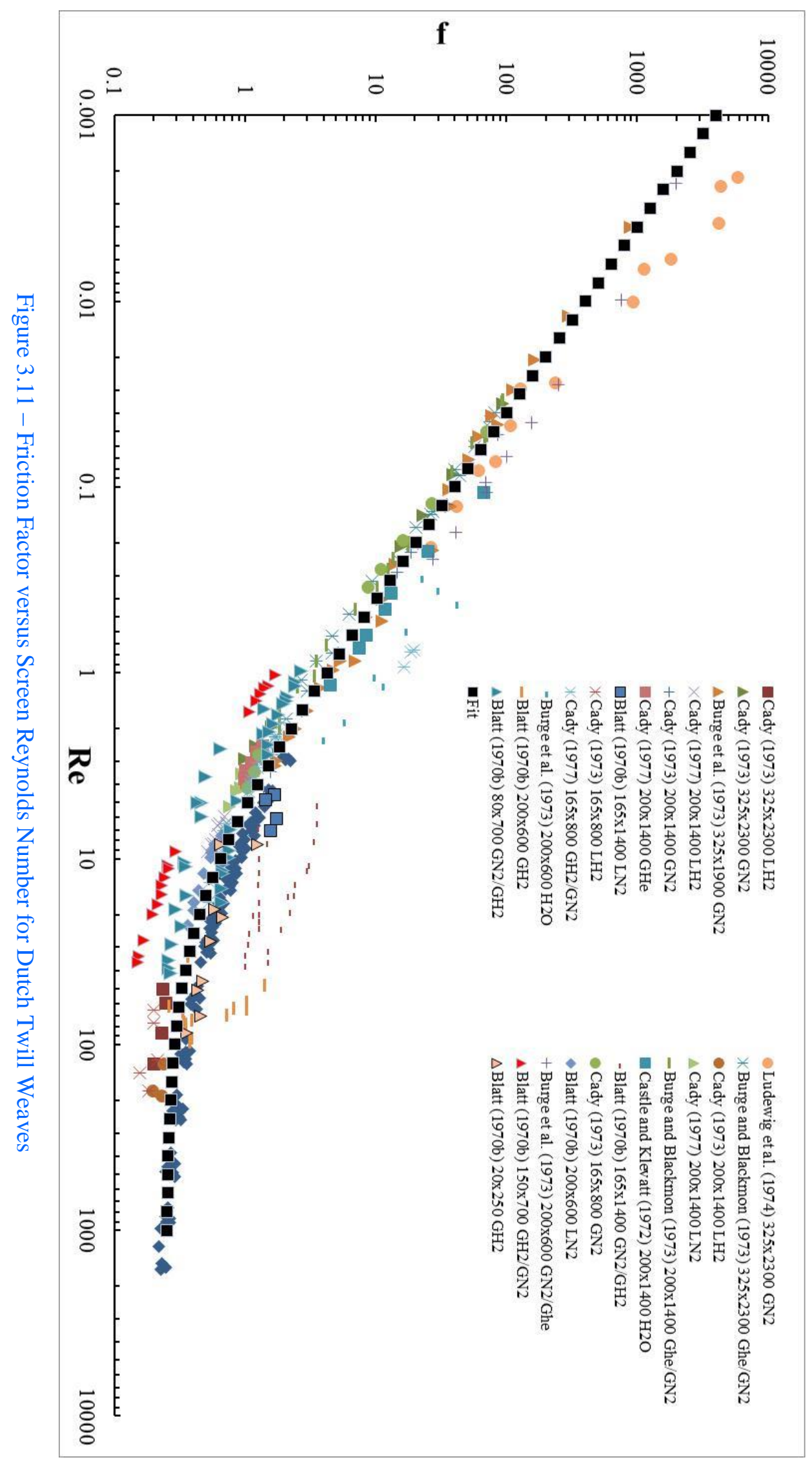




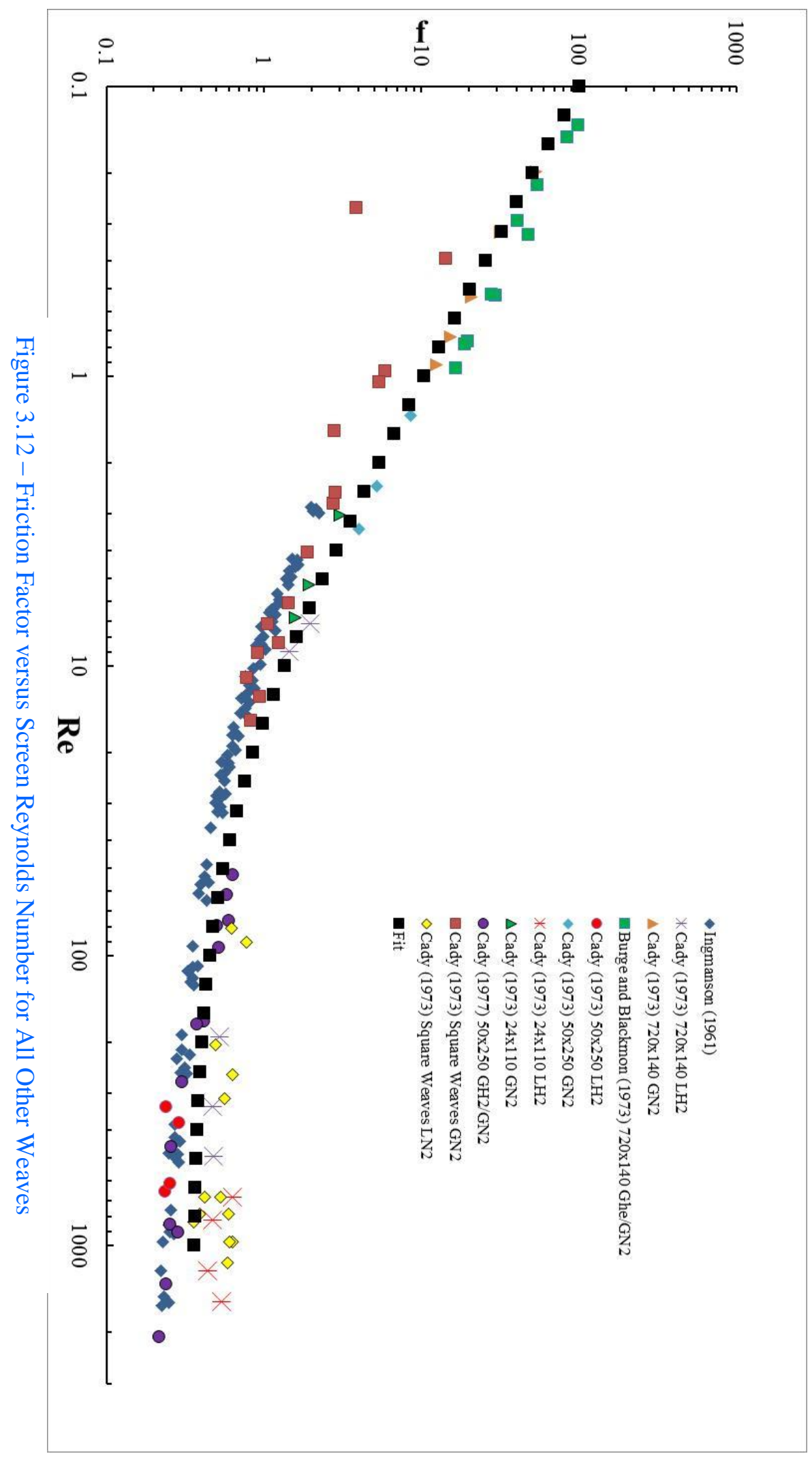




\begin{tabular}{|c|c|c|}
\hline Weave & $\boldsymbol{\alpha}$ & $\boldsymbol{\beta}$ \\
\hline Dutch Twills & 7.22 & 0.217 \\
All Others & 10 & 0.35 \\
\hline
\end{tabular}

Table 3.4 - Fitting Parameters for Flow-through-Screen Pressure Drop Model

\subsection{Frictional and Dynamic Pressure Drop}

Viscous and inertial frictional pressure losses and dynamic head pressure losses arise due to propellant flowing down the channel to the exit as indicated in the red arrows in Figure 3.13. Consider the vertically oriented LAD channel as shown in Figure 3.1 and 3.13, with the origin attached to the bottom center of the channel. The list of assumptions and corresponding implications used to solve for the viscous pressure drop inside the channel are as follows:

1. Constant and uniform properties $\therefore \rho, \mu$ constant

2. Isotropic fluid

3. Newtonian fluid

4. Stoke's hypothesis holds $\quad \therefore$ Navier-Stokes equations apply

5. Steady state

$$
\therefore \frac{D}{D t}() \rightarrow 0
$$

6. Incompressible fluid $\quad \therefore \frac{D \rho}{D t} \rightarrow 0$

7. Gravity force is accounted for in the hydrostatic term and is therefore ignored $\therefore F_{B}=0$ 
8. Flow in the $\mathrm{x}$ direction is small $\therefore v_{x}<<v_{z}$

9. Flow in the y direction is small $\therefore v_{y}<<v_{z}$

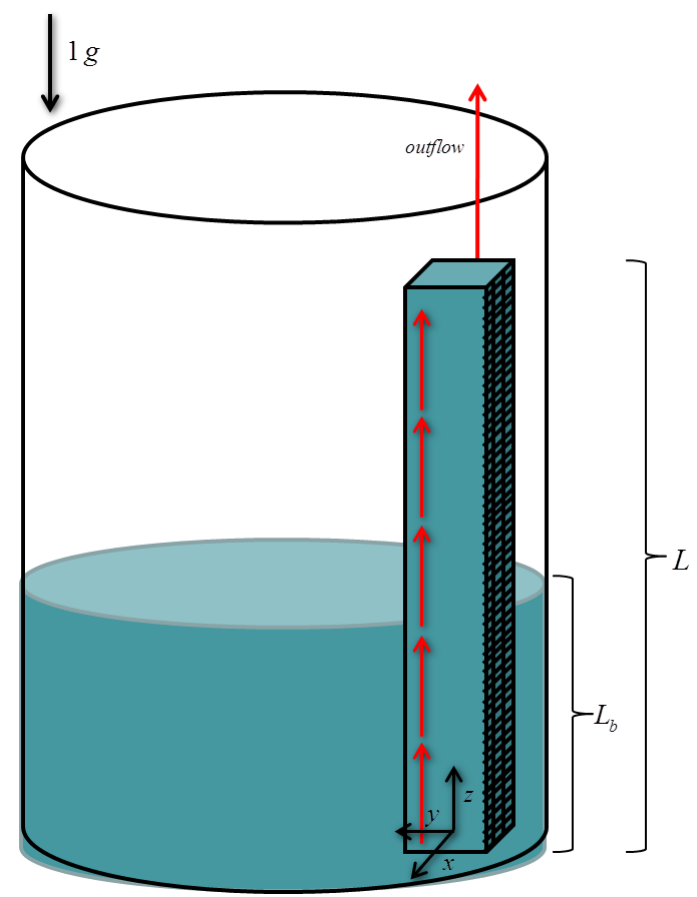

Figure 3.13 - Frictional and Dynamic Pressure Losses down Liquid Acquisition Device Channel

The set of governing equations of continuity and $\mathrm{x}, \mathrm{y}$, and $\mathrm{z}$ momentum:

$\frac{D \rho}{D t}=0$

$\frac{\partial(\rho \underset{\sim}{\rho})}{\partial t}+\underset{\sim}{v} \nabla \bullet \underset{\sim}{\rho} \underset{\sim}{v}+\underset{\sim}{\rho} \bullet \underset{\sim}{\nabla} \underset{\sim}{v}=\nabla P+\mu \nabla^{2} \underset{\sim}{v}$

reduce to the following set of equations after applying the assumptions:

$\frac{d v_{z}}{d z}=0$ 


$$
\begin{aligned}
& \frac{\partial P}{\partial x}=0 \\
& \frac{\partial P}{\partial y}=0 \\
& \frac{\partial^{2} v_{z}}{\partial x^{2}}+\frac{\partial^{2} v_{z}}{\partial y^{2}}=\frac{1}{\mu} \frac{\partial P}{\partial z}
\end{aligned}
$$

Continuity implies $v_{z}(x, y, z) \rightarrow v_{z}(x, y) ; \mathrm{x}, \mathrm{y}$-momentum equations imply $P(x, y, z) \rightarrow P(z)$. Equations 3.38d thus simplifies to:

$$
\frac{\partial^{2} v_{z}}{\partial x^{2}}+\frac{\partial^{2} v_{z}}{\partial y^{2}}=\frac{1}{\mu} \frac{d P}{d z}
$$

The right hand side of Equation 3.39 is a constant that depends on $\mathrm{z}$ alone. This is a second order, constant coefficient partial differential equation (PDE) in $\mathrm{x}$ and $\mathrm{y}$. Consistent with Figures 3.1 and 3.13, the boundary conditions (BCs) necessary to solve the PDE are:

$$
\begin{aligned}
& v_{z}\left( \pm \frac{W}{2}, y\right)=0 \\
& v_{z}\left( \pm x, \frac{H}{2}\right)=0
\end{aligned}
$$

$$
\begin{aligned}
& \left.\frac{\partial v_{z}}{\partial x}\right|_{x=0}=0 \\
& \left.\frac{\partial v_{z}}{\partial y}\right|_{y=0}=0
\end{aligned}
$$

The first two are the no slip conditions and the second two are symmetry conditions. The method of solution is to break Equation 3.39 into a homogenous PDE with one nonhomogeneous $\mathrm{BC}$ and a nonhomogeneous ordinary differential equation (ODE) with homogeneous BCs as follows: 
$\mathrm{v}_{z}(\mathrm{x}, \mathrm{y})=\phi(\mathrm{y})+\psi(\mathrm{x}, \mathrm{y})$

Plugging Equation 3.41 into Equations 3.39 and 3.40a - d, Equations 3.42a - e and 3.43a $-c$ are obtained:

$\frac{\partial^{2} \psi}{\partial x^{2}}+\frac{\partial^{2} \psi}{\partial y^{2}}=0$

$\psi\left(\mathrm{x}, \frac{H}{2}\right)=0$

$\left(\frac{\partial \psi}{\partial x}\right)_{x=0}=0$

$$
\frac{\partial^{2} \phi}{\partial y^{2}}=0
$$

$\left(\frac{\partial \psi}{\partial y}\right)_{y=0}=0$

$\phi\left(\frac{H}{2}\right)=0$

$\left(\frac{\partial \phi}{\partial y}\right)_{y=0}=0$

$\psi\left(\frac{W}{2}, \mathrm{y}\right)=-\phi(y)$

The solution of the PDE is straightforward using separation of variables and solving for the eigenvalues. The solution of the ODE is straightforward as well. Details are omitted. The final solution for steady state velocity inside the channel becomes:

$v_{z}(x, y)=\frac{1}{2 \mu} \frac{d P}{d z}\left(y^{2}-\frac{H^{2}}{4}\right)+\sum_{n=0}^{\infty} \frac{4 \frac{d P}{d z}(-1)^{n}}{H \lambda_{n}^{3} \mu \cosh \left(\lambda_{n} \frac{W}{2}\right)} \cosh \left(\lambda_{n} y\right) \cos \left(\lambda_{n} y\right)$

The volumetric flow rate is calculated by integrating Equation 3.44:

$\dot{V}=\int_{-W / 2}^{W / 2} \int_{-H / 2}^{H / 2} v_{z}(x, y) d x d y$

Integrating, 
$\dot{V}=-\frac{H^{3} W}{12 \mu} \frac{d P}{d z}-\sum_{n=0}^{\infty} \frac{16}{H \lambda_{n}^{5} \mu} \frac{d P}{d z} \tanh \left(\frac{\lambda_{n} W}{2}\right)$

Assuming an incompressible fluid,

$\dot{m}=\rho \dot{V}$

Equations 3.46 and 3.47 can then be used to solve for the viscous pressure drop in terms of the mass flow rate up the channel:

$-\frac{d P}{d z}=\frac{\dot{m}}{\rho}\left\{\frac{H^{3} W}{12 \mu}+\sum_{n=0}^{\infty} \frac{16}{\mu H \lambda_{n}^{5}} \tanh \left(\frac{W \lambda_{n}}{2}\right)\right\}^{-1}$

Since the viscous pressure drop is linear with $\mathrm{z}$ :

$\Delta P_{\text {viscous }}(z)=\frac{\dot{m} z}{\rho}\left\{\frac{H^{3} W}{12 \mu}+\sum_{n=0}^{\infty} \frac{16}{\mu H \lambda_{n}^{5}} \tanh \left(\frac{W \lambda_{n}}{2}\right)\right\}^{-1}$

Meanwhile the inertial pressure drop obeys the relation:

$\Delta P_{\text {ineritial }}(z)=f \frac{z}{D_{H}} \frac{\rho v_{z}^{2}}{2}$

For high Re flows, the friction factor is independent of Reynolds number and depends on roughness, $e$ (Cady 1973):

$$
f=\frac{1}{4\left(\log \left(\frac{3.7}{e / D_{H}}\right)\right)^{2}}
$$

The hydraulic diameter is defined as: 


$$
D_{H}=\frac{4 A}{P e}
$$

For Dutch Twill screens, an appropriate hydraulic diameter is taken as:

$$
D_{H}=\frac{4(W H)}{2(H+W)}
$$

The roughness can be approximated as twice the shute wire diameter. Plugging Equations 3.51 and 3.53 into Equation 3.50 and rewriting in terms of mass flow rate:

$$
\Delta P_{\text {inertial }}(z)=\frac{z(\dot{m})^{2}(H+W)}{16 \rho W^{3} H^{3}\left(\log \left(\frac{14.8 W H}{d_{s}(W+H)}\right)\right)^{2}}
$$

The total frictional pressure drop inside the channel is the sum of the viscous and inertial pressure drops:

$$
\Delta P_{\text {frictional }}(z)=\frac{\dot{m} z}{\rho}\left\{\frac{H^{3} W}{12 \mu}+\sum_{n=0}^{\infty} \frac{16}{\mu H \lambda_{n}^{5}} \tanh \left(\frac{W \lambda_{n}}{2}\right)\right\}^{-1}+\frac{(\dot{m})^{2} z(H+W)}{16 \rho W^{3} H^{3}\left(\log \left(\frac{14.8 W H}{d_{s}(W+H)}\right)\right)^{2}}
$$

which is of the general form of $\Delta P_{\text {frictional }}=C_{1} \dot{m}+C_{2}(\dot{m})^{2}$, as expected. Thus the expression appearing in Van Dyke (1998) is incorrect, as the pressure drop proposed there is not proportional to channel length, $z$ or $L$, as is the case in Equation 3.55. Because the 1D steady state model assumes a constant inflow into the channel due to 
constant FTS pressure drop across the screen, to satisfy continuity, the frictional pressure drop and thus flow rate inside the channel, will vary linearly with $z$ :

$$
\dot{m}=\dot{m}_{\text {demand }}\left[\left(1-\frac{H}{L}\right) \frac{z}{L}+\frac{H}{L}\right]
$$

where $\dot{m}_{\text {demand }}$ is the desired demand mass flow rate at the channel exit. Equation 3.55 can be used to evaluate the frictional pressure drop at the point of maximum pressure difference across the screen, located at the top of the channel:

$$
\Delta P_{\text {frictional }}=\frac{\dot{m} L}{\rho}\left\{\frac{H^{3} W}{12 \mu}+\sum_{n=0}^{\infty} \frac{16}{\mu H \lambda_{n}^{5}} \tanh \left(\frac{W \lambda_{n}}{2}\right)\right\}^{-1}+\frac{(\dot{m})^{2} L(H+W)}{16 \rho W^{3} H^{3}\left(\log \left(\frac{14.8 W H}{d_{s}(W+H)}\right)\right)^{2}}
$$

Finally, the dynamic head pressure inside the channel is simply expressed as:

$$
\Delta P_{\text {dynamic }}=\frac{\dot{m}^{2}}{2 \rho H^{2} W^{2}}
$$

There is no known frictional LAD channel loss data reported in the literature. Equations 3.23, 3.33, 3.57 and 3.58 can be substituted into Equation 3.2 for a given LAD system to determine the total steady state pressure drop for the LAD system for any configuration. $1 \mathrm{D}$ model results are compared to 1 -g inverted vertical outflow test results in $\mathrm{LH}_{2}$ in Chapter 9. 


\subsection{Wicking Rate}

Screen channel LADs can support high tank outflow rates across a much wider range of thermal conditions and gravitational levels relative to other LADs. Another advantage of screen channel LADs is the ability to wick liquid to areas of the screen that dry out due to evaporation during tank pressurization and outflow. While not a pressing issue with storable liquid systems, evaporation can greatly diminish the performance of LADs with cryogenic liquids. When relatively warm vapor touches a fully wetted screen, some of the liquid in the pores may evaporate, reducing the capillary pressure and thus bubble point of the screen. While this reduction in capillary pressure does not imply breakdown or failure of the $\mathrm{LAD}$, it can reduce the bubble point and thus diminish the performance of the LAD. However, if the propellant in a section of a screen starts to dry out, the fluid from the fully wetted screen surrounding it will naturally wick towards that

area so that it is rewetted. Therefore the wicking rate is an important secondary factor and the third influential factor in LAD screen selection for cryogenic systems.

The wicking rate is defined as the speed at which the liquid propellant rewets the screen. The higher the wicking rate, the quicker the dried out area is rewetted. Wicking flow is driven by the capillary pressure, similar to the phenomena governing the bubble point. For wicking to occur, the liquid must reside in a capillary tube or a similar enclosure to generate the capillary pressure. Dutch Twill and Plain Dutch meshes have a geometry that enables wicking to occur within the screen. Square meshes, however, do not have a geometry that generates any capillary pressure. Therefore, for screen channel LADs, wicking rate is highly dependent on the specific mesh. The wicking rate is also dependent on the direction of wicking relative to the orientation of the wires. For any 
given Dutch Twill or Plain Dutch screen, the liquid may wick faster along the warp wire direction or along the shute wire direction. This is due the warp and shute wires having different diameters, thus creating different shaped flow paths in either direction.

\subsubsection{Model Derivation}

A derivation of the wicking rate is as follows: The incompressible liquid assumption is valid for cryogenic and storable propellants because the wicking process typically generates very low speeds. Consider a screen with one section completely wetted within a liquid reservoir while the remaining portion is completely dry as depicted in Figure 3.14. The liquid immediately begins to wick along the screen away from the liquid reservoir at speed $v_{W}$. At some time later the wicking front, or the dividing line between the wetted and dry screen portions, has traveled a distance $L_{W}$ from the liquid reservoir, and the wicking velocity $v_{W}$ has diminished to a smaller value.

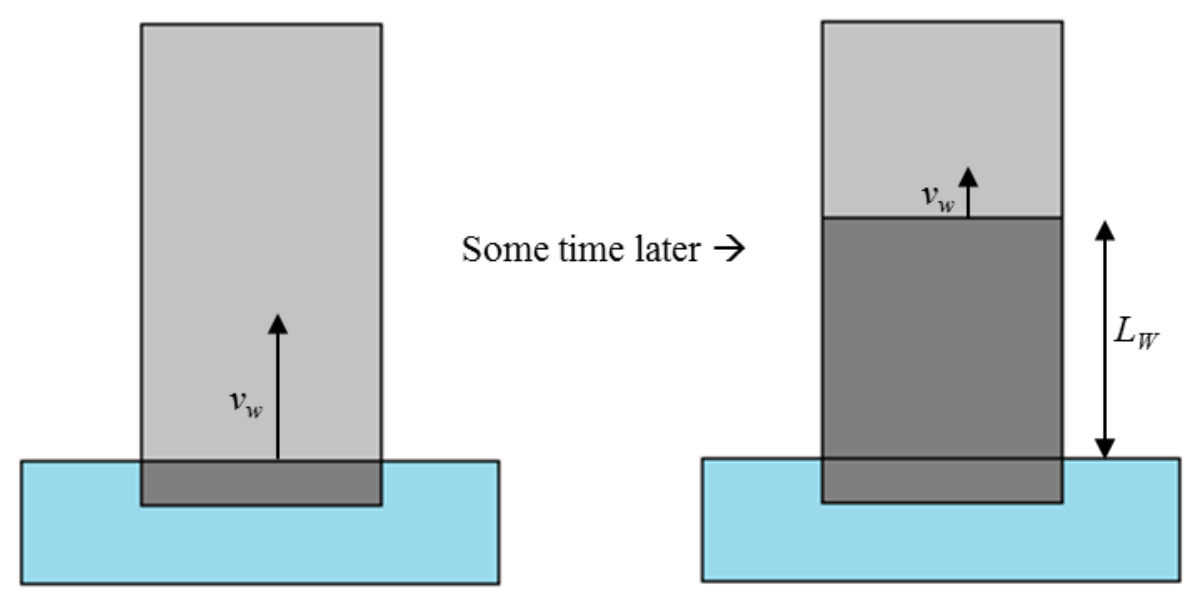

Figure 3.14 - Illustration of the Wicking Phenomena 
The screen is modeled as a sheet of capillary tubes lying parallel to the direction of wicking, which allows the following equation for pressure difference in a capillary tube to be applied to the screen:

$$
\Delta P_{C}=\frac{4 \gamma_{L V} \cos \theta_{C}}{D_{C}}
$$

The capillary diameter, $D_{C}$ takes into account the complex geometry within the Dutch Twill and Plain Dutch meshes. This equation looks similar to the bubble point equation (Equation 3.16), but $D_{C}$ is not necessarily the same as $D_{P}$ since the bubble point pressure is directed normal to the screen plane and the wicking capillary pressure is directed along the screen plane. Second, "capillary tubes" within the screen, which can be thought of as capillary pathways, are so small that the flow can be modeled by pressure driven flow in a channel with circular cross section, or Poiseuille flow. In this type of flow, the driving pressure is balanced by the frictional resistance generated at the tube surface. In real capillary flow, there is an entrance region where inertial effects are important and the equation for Poiseuille flow is not appropriate. However, it has been shown that for small diameter capillary tubes this entrance region is negligibly small (Washburn 1921). With this assumption, the equation for volumetric flow rate in pressure driven circular channel flow is used to describe the volumetric flow rate $\dot{V}$ at which liquid within the screen is wicked away from the liquid reservoir:

$\dot{V}=\frac{d V}{d t}=\frac{\pi}{128 \mu} D_{H}^{4} \frac{\Delta P}{L_{W}}$ 
where $V$ is the volume of liquid in a capillary pathway, $\Delta P$ is the driving pressure, and $L_{W}$ is the length of the flow path. Here again it is assumed that the screen is made of circular tubes parallel with the flow direction, this time with hydraulic diameter $D_{H}$. Since the screen pore geometry is quite different than a row of capillary tubes, $D_{H}$ is not necessarily equal to $D_{C}$. Therefore they both will be treated as unknowns. The differential volume, $d V$ can be expressed as the cross sectional area of the capillary pathway, $\frac{\pi D_{H}^{2}}{4}$, multiplied by the differential flow path length, $d L_{W}$. Substituting and solving for $\frac{d L_{W}}{d t}$ and noting that $\frac{d L_{W}}{d t}$ is equal to the wicking velocity $v_{W}$, $v_{W}=\frac{d L_{W}}{d t}=\frac{1}{32 \mu} D_{H}^{2} \frac{\Delta P}{L_{W}}$

In general, $\Delta P$ consists of two sources - the capillary pressure, which always drives the flow towards the dry area of the screen, and the hydrostatic head, which drives the flow in the direction of the gravity vector. For simplicity, a case for gravity pointing in the opposite direction of the capillary pressure driving force and a case for zero gravity force is considered separately. The second condition is appropriate for both microgravity environments and for when the screen is positioned horizontally so that the gravity vector is perpendicular to the screen plane. Therefore for the vertical case, Equation 3.61 becomes

$$
v_{W}=\frac{d L_{W}}{d t}=\frac{D_{H}^{2}}{32 \mu L_{W}}\left(\frac{4 \gamma_{L V} \cos \theta_{C}}{D_{C}}-\rho g L_{W}\right)
$$


For the horizontal case, the hydrostatic term drops and the equation simplifies to:

$$
v_{W}=\frac{d L_{W}}{d t}=\frac{D_{H}^{2}}{32 \mu L_{W}}\left(\frac{4 \gamma_{L V} \cos \theta_{C}}{D_{C}}\right)
$$

Equations 3.62 and 3.63 are the wicking rate equations for the vertical and horizontal cases, respectively. Both equations can be integrated to determine the wicking distance $L_{W}$, as a function of time. Integrating Equations 3.62 and 3.63 and applying the initial condition $L_{W}(t=0)=0$ :

$$
\begin{aligned}
& -\frac{4 \gamma_{L V} \cos \theta_{C}}{\rho g D_{C}} \ln \left(1-\rho g \frac{D_{C}}{4 \gamma \cos \theta_{C}} L_{w}\right)-L_{w}=\rho g \frac{D_{H}^{2}}{32 \mu} t \\
& L_{w}=\sqrt{\left(\frac{D_{H}^{2}}{D_{C}}\right) \frac{\gamma_{L V} \cos \theta_{C}}{4 \mu} t}
\end{aligned}
$$

The solution for the wicking distance in the horizontal case is clearly much simpler, and therefore it is useful to perform wicking experiments in a horizontal direction to cancel out the hydrostatic head term. The complexity of the screen geometries makes it difficult to analytically and directly determine $D_{C}$ and $D_{P}$. Therefore the effective wicking diameter $D_{E}$ is defined:

$$
D_{E} \equiv \frac{D_{H}^{2}}{D_{C}}
$$

$D_{E}$ can be considered as a ratio of the geometrical effect of the screen on the capillary pressure retention in the wicking direction to the geometrical effect of the screen on the viscous resistance in the wicking direction. It is denoted a "diameter" instead of a ratio 
because it has the units of length. However it does not physically correspond to any physical length of the screen weaves. It must be determined experimentally for each screen.

\subsubsection{Wicking Rate Experiment}

Wicking tests are conducted in the following manner: A portion of screen is dipped in a liquid reservoir, much like the concept from Figure 3.14, and then the wicking distance $L$ is measured over time $t . D_{E}$ is then determined from a curve fit to Equation 3.65 with wicking distance as a function of time data using a liquid with known surface tension and viscosity in the horizontal testing orientation. Since the wicking rate depends on the direction of the wicking rate relative to the orientation of the wires, the value of $D_{E}$ should be measured parallel and perpendicular to the warp wires.

Evaporation is minimized by using a liquid with a low vapor pressure and by keeping the volume of the experiment as enclosed from the ambient as possible.

\subsubsection{Historical Data and Trends}

To validate to the wicking rate model, wicking rate data is compiled from the 4 sources available in the literature (Symons 1974, Dodge 2000, Fries et al. 2007, and Hastings et al. 2011). Results are listed in Table 3.5. Some of the studies used different parameters than $D_{E}$ based off of different derivations of the wicking rate. Information from these studies were converted into $D_{E}$ values to permit easy comparison between screens. Hastings et al. (2011) was the only study to obtain wicking data with a cryogenic 
liquid, in nitrogen. Bingham and Tegart (1977) report bubble point data for ethyl alcohol as a function of time that cannot be converted into an effective $D_{E}$.

\begin{tabular}{|c|c|c|c|c|c|c|}
\hline \multirow[b]{2}{*}{ Screen Type } & \multirow[b]{2}{*}{ Weave Type } & \multirow[b]{2}{*}{ Orientation } & \multicolumn{3}{|c|}{ Wicking Effective Diameter, De $(\mu \mathrm{m})$} & \multirow[b]{2}{*}{ Hastings et al. 2011} \\
\hline & & & Symons 1974 & Dodge 2000 & Fries et al. 2007 & \\
\hline $325 \times 2300$ & Twilled Dutch & Perpendicular & 0.88 & - & - & 1.77 \\
\hline ' & & Parallel & 1.36 & - & - & 2.74 \\
\hline $200 \times 1400$ & Twilled Dutch & Perpendicular & 0.88 & 0.88 & - & 2.11 \\
\hline ' & ' & Parallel & 2.72 & 2.72 & 3.38 & 4.15 \\
\hline $165 \times 1400$ & Twilled Dutch & Perpendicular & 1.52 & 1.52 & - & - \\
\hline ' & ' & Parallel & 3.04 & 3.04 & - & - \\
\hline $200 \times 600$ & Twilled Dutch & Perpendicular & 7.76 & 7.76 & - & - \\
\hline ' & ' & Parallel & 4 & 4 & - & - \\
\hline $165 \times 800$ & Twilled Dutch & Perpendicular & 12.8 & 12.8 & - & 14.18 \\
\hline ' & ' & Parallel & 3.84 & 3.84 & - & 4.2 \\
\hline $80 \times 700$ & Twilled Dutch & Perpendicular & 3.36 & - & - & - \\
\hline ' & ' & Parallel & 6.96 & 6.96 & - & - \\
\hline $30 \times 500$ & Twilled Dutch & Perpendicular & 5.68 & - & - & - \\
\hline ' & ' & Parallel & 29.6 & - & - & - \\
\hline $30 \times 250$ & Twilled Dutch & Perpendicular & - & 1.83 & - & - \\
\hline ' & ' & Parallel & - & - & - & - \\
\hline $20 \times 250$ & Twilled Dutch & Perpendicular & 13.6 & 13.6 & - & - \\
\hline 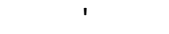 & ' & Parallel & 27.2 & 27.2 & - & - \\
\hline $50 \times 250$ & Plain Dutch & Perpendicular & 12.8 & - & & \\
\hline ' & ' & Parallel & 5.36 & - & - & - \\
\hline $24 \times 110$ & Plain Dutch & Perpendicular & 20 & - & - & - \\
\hline 1 & ' & Parallel & 12 & - & - & - \\
\hline
\end{tabular}

Table 3.5 - Effective Wicking Diameters for Several Dutch Twill and Plain Dutch

Weaves. Plain Square and Twilled Square weaves do not wick. The orientation column signifies how the wicking front is oriented relative to the warp wire direction.

The most important trend to note is that $D_{E}$ tends to decrease for finer meshes, implying that finer meshes will have slower wicking rates than the coarser meshes. This is due to the finer meshes having a more obstructed flow path and thus a higher resistance to flow. The coarser meshes have less obstruction so that the liquid is able to flow with less required capillary driving pressure. 


\subsection{Screen Compliance}

At the transient start of flow from the propellant tank to the transfer line through a LAD screen, a large inward deflection of the screen can cause a pressure loss large enough to cause vapor ingestion to a portion of the screen in contact with pressurant gas. In addition, large deflections can cause the screen wires to yield or even rupture if the flow demand is high enough. In flight design, often times this pressure drop contribution is ignored. However, the transient pressure drop at the start of flow can exceed all other terms in Equation 3.2 due to expansion and distortion of the LAD screen pores. Therefore the rate at which a screen deflects or bends, known as screen compliance, is an important secondary factor and fourth influential factor affecting screen selection for LAD systems.

\subsubsection{Model Derivation and Screen Compliance Experiment}

Through experiments, the following relationship to quantify screen compliance for circular samples of fine mesh LAD screens has been established by Paynter (1973a):

$W_{0}=K_{d} \Delta P_{d}^{1 / 3}$

where $W_{0}$ is the screen deflection at the center of the circular screen sample for the given pressure difference across the screen, $\Delta P_{d}$, and $K_{d}$ is a constant. Higher deflection constants indicate that the screen bends or flexes more for the same pressure difference across the screen. $K_{d}$ is a constant that depends both on the fineness of the screen as well as the screen metal type, since certain metals are more flexible than others.

A brief explanation of a potential screen compliance test is given here. A screen is clamped to the top of a liquid reservoir, as shown in Figure 3.15. The liquid reservoir is 
completely filled so that the screen is entirely wetted. A syringe is connected to the liquid reservoir so that it can draw liquid out of the reservoir. As liquid is removed, the screen deflects inward towards the reservoir. A screen deflection will not be directly measured, but the volume of liquid removed from the reservoir will be measured by the syringe. The higher the volume removed, the more the screen has deflected. The pressure difference across the screen that causes the screen deflection can be measured by a DPT. The liquid volume removed vs. pressure drop yields an accurate measure of screen compliance.

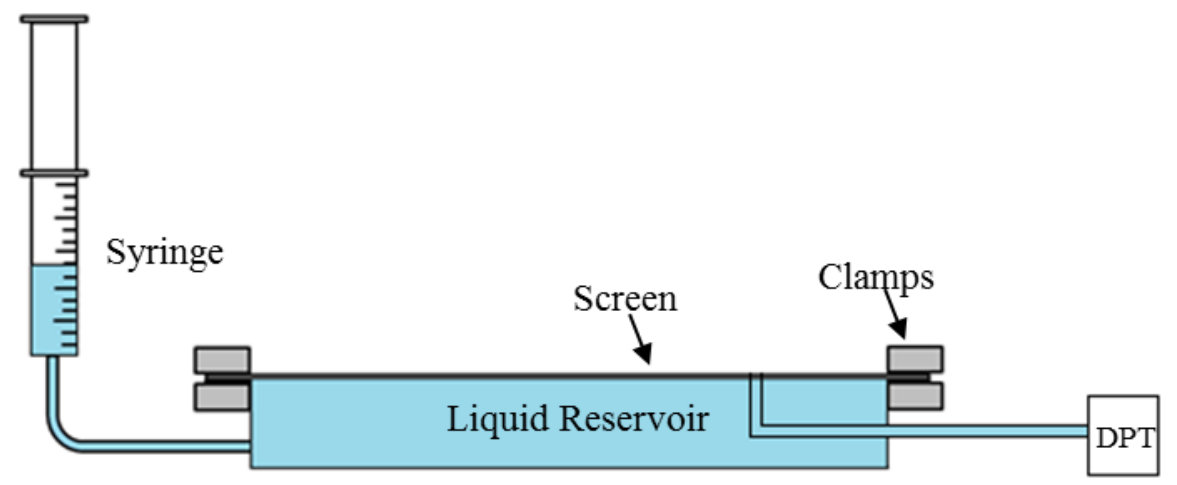

Figure 3.15 - Ideal Experimental Setup for a Screen Compliance Experiment

\subsubsection{Historical Data and Trends}

The constant $K_{d}$ was determined experimentally for 3 different Dutch Twill screens - 325x2300 SS, 200x1400 SS, and 200x1400 Al from Paynter (1973a). The $K_{d}$ values are listed in Table 3.6. For a given $\Delta P_{d}$ the maximum deflection occurred for the 200x1400 Al screen, as expected, since it is made of a more elastic material than the other two screens. When comparing meshes of the same metal, the 200x1400 SS had a smaller $K_{d}$ value than the $325 \times 2300$ SS mesh. This supports the trend that finer meshes experience more deflection, or compliance, than coarser meshes for the same pressure 
difference and same metal. Since fine mesh screens are generally desirable for $\mathrm{LH}_{2}$ systems, additional screen compliance tests with these fine mesh Dutch Twill screens would help to verify this trend.

\begin{tabular}{|c|c|c|}
\hline Screen Type & Material & Kd \\
\hline $325 \times 2300$ & Stainless Steel & 0.223 \\
$200 \times 1400$ & Stainless Steel & 0.157 \\
$200 \times 1400$ & Aluminum & 0.33 \\
\hline
\end{tabular}

Table 3.6 - Screen Compliance Model Parameters for Three Different Screens

\subsection{Material Compatibility}

Flight hardware mass is probably the most important factor when it comes to launch cost, because the smaller the overall mass used by the vehicle, engine, propellant, and propellant tank, the greater the payload mass that can be launched into orbit. In addition to lower overall system mass, material compatibility issues may arise. For example, LAD screen meshes may be constructed out of lightweight Ti, but the channel itself is made of SS. This difference in metals may cause premature screen breakdown at the location where the screen meets the channel due to differing rates of thermal contraction with reduced cryogenic temperatures. The screen metal type generally controls the metal type for the channel, and so less dense metals like $\mathrm{Al}$ are desired to reduce the overall mass of the PMD, since tanks can be constructed out of these same lightweight metals (Bailey et al. 1986 and Tam et al. 2008b). Therefore, another consideration and the fifth influential design factor for cryogenic LAD screen selection is materials and material compatibility. 
In general, less dense materials like $\mathrm{Al}$ or $\mathrm{Ti}$ are desirable to be used for the screen as opposed to SS. However manufacturers can only construct the finest Dutch Twill meshes out of SS. After doing a comprehensive survey of porous screen manufacturers it was found that construction of fine $\mathrm{Al}$ or Ti meshes were limited by the size of the wire diameter. At a certain diameter, a wire becomes too thin to weave properly. For suitable weaving, a wire needs to have a certain strength and elongation capability. Aluminum lacks the required strength below a particular wire diameter, and $\mathrm{Ti}$, a relatively brittle material, shows insufficient elongation below a particular wire diameter. Results are summarized in Table 3.7. A popular candidate for storable propulsion systems is to use a lightweight Ti $165 \times 800$ screen and channel and a Ti propellant tank (Leach and Szpakowski 1982).

\begin{tabular}{|c|c|}
\hline Dutch Twill Screen Type & Material Compatibility \\
\hline $325 \times 2300$ and finer & $\mathrm{SS}$ \\
$250 \times 1370$ & $\mathrm{SS}, \mathrm{Ti}, \mathrm{Al}$ \\
$200 \times 1400$ and coarser & $\mathrm{SS}, \mathrm{Ti}, \mathrm{Al}$ \\
\hline
\end{tabular}

Table 3.7 - Manufacturability of Liquid Acquisition Device Screen Meshes

Depending on the mission requirements, it may be appealing from a design standpoint to trade the higher bubble point for a coarser mesh that can be made of lighter material. For example, one study on the design of a dual-screen liner for the Shuttle OMS/RCS system found that two layers of Al 200x1400 screen were found to have less mass and still outperform a single SS 325x2300 mesh (Paynter 1970). Note that mass savings can even be achieved by using coarser meshes that are less dense for the same material. To illustrate this, Cady (1973) tested the design of a single-screen complete tank wall liner. Ten meshes were tested, and the finest mesh that was tested, the $325 \times 2300$, 
was shown to be the optimum mass design for relatively high flow rates. However, if the mission duration were extended so that lower flow rates were allowable, the $150 \times 150$ Square weave screen was the optimum mass design.

\subsection{The Room Temperature Reseal Pressure Model}

Once the bubble point pressure is exceeded and pressurant gas penetrates the wetted portion of the screen, the screen is said to have failed. However, this is not the final failure point for the LAD system. By reducing the pressure differential across the LAD screen, it is possible for the screen to rewet and essentially reseal itself by suppressing gas ingestion. In ground experiments, this can be accomplished by simply lowering the pressurant gas flow rate on the gas side of the screen sample. In a flight propellant tank, the pressure differential across a full scale screen channel LAD can be reduced by either reducing outflow rate or by increasing tank pressure. Eventually, the screen will break down again, but the existence of the reseal point allows the LAD to drain the tank farther and achieve slightly higher overall expulsion efficiency. Therefore understanding and quantifying the behavior of this reseal point is essential in fully characterizing the LAD system.

To illustrate the reseal pressure, and to compare this pressure with the breakthrough pressure, Figure 3.16 illustrates both a cross-section of the screen and a simplified image of the complex 3D screen pore. A pore throat is defined at the location within the screen pore where the cross sectional area is the smallest. Likewise a pore mouth is defined at the location of maximum cross sectional area on the liquid side of the interface. Pressurant gas enters from the underside of the screen, slowly pushing the 
liquid through the wetted pore. Eventually, a pressurant gas bubble passes through the pore throat, and the screen breaks down. Thus, the effective pore diameter physically corresponds to the largest pore throat, since the screen will break down once pressurant gas passes the point of least resistance through the screen. Likewise, the screen will reseal once liquid rewets every single pore mouth. Therefore, an effective reseal diameter can also be also defined, which corresponds to the largest pore mouth of the LAD screen. The effective pore diameter and effective reseal diameter correspond to the largest pore throat and largest pore mouth of the LAD screen, respectively.

\subsubsection{Model Derivation}

Analogous to the bubble point pressure, the reseal pressure can be defined from a simplification of the general 3D YLE for the pressure drop across a curved L/V interface embedded within the 3D space of the mesh. Consider the L/V interface formed within the LAD mesh screen as shown in Figures 3.16 and 3.17. Retaining assumptions $1-4$ from the bubble point model in Section 3.2.2, the following additional assumptions are required to solve for the reseal pressure:

1. The continuum hypothesis is invoked; the pressure on the gas side of the screen is reduced in slow, quasi-static steps.

2. The receding contact angle that is pinned at the pore mouth is the same receding contact angle that is measured on the macroscopic screen surface.

3. The receding contact angle between liquid and pore mouth is the same receding contact angle between liquid and pore throat as the pressurant gas bubble creeps back into the pore. 


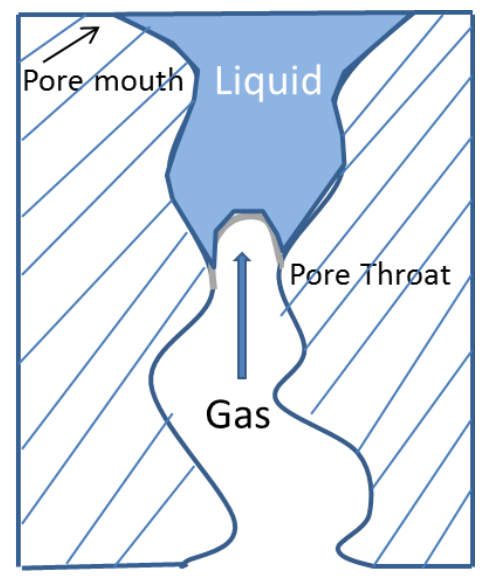

Figure 3.16 - Conceptual Image of a Liquid Acquisition Device Screen Pore Illustrating the Pore Mouth and Pore Throat

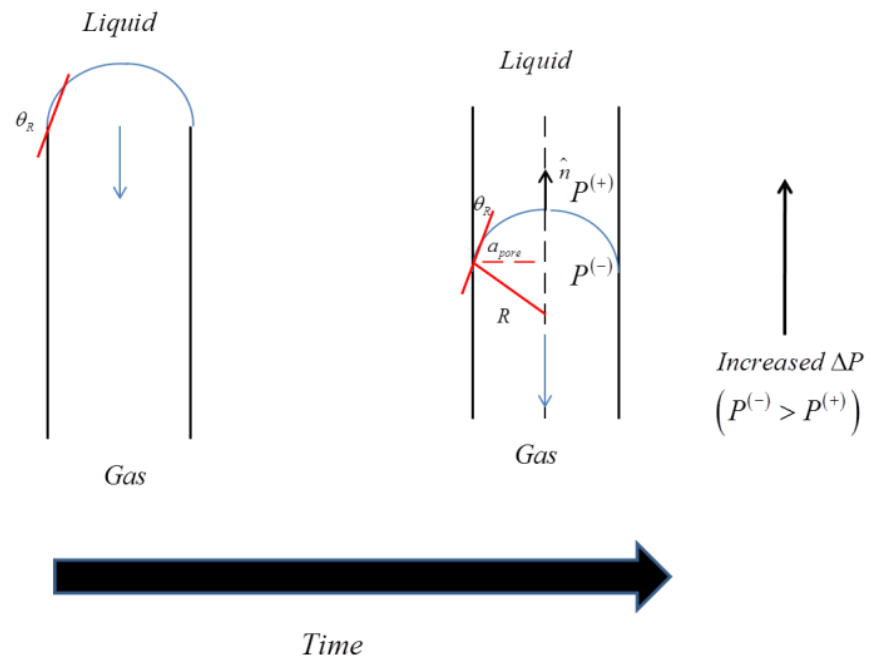

Figure 3.17 - Time Evolution of the Screen Reseal

Beginning with the pressure jump across the interface for the vertical capillary tube from Equation 3.13 shown in Figure 3.17:

$$
\Delta P=\frac{2 \gamma_{L V} \cos \theta_{C}}{a_{\text {pore }}}
$$


To relate the diameter at which the screen reseals at the pore mouth to the vertical tube radius $a_{\text {pore }}$, invoking assumption 1:

$\Delta P=\frac{4 \gamma_{L V} \cos \theta_{C}}{D_{R}}$

where $D_{R}$ is the effective diameter at which the screen first reseals. The vertical tube radius, $a_{\text {pore }}$ is again assumed isomorphic with the mean curvature, and can be approximated using the hydraulic diameter of a circle inscribed within the complex triangular pore.

To achieve a reseal, the pressure underneath the screen is slowly reduced so that the column of gas emitting from the screen pore creeps back down into the pore. Eventually the gas bubble will become pinned at the pore mouth. Subsequent reduction in gas pressure beneath the screen allows the gas bubble to creep back down the pore.

Figure 3.17 illustrates this evolution. Invoking assumptions $1-3$ allows the macroscopic measured contact angle on the screen to be related to the contact angle for all other times. To be precise, the measured contact angle is actually the receding contact angle, since the pressurant gas column eventually decreases, the bubbles then recedes at the screen, becomes attached to the pore mouth, and then creep back into the screen pore. Experimentally, the reseal pressure is defined as the differential pressure across the screen at the moment when the screen mouth becomes rewetted. Therefore using the receding contact angle, Equation 3.69 becomes:

$$
\Delta P_{R S}=\frac{4 \gamma_{L V} \cos \theta_{R}}{D_{R}}
$$


Equation 3.70 is the simplified room temperature reseal pressure model.

Equations 3.16 and 3.70 represent the breakthrough and reseal pressure models for screen channel LADs operating in room temperature storable liquids, respectively. Comparing the two equations, the measured breakthrough and reseal pressures are thus different on account of differences between advancing and receding contact angles as well as differences between the size of the pore throat and pore mouth.

The same inverted bubble point test configuration used to measure the bubble point is the ideal test configuration for measuring the reseal pressure for a given screen. Each controlled bubble breakthrough test allows for a controlled reseal pressure test. Details of the inverted reseal pressure test are reserved for Chapter 4 along with the IBP test configuration.

\subsubsection{Historical Data and Trends}

A rigorous review of the literature reveals only three previous studies which report reseal pressure data along with the bubble point data. Heckman (1971) reported a single reseal pressure value for eight different screens, including Dutch Twilled, Plain Dutch, and Square weaves using IPA. Paynter (1973a) reported a single reseal data point for three different Dutch Twill meshes (200x1400, 250x1370, and 325x2300) in methanol. Meanwhile Chato and Kudlac (2002) reported IPA and $\mathrm{LN}_{2}$ reseal pressure data for a $200 \times 1400$ screen and IPA, $\mathrm{LN}_{2}$, and $\mathrm{LH}_{2}$ reseal pressures for a $325 \times 2300$ screen. There was difficulty even achieving a controlled reseal pressure measurement in $\mathrm{LH}_{2}$ with the coarser 200x1400 screen. Physically, if the screen cannot reseal, then the reseal pressure is 0 . 


\subsubsection{Specifying the Reseal Diameter}

Effective reseal diameters can be estimated using the three methods outlined in Section 3.2.5 for specifying the effective pore diameters. Reseal diameters can be determined through room temperature reseal pressure experiments with a liquid of known surface tension using a modification of Equation 3.70 (Method 1):

$D_{R}=\frac{4 \gamma_{r e f} \cos \theta_{R}}{\Delta P_{\mathrm{RS}, r e f}}$

For a more statistically accurate value, all historical data for a given LAD mesh can be taken into account into the reseal pore diameter calculation (Method 2). SEM analysis can also be used (Method 3)

Table 3.8 lists the room temperature reseal diameters for screens where data is available. In general, the reseal diameter scales with the fineness of the screen in the same way that the pore diameter does. Comparing Table 3.2 and Table 3.8, for all screens, the reseal diameter is larger than the pore diameter, as expected.

\begin{tabular}{|c|c|}
\hline Mesh & Average Reseal Diameter $[\boldsymbol{\mu m}]$ \\
\hline $\mathbf{3 2 5 \times 2 3 0 0}$ & 18 \\
\hline $\mathbf{2 0 0 \times 1 4 0 0}$ & 26.2 \\
\hline $\mathbf{2 5 0 \times 1 3 7 0}$ & 24.4 \\
\hline $\mathbf{2 0 0 \times 6 0 0}$ & 56.9 \\
\hline $\mathbf{8 0 \times 7 0 0}$ & 66.76 \\
\hline $\mathbf{5 0 \times 2 5 0}$ & 118.02 \\
\hline $\mathbf{3 0 \times 2 5 0}$ & 159.77 \\
\hline $\mathbf{2 4 \times 1 1 0}$ & 242.3 \\
\hline $\mathbf{3 2 5 \times 3 2 5}$ & 70.36 \\
\hline $\mathbf{1 2 0 \times 1 2 0}$ & 203.26 \\
\hline $\mathbf{2 3 0 \times 2 3 0}$ & 101.63 \\
\hline
\end{tabular}

Table 3.8 - Room Temperature Reseal Diameters using Method 2 


\subsection{Pressurant Gas Type}

The sixth and final influential factor for cryogenic LAD design is the type of gas used to pressurize the propellant tank. This parameter has implications on both LAD and pressurization subsystems. Effects on LAD performance due to pressurant gas type have minimal effects in storable propulsion systems; for cryogenic systems, the type of gas will affect heat and mass transfer during tank drain.

For cryogenic systems, there are two classical methods to pressurize a flight propellant tank. Autogenous pressurization, which is pressurization of the liquid with the condensable vapor like gaseous hydrogen $\left(\mathrm{GH}_{2}\right) / \mathrm{LH}_{2}$, is the simplest to implement due to reduced system complexity. Meanwhile pressurization with a non-condensable gas such as helium requires onboard helium bottles. There are advantages and disadvantages to both schemes.

For example, autogenous pressurization is more attractive due to lower overall system mass, but pressurization is complicated through heat and mass transfer across the tank L/V interface during outflow, which may prematurely warm the liquid and cause the LAD to break down early (Stochl et al. 1991). As such, autogenous pressurization may not be sufficient to sustain larger outflow rates. In addition, heaters or pressure building circuits may be required to pressurize a liquid with its own vapor. Non-condensable pressurization is attractive due to less complexity in heat and mass transfer; helium has low solubility in all major cryogens (Zimmerli et al. 2010), and heat and mass transfer is minimized during outflow. Helium is likely sufficient to cover and sustain all anticipated outflow rates for future missions (Smith et al. 2010). However, pressurization with 
helium requires onboard helium tanks, thus increasing system mass. In addition, it may be the more costly option of the two. The type of pressurant gas affects both total system performance and should also affect LAD subsystem performance.

Related to LADs, studies investigating the effects of autogenously pressurizing a liquid hydrogen system found that the changes in surface tension may be due to thermocapillary flows which affect the meniscus within the screen pore (Schmidt 1994 and Schmidt et al. 1995). Pressurizing liquid hydrogen with heated hydrogen vapor lead to condensation and induced temperature gradients along the liquid surface. The ensuing thermocapillary flow established a pressure distribution within the interface which deformed the center of the surface into the liquid. The meniscus expanded into the liquid and eventually detached from the screen wires. Similarly, it was observed in (Schmidt 1994 and Schmidt et al. 1995) that pressurizing liquid hydrogen with heated helium lead to evaporation and a thermocapillary flow structure which was opposite to that seen with autogenous pressurization.

\subsection{Concluding Remarks and Implications for Cryogenic Propulsion Systems}

Clearly the optimal mesh for a particular mission requires trading all of the aforementioned six influential factors against one another. Space flight requirements which include mass flow rate, acceleration level and direction, and thermal environment dictate selection of the screen for a particular mission. The primary performance parameters governing screen channel LAD design are the bubble point (and reseal pressure) and FTS pressure drop, while secondary parameters are the wicking rate, screen compliance, material compatibility, and type of pressurant gas.. 
All cryogenic propellants like $\mathrm{LH}_{2}$ have extremely low surface tension. Therefore, for $\mathrm{LH}_{2}$ propulsion systems, fine mesh screens are highly desirable to counteract the low surface tension. In general, a higher bubble point translates into higher margin in system design. However, a high bubble point is not the only consideration in system design, since fine mesh screens may generate higher flow losses and become clogged by particulate matter.

For FTS pressure drop, implications for an $\mathrm{LH}_{2}$ system are as follows: at high mass flow rates the second term in Equation 3.33, which contains $\rho^{-1}$, dominates the pressure drop. Since the density of $\mathrm{LH}_{2}$ is an order of magnitude less than the density of storable propellants, it is expected that $\Delta P_{F T S}$ for $\mathrm{LH}_{2}$ will actually be higher than $\Delta P_{F T S}$ for storable propellants for the same mass flow rate. This is an important aspect from a LAD design standpoint since the bubble point, the safeguard against vapor ingestion due to FTS pressure drop, is much smaller for $\mathrm{LH}_{2}$ than for storable propellants. Therefore vapor ingestion can occur much easier for an $\mathrm{LH}_{2}$ system than a storable system, even without considering the complications due to heat transfer at cold $\mathrm{LH}_{2}$ temperatures. A balance must exist between bubble point and FTS pressure drop.

In regard to wicking rate, implications for an $\mathrm{LH}_{2}$ system are as follows: Due to the temperature variation of the surface tension and viscosity of $\mathrm{LH}_{2}$, the wicking velocity should increase slightly for increasing liquid temperature. Therefore, from a LAD design standpoint, the gain in wicking velocity for higher $\mathrm{LH}_{2}$ temperatures may be an influential factor when determining the operating temperature of the $\mathrm{LH}_{2}$ storage and transfer system. 
For screen compliance, implications for an $\mathrm{LH}_{2}$ system are as follows: Recalling from Section 3.4, colder temperature $\mathrm{LH}_{2}$ will experience a higher pressure drop for the same mass flow rate. Therefore $\mathrm{LH}_{2}$ transfer systems operating at colder temperatures will see higher LAD screen deflections. Finer mesh screens are desirable to counter the low surface tension of $\mathrm{LH}_{2}$, but finer mesh screens are expected to have higher screen compliance.

In regard to material compatibility, implications for an $\mathrm{LH}_{2}$ system are as follows: The $\mathrm{LH}_{2}$ surface tension is at least one order of magnitude less than other propellants. With such a small surface tension, the bubble point for any screen type is relatively very low. Therefore, the sacrifice of using the heavier SS material in order to gain the highest bubble point possible, and thus highest protection against adverse accelerations, may be necessary in order to effectively prevent vapor ingestion across a range of mission requirements.

Because the purpose of this dissertation is to develop the most flexible and robust technology for low surface tension liquid acquisition devices operating in low gravity, specifically for in-space cryogenic engines and cryogenic fuel depots, emphasis in this work will be on developing and testing finer LAD screen meshes. Review of the literature shows that the finest mesh where data is available is the $325 \times 2300$ Dutch Twill screen. While the $325 \times 2300$ has flight heritage in storable propulsion systems as well as the SHOOT SFHe experiment, a finer 450x2750 mesh Dutch Twill was mentioned in the literature, but never fully tested. 
But there is even more promise for improving $\mathrm{LH}_{2}$ screen channel LAD technology. Due to recent improved techniques in metallic wire cloth weaving, it is possible to fabricate an even finer mesh screen. During a recent comprehensive survey of particulate filtration techniques, a 510x3600 wire mesh was found in use in the filtration community. No bubble point data or information on the pore diameter yet exists in the literature. Given the expectation that this mesh screen should have a larger bubble point, this screen will be tested along with a 200x1400, 325x2300, and 450x2750 screen across a range of thermodynamic conditions, in room temperature and cryogenic liquids, using both pressurization schemes in order to validate models outlined in this chapter.

The chronology of cryogenic testing conducted that is most relevant to this dissertation is presented in Table 3.9. In the early 2000s, a small amount of static bubble point data was reported for a 200x1400 and 325x2300 screen in $\mathrm{LH}_{2}, \mathrm{LN}_{2}, \mathrm{LOX}$, and $\mathrm{LCH}_{4}$ to determine the baseline values. High pressure testing capability in $2009-2011$ allowed examination of both saturated and subcooled states in $\mathrm{LOX}$ and $\mathrm{LCH}_{4}$ for a $200 \times 1400$ and 325x2300 screen. Full scale LAD outflow tests were conducted in LOX in 2010. The three finest Dutch Twill screens were then tested in $\mathrm{LH}_{2}$ and $\mathrm{LN}_{2}$ in 2011 across a wide range of conditions in a parametric fashion. Finally, cryogenic experiments culminated in full scale LAD channel outflow tests using a 325x2300 screen in $\mathrm{LH}_{2}$. Note that $\mathrm{LH}_{2}$ tests from 2011 are first discussed before the high pressure tests in LOX and $\mathrm{LCH}_{4}$ in order to systematically present the functional dependencies of the bubble point. 


\begin{tabular}{|c|c|c|c|c|c|c|}
\hline Year & Authors & Screen & Fluid & $\begin{array}{l}\text { Liquid } \\
\text { State }\end{array}$ & $\begin{array}{c}\text { Conditions } \\
\mathrm{T}[\mathrm{K}] \quad \mathrm{P} \text { [psia] }\end{array}$ & Gas \\
\hline 2002 & Chato, Kudlac & $325 \times 2300$ & $\begin{array}{c}\mathrm{LH} 2 \\
(\& \mathrm{LN} 2)\end{array}$ & Sat & $\begin{array}{l}T=21.9 \\
P=18\end{array}$ & $\mathrm{He}$ \\
\hline 2005 & Kudlac, Jurns & $\begin{array}{l}200 \times 1400 \\
325 \times 2300\end{array}$ & LOX & Sat & $\begin{array}{l}T=93.4 \\
P=20\end{array}$ & $\mathrm{He}$ \\
\hline 2006 & Jurns et al. & $\begin{array}{l}200 \times 1400 \\
325 \times 2300\end{array}$ & $\mathrm{LCH} 4$ & Sat & $\begin{array}{c}113<\mathrm{T}<116 \\
13<\mathrm{P}<16\end{array}$ & $\mathrm{He}$ \\
\hline 2007 & Jurns, McQuillen & $\begin{array}{l}200 \times 1400 \\
325 \times 2300\end{array}$ & LOX & Sub & $\begin{array}{l}86<T<94 \\
14<P<22\end{array}$ & $\mathrm{He}$ \\
\hline $\begin{array}{c}2009 \\
10\end{array}$ & Hartwig, Jurns & $\begin{array}{l}200 \times 1400 \\
325 \times 2300\end{array}$ & $\begin{array}{l}\text { LOX } \\
\text { (\&LN2) }\end{array}$ & $\begin{array}{l}\text { Sat, } \\
\text { Sub }\end{array}$ & $\begin{array}{c}93.5<\mathrm{T}<130.8 \\
18<\mathrm{P}<262\end{array}$ & $\begin{array}{l}\text { He, } \\
\text { GOX }\end{array}$ \\
\hline 2010 & $\begin{array}{c}\text { Chato, McQuillen, } \\
\text { Rame }\end{array}$ & $\begin{array}{l}325 \times 2300 \\
\text { outflow }\end{array}$ & LOX & $\begin{array}{l}\text { Sat, } \\
\text { Sub }\end{array}$ & $\begin{array}{l}95<\mathrm{T}<120 \\
50<\mathrm{P}<250\end{array}$ & $\mathrm{He}$ \\
\hline $\begin{array}{c}2010 \\
11\end{array}$ & Hartwig et al. & $325 \times 2300$ & $\begin{array}{l}\text { LCH4 } \\
\text { (\&LN2) }\end{array}$ & $\begin{array}{l}\text { Sat, } \\
\text { Sub }\end{array}$ & $\begin{array}{c}105<\mathrm{T}<160 \\
14<\mathrm{P}<250\end{array}$ & $\begin{array}{c}\mathrm{He}, \\
\mathrm{CH} 4, \\
\mathrm{~N} 2\end{array}$ \\
\hline 2011 & Hartwig et al. & $\begin{array}{l}325 \times 2300 \\
450 \times 2750 \\
510 \times 3600\end{array}$ & $\begin{array}{c}\text { LH2 } \\
(\& L N 2)\end{array}$ & $\begin{array}{l}\text { Sat, } \\
\text { Sub }\end{array}$ & $\begin{array}{c}16.8<\mathrm{T}<21.5 \\
4.5<\mathrm{P}<22\end{array}$ & $\begin{array}{l}\mathrm{He}, \\
\mathrm{H} 2 \\
\mathrm{~N} 2\end{array}$ \\
\hline 2012 & $\begin{array}{l}\text { Hartwig, Chato, } \\
\text { McQuillen, Rame }\end{array}$ & $\begin{array}{l}325 \times 2300 \\
\text { outflow }\end{array}$ & LH2 & $\begin{array}{l}\text { Sat, } \\
\text { Sub }\end{array}$ & $\begin{array}{c}20.3<\mathrm{T}<24.2 \\
15<\mathrm{P}<75\end{array}$ & $\mathrm{He}$ \\
\hline
\end{tabular}

Table 3.9 - Chronology of Recent Liquid Acquisition Device Technology Development Program Conducted at NASA Glenn Research Center 


\section{Chapter 4}

\section{Room Temperature Liquid Acquisition Device Performance Experiments}

The purpose of this chapter is to present the liquid acquisition device performance experiments carried out in room temperature liquids. Bubble point and reseal pressure tests for a 325x2300, 450x2750, and 510x3600 Dutch Twill screen are conducted in storable liquids, methanol, acetone, IPA, water, and binary methanol/water mixtures of various methanol concentrations spaced over the intermediate surface tension range. First screen pore diameters are estimated based on analysis from scanning electron microscopy and historical data. Second, the experimental hardware and procedure for conducting a bubble point test is outlined. Third, contact angles are measured for both pure and binary mixture fluids using a modified version of the Sessile Drop technique. Fourth, Langmuir isotherms are used to make pretest predictions for the binary mixture tests. Fifth, experimental results are used to compare methods for determining effective pore diameter. Sixth, the equation of state analysis from Neumann and Good (1979) is used to determine the critical Zisman surface tension for stainless steel LAD screens, which defines the point of total wettability. Eighth, room temperature reseal test methodology and experiments are presented. Finally, room temperature wicking tests are performed in IPA for several different LAD meshes. Appendices C and D report governing physics for 
generating the Langmuir isotherm fits to the surface tension data, and also report the computed solid/liquid and solid/vapor interfacial tensions for the methanol/water mixture. All testing in this chapter was performed at the Cryogenics Components Lab-7 (CCL-7) at the NASA Glenn Research Center (GRC) in Cleveland, Ohio (Jurns and Kudlac 2006).

\subsection{Pure Fluid Tests}

Due to the high degree of scatter in historical room temperature bubble point data and relatively poor quality experiments reported in several historical works, it was highly desirable to conduct high accuracy room temperature performance tests. Room temperature tests will also be used to validate the phenomenological bubble point model given in Chapter 3. From Equation 3.16, bubble point pressure is theoretically proportional to liquid surface tension. This relationship dictated the selection of reference fluids, since it was desired to obtain bubble breakthrough data across the widest possible range of surface tension values. Acetone, IPA, and methanol were chosen because the surface tension of these pure liquids is roughly one order of magnitude higher than cryogenic liquids $(20-30 \mathrm{mN} / \mathrm{m})$ and thus would provide a good calibration range for pretest predictions in cryogenic liquids. These liquids were also readily available, easy to handle, and all had low projected contact angles. Tests were also conducted in water (70 $\mathrm{mN} / \mathrm{m}$ ) despite complications that could arise due to minute surfactants present in the liquid.

\subsubsection{Scanning Electron Microscopy Analysis}

All three screen samples that were tested were analyzed under an SEM microscope to check for defects, dirt and debris, and to ensure consistency in weave, 
warp and shute wire diameters, and pore diameters. Displayed in Figures 4.1a and b are SEM images of the new 450x2750 and 510x3600 screens, respectively. Shown in both figures are the $2 \mathrm{D}$ triangular projections of the 3D pore based on Equations 3.21 and 3.22 .

Table 4.1 lists the predictions for the pore diameters based on image analysis from the SEM along with measured diameters of warp and shute wires and absolute micron ratings (smallest size of the hole opening per the manufacturer specification). Wire diameters were measured using advanced SEM software. Applying a basic uncertainty analysis on Equation 3.22 using known uncertainties in wire diameters, pore diameters based on SEM analysis are good to within $0.05 \mu \mathrm{m}$.

As shown, the effective pore diameter should scale with the mesh of the screen, and thus Equation 3.16 predicts that the finest mesh screen will have the highest bubble point. Naturally, for pore diameters based on SEM photos, rigorous statistical analysis would need to be implemented to account for pore to pore variations within an individual sample such that an "average" pore diameter would again be defined. During analysis, however, images were taken at numerous locations everywhere along the screen to verify repeatability in the measured dimensions. Effective pore diameters from the current work scale much better with manufacture supplied absolute micron ratings versus those from previous work (Armour and Cannon 1968), which used simple statistical methods to determine the pore diameter. Recent advances in SEM technology have allowed much more accurate measurements of the warp and shute wire diameters and thus pore diameter. In addition, variation in performance from screen sample to sample is minimized due to advances in woven wire technology. 

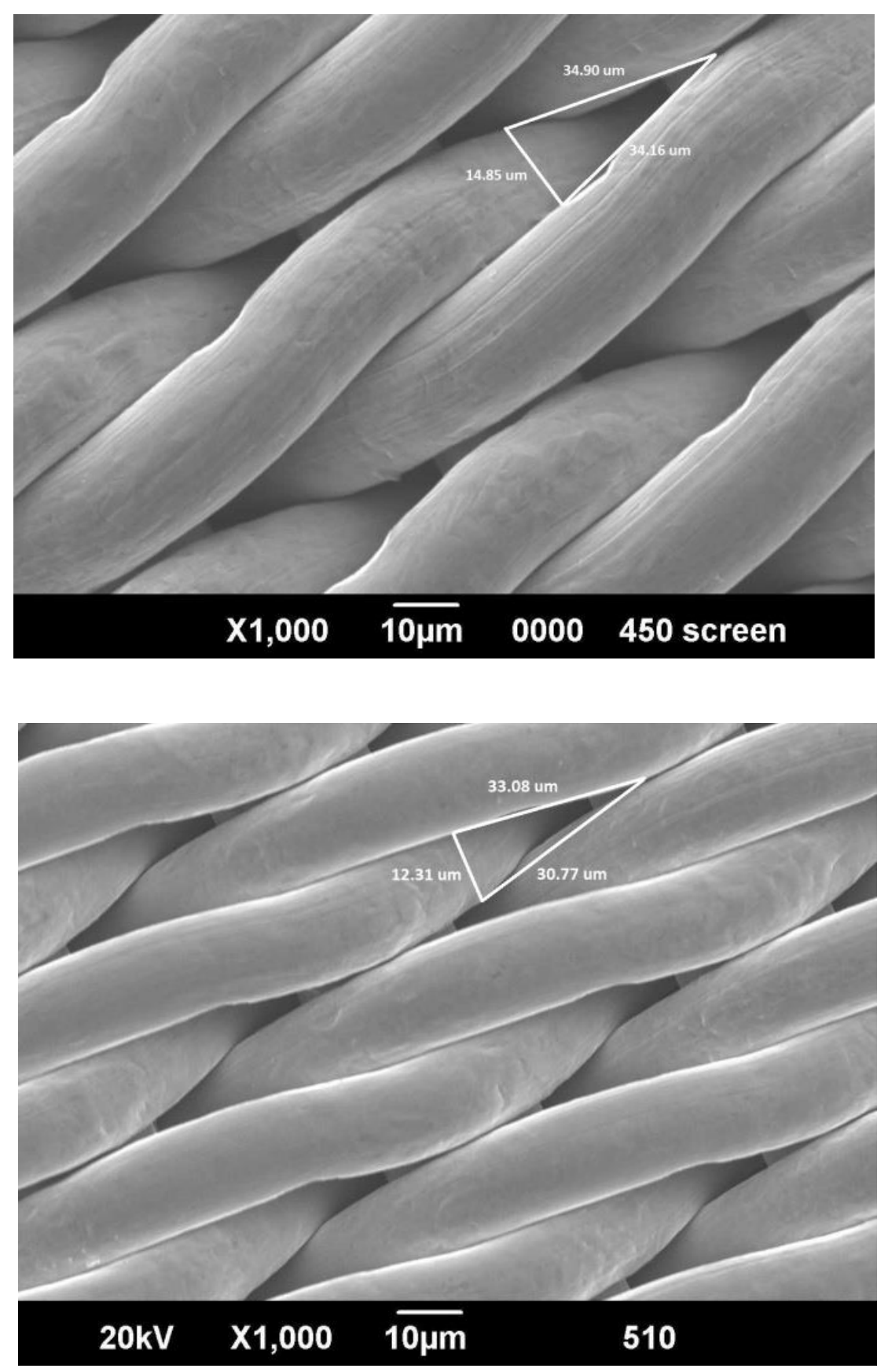

Figure 4.1 - Scanning Electron Microscopy Image of a) 450x2750 Mesh and b) 510x3600 Mesh Dutch Twill Screen Samples. Triangles are drawn to represent a simplified geometry of the complex screen pore. 


\begin{tabular}{|c|c|c|c|c|c|c|}
\hline \multirow[b]{2}{*}{ Mesh } & \multicolumn{3}{|c|}{ Screen Characteristics } & \multirow[b]{2}{*}{ d shute $[\mu \mathrm{m}]$} & \multirow{2}{*}{\begin{tabular}{|c|} 
Absolute \\
micron rating*
\end{tabular}} & \multirow{2}{*}{$\begin{array}{c}\text { Pore Diameter [ } \mu \mathrm{m}] \\
\text { Based on SEM }\end{array}$} \\
\hline & n warp & n shute & d warp $[\mu \mathrm{m}]$ & & & \\
\hline $325 \times 2300$ & 325 & 2300 & 38.1 & 25.4 & 8 to 9 & $14.8 \pm 0.05$ \\
\hline $450 \times 2750$ & 450 & 2750 & 25.4 & 20.3 & 6 to 7 & $11.9 \pm 0.05$ \\
\hline $510 \times 3600$ & 510 & 3600 & 25.4 & 15.2 & 5 to 6 & $9.95 \pm 0.05$ \\
\hline
\end{tabular}

\section{Table 4.1 - Screen Parameters from Scanning Electron Microscopy Image Analysis}

Stainless steels are complex mixtures of various components. During screen SEM analysis, qualitative composition measurements of each of the three meshes were made using Energy Dispersive X-Ray Microanalysis (EDAX) on both warp and shute wires. For all three screens, analysis showed that both wires were comprised of similar steel components, and that the ratios of metal components of the porous LAD screen, such as Carbon, Oxygen, Nickel (Ni), and Iron, were in direct proportion to a bulk 304SS sample, although not shown here.

\subsubsection{Bubble Point Experimental Setup}

Figures 4.2 and 4.3 display the experimental set up for the room temperature bubble point tests. For all three weaves, $9 \mathrm{~cm}$ (3.5 inch) outer diameter (OD) 304SS screen samples were cut and fabricated to fit on top of a heavy flange. A single fillet weld was used to bond the screen to the flange and to bond a $0.318 \mathrm{~cm}(1 / 8 \mathrm{in})$ thick cover ring on top of the screen as shown in Figure 4.2. The resultant OD and surface area of the screen exposed to the liquid was $6.35 \mathrm{~cm}(2.5 \mathrm{in})$ and $20.25 \mathrm{~cm}^{2}\left(3.14 \mathrm{in}^{2}\right)$, respectively. Visual observations during tests indicated adequate welding of the screen to the flange; bubble breakthrough locations were distributed randomly across the surface of the sample (i.e. there was no preferential bubble breakthrough at the edges). 


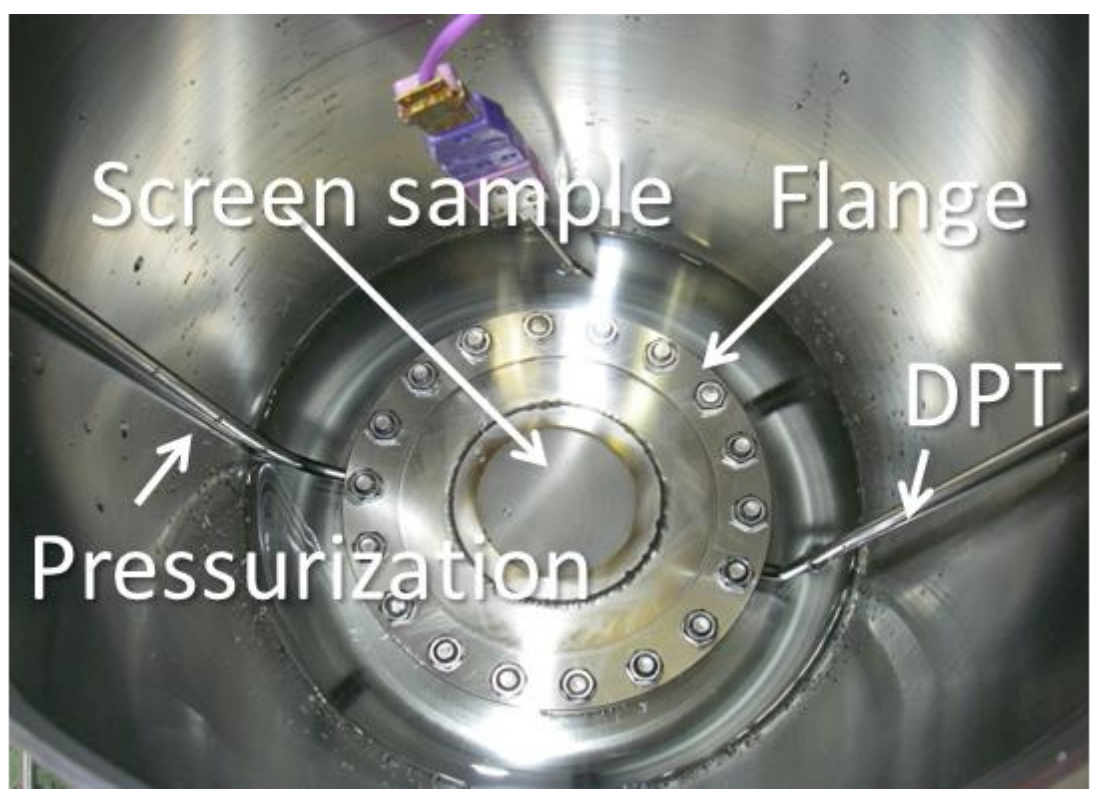

Figure 4.2 - Submerged Screen Sample Mounted within the Holding Cylinder

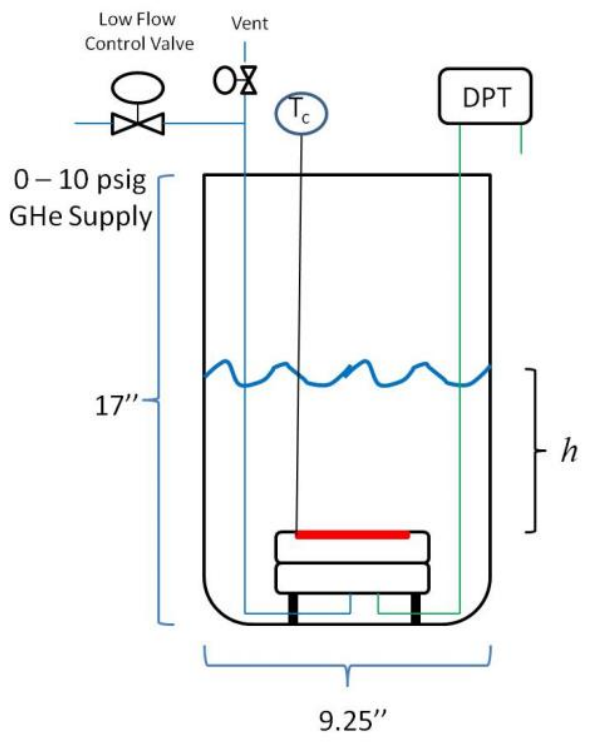

Figure 4.3 - Schematic of Room Temperature Bubble Point Experimental Setup

Bubble point testing was performed in an inverted configuration with the liquid on top and gas on the bottom. The flange was mated with a bottom "blind" flanged section to create a tight seal at the edges and to allow a small gap beneath the screen for 
pressurant gas to build up. The bottom section was fitted with pressurization and sensing port lines to regulate gas flow and to measure the differential pressure across the screen as shown in Figures 4.2 and 4.3. A $101-170 \mathrm{kPa}(0-10 \mathrm{psig})$ GHe supply line was connected in series to a low flow control valve to slowly ramp the pressure beneath the screen sample. Helium was chosen over other inert gases due to its frequency in usage in flight pressurization systems. To relieve pressure underneath the screen in between successive bubble breakthroughs, a vent valve was connected to the pressurization line. The screen sample and flanged section were mounted inside of a $44 \mathrm{~cm}$ (17 in) tall, 23.4 cm (9.2 in) OD SS vertical cylinder as shown in Figure 4.3. The purpose of the cylinder was to submerge the screen sample within the room temperature liquid, thus creating an inverted $\mathrm{L} / \mathrm{V}$ interface within the screen pores.

A $101-120 \mathrm{kPa}$ (0-3 psid) DPT measured the pressure beneath the screen with respect to atmospheric pressure. A Type-E thermocouple was used to measure the temperature of the liquid side of the screen. Pressure of the incoming pressurant gas was measured using a $0-345 \mathrm{kPa}(0-50 \mathrm{psia})$ transducer. A ruler was used to determine the height of the liquid on top of the LAD screen sample before each test.

Acetone, IPA, and methanol were purchased on lab or from local chemical vendors and were used, as received. All chemicals were of high grade purity (> 99.5\% pure). Standard distilled water was used for the pure water and diluted water/methanol tests. To reduce uncertainty in the data due to a reduction in water surface tension due to the presence of impurities, the water bottles were subjected to the "shake test" to ensure minimum surfactant concentration. The shake test uses the property that foam fraction is an effective separation process for surfactants in aqueous solutions. Simply shake a 
sample of the water in a clean volumetric flask. Any bubbles will immediately break if the surfactant concentration due to contamination is ignorable.

\subsubsection{Bubble Point Experimental Methodology and Data Reduction}

The screen and flange were mounted inside the cylinder. Next a gas flow purge was established to prevent flooding of the screen. Graduated cylinders were then used to measure and add liquid into the holding vessel by slowly pouring liquid down the side wall of the vessel until the screen was submerged. Bubble point tests would then commence by slowly ramping the pressure underneath the screen relative to atmospheric pressure. Once the differential pressure across the screen was sufficiently high, bubbles would break through the screen as indicated through visual inspection and the spike in the DPT signal. At the exact moment of bubble breakthrough, the time stamp in the data acquisition was noted to compare with the time stamp in the data file during post processing. As soon as a bubble broke through the screen, the differential pressure was immediately decreased to reseal the screen. The average time between initial ramp in pressure and bubble breakthrough was approximately 25 - 35 seconds. The slow ramp rate for this and all subsequent bubble point tests renders the correction factor for gas pressurization ramp rate on pore diameter to be less than $1 \%$ (Hernandez et al. 1996):

$$
D_{P}=\frac{4 \gamma_{r e f} \cos \theta_{c}}{\Delta P_{B P, r e f}}\left(1+\frac{2 B}{\gamma_{L V}} \sqrt{\frac{d P_{r a m p}}{d t} \mu}\right)
$$

Bubble point tests were then repeated numerous times at identical conditions to ensure quality results, repeatability, and consistency and to null out the possibility of defects by ensuring bubbles would break through various locations on the screen. A sample test run 
is depicted in Figure 4.4 where the pressure differential at break through and at reseal is superimposed on the raw DPT signal. Note that the reseal pressure is always lower than the breakthrough pressure.

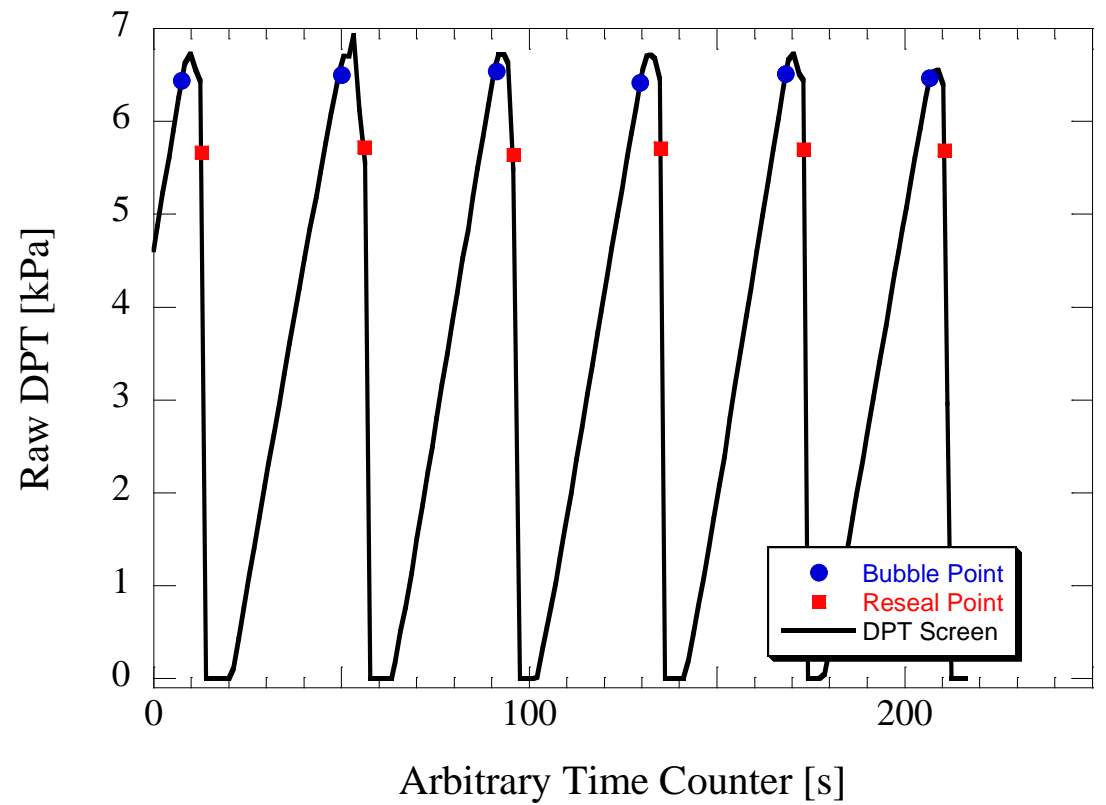

Figure 4.4 - Raw Differential Pressure Transducer Signal, Bubble Point, and Reseal Pressure as a Function of Time

Before removing the screen sample and flange from the holding cylinder, a purge gas flow was established for $5-10$ minutes to remove any trace liquid from the pressurization line and from beneath the screen. The screen sample and flange were removed from the holding cylinder and disconnected from the pressurization and sensing lines. Liquids were properly removed and disposed from the holding cylinder, and the vessel was chemically cleaned, wiped, and allowed to dry overnight in a dry oven.

Proper cleaning and handling of screen samples between tests was extremely important to ensure consistent bubble points and wettability of the screens (Arifvianto et 
al. 2011). Experimental results of Thongyai (2005) and Niemi et al. (2010) indicated that simple chemical cleaning of the screens would be sufficient to obtain repeatable results. Once testing was completed, screen samples were immediately removed from the flanged section and allowed adequate time to dry with a high flow GHe purge gas. Once the sample was dry, it was chemically cleaned in 2-3 acetone baths to remove any possible debris or residue from previous tests. The screens were then allowed to dry in a warm environment for a full day before commencement of the next reference fluid test.

For data reduction, the raw DPT pressure at breakthrough was extracted from the file and superimposed on the raw signal as a function of time as shown in Figure 4.4. Any small residual baseline due to trapped liquid beneath the screen was subtracted from the raw DPT signal. The signal was corrected for the pressure from the liquid head on top of the screen through knowledge of liquid density calculated from thermocouple temperature readings and liquid height on top of the screen to obtain the experimental bubble point pressure values for pure reference fluids:

$$
\Delta P_{B P}=\left(\Delta P_{D P T}-\text { baseline }\right)-\rho_{\text {liquid }} g h
$$

The thermocouple measured liquid temperature to within $+/-1 \mathrm{~K}$. The helium line pressure was measured to within $4.6 \mathrm{kPa}(0.667 \mathrm{psia})$. The height of liquid on top of the screen was measured to within $0.318 \mathrm{~cm}(1 / 8$ in). The uncertainty in the raw DPT measurement was less than $+/-34.5 \mathrm{kPa}(0.005 \mathrm{psia})$. However, due to read off errors, interpolating between recorded values, and due to uncertainty in liquid head pressure, the total uncertainty in reported bubble point values was $+/-62 \mathrm{kPa}(0.009$ psia), which was no greater than $1.2 \%$ at the smallest measured value. 


\subsubsection{Contact Angle Measurements}

After all bubble point testing was completed, the desired method for measuring contact angles (Lander et al. 1993) for each combination of fluid and screen were performed using the Sessile Drop Method (Mack and Lee 1936, Smolders and Duyvis 1961, Sangiorgi et al. 1982, Cheng et al. 1990, Rio and Neumann 1997), to correct theoretical predictions. To the author's knowledge, there was only one attempt at measuring the contact angle of a methanol/water binary mixture (Addesso and Lund 1997) as well as other binary mixtures (Li et al. 1992) on normal SS, but no known attempts at measuring pure fluid or binary mixture fluids with porous LAD screens. A priori knowledge of the free surface energy for each reference fluid permits the use of a modified version of the Sessile Drop method to determine the advancing contact angle for pure fluids and as a function of methanol mass fraction for mixtures (Johnson and Dettre 1964, Neumann and Good 1979). Advancing contact angles can be measured through knowledge of both the volume and diameter of deposited oblate semi-spheroidal liquid drops on top of the screen surface as a function of time (Holdich et al. 2006). The radius of the droplet as a function of time can be determined through visualization with an appropriate length scale on the screen surface in the video, and the height of the spheroid can be determined through droplet volume. Through geometrical arguments, one can then solve for the inner angle of the oblate spheroidal droplet and thus contact angle (Woodward 2010). For repeatable and consistent results, cautionary techniques from previous contact angle measurements were employed in these measurements (Johnson and Dettre 1969 and Drelich et al. 1996). 
A hypodermic syringe needle equipped with a 19 gauge needle was used to extract liquid from the cylinder and deposit liquid on the screen surface. A 10 megapixel camera imaged the screen and recorded small video clips of the technique in real time. A fine ruler was positioned in the background of the image to accurately measure the growing radius of the oblate spheroidal drops. Each liquid was tested with each screen sample to rule out possible differences in contact angles that may arise between the different screen meshes. While this added to total testing time (one data point per screen per day), it further ensured accurate results. Uncertainty in reported contact angles is estimated to be no greater than $1^{\circ}$ for pure fluids and $1.67^{\circ}$ for mixtures.

Figure 4.5 shows water droplets on a 510x3600 screen during Sessile Drop measurements. Measured contact angles for the $325 \times 2300$ screen using acetone, methanol, IPA, and water are $5.97^{\circ}, 5.4^{\circ}, 6.3^{\circ}$, and $49.5^{\circ}$ respectively. Contact angles for these fluids on porous fine mesh screens, which might be assumed to be "rough", compare well with solid SS304 surfaces where data is available in the literature (Addesso and Lund 1997). Although not reported here, variations in contact angles between the three meshes were less than the uncertainty in the measurement, and therefore contact

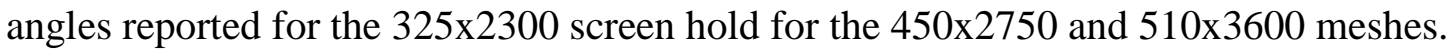




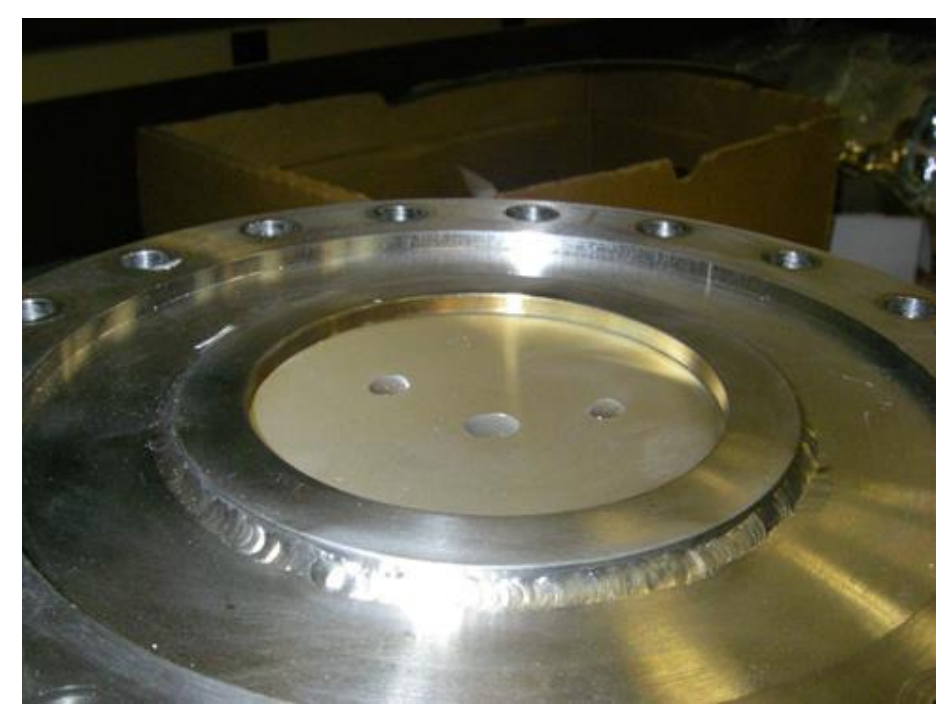

Figure 4.5 - Water Droplets on a 510x3600 Screen Sample during Sessile Drop Measurements

\subsubsection{Experimental Bubble Point Results}

Figures $4.6 \mathrm{a}-\mathrm{c}$ plot experimental results for the pure reference fluid bubble point tests for the $325 \times 2300,450 \times 2750$, and 510x3600 screen samples, respectively. To compare with pore diameters based on SEM analysis and historical data, also plotted for reference is a best fit linear curve to the data. As shown, for all three screens, bubble point is directly proportional to the contact angle corrected surface tension of each pure reference fluid, thus validating the simplified model in Chapter 3. From the bubble point versus surface tension plots, one can easily determine a best fit effective pore diameter (Method 1) for each screen sample. These pore diameters, along with those based on SEM analysis and historical data, are listed in Table 4.2 for comparison of the three methods proposed earlier. Uncertainties for pore diameters based on reference fluid tests here are based on uncertainty in the DPT reading. Uncertainties for historical values are estimated. 
A few key points are noted from this comparison. First, the plots of $\Delta P_{B P} v s \gamma_{L V}$ are linear. But, the pore diameters based on experimental data show that bubble point pressure does not scale inversely with the mesh of the screen: the highest bubble point pressures were obtained using the second finest $450 \times 2750$ mesh screen. Surprisingly, the coarser 325x2300 screen also outperforms the finest 510x3600 mesh at room temperature.

Second, experimental results reported here compare very well with data available in the literature for the $325 \times 2300$ screen reported in Chapter 3. Miniscule differences between Methods 1 and 2 are attributed to small variations in screen batches during the manufacturing process. In addition, most historical data was not corrected for nonzero contact angle.

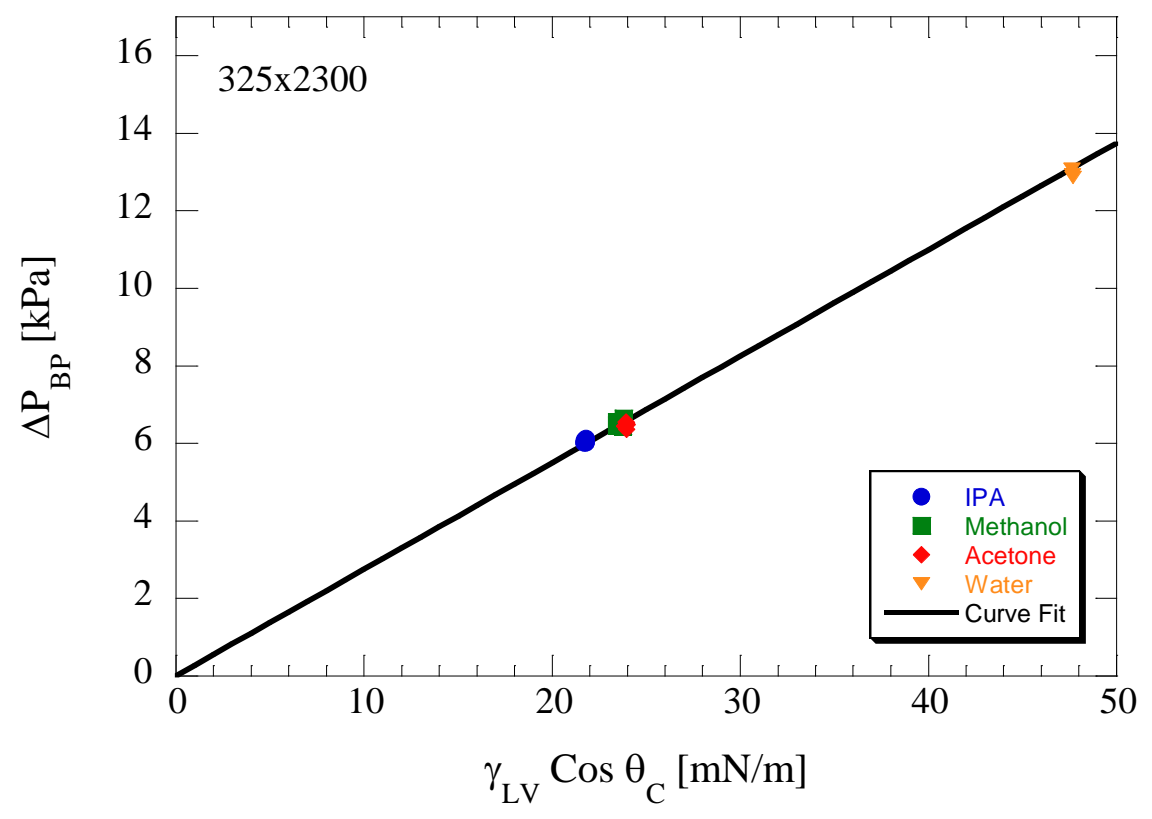



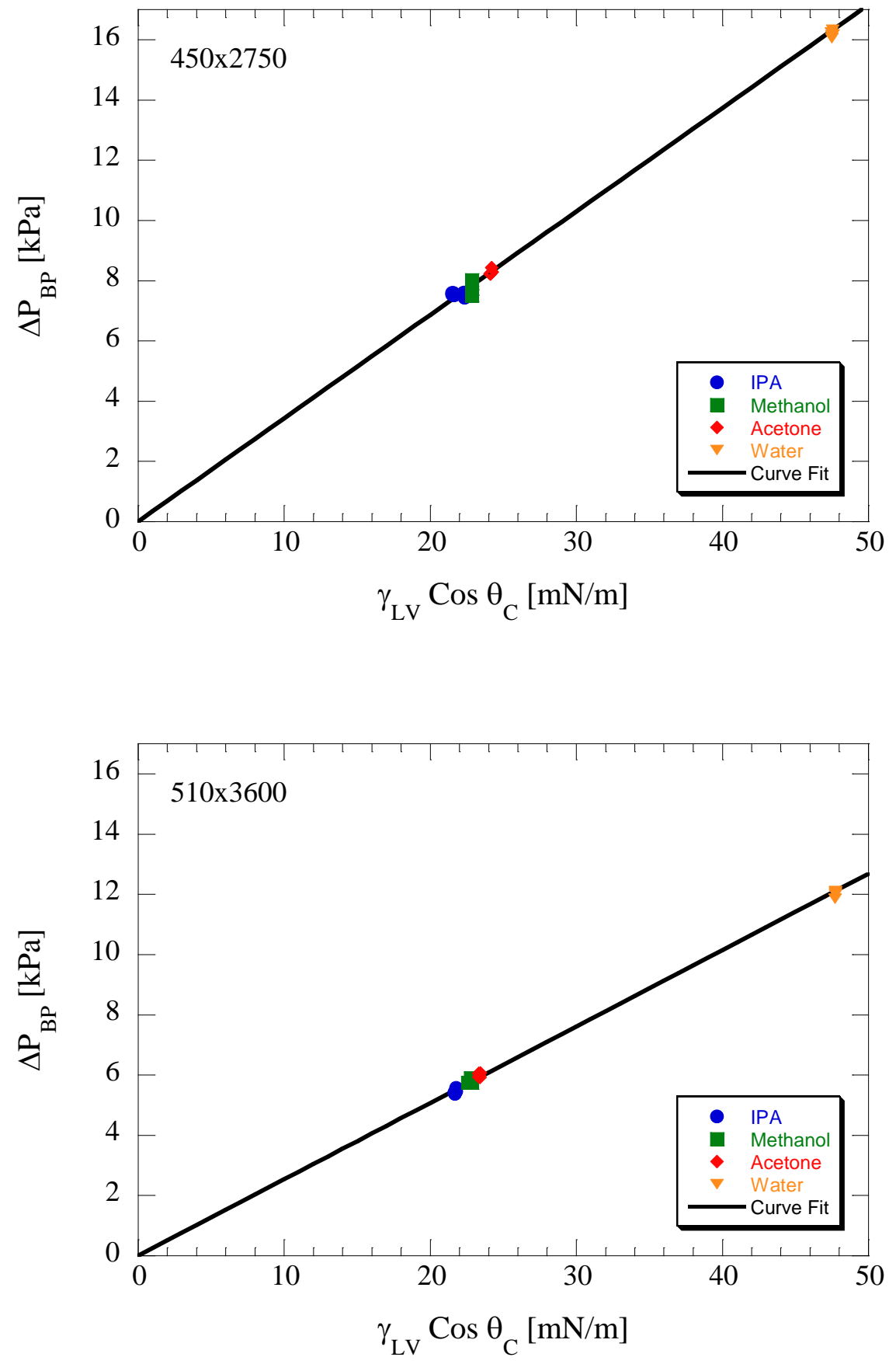

Figure 4.6 - Room Temperature Bubble Point Pressure as a Function of Surface Tension of Pure Fluids for the a) 325x2300, b) 450x2750, and c) 510x3600 Mesh Screen Samples 


\begin{tabular}{|c|c|c|c|}
\cline { 2 - 4 } \multicolumn{1}{c|}{} & More Diameter $[\boldsymbol{\mu m}]$ & \multicolumn{1}{c|}{} \\
\cline { 2 - 4 } & $\begin{array}{c}\text { Method 1 } \\
\text { Current Work }\end{array}$ & $\begin{array}{c}\text { Method 2 } \\
\text { Historical }\end{array}$ & Method 3 \\
\hline $\mathbf{3 2 5 \times 2 3 0 0}$ & $14.55 \pm 0.3225$ & $14.6 \pm 0.55$ & $14.8 \pm 0.05$ \\
\hline $\mathbf{4 5 0 \times 2 7 5 0}$ & $11.65 \pm 0.3225$ & $11.8 \pm 0.55$ & $11.9 \pm 0.05$ \\
\hline $\mathbf{5 1 0 \times 3 6 0 0}$ & $15.77 \pm 0.3225$ & - & $9.95 \pm 0.05$ \\
\hline
\end{tabular}

Table 4.2 - Comparison of Three Methods for Determining the Effective Pore Diameter: 1. Fit to Equation 3.20 Using Pure Fluid Contact Angles, 2. Historical Data, 3. Scanning Electron

Microscopy Image Analysis

Third, pore diameters based on SEM analysis are slightly higher than those obtained via experimental bubble point tests for the $325 \times 2300$ and $450 \times 2750$ meshes. Therefore, SEM analysis tends to underpredict the bubble point pressure. Differences in pore diameters between SEM image analysis and experiments are attributed to the fact that the $\mathrm{L} / \mathrm{V}$ interface forms a complex 3D structure within the screen. The SEM pores are purely a $2 \mathrm{D}$ projection onto the top of the screen. Recent simulations from PMD Technology (Jaekle 2011) indicate that the actual L/V interface at bubble breakthrough may form a $60-70^{\circ}$ angle with respect to the screen face, deep within the screen mesh itself. This would result in a much smaller hole size than the 2D planar triangular pore.

Fourth, for all three screens and each room temperature liquid, the bubble point pressure is accurately linear with $\gamma_{L V} \cos \theta_{C}$ for each liquid. The effective pore diameter $D_{p}$, computed from Equation 3.20 was consistent with the effective diameter estimated from SEM images of the weaves for the two coarser screens. However, for the 510x3600 weave $D_{p, \text { SEM }}=9.95 \pm 0.05 \mu \mathrm{m}$ while $D_{p}=15.77 \pm 0.32 \mu \mathrm{m}$. It may be that the contribution of the pore structure of the weave to $F\left(\cos \theta_{c}, D_{p}\right)$ can be sharpened. 
Finally, results indicate that there are other factors that control bubble point pressure beyond the fineness of the screen, such as the actual shape of the L/V interface within the pores, the path length that a gas bubble must travel before breaking through the wetted portion of the screen, as well as the geometry of the actual pore formed by the intersection of the wires. Interestingly, results show that there may be an optimal mesh to maximize the bubble point pressure.

\subsection{Binary Mixture Tests}

\subsubsection{Experimental Setup, Methodology, and Data Reduction}

The same experimental setup and methodology from pure fluid tests was used here for binary mixture reference fluid testing. The following modifications were employed: Using graduated cylinders, water or methanol was added in known quantities to create a mixture at a known mass fraction of methanol, to submerge the LAD screen. Stirring rods were used to ensure uniform mixture composition. To change the mass fraction, a known amount of either methanol or water was simply added and mixed within the holding cylinder. Mixture tests would cease when the holding cylinder was nearly full. Cold ambient temperatures less than $277 \mathrm{~K}\left(40^{\circ} \mathrm{F}\right)$ in the test cell during bubble point tests ensured minimal evaporation of methanol from the mixtures. Then bubble point tests would commence; GHe was introduced beneath the LAD screen until breakthrough. The time at breakthrough was noted to compare with the time stamp in the data acquisition recording of the differential pressure across the screen. Then the GHe supply was decreased until screen reseal, and the process repeated again. At each methanol mass fraction, for each screen mesh, multiple points were recorded to ensure 
repeatability and consistency. At the end of each test, the screen and flange assembly was purged with dry GHe, the LAD screen was subject to a chemical bath, and the vertical holding cylinder was wiped clean and allowed to dry overnight.

To report bubble point values as a function of methanol mixture mass fraction, the following equation was used:

$\Delta P_{B P}=\Delta P_{D P T}-\rho_{\text {mix }} g h$

where $\rho_{m i x}$ was the binary fluid mixture density. The mixture density was calculated through precise knowledge of the added volume of methanol and water:

$\rho_{\text {mix }}=Y_{\text {methanol }} \rho_{\text {meth }}+\left(1-Y_{\text {methanol }}\right) \rho_{\text {water }}$

where methanol mass fraction was calculated from:

$Y_{\text {methanol }}=\frac{\rho_{\text {meth }} V_{\text {meth }}}{\rho_{\text {meth }} V_{\text {meth }}+\rho_{\text {water }} V_{\text {water }}}$

The pressure underneath the screen was increased in fixed, quasi-static increments for precise pressure measurements and to allow a quasi-static equilibrium for the contact angle between the pore throat and solution and for the contact angle that is eventually pinned at the pore mouth. Bubble point was thus taken as the point when a visible gas bubble detached and broke away from the screen.

\subsubsection{Theoretical Predictions}

Pre-test predictions were performed based on Equation 3.16. To do so, knowledge of mixture surface tension, mixture contact angle, and effective pore diameters is 
required. Here, pore diameters were based on Method 1 in Table 4.2, pure reference fluid bubble point tests. Mixture surface tension for all mass fractions was estimated from an equation of state and Langmuir isotherm (1916) fit to data available in the literature. Mixture contact angles were measured as a function of methanol mass fraction.

\subsubsection{Liquid/Vapor Surface Tension of the Methanol/Water Mixture}

Surface tension values were previously measured at different mass fractions of methanol in water at constant temperature from $293 \mathrm{~K}$ to $323 \mathrm{~K}$, and are available in the literature (Vazquez et al. 1995). Bubble point tests here were conducted over a colder range of temperatures $(275-295 \mathrm{~K})$, so measurements from Vazquez et al. (1995) at $293 \mathrm{~K}$ are used to predict all surface tension values for colder liquid. Surface tension data as a function of mass fraction of surfactant (methanol) at $293 \mathrm{~K}$ is plotted in Figure 4.7. Also plotted for comparison is the data from Addesso and Lund (1997).

A Langmuir isotherm, Equation C.26, is employed to obtain a curve fit to the data for the purposes of predicting bubble point pressures at any mass fraction. The Gibbs equation using the convention that the excess volume and the excess of the solvent (water in this case) are set to zero in order to satisfy the Gibbs phase rule that specifies the number of independent variables allowed in the representation of the surface tension of the binary solution of water and methanol. A derivation is presented in Appendix C; details are also available in Hansen (1962) and Turkevich and Mann (1990a). Figure 4.7 plots the Langmuir isotherm to the data of Vazquez et al. (1995) using these fitting parameters. Only the data from Vazquez et al. (1995) is included in the fit because data 
from Addesso and Lund (1997) do not report a binary liquid temperature for their experiments.

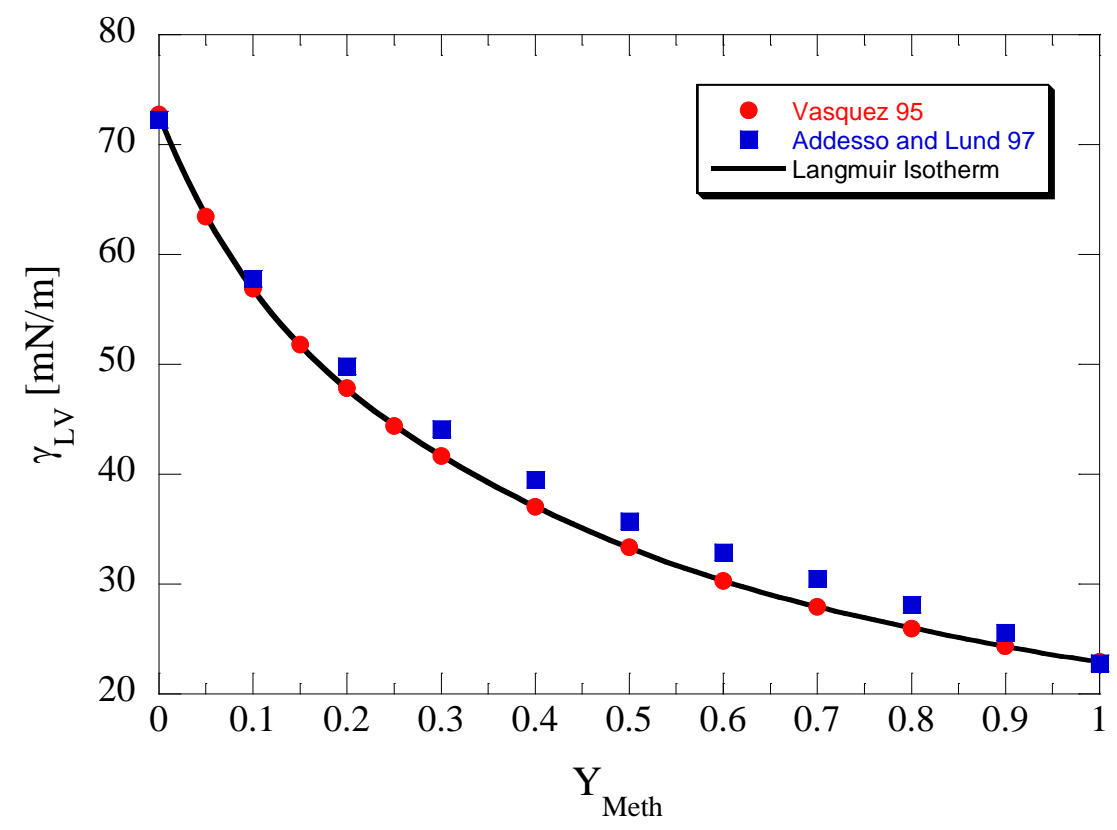

Figure 4.7 - Experimental Data and Langmuir Isotherm Fit to the $\mathrm{T}=20^{\circ} \mathrm{C}$ Methanol/Water Surface Tension Data from Vazquez et al. (1995)

\subsubsection{Contact Angle Measurements}

The same modified version of the Sessile Drop method was used to measure the advancing contact angle for mixtures with various methanol mass fractions. All solutions were prepared and mixed within a 100 milliliter glass graduated cylinder to measure liquid volume to within $1 \%$. Figure 4.8 plots the contact angle of methanol/water mixtures as a function of methanol mass fraction. 


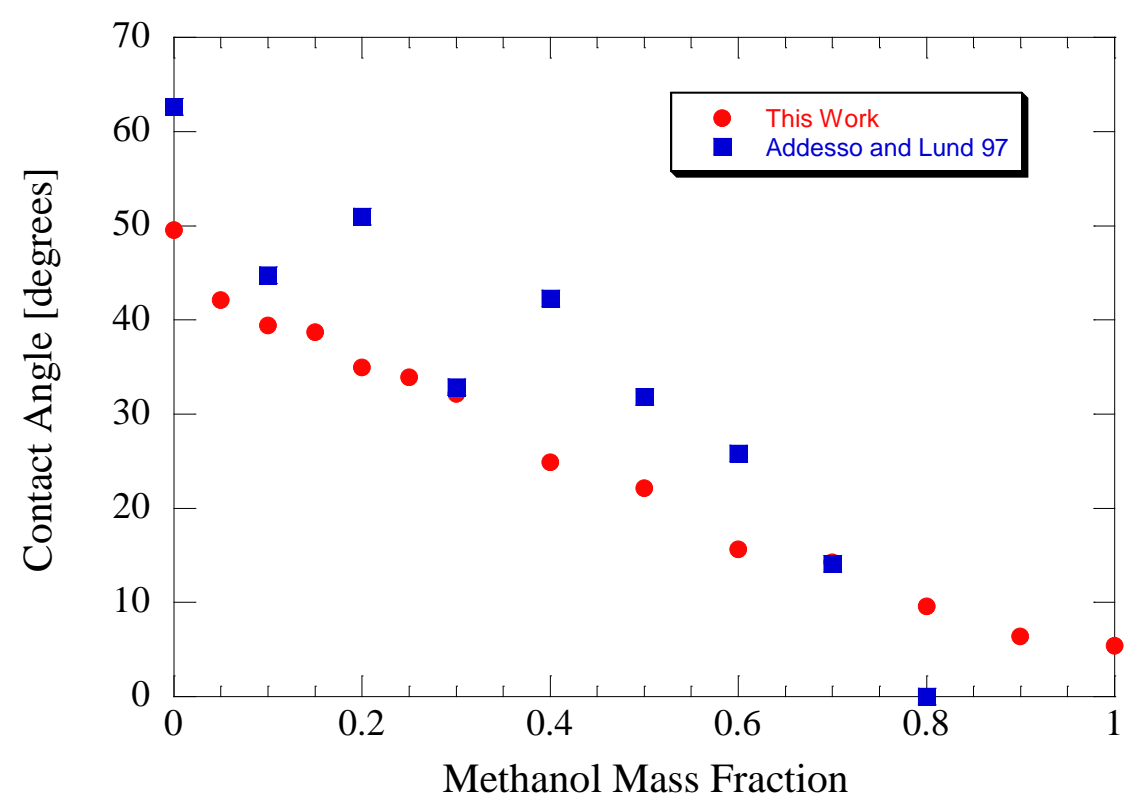

Figure 4.8 - Contact Angle Measurements of Methanol/Water Mixtures on the 325x2300 Screen

Each data point represents the average of measurements at three independent, random locations on the screen surface. Also plotted is the methanol/water contact angle data from Addesso and Lund (1997) obtained using the Whilhelmy plate method. Reasonable agreement exists between the data sets. Methanol mass fraction values in this work were chosen consistent with mass fractions from bubble point measurements. Contact angle measurements for mixtures here are also in qualitative agreement with previously reported results from Fan et al. (2011) for ethanol and n-propanol aqueous solutions. Note that mixture contact angles did not deviate outside of experimental uncertainty between the three screens as in the pure fluids contact angle measurements.

Pretest predictions for the methanol/water binary mixture are plotted in Figure 4.9. As shown in Figure 4.9a, bubble point pressure scales with the contact angle corrected L/V surface tension of the liquid. The curvature tracks the surface tension data 
and contact angle measurements from Figures 4.7 and 4.8, respectively. Meanwhile, the predicted bubble point pressure scales with methanol mass fraction according to the curves in Figure 4.9b. Note that the $450 \times 2750$ mesh is predicted to outperform both 325x2300 and 510x3600 screen when using Method 1 to specify the effective pore diameter.

\subsubsection{Experimental Results}

\subsubsection{Bubble Point Pressure}

Figures $4.10 \mathrm{a}-4.12 \mathrm{~b}$ plot the experimental results for the binary mixture bubble point tests. Figures 4.10a, 4.11a, and 4.12a plot bubble point pressure as a function of the contact angle corrected surface tension, and Figures $4.10 \mathrm{~b}, 4.11 \mathrm{~b}$, and $4.12 \mathrm{~b}$ plot the bubble point pressure as a function of the methanol mass fraction for the $325 \times 2300$, 450x2750, and 510x3600 screens, respectively. For each screen, tests were conducted beginning with pure methanol, then diluting with water, as well as tests beginning with pure water then diluting with methanol. The small overlap in methanol mass fractions allowed direct comparison between the two dilution techniques and ensured that the data was independent of the chosen starting point. As shown, excellent agreement is obtained in the intermediate methanol mass fraction range because the data compare well between the two marching schemes. Theoretical predictions are based on pore diameters based on Method 1 from Table 4.2. 

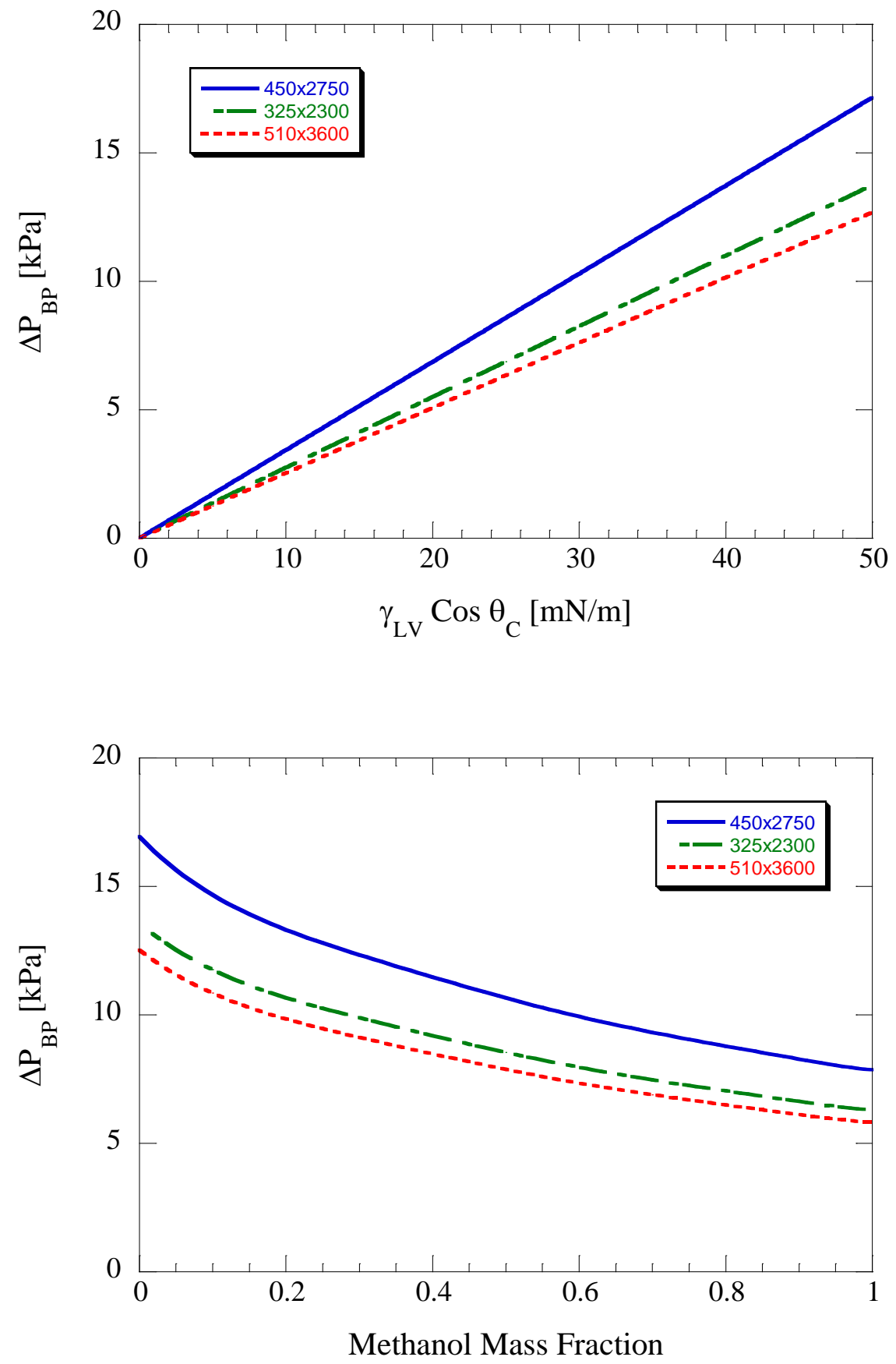

Figure 4.9 - Binary Mixture Bubble Point Predictions as a Function of a) Mixture Surface Tension and b) Methanol Mass Fraction 

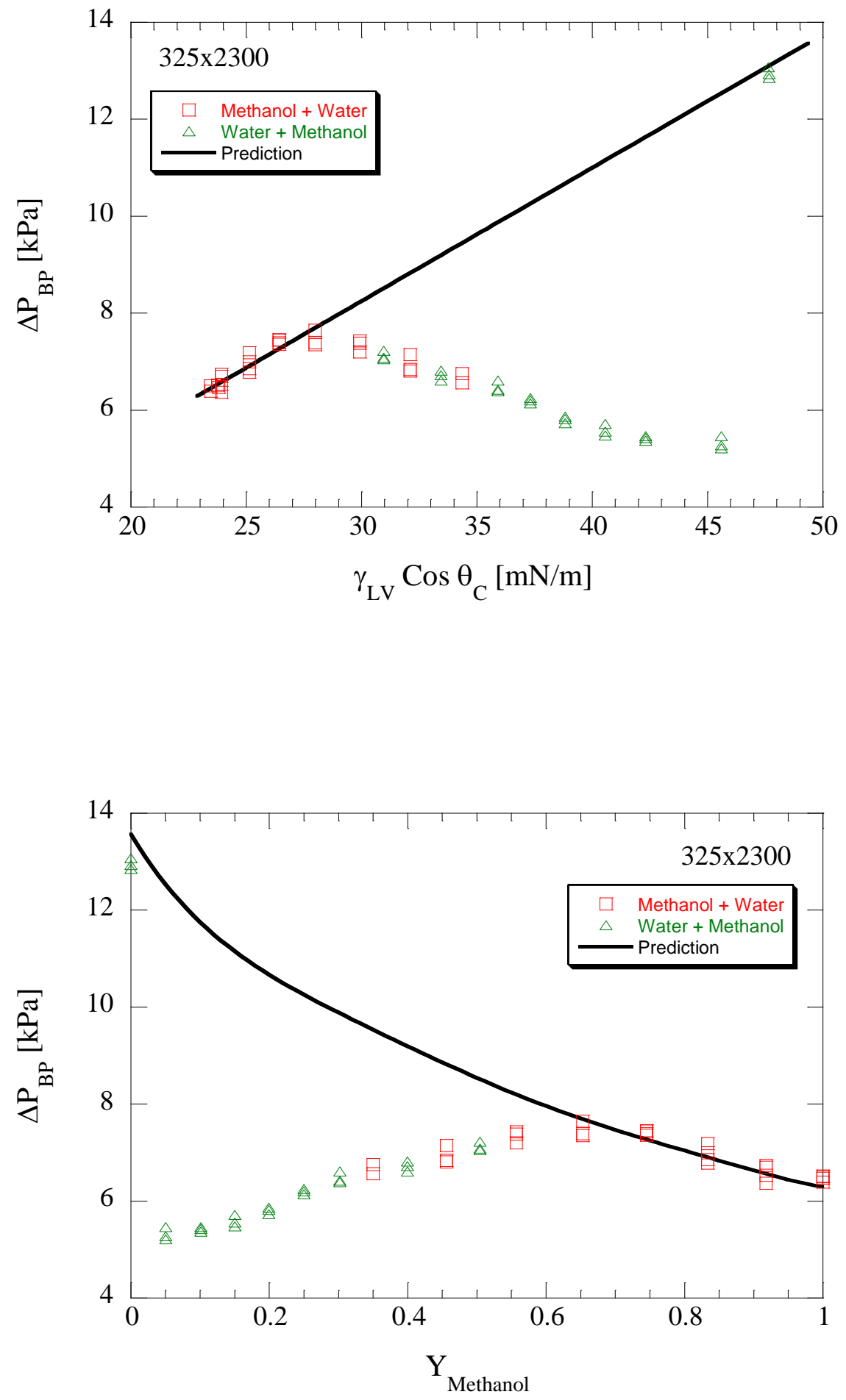

Figure 4.10 - Bubble Point Pressure as a Function of Methanol/Water Mixtures as a Function of

a) Surface Tension and b) Methanol Mass Fraction for the 325x2300 Screen 

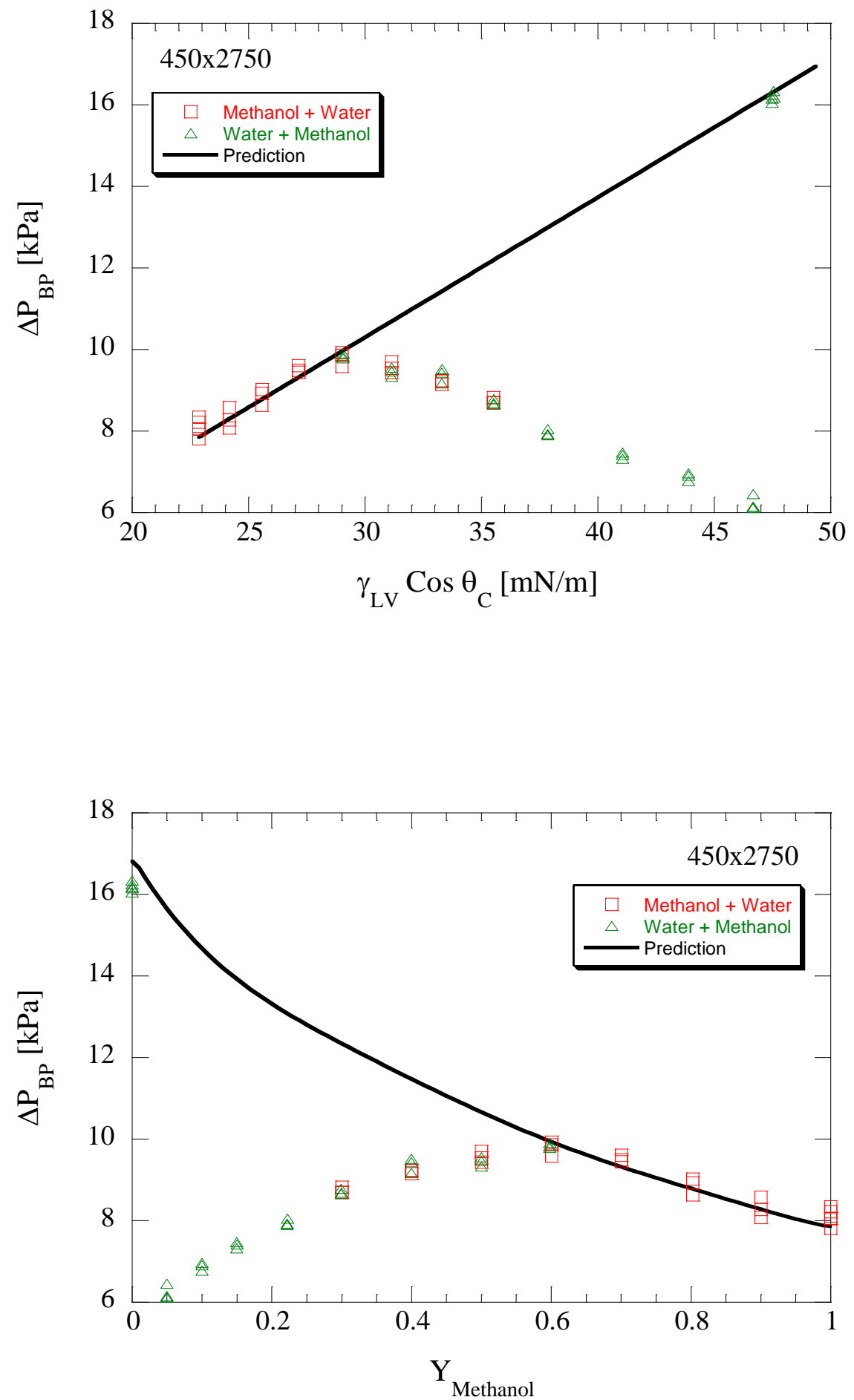

Figure 4.11 - Bubble Point Pressure as a Function of Methanol/Water Mixtures as a Function of a) Surface Tension and b) Methanol Mass Fraction for the 450x2750 Screen 

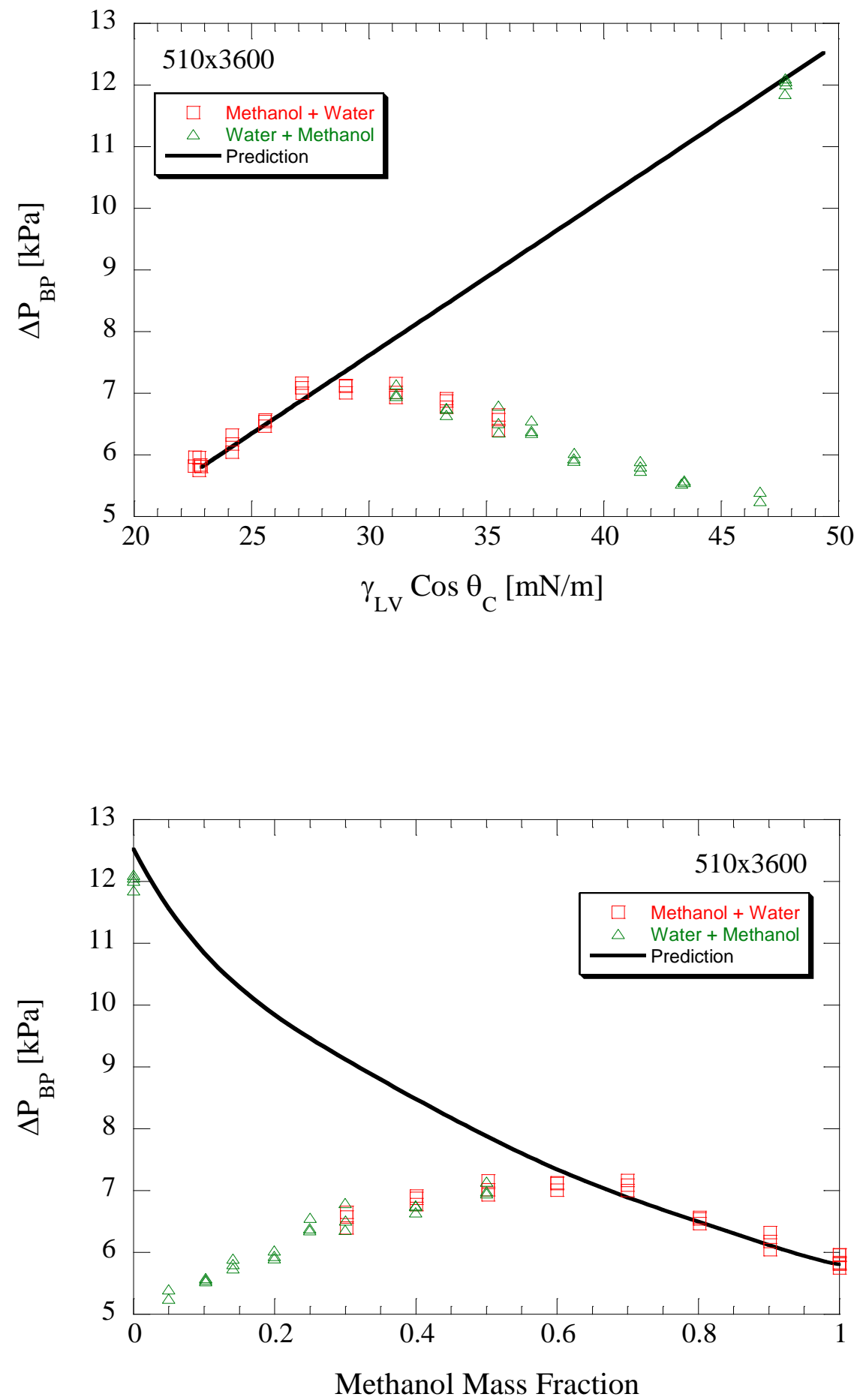

Figure 4.12 - Bubble Point Pressure as a Function of Methanol/Water Mixtures as a Function of

a) Surface Tension and b) Methanol Mass Fraction for the 510x3600 Screen 
As shown, the $450 \times 2750$ produces the highest bubble point pressure across the range of conditions. The coarser 325x2300 mesh outperforms the finest 510x3600 mesh, confirming the fact that the bubble point pressure does not scale inversely with the fineness of the screen. This reiterates the fact that the effective pore diameter is not only affected by the fluid, metal, and contact angle, but also the specific geometry of the L/V interface within the screen pores. Nonetheless, a simple 2D projection of a complex 3D interface does not model the binary mixture or the pure fluid data, and thus Method 3 based on SEM analysis is again rejected.

To confirm that the performance gain using the 450 screen over the 510 screen was indeed true, and not due to faulty equipment or experimental apparatus, bubble point tests were again repeated in both pure and mixture fluids a second round to ensure repeatability. No significant deviations in the results were noted. In addition, test hardware was inspected again. The screen samples were again analyzed under the SEM microscope to ensure consistency in warp and shute wire diameters all along the surface of the sample. No noticeable defects or deviations from the original measurements were noticed.

For all three screens, the data clearly deviates from the theoretical predicted value at methanol mass fractions less than $65 \%$, even when the surface tension data is corrected for contact angle. Below methanol mass fractions of $65 \%$, the only data point that matches with theoretical predictions is the pure water case. Figure 4.13 plots methanol mass fraction vs. methanol mole fraction. Comparing mass to mole fraction indicates that this apparent peak in bubble point at a mass fraction of $65 \%$ corresponds to a mole fraction of $\sim 50 \%$ for the methanol/water mixtures from this work. 


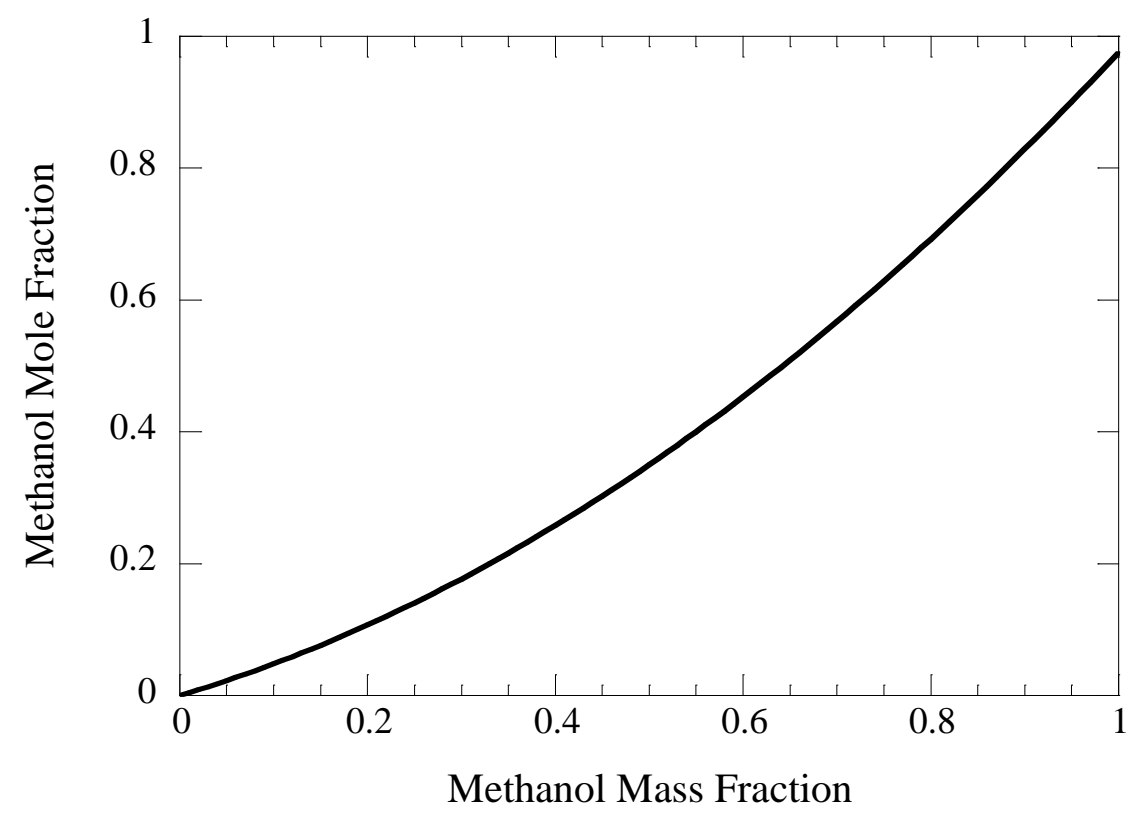

Figure 4.13 - Methanol Mole Fraction versus Mass Fraction

The disagreement between data and theory is attributed to the following rationale: The screen is a porous object with millions of micron sized holes, each with its own L/V interface. Statistically speaking, it is unlikely that every pore will have the same mixture composition at equilibrium. During pressurization underneath the screen, a bubble will pass through the largest pore that is occupied by the liquid with the lowest surface tension relative to other pores (i.e. simple path of least resistance). Results here indicate that the highest bubble point for mixture fluids occurred when there was an equal distribution of methanol and water molecules at the liquid side of the interface. As the mixture dilutes toward pure water, the high contact angle of water and low surface tension of methanol dominate to produce lower than expected breakthrough pressures. Therefore, the data seems to indicate that methanol has a higher probability of populating an interface for this particular mixture. The paper by Dougan et al. (2004) provided a study that demonstrated 
the complex character of the structures that are found in methanol/water solutions at various mole fractions. They provided molecular dynamic simulations (MDS) that suggest the possibility of doing simulations of the equilibrium states on a well-defined surface for a range of methanol concentrations. The implication of inhomogeneous solution of these room temperature liquids at the screen thankfully has no effect on LAD performance in cryogenic liquids, since propellants are always stored in a homogenous, pure liquid state inside the propellant tank.

\subsubsection{Critical Zisman Surface Tension}

$\mathrm{L} / \mathrm{V}$ surface tension values can be used to estimate solid/liquid (S/L) and solid/vapor (S/V) surface tensions of binary liquids from the classical force balance projected on the helium/binary liquid mixture/SS304 screen pore interface as follows:

$$
F_{S L}-F_{S V}=-F_{L V} \cos \theta_{C}
$$

This force balance assumes that the vertical component of the force is ignored, which resulted in a long and tangled history of dispute (Adamson and Gast 1997). For the YLE, these forces are in direct proportion to the respective surface tensions:

$\gamma_{S L}-\gamma_{S V}=-\gamma_{L V} \cos \theta_{C}$

where $\gamma_{S L}$ and $\gamma_{S V}$ are the SS304/solution and SS304/vapor surface tensions, respectively. The YLE is considered valid for surfaces that are sufficiently hard so that there is a small normal displacement at the three phase contact line. Although it is not possible to directly measure $\gamma_{S L}$ or $\gamma_{S V}$, several algorithms based on the work of adhesion 
and the work of cohesion have been formulated to allow computation of $\gamma_{S L}$ and $\gamma_{S V}$ as a function of composition.

Consider the free energy change per unit area, known as the work of cohesion, defined as:

$\Delta G_{C}=2 \gamma_{S V}$

The work of adhesion is defined as:

$W_{a}=\Delta G_{a}=\gamma_{L V}+\gamma_{S V}-\gamma_{S L}$

Substituting Equation 4.7 into Equation 4.9:

$W_{a}=\gamma_{L V}\left(1+\cos \theta_{C}\right)$

which permits direct computation of the work of adhesion using the known, measured binary mixture surface tension $\gamma_{L V}$ and contact angle $\theta_{C}$. The empirical equation of state analysis of Neumann and Spelt (1996) is used to estimate $\gamma_{S V}$ :

$\overline{W_{a}}=2 \sqrt{x} \exp \left(-\beta_{M} \gamma_{L V}^{2}(1-x)^{2}\right)$

where $\overline{W_{a}}=\frac{W_{a}}{\gamma_{L V}}$ and $x=\frac{\gamma_{S V}}{\gamma_{L V}}$. Certain authors, for example Li et al. (1992) and Good (1977), comment that $\gamma_{S V}$ should be independent of $\gamma_{L V}$, but this is often not observed.

Since the contact angle necessarily involves the three-phase contact line, the assumption of uniform chemical potentials requires that the composition of the vapor phase and therefore the magnitude of $\gamma_{S V}$ must depend on $Y_{2}$. 
Figure 4.14 plots the normalized work of adhesion $\bar{W}_{a}$, of the SS304 system as a function of the $\mathrm{L} / \mathrm{V}$ surface tension, $\gamma_{L V}$. Also plotted for comparison is the methanol/water data from Addesso and Lund (1997). As shown in both data sets, the normalized work of adhesion varies with $\mathrm{L} / \mathrm{V}$ surface tension; results from the current work show smoother variation to the contact angle data than that from Addesso and Lund (1997).

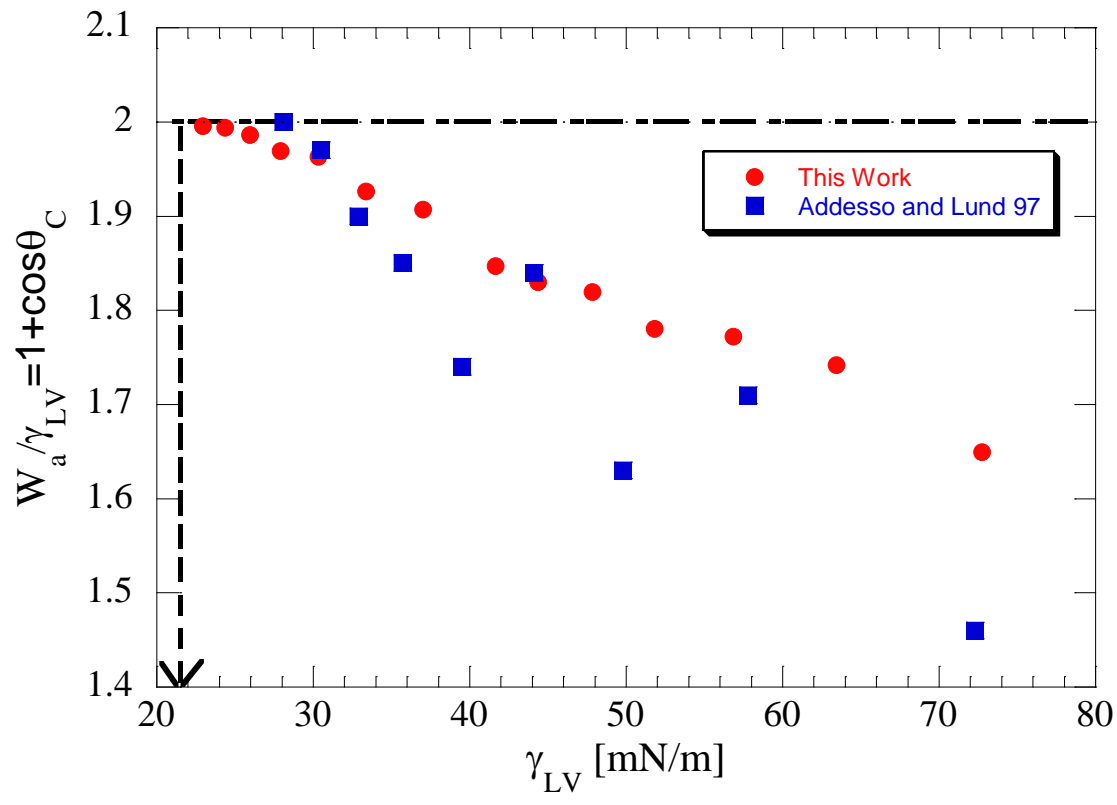

Figure 4.14 - Zisman Plot for Binary Methanol/Water and Stainless Steel 304 System

Figure 4.14 is otherwise known as a Zisman plot (1964). By extrapolating to $1+\cos \theta_{C} \rightarrow 2$, one can obtain the so-called "critical" Zisman (CZ) surface tension for a given solid material, where the contact angle approaches 0 . This condition defines the point of total wettability at the surface of the solid. Therefore, for $\gamma_{L V}<\gamma_{C Z}$, the contact angle is not expected to deviate from $\theta_{C}=0$, and the surface can be assumed to be 
completely wetted. Examination of data from this work indicates $\gamma_{C Z}=23.2 \pm 2 \frac{\mathrm{mN}}{\mathrm{m}}$ for the porous LAD screen. This compares to $\gamma_{C Z}=24.8 \pm 5 \frac{m N}{m}$ for a solid SS304 surface (Addesso and Lund 1997). This implies LAD screens demonstrate slightly improved wettability over nonporous SS due to the presence of the LAD screen pore.

The critical Zisman surface tension is anticipated to be only slightly dependent on temperature between room and $\mathrm{LH}_{2}$ temperatures. The temperature dependent coefficient for the surface tension of the liquid and solid materials is negative so that the surface tension increases as the temperature is cooled below the critical temperature (Adamson and Gast 1997). This property is difficult to measure in solids but is most probable that the S/V surface tension of SS is at most somewhat larger at $\mathrm{LH}_{2}$ temperatures than at room temperatures.

Critical Zisman surface tension values obtained here have direct implications for predicting LAD behavior for cryogenic propellants. All cryogenic fluids of interest, including liquid helium, hydrogen, nitrogen, oxygen, and methane, have surface tension values less than the critical Zisman surface tension reported here. Results imply total wettability and that $\theta_{C}=0$ for all cryogenic liquids with SS304 LAD screens, which further simplifies the bubble point model presented earlier. While bubble point data exists for LAD screens of different metals, contact angle between cryogenic liquids and $\mathrm{Al}$ and Ti screens is not anticipated to deviate from $0 . \mathrm{S} / \mathrm{V}$ and $\mathrm{S} / \mathrm{L}$ surface tension values are given in Appendix D. 


\subsection{Reseal Pressure Tests}

Room temperature reseal pressure data was collected simultaneously with bubble point data. The same inverted bubble point test configuration used to measure the bubble point was used to measure reseal pressures. A bubble point test ceases once pressurant gas breaks through the wetted pore. To commence a reseal point test, the pressurant gas flow rate beneath the screen is slowly reduced in fixed quasi-static increments to slowly encroach upon the differential pressure across the screen at which the screen reseals. Eventually the screen rewets/reseals itself as evident in visualization of no more bubbles across the screen. The raw DPT at reseal is then corrected for liquid head pressure on top of the screen. Reseal tests can then be simultaneously collected along with bubble point data, and repeated at different thermodynamic conditions.

Figure 4.4 illustrates this effect for a room temperature bubble point and reseal test where the corrected reseal pressure is superimposed on the raw DPT signal. As shown, the reseal pressure is always lower than the bubble point pressure. Unlike bubble point data reduction, the DPT across the screen cannot alone be used to determine screen reseal. Rather, time synchronization with the visualization system is required to determine the exact differential pressure across the screen at reseal. Sole reliance on visualization makes reseal point data inherently noisier than bubble point data.

Room temperature reseal pressure data is shown in Figure 4.15 for a $325 \times 2300$, 450x2750, and a 510x3600 screen in IPA, methanol, acetone, and water. Also plotted in Figure 4.15 is a best fit line to the data. The exact same trends in bubble point pressure are seen in reseal pressures. Comparing Figure 4.6 to Figure 4.15, all reseal pressures 
clearly lie below the corresponding bubble points. For all data collected here, reseal pressures are approximately $90 \%$ of the bubble point data.
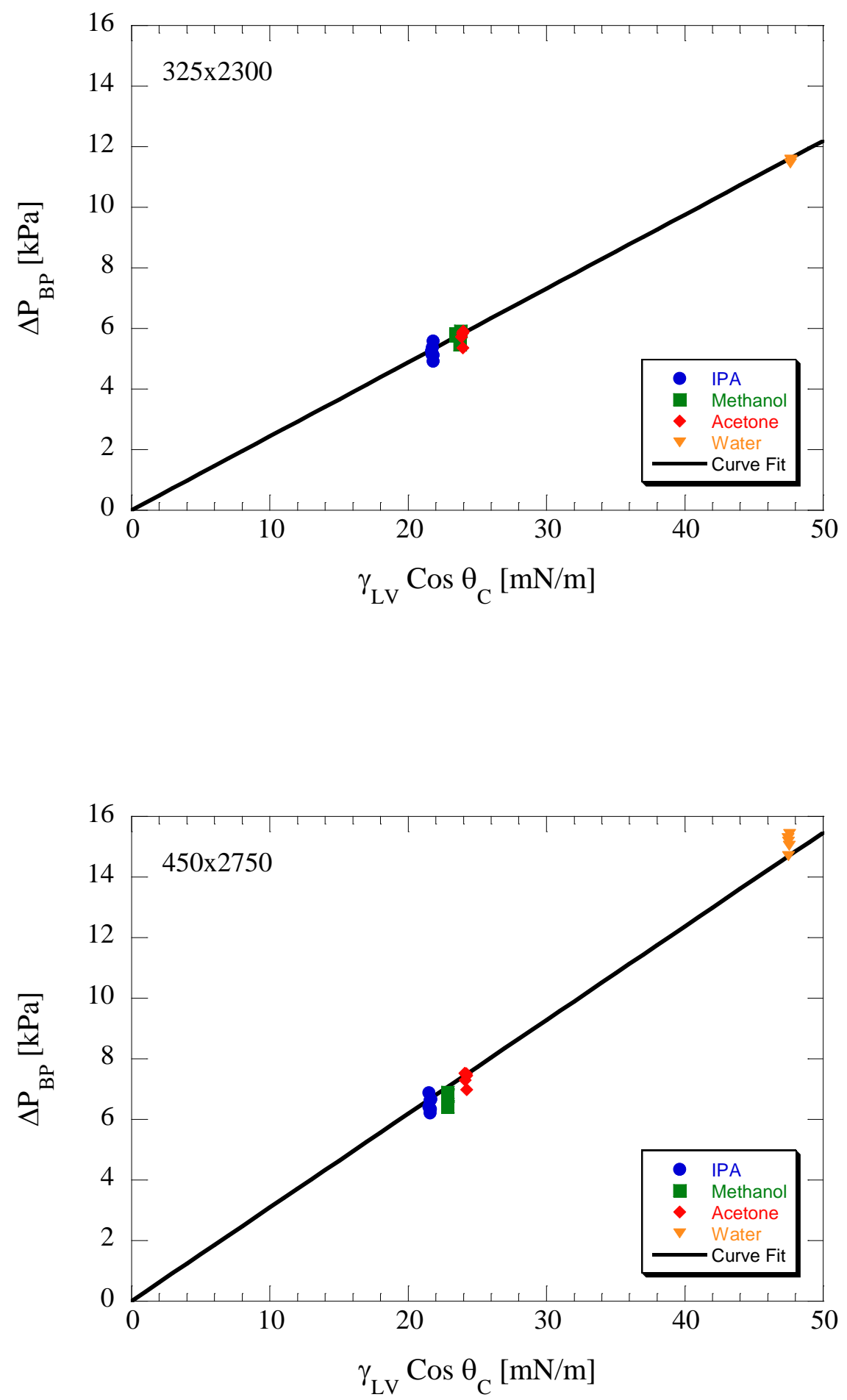


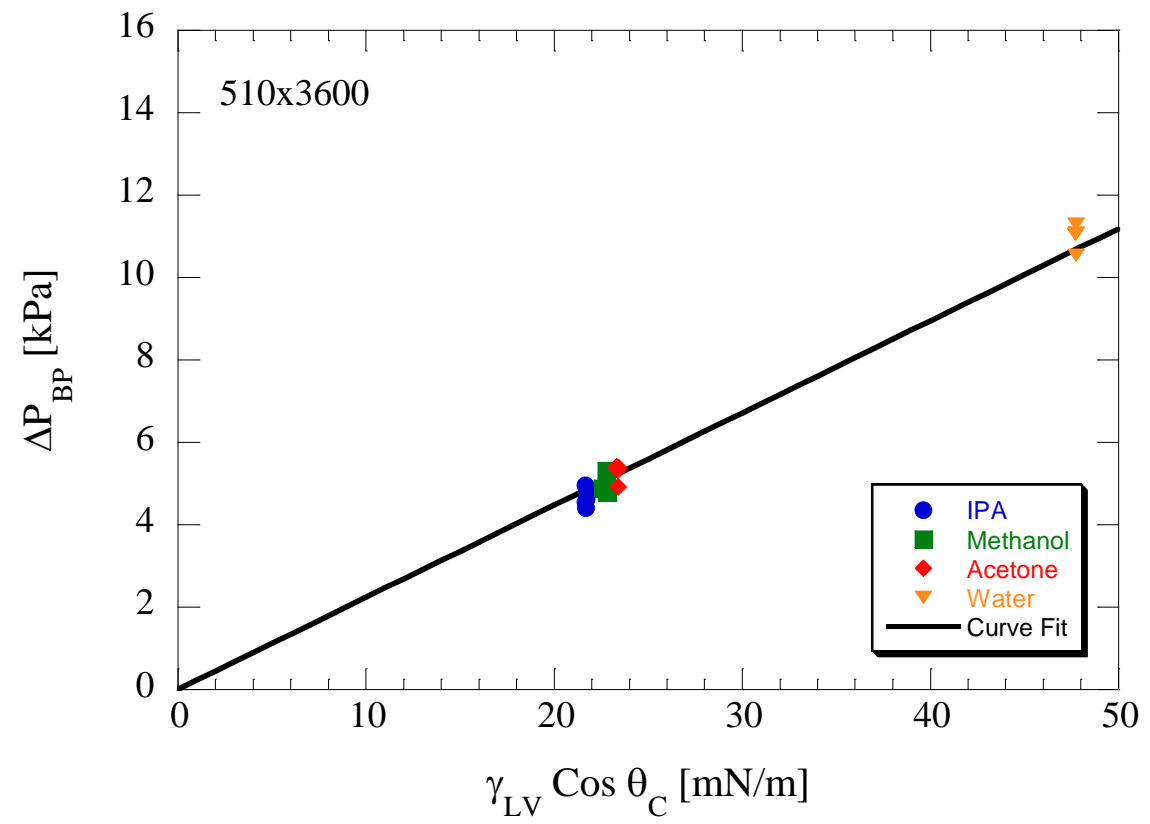

Figure 4.15 - Room Temperature Reseal Point Pressure as a Function of Surface Tension of Pure Fluids for the a) 325x2300, b) 450x2750, and c) 510x3600 Mesh Screen Samples

\subsection{Wicking Rate Tests}

Using the testing methodology outlined in Section 3.6.2, a simple horizontal wicking experiment was performed with a 30x150, 325x2300, 450x2750, and 510x3600 LAD mesh sample at the CCL-7. A picture of the experimental setup is shown in Figure 4.16. In the experiment, each screen sample was cut into a $7.62 \mathrm{~cm} \times 20.3 \mathrm{~cm}(3$ in $\times 8$ in) rectangle. Each sample was cut such that there was a single razor sharp straight edge to ensure a uniform wicking front along the screen. A non-straight edge would cause the wicking front to proceed at different rates at different points of the screen. This can be corrected by averaging the wicking distance at multiple coordinates, but it is preferable to simply eliminate the source of error. 


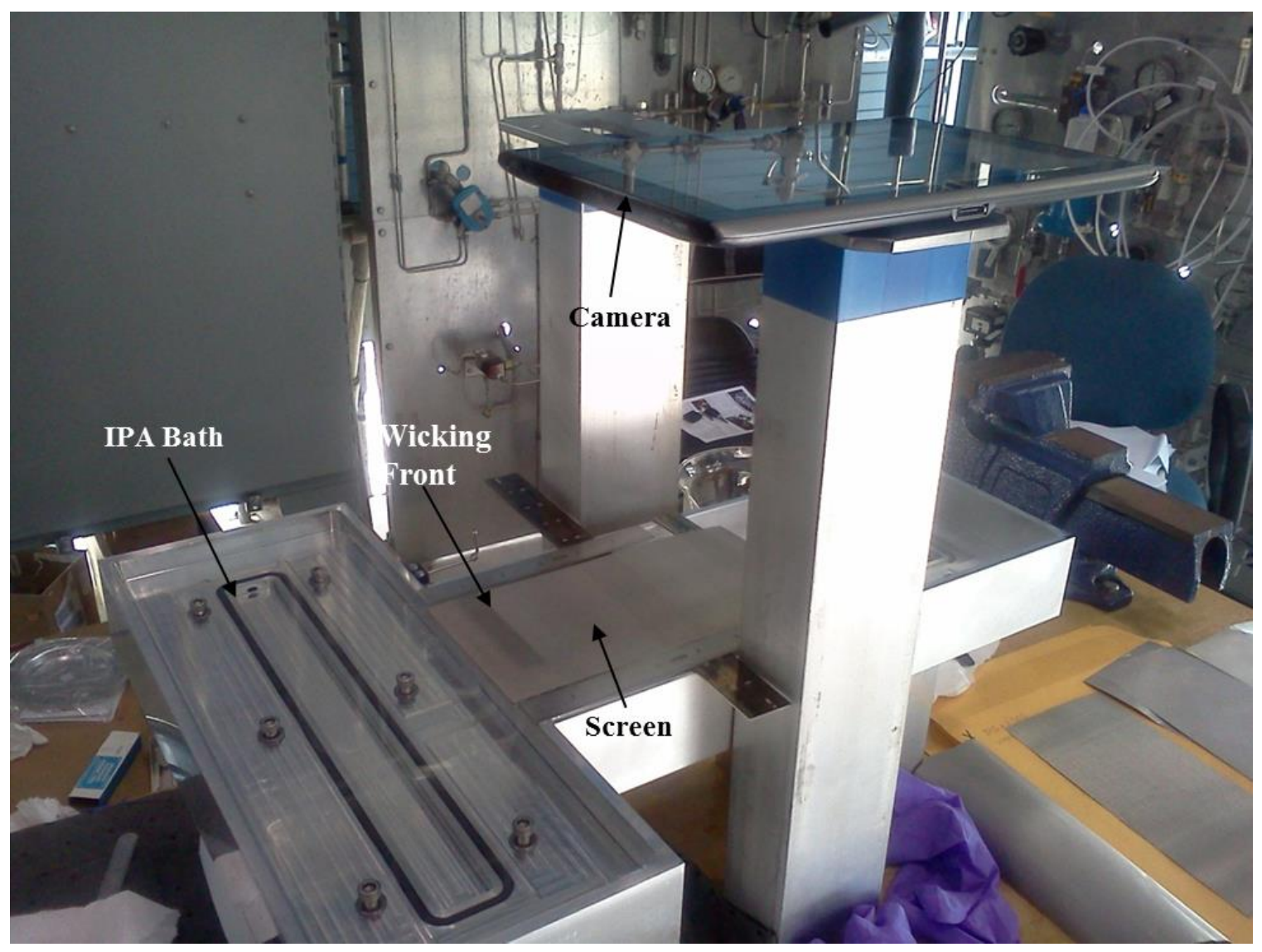

Figure 4.16 - Experimental Setup for Wicking Tests. Shown in the picture is the 30x150 screen.

IPA was the test fluid of choice. For each test, a screen sample was placed horizontally over the edge of the liquid bath shown on the left of Figure 4.16. The IPA bath was filled to the top so that the overhanging portion of the screen became wet. A camera from above then recorded video of the screen as the liquid wicked to the dry portion of the screen. Rulers were placed next to the screen so that wicking distance could be monitored with time. From the wicking front versus time video, it was possible to determine the effective wicking diameter for each screen. Tests were conducted parallel and perpendicular to the warp wires, for comparison. 
Figure 4.17 shows a sample of the wicking distance as a function of time for the three fine mesh Dutch Twill samples. As shown, the wicking distance is proportional to the square root of time, as predicted by Equation 3.65. The fastest wicking distance occurs with the coarsest screen, consistent with historical trends.

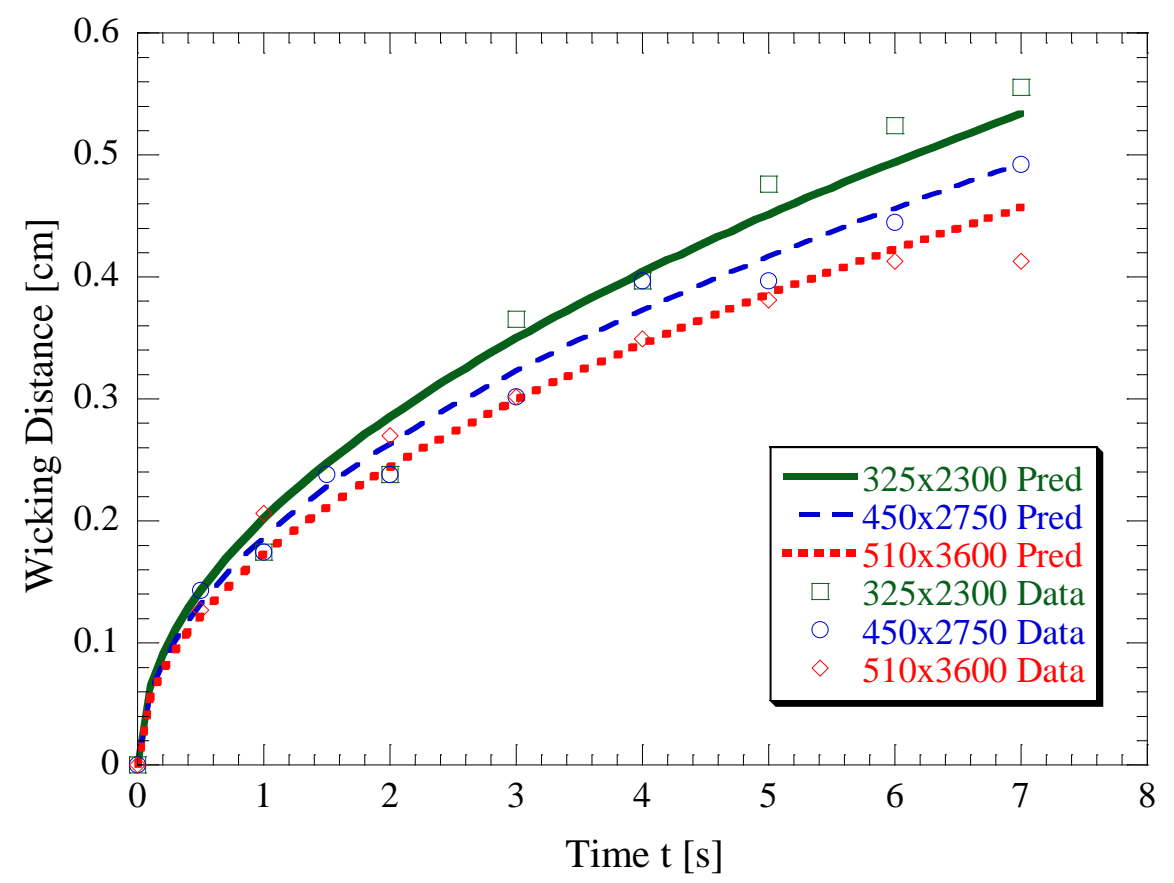

Figure 4.17 - Wicking Distance as a Function of Time for a 325x2300, 450x2750, and 510x3600 Dutch Twill Screen in Isopropyl Alcohol

Fitting a curve to the data sets, effective wicking diameters $D_{E}$, can be obtained for the four screens. Table 4.3 lists the updated wicking diameters from historical and current work, for comparison. Examination of Table 4.3 and Equation 3.65 indicates that the flow resistance becomes stronger more so than the capillary pressure does as the screen becomes finer. Therefore, $D_{H}^{2}$ becomes smaller more so than $D_{C}$ does as the screen becomes finer. Examination of the data indicates for screens with an $n_{s}$ to $n_{w}$ 
ratio greater than 5, the wicking rate is faster when the warp wires are parallel to the wicking front than when the warp wires are perpendicular to the wicking front. The opposite is true for screens with an $n_{s}$ to $n_{w}$ ratio less than 5. In general, coarser screens wick faster than finer meshes.

\begin{tabular}{|c|c|c|c|c|c|c|c|}
\hline \multirow[b]{2}{*}{ Screen Type } & \multirow[b]{2}{*}{ Weave Type } & \multirow[b]{2}{*}{ Orientation } & \multicolumn{4}{|c|}{ Wicking Effective Diameter, De $(\mu \mathrm{m})$} & \multirow[b]{2}{*}{ Current Work } \\
\hline & & & Symons 1974 & Dodge 2000 & Fries et al. 2007 & Hastings et al. 2011 & \\
\hline $510 \times 3600$ & Twilled Dutch & Perpendicular & - & - & - & - & $0.54 \pm 0.13$ \\
\hline ' & ' & Parallel & - & - & - & - & $1.09 \pm 0.10$ \\
\hline $450 \times 2750$ & Twilled Dutch & Perpendicular & - & - & - & - & $1.24 \pm 0.08$ \\
\hline ' & ' & Parallel & - & - & - & - & $1.27 \pm 0.09$ \\
\hline $325 \times 2300$ & Twilled Dutch & Perpendicular & 0.88 & - & - & 1.77 & $1.24 \pm 0.10$ \\
\hline ' & & Parallel & 1.36 & - & - & 2.74 & $1.49 \pm 0.10$ \\
\hline $200 \times 1400$ & Twilled Dutch & Perpendicular & 0.88 & 0.88 & - & 2.11 & \\
\hline ' & ' & Parallel & 2.72 & 2.72 & 3.38 & 4.15 & \\
\hline $165 \times 1400$ & Twilled Dutch & Perpendicular & 1.52 & 1.52 & - & - & \\
\hline ' & ' & Parallel & 3.04 & 3.04 & - & - & \\
\hline $200 \times 600$ & Twilled Dutch & Perpendicular & 7.76 & 7.76 & - & - & \\
\hline ' & ' & Parallel & 4 & 4 & - & - & \\
\hline $165 \times 800$ & Twilled Dutch & Perpendicular & 12.8 & 12.8 & - & 14.18 & \\
\hline ' & ' & Parallel & 3.84 & 3.84 & - & 4.2 & \\
\hline $80 \times 700$ & Twilled Dutch & Perpendicular & 3.36 & - & - & - & \\
\hline ' & ' & Parallel & 6.96 & 6.96 & - & - & \\
\hline $30 \times 500$ & Twilled Dutch & Perpendicular & 5.68 & - & - & - & \\
\hline ' & ' & Parallel & 29.6 & - & - & - & \\
\hline $30 \times 250$ & Twilled Dutch & Perpendicular & - & 1.83 & - & - & \\
\hline ' & ' & Parallel & - & - & - & - & \\
\hline $20 \times 250$ & Twilled Dutch & Perpendicular & 13.6 & 13.6 & - & - & \\
\hline ' & ' & Parallel & 27.2 & 27.2 & - & - & \\
\hline $50 \times 250$ & Plain Dutch & Perpendicular & 12.8 & - & & & \\
\hline ' & ' & Parallel & 5.36 & - & - & - & \\
\hline $30 \times 150$ & Plain Dutch & Perpendicular & & & & & $13.36 \pm 0.15$ \\
\hline ' & ' & Parallel & & & & & $0.54 \pm 0.12$ \\
\hline $24 \times 110$ & Plain Dutch & Perpendicular & 20 & - & - & - & \\
\hline ' & ' & Parallel & 12 & - & - & - & \\
\hline
\end{tabular}

Table 4.3 - Updated Effective Wicking Diameters for Dutch Twill and Plain Dutch Weaves. The orientation column signifies how the wicking front is oriented with the warp wire direction.

\subsection{Concluding Remarks}

The simplified bubble point model can be used to interpolate predictions to any pure fluid when pore diameters are based on reference fluid testing, and not SEM analysis. Where available, using historical data also results in reasonable predictions. For pure reference fluids, bubble point pressure scales linearly with surface tension only 
when correcting for contact angle. The long held assumption that bubble point pressure scales inversely with effective pore diameter is shown to be invalid, because the second finest 450x2750 screen produced the highest bubble point of the three screens. Pore diameters based on reference fluid bubble point tests conducted here are in excellent agreement with historical data, but do not match with pore diameters based on SEM analysis for the 510x3600 mesh. This discrepancy is attributed to the assumption that the equilibrium L/V interface cannot be approximated by a simple 2D planar triangular pore at the surface.

Bubble point tests conducted in methanol/water mixtures are in agreement with pure reference fluid tests in that the $450 \times 2750$ again outperformed the $325 \times 2300$ and 510x3600 meshes. Experimental data and prediction values based on pore diameters based on the pure reference fluids match well for methanol mole fractions greater than $50 \%$; for methanol mole fractions less than $50 \%$, the data deviates from theory. Care was taken during testing to ensure adequate bulk mixing of the fluids, but this discrepancy is likely attributable to small differences in mixture composition within the micron sized pores. As the mixture tends toward higher water concentrations, the low surface tension of methanol and high contact angle of water dominate and occupy the breakthrough sites.

A critical Zisman L/V surface tension value of $\gamma_{C Z}=23.2 \pm 2 \frac{\mathrm{mN}}{\mathrm{m}}$ is defined for these screen channel LADs. All cryogenic L/V surface tensions lie well below this critical value. Therefore the implication is that one can confidently assume zero contact angle between SS304 LAD screens and all cryogenic liquids. 
Trends in room temperature reseal pressure data mirrors trends in bubble point pressure data. All reseal pressures collected here are about $90 \%$ of the corresponding bubble point values. Operationally, this implies that only a $\sim 10 \%$ reduction in differential pressure across the screen is required to reseal the screen and prolong the point of total LAD failure to yield a higher overall expulsion efficiency. Wicking rate test results performed in IPA align nicely with historical trends as coarser meshes outperform finer meshes. 


\section{Chapter 5}

\section{Parametric Analysis of the Liquid Hydrogen and Nitrogen Bubble Point Pressure}

The purpose of this chapter is to present the parametric experimental investigation of the influential factors which govern screen channel liquid acquisition device performance in a low pressure propellant tank. Five of the six test parameters that were varied include the screen mesh, liquid, liquid temperature and pressure, and type of pressurant gas. Static cryogenic bubble point tests were performed in liquid hydrogen and nitrogen on three fine mesh stainless steel screen channel LAD samples $(325 \times 2300$, $450 \times 2750$, and 510x3600) over a range of liquid temperatures $(16.7 \mathrm{~K}<\mathrm{T}<21.1 \mathrm{~K})$ and pressures $(31.5 \mathrm{kPa}<\mathrm{P}<155 \mathrm{kPa})$, in saturated and subcooled liquid states, using both non-condensable (helium) as well as autogenous (hydrogen) pressurization schemes to examine functional dependencies of each of these five parameters. Details on the experimental design and methodology are discussed, including the facility, test article, instrumentation, and how to conduct a cryogenic bubble point test. Experimental results are divided among the five parameters for clarity. 


\subsection{Test Purpose and Motivation}

The primary purpose of this test series is to determine how the bubble point pressure varies with operational and thermodynamic conditions inside a low pressure cryogenic propellant tank. Parameters were thus varied in a parametric fashion across the trade space to systematically understand the individual functional dependencies on bubble point. Prior to the current work, the majority of cryogenic bubble point experiments were conducted in saturated or near saturated states at equal liquid and vapor chemical potentials, $\mu_{1}^{L} \cong \mu_{1}^{V}$, where the liquid, component 1 , was near the NBP. Previous bubble point tests conducted in subcooled liquid and at warm pressurant gas states show the data deviates significantly from simplified theory presented in Chapter 3 . Only recent high pressure capabilities have allowed the independent examination of saturated and subcooled states on the bubble point pressure (Chapters 6 and 7). Chronologically, high pressure tests were completed before the low pressure tests, and the high pressure tests show that the bubble point equation (Equation 3.16) only holds for saturated or near saturated cryogenic liquid states. Only precisely controlled parametric test conditions can elucidate underlying model discrepancies.

The secondary purpose of this test series was to demonstrate improved screen retention in several ways over the baseline $325 \times 2300$ NBP bubble point pressure. Since the $325 \times 2300$ screen has rich flight heritage in storable propulsion systems, and because data at cryogenic hydrogen exists, the $325 \times 2300$ is considered the baseline. First, choosing a finer mesh screen can theoretically decrease the effective pore diameter. While the $325 \times 2300$ has been extensively used in ground tests, examination of Figure $3.8 \mathrm{~b}$ shows that the average bubble point at the NBP at $20.3 \mathrm{~K}$ is approximately $568 \mathrm{~Pa}$. 
This low baseline reference value severely hinders operating conditions for future $\mathrm{LH}_{2}$ LAD fueled propulsion systems. This is precisely why it was desired to find and test two finer mesh screens.

The second method to increase the low $\mathrm{LH}_{2}$ baseline bubble point is to simply operate at a colder liquid temperature. L/V surface tension is an inverse function of temperature, so at colder liquid temperatures L/V surface tension is larger, resulting in higher resistance to vapor ingestion. Thus, it was desired to obtain bubble point data over a range of liquid temperatures representative of the thermal environment for $\mathrm{LH}_{2}$ propellant tank. Finally, historical bubble point data shows that bubble point may be affected by the type of gas in contact with the screen; experiments conducted in this chapter will quantify this effect.

\subsection{Experimental Design}

\subsubsection{Test Article and Facility}

Testing was performed at the CCL-7 at GRC. The exact same three samples from room temperature tests were used for cryogenic tests here. A LAD screen sample is shown in Figure 5.1. Screen samples were cut, mounted, and welded to a heavy flange with a $0.32 \mathrm{~cm}(1 / 8 \mathrm{in})$ cover ring on top of the screen. The purpose of the cover ring was to allow space for a fillet weld between flange and screen to create a leak tight seal at the screen edges and prevent premature screen breakdown due to manufacturing. The

effective exposed OD and surface area was $6.5 \mathrm{~cm}(2.5 \mathrm{in})$ and $31.7 \mathrm{~cm}^{2}\left(4.9 \mathrm{in}^{2}\right)$, respectively. Each screen was mounted into its own flange to permit rapid change out between tests. 


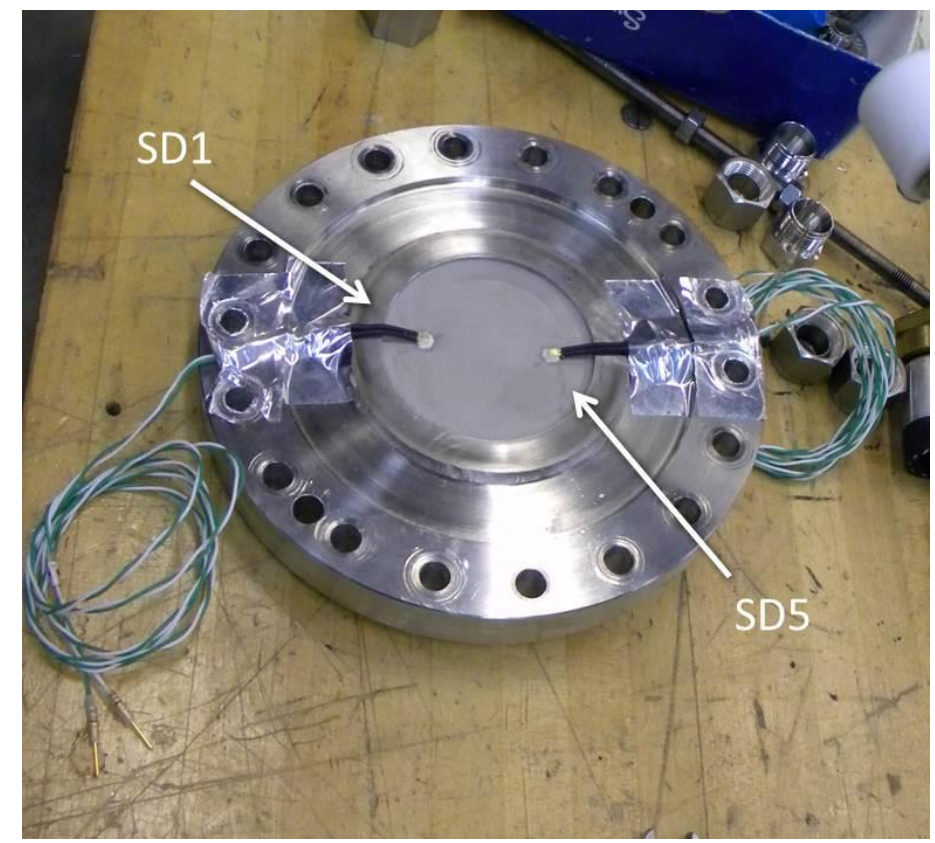

Figure 5.1 - Screen Sample and Flange

The flange was mated with a cylindrical cup shown in Figure 5.2. The purpose of the cup was to create the $\mathrm{L} / \mathrm{V}$ interface at the screen by pressurizing from underneath. Bubble point tests were conducted in an inverted fashion with liquid on top and vapor or gas on the bottom. The cup was equipped with a custom built cross to allow uniform injection of pressurant gas and slow pressure rise inside the cup. The cup was equipped with a custom fabricated central rod to support three doughnut style heaters which were mounted on the bottom of the disks in Figure 5.2. The purpose of the heaters was to warm the incoming pressurant gas, if desired. A sense line was mounted inside the cup for a DPT measurement across the screen as shown in Figure 5.2.

The completed LAD screen/cup assembly is shown in Figure 5.3. Threaded rods were used to support an angled aluminized mirror plate, which was used to reflect the top 
of the LAD screen to an externally mounted camera. A fiber optic light source was used to illuminate the mirror to enhance the visualization.

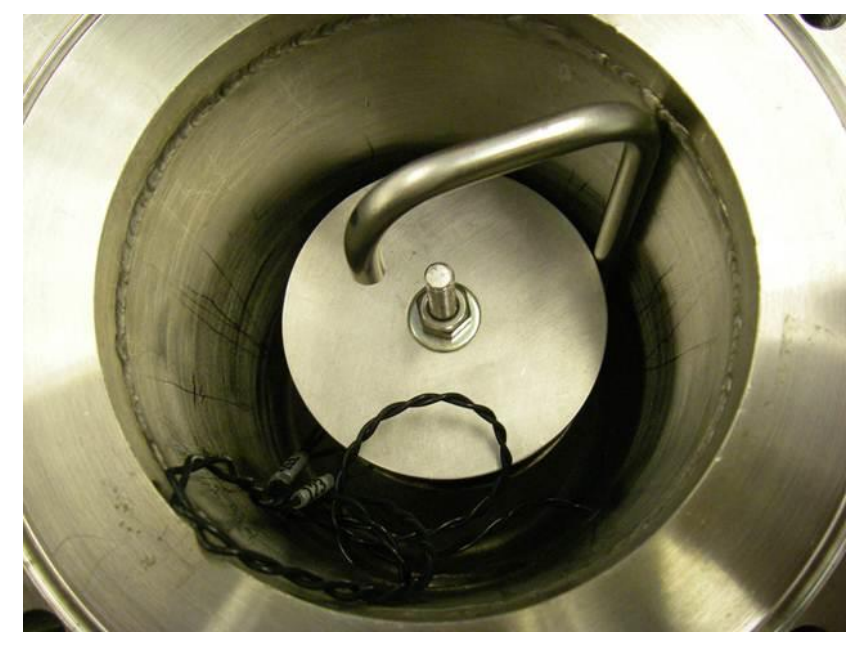

Figure 5.2 - Low Pressure Liquid Acquisition Device Cup

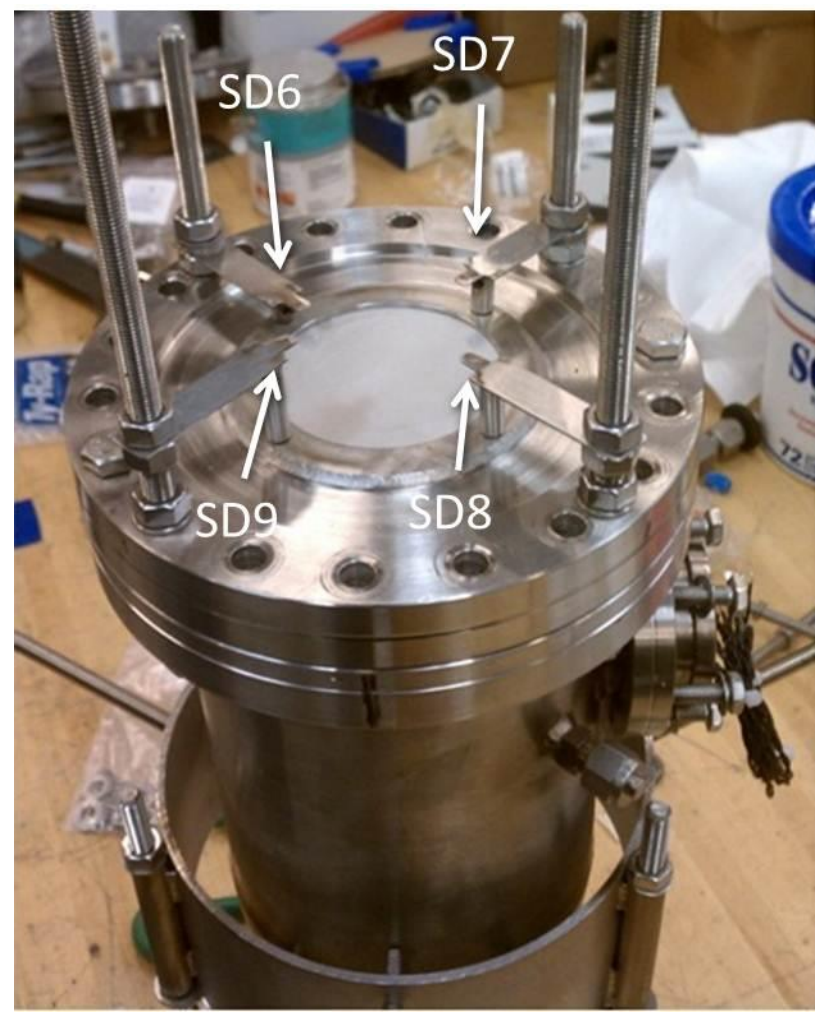

Figure 5.3 - Completed Low Pressure Screen/Cup Assembly 
The LAD screen/cup assembly was mounted inside a $56 \mathrm{~cm}$ (22 in) OD, $107 \mathrm{~cm}$ (42 in) tall, $0.229 \mathrm{~m}^{3}\left(8.1 \mathrm{ft}^{3}\right)$, optically accessible vacuum jacketed (VJ) receiver dewar (RD) as shown in Figure 5.4. The purpose of the RD was to house the cryogenic liquid on top of the LAD screen. The RD was equipped with seven ports for liquid fill and drain, vent and burst disc (BD) (x2), power, light source, and instrumentation for both RD and LAD cup, and three side ports for pressurant gas fill, drain, and DPT measurements. A viewport on the RD permitted a real time view of the LAD screen. A charge coupled device (CCD) camera located inside a purge box was connected to the viewport to view the LAD screen. The images were time stamped and recorded in real time, and sent directly to a monitor inside the Cell 7 control room used to remotely control the experiment to synchronize with all other data. The RD was rated to a maximum pressure of $170 \mathrm{kPa}$ (24.7 psia), which permitted data collection up to about $152 \mathrm{kPa}(22 \mathrm{psia})$. Air ejectors were used to evacuate the ullage space of the RD to sub-atmospheric pressures as low as $31 \mathrm{kPa}(4.5 \mathrm{psia})$.

The $\mathrm{LH}_{2}$ supply line was first routed through a supply tank (ST) as shown in Figure 5.5. The purpose of the ST was to condition the liquid cryogen for the RD. The ST contained a dedicated cooling coil that was used to supply and/or subcool the liquid before entering the RD. For testing here, the ST itself was never filled with liquid; it was simply pumped down to $13.8 \mathrm{kPa}$ to minimize heat leak into the liquid feed line. VJ lines connected the dewars in series. 


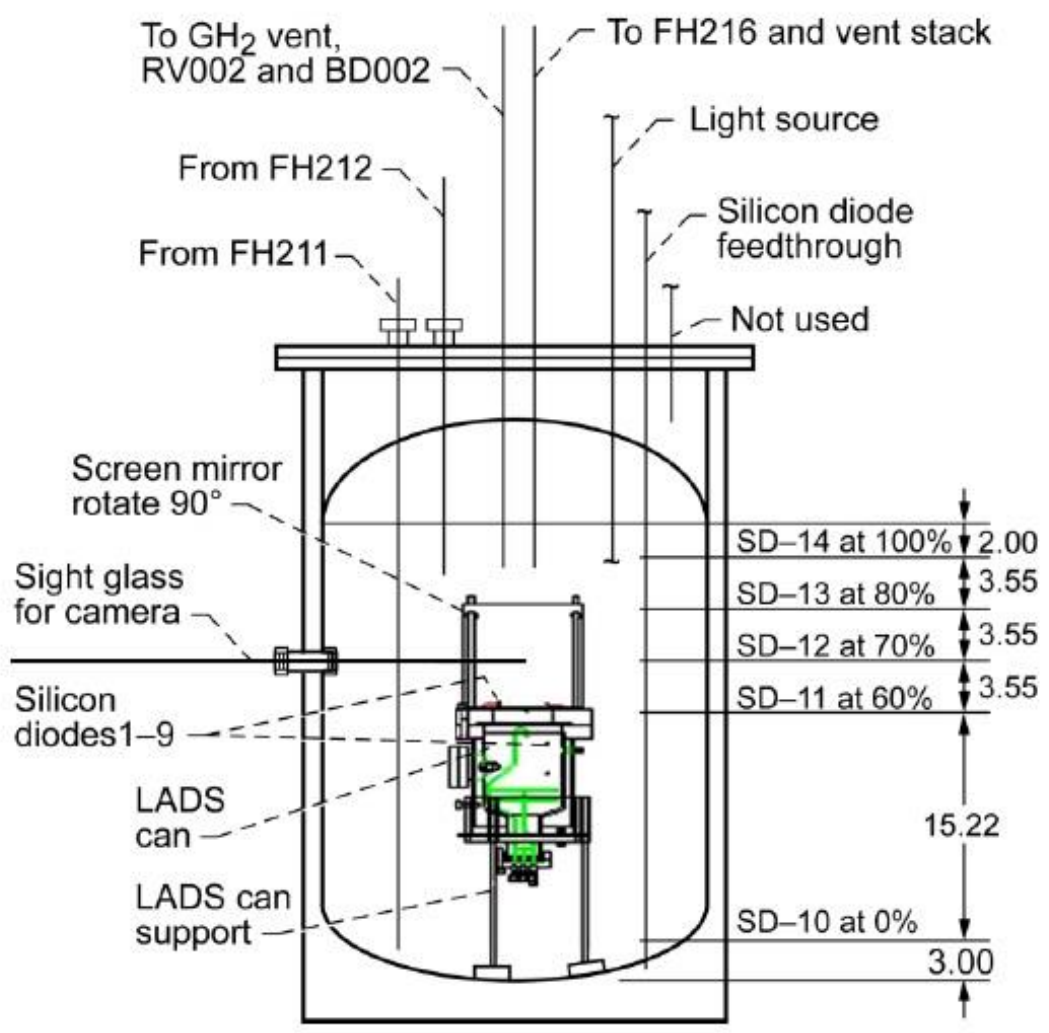

Figure 5.4 - Receiver Dewar for Low Pressure Liquid Hydrogen and Nitrogen Bubble Point Tests

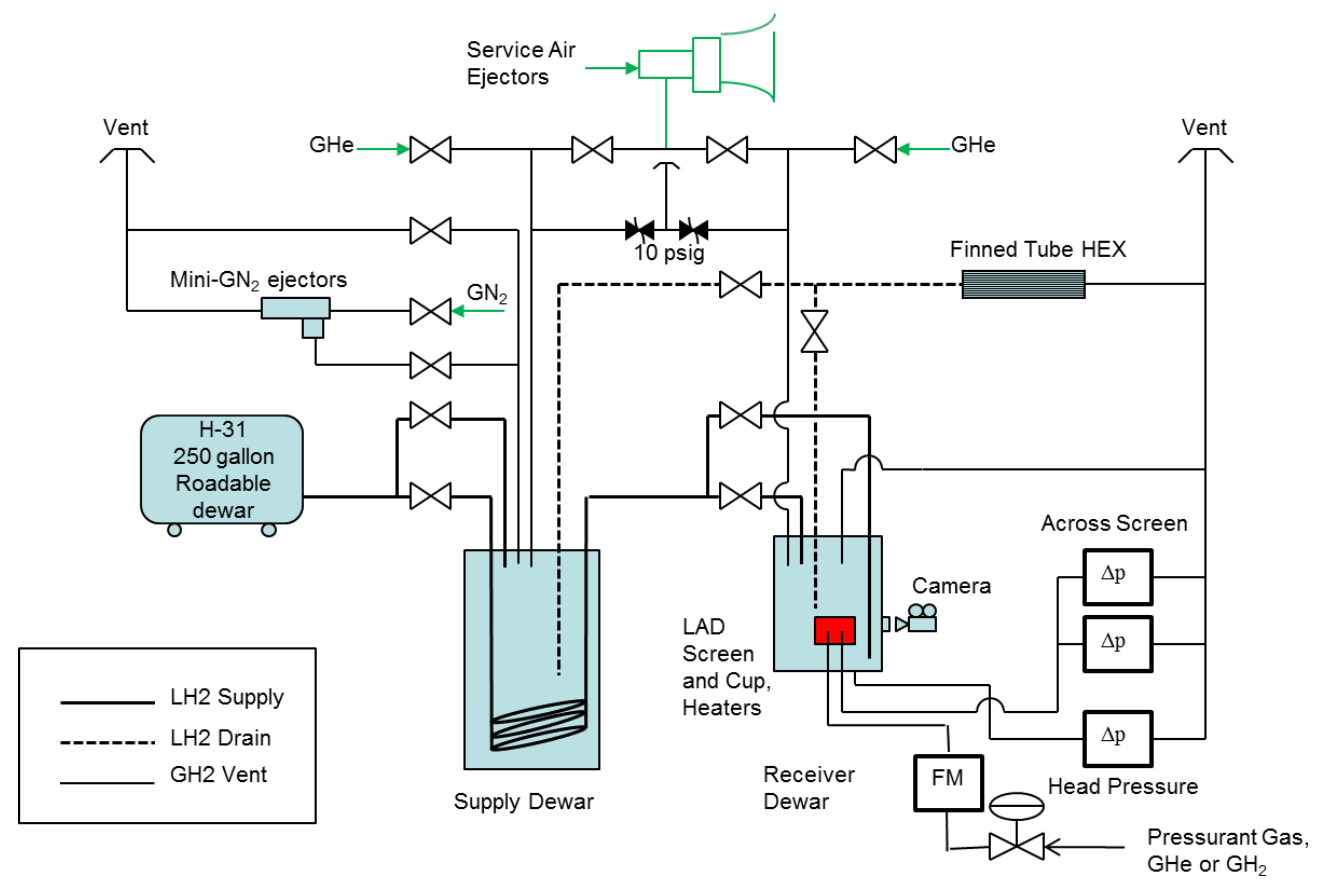

Figure 5.5 - Cell 7 Liquid Hydrogen Flow System 
Several facility modifications were required before flowing $\mathrm{LH}_{2}$ at Cell 7 , and to ensure higher accuracy results over previous bubble point tests. All critical pressure control and regulation valves were remotely controlled to allow complete remote operation of Cell 7 from a control room located approximately 800 meters away. Second, amendments were made to the flow system to remotely flow three different pressurant gases beneath the screen: $\mathrm{GHe}, \mathrm{GH}_{2}$, and gaseous nitrogen $\left(\mathrm{GN}_{2}\right)$. Third, a higher accuracy pressurant gas flow control system was implemented to allow slow ramp in pressurization beneath the screen. Fourth, dual range high accuracy DPTs were installed to measure the differential pressure across the screen as shown in Figure 5.5. Fifth, smaller $0.32 \mathrm{~cm}(1 / 8 \mathrm{in})$ lines were used for all pressure sensing to improve signal response time. Fifth, extra silicon diodes (SDs) were installed to resolve temperature close to the screen $\mathrm{L} / \mathrm{V}$ interface. Diodes are used to measure temperature at cryogenic temperatures, and can also be used as point sensors to determine the liquid level in the tank (Dempsey and Fabik 1992). Finally, a new Labview data acquisition system with precise time synchronization between data file and camera system was installed to eliminate any errors due to time delays.

$\mathrm{LH}_{2}$ was filled offsite in portable $0.946 \mathrm{~m}^{3}$ (250 gallon) dewars and was connected to the flow system through a flexible VJ line. $\mathrm{LN}_{2}$ was connected through the same VJ line via a roadable $1.89 \mathrm{~m}^{3}$ (500 gallon) dewar, and was used to perform all pretest check outs and cold shocking of the hardware, and to collect bubble point data. GHe was available from a portable tuber trailer, while $\mathrm{GN}_{2}$ and $\mathrm{GH}_{2}$ were available via a high pressure K-bottle. 
Pressure of the liquid inside the RD was primarily controlled through back pressure control using a proportional-integral-derivate (PID) loop. To increase pressure, gas was added to the ullage space in the RD. The pressure would also naturally climb due to heat leak into the RD. The vent valve was opened to reduce pressure back to ambient. To reduce pressures below atmospheric, Cell 7 ejectors were used to pump down on the $\mathrm{RD}$. Temperature of the $\mathrm{LH}_{2}$ was primarily controlled by initial conditioning of the liquid inside the roadable dewar. Cell 7 air ejectors could again be used to reduce the temperature of the liquid by pumping down on the ullage space. Parasitic heat leak into the $\mathrm{RD}$ provided more than sufficient thermal energy to increase liquid temperature, if desired. The pressure of the incoming pressurant gas was controlled by a set of low flow control valves. The temperature of the pressurant gas was controlled by switching on or off the heaters inside of the LAD cup, which were PID controlled using a diode located inside the cup (SD3).

\subsubsection{Instrumentation and Data Acquisition}

Temperature instrumentation is outlined in Figures 5.1, 5.3, 5.4 and 5.5, where each number corresponds to the location of a specific diode. Diodes were mounted directly on top and bottom of the screen. SD1 and SD5 provided redundant measure of the temperature of the liquid side of the screen while SD2 measured gas side screen temperature. SD3 and SD4 were mounted inside the LAD cup, which measured the temperature of the incoming pressurant gas. During tests, the temperature difference between these two diodes was less than the experimental uncertainty in the measurement. Temperature difference between SD1 and SD5 was also within experimental uncertainty. SD6 - 9 were mounted on top of the LAD screen in a vertically upward staircase fashion, 
spaced apart $0.635 \mathrm{~cm}(0.25 \mathrm{in})$ upward and $90^{\circ}$ radially from each other, as shown in Figure 5.3. The purpose of the staircase diode rake was to obtain fine resolution of the temperature gradients within the bulk liquid close to the LAD screen at breakdown in order to better correlate experimental measurements with theory. SD10 - 14 were located along a vertical rake within the bulk liquid inside the RD and were used to measure both temperature and liquid level in the dewar. Additional diodes were added later to provide more accurate measure of liquid level and are not shown.

The ullage pressure in the RD and absolute pressure inside the LAD cup were measured using a $0-345 \mathrm{kPa}(0-50 \mathrm{psia})$ pressure transducer $(\mathrm{PT})$. A $0-34.5 \mathrm{kPa}(0-$ 5 psia) DPT provided redundant measure of the liquid level inside the RD. Head pressure was determined by using silicon diodes as level sensing probes, which were spaced out on a vertical rake. The pressure at the LAD screen was determined by adding ullage pressure and head pressure on top of the LAD screen. For $\mathrm{LH}_{2}$ bubble point tests, the differential pressure across the LAD screen was measured using a $0-1245 \mathrm{~Pa}$ and $0-$ $2490 \mathrm{~Pa}\left(0-5\right.$ and $0-10$ in $\left.\mathrm{H}_{2} \mathrm{O}\right)$ high accuracy DPT while a $0-7470 \mathrm{~Pa}(0-30$ in $\mathrm{H}_{2} \mathrm{O}$ ) DPT was used for $\mathrm{LN}_{2}$ bubble point tests. Pressurant gas flow rates were measured using a $0-50$ or $0-100$ standard litres per minute (SLPM) flow meter upstream of the dewar to allow comparison of mass flow rates into the cup prior to and during screen breakdown.

All data channels were recorded at $5 \mathrm{~Hz}$ using a computer data acquisition system (DAQ). Live videos of the LAD screen were recorded and sent to the control room to allow real time display of the LAD screen during testing. Videos were time stamped with a resolution of 0.05 seconds and recorded on a digital video recording (DVR) player. An 
Inter-Range Instrumentation Group (IRIG-B) signal was used to time synchronization between DAQ and camera to within a few milliseconds.

SD1 - SD9 measured temperature to within +/- 0.1 K while SD10 - SD14 measured temperature to within $+/-0.5 \mathrm{~K}$. The ullage pressure and thus the pressure at the screen was within $+/-2.3 \mathrm{kPa}(0.333 \mathrm{psia})$ of the measured value. Liquid height in the dewar was determined to within $+/-5 \mathrm{~cm}$ ( 2 in). For $\mathrm{LH}_{2}$ tests, the raw DPT reading across the screen was good to within 0.32 and $0.62 \mathrm{~Pa}\left(0.0013\right.$ and 0.0025 in $\left.\mathrm{H}_{2} \mathrm{O}\right)$, respectively, using the two DPTs. However, due to read off errors, interpolating between recorded values, and due to uncertainty in liquid level measurement, maximum total uncertainty in $\mathrm{LH}_{2}$ bubble points is estimated to be $2.31 \mathrm{~Pa}\left(0.093\right.$ in $\left.\mathrm{H}_{2} \mathrm{O}\right)$ which is less than $2.2 \%$ of the lowest measured bubble point.

\subsubsection{Data Reduction}

Experimental bubble point values were deduced by correcting the raw DPT reading for head pressure:

$$
\left.\Delta P_{B P}(T, P)\right|_{\exp }=\Delta P_{D P T}-\rho_{L H 2} g L L
$$

where $L L$ is the liquid level above the screen in the RD as determined by the vertical silicon diode rake. The data reduction procedure was as follows: First the liquid level in the RD was manually extracted through examination of the LAD videos and plots of SD voltage as a function of time. Interpolating fits were used to determine the liquid level between the point sensing diodes to deduce liquid level for all time. Second, exact bubble breakthrough times were manually extracted through careful examination of the videos. 
Third, the row of data at bubble breakthrough was manually extracted from the data file. Fourth, thermodynamic properties of interest, such as surface tension, density, and saturation temperature, were calculated using National Institute of Standards and Technology’s (NIST) Reference Fluid Thermodynamic and Transport Properties Database (REFPROP). Fifth, head pressure was subtracted from the raw bubble point pressure. Finally, any other desired parameters of interest were deduced, such as mass flow rate, temperature gradients across the screen, etc.

\subsubsection{Test Matrix}

The overall test plan is outlined on the left hand side in Figure 5.6. Three fine mesh screen channel LAD samples $(325 \times 2300,450 \times 2750,510 \times 3600)$ were tested in two cryogenic liquids $\left(\mathrm{LH}_{2}\right.$ and $\left.\mathrm{LN}_{2}\right)$, using two pressurization schemes (autogenous $\mathrm{GH}_{2} / \mathrm{LH}_{2}$ and $\mathrm{GN}_{2} / \mathrm{LN}_{2}$ and non-condensable $\mathrm{GHe} / \mathrm{LH}_{2}$ and $\mathrm{GHe} / \mathrm{LN}_{2}$ ). Bubble point data was collected across the widest possible range of liquid temperatures and pressures consistent within facility limitations. The lower limit of data collection was limited by the ability of the Cell 7 air ejectors to pull vacuum on the RD to chill the liquid to the saturation temperature at $31 \mathrm{kPa}(4.5 \mathrm{psia})$. The upper limit of $152 \mathrm{kPa}(22.5 \mathrm{psia})$ was set by the maximum allowable working pressure (MAWP) on the RD. This allowed independent examination of both liquid temperature and pressure effects on bubble point using two different pressurant gases, as both saturated and subcooled liquid states were allowable; this is indicated in the blue shaded region in Figure 5.6. This corresponds to a range in $\mathrm{LH}_{2}$ temperatures of $16.7<\mathrm{T}<21.1 \mathrm{~K}$, which faithfully represents thermal conditions within a low pressure $\mathrm{LH}_{2}$ propellant tank. More bubble point data was collected during this test than the sum total of all previous LADs tests to date. 
Three screens:

$325 \times 2300$,

$450 \times 2750$,

$510 \times 3600$

Two liquids:

$\mathrm{LH}_{2}, \mathrm{LN}_{2}$

Three

Pressurization

Gases:

$\mathrm{GHe}, \mathrm{GH}_{2}, \mathrm{GN}_{2}$

Several

Pressurization

Gas Temps

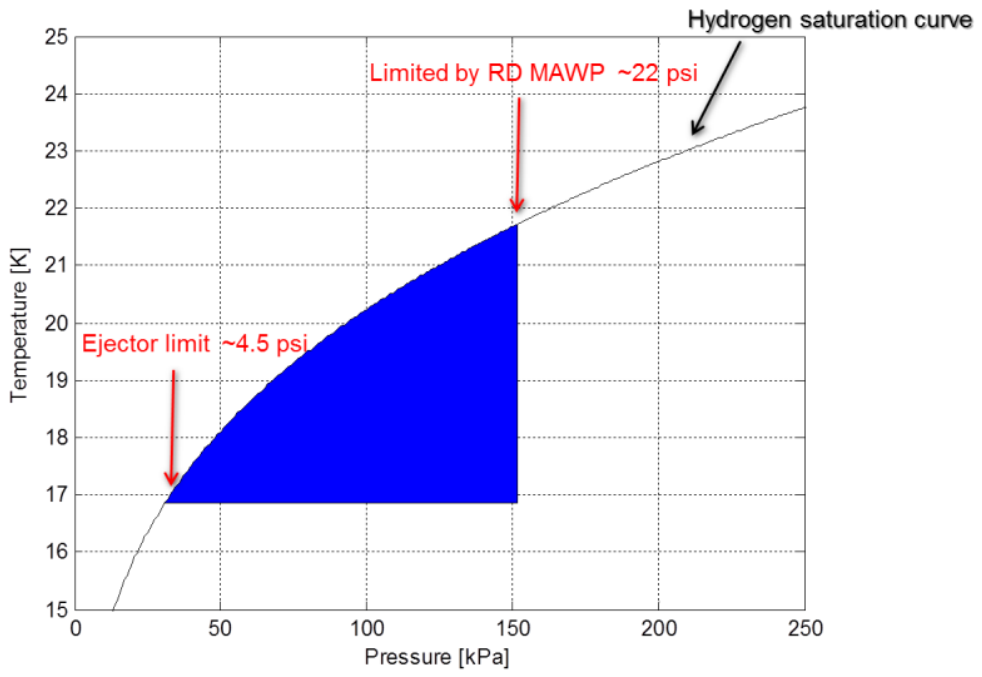

Figure 5.6 - Test Matrix for the Low Pressure Cryogenic Bubble Point Tests

An example test matrix is outlined in Table 5.1 for the $450 \times 2750$ screen in $\mathrm{LH}_{2}$.

Excellent temperature stability of the liquid during testing permitted an isothermal marching scheme in data collection. For temperature variation, the blue shaded region in Figure 5.6 was divided into six equally spaced isothermal slices across the temperature range. For pressure variation, controlled breakthroughs were spaced in $6.89 \mathrm{kPa}$ (1.0 psia) intervals from the saturation pressure up to the MAWP. Data was collected across the entire grid of liquid temperatures in Table 5.1 using both $\mathrm{GHe}$ and $\mathrm{GH}_{2}$ as pressurant gases in $\mathrm{LH}_{2}$ and using GHe and a similar grid using $\mathrm{GHe}$ and $\mathrm{GN}_{2}$ to pressurize $\mathrm{LN}_{2}$.

For pressurant gas variation, the entire grid in Table 5.1 was repeated for GHe and $\mathrm{GH}_{2}$ at a single baseline pressurant gas temperature. The pressurant gas feed line was embedded within the liquid inside the RD and the residence time of the gas inside the LAD cup was long enough such that the baseline gas temperature was approximately the liquid temperature. To collect data at elevated gas temperatures, the heaters were engaged 
and the entire grid in Table 5.1 was repeated for two more pressurant gas temperatures. Heated gas results are presented in Chapter 8. Then the entire process was repeated for the other two meshes. Parametric bubble point testing for a single mesh in both $\mathrm{LH}_{2}$ and $\mathrm{LN}_{2}$ required approximately 3 weeks of continuous testing with a total test time of approximately four months for cold shocks, check outs, testing, and screen change out.

\begin{tabular}{|c|c|c|c|c|c|c|c|c|c|c|c|c|c|c|c|c|c|c|}
\hline LH2 & $450 \times 2750$ & & & & & & & & & & & & & & & & & \\
\hline Psat & & & & & & & & & & & & & & & & & & \\
\hline kPa [psia] & $\mathbf{T}[\mathrm{K}]$ & P screen [psia] & & & & & & & & & & & & & & & & \\
\hline 29.3 [4.25] & 16.7 & 4.25 & 56 & 67 & 8 & 91 & 1011 & 112 & 13 & 14 & 15 & 16 & 17 & 18 & 19 & 20 & 21 & 22 \\
\hline $41.4[6]$ & 17.5 & & & 67 & 8 & 91 & 1011 & 112 & 13 & 14 & 15 & 16 & 17 & 18 & 19 & 20 & 21 & 22 \\
\hline $55.2[8]$ & 18.4 & & & & 8 & 91 & 1011 & 112 & 13 & 14 & 15 & 16 & 17 & 18 & 19 & 20 & 21 & 22 \\
\hline $75.8[11]$ & 19.3 & & & & & & & 12 & 13 & 14 & 15 & 16 & 17 & 18 & 19 & 20 & 21 & 22 \\
\hline 101.3 [14.5] & 20.3 & & & & & & & & & 14.5 & 15 & 16 & 17 & 18 & 19 & 20 & 21 & 22 \\
\hline $131+[19+]$ & $21.1+$ & & & & & & & & & & & & & & 19 & 20 & 21 & 22 \\
\hline
\end{tabular}

Table 5.1 - 450x2750 Test Matrix

\subsection{Experimental Methodology}

Consistent with Figure 5.5, the methodology for conducting the $\mathrm{LH}_{2}$ bubble point tests was as follows: Prior to bubble point tests, the system was purged with GHe. Then a GHe flow was established across the LAD screen to prevent flooding of the cup during $\mathrm{LH}_{2}$ fill. Next $\mathrm{LH}_{2}$ was transferred to condition the facility plumbing, $\mathrm{RD}$, and eventually fill the RD to the desired liquid level. Once the RD was filled, the liquid was conditioned to the desired temperature and pressure at the LAD screen using methods outlined in Section 5.2.1.

To conduct a bubble point test, the screen was resealed by slowly decreasing pressurant gas flow underneath the screen. The desired pressurant gas type and pressurant gas temperature was then selected. When switching over to autogenous pressurization, 
the vapor was allowed to flow through the lines, LAD cup, and screen for up to 10 minutes to ensure single component gases inside the network prior to controlled bubble point tests. Then the pressure underneath the LAD screen was slowly increased using the low flow control valves. Eventually a bubble would break through the wetted screen, breaking the screen down, as indicated through visual observation and rise in the DPT signal. The raw bubble point was taken as the moment when a visible bubble penetrated the liquid laden screen. The time stamp at breakthrough was noted and compared with the time stamp in the data file to extract the corrected bubble point pressure and any other relevant parameters. Bubble point measurements were then repeated at similar pressures and/or temperatures for repeatability and consistency before moving on to the next data point per the test matrix in Table 5.1. It is worth noting that bubbles broke through various locations on the screen, indicating that there was no preferential breakdown spot due to screen defects.

Once testing was completed, the RD was drained to atmosphere through the Cell 7 heat exchanger (HEX) and vent stack. Residual vapors in the flow system were purged using dry GHe. Then both the ST and RD were brought back to ambient pressure. Videos of the LAD screen were transferred to an external hard drive to be analyzed post-test.

\subsection{Experimental Results and Discussion}

Figure 5.7 depicts a screen shot of an ideal bubble point test. Plotted in the figure is the raw DPT signal across the screen, the liquid screen side temperature (SD1), bulk liquid temperature (SD11), and RD ullage pressure during a run of six successive controlled breakthroughs for a 450x2750 screen using GHe to pressurize in $\mathrm{LH}_{2}$. 
Corrected breakthrough pressures occurred at roughly $90-95 \%$ of the maximum DPT signal. As shown, screen side and bulk liquid temperature gradually rose in time as ullage pressure was increased through the test matrix grid in Table 5.1. Good signal to noise (SNR) ratio was also achieved, even in warmer liquid temperatures as the DPT signals decreased due to decreased surface tension of the liquid.

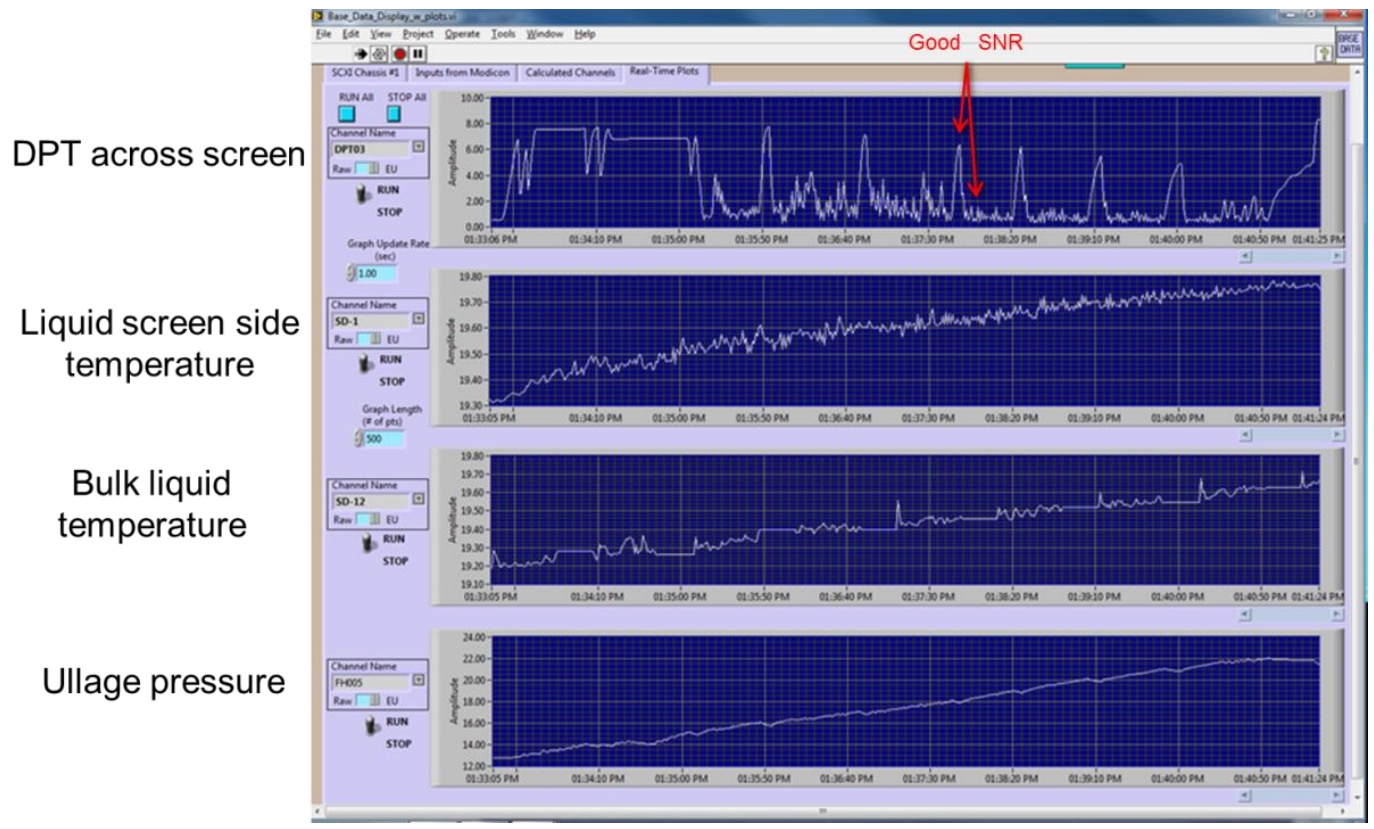

Figure 5.7 - Screenshot of an Ideal Test Run for a 450x2750 Screen with Gaseous Helium/Liquid Hydrogen Testing

Figures $5.8 \mathrm{a}-\mathrm{c}$ display the thermodynamic state of the screen at a controlled $\mathrm{LH}_{2}$ bubble breakthrough, in terms of the liquid screen side temperature (SD1) and the calculated pressure of the liquid at the LAD screen $\left(\mathrm{P}_{\text {SCREEN }}\right)$, for the $325 \times 2300$, 450x2750, and 510x3600 screens, respectively. The black curve is the saturation line, where points above are in the gaseous phase and points below are in the liquid phase. Therefore each point represents the local liquid conditions at the screen during a 
controlled breakthrough. Figures $5.9 \mathrm{a}-\mathrm{c}$ show the thermodynamic conditions at breakdown for the liquid nitrogen tests.
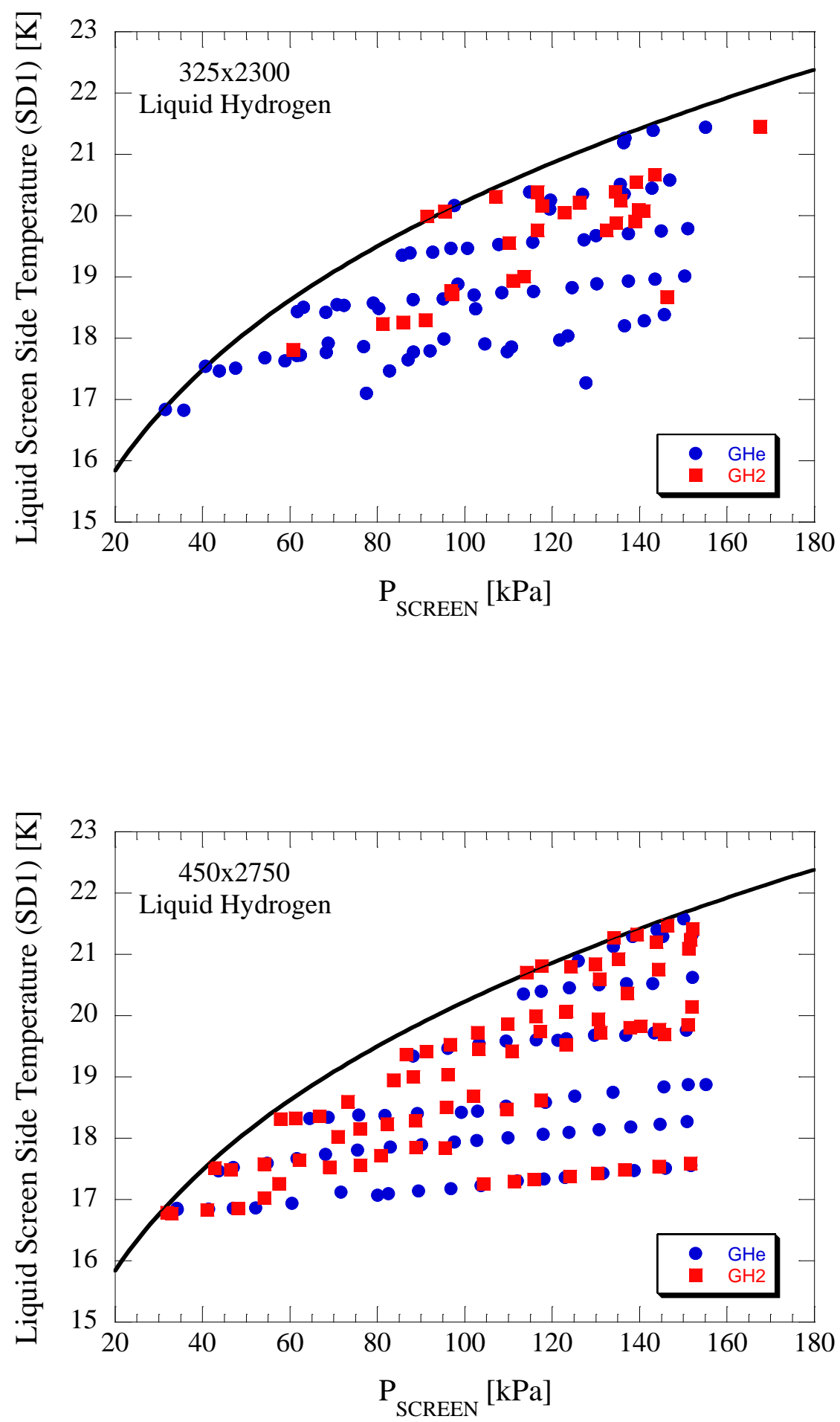


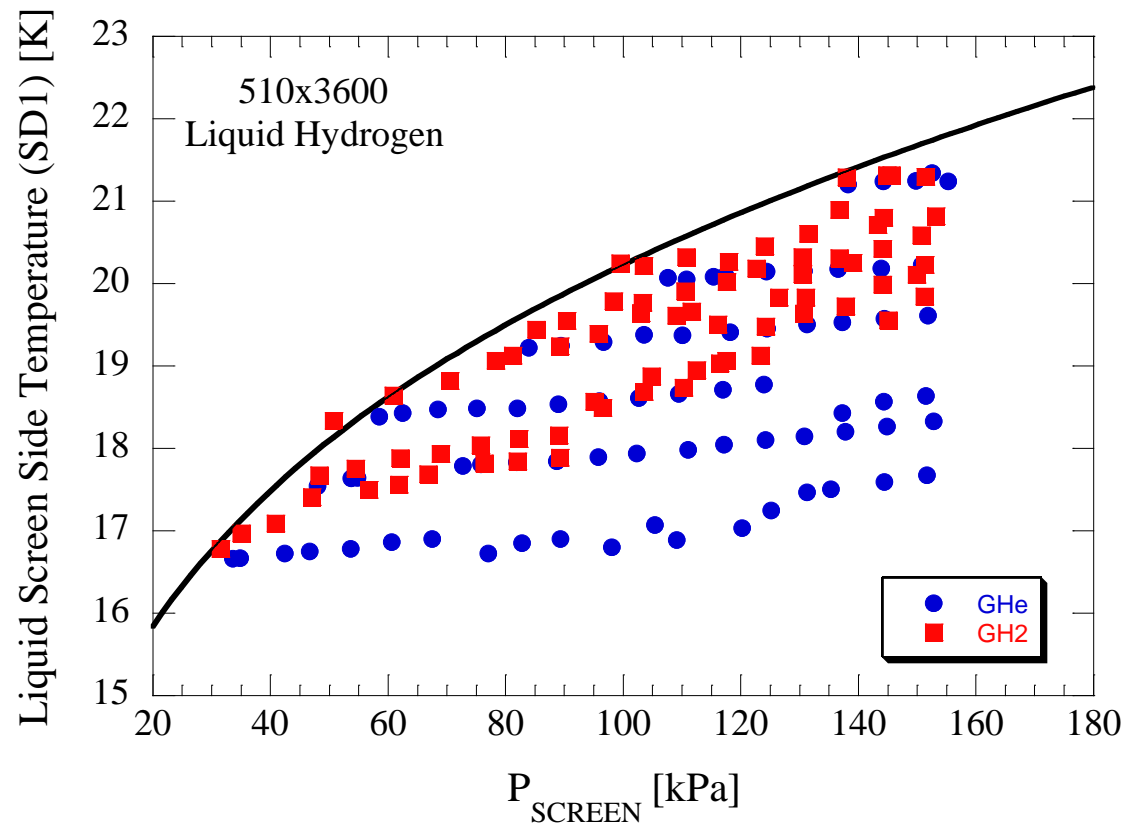

Figure 5.8 - Thermodynamic State of the Liquid Hydrogen at Screen Breakdown for a) $325 \times 2300$, b) $450 \times 2750$, and c) $510 \times 3600$ Screen. The black curve is the hydrogen saturation line. Associated with each point is a value of $\Delta \mathrm{P}_{\mathrm{BP}}$ that satisfies the Young-LaPlace Equation.

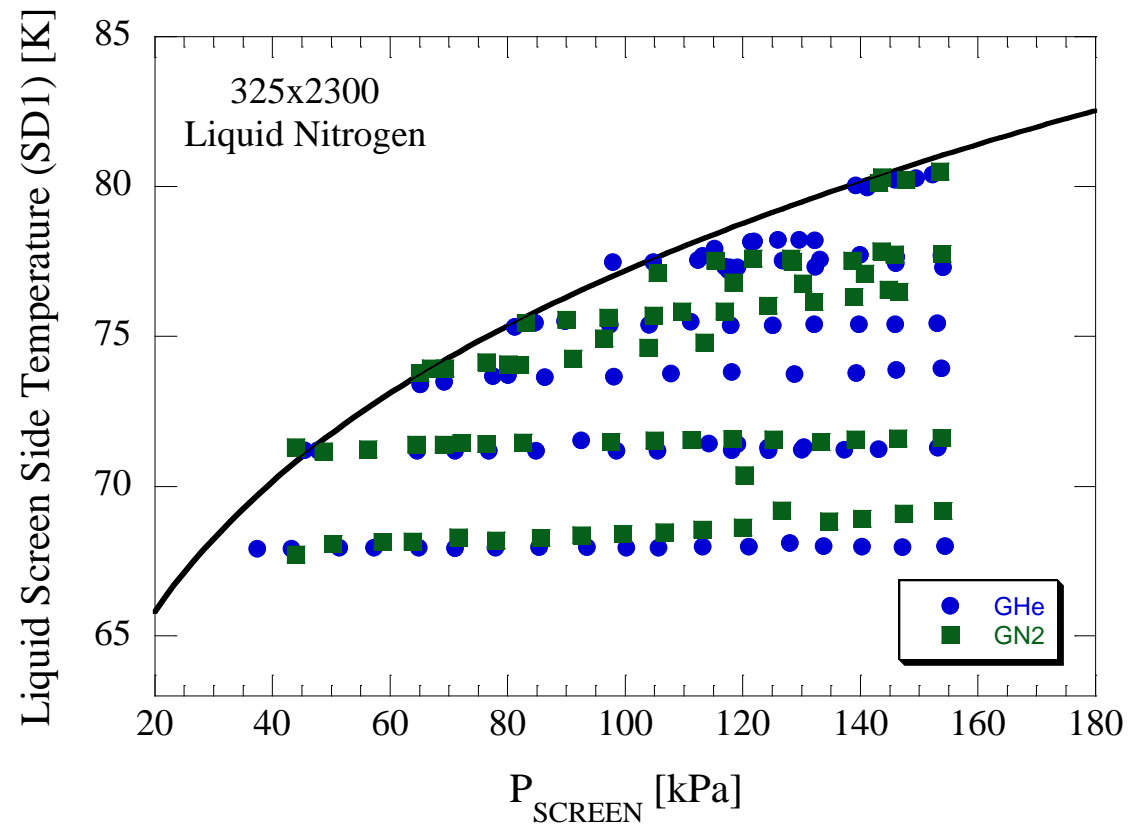



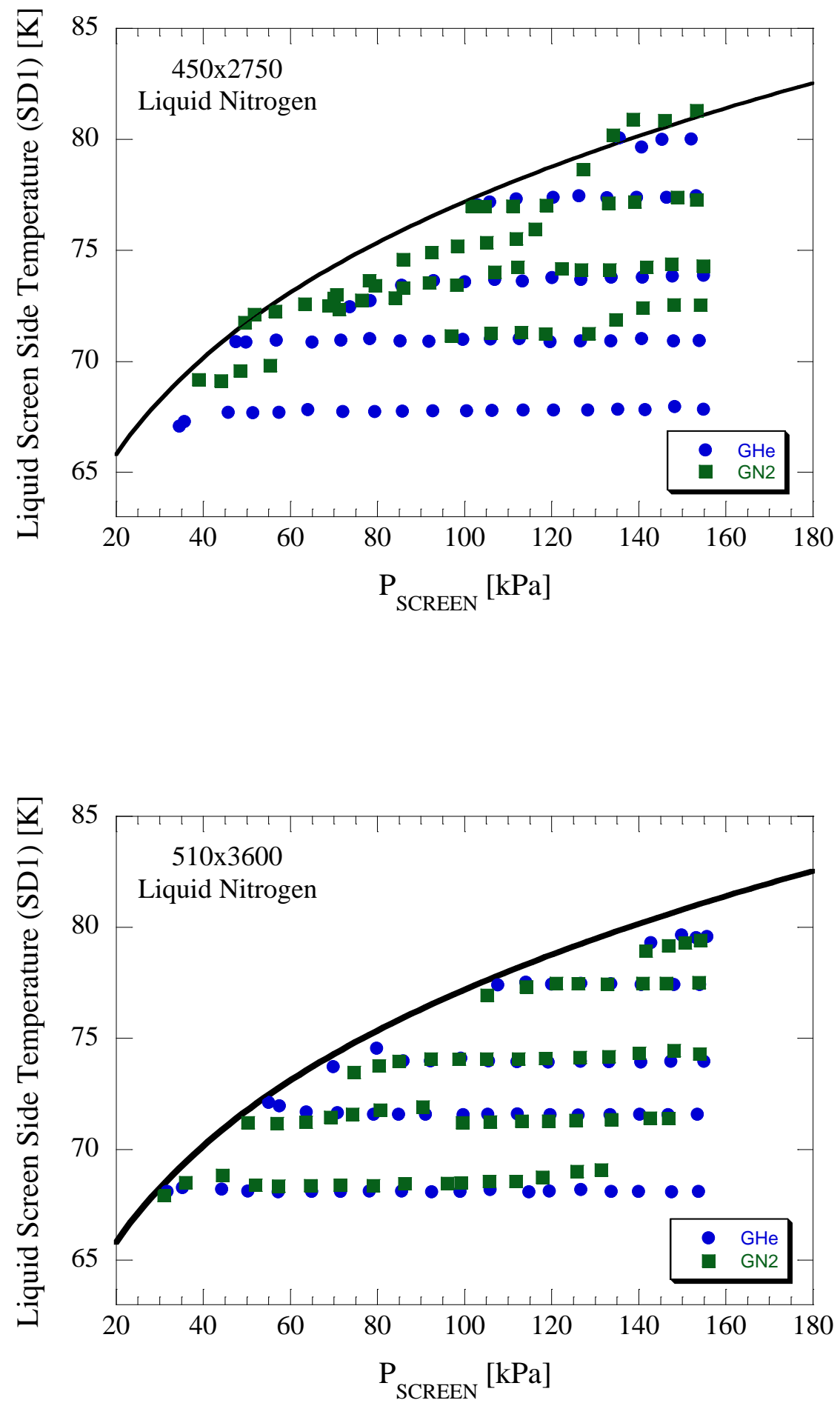

Figure 5.9 - Thermodynamic State of the Liquid Nitrogen at Screen Breakdown for a) 325x2300, b) 450x2750, and c) 510x3600 Screen. The black curve is the nitrogen saturation line. Associated with each point is a value of $\Delta \mathrm{P}_{\mathrm{BP}}$ that satisfies the Young-LaPlace Equation. 
As shown, reasonable temperature stability of the liquid cryogen permitted data collection over the full range of conditions consistent within facility limitations. Greater stability was achieved in $\mathrm{LN}_{2}$ over $\mathrm{LH}_{2}$ due to the warmer saturation temperature. Greater temperature stability of the liquid was achieved using the non-condensable pressurant over the condensable pressurant. Continuous condensable pressurant gas operation tended to warm the liquid more rapidly due to warm $\mathrm{GH}_{2}$ vapor bubbles condensing into the liquid as testing proceeded.

\subsubsection{Screen Weave Dependence}

The first parameter to be varied was the screen mesh, and it was anticipated that the effective pore diameter should order with the fineness of the mesh. To isolate the screen mesh dependence, only data from Figures 5.8a - c and 5.9a - c taken close to the NBP (at or near liquid saturation conditions) are included for comparison, and also to allow direct comparison with Equation 3.16, which only holds for saturated liquid states. Pure saturated liquid states are impossible to achieve using a gas to pressurize other than the vapor. Therefore, "near saturated" liquid states are defined as points which lie very close (less than $2 \%$ away of the difference in pressure between pressure and saturation pressure) to the saturation line. This definition confines the comparison of data points from Figures $5.8 \mathrm{a}-\mathrm{c}$ and $5.9 \mathrm{a}-\mathrm{c}$ to those located near the black saturation curve and minimizes the effect of subcooling the liquid on bubble point pressure.

Figures 5.10a and b plot the screen mesh dependence using GHe to pressurize in $\mathrm{LH}_{2}$ and $\mathrm{LN}_{2}$, respectively, by plotting bubble point data as a function of the liquid surface tension. Solid lines are model predictions using Equation 3.16 evaluated using 
pore diameters from room temperature bubble point tests from Chapter 4. Error bars are plotted, but are barely distinguishable. For all three meshes, results show that all data collected lies above the room temperature model predicted value. As shown in Figure 5.10a, for all surface tensions and thus liquid temperatures, higher bubble points are achieved using the finer 450 mesh, as the average gain in $\mathrm{LH}_{2}$ bubble point above the 325 data is approximately $25 \%$ higher. Higher bubble points are also achieved using the finest 510 mesh, with a modest average gain of $3 \%$ over the $325 \times 2300$ baseline screen. Trends are similar in the $\mathrm{LN}_{2}$ data.

The implications are as follows: the deviation from the room temperature prediction can be explained by taking into account that the screen pore itself is temperature dependent, since the effect of both liquid subcooling and pressurant gas type is null here. Table 5.2 presents pore diameters obtained in room temperature bubble point tests from Chapter 4 versus pore diameters obtained by fitting a curve to the data in Figure 5.10a. Uncertainties for the $20 \mathrm{~K}$ pore diameters factor in both measurement uncertainty and statistical uncertainty. As shown for all three screen meshes, there is an obvious gain in bubble point at colder $\mathrm{LH}_{2}$ temperatures, with the 510 mesh achieving a $23 \%$ gain over its room temperature prediction. Screen pores are shrinking at colder temperatures due to the thermal coefficient of contraction of the warp and shute wires. Because the gain is different for each screen, geometrical considerations of the actual pore structure must also be taken into account. Nonetheless, extra margin is obtained for all three meshes at cryogenic hydrogen temperatures. 

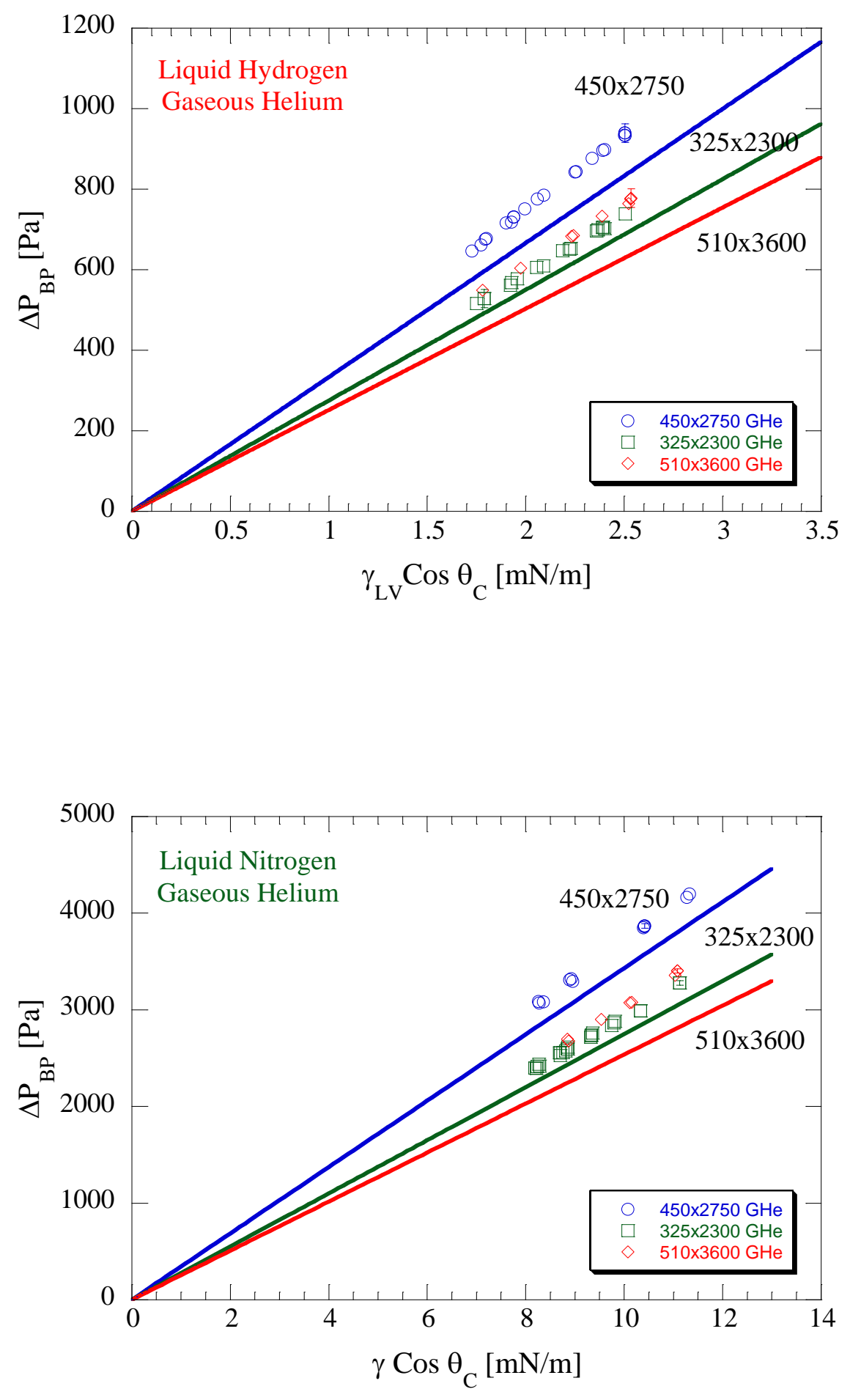

Figure 5.10 - Bubble Point Pressure Dependence on Screen Mesh in a) Liquid Hydrogen and b) Liquid Nitrogen. Solid lines are computed from Equation 3.16 using pore diameters determined at room temperature. 


\begin{tabular}{|c|c|c|c|}
\cline { 2 - 4 } \multicolumn{1}{c|}{} & Pore Diameter $[\boldsymbol{\mu m}]$ & Pore Diameter $[\boldsymbol{\mu m}]$ & \% Gain \\
\hline Mesh & 300 Kelvin & 20 Kelvin & \\
\hline 325x2300 & $14.55 \pm 0.323$ & $13.36 \pm 0.090$ & 8.91 \\
\hline $\mathbf{4 5 0 \times 2 7 5 0}$ & $12.01 \pm 0.323$ & $10.67 \pm 0.426$ & 12.56 \\
\hline $\mathbf{5 1 0 \times 3 6 0 0}$ & $15.91 \pm 0.323$ & $12.99 \pm 0.294$ & 22.48 \\
\hline
\end{tabular}

Table 5.2 - Temperature Dependence of the Effective Screen Pore Diameter

Second, Figure 5.10 confirms that bubble point pressure is not inversely proportional to the fineness of the screen, in either room temperature or cryogenic liquids, because the 450 screen outperforms the finest 510 screen in both cold and warm temperature regimes. The 510 slightly outperformed the 325 screen in $\mathrm{LH}_{2}$, which contradicts room temperature results from Chapter 4 where the 325 screen actually outperformed the 510 screen. In addition, the relative magnitude of disparity between room temperature and cryogenic bubble points is different for each of the three meshes. At $\mathrm{LH}_{2}$ temperatures, the 325,450 , and 510 screens deviate from room temperature behavior by $8.9 \%, 12.6 \%$, and $22.5 \%$, respectively. Therefore results indicate that the screen pore is both temperature and screen dependent. In other words, differences in performance between screens at the same liquid temperature using the same pressurant gas is due to differences in screen pore shrinkage as well as geometrical differences in the $\mathrm{L} / \mathrm{V}$ interface due to different contraction of screen wires and pore diameters. Thus the geometry of the L/V interface at breakdown, may also affect the pore diameter and thus performance of the screen. Third, results here imply that there may be an optimal LAD screen weave, at least in terms of maximizing bubble point, because the 450 mesh outperforms the other two mesh screens. This implies that this $450 \times 2750$ screen is capable of delivering a much higher flow rate over the $325 \times 2300$ screen since bubble point pressure translates into total allowable flow rate from a LAD to a transfer line. The 
LAD screen wires will shrink at cryogenic temperatures, and for a fixed OD sample, the effective pore diameters will thus shrink as the entire LAD screen/cup assembly shrinks. As will be shown in Section 5.4.5, some of this gain is due to enhanced interfacial cooling due to the presence of the non-condensable helium gas. However, it can be confidently deduced that the total gain in bubble point at reduced temperatures cannot solely be due to enhanced interfacial cooling, since the gain is different for each screen. Interfacial cooling with helium gas is strictly a function of type and temperature of the liquid, and not the screen type. Therefore, results imply that the pores are contracting at different rates relative to one another.

Fourth, results here also show that Equation 3.16 based on pore diameters based on room temperature tests can still be used to predict performance in cryogenic liquid when using GHe since all cryogenic bubble points are higher for all temperatures tested here. Using room temperature pore diameters is valuable to mission designers who desire cryogenic performance predictions on new screens which have never been tested; room temperature bubble point tests are fairly easy to conduct, and do not require the complexities of cryogenic bubble point tests. Nonetheless, for the most accurate results, pore diameters should be determined at each liquid temperature to account for changes in the screen and/or pore itself. Using a 450x2750 does in fact increase margin over the low baseline value for the $325 \times 2300$ screen.

\subsubsection{Liquid Dependence}

Equation 3.16 clearly predicts that bubble point pressure should scale linearly with the surface tension of the liquid when corrected for the contact angle between liquid 
and screen pore. Binary mixture bubble point test results from Chapter 4 showed that the critical Zisman surface tension value for liquids with these SS304 LAD screens was $\gamma_{C Z}=23.2 \pm 2 \frac{m N}{m}$, which is the critical surface tension value where contact angle is zero, below which contact angle will not deviate from zero. Since $\gamma_{L H 2}$ and $\gamma_{L N 2}<\gamma_{C Z}$ for SS304 LAD screens, it is assumed that $\theta_{C}=0$ for both $\mathrm{LH}_{2}$ and $\mathrm{LN}_{2}$.

Figures 5.11a - c plot the bubble point pressure dependence on the liquid. The black line is the prediction curve based on room temperature pore diameters. Pure liquid test results from Chapter 4 are plotted along with $\mathrm{NBP} \mathrm{LH}_{2}$ and $\mathrm{LN}_{2}$ data from the current work. Again, to isolate the liquid dependence, only cryogenic data collected using GHe to pressurize the screen is plotted.

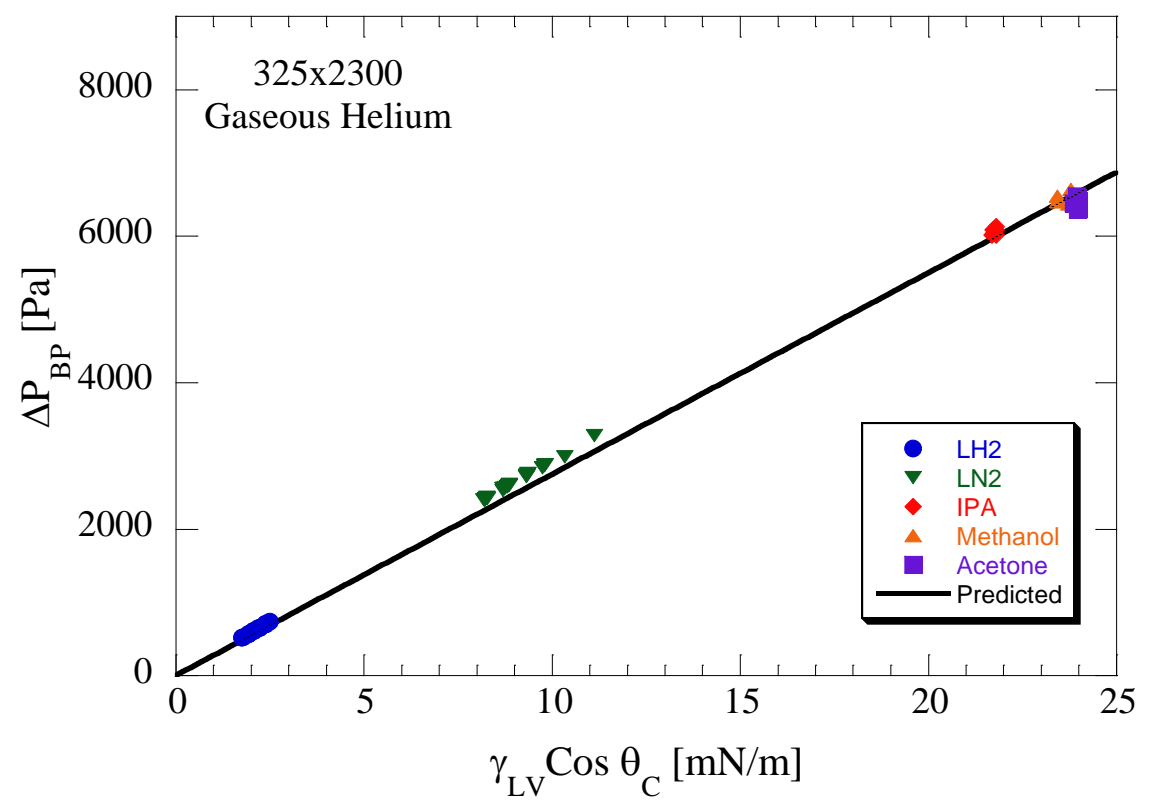



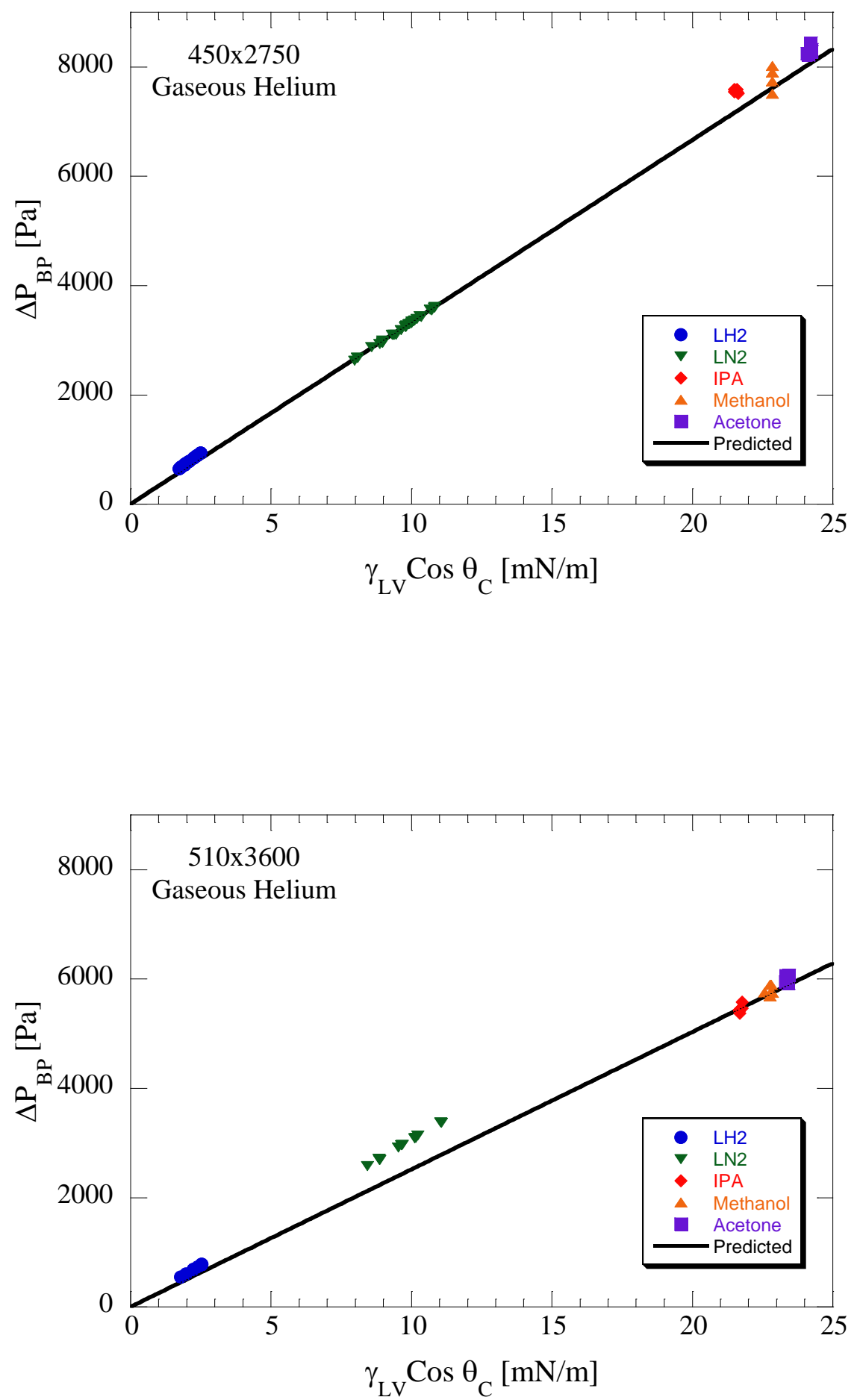

Figure 5.11 - Bubble Point Pressure Dependence on Liquid for a) 325x2300, b) 450x2750, and c) 510x3600 Screen Mesh Samples 
As shown for both room temperature and cryogenic liquids tested, bubble point pressure is indeed directly proportional to the contact angle corrected surface tension of the liquid. Deviation from theory for the three different meshes is strictly due to the temperature dependence of the pore diameter. Although it is difficult to see, the $\mathrm{LH}_{2}$ data lies farther away from the black prediction curve than the $\mathrm{LN}_{2}$ data, implying that the deviation from room temperature prediction worsens as the temperature decreases. Nonetheless, Equation 3.16 is in good agreement with experimental results across a wide range of liquids. Results here are also in excellent agreement with historical results collected in saturated and near saturated liquid states.

\subsubsection{Liquid Temperature Dependence}

The second way to improve upon the $325 \times 2300$ reference bubble point value is to increase the surface tension of the liquid. Figures 5.12a and b plot $\mathrm{LH}_{2}$ and $\mathrm{LN}_{2}$ bubble point data using GHe to pressurize as a function of the liquid side screen temperature, SD1 for all three screens, respectively. Again, only bubble points taken in saturated or near saturated liquid states are plotted to permit direct comparison between screen meshes. As shown for all three meshes, higher bubble points are always achieved in colder liquid temperatures, since surface tension is an inverse function of temperature. Across the entire temperature range, for both cryogens, the $450 \times 2750$ outperforms both the $325 \times 2300$ and $510 \times 3600$ screens. Because the gain is different for each screen, geometrical considerations of the actual pore structure must also be taken into account. Extra margin is always obtained by simply operating at a colder liquid temperature. For example, at an $\mathrm{LH}_{2}$ operating temperature of $16.7 \mathrm{~K}$, there is a $165 \%$ difference in bubble point for the $450 \times 2750$ mesh over the NBP $325 \times 2300$ value at $20.3 \mathrm{~K}$. 

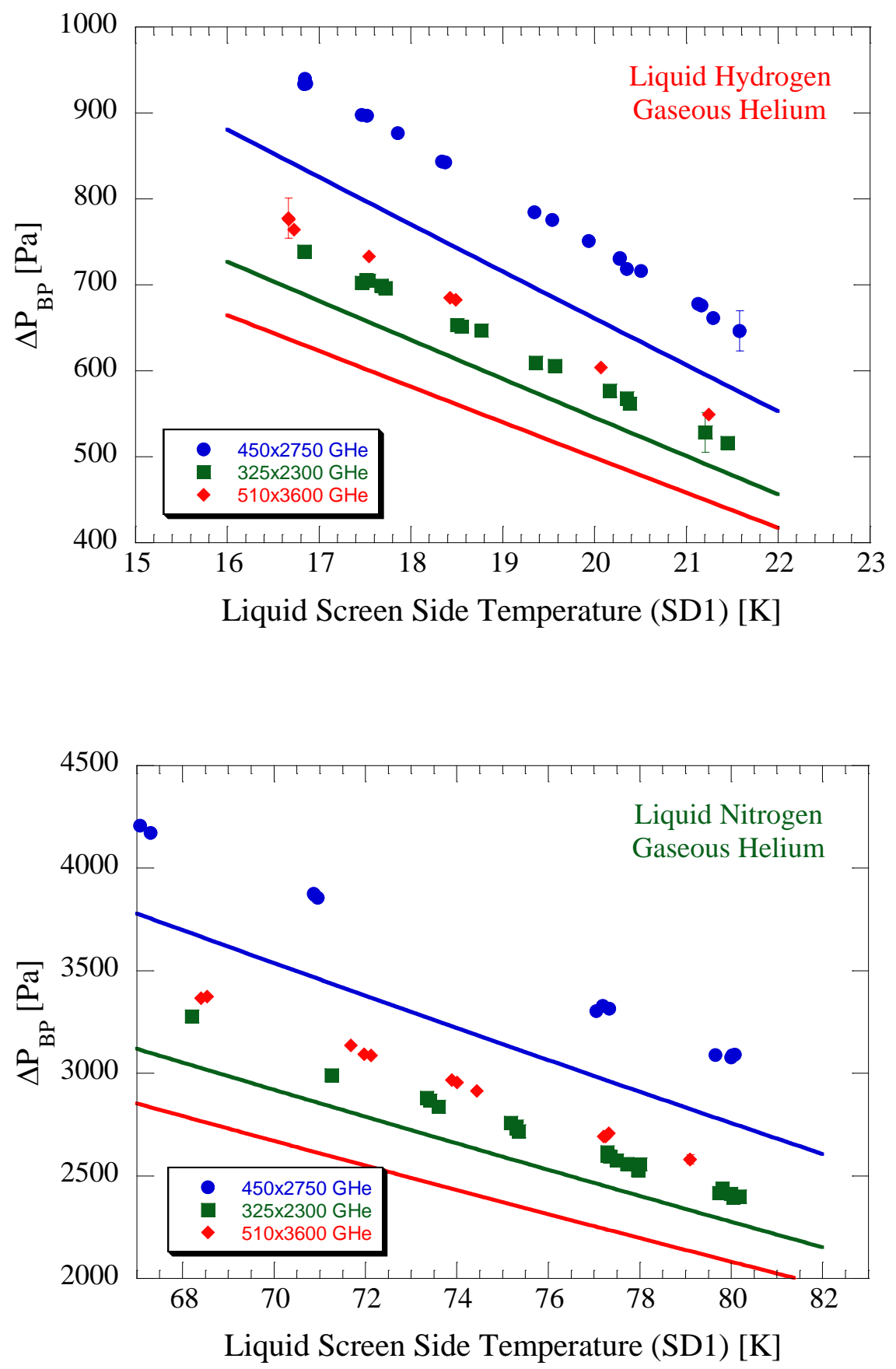

Figure 5.12 - a) Liquid Hydrogen and Liquid Nitrogen Bubble Point as a Function of Liquid Screen Side Temperature (SD1). Solid lines are model predictions based on Equation 3.16 evaluated using room temperature pore diameters. 


\subsubsection{Liquid Pressure Dependence}

The next parameter varied was the liquid pressure. Typically a mission designer will have control over the temperature and pressure and thus thermodynamic state of the liquid propellant prior to transfer, and so it was highly desirable to obtain bubble point data across a wide range of thermal conditions within a low pressure propellant tank to bound screen channel LAD behavior. Figures 5.13a $-\mathrm{c}$ and $5.14 \mathrm{a}-\mathrm{c}$ plot the $\mathrm{LH}_{2}$ and $\mathrm{LN}_{2}$ bubble point pressure using GHe to pressurize as a function of the temperature of the liquid side of the screen (SD1) for the $325 \times 2300,450 \times 2750$, and 510x3600 screens, respectively. To minimize the effect of pressurant gas type, only GHe data is presented in these plots. Each data point is colorized in terms of the difference between the pressure at the screen and the saturation pressure based on SD1. Therefore this pressure difference is a measure of the distance away from the saturation curve from Figures 5.8 and 5.9; the higher the pressure difference, the farther away the data point lies from the saturation state and the higher the level of subcooling at the screen L/V interface. The black line is again the prediction curve based on the room temperature pore diameter. Error bars are plotted but are barely distinguishable. All three $\mathrm{LH}_{2}$ and $\mathrm{LN}_{2}$ figures are plotted on the same scales, respectively, for comparison. 

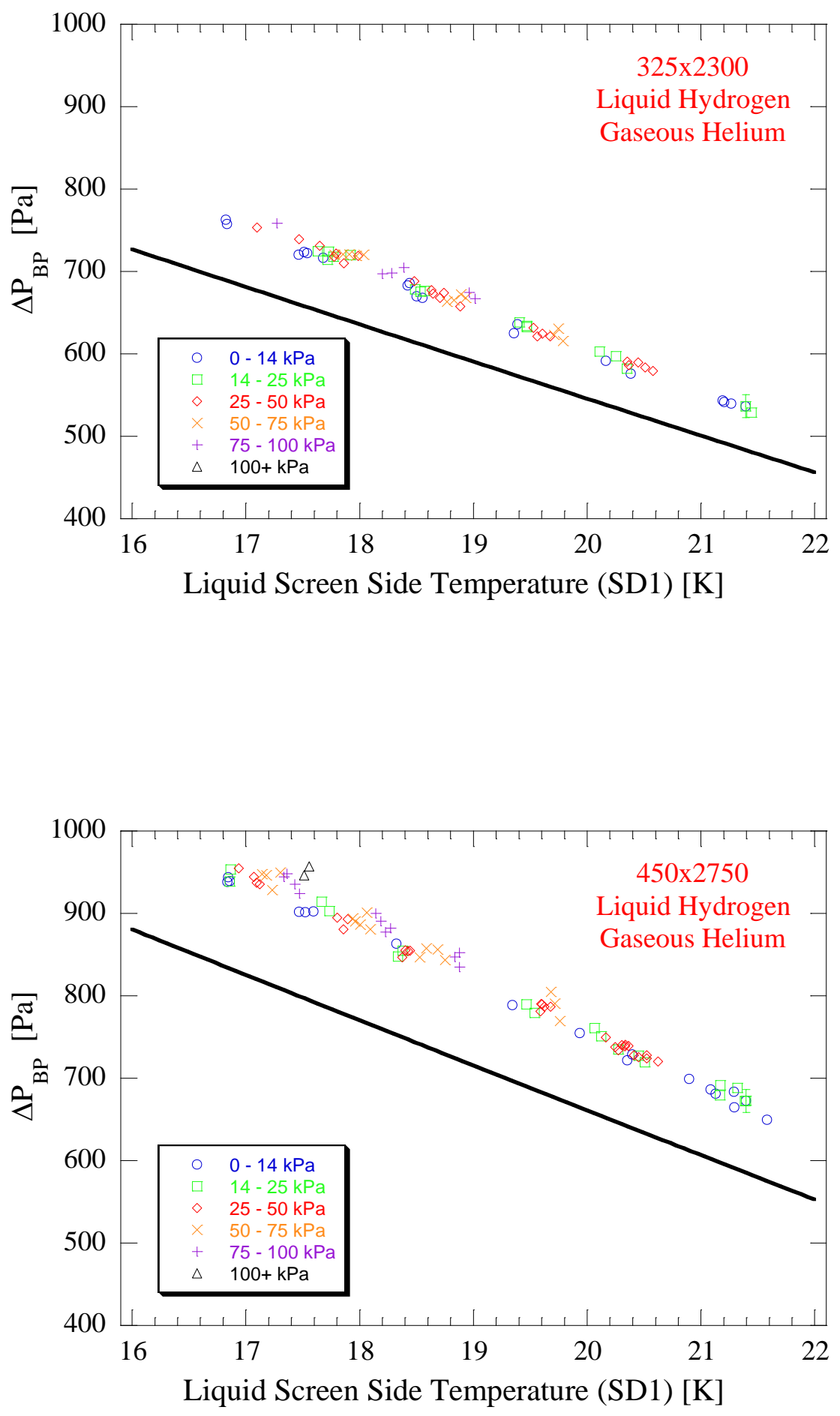


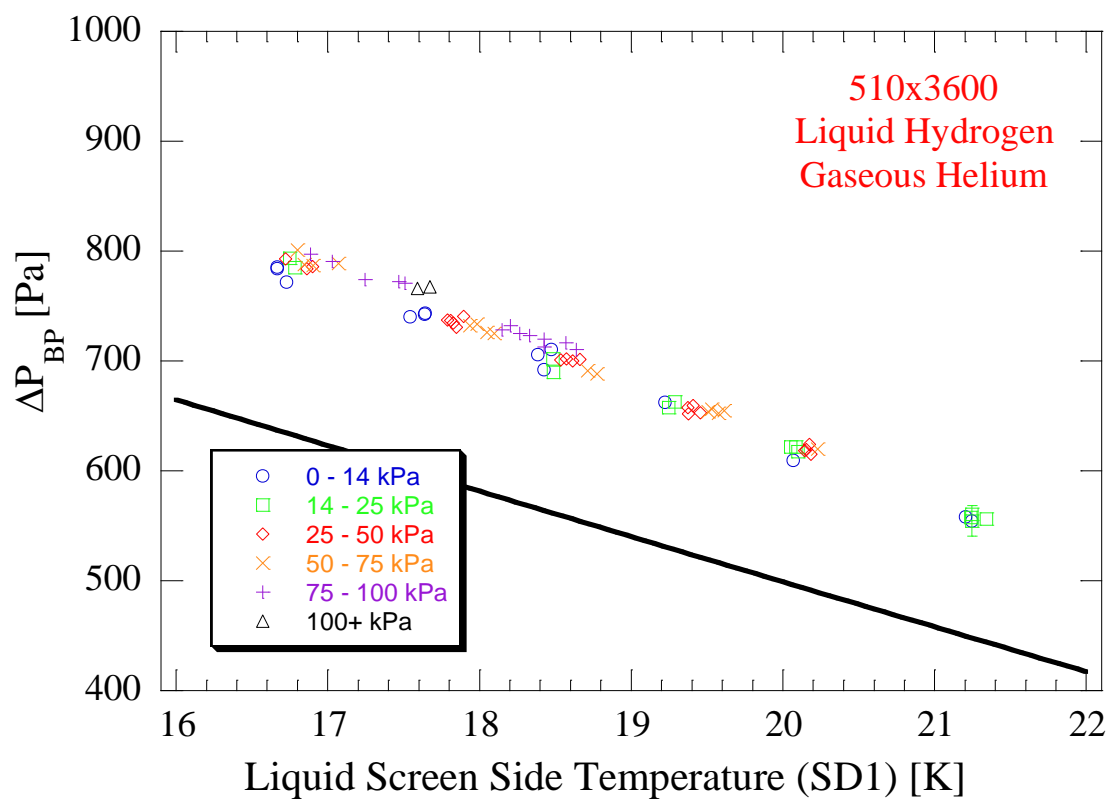

Figure 5.13 - Liquid Hydrogen Bubble Point Pressure Dependence on Liquid Temperature and Pressure using Helium as a Pressurant Gas for a) 325x2300, b) 450x2750, and c) 510x3600 Screens. The black line is the prediction curve based on the room temperature pore diameter.

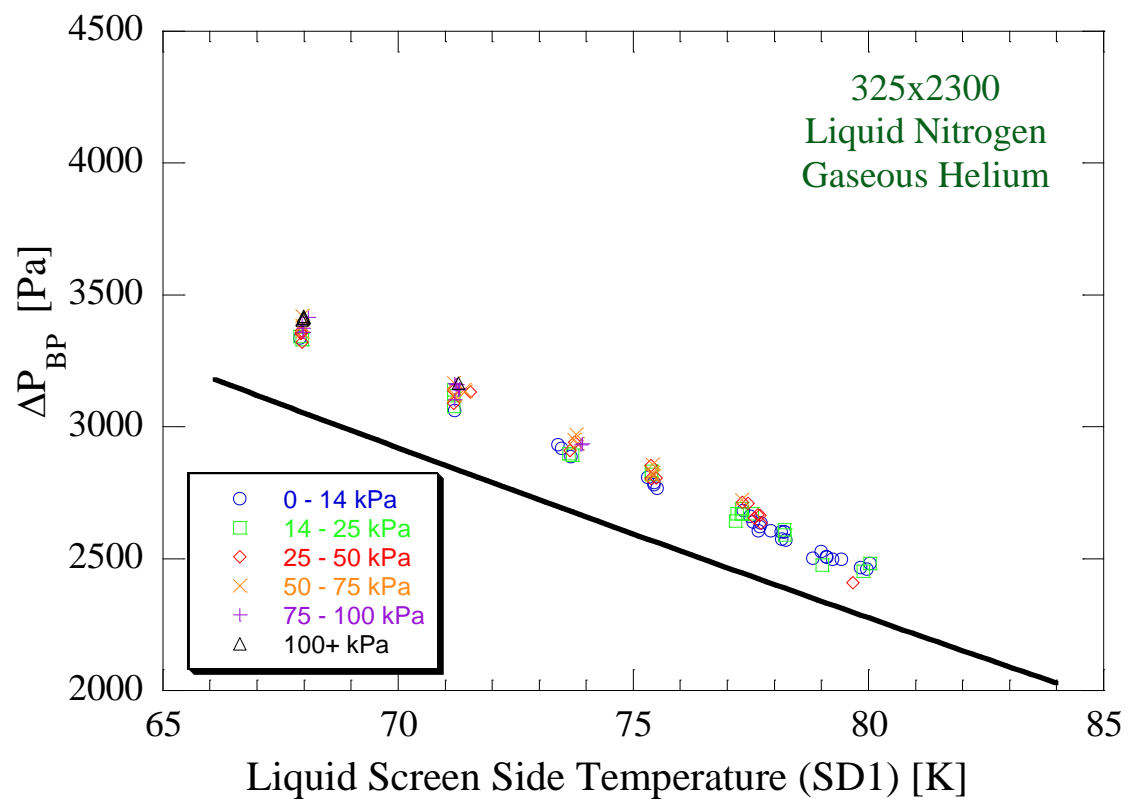



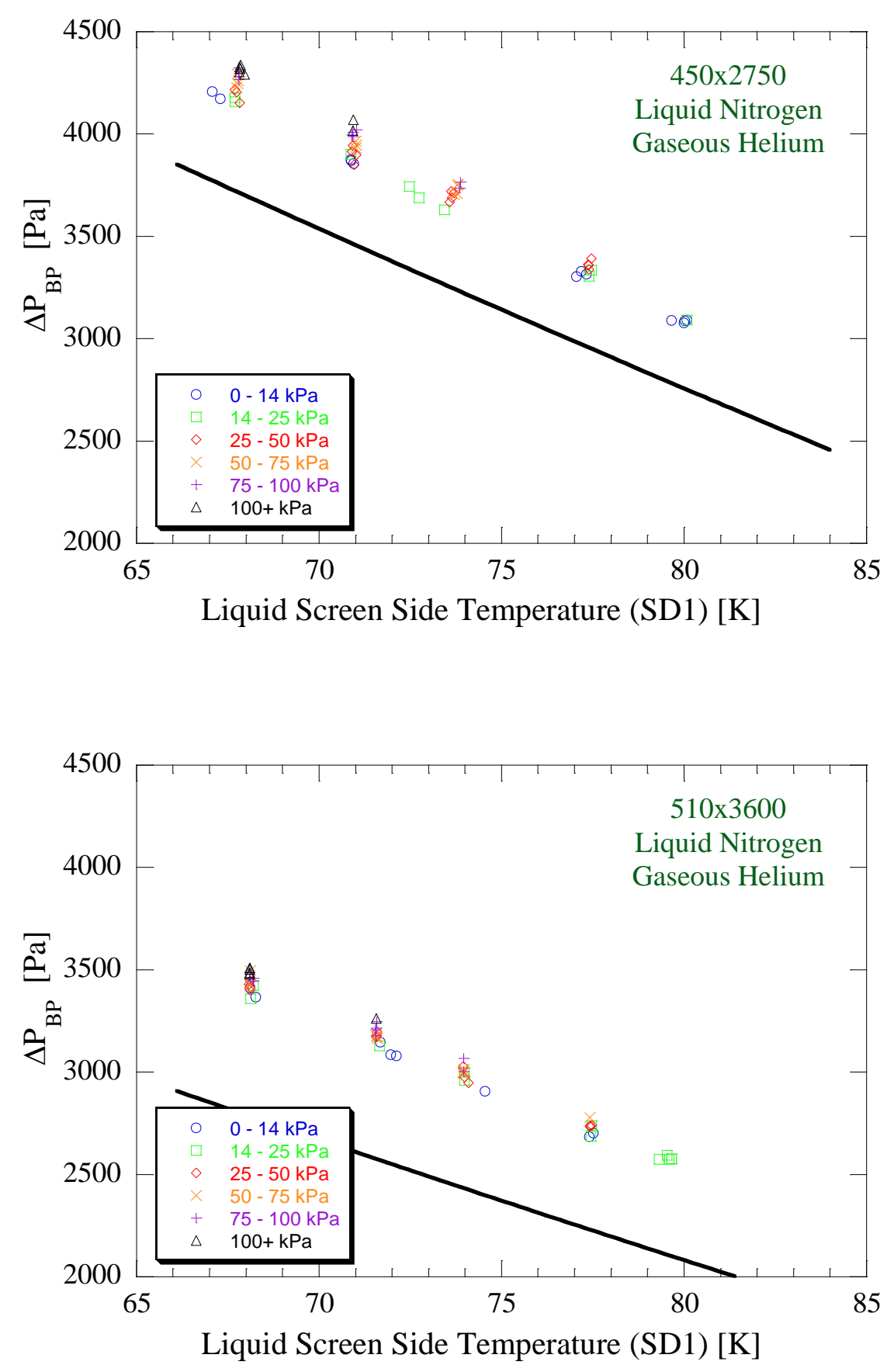

Figure 5.14 - Liquid Nitrogen Bubble Point Pressure Dependence on Liquid Temperature and Pressure using Helium as a Pressurant Gas for a) 325x2300, b) 450x2750, and c) 510x3600 Screens. The black line is the prediction curve based on the room temperature pore diameter. 
Results are as follows. For all three meshes and both liquids, bubble point pressure decreases with increasing temperature. Higher bubble point pressures are always achievable in colder liquid temperatures. The model qualitatively tracks the data but underpredicts the data for all three meshes in both liquids, with more discrepancy between model and data for the 510x3600 screen, followed by the 450x2750, and $325 \times 2300$ meshes. Data taken in saturated or near-saturated liquid states lie closest to the prediction curve for all screens and liquids as indicated by the proximity of the blue circles. For all three meshes, as the pressure difference is increased, as the thermodynamic state of the liquid becomes more subcooled, bubble point pressure increases as indicated by the data deviating farther from the room temperature prediction. Deviation between the model and data for subcooled data appears to grow proportionally with level of subcooling over the range of temperatures and pressures tested here. The existing simplified room temperature model does not account for changes in liquid pressure. The same trends of increased bubble point with pressure are shown in the $\mathrm{LN}_{2}$ data in Figure 5.14 where experimental uncertainty is negligible (i.e. $<0.6 \%$ at the lowest reported value), and it can thus be concluded that there is a weak gain in bubble point due to subcooling the screen pore L/V interface for these two cryogenic liquids. This subcooled trend is further examined in high pressure tests in Chapters 6 and 7.

\subsubsection{Pressurant Gas Dependence}

The fifth parameter varied in this study was the type of pressurant gas. This parameter has implications on both LAD and pressurization subsystems as mentioned previously in Chapter 3. To determine the effect of pressurant gas type on the LAD subsystem, bubble point tests were conducted for the three different meshes across the 
same set of thermodynamic states of the liquid using both non-condensable $\left(\mathrm{GHe} / \mathrm{LH}_{2}\right)$ and autogenous $\left(\mathrm{GH}_{2} / \mathrm{LH}_{2}\right)$ pressurization schemes. Results are plotted in Figures 5.15a$\mathrm{c}$ and Figures 5.16a - c for the 325x2300, 450x2750, and 510x3600 screens in $\mathrm{LH}_{2}$ and $\mathrm{LN}_{2}$, respectively. All the bubble point data collected in this experiment from Figures 5.8 and 5.9 is thus plotted for comparison. Solid lines are again model predictions based on room temperature pore diameters. Error bars are plotted but are barely discernible.

Although the autogenous data in Figures 5.15 and 5.16 are not colorized to the degree of subcooling as in Figures 5.13 and 5.14, identical trends are seen for the autogenous data as in the GHe data; pressurizing and subcooling the screen L/V interface always increases the bubble point pressure. The highest autogenous bubble points are again obtained in the coldest liquid temperatures at the highest screen pressures. The 450x2750 screen again produces the highest bubble points, followed by the finest $510 \times 3600$ and then the coarsest $325 \times 2300$ mesh.

Examination of Figures 5.15 and 5.16 shows a clear trend in both cryogens. Pressurization with GHe acts as a gain factor from room temperature behavior on bubble point pressure for all three screens and all liquid temperatures tested here. Therefore the third way to improve margin over the baseline $325 \times 2300 \mathrm{NBP}$ bubble point pressure is to pressurize the screen with a non-condensable gas such as helium. Meanwhile, autogenous pressurization acts as a degradation factor from room temperature across the parameter space. Differences in bubble point pressure between the two pressurization schemes at both $\mathrm{LH}_{2}$ and $\mathrm{LN}_{2}$ temperatures is far outside experimental uncertainty. Interestingly, all non-condensable pressurant bubble point pressures lie above the room temperature prediction curve for all three meshes. However, only the 510x3600 screen yielded 
autogenous bubble points higher than the corresponding room temperature prediction values, while the 325 and 450 data on or below the room temperature prediction. This crossover in performance is likely due to a competition between loss in performance from autogenous pressurization and gain in performance due to screen pore shrinkage. Results and trends here between the two pressurization schemes are in excellent agreement with all previously reported $\mathrm{LH}_{2}$ and $\mathrm{LN}_{2}$ bubble point pressures (Burge et al. 1973, Paynter 1973a, Cady 1973, 1975, and 1977, Chato and Kudlac 2002, and Kudlac and Jurns 2005).

To further illuminate the trends between varying screen type, liquid temperature and pressure, and type of pressurant gas, Figures $5.17-5.19$ and $5.20-5.22$ plot all of the bubble point data collected in this experiment for $\mathrm{LH}_{2}$ and $\mathrm{LN}_{2}$, respectively. Figures $5.17-5.19$ plot $\mathrm{LH}_{2}$ data for a 325x2300, 450x2750, and 510x3600 screen, respectively while Figures 5.20 - 5.22 plot $\mathrm{LN}_{2}$ data for a $325 \times 2300$, 450x2750, and 510x3600 screen, respectively. Data is plotted as a function of the thermodynamic state of the liquid at the screen at bubble breakthrough, in terms of liquid temperature and pressure. Data is colorized proportional to the magnitude of bubble point pressure. The black lines in Figures 5.17 and 5.18 are the saturation lines for hydrogen and nitrogen, respectively. For each liquid, data is colorized to the same color scale to permit easy comparison between different screens and pressurant gas types. The above mentioned trends, namely that decreasing temperature increases bubble point, increasing pressure improves margin, operating with the second finest 450x2750 mesh, and using a non-condensable pressurant gas to pressurize the LAD all result in improved performance. 

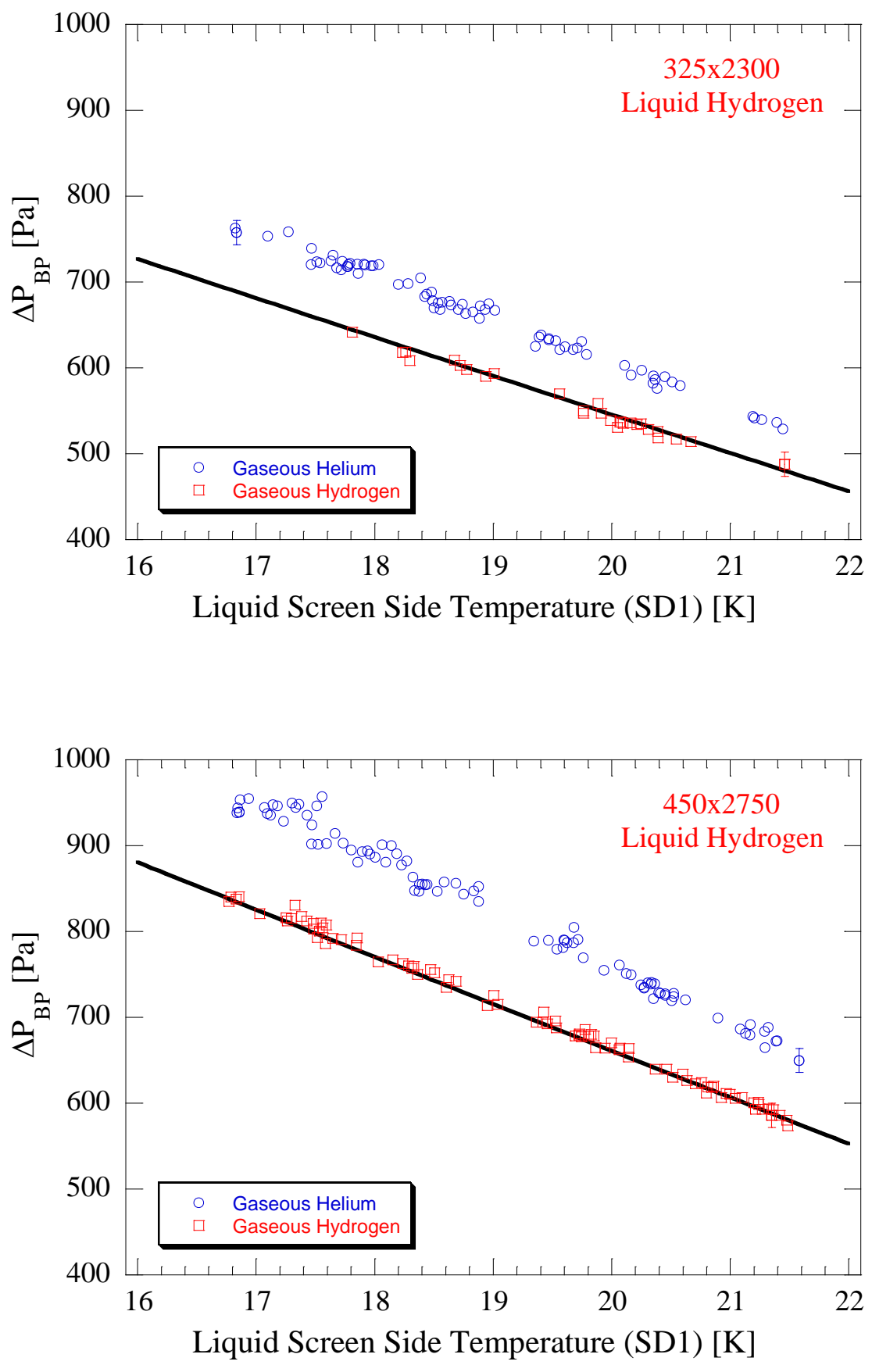


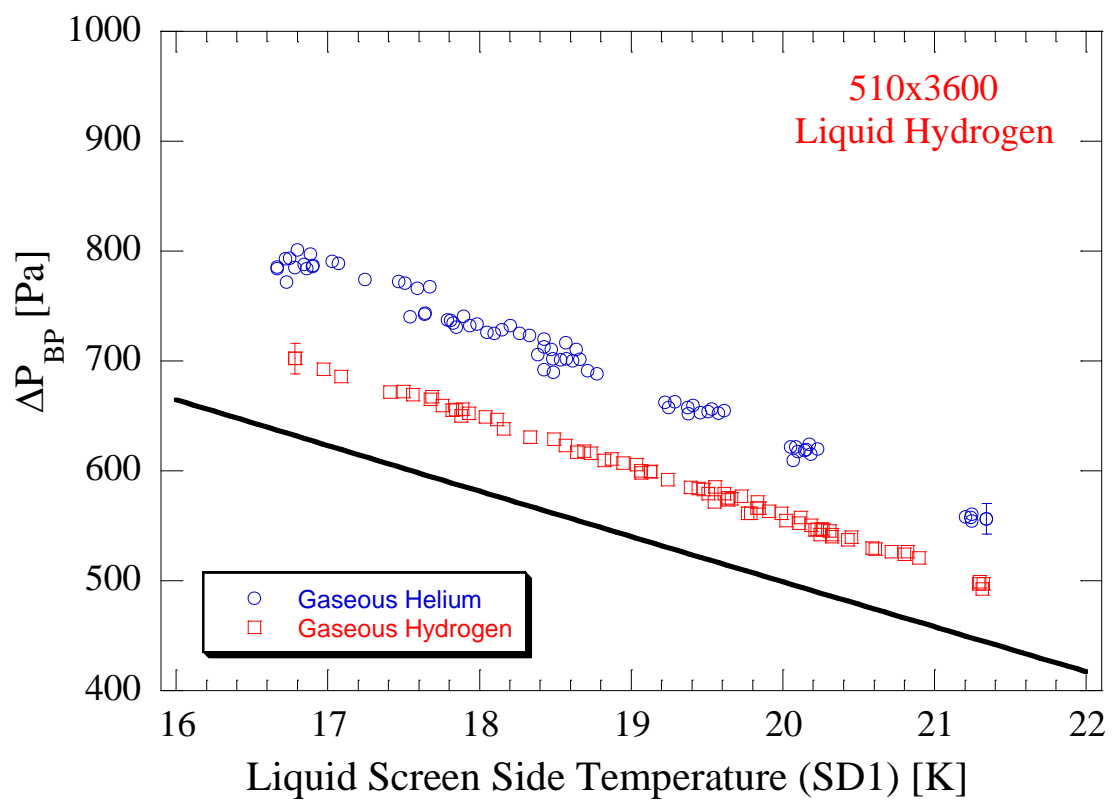

Figure 5.15 - Liquid Hydrogen Bubble Point Pressure Dependence on Pressurant Gas for a) $325 \times 2300$, b) $450 \times 2750$, and c) 510x3600 Screens. The black line is the prediction curve based on the room temperature pore diameter.

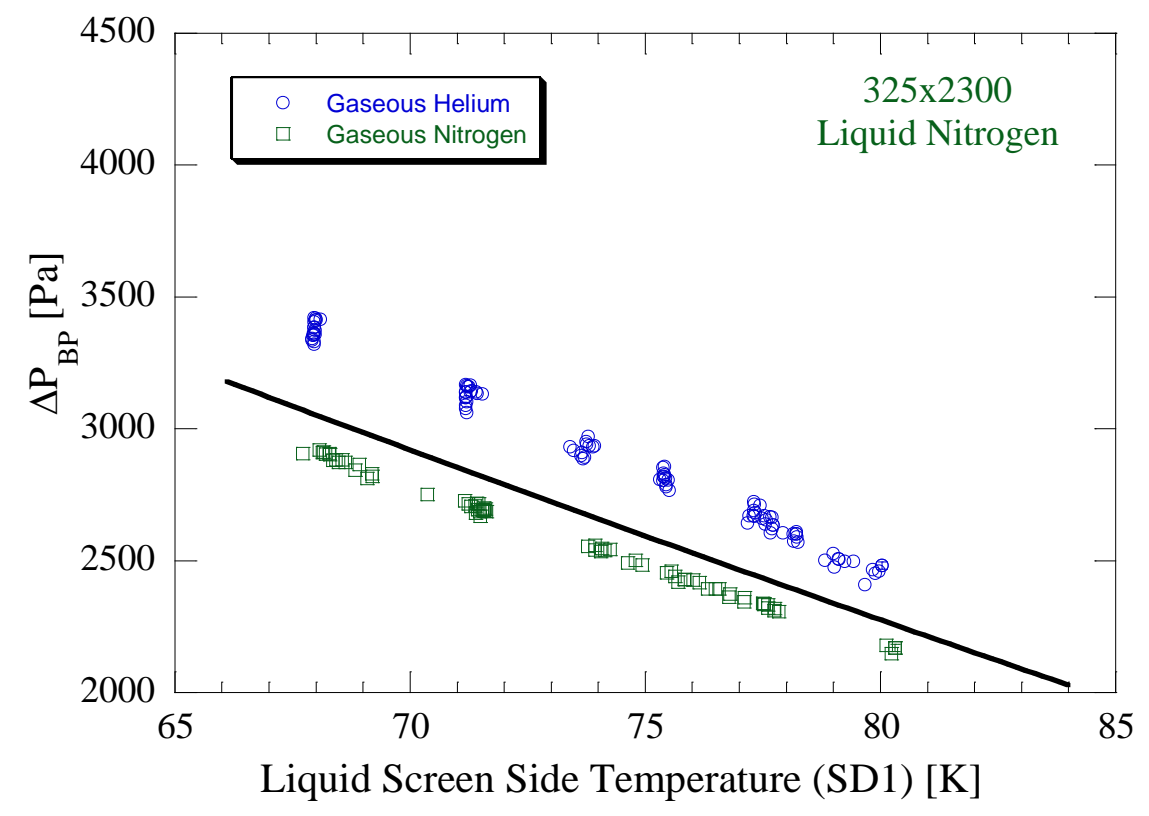



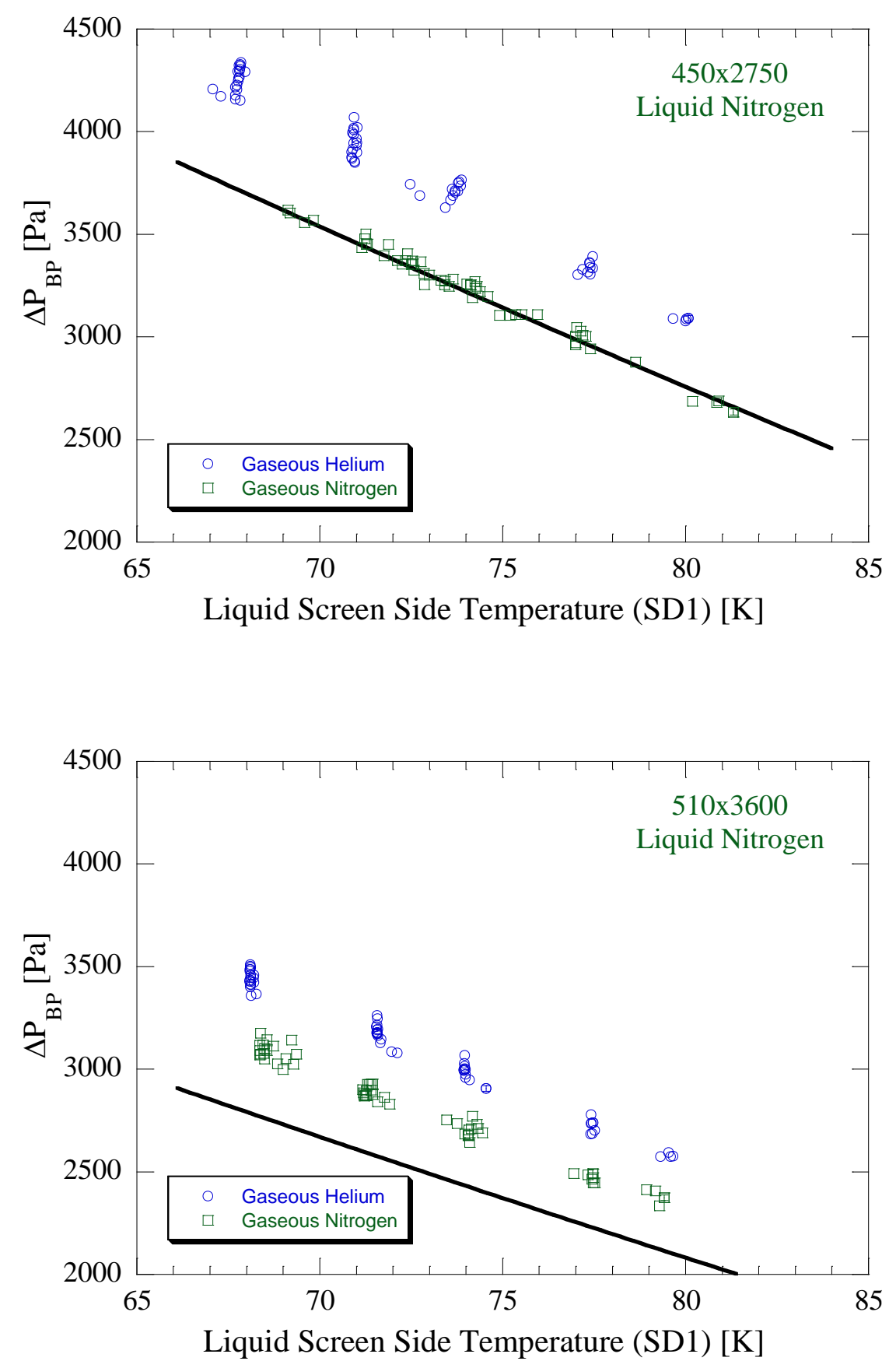

Figure 5.16 - Liquid Nitrogen Bubble Point Pressure Dependence on Pressurant Gas for a) $325 \times 2300$, b) $450 \times 2750$, and c) $510 \times 3600$ Screens. The black line is the prediction curve based on the room temperature pore diameter. 

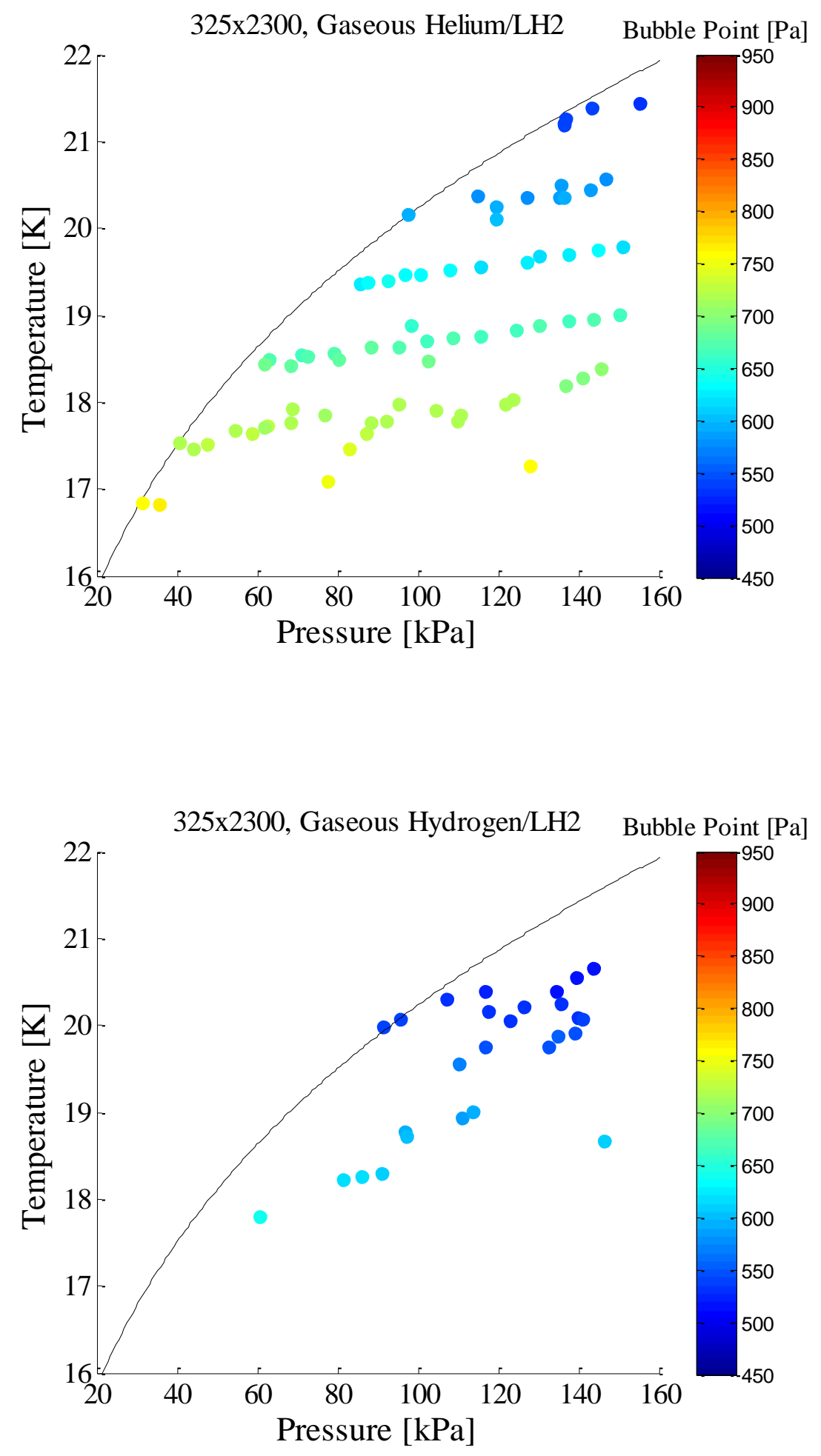

Figure 5.17 - 325x2300 Liquid Hydrogen Bubble Point Pressure as a Function of Liquid Temperature and Pressure using a) Gaseous Helium and b) Gaseous Hydrogen as a Pressurant. The black line is the prediction curve based on the room temperature pore diameter. 

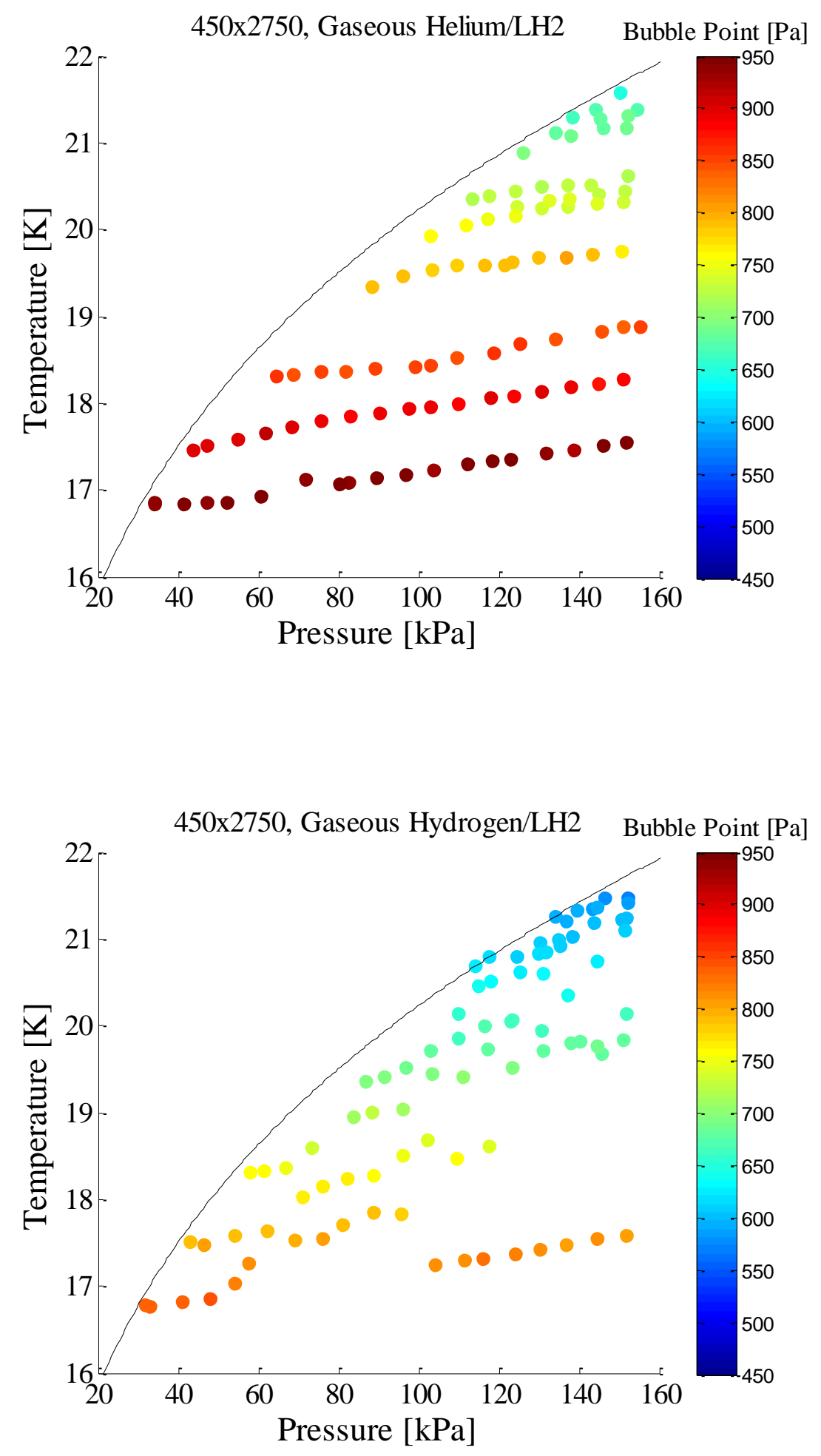

Figure 5.18 - 450x2750 Liquid Hydrogen Bubble Point Pressure as a Function of Liquid Temperature and Pressure using a) Gaseous Helium and b) Gaseous Hydrogen as a Pressurant. The black line is the prediction curve based on the room temperature pore diameter. 

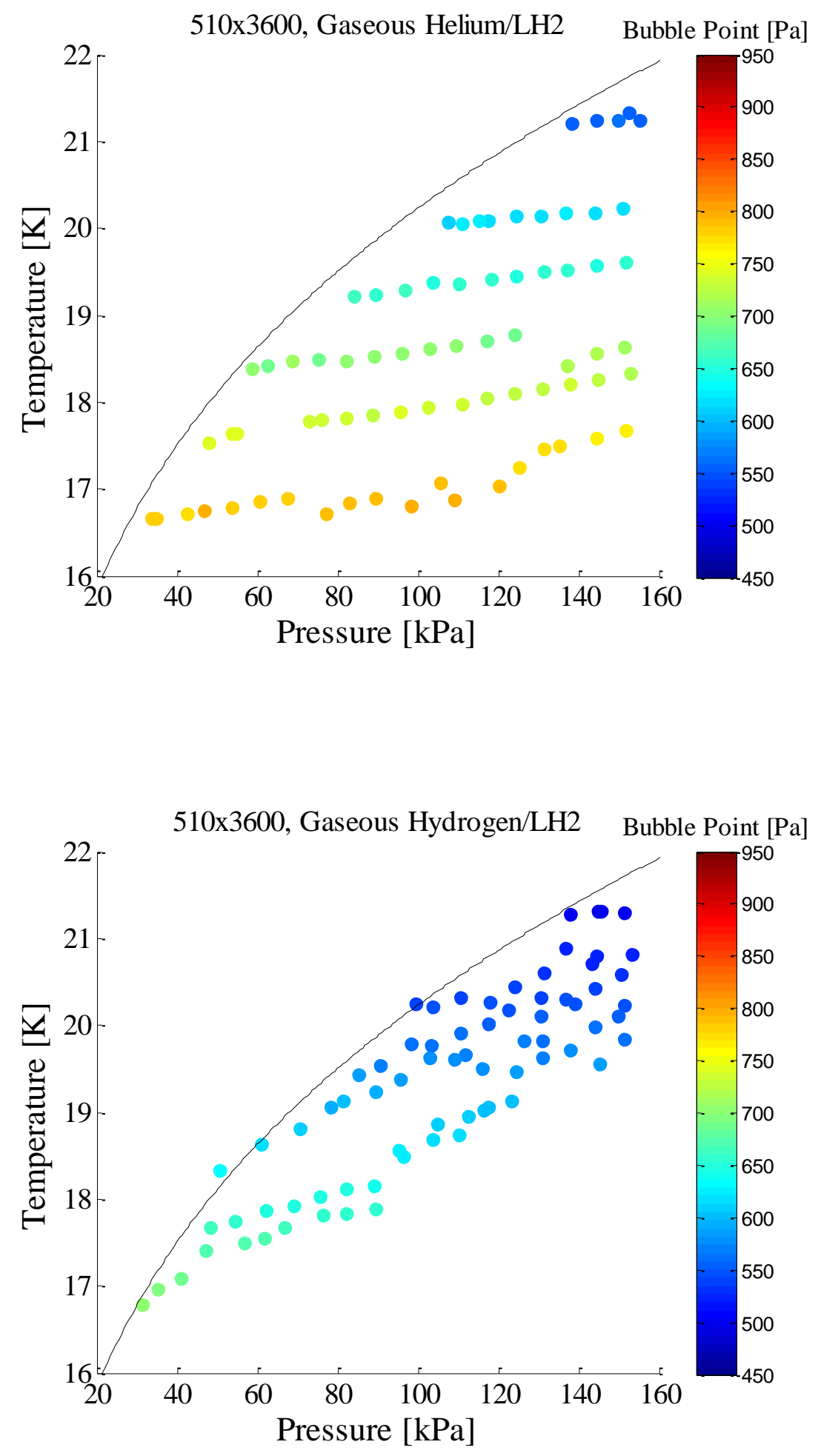

Figure 5.19 - 510x3600 Liquid Hydrogen Bubble Point Pressure as a Function of Liquid Temperature and Pressure using a) Gaseous Helium and b) Gaseous Hydrogen as a Pressurant. The black line is the prediction curve based on the room temperature pore diameter. 

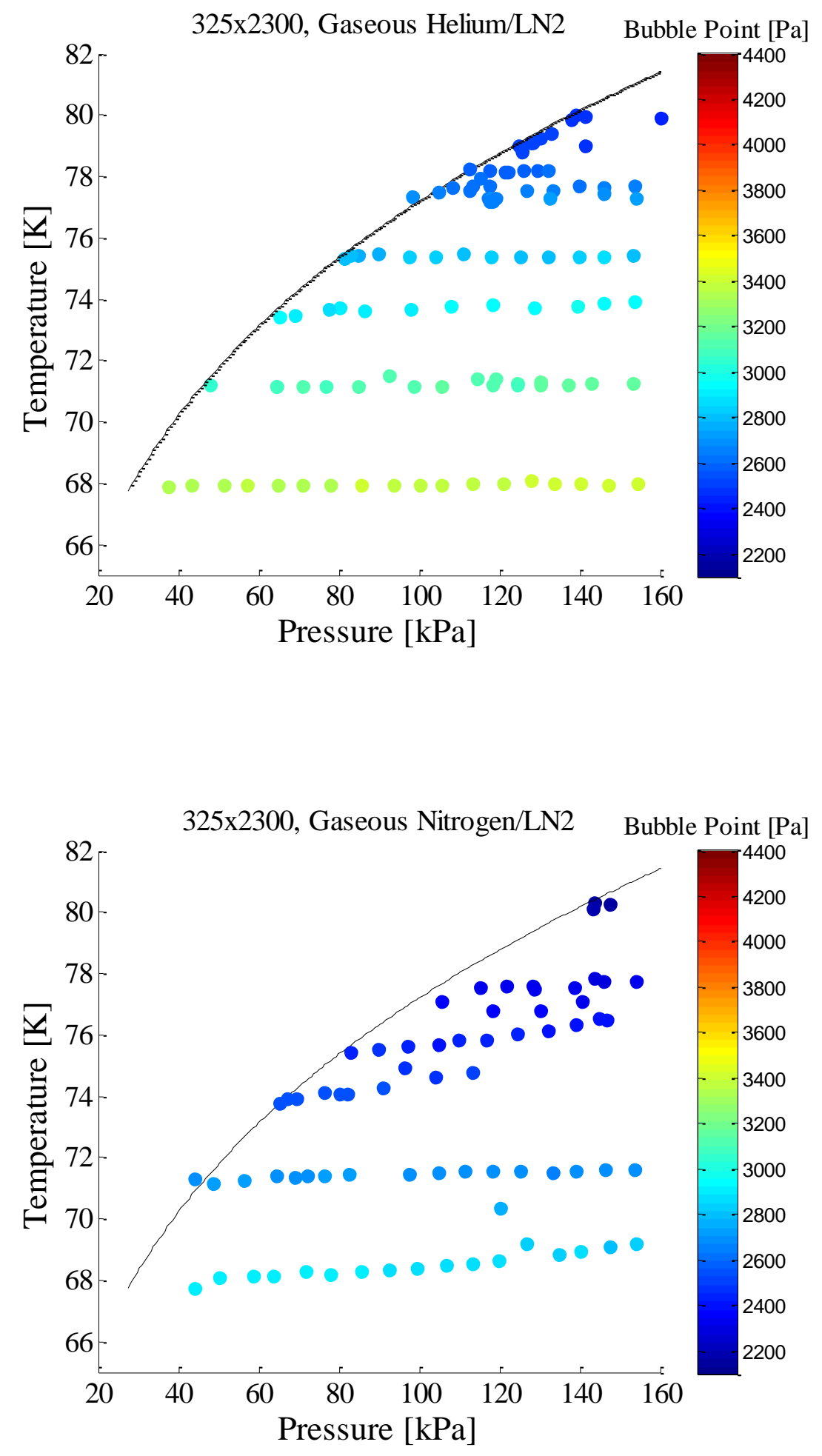

Figure 5.20 - 325x2300 Liquid Nitrogen Bubble Point Pressure as a Function of Liquid Temperature and Pressure using a) Gaseous Helium and b) Gaseous Nitrogen as a Pressurant. The black line is the prediction curve based on the room temperature pore diameter. 

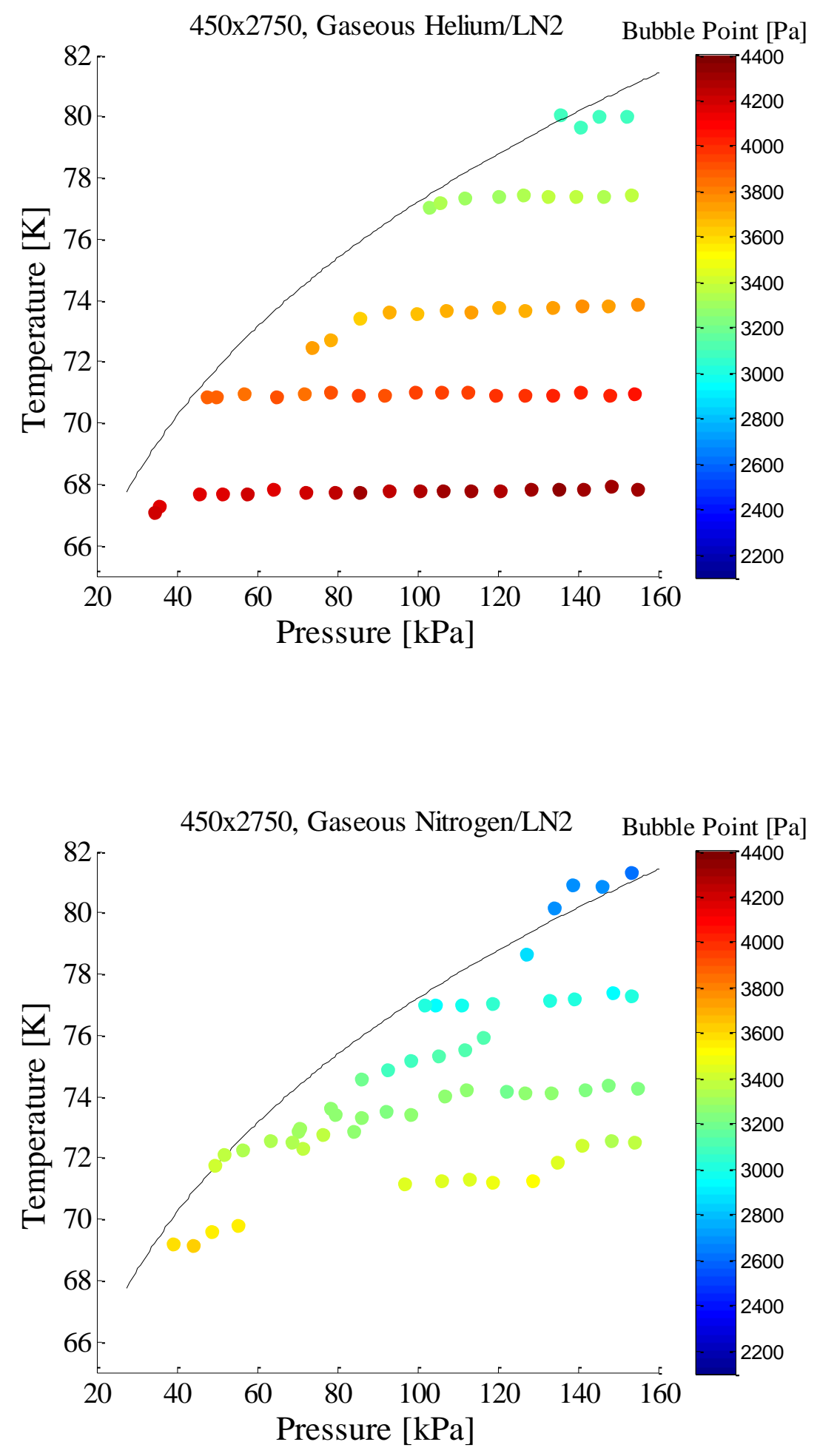

Figure 5.21 - 450x2750 Liquid Nitrogen Bubble Point Pressure as a Function of Liquid Temperature and Pressure using a) Gaseous Helium and b) Gaseous Nitrogen as a Pressurant. The black line is the prediction curve based on the room temperature pore diameter. 

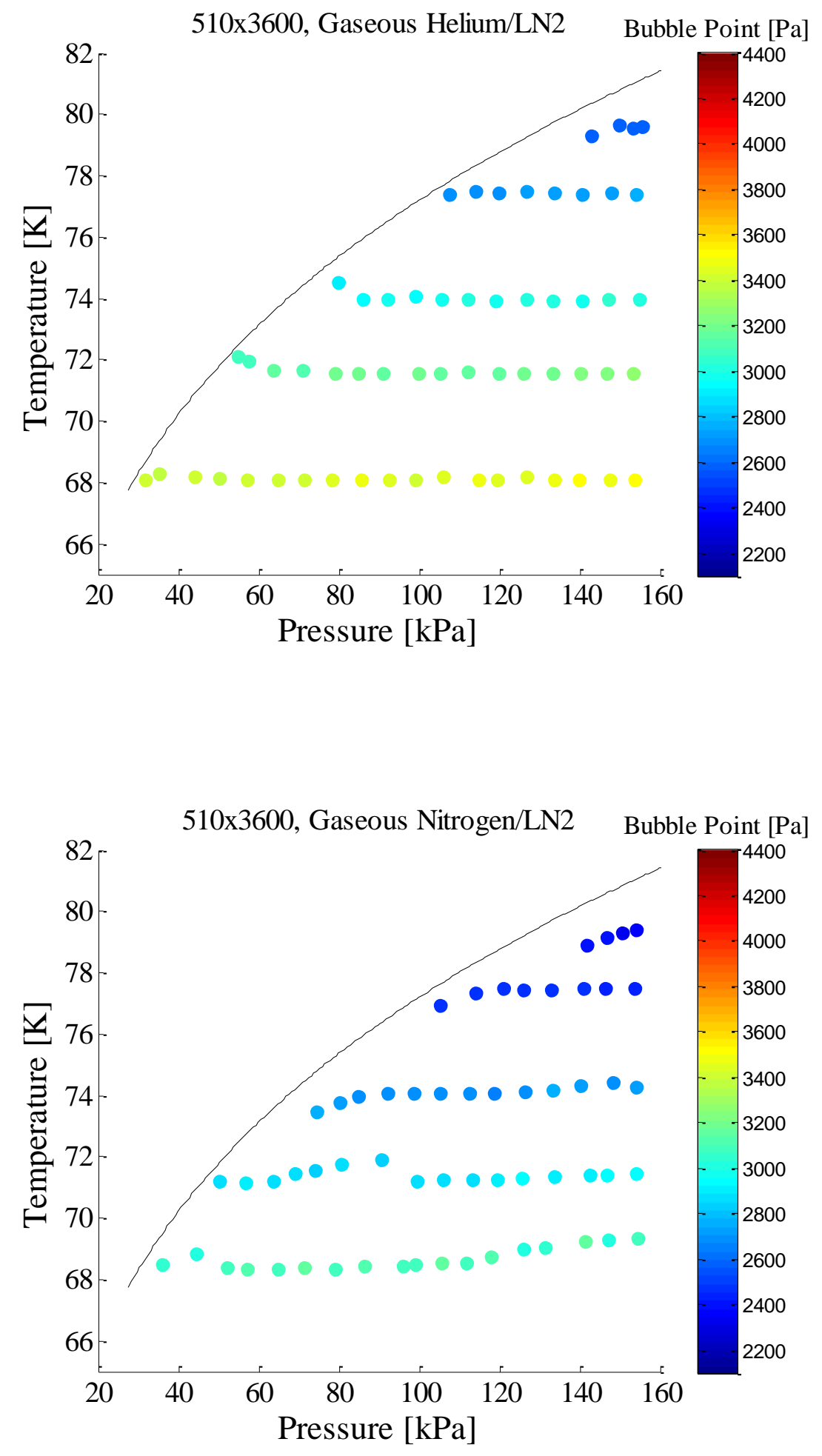

Figure 5.22 - 510x3600 Liquid Nitrogen Bubble Point Pressure as a Function of Liquid Temperature and Pressure using a) Gaseous Helium and b) Gaseous Nitrogen as a Pressurant. The black line is the prediction curve based on the room temperature pore diameter. 


\subsection{Concluding Remarks}

The low bubble point pressure of the $325 \times 2300$ screen hinders the operating range over which vapor free liquid may be extracted from an $\mathrm{LH}_{2}$ propellant tank. Parametric bubble point tests in liquid hydrogen and nitrogen demonstrate three ways to increase margin over the low baseline reference value. By using the finer 450x2750 mesh, operating at a colder liquid temperature inside the propellant tank, and pressurizing and subcooling the screen $\mathrm{L} / \mathrm{V}$ interface with a non-condensable pressurant gas, a higher margin in bubble point pressure is achieved over the $325 \times 2300$, increasing the total allowable flow rate or maximum allowable adverse acceleration against which liquid must be withdrawn from a storage tank in LEO. Experiments show that bubble points in excess of $950 \mathrm{~Pa}$ are achievable with the $450 \times 2750$ mesh, representing a $165 \%$ increase over the baseline NBP value for a $325 \times 2300$ screen.

The five test parameters that govern screen channel liquid acquisition device behavior in a low pressure propellant tank that were varied included the screen mesh, liquid, liquid temperature and pressure, and type of pressurant gas. Bubble point pressure does not scale inversely with the mesh of the screen, as the middle $450 \times 2750$ screen produced the highest pressures in both cryogenic and room temperature liquids. Bubble point pressure is shown to scale with the surface tension of the liquid for both cryogenic and room temperature liquids. Experiments also show that the effective pore diameter is itself temperature dependent, and that the pore shrinks at different rates depending on the specific screen mesh. Bubble point is inversely proportional to the temperature of the liquid, and pressurizing and subcooling the screen pore $\mathrm{L} / \mathrm{V}$ interface is shown to increase margin for all meshes and liquids tested in this work. Pressurization with GHe adds 
margin to bubble point pressure while autogenous pressurization acts as a degradation factor in performance. The highest bubble point pressures are obtained with the middle 450x2750 screen in the coldest liquid temperatures using GHe to pressurize the screen. 


\section{Chapter 6}

\section{High Pressure Liquid Oxygen Bubble Point Experiments}

The purpose of this chapter is to present the high pressure and temperature liquid oxygen static bubble point experiments. The purpose of the tests was to examine the key parameters which affect the bubble point pressure for screen channel LADs across a very wide range of thermal and operational conditions applicable for a high pressure cryogenic propellant tank. Two fine mesh Dutch Twill screens (200x1400 and 325x2300) were tested across a wide range of liquid temperatures $(92 \mathrm{~K}<\mathrm{T}<130 \mathrm{~K})$ and pressures $(0.138$ $\mathrm{MPa}<\mathrm{P}<1.79 \mathrm{MPa}$ ) using both a non-condensable helium and condensable gaseous oxygen $(\mathrm{GOX})$ vapor to pressurize the screen to further examine the functional dependencies of each parameter. Details on the high pressure experimental design and methodology are also discussed, including the facility, new test article, and instrumentation. Specifically, high pressure testing capability allows detailed assessment of the importance of subcooling the liquid on LAD performance, and the additional associated heat transfer effects that arise due to high pressure subcooling and due to different pressurant gases in contact with the screen. Basic image analysis of the screens at LAD breakdown is also performed to compare performance between the two pressurant gases. 


\subsection{Test Purpose and Motivation}

For traditional cryogenic propulsion systems, $\mathrm{LOX} / \mathrm{LH}_{2}$ has been the preferred choice, owing to its high ISP relative to other propellant combinations. However, in the optimal design of any spacecraft, high performance is not the only consideration. From Chapter 1, long term storage of $\mathrm{LH}_{2}$ would require large and thick walled propellant tanks, owing to its low density and low NBP. The ability to manufacture propellant in situ at the destination is another factor in the propellant choice.

Recently, there has been a growing desire to develop technology to enable higher pressure fed cryogenic propellant engines to take advantage of potential higher engine performance at higher operating pressures. Principally, operating at a higher tank pressure would minimize boil off losses by allowing the cryogen to absorb parasitic heat leak. Pressure-fed engines are also attractive in potentially eliminating the requirement for the turbopump between propellant tank and engine, which is used to pressurize the liquid prior to injection. Elimination of the turbopump by using the natural pressure gradient between the liquid in the storage tank and the engine leads to an inherently simpler design. As an alternative to using storable propellants, initial trade studies were conducted to evaluate the feasibility of utilizing $\mathrm{LOX} / \mathrm{LCH}_{4}$ for the Altair AME and RCS engines. This option considered the use of high pressure (up to 2.4 MPa) propellant tanks which used the natural driving pressure gradient between propellant tank and engine to feed the AME (Dickens 2010).

A LOX/LCH $\mathrm{Lueled}_{4}$ system is attractive because it does not have the issues associated with $20 \mathrm{~K}$ storage and transfer. Even though $\mathrm{LOX} / \mathrm{LH}_{2}$ systems have a higher 
ISP, $\mathrm{LOX} / \mathrm{LCH}_{4}$ systems are more "storable" because the NBP of $\mathrm{LOX}$ and $\mathrm{LCH}_{4}$ are much warmer, 90K and 112K, respectively. Second, transfer systems would be less complex because the two fluids exist as liquids under similar thermodynamic conditions. Third, methane exists as a liquid over a much broader range than $\mathrm{LH}_{2}$ giving mission designers much more flexibility in design. Fourth, the higher liquid density of $\mathrm{LCH}_{4}$ over $\mathrm{LH}_{2}$ implies lighter and smaller storage tanks. Fifth, methane exists in Martian atmospheres, thus promoting the use of $\mathrm{LCH}_{4}$ fueled return systems to Earth for Mars missions through ISRU. To enable future higher pressure-fed engine capabilities for longer duration space missions, such as the proposed Lunar and Martian ascent stages or Earth Departure Stage (ESAS study, Anonymous 2005), ground tests are first required to fully characterize these LADs over a wide range of thermodynamic conditions. Given that some design reference missions proposed the use of pressurized cryogenic propellant tanks, and due to the paucity of data at these conditions, the CFM program at NASA GRC opted to pursue experiments to measure the bubble point for LOX at elevated pressures and temperatures representative of the proposed Lunar Ascent Stage for Altair.

In terms of the $\mathrm{LAD}$, there were reported model discrepancies when attempting to predict performance at subcooled liquid states. While the bubble point equation correlated reasonably well when using the liquid temperature to predict the bubble point for conditions near atmospheric pressure, there was deviation when liquid was subcooled. Initial efforts to predict the bubble point for a subcooled liquid based on the bulk liquid temperature were attempted by Jurns et al. (2007) for $\mathrm{LCH}_{4}$ at temperatures slightly below atmospheric conditions. The original bubble point equation was modified by taking into account the fluid viscosity and density using a relationship developed by 
Bretherton (1961) for the slow motion of an elongated bubble through a capillary tube. While the analysis did correlate well for subcooled $\mathrm{LCH}_{4}$, Jurns and McQuillen (2008) found that this correction term did not extend to results for subcooled LOX also over the small subcooled temperature range. Rather than extrapolate screen channel LAD performance to these elevated pressure conditions in subcooled states, it was determined that bubble point measurements needed to be conducted at or near these operating conditions, since there was discrepancy of up to $10 \%$ in surface tension data for LOX and $\mathrm{LCH}_{4}$ from different published sources (e.g. Fuks and Bellemans 1966, Sprow and Prausnitz 1966, and Roder and Weber 1972). Only three studies in the literature report LOX bubble point data (Paynter 1973a, Kudlac and Jurns 2006, and Jurns and McQuillen 2008), and the majority of this data was collected in saturated or near saturated liquid states at low pressure.

Screen channel LAD performance in high pressure propellant tanks may be affected by the degree of propellant subcooling and type of gas used during pressurization or liquid expulsion. Therefore it was ensured that bubble point data was collected over the widest possible range of thermal conditions inside a LOX propellant tank, consistent with the limitations of the test hardware. Therefore, the purpose of this chapter is to conduct an in-depth analysis on these high pressure LOX bubble point tests to understand parameters that affect LAD performance in an elevated pressure environment. 


\subsection{Experimental Design}

\subsubsection{Test Article and Facility}

Testing was conducted in the CCL-7 facility at GRC. Two LAD screen samples were tested, a 200x1400 and 325×2300 Dutch Twill screen. The thickness of the 200x1400 and 325 x 2300 screen samples were $152 \mu \mathrm{m}$ and $89 \mu \mathrm{m}$, respectively. Screen samples were each mounted onto a $2.38 \mathrm{~cm}$ tall, $5.08 \mathrm{~cm}(2 \mathrm{in})$ OD cup as shown in Figure 6.1. Note that the LAD cup used in the high pressure tests was significantly smaller than the screen and cup assembly used in the low pressure $\mathrm{LH}_{2}$ bubble point tests. As in previous tests, an inverted bubble point test configuration was implemented for the high pressure LOX tests. The cup was equipped with a pressurization port and a port for purging, in the event of liquid accumulation inside the cup due to high pressure.

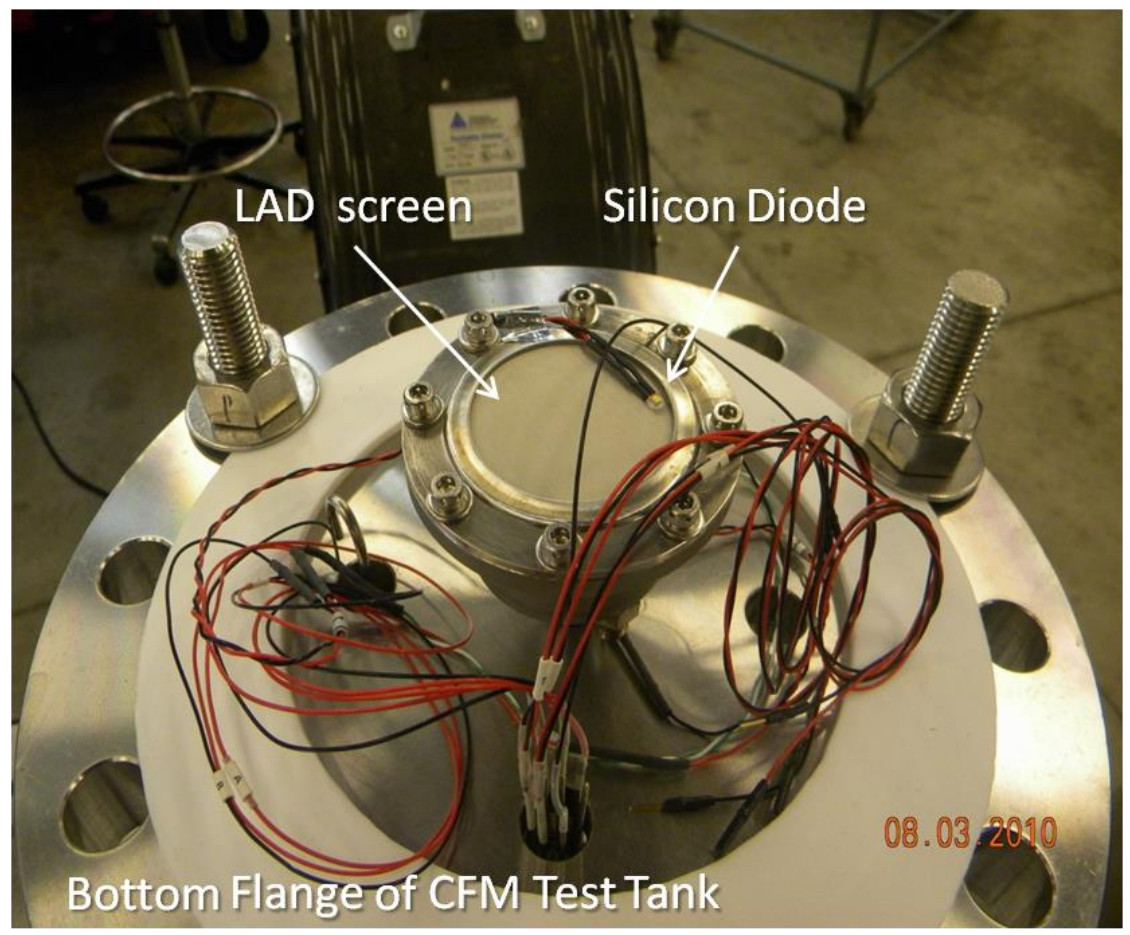

Figure 6.1 -Screen/Cup Assembly for High Pressure Bubble Point Experiments 
The LAD screen and cup assembly was mounted inside of a custom fabricated $3.55 \mathrm{MPa}$ (500 psig) rated, $15 \mathrm{~cm}$ (6 in) OD, $33.65 \mathrm{~cm}$ (13.25 in) high $6000 \mathrm{~cm}^{3}$, optically accessible CFM test tank as illustrated in Figure 6.2. Design details of how the CFM test tank was constructed are omitted for brevity. The purpose of the CFM test tank was to house the high pressure LOX on top of the LAD screen. The CFM test tank was then mounted inside a $56 \mathrm{~cm}$ (22 in) OD, $107 \mathrm{~cm}$ (42 in) tall, $0.229 \mathrm{~m}^{3}\left(8.1 \mathrm{ft}^{3}\right) \mathrm{RD}$ shown in Figure 6.3. There was no active external control of the temperature of the test tank, therefore the purpose of the RD was to minimize parasitic heat leak into the CFM test tank. The vacuum jacket for the dewar was fully evacuated prior to every test. During testing, Cell 7 air ejectors pulled the pressure inside the RD down to $0.10 \mathrm{kPa}$. In spite of these efforts, there still was tendency for the pressure and temperature of the liquid to increase over time that was obviated through the use of multiple chill down cycles of the hardware prior to measurements. The lid on the dewar was equipped with feedthrough ports for vacuum, relief, instrumentation, and electrical wiring.

The CFM test tank was equipped with three $7.62 \mathrm{~cm}(3.0 \mathrm{in}) \mathrm{OD}, 3.81 \mathrm{~cm}$ (1.5 in) thick quartz windows to view the LAD screen with a single CCD camera, illuminated by two light sources mounted inside the vacuum space as shown in Figure 6.2. The image was transmitted, time stamped, and recorded during testing. A heater mounted on the back of the camera maintained the camera in its operational temperature band of $4^{\circ} \mathrm{C}-$ $15.5^{\circ} \mathrm{C}$ and prevented condensation of any moisture on the camera. The camera image was sent to a monitor to provide real time imaging of the LAD screen during testing.

LOX tests were conducted inside Cell 7 and not performed remotely like the $\mathrm{LH}_{2}$ tests. All critical hardware was controlled via a computer located within the cell. 
Modifications to the cell were made to flow GOX. A pressure regulator controlled incoming pressure of the gas, and flow rate was controlled by a set of flow control valves located directly upstream of the CFM test tank.

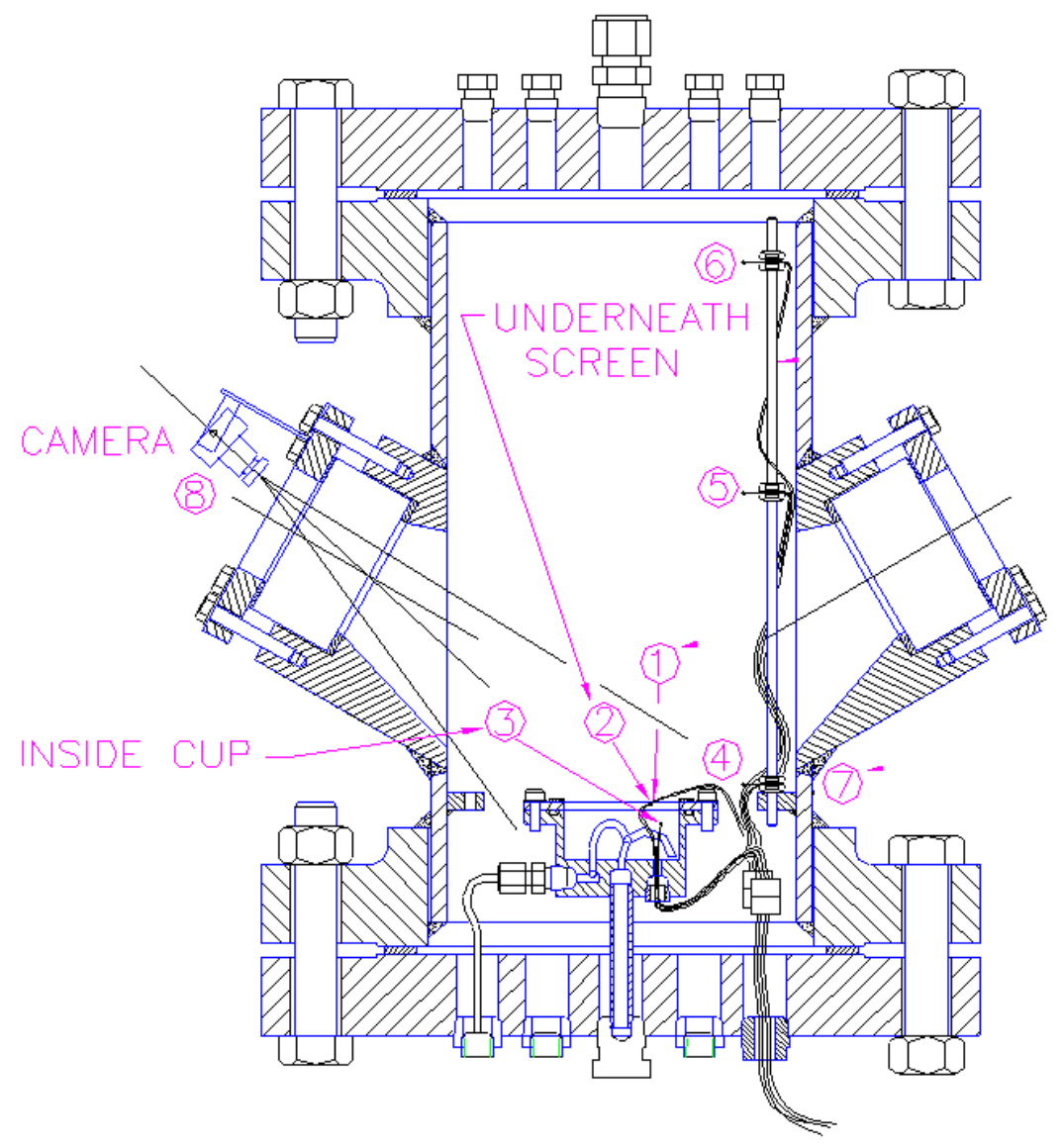

Figure 6.2 - High Pressure Cryogenic Fluid Management Test Tank

Liquid pressure inside the CFM test tank was controlled through a back pressure control valve. The ejectors were used to continually pump down on the reduced pressure space of the dewar, thus minimizing heat leak into the liquid inside the CFM test tank. Temperature of the liquid inside the CFM test tank was initially determined by the liquid temperature inside the portable LOX storage dewar. Cold liquid was achieved by venting 
the dewar down to atmospheric pressure while warmer liquids were achieved by allowing the dewar to warm over a period of days. During testing, small adjustments in liquid temperatures were made by chilling down the hardware, by simply venting, or by allowing the liquid to absorb parasitic heat leak over time. Gas pressure and flow rate into the LAD screen/cup assembly were manually set using flow control valves to slowly increase the pressure. GHe and GOX were available via a portable tuber trailer and $\mathrm{K}$ bottles, respectively.

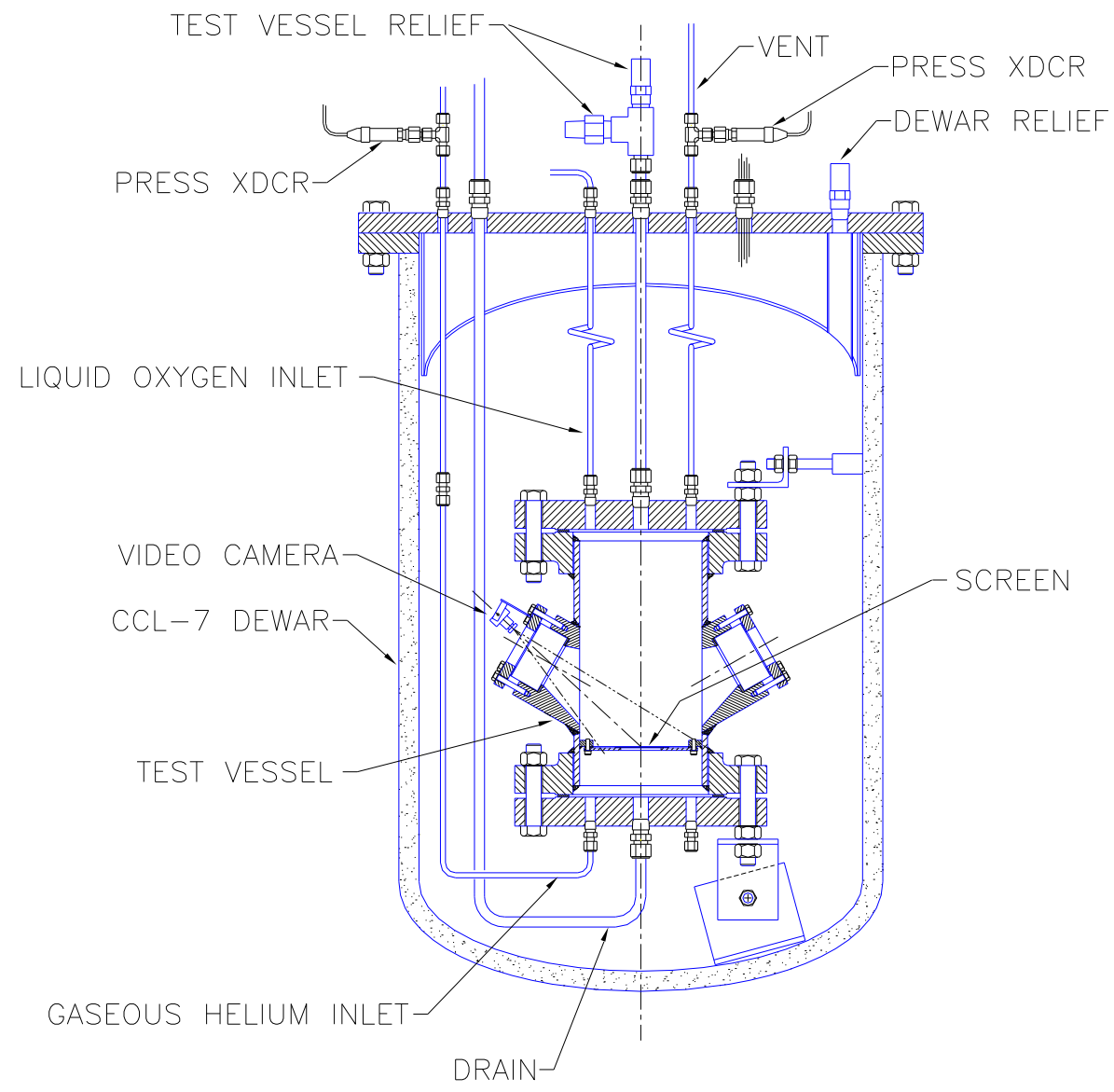

Figure 6.3 -Screen and Cup Assembly, High Pressure Test Tank, and Dewar 


\subsubsection{Instrumentation and Data Acquisition}

All data was recorded at $2 \mathrm{~Hz}$. Video images of the LAD screen were transmitted and recorded during testing. Videos were time stamped with a resolution of 0.1 seconds and recorded on a DVR player to correlate with the time stamped data files to determine the exact raw pressure (and all other desired measurements) at screen breakdown.

Key temperature instrumentation is shown in Figure 6.2 where each number corresponds to the location of a specific silicon diode. SD1 was mounted directly to the LAD screen on the liquid side of the interface while SD2 was attached to the vapor side of the screen within the cup assembly. Both diodes were directly mounted to the LAD screen using a low thermal conductivity epoxy. SD3 measured the temperature of incoming pressurant gas. SD4 - 6 were located along a vertical rake within the test tank and were used to measure both temperature and height of the bulk liquid. The pressure in the ullage space was measured using a 3.45 MPa (500 psia) pressure transducer while two DPTs were used to deduce the liquid head pressure on top of the screen and differential pressure across the LAD screen sample. DPT03 measured over a range of 0 $7.5 \mathrm{kPa}\left(0-30\right.$ in $\left.\mathrm{H}_{2} 0\right)$ the pressure at the bottom of the screen/cup assembly with respect to the ullage pressure, while DPT01 measured over a range of $0-34 \mathrm{kPa}(0-5 \mathrm{psid})$ the pressure at the bottom of the CFM test tank referenced to the ullage pressure. Bubble point was deduced by correcting the DPT03 measurement for head pressure using the DPT01 signal. While the CFM test tank was rated up to $3.45 \mathrm{MPa}$ (500 psia), data collection was limited to $1.725 \mathrm{MPa}$ (250 psia) as limited by the maximum allowable DPT line pressure. 
All silicon diodes measured temperature to within $+/-0.5 \mathrm{~K}$. The ullage pressure and thus the pressure at the screen was within $+/-4.6 \mathrm{kPa}(0.67 \mathrm{psi})$ of the measured value. The DPT across the LAD screen sample measured pressure to within $+/-0.0104$ $\mathrm{kPa}\left(0.042\right.$ in $\left.\mathrm{H}_{2} \mathrm{O}\right)$, which was less than $1 \%$ at the lowest reported bubble point. However, due to read off errors, interpolating between recorded values, and due to uncertainty in liquid level (and thus liquid head pressure), the total uncertainty in reported bubble point values is estimated to be $+/-0.08 \mathrm{kPa}\left(0.32\right.$ in $\left.\mathrm{H}_{2} \mathrm{O}\right)$, which was less than $8 \%$ at the warmest liquid temperature. Data was reduced in the same manner as reported in Section 5.2.3 except liquid head pressure was determined by using the DPTs and not the silicon diodes.

\subsubsection{Test Matrix}

Figure 6.4 summarizes the test conditions over which LOX bubble point data was collected in terms of the liquid temperature and pressure at the LAD screen. The goal was to collect bubble breakthrough values over as wide a range as possible to give future mission designers direct bubble point data to characterize the LAD subsystem at any elevated temperature or pressure within the propellant tank. Also plotted are test conditions from the recent low pressure LOX bubble point tests from Jurns and McQuillen (2008), analytical work from the Lunar Ascent Propulsion System Study (LAPS) for the anticipated Altair engine start box conditions (Dickens 2010), and altitude combustion testing conditions of a small scale $440 \mathrm{~N}$ (100 lbf) RCS engine at GRC and the $24.5 \mathrm{kN}$ (5500 lbf) AME engine at White Sands Test Facility (WSTF) (Stiegemeier and Marshall 2010 and Stiegemeier et al. 2010). 


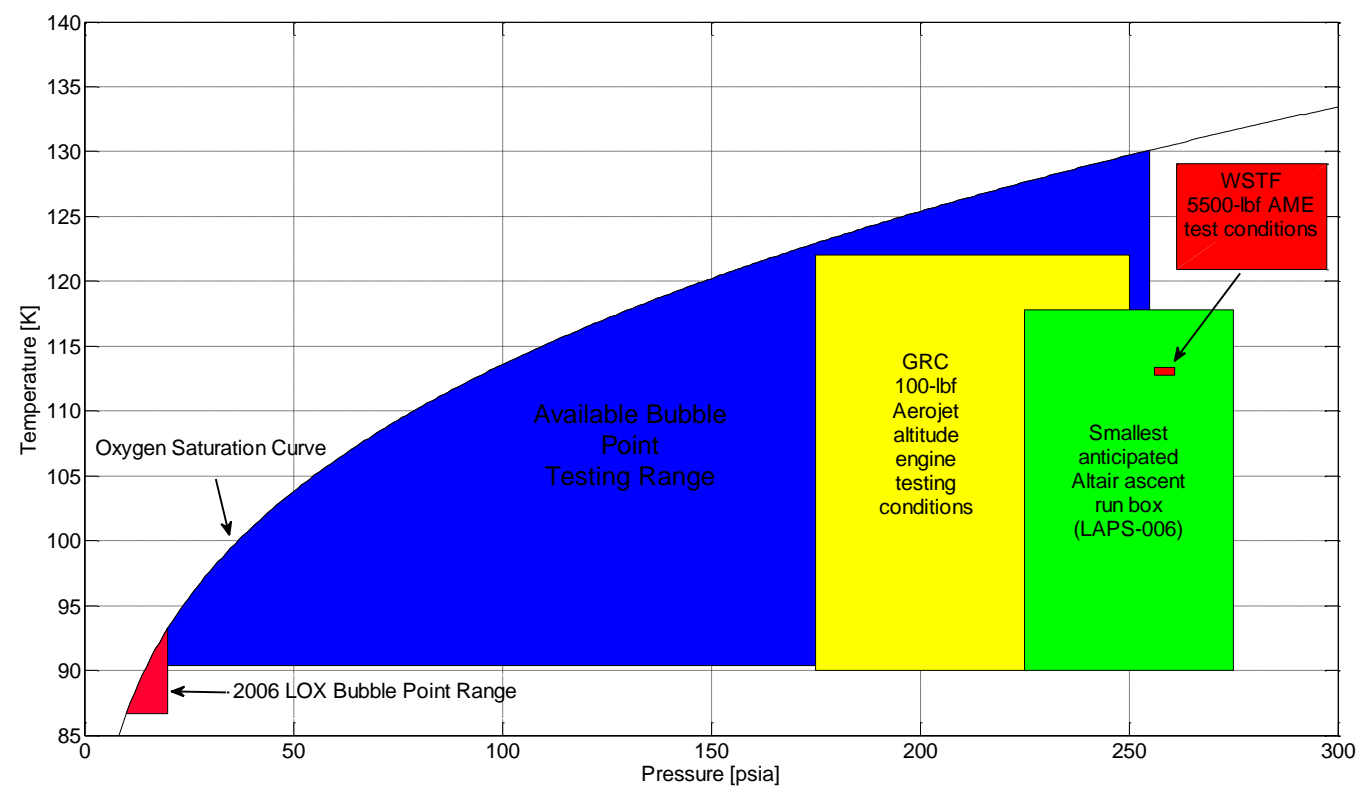

Figure 6.4 - Summary of Test Conditions for Current High Pressure Liquid Oxygen Bubble Point Tests and Other Related Liquid Oxygen Technology Development Programs

\subsection{Experimental Methodology}

The methodology for conducting a high pressure bubble point test was as follows: Before flowing propellant, the RD was evacuated to minimize heat leak into the CFM test tank. All the flow lines and test hardware were purged with GHe. Next the cup was pressurized using GHe to prevent flooding of the cup during liquid transfer. The hardware was pre-chilled several times using $\mathrm{LN}_{2}$ to condition the system to the desired temperature. When the hardware was sufficiently cold, LOX was transferred into the CFM test tank with the vent value open. When the tank was full, the vent valve was closed and the system was pressurized to the desired state. Before data collection, the LAD screen/cup assembly was periodically purged with high pressure GHe to prevent flooding of the cup and instrumentation lines. 
To conduct a bubble point test, the screen was resealed by slowly decreasing the GHe flow into the cup. Measurements would commence if ullage pressure and liquid temperature were constant. The pressure underneath the screen was gradually increased relative to the ullage pressure. Eventually, a bubble would break through the screen as seen on the video monitor and by the spike in the DPT03 signal. The bubble point was taken as the moment that visible gas or vapor bubbles penetrated the screen mesh. The time stamp at breakthrough was noted and compared with the time stamp in the data file to extract the exact bubble point pressure.

Bubble point measurements were repeated at similar tank pressures and/or temperatures for repeatability and consistency. Because screen pore sizes may vary slightly due to screen manufacturing variations or defects, it was also desired to repeat measurements to verify that bubble breakthrough would occur at different locations on the screen. To repeat bubble point tests at a specific temperature or pressure, the screen was carefully resealed by slowly decreasing pressurant gas flow into the cup until no more bubbles penetrated the screen. The liquid on top of the LAD screen was conditioned to the desired pressure and/or temperature and the pressurant gas flow rate was increased again until the next bubble breakthrough. The average time between initial ramp and final bubble breakthrough was $30-40$ seconds.

Once testing was complete, any residual vapor was directly vented through a vent stack to atmosphere. The tank and flow lines were purged with GHe. The pressure in the $\mathrm{RD}$ was brought back to ambient conditions. Videos of the LAD screen were then transferred to an external hard drive. 
For this test, the high pressure CFM test tank allowed examination of both subcooled and saturated liquid states at the LAD screen. Subcooled states were achieved by pressurizing the ullage space in the test tank. Bubble points were also obtained close to the saturation curve over a range of temperatures by allowing the hardware to warm over a period of several days, and immediately flowing cold liquid, thus maintaining the propellant near the boiling state. Thus it was possible to independently examine the temperature and pressure dependence of the bubble point for two different pressurant gases over the whole blue shaded region from Figure 6.4 using this flexible system.

\subsection{Experimental Results and Discussion}

Testing covered 8 weeks from February to April 2010. Numerous bubble point tests were conducted over the temperature range from 92 to $130 \mathrm{~K}$ and the pressure range from 0.138 to $1.79 \mathrm{MPa}$ for both screens. Bubble point data was collected using GHe for the 200x1400 screen and GHe and GOX for the 325x2300 screen.

\subsubsection{Test Conditions}

Figures 6.5a and b plot the thermodynamic state of the 200x1400 LAD screen at bubble breakthrough based on the pressure at the liquid side of the LAD screen and the temperature at the liquid side of the screen and in the bulk liquid, respectively. Similarly, Figures 6.6a and b plot the same test conditions for the $325 \times 2300$ screen. The saturation curve for oxygen is plotted for reference. 

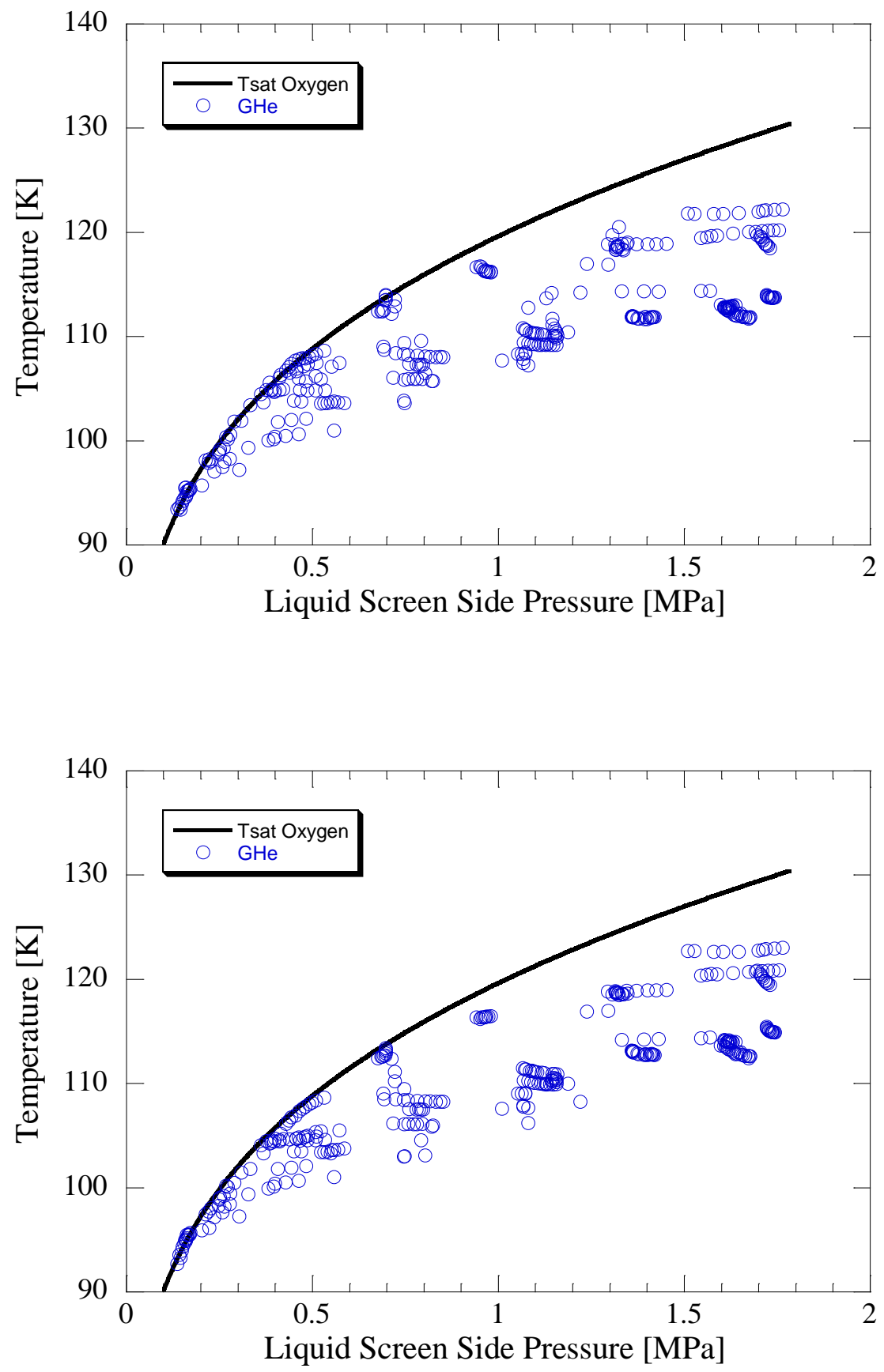

Figure 6.5 - Liquid Oxygen Thermodynamic Conditions at Bubble Breakthrough as a Function of the Liquid Screen Side Pressure and a) Liquid Screen Side Temperature (SD1) and b) Bulk Liquid Temperature (SD4) for the 200x1400 Screen 

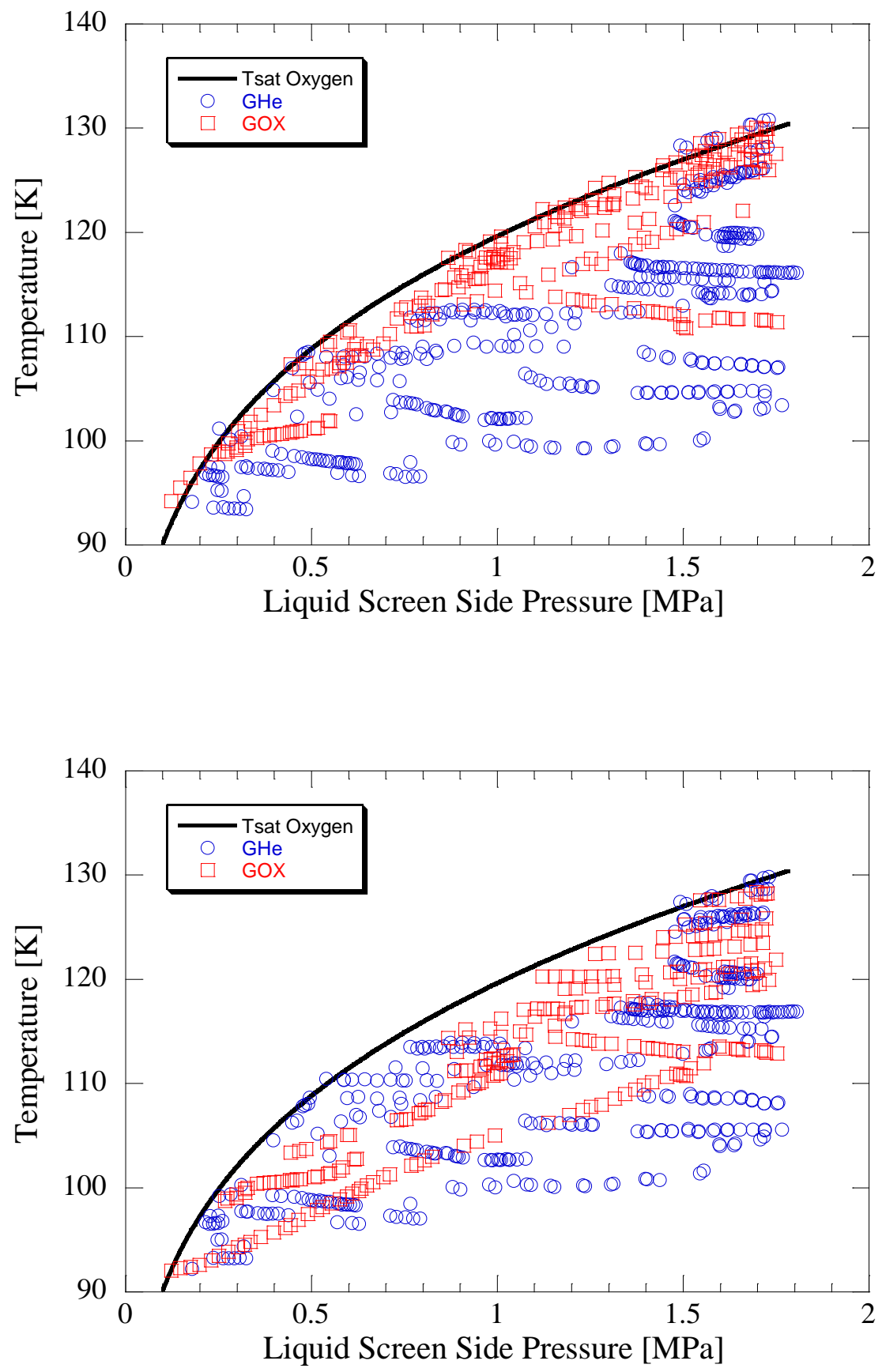

Figure 6.6 - Liquid Oxygen Thermodynamic Conditions at Bubble Breakthrough as a Function of the Liquid Screen Side Pressure and a) Liquid Screen Side Temperature (SD1) and b) Bulk Liquid Temperature (SD4) for the $325 \times 2300$ Screen 
As shown, using GHe as a pressurant gas, it was possible to obtain controlled breakthroughs over the full range of conditions with little disparity between screen and bulk liquid temperature. However, when GOX was the pressurant gas, there was considerable difference between reporting bubble breakthrough values using the liquid screen side temperature (SD1) vs. the bulk liquid temperature (SD4). The data clustering around the saturation curve for GOX in Figure 6.6a indicates that the temperature of the $\mathrm{L} / \mathrm{V}$ interface within the screen pores agreed better with the local saturation temperature based on the pressure at the screen.

Results imply that bubble breakthrough is relatively insensitive to changes in the bulk liquid temperature with autogenous (GOX) tank pressurization and relatively sensitive to bulk liquid temperature when using a non-condensable $(\mathrm{GHe})$ pressurization scheme. This means that when using helium to pressurize, conditions at the LAD screen are coupled to the bulk fluid conditions inside the propellant tank during expulsion. Meanwhile, when using GOX to pressurize, conditions at the screen track saturation conditions and are thus relatively independent of the bulk fluid.

\subsubsection{Elevated Temperature Dependence}

Figures $6.7 \mathrm{a}$ and $\mathrm{b}$ and $6.8 \mathrm{a}$ and $\mathrm{b}$ plot the experimentally obtained bubble point pressure as a function of the liquid screen side temperature and bulk liquid temperature, respectively for the $200 \times 1400$ and $325 \times 2300$ screens. The room temperature bubble point prediction curve is also plotted in these figures. The 200x 1400 room temperature pore diameter is taken from Table 3.2. The results from Jurns and McQuillen (2008) are plotted in Figures $6.7 \mathrm{~b}$ and $6.8 \mathrm{~b}$ for comparison to the current data. 

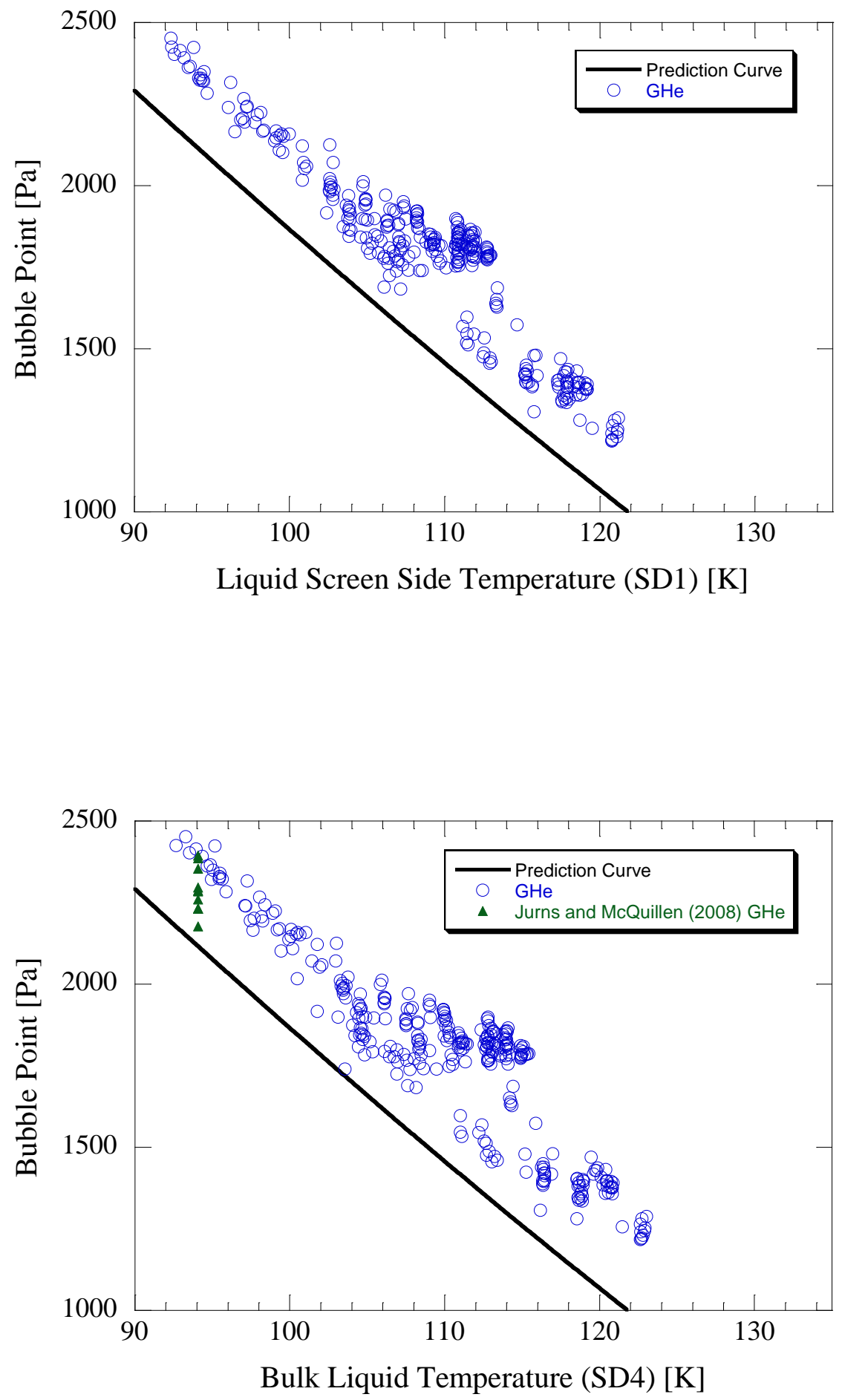

Figure 6.7 - Liquid Oxygen Bubble Point Pressure as a Function of a) Temperature of the Liquid Side of the Screen and b) Bulk Liquid Temperature for the 200x1400 Screen 

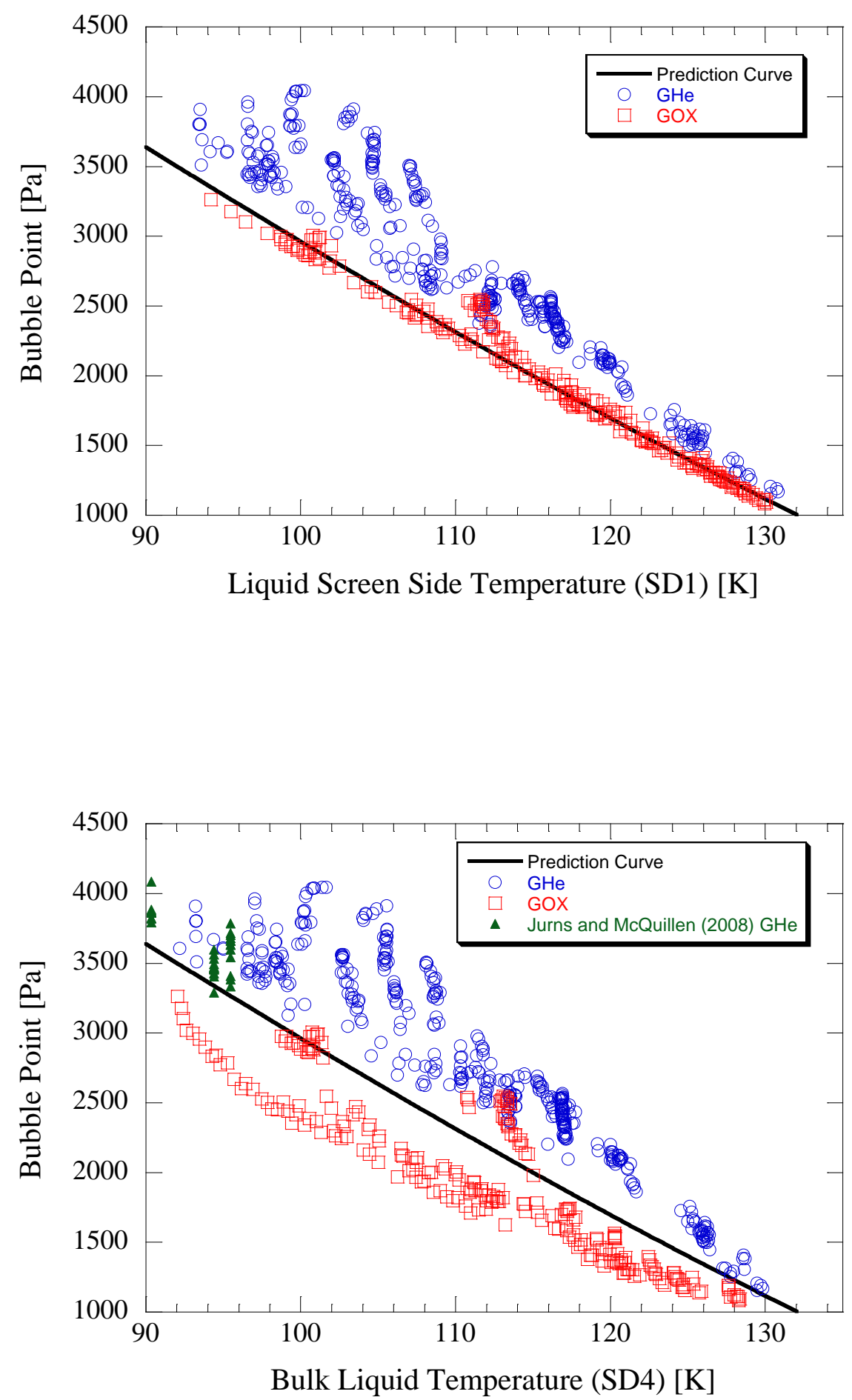

Figure 6.8 - Liquid Oxygen Bubble Point Pressure as a Function of a) Temperature of the Liquid Side of the Screen and b) Bulk Liquid Temperature for the 325x2300 Screen 
As can be seen, the finer $325 \times 2300$ mesh screen yields higher bubble points than the 200x 1400 mesh screen across the entire range of cryogenic temperatures. In both cases, the bubble point pressure decreases with increasing liquid temperature, due to decreasing surface tension of the liquid. The prediction curve again qualitatively follows this trend but underpredicts the data, especially at colder liquid temperatures. At identical liquid temperatures, bubble point pressures using GHe as a pressurant are generally higher than those obtained using GOX. The model curve underpredicts the data by as much as $30 \%$ for GHe, while better agreement is obtained when using GOX as a pressurant.

For a system designer, the data implies that using GHe to pressurize and subcool the LOX during expulsion results in higher margin in the total allowable pressure loss for the LAD system. Comparing results in $\mathrm{LOX}$ to results in $\mathrm{LH}_{2}$, greater deviation from theory is shown at the colder $\mathrm{LH}_{2}$ temperatures. High pressure results in LOX here confirm near linear correlation between bubble point pressure taken in saturated states and liquid surface tension across a very wide range of cryogenic liquid temperatures.

Comparing Figures 6.8a and 6.8b, the data and model appear to correlate better when using the liquid screen side temperature (SD1) instead of the bulk liquid temperature (SD4), especially for GOX pressurization. For a single component system (gaseous and liquid oxygen), the saturation temperature based on the pressure at the screen and SD1 should be nearly identical at bubble breakthrough. For a multiple component system (helium and oxygen), the greater the amount of liquid subcooling at the screen, the greater the deviation will be from the simple prediction curve. Examination of Figures 6.9a and $\mathrm{b}$ illustrate this trend for the $325 \times 2300 \mathrm{GOX}$ data. The 
majority of the SD1 values are nearly identical to their corresponding saturation temperatures whereas the SD4 data is scattered throughout the temperature range. At bubble breakthrough, when using GOX, the L/V interface at the LAD screen tends towards saturation, regardless of the conditions in the bulk liquid surrounding the screen.

\subsubsection{Liquid Subcooling and Pressurant Gas Dependence}

The scatter in Figures $6.7 \mathrm{~b}$ and $6.8 \mathrm{~b}$ from previous bubble point measurements made by Jurns and McQuillen (2008) could be explained by potential temperature differences between the bulk fluid and the screen; however, temperature sensors directly mounted on the screen were not incorporated for those tests. None of the historical measurements used screen side temperatures to deduce bubble point. In Figures 6.7 and 6.8 , the apparent scatter in the data is not a result of experimental uncertainty. Rather, it is due to the fact that bubble point pressure depends not just on liquid temperature, but also the level of subcooling, as shown in the $\mathrm{LH}_{2}$ tests in Chapter 5. Because of facility limitations, there is more variation in bubble points at colder liquid temperatures under a broad range of pressures and less variation at warmer temperatures that could only be achieved at higher pressures. In other words, the apparent converging of the data at warmer temperatures is due to inability to achieve highly subcooled states at warm temperatures. Figure 6.10 plots the bubble point as a function of SD1 and the pressure difference between screen and saturation pressure based on SD1 for the $325 \times 2300 \mathrm{GHe}$ data. As shown, bubble points achieved in near saturated liquid states lie close to the room temperature prediction line indicated by blue colored points. Meanwhile bubble points obtained at the highest levels of subcooling deviate the most from NBP predictions as indicated by the red colored points. 

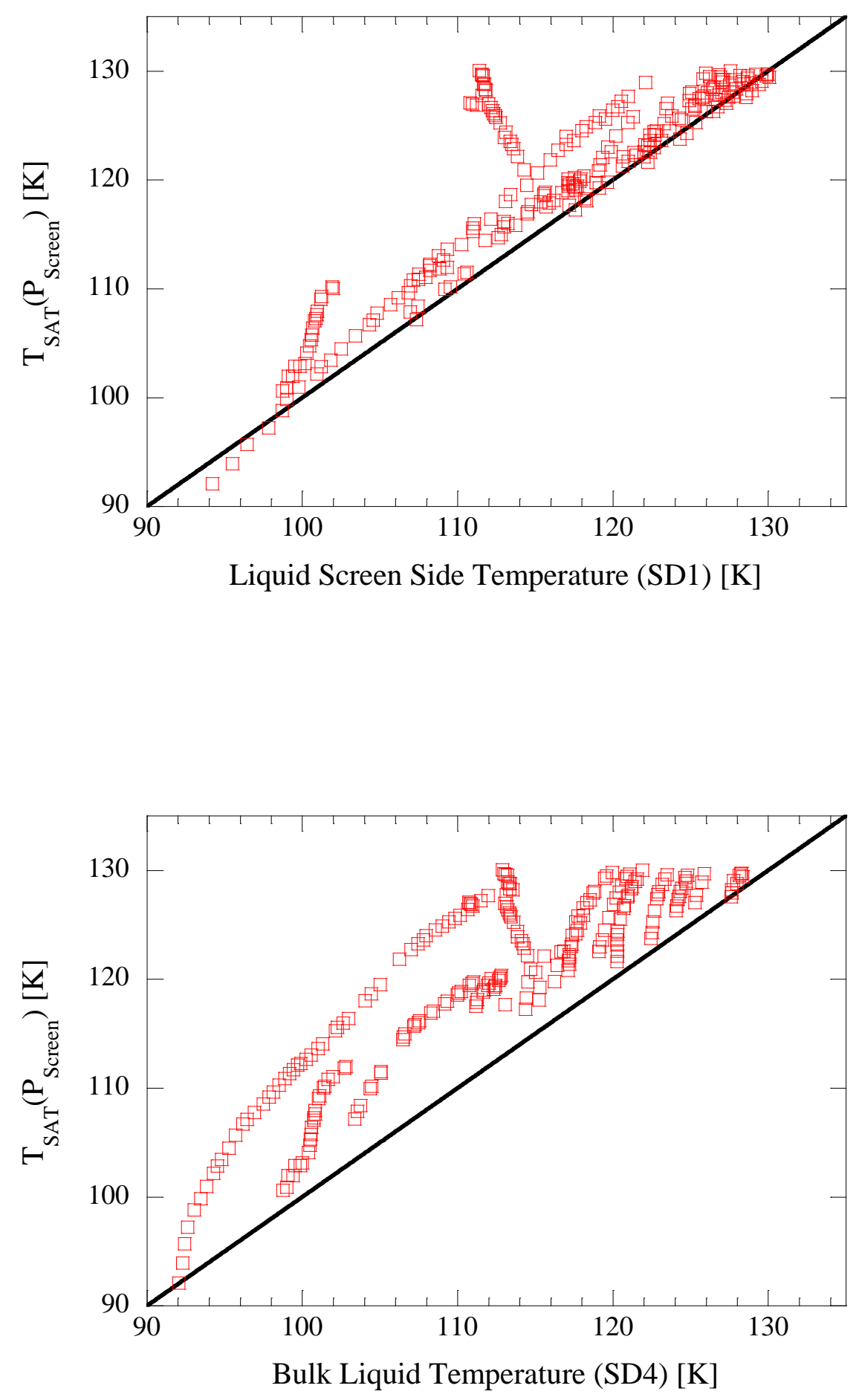

Figure 6.9 - Oxygen Saturation Temperature vs. a) Liquid Screen Side Temperature and b) Bulk Liquid Temperature at Bubble Breakthrough When Using Gaseous Oxygen for the 325x2300 Screen 


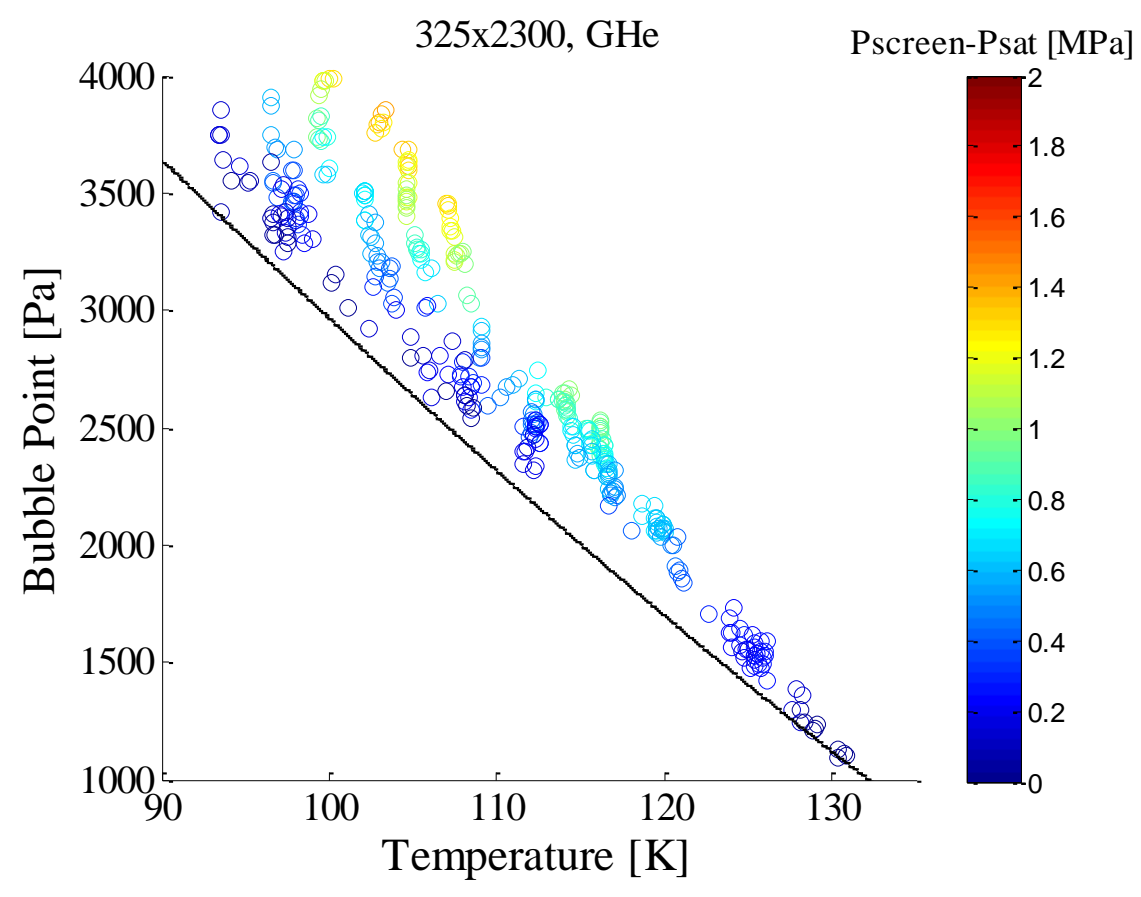

Figure 6.10 - Liquid Oxygen Temperature Correlation for Gaseous Helium at Bubble Breakthrough for the $325 \times 2300$ Screen. Color represents $\mathrm{P}_{\text {SCREEN }}-\mathrm{P}_{\mathrm{SAT}}$ in units of $[\mathrm{MPa}$.

This variation in bubble points at colder liquid temperatures is illustrated in Figure 6.11 where the bubble point data for the $325 \times 2300$ screen is plotted as a function of liquid side screen pressure. The prediction values are calculated from surface tension using the saturation temperature based on the pressure at the screen. Elevated bubble points are achievable using GHe for all pressures (depending on the liquid temperature) while bubble points obtained using GOX fall off with the normal boiling point prediction curve, regardless of tank pressure. For a multiple component system (helium and oxygen), the greater the amount of liquid subcooling at the screen, the greater the deviation will be from room temperature predictions. 


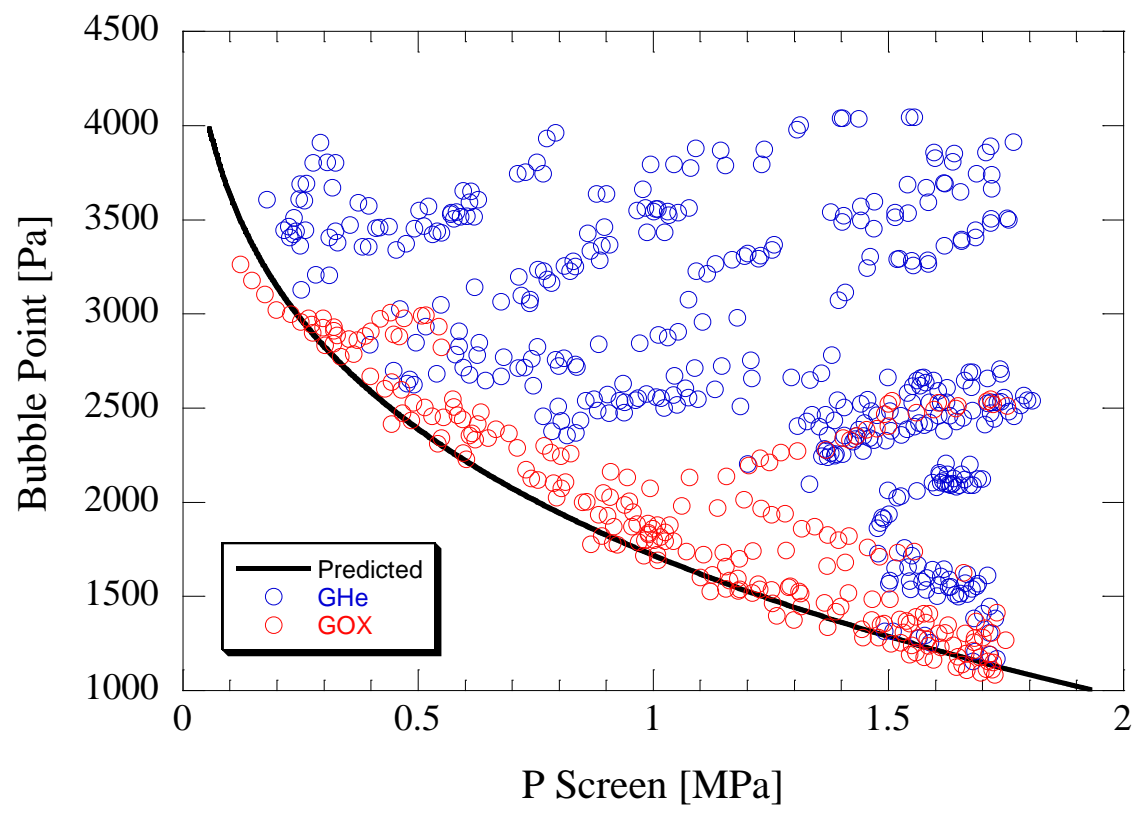

Figure 6.11 - Liquid Oxygen 325x2300 Bubble Point as a Function of Liquid Screen Side Pressure

Surface tension is generally known to be a function of temperature only (Ferguson and Kennedy 1936, Turkevich and Mann 1990a and b). Incorporating the temperature dependence of surface tension into Equation 3.16 indicates that the bubble point pressure has no pressure dependence. However, during transfer of liquid from the propellant tank through the LAD to the transfer line, the liquid temperature and pressure difference between engine and propellant tank govern the flow rate through the LAD and the amount of vaporization that may occur at the LAD screen.

To further investigate this variation in bubble point with pressure, Figures $6.12 \mathrm{a}-$ c superimpose the bubble point data as a function of the temperature (SD1) and pressure at the LAD screen on the saturation curve for the 200x1400 and $325 \times 2300$ screen using GHe, and 325x2300 using GOX, respectively. All data is plotted on the same color axes 
for comparison. These figures are essentially contour plots of Figures 6.5a and 6.6a where the color scale is used to represent the magnitude of the bubble point. As expected, higher bubble points are obtained at colder liquid temperatures for both pressurant gases. However, there is also a weak secondary effect of liquid subcooling on the bubble point. The highest bubble points are obtained in the coldest liquid at the highest level of subcooling. Although not shown, large discrepancies were again observed when using the temperature of the bulk liquid to correlate the data. The subcooling trends evident in the low pressure $\mathrm{LH}_{2}$ data are magnified here in the high pressure LOX data. For example, for the $325 \times 2300$ screen with GHe pressurant, pressurizing at the NBP from room pressure up to $1.75 \mathrm{MPa}$ yields almost $140 \%$ gain in margin in bubble point pressure. Comparing Figures $6.12 \mathrm{~b}$ to $6.12 \mathrm{c}$, for the same level of subcooling, there is more gain in bubble point using the non-condensable over the condensable gas to pressurize the screen. Therefore not only the high pressure but also the type of gas in contact with the screen affects performance.

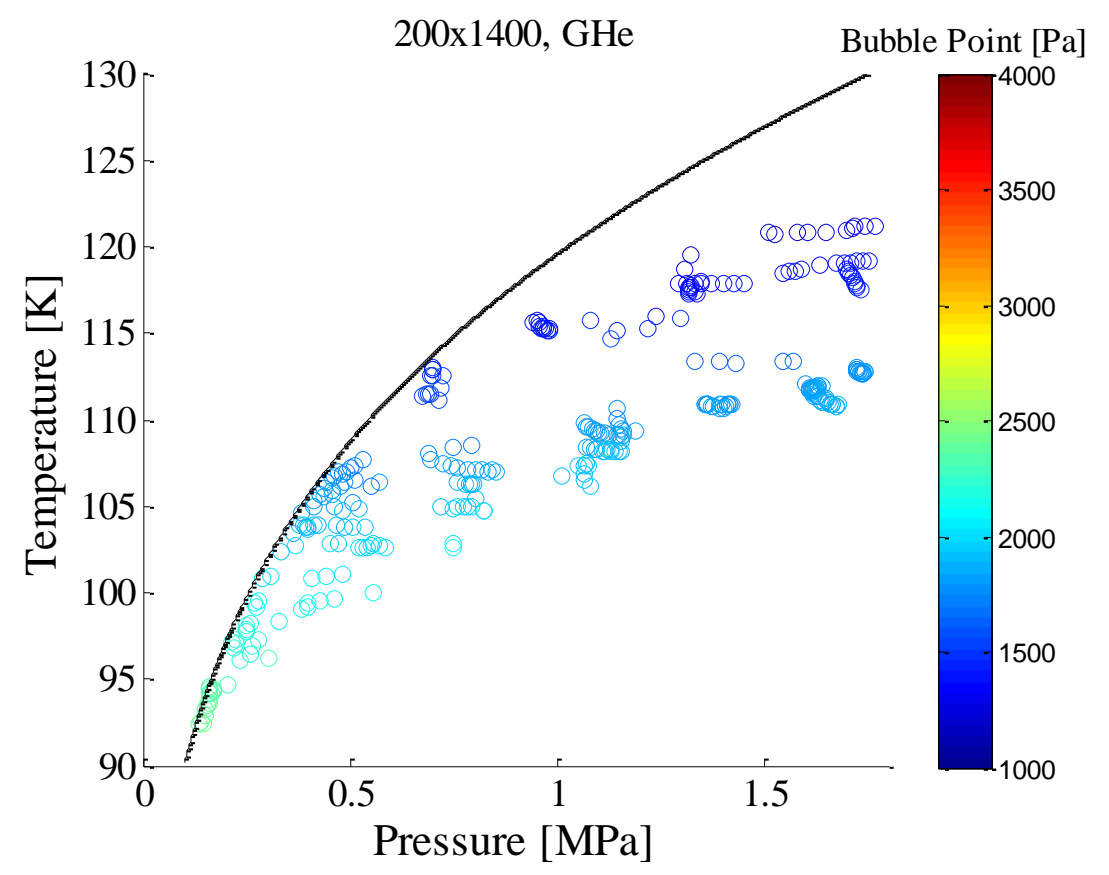



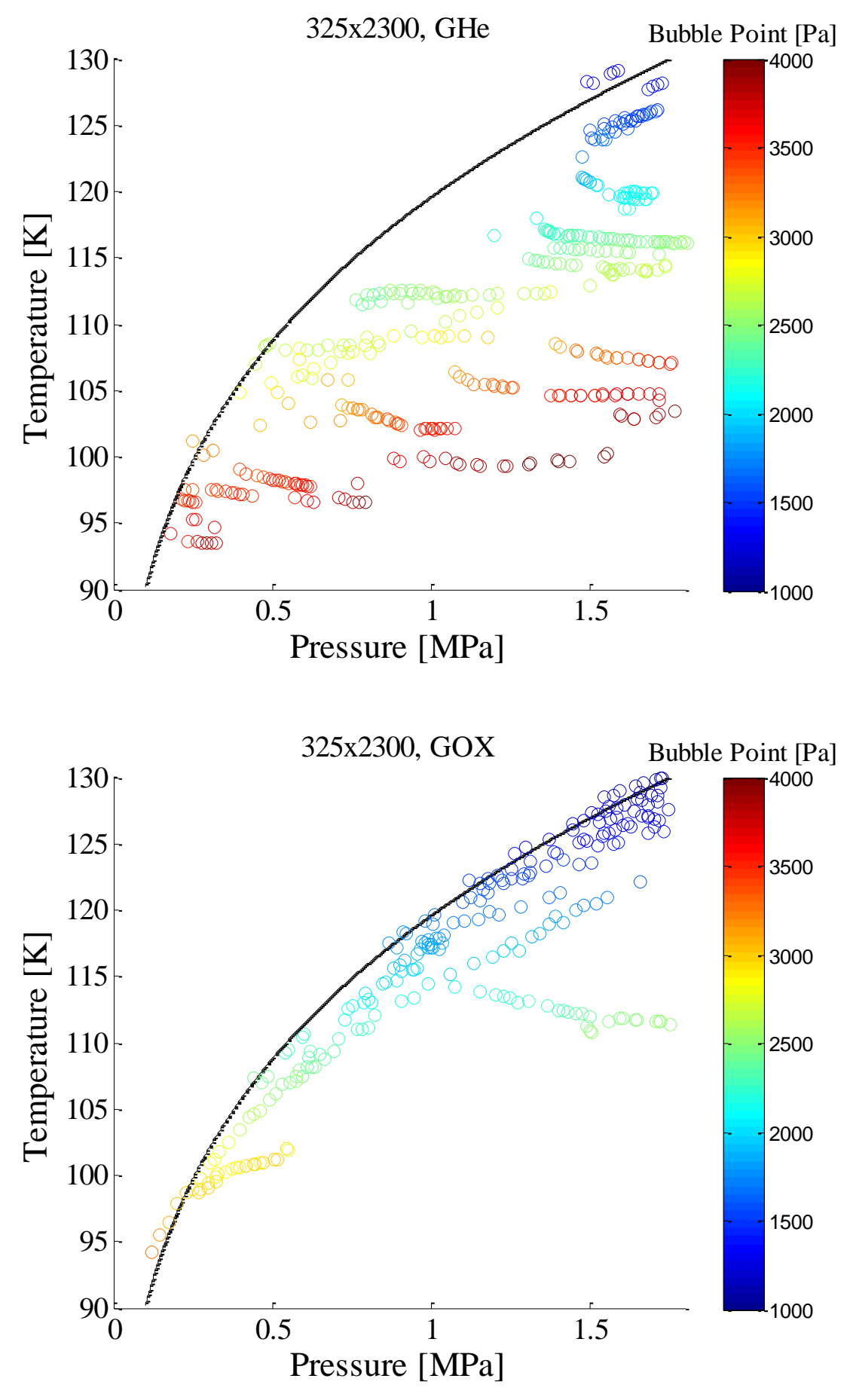

Figure 6.12 - Liquid Oxygen Bubble Point Pressure as a Function of the Thermodynamic State of the Liquid at the Screen for a) 200x1400 Using Gaseous Helium b) 325x2300 Using Gaseous Helium and c) 325x2300 Using Gaseous Oxygen. Color represents bubble point in units of [Pa]. 


\subsubsection{Heat Transfer Effects at Elevated Temperature}

Closer examination of the temperature measurements at bubble breakthrough revealed some unusual, but not totally unexpected results. Figures $6.13 \mathrm{a}$ and $\mathrm{b}$ plot the bubble point pressure for the $200 \times 1400$ screen as a function of the temperature difference across the screen (SD2 - SD1) and the temperature difference within the liquid from the liquid screen side to the bulk liquid measurement location (SD1 - SD4), respectively. Figures $6.14 \mathrm{a}$ and $\mathrm{b}$ plot these features for the $325 \times 2300$ screen. As shown in Figures 6.13a and 6.14a, the gas was always slightly warmer than the liquid across the screen at breakthrough for both screens. The pressurant gas had sufficient exposure to cryogenic temperatures within the piping and the LAD screen/cup assembly that the gas was nearly at cryogenic temperatures; however, the gas was still always warmer at the screen.

Figures 6.13a and 6.14a imply minimal heat transfer across the 200x1400 and 325x2300 screens during breakthrough with GHe, because the temperature difference is no more than $2 \mathrm{~K}$ across the entire range of liquid temperatures for the $325 \times 2300$ while temperature differences across the $200 \times 1400$ screen are as high as $4 \mathrm{~K}$.

In contrast, when GOX is the pressurant, the temperature differentials were in excess of $9 \mathrm{~K}$. Heat transfer across the screen is relatively constant for GHe across the bubble point range but reaches a maximum value in the intermediate bubble point range for GOX. This trend is not intuitive, as it was initially expected that heat transfer between gas and liquid would be a maximum at the coldest liquid temperatures and thus highest bubble points. 

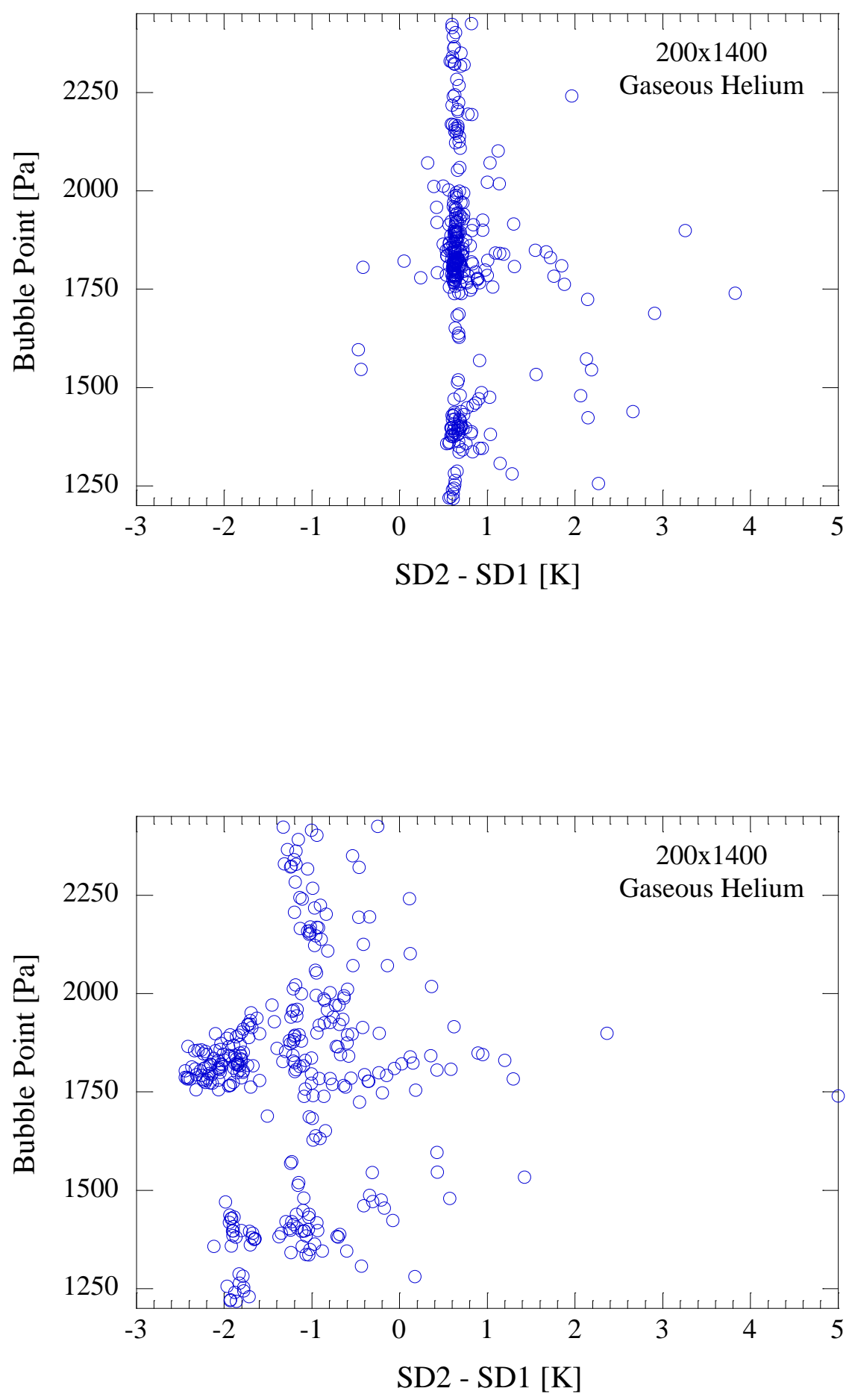

Figure 6.13 - Liquid Oxygen Bubble Point Pressure as a Function of the Temperature Difference a) Across the Screen and b) Between Liquid at Screen and Bulk Liquid in the Tank for the 200x1400 Screen 

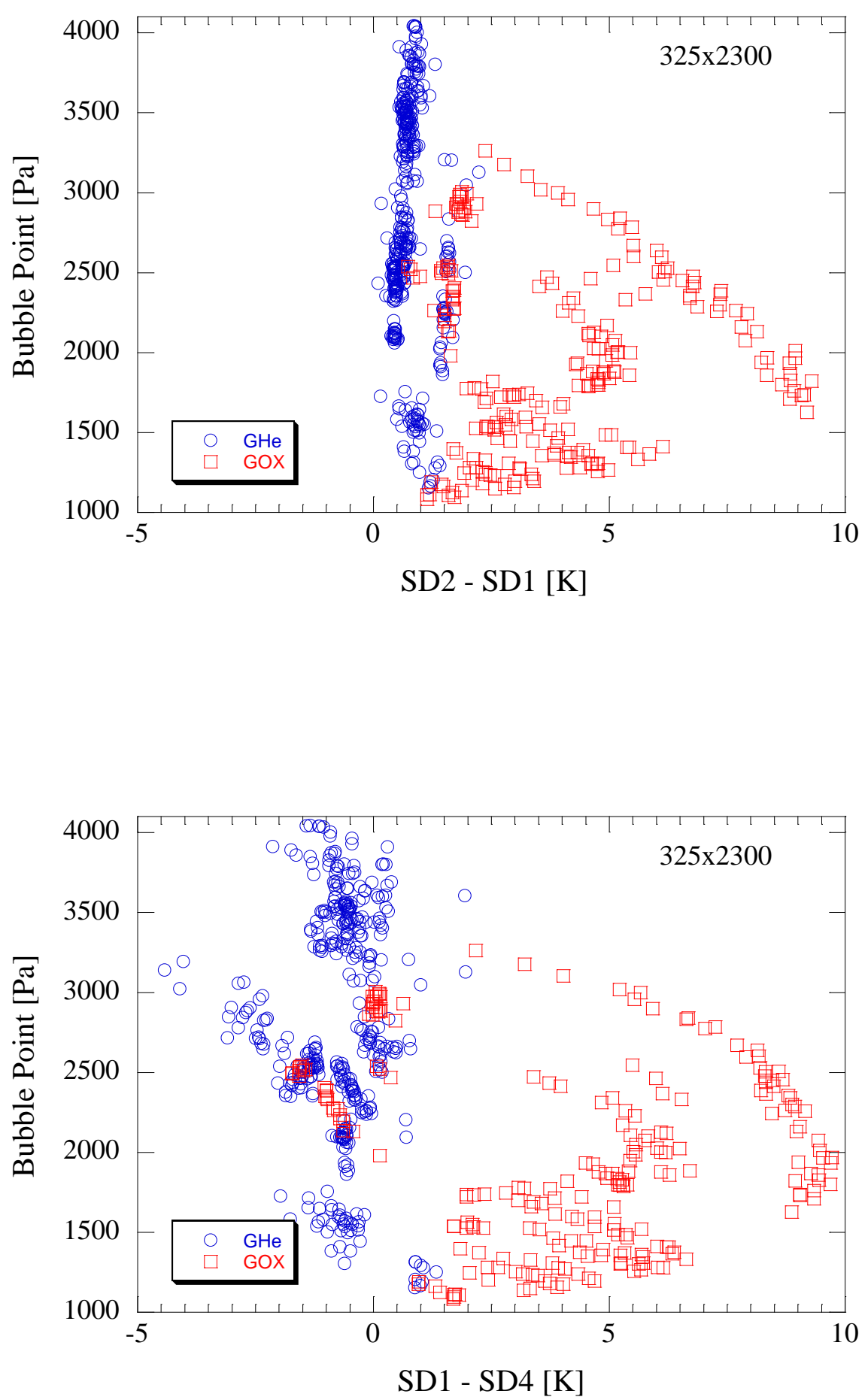

Figure 6.14 - Liquid Oxygen Bubble Point Pressure as a Function of the Temperature Difference a) Across the Screen and b) Between Liquid at Screen and Bulk Liquid in the Tank for the $325 \times 2300$ Screen 
It is evident that pressurization with the vapor causes a significant temperature difference across the screen over pressurizing with the non-condensable GHe. This is consistent with findings by Rahimi and Ward (2005) and Sefiane and Ward (2007) who found large temperature differences across a gas-liquid interface where there was mass transfer between phases. Note that pressurant gas ramp rates for both gases were nearly constant across the range of tests.

Figure $6.14 \mathrm{~b}$ indicates a minimal temperature difference within the liquid at the screen and the bulk liquid during breakthrough with GHe for the 325 screen. The bulk liquid is warmer than the top of the screen for the majority of test cases. For the coarser 200x 1400 mesh screen, there is significantly higher heat transfer, as the temperature difference between screen and bulk liquid reached a maximum of $2.5 \mathrm{~K}$ with a single outlier at $5 \mathrm{~K}$ in Figure 6.13b. Meanwhile, temperature differences in excess of $9 \mathrm{~K}$ are present when using GOX. The bulk liquid is shown to be significantly colder than the top of the screen.

This implies that pressurizing with GHe causes LOX to evaporate away from the screen, cooling the screen, while pressurizing with GOX causes condensation of the gaseous oxygen into the liquid, warming the screen. This explains why bubble point pressures in GHe are higher than in GOX. Pressurization with GHe causes the localized liquid temperature at the screen to decrease, increasing the surface tension of the LOX at the screen and the bubble point. Pressurization with GOX causes the liquid temperature at the screen to rise, decreasing the surface tension and the bubble point. 
To illustrate this trend, heat transfer and bubble point pressure data at breakthrough are combined in color plots in Figures $6.15-6.17$ where bubble point is plotted as a function of SD1 and the temperature gradient across the screen and between the liquid at the screen and bulk liquid using GHe for the 200x1400 screen, and using GHe and GOX for the $325 \times 2300$ screen, respectively. It was initially anticipated that larger temperature differences proportional to the level of subcooling would exist and that maximum heat transfer across the screen would occur at the coldest liquid temperatures at the highest pressure, and decrease as the liquid temperature approached the saturation temperature. However, Figures 6.15 and Figures 6.16 show relatively uniform temperature differences over the whole range of liquid states tested for $\mathrm{GHe}$ pressurization.

Differences in heat transfer between the two screens are attributed to differences in the actual meshes. The coarser 200x 1400 screen has a larger effective open area and a longer path length through the screen making it more likely to build temperature gradients across the screen prior to breakdown. For GOX pressurization, the colored plots in Figure 6.17 exhibit a peak in the temperature difference across the screen and between screen and bulk liquid that is centered on the predicted value. 

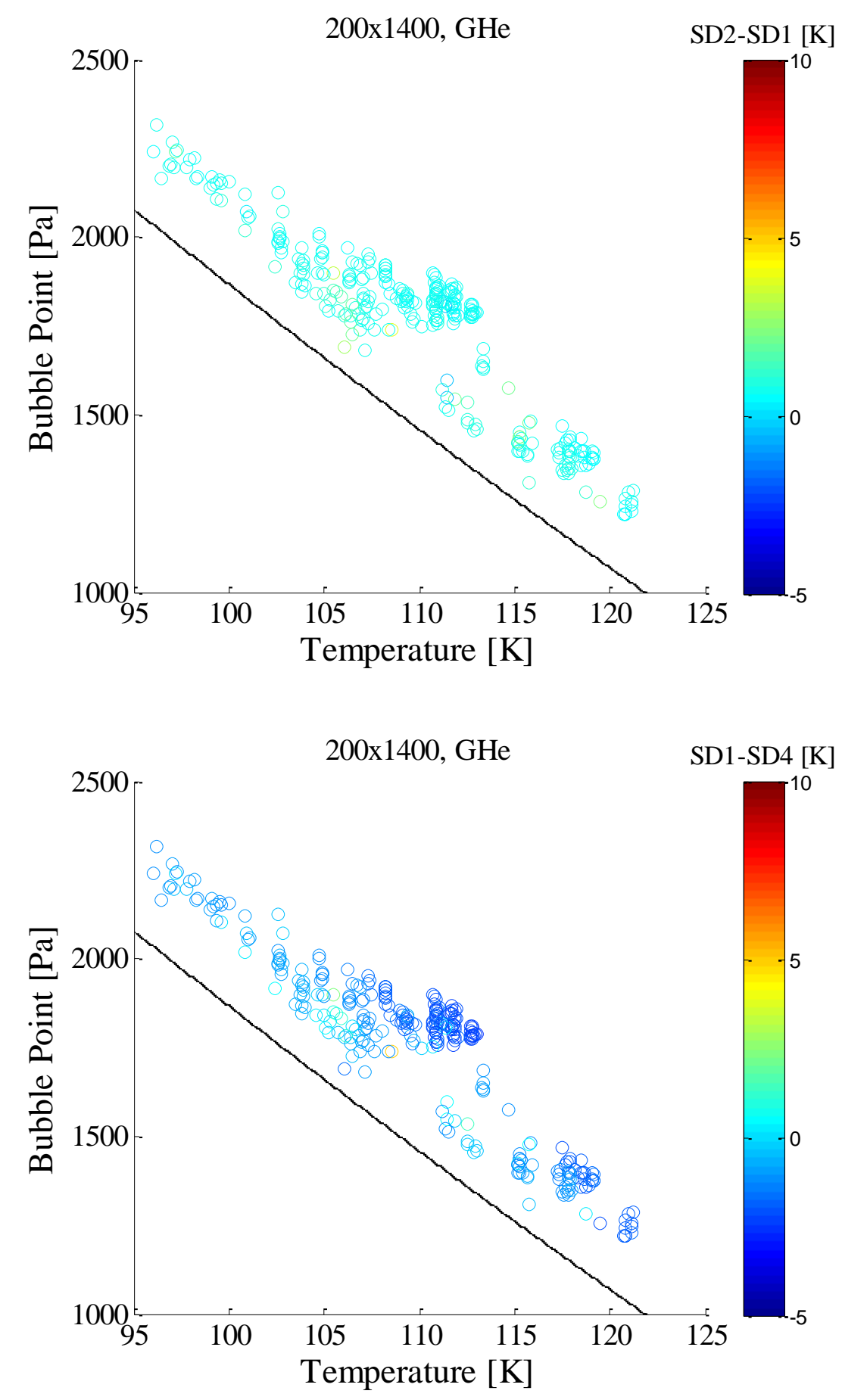

Figure 6.15 - Liquid Oxygen Bubble Point Pressure as a Function of Liquid Screen Side Temperature and a) Temperature Difference across the Screen and b) Temperature Difference between Liquid at Screen and Bulk Liquid in Tank at Breakdown for the 200x1400 Screen Using Gaseous Helium. Color represents temperature differences in units of [K]. 

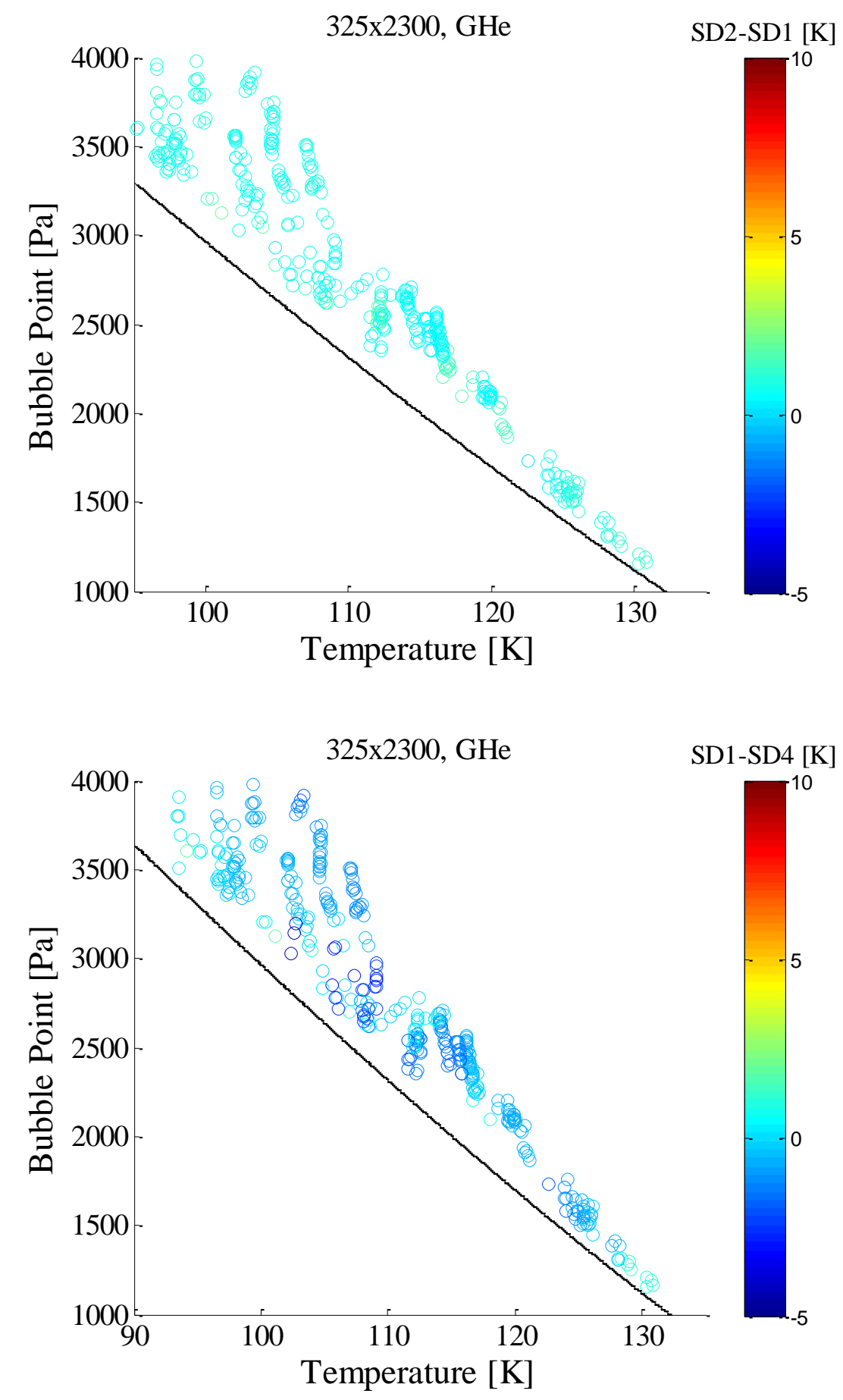

Figure 6.16 - Liquid Oxygen Bubble Point Pressure as a Function of Liquid Screen Side Temperature and a) Temperature Difference across the Screen and b) Temperature Difference between Liquid at Screen and Bulk Liquid in Tank at Breakdown for the 325x2300 Screen Using Gaseous Helium. Color represents temperature difference in units of [K]. 

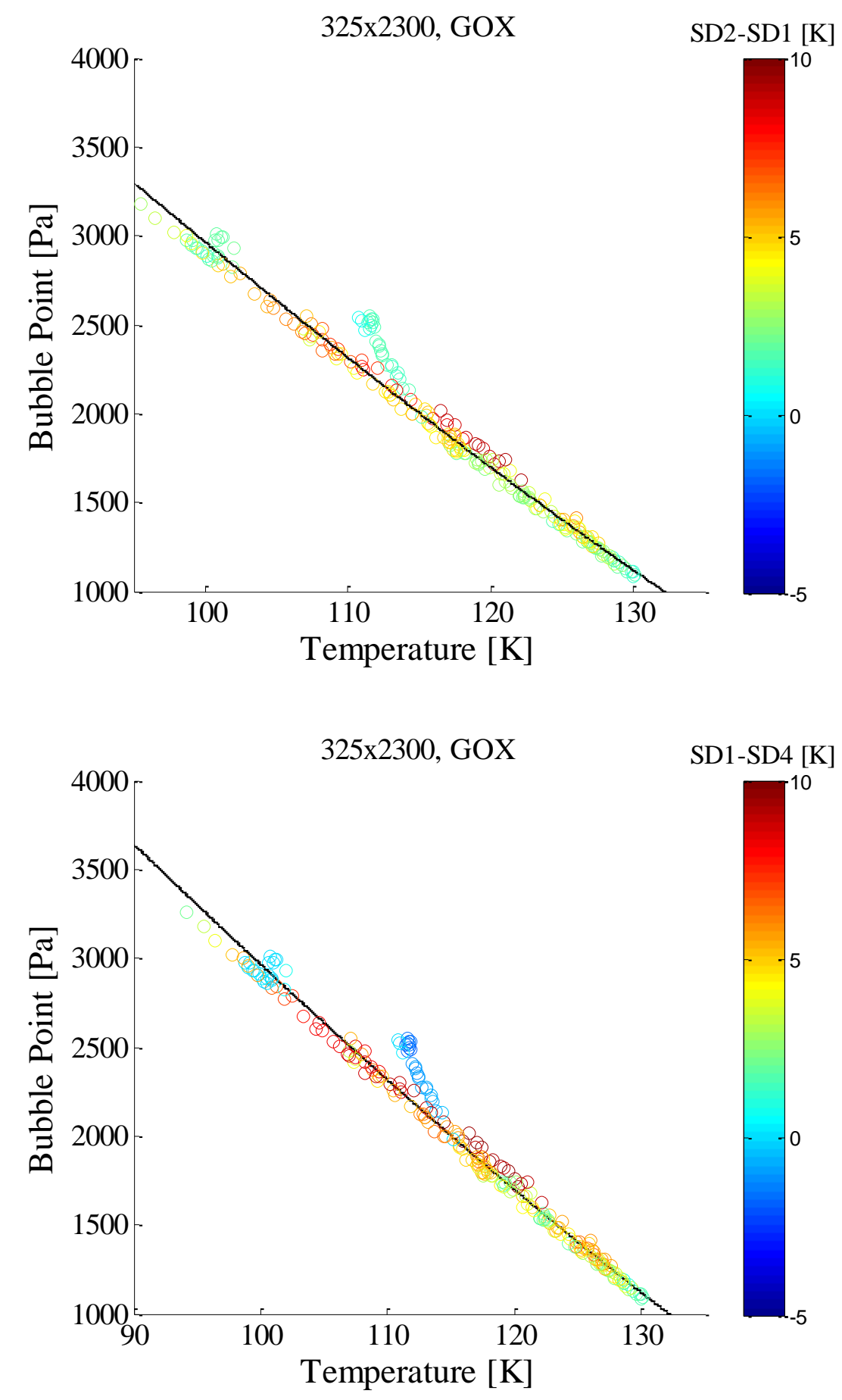

Figure 6.17 - Liquid Oxygen Bubble Point Pressure as a Function of Liquid Screen Side Temperature and a) Temperature Difference across the Screen and b) Temperature Difference between Liquid at Screen and Bulk Liquid in Tank at Breakdown for the 325x2300 Screen Using Gaseous Oxygen. Color represents temperature difference in units of [K]. 


\subsubsection{Analysis of Videos}

To attempt to find a correlation between bubble point and heat transfer across the screen for both gases, typical time traces of temperature (SD1-4) and differential pressure (DPT03) across the $325 \times 2300$ screen transients are analyzed from initial ramp in pressure until bubble breakthrough, as plotted in Figures 6.18a and b for SD1 - 4 and DPT03 using GHe and GOX, respectively. Snapshot images from the camera are superimposed upon their corresponding time. Image subtraction and enhancement was used to show differences between the screen at initial pressurization and the screen as the test evolved.

For Figure 6.18a, image subtraction equates to the visible bubbles. For Figure $6.18 \mathrm{~b}$, this analysis shows the initial thermal distortions at an intermediate time and the bubbles at the end of pressurization. For the GHe case (Figure 6.18a), the imagery at bubble breakthrough is apparent because there is obvious mass transfer across the screen that corresponds to the single spike in DPT03 pressure. For this particular test, the temperature difference among all four sensors was less than $2 \mathrm{~K}$.

However, for the bubble breakthrough conditions for GOX (Figure 6.18b), the visual imagery was less than certain. Careful examination of the distortion in this video indicates the possibility that mass transport between the phases and across the screen occurs before visible bubbles break through the screen. In addition, at high heat transfer rates across the screen, due to the rapid condensation of the GOX into the cold LOX, bubbles were not readily detectable after they had risen any appreciable distance from the screen. Thus Figure $6.18 \mathrm{~b}$ also shows that, during an intermediate time frame, from approximately 5 to 22 seconds, the differential pressure increase was gradual and that 
bubbles may have broken through the screen but were immediately condensed into the liquid. While the temperature difference between the gas/vapor phase temperature sensors (SD3 - SD2) was minimal, the temperature difference across the screen was about 4K. There was an additional $5 \mathrm{~K}$ difference between the bulk liquid temperature and the liquid temperature at the screen.

Therefore, it is evident that oxygen vapor that initially permeates the screen may initially condense and release latent heat into the surrounding liquid oxygen long before a visible bubble breaks through the screen. Eventually, the local liquid temperature at the screen warms, and bubbles break through. Thus, there is minimal heat transport across the screen at bubble breakthrough using GHe, but a considerable amount of both interfacial mass and heat transport across the screen when using GOX.

\subsection{Concluding Remarks}

Better agreement between data and theory is obtained when using the liquid temperature at the screen to report the bubble point for a screen channel LAD. This negates the previous assumption that the bubble point scales with the bulk liquid temperature inside the propellant tank. Bubble point predictions based on room temperature pore diameters nearly hold for saturated liquid states but fail to predict for subcooled liquid states. Across the full temperature range, bubble point is shown to be a strong function of liquid temperature that qualitatively scales with the surface tension of the liquid. 

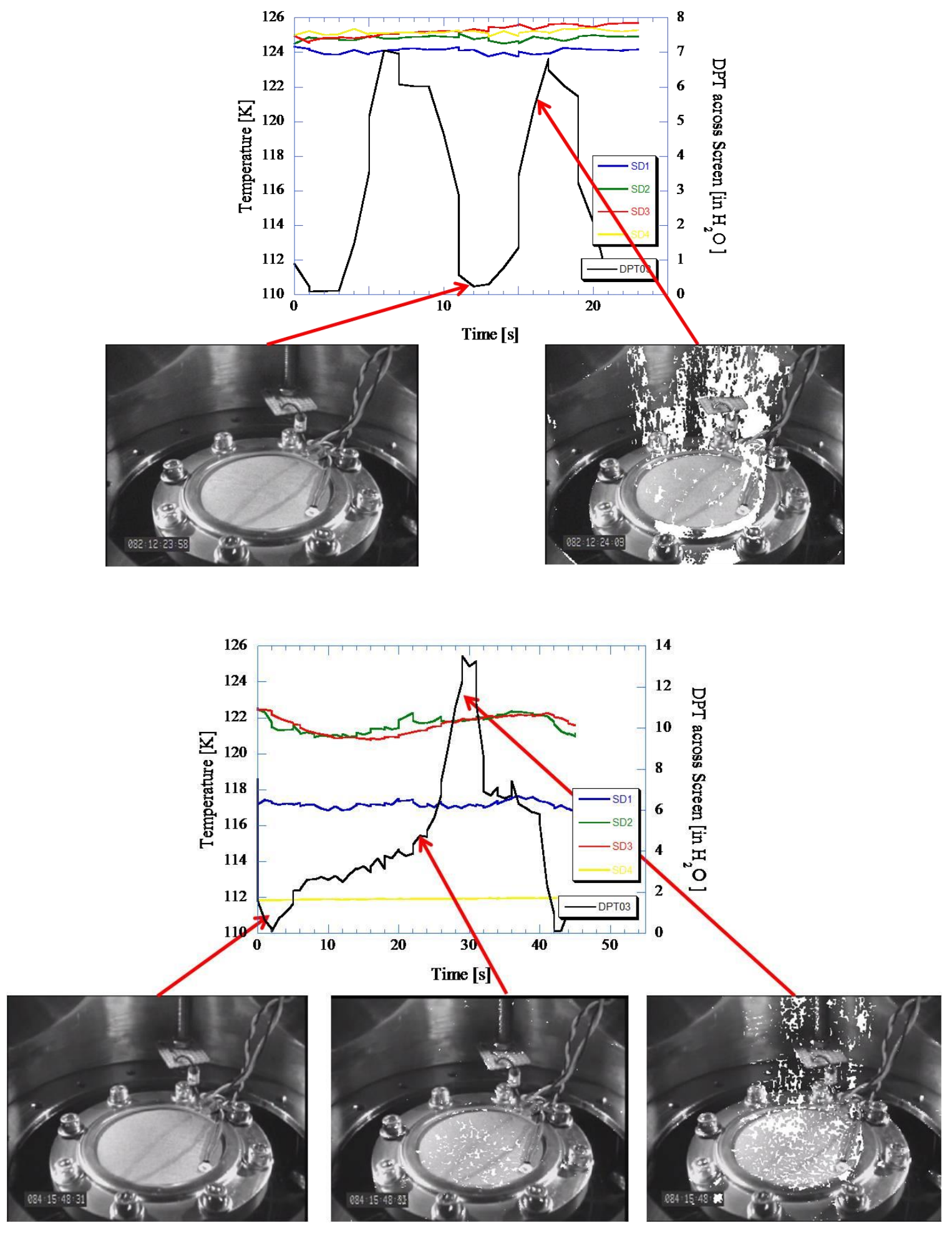

Figure 6.18 - Time Trace of Temperature Sensors and Differential Pressure Transducer with Corresponding Images of Screen using a) Gaseous Helium and b) Gaseous Oxygen as Pressurants 
The implication for mission design is that pressurizing with helium gas increases margin for the bubble point at a given operating temperature. Using GHe to pressurize also allows margin in liquid temperature in that one can operate at temperatures warmer than the design point and still retain a high bubble point if the liquid inside the propellant tank is gradually and sufficiently pressurized with helium. Pressurizing with oxygen gas has a weak effect on the margin in bubble point.

For all pressures, the bubble point is higher than the predicted value when using GHe to pressurize the system. Minimal heat transfer occurs across the screen. GHe causes LOX to evaporate away from the screen, cooling the interface temperature, increasing the surface tension and thus bubble point. Meanwhile, good agreement exists between theoretical predictions and experimentally obtained bubble points using GOX. During breakthrough, the temperature at the screen tends towards the saturation temperature based on the pressure at the screen regardless of the bulk liquid temperature. Considerable heat transfer across the screen occurs prior to and during breakthrough, causing warm GOX bubbles to condense into the liquid, warming the screen, and decreasing the local surface tension and bubble point pressure. As is evident from image subtraction techniques, initial heat transfer across the screen associated with condensation of vapor during the initial pressure ramp is followed by the eventual breakthrough of a visible bubble. Therefore the supposed pressure dependence may simply be an enhancement of the temperature dependence as the temperature at the screen is modified through added heating or cooling of the local liquid oxygen and screen through variable condensation or evaporation during breakthrough. Comparing low pressure $\mathrm{LH}_{2}$ results to 
high pressure LOX results, the gain in margin due to subcooling the liquid is enhanced by as much as $140 \%$ at elevated pressures up to $1.79 \mathrm{MPa}$. 


\section{Chapter 7}

\section{High Pressure Liquid Methane Bubble Point Experiments}

The purpose of this chapter is to present the high pressure and temperature liquid methane static bubble point experiments. The purpose of the tests was to augment the high pressure liquid oxygen tests and to further investigate the parameters which affect LAD performance in a high pressure cryogenic propellant tank. The same $325 \times 2300$ Dutch Twill screen is tested in liquid methane across a wider range of thermal and operational conditions $(106 \mathrm{~K}<\mathrm{T}<160 \mathrm{~K}, 0.0618<\mathrm{P}<1.78 \mathrm{MPa})$ using three different pressurant gases. The warmer saturation temperature of methane allows examination of the effect of two different non-condensable pressurant gases (nitrogen and helium) in addition to the condensable methane vapor, on bubble point pressure. Details are presented on facility modifications and updates to instrumentation. Thermal analysis is conducted to quantify heat and mass transfer effects associated with the high pressure cryogenic environment. Additionally, LAD performance is compared and contrasted for the three pressurant gas types by computing interfacial temperatures, screen Reynolds numbers, condensation and evaporation rates, and heat conduction into the liquid prior to and during LAD breakdown. 


\subsection{Test Purpose and Motivation}

The motivation for conducting high pressure methane bubble point tests are generally the same as those cited for the high pressure LOX experiments in Section 6.1. Research and technology development for high pressure-fed engines requires ground tests on both component level as well as full scale systems relevant to $\mathrm{LOX} / \mathrm{LCH}_{4}$ propulsion systems. A survey of most of the work completed under the CFM and Propulsion and Cryogenics Advanced Development (PCAD) programs is available in the literature (Smith et al. 2010).

Preliminary system concepts envision that both the main propulsion system (MPS) and RCS will both be fed from the same cryogenic propellant tank for the Lunar Ascent Stage. Therefore, single phase propellant management and propellant delivery become critical design and safety factors to ensure mission success. In order to give mission designers confidence in their propulsion system design, it will be necessary to demonstrate single phase propellant delivery under all anticipated thermal conditions. Again, rather than extrapolate sparse low pressure data to elevated pressure and temperature conditions, NASA chose to fund tests at the conditions representative of the high pressure environment. Testing on the RCS and MPS subsystems were recently concluded at Johnson Space Center (JSC) and WSTF facilities (Collins et al. 2010 and Stiegemeier et al. 2010). Because NASA was also interested in densifying the $\mathrm{LCH}_{4}$ by cooling it below its NBP (Tomsik et al. 2010), it was also determined that measurements also needed to be conducted below ambient pressure. 
While there are reported bubble point values for $\mathrm{LH}_{2}, \mathrm{LN}_{2}$, and $\mathrm{LOX}$ data from several independent sources, the only known previously reported bubble points in $\mathrm{LCH}_{4}$ are reported in Jurns et al. (2007). As shown in Figure 1.1, methane has the highest surface tension and thus the highest bubble point relative to other cryogenic liquids. With an order of magnitude higher bubble point over the traditional cryogenic propellant fuel $\mathrm{LH}_{2}$, it may be possible to support much higher flow rates to a methane fueled engine using these screen channel LADs. Tests using LOX to characterize the performance of the screen channel LAD were reported in Chapter 6; thus it is the primary purpose of this chapter to completely characterize the same style PMD in $\mathrm{LCH}_{4}$ over a similar wide range of fluid conditions, as well as augment the investigation on the effect of liquid subcooling and pressurant gas type on LAD performance in an elevated pressure environment.

\subsection{Experimental Design}

Testing was again conducted in the CCL-7 facility at NASA GRC. The same 325x2300 Dutch Twill screen sample from LOX tests was tested here in $\mathrm{LCH}_{4}$. The same screen and cup assembly, high pressure CFM test tank, dewar, imaging system, and DAQ from LOX tests was used here. Several modifications were made to the existing hardware and instrumentation to facilitate more accurate results over a wider range of experimental conditions.

\subsubsection{Modifications to Facility, Test Article, and Instrumentation}

The CFM test tank used in the high pressure $\mathrm{LCH}_{4}$ bubble point tests is shown in Figure 7.1. First, manual pressure control and regulation valves were replaced with 
remotely actuated valves to provide a safe distance between CCL-7 and the control room. Remote operation was required for this test. Second, amendments to the flow system were made to remotely flow three different pressurant gases underneath the screen: GHe, $\mathrm{GN}_{2}$, and gaseous methane $\left(\mathrm{GCH}_{4}\right)$. Third, an additional ejector was installed to subcool the liquid in the CFM test tank down below the NBP to an $\mathrm{LCH}_{4}$ saturation temperature at $62 \mathrm{kPa}$ (9 psia). Fourth, the existing RD lid with seven feedthroughs was replaced with a lid with ten feedthroughs for the RD vacuum, liquid fill and drain (single line), vent, the subcooling ejector, pressurant gas inlet, three optical ports (camera and light source $\mathrm{x} 2$ ), and silicon diode wires (x2), as shown in Figure 7.2. The CFM test tank was also equipped with several ports on top and below for liquid fill and drain, pressurization, back pressure control, instrumentation, and relief, also shown in Figure 7.2. Fifth, the existing pressurant gas flow control was replaced with a higher accuracy, dual low flow control valve system to allow slower ramp in pressure into the LAD cup.

$\mathrm{LN}_{2}$ was used for all pre-test check outs and cold shocking of the hardware. Liquid methane was transported to CCL-7 in portable $0.45 \mathrm{~m}^{3}(450 \mathrm{~L})$ dewars that were connected to the flow system through a flexible VJ line. To pressurize beneath the LAD screen, GHe was supplied from a portable tuber trailer while $\mathrm{GCH}_{4}$ and $\mathrm{GN}_{2}$ were available via a manifold of 12 high pressure K-bottles. A special remote operated pressure regulator controlled incoming pressure of the gas between $0-1.723 \mathrm{MPa}(0-$ 250 psia). Flow rate was controlled by a set of two low flow control valves located directly upstream of the CFM test tank. 


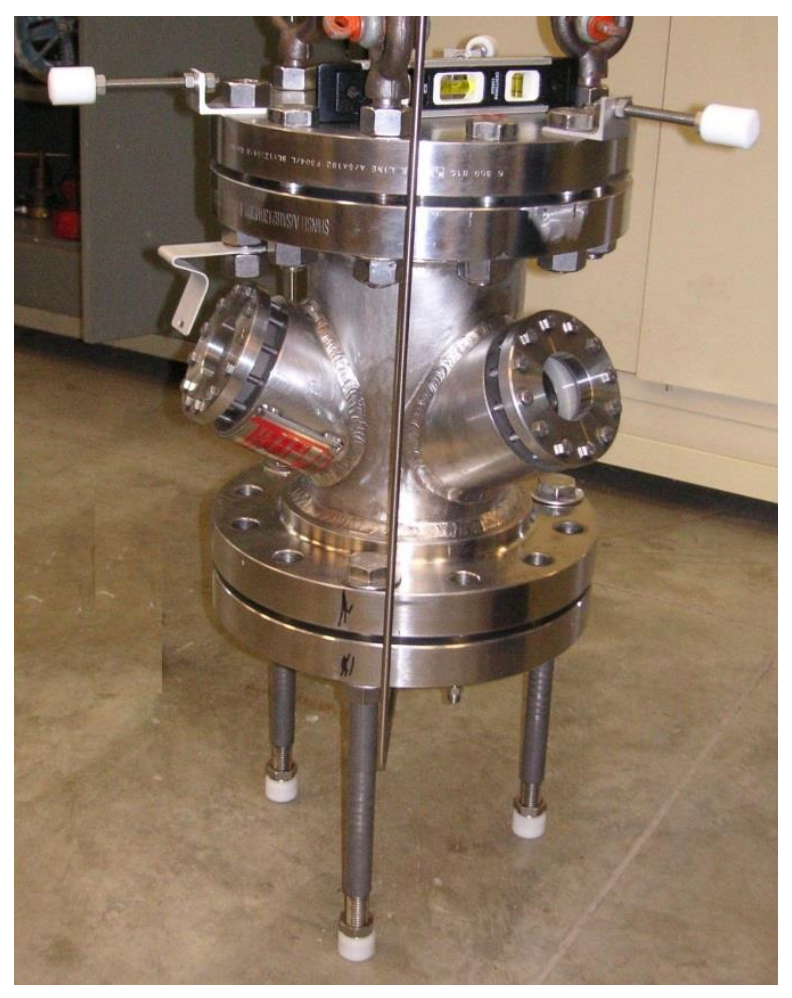

Figure 7.1 -Screen/Cup Assembly for High Pressure Methane Experiments

Liquid pressure inside the CFM test tank was controlled either using a back pressure control valve or the mini-ejector. The ejectors were used to obtain pressures below atmospheric. Temperature of the liquid inside the CFM test tank was initially determined by the liquid temperature inside the portable $\mathrm{LCH}_{4}$ dewar. Cold liquid (< $110 \mathrm{~K})$ was achieved by venting the dewar down to atmospheric pressure while warmer liquid (> 150K) was achieved by allowing the dewar to warm over a period of days. During testing, small adjustments in liquid temperatures were made by using the mini ejector to chill the liquid while warmer liquid states were achieved by injecting warm slugs of residual $\mathrm{GCH}_{4}$ that were trapped in the liquid fill line, or by allowing the liquid to absorb parasitic heat leak. 


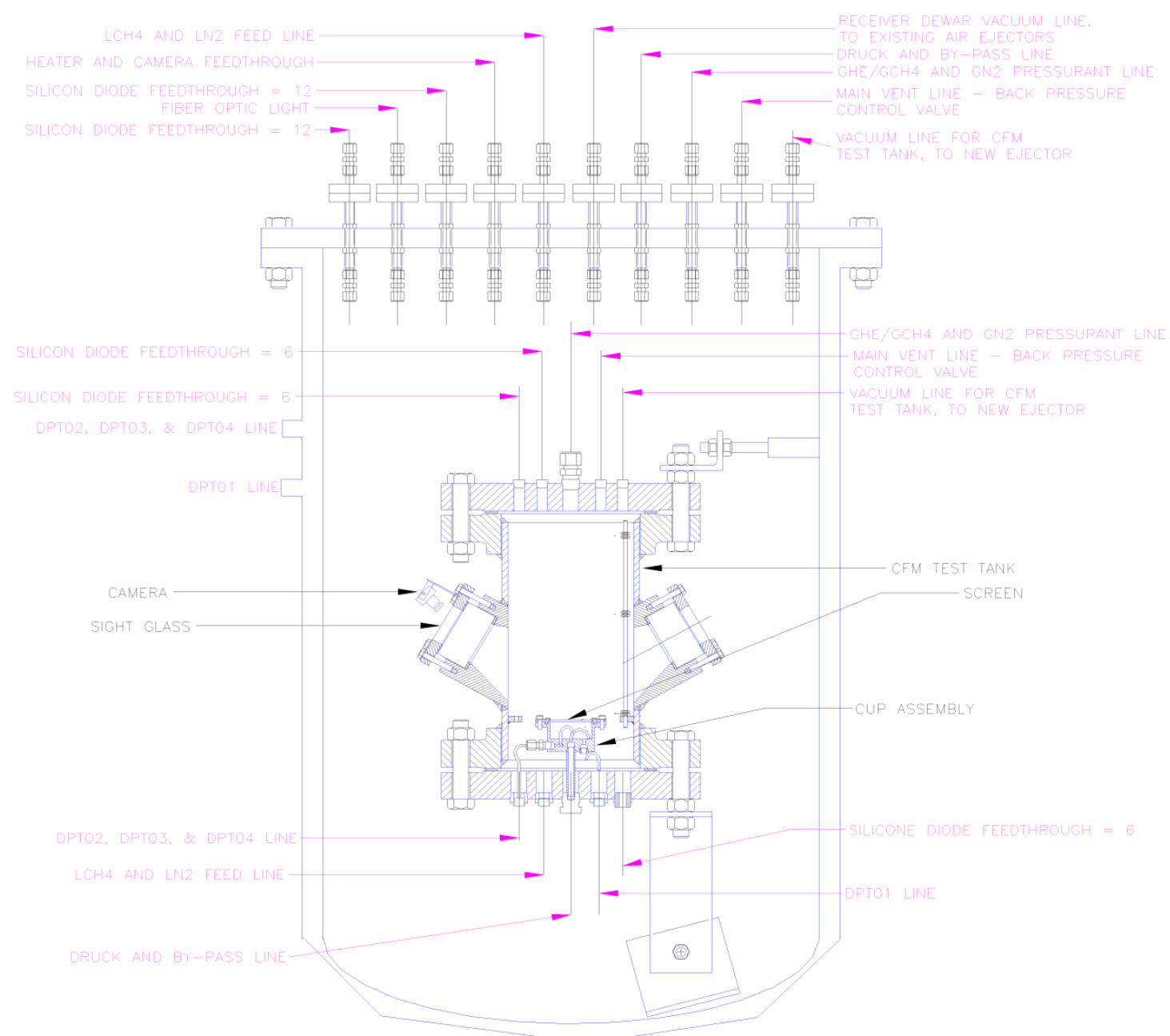

Figure 7.2 - Updated Screen and Cup Assembly, High Pressure Test Tank, and Dewar

Temperature instrumentation is outlined in Figure 7.3, where each number corresponds to the location of a specific diode. SD1 - 3 were the same from LOX tests. The sixth design modification involved adding the maximum number of diodes allowable with two electrical feedthroughs on the RD lid. SD4 - 17 were located along a vertical rake spaced $1.27 \mathrm{~cm}(0.5 \mathrm{in})$ apart, and these diodes measured both temperature and height of the bulk liquid as shown in Figure 7.4. Doing so reduced uncertainty in the bubble point values by a factor of nearly 2 over previous measurements in LOX. A single 
diode (SD18) was also mounted in the center of the CFM test tank to sense bulk liquid changes precluding screen breakdown. SD19 was mounted on the back side of the camera and SD20 was mounted horizontal to SD1 and SD4 to measure a radial heat flux during tests. Diodes (SD21 - SD24) were mounted inside the DPT lines to indicate if liquid was entering the DPT lines as testing evolved. Thermocouples were used to determine the temperature of the liquid (either $\mathrm{LCH}_{4}$ or $\mathrm{LN}_{2}$ ) during filling.

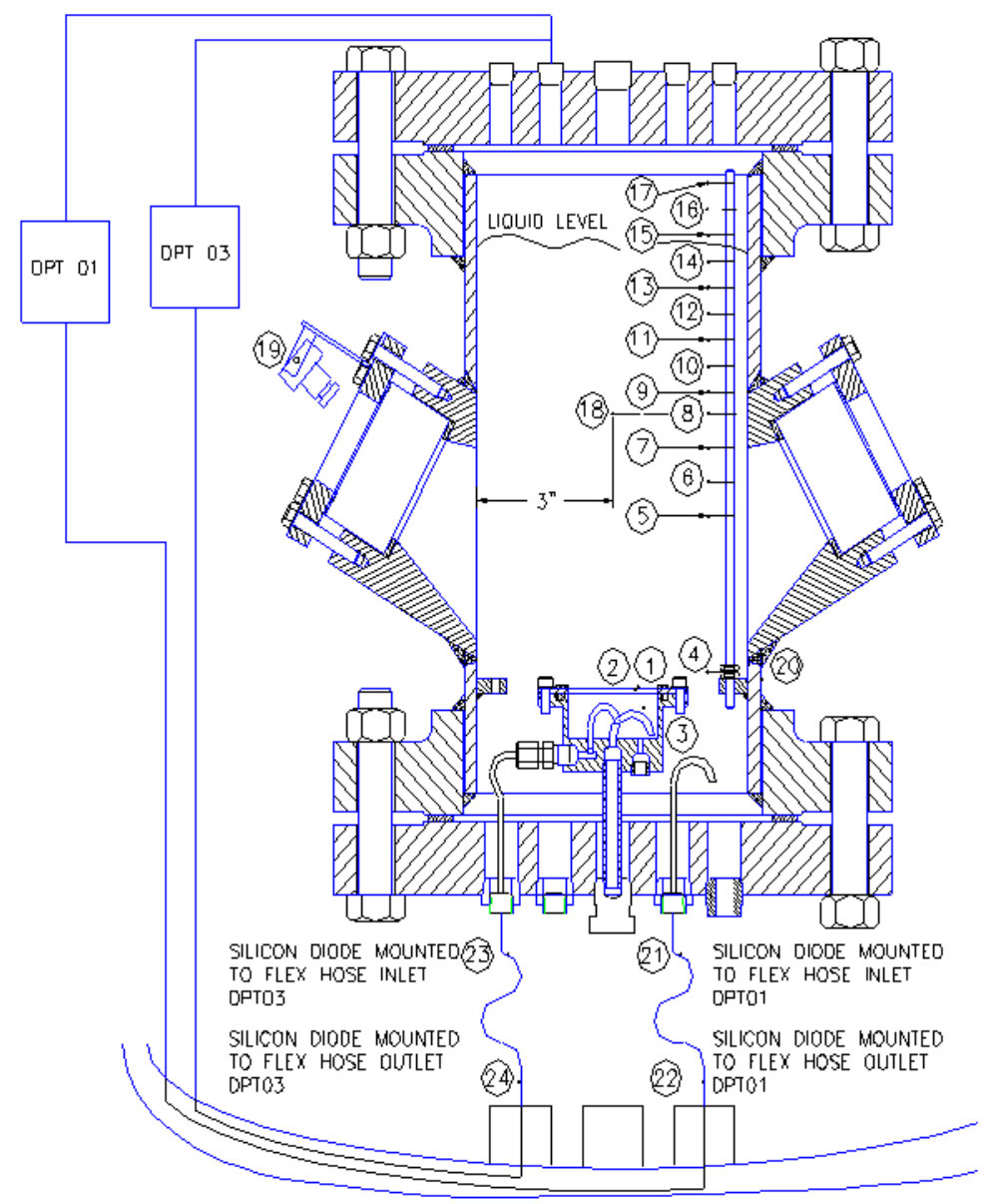

Figure 7.3 - Location of Temperature Measurements inside Cryogenic Fluid Management Test Tank 


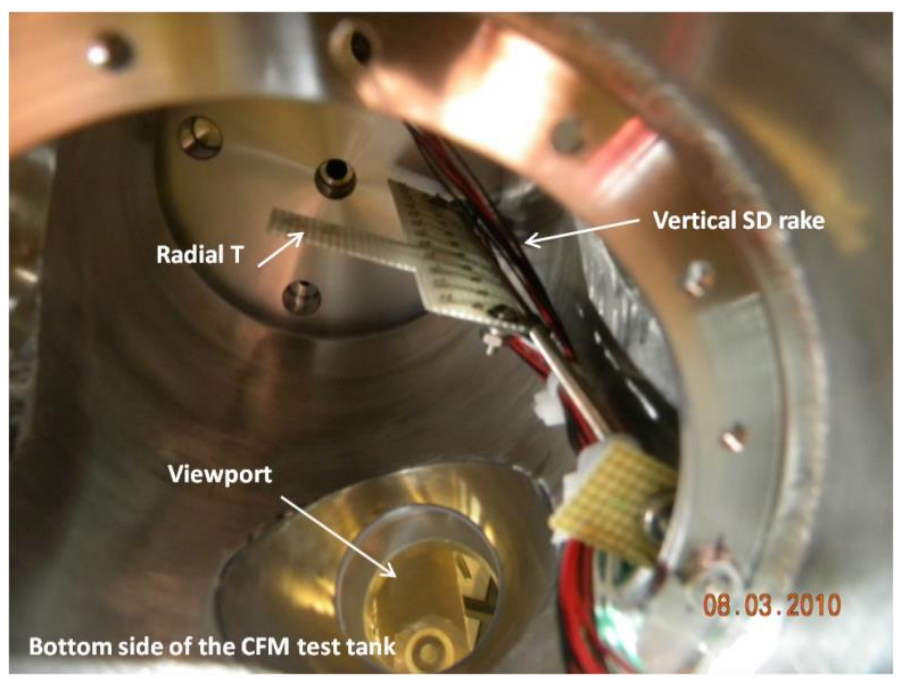

Figure 7.4 - Temperature and Liquid Level Measurement of the Bulk Liquid in the Cryogenic Fluid Management Test Tank as Viewed from the Bottom of the Tank

The pressure in the ullage space of the CFM test tank was measured using a 3.45 MPa (500 psia) pressure transducer, and three DPTs were used to deduce the differential pressure across the LAD screen sample and liquid head pressure above the screen. DPT02 and DPT03 had a range of $0-7.5 \mathrm{kPa}\left(0-30\right.$ in $\left.\mathrm{H}_{2} \mathrm{O}\right)$ and provided redundant measurement of the pressure inside the LAD screen/cup assembly with respect to the ullage pressure, while DPT01 had a range of 0-34.5 $\mathrm{kPa}$ (5 psid) and measured the pressure of the bottom of CFM test tank with respect to the ullage. The seventh and final design modification was the installation of high and low range flow meters (FMs) to allow comparison of mass flow rates into the cup prior to and during screen breakdown; LOX tests only employed a single FM. All data was recorded at $4 \mathrm{~Hz}$. Video images of the LAD screen were taken in a similar manner as in previous LOX tests. The methodology for conducting an $\mathrm{LCH}_{4}$ bubble point test was the same as the LOX tests. 
All silicon diodes measured temperature to within +/- $0.5 \mathrm{~K}$. The ullage pressure and thus the pressure at the screen was within $+/-4.6 \mathrm{kPa}(0.667 \mathrm{psia})$ of the measured value. It is worth noting that there was less than $0.05 \mathrm{kPa}\left(0.2\right.$ in $\left.\mathrm{H}_{2} \mathrm{O}\right)$ difference between DPT01 and DPT02 in all measured values. Liquid level measurements were good to within $6.35 \mathrm{~mm}(0.25 \mathrm{in})$ using the SDs. The flow meter read volumetric flows to within +/- 1 SLPM. The DPT across the LAD screen sample measured pressure to within +/$0.0104 \mathrm{kPa}\left(0.042\right.$ in $\left.\mathrm{H}_{2} \mathrm{O}\right)$, which was less than $1 \%$ at the lowest reported bubble point. However, interpolating between recorded values in time stamps, and due to uncertainty in liquid level (and thus liquid head pressure), the total uncertainty in reported bubble point values is estimated to be $+/-0.034 \mathrm{kPa}\left(0.138\right.$ in $\left.\mathrm{H}_{2} \mathrm{O}\right)$, which was no larger than $3.1 \%$ at the warmest liquid temperature.

\subsubsection{Test Matrix}

Figure 7.5 provides a summary of recent analysis and experiments conducted at NASA GRC and WSTF for $\mathrm{LCH}_{4}$ work. In addition to engine testing, propellant tank conditions from the Methane Lunar Surface Thermal Control (MLSTC) boil off and pressure rise tests are plotted in Figure 7.5 (Plachta et al. 2012 and Johnson et al. 2012) along with previous low pressure methane bubble points (Jurns et al. 2007). The black line is the saturation curve for methane.

The blue shaded region represents available thermodynamic conditions at the LAD screen over which the current bubble point tests were conducted. The same goal was to collect bubble breakthrough values over as wide a range as possible to give future mission designers direct bubble point data to characterize the LAD subsystem at any 
elevated methane temperature or pressure within the propellant tank. The low temperature limit on data collection for $\mathrm{LCH}_{4}$ tests was extended below the LOX limit due to the mini-ejector system while the high temperature limit was the same.

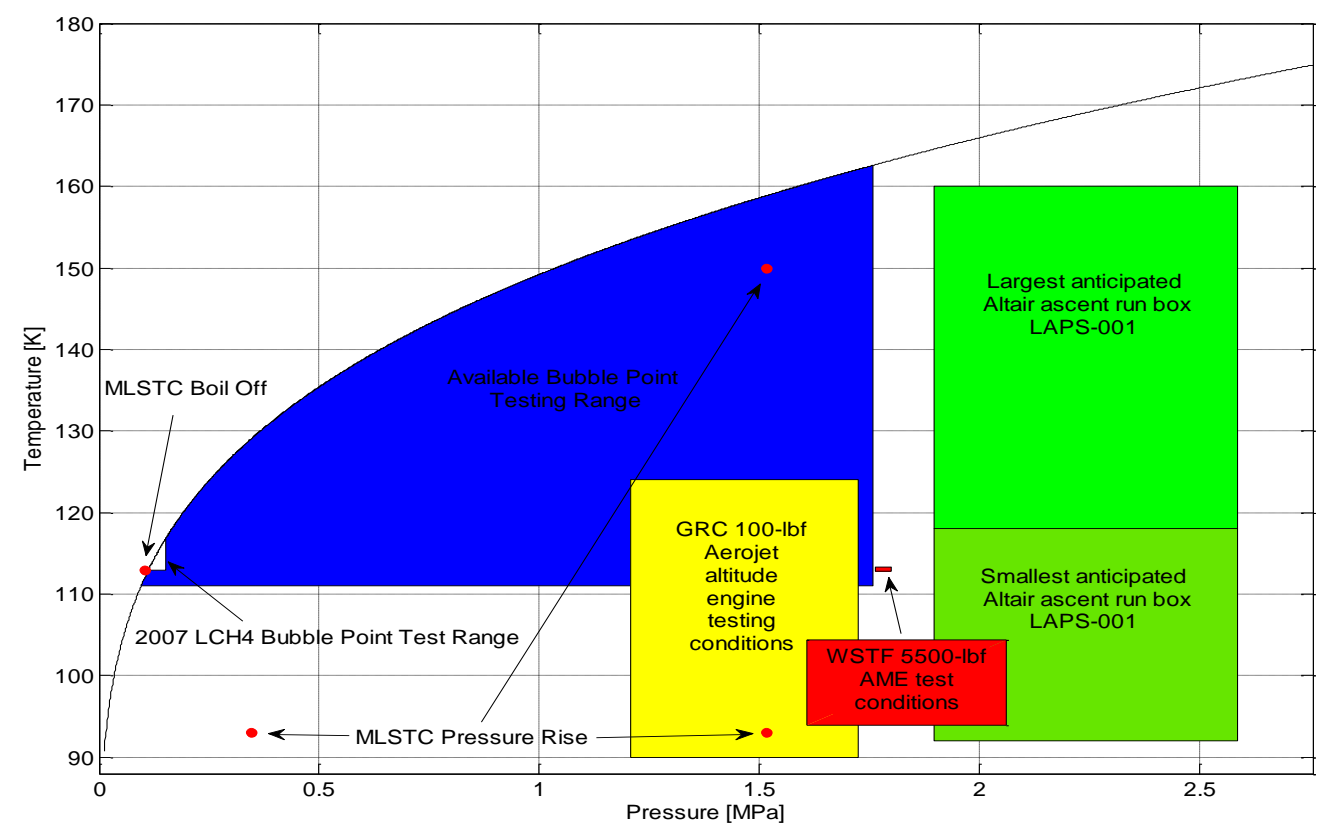

Figure 7.5 - Summary of the Primary Liquid Methane Analytical and Experimental Propellant Tank Test Conditions under NASA’s Liquid Oxygen/Liquid Methane Technology Development Program

\subsection{Experimental Results and Discussion}

\subsubsection{Test Conditions}

From December 2010 to January 2011, over the course of only a few weeks, numerous bubble point tests were conducted over the range of conditions in the blue shaded region in Figure 7.5. Figure 7.6 plots the thermodynamic state of the liquid at the LAD screen during bubble breakthrough in terms of the pressure and temperature of the 
bulk liquid. As shown, bubble breakthrough across the LAD screen was achieved across the entire range of conditions for the proposed high pressure $\mathrm{LCH}_{4}$ propellant tank for GHe. Data was also collected over a wide range of conditions for the other two pressurant gases.

\subsubsection{Elevated Temperature Dependence}

Figures $7.7 \mathrm{a}$ and $\mathrm{b}$ plot the experimental versus predicted bubble point pressure as a function of the liquid screen side temperature and bulk liquid temperature. The predicted value of the bubble point is calculated using the surface tension based on SD1 using the room temperature pore diameter for the $325 \times 2300$ screen. Data from Jurns et al. (2007) is also plotted in Figure 7.7b. The error bar near 104K in Fig. 7.7a is barely distinguishable. As expected, the bubble point pressure decreases with increasing liquid temperature, due to decreasing surface tension of the liquid. The trend hold true across the wide $60 \mathrm{~K}$ range of temperature over which data was collected. The prediction qualitatively tracks the experimental data but under predicts the $\mathrm{SD} 1 \mathrm{GHe}$ and $\mathrm{GN}_{2}$ data by as much as $37 \%$ and $25 \%$, respectively, especially at colder liquid temperatures. The prediction matches the SD1 $\mathrm{GCH}_{4}$ data well. Comparing Figures 7.7a and b, the data and prediction again correlate better when using the liquid screen side temperature over the bulk liquid temperature, especially for $\mathrm{GCH}_{4}$ pressurization, agreeing with $\mathrm{LOX}$ test results. Therefore, the bubble point correlates best with surface tension based on the liquid screen side temperature based on the partial pressure of $\mathrm{LCH}_{4}$ at the screen. Again, the apparent convergence of the data at high pressure is an anomaly of the fact that only a small range of thermodynamic states of the liquid are achievable at warm liquid temperatures, consistent with facility limits on pressure. 

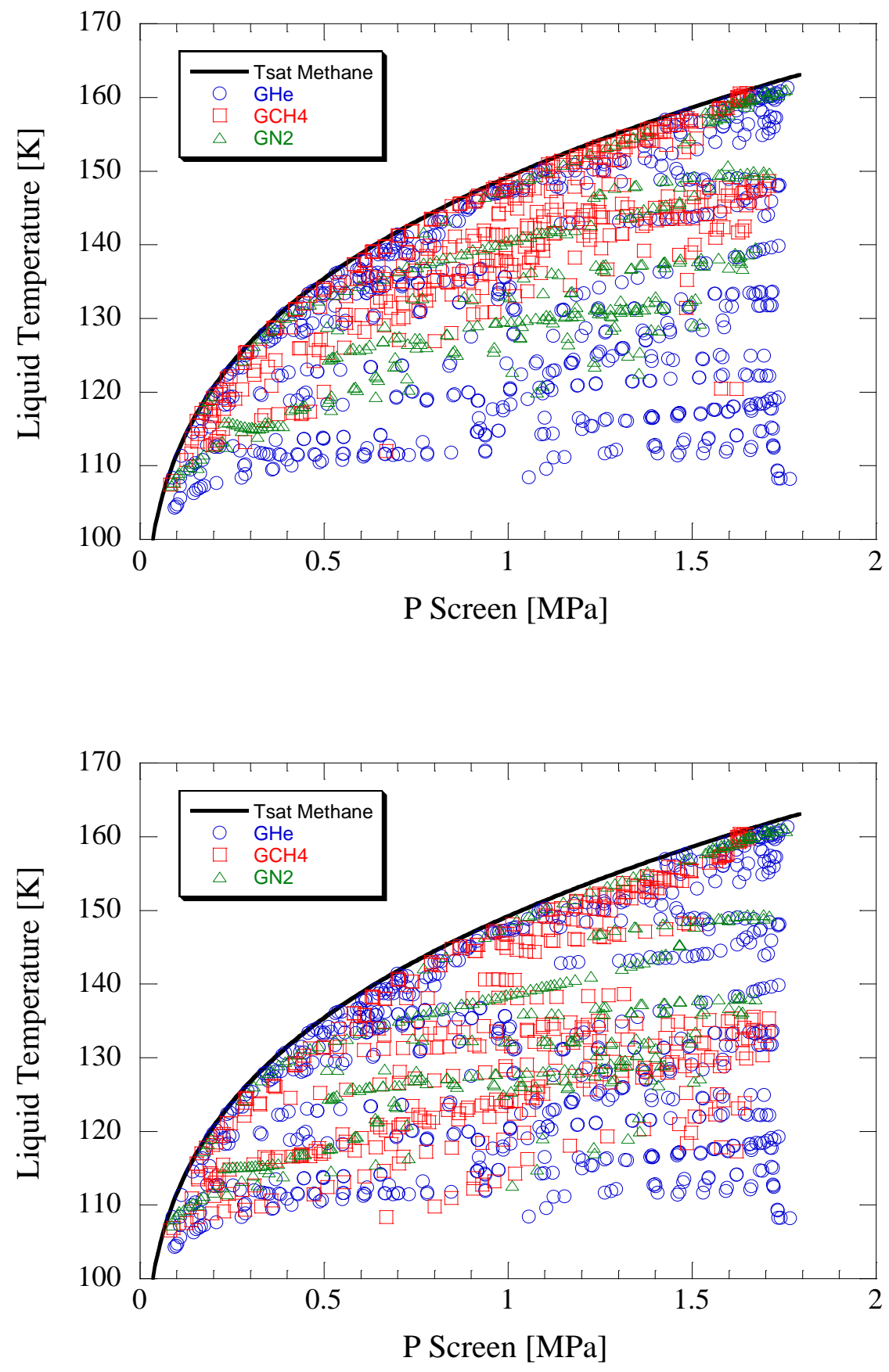

Figure 7.6 - Liquid Methane Thermodynamic Conditions at Bubble Breakthrough as a Function of the Liquid Screen Side Pressure and a) Liquid Screen Side Temperature (SD1) and b) Bulk Liquid Temperature (SD4) 

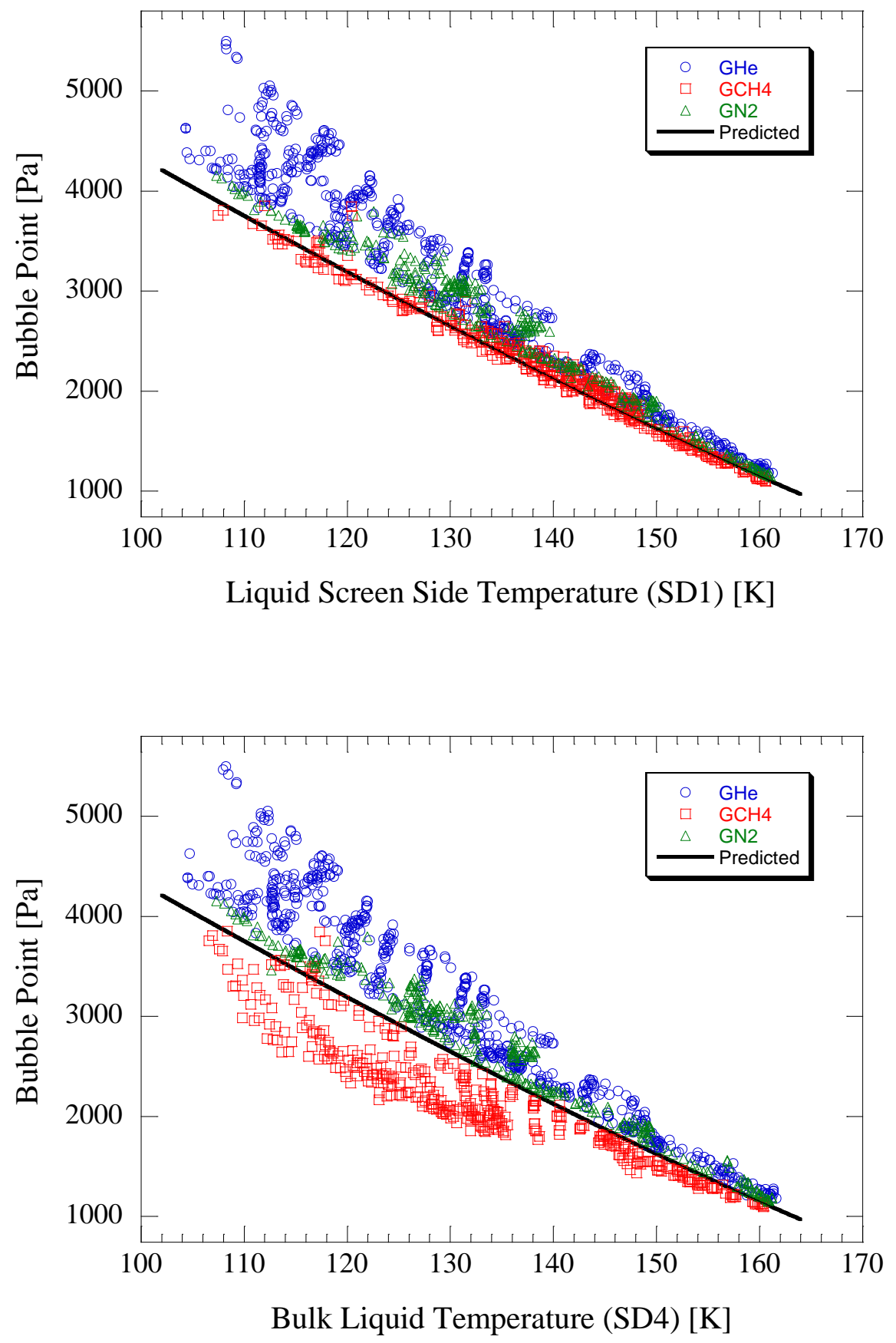

Figure 7.7 - 325x2300 Liquid Methane Bubble Point Pressure as a Function of a) Temperature at the Liquid Side of the Screen and b) Bulk Liquid Temperature

The implication is that, for a single component propellant tank (gaseous and liquid methane), SD1 and the saturation temperature based on the pressure at the screen 
are nearly identical at breakthrough. Meanwhile for a multiple component system using either $\mathrm{GHe}$ or $\mathrm{GN}_{2}$, deviation from the NBP prediction curve should be proportional to the level of subcooling. Comparing $\mathrm{LH}_{2}, \mathrm{LOX}$, and $\mathrm{LCH}_{4}$ data, deviation between room temperature prediction and data worsens as the saturation temperature decreases.

\subsubsection{Liquid Subcooling and Pressurant Gas Dependence}

The temperature trends are shown in Figures 7.8 and 7.9a and $\mathrm{b}$ for methane, helium, and nitrogen gas, respectively. As shown in Fig. 7.8, most of the SD1 values are identical to the corresponding saturation temperatures. The remaining data lies above the curve and implies relatively small subcooling at the screen was possible using autogenous pressurization. Meanwhile in Figure 7.9a and b, the trend is obvious; bubble point increases with distance away from the prediction as the pressure of the liquid is increased. Comparing Figures $7.7 \mathrm{a}$ to $7.7 \mathrm{~b}$ and $7.9 \mathrm{a}$ to $7.9 \mathrm{~b}$, it is also clear that a higher bubble point is achievable using $\mathrm{GHe}$ over $\mathrm{GN}_{2}$ and $\mathrm{GCH}_{4}$, with $\mathrm{LCH}_{4}$ bubble points in excess of $5.5 \mathrm{kPa}$ for GHe pressurant. Nonetheless, higher bubble points are achieved using $\mathrm{GN}_{2}$ over the vapor pressurant.

Despite the under prediction of both $\mathrm{GHe}$ and $\mathrm{GN}_{2}$ data, the bubble point equation may again be used as a lower bound to predict screen channel LAD performance for a flight system, since the actual breakdown pressure is higher for all $\mathrm{LCH}_{4}$ temperatures tested here. Based on these results, using $\mathrm{GHe}$ or $\mathrm{GN}_{2}$ to pressurize and subcool $\mathrm{LCH}_{4}$ during expulsion increases the margin for the total allowable pressure loss for the LAD. In a reduced gravity environment, where the leading order hydrostatic pressure loss is negligible and the flow through screen pressure loss dominates in Equation 3.3, higher 
bubble point pressures translate into higher propellant flows to the engine. Using empirical correlations for the flow through screen pressure drop from Chapter 3, $\mathrm{GHe} / \mathrm{LCH}_{4}$ bubble point pressures imply that screen channel LADs are capable of supplying an AME or RCS with flows in excess of $1.82 \mathrm{~kg} / \mathrm{s}(4.0 \mathrm{lbm} / \mathrm{s})$ for methane fuel and $5 \mathrm{~kg} / \mathrm{s}(11 \mathrm{lbm} / \mathrm{s})$ for oxidizer, which exceeds mission requirements (Dickens 2010).

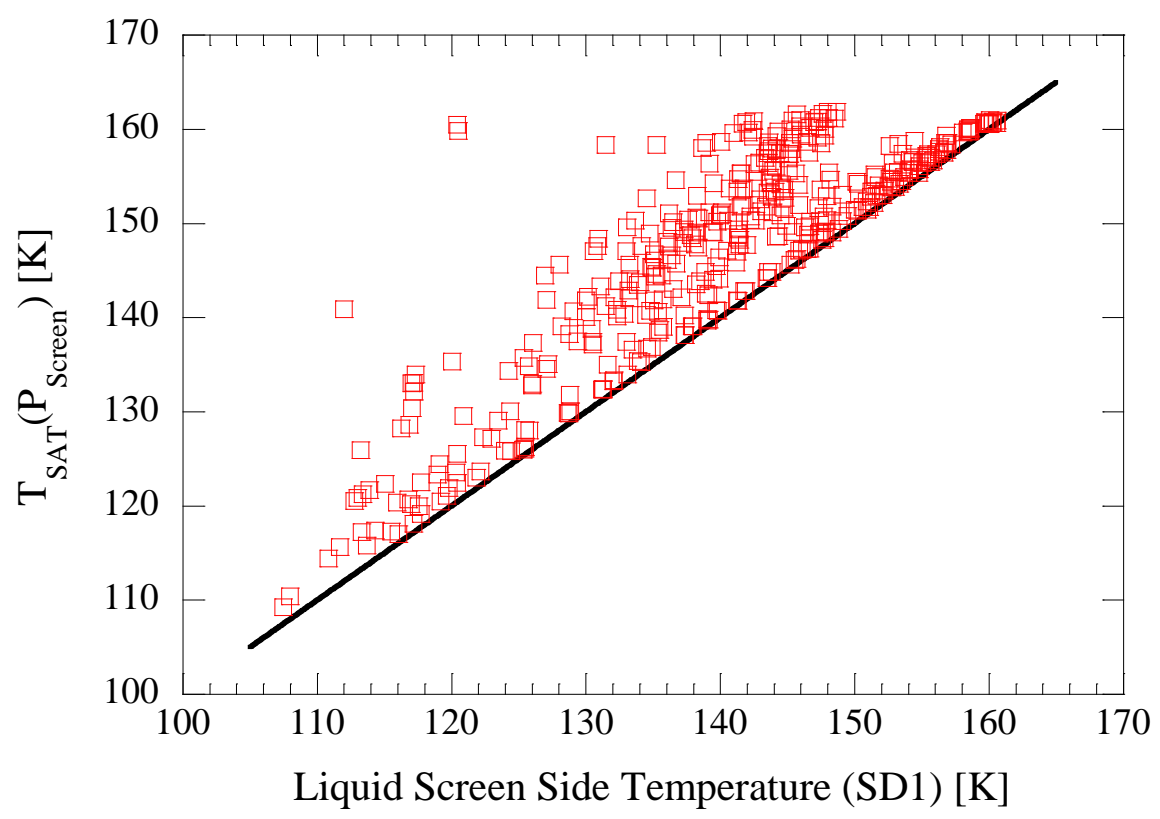

Figure 7.8 - Methane Saturation Temperature versus Liquid Screen Side Temperature at Bubble Breakthrough 

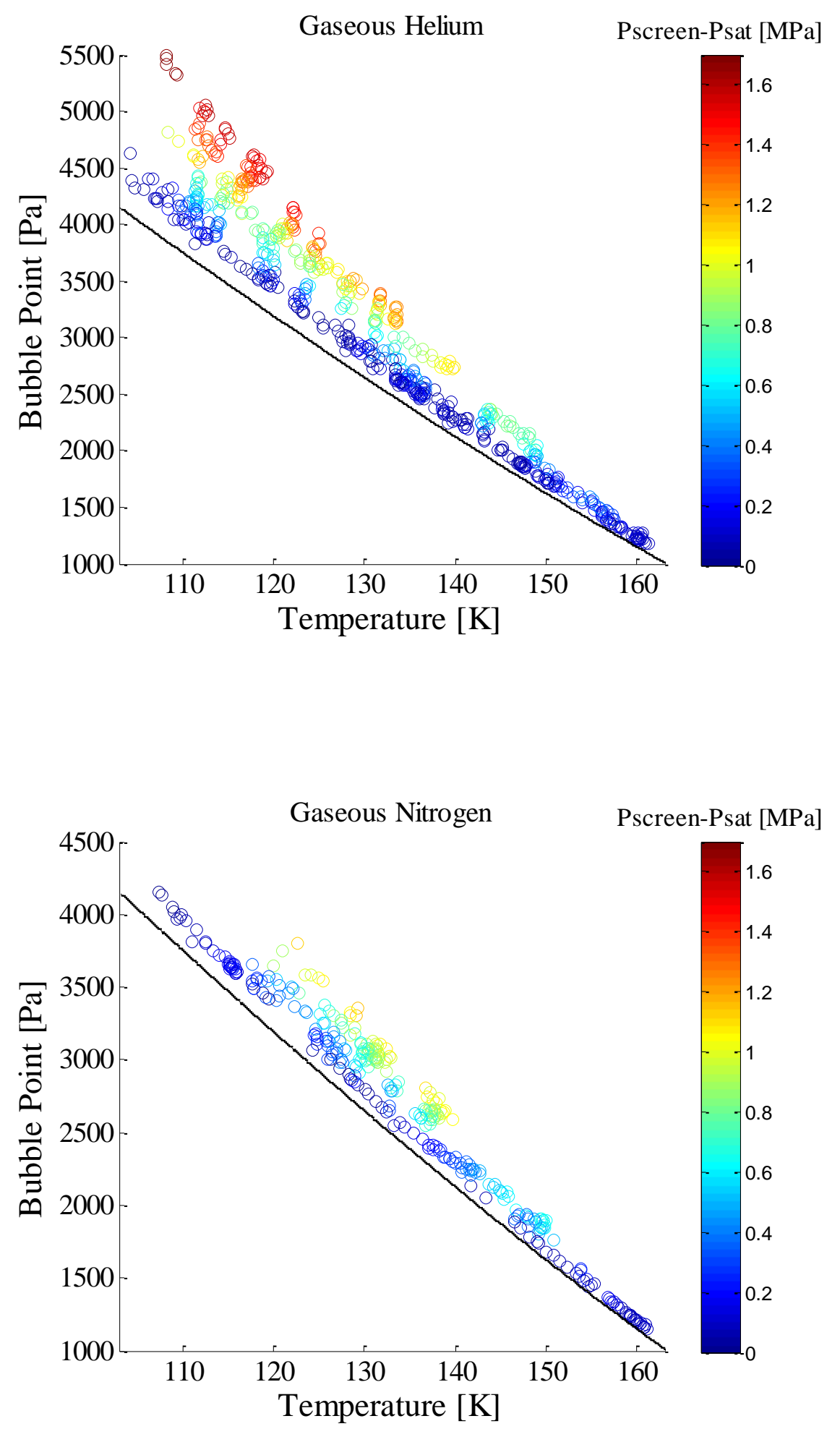

Figure 7.9 - Liquid Methane Temperature Correlation for a) Gaseous Helium and b) Gaseous Nitrogen at Breakthrough for a 325x2300 Screen 
To further illuminate the gain in margin due to pressurizing the liquid, Figures $7.10 \mathrm{a}-\mathrm{c}$ map the bubble point value on a $2 \mathrm{D}$ surface plot as a function of the thermodynamic state of the liquid (SD1 and the pressure at the screen) at breakthrough for gaseous helium, methane, and nitrogen, respectively. Figures $7.10 \mathrm{a}-\mathrm{c}$ are color plots of Figure 7.6a, where color indicates bubble point in units of [Pa]. For all three gases, there is a distinct trend that subcooling the liquid increases margin in LAD system design: pressurizing the liquid at constant temperature yields a higher bubble point, while pressurizing a system in which the liquid temperature is allowed to gradually increase over time, one can still retain a constant, elevated bubble point. In this way, subcooling the liquid adds flexibility in the system design.

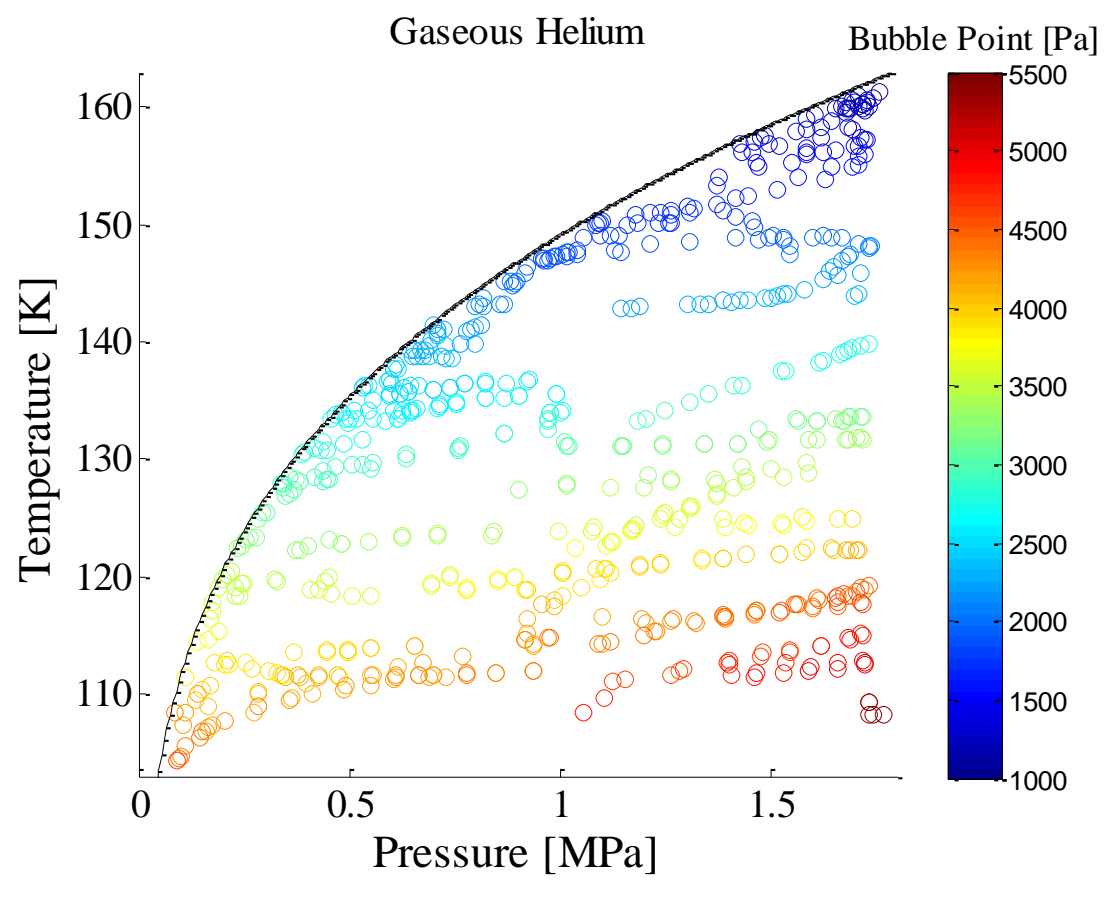



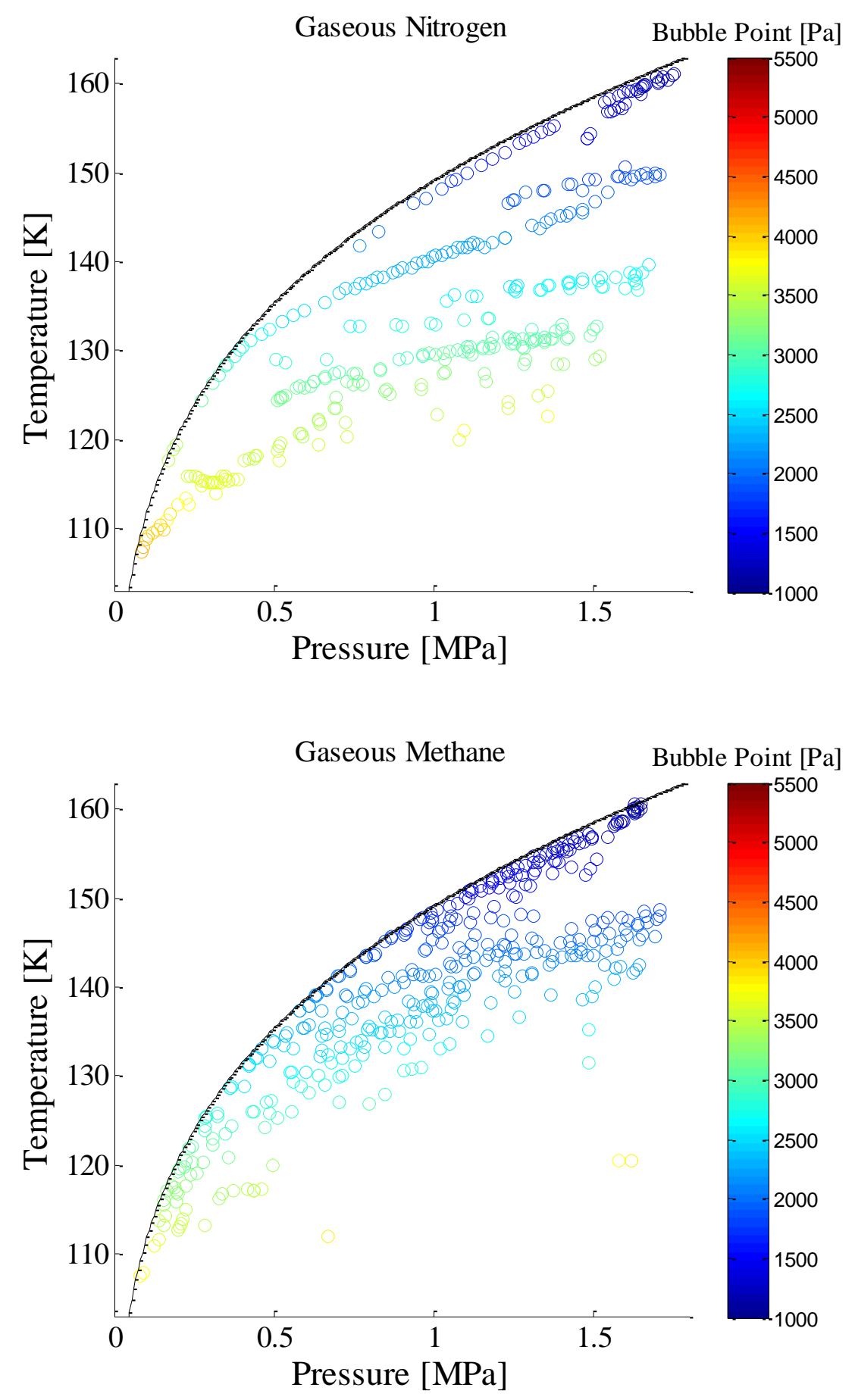

Figure 7.10 - Combined Liquid Temperature, Pressure, and Pressurant Gas Type Dependence on 325x2300 Liquid Methane Bubble Point Pressure using a) Helium, b) Nitrogen, and c) Methane as Pressurants. Color indicates bubble point in units of [Pa]. 
A mission designer can thus use Figures 7.10a - c to simply "dial in" the bubble point for a given thermodynamic state of the liquid at the LAD screen inside the propellant tank. The maximum breakthrough pressures always occur at the highest level of liquid subcooling at the coldest liquid temperatures. The rate that bubble point increases with the pressure along an isotherm is slightly higher using helium as a pressurant as opposed to nitrogen or methane. Obtaining data using $\mathrm{GCH}_{4}$ as a pressurant in $\mathrm{LCH}_{4}$ was difficult, due to rapid interaction between liquid and vapor phases at the screen interface. This indicates that the liquid subcooling at the screen was purely metastable and would likely not be sustained over long durations in a flight system during liquid expulsion.

Figure 7.11 plots the bubble point as a function of the liquid screen side pressure at the LAD screen. The prediction values use the surface tension calculated using the saturation temperature based on the liquid pressure at the screen with the room temperature pore diameter for the $325 \times 2300$ screen. Elevated bubble points were

achieved using GHe and $\mathrm{GN}_{2}$ over the entire pressure range, while bubble points obtained using $\mathrm{GCH}_{4}$ are in reasonable agreement with the prediction curve. Depending on the amount of subcooling, higher bubble points and thus higher flow rates are sustainable using non-condensable pressurant gases with screen channel LADs.

\subsection{Thermal Analysis}

The availability of bubble point data using multiple pressurant gases over such a wide range of thermodynamic conditions warrants further thermal analysis. To compare between pressurization schemes, screen interfacial temperatures, screen Re numbers, 
condensation and/or evaporation mass flow rates at breakdown, and heat fluxes produced at the screen are computed as a function of liquid temperature and pressure. Condensation and evaporation rates are also computed using kinetic theory to allow comparison.

Calculations are then used to determine optimal propellant pressure and temperature operating regimes and to assess the feasibility of using autogenous pressurization in future space missions. Previous heat entrapment inside a LAD was quantified analytically (Cady and Blackmon 1974) and experimentally in water and $\mathrm{LN}_{2}$ (Bolshinskiy et al. 2007).

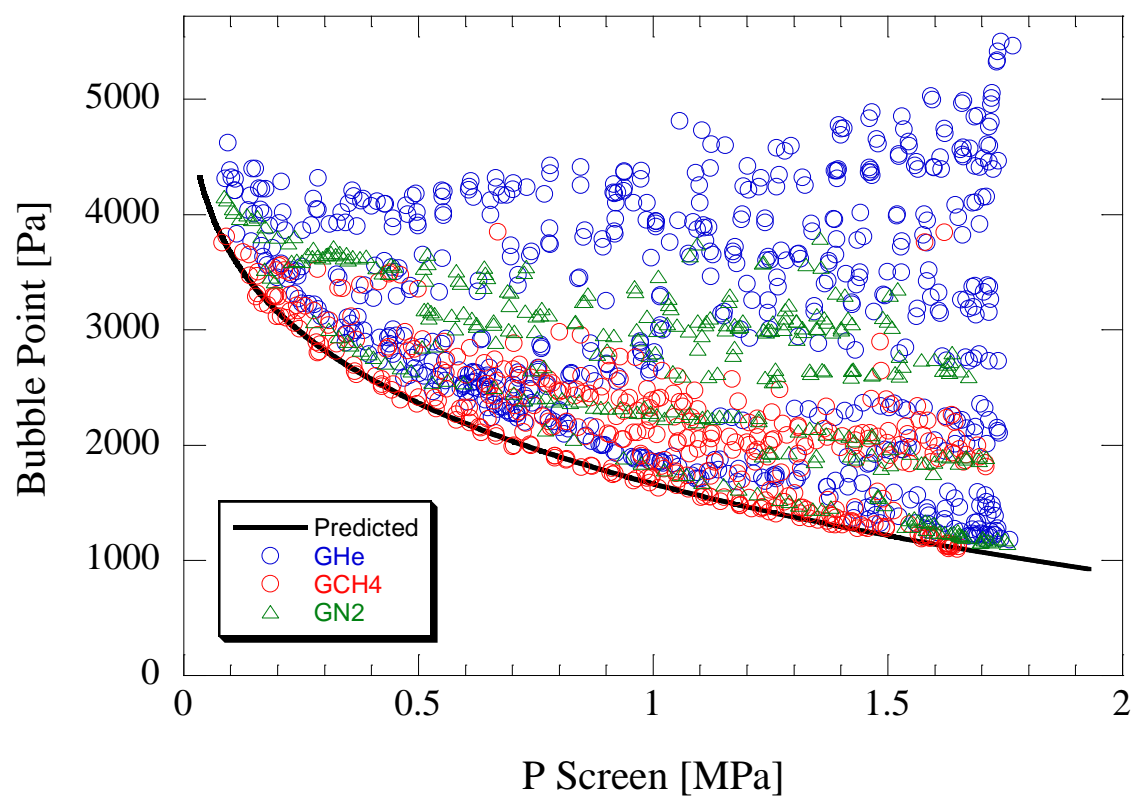

Figure 7.11 - Pressure Dependence on Liquid Methane Bubble Point Pressure for 325x2300 Screen 


\subsubsection{Heat Transfer at Breakdown}

Examination of the temperature measurements at bubble breakthrough provide insight into possible causes for reduced bubble points in the autogenous pressurization case. Figures $7.12 \mathrm{a}$ and $\mathrm{b}$ plot the bubble point as a function of the temperature difference across the screen and between the liquid screen side temperature and bulk liquid, respectively. As shown by the cluster of data points in Figure 7.12a, there is negligible heat transfer across the screen when helium and nitrogen are the pressurants. Heat transfer across the screen is relatively constant over the range of test conditions. However, when gaseous methane is used as the pressurant, temperature differences in excess of $17 \mathrm{~K}$ across the $89 \mu \mathrm{m}$ screen exist at bubble breakthrough. The largest temperature differences across the screen occur in the intermediate bubble point range, which corresponds to intermediate temperatures over a range of pressures at the LAD screen. While there was sufficient residence time for the pressurant gas within the dewar walls and piping within the dewar en route to the LAD screen/cup assembly to ensure the gas was at cryogenic temperatures, the gas was always slightly warmer than the liquid.

For temperature differences within the liquid, the trends in Figure 7.12b mirror Figure 7.12a with subtle variations. Most of the helium data indicate that the liquid at the screen was colder than the bulk liquid at breakthrough while the nitrogen data shows warmer screen temperatures than bulk liquid temperatures. Small differences in temperature gradients between $\mathrm{GHe}$ and $\mathrm{GN}_{2}$ are possibly attributable to the difference in solubility of the pressurants in the $\mathrm{LCH}_{4}$ (Miyamoto 1932, Sinor 1965, Sinor and Kurata 1966, and DeWitt and McIntire 1974). The gaseous methane data shows significantly higher temperature differences of almost $20 \mathrm{~K}$ between screen and bulk liquid. The heat 
transfer again reaches a peak in the same intermediate bubble point range corresponding to the intermediate temperature range. Highly subcooled states were not achievable, despite numerous attempts, because the warm $\mathrm{GCH}_{4}$ pressurant would immediately condense into subcooled liquid. These trends are consistent with Figure 7.6; regardless of the conditions of the liquid in the propellant tank, the liquid temperature at the screen approaches the saturation temperature for $\mathrm{GCH}_{4}$ pressure in the LAD cup as the system attempted to approach thermodynamic equilibrium. For non-condensable pressurization, the conditions at the screen matched the bulk liquid, and this is supported by the evidence of a minimal temperature difference across the screen and into the bulk liquid.

Based on the temperature differences in Figure 7.12, pressurization with gaseous methane vapor results in considerably higher heat transfer across the screen and into the bulk liquid over pressurizing with non-condensable helium or nitrogen. This is consistent with findings by Sefiane and Ward (2007) and with $\mathrm{LH}_{2}$ and LOX tests. The temperature difference between the gas side of the screen and the bulk liquid was as high as $36 \mathrm{~K}$ over a distance of approximately $6 \mathrm{~cm}$ when using gaseous methane at bubble breakthrough despite the fact that the gas temperature was nearly equal to the surrounding liquid temperature before the start of the next bubble point test. 

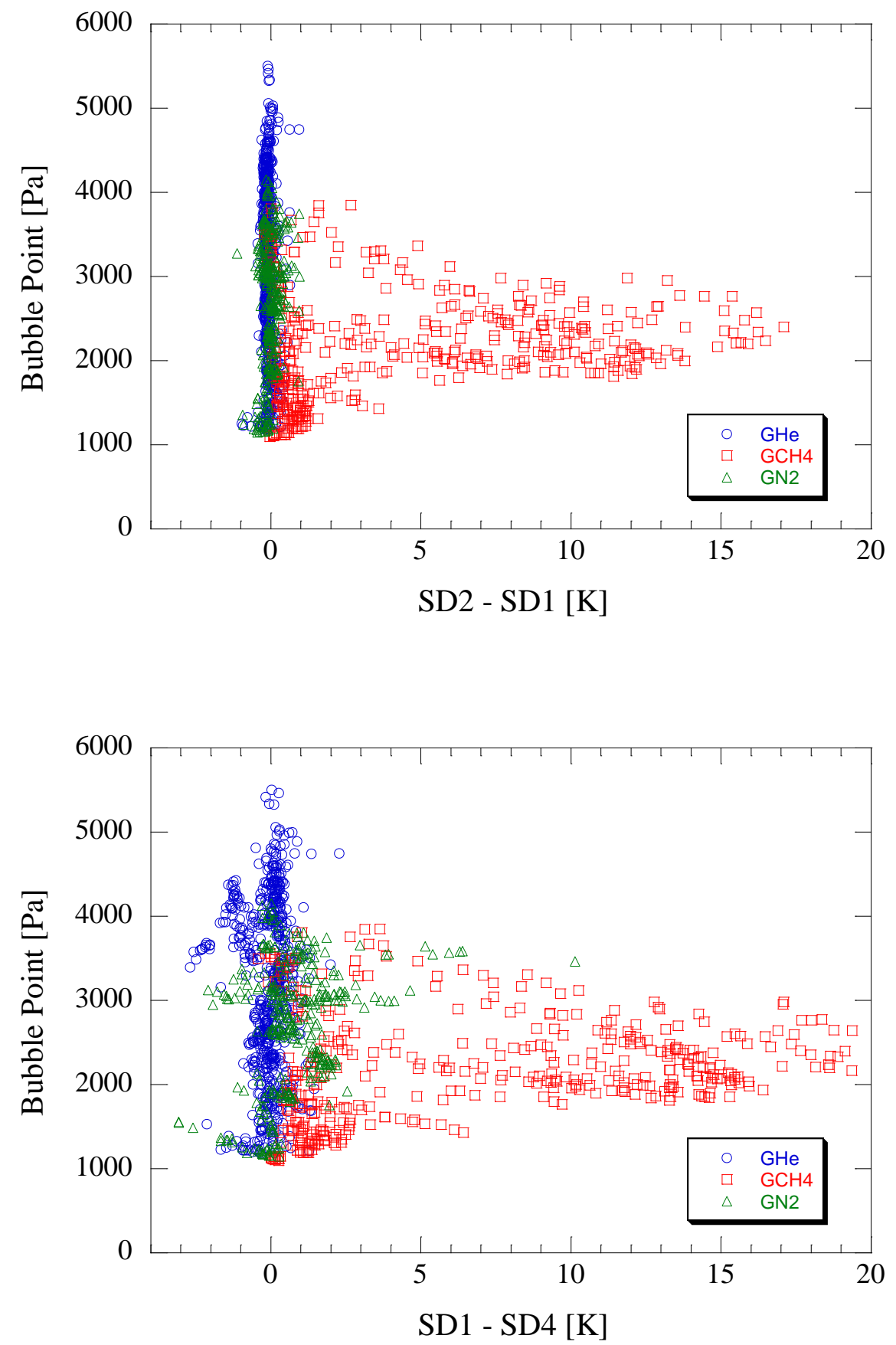

Figure 7.12 - Liquid Methane Bubble Point Pressure as a Function of the Temperature Difference a) Across the Screen and b) Between Liquid at Screen and Bulk Liquid in Tank for the $325 \times 2300$ Screen 
Figures 7.13a - 7.15a plot the bubble point as a function of SD1 and the temperature gradient across the screen using helium, nitrogen, and methane, respectively. Similarly, Figures $7.13 \mathrm{~b}-7.15 \mathrm{~b}$ plot the bubble point as a function of SD1 and the temperature gradient from the liquid screen side to the bulk liquid for gaseous helium, methane, and nitrogen, respectively. Examination of Figures 7.13a and 7.14a shows relatively uniform temperature differences over the entire range of bubble point data for GHe and $\mathrm{GN}_{2}$. While the subcooled data exhibits slightly higher heat transfer rates across the screen over saturated states, the difference is negligible for these two gases. Meanwhile, Figure 7.15a shows that the peak in the temperature difference across the screen occurs in the intermediate bubble point range where highly subcooled states were achievable using $\mathrm{GCH}_{4}(135$ - 145K). Rapid warming of the liquid during autogenous pressurization prevented testing at subcooled states with the colder liquid temperatures. Therefore it is shown that, for a given screen liquid temperature, that the largest temperature difference across the screen occurred when the temperature at the screen liquid/vapor interface approached the saturation temperature based on the liquid side screen pressure. Figures $7.13 \mathrm{~b}-7.15 \mathrm{~b}$ reflect these same trends. There is relatively uniform temperature difference between the screen and bulk liquid over the range of test conditions using $\mathrm{GHe}$ and $\mathrm{GN}_{2}$. Maximum heat transfer between screen and bulk liquid at bubble breakthrough occurs in the intermediate bubble point data range. The peak in the heat transfer data occurred over a wider range of $130-148 \mathrm{~K}$. 

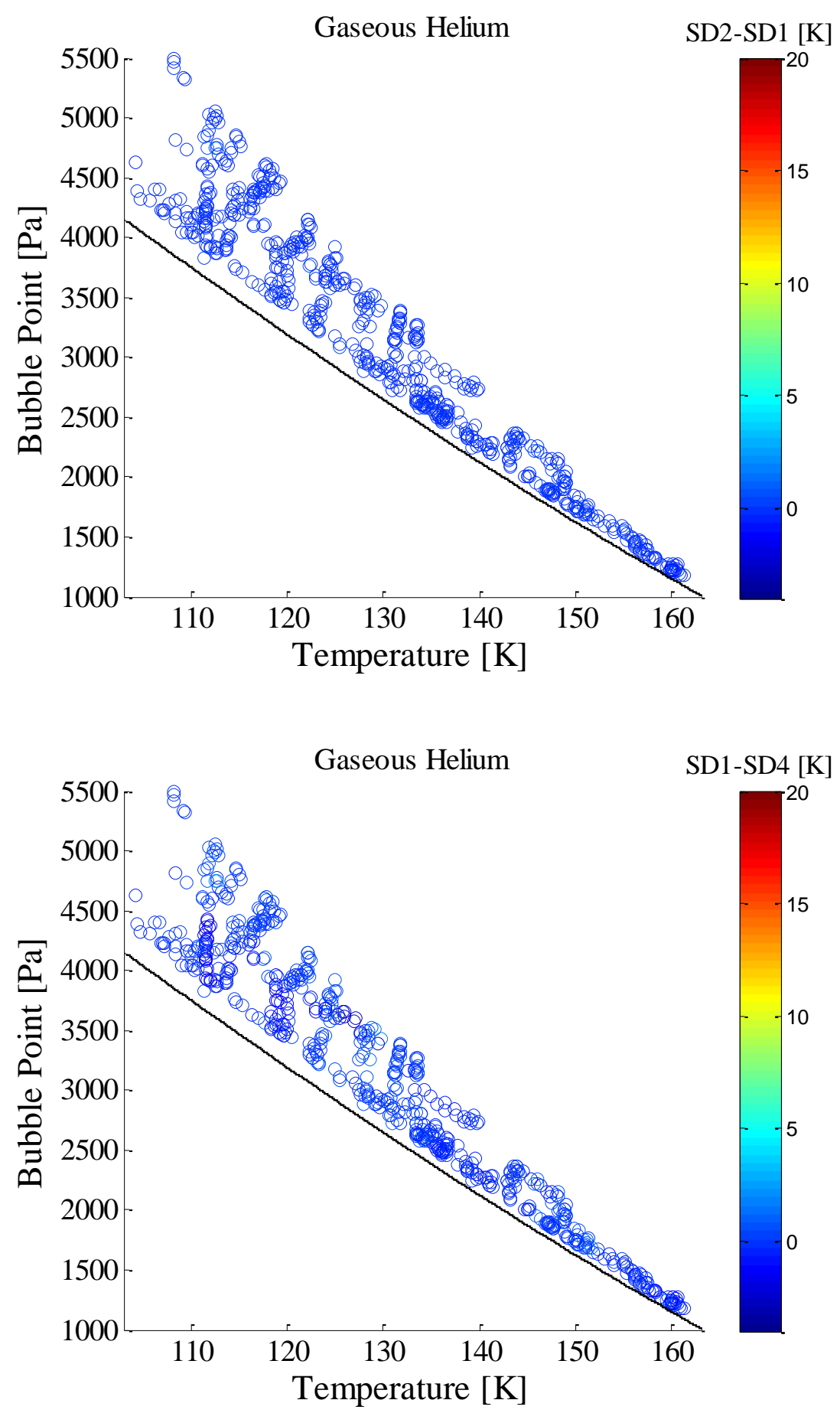

Figure 7.13 - Liquid Methane Bubble Point Pressure as a Function of Liquid Screen Side Temperature and a) Temperature Difference across the Screen and b) Temperature Difference between Liquid at Screen and Bulk Liquid in Tank at Breakdown for the 325x2300 Screen using Gaseous Helium. Color represents temperature differences in units of [K]. 

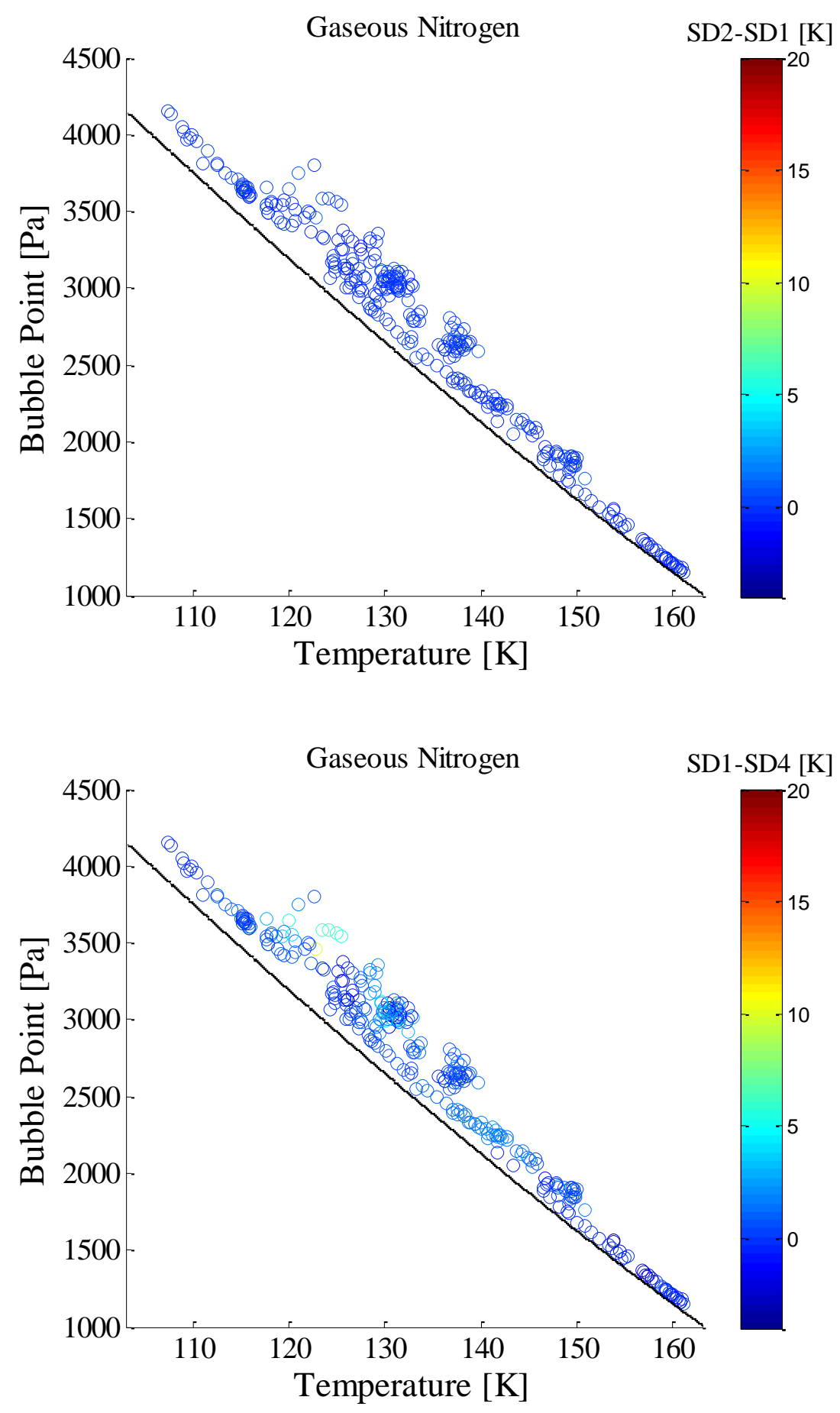

Figure 7.14 - Liquid Methane Bubble Point Pressure as a Function of Liquid Screen Side Temperature and a) Temperature Difference across the Screen and b) Temperature Difference between Liquid at Screen and Bulk Liquid in Tank at Breakdown for the 325x2300 Screen using Gaseous Nitrogen. Color represents temperature differences in units of [K]. 

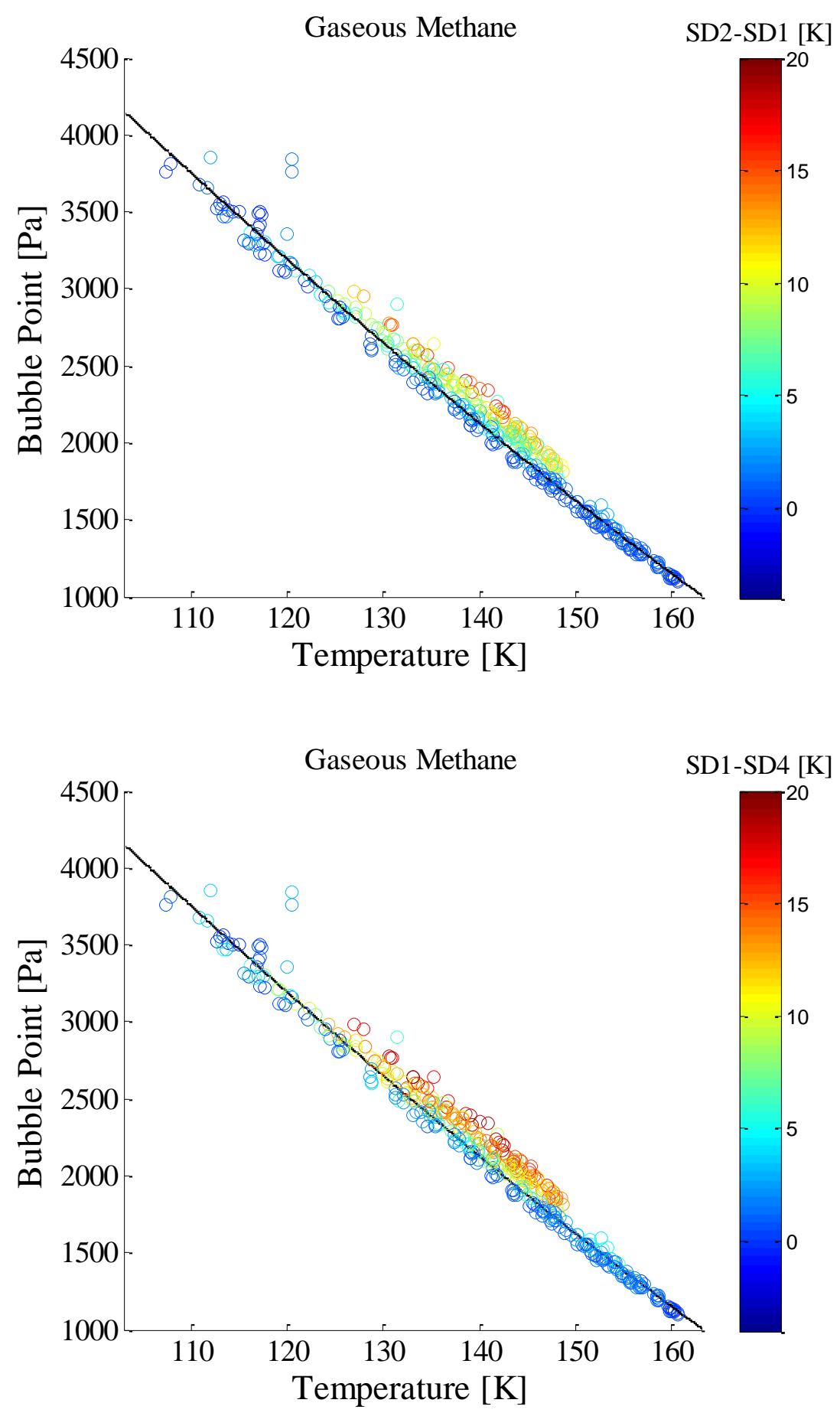

Figure 7.15 - Liquid Methane Bubble Point Pressure as a Function of Liquid Screen Side Temperature and a) Temperature Difference across the Screen and b) Temperature Difference between Liquid at Screen and Bulk Liquid in Tank at Breakdown for the 325x2300 Screen using Gaseous Methane. Color represents temperature differences in units of [K]. 
Combined bubble point and temperature difference plots explain why bubble points using gaseous methane are lower than those obtained with gaseous helium or nitrogen. The substantial temperature difference across the screen with $\mathrm{GCH}_{4}$ is caused by rapid condensation of vapor bubbles across the screen up to and including screen breakdown. Warm vapor bubbles conduct heat across the screen and release latent heat into surrounding liquid. Therefore pressurization with $\mathrm{GCH}_{4}$ forced the temperature at the liquid side of the screen to drop, causing the surface tension and thus bubble point to decrease.

The total pressure is a sum of the partial pressures of components as given below.

$$
P_{\text {Total }}=\sum_{i=1}^{n} P_{i}=P_{C H_{4}}+P_{\text {Non-Condensables }}
$$

Pressurization with the non-condensable gases, such as helium or nitrogen, lowers the pressure contribution of the methane, which is achievable by lowering its saturation temperature. The lower saturation temperature increases the surface tension, thus increasing bubble point.

Differences in bubble point performance are also attributable to differences in evaporation rates of the $\mathrm{LCH}_{4}$ away from the interface (Pong and Moses 1986). The noncondensable gas with the higher atomic mass causes a larger decrease in the condensation rate because the heavier gas accumulates more near the interface. The heavier $\mathrm{GN}_{2}$ molecules may accumulate at the interface, partially blocking evaporation, thus lowering bubble point pressure relative to GHe. In addition, GHe has a very low solubility in $\mathrm{LCH}_{4}$ as verified by Zimmerli et al. (2010). $\mathrm{GN}_{2}$ is more soluble in $\mathrm{LCH}_{4}$ and exerts a lower 
partial pressure than GHe under similar conditions, thus its effect on raising the bubble point pressure is not as dramatic.

\subsubsection{Interfacial Temperature}

Based on cryogenic bubble point tests in $\mathrm{LH}_{2}, \mathrm{LN}_{2}, \mathrm{LOX}$, and $\mathrm{LCH}_{4}$ from the current work, the bubble point equation correlates best with experimental data when surface tension is based on the liquid screen side temperature. Therefore, in cryogenic liquids, Equation 3.16 holds for saturated liquid states only, when taking into account the temperature dependence of the effective pore diameter. Ideally, the bubble point equation would be evaluated at the exact location of the $\mathrm{L} / \mathrm{V}$ interface temperature deep within the screen. However the micron size of the screen pores renders it impossible to measure temperature within the pore using conventional thermocouples or diodes. In addition, even though there were silicon diode measurements on both the liquid and gas sides of the screen, one cannot assume an interfacial temperature at the mean of these two values. This is because of the interfacial temperature jump condition which has been reported in previous work (Sefiane and Ward 2007) where experiments have shown that a temperature discontinuity of as much as $7.8 \mathrm{~K}$ could exist between the liquid and vapor sides of the interface for a water/water vapor system.

The bubble point equation can be used to back calculate interfacial temperature. Using the effective pore diameter for a $325 \times 2300$ screen from Chapter 4 , the methane surface tension curve fitting parameters, and the experimental bubble point data using the three different pressurant gases from the current chapter, one can estimate the interfacial temperature: 
$T_{\text {interface }}=T_{C}\left[1-\left(\frac{\Delta P_{B P} D_{P}}{\gamma_{0}}\right)^{a_{0}^{-1}}\right]$

The assumption that the surface tension is independent of the pressurant gas is corroborated by the work in Turkevich and Mann (1990a and b), which concludes that the effect of pressure on the interfacial tension between fluids is small. The effect of pressurant gas type on bubble point has been shown to be a modification of the effective interfacial temperature through enhanced heating and/or cooling due to condensation and/or evaporation in both the $\mathrm{LOX}$ and $\mathrm{LCH}_{4}$ tests. Therefore, any observed changes in the surface tension for this experiment are due to changes in the interfacial temperature, rather than due to pressurization effects.

Experiments carried out in Conrath and Dreyer (2009 and 2012) reveal that the measured bubble point pressure may depend on the pressure gradients caused by the supply gas. If the gas is supplied at a high enough volumetric flow rate, then the incoming gas could cause premature bubble breakthrough before the true bubble point pressure differential is reached, resulting in incorrectly measured bubble point pressures.

A quantity of interest is the Fritz volume (1936), which is the bubble volume at which the buoyancy force overcomes the surface tension force keeping the bubble from breaking through the screen:

$V_{F R I T Z}=\frac{4}{3} \pi R a_{F}^{3}$

where $R a_{F}$ is the Fritz radius defined as: 
$R a_{F}=\left(\frac{3 \gamma_{L V} D_{P}}{4 \rho g}\right)^{1 / 3}$

A bubble cannot detach from the screen before the Fritz volume is reached, which can happen either in the inertial time:

$t_{\text {inertial }}=R a_{F}\left(\frac{\rho}{P-P_{\text {bubb }}}\right)^{1 / 2}$

where $P$ is the pressure inside the tank and $P_{b u b b}$ is the pressure in the liquid surrounding the bubble, or in the Fritz time:

$t_{F}=\frac{V_{F R I T Z}}{Q_{G}}$

where $Q_{G}$ is the volumetric supply rate of the pressurant gas.

A ratio of the Fritz time to the inertial time is therefore indicative of the nature of the bubble breakthrough, with ratios greater than 1.0 signifying that bubble growth is driven by the self-expansion of the meniscus rather than by the pressurant gas "blowing up" the bubble. Such high ratios would suggest that the correct bubble point is being measured and would affirm the validity of using the bubble point equation to calculate the interfacial temperatures.

For this experiment, the incoming pressurant gas impinged on the bottom of the cup and did not directly flow into the wire screen; thus, $Q_{G}$ needed to be estimated. Detailed SEM analysis of the screens revealed that there were no visible flaws and therefore no preferential breakthrough point. Thus, it was reasonable to assume that all of 
the screen pores were roughly the same size and that the gas would uniformly pressurize over the entirety of the screen before breakthrough occurred. A highly conservative approach was taken and the gas was assumed to pressurize over only $10 \%$ of the available screen pores. With this assumption, the experimentally determined volumetric flow rate of the incoming gas was divided among $10 \%$ of the total number of pores to extract the estimated flow rate into each pore and, thus, into each forming bubble for calculation of the Fritz time. An estimate for the total number of pores in the $325 \times 2300$ Dutch Twill screen used in this study can be found by calculating the total number of wires per unit area and then multiplying by the total screen area. This assumes that there is approximately one pore for each wire crossing:

\# Pores $=n_{w} n_{s}\left(\frac{\pi D_{S}^{2}}{4}\right)$

where $D_{S}$ is the diameter of the screen.

The results of this analysis are shown in Figure 7.16. The important result is that this ratio is much greater than 1.0 for all of the data points in this study, indicating that the bubble point pressure has not been misrepresented and that breakthroughs have occurred as a result of overcoming the surface tension force. The smallest calculated time ratio was approximately 1200 while the largest value is greater than $1 \times 10^{6}$. While not shown here, calculated time ratios for the $\mathrm{LH}_{2}, \mathrm{LN}_{2}$, and LOX tests show similar results, with the time ratio no less than 1000, even after applying such an extremely conservative approach. This implies that the entire set of bubble breakthrough data presented in this dissertation is entirely valid applying the check of Conrath and Dreyer (2012). Thus, the 
interfacial temperatures that are present at bubble breakthrough can be solved using Equation 7.2. In addition, corrections to the pore diameter due to dynamic gas flow from Hernandez et al. (1996), Schutz et al. (2008), and Equation 4.1 are not required since the ramp rate for all tests was sufficiently slow to render this correction factor less than $0.1 \%$ :

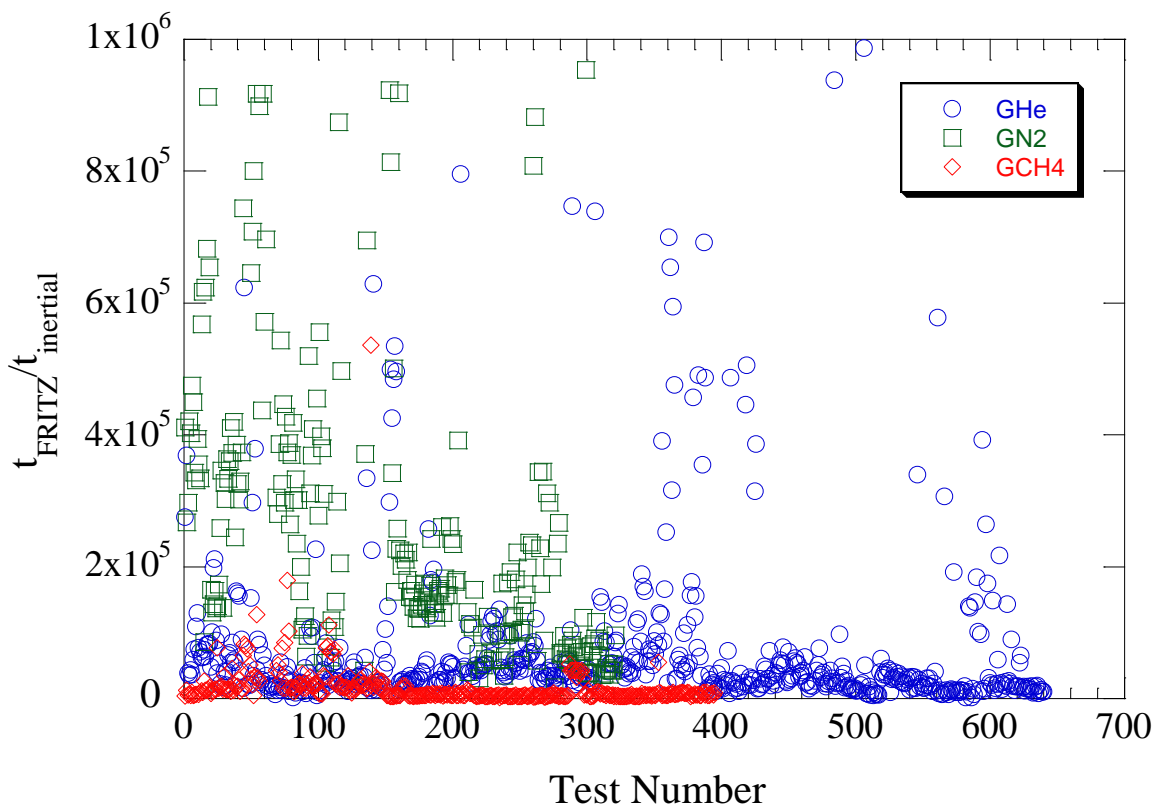

Figure 7.16 - Ratio of the Computed Fritz Time to Inertial Time as a Function of the Test Number for Gaseous Helium, Nitrogen, and Methane

Figure 7.17 plots the results of the interfacial temperature analysis. The color gradient represents the difference between the calculated interfacial temperature and the bulk liquid temperature from SD4. This temperature difference is plotted as a function of the pressure at the screen and bulk liquid temperature inside the test tank. A positive value indicates that the interfacial temperature has warmed up relative to the bulk liquid, while a negative value indicates cooler interface temperatures relative to the bulk liquid. Figure 7.17a shows that for temperatures and pressures near the methane saturation 
curve, the interfacial temperature is colder than that of the bulk liquid (up to nearly $5 \mathrm{~K}$ colder). This is evidence of evaporation occurring at the screen, which would tend to cool the interface. However, as the $\mathrm{LCH}_{4}$ is subcooled, the interfacial temperature rises relative to that of the bulk liquid until it eventually becomes significantly warmer. This corresponds to the previous result for autogenous pressurization that condensation effects become more significant as the liquid is subcooled and causes the interfacial temperature to rise.

This subcooling trend is not seen for pressurization with GHe. Instead, a trend with respect to the pressure of the liquid at the screen appears to exist. At low screen pressures the interfacial temperature is slightly warmer than the bulk liquid temperature, with the largest difference being an interfacial temperature about $2 \mathrm{~K}$ warmer than the bulk liquid. As the screen pressure rises, the interfacial temperature cools below that of the bulk liquid and becomes nearly $19 \mathrm{~K}$ colder at the most severe point. While this trend is strongest with pressure, there is a weak temperature dependence as well which appears to create regions of similar temperature gradient that loosely follow the contour of the methane saturation curve. The implication of Figure $7.17 \mathrm{~b}$ is that pressurizing with helium dramatically subcools the screen interface, which significantly improves surface tension and thus bubble point pressure.

Meanwhile Figure 7.17c plots results for interface temperatures from $\mathrm{GN}_{2}$ pressurization. The subcooling regime is apparent for screen pressures below roughly 1 $\mathrm{MPa}$; however, above this pressure the interfacial temperature seems to be strongly pressure dependent. The interfacial temperature ranges from about $5 \mathrm{~K}$ warmer than the bulk liquid to nearly $11 \mathrm{~K}$ colder than the bulk liquid. 

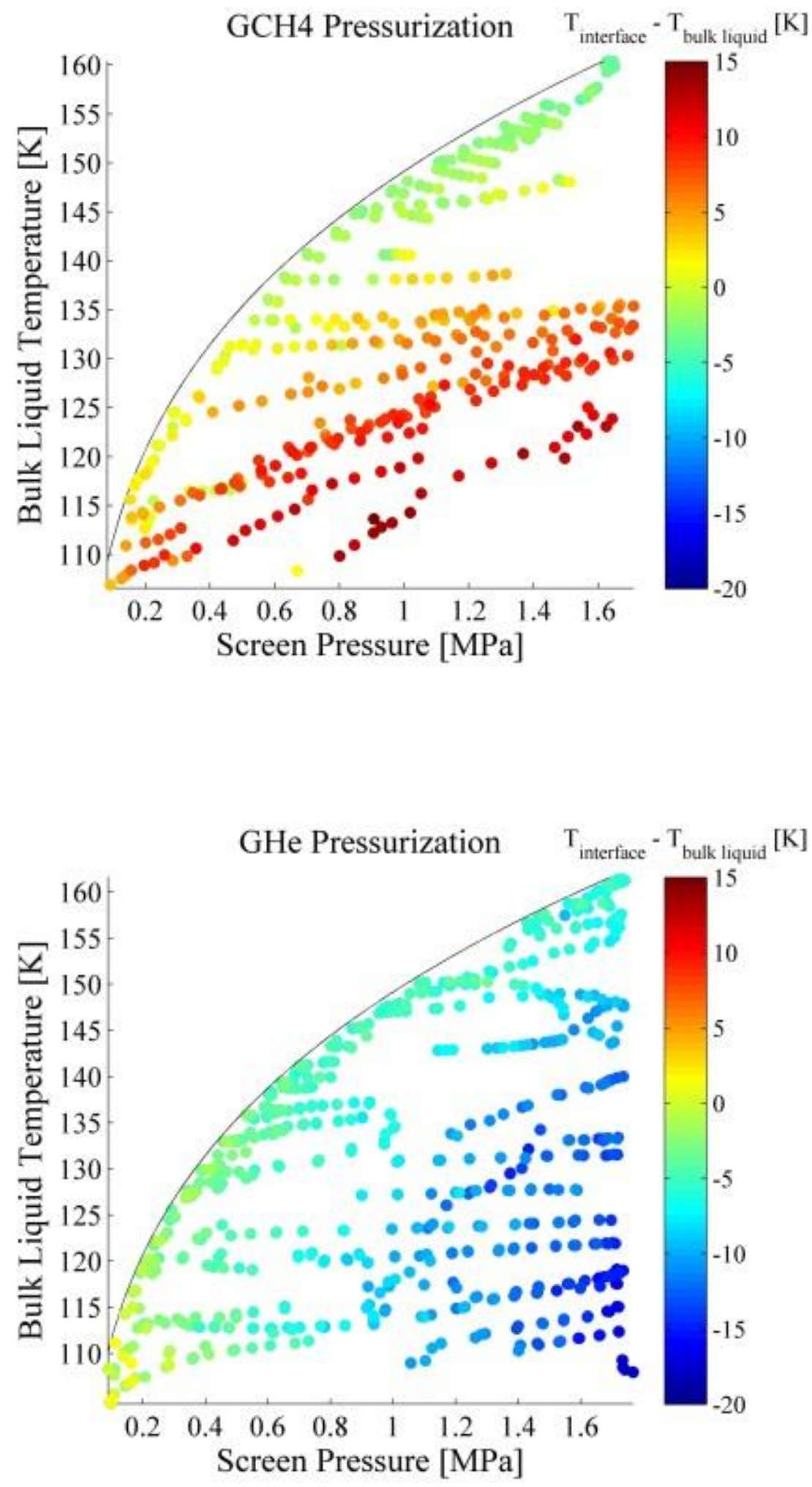


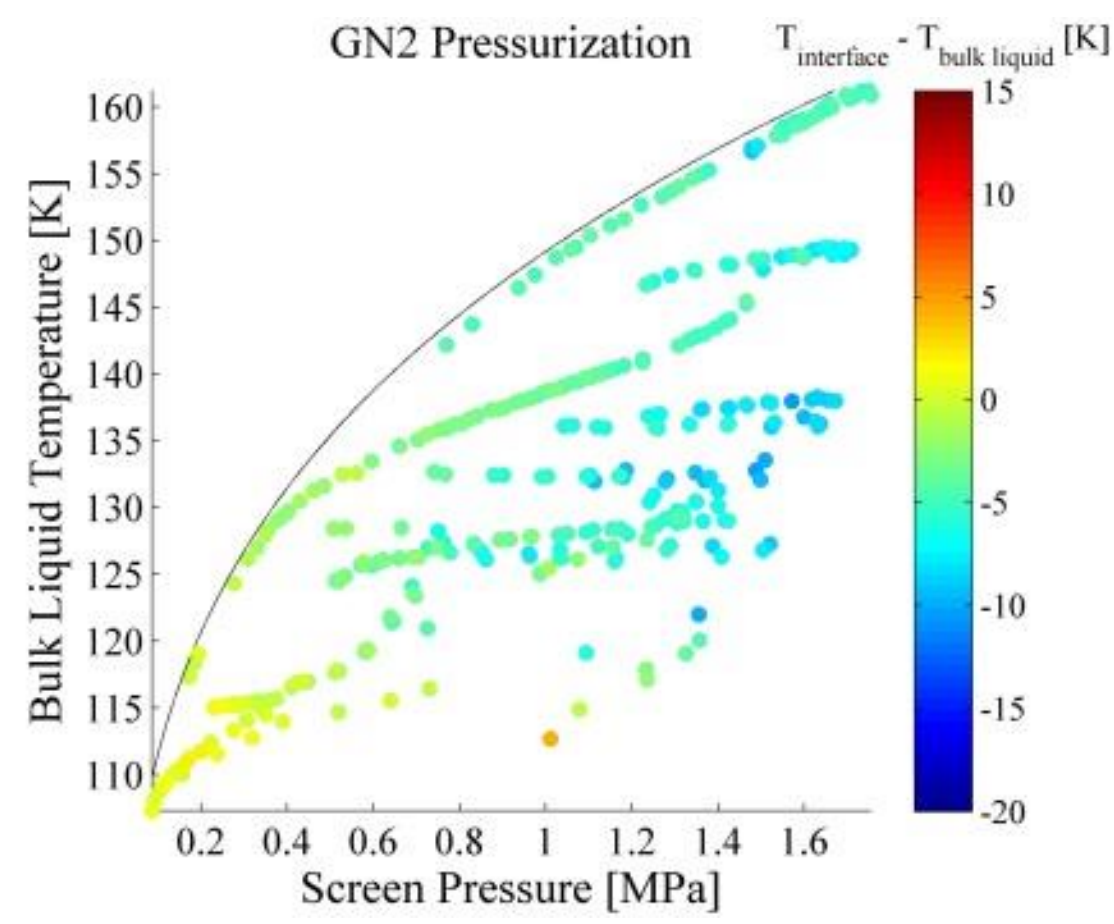

Figure 7.17 - Difference between the Calculated Interfacial Temperature and the Measured Bulk Liquid Temperature at Breakthrough for Pressurization with a) Gaseous Methane b) Gaseous Helium and c) Gaseous Nitrogen

It is interesting to note that the solubility of $\mathrm{GN}_{2}$ in $\mathrm{LCH}_{4}$ has been observed to increase as the liquid is subcooled (Hibbard and Evans 1968). The higher solubility of $\mathrm{GN}_{2}$ in $\mathrm{LCH}_{4}$ at higher degrees of liquid subcooling corresponds to the observed trend of interfacial heating in Figure 7.17c. When the $\mathrm{GN}_{2}$ dissolves there will be an associated enthalpy release. Since the interface will be heated by the enthalpy contribution of the dissolved $\mathrm{GN}_{2}$, the largest amount of heating may occur when there is the largest drive for dissolution to occur.

To illuminate the aforementioned trends, Figure 7.18 shows the difference between the interface temperature and the bulk liquid temperature as a function of the level of subcooling of the liquid for pressurization with $\mathrm{GCH}_{4}, \mathrm{GHe}$, and $\mathrm{GN}_{2}$. The 
autogenous case shows a strong trend of interfacial warming as the liquid is subcooled. Pressurization with GHe shows a slight amount of heating at low pressures, but there is an overall trend of interfacial cooling as the liquid is subcooled. Pressurization with $\mathrm{GN}_{2}$ shows elements of both autogenous and GHe pressurization with a stronger trend toward interface cooling.

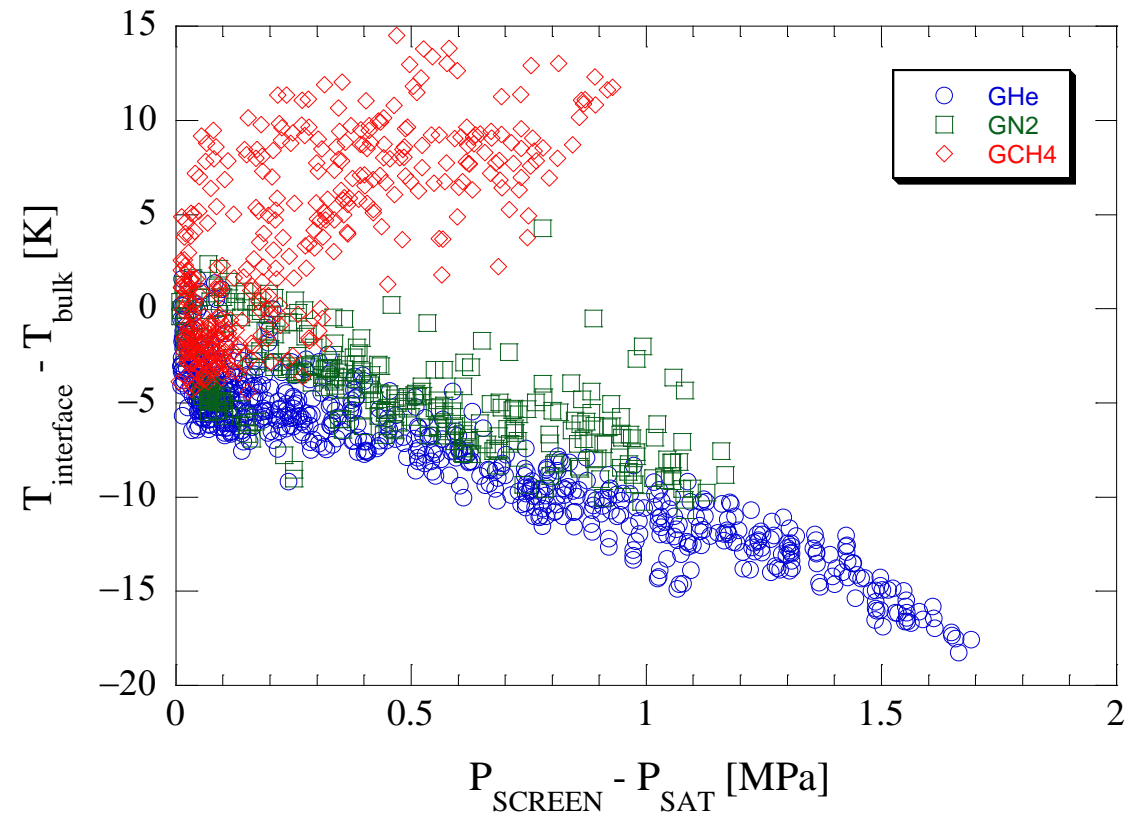

Figure 7.18 - Difference between the Calculated Interfacial Temperature and the Bulk Liquid Temperature at Bubble Breakthrough as a Function of Liquid Subcooling

\subsubsection{Condensation and Evaporation Mass Flux}

Before a mass flow rate across the screen can be quantified, the overall mass flow rate of the gas must be computed. A control volume $(\mathrm{CV})$ is defined with boundaries that span the walls of the cup and end at the half-width level of the screen, which for simplification, is the assumed location of the L/V interface. If the overall gaseous mass 
flow rate for this $\mathrm{CV}$ is denoted as $\dot{m}_{G}$, an expression for this flow rate can be determined by starting with the compressible gas law and differentiating:

$$
\begin{aligned}
& m=\frac{P V}{R_{G} T Z} \\
& \dot{m}_{G}=\frac{V}{R_{G} T Z} \frac{d P}{d t}-\frac{P V}{R_{G} T^{2} Z} \frac{d T}{d t}
\end{aligned}
$$

where $R_{G}$ is the gas constant and $Z$ is the compressibility factor.

Equation 7.9 can be used along with the experimental data to obtain a quantitative value for the gaseous mass flow rate in the $\mathrm{CV}$ at all times. However, it must be noted that the value of $\dot{m}_{G}$ is heavily dependent on the calculated values for the pressure and temperature derivatives. For this analysis, the derivatives were calculated at each time step using the finite difference method. A complication thus arises when evaluating these derivatives at the breakthrough point because the data immediately after breakthrough may no longer be representative of the predefined $\mathrm{CV}$ due to possible liquid ingestion into the cup. To account for this, the pressure and temperature derivatives at breakthrough are taken to be those for the data point immediately preceding it. While this is a source of error, the data acquisition time steps are small enough such that the incurred error is assumed to be negligible. Additionally, taking into account the various experimental uncertainties detailed in Section7.2.1, the calculated pressure derivatives are estimated to within $\pm 300 \mathrm{~Pa} / \mathrm{s}$ and the calculated temperature derivatives are estimated to within $\pm 2 \mathrm{~K} / \mathrm{s}$. 
Another important parameter for cryogenic LAD screen systems is the condensation and/or evaporation mass flux that is present across the screen interface up to and including bubble breakdown. This mass flux has a strong effect on the surface tension of the liquid and as a result, can enhance or degrade the bubble point pressure. For example, condensation will heat up the liquid due to the introduction of warmer gas. Since surface tension is inversely proportional to liquid temperature, the surface tension will decrease at the $\mathrm{L} / \mathrm{V}$ interface and the LAD screen will be more susceptible to bubble ingestion into the liquid. The opposite is true for evaporation, where an increase in surface tension would occur due to the cooling of the liquid. For pressurization with noncondensable $\mathrm{GHe}$ or $\mathrm{GN}_{2}$, a positive value for the mass flux across the screen interface could represent condensation as well as non-condensable gas transfer, or small amounts of gas dissolution. There is no way to separate these effects using this type of analysis, but the results still hold significance as a comparison tool among the different pressurant gases.

\subsubsection{Temperature and Pressure Data-Based}

The first method employed to quantify the amount of condensation and evaporation at bubble breakthrough is based on a simple mass balance for the CV. The mass flow across the screen interface is defined as positive for flow out of the cup. Applying conservation of mass for the $\mathrm{CV}$ yields an expression for this mass flow rate:

$\dot{m}_{S}=\dot{m}_{I N}-\left(\frac{V}{R_{G} T Z} \frac{d P}{d t}-\frac{P V}{R_{G} T^{2} Z} \frac{d T}{d t}\right)$ 
where $\dot{m}_{S}$ and $\dot{m}_{I N}$ are the mass flow rates across the screen and into the LAD cup, respectively.

The mass flow rate of the inflowing gas is directly measured, and the other parameters in Equation 7.10 are all obtained from experimental data. The uncertainty in the mass flow across the screen is calculated to within $\pm 0.02 \mathrm{~g} / \mathrm{s}$ for $\mathrm{GCH}_{4}$ pressurization, within $\pm 0.03 \mathrm{~g} / \mathrm{s}$ for $\mathrm{GHe}$ pressurization, and within $\pm 0.02 \mathrm{~g} / \mathrm{s}$ for $\mathrm{GN}_{2}$ pressurization. While it is instructive to obtain mass flow rates, it is even more attractive to determine mass fluxes across the screen, because these are normalized and the results can be applied to a variety of scenarios. To calculate the effective screen area for condensation and evaporation, porosity and screen thickness values from Chapter 3 are taken for a $325 \times 2300$ screen. For the $325 \times 2300$ Dutch Twill screen used in this study, the uncertainties in warp and shute wire diameters reported in Chapter 4 translate to an uncertainty in porosity of $0.245 \pm 0.007$. For porous LAD screens the area for condensation and evaporation is just the $2 \mathrm{D}$ area of the screen multiplied by this porosity factor. Dividing Equation 7.10 by this condensation area yields the desired mass fluxes across the screen. Such an analysis was performed for $\mathrm{GCH}_{4}, \mathrm{GHe}$, and $\mathrm{GN}_{2}$, and the results are shown in Figure 7.19. Here the mass flux across the screen at breakdown is plotted as a function of the amount of liquid subcooling at the screen. Uncertainty analysis reveals that the mass fluxes across the screen are estimated to within \pm 0.09 $\mathrm{kg} /\left(\mathrm{m}^{2} * \mathrm{~s}\right)$ for $\mathrm{GCH}_{4}$ pressurization, within $\pm 0.03 \mathrm{~kg} /\left(\mathrm{m}^{2} * \mathrm{~s}\right)$ for GHe pressurization, and within $\pm 0.04 \mathrm{~kg} /\left(\mathrm{m}^{2} * \mathrm{~s}\right)$ for $\mathrm{GN}_{2}$ pressurization.

As shown for the autogenous case, there is a direct correlation between level of subcooling and condensation mass flux at breakthrough. This suggests that there is an 
inherent tradeoff involved when pressurizing autogenously: higher levels of subcooling allow for a greater margin in bubble point pressure before bubble breakthrough occurs, but this also leads to higher condensation levels and therefore lower liquid surface tension. This subcooling effect on condensation rates has been previously documented (e.g. large condensation rates have also been observed when injecting steam into subcooled water) (Class et al. 1987 and Lee and No 1998).

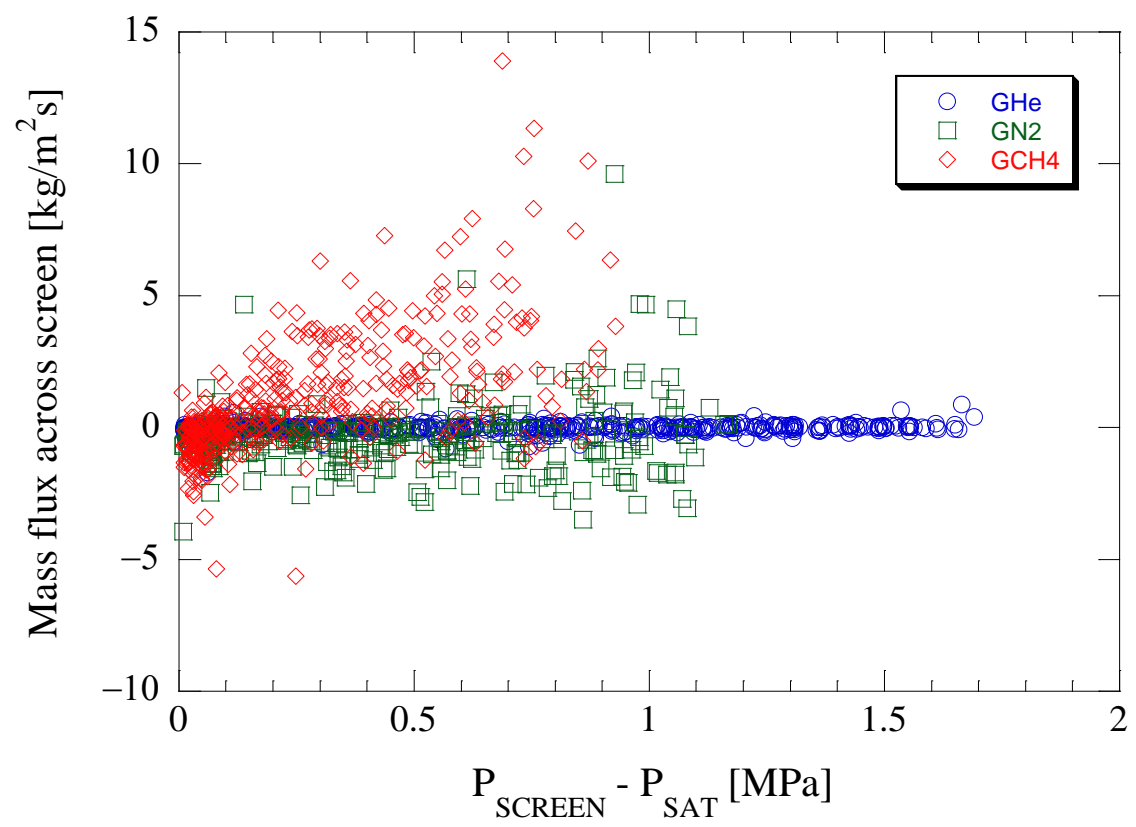

Figure 7.19 - Mass Flux across the Screen at Bubble Breakthrough as a Function of Liquid Methane Subcooling

Meanwhile for GHe pressurization, mass fluxes across the screen are relatively insensitive to the amount of liquid subcooling, and the magnitude of the screen mass flux is very near zero. This is to be expected for GHe pressurization since it is a noncondensable gas, and even small amounts of non-condensable gas may reduce condensation rates for cryogenic storage (Pong and Moses 1986 and Panzarella and 
Kassemi 2009). Additionally, the vapor fraction of $\mathrm{GCH}_{4}$ in the cup will be relatively small during GHe pressurization, leading to a state favoring evaporation. For $\mathrm{GN}_{2}$ pressurization there is also a slight bias toward evaporation, but only for low levels of subcooling. As the level of subcooling increases, there is a noticeable increase in the magnitude of the screen mass flux compared to GHe pressurization with net flow both into and out of the pressurant cup being observed at bubble breakthrough for various subcooled conditions. The higher magnitude screen mass fluxes for $\mathrm{GN}_{2}$ are attributed to the larger atomic mass of nitrogen compared to that of helium. To make this information more tractable for design purposes, mass fluxes across the screen interface are plotted as a function of the liquid thermodynamic state in terms of the temperature and pressure at the LAD screen in Figure 7.20. The black line is the methane saturation curve. This color plot further illustrates the points mentioned earlier; namely, the subcooling effect is very noticeable for $\mathrm{GCH}_{4}$, essentially nonexistent for $\mathrm{GHe}$, and weak for $\mathrm{GN}_{2}$.

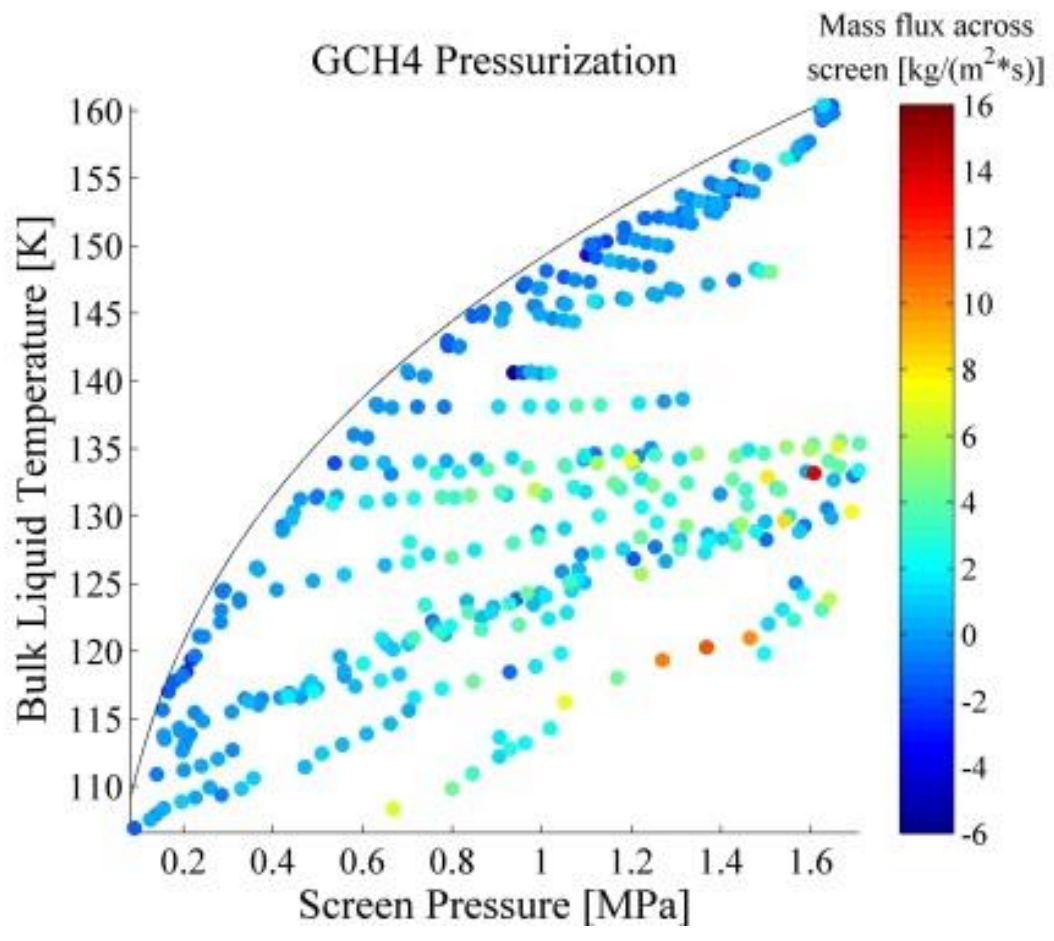



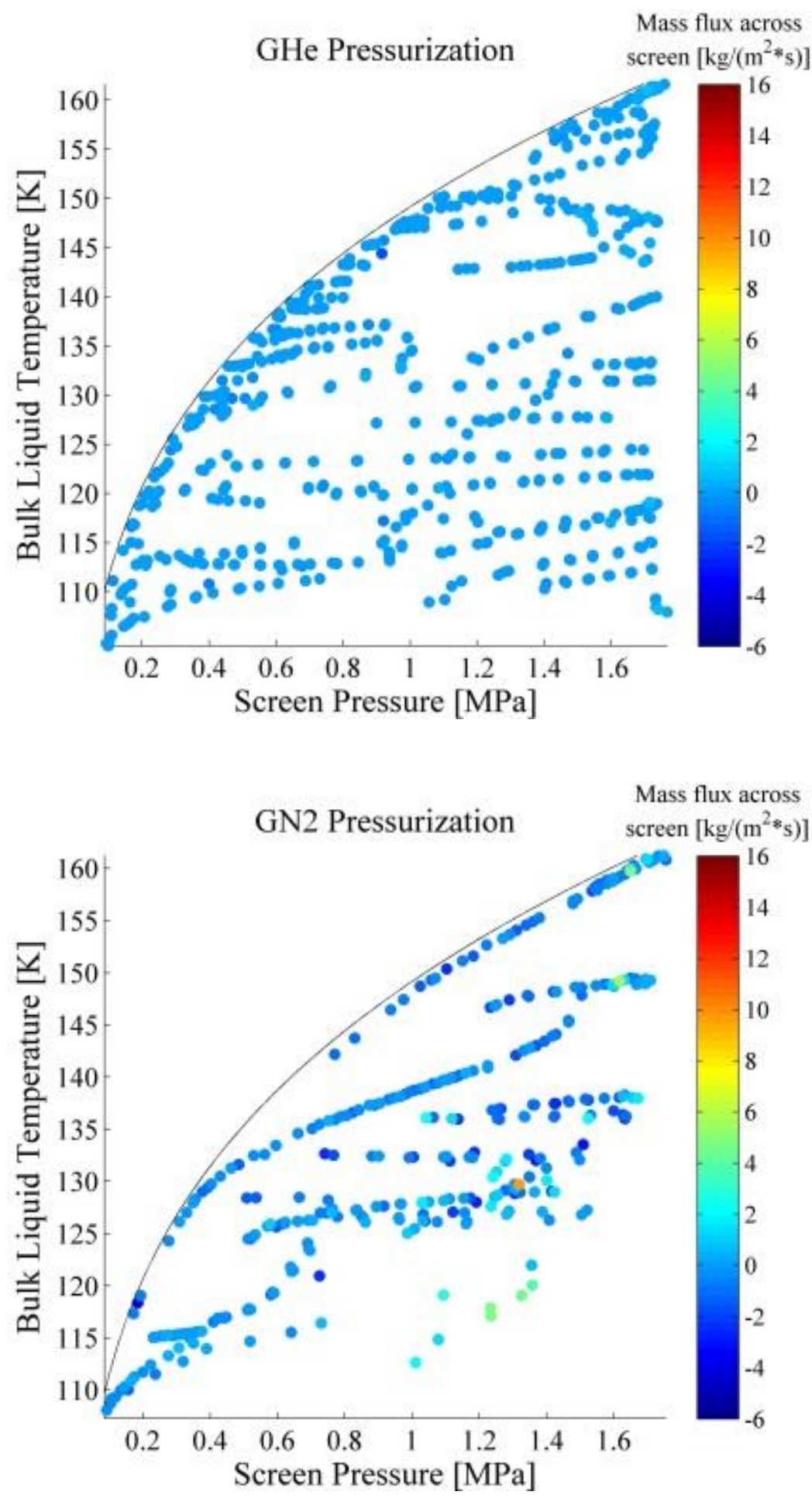

Figure 7.20 - Mass Flux across the Screen at Bubble Breakthrough as a Function of the Bulk Liquid Temperature and Pressure using a) Gaseous Methane, b) Gaseous Helium, and c) Gaseous Nitrogen as a Pressurant 


\subsubsection{Kinetic Theory}

To compare the experimental trends to analytical calculations, the model developed by Schrage (1953) for the net mass flux due to condensation $J_{C}$ is used:

$J_{C}=-\frac{2}{2-\sigma_{C}} \frac{1}{\sqrt{2 \pi R_{G}}}\left(\sigma_{E} \frac{P_{S A T}\left(T_{I}\right)}{\sqrt{T_{I}}}-\sigma_{C} \frac{P_{G}}{\sqrt{T_{G}}}\right)$

where $\sigma_{C}$ and $\sigma_{E}$ are condensation and evaporation coefficients, respectively, the saturation pressure of the liquid is determined based on the interface temperature, and $P_{G}$ and $T_{G}$ are the pressure and temperature of the gas inside the LAD cup. This equation can only be used to calculate the net mass flux due to condensation for the autogenous pressurization case. If it is to be extended to the cases with non-condensable pressurization, then relevant partial pressures of the autogenous gas compared to the noncondensable gas would need to be known, and this data is not available for these experiments.

Parameters in Equation 7.11 which are not immediately obtainable from experimental data are the condensation and evaporation coefficients as well as the interfacial temperature. Ideally, $\sigma_{C}$ and $\sigma_{E}$ would be determined experimentally. However, since there have never been any experiments with the exact same experimental setup as this one, the empirical data is nonexistent. Nevertheless, current computational fluid dynamics (CFD) simulations commonly assign these coefficient values around 0.01 for cryogenic conditions (Moder 2011). Furthermore, the interfacial temperature is quantified using the methods outlined in Section 7.4.2. Mass flux was calculated, and the 
results are shown in Figure 7.21. One thing that is immediately noticeable is the pronounced subcooling effect. The condensation mass flux is more severe the higher the level of subcooling. The magnitudes of the mass fluxes shown in Figure 7.21 are not as important as the predicted trend. Rather, the important result is independent confirmation of the subcooling effect. Using a modified version of Equation 7.11 would be ideal to corroborate the trends seen for $\mathrm{GHe}$ and $\mathrm{GN}_{2}$ pressurization, but there was not enough information to properly determine the vapor fraction of $\mathrm{GCH}_{4}$ that was in the cup as a function of time.

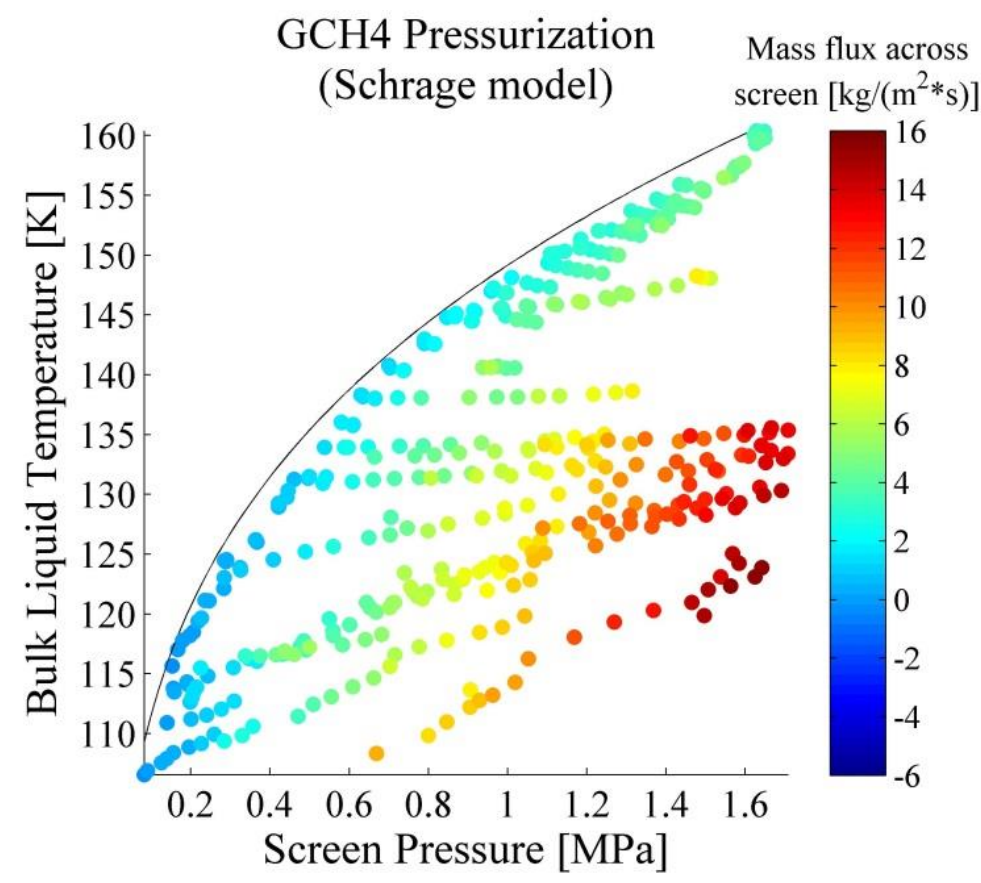

Figure 7.21 - Mass Flux across the Screen at Bubble Breakthrough using Gaseous Methane to Pressurize as a Function of the Liquid Temperature and Pressure using the Schrage Model from Kinetic Theory 


\subsubsection{Screen Reynolds Number}

An estimate of the Reynolds number of the pressurant gas as it breaks through the screen can be obtained by defining the Reynolds number in the following way:

$$
R e_{S}=\frac{\rho_{G} U_{G} S}{\mu_{G}}
$$

where

$U_{G}=\frac{\dot{m}_{S}}{\rho_{G} A_{C}}$

$$
A_{C}=\frac{\pi}{4} D_{S}^{2} \varepsilon
$$

and $S_{C}$ is a characteristic screen length defined as the radius of the circle with equivalent area to the porous region of the screen:

$S_{C}=\frac{D_{S}}{2} \sqrt{\varepsilon}$

Additionally, the speed of the gas as it passes through the screen is estimated using the calculated mass flow rate through the screen and the porous area of the condensation area as shown in Equations 7.13 and 7.14. Results are plotted in Figure 9.22.

For the autogenous pressurization case, as the level of subcooling increases, the flow across the screen transitions into the turbulent regime with screen Re numbers as high as 28,000 . It is clear that GHe pressurization results in laminar flow across the screen over the entire range, whereas $\mathrm{GN}_{2}$ pressurization leads to some turbulence at 
higher levels of subcooling. These trends are even more tractable as color plots relating the bulk liquid temperature, screen pressure, and screen Re number as shown in Figure 7.23, where a much higher degree of turbulence is associated with autogenous pressurization compared to non-condensable pressurization.

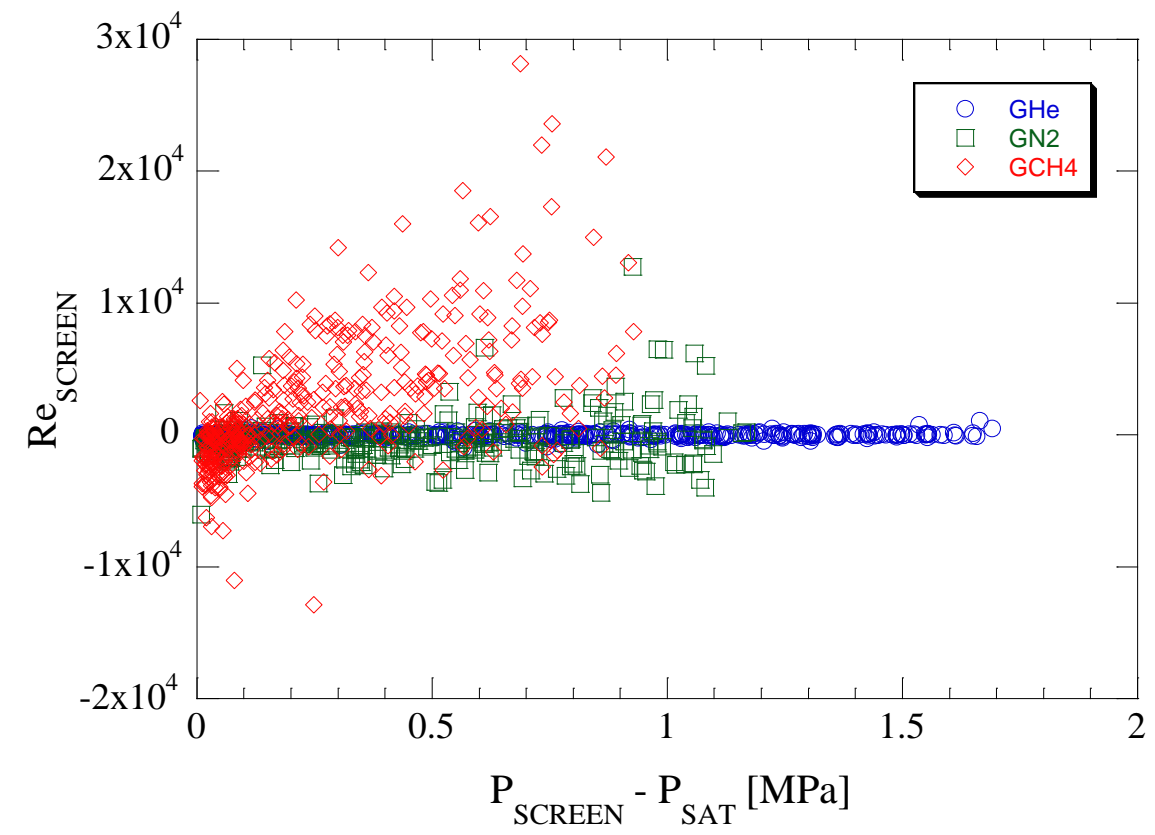

Figure 7.22 - Screen Reynolds Number at Bubble Breakthrough as a Function of Liquid Subcooling 

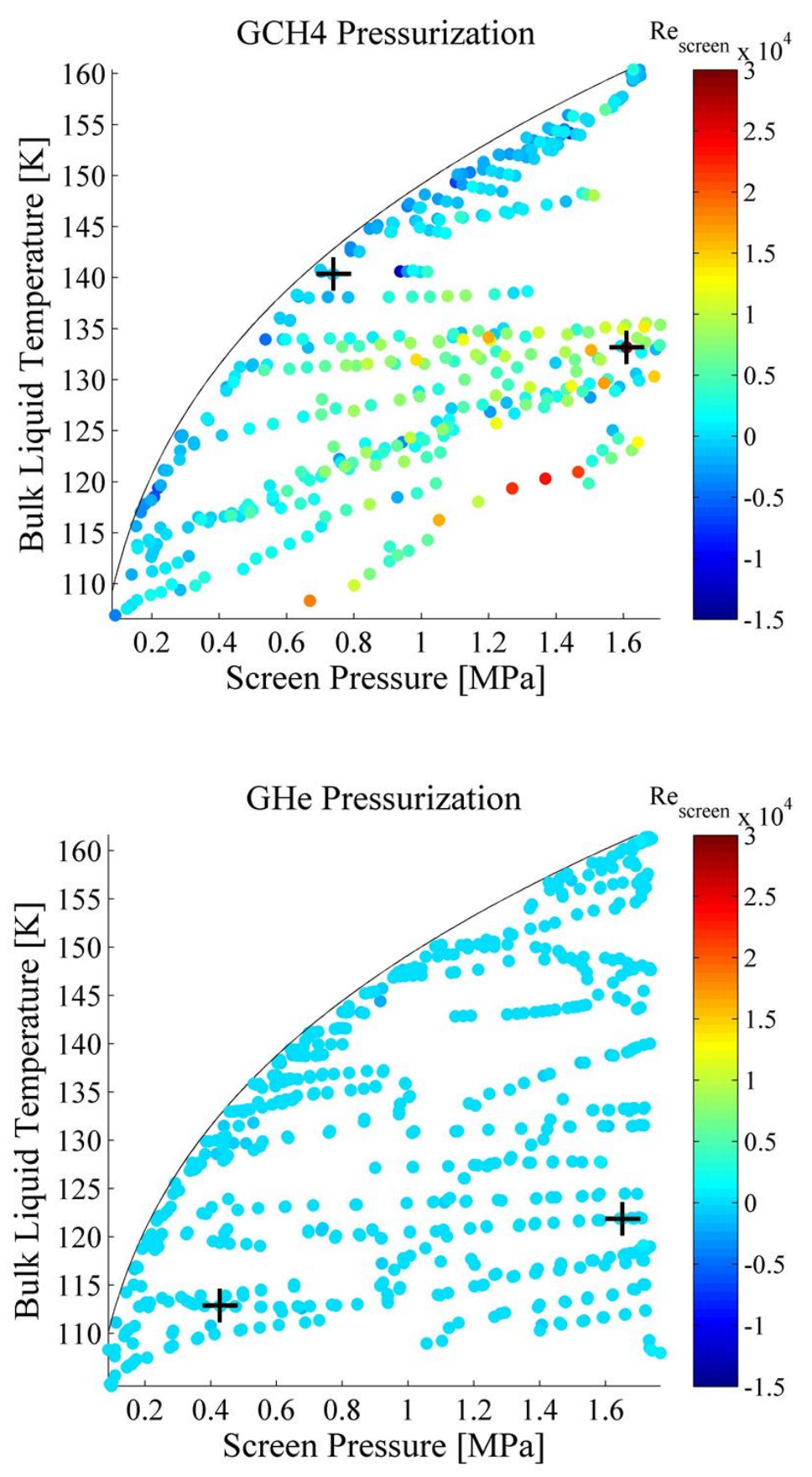


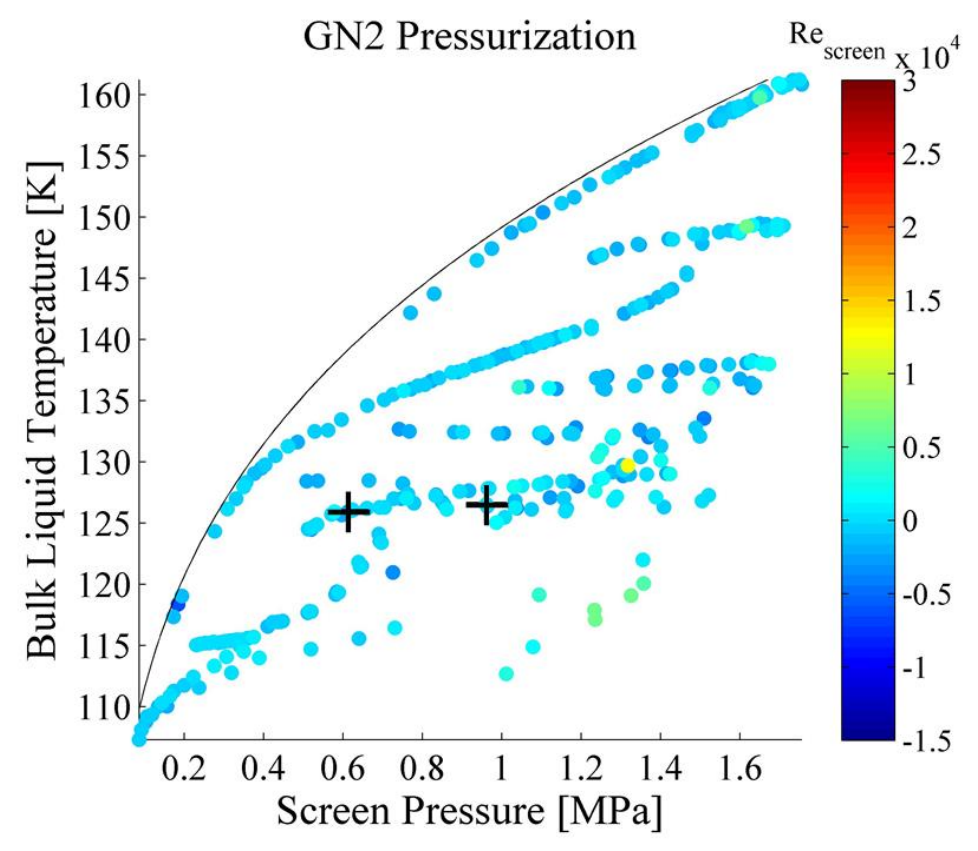

Figure 7.23 - Screen Reynolds Number at Bubble Breakthrough as a Function of Liquid Pressure and Temperature using a) Gaseous Methane, b) Gaseous Helium, and c) Gaseous Nitrogen to pressurize. Crosses indicate data points for visualization images in Figures $7.24 \mathrm{a}-\mathrm{f}$.

Screen captures taken at bubble breakthrough for pertinent liquid thermodynamic states (marked by crosses in Figure 7.23) further illustrate this claim. Bubble breakthroughs for autogenous pressurization are shown in Figures 7.24a and b. Massive amounts of vapor bubbles pass across the screen, up to and during bubble breakdown. Large convection currents in the bulk liquid are shown, which is indicative of heat and mass transfer across the screen. In stark contrast are Figures $7.24 \mathrm{c}$ and d, which show bubble breakthroughs for GHe pressurization. Here there is no visible churning of the $\mathrm{LCH}_{4}$, and all that can be seen is small streams of bubbles passing through the screen. $\mathrm{GN}_{2}$ pressurization shown in Figures $7.24 \mathrm{e}$ and $\mathrm{f}$, is an intermediary case between autogenous and GHe pressurization. There is a small amount of churning at breakthrough and the bubble motion is significantly more violent than that for GHe pressurization. The 
implication here is that autogenous pressurization results in added mass transport to the bulk liquid through turbulent mixing of the gas and liquid up to and including screen breakdown, which will prematurely heat the liquid. Both non-condensable gases result in little mass transport across the screen due to laminar flow.

\subsubsection{Heat Conduction into Liquid}

Because the main goal of LADs is to provide vapor free liquid propellant to an engine, a vital object of consideration is the amount of heat that is produced within the LAD channel from external sources during operation. The heat production must remain low enough to avoid excessive boil off of the liquid and to keep the liquid subcooled so that cavitation or vapor formation does not occur during propellant outflow to the engine. Studies have shown that the primary mode of heat transfer across LAD screens is thermal conduction (from the gas to the screen, through the screen, and from the gas to the liquid), and that all natural convection effects through the screens are negligible (Bolshinskiy et al. 2008). Furthermore, experimental and analytical results show that the thermal conductivity of wire screens is highest in the direction parallel to the plane of the wire screen layers, but that the conductivity in the direction normal to this plane can be significantly higher if the contact conditions between the individual wires and layers are good (Li and Peterson 2006). This implies that heat created from vapor condensation across the screen at the portion of the LAD screen exposed to pressurant gas could be transferred along the LAD screen into the liquid. 


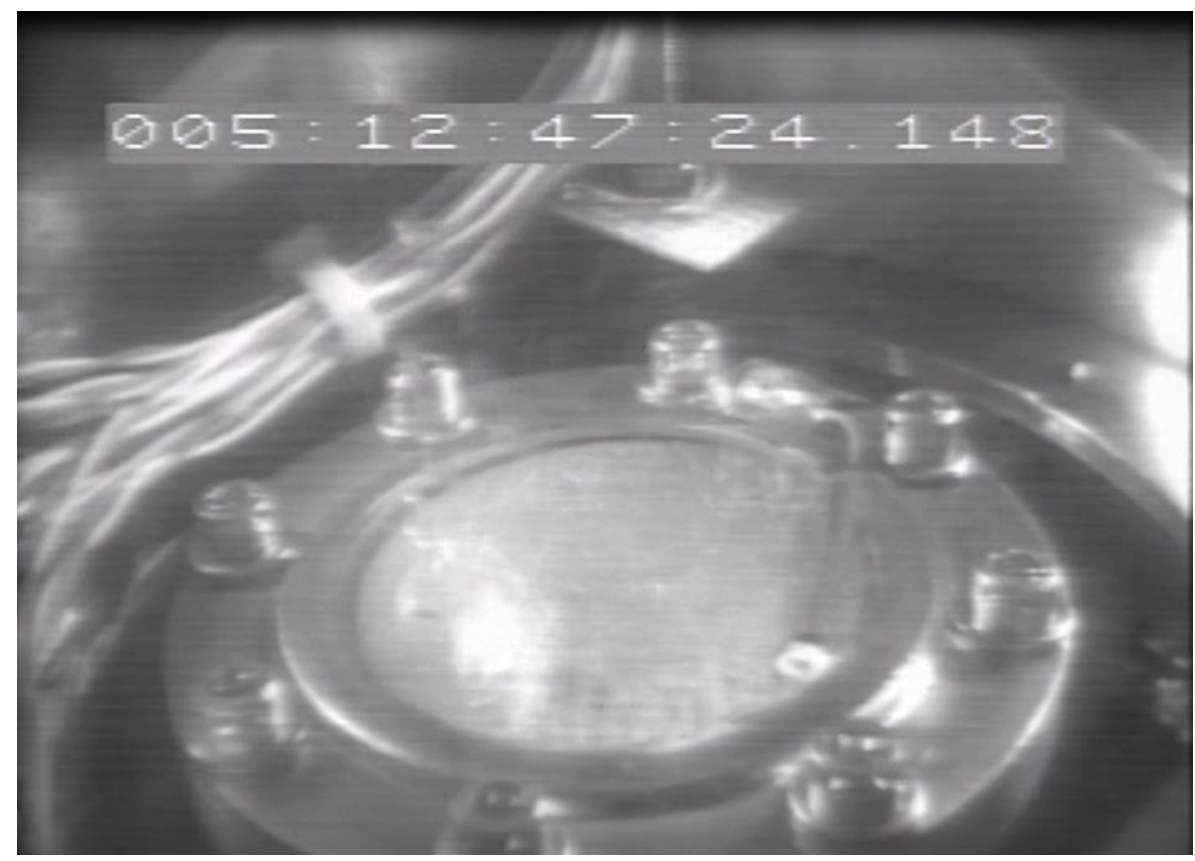

Figure 7.24 - Bubble Breakthroughs for: a) Gaseous Methane Pressurization at a Low Screen Reynolds Number $(\approx 400)$

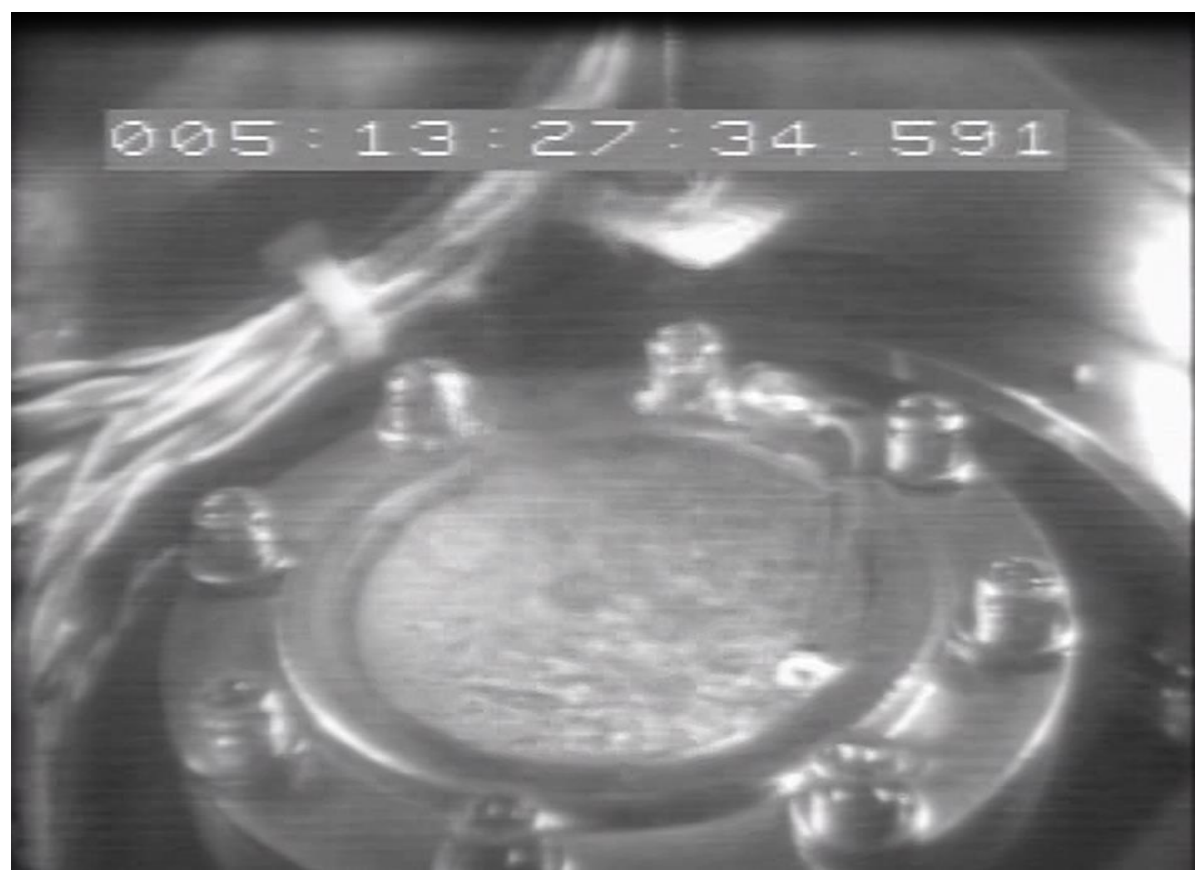

b) Gaseous Methane Pressurization at a High Screen Reynolds Number $(\approx 28,000)$ 


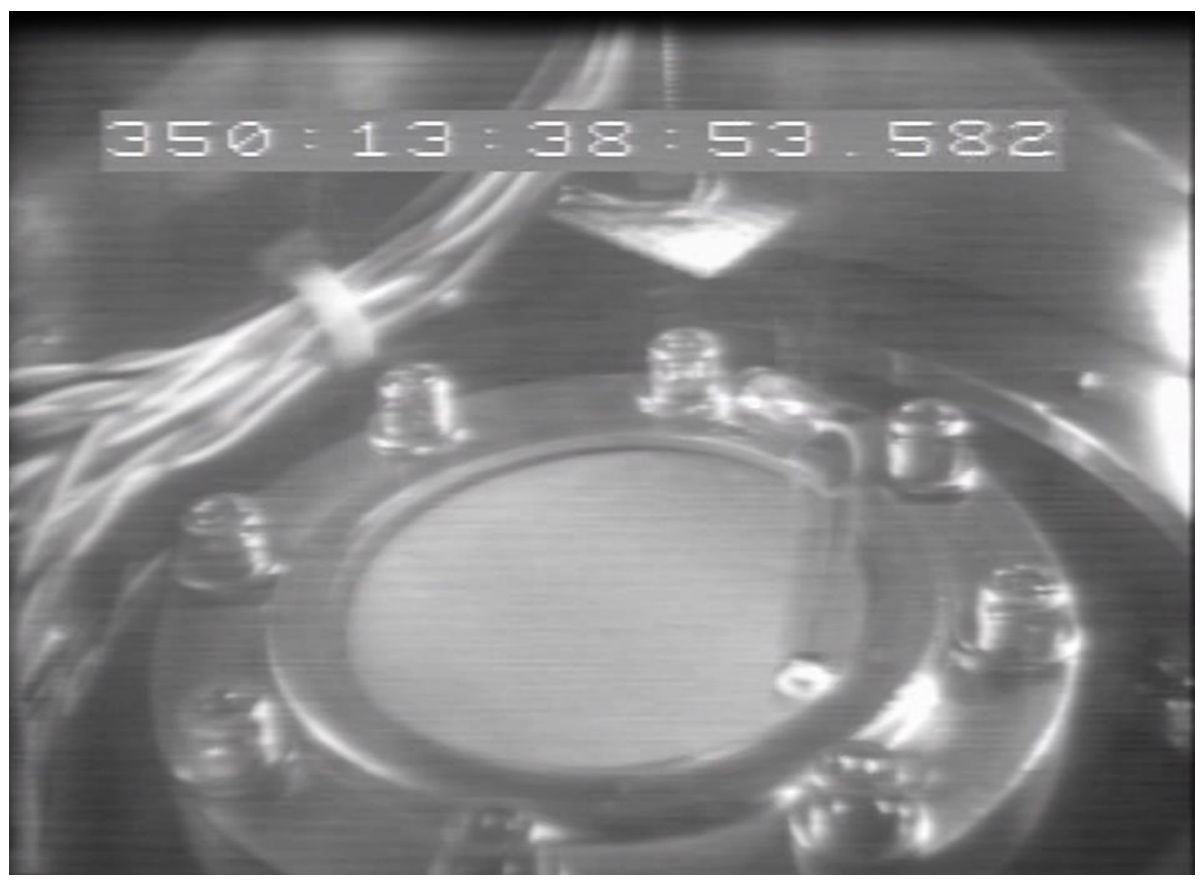

c) Gaseous Helium Pressurization at a Low Screen Reynolds Number $(\approx 20)$

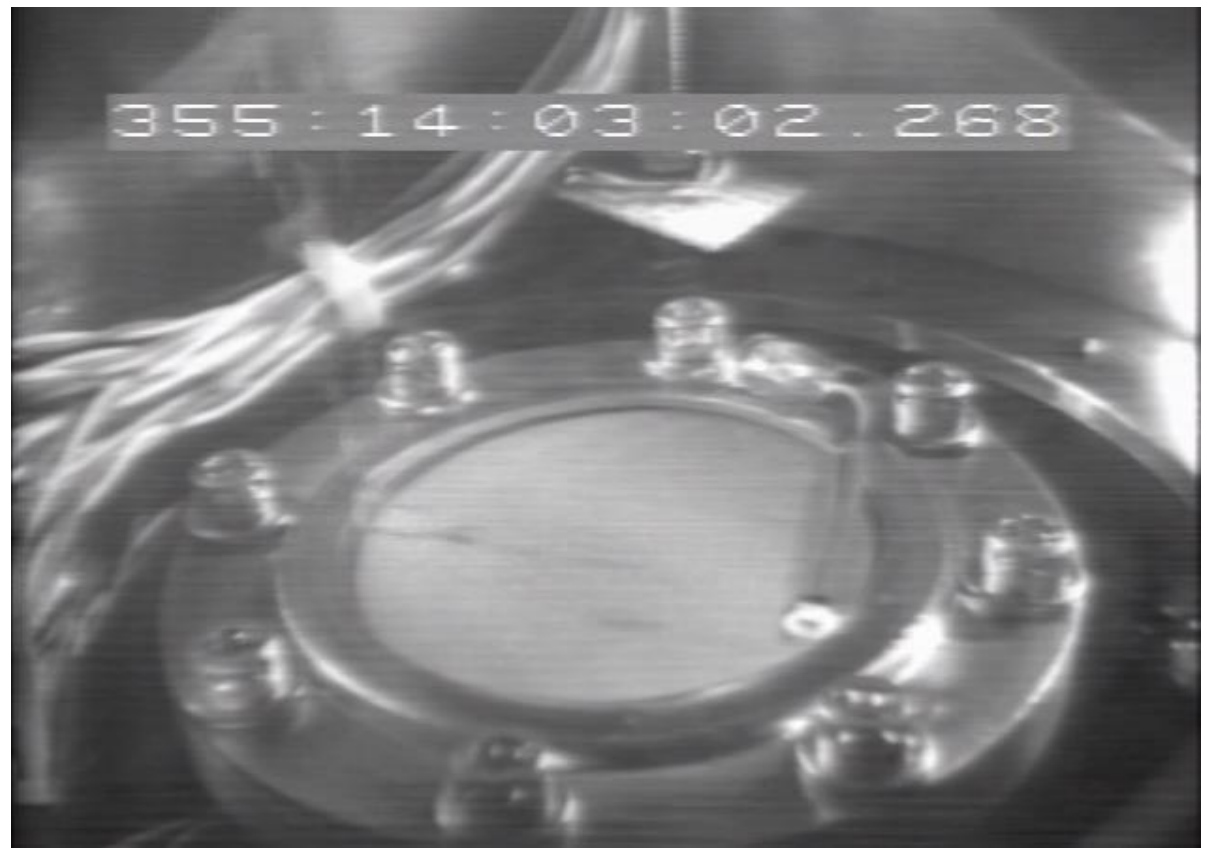

d) Gaseous Helium Pressurization at a High Screen Reynolds Number $(\approx 40)$ 


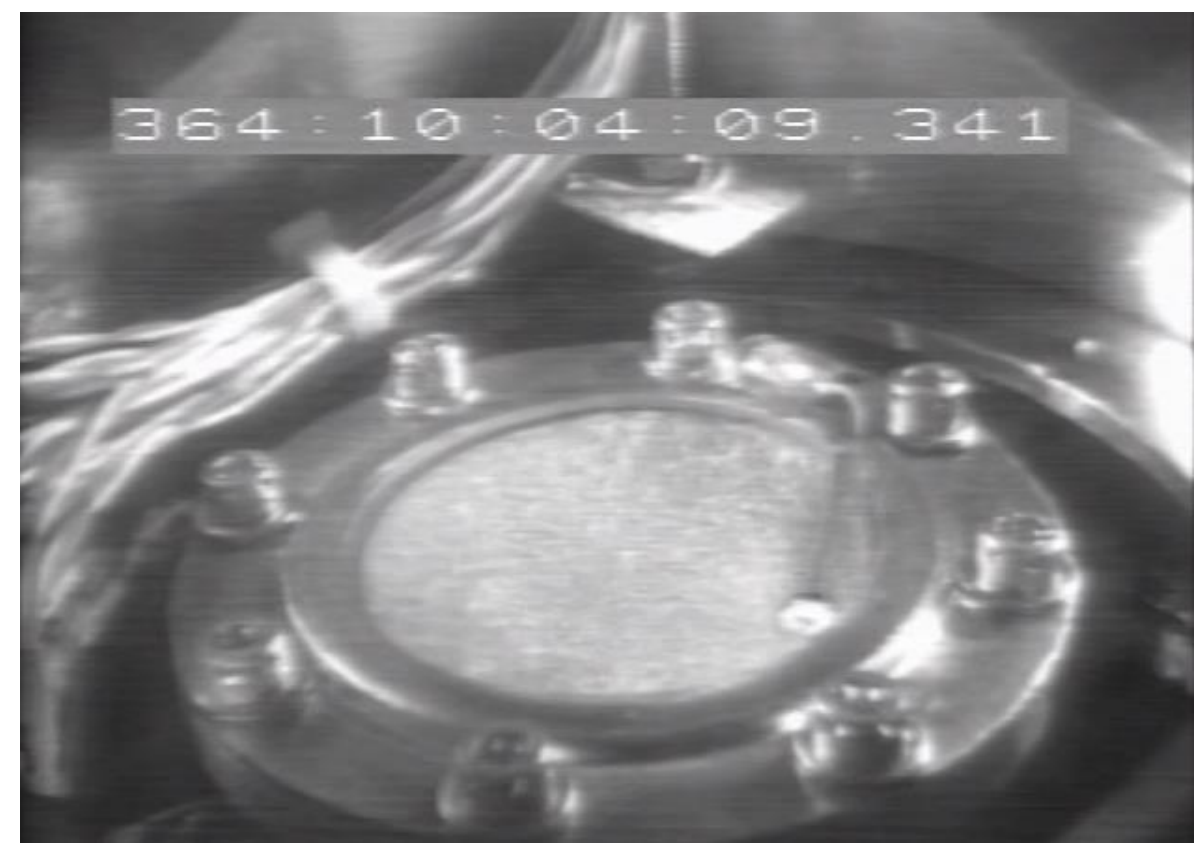

e) Gaseous Nitrogen Pressurization at a Low Screen Reynolds Number $(\approx 250)$

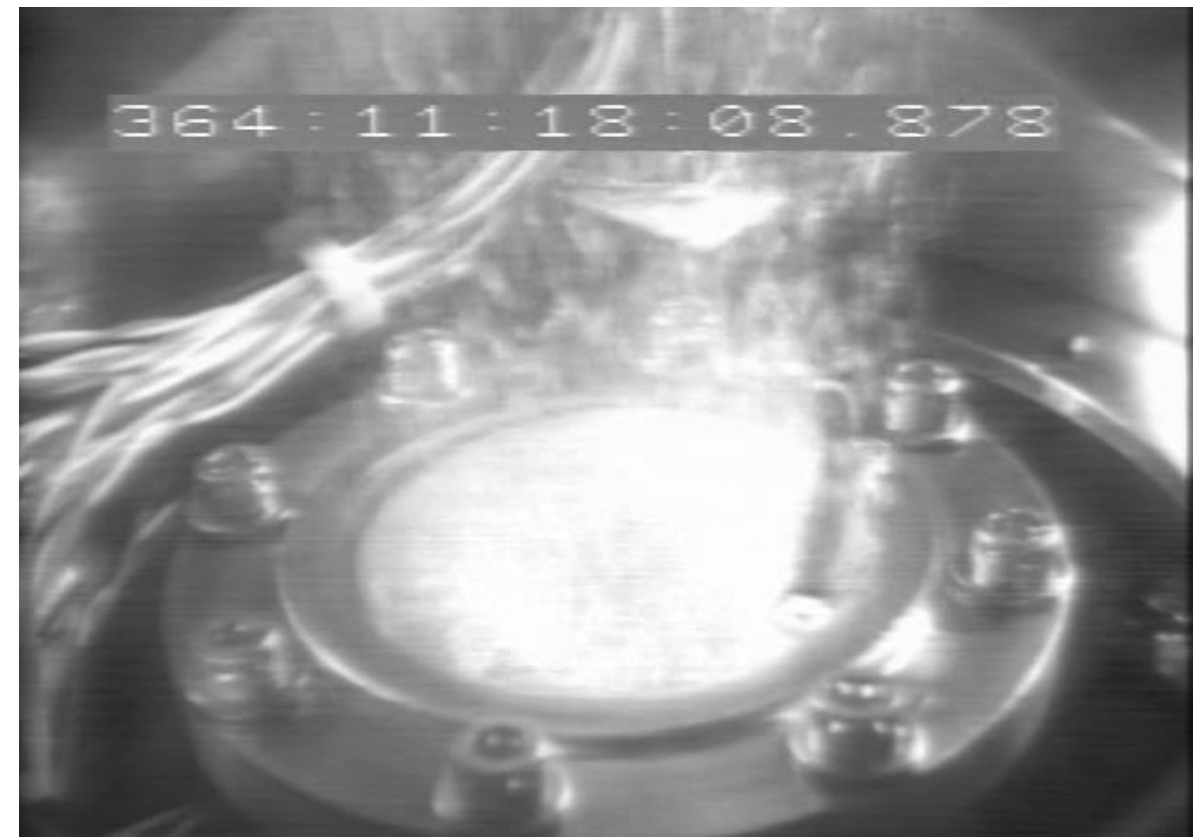

f) Gaseous Nitrogen Pressurization at a High Screen Reynolds Number $(\approx 2400)$

Evidence that the LAD screen is conducting heat is observed by looking at the difference between the calculated interfacial temperature and the temperature of the 
screen on the liquid side for the autogenous pressurization case, as shown in Figure 7.25 for $\mathrm{GCH}_{4}$ pressurization. This temperature difference is a measure of how much heat is being conducted into the liquid at the screen. For low screen pressures the interfacial temperature is slightly warmer than the screen temperature, but as the pressure and the level of subcooling increases the screen becomes hotter than the interface, reaching a maximum temperature difference of $8 \mathrm{~K}$ at the highest level of subcooling. This indicates that as the level of condensation increases, the screen absorbs more heat compared to the interface. It is therefore important to quantify the heat transfer occurring at the interface because it could be transferred along the LAD screen, leading to unwanted heating of the liquid and premature breakdown of the LAD.

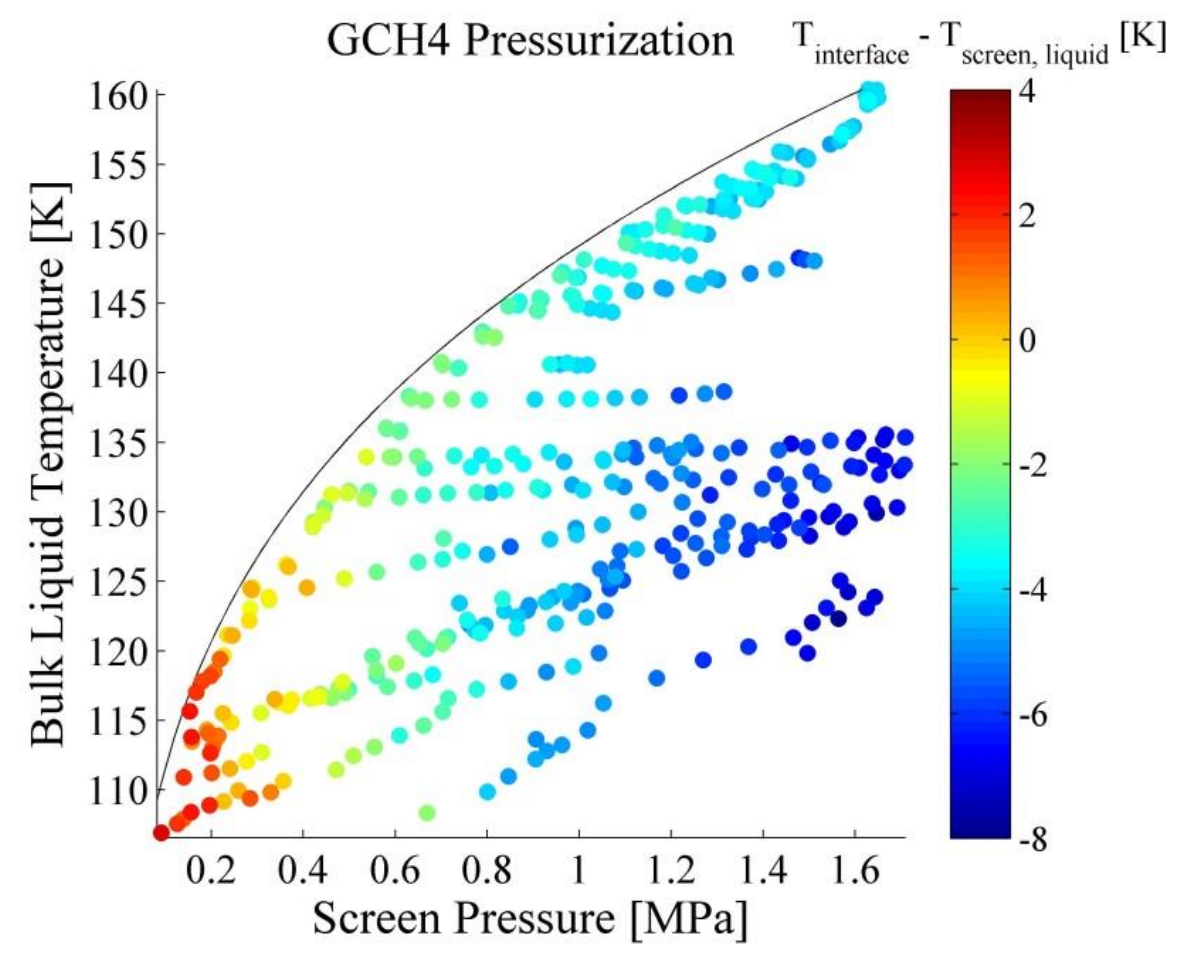

Figure 7.25 - Calculated Difference between Interface and Screen Liquid Side Temperature as a Function of Bulk Liquid Pressure and Temperature using Gaseous Methane to Pressurize 
The net heat flux that is transferred into the interface due to phase change (for the autogenous pressurization case) is

$$
\Phi_{I}=\frac{\dot{m}_{S} h_{f g}}{A_{C}}
$$

The plot is shown in Figure 7.26, with the highest heat flux being approximately 5 $\mathrm{MW} / \mathrm{m}^{2}$ and the lowest being $-2 \mathrm{MW} / \mathrm{m}^{2}$ (negative flux signifying evaporation and, therefore, energy leaving the interfacial location). Compare these high heat flux values with those found in the literature associated with boiling heat transfer (e.g. Mudawar 2001). While the calculated heat fluxes are specific to the experimental design of this study, the trends that are elucidated are applicable to a flight scenario. The regions of high heat flux into the interface shown in Figure 7.26 are governed by the screen properties, the liquid propellant, and the pressurant gas. As long as the same pressurant/propellant combination is used with the same type of screen weave, the same subcooling effect will occur, even for a full-sized flight LAD.

\subsection{Concluding Remarks}

Bubble point data for a 325x2300 Dutch Twill screen was obtained over a wide range of thermal conditions inside an elevated pressure liquid methane propellant tank using three different pressurant gases. Results here in $\mathrm{LCH}_{4}$ echo results in $\mathrm{LOX}$ in that bubble point is a strong function of liquid temperature and a weak function of the level of subcooling over a $60 \mathrm{~K}$ temperature range. The simplified model agrees well with experimental bubble points obtained in a saturated liquid state, but deviates from data for subcooled states. Subcooling the liquid always adds margin in bubble point, but the 
specific gain in performance is dependent on the type of gas in contact with the screen.

The highest bubble points are always obtained in the coldest liquid states, when

pressurizing and subcooling with helium. The bubble point data for saturated liquid states is highly linear with surface tension in liquid methane over a $60 \mathrm{~K}$ interval.

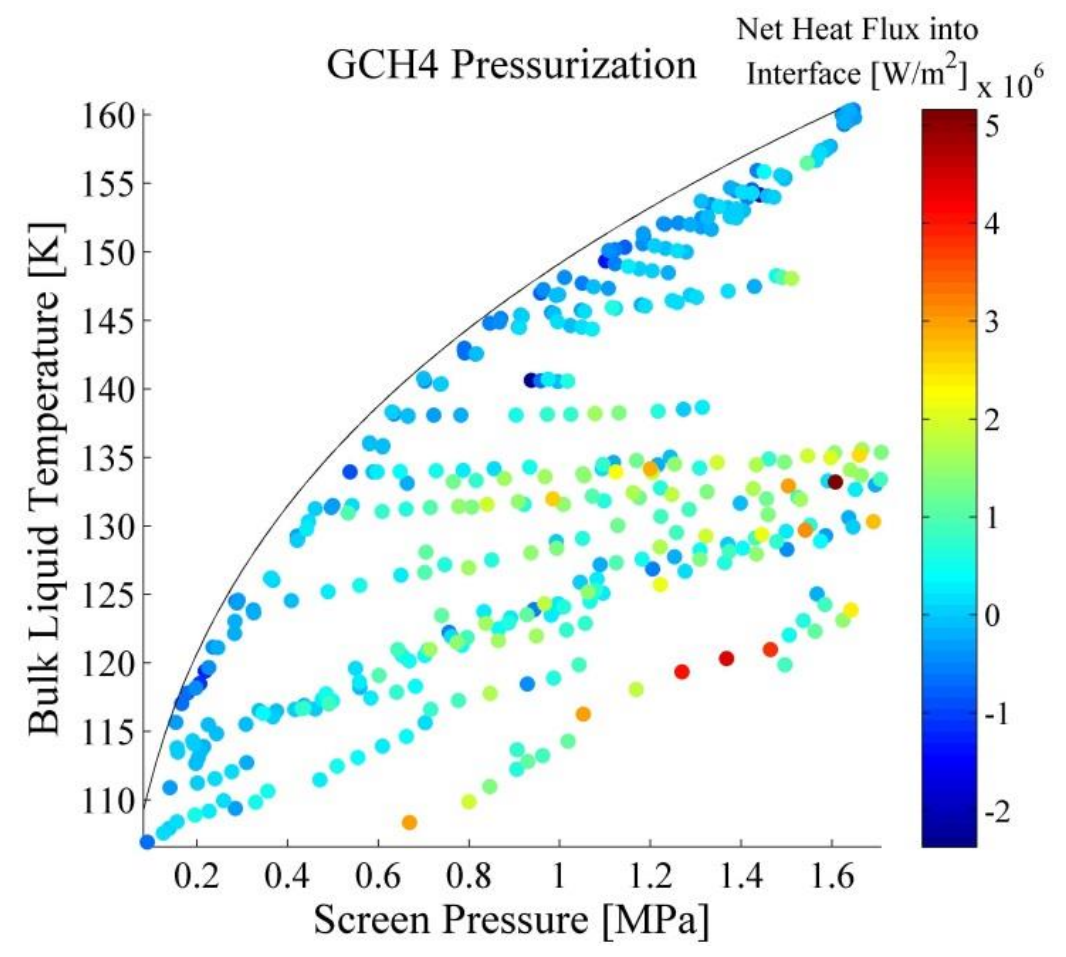

Figure 7.26 - Net Heat Flux into the Interface at Bubble Breakthrough as a Function of Liquid Pressure and Temperature using Gaseous Methane to Pressurize

The pressure dependence (subcooling dependence) on bubble point modifies the temperature dependence through added heating or cooling of the liquid/vapor interface through condensation and/or evaporation. Pressurization with a non-condensable like helium and nitrogen causes evaporation at the screen, lowering the interfacial temperature, increasing surface tension and thus bubble point. Meanwhile pressurization with the condensable vapor causes bubbles to condense into the liquid, raising the 
interfacial temperature, decreasing methane surface tension and thus bubble point. Differences in performance between the two non-condensable gases is likely due to differences in solubility in $\mathrm{LCH}_{4}$ as well as the ability of the pressurant gas to effectively evaporate liquid away from the screen, thus lowering the effective interfacial temperature. All of these trends are validated through flow visualization of the LAD screen. Experiments, thermal analyses, and flow visualization can be combined to qualitatively assess optimal operating ranges for a high pressure liquid methane propellant tank.

\subsubsection{Pressurization with Gaseous Methane}

Thermal analysis and flow visualization show that large amounts of condensation occurred with autogenous pressurization, and that the amount of condensation increased as the liquid was subcooled. Figure 7.20a shows that condensation fluxes were most severe for screen pressures above $1 \mathrm{MPa}$ with the largest calculated condensation flux being approximately $16 \mathrm{~kg} /\left(\mathrm{m}^{2} * \mathrm{~s}\right)$ at screen breakdown. Condensation fluxes were very low, however, for bubble point data collected at liquid conditions close to the saturation curve. Furthermore, autogenous pressurization led to a high degree of interfacial heating which corresponded with the observed condensation fluxes. Additionally, autogenous pressurization yielded turbulent screen Re numbers as high as 28,000 . This shows that a large amount of convective mixing and mass transfer occurs at the screen near the time of bubble breakthrough. Therefore, if an autogenous pressurization scheme is used for $\mathrm{LCH}_{4}$, to optimize operation for the LAD, operating conditions in the propellant tank should be kept below $1 \mathrm{MPa}$ and at temperatures as close to the saturation curve as possible, while retaining the amount of subcooling that is desired. The regions nearest the 
saturation curve had the lowest condensation fluxes as well as the lowest amount of interfacial heating. Qualitatively analyzing Figure 7.17a, it is recommended to keep the bulk liquid temperature above $135 \mathrm{~K}$ to minimize the amount of interfacial heating. The optimal operating region for $\mathrm{GCH}_{4}$ pressurization in the propellant tank is outlined in red in Figure 7.27.

Suggested Operating Regimes for Pressurization of

LCH4 with GCH4, GHe, and GN2

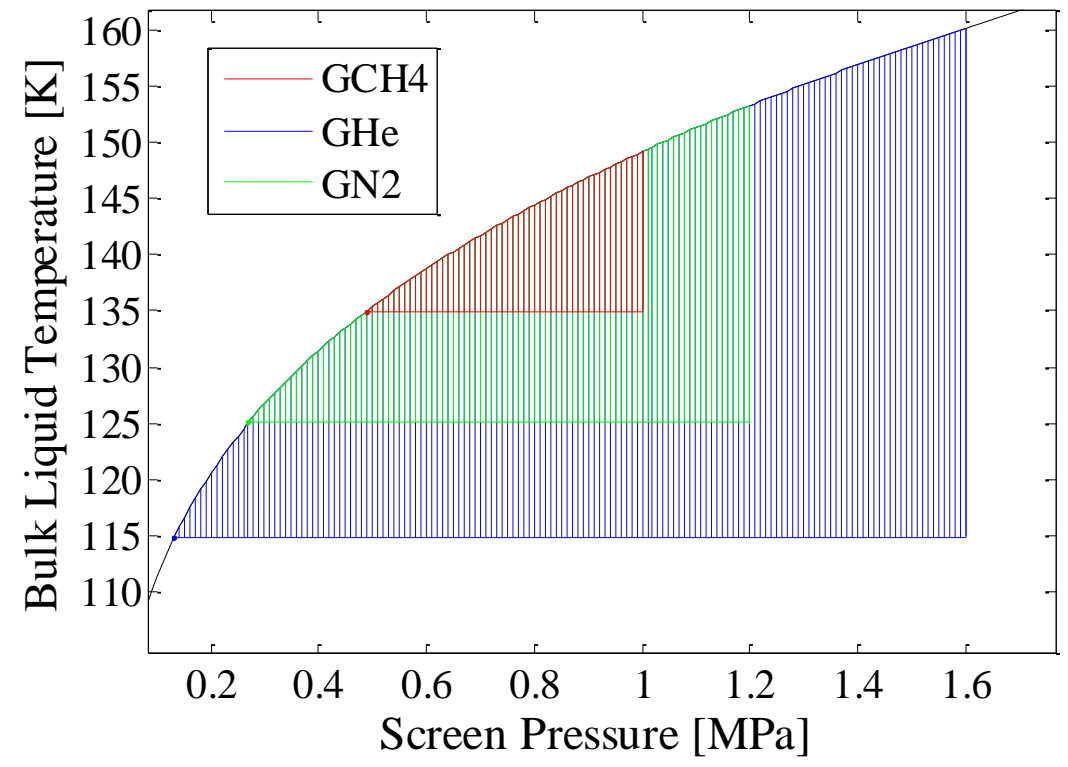

Figure 7.27 - Qualitative Operating Regimes for Different Pressurant Gases with Liquid Methane

\subsubsection{Pressurization with Gaseous Helium}

When pressurizing with non-condensable GHe, evaporation was the dominant effect. Figure $7.20 \mathrm{~b}$ shows that the condensation fluxes, while marginal at most temperatures and pressures, did increase slightly at screen pressures around 1.6 MPa and above. Nevertheless, even at these pressures, the condensation flux remained at or below $2 \mathrm{~kg} /\left(\mathrm{m}^{2} * \mathrm{~s}\right)$. Additionally, GHe pressurization led to screen Re numbers on the orders of 
10-100. This shows that not much mass transfer or convective heat transfer was occurring near the LAD screen during bubble breakthrough. Figure $7.17 \mathrm{~b}$ clearly shows that the least amount of interfacial heating occurred with GHe pressurization. The interface cooled off compared to the bulk liquid temperature due to evaporation effects. However, Figure $7.17 \mathrm{~b}$ also shows that for GHe pressurization the largest degree of interfacial heating occurred at bulk liquid temperatures below roughly $115 \mathrm{~K}$. Taking these results into account, a suggested operating range for pressurizing $\mathrm{LCH}_{4}$ with $\mathrm{GHe}$ is at bulk liquid temperatures above $115 \mathrm{~K}$ and screen pressures below 1.6 MPa. This region is also plotted in blue in Figure 7.27.

A benefit to pressurizing $\mathrm{LCH}_{4}$ with $\mathrm{GHe}$ is the large amount of interfacial cooling due to evaporation at the interface. Even though evaporation also occurs with $\mathrm{GN}_{2}$ pressurization, the higher molecular weight nitrogen molecules serve as a greater barrier to the evaporating methane molecules than do the lighter helium atoms. This results in more evaporation taking place with GHe pressurization, which leads to a higher degree of interfacial cooling. This can be seen in Figure 7.17. However, there are cons to GHe pressurization as well, with one being its higher cost compared to that of $\mathrm{GN}_{2}$. Furthermore, since GHe has less drive to liquefy in $\mathrm{LCH}_{4}$ compared to $\mathrm{GCH}_{4}$ and $\mathrm{GN}_{2}$, if vapor ingestion does occur, it would be extremely difficult to condense out GHe bubbles. Nonetheless, GHe pressurization leads to the widest range of operating conditions as well as the highest bubble point pressures. 


\subsubsection{Pressurization with Gaseous Nitrogen}

Pressurization with $\mathrm{GN}_{2}$ had trends which were intermediary between autogenous pressurization with $\mathrm{GCH}_{4}$ and $\mathrm{GHe}$. The calculated condensation fluxes were nowhere near those seen with autogenous case, but mass flow from the gaseous side of the screen to the liquid side was much more significant for $\mathrm{GN}_{2}$ pressurization than for $\mathrm{GHe}$ pressurization. Figure $7.20 \mathrm{c}$ shows that condensation fluxes were most severe at screen

pressures above 1.2 MPa. While condensation fluxes were mostly around $2-4 \mathrm{~kg} /\left(\mathrm{m}^{2} * \mathrm{~s}\right)$, at high screen pressures fluxes in excess of $8 \mathrm{~kg} /\left(\mathrm{m}^{2} * \mathrm{~s}\right)$ were seen. Additionally, $\mathrm{GN}_{2}$ pressurization resulted in interfacial cooling slightly less than that of GHe. A qualitative assessment of Figure 7.17c shows that most of the interfacial heating occurred at bulk liquid temperatures below $125 \mathrm{~K}$. Furthermore, $\mathrm{GN}_{2}$ pressurization yielded screen Reynolds numbers on the order of 100-1000. This implied that, depending on the thermodynamic state of the liquid propellant, convective heat transfer and mass transfer could be significant during bubble breakthrough. Thus, a suggested operating regime for pressurization of $\mathrm{LCH}_{4}$ with $\mathrm{GN}_{2}$ is at screen pressures below $1.2 \mathrm{MPa}$ and bulk liquid temperatures above $125 \mathrm{~K}$. This region is plotted in green in Figure 7.27.

Benefits involved with $\mathrm{GN}_{2}$ pressurization are its lower cost compared to $\mathrm{GHe}$ and its higher bubble point pressure compared to $\mathrm{GCH}_{4}$. However, $\mathrm{GN}_{2}$ pressurization cannot provide as high of a bubble point pressure as GHe pressurization. In addition, interfacial heating does occur at high levels of liquid subcooling for pressurization with $\mathrm{GN}_{2} . \mathrm{GN}_{2}$ pressurization seems to be an intermediary case and has elements of both of the other pressurization schemes outlined in this study; therefore, it has some of their benefits and drawbacks as well. 
Results presented here have implications in the design of LADs for in-space cryogenic propellant tanks and the type of pressurization system considered for a $\mathrm{LOX} / \mathrm{LCH}_{4}$ propulsion system. The $325 \times 2300$ screen channel LAD is capable of delivering and sustaining the high flow rates required to feed liquid to a pressure-fed AME or RCS through elevated bubble points. Pressurization with a non-condensable gas may be preferable over autogenous pressurization in a high pressure propellant tank if a higher bubble point is desired. In either pressurization scheme, subcooling the liquid acts to increase the total allowable flow rate to the engine by increasing the margin in the bubble point of the screen, but the rate of gain is dependent on which gas is in contact with the LAD screen. Pressurizing and subcooling the 325x2300 screen liquid/vapor interface with GHe from the NBP at room pressure up to a pressure of $1.72 \mathrm{MPa}(250$ psia) results in an increase in bubble point of approximately $38 \%$ over the baseline NBP value. 


\section{Chapter 8}

\section{Warm Pressurant Gas Effects on the Static}

\section{Bubble Point Pressure for Cryogenic Liquid}

\section{Acquisition Devices}

The purpose of this chapter is to present the experimental results for the static liquid hydrogen and nitrogen bubble point tests using warm pressurant gas at the screen interface. The purpose of the test series was to determine the effect of elevating the temperature of the pressurant gas on LAD performance. The gas temperature represents the sixth and final parameter to be varied in the parametric investigation of the parameters that affect LAD performance in a low pressure cryogenic propellant tank. Three Dutch Twill screens (325x2300, 450x2750, and 510x3600) were tested in liquid hydrogen and liquid nitrogen using cold and warm non-condensable (helium) and condensable (hydrogen or nitrogen) pressurant gases. The gases were conditioned $0-$ $120 \mathrm{~K}$ above the liquid cryogen temperature. Details are also presented on hardware modifications from cold gas bubble point testing. 


\subsection{Test Purpose and Motivation}

The purpose for conducting these tests is to experimentally determine the effect of elevating the temperature of the pressurant gas above the cryogenic liquid temperature on LAD performance in a relevant thermal environment. All bubble points collected in Chapters $4-7$ were taken with the gas temperature approximately equal to the liquid temperature. Most historical bubble point data was also taken with nearly equivalent gas and liquid temperatures. However, in a real flight application, without the presence of thermal links between Composite Overwrap Pressure Vessel (COPV) pressurant gas tank and cryogenic propellant tank, it is anticipated that the pressurant gas temperature may be warmer than the cryogenic propellant liquid temperature. Therefore warm pressurant gas data will yield valuable information for a system designer desiring knowledge on both the LAD and pressurization subsystems.

The background and motivation for obtaining warm pressurant gas bubble point data is as follows: During spaceflight, there are two primary sources of heat leak into the tank as indicated by the red arrows in Figure 8.1; one associated with storage and one with transfer of the propellant. Radiation and conduction heat leak enters the tank through the support struts, fill, vent, and instrumentation penetrations. Mitigation strategies to reduce heat leak are straightforward; passive thermal control is used to reduce heat leak by using thick MLI blankets, composite struts, and optimizing the MLI layer density, while active thermal control techniques use a HEX to reduce or eliminate propellant boil off. A second source of heat leak into the tank is from warm pressurant gas contacting the cold liquid propellant during liquid transfer. Mitigation strategies here are complicated. From a systems level standpoint, it is highly desirable to use warm gas as a pressurant 
because less mass is required to thermally condition the gas to tank conditions. From the LAD subsystem standpoint however, it is desirable to use cold gas because LADs are surface tension driven devices, and as shown for all of the four primary cryogenic liquids, colder temperatures always result in better performance.

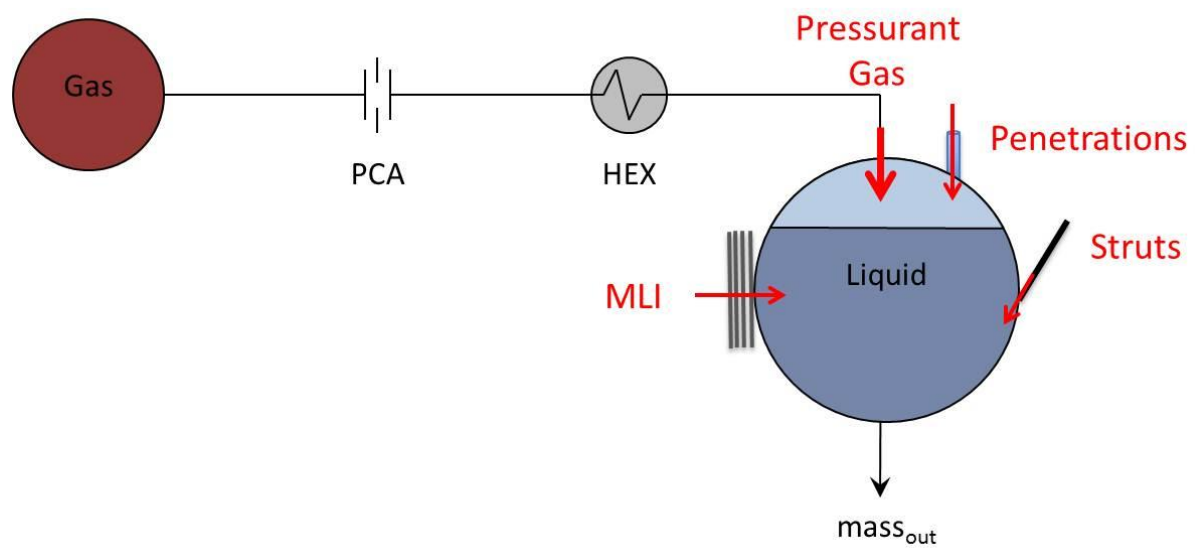

Figure 8.1 - Sources of Heat Leak into a Cryogenic Propellant Tank. Shown in the pressurant gas transfer lines are high pressure Composite Overwrapped Pressure Vessel, Pressure Control Assembly (PCA), and HEX.

During spaceflight, the pressurant gas typically assumes the environmental temperature, which is warmer than the saturation temperature for cryogenic propellants at atmospheric pressure. Even if the pressurant tank bottle is thermally linked to the propellant tank, the pressurant gas temperature will still be warmer than the liquid temperature. As will be shown later, warm pressurant will always decrease the surface tension of the cryogen, consequently, degrading the LAD performance. Clearly an optimal design point between the pressurization and LAD subsystems exists for each mission. In low gravity, warm gas will not impinge on the LAD screen until low tank fill levels, but tests are warranted to quantify the effect of heat absorption into the liquid on 
the LAD performance because warm gas will adversely affect the expulsion efficiency of the LAD.

In regard to the pressurization subsystem, there are two ways to pressurize a cryogenic propellant tank during liquid transfer, the vapor case and the non-condensable case. The advantages and disadvantages of both pressurization schemes were previously addressed in Sections 3.10 and 7.5. The goal is thus to give mission designers direct insight into the combined LAD and pressurization subsystem performance and design for future cryogenic engines and cryogenic fuel depots.

Screen channel LADS have flight heritage with storable propellants where heat transfer effects are not as severe as they are for cryogenic propellants. Before these LADs can be routinely used in cryogenic propulsion systems, the effects of undesirable heat on the LAD must be fully quantified. This environmental parasitic heat leak into the tank or heat input from warm pressurant gas may adversely affect LAD performance by vaporizing the liquid and drying out the LAD screen.

In addition, examination of Equation 3.16 shows that the current bubble point model does not account for variations in pressurant gas temperature, so warm gas data will be valuable for improving the prediction for cryogenic systems. Previous attempts were made to quantify the effect of warm gas on LAD degradation in the literature, but there were numerous reported inconsistencies in results as well as issues reported with the test apparatus. Historical attempts to quantify this effect are reserved for Appendix E. Clearly, carefully designed and controlled tests are required to accurately quantify the effect of warm pressurant gas on LAD performance. 


\subsection{Design Modifications}

Warm pressurant gas testing was performed at the CCL-7 at NASA GRC. The same exact 325x2300, 450x2750, and 510x3600 Dutch Twill screen samples from Chapters 4 and 5 were used here. Tests were conducted in both $\mathrm{LH}_{2}$ and $\mathrm{LN}_{2}$ using both autogenous and non-condensable pressurization schemes. Testing was performed in a parametric fashion as outlined in Chapter 5.

The same screen and cup assembly and low pressure dewar that were used in cryogenic testing in Chapter 5 were used here. The cup was equipped with a central support rod as shown in Figure 8.2 which supported three donut style thin film Kapton heaters, which warmed the incoming pressurant gas to the desired temperature before incidence on the LAD screen. A heater was placed on the underside of the bottom and top disc, and the top of the bottom disc, which eliminated view factors between heating source and LAD screen, and ensured that all of the heating was via conduction and convection of the incoming pressurant gas. This design forced uniform heating and pressure rise within the cup. It also eliminated direct warm gas impingement on the screen. $250 \mathrm{~W}$ of power were available for warming the pressurant gas. Bubble point pressure was deduced from the raw DPT measurement from the sense line shown in Figure 5.2. The sensing port pointed away from the screen to eliminate flooding of the line and to eliminate two phase DPT signals. 


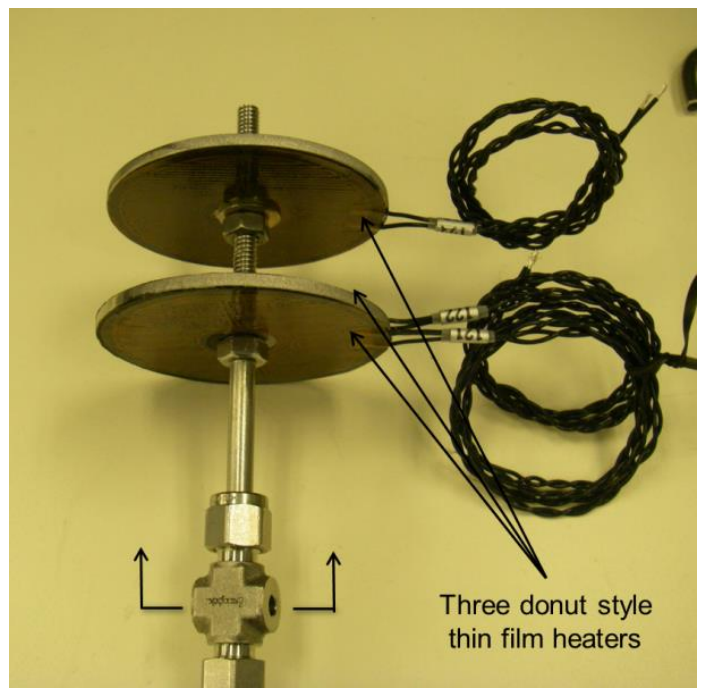

Figure 8.2 - Warm Pressurant Gas Heater Bank

Liquid temperature and pressure were controlled in a similar manner as outlined in Chapter 5. Temperature of the pressurant gas was monitored using a silicon diode inside the LAD cup, and was controlled by cycling on or off the heaters, which were controlled through a tight PID loop using a silicon diode (SD3) inside the LAD cup. All temperatures were measured using silicon diodes as point sensors. Critical measurements for heated pressurant gas tests were the temperature of the liquid and gas side of the LAD screen, temperature of the pressurant gas, pressure of the ullage, DPT across the LAD screen, and pressurant gas mass flow rates into the cup. All data was recorded at $5 \mathrm{~Hz}$ with a computer DAQ. Videos of the LAD screens were again time stamped and recorded to compare with the time stamp in the data. Uncertainties for all measurements are listed in Chapter 5. The total uncertainty in the corrected bubble point pressure is estimated to be $12 \mathrm{~Pa}$, which is $10 \%$ uncertainty in the lowest reported bubble point pressure for the heated gas runs. This low absolute error in measurement relative to previously reported cold gas tests was made possible by modifications in the DAQ system. 


\subsection{Experimental Methodology}

To conduct a heated pressurant gas bubble point test, a GHe flow was first established across the screen during liquid fill of the dewar to prevent flooding of the cup. Heated pressurant gas testing here was always conducted in above atmospheric pressure conditions, which eliminated the need to pre-condition the liquid. The liquid temperature only varied between a small range of $20.5 \mathrm{~K}-21.0 \mathrm{~K}$, which allowed independent examination of the effect of heated pressurant gas on LAD performance. When the dewar was filled to the desired liquid level, the pressurant gas type was selected and allowed to flow through the flow network for a period of 10 minutes or more. The screen was then allowed to reseal. Next, the heaters were engaged until the desired temperature of the pressurant gas was achieved. The gas was always allowed several minutes residence time to come to equilibrium at the desired gas temperature before attempting a controlled breakthrough. Then the pressure underneath the LAD screen was slowly increased until a bubble broke through the wetted screen as indicated on the live video and in the sharp rise in the DPT signal. The screen was then resealed. Bubble points were repeated at similar pressurant gas temperatures for repeatability before moving on to the next gas temperature. Due to the low surface tension of $\mathrm{LH}_{2}$ and low baseline cold gas bubble point values, it was sometimes difficult during testing to even reseal the screens. Raw bubble point measurements were again corrected for liquid head pressure using the diode rake inside the dewar used to sense liquid level. 


\subsection{Test Matrix}

The test matrix consisted of the following: Three fine mesh screen channel LAD samples $\left(325 \times 2300,450 \times 2750\right.$, and 510x3600) were tested in two cryogenic liquids $\left(\mathrm{LH}_{2}\right.$ and $\mathrm{LN}_{2}$ ), with two pressurization schemes (autogenous $\mathrm{GH}_{2} / \mathrm{LH}_{2}$ and $\mathrm{GN}_{2} / \mathrm{LN}_{2}$ and noncondensable $\mathrm{GHe} / \mathrm{LH}_{2}$ and $\mathrm{GHe} / \mathrm{LN}_{2}$ ) at several different pressurant gas temperatures. Bubble point data was first collected using cold pressurant gases. The cold baseline temperature was approximately equal to the liquid temperature to minimize the temperature gradient across the screen at breakdown. This was only possible because the LAD cup was immersed in the liquid. To collect data at elevated temperatures, the heaters were engaged to several different fixed gas temperatures over the range of $0-$ $120 \mathrm{~K}$ above the liquid temperature, and measurements repeated to allow comparison with the cold gas data using Equation 3.16.

\subsection{Warm Pressurant Gas Liquid Hydrogen Experiments}

Figure 8.3 summarizes the effect of changing the screen weave, liquid temperature, and pressurant gas on the $\mathrm{LH}_{2}$ bubble point pressure by plotting data for all three screen meshes and both pressurant gases from Chapter 5. Error bars are plotted. The trends are as follows: First, for all three meshes, and for both pressurant gases, bubble point decreases with increasing liquid temperature, due to decreasing surface tension. The highest bubble point pressures are always obtained in the coldest liquid temperatures, regardless of screen or gas type. Second, for all three meshes, regardless of liquid temperature, bubble points obtained using the non-condensable gas are always higher than those obtained using the condensable gas. Gaseous helium adds margin to the bubble 
point pressure while $\mathrm{GH}_{2}$ acts like a degradation factor. The disparity between pressurization schemes is relatively fixed for all three LAD screen meshes tested here.

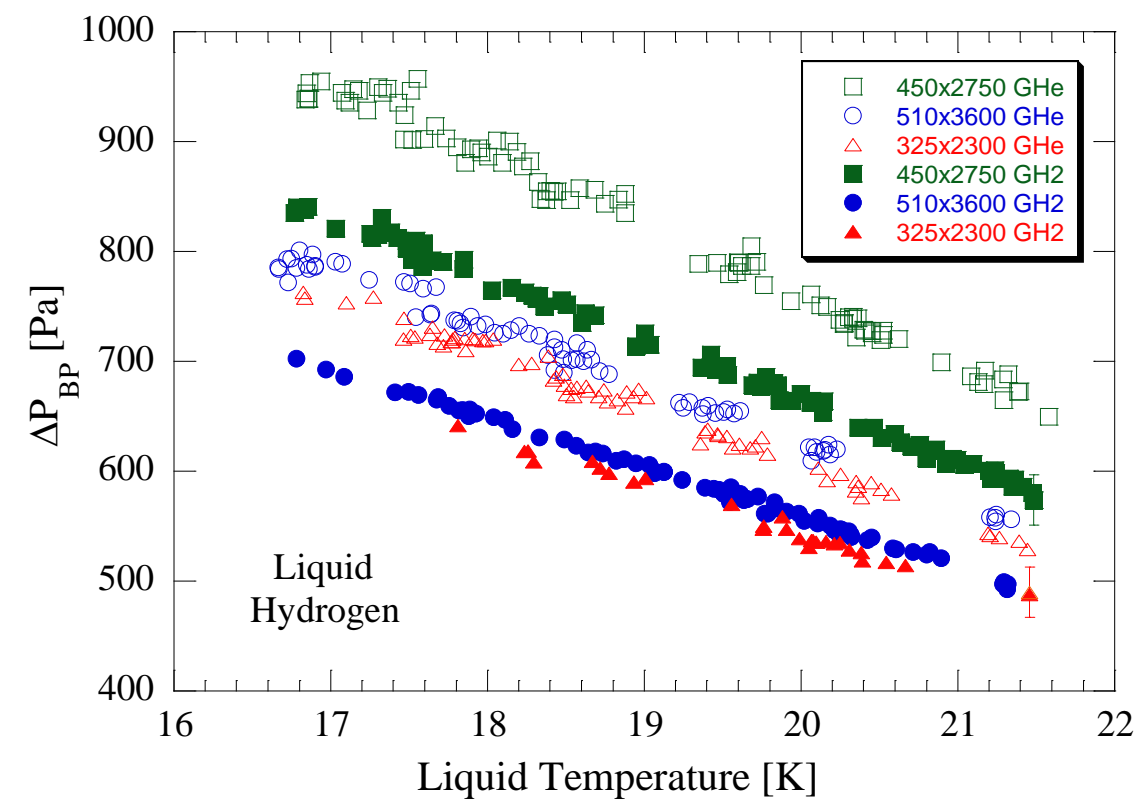

Figure 8.3 - Cold Gas Liquid Hydrogen Bubble Point as a Function of the Liquid Screen Side Temperature

Third, for all liquid temperatures and for both pressurant gases, bubble point pressure does not scale with the mesh of the screen. This is the most complex trend of the original five parameters tested. The second finest 450x2750 mesh produced the highest bubble points, for both $\mathrm{GHe}$ and $\mathrm{GH}_{2}$. The 510x3600 mesh outperformed the 325x2300 mesh at $\mathrm{LH}_{2}$ temperatures, but the $325 \times 2300$ yielded higher pressures in room temperature liquids. The reason for this crossover in performance is due to the temperature dependence of the screen pore diameter and geometry of the actual $\mathrm{L} / \mathrm{V}$ interface at breakdown, as mentioned previously. The 510 screen has the largest gain at $\mathrm{LH}_{2}$ temperatures over the room temperature bubble point. 
The sixth and final parameter that was investigated was the temperature of the pressurant gas. Data is first presented on an absolute scale to allow direct comparison to cold gas data. Figure 8.4 plots all $\mathrm{LH}_{2}$ bubble point data collected using warm pressurant gas. Data is plotted as a function of the temperature difference between warm gas and cold liquid at breakthrough. Controlled breakthroughs and reseals were achieved for gas temperatures between $30 \mathrm{~K}<\mathrm{T}_{\mathrm{GAS}}<116 \mathrm{~K}$ which correspond to a temperature difference across the screen of $10 \mathrm{~K}<\Delta \mathrm{T}<95 \mathrm{~K}$. Error bars are plotted.

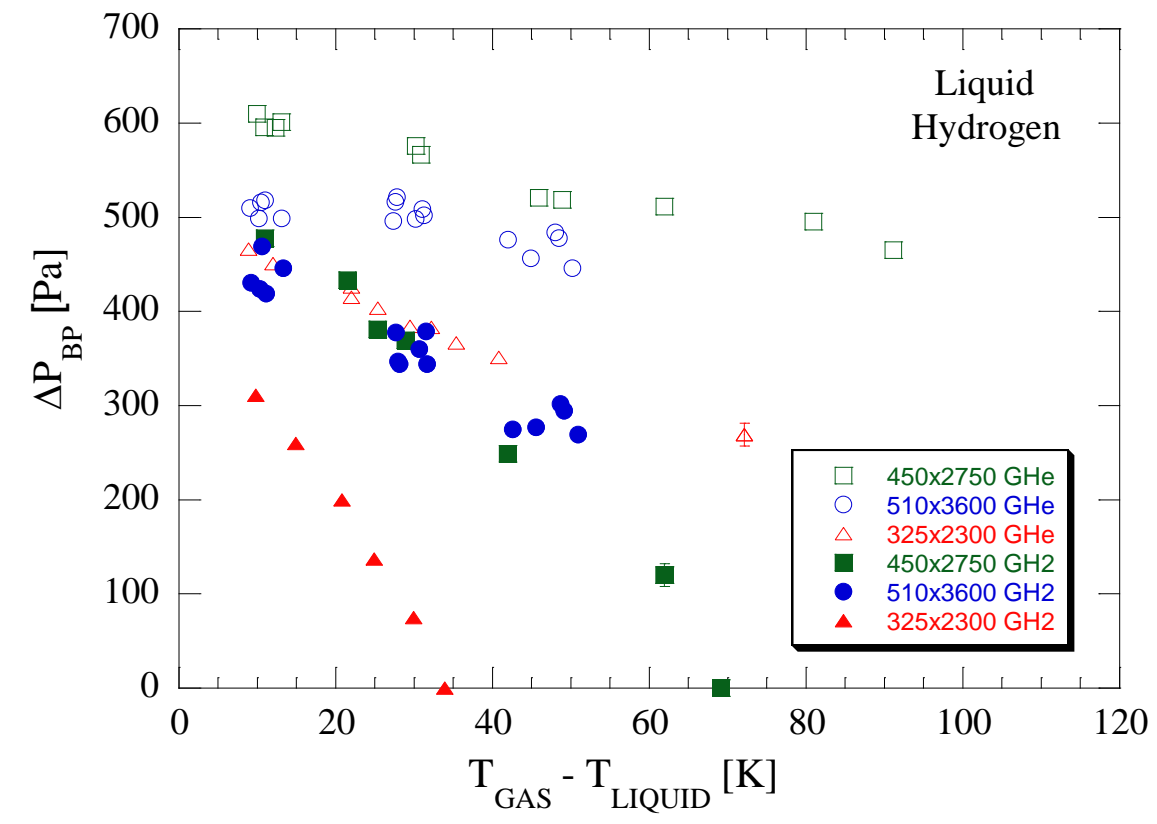

Figure 8.4 - Heated Pressurant Gas Liquid Hydrogen Bubble Point Pressure as a Function of the Temperature Difference between Pressurant Gas and Liquid

As shown for all three screens, the bubble point pressure decreases approximately linearly with increasing pressurant gas temperature. The degradation in bubble point is more pronounced using $\mathrm{GH}_{2}$ vs. pressurizing with $\mathrm{GHe}$ because the $325 \times 2300$ and $450 \times 2750$ screens failed to reseal at temperature differences of $34 \mathrm{~K}$ and $69 \mathrm{~K}$, 
respectively using the condensable gas. Meanwhile, controlled breakthroughs were achieved with all three meshes using GHe beyond temperature differences of $50 \mathrm{~K}$. Regardless of the pressurization method, the onset of degradation is immediate for gas temperatures greater than the liquid temperature. This is in contrast to previously reported $\mathrm{LH}_{2}$ heated gas bubble point pressures where the onset of degradation would be delayed and occur at a certain gas temperature.

To allow comparison between the four different LAD test methods in Appendix E and all of the historical heated gas bubble point data, a normalized bubble point ratio is defined:

$$
\frac{\Delta P_{B P}(\Delta T)}{\Delta P_{B P}(\Delta T=0)}
$$

where $\Delta T$ is the temperature difference between liquid propellant and pressurant gas. Therefore the denominator is the normal breakdown value for equal gas and liquid temperatures, and the numerator is the breakdown value when the gas is sufficiently heated above the liquid temperature (i.e. the temperature difference across the screen). The normalized ratio is plotted as a function of the temperature difference across the screen for all the historical data in Appendix E.

Figure 8.5 plots heated bubble point pressures using Equation 8.1 to normalize the data to the cold gas value obtained at the liquid temperature. Since each controlled breakthrough/reseal pair occurred at slightly different liquid temperatures from $20.5 \mathrm{~K}<$ $\mathrm{T}<21.1 \mathrm{~K}$, each point was normalized to its own cold gas bubble point pressure, as opposed to normalizing to a single value. Therefore, at a temperature difference across 
the screen of $0 \mathrm{~K}$, there is no deviation from the unheated pressurant gas bubble point ratio, by definition. Data is again plotted as a function of the $\Delta \mathrm{T}$ across the screen. Lines are simple linear fits to the data.

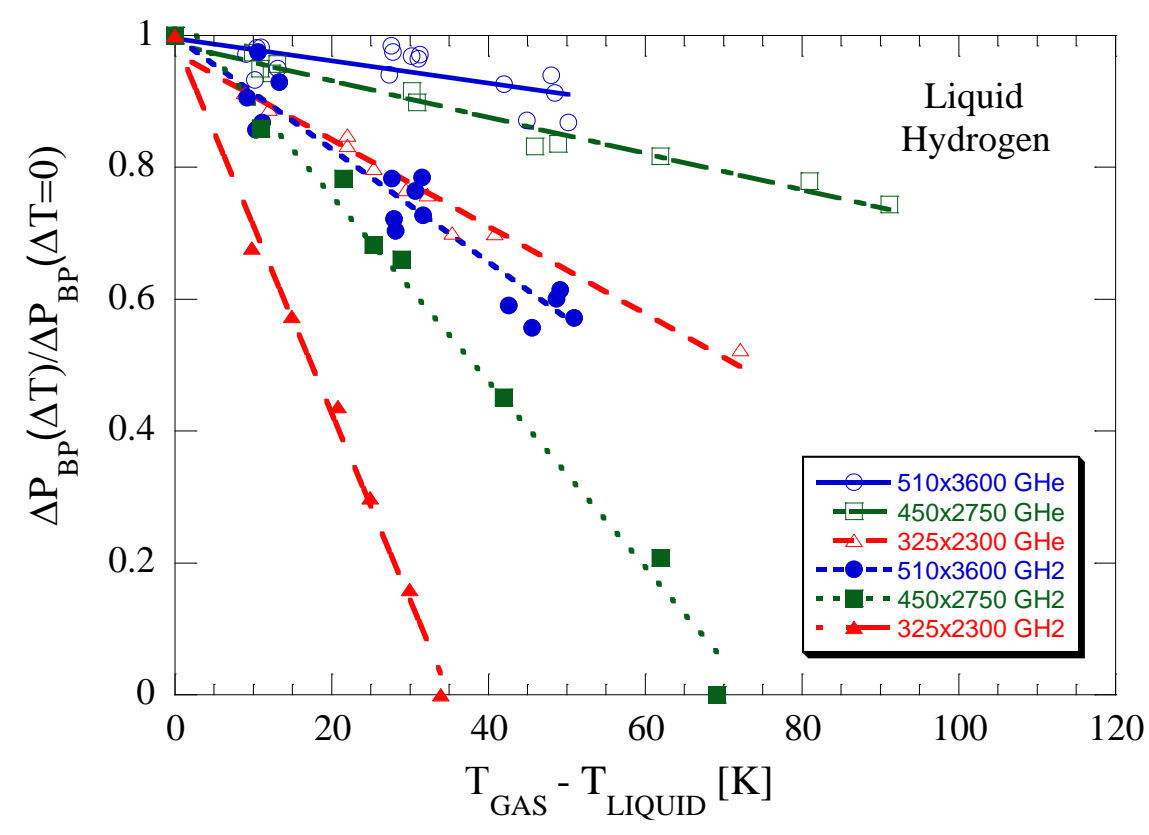

Figure 8.5 - Normalized Heated Pressurant Gas Liquid Hydrogen Bubble Point Pressure as a

Function of the Temperature Difference between Pressurant Gas and Liquid at the Screen

Normalizing the data shows three distinct trends. First, for all 3 screens and both pressurant gases, heating the gas above the liquid temperature acts as a degradation factor on the cold gas bubble point pressure. The larger the temperature difference across the screen, the earlier the screen will break down. Second, for all three screens, bubble point pressure degrades from the cold gas value much more rapidly using condensable gas than using non-condensable gas as indicated by steeper slopes in the linear curve fits. Third, for both pressurization schemes, degradation in bubble point is inversely proportional to the porosity of the screen. 
As defined in Chapter 3, porosity is the ratio of open area for fluid to flow through the screen divided by the total screen area. Computed screen thicknesses and porosities are shown in Table 3.2. Therefore higher porosities indicate more open area for the gas to interact directly with the liquid. As shown in Figure 8.5, the finest 510x3600 mesh exhibited the smallest degradation in performance over the 450x2750 and $325 \times 2300$ screen, as indicated by the slopes of the linear curve fits. This trend holds for both pressurization schemes. As is evident in Figures 8.4 and 8.5, although the 450x2750 screen outperforms the $510 \times 3600$ on an absolute basis, degradation in performance for the $510 \times 3600$ screen is less.

Coarser screens are likely to build up higher temperature differences across the screen prior to bubble breakthrough, causing the local interfacial temperature to increase significantly higher than finer meshes. Coarser Dutch Twills are thicker and have lower porosities relative to finer Dutch Twills. These longer path lengths for warm gas or vapor to travel through the screen coupled with less overall open area for gas and liquid to exchange heat and mass causes larger temperature differences to build across the screen before the actual visible warm gas bubble breaks through the screen. The finest 510 mesh is the thinnest screen and has the smallest absolute micron rating and largest porosity, making it easier for heat and mass to transfer across the screen between liquid and gas prior to breakdown. Since mass is more easily transferrable across the screen, the local gas at the screen pore will cool slightly, cooling the interface temperature relative to the coarser meshes long before the bubble breaks through the screen. Meanwhile, for the coarser mesh, the larger pore size, thicker screen, and lower porosity causes larger temperature differences across the screen to build prior to breakdown. The larger pore 
sizes and less contact area between warm gas and cold liquid prevent heat transfer from the gas into the liquid.

Coarser screens like the 200x1400 and 325x2300 act more like an insulator, resulting in large temperature differences across the screen ( $\mathrm{Li}$ and Peterson 2006). The dominate mode of heat transfer for the finer mesh screens is primarily parallel path heat conduction: conduction within the metal and conduction within the liquid phase trapped initially in the mesh. Except at breakthrough, there is no convective motion through the screen, because the tiny pore size and the liquid viscosity limit the motion.

The degradation in performance using condensable gas can also be explained as follows: As warm condensable vapor passes through the screen, it condenses into the liquid and warms the $\mathrm{L} / \mathrm{V}$ interface, reducing the surface tension and thus bubble point. At high rate phase transitions, the interface becomes unstable due to the sudden decrease of surface tension (Malyshenko and Dunikov 2002). For GHe pressurization, the small additional margin in bubble point pressure is due to the suppression of the local partial pressure of $\mathrm{GH}_{2}$ within the screen pores. Free mass transport is minimized with helium present, but heat conduction between warm gas and cold liquid still occurs at a slightly lower rate relative to $\mathrm{GH}_{2}$ pressurization. Meserole and Jones (1993) speculated that for $\mathrm{GH}_{2}$ pressurization, the liquid may be in a locally superheated state at the screen, which may cause any heat input into the liquid to produce bubbles immediately and lead to the liquid detaching from the screen. Meanwhile, for GHe pressurization, the liquid at the screen may be in a slightly subcooled state, since GHe has been shown to evaporate liquid away from the screen. 
Results are in fair agreement with Cady's (1975) results using a 325x2300 mesh and GHe; he showed a smaller degradation factor (despite higher heat transfer rates) of only $10 \%$ at a gas temperature $55 \mathrm{~K}$ above the liquid temperature. However, Cady also reported near identical degradation in performance for several different meshes, which is in disagreement with the current study. The current study is also in agreement with Burge and Blackmon's (1973b) heated $\mathrm{GH}_{2}$ NIBP data for a 250x1370 that showed steeper degradation relative to the $325 \times 2300 \mathrm{GH}_{2}$ here. Note that the $250 \times 1370$ screen has a lower porosity relative to the $325 \times 2300$ and would be expected to degrade more relative to the finer meshes.

\subsection{Warm Pressurant Gas Liquid Nitrogen Experiments}

Heated pressurant gas tests were also conducted in $\mathrm{LN}_{2}$ using the same exact screens, experimental design, and procedure. Only the DPT that was used to measure raw bubble point pressure was changed to a higher range DPT to account for the higher $\mathrm{LN}_{2}$ surface tensions, and thus bubble point, relative to $\mathrm{LH}_{2}$. Results are plotted in Figures 8.6 - 8.8. First, the cold pressurant gas bubble point pressure as a function of the liquid screen side temperature is plotted to establish the baseline reference value. Error bars are plotted but are barely distinguishable. The same trends visible in $\mathrm{LH}_{2}$ data in Figure 8.3 are reflected in the $\mathrm{LN}_{2}$ data in Figure 8.6. For all three meshes and both pressurant gases, $\mathrm{LN}_{2}$ bubble point pressure decreases with increasing liquid temperature. For all temperatures and meshes tested, pressurization with the non-condensable GHe yields higher performance over pressurization with the condensable $\mathrm{GN}_{2}$. For both pressurant gases and all liquid temperatures tested, the same trend between screen meshes is evident 
with $\mathrm{LN}_{2}$ as in $\mathrm{LH}_{2}$ tests. The second finest $450 \times 2750$ yields the highest bubble points, followed by the finest $510 \times 3600$ screen, followed by the coarsest $325 \times 2300$ mesh.

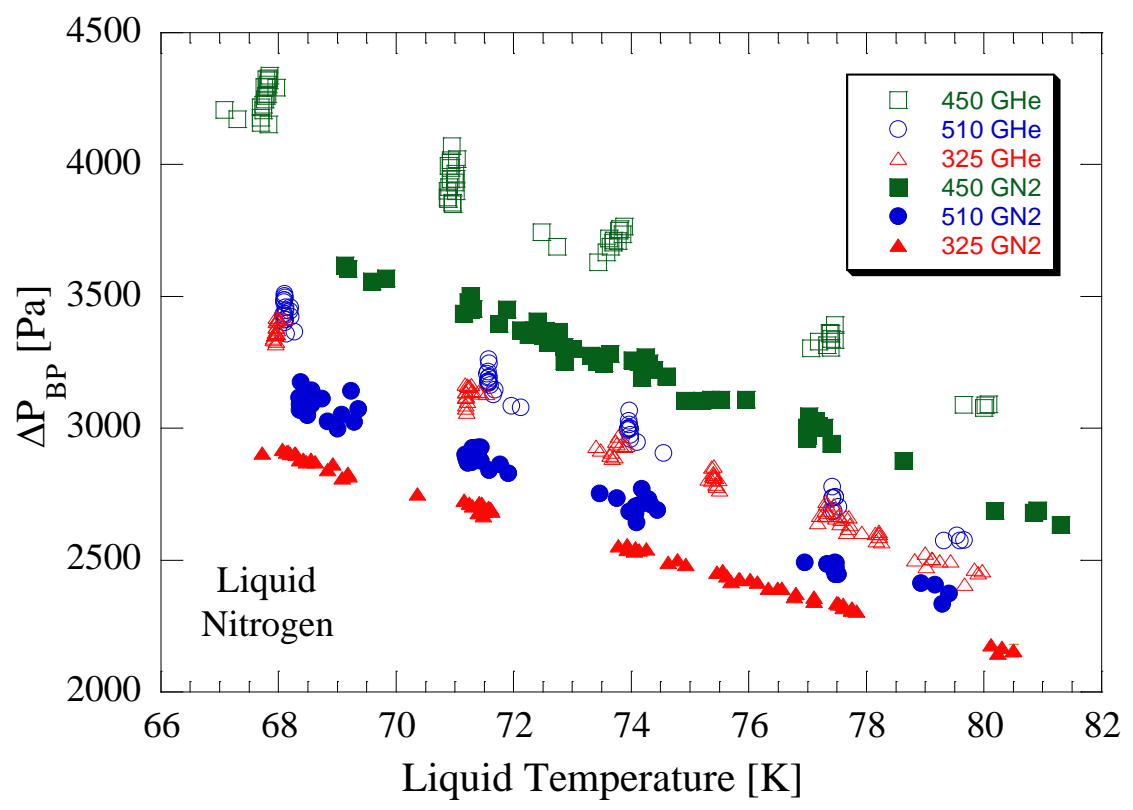

Figure 8.6 - Cold Gas Liquid Nitrogen Bubble Point as a Function of the Liquid Screen Side Temperature

Figure 8.7 plots heated pressurant gas bubble point data, on an absolute scale, as a function of the temperature difference across the screen at breakthrough. Error bars are plotted but are barely discernable. The liquid temperature for all of these tests was between the range of $79 \mathrm{~K}<\mathrm{T}_{\text {liquid }}<81 \mathrm{~K}$. Controlled breakthroughs were achieved for gas temperatures between $80 \mathrm{~K}<\mathrm{T}_{\mathrm{GAS}}<211 \mathrm{~K}$ which correspond to a temperature difference of $0 \mathrm{~K}<\Delta \mathrm{T}<131 \mathrm{~K}$. Higher gas temperatures using $\mathrm{GHe}$ and $\mathrm{GN}_{2}$ in $\mathrm{LN}_{2}$ were achieved due to greater temperature stability of the $\mathrm{LN}_{2}$ during testing; more controlled breakthroughs and reseals were achievable due to the higher $\mathrm{LN}_{2}$ surface tension. As shown for all three screens, bubble point pressure decreases with increasing pressurant 
gas temperature. The degradation is again more pronounced using the condensable gas versus the GHe case. However, examination of Figure 8.7 shows that the slopes in degradation for $\mathrm{LN}_{2}$ are not as sharp as those for $\mathrm{LH}_{2}$. In addition, for the $450 \times 2750$ and 510x3600 screens using GHe pressurization, the onset of degradation does not occur immediately.

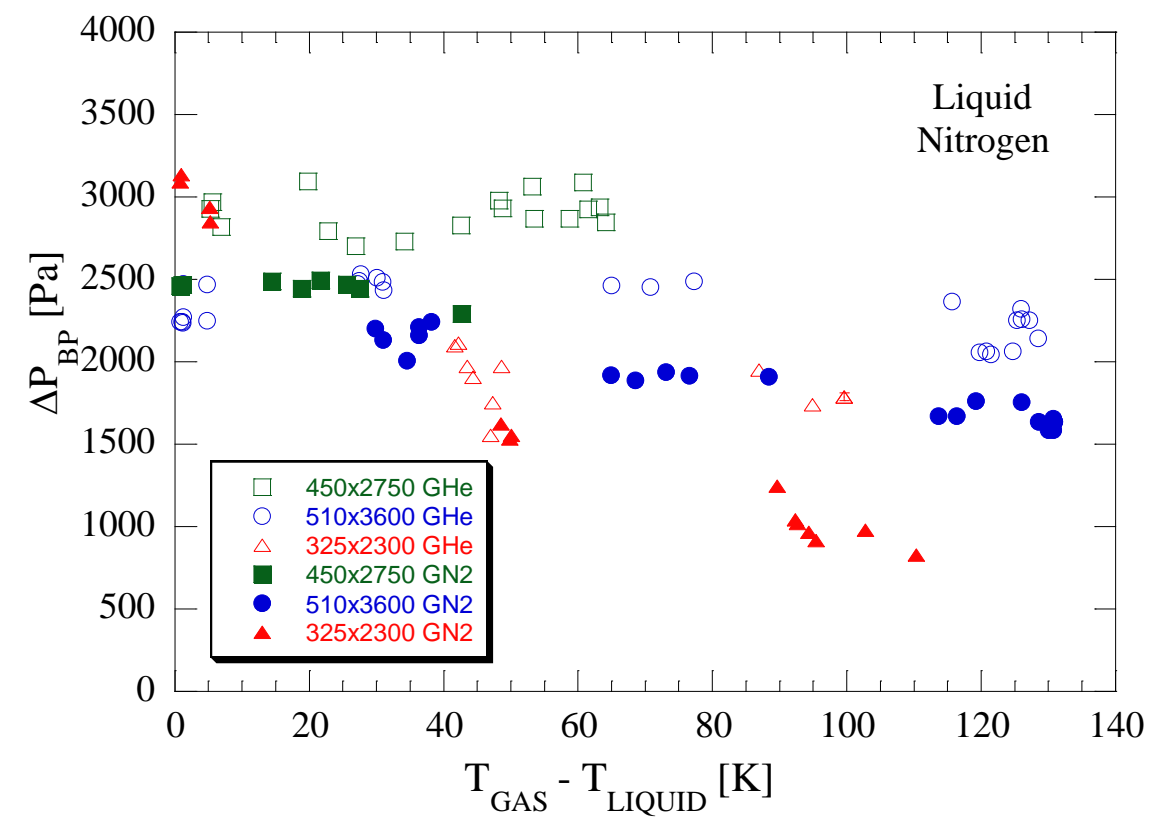

Figure 8.7 - Heated Pressurant Gas Liquid Nitrogen Bubble Point Pressure as a Function of the Temperature Difference between Pressurant Gas and Liquid

To elucidate this apparent trend, Figure 8.8 plots heated bubble point pressures using Equation 8.1 to normalize the data to each cold gas bubble point value. Data is plotted as a function of the temperature difference between liquid and gas. Lines are fits to the data from the onset of degradation. The same trends in previous $\mathrm{LH}_{2}$ data are reflected here in $\mathrm{LN}_{2}$. Warmer pressurant gas temperatures degrade the bubble point ratio. Bubble point pressures degrade quicker using $\mathrm{GN}_{2}$ over $\mathrm{GHe}$ pressurization as 
indicated by the steeper rate slopes. Degradation in bubble point is again inversely proportional to the porosity of the screen for both pressurization schemes. However, unlike $\mathrm{LH}_{2}$ results, where the onset of degradation occurred immediately, onset of degradation in performance for the 450x2750 using GHe and 510x3600 using both $\mathrm{GHe}$ and $\mathrm{GN}_{2}$ does not occur until a minimum temperature difference is achieved $(41 \mathrm{~K}, 77 \mathrm{~K}$, 21K, respectively). Compared to historical NIBP results from Castle (1972), no degradation in performance was noticed for any of the fine Dutch Twill screens, including the $200 \times 1400$ and $325 \times 2300$ screens over a much larger temperature range using $\mathrm{GN}_{2}$ pressurant.

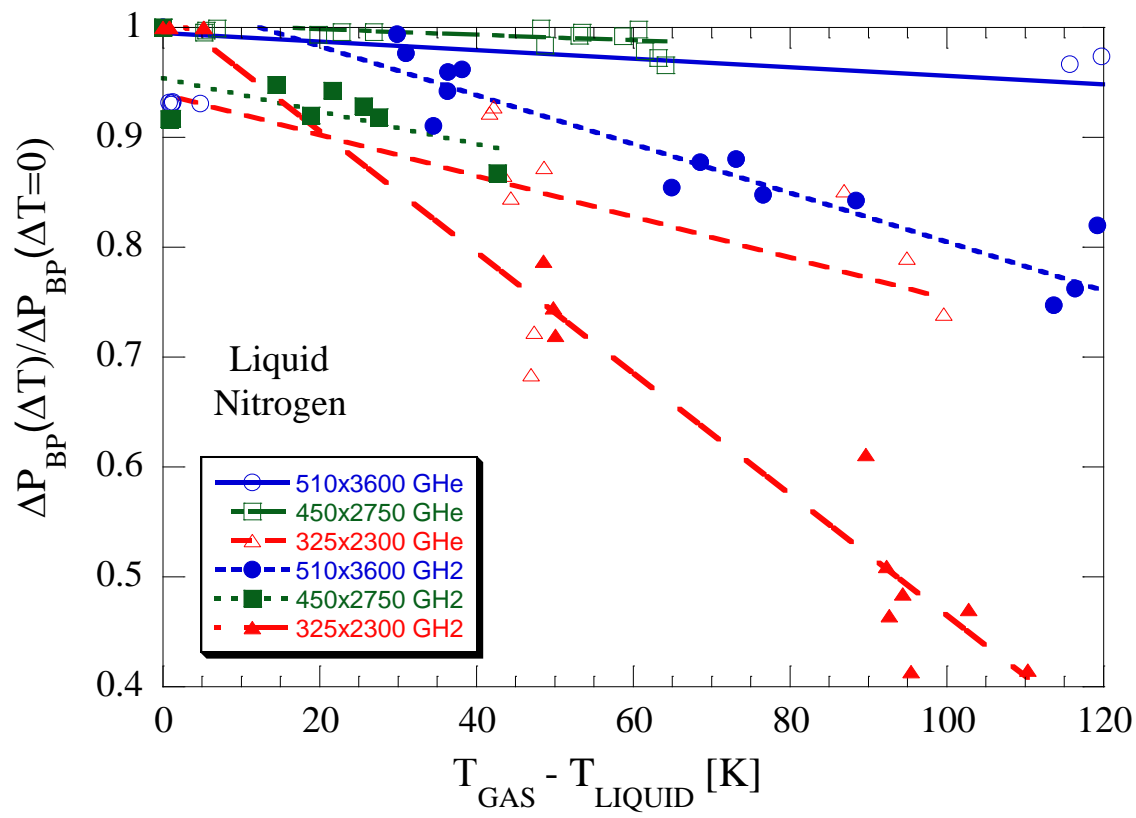

Figure 8.8 - Normalized Heated Pressurant Gas Liquid Nitrogen Bubble Point Pressure as a Function of the Temperature Difference between Pressurant Gas and Liquid at the Screen

$\mathrm{LN}_{2}$ bubble points degrade less than $\mathrm{LH}_{2}$ over the same range of $\Delta \mathrm{T}$. This is due to the fact that $\mathrm{LN}_{2}$ has a higher surface tension and higher heat capacity relative to $\mathrm{LH}_{2}$, 
making it harder to alter the effective interfacial temperature and thus surface tension of the liquid at the screen pore. Castle (1972) claimed that differences in degradation between $\mathrm{LH}_{2}$ and $\mathrm{LN}_{2}$ are because $\mathrm{LH}_{2}$ is more susceptible to drying out because of the lower superheat required to initiate boiling.

\subsection{Concluding Remarks}

Experimental results here confirm that elevating the pressurant gas temperature above the liquid temperature always acts as a degradation factor on the performance of the LAD. For all three meshes, both liquids, and both pressurization schemes, normalized bubble point pressure ratio decreases linearly with increasing gas temperatures.

Degradation in performance is much sharper using condensable gases versus using noncondensable gases. The reduction in LAD performance scales inversely with the porosity of the screen for both liquids and pressurization schemes, as the finest 510x3600 screen exhibited the least amount of degradation, and the coarsest $325 \times 2300$ had the highest reduction in performance. For heated $\mathrm{LH}_{2}$ static bubble point tests, the onset of degradation always occurred immediately with warm gas for all three meshes, whereas for heated $\mathrm{LN}_{2}$ tests, the onset of degradation did not occur below a minimum temperature difference between the pressurant gas and liquid for the $450 \times 2750$ and $510 \times 3600$ meshes.

Differences in performance between the three different screens are due to the effect of the screen thickness and porosity on the overall heat transfer across the LAD screen. Differences in performance between pressurants are due to modified heat and mass transport at the screen pore $\mathrm{L} / \mathrm{V}$ interface through evaporation $(\mathrm{GHe})$ and/or 
condensation $\left(\mathrm{GH}_{2}\right.$ or $\left.\mathrm{GN}_{2}\right)$. Differences in performances between the two liquids are explained through the differences in superheats required to initiate boiling in the liquid.

Results here have direct impact on future LAD and pressurization system design for low surface tension liquids, especially the CPST TDM and future cryogenic hydrogen fueled depots. Higher bubble points are always obtained when the gas used to pressurize the propellant tank, and thus flow liquid through the LAD, is as cold as possible relative to the liquid, with the highest values obtained when the pressurant gas and liquid temperature are approximately equal. However, unless the pressurant gas bottles and propellant tanks are thermally linked, or subsurface pressurization methods are employed, the gas will always be significantly warmer than the cryogenic propellant, and some degradation in LAD performance will be expected for all real mission scenarios. This chapter quantifies this effect. 


\section{Chapter 9}

\section{Full Scale Liquid Acquisition Device Outflow}

\section{Tests in Liquid Hydrogen}

The purpose of this chapter is to present the experimental results for the full scale LAD outflow tests in liquid hydrogen. Two full scale LAD channels were designed and constructed and the purpose of the test series was to examine the factors that affect LAD channel performance in a thermally representative, full scale, dynamic outflow environment. First the motivation for testing is presented. Three separate experiments were conducted in this test series, horizontal LAD outflow tests, a FTS pressure drop test, and full scale 1-g inverted vertical LAD outflow tests. Then, the test purpose, experimental hardware, instrumentation, methodology, and test results are discussed for each experiment. Finally, model predictions from Chapter 3 are compared to each set of experimental data.

All tests were performed in $\mathrm{LH}_{2}$. Test conditions were taken over a wide range of liquid temperatures $(20.3 \mathrm{~K}-24.2 \mathrm{~K})$, tank pressures $(100 \mathrm{kPa}-350 \mathrm{kPa})$, and outflow rates $(0.010-0.055 \mathrm{~kg} / \mathrm{s})$ thermally and operationally representative of an in-space propellant transfer from a depot storage tank or to a smaller scale in-space engine. Horizontal LAD tests were conducted to independently measure the frictional and 
dynamic losses down the channel. Flow-through-screen tests were performed to independently measure the dominate pressure loss in LEO, the FTS resistance. Meanwhile, 1-g inverted vertical LAD outflow tests were conducted to obtain performance data for two full scale $325 \times 2300 \mathrm{LAD}$ channels. One of the channels had a perforated plate and a custom built internal thermodynamic vent system to enhance performance.

\subsection{Test Purpose and Motivation}

Before LADs can be considered a viable option for cryogenic liquid acquisition systems in microgravity, they must first be qualified in ground testing. The purpose of the current work is thus to design, characterize, and parametrically test two $325 \times 2300$ full scale $\mathrm{LAD}$ channels in $\mathrm{LH}_{2}$ in an inverted outflow configuration over a wide range of liquid temperatures $(20.3-24.2 \mathrm{~K})$, pressures $(100-350 \mathrm{kPa})$, and flow rates $(0.010-$ $0.055 \mathrm{~kg} / \mathrm{s}$ ) representative of a depot propellant tank and transfer. Test results will influence the design and operating ranges of flight LADs for future cryogenic depots. Tests here will be instrumental in determining the effectiveness and suitability of applying LAD channel designs to all future in-space cryogenic fueled systems.

The primary motivation for the planned test was to address Needs, Goals, and Objectives (NGO) for the CPST TDM. However, the ultimate goal is to provide data applicable to multiple $\mathrm{LH}_{2}$ flight system architectures. Therefore, a survey was conducted of historical and proposed architectures to determine what operational conditions would be best applicable. It is noted that the most challenging condition for a LAD would be operating in a microgravity environment under large flow rates for an in-space cryogenic 
propellant depot. $\mathrm{LH}_{2}$ depots would likely be limited to less than $345 \mathrm{kPa}$ (50 psi) working pressure, assuming that tank mass would be minimized by keeping pressures low.

Table 9.1 summarizes the four architectures referenced to determine test conditions, in terms of maximum expected operating pressure (MEOP), flow rate, and transfer line size and velocity, including the two original CPST TDM Point of Departure (POD) reference points (CPST 2011), the proposed Centaur upper stage to the Cryogenic Orbital Testbed-LITE (CRYOTE-LITE) transfer (Gravlee et al. 2010), as well as estimated, scaled parameters for a full scale depot. Depot parameters are estimated based on a Martian surface/return mission. Additional historical references, which include previously attempted cryogenic propellant technology demonstration missions is reserved in Appendix A. Recent depot design concepts are in Section 1.6.2. Note that for all missions referenced, working pressure typically falls in the range of $200-345 \mathrm{kPa}$ (3050 psia). Depending on the pressurization system used, either with a non-condensable gas such as $\mathrm{GHe}$ or condensable with $\mathrm{GH}_{2}$, the LAD may need to operate in liquid as warm as the saturation temperature at $275 \mathrm{kPa}$ (40 psia). Therefore the facility and test hardware here was modified to service $\mathrm{LH}_{2}$ across the range of temperatures, pressures, and flow rates listed to eclipse the first two architectures and reach the third architecture in Table 9.1 (assuming a four LAD arm configuration for Centaur to CRYOTE-LITE transfer). 


\begin{tabular}{|l|c|c|c|c|c|}
\cline { 3 - 6 } & & & Transfer Line & Transfer Line & Transfer Line \\
\hline & Reference Point & MEOP [kPa] & Flow Rate [kg/s] & ID (OD) [cm] & Velocity [m/s] \\
\hline 1 & CPST Reference Point \#1 & $207-345$ & $0.01^{*}$ & $1.1(1.3)$ & $3.48-3.66$ \\
\hline 2 & CPST Reference Point \#2 & $207-345$ & $0.02^{*}$ & $1.1(1.3)$ & $6.64-6.99$ \\
\hline 3 & Centaur to CRYOTE-LITE & $207-276$ & 0.182 & $2.2(2.5)$ & $7.03-7.39$ \\
\hline 4 & Full Scale Depot & $207-276$ & $2.73 * *$ & $5.8(7.6)$ & $15.08-15.87$ \\
\hline \multirow{2}{*}{$\begin{array}{c}\text { Assumptions } \\
\text { *Pre-Phase A POD, 27, July 2011 }\end{array}$} & & & \\
\hline & **Based off scaling and Kutter (2011) & & & \\
\hline
\end{tabular}

Table 9.1 - Summary of Reference Missions for Full Scale Outflow Experiment

\subsection{Test Plan}

Four tests were planned using the same facility and propellant tank using $\mathrm{LH}_{2}$. First, a horizontal LAD outflow test was conducted to single out frictional and dynamic losses down a LAD channel. A single horizontal 325x2300 LAD channel from a previously conducted test in LOX was used here to provide a direct comparison between LAD dynamic performance in $\mathrm{LOX}$ versus $\mathrm{LH}_{2}$. This LAD also served as the inlet to the outflow transfer line, which was the second test conducted using this hardware. The purpose of the line chill down test was to investigate optimal chill down methods for a thermally representative tank to tank transfer line, to characterize the dynamic response of the transfer line, as well as perform fundamental flow visualization of the $\mathrm{LH}_{2}$ chill down process. Details and results of the chill down test are presented elsewhere; see for example (Rame et al. 2014). The third planned test was the FTS pressure drop test to single out the FTS pressure drop as measured in $\mathrm{LH}_{2}$ flows. $325 \times 2300$ and $450 \times 2750$ circular sample coupons were used to evaluate the pressure drop. Finally the fourth test was a 1-g inverted vertical outflow test to single out the leading order hydrostatic pressure drop. A standard 325×2300 and TVS cooled 325x2300 channel were tested in $\mathrm{LH}_{2}$. 


\subsection{Facility and Test Article}

All dynamic LAD testing was conducted at the Small Multipurpose Research Facility (SMiRF) at NASA GRC (Bamberger et al. 2011). All of the research hardware was mounted inside a $1.84 \mathrm{~m}^{3}$ (487 gallon), $800 \mathrm{kPa}$ (115 psi) rated, SS tank, with a MEOP of $550 \mathrm{kPa}(80 \mathrm{psi})$ as shown in Figure 9.1. A silicon diode rake was used as point sensors to sense liquid level in the tank. Load cells were used to interpolate the liquid level in between consecutive diodes. The diode rake, along with the FTS test assembly hung from the test tank lid as shown in Figure 9.2. Orange Kapton strip heaters were mounted to the tank walls and were used to condition the propellant to warmer liquid temperatures, as shown in Figure 9.1. 69W were available to warm the propellant overnight to higher saturation temperatures. Although not shown, several layers of MLI were then added to the tank after hardware assembly to reduce heat leak into the tank.

The research hardware was mounted inside the test tank as shown in Figure 9.3. Shown from a top down view is the horizontal $325 \times 2300$ LAD channel, two vertical outflow LAD channels, the DPT sense lines for measurement, and sight glasses which were used to visualize the output of the vertical channels. An axial jet is also shown, which was used to de-stratify the liquid propellant, if needed.

After all test hardware was assembled and mounted inside the test tank, the tank was mounted from a vacuum chamber (VC) lid as shown in Figures 9.1 and 9.4. As shown, multiple feedthroughs were required to conduct this test. Each test had a dedicated liquid outflow line, along with feedthroughs for liquid fill, drain, and vent, pressure, temperature, and flow rate instrumentation, two cameras and light sources, and 
heaters. The outflow lines for FTS and vertical outflow tests were VJ lines to minimize heat leak and reduce complications due to two phase flow measurement. As of June 2014, this was by far the most complex test series ever performed at the SMiRF facility.

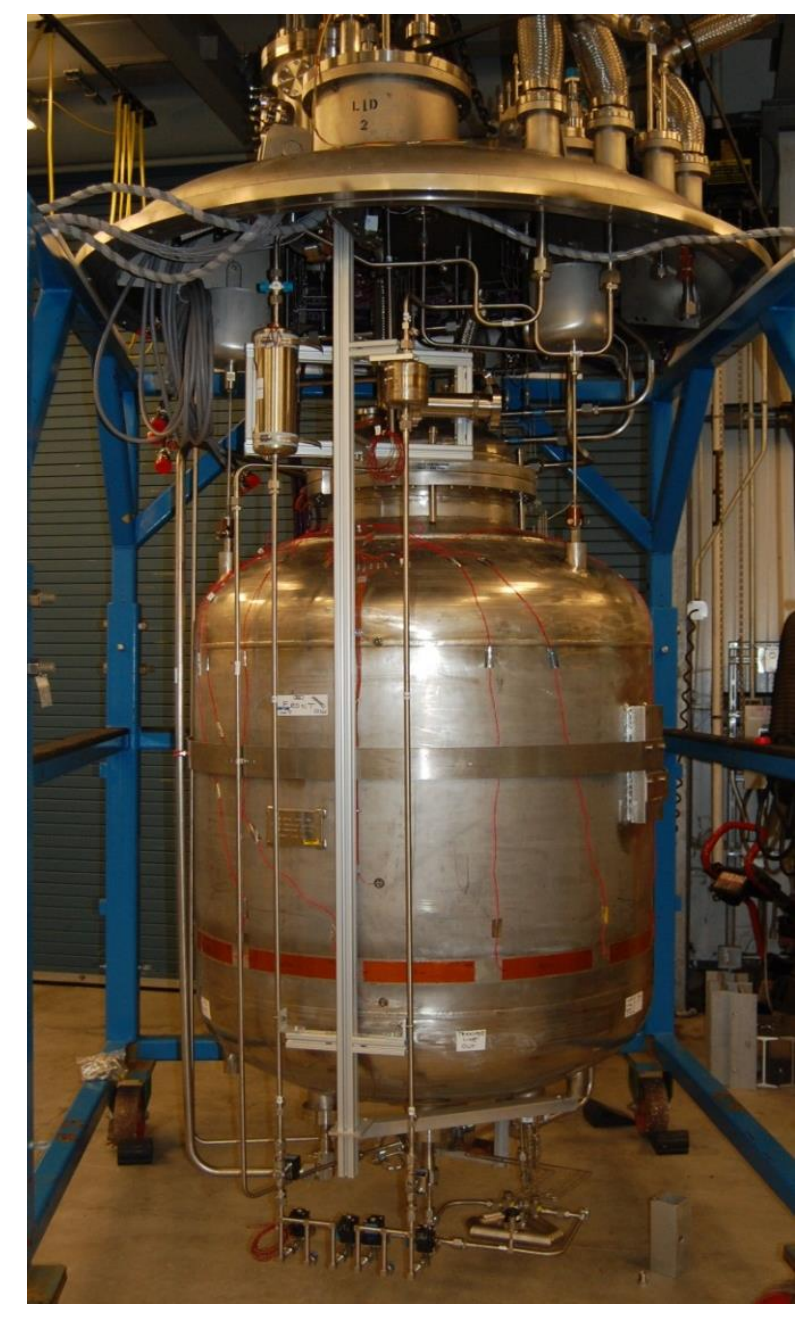

Figure 9.1 - Liquid Hydrogen Test Tank

Once assembly was completed, The VC lid and test tank were then inserted inside the cryoshroud inside the SMiRF VC. The purpose of the cryoshroud was to control the ambient environment for the test tank. Prior to every test, the chamber was pumped down to a pressure of $1 \times 10^{-6}$ torr to simulate the vacuum of LEO. The cryoshroud could also 
control the ambient temperature through the use of a dual HEX system, which circulated $\mathrm{GN}_{2}$ and $\mathrm{LN}_{2}$ through cooling coils embedded within the shroud. A temperature controlled PID scheme was used to regulate the amount of $\mathrm{LN}_{2}$ that was distributed into the coils, which in turn controlled the ambient cryoshroud temperature. For this test, a background temperature of $250 \mathrm{~K}$ was chosen to simulate the cold solar inertial orbit that was predicted for the CPST TDM flight system in LEO (Sutherlin 2011).

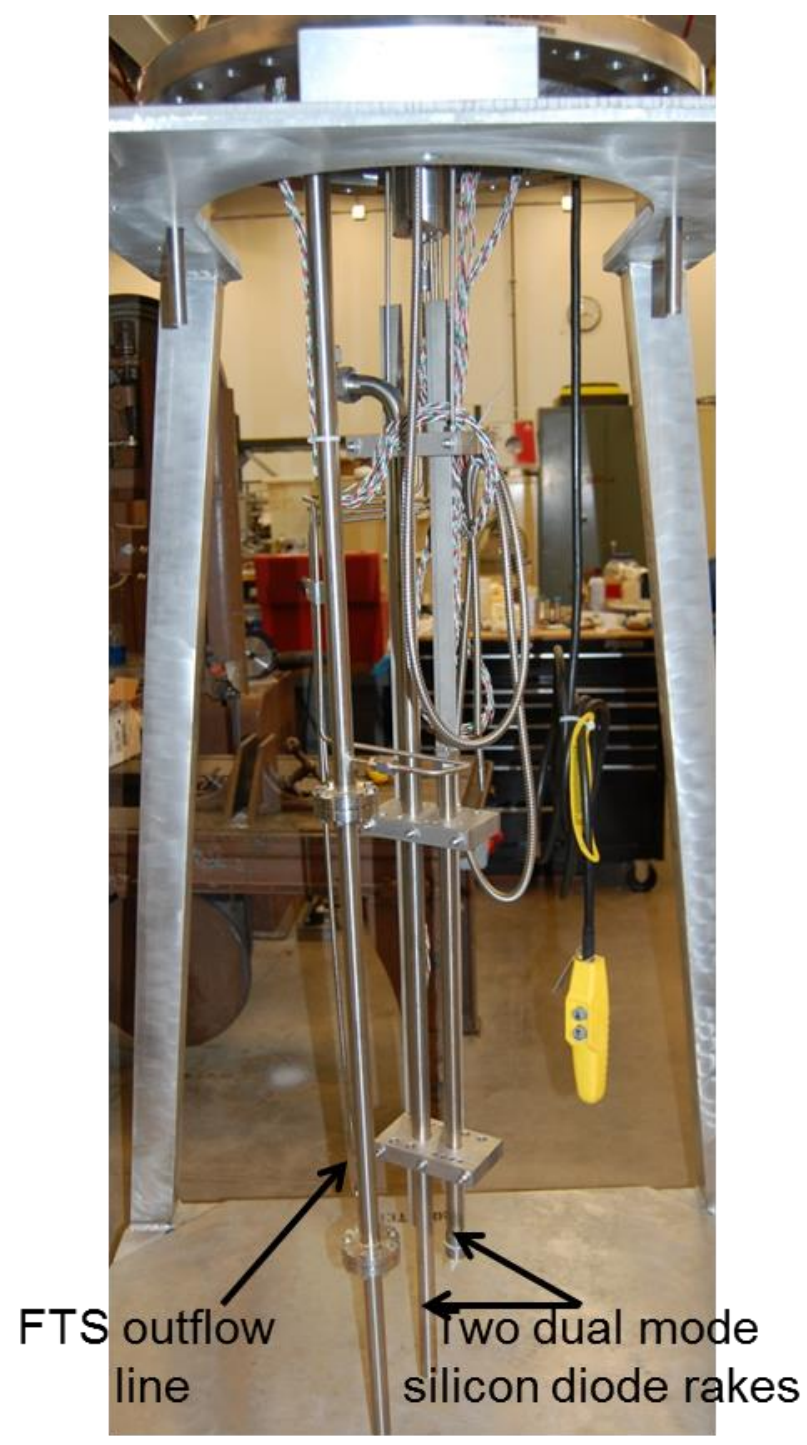

Figure 9.2 - Test Tank Lid 


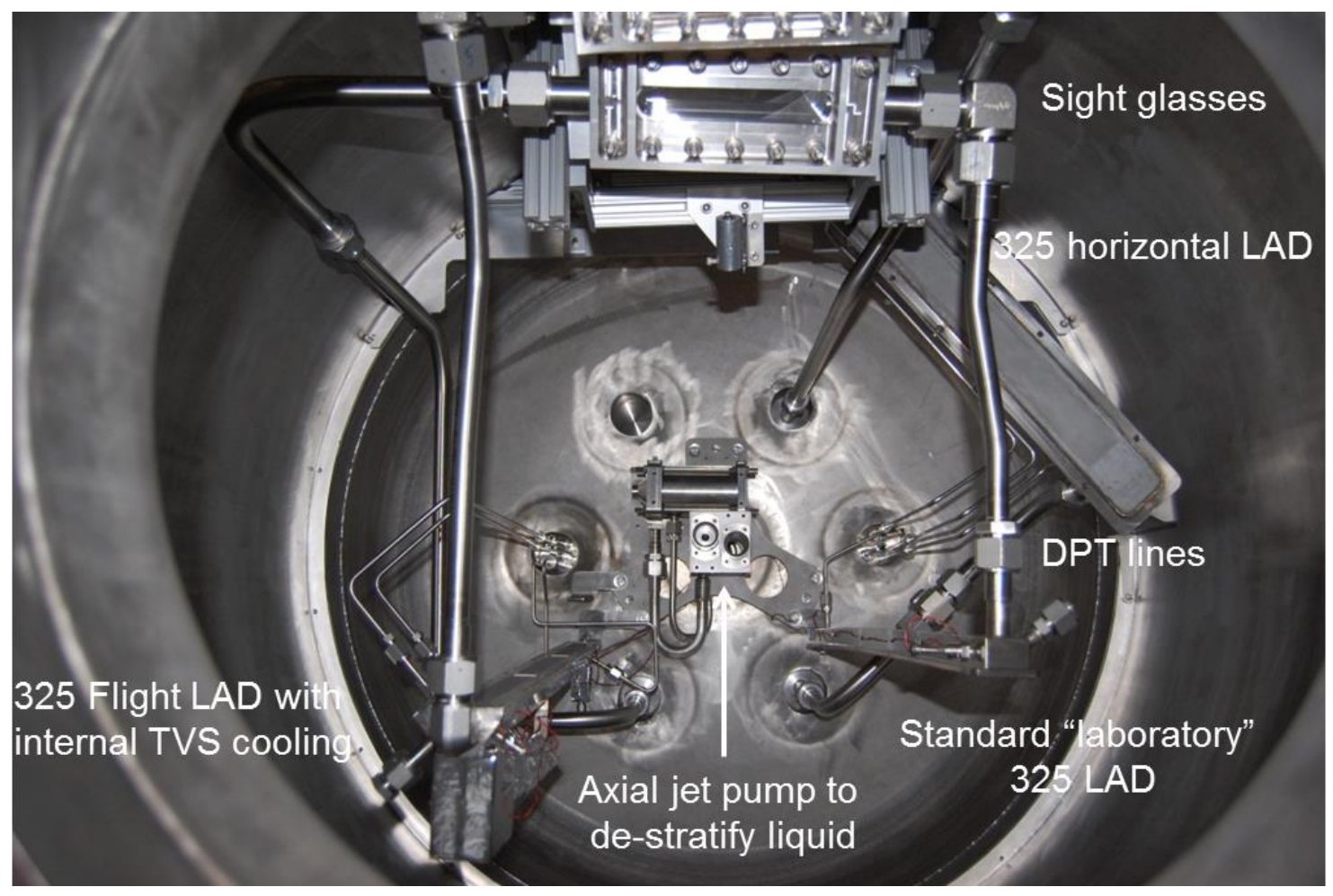

Figure 9.3 - Test Tank Internal Hardware
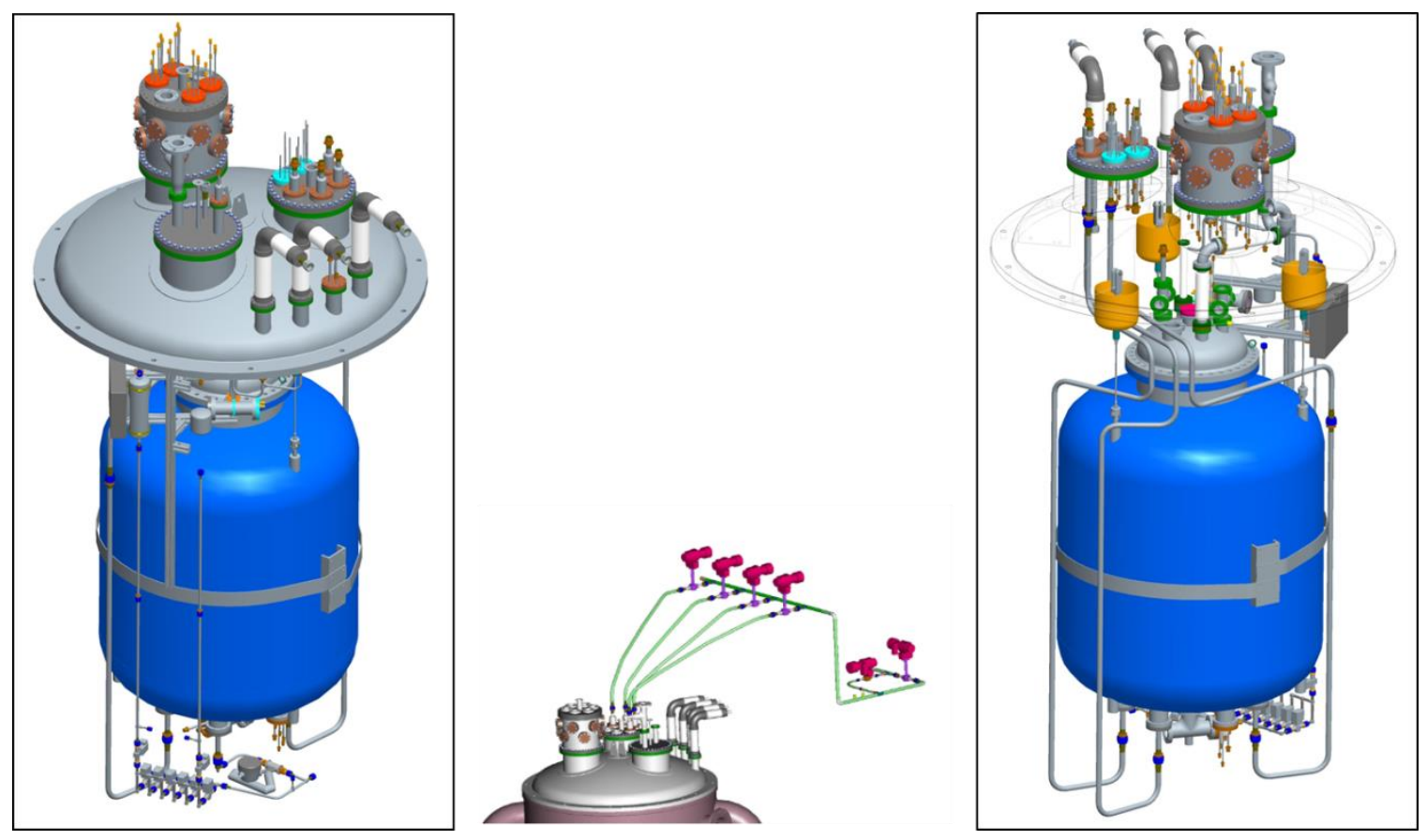

Figure 9.4 - Vacuum Chamber Lid and Feedthroughs 
The completed test tank and VC lid were then integrated into the SMiRF facility, as shown in Figure 9.5. Shown in the figure is the FTS DPT panel, VC lid, VJ piping, and back pressure (BP) flow control manifold. The $\mathrm{LH}_{2}$ outflow lines were routed out of the top of the test tank, through the VJ lines, through the top of the $\mathrm{VC}$ lid and out of the facility to vent. For flow control, isolation valves were used to select outflow from one of the four tests while a bank of PID BP flow control valves were used to regulate and control flow. For flow measurement, each outflow line had a dedicated Venturi FM. A common two stage DPT measurement system was used to measure the flow through the Venturis. To minimize complications due to sensing two phase flow, the Venturis were all mounted outside of the test tank, but still inside the VC.

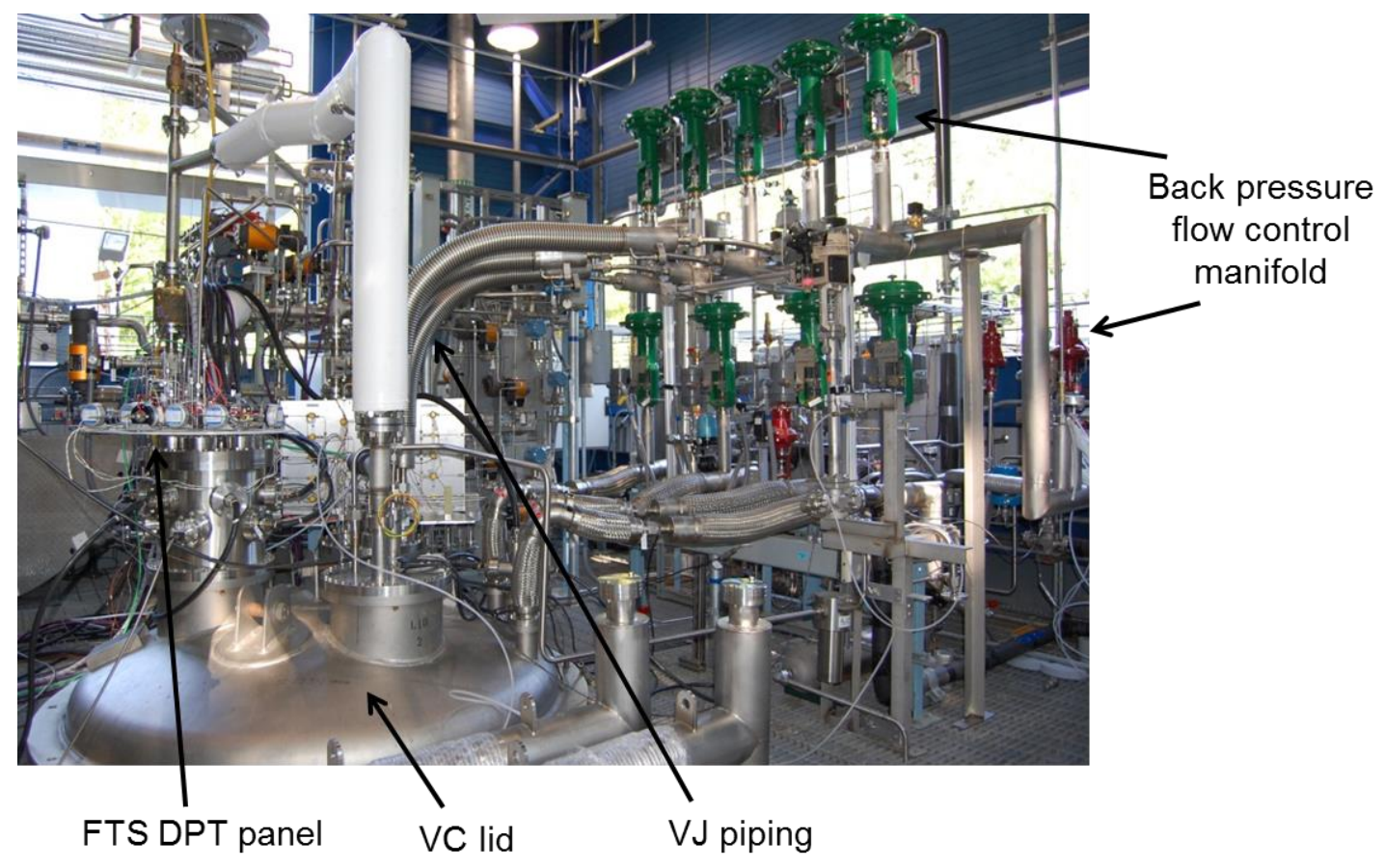

Figure 9.5 - Integration of Test Tank into Facility

Figure 9.6 shows an overview of the SMiRF $\mathrm{LH}_{2}$ flow control system. Liquid hydrogen was routed from the H-16 $5.68 \mathrm{~m}^{3}$ (1500 gallon) supply dewar into the facility 
for testing. GHe was used to pressurize the tank and to purge the system after testing. All flows were eventually routed out of the tank and through a HEX before venting to atmosphere. The vacuum chamber was back filled with GHe and then pumped down prior to every test.

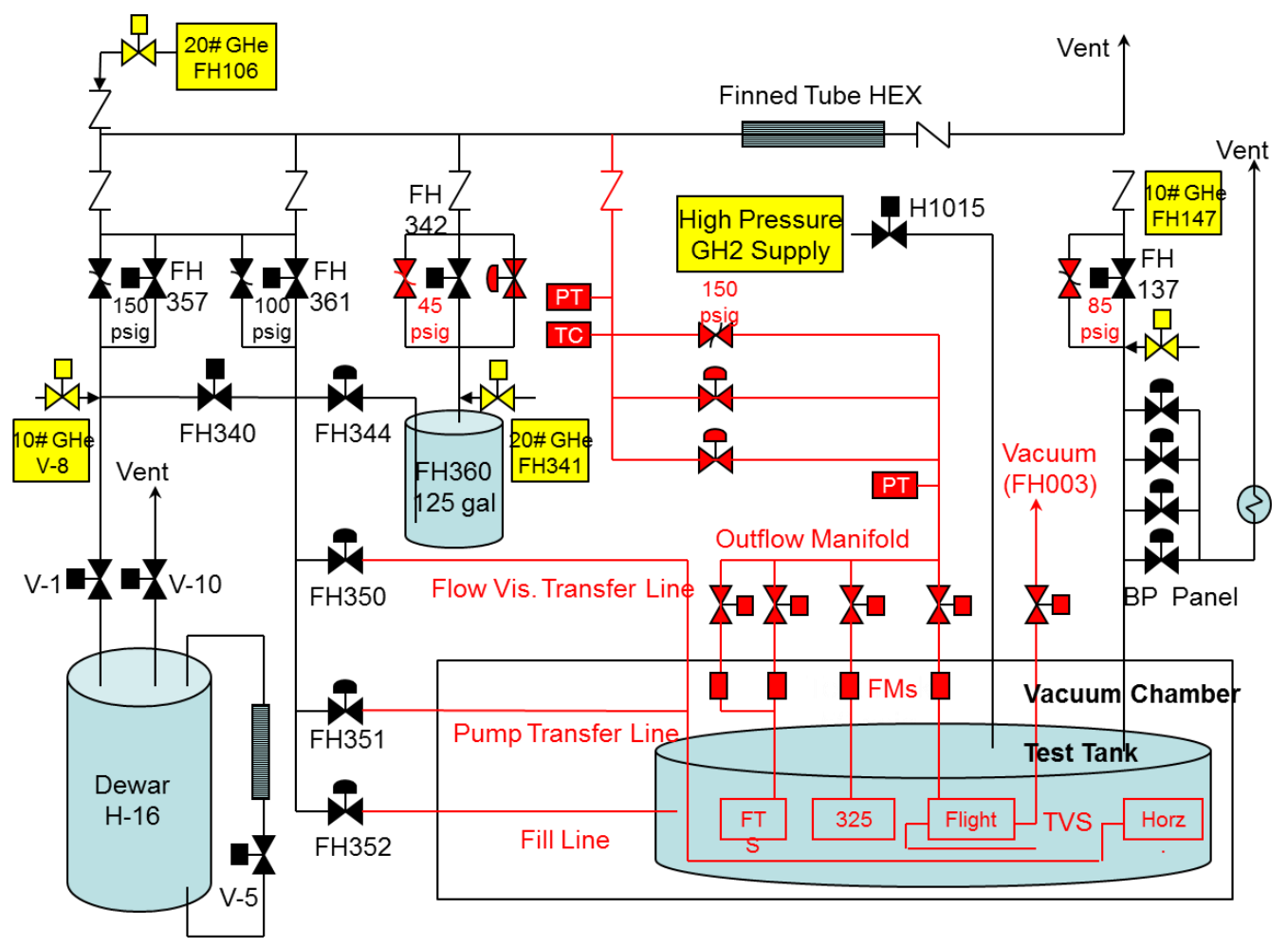

Figure 9.6 - Small Multipurpose Research Facility Liquid Hydrogen Flow Control System

All tests were remotely controlled from a control room located approximately 800 meters away from the test cell. All pressure, temperature, and flow data was recorded using a computer DAQ. A picture of the screen used to determine the LL in the tank is shown in Figure 9.7. Diodes were used in dual sense mode as temperature or liquid level sensors, as controlled on the right hand side of Figure 9.7. All data was scanned and recorded at $2 \mathrm{~Hz}$. A camera was used to detect LAD breakdown, and the video feed was sent to the control room monitor to view the LAD output in real time. 


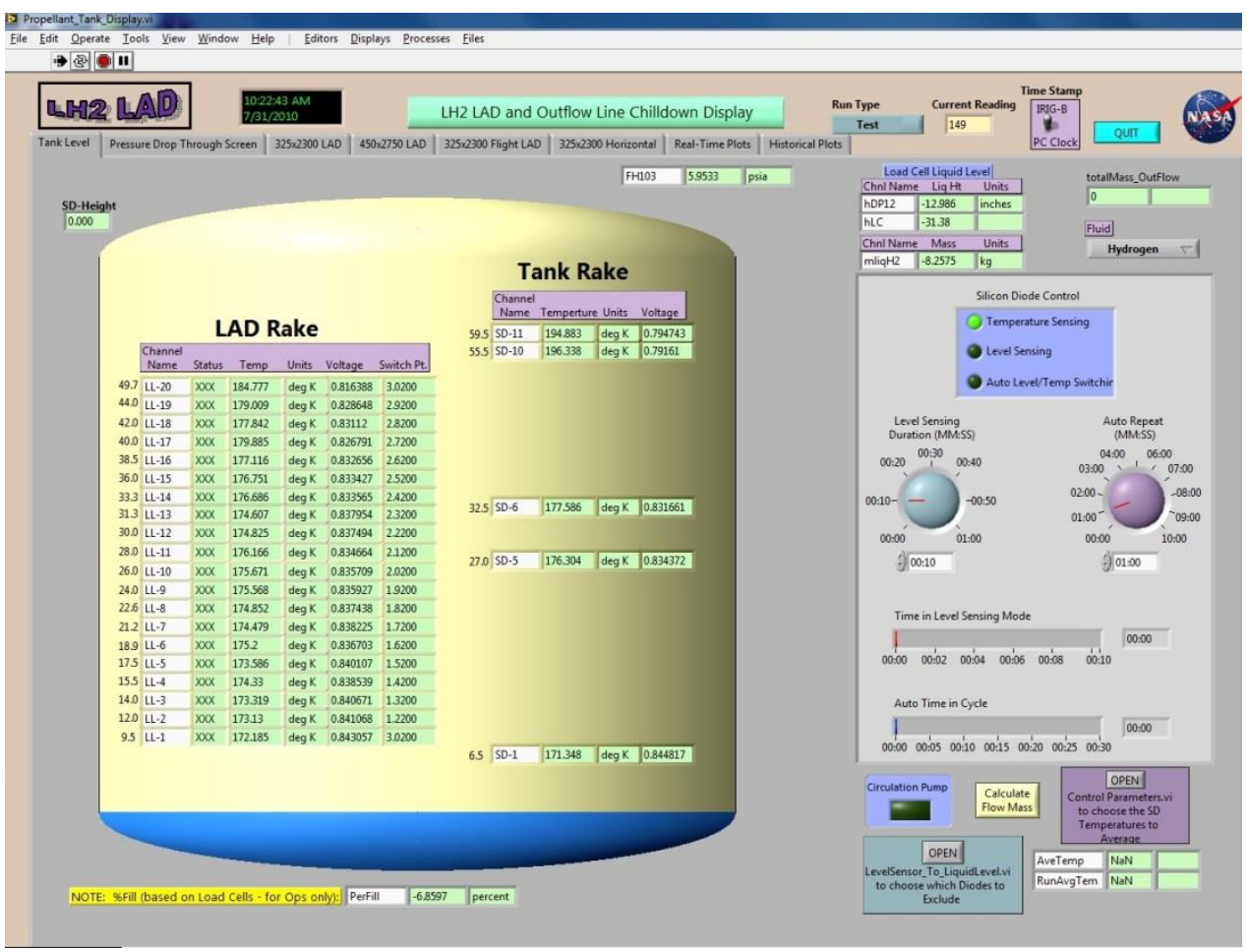

Figure 9.7 - Computer Data Acquisition System

\subsection{Horizontal Liquid Acquisition Device Tests}

The purpose of the horizontal LAD tests was to measure the frictional and

dynamic pressure losses down the LAD channel in $\mathrm{LH}_{2}$. While frictional losses are small relative to hydrostatics and FTS losses, they scale with the size of the LAD channel.

Experiments are required to determine the magnitude of this contribution and to validate models from Chapter 3 at cryogenic temperatures.

\subsubsection{Test Description}

Examination of Equations 3.57 and 3.58 for the frictional and dynamic pressure losses indicates that both are functions of length and width of the channel, liquid temperature, and demand flow rate. Because both of these contributions are essentially third order 
effects in a 1-g environment, the horizontal configuration was chosen to null out hydrostatics to even be able to measure these small pressure drops. At all points within the channel the hydrostatic pressure is constant, and according to the FTS model, pressure drop across the screen would be constant as well.

The horizontal LAD testing methodology is depicted in Figure 9.8. To measure the frictional and dynamic pressure drops, the channel is mounted inside a test tank and flow is routed through the screen and out through a port at the end of the channel. Pressure drop as a function of mass flow rate down the LAD is measured at multiple locations to quantify performance.

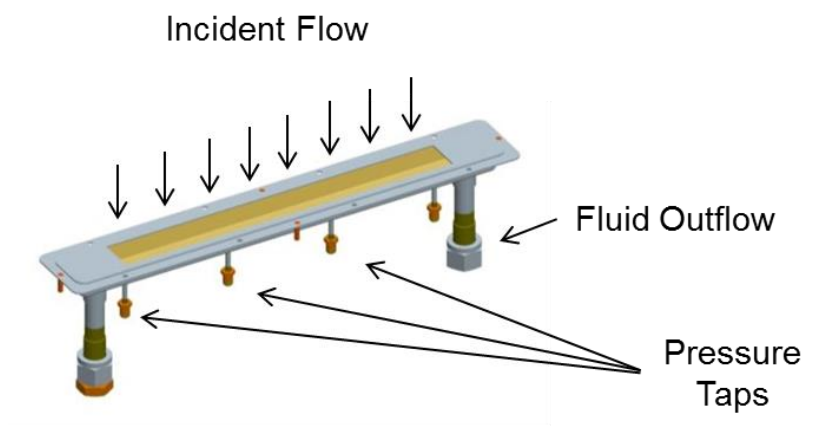

Figure 9.8 - Illustration of Horizontal Liquid Acquisition Device Testing

\subsubsection{Research Hardware}

A picture of the $325 \times 2300$ horizontal LAD channel used for testing is shown in Figure 9.9. The same exact LAD channel used from previous 2010 LOX LADs outflow tests was used here. Total channel length was $0.61 \mathrm{~m}$ (24 in), but the available screen area for flow was $0.46 \mathrm{~m}$ (18 in). The width of the screen was $5 \mathrm{~cm}$ (2 in). Four pressure taps were spaced out $15 \mathrm{~cm}$ ( 6 in) apart to measure pressure inside the channel. To deduce the actual frictional and dynamic pressure drop between any two of the taps, the absolute 
pressure measurements at successive taps can be corrected for FTS across the screen using CFD simulations from Zhang et al. (2009). The LAD was used to feed the line chill down assembly, so flow was routed out of the channel to the bottom of the tank. A Coreolis FM was used to measure flow rate downstream of the channel outlet just outside the bottom of the tank, and the FM was close coupled to the tank to ensure single phase liquid measurement at all times. As in the FTS tests, the channel was bubble point tested in IPA to ensure that the screen was defect free. Multiple differential pressure transducers were used to cover a wide flow range.

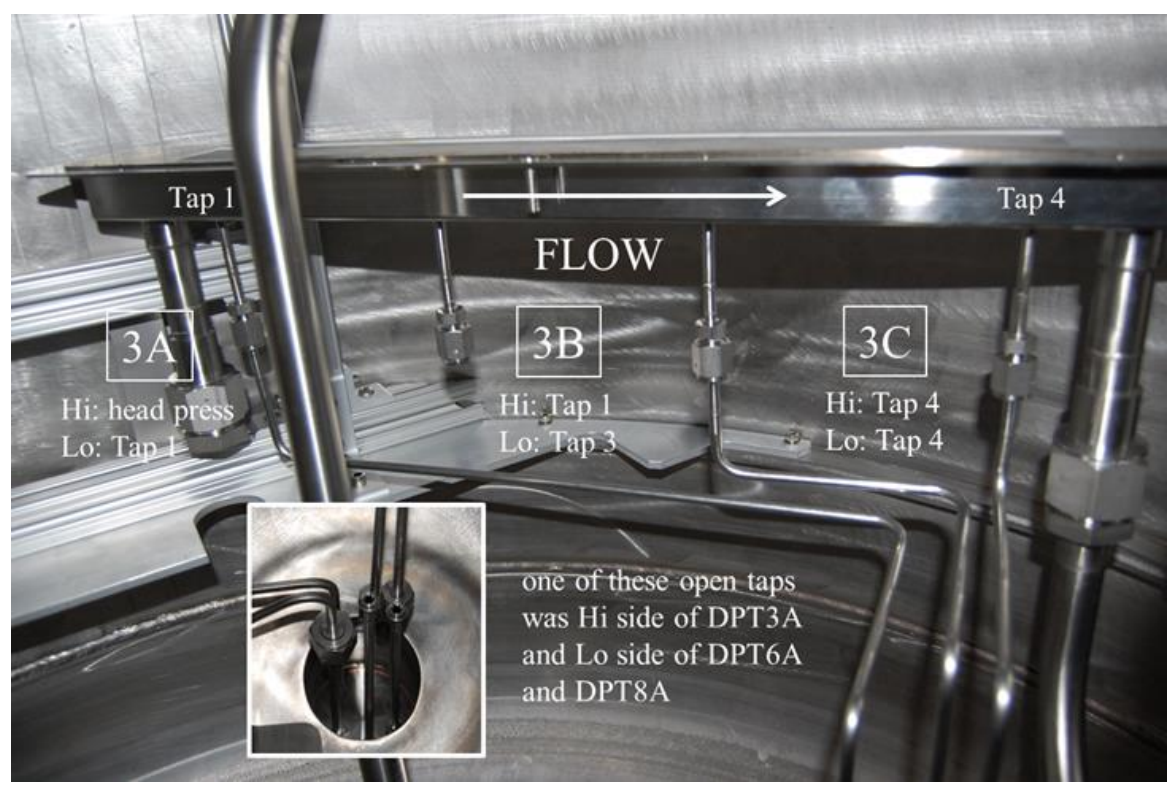

Figure 9.9 - Horizontal Liquid Acquisition Device Research Hardware

\subsubsection{Instrumentation and Test Methodology}

Instrumentation used for the horizontal LAD tests is shown in Table 9.2. Multiple ranged DPTs were used to measure pressure between various taps in Figure 9.9. Tank pressure is used to correct the DPT readings for hydrostatic and FTS pressures. Silicon diodes are used to sense inlet liquid temperature. 


\begin{tabular}{|c|c|c|c|c|}
\hline Channel & Units & & Uncertainty & Purpose \\
\hline PTs & & & & Tank ullage pressure \\
\hline FH103 & $0-690$ & $\mathrm{kPa}$ & $\pm 4.6 \mathrm{kPa}$ & Upstream of valve manifold \\
PT05 & $0-2070$ & $\mathrm{kPa}$ & $\pm 5.17 \mathrm{kPa}$ & \\
DPTs & & & & Between bottom of tank and channel \\
\hline DPT03A & $0-2490$ & $\mathrm{~Pa}$ & $\pm 1.82 \mathrm{~Pa}$ & Between first and second taps \\
\hline DPT03B & $0-1245$ & $\mathrm{~Pa}$ & $\pm 0.92 \mathrm{~Pa}$ & Between second and third taps \\
\hline DPT03C & $0-2490$ & $\mathrm{~Pa}$ & $\pm 1.82 \mathrm{~Pa}$ & \\
\hline Diodes & & & & Bulk liquid temp. at LAD height \\
\hline SD5,6 & $15-300$ & $\mathrm{~K}$ & $\pm 0.5 \mathrm{~K}$ & Stream temp. upstream of valve manifold \\
\hline SD15 & $15-300$ & $\mathrm{~K}$ & $\pm 0.1 \mathrm{~K}$ & \\
\hline FM & & & & Coriolis flow meter \\
FM1 & $0-0.045 \mathrm{~kg} / \mathrm{s}$ & $1 \%$ of reading & \\
\hline
\end{tabular}

Table 9.2 - Horizontal Liquid Acquisition Device Instrumentation

Horizontal LAD tests in $\mathrm{LH}_{2}$ were conducted across the a range of liquid

temperatures $(20.3 \mathrm{~K}<\mathrm{T}<24.2 \mathrm{~K})$ and outflow rates $(0.002 \mathrm{~kg} / \mathrm{s}<\dot{m}<0.037 \mathrm{~kg} / \mathrm{s})$. The procedure for running a test was as follows: The tank was filled with liquid cryogen, submerging the entire LAD channel and piping downstream. Liquid was conditioned to the desired saturation temperature. A control valve controlled ullage pressure while orifices downstream of the horizontal LAD channel located outside of the channel controlled flow rate. Test conditions were held at a given flow rate for several minutes to ensure accurate readings. Then flow was routed through a different orifice and repeated at the next flow rate. Tests were then repeated at multiple liquid temperatures. Note that for these tests, the horizontal LAD is always submerged in liquid.

\subsubsection{Experimental Results and Comparison to Model}

There is no known previously reported frictional LAD channel pressure loss data in the literature. Horizontal LAD channel data for a 325x2300 screen was collected in $\mathrm{LH}_{2}$ along with the FTS data. Unfortunately the frictional pressure signal levels in $\mathrm{LH}_{2}$ 
were too low compared to the random noise in the signal to discern any noticeable trends. Signal levels were anticipated to be low, but the noise overran the raw signals.

Fortunately, unreported horizontal LAD channel data from testing in 2010 was collected in LOX over an order of magnitude wider flow rate range. LOX LADs tests used the exact same $325 \times 2300$ horizontal LAD channel, with the exact same pressure taps. Only different ranged DPTs were used to cover high signal ranges.

In attempt to validate the model, two representative samples of LOX data are plotted against model prediction curves. Shown in Figure 9.10a is the pressure difference inside the channel between the last two taps, a distance of $15 \mathrm{~cm}$ apart, for tank conditions of $90.6 \mathrm{~K}$ and $1.03 \mathrm{MPa}$; Figure $9.10 \mathrm{~b}$ plots this pressure difference at tank conditions of $107 \mathrm{~K}$ and 1.65 MPa. Conditions represent a "cold" and "warm" liquid run. Both sets of raw data sets were corrected for a nonzero offset at zero flow and also corrected for FTS pressure drop across this distance in the channel. Solid lines are the model prediction curves while dotted lines are curves fit to the data. Raw uncertainties in the pressure and flow rate measurements are estimated to be no greater than $7 \%$ each.

As shown, there is quite a bit of scatter in the data. These differential pressure signals are less than $0.25 \%$ of the ullage pressure, therefore small fluctuations in tank pressure can cause large fluctuations in the pressure taps inside the channel, and thus the measured frictional pressure drops. Nonetheless, a smooth curve is fit to the data to compare directly with the model. 

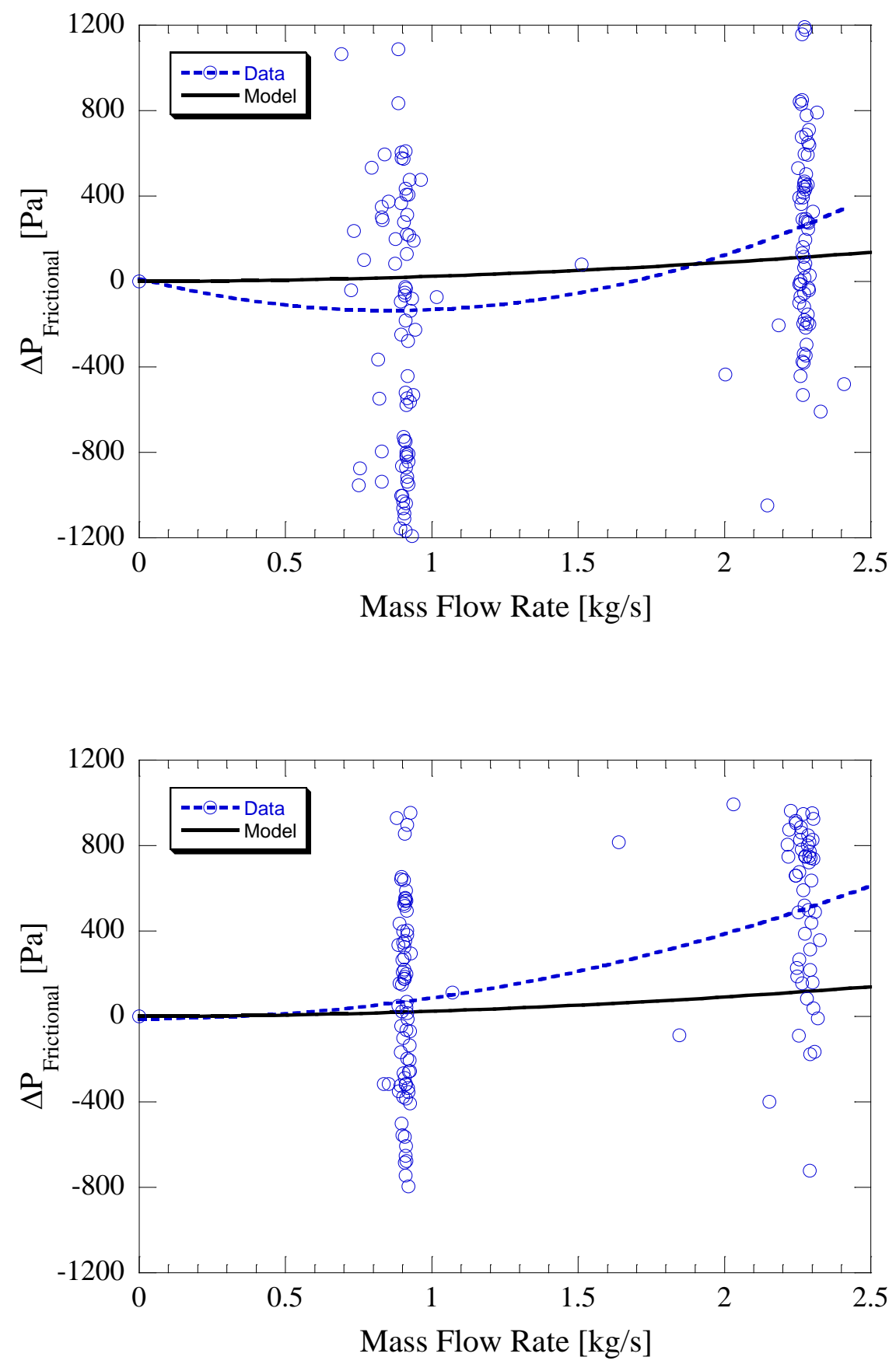

Figure 9.10 - Liquid Oxygen Frictional and Dynamic Pressure Loss Data versus Model Predictions in a) $90.6 \mathrm{~K}, 1.03 \mathrm{MPa}$ and b) $107 \mathrm{~K}, 1.65 \mathrm{MPa}$ Liquid

At the same flow rates, the data shows higher losses down the channel in warmer liquid over the colder liquid runs, as expected. As shown, the model underpredicts the 
data, with more disparity between data and model in the warmer liquid runs. The disparity is attributed to the scatter in the data, in the correction for FTS pressure drop across the screen between the two pressure taps, and the likelihood that the model assumption of constant fluid velocity in the channel is invalid. The data shows that the fluid between the last two taps accelerates much more rapidly than the model predicts which may be due to a non-uniform injection of fluid across the screen. Therefore the model assumption of fully developed flow along the channel requires refinement. Nonetheless, horizontal LOX LAD channel pressure drop data does confirm that the frictional and dynamic pressure drop terms are significantly smaller than the FTS pressure drop.

\subsection{Flow-through-Screen Tests}

The purpose of the FTS tests was to measure the FTS pressure loss associated with liquid flow through a $\mathrm{LAD}$ screen in $\mathrm{LH}_{2}$. While the FTS pressure drop is second order in 1-g, it becomes the dominate pressure loss in flight, in LEO, where hydrostatics are minimal. Therefore, experiments are required to determine the magnitude of this contribution as well as validate the FTS model from Chapter 3.

\subsubsection{Test Description}

Examination of Equation 3.33 indicates that the FTS pressure drop is dependent on both mass flow rate through the screen as well as liquid temperature. To maximize signal levels, a simple pipe flow is best used to measure the FTS contribution. For a circular screen sample, the FTS pressure drop obeys the following relationship: 
$\Delta P_{F T S}=\frac{4 Q \alpha B \mu a^{2}}{\pi \rho \varepsilon^{2}} \frac{\dot{m}}{D_{S}^{2}}+\frac{16 Q \beta B}{\pi^{2} \rho \varepsilon^{2} D_{p} D_{S}^{4}} \dot{m}^{2}$

where $D_{S}$ is the diameter of the screen sample within the pipe. To measure FTS pressure drop, a circular screen sample is simply mounted inside a pipe and the flow is routed through the screen. Differential pressure and mass flow rate are measured across the screen to quantify performance.

\subsubsection{Research Hardware}

A picture of the pipe assembly used for FTS testing is shown in Figure 9.11. Two $5.08 \mathrm{~cm}$ ( 2 in) OD screen samples were mounted inside the pipe assembly as shown. Pressure taps were mounted to measure the differential pressure across the screen as a function of flow rate. The second screen was spaced far enough from the first sample to minimize entrance effects at the highest flow rates tested, thus ensuring single phase liquid flow at each screen. The assembly was mounted from the top of the tank and so the flow was routed vertically upward. Both screens passed pretest IPA bubble point tests to ensure that they were defect free.

\subsubsection{Instrumentation}

Instrumentation used for the FTS tests is shown in Figure 9.12 and Table 9.3. Multiple DPTs were used to cover a wide flow rate range. Two DPTs were mounted in parallel across each screen sample to sense low and high flow rates. A DPT measured the total DPT across both screen samples. An additional DPT measured the differential pressure between the screen samples to ensure single phase flow at the second screen 
sample. A Venturi FM was mounted downstream to measure the flow rate through the assembly.

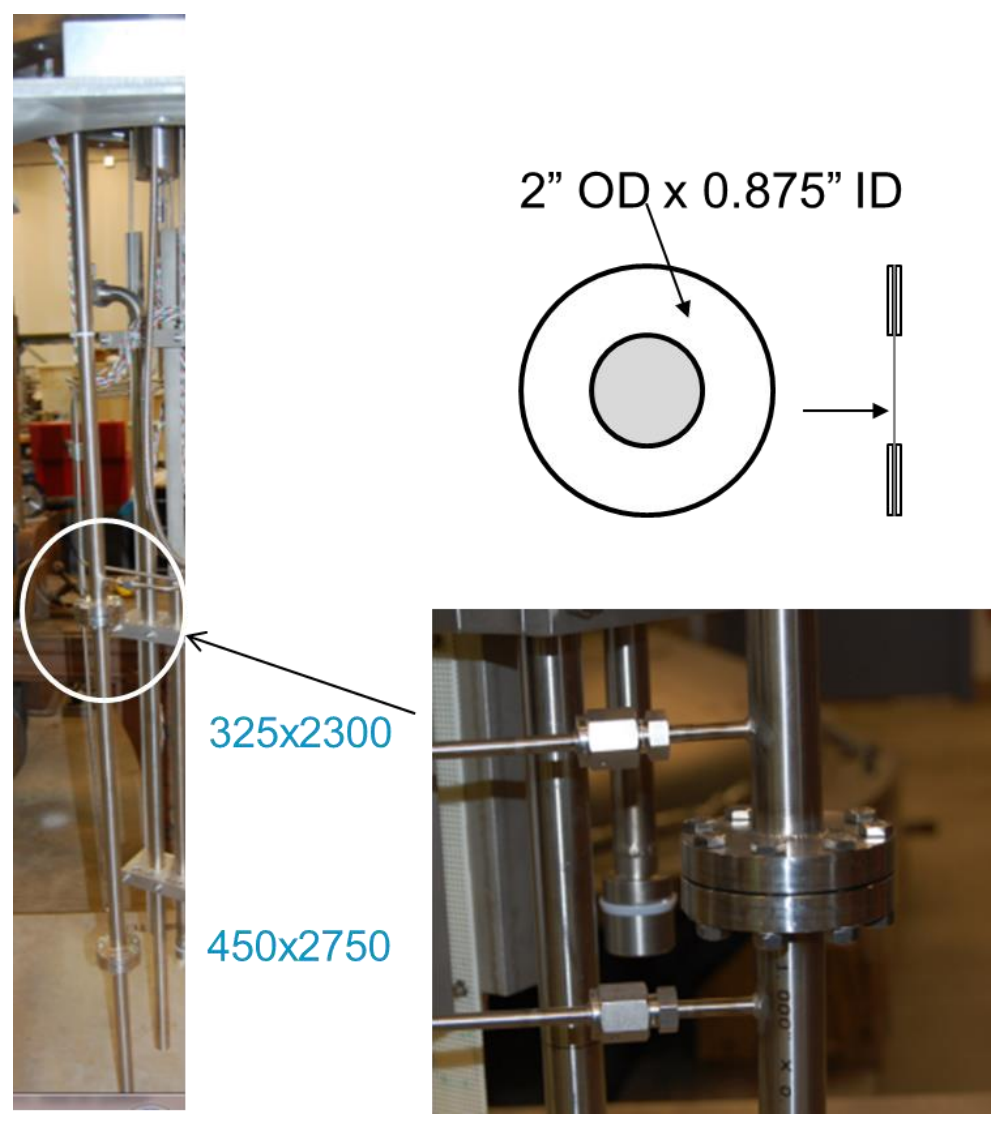

Figure 9.11 - Flow-through-Screen Research Hardware 


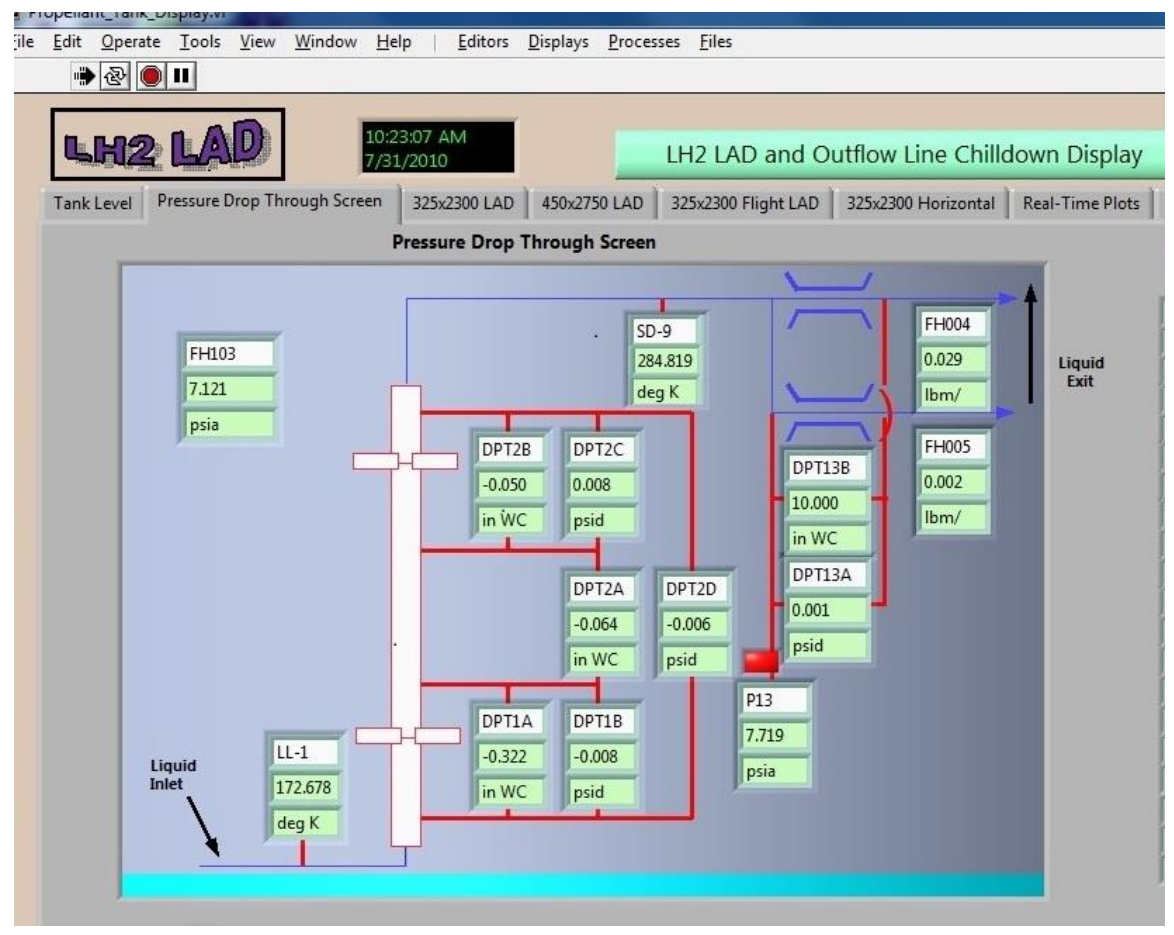

Figure 9.12 - Screenshot of Flow-through-Screen Data Acquisition

\begin{tabular}{|c|c|c|c|c|}
\hline Channel & Units & & Uncertainty & Purpose \\
\hline PTs & & & & \\
\hline FH103 & $0-689$ & $\mathrm{kPa}$ & $\pm 4.6 \mathrm{kPa}$ & Tank ullage pressure \\
\cline { 2 - 4 } P12 & $0-689$ & $\mathrm{kPa}$ & $\pm 4.6 \mathrm{kPa}$ & VJ manifold pressure \\
\cline { 2 - 4 } P13 & $0-689$ & $\mathrm{kPa}$ & $\pm 4.6 \mathrm{kPa}$ & Venturi static P \\
\hline DPTs & & & & \\
\hline DPT01A & $0-7473$ & $\mathrm{~Pa}$ & $\pm 5.5 \mathrm{~Pa}$ & FTS DPT across 450 \\
DPT01B & $0-69$ & $\mathrm{kPa}$ & $\pm 0.69 \mathrm{kPa}$ & FTS DPT across 450 \\
DPT02A & $0-7473$ & $\mathrm{~Pa}$ & $\pm 5.5 \mathrm{~Pa}$ & FTS DPT between 450 and 325 \\
DPT02B & $0-7473$ & $\mathrm{~Pa}$ & $\pm 5.5 \mathrm{~Pa}$ & FTS DPT across 325 \\
DPT02C & $0-69$ & $\mathrm{kPa}$ & $\pm 0.69 \mathrm{kPa}$ & FTS DPT across 325 \\
DPT02D & $0-103$ & $\mathrm{kPa}$ & $\pm 1.03 \mathrm{kPa}$ & FTS DPT across 450 and 325 \\
\hline DPT13A & $0-138$ & $\mathrm{kPa}$ & $\pm 1.38 \mathrm{kPa}$ & Venturi DPT high \\
\hline DPT13B & $0-2491$ & $\mathrm{~Pa}$ & $\pm 18.2 \mathrm{~Pa}$ & Venturi DPT med \\
\hline SDs & & & & LL in tank [in] \\
\hline SD9 & $15-300$ & $\mathrm{~K}$ & $\pm 0.5 \mathrm{~K}$ & FTS temperature @ venturi \\
\hline FMs & & & & \\
\hline FH004 & - & $\mathrm{lbm} / \mathrm{s}$ & $\sim 2 \%$ reading & High leg outflow for FTS \\
\hline FH005 & - & $\mathrm{Ibm} / \mathrm{s}$ & $\sim 2 \%$ reading & Low leg outflow for FTS \\
\hline
\end{tabular}

Table 9.3 - Flow-through-Screen Instrumentation 


\subsubsection{Test Methodology}

FTS tests in $\mathrm{LH}_{2}$ were conducted across a wide range of liquid temperatures (20.3 $<\mathrm{T}<24.2 \mathrm{~K})$ and mass flow rates $(0.005<\dot{m}<0.05 \mathrm{~kg} / \mathrm{s})$. The methodology for running a FTS test in $\mathrm{LH}_{2}$ was as follows: The test tank holding the FTS assembly was filled with $\mathrm{LH}_{2}$. The liquid was conditioned to the desired saturation conditions. Then flow was routed through the FTS assembly to prechill the FM downstream to ensure single phase liquid at the FM. A back pressure control valve controlled flow rate, and a tank pressure control valve controlled the ullage pressure in the tank. Once the hardware was chilled in, testing commenced at the desired flow rate. Tank and back pressure were held at 20-30 seconds to ensure an accurate reading and constant flow rate through both screen samples. Then the valves were adjusted to the new flow rate. Tests were then repeated at the next liquid temperature. Multiple repeat tests were conducted at the same flow rate and/or liquid temperature.

\subsubsection{Experimental Results and Comparison to Model}

\subsubsection{Screen Weave Dependence}

FTS pressure drop data for a 325x2300 and 450x2750 screen was collected in $\mathrm{LH}_{2}$, and also in GHe, to validate the room temperature model. In addition, unreported LOX FTS data from tests in 2010 using the exact same $325 \times 2300$ screen sample is also included for comparison. Figure 9.13 plots a typical test run in $\mathrm{LH}_{2}$ for the $325 \times 2300$ and 450x2750 screens where the DPT reading across the screen is plotted as a function of liquid hydrogen flow rate. Error bars are plotted but are indistinguishable. Solid curves 
are model generated curves using room temperature prediction values for Dutch Twill screens in Equation 9.1. Parameters for the 450x2750 screen are taken from Chapter 4.

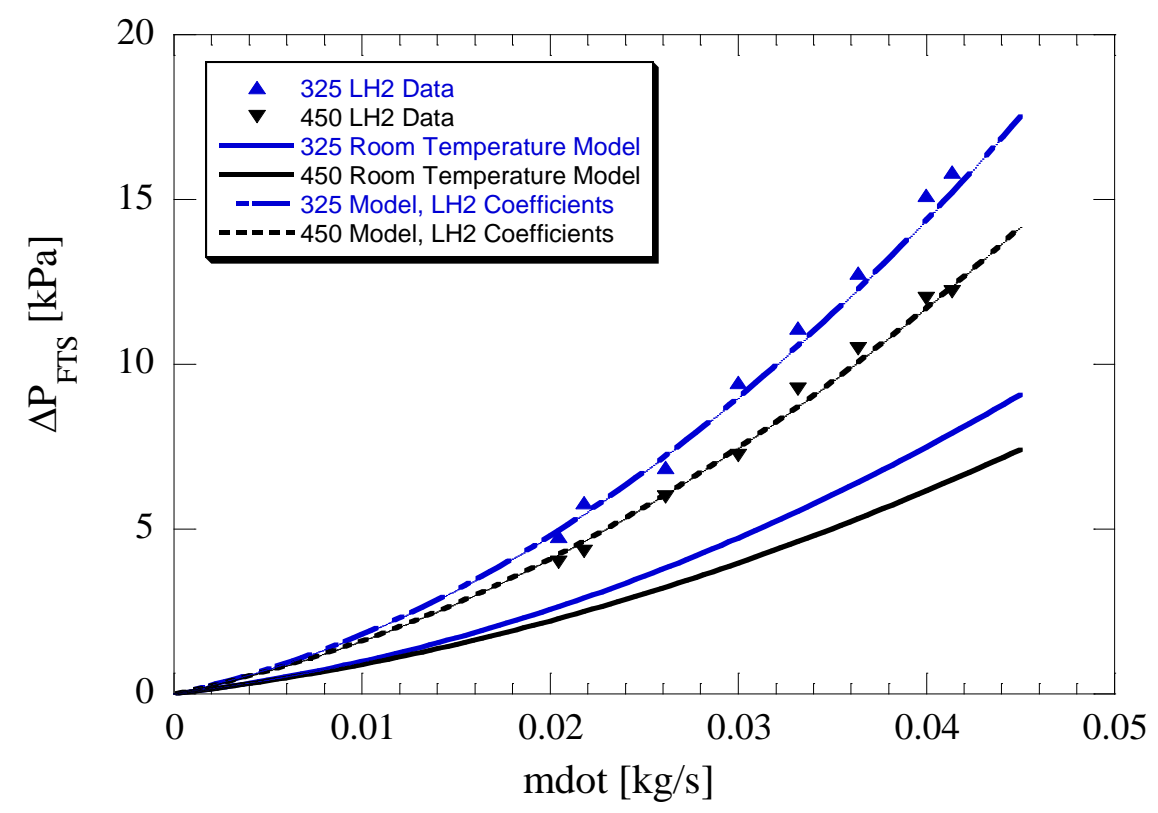

Figure 9.13 - Typical Flow-through-Screen Test Run for the 325x2300 and 450x2750 Screen Samples in Liquid Hydrogen. Lines are model generated curves using Equation 3.33.

As shown in Figure 9.13, the data follows the standard parabolic profile for flow through a porous element. Both the room temperature model and the cryogenic data show that the $325 \times 2300$ screen has higher flow losses over the $450 \times 2750$ screen. This is due to the larger warp and shute wires and smaller porosity of the $325 \times 2300$ screen.

Interestingly, both data sets lie above the corresponding room temperature prediction curve. This implies that there is higher FTS resistance at cryogenic liquid temperatures. The data deviates by as much as $50 \%$ over the room temperature model.

The general trends for cryogenic tests in $\mathrm{LH}_{2}$ are as follows: First, for fixed flow area and liquid temperature, increasing the flow rate through the screen increases the FTS 
resistance. Second, for fixed flow rate and flow area, increasing liquid temperature also increases FTS resistance. Although other data sets at warmer liquid temperatures are not shown, increasing the liquid temperature increases the FTS pressure drop slightly. For the $325 \times 2300$ screen in $\mathrm{LH}_{2}$, there is approximately $4 \%$ increase in FTS resistance at $24.2 \mathrm{~K}$ versus $20.3 \mathrm{~K}$. Both liquid density and viscosity decrease with increased temperature, and examination of Equation 3.33 shows that the increase in FTS resistance is attributed to the $\rho^{-1}$ term in both viscous and inertial pressure terms. Meanwhile, for a fixed flow rate and liquid temperature, increasing the available flow area obviously reduces the FTS resistance. Differences in flow rate cause significantly higher changes in the pressure drop over the liquid temperature. Trends here agree very well with historical trends outlined in Chapter 3 where finer mesh screens generally produce higher FTS losses.

Figure 9.14 plots a typical test run for a FTS test with room temperature GHe for the $325 \times 2300$ screen. The model prediction curve is again evaluated using the lumped set of $\alpha, \beta$ for all of the historical Dutch Twill data in Chapter 3. The same general parabolic profile is again evident in the GHe data. The model prediction curve is nearly linear in this flow regime, due to laminar flow. As shown, the room temperature model curve lies above the data. This is attributed to the fact that the $\alpha, \beta$ from Chapter 3 was generated over a wide range of Dutch Twill screens and fluids, which would lead to slight deviation between model and data for a specific single screen. If the $\alpha, \beta$ were only generated for the $325 \times 2300$ Dutch Twill, then excellent agreement between model and data would exist. Nonetheless, the difference between mode and data is less than $2 \%$. 


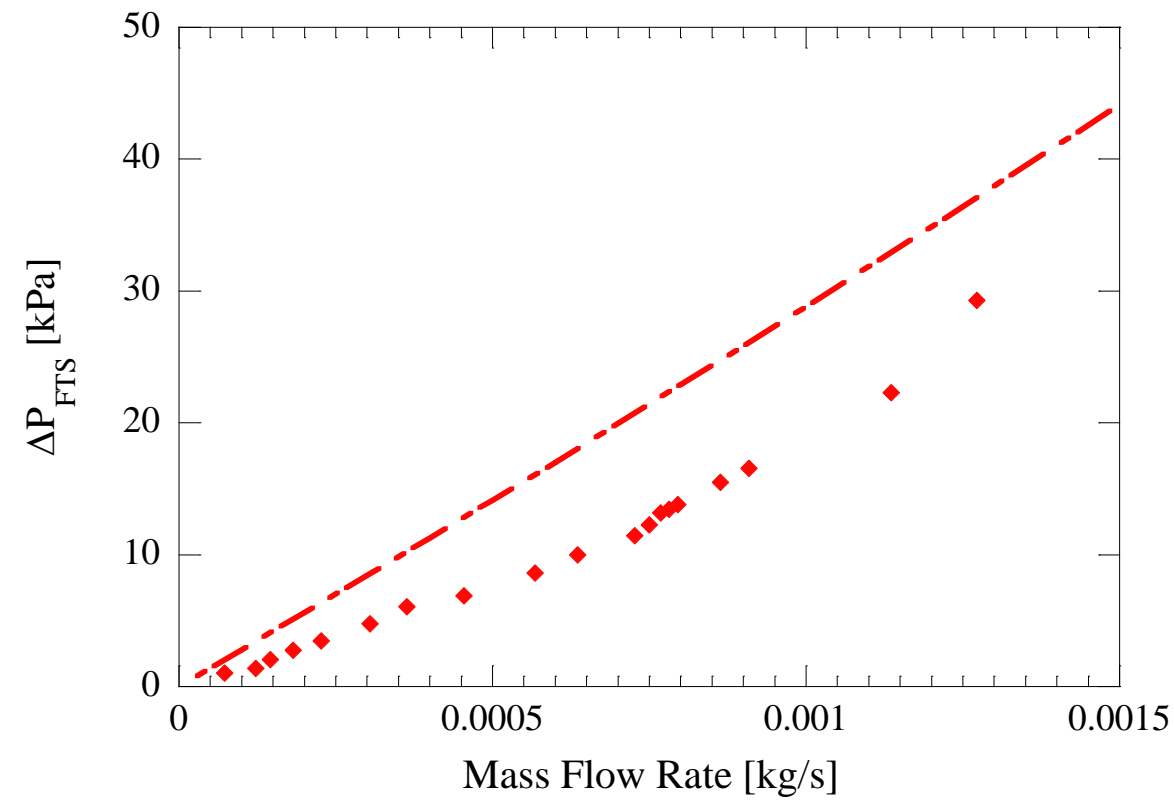

Figure 9.14 - Typical Flow-through-Screen Test Run for the 325x2300 Screen Sample in Gaseous Helium. The line is a model generated curve using Equation 3.33.

\subsubsection{Temperature Dependence}

To compare the $325 \times 2300 \mathrm{LH}_{2}$, LOX, and GHe FTS test data, all the data sets can be cast into a non-dimensional form in the same way as the historical data in Chapter 3 using Equations 3.34 - 3.36 for friction factor and modified LAD screen Re number. FTS data for the exact same $325 \times 2300$ screen sample are plotted in $\mathrm{LH}_{2}, \mathrm{LOX}$, and $\mathrm{GHe}$ in Figure 9.15. Data is plotted on a log/log scale as friction factor versus screen Re number. Error bars are also plotted, but are indistinguishable. The average uncertainty in mass flow rate and delta $\mathrm{P}$ across the screens was less than $1 \%$ at the lowest possible reading for all data taken here. Also plotted for reference is the curve fit using the room temperature fitting parameters for Dutch Twills from Chapter 3. The nominal 
temperatures for $\mathrm{LH}_{2}, \mathrm{LOX}$, and GHe data sets were $24 \mathrm{~K}, 91 \mathrm{~K}$, and $296 \mathrm{~K}$, respectively, though the data sets include measurements taken at different $\mathrm{LOX}$ and $\mathrm{LH}_{2}$ temperatures.

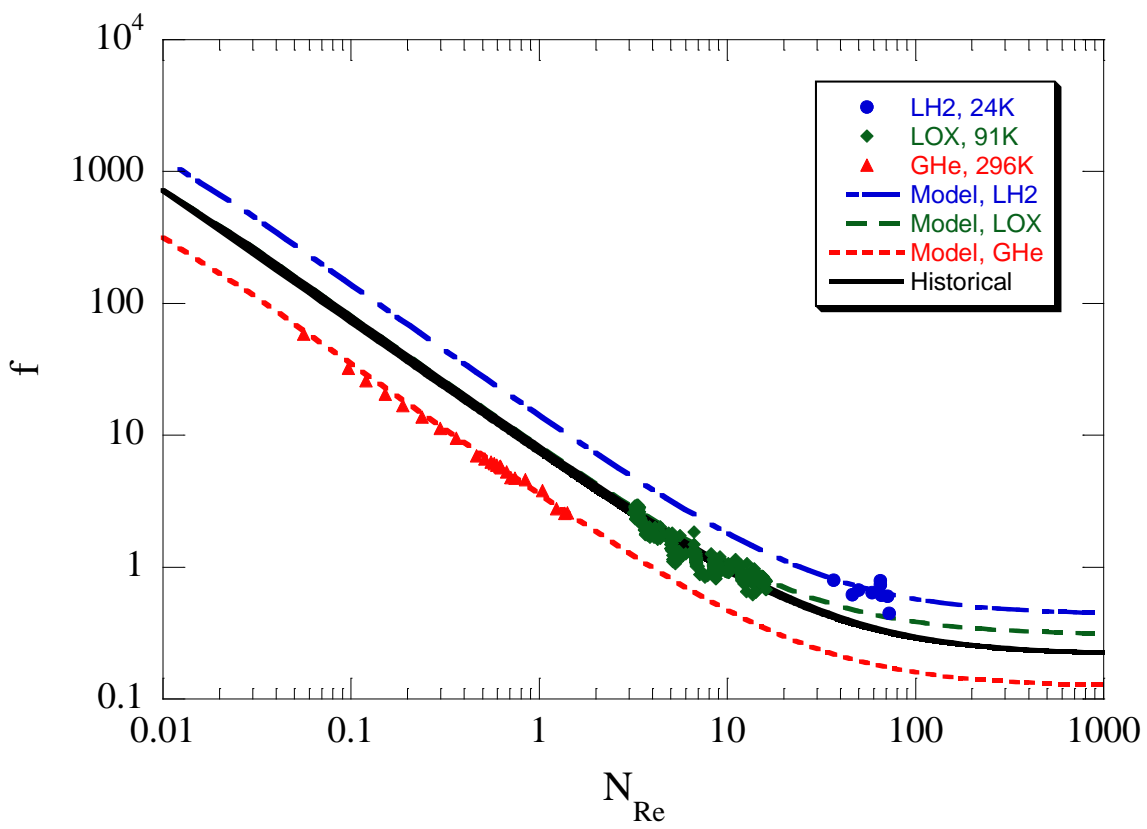

Figure 9.15 - Temperature Dependence of the Flow-through-Screen Pressure Drop

The trends are quite clear. As shown in Figure 9.15, the three data sets all fall on three distinct curves. For a given flow rate (or modified Re number), the warmest fluid (GHe) yields the smallest pressure drop (or friction factor). FTS LOX data from 2010 yields the second highest friction factor, and $\mathrm{LH}_{2}$ data from the current work yields the highest friction factor. This indicates that the FTS pressure drop is temperature dependent.

To further illustrate this temperature dependence, separate curves were fit to the GHe, $\mathrm{LOX}, \mathrm{LH}_{2}$ data using a weighted least squares method, and the fitting parameters from the curves in Figure 9.15 are listed in Table 9.4. As shown in Figure 9.15, the curves all line up in order of increasing flow resistance with decreasing temperature. 
While the lumped room temperature prediction curve and parameters $\alpha=7.22, \beta=0.217$ coincide with the LOX data, they clearly underpredict flow resistance at $\mathrm{LH}_{2}$ temperatures.

\begin{tabular}{|c|c|c|c|}
\hline Fluid & Nominal Temperature [K] & $\boldsymbol{\alpha}$ & $\boldsymbol{\beta}$ \\
\hline GHe & 296 & 3.16 & 0.12 \\
LOX & 91 & 7.22 & 0.31 \\
LH2 & 24 & 12.54 & 0.44 \\
\hline
\end{tabular}

Table 9.4 - Flow-through-Screen Fitting Parameters for Various Fluids for a 325x2300 Screen

The increase in flow resistance with decreasing temperature is likely attributed to the change in screen properties with reduced temperatures. As the temperature drops, the metal contracts, which causes the pores to shrink. For a fixed sample, this will produce a larger resistance to flow. This claim is substantiated by the recent findings that the screen pore diameter actually does shrink at cryogenic temperatures; when comparing $325 \times 2300$ static room temperature bubble point data from Chapter 4 to cryogenic bubble point data in $\mathrm{LH}_{2}$ and $\mathrm{LN}_{2}$ from Chapter 5, the pore diameter shrunk by a factor of $9 \%$ for the same $325 \times 2300$ screen sample. Other screen parameters in Equation 3.33 that are assumed constant, such as porosity and surface area to volume ratio, are also likely to change at cryogenic temperatures. For simplicity however, the temperature dependence of the FTS was simply lumped into changes in $\alpha, \beta$.

To confirm that the temperature dependence is statistically significant, Figure 9.16 plots the curve fit to both the historical data and $\mathrm{LH}_{2}$ data from the current work, as well as curves for the $+1 /-1$ standard deviation of both. $\mathrm{LH}_{2}$ data is also plotted for reference. As shown, the solid model $\mathrm{LH}_{2}$ curve does not intersect the standard deviation 
curve for the room temperature data, so one can reasonably assume that the difference between solid curves is statistically significant and that the friction factor is higher at $\mathrm{LH}_{2}$ temperatures over the room temperature behavior.

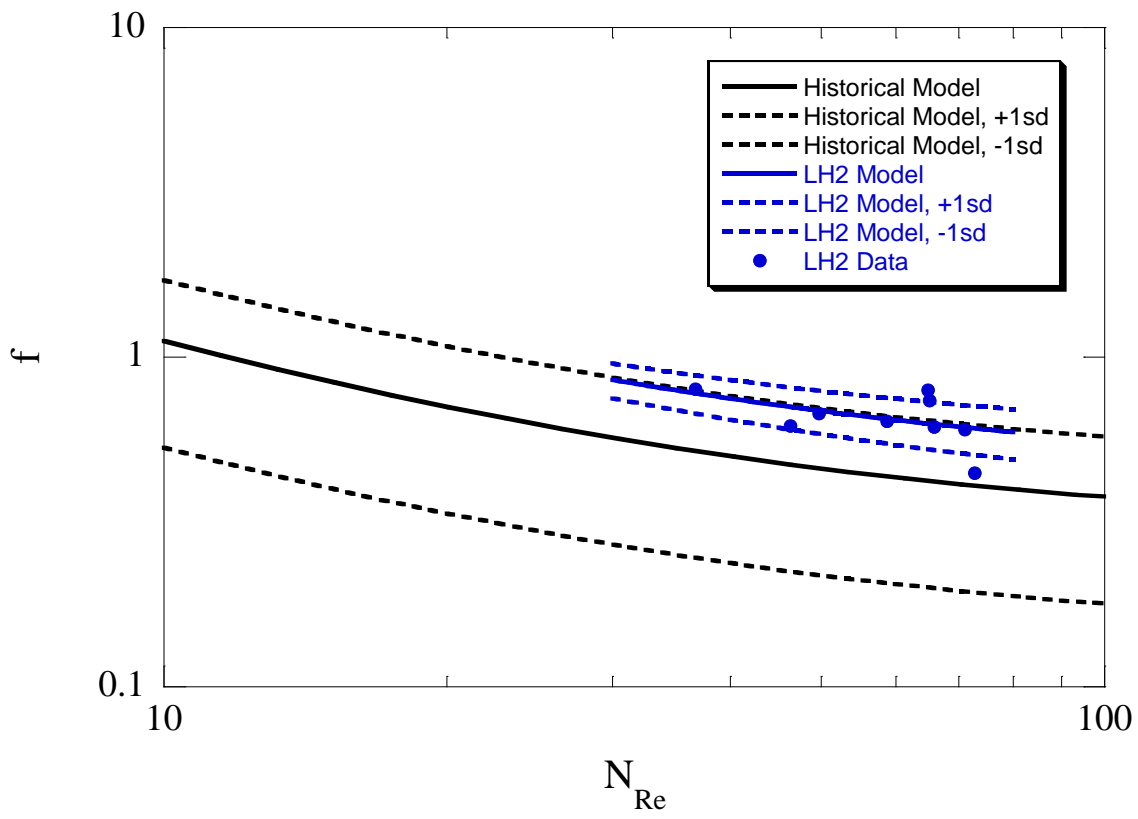

Figure 9.16 - Standard Deviation Plots of Room Temperature and Liquid Hydrogen Flowthrough-Screen Model Curves

To better illustrate the magnitude of this temperature dependence effect on the FTS pressure drop, Figure 9.17 plots the percent difference between the FTS pressure drop using fitting parameters at $\mathrm{LH}_{2}$ vs. fitting parameters at room temperature $\left(\frac{\Delta P_{F T S, L H 2}-\Delta P_{F T S, 295 \mathrm{~K}}}{\Delta P_{F T S, L H 2}} \times 100\right)$ as a function of the mass flux across the LAD screen. At low mass fluxes, in the laminar flow regime, there is $>42 \%$ higher FTS pressure drop across the screen at $\mathrm{LH}_{2}$ temperatures while at higher mass fluxes in the turbulent regime, there is $>50 \%$ higher FTS pressure drop at $\mathrm{LH}_{2}$ temperatures over the room temperature value. 
The $\%$ difference in predictions approaches as asymptote at high mass fluxes, which simply reflects the asymptote in the friction factor at high Re numbers.

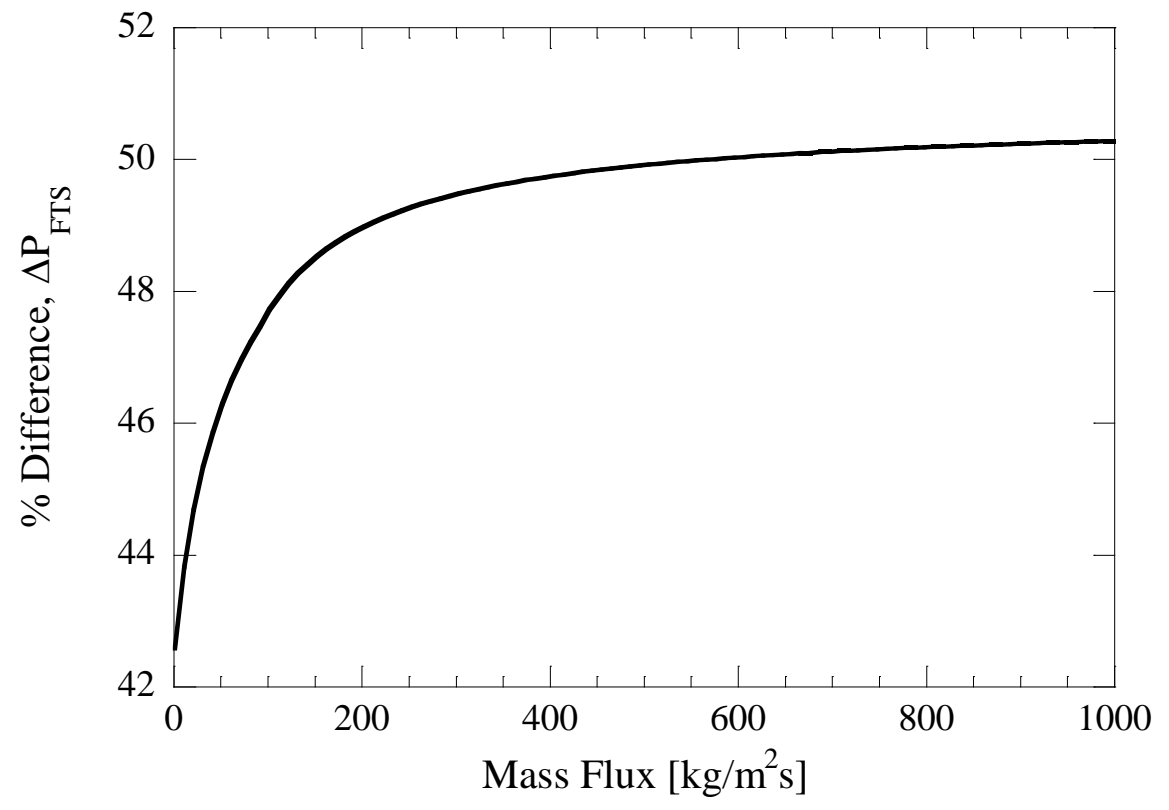

Figure 9.17 - Percent Difference in Flow-through-Screen Pressure Drop Models between Liquid Hydrogen and Room Temperature as a Function of Mass Flux across the Screen

These results suggest that although a simple room temperature prediction curve may be used to predict FTS performance for cryogens at or warmer than LOX temperatures (ex. for $\mathrm{LCH}_{4}$ ), it cannot be used to predict FTS performance below LOX temperatures (ex. $\mathrm{LN}_{2}, \mathrm{LH}_{2}$, and $\mathrm{LHe}$ ) where the metallic screens may shrink considerably from the room temperature setting. Findings here negate the long-lived assumption that the flow resistance of porous screens does not change with reduced temperature.

While there is gain in bubble point at cryogenic temperatures due to pore shrinkage and enhanced evaporation using a non-condensable pressurant gas, this gain is 
somewhat offset by the fact that the FTS resistance also increases at cryogenic temperatures. This reiterates the fact that both bubble point and FTS pressure drop are considered the primary influential factors in cryogenic LAD screen selection from Chapter 3, rather than simply desiring the highest possible bubble point. However, in LAD system design, one can always increase the total available screen area to decrease the FTS resistance, whereas bubble point depends on the screen type and not amount of screen available. Nevertheless, each mission design requires careful optimization between these two influential factors, whether in storable or cryogenic propulsion systems.

\subsection{1-g Inverted Vertical Liquid Acquisition Device Outflow Tests}

The purpose of the 1-g inverted vertical outflow tests was fourfold. First these tests are required to measure the leading order hydrostatic pressure loss. Second, experiments can be used to examine the factors that affect LAD channel performance in a thermally representative, full scale, dynamic outflow environment. Third, outflow data can be used to compare against the simplified 1D model predictions to assess the accuracy of the model. Finally, tests here will quantify the efficiency of a TVS heat exchanger on dynamic LAD performance.

\subsubsection{Test Description}

Consistent with Figure 9.18, the description of an inverted LAD outflow test is as follows: The tank is drained through the LAD screen and out of the top of the channel by pressurizing the ullage space. As the liquid level in the tank is decreased, the screen is uncovered and exposed to pressurant gas. Eventually the LAD breaks down at a 
particular liquid level corresponding to the point when the differential pressure across the screen exceeds the bubble point pressure. A sight glass and camera located within the test tank, downstream of the top of the LAD channel, is used to detect the moment a visible bubble breaks through the screen. The inverted outflow configuration is preferred for dynamic outflow tests because the highest pressure differential occurs at the top of the LAD screen, consistent with analysis in Section 3.1, and bubbles that break through the screen are immediately forced to rise to the top and out of the channel to the sight glass. However, the sight glass itself resides in warmer ullage space, which could potentially complicate post-test analysis.

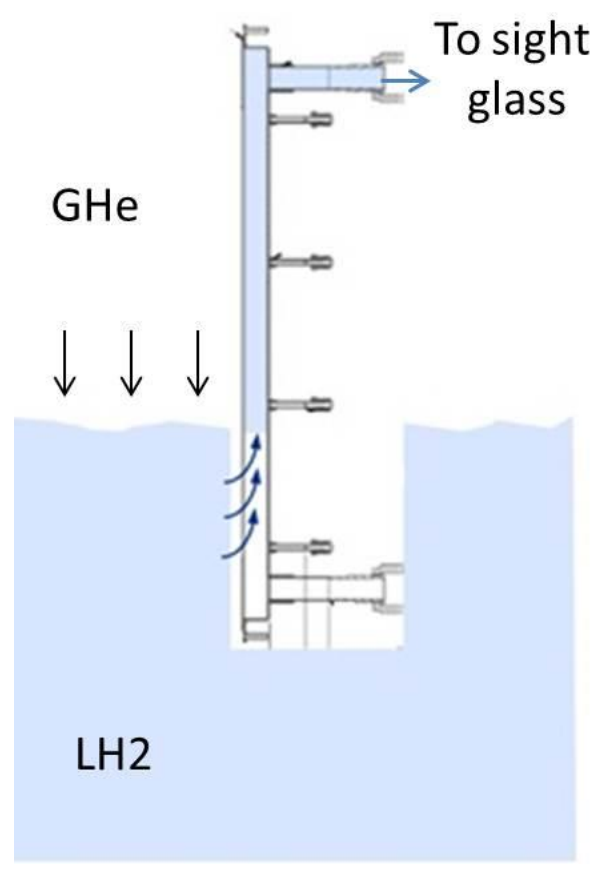

Figure 9.18 - Illustration of Inverted Liquid Acquisition Device Outflow Test 


\subsubsection{Research Hardware}

\subsubsection{Standard 325x2300 Channel}

Two $91 \mathrm{~cm}$ long by $2.54 \mathrm{~cm}$ wide by $2.54 \mathrm{~cm}$ deep (36.2 in $\mathrm{x} 1$ in $\mathrm{x}$ 1in) LAD channels were custom built, a standard $325 \times 2300$ channel and a TVS cooled $325 \times 2300$ channel. The width and depth of both channels were sized based off analysis of Jaekle's (1997) steady state pressure drop model from Chapter 3 to achieve a broad range of breakdown locations, and also to maximize the FTS and frictional pressure losses. The 91 cm (36 in) height was chosen to overcome the relatively large $\mathrm{LH}_{2}$ hydrostatic pressure drop over the range of liquid temperatures tested.

A cutout of the standard and TVS cooled channels is shown in Figures 9.19 and 9.20, respectively. The specific design and manufacturing details are omitted for brevity, only general details are presented. Channels were constructed in a piecewise fashion in the following procedure: First, 304SS 325x2300 LAD screens were diffusion bonded to a frame. A "sandwich" approach was used to bond the screens between two SS plates. Bonding was performed in a vacuum furnace at $1422 \mathrm{~K}$ using hydraulic rams to apply the compressive forces to the mating plates to achieve the bond. To promote sealing of the screen at the edges, a thin bead of $\mathrm{Ni}$ was deposited at the plate/screen interface. This was done to ensure breakdown did not occur at the screen edges where some of the screen pores could have been prematurely deformed due to the compressive forces. SEM imaging of the pieces indicated that the Ni coating was not wicked appreciably onto the screen away from the pores. Although it was desirable to fabricate a single $91 \mathrm{~cm}$ screen piece, sample length was limited to approximately $29 \mathrm{~cm}(11.4 \mathrm{in})$ due to the size of the 
furnace. To achieve the desired total length of screen area, three individual $2.54 \mathrm{~cm}(1.0$ in) wide by $29 \mathrm{~cm}$ (11.4 in) long samples were diffusion bonded to the backing plates with a $1.3 \mathrm{~cm}(0.5 \mathrm{in})$ frame all around as shown in Figure 9.19. This created a $2.54 \mathrm{~cm} \mathrm{x}$ $2.54 \mathrm{~cm}$ ( 1 in $x 1$ in) flow path, or hydraulic diameter inside the channel.

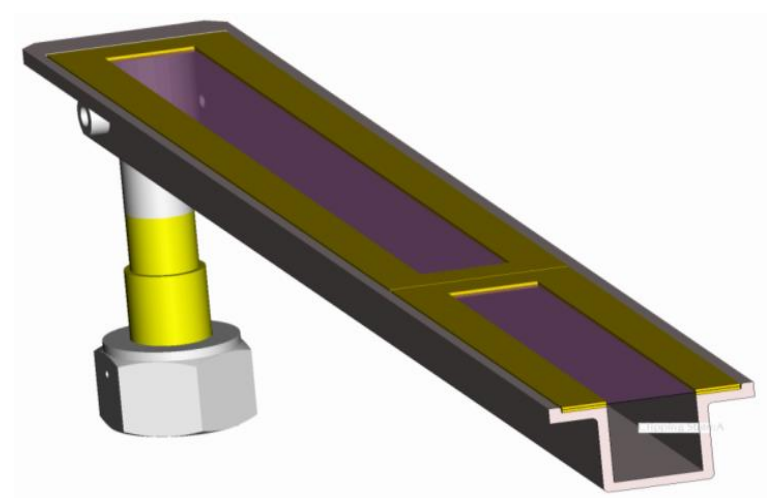

Figure 9.19 - 325x2300 Standard Channel

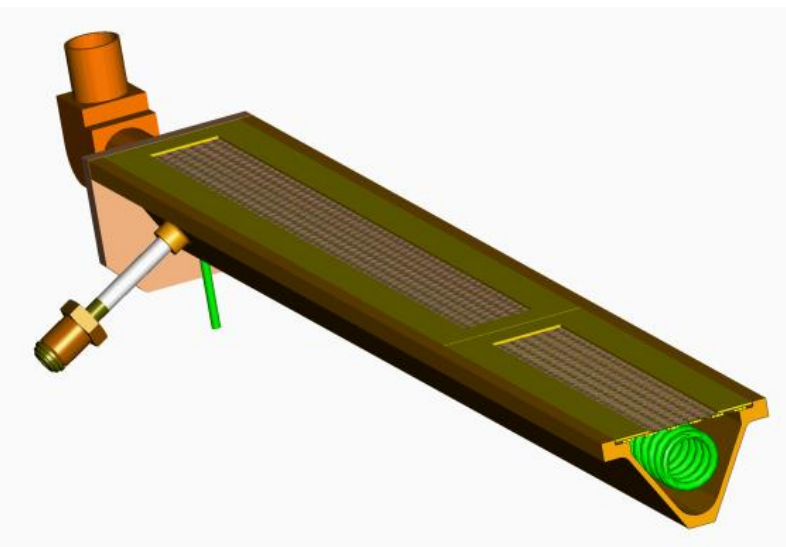

Figure 9.20 - 325x2300 Thermodynamic Vent System Cooled Channel

Second, each $325 \times 2300$ screen piece/backing plate was individually bubble point tested in IPA for performance and acceptance testing. Third, the screen sample/backing plates were then welded on top of the channel as shown in Figures 9.19 and 9.20. The seams between plates were also welded. The channel was fabricated with a liquid outflow 
port at the top of the channel and with instrumentation ports to measure two differential pressures: across the screen and from the bottom to top of the channel. A single silicon diode was epoxy bonded to the screen side facing internal to the channel in order to correlate breakdown height with predicted bubble point pressure based off this temperature as in all component level cryogenic bubble point tests from Chapters $5-8$. Fourth and finally, after completed assembly, the channel was IPA bubble point tested for final acceptance. Figure 9.21 shows a picture of the IPA testing of the completed channel. Test results are reserved in Section 9.6.8.1. Detailed visual inspection of the screen/plate edge were also performed under a microscope for quality control.

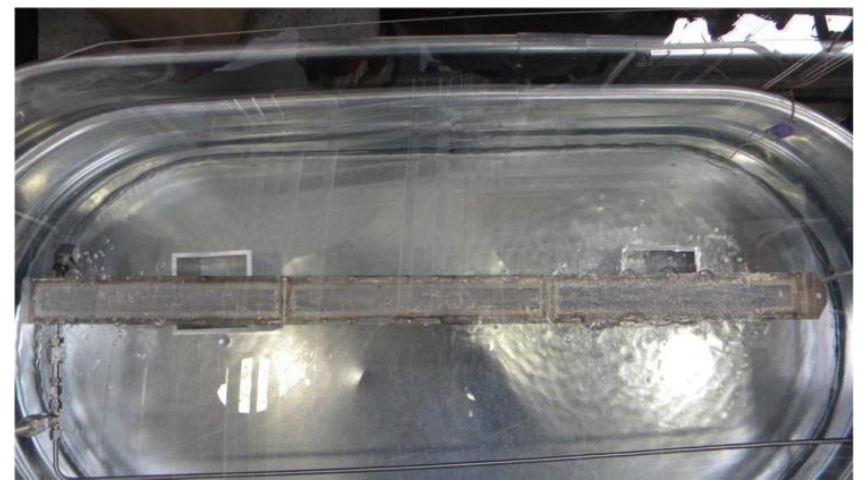

Figure 9.21 - Isopropyl Alcohol Bubble Point Testing of the Completed 325x2300 Channel

\subsubsection{Thermodynamic Vent System Cooled 325x2300 Channel}

The TVS cooled $325 \times 2300$ channel was fabricated in much the same way as the $325 \times 2300$ channel, but with a few differences. The individual screen samples had a perforated plate as the lower support plate as shown in Figure 9.20. The purpose of the plate was to provide additional structural support for the LAD screen, since launch loads may impose unwanted/unfavorable stresses on the screen. The plates cut down on the total area available for liquid flow to approximately $63 \%$ of the flow area of the standard 
$325 \times 2300$ channel. The LAD channel itself was triangular backed, as opposed to the straight back of the regular channel. As shown in Figure 9.20, the purpose of the triangular backing was to allow room for an internal TVS heat exchanging coil while maintaining the same hydraulic diameter of the standard channel.

The final assembled TVS cooled $325 \times 2300$ channel is shown in Figure 9.22. The purpose of the heat exchanger was to cool the liquid inside the channel, potentially raising the surface tension of the liquid at the screen, and thus increase the duration of liquid outflow before gas ingestion. As indicated in Equation 3.16, lowering the liquid temperature inside the channel will increase bubble point pressure.

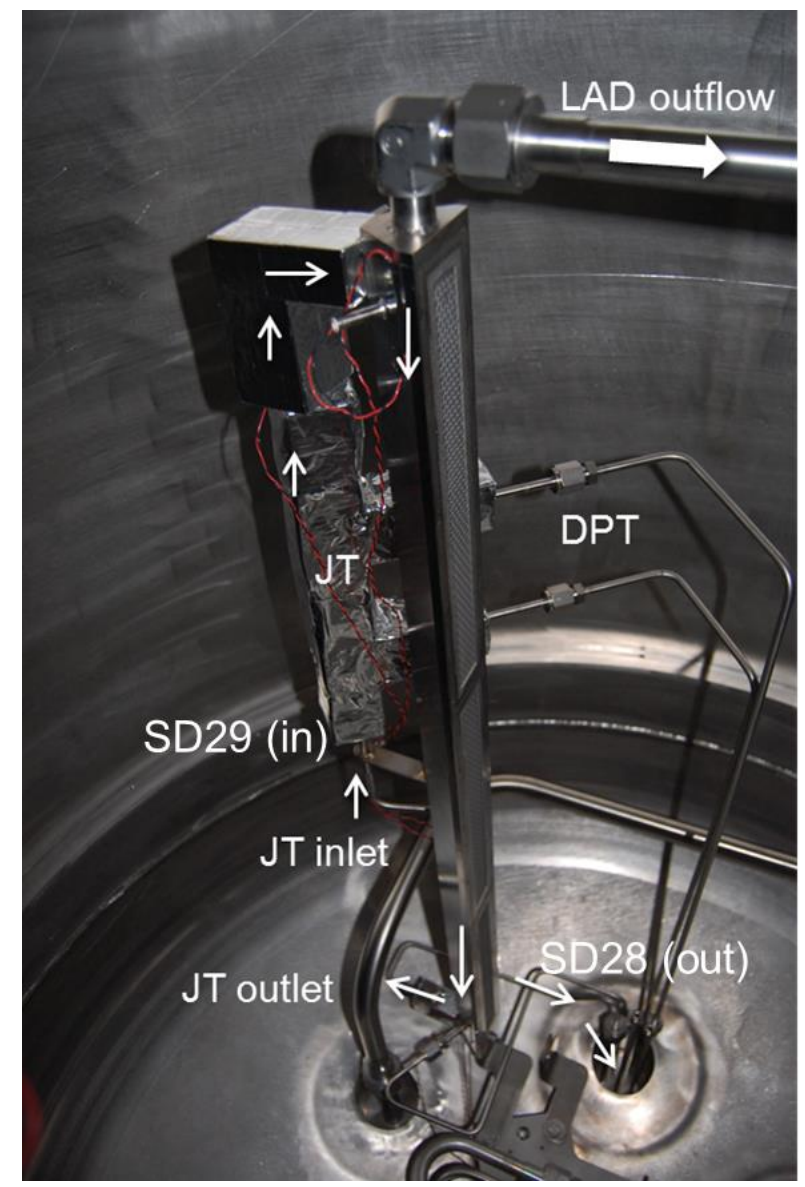

Figure 9.22 - Completed 325x2300 Thermodynamic Vent System Cooled Channel 
The basic principle of operation for the TVS cooled LAD channel is as follows: To provide cooling of the liquid flow through the LAD, liquid from the bottom of the tank is routed through a Joule-Thompson (JT) device. Liquid expands across the JT orifice at constant enthalpy, which causes a dramatic decrease in temperature. The liquid is then routed into the top of the LAD channel through a cooling coil, which acts as a counter flow heat exchanger for the liquid entering the channel through the LAD screen. Liquid is circulated through the cooling coil and then routed to a reduced vacuum environment $(<14 \mathrm{kPa})$ to increase pressure drop and thus enhance performance of the JT orifice.

\subsubsection{Thermodynamic Vent System Heat Exchanger Analysis}

The purpose of the TVS heat exchanger is to decrease the temperature of the liquid inside the LAD channel in an attempt to increase the local surface tension of the liquid in the screen pores to improve resistance to vapor ingestion and thus prolong the duration over which the LAD can drain the tank before breakdown. The heat exchanger utilizes the cooling capacity of the tube fluid to absorb heat from the warmer liquid inside the channel. The heat exchanger is composed of a coiled tube-in-shell counter-flow design as shown in Figure 9.20. Liquid hydrogen is routed from the tank through a tube to the top of the vertical LAD through a JT orifice, which expands the fluid to a state of lower temperature and serves as the cold side of the heat exchanger. The liquid travels down through a coil to maximize the heat transfer surface area of the coil with the liquid inside the channel. The shell side of the heat exchanger is the warmer liquid flowing from the tank across the screen into the channel. The warm fluid enters from the side walls of 
the LAD, in and around the coils, and exits through an outflow line at the top as shown in Figure 9.22.

A ViscoJet, which is a fluid-resistive device composed of multiple orifices and internal flow paths, is used as the JT orifice. The ViscoJet is designed to allow the control of flow rate based on pressure differential. The tube flow exits to a pressure of $14 \mathrm{kPa}$, so the anticipated pressure differential for this system, when the tank is pressurized to 207 $\mathrm{kPa}$ is nearly $193 \mathrm{kPa}$, with losses expected due to tube outflow. After the fluid passes through the ViscoJet, isenthalpic expansion occurs, and the drop in local pressure is met with a drop in local saturation temperature, cooling the fluid. This cool fluid runs inside a long $0.318 \mathrm{~cm}$ (1/8 in) diameter coil referred to as the TVS tube, with $0.05 \mathrm{~cm}(0.02 \mathrm{in})$ walls. The coil winding internal diameter is $1.59 \mathrm{~cm}(0.625 \mathrm{in})$, with a pitch of $0.635 \mathrm{~cm}$ (0.25 in). With these dimensions, approximately $8.53 \mathrm{~m}(28 \mathrm{ft})$ of wound coil is fit into the channel length as shown in Figure 9.20. This analysis was performed assuming a counter flow condition where a majority of the warm fluid entered from the bottom of the LAD, and out through the top.

The overall heat transfer coefficient $U_{0}$ of the heat exchanger was calculated based on three components of thermal resistance: the thermal resistance of the internal tube flow, the thermal resistance of the wall, and the thermal resistance of fluid flowing in the LAD on the outside of the tube:

$$
U_{o}=\frac{1}{A_{o} R_{T}}
$$

where 


$$
R_{T}=\frac{1}{A_{i} h_{i}}+\frac{t_{C}}{A_{m} k}+\frac{1}{A_{o} h_{o}}
$$

where the subscripts $i$ and $o$ reference inside and outside of the coil, respectively, and $t_{C}$ and $k$ are the thickness and thermal conductivity of the coil tube. The overall heat transfer coefficient is related to the overall performance of the heat exchanger using the NTU (number of heat transfer units) method, where

$$
N T U=\frac{A_{o} U_{o}}{C_{\min }}
$$

where $C_{\min }$ is the minimum heat capacity of the hot and cold fluids. As the cold fluid makes contact with the relatively warmer walls of the coil, nucleate boiling occurs and induces a phase change inside the tube, which increases the fluid cooling capacity. To model this two-phase fluid convection, the Forster-Zuber correlation (Chen 1966) is used, along with a nucleate boiling suppression factor $S_{N}$ and a two-phase multiplier $F$ where $h_{i}=h_{N B} S_{N}+h_{L} F$

where $h_{N B}$ is the nucleate boiling heat transfer coefficient and $h_{L}$ is the heat transfer coefficient assuming all liquid flow (Thome 2004). The two heat transfer coefficients are defined as:

$$
\begin{aligned}
& h_{N B}=0.00122 \frac{k_{L}^{0.79} c_{p, L}^{0.45} \rho_{L}^{0.49} \Delta T_{e}^{0.24} \Delta P_{s a t}^{0.75}}{\gamma_{L V}^{0.5} \mu_{L}^{0.29} h_{f g}^{0.24} \rho_{V}^{0.24}} \\
& h_{L}=0.023 \operatorname{Re}_{L}^{0.8} \operatorname{Pr}_{L}^{0.4}\left(\frac{k_{L}}{d_{i}}\right)
\end{aligned}
$$


$S_{N}=\frac{1}{1+0.00000253 \operatorname{Re}_{t p}^{1.17}}$

where $\Delta T_{e}$ is the temperature difference between coil wall and saturation temperature, $\Delta P_{\text {sat }}$ is the difference between saturation pressure based on the wall and the fluid, $\operatorname{Pr}$ is the Prandtl number, $d_{i}$ is the inner tube diameter, and subscripts $G$ and $L$ implies properties are evaluated assuming gaseous and liquid saturated phases, respectively, and the two-phase Reynolds number is defined as:

$\operatorname{Re}_{t p}=\operatorname{Re}_{L} \mathrm{~F}^{1.25}$

where the liquid Re number is defined as:

$\operatorname{Re}_{L}=\frac{\dot{m}_{\text {demand }}(1-x) d_{i}}{\mu_{L}}$

where $x$ is the quality. The two phase flow multiplier is:

$F=\left(\frac{1}{X_{t t}}+0.213\right)^{0.736}$

where the Martinelli parameter is used:

$X_{t t}=\left(\frac{1-x}{x}\right)^{0.9}\left(\frac{\rho_{G}}{\rho_{L}}\right)\left(\frac{\mu_{L}}{\mu_{G}}\right)^{0.1}$

This convective heat transfer coefficient is used with $A_{i}$, the area of the surface inside the tubes, to determine the thermal resistance between the cold fluid and the TVS tube walls in Equation 9.3. The heat transfer resistance across the tube is simply a function of 
the mean cross sectional area $A_{m}$ across the tube wall, the thickness of the wall $0.05 \mathrm{~cm}$ (0.020 in) and its thermal conductivity $k$, as shown in Equation 9.3. The convective heat transfer $h_{o}$ of the warmer LAD fluid outside the tube is calculated using the Churchill and Bernstein relation of fluid flow around a bank of tubes from Incropera and DeWitt (1996).

In such heat exchangers, where boiling occurs inside the tubes, the heat capacity rate of the boiling side is regarded as infinite, and the limiting factor of the heat exchanger becomes the heat capacity rate of the non-boiling side. In this case, this is the warm LAD fluid (outflow rate in the Test Matrix in Table 9.6). The heat capacity rate is the product of the flow rate and specific heat:

$C_{h}=\dot{m}_{\text {demand }} c_{p, L A D}=C_{\text {min }}$

where $c_{p, L A D}$ is the specific heat of the liquid in the channel. This value for $C_{\min }$ is used in Equation 9.4 to determine the NTU of the heat exchanger, which, in turn, is used to determine the heat exchanger effectiveness, $\varepsilon_{H E X}$. For counter-flow heat exchangers where boiling is occurring on one side, the effectiveness is calculated with the relation:

$\varepsilon_{H E X}=1-e^{-N T U}$

Knowledge of the effectiveness, along with the hot (LAD channel liquid) and cold (liquid inside TVS coil) fluid temperatures, allows one to calculate the overall heat transfer:

$\dot{Q}_{H E X}=\varepsilon_{H E X} C_{\min }\left(T_{h, i n}-T_{c, i n}\right)$ 
Finally, after obtaining the heat transfer rate $\dot{Q}_{H E X}$, the resultant temperature of the liquid inside the LAD channel at the LAD channel outlet is:

$$
T_{h, \text { out }}=T_{h, \text { in }}-\frac{\dot{Q}_{H E X}}{C_{h}}
$$

Testing is conducted with the LAD vertically oriented inside the tank. Before the liquid level in the tank drops below the top of LAD, the efficiency of the heat exchanger is negligible. When the liquid level drops below the top of the LAD channel, the efficiency of the heat exchanger will increase as a function of tank level drain. This is because the temperature of the liquid inside and outside of the channel is equal below the tank $\mathrm{L} / \mathrm{V}$ interface. Above the tank $\mathrm{L} / \mathrm{V}$ interface, where one side of the channel is exposed to warm ullage gas, the temperature of the liquid inside the channel will rise relative to the temperature of the liquid below the $\mathrm{L} / \mathrm{V}$ interface. Therefore, as the LAD screen is exposed to warm ullage gas, the overall effective heat transfer length of the coil increases. Expected versus actual performance is presented in Section 9.6.8.4.

\subsubsection{Instrumentation}

Figure 9.23 and Table 9.5 lists critical instrumentation for the inverted vertical LAD outflow tests. Ullage pressure was measured in order to deduce the pressure at the LAD screen. The stream pressure and temperature inside the LAD outflow line downstream of the channel but upstream of the Venturi flow meter was measured to ensure that there was vapor free liquid flow at the Venturi prior to the start of the test. To cover a broad range of liquid outflow rates, a set of two DPTs were used to calculate flow rate for the Venturi flow meters. The DPT between the inside of the channel and the 
ullage pressure was used to determine the FTS pressure drop across the screen as a function of the changing liquid level in the tank. Differential pressure across the JT orifice was also measured to determine the flow rate through the heat exchanger. A diode was epoxy bonded to the inner side of the topmost screen in the channel near the location of the highest pressure difference across the screen at breakdown in order to correlate with bubble point pressure models. Stream diodes were mounted upstream of the JT orifice and downstream of the cooling coil to assess the effectiveness of the TVS heat exchanger. Silicon diodes were spaced approximately $5.0 \mathrm{~cm}$ apart on a vertical rake and were used to measure both the bulk liquid temperature as well as height of the liquid level in the tank. Load cells were also used to measure mass and thus served as a redundant measure of the liquid level. The vertical diode rake was used as the primary liquid level sense method at LAD breakdown while the load cells were used to interpolate liquid level in between the diodes.

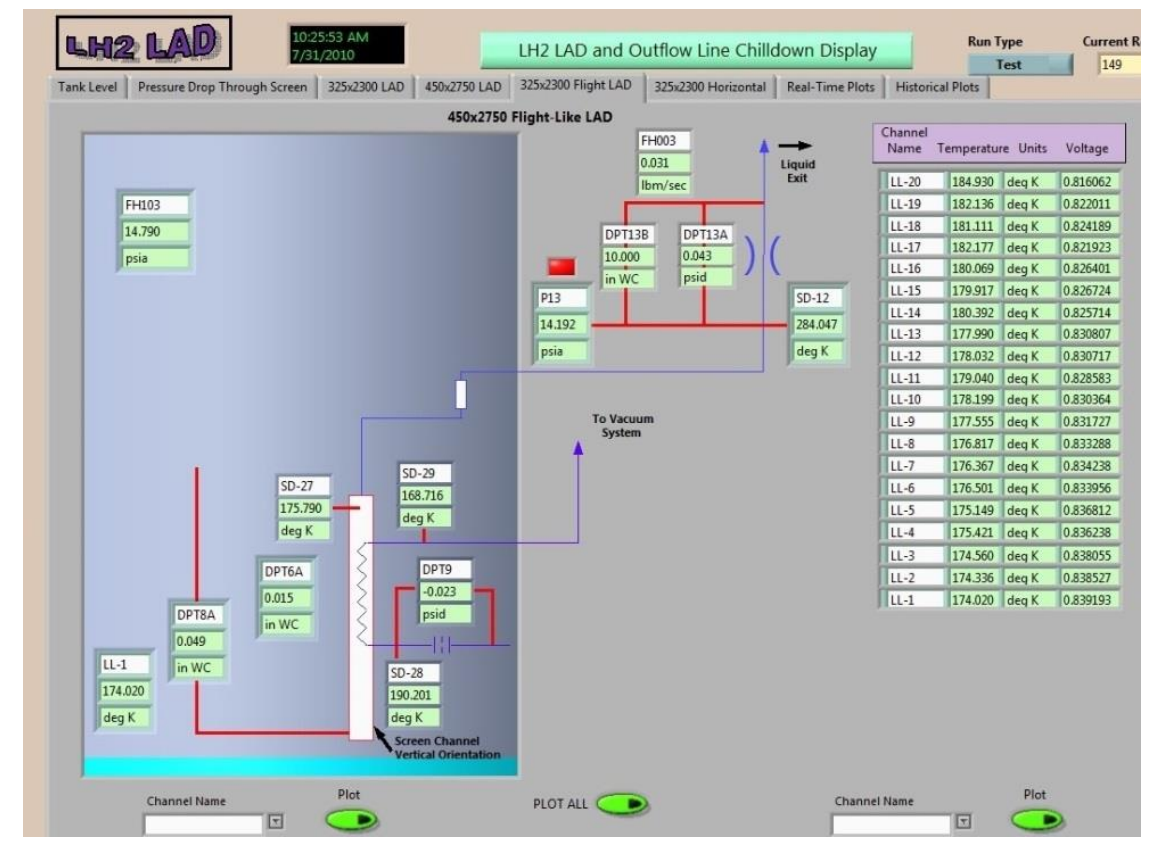

Figure 9.23 - Screenshot of Vertical Liquid Acquisition Device Outflow Data Acquisition 


\begin{tabular}{|c|c|c|c|c|}
\hline Channel & Units & & Uncertainty & Purpose \\
\hline \multicolumn{5}{|l|}{ PTs } \\
\hline FH103 & $0-689$ & $\mathrm{kPa}$ & $\pm 4.6 \mathrm{kPa}$ & Tank ullage pressure \\
\hline P12 & $0-689$ & $\mathrm{kPa}$ & $\pm 4.6 \mathrm{kPa}$ & VJ manifold pressure \\
\hline P13 & $0-689$ & $\mathrm{kPa}$ & $\pm 4.6 \mathrm{kPa}$ & Venturi static $P$ \\
\hline \multicolumn{5}{|l|}{ DPTs } \\
\hline DPT06A & $0-2491$ & $\mathrm{~Pa}$ & $\pm 1.82 \mathrm{~Pa}$ & 325 LAD head pressure \\
\hline DPT08A & $0-2491$ & $\mathrm{~Pa}$ & $\pm 1.82 \mathrm{~Pa}$ & 325F LAD head pressure \\
\hline DPT9 & $0-345$ & $\mathrm{kPa}$ & $\pm 4.6 \mathrm{kPa}$ & Joule Thompson DPT \\
\hline DPT13A & $0-138$ & $\mathrm{kPa}$ & $\pm 4.6 \mathrm{kPa}$ & Venturi DPT high \\
\hline DPT13B & $0-2491$ & $\mathrm{~Pa}$ & $\pm 1.82 \mathrm{~Pa}$ & Venturi DPT med \\
\hline \multicolumn{5}{|c|}{ SILICON DIODES } \\
\hline SD25 & $15-300$ & K & $\pm 0.1 \mathrm{~K}$ & 325 liquid screen side temp \\
\hline SD27 & $15-300$ & K & $\pm 0.1 \mathrm{~K}$ & 325F liquid screen side temp \\
\hline SD28 & $15-300$ & K & $\pm 0.1 \mathrm{~K}$ & TVS in \\
\hline SD29 & $15-300$ & K & $\pm 0.1 \mathrm{~K}$ & TVS out \\
\hline SD12 & $15-300$ & K & $\pm 0.1 \mathrm{~K}$ & Flight 325 LAD temperature @ venturi \\
\hline SD14 & $15-300$ & K & $\pm 0.1 \mathrm{~K}$ & 325 LAD temperature @ venturi \\
\hline \multicolumn{5}{|c|}{ FLOW METERS } \\
\hline FH001 & - & $\mathrm{Ibm} / \mathrm{s}$ & $\sim 2 \%$ of reading & Outflow for $325 \times 2300$ vertical LAD \\
\hline $\mathrm{FHOO3}$ & - & $\mathrm{lbm} / \mathrm{s}$ & $\sim 2 \%$ of reading & Outflow for $325 \times 2300$ flight vertical LAD \\
\hline
\end{tabular}

Table 9.5 - Instrumentation List for Inverted Vertical Outflow Tests

All pressures, DPTs, temperatures, and flows were recorded at $2 \mathrm{~Hz}$ using a computer DAQ. A 640x480 resolution, 30 frames per second, monochromatic camera was used to view the sight glass in real time. The camera system was modified to operate safely at $\mathrm{LH}_{2}$ temperatures. The pressure in the ullage and $\mathrm{VJ}$ manifold was measured to within $4.6 \mathrm{kPa}(0.667 \mathrm{psia})$. All DPTs measured pressure to within $1 \%$ of the full scale range. SD12, 14, and 25 - 29 measured temperature to within $0.1 \mathrm{~K}$; all diodes in the vertical rake measured temperature to within $0.5 \mathrm{~K}$. Liquid height was measured to within $2.7 \mathrm{~cm}$ (1.067 in) using the diodes. Uncertainty analysis was performed on the load cell calculated liquid level based on the internal volume of the tank and mass of the $\mathrm{LH}_{2}$. The uncertainty in liquid level between consecutive diodes is estimated to be 0.40 $\mathrm{cm}(0.154 \mathrm{in})$ in the coldest liquid temperatures and $1.27 \mathrm{~cm}(0.5 \mathrm{in})$ in the warmest liquid temperatures. Uncertainty in the LAD liquid flow rate is estimated based on the 
Venturi equation and was approximately $2-3 \%$ of the measured value across the whole range of tests.

\subsubsection{Test Methodology}

To conduct an inverted LAD outflow test, the tank was first filled with $\mathrm{LH}_{2}$, saturated to ambient pressure. Then the desired liquid temperature was achieved by simply setting the ullage pressure to the desired saturation pressure under back pressure control, and allowing the liquid to naturally absorb parasitic heat leak. Wall mounted heaters on the tank wall were used to elevate the liquid temperature by allowing the liquid to absorb heat over night. Once the liquid was conditioned, the ullage was

pressurized with GHe to the desired tank pressure in order to subcool the liquid for LAD outflow. The desired LAD channel was then chosen. The tank was drained through the LAD while the liquid level was still sufficiently above the top of the channel in order to prechill the outflow lines and Venturi FM located downstream of the LAD. The desired LAD mass flow rate was chosen by adjusting the control valves located downstream of the flow meter. When the flow rate was constant and steady for a period of at least 5 minutes, outflow tests commenced. If the lines downstream were not sufficiently prechilled before the tank liquid level was below the top of the channel, the test was aborted because the warm plumbing downstream of the channel would produce bubbles inside of the sight glass, complicating results.

LAD outflow was sampled in real time using a monitor inside of the control room; eventually a GHe bubble would break through the screen at a particular liquid fill level in the tank, corresponding to both visualization in the sight glass and change in the 
differential pressure measurement across the screen. Temperature readings downstream of the sight glass ensured that visible bubbles were helium, rather than gaseous hydrogen bubbles caused by liquid boiling due to heat leak into the line. The time at breakthrough was noted and synchronized with the video to compare with the data during post-test analysis. Two breakdown times were recorded, once at the first GHe visible bubble, and once at total LAD channel breakdown where a constant stream of pressurant gas bubbles were visible in the sight glass. Once the LAD was sufficiently broken down, flow through the LAD ceased, the tank was refilled above the top of the channel, and testing was conducted at a different mass flow rate or liquid temperature.

\subsubsection{Test Matrix}

Test conditions for the inverted 1-g vertical outflow tests are shown in Table 9.6. The test matrix is divided between the TVS cooled $325 \times 2300$ and standard $325 \times 2300$ channel test runs. For clarity, the table is organized and color coded by saturation temperature (and pressure), and then organized by flow rate.

As shown, vertical outflow tests were conducted at fixed fluid saturation temperatures of $20.3 \mathrm{~K}, 21.4 \mathrm{~K}, 22.9 \mathrm{~K}$, and $24.2 \mathrm{~K}$. Breakdowns were attempted at several different controlled mass flow rates representative of the previously referenced mission architectures, as shown in Table 9.1. Tank pressure was generally chosen to be sufficiently greater than saturation pressure to suppress liquid boiling at the LAD screen. For the TVS cooled 325x2300 LAD, three tests were repeated at the same liquid temperature to directly compare performance with and without the TVS engaged. To examine the effect of LAD performance gain in bubble point pressure due to pressurizing 
and subcooling with the non-condensable pressurant, three tests were repeated for the

TVS cooled $325 \times 2300$ channel at elevated tank pressure.

\begin{tabular}{|c|c|c|c|c|c|c|c|}
\hline & & & Ptank & Psat & Tsat & Flow Rate & Notes \\
\hline Test \# & Day & Date & kPa [psia] & kPa [psia] & [K] & $\mathrm{kg} / \mathrm{s}[\mathrm{lbm} / \mathrm{s}]$ & \\
\hline \multicolumn{8}{|l|}{$325 \times 2300$} \\
\hline 5 & 3 & $8 / 1 / 2012$ & $204.1[29.6]$ & 103.4 [15] & 20.3 & $0.0241[0.053]$ & \\
\hline 4 & 3 & 8/1/2012 & 202.7 [29.4] & $103.4[15]$ & 20.3 & 0.0364 [0.08] & \\
\hline 9 & 5 & $8 / 3 / 2012$ & $202.0[29.3]$ & $103.4[15]$ & 20.3 & $0.0414[0.091]$ & \\
\hline 22 & 15 & $8 / 17 / 2012$ & 240.0 [34.8 ] & $137.9[20]$ & 21.4 & $0.0418[0.092]$ & \\
\hline 14 & 9 & $8 / 9 / 2012$ & 233.7 [33.9] & $137.9[20]$ & 21.4 & $0.0486[0.107]$ & \\
\hline 20 & 13 & $8 / 15 / 2012$ & 282.0 [40.9] & 206.8 [30] & 22.9 & $0.0123[0.027]$ & \\
\hline 27 & 19 & $8 / 23 / 2012$ & 270.3 [39.2] & 206.8 [30] & 22.9 & $0.0491[0.108]$ & \\
\hline 15 & 10 & $8 / 10 / 2012$ & 344.7 [50] & $275.8[40]$ & 24.2 & $0.0167[0.0367]$ & \\
\hline 24 & 18 & $8 / 22 / 2012$ & $343.4[49.8]$ & $275.8[40]$ & 24.2 & $0.0223[0.049]$ & \\
\hline 18 & 12 & $8 / 14 / 2012$ & $348.2[50.5]$ & $275.8[40]$ & 24.2 & $0.034[0.0747]$ & \\
\hline \multicolumn{8}{|c|}{$325 \times 2300$ TVS Cooled } \\
\hline 7 & 4 & $8 / 2 / 2012$ & 204.8 [29.7] & 103.4 [15] & 20.3 & $0.019[0.042]$ & \\
\hline 2 & 1 & $7 / 30 / 2012$ & $173.1[25.1]$ & $103.4[15]$ & 20.3 & $0.0227[0.05]$ & \\
\hline 1 & 1 & $7 / 30 / 2012$ & $139.3[20.2]$ & $103.4[15]$ & 20.3 & $0.0364[0.08]$ & \\
\hline 19 & 12 & $8 / 14 / 2012$ & $242.7[35.2]$ & $137.9[20]$ & 21.4 & $0.015[0.033]$ & \\
\hline 3 & 2 & $7 / 31 / 2012$ & 236.5 [34.3] & $137.9[20]$ & 21.4 & $0.02[0.044]$ & \\
\hline 21 & 14 & $8 / 16 / 2012$ & 236.5 [34.3] & $137.9[20]$ & 21.4 & $0.0286[0.063]$ & \\
\hline 12 & 7 & $8 / 7 / 2012$ & $271.7[39.4]$ & $206.8[30]$ & 22.9 & $0.0414[0.091]$ & \\
\hline 26 & 19 & $8 / 23 / 2012$ & $273.0[39.6]$ & $206.8[30]$ & 22.9 & $0.0495[0.109]$ & \\
\hline 23 & 17 & $8 / 21 / 2012$ & $273.7[39.7]$ & $206.8[30]$ & 22.9 & $0.05[0.11]$ & \\
\hline 8 & 5 & $8 / 3 / 2012$ & 339.9 [49.3] & $275.8[40]$ & 24.2 & $0.0132[0.029]$ & \\
\hline 6 & 4 & $8 / 2 / 2012$ & $339.2[49.2]$ & $275.8[40]$ & 24.2 & $0.025[0.055]$ & \\
\hline 17 & 11 & $8 / 13 / 2012$ & $344.7[50]$ & $103.4[15]$ & 20.3 & $0.0141[0.031]$ & Subcooled run \\
\hline 16 & 10 & $8 / 10 / 2012$ & $344.7[50]$ & $103.4[15]$ & 20.3 & $0.0263[0.058]$ & Subcooled run \\
\hline 25 & 18 & $8 / 22 / 2012$ & $332.3[48.2]$ & $103.4[15]$ & 20.3 & $0.055[0.121]$ & Subcooled run \\
\hline 13 & 7 & $8 / 7 / 2012$ & $204.1[29.6]$ & $103.4[15]$ & 20.3 & $0.03[0.066]$ & TVS off \\
\hline 11 & 6 & $8 / 6 / 2012$ & 203.4 [29.5] & 103.4 [15] & 20.3 & $0.0386[0.085]$ & TVS off \\
\hline 10 & 5 & $8 / 3 / 2012$ & $202.7[29.4]$ & 103.4 [15] & 20.3 & $0.0414[0.091]$ & TVS off \\
\hline
\end{tabular}

Table 9.6 - Inverted Outflow Test Matrix

\subsubsection{One Dimensional Steady State Pressure Drop Model General Trends}

Substituting the hydrostatic, new temperature dependent FTS, and frictional and dynamic models into Equation 3.3, the total 1D pressure drop for the LAD channel in 1-g steady flow is determined, and the model can be used to predict the breakdown point as a function of the liquid temperature and mass flow rate through the LAD. To demonstrate general model trends and predictions, setting Equation 3.3 equal to the bubble point 
pressure in Equation 3.16, one can then simulate LAD outflow in an inverted 1-g configuration in $\mathrm{LH}_{2}$ for the $\mathrm{LAD}$ channels and test conditions that would be typical of an in-space propellant transfer. For a fixed 325x2300 screen mesh and LAD channel geometry, examination of Equations 3.23, 3.33, 3.55, and 3.58 show that the steady state pressure drop is a function of LAD dimensions, liquid temperature, pressure, and mass flow rate only.

The model simulates tank drain through the vertically mounted LAD in 1-g. It can be used to calculate the total pressure drop across the screen as a function of the pressure and temperature inside the tank by computing each of the four terms at each liquid level. In an iterative fashion, the code continues to run as the tank is drained until $\Delta P_{\text {total }}>\Delta P_{B P}$ to compare the model predicted breakdown height against the actual breakdown height. The model assumes that liquid flow enters the channel uniformly through the available wetted screen area. The model also assumes uniform flow down the channel.

Figure 9.24 plots general model trends. To gauge LAD performance, the breakdown point is plotted as exposed screen length. Therefore, longer exposed screen lengths equate to better performance. Figure 9.24 plots exposed screen length as a function of $\mathrm{LH}_{2}$ saturation temperature and mass flow rate through the LAD.

As shown, the model predicts that at a constant mass flow rate, increasing the fluid temperature decreases the exposed screen height in a near linear fashion. This is primarily due to the decrease in surface tension, and thus bubble point, at warmer liquid temperatures. Meanwhile, at a constant fluid temperature, increasing the mass flow rate through the LAD also causes the LAD to break down earlier. This is primarily due to the 
increase in FTS pressure drop caused by reduced available area for liquid to flow through the screen into the channel. At a flow rate of $0.1 \mathrm{~kg} / \mathrm{s}$, the initial FTS pressure drop is large enough to cause the LAD to break down immediately (i.e. zero exposed screen length) for nearly all liquid temperatures shown in Figure 9.24.

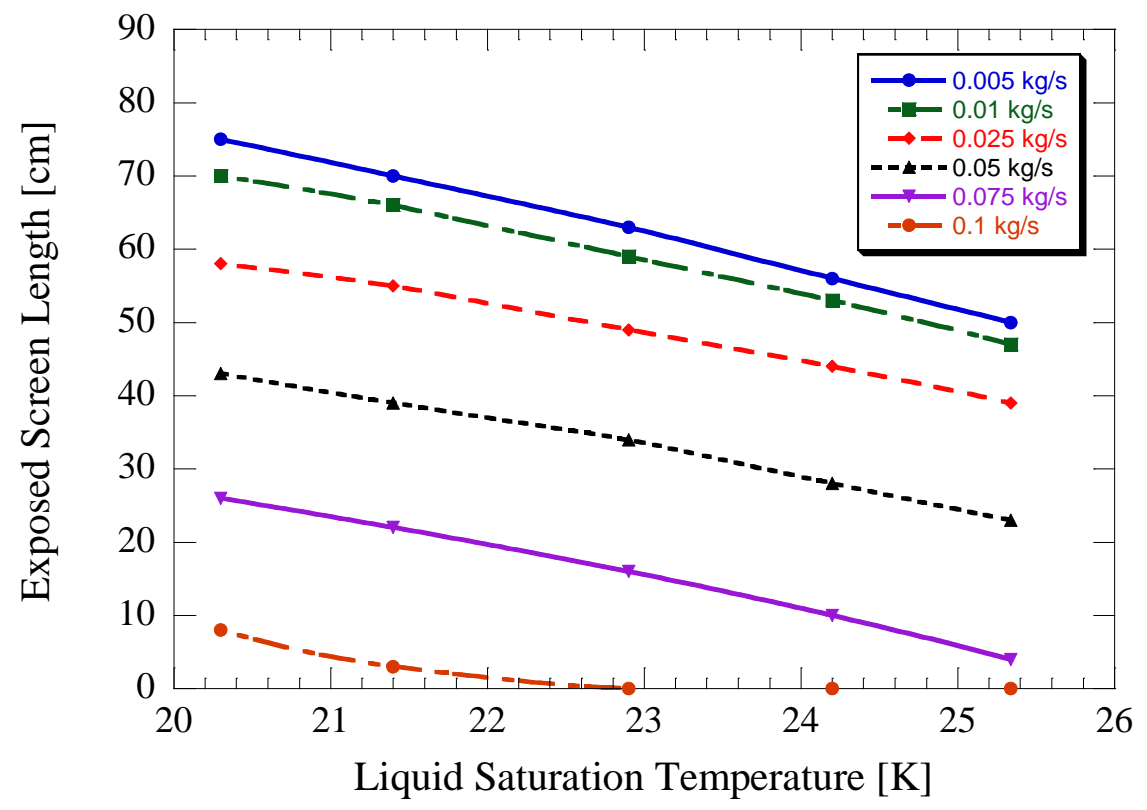

Figure 9.24 - General One Dimensional Steady State Pressure Drop Model Trends - Exposed Screen Length as a Function of Liquid Temperature and Mass Flow Rate through the Channel

To illustrate the relative magnitudes of the hydrostatic and FTS pressure drops in a 1-g environment, Figure 9.25 plots these two terms along with the total pressure drop at the point in the LAD where the pressure differential across the screen is highest, at the top of the channel, at bubble breakdown. Figure 9.25a shows model predictions for a low flow of $0.005 \mathrm{~kg} / \mathrm{s}$ while Figure $9.25 \mathrm{~b}$ shows model predictions for a higher flow of 0.025 $\mathrm{kg} / \mathrm{s}$. Pressure drops are plotted as a function of increased exposed screen length. 

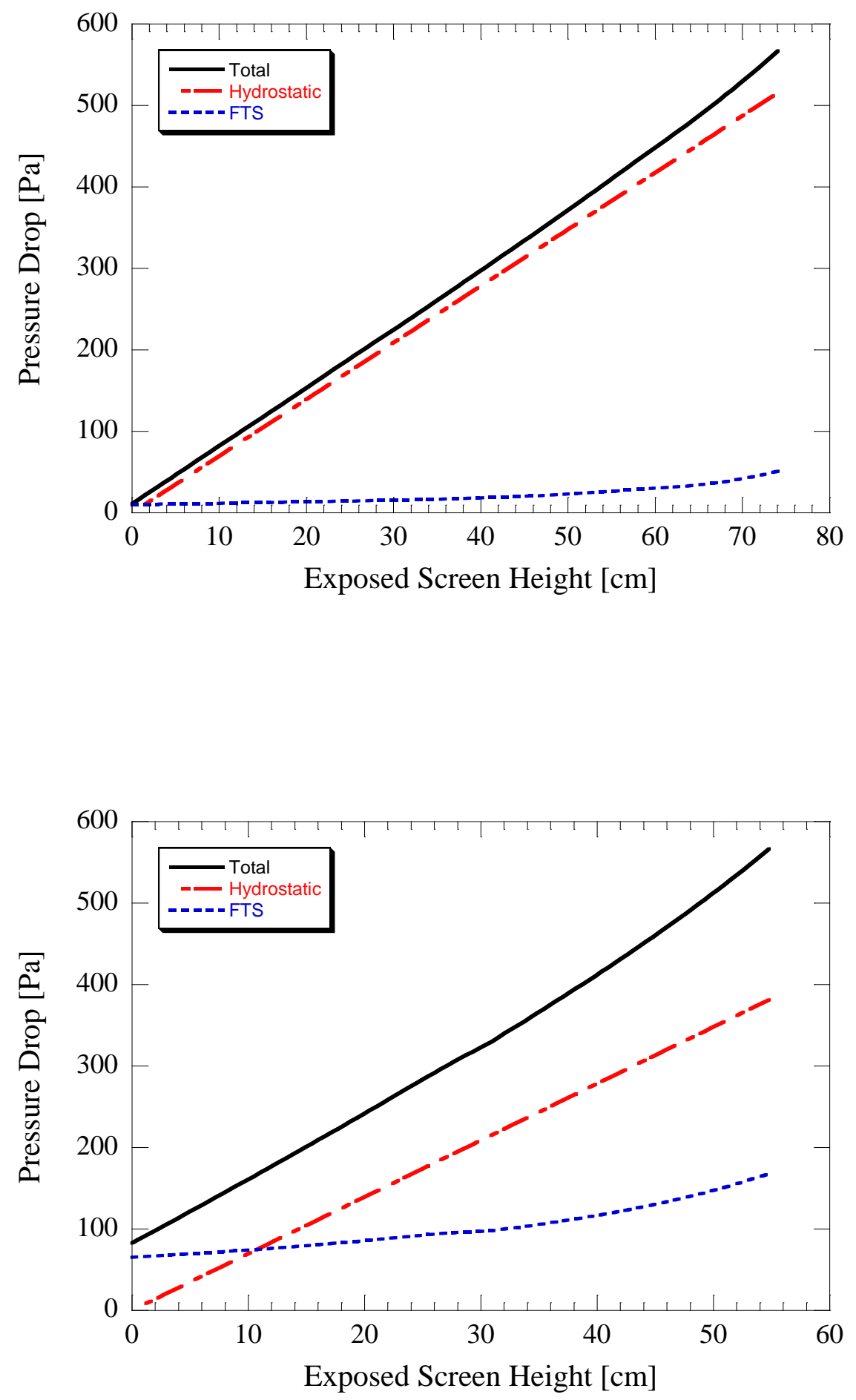

Figure 9.25 - Relative Magnitude of Pressure Drop Contributions as a Function of Exposed Channel Length for a) Low Flow $(0.005 \mathrm{~kg} / \mathrm{s})$ and b) High Flow $(0.025 \mathrm{~kg} / \mathrm{s})$ 
As shown for the low flow case, hydrostatic pressure drop is the leading order term for the duration of the tank drain, while FTS pressures drop is a second order effect. Meanwhile at the high flow case, the FTS contribution increases proportionally; initially this contribution is higher than the hydrostatic term, but eventually is overcome by hydrostatics. To further illustrate, Table 9.7 shows the percent contribution of hydrostatic, FTS, and the sum of frictional and dynamic pressure drops at LAD breakdown for both of these model runs.

\begin{tabular}{|c|c|c|c|c|}
\hline Tliq [K] & mdot [kg/s] & \% Hydrostatics & \% FTS & \% Frictional + Dynamic \\
\hline 20.3 & 0.005 & 90.89 & 8.99 & 0.12 \\
\hline 20.3 & 0.025 & 67.3 & 29.68 & 3.01 \\
\hline
\end{tabular}

Table 9.7 - Pressure Drop Contributions at Breakdown for Low and High Flows

\subsubsection{Experimental Results}

\subsubsection{Screen Channel Bubble Point Tests in Isopropyl Alcohol}

Before submersion in $\mathrm{LH}_{2}$, both channels were cold shocked in $\mathrm{LN}_{2}$ and then bubble point tested in IPA as shown in Figure 9.21. The method of bubble point testing is outlined in Chapter 4. Test results are shown in Figure 9.26. The black line is the prediction curve based on surface tension values based on the local temperature of the IPA during tests and $325 \times 2300$ pore diameters from room temperature tests in Chapter 4 . Error bars are also plotted for reference.

As shown, the standard $325 \times 2300$ channel exceeded predicted performance before testing; but after the channel was tested in $\mathrm{LH}_{2}$, the performance degraded by $4.9 \%$. Meanwhile, the TVS cooled LAD did not exceed the expectation value before or after $\mathrm{LH}_{2}$ testing, with a degradation factor of $19 \%$. Note from Figure 9.26 that the two 
channels did not have the same exact bubble point pressure, before or after $\mathrm{LH}_{2}$ tests, despite both being manufactured from the same raw batch of $325 \times 2300$ screen material. The difference in performance pre-test was just outside experimental uncertainty of $3 \%$. However the difference in performance post-test was greater than $15 \%$.

The change in performance of both channels is likely due to the differential contractions and expansions of the screen wires after being exposed to multiple thermal cycles during $\mathrm{LH}_{2}$ testing. The reduction in performance could also be due to defects at the $\mathrm{Ni} / \mathrm{SS}$ interface at the screen/plate interface. It is fairly certain that degradation in bubble point occurred after $\mathrm{LH}_{2}$ tests, and not before, since both channels performed relatively similar at similar $\mathrm{LH}_{2}$ test conditions as will be shown, and because experimental performance compared well with model predictions.

Nonetheless, since the channels had slightly different pretest bubble points, it is not advisable to compare the breakdown points on an absolute scale. Rather, relative trends in performance between the two channels are considered. This is also precisely why it was desired to conduct tests with the TVS cooled LAD channel with the TVS engaged and disengaged in order to quantify TVS cooling performance, rather than compare the two channels. 

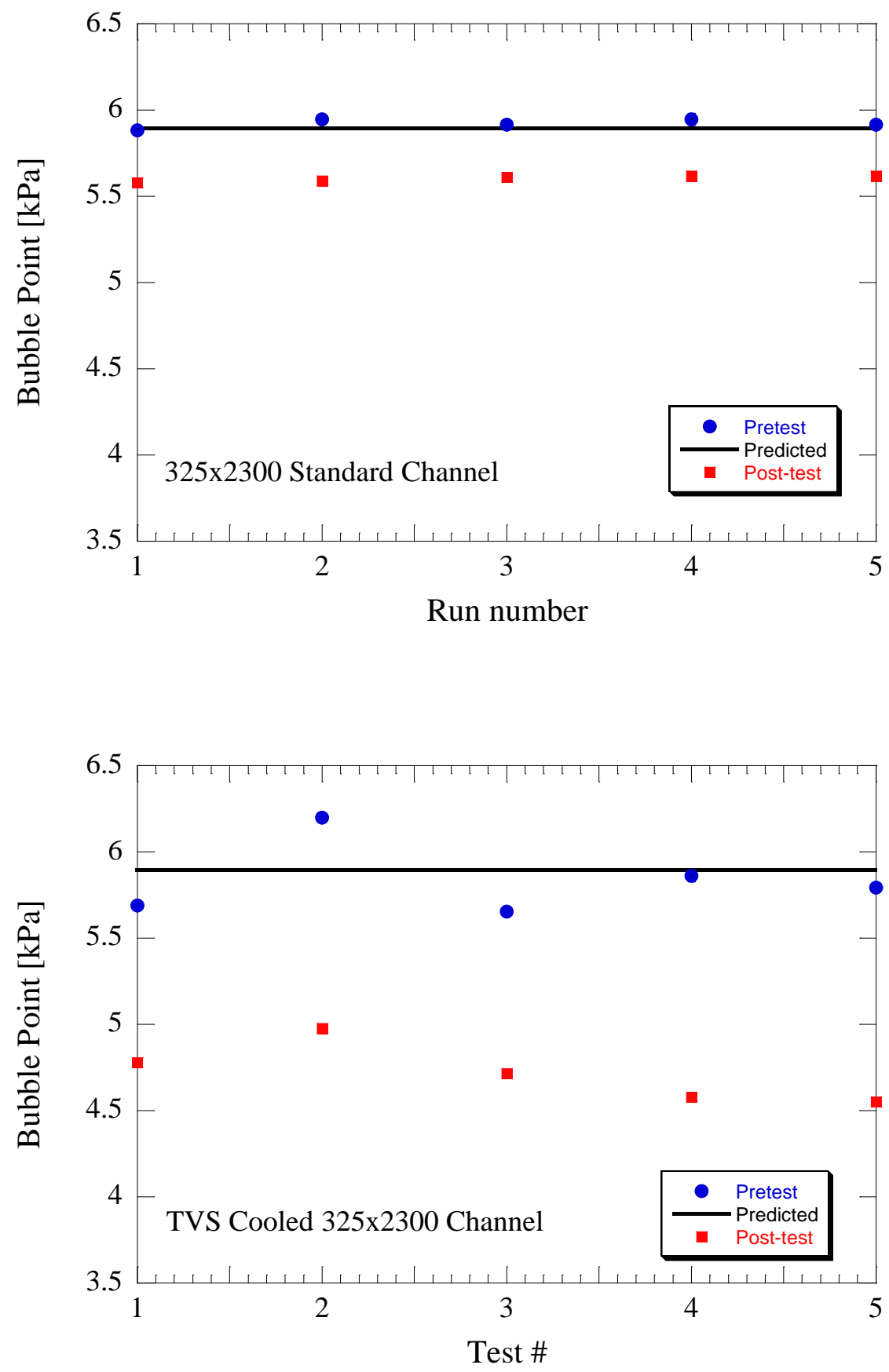

Figure 9.26 - Pre and Post Isopropyl Alcohol Bubble Point Testing of the a) 325x2300 Standard Channel and b) Thermodynamic Vent System Cooled Channel. The black line is the prediction curve based on the temperature of the IPA and $325 \times 2300$ pore diameter from Chapter 4. 


\subsubsection{Standard 325x2300 Channel Performance}

Inverted 1-g LAD outflow test results are plotted in Figures 9.27 and 9.28. Performance is measured in terms of the vertical exposed screen height as a function of liquid temperature and mass flow rate. Therefore, longer exposed screen heights equate to higher performance, since the LAD is able to sustain outflow for a longer period of time before gas ingestion. Alternatively, one could also use distance from the bottom of the LAD channel to gauge performance, which is simply the total channel length minus the exposed screen height. Ideally, one would compare the measured differential pressure across the screen at the point of bubble breakthrough, but examination of the DPT readings across the screen yielded data that was too noisy. It is likely that small fluctuations in the tank L/V interface during outflow caused dramatic changes in the DPT signal levels, nulling the raw DPT measurements. The next best way to compare LAD performance between data and model is to simply report the liquid level (or exposed screen length) at breakdown, and then use the 1D model to compute the liquid level when the differential pressure across the screen exceeds the bubble point pressure.

Figure 9.27 plots the exposed screen length for the $325 \times 2300$ standard channel as a function of tank liquid temperature and LAD outflow rate. Figure 9.27a plots the breakdown point the moment a pressurant gas bubble was visible in the sight glass while Figure $9.27 \mathrm{~b}$ plots the breakdown point when the LAD had ingested a steady stream of bubbles (i.e. complete failure). Error bars are plotted for reference. 

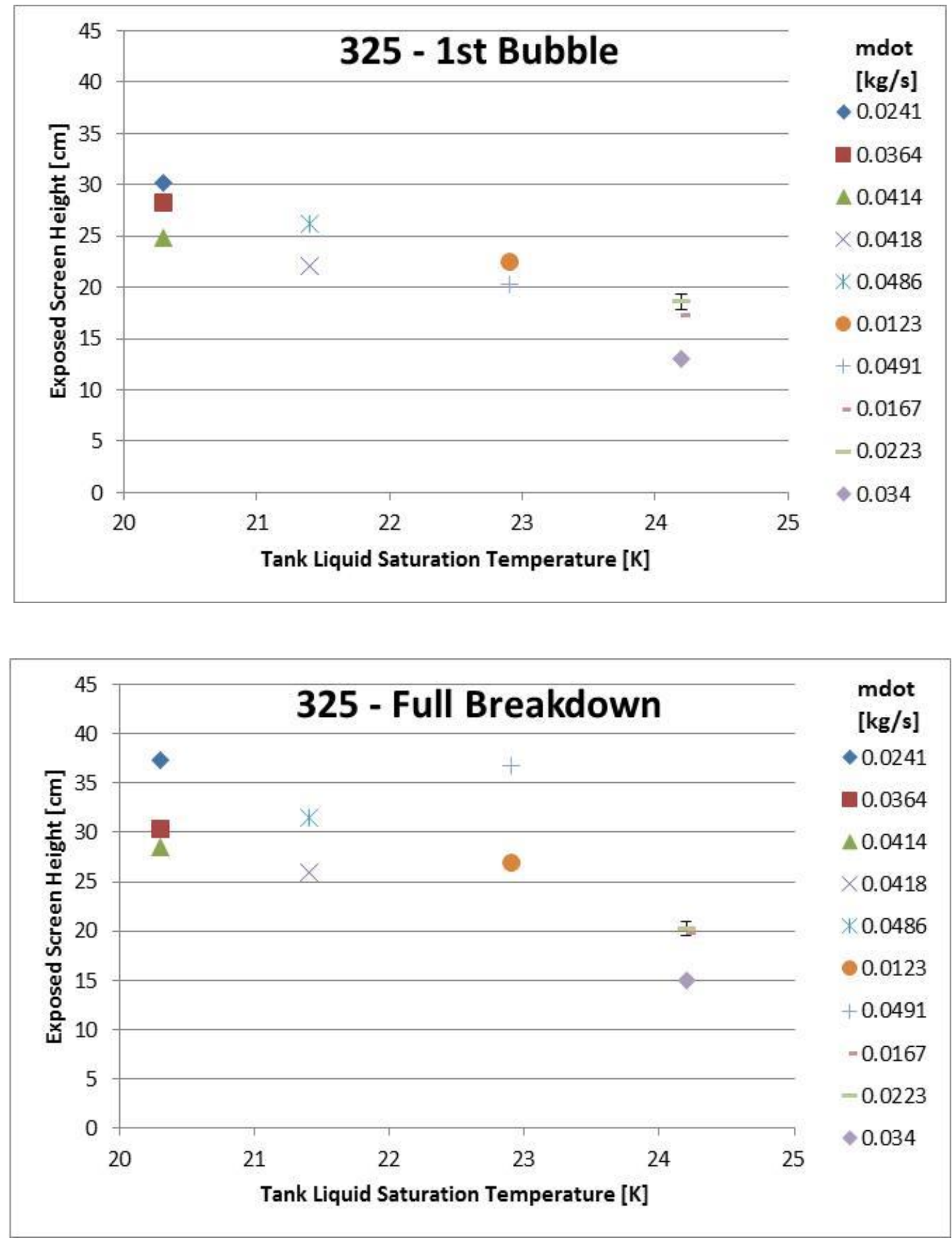

Figure 9.27 - Exposed Screen Height as a Function of Tank Liquid Conditions and Mass Flow Rate through the Standard 325x2300 Channel at a) First Helium Bubble Ingestion and b) Total Breakdown 

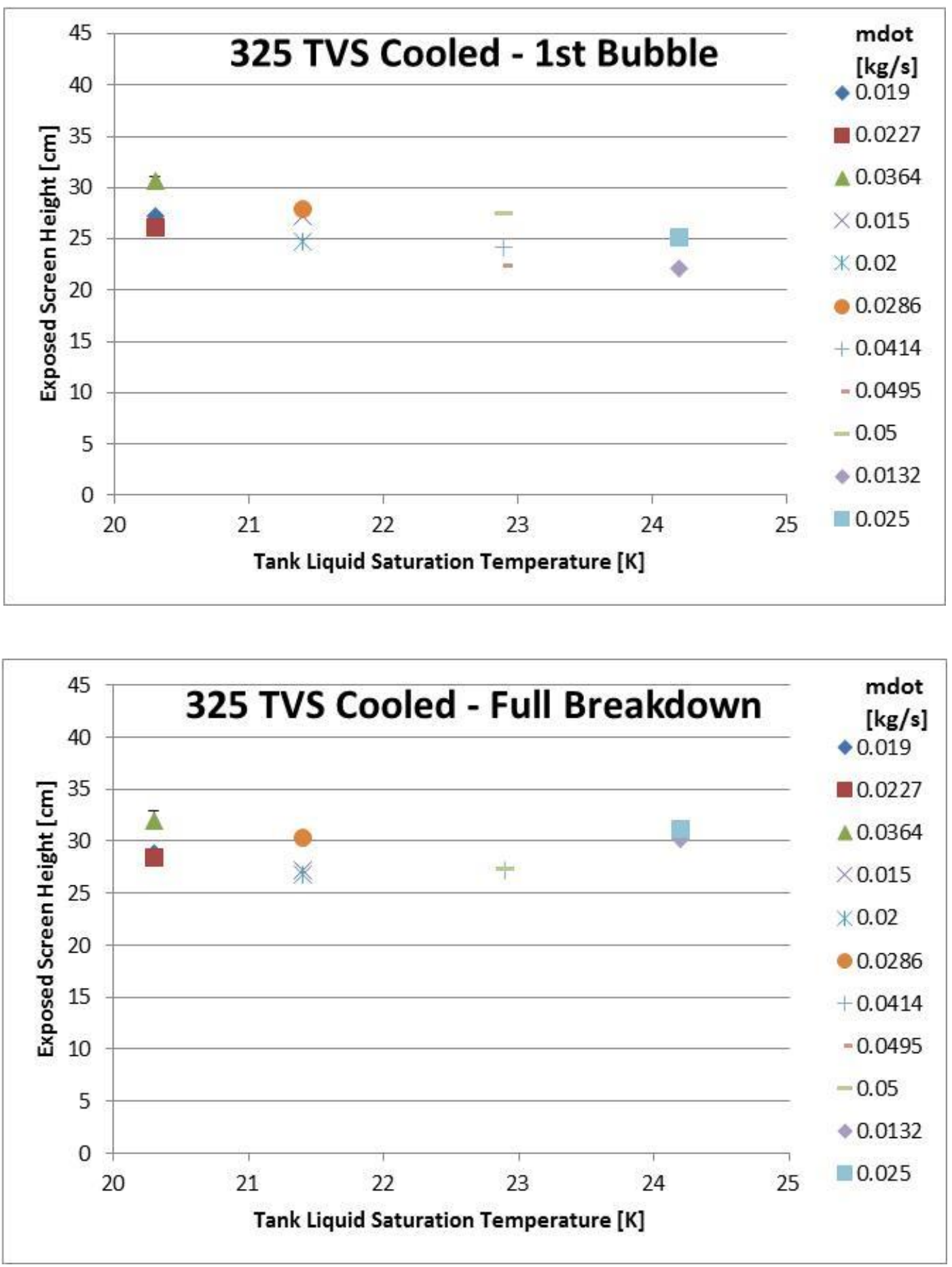

Figure 9.28 - Exposed Screen Height as a Function of Tank Liquid Conditions and Mass Flow

Rate through the Thermodynamic Vent System Cooled 325x2300 Channel at a) First Helium Bubble Ingestion and b) Total Breakdown 
The three trends are as follows: First, the breakdown point is dominated by liquid temperature, because longer exposed screen lengths are achievable in colder liquid temperatures, since higher surface tensions are achievable in colder temperatures. The amount of exposed screen length decreases linearly with increasing tank liquid temperature. At the warmest liquid temperature tested of $24.2 \mathrm{~K}$, the LAD breakdown point has shifted by about $15 \mathrm{~cm}$, or about $16 \%$ of the total LAD channel height. Second, the breakdown point is also dependent on the mass flow rate through the LAD. At a given fixed liquid temperature, the standard 325x2300 channel broke down at higher flow rates. Over a change in flow rate from $\sim 0.01 \mathrm{~kg} / \mathrm{s}$ to $0.04 \mathrm{~kg} / \mathrm{s}$, the breakdown point shifts by approximately $4 \%$ of the total channel length. This shift in the breakdown point with increased mass flow rate is simply due to an increase in the FTS pressure drop across the channel, which increases as the amount of screen is exposed to vapor (i.e. less cross sectional area for the liquid to flow through the screen). The effect of mass flow rate is consistent across the range of liquid temperatures tested here. Third, when comparing Figure 9.27 a to $9.27 b$, the amount of exposed screen length increases from the point at which the channel ingests a single GHe bubble to the total breakdown point, as expected. The average gain in performance from single bubble ingestion to total breakdown is approximately $4.8 \mathrm{~cm}$ or $5 \%$ of the total LAD height. The trends here agree well with the general model predicted trends in Figure 9.24.

\subsubsection{Thermodynamic Vent System Cooled 325x2300 Channel Performance}

Figure 9.28 plots the exposed screen length for the TVS cooled 325x2300 channel as a function of tank liquid temperature and LAD outflow rate, and Table 9.8 summarizes TVS performance. For all points shown in Figure 9.28, the TVS was engaged, and the 
flow rate through the TVS coil was approximately the same, $1.14 \mathrm{~g} / \mathrm{s}$. However the amount of cooling was slightly different due to different TVS inlet liquid temperatures. The TVS inlet pressure was the tank pressure, and the outlet pressure was $14 \mathrm{kPa}$.

\begin{tabular}{|c|c|c|c|c|c|c|c|}
\hline \multicolumn{1}{|c|}{} & Ptank & Psat & T LAD in & T LAD Out & mdot LAD & delta T JT & delta P JT \\
\hline \multicolumn{1}{|c|}{ Test \# } & {$[\mathbf{k P a}]$} & {$[\mathrm{kPa}]$} & {$[\mathrm{K}]$} & {$[\mathrm{K}]$} & {$[\mathbf{k g} / \mathbf{s}]$} & {$[\mathbf{K}]$} & {$[\mathbf{k P a}]$} \\
\hline 7 & 204.77 & 103.42 & 20.3 & 20.3 & 0.0191 & 4.61 & 44.4 \\
\hline 2 & 173.06 & 103.42 & 20.3 & 20.3 & 0.0227 & 5.28 & 31.72 \\
\hline 1 & 139.27 & 103.42 & 20.3 & 20.3 & 0.0364 & 4.88 & 41.71 \\
\hline 17 & & & & & & & \\
\hline 16 & 344.74 & 103.42 & 20.3 & 20.3 & 0.0141 & 5.22 & 68.26 \\
\hline 25 & 334.74 & 103.42 & 20.3 & 20.3 & 0.0263 & 4.51 & 4.96 \\
\hline 19 & 242.70 & 103.42 & 20.3 & 21.2 & 0.0550 & 5.03 & 5.12 \\
\hline 3 & 236.49 & 137.90 & 21.4 & 21.4 & 0.0200 & 6.08 & 41.37 \\
\hline 21 & 236.49 & 137.90 & 21.4 & 21.2 & 0.0286 & 6.43 & 39.85 \\
\hline 12 & 271.65 & 206.84 & 22.9 & 22.8 & 0.0414 & 6.18 & 168.23 \\
\hline 26 & 273.03 & 206.84 & 22.9 & 22.9 & 0.0495 & 7.31 & 32.68 \\
\hline 23 & 273.72 & 206.84 & 22.9 & 22.8 & 0.0500 & 7.25 & 34.2 \\
\hline 8 & 339.91 & 275.79 & 24.2 & 23.3 & 0.0132 & 7.05 & 43.3 \\
\hline 6 & 339.22 & 275.79 & 24.2 & 23.8 & 0.0250 & 7.38 & 39.58 \\
\hline
\end{tabular}

Table 9.8 - Thermodynamic Vent System Cooled 325x2300 Channel Performance

The four trends are as follows: First, the breakdown point is again dominated by liquid temperature. Second, there again is a secondary dependence on mass flow rate. However, unlike the standard $325 \times 2300$ screen channel, higher flow rates lead to longer exposed screen lengths. Higher flow rates cause the TVS cooled channel to break down later. The spread in data is approximately $3 \%$ of the total channel length. This difference in performance with flow rate between the two channels is attributed to the presence of the perforated plate in the TVS cooled channel. Previous studies have shown that the perforated plate enhanced wicking of liquid (Paynter 1973a). As liquid is routed up through the top of the TVS cooled channel, the plate acts to rewet areas of the screen 
which would dry out, thus extending the point of breakdown. So the presence of the plate is beneficial both structurally and thermally.

Third, when comparing Figure 9.28a to 9.28b, the amount of exposed screen length increased from the point at which the channel ingests a single GHe bubble to the total breakdown point, as expected. The average gain in performance from single bubble ingestion to total breakdown is approximately $2.9 \mathrm{~cm}$ or $3 \%$ of the total TVS cooled LAD height. Fourth, comparing Figure 9.28a to 9.27a, the slope of the breakdown points flattens out for the TVS cooled channel relative to the standard channel. This is proof that the TVS cooling enhanced performance of the LAD in warmer liquid temperatures. This implies that the TVS cooling can provide nearly the same performance in warmer liquid temperatures where the channel has a higher tendency to break down than it does in colder liquid temperatures. In addition, when comparing Figures 9.28a to 9.28b, the perforated plate and TVS cooling also lead to longer exposed screen heights at the warmest liquid temperatures. The trends here do not agree well with the model predicted trends from Figure 9.24 because the 1D model does not account for the additional wicking.

\subsubsection{Thermodynamic Vent System Efficiency}

To isolate and test the efficiency of the TVS cooling system, six tests were conducted with the TVS cooled LAD channel in 20.3K saturated liquid with identical tank pressures of $207 \mathrm{kPa}$ as shown in Table 9.8. For three of the tests, the TVS cooling was engaged; for three of the tests the TVS was disengaged. Only liquid flow rate through the LAD varied for these tests. Since the two channels had different pretest 
bubble points, this testing strategy was the only way to directly measure the efficiency of the TVS cooling. Results are plotted in Figure 9.29. As shown, longer exposed screen lengths were achievable with the heat exchanger engaged, as expected. The average difference in performance is approximately $4.9 \%$ of total channel length, or $31 \mathrm{~Pa}$ of hydrostatic head pressure in $\mathrm{LH}_{2}$, which from examination of $325 \times 2300 \mathrm{LH}_{2} / \mathrm{GHe}$ bubble point pressures, Figure 5.11, translates into approximately $0.7 \mathrm{~K}$ of extra margin in bubble point pressure. The largest difference in performance was at higher flow rates where the perforated plate was able to wick colder fluid into the screen pores at the top of the channel, thus prolonging the breakdown point relative to slower flow rates.

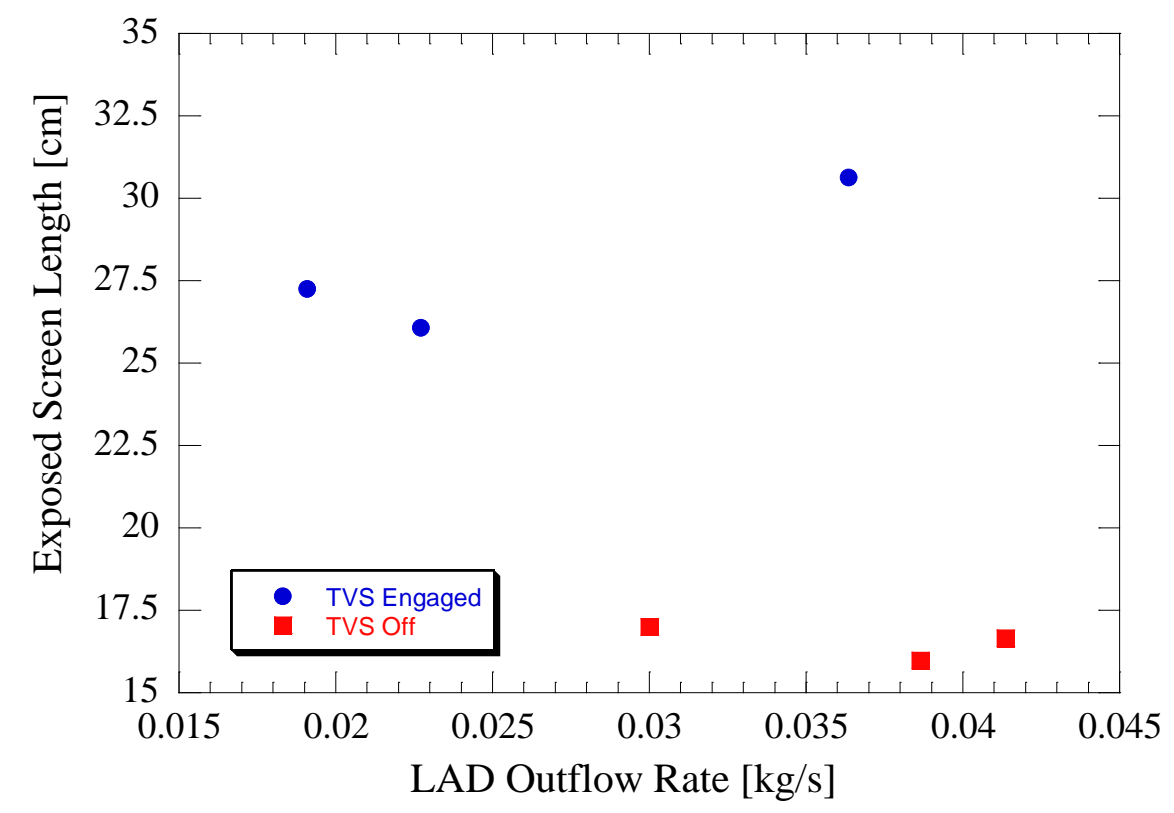

Figure 9.29 - Exposed Screen Height as a Function of Mass Flow Rate through the Thermodynamic Vent System Cooled 325x2300 Channel with the Thermodynamic Vent System Engaged and Disengaged 
The primary purpose of the TVS heat exchanger was to lower the liquid temperature internal to the LAD channel. Therefore, a second way to assess the efficiency of the TVS heat exchanger is to directly compare the measured liquid temperature inside of the channel to the tank bulk liquid temperature during steady outflow. Of the primary 11 tests run with the TVS cooled LAD channel, only the two runs in the warmest liquid temperature of $24.2 \mathrm{~K}$ showed noticeable differences outside the experimental uncertainty between the two temperatures; these runs are highlighted in Table 9.8. These two points are plotted in Figure 9.30 along with heat exchanger model predicted performance from Equation 9.16.

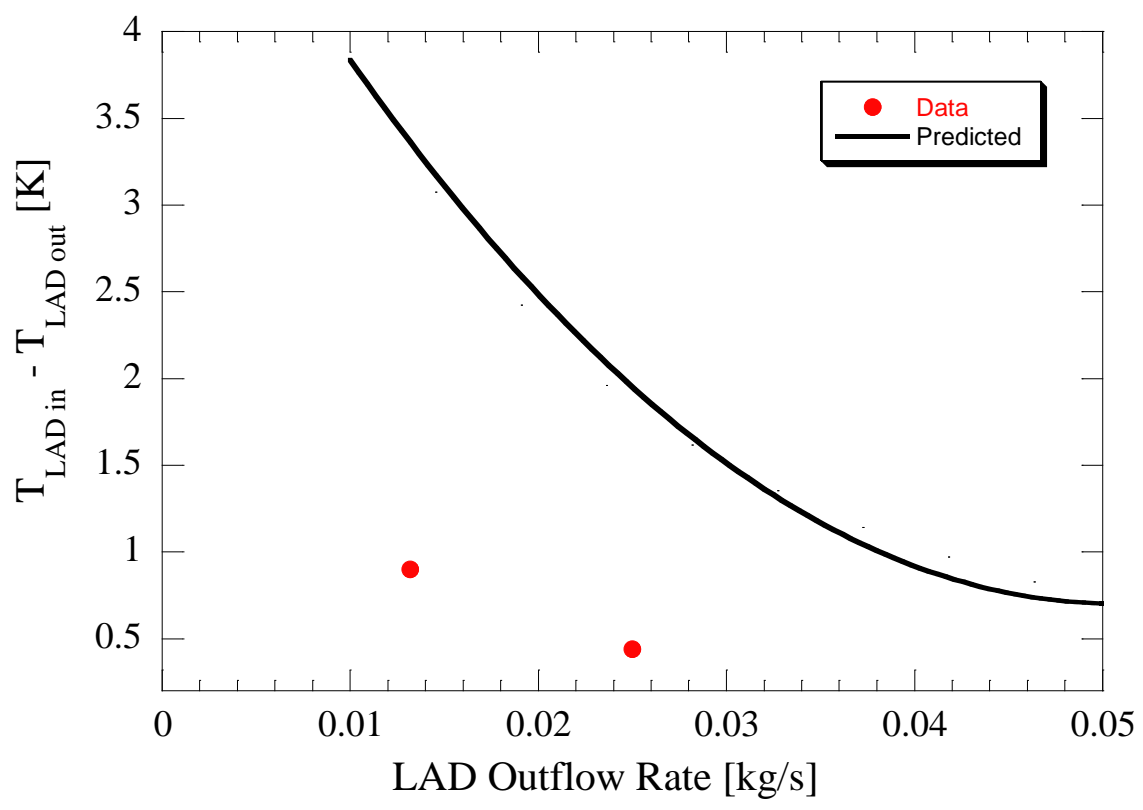

Figure 9.30 - Thermodynamic Vent System Heat Exchanger Efficiency in Terms of the Temperature Difference between Bulk Liquid in the Tank and the Liquid inside the Channel. Data points are for $24.2 \mathrm{~K}$ liquid temperature tests. 
As shown there was at most $1 \mathrm{~K}$ (or $4 \%$ of bulk liquid temperature) difference between the liquid temperatures when the TVS was engaged during steady state outflow. Meanwhile the idealized model predicted a higher temperature difference of 2-3K. The disparity is due to the model assumptions and idealizations. The model prediction curves assume a full $91 \mathrm{~cm}$ exposed length for heat exchange, but examination of Figures 9.28a and $b$ indicate that at most $1 / 3$ of the LAD was exposed during outflow tests. This would limit the actual efficiency of the heat exchanger. The LADs broke down earlier than anticipated because the FTS pressure drop was $\sim 50 \%$ higher than anticipated at $\mathrm{LH}_{2}$ temperatures. Nonetheless, the TVS heat exchanger is still shown to reduce the effective liquid temperature inside of the channel and prolong breakdown by as much as $5 \%$ of the total channel length.

\subsubsection{Subcooling Effect}

Previous bubble point tests conducted in $\mathrm{LH}_{2}$, $\mathrm{LOX}$, and $\mathrm{LCH}_{4}$ showed an increase in bubble point pressure proportional to the level of subcooling the liquid at the screen interface. Thus the gain in bubble point is proportional to the difference between the total pressure $P$ and saturation pressure $P_{S A T}$ of the liquid at the screen:

$$
\frac{\Delta P_{B P}(P)}{\Delta P_{B P, N B P}} \propto\left(P-P_{S A T}\right)
$$

where $\Delta P_{B P}(P)$ and $\Delta P_{B P, N B P}$ are the bubble point pressures at pressure $\mathrm{P}$, and the NBP, respectively. The gain in bubble point pressure appears to be linear with the pressure difference, so higher partial pressures of GHe equate to higher bubble points. Thus it was highly desired to demonstrate whether or not the gain in the static bubble point pressure 
would translate into a gain in performance in a dynamic outflow environment. Therefore, higher bubble point pressures should translate into longer exposed screen lengths.

To test this hypothesis, six tests were conducted in normally saturated $\mathrm{LH}_{2}$ $(20.3 \mathrm{~K})$ using the TVS cooled $325 \times 2300$ channel. Three tests were performed with a total tank pressure of $207 \mathrm{kPa}$ (30 psia), $102 \mathrm{kPa}$ of subcooled margin, and three with tank pressure of $345 \mathrm{kPa}$ (50 psia), $244 \mathrm{kPa}$ subcooled margin. TVS flow rate was identical for all six tests. As shown in Figure 9.31, longer exposed screen lengths were achievable in higher tank pressures; there is approximately $7 \%$ difference of total channel length in exposed screen height when subcooling the liquid by an additional $138 \mathrm{kPa}$.

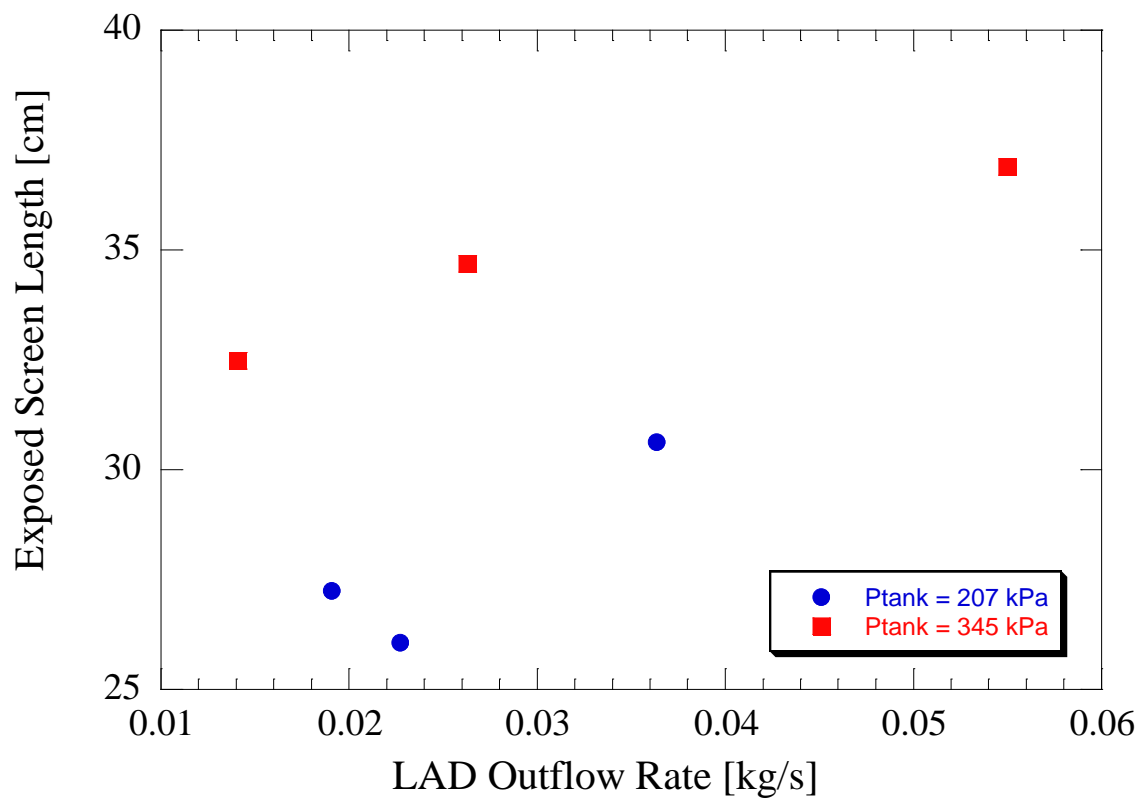

Figure 9.31 - Gain in Dynamic Liquid Acquisition Device Performance Due to Subcooling the Bulk Liquid Hydrogen in the Tank

To compare this gain to the static bubble point gain, a curve was fit to $325 \times 2300$ $\mathrm{GHe} / \mathrm{LH}_{2}$ subcooled bubble point data from Chapter 5. Figure 9.32 plots Equation 9.17 fit 
to the $\mathrm{LH}_{2}$ bubble point data against the difference in pressure between tank pressure and saturation pressure based on the temperature at the screen, along with the two dynamic outflow test conditions in red. As shown, the data only extends to a pressure difference of about $100 \mathrm{kPa}$, so the curve fit extrapolates to tank pressures of $207 \mathrm{kPa}$ and $345 \mathrm{kPa}$ under which dynamic outflow tests were conducted.

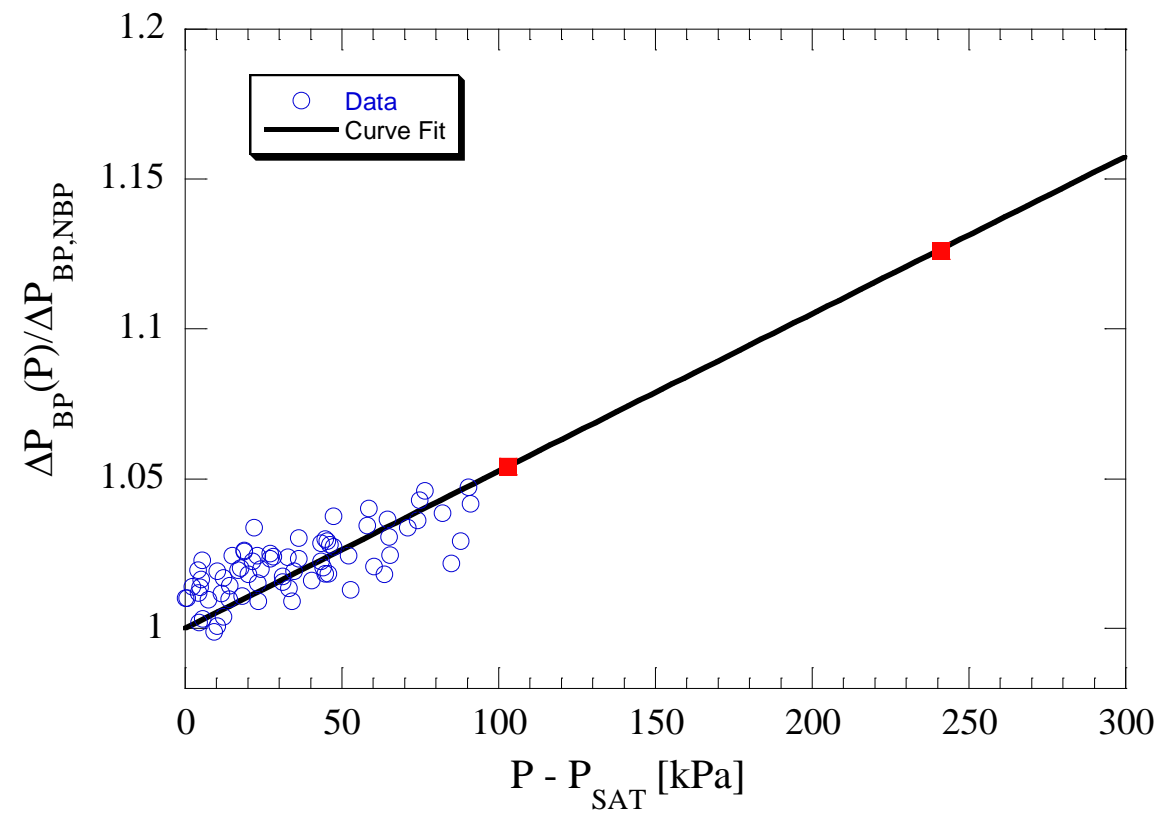

Figure 9.32 - Gain in Static Liquid Acquisition Device Performance due to Subcooling the Bulk Liquid Hydrogen at the Screen Liquid/Vapor Interface

Table 9.9 summarizes results; at a total tank pressure of $207 \mathrm{kPa}$, there is a $5.4 \%$ gain in bubble point pressure from the normal boiling point (saturated state) value $\Delta P_{B P, N B P}$; at a tank pressure of $345 \mathrm{kPa}$, there is an $12.6 \%$ gain over the NBP value. Between the two pressures, the relative gain in static bubble point pressure is $7.2 \%$, which compares nicely with the $7 \%$ gain during dynamic outflow tests shown in Figure 
9.31. Therefore, the gain in exposed screen length in the dynamic environment is indeed directly proportional to the gain in static bubble point pressure.

\begin{tabular}{|c|c|c|c|}
\hline $\begin{array}{c}\text { Psat } \\
{[\mathrm{kPa}]}\end{array}$ & $\begin{array}{c}\text { Ptank } \\
{[\mathrm{kPa}]}\end{array}$ & $\begin{array}{c}\text { delta P } \\
{[\mathrm{kPa}]}\end{array}$ & $\begin{array}{c}\text { Gain from NBP } \\
\text { \% }\end{array}$ \\
\hline 103.42 & 206.84 & 103.42 & 1.054 \\
\hline 103.42 & 344.74 & 241.32 & 1.126 \\
\hline
\end{tabular}

Table 9.9 - Gain in Bubble Point Pressure as a Function of the Level of Subcooling

\subsubsection{Comparison to One Dimensional Model}

To compare against the specific experimental data collected in this chapter, tank drain through the LAD was simulated using the steady state 1D model code. First the liquid temperature, gas temperature, tank pressure, and mass flow rate data is fed into the code. Then, the 1-g inverted outflow model calculates the $\mathrm{LH}_{2}$ bubble point pressure as a function of the pressure and temperature inside the tank. Degradation in bubble point due to the presence of heated gas is also taken into account (see Chapter 10). Initially the liquid level in the tank is above the LAD. As the liquid level drops, the LAD is exposed and the pressure difference across the screen rises in time. Next, for a given liquid level in the tank each pressure drop term is computed. The total pressure drop in the LAD is calculated to compare with the bubble point pressure. The liquid level is decreased, and the code iterates until the total pressure drop exceeds the bubble point pressure. In light of the increased FTS pressure drop at $\mathrm{LH}_{2}$ temperatures, the new set of flow coefficients are used to update the FTS pressure drop at $\mathrm{LH}_{2}$ temperatures. For the TVS cooled channel, the code can take into account the reduction in available FTS area due to the perforated plate. The code can also take into account the effect of TVS cooling by using the measured internal screen temperature of the liquid to calculate bubble point pressure. The 
code does not take into account any enhanced wicking due to the presence of the perforated plate.

In 1-g outflow tests, longer exposed screen lengths equate to higher performance. Figure 9.33 plots the model simulated exposed screen height vs. the actual exposed screen height. The model is only run for the $325 \times 2300$ standard channel. As shown, the model predicts higher performance over the data for all of the tests. When comparing the percent difference between model and data, the model overpredicts the standard 325 data by an average of $18 \%$ of total channel length.

The disparity between the model and data is caused by the model assumption that the FTS pressure drop is uniform across the length of the wetted screen. Since the model predicts higher performance than the data, it stands to reason that it is underpredicting the total pressure drop across the channel, especially at the top where the total pressure drop is a maximum. A non-uniform FTS pressure drop would cause higher fluid velocities across the channel at the exit, thus causing higher pressure differences across the screen at the channel exit. If the fluid velocity inside the channel increases, the frictional pressure drop would also increase, causing premature breakdown. These two statements are substantiated by basic CFD simulations from Zhang et al. (2009) which indicate that liquid is brought into the channel over a small portion of the total available wetted screen area. If this is true, then the FTS pressure drop would not be constant along the length of the channel, but rather linear or even exponential along the length. Although not shown here, disparity between model predictions for the two channels is due to the enhanced cooling and wicking in the TVS cooled channel due to the internal heat exchanger and presence of the perforated plate, which would lessen differences between model and data. 
To show how sensitive the $1 \mathrm{D}$ model is to the screen flow area term $A_{C}$ in Equation 3.33, Figure 9.34 plots the model predictions against the data at a reduced FTS area that minimizes error between data and model (40\% flow area for 325 standard channel). With the reduced area, about half of the data lies above and below the predictions. A higher fidelity model would be required to accurately explain the FTS pressure distribution along the LAD. Nonetheless, the 1D model qualitatively tracks the trends in the data.

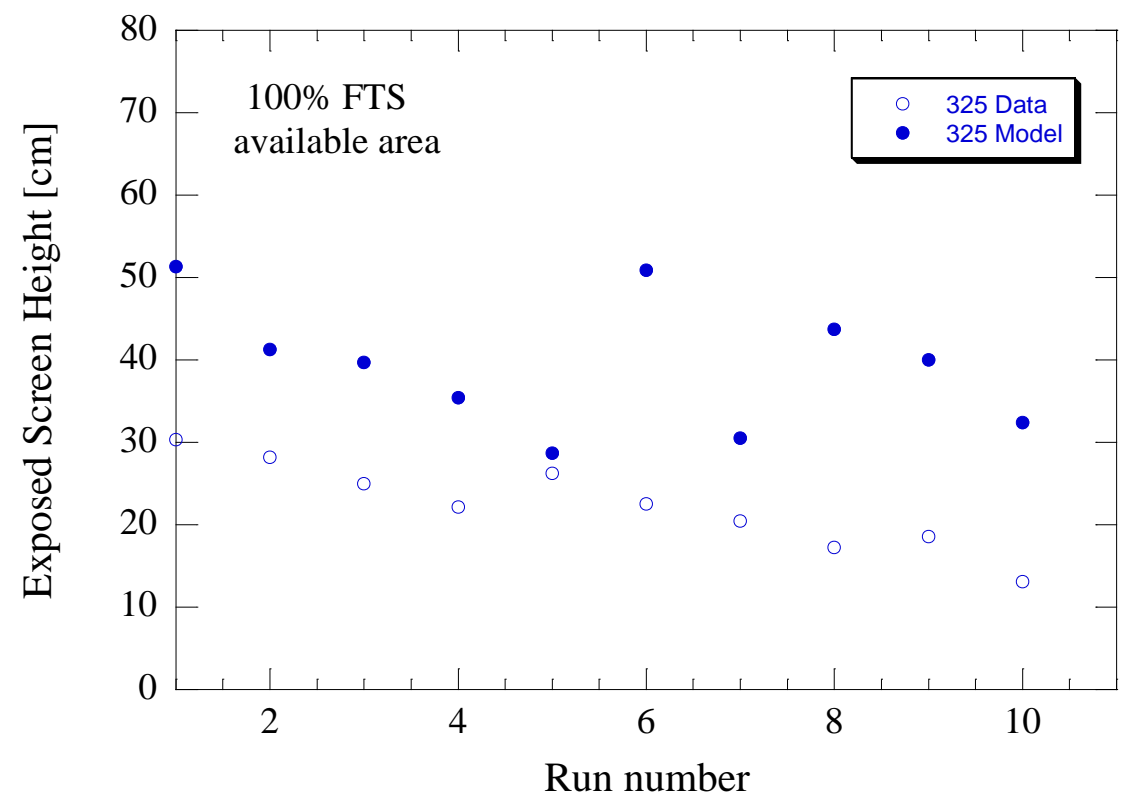

Figure 9.33 - One Dimensional Pressure Drop Model Simulations versus Data for Exposed Screen Height at Channel Breakdown Assuming 100\% Available Flow-through-Screen Flow Area 


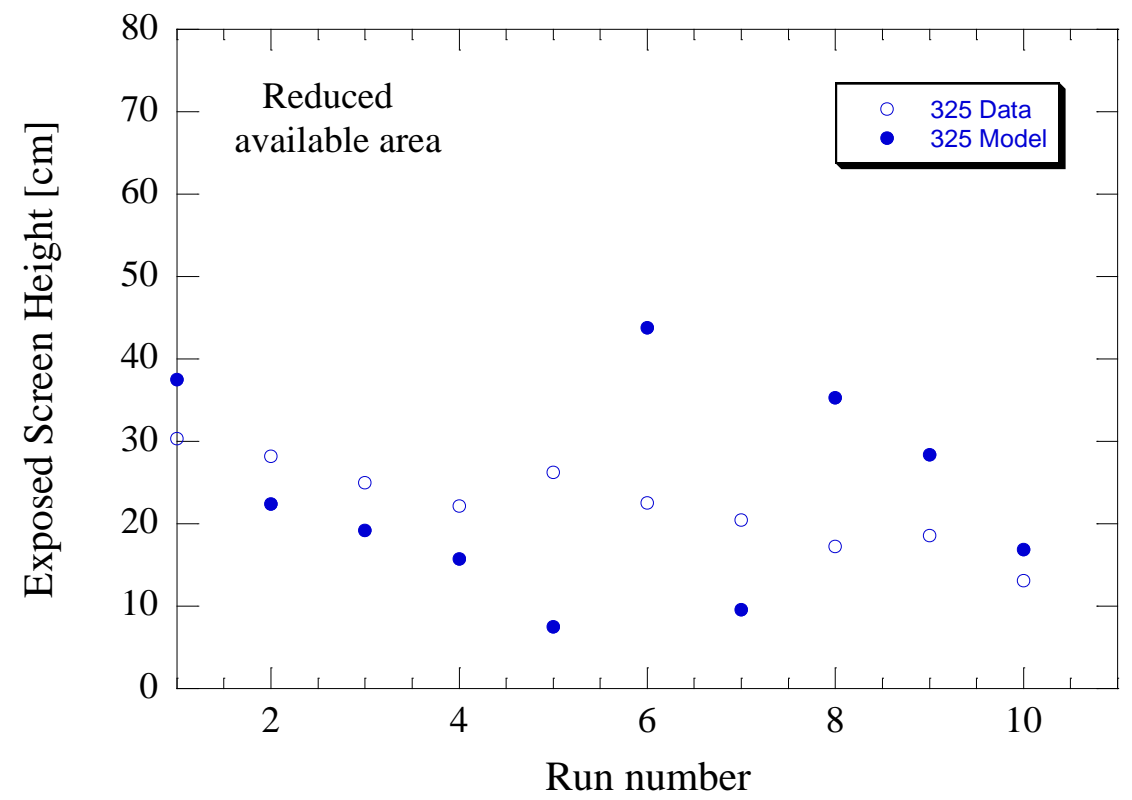

Figure 9.34 - One Dimensional Pressure Drop Model Simulations versus Data for Exposed Screen Height at Channel Breakdown Assuming Reduced Available Flow-throughScreen Flow Area

Another factor which may cause disparity between model and data is that the simplified model does not take into account possible gains in performance due to forced convection of the liquid on the screen. As warm pressurant gas impinges on the LAD screen, cold liquid is brought into the bottom of the channel and routed up to the top of the channel where the largest temperature gradient exists between ullage gas and liquid. While the bubble point equation can be corrected for degradation due to warm pressurant gas, it only applies for a static testing environment over which the data was collected, and not the dynamic outflow environment in the current experiments. A third factor for disparity is that the model assumed fully developed velocity profile is likely inaccurate. To investigate these effects, Figure 9.35 plots the difference in exposed screen length between data and model as a function of the flow rate through the LAD. As shown, there 
is a direct correlation with flow rate, hinting at the possibility of performance enhancement with convective cooling not currently tracked by the simplified 1D model.

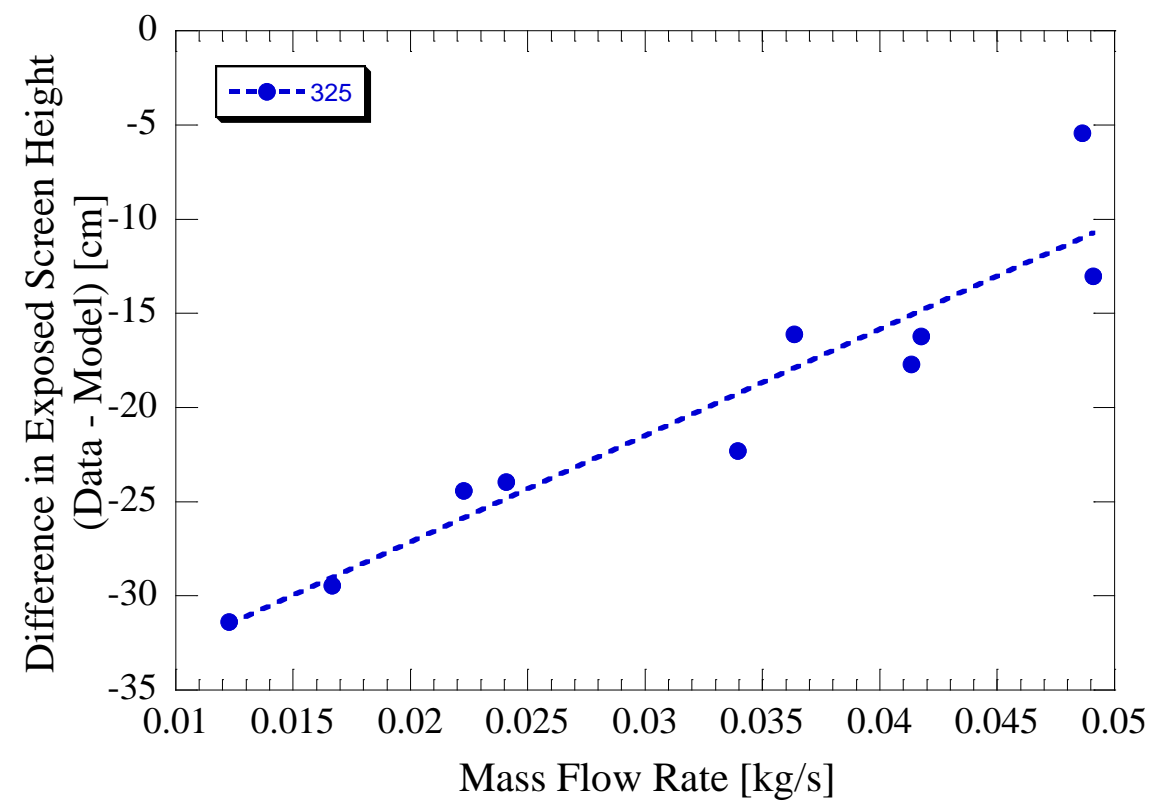

Figure 9.35 - Disparity between Data and One Dimensional Model Predicted Exposed Screen Height as a Function of Flow Rate through the Channel

\subsection{Concluding Remarks}

This chapter presented experimental data that characterizes a LAD channel in a dynamic outflow environment during propellant transfer from a cryogenic propellant tank over a wide range of liquid temperatures, pressures, and outflow rates. Results of the FTS $\mathrm{LH}_{2}$ pipe flow for a $325 \times 2300$ and $450 \times 2750$ screen sample shows that the FTS pressure drop is temperature dependent because the pressure drop is at least $50 \%$ higher at $\mathrm{LH}_{2}$ temperatures over the old room temperature prediction value. FTS test results are consistent with static bubble point tests which suggest that LAD screen pores shrink with decreasing temperatures and yield higher static bubble points at the cost of higher flow 
resistance. Additionally, the $450 \times 2750$ mesh is shown to have lower flow losses than the $325 \times 2300$ screen, consistent with theory.

Horizontal LAD channel tests confirm that the frictional and dynamic pressure losses within the channel are quite small. The signals are barely measurable in $\mathrm{LH}_{2}$, and the signals are small for LOX flows in excess of $2.25 \mathrm{~kg} / \mathrm{s}$. The 1D model also correlates with the single set of LOX channel frictional pressure drop data. Disparity is due to higher than expected screen injection velocities at the channel exit and scatter in the data.

Two full scale LAD channels were designed and tested over a range of operational and thermodynamic conditions for the CPST TDM as well as future in-space propellant transfers. One of the channels was thermally flight representative due to the presence of a custom built internal TVS HEX and a perforated plate. Results indicate that the breakdown point of the LAD is dominated by the temperature of the liquid, with a second order effect on the mass flow rate through the LAD channel. For both channels, the highest performance is always obtained in colder liquid states. For the standard $325 \times 2300$ channel, higher flow rates caused the LAD to breakdown earlier, while for the TVS cooled $325 \times 2300$ channel, higher flow rates prolonged the breakdown point. For a fixed mass flow rate, elevating the temperature of the propellant from $20.3 \mathrm{~K}$ to $24.2 \mathrm{~K}$ caused the standard $325 \times 2300$ channel to breakdown earlier by a factor of $11 \%$ of the total channel height while the $325 \times 2300$ TVS cooled channel by a factor of $5 \%$ of the total channel height. Meanwhile, varying the flow rate from 0.02 to $0.04 \mathrm{~kg} / \mathrm{s}$ changed the breakdown location by approximately $4-5 \%$ for both LAD channels. The presence of the perforated plate in the TVS cooled LAD is believed to enhance wicking and thus screen retention during outflow. 
For all operating conditions, the TVS cooled LAD demonstrated higher performance over the standard channel, due to the presence of the internal heat exchanger. Higher exposed screen lengths were achievable with the TVS system engaged. The TVS system cooled the liquid inside the channel by $1 \mathrm{~K}$ in $24.2 \mathrm{~K}$ liquid, and prolonged breakdown by as much as $4.9 \%$ of the total channel length. In addition, the TVS cooling provided nearly the same performance in warm liquid as in colder liquid as shown by the flattening of the slope in Figure 9.28b. Subcooling the liquid at the screen is also shown to improve performance proportional to the difference between total pressure and liquid saturation pressure.

In addition, the simplified 1D steady state pressure drop model for screen channel liquid acquisition devices has been developed and validated using the FTS, horizontal LAD, and full scale LAD outflow experiments. Both experimental data and model confirm that, in 1-g outflow from a cryogenic propellant tank, the hydrostatic pressure drop is the leading order term, followed by the FTS pressure drop, and frictional and dynamic losses down the channel. All three components of the steady state pressure drop model have been individually validated through the set of three experiments.

The model qualitatively tracks the $\mathrm{LH}_{2} 1-\mathrm{g}$ inverted outflow test results. Discrepancies between 1-g model and data are primarily attributed to a nonuniform FTS pressure distribution along the channel. Results show that both LAD channels behaved close to anticipated performance and that this simplified 1D model can be used to qualitatively track LAD performance in a dynamic outflow environment. 
Results here again have direct implications for future LAD design for the proposed in-space $\mathrm{LH}_{2}$ fuel depots. Both the presence of the internal LAD heat exchanger and the perforated plate prolonged the breakdown point, which implies that the LAD can sustain higher flow rates or flow for longer durations before gas ingestion, leading to higher overall expulsion efficiencies. When compared to updated model predictions, the screen channel LADs performed well within design expectations and are thus capable of delivering high flow rates for low surface tension cryogenic propellants. 


\section{Chapter 10}

\section{The Bubble Point Pressure Model for Cryogenic}

\section{Propellants}

The purpose of this chapter is to derive and validate a robust semi-analytical predictive equation based on 45 years of historical bubble point data, as well as the current set of room temperature and cryogenic experiments from Chapters $4-8$, which can be used to accurately predict the bubble point pressure for any screen channel LAD operating in cryogenic liquids by extending the existing simplified room temperature model to cryogenic temperatures. The seven parameters which affect the bubble point pressure include the surface tension (liquid type), contact angle, screen pore diameter, liquid temperature, degree of subcooling, and pressurant gas type and temperature.

First the current model discrepancies are discussed through examination of the trends in the data. Then the functional model components are systematically formulated based on trends in the data. Finally, each functional component is validated by experimental data. Additionally, a new model to predict bubble point for any mesh, which relates the physical number of warp and shute wires of the screen to the nonphysical "effective" pore diameter, is presented and validated with new bubble point data. 


\subsection{Current Model Limitations}

The current bubble point model, which is a simplification of the 3D YLE that was derived in Chapter 3, predicts bubble point pressures accurately for storable propellants and room temperature liquids. Effective pore diameters of a particular screen mesh must either be based on reference fluid bubble point tests (Method 1) or through summation of historical data (Method 2):

$D_{P}=\frac{4 \gamma_{r e f} \cos \theta_{c}}{\Delta P_{B P, r e f}}$

since SEM analysis proved insufficient. If pore diameter is known, the bubble point equation can theoretically be used to determine the bubble point of any fluid with a known surface tension:

$\Delta P_{B P_{2}}=\frac{\gamma_{2} \cos \theta_{C_{2}}}{\gamma_{1} \cos \theta_{C_{1}}} \Delta P_{B P_{1}}$

where subscript 1 is the liquid with known bubble point and subscript 2 is the liquid with the unknown bubble point. However, this method only works when liquids are relatively close in temperature; the linearity between $\Delta P_{B P}$ and $\gamma_{L V} \cos \theta_{C}$ is shown to break down over wide spans of temperatures when attempting to predict bubble points at cryogenic temperatures. While the simplified model compares well with room temperature data, Equation 3.16 fails to match cryogenic data. To illustrate this disparity, Figure 10.1 plots the $325 \times 2300 \mathrm{LH}_{2}$ bubble point data using $\mathrm{GHe}$ and $\mathrm{GH}_{2}$ to pressurize. As shown, the solid room temperature prediction curve (based on Method 2) matches neither pressurization case, with the GHe data all lying above the curve and most of the $\mathrm{GH}_{2}$ data 
lying below the curve (when factoring in room temperature bubble point from other sources into the room temperature pore diameter calculation). The current model only tracks the temperature dependence of the surface tension; it does not take into account temperature effects of the screen pore diameter itself or differences in performance between different pressurant gases at cryogenic temperatures from Chapters $5-7$. In addition, high temperature $\mathrm{LOX}$ and $\mathrm{LCH}_{4}$ bubble point data in Chapters 6 and 7 indicates a gain in performance due to subcooling the liquid, and bubble point data conducted at pressurant gas temperatures warmer than the liquid temperature in Chapter 8 indicate a degradation in performance at warm gas temperatures.

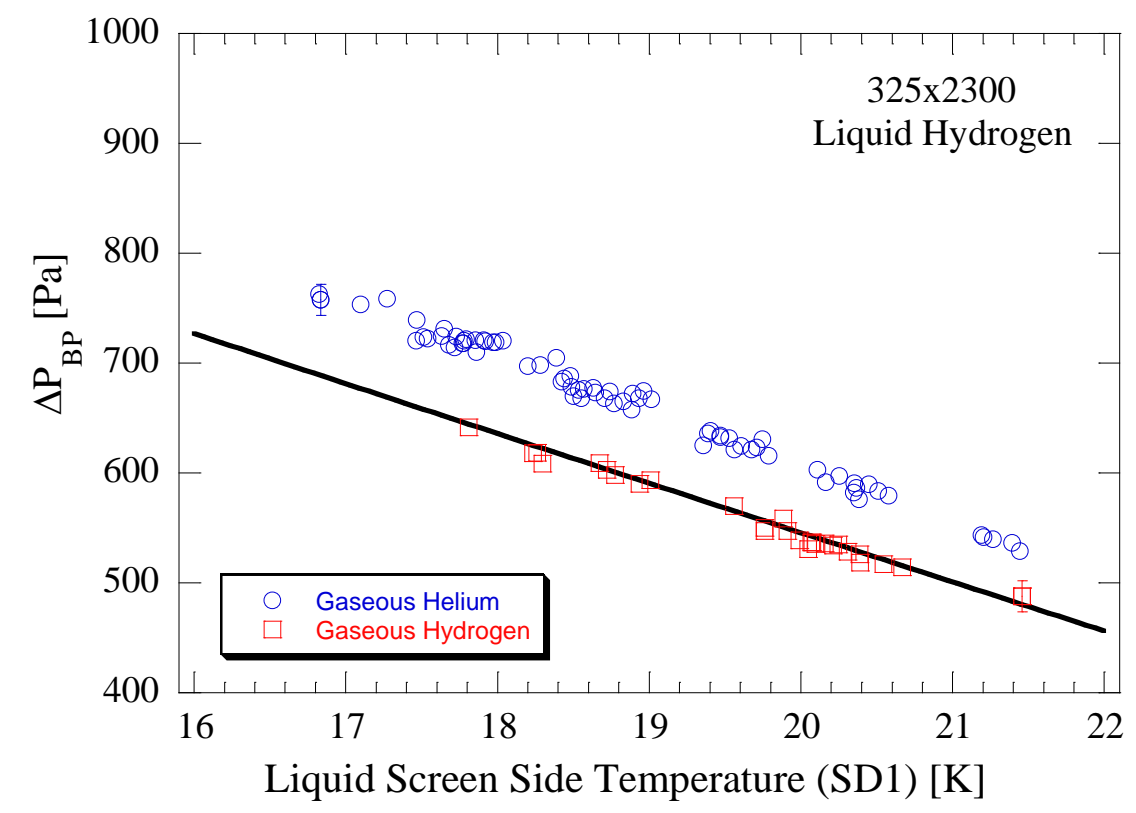

Figure 10.1 - Liquid Hydrogen Bubble Point Pressure Dependence on Pressurant Gas for the $325 \times 2300$ Screen

The new model proposed here will therefore address the following four discrepancies that exist between cryogenic bubble point data and simplified room temperature model: 
1. The temperature dependence of the effective screen pore diameter.

2. The effect of pressurant gas type.

3. The effect of subcooling the liquid.

4. The effect of elevating the temperature of the pressurant gas above the liquid temperature.

The seven known parameters that affect the bubble point pressure include the surface tension (liquid type), contact angle, effective pore diameter (which takes into account screen style, mesh, and metal type), liquid temperature, degree of subcooling (pressure), and pressurant gas type and temperature. The model will be validated through data collected over the past half-decade, as well as with data from the current work, which spans the space of these seven parameters. Ultimately, to be of topmost relevance for all future cryogenic propulsion missions, the model will be formulated in such a way that typical mission parameters like screen mesh, liquid properties, and pressurant gas properties can be input into the equation to easily and quickly obtain bubble point pressure at any desired condition.

\subsection{Summary of Data}

A summary of all of the bubble point data, which includes the screen meshes tested, liquids, and pressurant gases, is presented in Appendix B. There is data characterizing 40 different LAD screens, which includes all 5 different LAD mesh types, in 20 different liquids, using both pressurization schemes. In all, over 5100 bubble point data points were gathered, processed, and compiled. 
Before proceeding with the model, the data was first analyzed to identify potential outliers. Many of the room temperature results did not report a liquid temperature. Most of the historical cryogenic bubble point references did not report a tank pressure. Based on a rigorous uncertainty analysis, some of the data sets were excluded, due to high error. The 325x2300 LOX data from Paynter (1973a) was eliminated because the data was erratic and the results were suspect, as mentioned in their paper. The $325 \times 2300 \mathrm{LH}_{2}$ data from Burge et al. (1973) was discarded because the bubble point data had too wide a span for the liquid temperature to have been constant, as was reported in the paper, leading to erroneous and inconsistent results. Inclusion of this data would have significantly increased the uncertainty in the effective pore diameter calculations. The $325 \times 2300 \mathrm{LH}_{2}$ data from Cady (1973) was excluded because the error in the data was too high, and the data were significant outliers relative to all other reported data at $\mathrm{LH}_{2}$ temperatures. The Freon-114 data from Cady (1977) was discarded because the pore diameters calculated at Freon-114 temperatures were smaller than those at $20 \mathrm{~K}$, which is physically impossible. The $165 \times 800 \mathrm{GH}_{2} / \mathrm{LH}_{2}$ data from Cady (1973) were discarded because they were statistically significant outliers relative to the remainder of the $165 \times 800 \mathrm{GH}_{2} / \mathrm{LH}_{2}$ data. The room temperature 200x200 data from Paynter (1973b) was excluded because the calculated pore diameter at room temperature was exceedingly too low. The $325 \times 2300$ GHe/IPA from Cady (1975) and GHe/IPA data from Burge and Blackmon (1973b) were discarded because they were statistically significant outliers. In addition, some of the screens in Appendix B do not have bubble point data, but rather only manufacturer specified ratings, which do not include effective pore diameter. Overall, the reduced data set of approximately 5000 bubble point pressures examined in this dissertation spans 
more than 45 years of testing, utilizing 26 different LAD screen meshes, 20 different liquids, and both pressurization schemes.

\subsection{Room Temperature Pore Diameter Model}

The room temperature bubble point pressure is directly proportional to the product of the surface tension and cosine of the advancing contact angle. The surface tension model was derived in Section 3.2.4 for all 20 fluids. Binary methanol/water mixture tests from Chapter 4 showed that the $\mathrm{CZ}$ surface tension value for the porous $\mathrm{SS}$ LAD screen was $\gamma_{C Z}=23.2 \pm 2 \mathrm{mN} / \mathrm{m}$. Since the surface tension of all cryogenic liquids is less than this critical value, the contact angle can be assumed to be zero for all cryogenic propulsion systems employing SS LAD screens. Contact angle also does not depend on the screen mesh type. While bubble point data exists for different metal screens like $\mathrm{Al}$ and $\mathrm{Ti}$, the $\mathrm{CZ}$ value is not anticipated to deviate much from the $\mathrm{SS} \mathrm{CZ}$ value. Therefore, for all cryogenic liquids and LAD screens, the bubble point equation simplifies to:

$$
\Delta P_{B P}=\frac{4 \gamma_{L V}}{D_{P}}
$$

Hence, a contact angle correction factor is only necessary for storable liquids. Contact angles were measured for common fluids such as water, IPA, methanol, and acetone in Chapter 4. To the author's knowledge, there is no contact angle data available for toxic storable propellants such as NTO and MMH. Except for water, all storable liquids studied in the current work are fully wetting with negligible contact angles.

\subsubsection{Model}


For any LAD mesh type, a pore throat defines the point within the pore where cross sectional area is a minimum for a gas or vapor bubble to pass through the wetted screen, while the pore mouth defines the point where area is a maximum. The effective pore diameter, and thus bubble point pressure, is related to the pore throat. Statistically, the screen breaks down when a gas bubble passes through the largest pore throat, and thus path of least resistance, of the LAD screen. Likewise the largest pore mouth controls the screen reseal pressure.

For a given screen, the effective pore diameter at room temperature is most easily determined using Equation 10.1, by fitting a curve to bubble point vs. contact angle corrected surface tension for all room temperature fluids. While this method requires experiment, it is straightforward and easy to implement. However when using this method, the pore diameter is not directly based on any physical properties of the screen.

To find the pore diameter at room temperature $(295 \mathrm{~K}) D_{P, 295 K}$, bubble point pressure was plotted against contact angle corrected surface tension for each screen, and the pore diameter was back calculated using Equation 10.1. For each point, uncertainty $C$ in pore diameter is estimated from the uncertainty in surface tension $A$ and reported bubble point pressure $B$ :

$$
C=\sqrt{\left(\frac{A}{\gamma}\right)^{2}+\left(\frac{B}{\Delta P_{B P}}\right)^{2}} * D_{P}
$$

For a given screen, all room temperature data was averaged into a single value using the standard uncertainty $E$, in the calculation: 
$E=\sqrt{\frac{\sum_{i=1}^{n} C_{i}^{2}}{n^{2}}}$

where $n$ is the number of data points. Because there is little to no disparity in bubble point due to different pressurant gases at room temperature, the room temperature pore diameter was averaged across different gases, including $\mathrm{GHe}, \mathrm{GN}_{2}$, and $\mathrm{GH}_{2}$. Table 10.1 lists the resultant room temperature pore diameters for the 26 available LAD screens.

\begin{tabular}{|c|c|c|}
\hline Mesh & Dp295 $[\boldsymbol{\mu m}]$ & $\boldsymbol{U}[\boldsymbol{\mu m}]$ \\
\hline $\mathbf{5 1 0 \times 3 6 0 0}$ & 15.91 & $3.40 \mathrm{E}-02$ \\
$\mathbf{4 5 0 \times 2 7 5 0}$ & 12.01 & $1.78 \mathrm{E}-02$ \\
\hline $\mathbf{3 2 5 \times 2 3 0 0}$ & 14.65 & $8.39 \mathrm{E}-02$ \\
\hline $\mathbf{2 5 0 \times 1 4 0 0}$ & 18.44 & $8.49 \mathrm{E}-02$ \\
\hline $\mathbf{2 5 0 \times 1 3 7 0}$ & 19.21 & $7.35 \mathrm{E}-02$ \\
\hline $\mathbf{2 0 0 \times 1 4 0 0}$ & 21.45 & $3.34 \mathrm{E}-01$ \\
\hline $\mathbf{1 6 5 \times 8 0 0}$ & 46.91 & $6.53 \mathrm{E}-02$ \\
\hline $\mathbf{8 0 \times 7 0 0}$ & 60.22 & $3.84 \mathrm{E}+00$ \\
\hline $\mathbf{2 0 0 \times 6 0 0}$ & 44.76 & $3.99 \mathrm{E}+00$ \\
\hline $\mathbf{5 0 \times 2 5 0}$ & 103.81 & $1.07 \mathrm{E}+01$ \\
\hline $\mathbf{3 0 \times 2 5 0}$ & 139.04 & $1.52 \mathrm{E}+01$ \\
\hline $\mathbf{2 4 \times 1 1 0}$ & 200.28 & $2.98 \mathrm{E}+01$ \\
\hline $\mathbf{7 2 0 \times 1 4 0}$ & 38.36 & $1.39 \mathrm{E}-01$ \\
\hline $\mathbf{5 0 0 \times 5 0 0}$ & 44.89 & $1.27 \mathrm{E}-01$ \\
\hline $\mathbf{3 2 5 \times 3 2 5}$ & 64.94 & $1.82 \mathrm{E}+01$ \\
\hline $\mathbf{1 8 0 \times 1 8 0}$ & 85.27 & $5.32 \mathrm{E}-03$ \\
\hline $\mathbf{1 2 0 \times 1 2 0}$ & 168.7 & $2.00 \mathrm{E}+01$ \\
\hline $\mathbf{1 0 0 \times 1 0 0}$ & 118.23 & $2.21 \mathrm{E}-04$ \\
\hline $\mathbf{2 3 0 \times 2 3 0}$ & 94.48 & $5.15 \mathrm{E}+01$ \\
\hline $\mathbf{2 0 0 \times 2 0 0}$ & 99.7 & $6.85 \mathrm{E}-03$ \\
\hline $\mathbf{1 5 0 \times 1 5 0}$ & 138.77 & $2.50 \mathrm{E}-01$ \\
\hline $\mathbf{8 0 \times 8 0}$ & 209.12 & $5.62 \mathrm{E}-04$ \\
\hline $\mathbf{6 0 \times 6 0}$ & 346.75 & $1.13 \mathrm{E}+00$ \\
\hline $\mathbf{5 0 \times 5 0}$ & 307.11 & $8.25 \mathrm{E}-04$ \\
\hline $\mathbf{4 0 \times 4 0}$ & 554.84 & $1.81 \mathrm{E}+00$ \\
\hline $\mathbf{3 0 \times 3 0}$ & 539.71 & $1.45 \mathrm{E}-03$ \\
\hline
\end{tabular}

Table 10.1 - Calculated Pore Diameters at Room Temperature 
While previous statistical attempts at using SEM analysis to relate a 2D projection of the 3D pore structure proved unsuccessful, correlations do exist between the pore diameter and screen properties for all meshes examined in the current work. Figures 10.2 and 10.3 plot the effective pore diameter against the shute wire diameter and the square root of the number of pores per square inch of screen $\left(\sqrt{n_{\text {warp }} n_{\text {shute }}}\right)$, respectively for the 26 screens that have room temperature bubble point data. Error bars are also plotted in both figures, but are barely distinguishable.

Based on unpublished analysis of the LAD screen geometry based on the work of Mason and Morrow (1984, 1986, and 1991) and Prodanovic and Bryant (2006), the gap between four adjacent shute wires may be the dominate site for bubble breakthrough. A measure of this gap is approximately $1 / 4^{*}$ shute wire diameter. Figure 10.3 is essentially Figure 3.9 updated with the new 450x2750 and 510x3600 data. Meanwhile, Table 10.1 is an update of Table 3.2, since the room temperature pore diameters in Table 10.1 are based on the entire data set of historical and current bubble point data. Notice that the pore diameter values for screens tested in the current work have changed slightly due to inclusion of more data. 


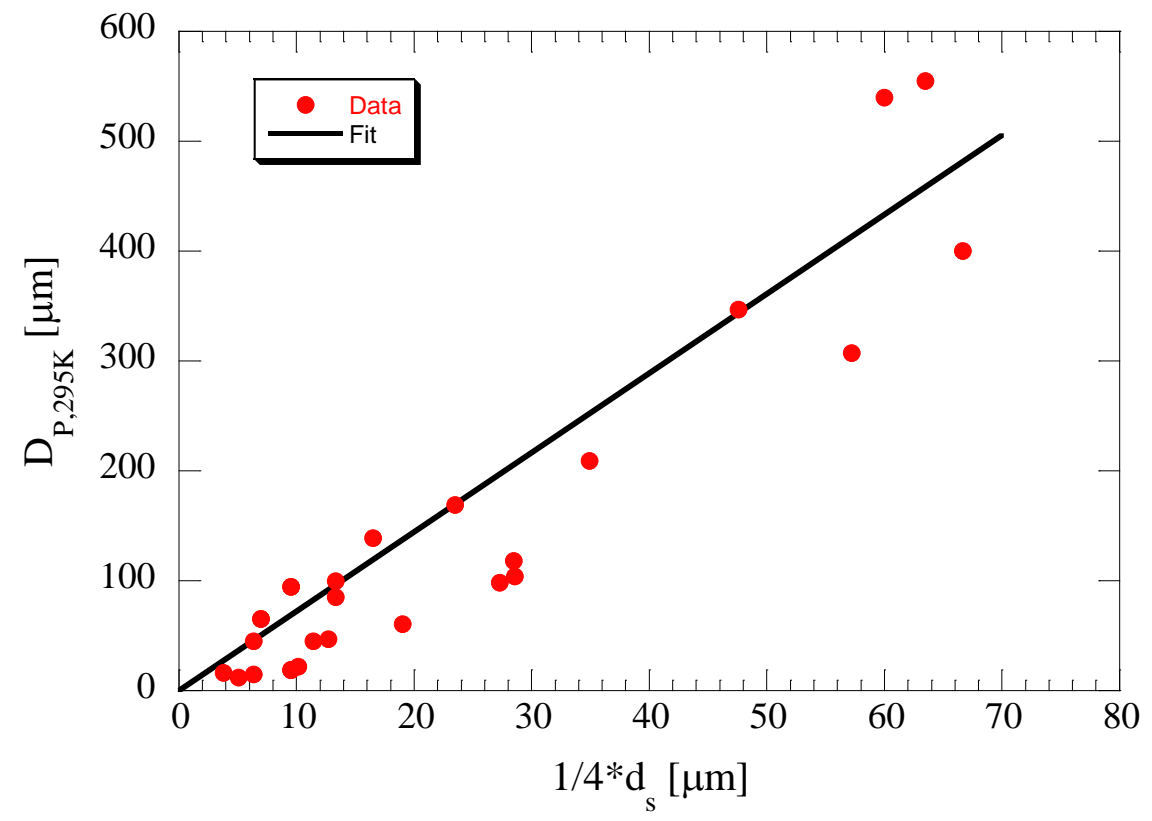

Figure 10.2 - Room Temperature Pore Diameter as a Function of Gap between Four Shute Wires

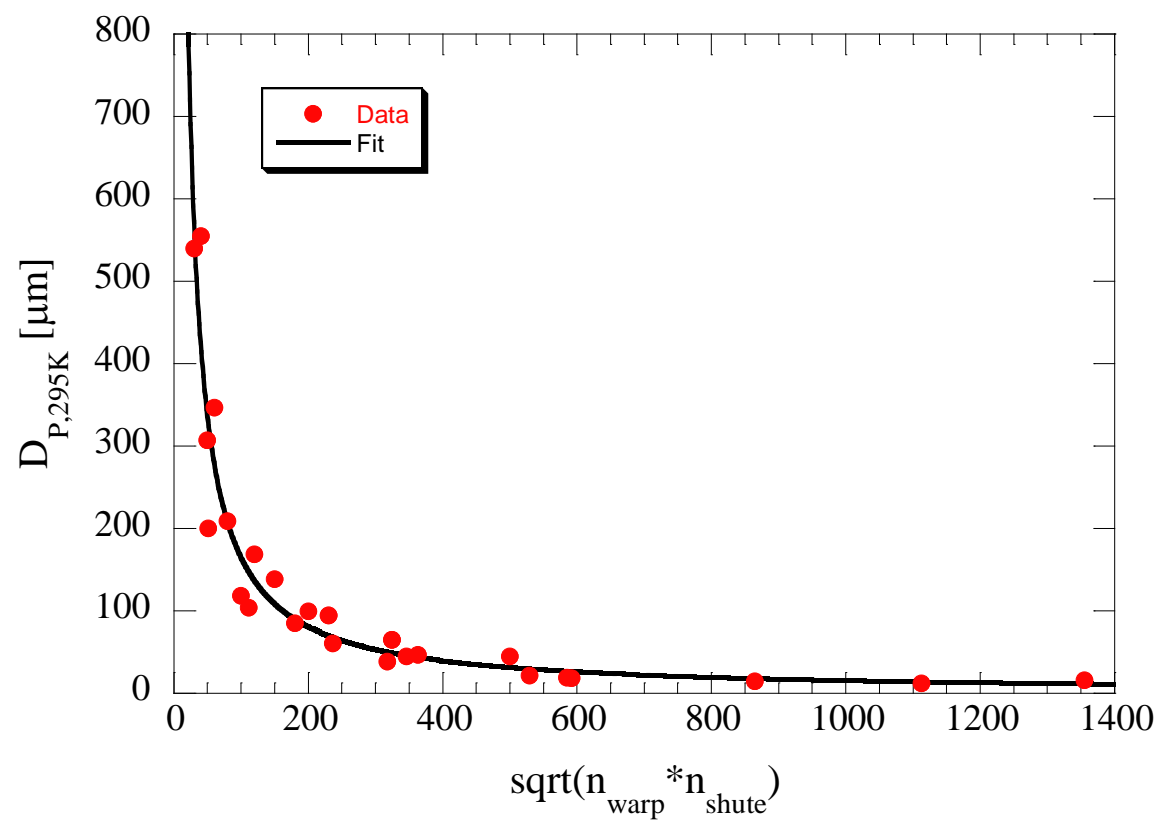

Figure 10.3 - Room Temperature Pore Diameter as a Function of the Fineness of the Mesh 
Figure 10.2 shows a near linear trend between the effective pore diameter and gap for all LAD meshes and screen styles. Meanwhile, there is also an interesting correlation between the fineness of the screen (square root of the number of holes per screen area) and the pore diameter, as shown in Figure 10.3. In general, the inverse relationship from Figure 10.3 can be used to interpolate effective pore diameters for new, coarser screens where data is not yet available:

$$
D_{P, 295 K}=\frac{19192}{\left(\sqrt{n_{\text {warp }} n_{\text {shute }}}\right)^{1.03}} \quad[\mu \mathrm{m}]
$$

These two physical parameters are fair at defining a correlation with the non-physical pore diameter.

\subsubsection{Maximum Bubble Point Pressure}

Examination of Figure 10.3 shows that, in general, increasing the fineness of the mesh decreases the effective pore diameter, thus increasing bubble point pressure. However, the curve fit to Equation 10.6 does not predict that the lowest effective pore diameter occurs for the second finest 450x2750 mesh as the data indicates. Since the finest mesh does not yield the highest bubble point pressure (in either room temperature or cryogenic liquids), further investigation is warranted into understanding the underlying cause for this discrepancy and also to create a better correlation that matches the data.

To rule out the possibility that the lower 510x3600 bubble point pressures reported in Chapter 4 were not caused by defects, repeat bubble point tests were 
conducted in IPA. No deviation was noticed from previously reported values. Additional 510x3600 screen samples were tested from different batches of raw materials, and still there was no deviation from previously reported bubble points. The screens were also reexamined under the SEM to detect for defects, and analysis revealed no complications. Therefore, the long standing assumption that the bubble point pressure scales with the fineness of the screen is in fact invalid. The combined bubble point data set indicates that there is an optimal mesh and pore geometry which maximizes the bubble point pressure.

The general trend that has been commonly assumed throughout historical LAD screen design is that the finer the screen, the higher the bubble point. This assumption is based on the view that higher mesh counts have smaller absolute micron ratings, and the smaller the micron rating, the higher the capillary pressure. In light of recent tests however, this assumption obviously does not hold true; the 510x3600 attains lower bubble points than the coarser 450x2750 mesh, even though it has the lowest absolute micron rating reported by manufacturers. As speculated in Chapters 4 and 5 it is believed that the shape of the actual pore structure is a secondary influence (the primary influence being the fineness of the screen) on the effective pore diameter. For example, for Dutch Twill meshes, these openings are complex 3D structures. The equilibrium L/V interface shape is just as complex and it depends on the size and packing of the wires. If it were possible to microscopically observe a L/V interface within a Dutch Twill weave, it might be possible to understand why the $450 \times 2750$ outperforms the $510 \times 3600$. Rather, it is much more efficacious to look at deeper correlations between the pore diameter and geometrical properties of the mesh like the warp and shute wire diameters. Since the 
warp and shute diameters are a major factor in determining the pore shape it is likely that they have a relationship with the pore diameter.

After performing rigorous statistical analysis and examining multiple possible correlations, a correlation between the pore diameter $D_{P}$, the number of screen wires per inch $n_{w}$ or $n_{s}$, the warp diameter $d_{w}$, and the shute diameter $d_{s}$ does in fact track the trends in the data. Specifically, it is the shute to warp diameter ratio which controls the maximum bubble point for LAD screens. The following relationship is used to establish the correlation between pore diameter and shape, size, and geometry of the pore. Pore diameter scales inversely with $X$, and not the fineness of the screen, where $X$ is:

$$
X=n^{r_{d}}
$$

where $n$ can be either the number of warp or shute wires per inch and

$$
r_{d}=\frac{d_{s}}{d_{w}}
$$

The two ways to increase $X$ are by increasing the shute to warp diameter ratio or by increasing the number of wires. The $450 \times 2750$ has a large $n$ value, and a relatively large $r_{d}$ value of 0.8 . For these reasons it has the best bubble point performance. The 510x3600 has the largest $n$ value, but one of the lowest $r_{d}$ values of all the Dutch Twill meshes: 0.6. So even though it has the most wires per inch, it does not outperform the 450x2750 because of its low wire diameter ratio.

This new correlation works very well at explaining bubble point performance of other LAD meshes as well. For example, similar results are seen by comparing the 
performance of the 200x1400 and the 250x1370. These two weaves have nearly the same number of wires per inch in both directions, yet the 250x1370 outperforms the 200x1400 with a lower $D_{P}$. The big difference between the two meshes is in comparing $r_{d}$ values. The $250 \times 1370$ has a higher ratio of 0.68 while the $200 \times 1400$ has a lower ratio of 0.57 ; Therefore the 250x1370 has a larger value of $X$. This matches with the trend that larger values of $X$ correspond to lower values of $D_{P}$.

Figure 10.4 plots effective pore diameter against $X$ using $n_{s}$. The trend clearly shows that as $X$ increases, $D_{P, 295 K}$ decreases, for all LAD meshes. Figure 10.4 indeed looks similar to Figure 10.3. However, the farthest point on the abscissa is the screen that has the lowest effective pore diameter, the $450 \times 2750$ mesh. Fitting a least squares curve to the data reveals the simple inverse relationship between $X$ and $D_{P}$ :

$$
D_{P, 295 K}=\frac{9633 \mu m}{X_{s}}
$$

where

$$
X_{s}=n_{s}^{r_{d}}
$$

A similar relationship can be derived using the number of warp wires per square inch as well. Thus the pore diameter indeed obeys an inverse dependence on the screen parameters, but it is the shute to warp diameter ratio, and not the number of pores, that controls the size of the effective pore diameter. This is because $\mathrm{x}$ takes into account both fineness of the screen and the actual geometry of the pore itself. Through interpolation, Equation 10.9 can be used to determine the pore diameter for any LAD screen. 


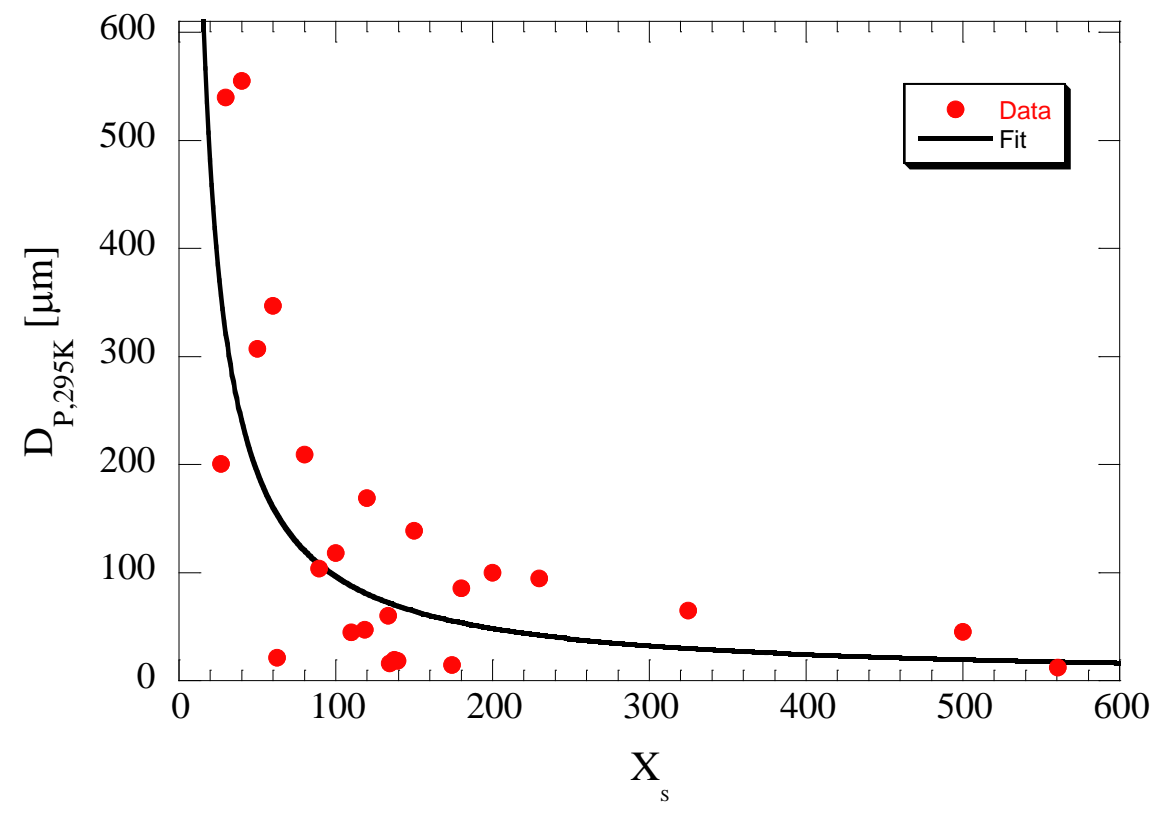

Figure 10.4- Room Temperature Pore Diameter as a Function of X. X is defined using the number of shute wires per inch.

Table 3.2 is updated with room temperature pore diameters based on historical data as shown in Table 10.2. Parameters for the two finest Dutch Twill 450x2750 and 510x3600 screens from Chapter 4 are included for the sake of completeness. For mission design, there is performance data available for 31 different LAD meshes. 


\begin{tabular}{|c|c|c|c|c|c|c|c|c|}
\hline Mesh & Mesh Type & dwarp [ $[\mu \mathrm{m}]$ & dshute [ $\mu \mathrm{m}]$ & Source, wire diameters & $\mathrm{Dp}[\mu \mathrm{m}]$ & $B[\mu \mathrm{m}]$ & $a[\mu \mathrm{m}-1]$ & $\varepsilon$ \\
\hline $510 \times 3600$ & Dutch Twill & 25.4 & 15.2 & Industry & 15.91 & 55.8 & 0.1676 & 0.29 \\
\hline $450 \times 2750$ & Dutch Twill & 25.4 & 20.3 & Industry & 12.01 & 66 & 0.1411 & 0.256 \\
\hline $325 \times 2300$ & Dutch Twill & 38.1 & 25.4 & Industry & 14.65 & 91.4 & 0.1102 & 0.245 \\
\hline $325 \times 1900$ & Dutch Twill & 35.6 & 30.5 & Armour \& Cannon (1968) & - & 96.5 & 0.1005 & 0.215 \\
\hline $250 \times 1400$ & Dutch Twill & 55.9 & 38.1 & Industry & 18.44 & 132 & 0.0721 & 0.255 \\
\hline $250 \times 1370$ & Dutch Twill & 55.9 & 38.1 & Burge \& Blackmon (1973b) & 19.21 & 132 & 0.0708 & 0.267 \\
\hline $200 \times 1400$ & Dutch Twill & 71.1 & 40.6 & Industry & 21.45 & 152 & 0.0654 & 0.248 \\
\hline $165 \times 1400$ & Dutch Twill & 71.1 & 40.6 & Industry & - & 152 & 0.0611 & 0.306 \\
\hline $165 \times 800$ & Dutch Twill & 71.1 & 50.8 & Industry & 46.91 & 173 & 0.0415 & 0.43 \\
\hline $80 \times 700$ & Dutch Twill & 102 & 76.2 & Industry & 60.22 & 254 & 0.0318 & 0.369 \\
\hline $200 \times 600$ & Dutch Twill & 62.2 & 45.7 & Industry & 44.76 & 154 & 0.0356 & 0.552 \\
\hline $30 \times 500$ & Dutch Twill & 250 & 110 & Industry & - & 470 & 0.0171 & 0.461 \\
\hline $30 \times 160$ & Dutch Twill & 229 & 178 & Cady (1973) & - & 584 & 0.0078 & 0.635 \\
\hline $20 \times 250$ & Dutch Twill & 102 & 63.5 & Industry & - & 229 & 0.0097 & 0.835 \\
\hline $50 \times 250$ & Plain Dutch & 140 & 114 & Industry & 103.81 & 368 & 0.0131 & 0.611 \\
\hline $30 \times 250$ & Plain Dutch & 254 & 203 & Heckman (1971) & 139.04 & 660 & 0.0122 & 0.36 \\
\hline $24 \times 110$ & Plain Dutch & 381 & 267 & Industry & 200.28 & 914 & 0.0058 & 0.572 \\
\hline $720 \times 140$ & Reverse Dutch & 35.6 & 109 & Cady (1977) & 38.36 & 254 & 0.0439 & 0.03 \\
\hline $500 \times 500$ & Twill Square & 25.4 & 25.4 & Cady (1977) & 44.89 & 50.8 & 0.0655 & 0.584 \\
\hline $325 \times 325$ & Twill Square & 27.9 & 27.9 & Heckman (1971) & 64.94 & 55.9 & 0.0414 & 0.711 \\
\hline $180 \times 180$ & Twill Square & 53.3 & 53.3 & Paynter (1970) & 85.3 & 107 & 0.023 & 0.693 \\
\hline $120 \times 120$ & Twill Square & 50.8 & 50.8 & Heckman (1971) & 169 & 102 & 0.0257 & 0.674 \\
\hline $100 \times 100$ & Square & 114 & 114 & Industry & 118 & 228 & 0.0136 & 0.614 \\
\hline $230 \times 230$ & Square & 38.1 & 38.1 & Heckman (1971) & 94.5 & 76.2 & 0.0301 & 0.713 \\
\hline $200 \times 200$ & Square & 53.3 & 53.3 & Industry & 99.7 & 107 & 0.0268 & 0.643 \\
\hline $150 \times 150$ & Square & 66 & 66 & Heckman (1971) & 139 & 132 & 0.0199 & 0.671 \\
\hline $80 \times 80$ & Square & 140 & 140 & Heckman (1971) & 209 & 279 & 0.0108 & 0.622 \\
\hline $60 \times 60$ & Square & 191 & 191 & Heckman (1971) & 347 & 381 & 0.0081 & 0.612 \\
\hline $50 \times 50$ & Square & 229 & 229 & Industry & 307 & 458 & 0.0068 & 0.612 \\
\hline $40 \times 40$ & Square & 254 & 254 & Heckman (1971) & 555 & 508 & 0.0053 & 0.662 \\
\hline $30 \times 30$ & Square & 240 & 240 & Armour \& Cannon (1968) & 540 & 480 & 0.0039 & 0.769 \\
\hline
\end{tabular}

Table 10.2 - Updated Set of Measured and Calculated Liquid Acquisition Device Screen

Parameters

\subsection{Temperature Dependent Pore Diameter and Pressurant Gas Model}

Of the four sub-models developed in this section, the hardest to model is the

combined effect of pressurant gas type and screen pore shrinkage. These two effects are coupled together because each cryogenic bubble point is conducted at a specific

temperature using a specific pressurant gas. Prior to the current work, the pore diameter of the screen was always assumed constant for LAD screens in contact with cryogenic liquids, despite the well-known behavior of metals shrinking with reduced temperature. 
Evidence for this temperature dependence is shown in comparing room temperature bubble point data from Chapter 4 to cryogenic bubble point data from Chapters $5-8$ in normally saturated cryogenic liquid for the exact same $325 \times 2300,450 \times 2750$, and 510x3600 screen sample where Equation 10.1 may be used for comparison of pore diameters at the two temperatures. For all screens, higher bubble points, and thus smaller pore diameters, are always obtained in colder liquid temperatures. To calculate pore diameters and uncertainties in pore diameters at reduced temperatures, Equations 10.1, 10.4 , and 10.5 can be used for cryogenic data taken only in saturated or near saturated liquid states. Saturated liquid states are defined in the usual sense, with the thermodynamic state taken as the liquid temperature and a quality equal to zero. Graphically, for example, saturated or near-saturated states are the data points located along the saturation line in Figures 5.8, 5.9, 6.5, 6.6, and 7.6.

Figures $10.5 \mathrm{a}-\mathrm{c}$ plot calculated pore diameters from all available normally saturated cryogenic bubble point data as a function of temperature for three of the most popular LAD screen meshes, the 325x2300, 450x2750, and 510x3600 Dutch Twill screens using both non-condensable and autogenous pressurization schemes. As shown for all 3 screens, the GHe data follows a distinct trend, in that the pore diameter decreases rapidly from room temperature down to $\mathrm{LN}_{2}$ temperatures $(77 \mathrm{~K})$, and then essentially bottoms out between $\mathrm{LN}_{2}$ and $\mathrm{LH}_{2}$ temperatures. The shape of the curves closely resembles that of the well-known coefficient of thermal contraction. Similar trends are also noted with other meshes, even though not shown here. Meanwhile, the pore diameters obtained using autogenous pressurization increase with decreased temperatures for the two coarser meshes and decrease with temperature for the finest 510x3600 screen. 

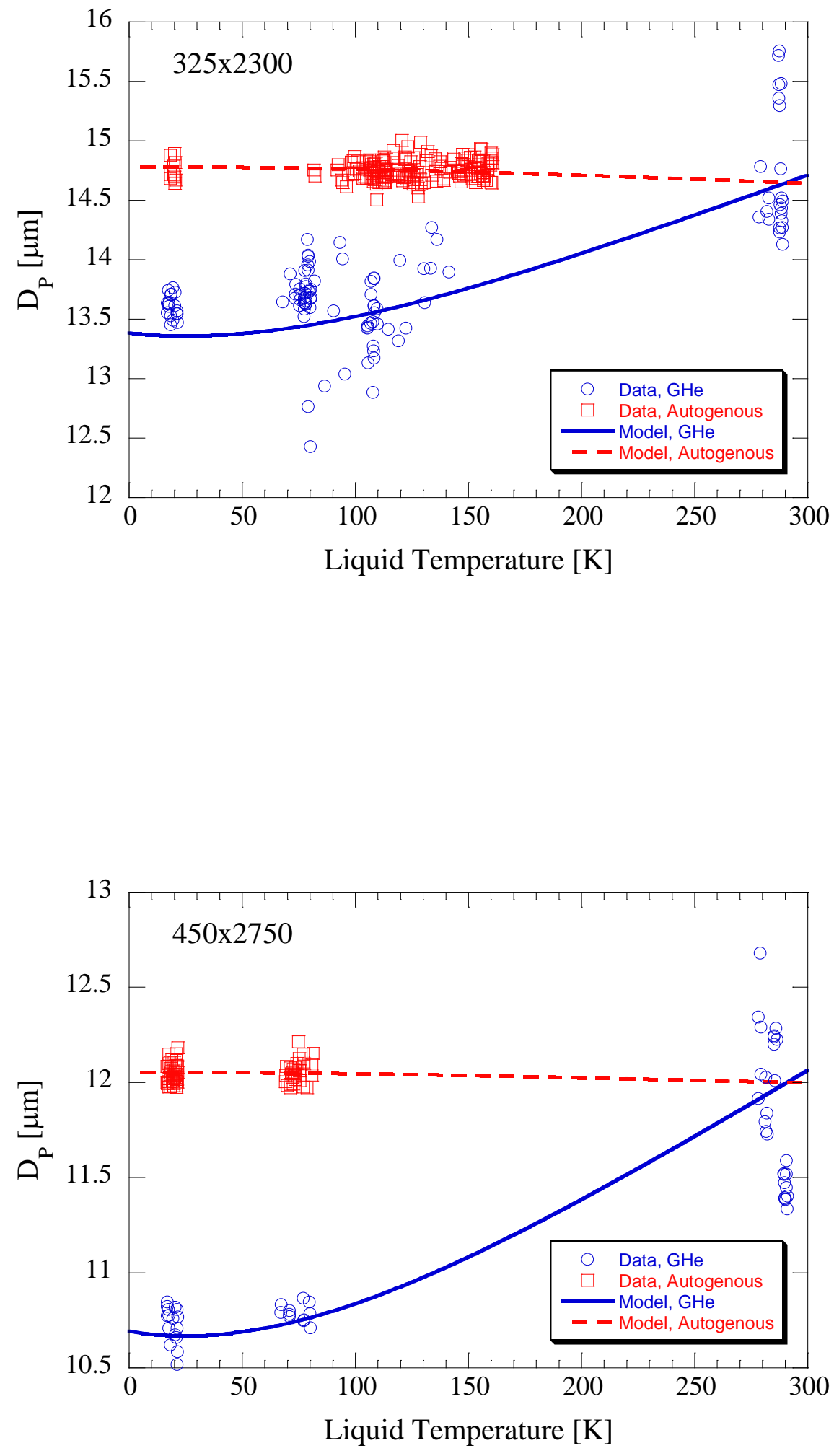


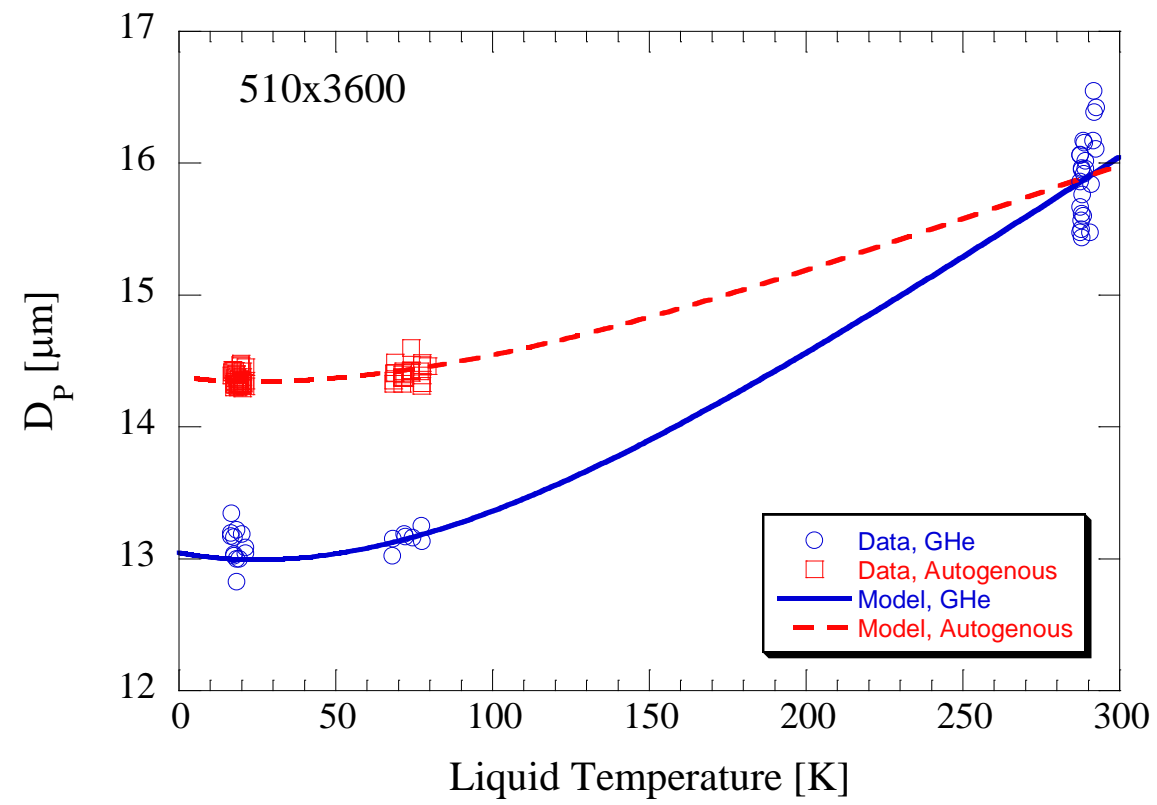

Figure 10.5 - Pore Diameter as a Function of Temperature for a) 325x2300, b) 450x2750, and c) 510x3600 Dutch Twill Screens. Solid lines represent model generated curves.

For clarity and comparison, Table 10.3 shows the gain or loss from the room temperature bubble point value to the bubble point value at $\mathrm{LH}_{2}$ temperatures $(20.3 \mathrm{~K})$. For example, using $\mathrm{GH}_{2}$ pressurant and a 450x2750 mesh, there is virtually no deviation from room temperature, but using GHe pressurant, there is a $12.5 \%$ gain. Examination of Figure 10.5 and Table 10.3 shows that there are different gains and losses depending on the screen.

\begin{tabular}{|c|c|c|c|}
\hline & $325 \times 2300$ & $\mathbf{4 5 0 \times 2 7 5 0}$ & $\mathbf{5 1 0 \times 3 6 0 0}$ \\
\hline Helium & 1.097 & 1.125 & 1.224 \\
\hline Autogenous & 0.991 & 0.996 & 1.109 \\
\hline
\end{tabular}

Table 10.3 - Gain or Loss in Bubble Point Pressure from Room Temperature Value at Liquid Hydrogen Temperatures 
Therefore the bubble point equation can be modified to account for screen contraction at lower temperatures as well as differences in performance between different pressurant gases at cryogenic temperatures:

$\Delta P_{B P}(T)=\frac{4 \gamma_{L V} \cos \theta_{C}}{D_{P}(T)}$

where $D_{P}(T)$ is the temperature dependent pore diameter, which is defined by:

$D_{P}(T)=D_{P, 295 K}\left(1+b_{0} \xi(T)\right)$

$\xi(T)$ is the volumetric coefficient of thermal contraction, which for an isotropic material, is defined in the traditional manner (Corruccini and Gniewek 1961):

$\xi(T)=3 \frac{L_{T}-L_{295 K}}{L_{295 K}}=3\left(c_{0}+c_{1} T+c_{2} T^{2}+c_{3} T^{3}+c_{4} T^{4}\right) * 10^{-5}$

where $L_{T}$ and $L_{295 K}$ is the length of the material at temperature $T$ and room temperature $(295 \mathrm{~K})$, respectively, and $\left(c_{0}, c_{1}, c_{2}, c_{3}, c_{4}\right)$ are coefficients fit to different metals. Table 10.4 lists contraction coefficients for 304SS, Al, and Ti LAD screen metals down to cryogenic temperatures (Corruccini and Gniewek 1961). $b_{0}$ is a dimensionless fitting parameter to the data which incorporates both the effect of screen shrinkage as well as pressurant gas type. Thus $b_{0}$ is essentially a weighting factor of how much the screen shrinks, how much the physical shape and geometry of the actual L/V interface changes at a given temperature, and whether or not there is gain or loss from the room temperature bubble point value due to pressurizing with a non-condensable or 
condensable gas. Since the coefficient of thermal contraction is negative, larger $b_{0}$ indicates greater deviation from the room temperature value. A positive $b_{0}$ indicates gain from room temperature while a negative $b_{0}$ indicates loss in bubble point from room temperature. Note that this analysis assumes pressurization gas ramp rate is sufficiently slow so that the correction for ramp rate need not be considered (Hernandez et al. 1996).

\begin{tabular}{|c|r|r|r|}
\cline { 3 - 4 } \multicolumn{1}{c|}{} & \multicolumn{1}{c|}{ Material } & \multicolumn{1}{c|}{} \\
\hline Coefficient & \multicolumn{1}{c|}{ 304 SS } & Aluminum & Titanium \\
\hline c0 & $-2.93 \mathrm{E}+02$ & $-4.12 \mathrm{E}+02$ & $1.50 \mathrm{E}+02$ \\
c1 & $-4.45 \mathrm{E}-01$ & $-4.37 \mathrm{E}-01$ & $-1.59 \mathrm{E}-01$ \\
c2 & $9.63 \mathrm{E}-03$ & $1.01 \mathrm{E}-02$ & $3.69 \mathrm{E}-03$ \\
c3 & $-2.17 \mathrm{E}-05$ & $-1.37 \mathrm{E}-05$ & $-5.20 \mathrm{E}-06$ \\
$\mathbf{c 4}$ & $1.94 \mathrm{E}-08$ & $2.68 \mathrm{E}-09$ & $1.39 \mathrm{E}-09$ \\
\hline
\end{tabular}

Table 10.4 - Linear Thermal Contraction Coefficients for Screen Channel Liquid Acquisition Device Metals

Table 10.5 lists the temperature dependent pore diameter model coefficients for all available LAD screens as a function of the screen type and pressurization scheme. For example, 325×2300 autogenous includes all data where the vapor was used as a pressurant gas, such as for $\mathrm{GH}_{2} / \mathrm{LH}_{2}, \mathrm{GN}_{2} / \mathrm{LN}_{2}, \mathrm{GOX} / \mathrm{LOX}$, and $\mathrm{GCH}_{4} / \mathrm{LCH}_{4}$. Very little data exists for screens coarser than the $80 \times 700$ mesh, some of which only contain 1-2 points from a single reference. Therefore, while the coefficients for these coarser meshes are suspect to scrutiny, they are nonetheless presented. For a conservative approach, a mission designer can simply use the room temperature pore diameter for those screens and neglect the temperature dependence. Figures $10.5 \mathrm{a}-\mathrm{c}$ plot the resultant model generated temperature dependent pore diameter curves as a function of temperature using both non-condensable as well as autogenous pressurization schemes to compare with 
both data sets. Examination of Figure 10.5 and Table 10.5 shows that the effective pore diameter decreases with temperature due to the combined effect of screen shrinkage and gain due to pressurization with GHe for all meshes where considerable data is available. Meanwhile the pore diameter generally increases with temperature, due to competition from gain in bubble point due to decreased pore size, but loss in bubble point due to pressurization with the condensable gas for the autogenous pressurization case.

Examining Figures $10.5 \mathrm{a}-\mathrm{c}$ for the autogenous pressurization case shows the evolution of this competition between the three meshes.

\begin{tabular}{|c|c|c|c|c|}
\hline Mesh & Metal & Pressurization Method & $\mathrm{bO}[\mu \mathrm{m}]$ & $r[\mu \mathrm{m}]$ \\
\hline $510 \times 3600$ & SS & $\mathrm{GHe}$ & 20.680 & 0.05412 \\
\hline $510 \times 3600$ & SS & Autogenous & 11.077 & 0.00498 \\
\hline $450 \times 2750$ & SS & $\mathrm{GHe}$ & 12.513 & 0.08855 \\
\hline $450 \times 2750$ & SS & Autogenous & -0.487 & 0.0161 \\
\hline $325 \times 2300$ & SS & $\mathrm{GHe}$ & 9.933 & 0.134 \\
\hline $325 \times 2300$ & SS & GN2 & 6.697 & 1.12 \\
\hline $325 \times 2300$ & SS & Autogenous & -0.987 & 0.995 \\
\hline $250 \times 1400$ & SS & Autogenous & 0.408 & 0.023 \\
\hline $250 \times 1370$ & SS & Autogenous & 17.100 & 3.146 \\
\hline $200 \times 1400$ & SS & $\mathrm{GHe}$ & 2.847 & 0.0352 \\
\hline $200 \times 1400$ & SS & Autogenous & -18.367 & 8.993 \\
\hline $200 \times 1400$ & Al & Autogenous & -26.307 & 12.258 \\
\hline $165 \times 800$ & SS & $\mathrm{GHe}$ & 5.880 & 14.382 \\
\hline $165 \times 800$ & SS & Autogenous & 6.493 & 2.4768 \\
\hline $80 \times 700$ & SS & $\mathrm{GHe}$ & 4.770 & 33.334 \\
\hline $80 \times 700$ & SS & Autogenous & -13.813 & 2.09E-05 \\
\hline $50 \times 250$ & SS & Autogenous & 7.783 & 0.0343 \\
\hline $24 \times 110$ & SS & Autogenous & 8.603 & 135.32 \\
\hline $720 \times 140$ & SS & $\mathrm{GHe}$ & -8.507 & 0.1038 \\
\hline $720 \times 140$ & SS & Autogenous & 1.823 & 0.1038 \\
\hline $500 \times 500$ & SS & $\mathrm{GHe}$ & -3.060 & 0.00473 \\
\hline $500 \times 500$ & SS & Autogenous & 11.610 & 4.83E-05 \\
\hline $325 \times 325$ & SS & Autogenous & -0.407 & 0.00013 \\
\hline $120 \times 120$ & SS & Autogenous & -96.800 & 0.0324 \\
\hline $120 \times 120$ & $\mathrm{Al}$ & Autogenous & 13.723 & 0.0532 \\
\hline $200 \times 200$ & SS & Autogenous & -1.693 & 1.439 \\
\hline $150 \times 150$ & SS & Autogenous & 1.233 & 16.11 \\
\hline $60 \times 60$ & SS & Autogenous & 19.497 & $6.56 \mathrm{E}-06$ \\
\hline $40 \times 40$ & SS & Autogenous & 33.567 & 0.0156 \\
\hline
\end{tabular}

Table 10.5 - Temperature Dependent Pore Diameter Fitting Parameters 
The differences in gain or loss between the different screens is attributed to differences in the geometry of the actual pore shape and shape of the L/V interface, which is a function of the ratio of the warp to shute diameter ratio from Section 10.3.2. The warp to shute diameter ratio is close for the 325 and 450 meshes, and thus these meshes yield near identical gains for GHe pressurization and loss for autogenous pressurization. The gain due to pore shrinkage is slightly outmatched by the loss due to pressurization with the condensable vapor. Meanwhile the warp to shute diameter ratio is quite different for the 510 mesh, which yields noticeably different gain and loss from room temperature. The gain due to pore shrinkage outmatches the loss due to pressurization with the condensable vapor. To examine this trend for other meshes, Figure 10.6 plots the $b_{0}$ values against the fineness of the mesh for screens where data is available using both pressurization schemes. The gain from room temperature generally becomes greater as the fineness of the screen increases for both pressurization schemes.

Figures $10.7 \mathrm{a}$ and $\mathrm{b}$ plot the model generated curves against data for normally saturated liquid states in $\mathrm{LH}_{2}$ and $\mathrm{LN}_{2}$, respectively. $\mathrm{LH}_{2}$ saturated state data is taken from Chapter 5, while the saturated $\mathrm{LN}_{2}$ data is taken from the pool of data collected alongside low pressure $\mathrm{LH}_{2}$ and high pressure LOX cryogenic bubble point data. Error bars are plotted but are barely distinguishable. Excellent agreement is seen between $\mathrm{LH}_{2}$ data and new temperature dependent pore diameter bubble point model. The model also matches $\mathrm{LN}_{2}$ data reasonably well, despite some scatter in the $\mathrm{GN}_{2} / \mathrm{LN}_{2}$ bubble point data taken in warmer liquid states. 


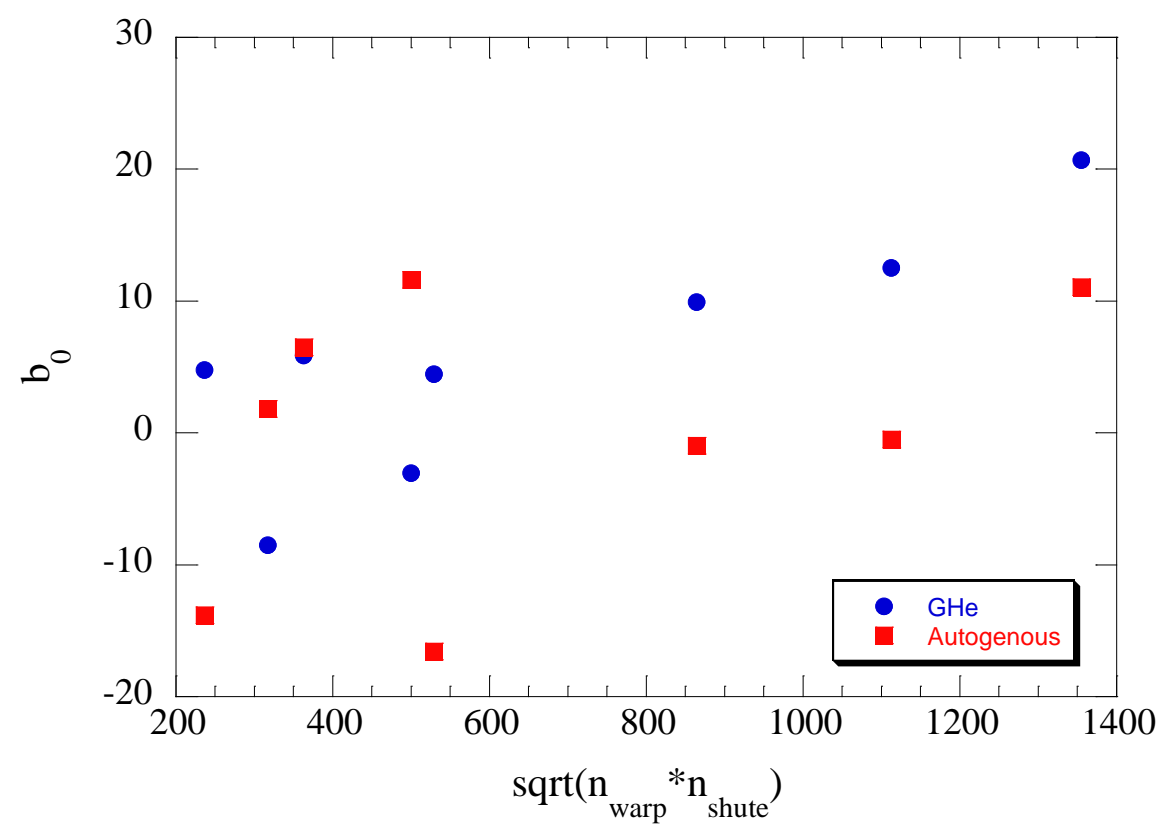

Figure 10.6 - Temperature Dependent Pore Diameter Fitting Coefficient versus the Fineness of the Mesh

For all screens, decreasing the temperature of the system acts to decrease the pore size. Overall, at cryogenic temperatures, autogenous pressurization acts as a degradation in performance and pressurization with a non-condensable gas such as GHe acts as a gain in performance. The physical explanation for this difference in performance is attributed to added heating and cooling of the $\mathrm{L} / \mathrm{V}$ interface due to added condensation and evaporation, respectively. Pressurization with the vapor acts to increase the interfacial temperature while pressurization with a non-condensable gas such as helium acts to reduce the interfacial temperature. 

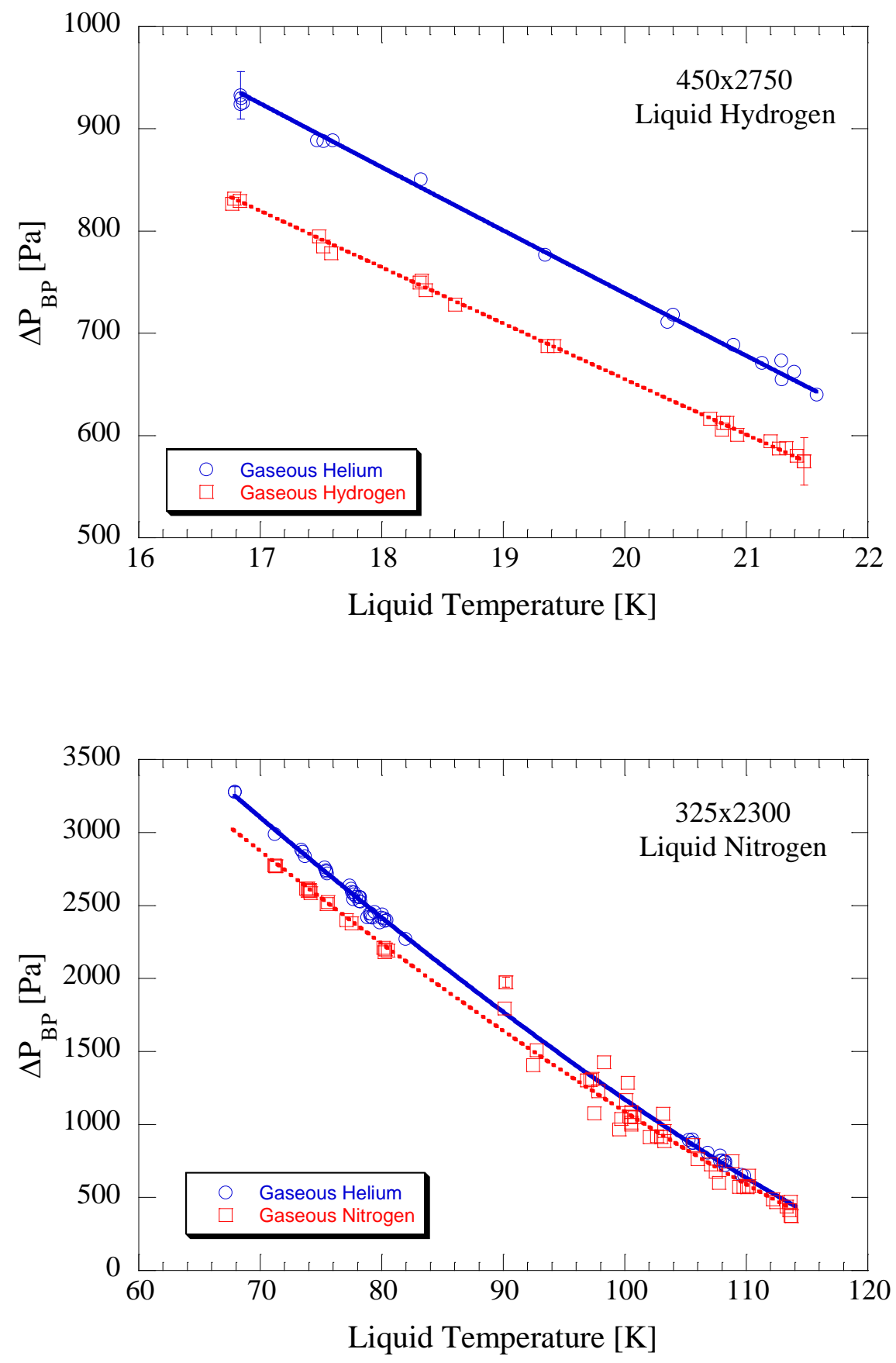

Figure 10.7 - Updated Model Performance versus Bubble Point Data Taken in Normally Saturated Liquid States: a) 450x2750 Liquid Hydrogen Data using Gaseous Helium and Gaseous Hydrogen and b) 325x2300 Liquid Nitrogen Data using Gaseous Helium and Gaseous Nitrogen. Solid lines are the new model generated curves. 
To compare the relative differences between pressurization schemes, Figure 10.8 plots the bubble point pressure obtained using GHe divided by the bubble point obtained using the vapor as a function of the reduced temperature over the range of experimental data for a 325x2300 Dutch Twill. Therefore the curves represent the relative difference in performance between the two schemes. The $325 \times 2300$ is the only LAD screen with data in four different cryogenic liquids using both pressurization schemes, thus facilitating a normalized comparison in performance between the two pressurization schemes.

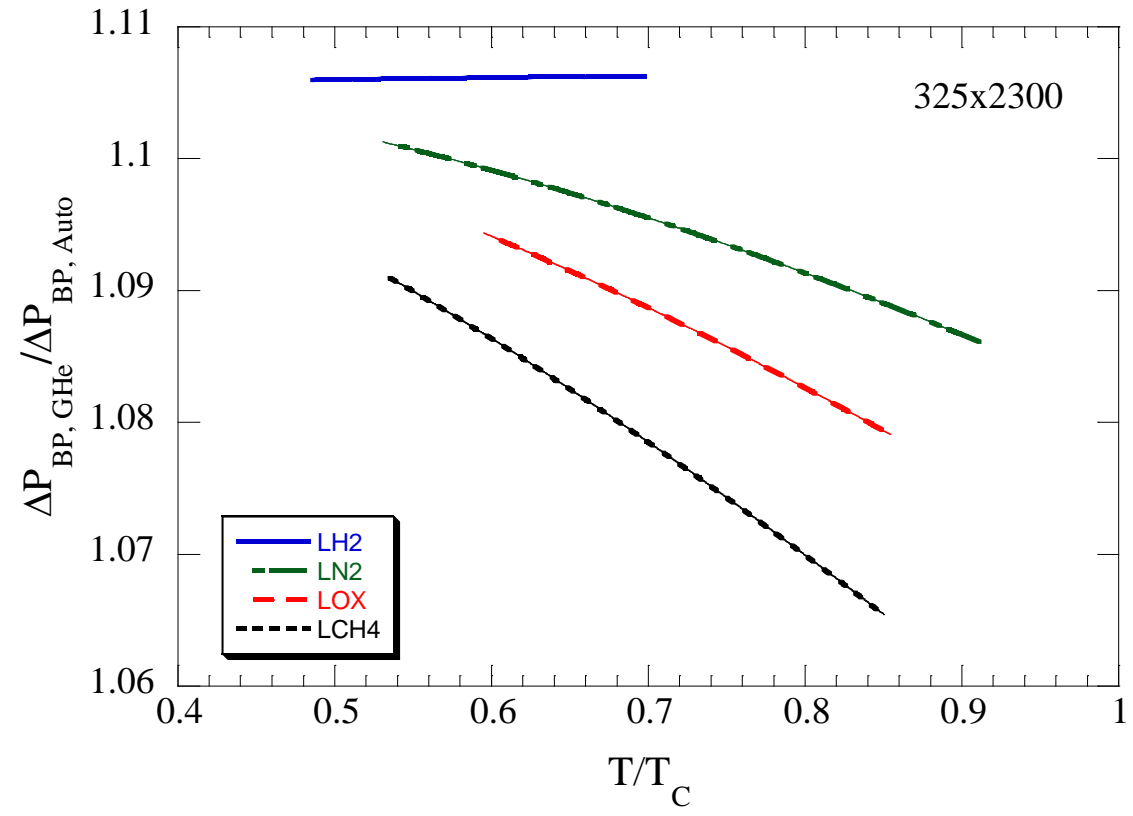

Figure 10.8 - Model Generated 325x2300 Bubble Point Ratio as a Function of the Reduced Temperature for Normally Saturated Liquid Taken Over the Range of Conditions of the Data

Comparing the curves reveals noticeable differences in performance between the liquids; as the saturation temperature decreases, the disparity in performance between pressurization schemes becomes greater. For example, the effect of adding helium to the interface improves performance over the baseline vapor pressurant in $\mathrm{LH}_{2}$ much more 
than it does in $\mathrm{LCH}_{4}$. As the temperature of the system increases towards the critical point, differences in performance between the two pressurization schemes diminishes, as expected, because surface tension diminishes to zero at the critical point.

The trends in the data and model can be explained through the following rationale. First, the diffusion coefficient of GHe is relatively the largest in hydrogen and smallest in methane (Sinor 1965 and Zimmerli et al. 2010). Therefore higher diffusion coefficients imply there will be a higher concentration of helium at the screen L/V interface in hydrogen over the other liquids. The higher probability of GHe diffusing to the interface in hydrogen over methane would promote more interfacial cooling and thus greater relative difference in bubble point performance between non-condensable and vapor cases, because GHe acts to evaporate liquid away from the screen L/V interface.

Second, higher concentrations of helium at the interface will act to block condensation mass transfer, enhancing evaporation rates, and will thus cause greater differences in relative performance between the non-condensable and vapor pressurant cases. It has been shown numerically that even relatively small concentrations of GHe will act to suppress condensation rates (Pong and Moses 1986 and Panzarella and Kassemi 2009). The Schrage (1953) model can be used to estimate the condensation rate $J_{C}$ for the vapor case from Equation 7.11. However, accommodation coefficients do not exist in the literature and must be determined experimentally. For the purposes of qualitative comparison, one can assume the coefficients are equal. Evaluating bubble point and temperature data for the $325 \times 2300$ screen at the NBP state for the four liquids, approximating the interface temperature as the liquid screen side temperature, the 
Schrage model predicts higher condensation rates for oxygen and nitrogen, followed by methane, and hydrogen.

The Schrage model cannot be used to estimate condensation or evaporation rates for the GHe case due to significant uncertainty in the interface temperature and partial pressure of the vapor when helium is present. However evaporation rates can be estimated from diffusion rates based on kinetic theory. Theory predicts the highest diffusion rates for $\mathrm{GHe} / \mathrm{LH}_{2}$, with $\mathrm{GHe} / \mathrm{LCH}_{4}$ yielding the lowest evaporation rates. Therefore, while the absolute magnitude of the condensation rate may be higher in $\mathrm{GCH}_{4} / \mathrm{LCH}_{4}$ over $\mathrm{GH}_{2} / \mathrm{LH}_{2}$, the relative difference in evaporation and condensation rates is higher in $\mathrm{LH}_{2}$ than in $\mathrm{LCH}_{4}$, which would lead to greater differences in interface temperatures and thus bubble point performance between the two pressurization schemes in $\mathrm{LH}_{2}$ than in $\mathrm{LCH}_{4}$. Once accommodation coefficients become available, Equation 7.11 can be used to substantiate or negate these claims.

\subsection{Liquid Subcooling Model}

In comparing bubble point pressures in saturated liquid to those taken in subcooled liquid, cryogenic bubble point tests from Chapters $5-7$ indicate a distinct gain in performance due to subcooling the liquid. A subcooled state can be achieved by either reducing the temperature of the liquid at the $\mathrm{LAD}$ screen below the saturation temperature based on the pressure at the screen or by elevating the pressure of the liquid at the screen above the saturation pressure based on the temperature of the liquid at the screen. Operationally in flight propellant tanks, the latter is more easily achievable, because it is significantly easier to pressurize a tank than to remove heat. This gain in 
performance is of particular interest, because all cryogenic flight engines (Santiago 1996 and Schuster et al. 1996) and proposed cryogenic fuel depots (see Kutter 2010 for example) will maintain some level of subcooling throughout any portion of the mission involving cryogenic liquid transfer to suppress boiling.

To model this gain, the bubble point formula for saturated cryogenic liquid states can be modified to incorporate subcooled liquid states. Examination of subcooled data indicates that the gain in bubble point pressure is approximately linearly proportional to the degree of subcooling, or distance away from the saturation curve, on a temperature/pressure plot. Therefore the proposed subcooled bubble point model takes the following form:

$\Delta P_{B P, S u b}=\Delta P_{B P, S a t}\left(1+n_{S u b}\left(\frac{P-P_{S a t}}{P_{C}}+\frac{T_{S a t}-T}{T_{C}}\right)\right)$

where $\Delta P_{B P, S a t}$ is the bubble point taken at the saturated liquid state from Equation 10.11, $P$ is the pressure of the liquid at the screen, $P_{S a t}$ is the saturation pressure of the liquid based on the temperature of the liquid at the screen, $T_{\text {Sat }}$ is the saturation temperature based on the pressure at the screen, and $n_{\text {Sub }}$ is a dimensionless fitting parameter which accounts for the gain in bubble point relative to the saturated state value. All pressures are in units of $[\mathrm{kPa}]$ and all temperatures are in units of $[\mathrm{K}]$. As will be shown, $n_{\text {Sub }}$ is only a function of the pressurant gas/cryogenic liquid pair and does not depend on the screen type. As the state of the liquid approaches the saturation state, Equation 10.14 collapses into Equation 10.11. 
The fitting coefficients were determined in the following manner: For each subcooled bubble point pressure data point, a corresponding model generated saturated bubble point was calculated at each state. The subcooled gain, defined as the ratio of bubble point pressures:

$$
\frac{\Delta P_{B P, S u b}}{\Delta P_{B P, S a t}}=1+n_{\text {Sub }}\left(\frac{P-P_{\text {Sat }}}{P_{C}}+\frac{T_{\text {Sat }}-T}{T_{C}}\right)
$$

was then plotted against the term in parenthesis in Equation 10.15 in order to determine $n_{\text {Sub }}$ using a linear least squares method: Each gas/liquid pair yielded a different $n_{\text {Sub }}$. Since it was determined that $n_{\text {Sub }}$ did not depend on the screen type, the $n_{\text {Sub }}$ for a given gas/liquid pair were then averaged across screens to yield a single value. Table 10.6 lists all the individual fitting parameters for each screen/gas/liquid triple as well as the uncertainty in each case. Table 10.7 lists the averaged $n_{\text {Sub }}$ values for each gas/liquid pair along with critical temperature and pressure. Examination of Table 10.6 indicates for both GHe and autogenous pressurization schemes, the $n_{\text {Sub }}$ values generally decrease with decreasing liquid saturation temperature. However, since the $n_{\text {Sub }}$ values in Equation 10.15 were obtained by normalizing the data by the respective critical point values, and not a standard reference value, sensitivity between liquids is suppressed. 


\begin{tabular}{|c|c|c|c|c|}
\hline Mesh & Liquid & Gas & nsub & r \\
\hline $\mathbf{2 0 0 \times 1 4 0 0}$ & LOX & GHe & 0.5245 & 0.000418 \\
\hline $\mathbf{3 2 5 \times 2 3 0 0}$ & LCH4 & GHe & 0.4531 & 0.0004032 \\
\hline $\mathbf{3 2 5 \times 2 3 0 0}$ & LCH4 & GN2 & 0.4043 & 0.0002687 \\
\hline $\mathbf{3 2 5 \times 2 3 0 0}$ & LCH4 & Autogenous & 0.388 & 0.00024 \\
\hline $\mathbf{3 2 5 \times 2 3 0 0}$ & LOX & GHe & 0.5308 & 0.0005297 \\
\hline $\mathbf{3 2 5 \times 2 3 0 0}$ & LOX & Autogenous & 0.3208 & 0.00020333 \\
\hline $\mathbf{3 2 5 \times 2 3 0 0}$ & LN2 & GHe & 0.3081 & 0.001272 \\
\hline $\mathbf{3 2 5 \times 2 3 0 0}$ & LN2 & Autogenous & 0.2094 & 0.00802 \\
\hline $\mathbf{3 2 5 \times 2 3 0 0}$ & LH2 & GHe & 0.2671 & 0.0000713 \\
\hline $\mathbf{3 2 5 \times 2 3 0 0}$ & LH2 & Autogenous & 0.1492 & 0.0001357 \\
$\mathbf{4 5 0 \times 2 7 5 0}$ & LN2 & GHe & 0.328 & 0.000053275 \\
$\mathbf{4 5 0 \times 2 7 5 0}$ & LN2 & Autogenous & 0.2118 & 0.000063174 \\
\hline $\mathbf{4 5 0 \times 2 7 5 0}$ & LH2 & GHe & 0.2535 & 0.00008973 \\
\hline $\mathbf{4 5 0 \times 2 7 5 0}$ & LH2 & Autogenous & 0.1413 & 0.00003724 \\
\hline $\mathbf{5 1 0 \times 3 6 0 0}$ & LN2 & GHe & 0.3006 & 0.00006851 \\
\hline $\mathbf{5 1 0 \times 3 6 0 0}$ & LN2 & Autogenous & 0.2115 & 0.000136 \\
\hline $\mathbf{5 1 0 \times 3 6 0 0}$ & LH2 & GHe & 0.2536 & 0.000046444 \\
\hline $\mathbf{5 1 0 \times 3 6 0 0}$ & LH2 & Autogenous & 0.1388 & 0.000023734 \\
\hline
\end{tabular}

Table 10.6 - Complete Coefficient Matrix for Subcooled Liquid Bubble Point Model

\begin{tabular}{|c|c|c|c|c|c|c|c|}
\hline & Tc [T] & Pc [kPa] & GHe & GCH4 & GOX & GN2 & GH2 \\
\hline LCH4 & 190.6 & 4606 & 0.453 & 0.388 & $x$ & 0.4043 & $x$ \\
\hline LOX & 154.6 & 5043 & 0.528 & $x$ & 0.3208 & $x$ & $x$ \\
\hline LN2 & 126.2 & 3396 & 0.312 & $x$ & $x$ & 0.2109 & $x$ \\
\hline LH2 & 32.94 & 1286 & 0.258 & $x$ & $x$ & $x$ & 0.1431 \\
\hline
\end{tabular}

Table 10.7 - Averaged Subcooled Liquid Gain Slopes as a Function of Gas/Liquid Pair

To compare between liquids, Figures 10.9a and b plot model generated subcooled gains for a 325x2300 screen using GHe and autogenous pressurization schemes, respectively, as a function of the pressure difference from the NBP pressure up to the critical pressure of each fluid. The curves are generated assuming a constant liquid temperature equal to the saturation temperature. This allows comparison of the effect of subcooling by a fixed delta $\mathrm{P}$ between liquids. 

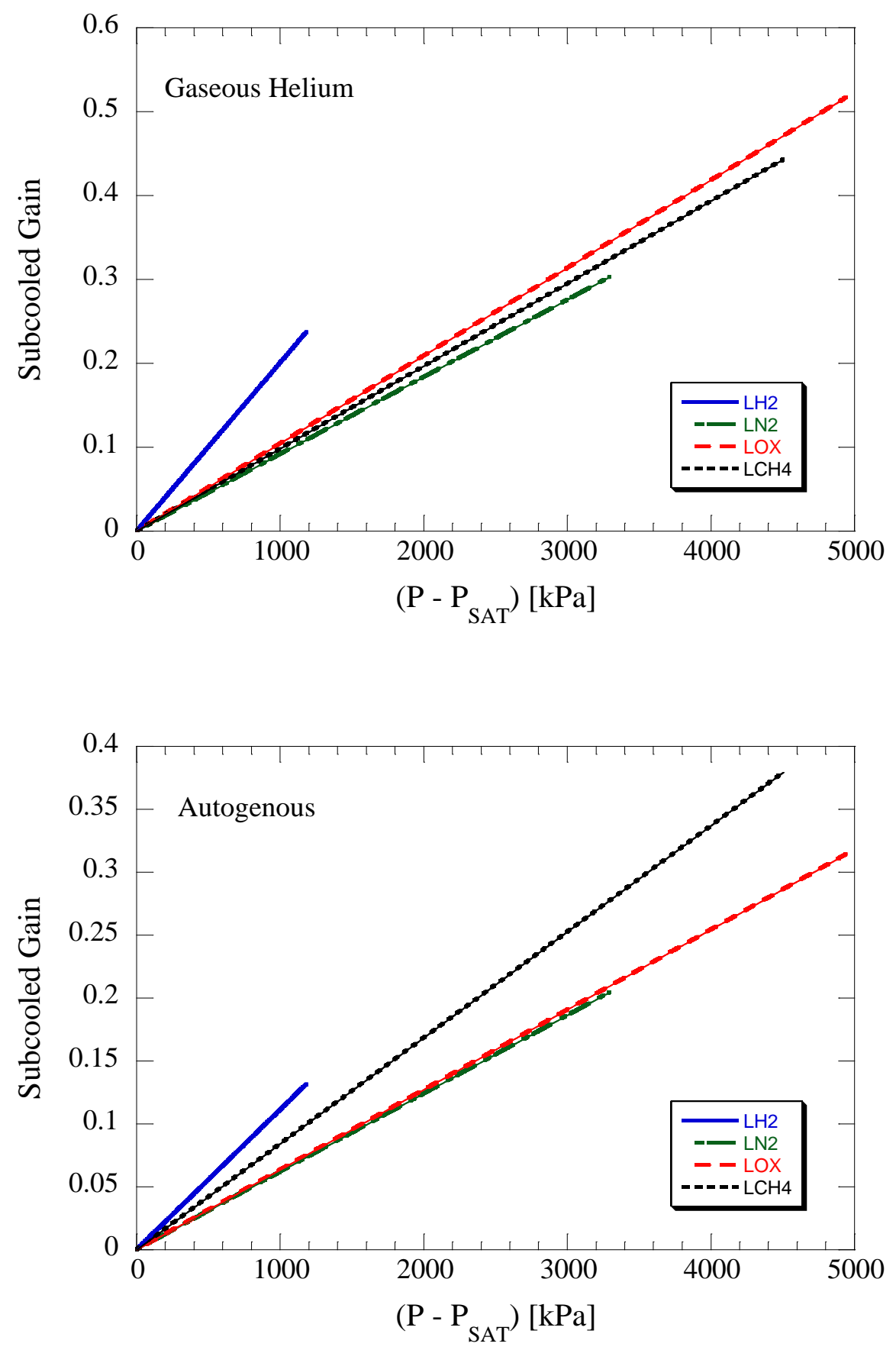

Figure 10.9 - Model Generated Subcooled Gain Curves for a 325x2300 Screen using a) Gaseous Helium and b) Autogenous Pressurization Schemes as a Function of Pressure Difference Above the Saturation Pressure at the Normal Boiling Point. Curves are generated using Equation 10.15 with a liquid temperature equal to the saturation temperature. 
Examination of Figure 10.9a shows that the highest gain using a non-condensable pressurant is achieved in $\mathrm{LH}_{2}$, as indicated by the steepest slope. The slope of subcooled gain scales with the saturation temperature of the fluid for hydrogen, oxygen, and methane, as $\mathrm{GHe} / \mathrm{LCH}_{4}$ yields the weakest slope. Meanwhile there is no definitive trend for autogenous pressurization from Figure 10.9b, but pressurization with the vapor yields the steepest gain in the coldest liquid $\left(\mathrm{LH}_{2}\right)$. For example, for a pressure difference of 1 MPa above saturation, there is a $21 \%$ and $12.5 \%$ gain in $\mathrm{LH}_{2}$ bubble point over the corresponding $\mathrm{NBP}$ value using $\mathrm{GHe}$ and $\mathrm{GH}_{2}$, respectively.

Figures 10.10a and $\mathrm{b}$ plot all of the higher pressure experimental $\mathrm{LN}_{2}$ bubble point data collected in this dissertation for a $325 \times 2300$ LAD screen using $\mathrm{GHe}$ and $\mathrm{GN}_{2}$ to pressurize, respectively. Similarly, Figures 10.11a and b plot model predictions for $\mathrm{GHe} / \mathrm{LN}_{2}$ and $\mathrm{GN}_{2} / \mathrm{LN}_{2}$ for a $325 \times 2300$ screen. At a constant temperature, elevating the pressure increases margin in bubble point; at a constant pressure, decreasing the temperature also increases the margin in bubble point as both indicated in the darkening in color. The new model matches the subcooled trends in the data quite well. 

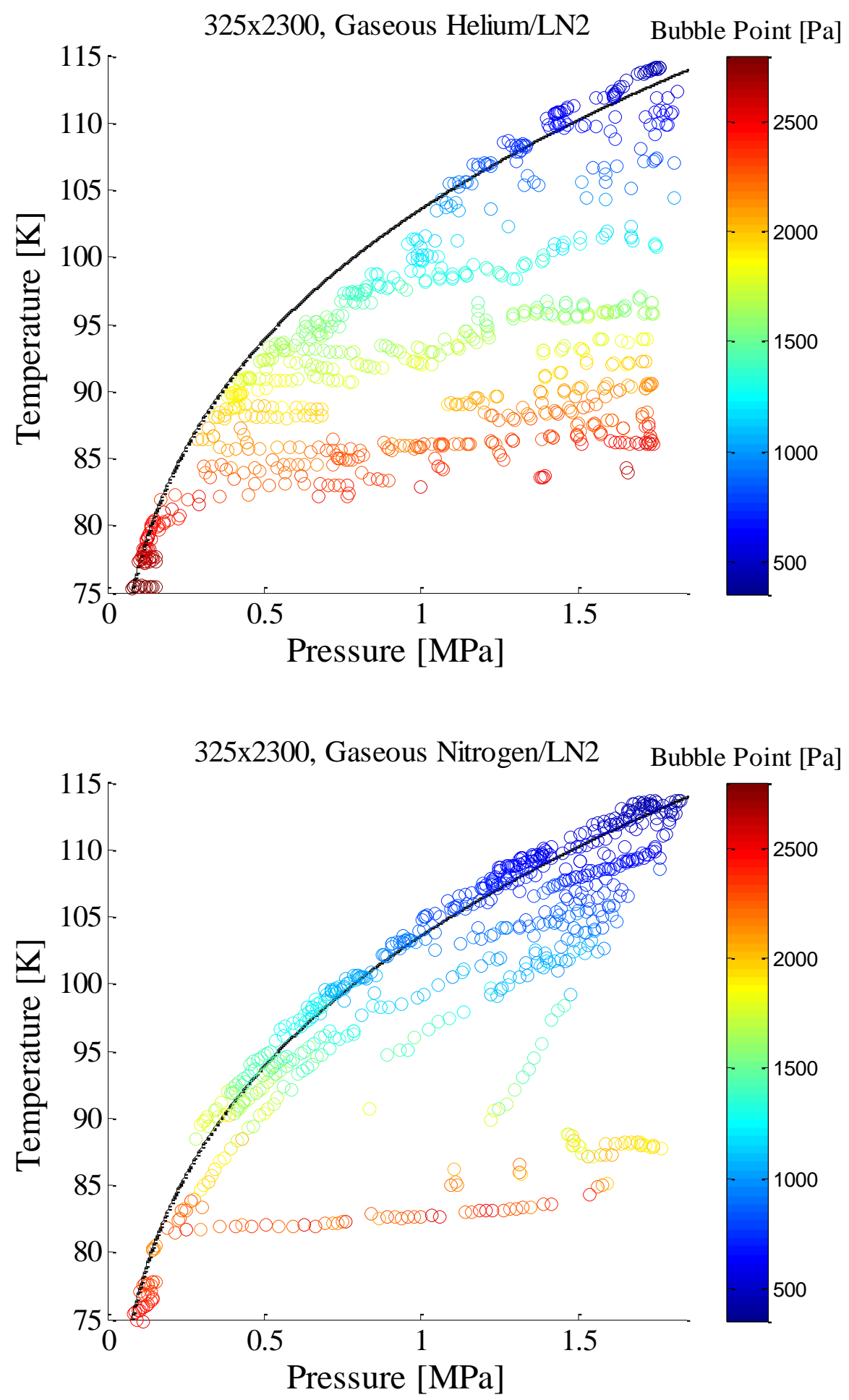

Figure 10.10 - 325x2300 Liquid Nitrogen Bubble Point Data as a Function of Liquid Pressure and Temperature using a) Gaseous Helium and b) Gaseous Nitrogen as Pressurants. The black line is the nitrogen saturation curve. 

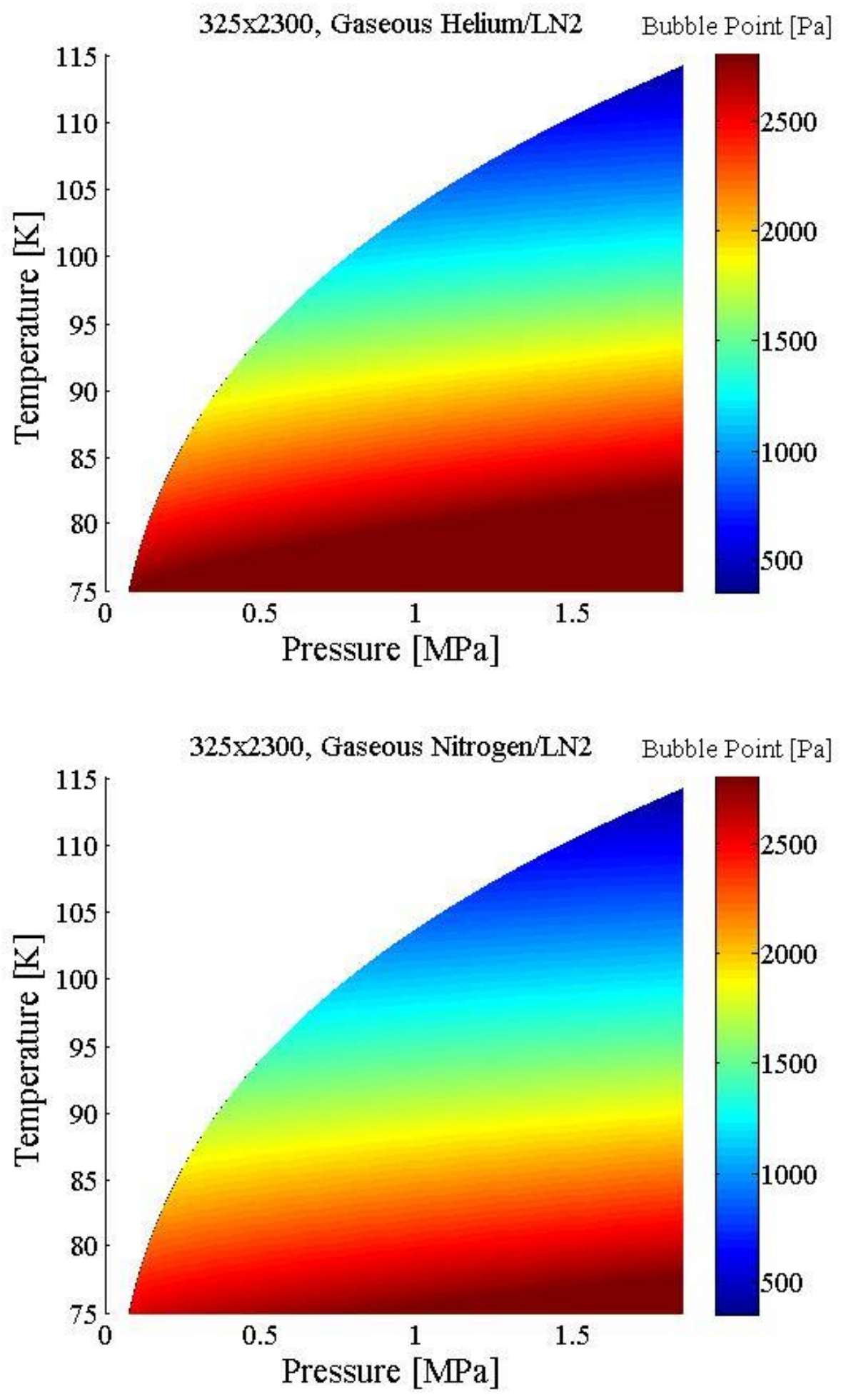

Figure 10.11 - Model Generated 325x2300 Liquid Nitrogen Bubble Point Pressure as a Function of the Liquid Pressure and Temperature using a) Gaseous Helium and b) Gaseous Nitrogen as Pressurants. The black line is the nitrogen saturation curve. 
Figures $10.12,10.13$, and 10.14 plot the model generated subcooled gain using Equation 10.14 versus the subcooled $\mathrm{LCH}_{4}$ data from Chapter 7 using three different pressurant gases, $\mathrm{GHe}, \mathrm{GN}_{2}$, and $\mathrm{GCH}_{4}$, respectively. Figures $10.12 \mathrm{a}-14 \mathrm{a}$ plot the data and Figures $10.12 \mathrm{~b}-10.14 \mathrm{~b}$ plot the model fits. As shown, regardless of pressurant gas, subcooling the $\mathrm{L} / \mathrm{V}$ interface at the LAD screen increases the bubble point pressure approximately linearly with distance away from the saturation line. However, the type of pressurant gas in contact with the screen does have an impact on the magnitude of the gain. As shown, GHe outperforms $\mathrm{GN}_{2}$, and both non-condensable gases yield higher subcooled gains relative to the vapor case $\left(\mathrm{GCH}_{4}\right)$. Differences between the two noncondensable pressurant gases is attributed to a higher molecular weight and higher rate of dissolution into the liquid for $\mathrm{GN}_{2}$ over $\mathrm{GHe}$, both of which act to limit the performance enhancing effect of evaporation on the bubble point pressure.

\subsection{Warm Pressurant Gas Model}

With the exception of warm pressurant gas bubble point tests conducted in Chapter 8 , the majority of data in the literature was taken with the temperature of the pressurant gas approximately equal to the liquid temperature. This is due to the standard inverted testing configuration used for measuring cryogenic bubble point pressures which forces uniform, constant, and cold pressurant gas temperature incident on the LAD screen. Warm pressurant gas data represents a much more realistic case and is quite possibly the most relevant to LEO, Lunar, and Martian mission design, since the temperature of the pressurant gas typically assumes the ambient temperature, which is generally much warmer than the saturation temperature of the cryogenic propellant. 


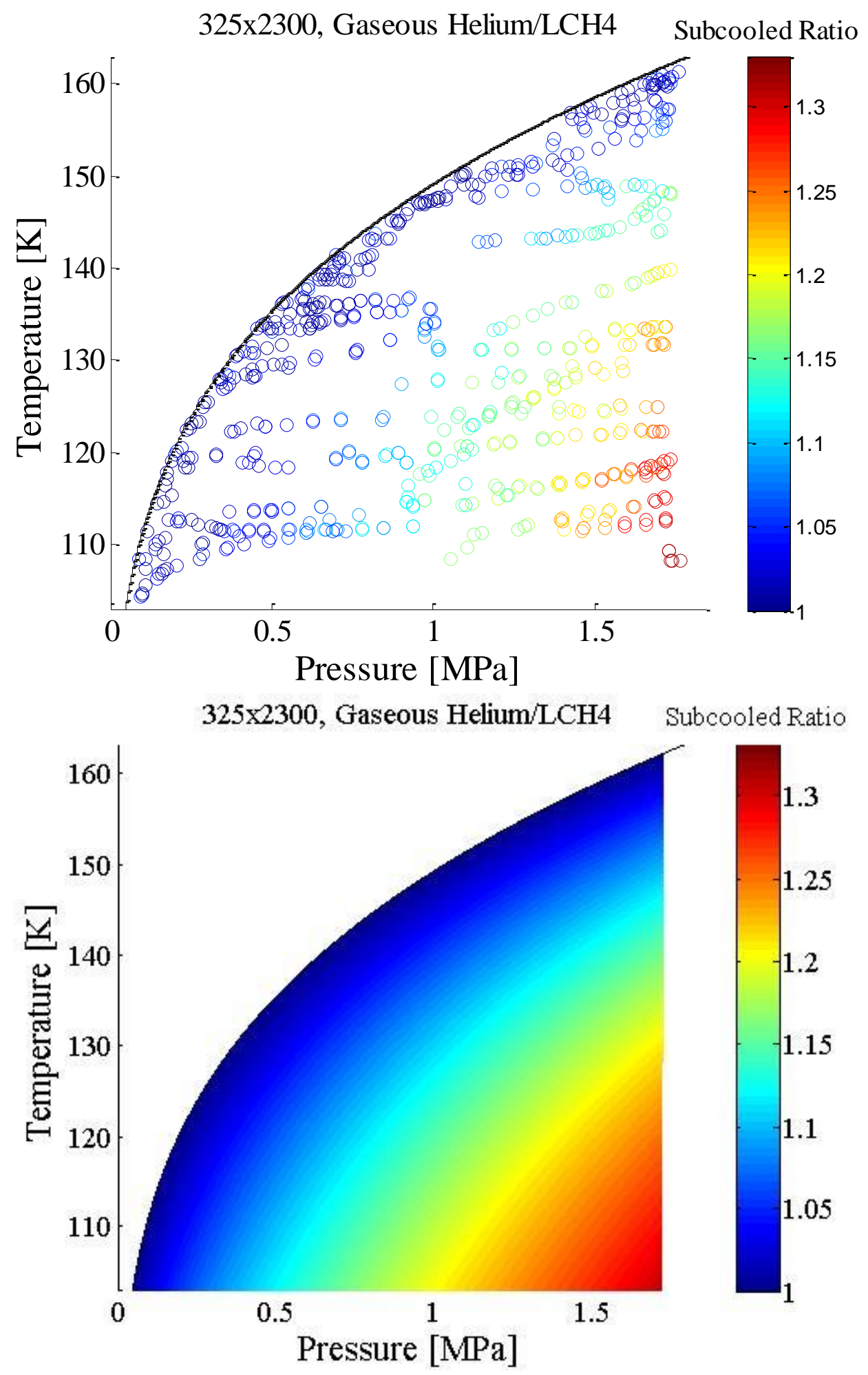

Figure 10.12 - Comparison of 325×2300 Gaseous Helium/Liquid Methane Subcooled Gain as a Function of Liquid Pressure and Temperature at the Screen between the a) Data and b) Model. The black line is the saturation curve. 


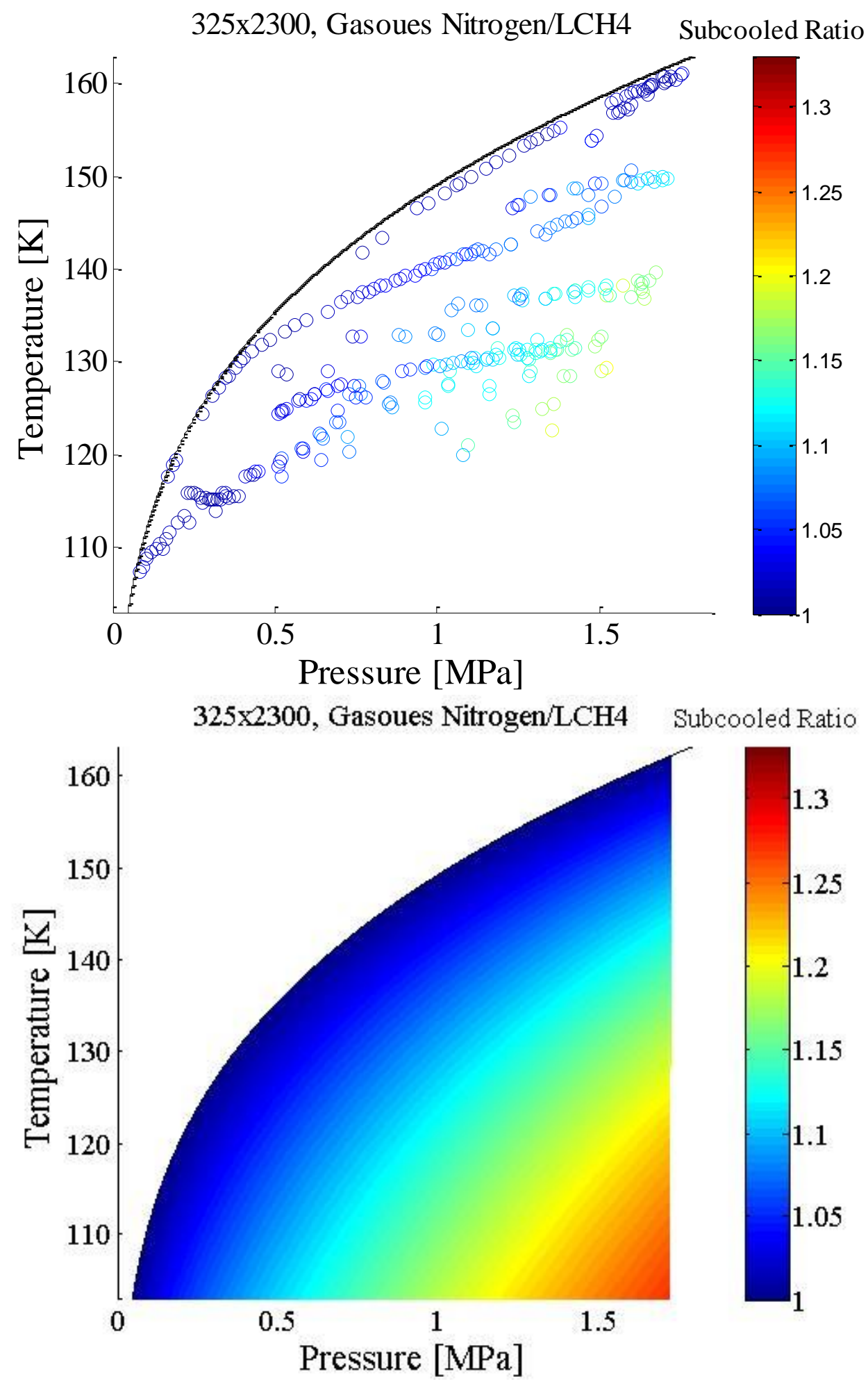

Figure 10.13 - Comparison of 325x2300 Gaseous Nitrogen/Liquid Methane Subcooled Gain as a Function of Liquid Pressure and Temperature at the Screen between the a) Data and b) Model. The black line is the saturation curve. 


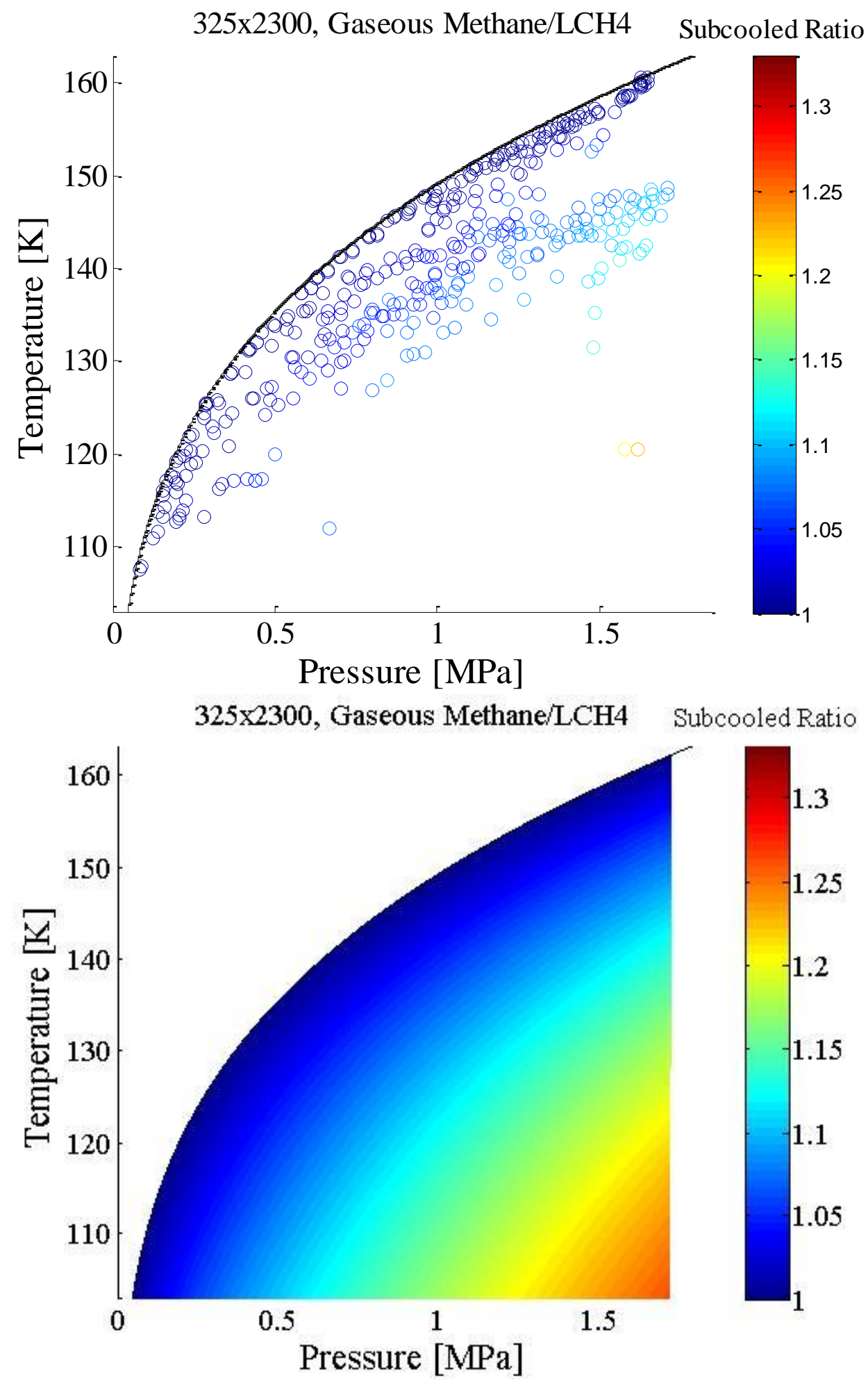

Figure 10.14 - Comparison of 325x2300 Gaseous Methane/Liquid Methane Subcooled Gain as a Function of Liquid Pressure and Temperature at the Screen between the a) Data and b) Model. The black line is the saturation curve. 
Warm pressurant gas bubble point tests from Chapter 8 were conducted in normally saturated liquid, essentially nulling out the subcooling effect. Examination of the heated gas data indicates that warm pressurant gas acts as a degradation factor on bubble point, and that the loss in performance is proportional to the difference between the liquid and pressurant gas temperatures. When comparing heated gas trends in $\mathrm{LH}_{2}$ to trends in $\mathrm{LN}_{2}$, the onset of degradation was immediate in $\mathrm{LH}_{2}$ for all screens and both pressurization schemes tested, while the onset of degradation was delayed in $\mathrm{LN}_{2}$ for some cases. Thus the proposed modification to the bubble point equation takes the following form:

$\Delta P_{B P, H o t}=\Delta P_{B P, S a t}\left[1-n_{H o t}\left(T_{G a s}-T\right)\right]$

where $\Delta P_{B P, S a t}$ is determined using Equation 10.11, $T_{G a s}$ is the temperature of the pressurant gas, $T_{\text {Onset }}$ is the gas temperature at which the heated gas bubble point begins to degrade from its cold gas value, and $n_{H o t}$ is a fitting parameter with dimensions of $[1 / \mathrm{K}]$. All temperatures are in SI units of Kelvin. $n_{H o t}$ is a function of each screen/liquid/gas triple. Note that $n_{H o t}$ is zero for all $\left(T_{\text {Gas }}-T\right)<T_{\text {Onset }}$. As the temperature of the pressurant gas approaches the liquid temperature, Equation 10.16 collapses into Equation 10.11.

The heated gas coefficients were determined in the same manner as the subcooled liquid coefficients. For each heated gas data point, a corresponding model generated saturated bubble point was calculated at each liquid temperature. For a given screen, 
pressurant gas, and cryogenic liquid, the heated gas loss, defined as the ratio of bubble point pressures:

$$
\frac{\Delta P_{B P, H o t}}{\Delta P_{B P, S a t}}=1-n_{H o t}\left(T_{G a s}-T\right)
$$

is fit against the temperature difference, and $n_{H o t}$ is determined using a linear least squares fit. Results for $\mathrm{LH}_{2}$ and $\mathrm{LN}_{2}$ heated pressurant gas model parameters are presented in Table 10.8. Uncertainties are tabulated for each case.

\begin{tabular}{|c|c|c|c|c|c|}
\hline Mesh & Liquid & Gas & nhot [1/K] & TOnset [K] & $\mathbf{r}$ \\
\hline $\mathbf{5 1 0} \times \mathbf{3 6 0 0}$ & Hydrogen & $\mathrm{GHe}$ & 0.0018 & 0 & 0.000783 \\
\hline $\mathbf{4 5 0} \times \mathbf{2 7 5 0}$ & Hydrogen & $\mathrm{GHe}$ & 0.003 & 0 & 0.000223 \\
\hline $\mathbf{3 2 5} \times \mathbf{2 3 0 0}$ & Hydrogen & $\mathrm{GHe}$ & 0.0073 & 0 & 0.00035 \\
\hline $\mathbf{5 1 0} \times \mathbf{3 6 0 0}$ & Hydrogen & $\mathrm{GH} 2$ & 0.0086 & 0 & 0.001757 \\
\hline $\mathbf{4 5 0} \times \mathbf{2 7 5 0}$ & Hydrogen & $\mathrm{GH} 2$ & 0.0133 & 0 & 0.002666 \\
\hline $\mathbf{3 2 5} \times \mathbf{2 3 0 0}$ & Hydrogen & $\mathrm{GH} 2$ & 0.0286 & 0 & 0.000805 \\
\hline $\mathbf{5 1 0} \times \mathbf{3 6 0 0}$ & Nitrogen & $\mathrm{GHe}$ & 0.00156 & 77 & 0.001252 \\
\hline $\mathbf{4 5 0} \times \mathbf{2 7 5 0}$ & Nitrogen & $\mathrm{GHe}$ & 0.00149 & 41 & 0.000298 \\
\hline $\mathbf{3 2 5} \times \mathbf{2 3 0 0}$ & Nitrogen & $\mathrm{GHe}$ & 0.0028 & 0 & 0.001193 \\
\hline $\mathbf{5 1 0} \times \mathbf{3 6 0 0}$ & Nitrogen & $\mathrm{GN} 2$ & 0.002 & 21 & 0.000746 \\
\hline $\mathbf{4 5 0} \times \mathbf{2 7 5 0}$ & Nitrogen & $\mathrm{GN} 2$ & 0.0031 & 0 & 0.000171 \\
\hline $\mathbf{3 2 5} \times \mathbf{2 3 0 0}$ & Nitrogen & $\mathrm{GN} 2$ & 0.0053 & 0 & 0.001784 \\
\hline
\end{tabular}

\section{Table 10.8 - Coefficient Matrix for Heated Pressurant Gas Bubble Point Model}

Figures 10.15a and b plot model generated curves using Equation 10.17 for $\mathrm{LH}_{2}$ and $\mathrm{LN}_{2}$, respectively. For all cases, elevating the temperature of the pressurant gas in contact with the screen acts as a degradation in performance of the LAD. Comparing Figures $10.15 \mathrm{a}$ to $10.15 \mathrm{~b}$, for all meshes and both pressurization schemes, there is steeper degradation in performance in $\mathrm{LH}_{2}$ over $\mathrm{LN}_{2}$. The onset of degradation is always immediate in $\mathrm{LH}_{2}$, but delayed for certain cases in $\mathrm{LN}_{2}$. For both liquids and all three meshes, degradation in performance is always much steeper using the autogenous 
pressurization scheme versus the non-condensable scheme. For both liquids and both pressurization schemes, the reduction in LAD performance scales inversely with the porosity of the screen, because the finest 510x3600 mesh degraded the least and the coarsest 325x2300 mesh degraded the most.

Differences in performance between the three different screens are due to the effect of screen thickness and porosity on the overall heat transfer across the screen. Differences in performance between pressurant gases are due to modification of the interfacial temperature due to added evaporation and/or condensation, as mentioned previously. Differences in performance between $\mathrm{LH}_{2}$ and $\mathrm{LN}_{2}$ are explained through differences in superheats required to initiate boiling on the liquid side of the LAD screen; the $\mathrm{LAD}$ screen is more susceptible to drying out in $\mathrm{LH}_{2}$ with warm pressurant gas due to the lower superheat relative to the $\mathrm{LN}_{2}$ case.

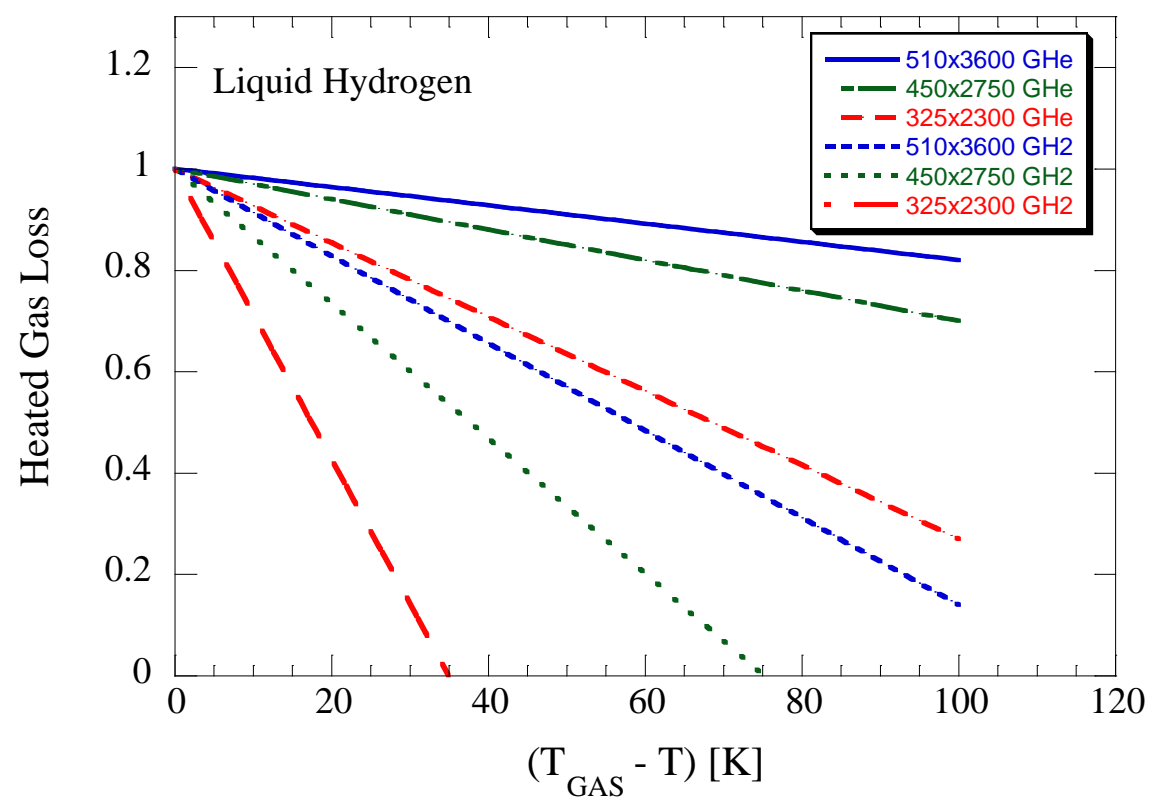




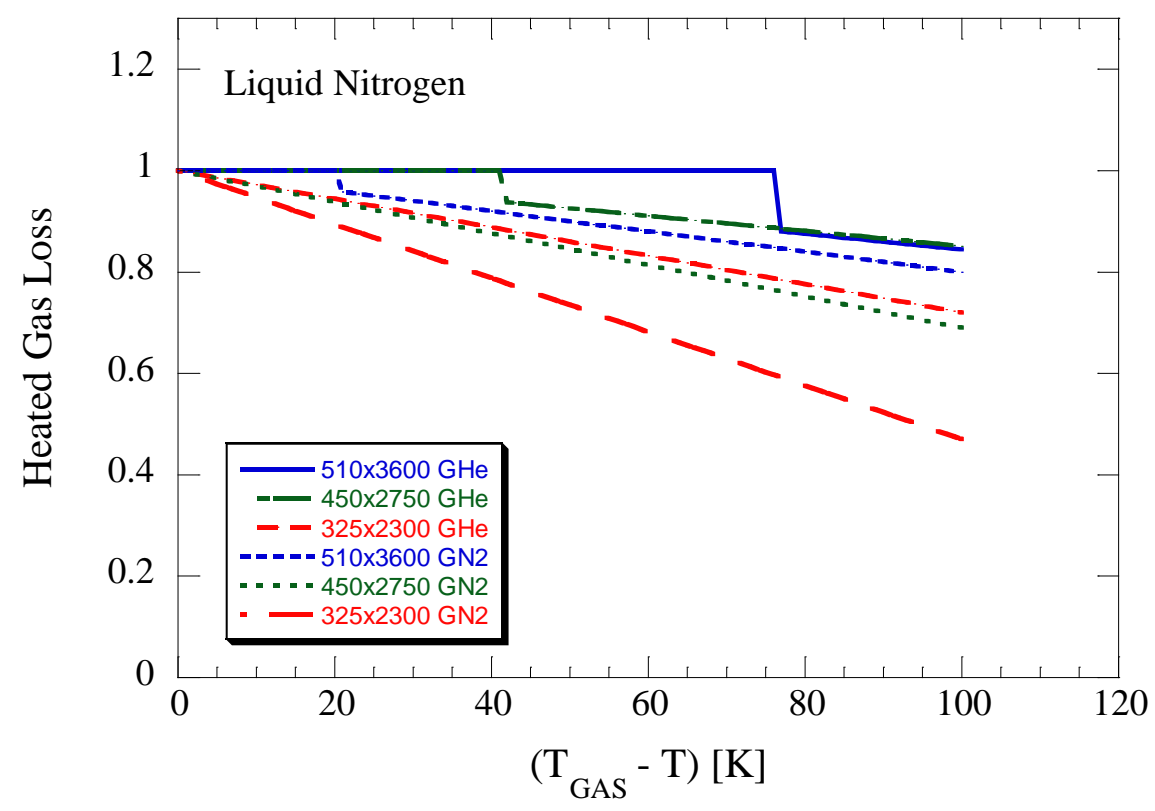

Figure 10.15 - Model Generated Curves of the Ratio of the Heated Pressurant Gas Loss from Equation 10.17 for a) Liquid Hydrogen and b) Liquid Nitrogen

\subsection{Concluding Remarks}

The primary performance parameter for characterizing porous screen channel LADs inside a propellant tank is the maximum bubble point pressure. The simplified room temperature bubble point equation based on Adamson and Gast (1997) was modified to predict performance in cryogenic liquids. The new cryogenic bubble point pressure model is constructed by compiling Equations 10.11, 10.14, and 10.16:

$\Delta P_{B P}=\frac{4 \gamma_{L V} \cos \theta_{C}}{D_{P}(T)}\left(1+n_{\text {Sub }}\left(\frac{P-P_{\text {Sat }}}{P_{C}}+\frac{T_{\text {Sat }}-T}{T_{C}}\right)\right)\left(1-n_{\text {Hot }}\left(T_{\text {Gas }}-T\right)\right)$

where $\gamma_{L V}$ is evaluated using Equation 3.19 with coefficients from Table 3.1 and $D_{P}(T)$ is evaluated using Equation 10.12 using fitting parameters from Tables 10.4 and 
10.5. The room temperature pore diameter can be determined for any LAD screen through simple knowledge of the diameter and number of warp and shute wires through the use of Equation 10.9. The temperature dependent pore diameter can be determined through simple bubble point tests in normally saturated liquids. $n_{S u b}$ is shown to only depend on the liquid/pressurant gas pair and does not depend on the screen mesh type. Meanwhile $n_{H o t}$ is dependent the screen/liquid/gas triplet. Table 10.9 compares the old simplified model and new cryogenic model performance against the data for the four most popular fine mesh LAD screens. The new model is accurate to within an average of $3 \%$ of the data, and matches well across the full range of thermodynamic conditions and meshes available in the literature.

\begin{tabular}{|c|c|c|c|c|c|c|c|c|}
\hline Screen & Liquid & Gas & \%min new & $\% \min$ old & \%max new & \%max old & \%avg new & \%avg old \\
\hline $200 \times 1400$ & LOX & $\mathrm{GHe}$ & -5.41 & 1.75 & 13.86 & 23.72 & -1.5 & 12.77 \\
\hline \multirow[t]{9}{*}{$325 \times 2300$} & LCH4 & $\mathrm{GHe}$ & -5.1 & 2.56 & 4.61 & 31.11 & -0.78 & 14.63 \\
\hline & & GN2 & -4.42 & 1.44 & 2.9 & 20.73 & -0.65 & 9.88 \\
\hline & & Autogenous & -2.71 & -1.69 & 4.9 & 20.34 & 0.87 & 4.87 \\
\hline & LOX & $\mathrm{GHe}$ & -7.56 & 1.59 & 6.64 & 30.14 & -0.19 & 16.75 \\
\hline & & Autogenous & -3.39 & -2.85 & 2.92 & 14.83 & -0.47 & 2.43 \\
\hline & LN2 & $\mathrm{GHe}$ & -7.7 & -23.47 & 9.43 & 34.56 & 1.23 & 16.07 \\
\hline & & Autogenous & -19.96 & -143.37 & 18.21 & 26.68 & 1.31 & 3.41 \\
\hline & LH2 & $\mathrm{GHe}$ & -4.06 & -72.17 & 6.47 & 11.15 & 0.34 & 5.55 \\
\hline & & Autogenous & -5.29 & -524.82 & 9.45 & 1.91 & 2.77 & -30.73 \\
\hline \multirow[t]{4}{*}{$450 \times 2750$} & LN2 & $\mathrm{GHe}$ & -3.77 & 1.36 & 2.3 & 11.82 & -0.52 & 8.38 \\
\hline & & Autogenous & -2.64 & -22.61 & 1.91 & 0.13 & 0.07 & -2.99 \\
\hline & $\mathrm{LH} 2$ & $\mathrm{GHe}$ & -2.56 & -25.84 & 3.23 & 14.44 & -0.06 & 8.54 \\
\hline & & Autogenous & -1.44 & -409.1 & 14.5 & 0.32 & 0.3 & -9.51 \\
\hline \multirow[t]{4}{*}{$510 \times 3600$} & LN2 & $\mathrm{GHe}$ & -11.05 & 0.51 & 2.67 & 20.19 & 0.03 & 16.99 \\
\hline & & Autogenous & -5.98 & -27.98 & 8.02 & 13.11 & 0.07 & 5.15 \\
\hline & $\mathrm{LH} 2$ & $\mathrm{GHe}$ & -5.24 & 6.18 & 4.09 & 20.91 & -0.06 & 17.92 \\
\hline & & Autogenous & -6.05 & -61.46 & 9.13 & 10.94 & 0.29 & 3.6 \\
\hline
\end{tabular}

Table 10.9 - Comparison of Performance between Old Room Temperature and New Cryogenic Bubble Point Pressure Model 
Equation 10.18 is the final modified bubble point pressure equation which predicts performance for both storable and cryogenic liquids. The equation is now a function of the seven parameters which affect bubble point pressure, which include liquid type, contact angle, screen mesh, liquid temperature, degree of subcooling, and pressurant gas type and temperature. Bubble point pressure is directly proportional to the liquid surface tension, implying there is always higher performance in storable over cryogenic liquids. Contact angle is shown to be zero for most storable and all cryogenic liquids in contact with SS LAD screens. In general, bubble point pressure increases with the fineness of the LAD screen. The second finest 450x2750 mesh has the highest bubble point pressure at both room temperature and cryogenic temperatures. The controlling parameter for minimizing effective pore diameter, and thus maximizing bubble point pressure, is the warp to shute diameter ratio. Curve fits to normally saturated bubble point data yield the combined effect of screen pore changes and type of pressurant gas on the bubble point pressure. Decreasing the temperature of the liquid in contact with the screen always increases bubble point due to increased surface tension and minor screen pore shrinkage. Higher bubble points are always obtained when the pressurant gas in contact with the screen is a non-condensable gas such as helium (due to enhanced evaporation) over the condensable vapor case (due to enhanced condensation). Subcooling the liquid/vapor interface at the LAD screen always increases margin in bubble point pressure. The rate of gain in performance is proportional to the level of subcooling, where the rate of gain depends on the gas in contact with the screen. Gain in margin due to subcooling increases as the saturation temperature decreases. Elevating the temperature of the pressurant gas in contact with the screen acts as a degradation factor, with higher 
rates of degradation with coarser meshes and using the vapor to pressurize. Degradation increases as the saturation temperature decreases. The new model has been constructed and validated based on over 45 years of experimental data and will remain an important design and analysis tool for predicting performance of screen channel LADs in both storable and cryogenic propulsion systems. 


\section{Chapter 11}

\section{The Reseal Point Pressure Model for Cryogenic}

\section{Propellants}

The purpose of this chapter is to derive and validate a predictive equation for the reseal pressure for liquid acquisition devices operating in cryogenic propulsion systems. Each controlled bubble point test allows for a controlled reseal pressure test. Liquid hydrogen, liquid nitrogen, liquid oxygen, and liquid methane reseal pressure data was thus collected alongside bubble point data from Chapters $5-8$, and the experimental results are presented here. Then the simplified room temperature reseal pressure model is extended to cryogenic temperatures through systematic examination of trends in the data. Much like the cryogenic bubble point pressure, the seven parameters which affect the reseal pressure inside a cryogenic propellant tank are the liquid type, contact angle, screen reseal diameter, liquid temperature and pressure, and pressurant gas type and temperature. Next the model components are derived in a similar fashion as the cryogenic bubble point derivation. Finally, each model component is validated by experimental data. The predictive equation derived in this chapter can be used to determine the reseal pressure of any LAD screen in both storable and cryogenic propellants. 


\subsection{Current Model Limitations}

The simplified reseal pressure model presented in Chapter 3 predicts reseal pressures well when reseal diameters are based on reference fluid tests (Method 1) or through summation of historical data (Method 2):

$D_{R}=\frac{4 \gamma_{r e f} \cos \theta_{R}}{\Delta P_{\mathrm{RS}, r e f}}$

If reseal diameter is known, the reseal pressure equation can theoretically be used to determine the reseal point of any fluid with a known surface tension. However, the same problem arises with cryogenic reseal data as with the cryogenic bubble point data. The room temperature prediction value matches neither the non-condensable or autogenous pressurant gas case. In addition, the room temperature model cannot be used to predict reseal pressures of subcooled cryogenic liquid states or elevated pressurant gases. Therefore, the new model must therefore address the following four discrepancies that exist between cryogenic reseal pressure data and simplified room temperature model:

5. The temperature dependence of the effective screen reseal diameter.

6. The effect of pressurant gas type.

7. The effect of subcooling the liquid.

8. The effect of elevating the temperature of the pressurant gas above the liquid temperature. 


\subsection{Summary of Data}

As mentioned in Section 3.9.2, there are only three previous studies where reseal data was reported. Reseal pressure data was collected alongside all bubble point test data from both room temperature as well as cryogenic bubble point tests for a 200x1400, 325x2300, 450x2750, and 510x3600 Dutch Twill LAD screen in IPA, methanol, acetone, water, $\mathrm{LH}_{2}, \mathrm{LN}_{2}, \mathrm{LOX}$, and $\mathrm{LCH}_{4}$. A total of 4768 reseal pressure data points were collected, processed, and analyzed to develop this model.

\subsection{Room Temperature Reseal Diameter Model}

The room temperature reseal pressure is directly proportional to the product of the surface tension and cosine of the receding contact angle. The surface tension model was derived in Section 3.2.4. The receding contact angle can be assumed to be zero for all cryogenic propulsion systems employing SS LAD screens. It can also be assumed that receding contact angle also does not depend on the screen mesh type. Therefore, for all cryogenic liquids and LAD screens, the room temperature reseal pressure equation simplifies to:

$$
\Delta P_{R S}=\frac{4 \gamma_{L V}}{D_{R}}
$$

The room temperature reseal diameters from Table 3.8 can be updated based on summation of room temperature data from Chapter 4 into the historical set of data. To determine the reseal diameter, the value was essentially back calculated from the plot of reseal pressure versus the product of surface tension times the contact of the receding 
contact angle for all fluids. Uncertainties for reseal diameters were estimated in the same way as effective pore diameters as outlined in Section 10.3.1.

Table 11.1 lists the room temperature reseal diameters for screens where data is available, along with calculated uncertainties for screens where more than a single data point was available. In general, the reseal diameter scales with the fineness of the screen in the same way that the pore diameter does; the second finest 450x2750 screen yields the smallest diameter over the finest $510 \times 3600$ screen. For all screens, the reseal diameter is larger than the pore diameter, as expected. Also shown in Table 11.1 is the ratio of room temperature reseal pressure divided by bubble point pressure. This ratio is plotted in Figure 11.1 as a function of the fineness of the screen in terms of the square root of the number pores per square inch of screen. As shown, the pressure ratio is quite constant across the whole range of LAD mesh types (Square, Plain Dutch, Twilled Square, Twilled Dutch), but does appear to be lower for coarser meshes and to level out for finer meshes. This implies that the hysteresis between breakthrough and reseal pressure levels out to a constant as the screen become finer. For most of the screens, the reseal pressure is roughly $90 \%$ of the breakthrough pressure at room temperature. Due to the paucity of reseal pressure data, room temperature reseal diameters based off the data are only available for 13 meshes, where 26 available meshes for the effective pore diameters. 


\begin{tabular}{|c|c|c|c|c|}
\hline Mesh & Dp293 $[\boldsymbol{\mu m}]$ & Dr288 $[\boldsymbol{\mu m}]$ & $\mathbf{U}[\boldsymbol{\mu m}]$ & $\boldsymbol{\Delta P}$ Ratio \\
\hline $\mathbf{5 1 0 \times 3 6 0 0}$ & 15.91 & 18.04 & $3.40 \mathrm{E}-02$ & 0.882 \\
\hline $\mathbf{4 5 0 \times 2 7 5 0}$ & 12.01 & 13.33 & $1.78 \mathrm{E}-02$ & 0.901 \\
\hline $\mathbf{3 2 5 \times 2 3 0 0}$ & 14.65 & 16.51 & $8.39 \mathrm{E}-02$ & 0.887 \\
\hline $\mathbf{2 0 0 \times 1 4 0 0}$ & 21.45 & 24.21 & $3.34 \mathrm{E}-01$ & 0.886 \\
\hline $\mathbf{2 5 0 \times 1 3 7 0}$ & 19.21 & 24.4 & - & 0.787 \\
\hline $\mathbf{2 0 0 \times 6 0 0}$ & 44.76 & 56.9 & - & 0.787 \\
\hline $\mathbf{8 0 \times 7 0 0}$ & 60.22 & 66.76 & - & 0.902 \\
\hline $\mathbf{5 0 \times 2 5 0}$ & 103.81 & 118.02 & - & 0.880 \\
\hline $\mathbf{3 0 \times 2 5 0}$ & 139.04 & 159.77 & - & 0.870 \\
\hline $\mathbf{2 4 \times 1 1 0}$ & 200.28 & 242.3 & - & 0.827 \\
\hline $\mathbf{3 2 5 \times 3 2 5}$ & 64.94 & 70.36 & - & 0.923 \\
\hline $\mathbf{1 2 0 \times 1 2 0}$ & 168.7 & 203.26 & - & 0.830 \\
\hline $\mathbf{2 3 0 \times 2 3 0}$ & 94.48 & 101.63 & - & 0.930 \\
\hline
\end{tabular}

Table 11.1 - Comparison of Breakthrough Pore Diameters with Reseal Diameters at Room Temperature

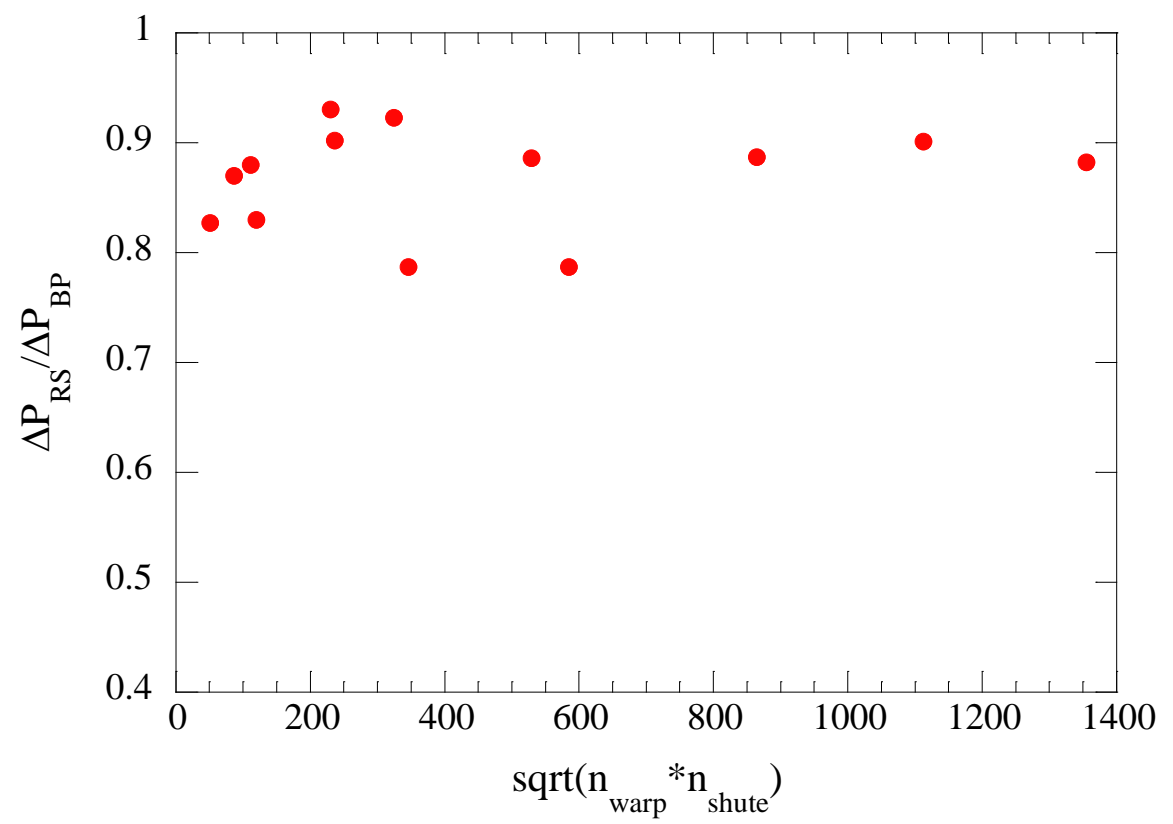

Figure 11.1 - Ratio of Room Temperature Reseal Pressure to Bubble Point Pressure versus the Fineness of the Screen

As with bubble point pressure, the controlling parameter for maximizing the reseal pressure is the warp to shute diameter ratio. Figure 11.2 plots effective reseal 
diameter against $X$ using $n_{s}$. Although there is more scatter in the reseal data, the trend clearly shows that as $X$ increases, $D_{\mathrm{R}, 288 K}$ decreases, for all LAD meshes. Fitting a least squares curve to the data reveals the inverse relationship between $X$ and $D_{R}$ :

$D_{\mathrm{R}, 288 K}=\frac{1463}{X_{s}^{0.669}}$

where

$X_{s}=n_{s}^{r_{d}}$

A similar relationship can be derived using the number of warp wires per square inch instead. Therefore both the pore and reseal diameters obey an inverse dependence on the shute to warp diameter ratio, and not the number of pores. Equation 11.3 can be used to determine the reseal pressure for any LAD screen through interpolation.

\subsection{Temperature Dependent Reseal Diameter Model}

Comparing reseal diameters in various normally saturated cryogenic liquid to reseal diameters at room temperature clearly indicates that the pore mouth changes with reduced temperature. LAD performance in cryogenic liquids is also highly dependent on the gas in contact with the screen. Note again that the effect of physical screen changes at reduced temperature and the effect of pressurant gas type are coupled together, since each reseal pressure data point in cryogenic liquid is attached to a specific pressurant gas. 


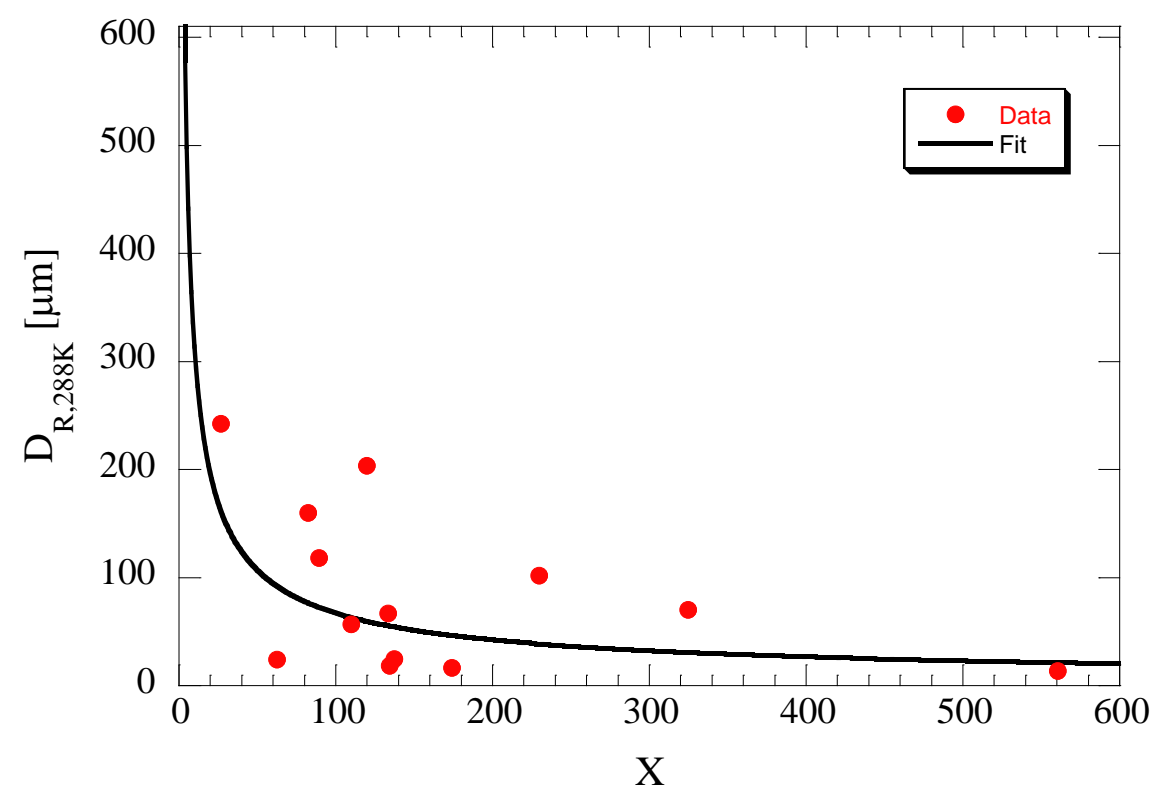

Figure 11.2 - Room Temperature Reseal Diameter as a Function of X. X is defined using the number of shute wires per inch.

Prior to the current work, designers always assumed that the pore and reseal diameters did not change with temperature. The temperature dependence of the pore and reseal diameters can be almost completely isolated by considering only data taken in saturated or near saturated states, such as those that lie along the black saturation curve in Figures 5.8, 5.9, 6.5, 6.6, and 7.6. Therefore Equation11.1 can be used to calculate reseal diameters at any temperature.

Figures $11.3 \mathrm{a}-\mathrm{c}$ plot calculated effective reseal diameters for all normally saturated cryogenic reseal pressure data as a function of temperature for a $325 \times 2300$, 450x2750, and 510x3600 LAD mesh, respectively. Data is grouped by pressurization scheme. All three figures are plotted on the same scale, for comparison. The data is clustered by the liquid saturation temperature of each fluid. Only $\mathrm{LH}_{2}$ and $\mathrm{LN}_{2}$ data is 
available for the 450x2750 and 510x3600 screens while data is available in $\mathrm{LH}_{2}, \mathrm{LN}_{2}$, LOX, and $\mathrm{LCH}_{4}$ for the $325 \times 2300$ mesh.
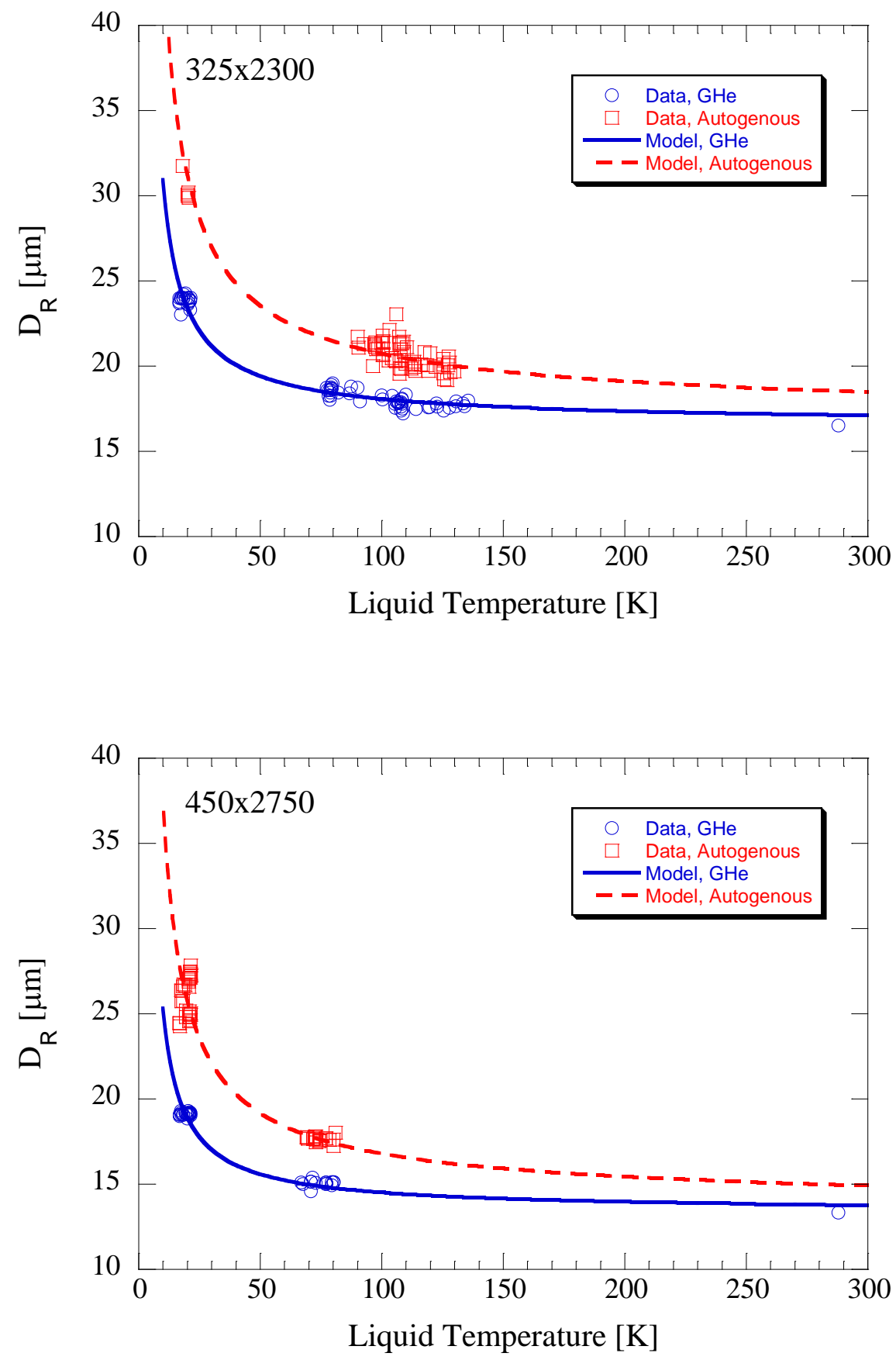


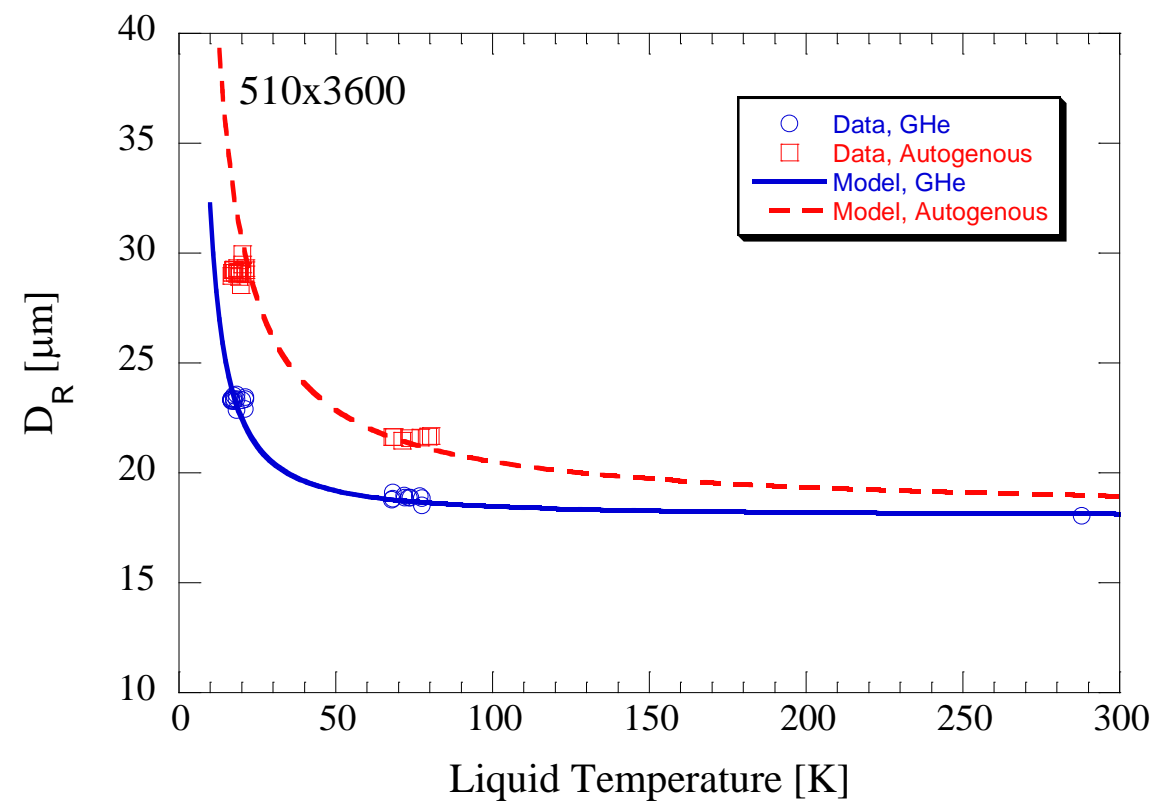

Figure 11.3 - Reseal Diameter as a Function of Temperature for a) $325 \times 2300$, b) 450x2750, and c) 510x3600 Dutch Twill Screen. Solid lines represent model generated curves.

As shown, there are several distinct trends evident in the data. First, for all three screens and both pressurization schemes, reseal diameter increases with decreasing temperature, which indicates reseal pressure reduces with reduced temperature. Bubble point pressure is shown to increase over the room temperature value with noncondensable pressurant and decrease over room temperature value with decreasing temperature. Second, for all three meshes, pressurization with the non-condensable yields higher performance than pressurization with the condensable vapor. This trend is also clearly evident in bubble point pressure. Third, for both pressurization schemes, the middle 450x2750 mesh outperforms the finer 510x3600 mesh, which outperforms the coarsest $325 \times 2300$ mesh. This crossover in performance is attributed to the warp to shute diameter ratio, and the effect of geometry of the L/V interface on the warp and shute 
diameters. The geometry of the pore throat and mouth of the 510x3600 screen change significantly more than the other meshes. The 450x2750 mesh yields the maximum reseal pressure at both room temperature and at cryogenic liquid temperatures.

For clarity and comparison, Table 11.2 plots the ratio of the reseal pressure at $\mathrm{LH}_{2}$ temperatures to the reseal pressure at room temperature for the three meshes and two pressurization schemes. For example, the reseal pressure reduces by $30 \%$ of the room temperature value for the 325 and 450 meshes, but only reduces to $80 \%$ of the value for the 510 mesh using the non-condensable gas. Meanwhile there is a higher degradation in performance using the autogenous pressurization scheme.

\begin{tabular}{|c|ccc|}
\cline { 2 - 4 } \multicolumn{1}{c|}{} & $\mathbf{3 2 5 \times 2 3 0 0}$ & $\mathbf{4 5 0 \times 2 7 5 0}$ & $\mathbf{5 1 0 \times 3 6 0 0}$ \\
\hline Helium & 0.704 & 0.705 & 0.802 \\
\hline Autogenous & 0.528 & 0.517 & 0.588 \\
\hline
\end{tabular}

Table 11.2 - Ratio of Reseal Pressure at 20.3K to Reseal Pressure at Room Temperature

Thus the simplified room temperature reseal pressure model can be modified to incorporate screen pore mouth changes and effects due to pressurant gas type by simply allowing the effective reseal diameter to vary with temperature:

$$
\Delta P_{R S}(T)=\frac{4 \gamma_{L V} \cos \theta_{R}}{D_{R}(T)}
$$

After attempting numerous nonlinear least squares curve fits to the data, the most appropriate function that tracks the temperature dependence of the reseal diameter is:

$$
D_{R}(T)=D_{R, 288 K} \exp \left(\frac{b_{1}}{T^{b_{2}}}\right)
$$


where $D_{R, 288 K}$ is the room temperature reseal diameter and $b_{1}$ and $b_{2}$ are fit to the data.

Table 11.3 lists the fitting parameters and errors between curve and data for all screens where cryogenic reseal data is available. The table is organized by pressurization scheme again, which means autogenous pressurization curve parameters can be fit to $\mathrm{GH}_{2} / \mathrm{LH}_{2}, \mathrm{GOX} / \mathrm{LOX}$ data, etc. Model generated curves are plotted in Figures $11.3 \mathrm{a}-\mathrm{c}$ to compare against the data. As shown, the temperature dependent function matches the data well; the exponential function approaches 1 as $T$ approaches room temperature and approaches infinity as $T$ approaches 0 . Physically, this is consistent with the data and with observed behavior in bubble point/reseal tests. Due to hysteresis between bubble point and reseal points, it is increasingly more difficult to achieve screen reseal with a liquid with low surface tension like $\mathrm{LH}_{2}$ than it is with a higher surface tension liquid such as LOX. $b_{2}$ essentially governs how quickly reseal pressure decays from the room temperature value to account for differences in pressurization scheme.

\begin{tabular}{|c|c|c|c|c|c|}
\hline Mesh & Metal & Pressurization Method & b1 & b2 & $\mathbf{r}[\boldsymbol{\mu m}]$ \\
\hline $\mathbf{5 1 0} \times \mathbf{3 6 0 0}$ & SS & GHe & 14.369 & 1.394 & 0.618 \\
\hline $\mathbf{5 1 0} \times \mathbf{3 6 0 0}$ & SS & Autogenous & 7.477 & 0.883 & 0.296 \\
\hline $\mathbf{4 5 0} \times \mathbf{2 7 5 0}$ & SS & GHe & 4.850 & 0.878 & 0.235 \\
\hline $\mathbf{4 5 0} \times \mathbf{2 7 5 0}$ & SS & Autogenous & 4.651 & 0.652 & 1.25 \\
\hline $\mathbf{3 2 5} \times \mathbf{2 3 0 0}$ & SS & GHe & 4.374 & 0.842 & 0.788 \\
\hline $\mathbf{3 2 5} \times \mathbf{2 3 0 0}$ & SS & GN2 & 8.090 & 1.075 & 0.00538 \\
\hline $\mathbf{3 2 5} \times \mathbf{2 3 0 0}$ & SS & Autogenous & 4.337 & 0.640 & 3.34 \\
\hline $\mathbf{2 0 0} \times \mathbf{1 4 0 0}$ & SS & GHe & 12.396 & 1.156 & 1.748 \\
\hline
\end{tabular}

Table 11.3 - Fitting Parameters for Temperature Dependent Reseal Diameter Model

Decreasing the temperature of the system increases surface tension but also increases the size of the effective reseal diameter. Differences in performance between screens is attributed to how the shrinkage of the warp and shute wires changes the actual 
$\mathrm{L} / \mathrm{V}$ interface within the screen, and is encompassed within the warp to shute diameter ratio. Differences in performance between pressurization schemes is again attributed to added heating and cooling of the L/V interface due to enhanced condensation and/or evaporation. Model generated curves are plotted in Figure 11.3 for the 325x2300, 450x2750, and 510x3600 screens.

To compare relative differences in performance between the two pressurization schemes as in the pore diameter case in Chapter 10, Figure 11.4 plots the model generated reseal pressure using GHe divided by the reseal pressure obtained using the vapor as a function of reduced temperature for a $325 \times 2300$ screen over the range of temperatures of experimental data. Comparing the curves reveals the same noticeable trend; as the saturation temperature of the liquid decreases, the relative difference in performance between the two pressurization schemes increases. At $\mathrm{LCH}_{4}$ temperatures there is a difference of $10-15 \%$ between $\mathrm{GHe}$ and $\mathrm{GCH}_{4}$ whereas at $\mathrm{LH}_{2}$ temperatures, there is a difference of over $30 \%$ between $\mathrm{GHe}$ and $\mathrm{GH}_{2}$. Therefore, adding helium gas at the interface has an increasingly positive effect on enhanced evaporative cooling at the interface as the saturation temperature decreases, with the biggest gain at $\mathrm{LH}_{2}$ temperatures. While the same general trend is evident in bubble point data, the trend is more pronounced in the reseal data. This may be attributed to the higher sensitivity in smaller $\mathrm{LH}_{2}$ reseal pressure values over the $\mathrm{LH}_{2}$ bubble point pressures. 


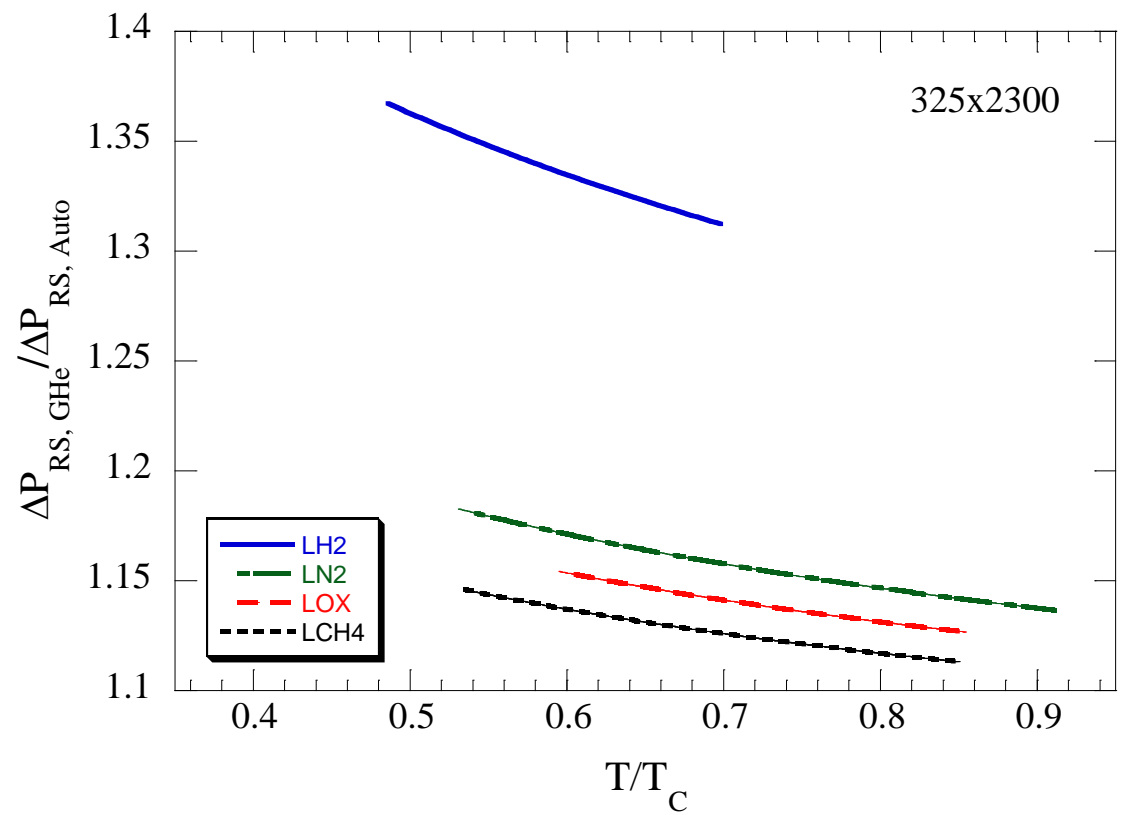

Figure 11.4 - Model Generated 325x2300 Reseal Pressure Ratio as a Function of the Reduced Temperature for Normally Saturated Liquid Taken Over the Range of Conditions of the Data

Explanation for the deviation in performance between the two schemes is attributed to the relative effect of GHe diffusing to the L/V interface, blocking condensation mass transfer, and enhancing evaporative cooling, where the Schrage model can be used to estimate condensation rates in the pure vapor case and kinetic theory can be used to estimate evaporation rates for the pure non-condensable case. While the absolute magnitude of the $\mathrm{GCH}_{4} / \mathrm{LCH}_{4}$ condensation rate is larger than the $\mathrm{GH}_{2} / \mathrm{LH}_{2}$ rate, the evaporation rate in $\mathrm{GH}_{2} / \mathrm{LH}_{2}$ is much larger than $\mathrm{GCH}_{4} / \mathrm{LCH}_{4}$. This would cause greater relative differences between the two rates in $\mathrm{GH}_{2} / \mathrm{LH}_{2}$ than in $\mathrm{GCH}_{4} / \mathrm{LCH}_{4}$, greater differences in interfacial temperature, and thus greater difference in reseal pressure between the two pressurization schemes in $\mathrm{LH}_{2}$ than in $\mathrm{LCH}_{4}$. 


\subsection{Liquid Subcooling Model}

The potential gain in performance in reseal pressure due to subcooling the liquid is as important as the gain in performance in bubble point pressure. Higher bubble points and higher reseal pressures equate to more margin in system design. An appropriate subcooling model can be used to determine the bubble point and reseal pressures through simple knowledge of the thermodynamic state of the liquid inside the propellant tank. Analysis of the data indicates that the gain in performance is linear with the level of subcooling and thus distance away from the saturation curve on a temperature/pressure plot as was the bubble point pressure. Therefore Equation 11.5 takes the form:

$$
\Delta P_{R S, S u b}=\Delta P_{R S, S a t}\left(1+n_{S u b}\left(\frac{P-P_{S a t}}{P_{C}}+\frac{T_{S a t}-T}{T_{C}}\right)\right)
$$

where $\Delta P_{R S, S a t}$ is the reseal pressure in the saturated liquid state from Equation 11.5. Pressures should be in units of $\mathrm{kPa}$ and temperatures taken in units of degrees Kelvin. As the thermodynamic state of the liquid at the LAD screen approaches a saturation state, Equation 11.7 collapses into Equation 11.5. The subcooled gain is thus defined as the ratio of reseal pressures:

$$
\frac{\Delta P_{R S, S u b}}{\Delta P_{R S, S a t}}=1+n_{S u b}\left(\frac{P-P_{S a t}}{P_{C}}+\frac{T_{S a t}-T}{T_{C}}\right)
$$

For each screen/pressurant gas/liquid triplet, $n_{S u b}$ was determined through linear least squares fitting of the pressure ratio in Equation 11.8 versus the term in parenthesis. As in the subcooled gain in bubble point pressure, analysis of the data indicates that the subcooled gain in reseal pressure does not depend on the mesh type either. Rather, it is 
purely dependent on the pressurant gas in contact with the liquid. Additionally, the fitting parameters for the subcooled reseal model, and thus gain due to subcooling, are identical to the fitting parameters in the subcooled bubble point model.

Table 11.4 lists subcooled fitting parameters where subcooled reseal pressure data is available. Following the previous method with bubble point pressure gain, to compare between liquids, Figures $11.5 \mathrm{a}$ and $\mathrm{b}$ plot model generated subcooled gains for reseal pressures for a $325 \times 2300$ screen using non-condensable and autogenous pressurization schemes, respectively, as a function of the pressure difference between the liquid pressure at the screen and the NBP. The curves terminate at the critical pressure. Therefore the curves are generated assuming a constant liquid temperature equal to the NBP of the liquid, which allows comparison of subcooled gain as a function of a fixed delta $\mathrm{P}$ between the liquids.

\begin{tabular}{|c|c|c|c|c|c|c|c|}
\cline { 2 - 9 } \multicolumn{1}{c|}{} & Tc [T] & Pc [kPa] & GHe & GCH4 & GOX & GN2 & GH2 \\
\hline LCH4 & 190.6 & 4606 & 0.453 & 0.388 & $x$ & 0.404 & $x$ \\
\hline LOX & 154.6 & 5043 & 0.528 & $x$ & 0.321 & $x$ & $x$ \\
\hline LN2 & 126.2 & 3396 & 0.312 & $x$ & $x$ & 0.211 & $x$ \\
\hline LH2 & 32.94 & 1286 & 0.258 & $x$ & $x$ & $x$ & 0.143 \\
\hline
\end{tabular}

Table 11.4 - Coefficient Matrix for Subcooled Liquid Reseal Pressure Model 

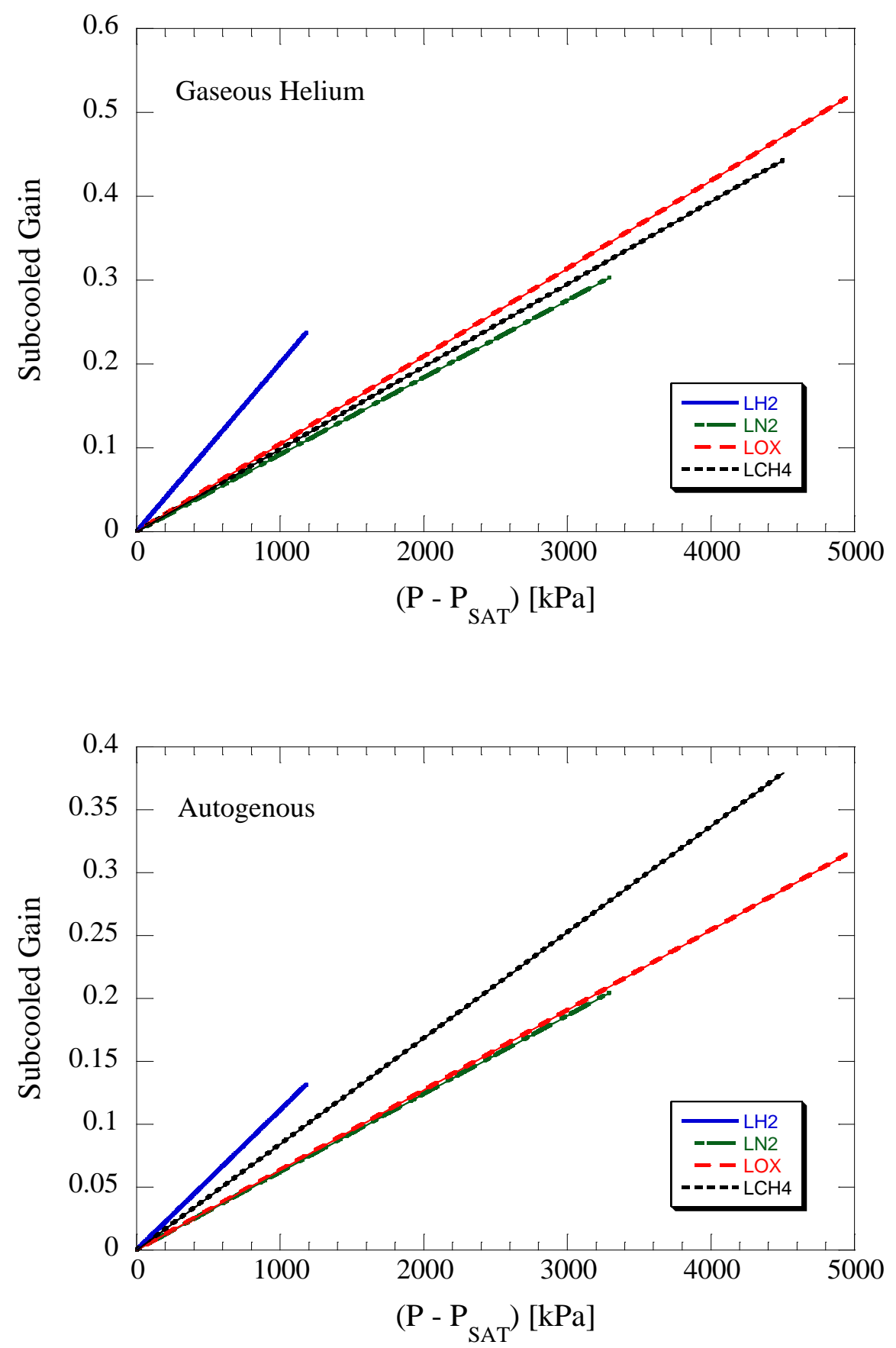

Figure 11.5 - Model Generated Subcooled Gain Curves for a 325x2300 Screen using a) Gaseous Helium and b) Autogenous Pressurization Schemes as a Function of Pressure Difference Above the Saturation Pressure at the Normal Boiling Point. Curves are generated using Equation 11.8 with a liquid temperature equal to the saturation temperature. 
Model trends here are identical to model trends in subcooled gain for bubble point pressure. There is a definitive trend in Figure 11.5a. Over a fixed pressure range, the highest subcooled gain is achieved in $\mathrm{GHe} / \mathrm{LH}_{2}$. The subcooled gain generally scales up with decreasing saturation temperature. For the autogenous pressurization case, there is no definitive trend, but the highest gain is achieved at the coldest saturation temperature. Comparing the two figures, it is clear that there is significantly higher gain in reseal pressure when the screen is pressurized and subcooled with the non-condensable gas. Subcooled gain is achieved in either pressurization case, but the magnitude of the gain is determined by the gas in contact with the screen.

Figure 11.6 plots subcooled gain reseal pressure data against the model generated subcooled data using Equation 11.7 as a function of the liquid temperature and pressure at the LAD screen for a 200x 1400 screen in LOX using GHe as a pressurant; Figure 11.7 plots this comparison for a $325 \times 2300$ screen. As shown, subcooled gain does not depend on the screen, but only the pressurant gas in contact with the liquid. Figures 11.8 and 11.9 plot $325 \times 2300 \mathrm{LN}_{2}$ reseal pressure data against model using $\mathrm{GHe}$ and $\mathrm{GN}_{2}$ to pressurize, respectively. The reseal model tracks the data well. As shown, both decreasing temperature below saturation and increasing pressure above saturation increases reseal pressure as indicated by the darkening in color. The subcooled gain in both bubble point and reseal pressures has implications for mission design, because a designer can simply "dial in" the value at a given thermodynamic state of the liquid in the tank using the models. Different subcooled gains with different pressurant gases are attributed to the effect of evaporative cooling at the LAD screen relative to the pure vapor case where condensation dominates. In either case, subcooling the liquid enhances performance. 

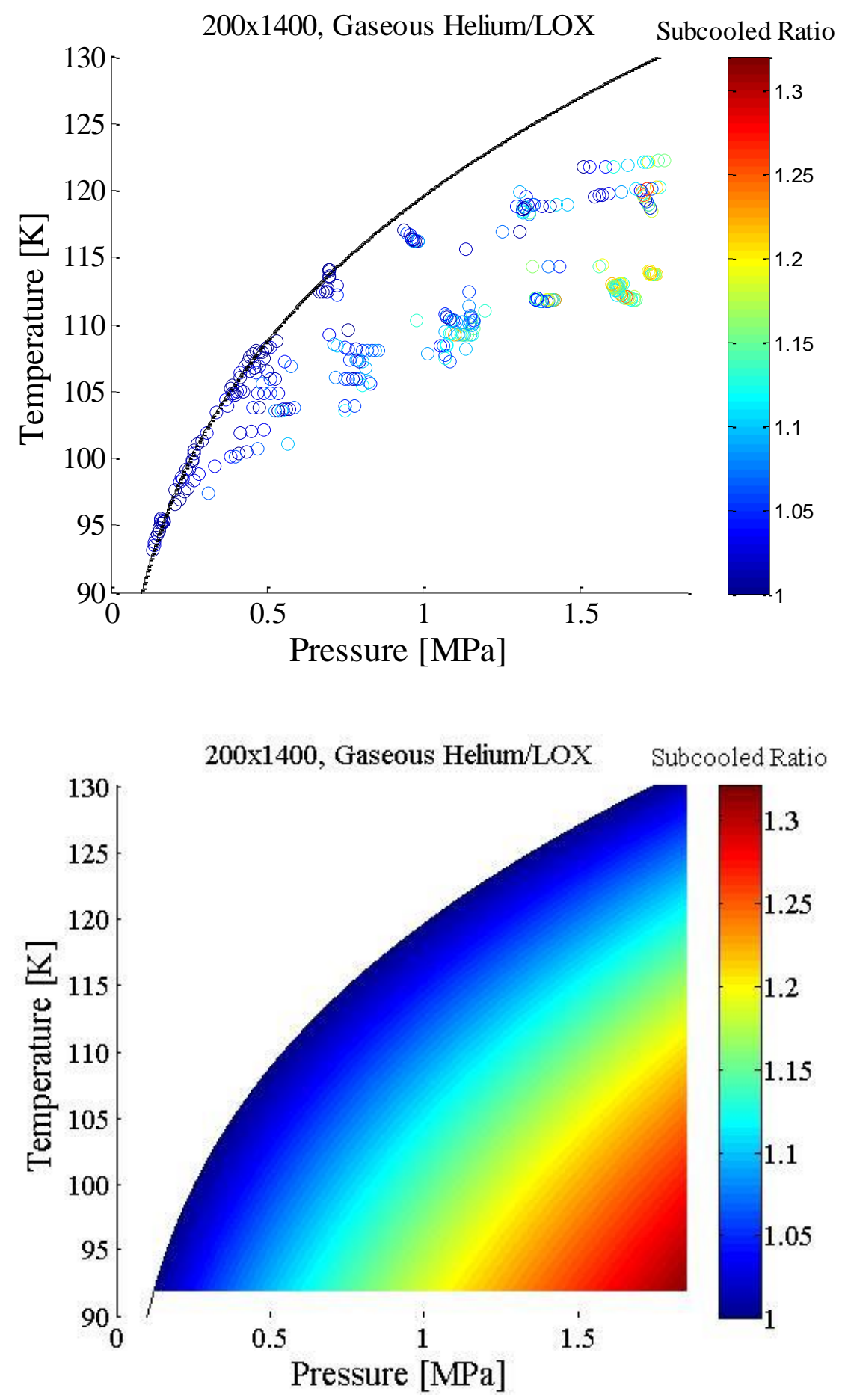

Figure 11.6 - a) Data and b) Model Generated Subcooled Gain as a Function of the Liquid Temperature and Pressure at the Screen for a 200x1400 Mesh in Liquid Oxygen using Gaseous Helium as a Pressurant. The black line is the oxygen saturation curve. Color indicates magnitude of subcooled gain. 

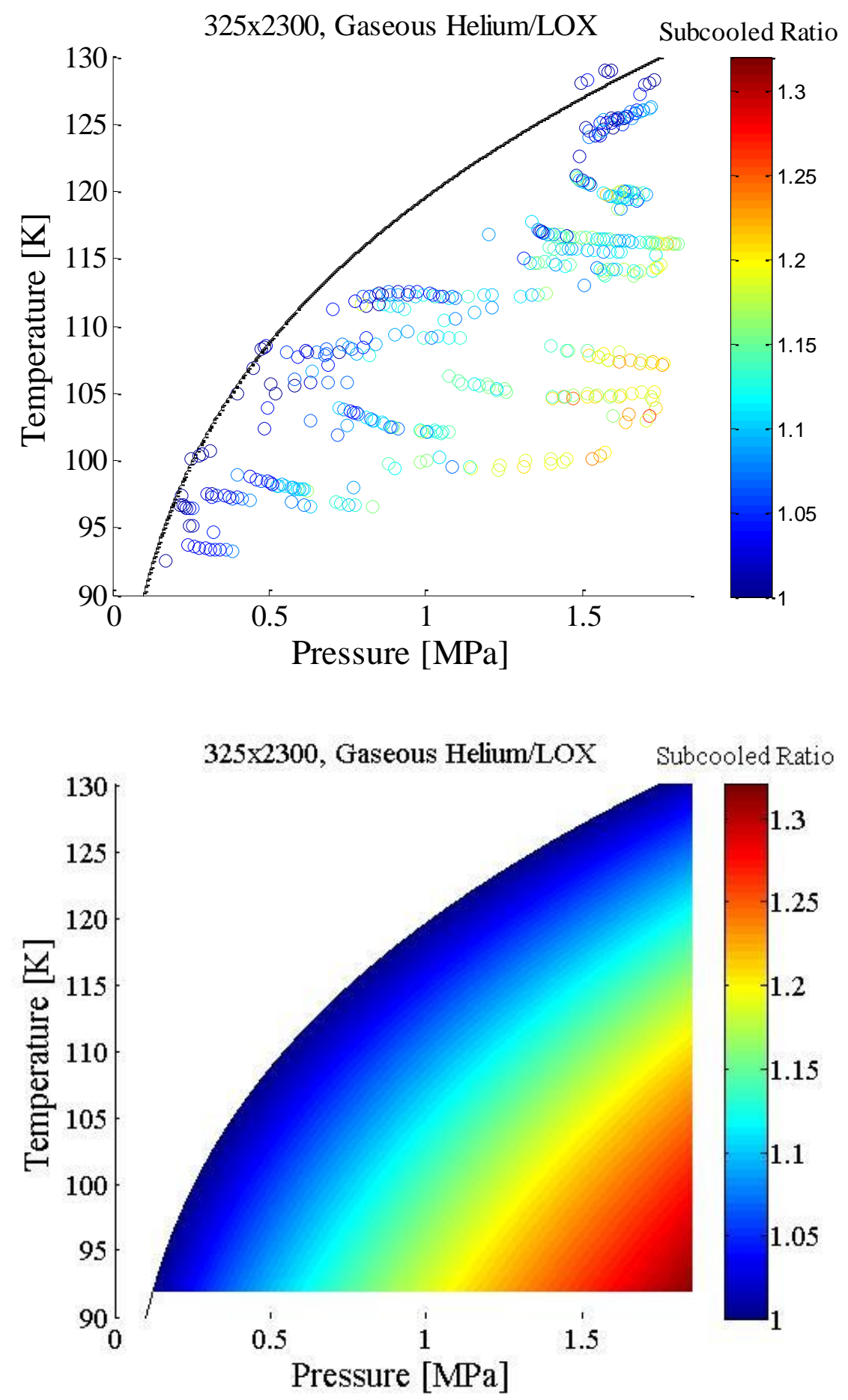

Figure 11.7 - a) Data and b) Model Generated Subcooled Gain as a Function of the Liquid Temperature and Pressure at the LAD Screen for a 325x2300 Mesh in Liquid Oxygen using Gaseous Helium as a Pressurant. The black line is the oxygen saturation curve. Color indicates magnitude of subcooled gain. 

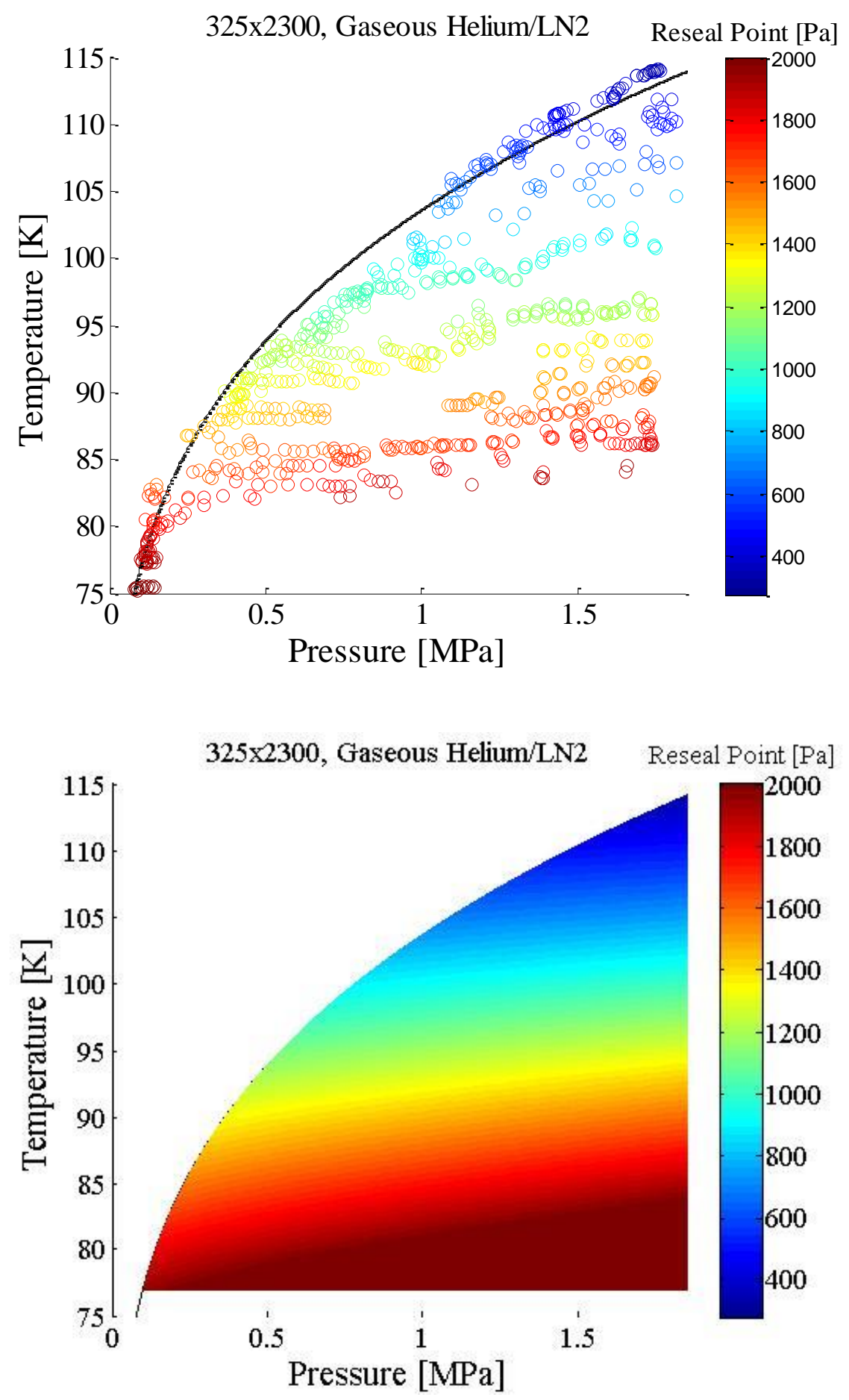

Figure 11.8 - a) Data and b) Model Generated Bubble Point as a Function of the Liquid Temperature and Pressure at the LAD Screen for a 325x2300 Mesh in Liquid Nitrogen using Gaseous Helium as a Pressurant. The black line is the nitrogen saturation curve. Color indicates magnitude of the reseal point. 

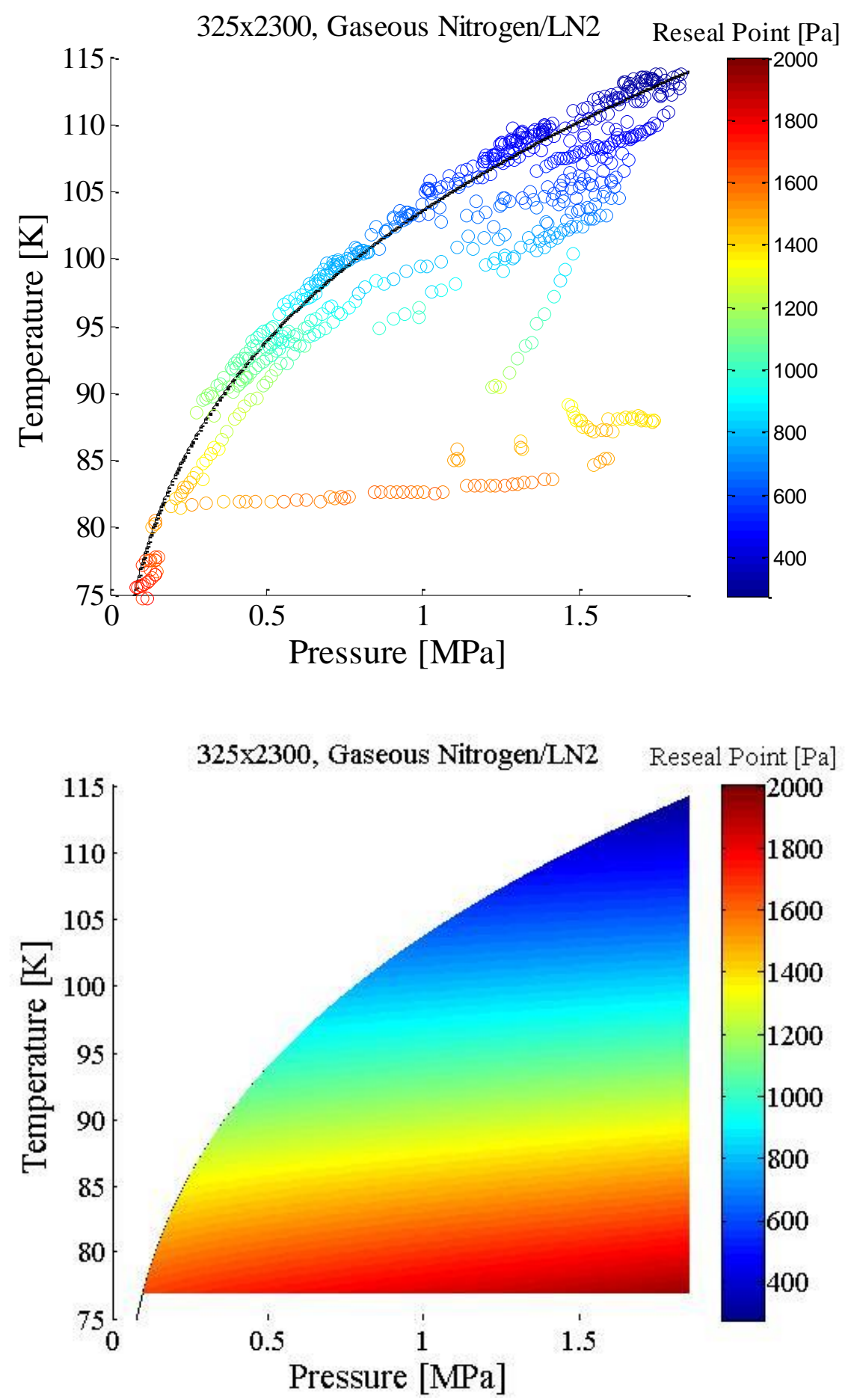

Figure 11.9 - a) Data and b) Model Generated Bubble Point as a Function of the Liquid Temperature and Pressure at the LAD Screen for a 325x2300 Mesh in Liquid Nitrogen using Gaseous Nitrogen as a Pressurant. The black line is the nitrogen saturation curve. Color indicates magnitude of the reseal point. 


\subsection{Warm Pressurant Gas Model}

Analysis of the heated pressurant gas reseal data for a $325 \times 2300,450 \times 2750$, and 510x3600 screen using both pressurization schemes in $\mathrm{LH}_{2}$ and $\mathrm{LN}_{2}$ shows that elevating the gas temperature acts as a degradation factor, proportional to the temperature difference between liquid and gas. The same general trends in heated pressurant gas bubble point data are seen in heated pressurant gas reseal data. Therefore, the reseal pressure model can be modified to account for degradation in reseal pressure due to elevating the temperature of the pressurant gas:

$$
\Delta P_{R S, H o t}=\Delta P_{R S, S a t}\left[1-n_{H o t}\left(T_{G a s}-T\right)\right]
$$

where $\Delta P_{R S, S a t}$ is the reseal pressure in the saturated liquid state from Equation 11.5. All temperatures are in degrees Kelvin. As the thermodynamic state of the liquid at the LAD screen approaches a saturation state, Equation 11.9 collapses into Equation 11.5. The heated gas loss is thus defined as the ratio of reseal pressures:

$$
\frac{\Delta P_{R S, H o t}}{\Delta P_{R S, S a t}}=1-n_{H o t}\left(T_{G a s}-T\right)
$$

Therefore Equation 11.10 is a ratio of reseal pressure at an elevated pressurant gas temperature to the reseal pressure taken when the gas temperature is approximately equal to the liquid temperature. Model coefficients were determined in the same way as the bubble point heated gas coefficients. It is determined that the fitting parameters for heated gas reseal pressures were identical to the parameters in the heated gas bubble point pressures, implying that for a given screen/pressurant gas/liquid triple, the rate of 
degradation in the bubble point pressure is approximately equal to the rate of degradation in the reseal pressure. Heated gas fitting parameters are shown in Table 11.5, for the sake of completeness. Since the trends are identical as the bubble point trends, Figure 10.15 can be used to illustrate relative performance between the three meshes, two liquids, and two pressurization schemes.

\begin{tabular}{|c|c|c|c|c|c|}
\hline Mesh & Liquid & Gas & nhot [1/K] & TOnset [K] & $r$ \\
\hline $510 \times 3600$ & Hydrogen & $\mathrm{GHe}$ & 0.0018 & 0 & 0.000783 \\
\hline $450 \times 2750$ & Hydrogen & $\mathrm{GHe}$ & 0.003 & 0 & 0.000223 \\
\hline $325 \times 2300$ & Hydrogen & $\mathrm{GHe}$ & 0.0073 & 0 & 0.00035 \\
\hline $510 \times 3600$ & Hydrogen & $\mathrm{GH} 2$ & 0.0086 & 0 & 0.001757 \\
\hline $450 \times 2750$ & Hydrogen & $\mathrm{GH} 2$ & 0.0133 & 0 & 0.002666 \\
\hline $325 \times 2300$ & Hydrogen & $\mathrm{GH} 2$ & 0.0286 & 0 & 0.000805 \\
\hline $510 \times 3600$ & Nitrogen & $\mathrm{GHe}$ & 0.00156 & 77 & 0.001252 \\
\hline $450 \times 2750$ & Nitrogen & $\mathrm{GHe}$ & 0.00149 & 41 & 0.000298 \\
\hline $325 \times 2300$ & Nitrogen & $\mathrm{GHe}$ & 0.0028 & 0 & 0.001193 \\
\hline $510 \times 3600$ & Nitrogen & GN2 & 0.002 & 21 & 0.000746 \\
\hline $450 \times 2750$ & Nitrogen & GN2 & 0.0031 & 0 & 0.000171 \\
\hline $325 \times 2300$ & Nitrogen & GN2 & 0.0053 & 0 & 0.001784 \\
\hline
\end{tabular}

Table 11.5 - Coefficient Matrix for Heated Pressurant Gas Reseal Model

Differences in the rate of degradation between the three different screens are due to differences in porosity and screen thickness. Differences in the rate of degradation between different pressurant gases are due to modification of the interfacial temperature due to condensation and/or evaporation at the LAD L/V interface. Differences in the rate of degradation between different liquids are due to differences in the superheats required to initiate boiling at the liquid side of the screen.

\subsection{Concluding Remarks}

The reseal pressure is an important performance parameter for porous screen channel liquid acquisition devices that defines the point at which a LAD screen can 
essentially reseal itself to pressurant gas ingestion. Knowledge of the reseal pressure as a function of thermodynamic conditions within a propellant tank will allow mission designers and operators to achieve higher expulsion efficiencies by draining the tank past the point of initial screen breakdown. The simplified room temperature reseal pressure model has been modified to account for effects due to screen pore mouth changes at reduced temperatures, relative differences in performance due to which type of gas is in contact with the screen, gain in performance due to subcooling the liquid, as well as degradation in performance due to warm pressurant gas.

The full reseal pressure point model takes the following final form:

$\Delta P_{R S}=\frac{4 \gamma_{L V} \cos \theta_{C}}{D_{R}(T)}\left(1+n_{\text {Sub }}\left(\frac{P-P_{S a t}}{P_{C}}+\frac{T_{S a t}-T}{T_{C}}\right)\right)\left(1-n_{\text {Hot }}\left(T_{\text {Gas }}-T\right)\right)$

where $\gamma_{L V}$ is determined from Equation 3.19 and the temperature dependent reseal diameter $D_{R}(T)$ is determined from Equation 11.6 using Tables 10.4 and 11.3. The reseal pore diameter can be determined at room temperature through simple reseal pressure tests while the temperature dependent reseal diameter can be determined through cryogenic reseal pressure tests where the liquid is near the saturation condition. As in the cryogenic bubble point pressure model, $n_{S u b}$ is dependent only the pressurant gas/liquid cryogen pair, while $n_{H o t}$ is dependent on the screen/gas/liquid triple. Statistical analysis was performed on the least squared difference between model generated reseal pressure and actual experimental reseal pressure, and this difference is on average no greater than $4 \%$ across the entire range of LAD meshes, liquids, and thermodynamic conditions of all data available in the literature. 
Equation 11.11 is the reseal pressure equation which can be used to predict performance for both storable and cryogenic liquids for any LAD mesh. The equation is a function of the seven parameters which govern LAD performance, including screen mesh, propellant type, contact angle, liquid temperature and pressure (degree of subcooling), and pressurant gas type and temperature. The reseal model trends are:

1. Reseal pressure scales inversely with the fineness of the mesh in general, however the second finest $450 \times 2750$ mesh outperforms the finest 510x3600 mesh. The controlling factor for maximum reseal pressure is the warp to shute diameter ratio.

2. Reseal pressure is directly proportional to the surface tension of the liquid.

3. Contact angle is zero for most storable and all cryogenic propellants.

4. Reducing liquid temperature always increases reseal pressure.

5. Subcooling the liquid at the LAD screen always improves margin in reseal pressure proportional to the level of subcooling. The rate of gain depends on the gas in contact with the liquid propellant. Across the range of liquids, gain in subcooled margin increases as the saturation temperature decreases because more margin is obtained in $\mathrm{LH}_{2}$ than $\mathrm{LCH}_{4}$ when subcooling over the same amount. This is attributed to a higher relative enhancement due to evaporative cooling.

6. Higher performance is always obtained using a non-condensable pressurant gas such as helium versus pressurizing with a condensable vapor.

7. Elevating the temperature of the pressurant gas always degrades reseal pressure, with the highest rates of degradation with coarser meshes using vapor as the 
pressurant gas. Across the range of liquids, degradation in reseal pressure increases as the saturation temperature decreases.

Except for differences between the temperature dependent pore throat and pore mouth, the equation governing reseal pressure is identical to the equation governing bubble point. This is illustrated in Figure 11.10, where the 450x2750 bubble point pressure and reseal pressures are plotted in $\mathrm{LH}_{2}$ using $\mathrm{GHe}$ as a pressurant from the triple line all the way up to the critical point. The relative trends are the same between bubble point and reseal for a fixed screen, liquid, and pressurant gas, but the absolute magnitude is different.

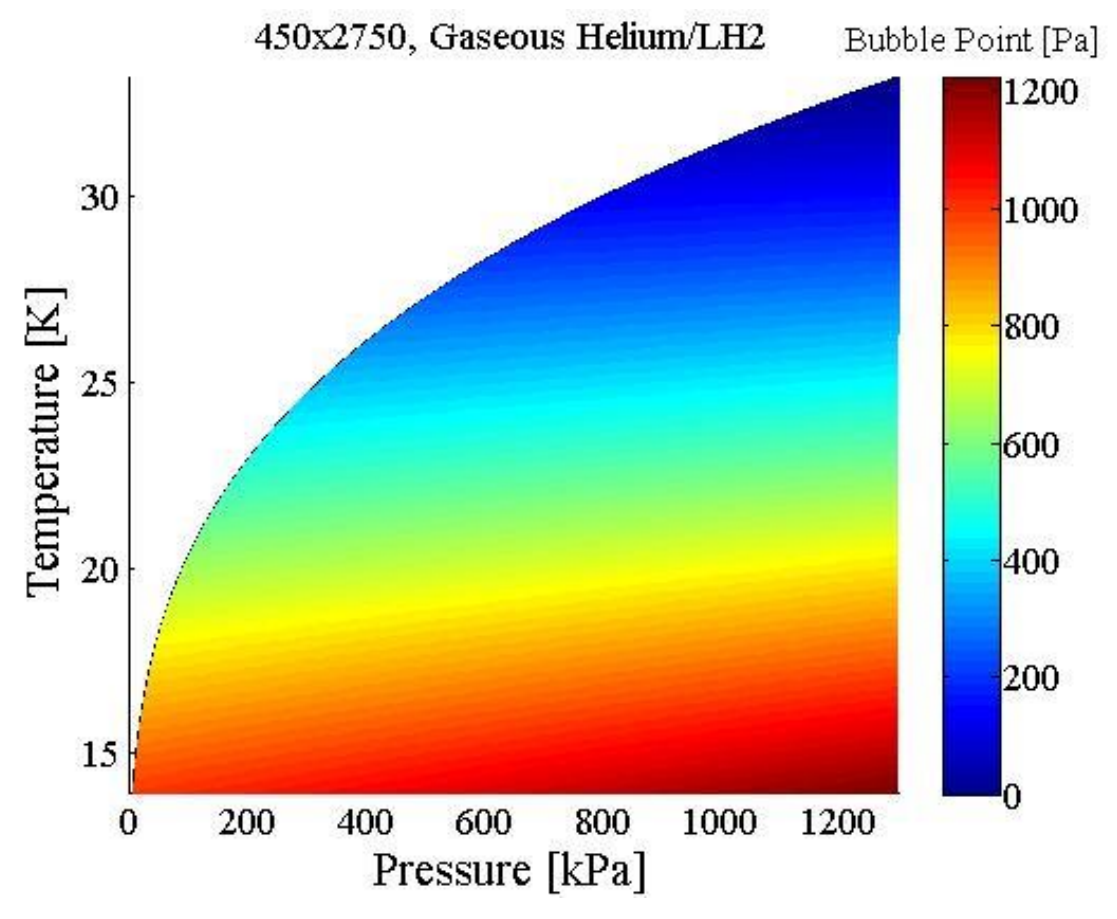




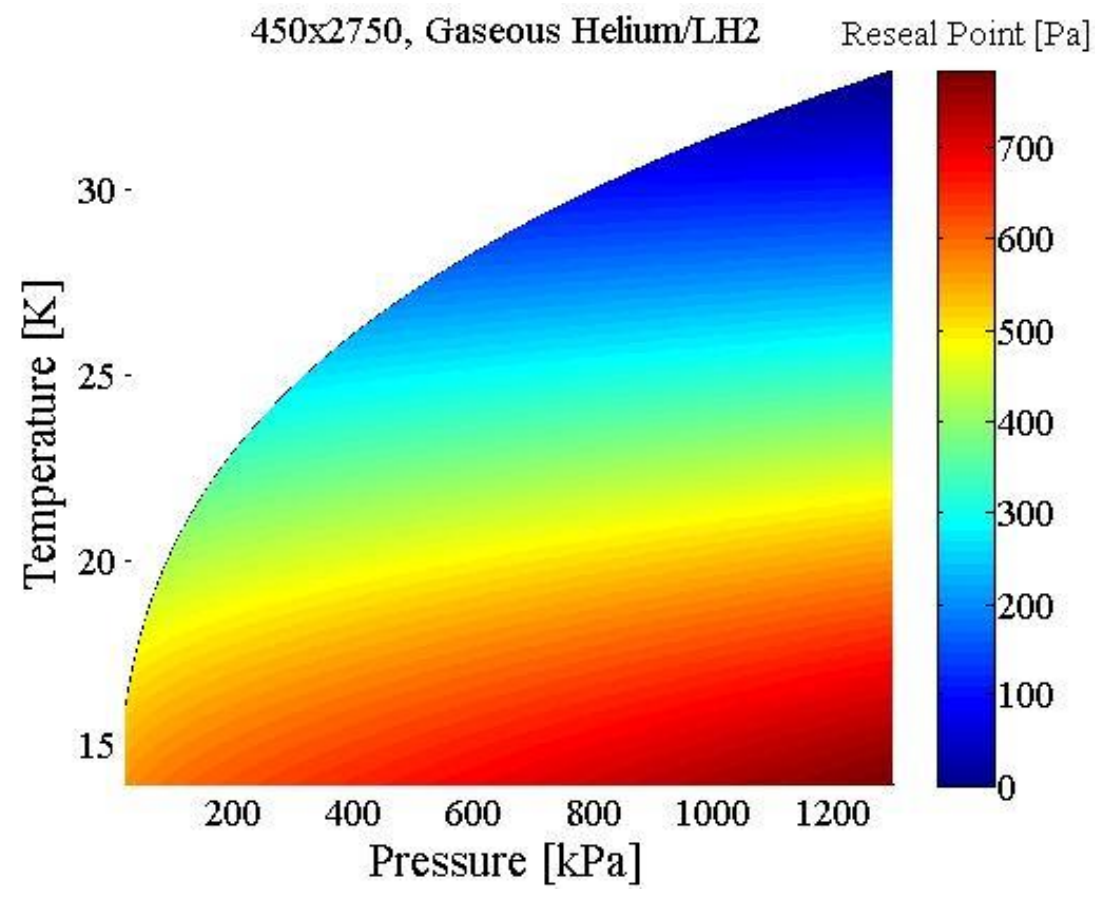

Figure 11.10 - Model Generated 450x2750 Liquid Hydrogen a) Bubble Point Pressure and b) Reseal Pressure using Gaseous Helium as a Pressurant. Color indicates magnitude of bubble point and reseal pressure, respectively. 


\section{Chapter 12}

\section{Analytical Model for Steady Flow through a \\ Porous Liquid Acquisition Device Channel}

The purpose of this chapter is to present an analytical solution of flow through a finite length channel with one porous wall. Of particular interest is the application of the analytical solution to model behavior of cryogenic propellant flow through a porous liquid acquisition device screen and channel in a 1-g environment. The purpose of the chapter is thus to derive from first principles a solution to cryogenic flow through the LAD and validate the model through recent cryogenic liquid outflow tests.

First a summary of the literature is given of previously reported analytical solutions pertinent to porous channel flow with one porous wall. Then the governing equations, boundary conditions, and model assumptions are used to derive the analytical flow solution. Next, the general results of the solution are summarized and compared to available experimental cryogenic oxygen and hydrogen LADs data. The model is first used to predict velocity and pressure fields across the screen and inside a LAD channel in liquid oxygen. Then the model is used to simulate propellant tank drain through the LAD channel in liquid hydrogen in a 1-g inverted outflow configuration. Finally, the static bubble point model is updated with a dynamic bubble point term, which factors in 
enhanced convection and cooling at the screen during propellant outflow. Convective heat transfer at the LAD screen during outflow is also quantified by comparing model and data.

\subsection{One Dimensional Pressure Drop Model Drawbacks}

An understanding of propellant flow through a porous LAD channel is required for designing in-space cryogenic engines as well as LEO cryogenic fuel depots that will enable all future deep space missions. In an unsettled environment in microgravity, where the location of the L/V interface may be unknown, a passive LAD is the easiest and most robust and reliable method to remove vapor free liquid from a propellant tank in both inspace engine and depot applications, therefore necessitating the fundamental understanding of velocity and pressure profiles within the LAD.

The total pressure drop in the LAD system was originally broken down into a sum of individual terms in Chapter 3:

$$
\Delta P_{\text {total }}=\Delta P_{\text {hydrostatic }}+\Delta P_{F T S}+\Delta P_{\text {frictional }}+\Delta P_{\text {dynamic }}
$$

where each pressure drop term is defined in Chapter 3. It is well known that the porous LAD screen pores shrink at cryogenic temperatures, causing a slight gain in bubble point performance due to decreased screen pore size. When compared against data in LOX and $\mathrm{LH}_{2}$ the FTS pressure drop was shown to be temperature dependent, with FTS flow losses at $\mathrm{LH}_{2}$ temperatures approximately $150 \%$ higher than the room temperature prediction value. The same expression (Equation 3.33) can be used to model the FTS 
losses by simply using the appropriate values of $\alpha, \beta$ at the given temperature. The FTS pressure drop term can be expressed as:

$\Delta P_{F T S}=C_{\text {lam }} \mu\left|v_{i n j}\right|+C_{t u r b} \rho v_{i n j}^{2}$

where $v_{i n j}$ is the injection velocity across the porous screen, and $C_{l a m}, C_{t u r b}$ are defined as:

$C_{\text {lam }}=\alpha \frac{Q B a^{2}}{\varepsilon^{2}}$

$C_{\text {turb }}=\beta \frac{Q B}{\varepsilon^{2} D_{p}}$

Equation 12.2 is defined to ensure positive injection velocity across the screen.

While Jaekle's 1D pressure drop model qualitatively matched the $\mathrm{LH}_{2}$ outflow data from Chapter 9, it failed to quantitatively track the data. Discrepancies between model and data are primarily due to two major assumptions that were used in formulating the $1 \mathrm{D}$ pressure drop, one each for the $\Delta P_{F T S}$ and $\Delta P_{\text {frictional }}$ pressure drops. The first is that $v_{i n j}$ is assumed to be constant along the wetted portion of the channel. This yielded a constant $\Delta P_{F T S}$ along the channel. CFD simulations by Zhang et al. (2009) and McQuillen et al. (2012) showed, however, that a pressure dependent boundary condition at the screen forces $v_{i n j}$ to increase along the channel to its maximum value near the outlet. The second assumption was that the flow through the channel was fully developed from channel end to end. This allowed for the formulation of an equation for the laminar and turbulent frictional pressure drop with a single flow rate. In actuality, the flow rate 
would likely increase from the channel dead end to the outlet as more flow is accumulated across the screen.

The difference between 1D model assumed flow and the actual outflow is illustrated in Figure 12.1. These two assumptions lead to unwanted errors in the solution which can be avoided by not separating the solution for $\Delta P_{F T S}$ and $\Delta P_{\text {frictional }}$. In order to do this, the 2D Navier-Stokes equations must be directly solved for flow through a finite channel with one porous wall.
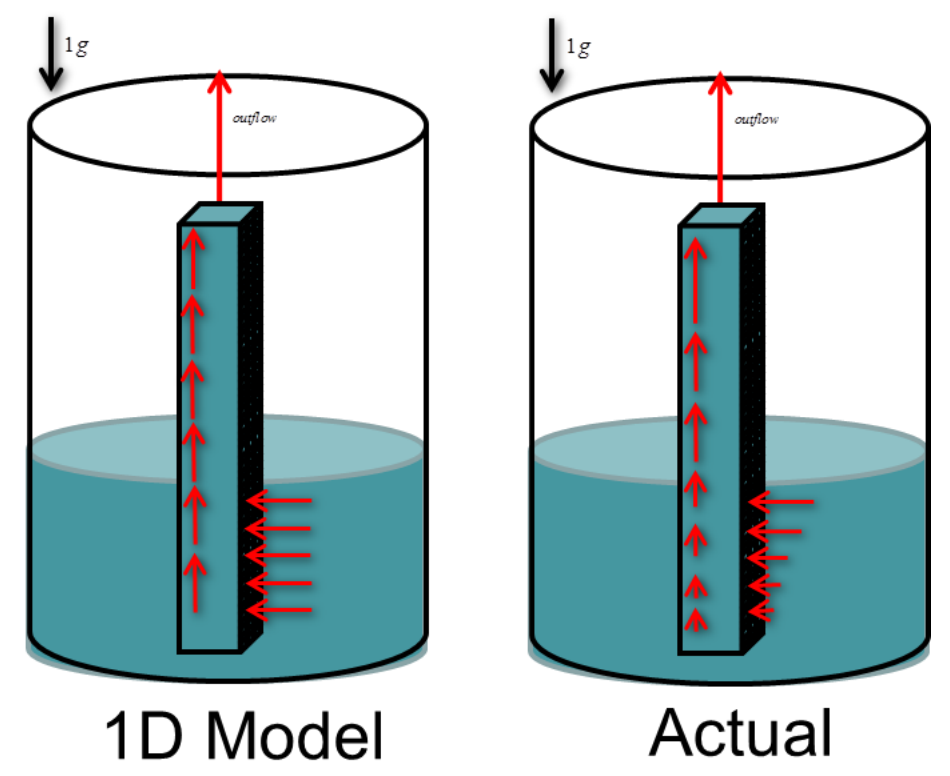

Figure 12.1 - Illustration of Discrepancies between One Dimensional Pressure Drop Model and Data for the 1-g Inverted Outflow Configuration

\subsection{Literature Review}

Flow through resistive porous elements has been studied by many in the particle filtration community to determine basic relations and empirical correlations (Ergun 1952, Jones and Krier 1972, Laws and Livesey 1978, Munson 1988, Brundrett 1993, Olbricht 
1996, Sodre and Parise 1997, Wakeland and Keolian 2003, Wu et al. 2005, and Valli et al. 2009). A detailed and rigorous review of previous analytical and numerical solutions in porous pipe, annulus, and channel flow is reserved in Appendix F; only highlights are presented here. Porous channel flow is classified by the size of flow within the channel (laminar or turbulent), the number of porous walls (one or two), the size (small, large, arbitrary) and nature (uniform or variable) of injection into the porous element, the type of transverse and axial boundary conditions at the porous surface (suction or injection), and whether or not there is heat transfer and/or electrical or magnetic component, where the injection Reynolds number is defined as:

$\operatorname{Re}_{i n j}=\frac{\rho v_{i n j} H}{\mu}$

where $H$ is the height of the channel. The transverse velocity is defined as the velocity normal to the porous surface whereas the axial velocity is defined as the velocity along the channel parallel to the porous surface. For the current derivation both heat transfer and effects due to an electric or magnetic field are ignored.

The first solution to porous channel flow was the similarity solution from Berman (1953), from which a nonlinear ODE was reported for channel flow with two porous walls and uniform velocity injection. Berman also reported a perturbation solution to this ODE for small $\mathrm{Re}_{i n j}$. A more mathematically challenging problem was the perturbation solution to the ODE for large $\operatorname{Re}_{i n j}$ which was solved for by both Yuan (1956) and Terrill (1965). By changing one boundary condition from the Berman (1953) problem, Green (1979) was able to obtain a small $\operatorname{Re}_{i n j}$ perturbation solution for a channel with one 
porous wall and uniform injection velocity. Other perturbation solutions were previously attempted (Singh and Laurence 1979a and b and Chellam et al. 1992). A large- $\operatorname{Re}_{i n j}$ perturbation solution for a single porous wall was not found in the literature. Solutions for arbitrary $\operatorname{Re}_{i n j}$ are provided by Sellars (1955), Morduchow (1957), White et al. (1958), White (1962), and Saad and Majdalani (2009), using different methods to obtain polynomial solutions for channels with two porous walls. Approaches taken by these authors applied to channel flow with one porous wall were not found in the literature.

None of these solutions were physically relevant since they did not take into account variation of $v_{i n j}$ along the channel due to the FTS pressure loss. As a result, a few authors began to investigate methods for solving the porous channel flow with variable $v_{i n j}$ (e.g. Galowin et al. 1974 and Haldenwang 2007). Accounting for variable $v_{i n j}$ adds complexity for two main reasons, however. First, a perturbation solution in terms of $\operatorname{Re}_{i n j}$ is not sufficient, since $\operatorname{Re}_{i n j}$ will change along the channel. Second, the transverse velocity boundary condition at the screen is no longer constant, but is pressure dependent.

It should be noted that all of the literature found on porous channel flow with variable $v_{i n j}$ only considered a boundary condition in which pressure drop across the screen was proportional to $v_{i n j}$. This is the first term on the right hand side of Equation 12.2. This is also known as Darcy's law. The solution in the current work includes a turbulent FTS term, the second term on the right hand side of Equation 12.2, in which pressure drop through the screen is proportional to the square of the injection velocity. 
This term was first given by Forchheimer (1901). It becomes more important for higher flow rates.

Oxarango et al. (2004) implemented the perturbation solution from Berman (1953) for the axial and transverse velocities, along with Darcy's Law in terms of velocity, back into the $\mathrm{x}$-momentum equation and then took the cross sectional average of each term. This created a second order nonlinear ODE in the axial direction so that boundary conditions on the axial velocity could be applied at the channel dead end and outlet. Haldenwang (2007) used a separation of variables approach in which the streamfunction was written as a function of the axial flow rate multiplied by a function that represented the solution from Berman (1953) at each differential slice of the channel. The corresponding axial and transverse velocities were input into the x-momentum equation, and a second order nonlinear ODE was obtained in terms of the axial flow rate, the injection Re number, and constants from the solution from Berman (1953). Galowin et al. (1974) assumed a parabolic axial velocity profile, also known as Poiseuille flow, and was able to obtain a solution for variable injection velocity using the KármánPohlhausen momentum integral technique. A second order nonlinear ODE was obtained, and a solution for the $2 \mathrm{D}$ velocity field was obtainable with a simple numerical method.

Each solution from Galowin et al. (1974), Oxarango et al. (2004), and Haldenwang (2007) showed the expected trend of increasing injection velocity, and thus increasing FTS pressure drop, towards the outlet. These solutions however still lacked the ability to enforce the no slip condition at the channel dead end. CFD from Zhang et al. (2009) and McQuillen et al. (2012) also did not address the no-slip condition. When solving with the flow field using each of their respective methods, $v_{i n j}$ and $\Delta P_{F T S}$ were 
nonzero at $\mathrm{x}=0$. This is because each solution provided a second order ODE, and only the boundary conditions on the axial velocity could be applied at the channel dead end and the outlet. Any boundary condition on the transverse velocity could not be enforced. The solution provided in this chapter follows the method offered by Galowin and Desantis (1971) and Galowin et al. (1974), with the additional step needed to enforce the no slip condition at $\mathrm{x}=0$. While $3 \mathrm{D}$ solutions have been attempted, they were only for uniform injection that did not vary along the length of the channel (e.g. Taylor et al. 1991 and Hewitt et al. 2003). The current solution is for a channel with one porous wall with arbitrary variable suction and injection, and builds upon previous solutions due to:

1. Satisfaction of the no slip condition at the channel dead end.

2. Addition of a turbulent term to the porous wall boundary condition to allow for arbitrary suction and injection.

3. Allowance of variable suction and injection along the porous surface.

4. Model anchoring/validation to cryogenic channel flow.

\subsection{Analytical Model Formulation}

Consider a rectangular LAD channel with length $L$, width $W$, and height $H$ as shown in Figure 12.2. Liquid flows through the screen in the negative y-direction and down the channel in the positive $\mathrm{x}$-direction as indicated by the blue arrows. The injection velocity across the screen is expected to increase from zero at the channel dead end $(x=0)$ to its largest value at the channel outlet $(x=L)$. 


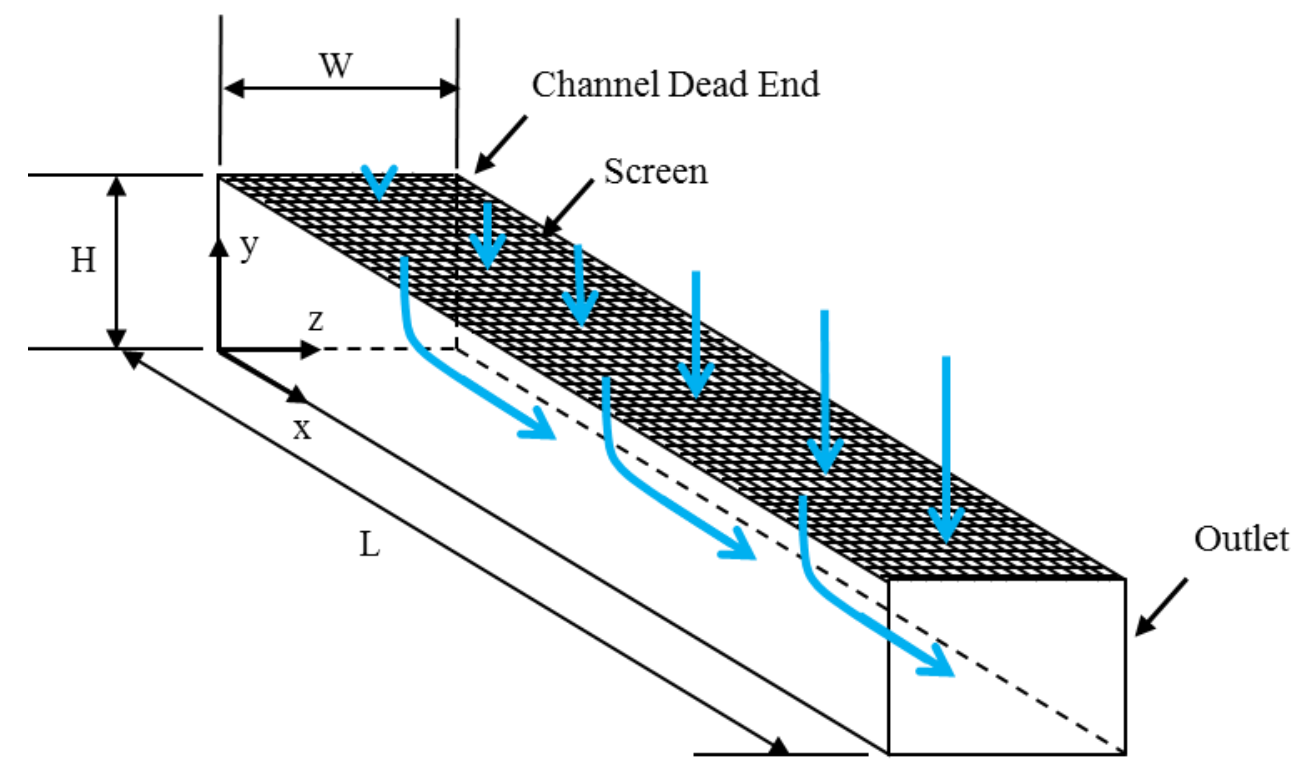

Figure 12.2 - Liquid Acquisition Device Channel with Flow through Screen and Out of the Channel

\subsubsection{Assumptions}

The list of assumptions and corresponding implications required to solve for the pressure and flow fields inside the porous channel are:

1. Constant and uniform properties $\quad \therefore \rho, \mu$ constant

2. Isotropic fluid

3. Newtonian fluid

4. Stokes' hypothesis holds true $\therefore$ Navier-Stokes equations hold true.

5. Steady state $\therefore \frac{D}{D t}() \rightarrow 0$

6. Isothermal fluid 
7. Incompressible fluid

$\therefore \frac{D \rho}{D t} \rightarrow 0$

8. Gravity force is uniform across channel $\therefore F_{B}=0$

9. Flow in the $\mathrm{z}$ direction is small $\quad \therefore v_{z}<\underset{\sim}{v_{x}, v_{y}}$

10. Flow does not change in $\mathrm{z}$ direction $\therefore \frac{\partial}{\partial z}() \rightarrow 0$

11. Flow inside channel is laminar. $\quad \therefore$ Effects of turbulence are neglected.

$\therefore v_{x}(y)$ can be modeled as Poiseuille flow.

\subsubsection{Governing Equations}

The set of governing equations of continuity and $\mathrm{x}$ and $\mathrm{y}$ momentum:

$$
\begin{aligned}
& \frac{D \rho}{D t}=0 \\
& \frac{D(\rho \underset{\sim}{v})}{D t}=\nabla P+\mu \nabla^{2} \underset{\sim}{v}
\end{aligned}
$$

reduce to the following set of equations after applying the assumptions:

$$
\begin{aligned}
& \frac{\partial v_{x}}{\partial x}+\frac{\partial v_{y}}{\partial y}=0 \\
& \rho\left(v_{x} \frac{\partial v_{x}}{\partial x}+v_{y} \frac{\partial v_{x}}{\partial y}\right)=-\frac{\partial P}{\partial x}+\mu\left(\frac{\partial^{2} v_{x}}{\partial x^{2}}+\frac{\partial^{2} v_{x}}{\partial y^{2}}\right) \\
& \rho\left(v_{x} \frac{\partial v_{y}}{\partial x}+v_{y} \frac{\partial v_{y}}{\partial y}\right)=-\frac{\partial P}{\partial y}+\mu\left(\frac{\partial^{2} v_{y}}{\partial x^{2}}+\frac{\partial^{2} v_{y}}{\partial y^{2}}\right)
\end{aligned}
$$

Scaling analysis can be used to simplify the y-momentum equation. Let 


$$
\begin{array}{ll}
\hat{x}=\frac{x}{L} & \hat{v}_{x}=\frac{v_{x}}{\bar{u}_{e}}
\end{array}
$$

where $\bar{u}_{e}$ is the average velocity at the exit of the channel and $\bar{v}_{i n j}$ is the average injection velocity across the porous screen. Substituting the scaled terms into continuity (Equation 12.7a):

$$
\frac{\bar{u}_{e}}{L} \frac{d \hat{v}_{x}}{d \hat{x}}+\frac{\bar{v}_{i n j}}{H} \frac{d \hat{v}_{y}}{d \hat{y}}=0
$$

Therefore, $\bar{u}_{e} \sim \frac{L}{H} \bar{v}_{i n j}$. Scaling x-momentum equation and simplifying,

$$
\frac{H}{L}\left(\hat{v}_{x} \frac{\partial \hat{v}_{x}}{\partial \hat{x}}+\hat{v}_{y} \frac{\partial \hat{v}_{x}}{\partial \hat{y}}\right)=-\frac{P_{r e f}}{\rho \bar{v}_{i n j}} \frac{H^{3}}{L^{3}}\left(\frac{\partial \hat{P}}{\partial \hat{x}}\right)+\frac{1}{\operatorname{Re}_{L}} \frac{H^{2}}{L^{2}}\left(\frac{\partial \hat{v}_{x}^{2}}{\partial \hat{x}^{2}}\right)+\frac{1}{\operatorname{Re}_{L}}\left(\frac{\partial \hat{v}_{x}^{2}}{\partial \hat{y}^{2}}\right)
$$

where $\mathrm{Re}_{L}$ is the Re number based on the length of the channel. Balancing the two leading order terms, $P_{r e f} \sim \rho v_{i n j}^{-2} \frac{L^{2}}{H^{2}}$. Scaling y-momentum equation and simplifying:

$$
\frac{H}{L} \hat{v}_{x} \frac{\partial \hat{v}_{y}}{\partial \hat{x}}+\frac{H^{2}}{L^{2}} \hat{v}_{y} \frac{\partial \hat{v}_{y}}{\partial \hat{y}}=-\left(\frac{\partial \hat{P}}{\partial \hat{y}}\right)+\frac{1}{\operatorname{Re}_{L}} \frac{H^{3}}{L^{3}}\left(\frac{\partial \hat{v}_{y}^{2}}{\partial \hat{x}^{2}}\right)+\frac{1}{\operatorname{Re}_{L}} \frac{H}{L}\left(\frac{\partial \hat{v}_{y}}{\partial \hat{y}^{2}}\right)
$$

Note that the pressure gradient term is order of magnitude 1. Using typical LAD channel dimensions to evaluate $\frac{H}{L}$, the magnitude of the second largest term is approximately $3 \%$ of the magnitude of the leading order term. Therefore retaining only the leading order term, 


$$
\frac{\partial \hat{P}}{\partial \hat{y}}=0
$$

Therefore, y-momentum implies $P(x, y) \rightarrow P(x)$. The result that pressure is a function of $\mathrm{x}$ only is also found in many of the previous solutions for flow through a channel with two porous walls, see Berman (1953) for example.

\subsubsection{Method of Solution}

Governing equations simplify to:

$$
\begin{aligned}
& \frac{\partial v_{x}}{\partial x}+\frac{\partial v_{y}}{\partial y}=0 \\
& \rho\left(v_{x} \frac{\partial v_{x}}{\partial x}+v_{y} \frac{\partial v_{x}}{\partial y}\right)=-\frac{d P}{d x}+\mu\left(\frac{\partial^{2} v_{x}}{\partial x^{2}}+\frac{\partial^{2} v_{x}}{\partial y^{2}}\right)
\end{aligned}
$$

Six BCs are required to solve the set of equations. An additional seventh $\mathrm{BC}$ is added to enforce the no slip condition (i.e. zero injection velocity) at the channel dead end.

\section{$\underline{\text { Boundary Conditions }}$}

1. $v_{x}(0, y)=0 \quad$ (no penetration)

2. $\quad v_{x}(\mathrm{~L}, y)=\bar{u}_{e} g(y) \quad$ (flow demand)

3. $v_{x}(\mathrm{x}, 0)=0 \quad$ (no slip)
4. $\quad v_{x}(\mathrm{x}, \mathrm{H})=0$
(no slip) 
5. $v_{y}(\mathrm{x}, 0)=0 \quad$ (no penetration)

6. $\quad v_{y}(\mathrm{x}, \mathrm{H})=v_{i n j}(x) \quad$ (mass injection)

7. $v_{y}(0, y)=0 \quad$ (no slip)

where $g(y)$ retains the shape of the $v_{x}(x)$ profile and $v_{i n j}(x)$ is the injection velocity across the screen. Before attempting an analytical solution, Equations 12.12 and 12.13 and the boundary conditions are partially scaled as follows:

$x^{*}=\frac{x}{H} \quad y^{*}=\frac{y}{H} \quad L^{*}=\frac{L}{H}$ such that:

$\frac{\partial v_{x}}{\partial x^{*}}+\frac{\partial v_{y}}{\partial y^{*}}=0$

$\rho\left(v_{x} \frac{\partial v_{x}}{\partial x^{*}}+v_{y} \frac{\partial v_{x}}{\partial y^{*}}\right)=-\frac{d P}{d x^{*}}+\frac{\mu}{H}\left(\frac{\partial^{2} v_{x}}{\partial x^{* 2}}+\frac{\partial^{2} v_{x}}{\partial y^{* 2}}\right)$

\section{$\underline{\text { Scaled Boundary Conditions }}$}

1. $v_{x}\left(0, y^{*}\right)=0 \quad$ (no penetration)

2. $v_{x}\left(\mathrm{~L}^{*}, y^{*}\right)=\bar{u}_{e} g\left(y^{*}\right)$ (flow demand)

3. $v_{x}\left(\mathrm{x}^{*}, 0\right)=0 \quad$ (no slip)

4. $v_{x}\left(\mathrm{x}^{*}, 1\right)=0 \quad$ (no slip)

5. $v_{y}\left(\mathrm{x}^{*}, 0\right)=0 \quad$ (no penetration) 

6. $v_{y}\left(\mathrm{x}^{*}, 1\right)=v_{i n j}\left(x^{*}\right) \quad$ (mass injection)
7. $v_{y}\left(0, y^{*}\right)=0 \quad$ (no slip)

For Dutch Twill LAD screens, the pressure drop across the screen is well known from Equation 12.2 from which the pressure drop inside the channel can be determined:

$$
\Delta P_{F T S}=P_{0}-P\left(x^{*}\right)
$$

where $P_{0}$ is the pressure outside of the channel, which is assumed constant. Plugging Equation 12.2 into Equation 12.16:

$P\left(x^{*}\right)=P_{0}-C_{l a m} \mu\left|v_{i n j}\left(x^{*}\right)\right|-C_{\text {turb }} \rho\left(v_{i n j}\left(x^{*}\right)\right)^{2}$

The function $g\left(y^{*}\right)$ characterizes the shape of the $v_{x}$ velocity profile in the y-direction. It has been shown by Morduchow (1957) and White et al. (1958) that for injection at both walls, $g\left(y^{*}\right)$ is almost invariant for different injection velocities. Here it is safe to assume that the same trend applies to injection through a single porous wall, since BC 6 contains demand flow rate information, and thus determines the injection velocity and injection location into the channel. The simplest $g\left(y^{*}\right)$ profile is to assume Poiseuille flow, or that the injection rate into the channel minimally affects the y portion of $v_{x}$ :

$g\left(y^{*}\right)=6 y^{*}\left(1-y^{*}\right)$

This is a reasonable assumption because the parabolic profile assumed in Equation 12.18 will be skewed by BC 2 to adjust for injection velocity. 
The approach taken by Galowin et al. (1974) is used to transform the set of partial differential governing equations into a single ODE, and the proposed solution for the $v_{x}$ velocity takes the form of:

$\mathrm{v}_{x}\left(x^{*}, y^{*}\right)=\bar{u}_{e}\left[1+f\left(x^{*}\right)\right]\left[6 y^{*}\left(1-y^{*}\right)\right]$

where $f\left(x^{*}\right)$, which takes into account the $\mathrm{x}$-variation of $v_{x}$, is to be determined. The proposed x-component of the $v_{x}$ solution takes the form $1+f\left(x^{*}\right)$, and not $f\left(x^{*}\right)$, for convenience in the numerical method used to solve the final ODE. First, continuity is used to solve for $v_{y}$. Plugging Equation 12.19 into continuity (Equation 12.14), integrating from $y^{*}=0$ to $y^{*}$, and solving for $v_{y}$ :

$$
\int_{y^{*}=0}^{y^{*}} \frac{\partial v_{y}}{\partial y^{*}} d y^{*}=\int_{y^{*}=0}^{y^{*}}-\bar{u}_{e} \frac{d f}{d x^{*}}\left(6 y^{*}\left(1-y^{*}\right)\right) d y^{*}
$$

Simplifying:

$v_{y}\left(x^{*}, y^{*}\right)-v_{y}\left(x^{*}, 0\right)=-6 \bar{u}_{e} \frac{d f}{d x^{*}}\left(\frac{1}{2} y^{* 2}-\frac{1}{3} y^{* 3}\right)$

Applying BC 5:

$v_{y}\left(x^{*}, y^{*}\right)=-\bar{u}_{e} \frac{d f}{d x^{*}}\left(3 y^{* 2}-2 y^{* 3}\right)$

Equation 12.22 and $\mathrm{BC} 6$ can be used to express pressure in terms of $f$ :

$v_{y}\left(x^{*}, 1\right)=v_{i n j}\left(x^{*}\right)=-\bar{u}_{e} \frac{d f}{d x^{*}}$ 
Substituting Equation 12.23 into the known pressure distribution (Equation 12.17):

$$
P\left(x^{*}\right)=P_{0}-C_{\text {lam }} \mu \bar{u}_{e} \frac{d f}{d x^{*}}-C_{\text {turb }} \rho \bar{u}_{e}^{2}\left(\frac{d f}{d x^{*}}\right)^{2}
$$

Equations $12.19,12.22$, and 12.24 for $v_{x}, v_{y}, P$ are now written in terms of a single unknown variable $f$. Before plugging in expressions for $v_{x}, v_{y}, P$ into $\mathrm{x}-$ momentum (Equation 12.15), the Kármán-Pohlhausen momentum integral technique is used, where $\rho v_{x}$ multiplied by continuity (Equation 12.14) is added to X-momentum:

$2 \rho v_{x} \frac{\partial v_{x}}{\partial x^{*}}+\rho v_{y} \frac{\partial v_{x}}{\partial y^{*}}+\rho v_{x} \frac{\partial v_{y}}{\partial y^{*}}=-\frac{d P}{d x^{*}}+\frac{\mu}{H}\left(\frac{\partial^{2} v_{x}}{\partial x^{* 2}}+\frac{\partial^{2} v_{x}}{\partial y^{* 2}}\right)$

Simplifying and integrating from $y^{*}=0$ to $y^{*}=1$ :

$\rho \int_{y^{*}=0}^{y^{*}=1} \frac{\partial\left(v_{x}^{2}\right)}{\partial x^{*}} d y^{*}+\rho \int_{y^{*}=0}^{y^{*}=1} \frac{\partial\left(v_{x} v_{y}\right)}{\partial y^{*}} d y^{*}=-\int_{y^{*}=0}^{y^{*}=1} \frac{d P}{d x^{*}} d y^{*}+\frac{\mu}{H} \int_{y^{*}=0}^{y^{*}=1} \frac{\partial^{2} v_{x}}{\partial x^{* 2}} d y^{*}+\frac{\mu}{H} \int_{y^{*}=0}^{y^{*}=1} \frac{\partial^{2} v_{x}}{\partial y^{* 2}} d y^{*}$

After taking the derivative, evaluating the integral and simplifying, the first term becomes:

$\rho \int_{y^{*}=0}^{y^{*}=1} \frac{\partial\left(v_{x}^{2}\right)}{\partial x^{*}} d y^{*}=\frac{12}{5} \rho \bar{u}_{e}^{2} f^{\prime}(1+f)$

where the prime symbol denotes a derivative. Evaluating the integral, the second term becomes:

$\rho\left[v_{x}\left(x^{*}, 1\right) v_{y}\left(x^{*}, 1\right)-v_{x}\left(x^{*}, 0\right) v_{y}\left(x^{*}, 0\right)\right]$ 
Applying BC 4 to the first term and BC 3 to the second term, Equation 12.28 simplifies to 0 . Since pressure only depends on $x$, the third term in Equation 12.26 simplifies to $-\frac{d P}{d x^{*}}$. Taking the derivative of Equation 12.24, the third term becomes:

$-\frac{d P}{d x^{*}}=C_{\text {lam }} \mu \bar{u}_{e} f^{\prime \prime}+2 C_{\text {turb }} \rho \bar{u}_{e}^{2} f^{\prime} f^{\prime \prime}$

Taking the derivative and evaluating the integral, the fourth term in Equation 12.26 becomes:

$$
\frac{\mu}{H} \int_{y^{*}=0}^{y^{*}=1} \frac{\partial^{2} v_{x}}{\partial x^{* 2}} d y^{*}=\frac{\mu \bar{u}_{e}}{H} f^{\prime \prime}
$$

Taking the derivative and evaluating the integral, the fifth term in Equation 12.26 becomes:

$$
\frac{\mu}{H} \int_{y^{*}=0}^{y^{*}=1} \frac{\partial^{2} v_{x}}{\partial y^{* 2}} d y^{*}=\frac{-12 \mu \bar{u}_{e}}{H}(1+f)
$$

Plugging Equations 12.27 - 12.31 back into x-momentum (Equation 12.26):

$$
\frac{12}{5} \rho \bar{u}_{e}^{2} f^{\prime}(1+f)=C_{\text {lam }} \mu \bar{u}_{e} f^{\prime \prime}+2 C_{\text {turb }} \rho \bar{u}_{e}^{2} f^{\prime} f^{\prime \prime}+\frac{\mu \bar{u}_{e}}{H} f^{\prime \prime}-\frac{12 \mu \bar{u}_{e}}{H}(1+f)
$$

Simplifying and rearranging,

$$
\left(\frac{\mu}{\rho \bar{u}_{e} H}+\frac{C_{\text {lam }} \mu}{\rho \bar{u}_{e}}+2 C_{\text {turb }} f^{\prime}\right) f^{\prime \prime}-\frac{12 \mu}{\rho \bar{u}_{e} H}(1+f)-\frac{12}{5} f^{\prime}(1+f)=0
$$


To non-dimensionalize Equation 12.33, let $\operatorname{Re}_{e}=\frac{\rho \bar{u}_{e} H}{\mu}$ and $N_{\text {lam }}=C_{\text {lam }} H$ :

$$
\left(\frac{1}{\operatorname{Re}_{e}}+\frac{N_{\text {lam }}}{\operatorname{Re}_{e}}+2 C_{\text {turb }} f^{\prime}\right) f^{\prime \prime}-\frac{12}{\operatorname{Re}_{e}}(1+f)-\frac{12}{5} f^{\prime}(1+f)=0
$$

The derivative of Equation 12.34 is taken to enforce no slip at the dead end:

$$
\left(\frac{1}{\operatorname{Re}_{e}}+\frac{N_{\text {lam }}}{\operatorname{Re}_{e}}+2 C_{\text {turb }} f^{\prime}\right) f^{\prime \prime \prime}+2 C_{\text {turb }}\left(f^{\prime \prime}\right)^{2}-\frac{12}{\operatorname{Re}_{e}} f^{\prime}-\frac{12}{5}\left(f^{\prime \prime}(1+f)+\left(f^{\prime}\right)^{2}\right)=0
$$

Transforming the remaining BCs:

$$
\begin{array}{lll}
\text { 1. } f(0)=-1 & \text { 2. } f\left(\mathrm{~L}^{*}\right)=0 & \text { 7. } f^{\prime}(0)=0
\end{array}
$$

Equation 12.35, with the remaining BCs, composes the exact solution to steady, incompressible, laminar flow through a channel with one porous wall. A fourth order Runge-Kutta method combined with the shooting method is used to solve Equation 12.35. After numerically solving for $f, v_{x}, v_{y}$ and $P$ are determined from Equations $12.19,12.21$, and 12.24 , respectively. For simulations in this chapter, the porous wall is a $325 \times 2300$ Dutch Twill screen and the LAD channel has a height $\mathrm{H}=2.54 \mathrm{~cm}$, width $\mathrm{W}=$ $2.54 \mathrm{~cm}$, and length $\mathrm{L}=1 \mathrm{~m}$, which are typical values for a LAD channel. A $325 \times 2300$ screen yields values of $C_{\text {lam }}=3.01 \times 10^{8} \mathrm{~m}^{-1}$ and $C_{\text {turb }}=58.2$. 


\subsection{Model Results, Sensitivities, and Comparison to One Dimensional Model}

The solutions for $f$ and $f^{\prime}$ are shown in Figure 12.3 for different $\operatorname{Re}_{e}$ numbers for a $325 \times 2300$ screen with LOX FTS temperature dependent coefficients $\alpha, \beta$. The $v_{x}$ velocity is proportional to $f$ whereas the injection velocity is proportional to $f^{\prime}$. At $\operatorname{Re}_{e}<10^{4}$, the solution for both $f$ and $f^{\prime}$ converges to one curve. For $f^{\prime}$, the low $\operatorname{Re}_{e}$ curve is actually linear, which shows that for low $\mathrm{Re}_{e}$, the injection velocity increases linearly to its largest value at the exit. For increasing $\operatorname{Re}_{e}$ above $10^{4}$, the solution for $f$ has a sharper slope change as it moves to the right of the low $\operatorname{Re}_{e}$ curve.

The physical interpretation is that, as $\operatorname{Re}_{e}$ is increased, the fluid accelerates more sharply near the outlet. In other words, for higher $\operatorname{Re}_{e}$ the majority of the fluid motion is concentrated near the channel outlet. This same trend is seen for $f^{\prime}$. For increasing $\operatorname{Re}_{e}$ above $10^{4}$, the curve for $f^{\prime}$ both skews to the right and becomes larger in magnitude at the outlet, which indicates that the injection velocity near the outlet becomes larger as $\operatorname{Re}_{e}$ is increased to keep up with the higher flow and sharper increase in $v_{x}$ near the outlet. 

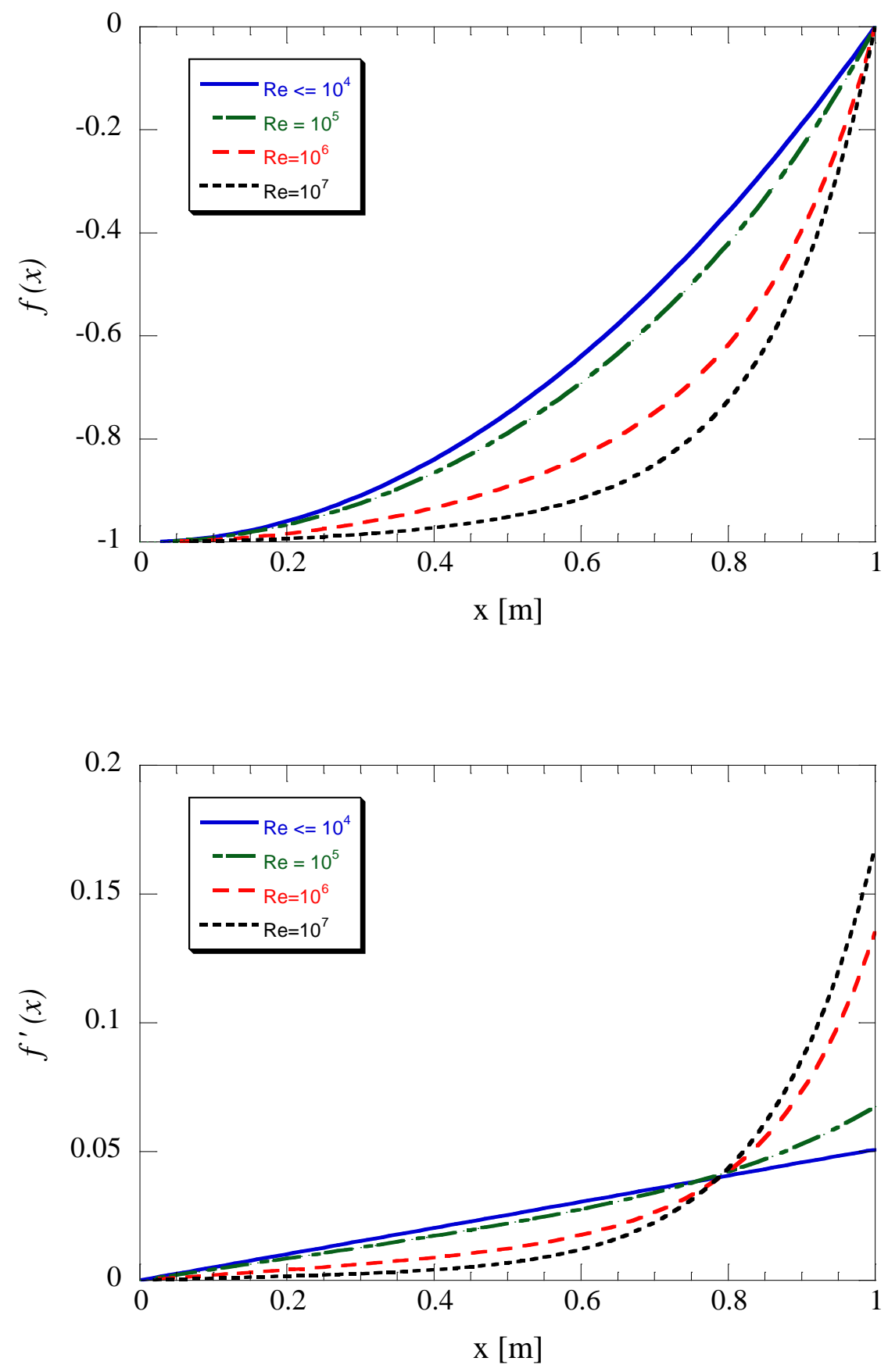

Figure 12.3 - Solution for a) $f$ and b) f' for Different Outlet Reynolds Numbers. The solution evaluates $C_{\text {lam }}$ and $C_{\text {turb }}$ for a $325 \times 2300$ Dutch Twill screen with liquid oxygen flow-throughscreen coefficients. 
Figure 12.4 illustrates the effect of channel height, width, and demand flow rate $\dot{m}_{e}=\rho \bar{u}_{e} H W$ on the distribution of velocity in the channel for a $325 \times 2300$ screen with LOX temperature $\alpha$ and $\beta$ coefficients by plotting 2D velocity vector plots inside the LAD channel. Flow rates were varied across two decades of demand flow rates typical of the demand for the proposed cryogenic fuel depots. The length of the channel is fixed to 1 meter. The width of the channel was kept equal to the channel height for each case, for simplicity. At low mass flow rates $\left(\dot{m}_{e}=0.01 \mathrm{~kg} / \mathrm{s}\right)$ the velocity vector plots are about the same for all channel heights. As flow rate increases, the disparity between the velocity vector plots for different heights becomes more apparent. For smaller channel heights, the outlet Re number is well above the $10^{4}$ mark so that the fluid motion becomes more concentrated towards the outlet, as illustrated in Figure 12.3. This equates to a larger injection velocity near the outlet for smaller channel heights. Since a larger injection velocity produces a larger pressure drop, this is an indicator to LAD designers that the channel height is an important and useful design parameter. For a given liquid and desired demand flow rate, Figure 12.4 shows that the channel should be designed to have a large height to promote linear $v_{i n j}$ variation so that the $\Delta P_{F T S}$ does not spike highly at the outlet, causing vapor ingestion and premature LAD breakdown. However, as channel height increases, the mass of the LAD channel also increases, indicating a tradeoff. 


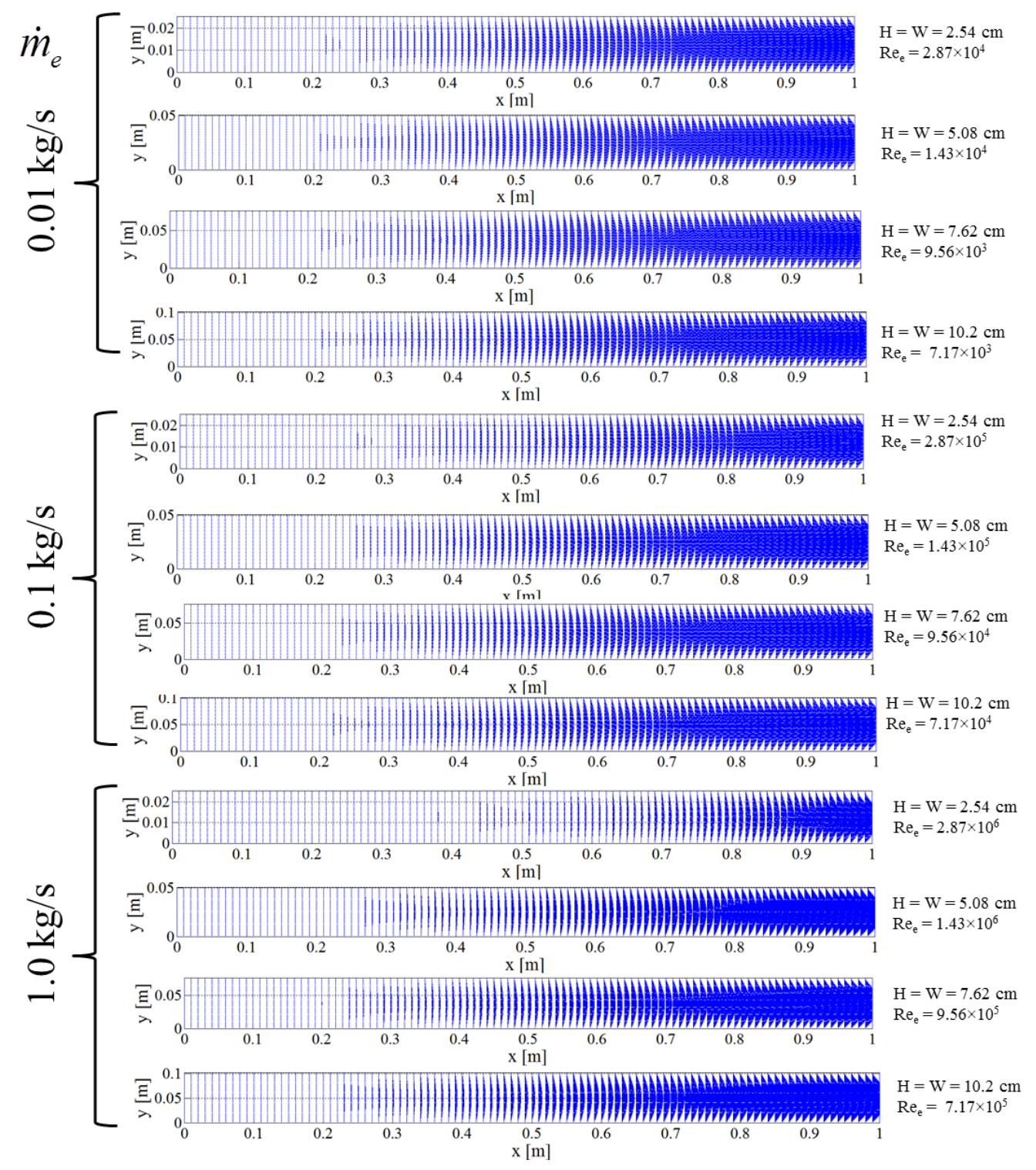

Figure 12.4 - Velocity Vector Plots for a Range of Liquid Oxygen Mass Flow Rates and Channel Dimensions. The solution evaluates $C_{\text {lam }}$ and $C_{\text {turb }}$ for a $325 \times 2300$ Dutch Twill Screen with Liquid Oxygen Flow-through-Screen Coefficients.

The FTS pressure drop is one of the primary factors in LAD channel design, along with the bubble point pressure of the screen. Previously, the 1D model assumed a constant FTS profile with distance along the LAD channel. Figure 12.5 plots the 
normalized FTS pressure drop for the new analytical model, $\frac{\Delta P_{F T S}}{\Delta P_{F T S-M A X}}$ against the distance along the channel for four different Dutch Twill screens, a 200x1400, 325x2300, $450 \times 2750$, and $510 \times 3600$ screen, where $\Delta P_{F T S-M A X}$ is the maximum FTS pressure drop in the channel. Values for the coefficients from Equation 12.35 for each screen are shown in Table 12.1. Each family of curves in Figure 12.5 is for a different $\operatorname{Re}_{e}$. The viscosity and density of saturated LOX at $90 \mathrm{~K}$ were used for calculating $\Delta P_{F T S}$ from Equation 12.17. As expected, for $\operatorname{Re}_{e} \leq 10^{4}$, the ratio is nearly linear for all curves due to the assumed Poiseuielle flow. This is physically consistent because for low $\operatorname{Re}_{e}$ there is a relatively small amount of velocity through the screen, approaching Poiseuielle flow. The pressure drop distribution then behaves similar to $f$, in that increasing $\operatorname{Re}_{e}$ results in skewing the curves to the right, increasing the magnitude of the slope near the outlet.

The variation between screens for each $\operatorname{Re}_{e}$ is a response to the three constants on the left hand side of Equation 12.35, $\mathrm{Re}_{e}^{-1}, N_{\text {lam }} \mathrm{Re}_{e}^{-1}$, and $C_{\text {turb }}$. Because $N_{\text {lam }}$ is large for each screen, as shown in Table 12.1, the $\mathrm{Re}_{e}^{-1}$ term is negligible for all Re numbers simulated in the current work. The $\mathrm{Re}_{e}^{-1}$ term is responsible for the frictional pressure drop whereas $N_{\text {lam }}$ determines the FTS pressure drop. Therefore the fact that the $\operatorname{Re}_{e}^{-1}$ term is negligible supports the fact that $\Delta P_{\text {frictional }}$ is significantly smaller than the $\Delta P_{F T S}$ as mentioned in Chapter 9. Furthermore, at lower Re numbers $N_{l a m} \operatorname{Re}_{e}^{-1}$ dominates over $C_{\text {turb }}$ and at higher flow rates $C_{\text {turb }}$ dominates over $N_{\text {lam }} \operatorname{Re}_{e}^{-1}$. Examining the values for $N_{\text {lam }}$ and $C_{\text {turb }}$ from Table 12.1 it is seen that the coarsest 200x1400 mesh has the lowest 
value for $N_{\text {lam }}$ while the finest $510 \times 3600$ mesh has the lowest value of $C_{\text {turb }}$. Therefore at lower $\operatorname{Re}_{e}\left(\operatorname{Re}_{e}=10^{4}-10^{5}\right)$, the laminar term $N_{\text {lam }} / \operatorname{Re}_{e}$ dominates and the $200 \times 1400$ has the most skewed curve; at high outlet Re numbers $\left(\operatorname{Re}_{e}=10^{7}\right), C_{\text {turb }}$ dominates and the 510x3600 has the most skewed curve. At middle range outlet Re numbers $\left(\operatorname{Re}_{e}=10^{6}\right.$ ), $N_{\text {lam }} \mathrm{Re}_{e}^{-1}$ and $C_{\text {turb }}$ are on the same order, and thus the 200x1400 and 510x3600 are equally skewed. Therefore, a designer of a LAD channel can select the appropriate screen for a particular mission, taking into account the relative effects of the $\Delta P_{F T S}$ spike effect at the outlet.

Now, with a means to calculate the pressure drop distribution, Equations 12.2 and 12.17 can be used to compare the current 2D model results with the former simplified 1D pressure drop model from Chapters 3 and 9. Figure 12.6 plots the maximum analytical model FTS pressure drop ( $\left.\triangle P_{F T S-M A X}\right)$, divided by the 1D model FTS pressure drop, ( $\left.\Delta P_{F T S, 1 D}\right)$ versus $\operatorname{Re}_{e}$. Over the full $\operatorname{Re}_{e}$ range, the $\Delta P_{F T S-M A X}$ is larger than $\Delta P_{F T S, 1 D}$ due to the 1D model assumptions. For low values of $\mathrm{Re}_{e}$ the maximum new model FTS pressure drop is twice that of the $1 \mathrm{D}$ model. This is due to the fact that at low $\operatorname{Re}_{e}$ the new model predicts a linear pressure drop. For higher values of $\mathrm{Re}_{e}, \Delta P_{F T S-M A X}$ becomes much larger than $\Delta P_{F T S, 1 D}$ due to the nonlinearity of the pressure drop distribution at the channel outlet, which was not accounted for in the 1D model. 

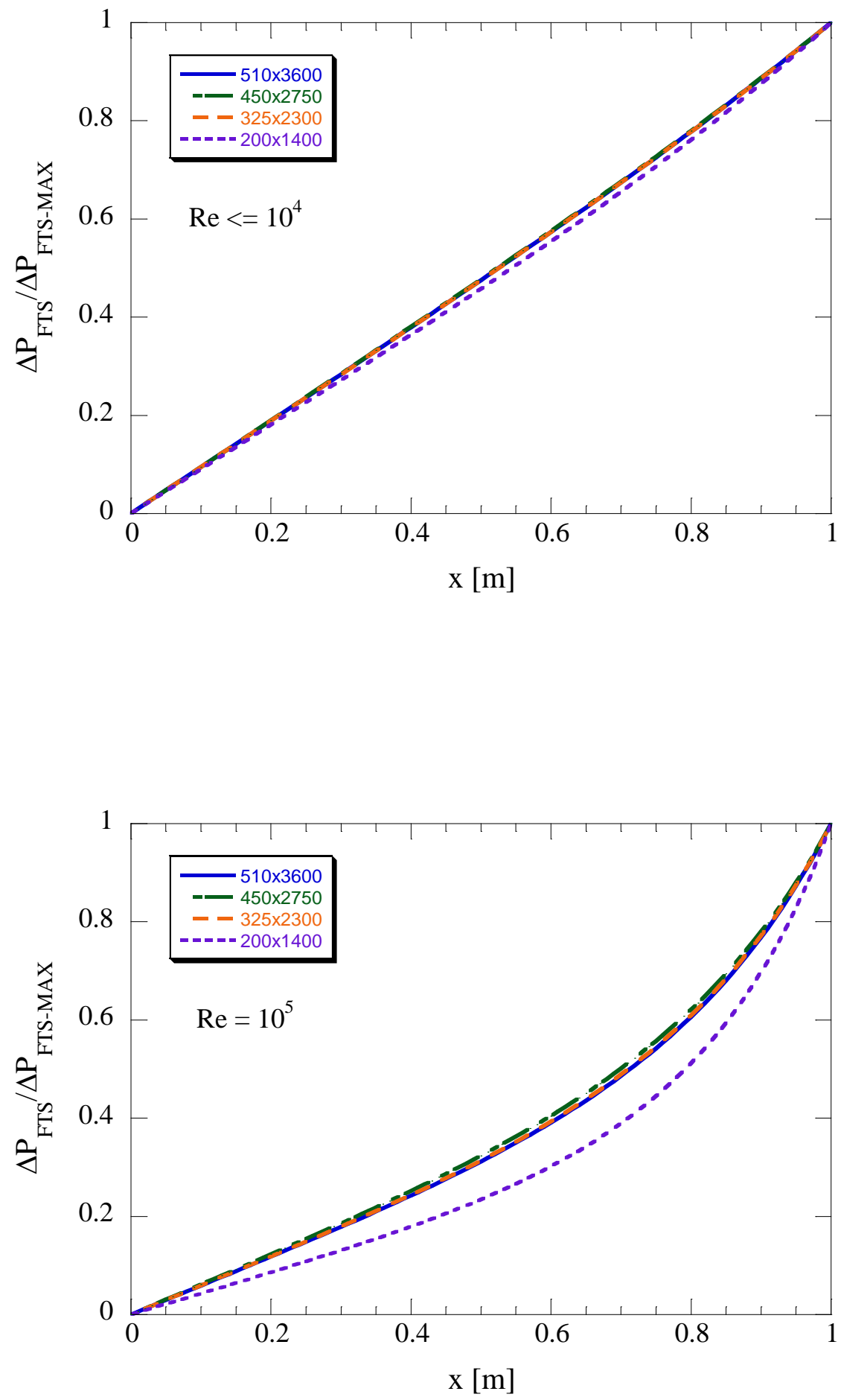

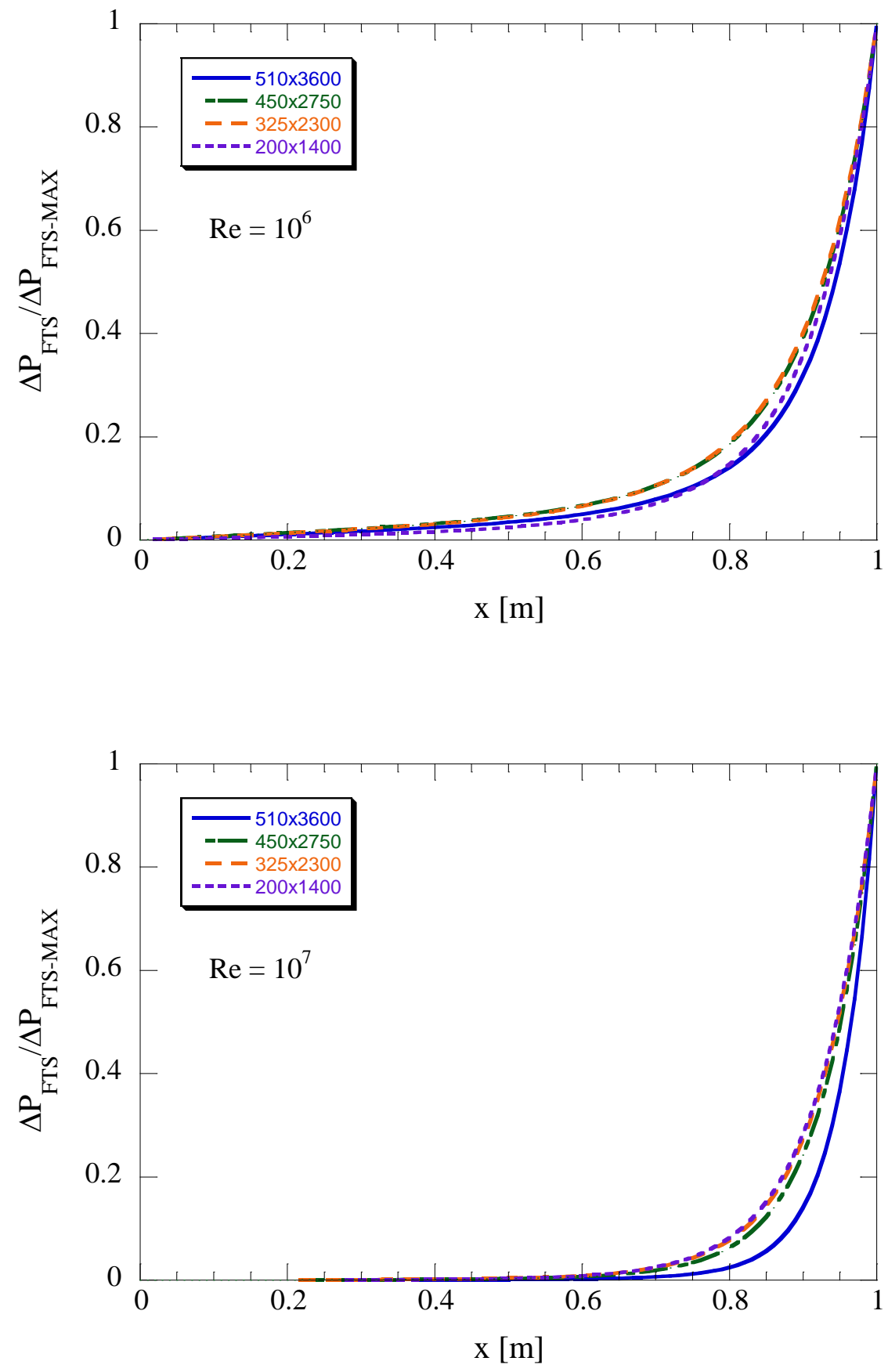

Figure 12.5 - Normalized Flow-through-Screen Pressure Drop along the Channel for Four Different Dutch Twill Screens for a) $\left.\operatorname{Re}_{\mathrm{e}}=10^{4}, \mathrm{~b}\right) \operatorname{Re}_{\mathrm{e}}=10^{5}$, c) $\operatorname{Re}_{\mathrm{e}}=10^{6}$, and d) $\operatorname{Re}_{\mathrm{e}}=10^{7}$. The solution evaluates $\mathrm{C}_{\mathrm{lam}}$ and $\mathrm{C}_{\text {turb }}$ for a $325 \times 2300$ Dutch Twill Screen with Liquid Oxygen Flowthrough-Screen Coefficients. 


\begin{tabular}{|l|c|c|c|}
\cline { 2 - 4 } \multicolumn{1}{c|}{} & Clam [1//m] & Nlam & Cturb \\
\hline $\mathbf{5 1 0 \times 3 6 0 0}$ & $3.04 \times 10^{\wedge} 8$ & $7.72 \times 10^{\wedge} 6$ & 23.6 \\
\hline $\mathbf{4 5 0 \times 2 7 5 0}$ & $3.27 \times 10^{\wedge} 8$ & $8.30 \times 10^{\wedge} 6$ & 48.2 \\
\hline $\mathbf{3 2 5 \times 2 3 0 0}$ & $3.01 \times 10^{\wedge} 8$ & $7.64 \times 10^{\wedge} 6$ & 58.2 \\
\hline $\mathbf{2 0 0 \times 1 4 0 0}$ & $1.72 \times 10^{\wedge} 7$ & $4.38 \times 10^{\wedge} 6$ & 64.4 \\
\hline
\end{tabular}

Table 12.1 - Flow-through-Screen Pressure Drop Coefficients for Different Screens

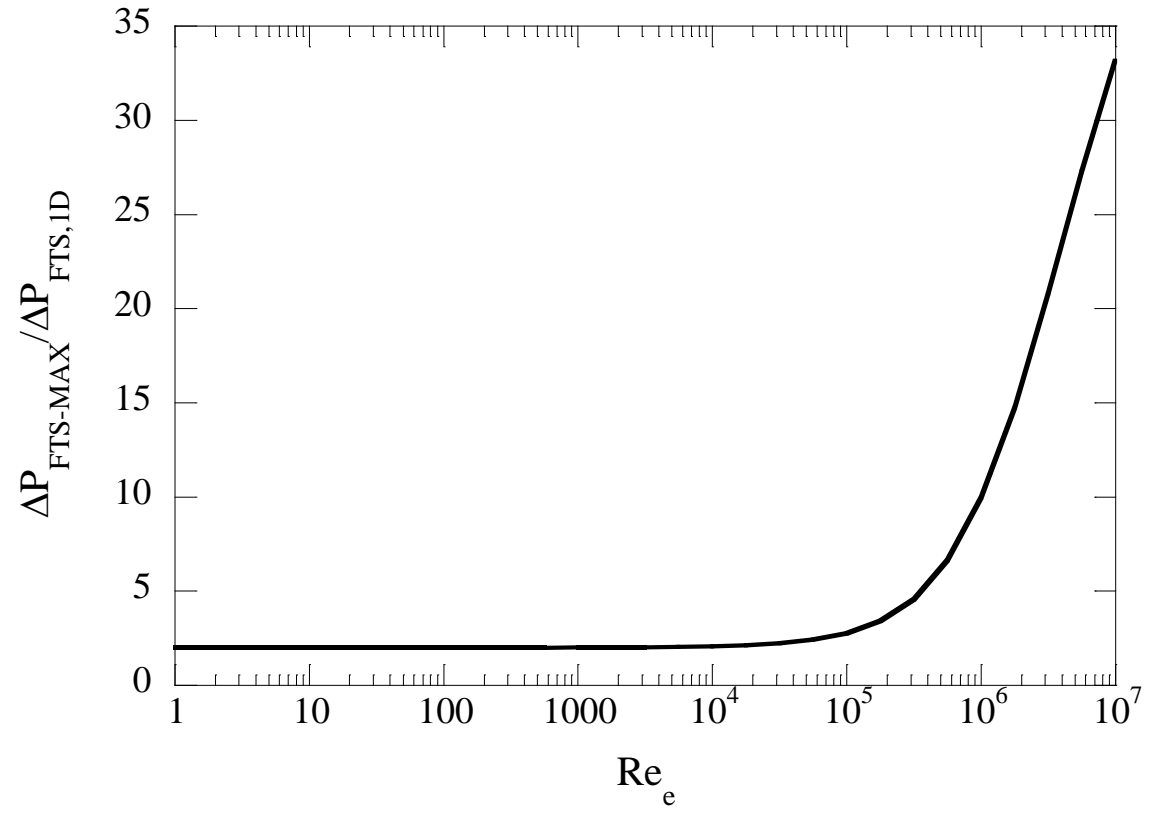

Figure 12.6 - Maximum Analytical Model Flow-through-Screen Pressure Drop Divided by the One Dimensional Model Flow-through-Screen Pressure Drop as a Function of Outlet Reynolds Number

\subsubsection{Validation of Laminar Channel Flow Assumption}

The turbulent portion of the fully-developed frictional pressure drop from Chapter 3 is written as:

$$
\Delta P_{\text {frictional,turbulent }}=f \frac{L}{D_{H}} \frac{\rho \bar{u}^{2}}{2}
$$


where $\bar{u}$ is the average velocity along the channel. Recall the corresponding friction factor and hydraulic diameter for the LAD screen are:

$$
\begin{aligned}
& f=\frac{1}{4\left\{\log \left[\frac{2(3.7)}{d_{s} / D_{H}}\right]\right\}^{2}} \\
& D_{H}=\frac{2 H W}{W+H}
\end{aligned}
$$

A worst-case scenario is if the average velocity along the channel is taken as the outlet average velocity:

$$
\Delta P_{\text {frictional, turbulent }}=f \frac{L}{D_{H}} \frac{\rho \bar{u}_{e}^{2}}{2}
$$

$\Delta P_{F T S-M A X}$ from the analytical solution is compared to the worst-case $\Delta P_{\text {frictional,turbulent }}$ for a range of $\operatorname{Re}_{e}$ in Figure 12.7. For all $\operatorname{Re}_{e}, \Delta P_{\text {frictional, turbulent }}$ is approximately $5 \%$ or less of $\Delta P_{F T S-M A X}$. Results show that the frictional pressure drop is significantly smaller than the FTS pressure drop in $\mathrm{LH}_{2}$. Results also confirm that ignoring the frictional pressure drop due to turbulent flow is valid, and that assumption 11 is valid. Also, the addition of the turbulent term to the porous boundary condition in Equation 12.2 is sufficient to explain the flow physics across the screen, and more complex log laws are not required to model the flow (Zagarola et al. 1997 and Afzal et al. 2007). 


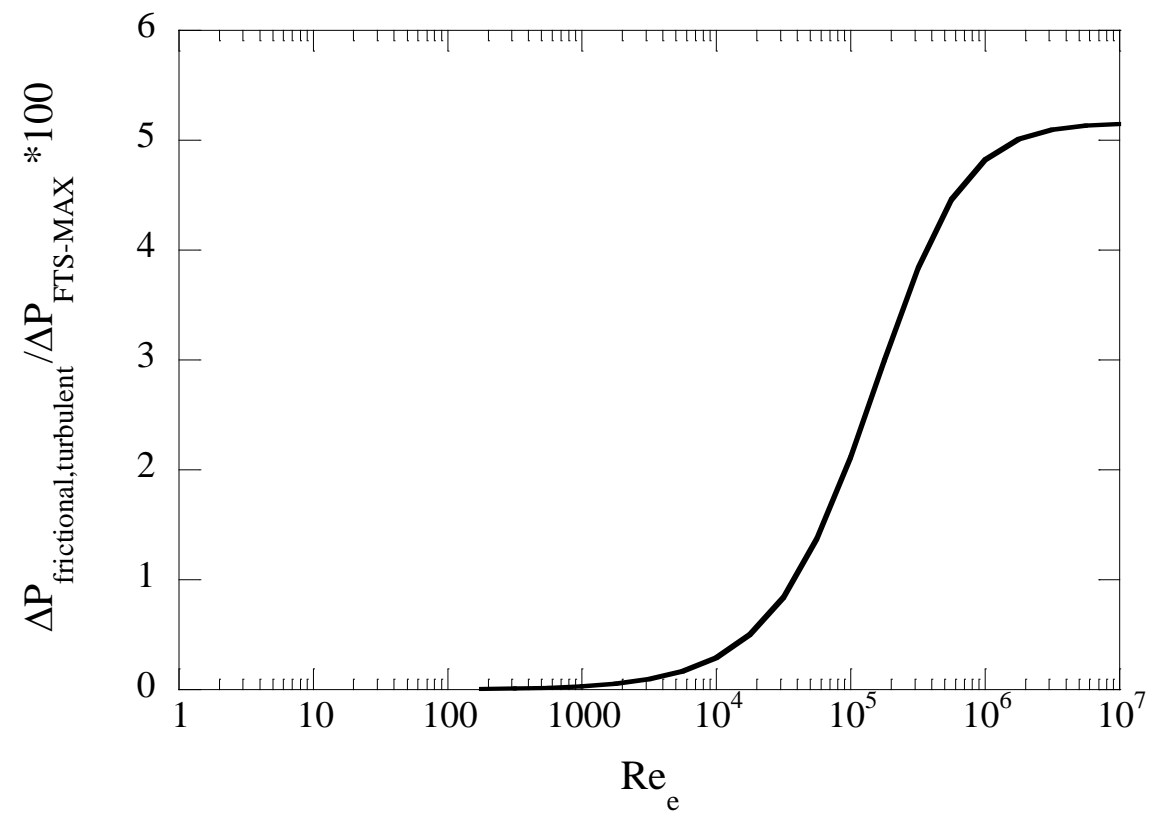

Figure 12.7 - Plot of the Worst-Case Turbulent Pressure Drop down the Channel as a Percentage of the Maximum Pressure Drop across the Screen

\subsubsection{Model Comparison to Liquid Oxygen Horizontal Liquid Acquisition Device Experiments}

The new analytical model can now be compared to the only two sets of available cryogenic LAD channel flow data. First, horizontal LAD channel data was collected in LOX and presented in Chapter 9. A picture of the LAD channel and dimensions is shown in Figure 12.8. The test setup consisted of a $61 \mathrm{~cm}$ long LAD channel with a height of $2.54 \mathrm{~cm}(1 \mathrm{in})$ and a width of $5.08 \mathrm{~cm}$ ( 2 in). The screen covered $48.2 \mathrm{~cm}$ of one side of the channel. Three pressure taps were placed at $15.2 \mathrm{~cm}$ (6 in) spacing within the channel. The pressure difference between the last two taps was recorded for different flow rates and two different tank LOX pressure and temperature conditions, at $1.034 \mathrm{MPa}$ and $90.6 \mathrm{~K}$ and at $1.655 \mathrm{MPa}$ and $107 \mathrm{~K}$. The analytical model was used to calculate the 
predicted pressure drop between these taps over a range of flow rates for the given liquid states. Figure 12.9 plots model results against the data for "cold" and "warm" LOX tests. Pressure drop is between the last two taps as shown in Figure 12.8.

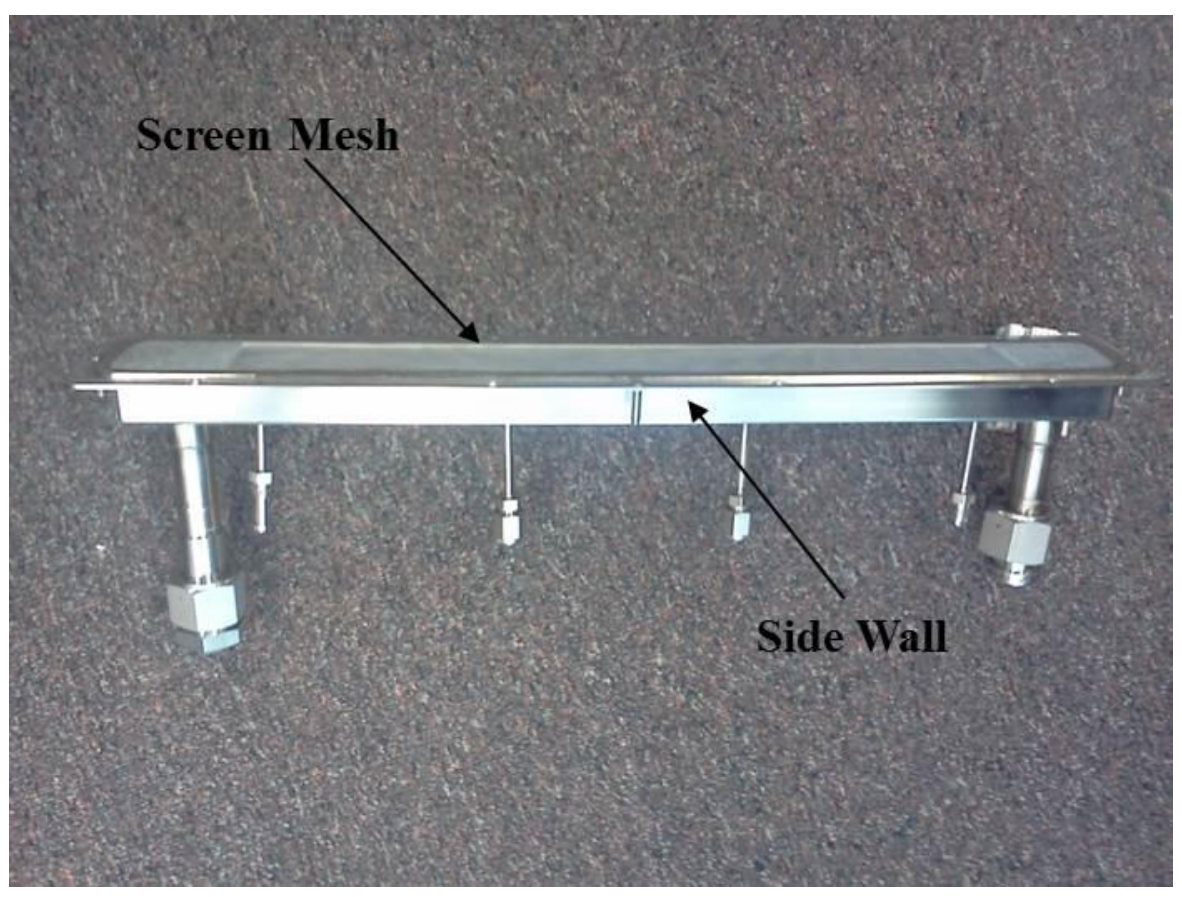

Figure $12.8-61.0 \mathrm{~cm} \times 2.54 \mathrm{~cm} \times 5.08 \mathrm{~cm}$ (24 in x 1 in x 2 in) Wide Horizontal Liquid Acquisition Device Channel

For the lower flow rate of $1 \mathrm{~kg} / \mathrm{s}$, the new model slightly underpredicts the average of the data for the first and second LOX LADs tests, respectively. This may seem like a large disagreement between data and model but it is primarily due to the large scatter in the data. The model predictions at $1 \mathrm{~kg} / \mathrm{s}$ are only 0.70 and 1.1 standard deviations away from the average of the data for the first and second clusters of data points, respectively. Another source of error may be the effects of the experimental setup on the data (i.e. the vortices at the outlets caused by placement of the outlet on the bottom of the channel) which are not accounted for in the new ideal analytical model (Zhang et 
al. 2009 and McQuillen et al. 2012). A third source of error is that there was an offset in the data, because the pressure drop does not go to zero at zero flow rate. Therefore the error between model and data at low flow rates is attributed to the data scatter, system effects, and the offset in the data.

Contrary to the case for lower flow rates, the model predicts a slightly larger pressure drop than the measured value at higher flow rates. The cause for disparity is due to the assumptions and boundary conditions: First, the actual $v_{x}$ profile diverges from the parabolic profile (assumption 11) as $\mathrm{Re}_{e}$ becomes larger; the assumed Poiseuille flow profile has a slightly steeper velocity gradient near the porous wall than for cases with large $\mathrm{Re}_{i n j}$ as shown by Morduchow (1957), White et al. (1958), and Chellam et al. (1995). A steeper velocity gradient predicted at the wall equates to a larger than actual shear stress and thus pressure gradient that opposes the shear stress. This would explain why the difference between model and data at a mass flow rate of $4.5 \mathrm{~kg} / \mathrm{s}$ is larger than the difference at a mass flow rate of $2.5 \mathrm{~kg} / \mathrm{s}$. Secondly, disparity between model and data at high flow rates may be due to $\mathrm{BC} 4$, the no slip condition at the LAD screen. As shown by Chellam et al. (1995), there may be a non-negligible slip velocity at the screen, which would cause less frictional resistance and thus cause pressure drop along the channel to be slightly lower than the model predictions. This slip velocity arises from flow inside the channel parallel to the screen mesh. While the actual x-velocity along the screen is nonzero in the real flow case, it is anticipated to be quite small. 

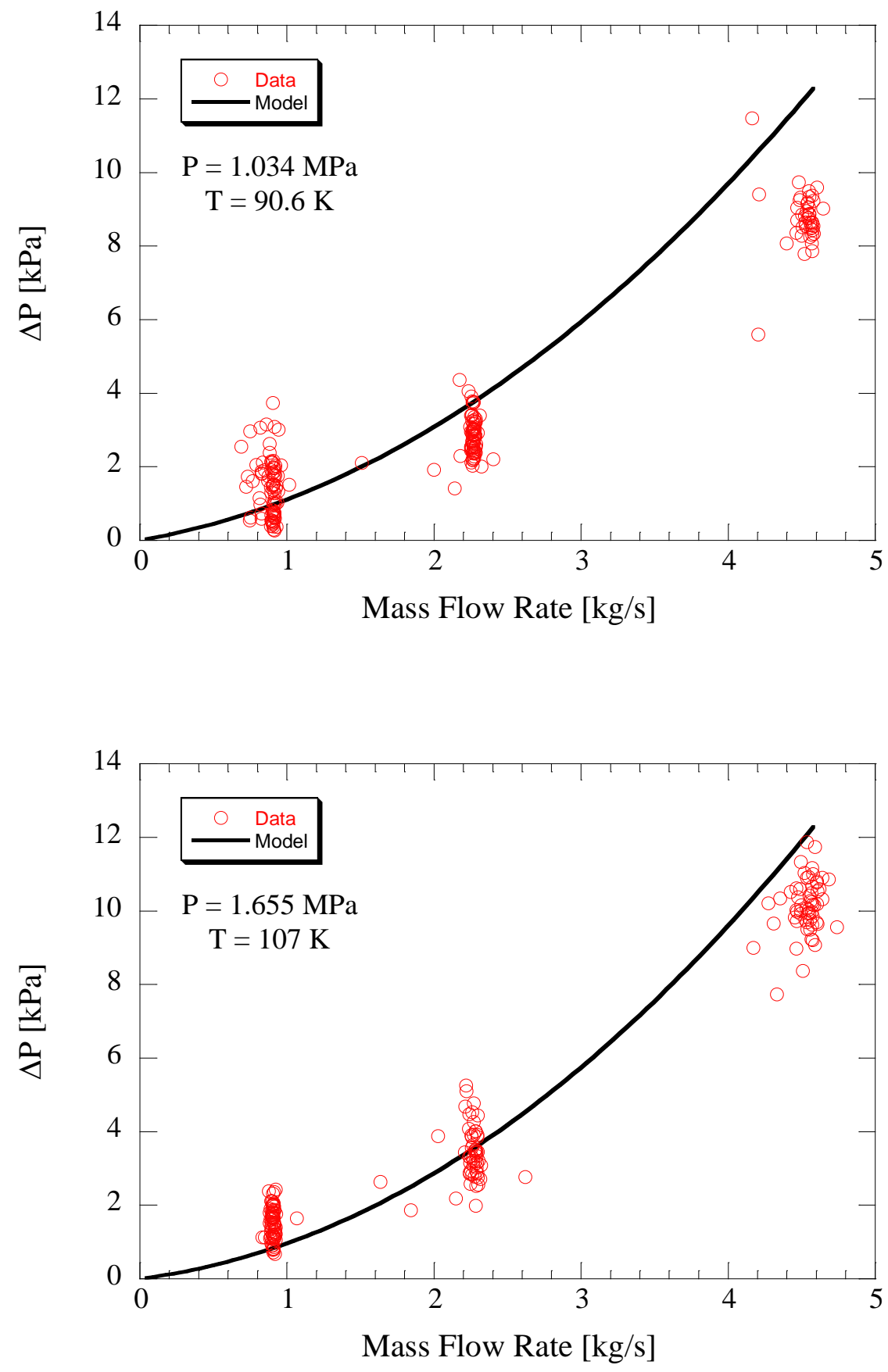

Figure 12.9 - New Analytical Model Comparison with Data for Liquid Oxygen Flow inside a Horizontal Liquid Acquisition Device Channel at a) 1.034 MPa and 90.6K and b) 1.655 MPa and 107K. Pressure difference is between the last two taps in Figure 12.8. 


\subsubsection{Model Comparison to Liquid Hydrogen 1-g Inverted Vertical Outflow Experiments}

The second set of cryogenic liquid LAD channel flow data that can be compared against model performance is the $\mathrm{LH}_{2} 1 \mathrm{~g}$ inverted outflow experiments reported in Chapter 9. The new analytical model can be used to simulate propellant tank drain to compare against the data. To model the system, the long axis of the LAD channel is aligned vertically, and the flow is routed vertically upward such that the hydrostatic pressure drop is accounted for, as shown in Figure 12.10. Only the 325x2300 standard channel is modeled. The channel was $91.4 \mathrm{~cm}$ long and $2.54 \mathrm{~cm}$ wide with a height of $2.54 \mathrm{~cm}$. There were two $2.54 \mathrm{~cm}$ gaps between three $29 \mathrm{~cm}$ long screen sections along the LAD, as opposed to one long, continuous screen piece. The LAD was mounted inside the propellant tank and the tank was drained through the LAD channel. Since DPT measurements were too noisy in those tests, the liquid level at breakdown, and thus length of the LAD channel that was exposed to pressurant gas $L_{\text {Exposed }}$, was recorded for each experiment to compare performance between model and experiment. Tank pressure, liquid temperature, and pressurant gas temperatures were all recorded at each test, at each flow rate, which allows calculation of the bubble point using the new cryogenic model from Equation 10.18. Note again that higher exposed screen lengths always equates to higher performance.

A computer program was written to simulate each test run using the new cryogenic bubble point model and new analytical LAD channel flow model. The raw analytical model was used to calculate the pressure drop in the channel portion that was submerged in liquid. The fully developed turbulent pressure drop expression from 
Equation 12.39 was used to calculate the pressure drop in the channel portion exposed to pressurant gas. In addition to the $\Delta P_{\text {frictional,turbulent }}$, due to flow against gravity, the hydrostatic pressure drop term was also included. The simulation proceeded using small time steps and assumed that the rate of tank liquid level depletion was slow enough that the steady state analytical model could be used at each time step. In general, time steps were no larger than 1 millisecond. At each time step, the pressure drop along the channel and across the screen was calculated to compare with the bubble point pressure. The simulation terminated when the pressure drop across the screen exceeded the bubble point pressure.

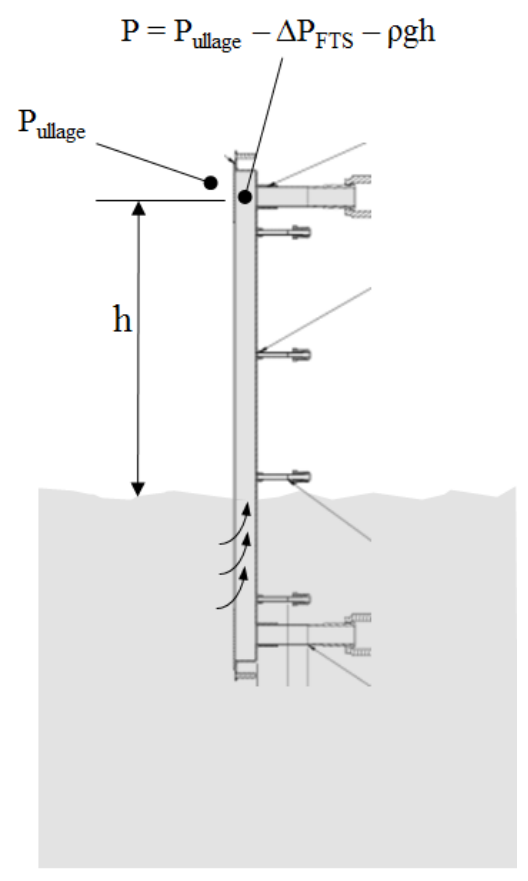

Figure 12.10 - Schematic of Liquid Acquisition Device Channel used to Model 1g Inverted Outflow Tests

Figure 12.11 plots the difference between experimental data and analytical model predicted breakdown heights as a function of demand mass flow rate from the $\mathrm{LH}_{2}$ 
outflow tests. $L_{\text {Exposed,data }}-L_{\text {Exposed,model }}<0$ implies that the model predicts a larger exposed screen length relative to the data, and thus better performance, and for $L_{\text {Exposed,data }}-L_{\text {Exposed,model }}>0$, the model predicts a smaller exposed screen length and thus worse performance compared to the data. Surprisingly, there is a distinct linear trend with flow rate.

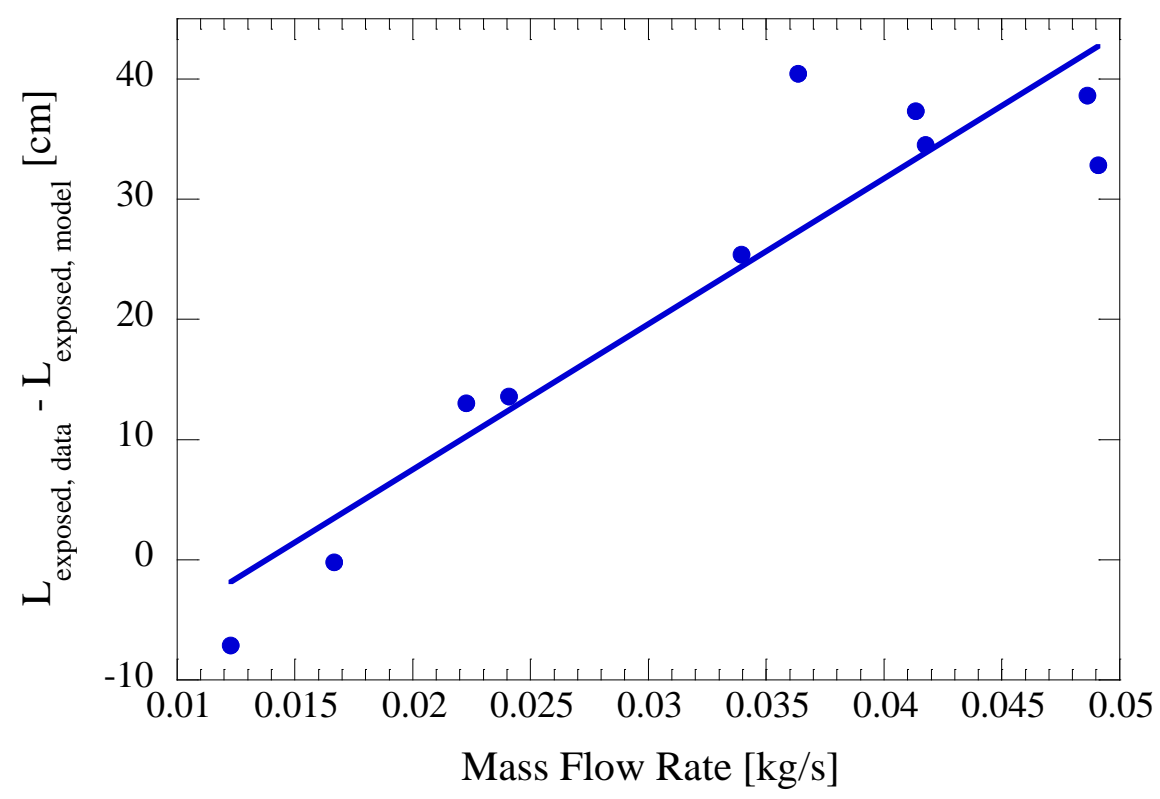

Figure 12.11 - Difference between Liquid Hydrogen Inverted Outflow Experimental Data from Chapter 9 and Analytical Model Predicted Exposed Screen Length at Breakdown as a Function of Mass Flow Rate using the New Model

Two system-dependent effects were included, one that reduced error in the data and one that reduced error in the raw analytical model. First, the effect of the area shrinkage at the channel exit was included using the Bernoulli Equation. The actual diameter of the exit tube was $1.4 \mathrm{~cm}$, which was significantly smaller than the hydraulic diameter of the channel, $2.54 \mathrm{~cm}$. This real system effect caused the model predicted 
pressure at the exit to decrease, thus lowering the predicted performance due to larger pressure drop across the screen near the outlet. The second system effect taken into account was the small performance loss between pre-test and post-test IPA channel bubble point tests as reported in Figure 9.26. The updated analytical model and experimental data which factor in these two real system effects is plotted in Figure 12.11. With real system effects factored in, the model is shown to underpredict, and thus predict worse performance relative to most of the experimental data.

\subsection{Dynamic Bubble Point Model}

In an attempt to reduce disparity between outflow data and model, further investigation was warranted to understand the underlying discrepancies. Recall from assumption 6 that the analytical pressure drop model was derived for an isothermal fluid, for simplicity. In general, any cryogenic fluid system may experience heat transfer effects due to temperature differences between fluids and ambient surroundings. In general the model predicts significantly lower performance than the data as shown in Figure 12.11. The static bubble point model from Chapter 10 was used to determine the point of breakdown in the dynamic outflow environment. Warm pressurant gas effects were factored in by inputting the measured ullage temperature just outside the LAD channel. However, the linear trend of the difference between experimental data and model with mass flow rate implies that there is an apparent performance enhancement due to convective cooling across the screen from the cool liquid side of the screen to the warm gas side of the screen as the liquid flows through the channel. An increase in convective heat transfer at the liquid side of the screen will most likely improve the screen retention capability in the dynamic outflow environment by cooling the liquid inside the LAD 
screen pores, in turn enhancing the static bubble point of the screen in order to prolong gas ingestion and eventual screen breakdown. Convection heat transfer will counteract the heated gas effect term from the cryogenic bubble point model in Equation 10.18. Therefore, the static cryogenic bubble point model, and thus full scale LAD outflow model, can be updated for the dynamic outflow case by using experimental results from Chapter 9. In other words, the flow rate of the liquid parallel to the LAD screen matters in the dynamic outflow environment.

The proposed update to the dynamic bubble point model takes the following form:

$$
\Delta P_{B P, \text { dynamic }}=\Delta P_{B P, \text { static }}\left[1+n_{d y n} \frac{\left(\dot{m}-\dot{m}_{\text {onset }}\right)}{W H}\right]
$$

where $\Delta P_{B P, \text { static }}$ is the static bubble point pressure calculated from Equation $10.18, n_{d y n}$ is the gain in static bubble point due to mass flow and thus convective cooling of the screen, and $\dot{m}_{\text {onset }}$ is the flow rate at which convective effects are essentially "turned on", at the lowest mass flow rate from the $\mathrm{LH}_{2}$ outflow data, $0.0123 \mathrm{~kg} / \mathrm{s}$. Since dynamic outflow data does not exist below this minimum value, the model assumes that the increase in performance due to convection is "turned off", and the bubble point pressure assumes the static value at the lowest flow rate. For all $\dot{m}<\dot{m}_{\text {onset }}$, the calculated bubble point is simply the static bubble point. In Equation 12.40, the flow rate is normalized by the cross sectional area to create a mass flux term. This makes it so that $n_{d y n}$ is independent of LAD geometry. 
This can be explained by looking at the case if $W$ or $H$ were doubled and $\dot{m}_{e}$ were held constant; the local velocity, and thus the local convection, at a point on the screen would actually reduce by one-half. For illustration this is shown in Figure 12.12 for a channel of height $H$ and a channel of height $2 H$. Heat is transferred from the gas to the liquid side of the screen. The channel of height $2 \mathrm{H}$ has half the velocity of the channel of height $H$. The same $n_{d y n}$ from the original LAD could still be applied with Equation 12.40 for the new larger LAD. While the value of $n_{d y n}$ is generated for a single $325 \times 2300$ screen in a single fluid $\left(\mathrm{LH}_{2}\right)$ using a single pressurant gas $(\mathrm{GHe})$, the value likely depends just on the screen type, liquid type, and the type of pressurization scheme (i.e. autogenous or non-condensable).
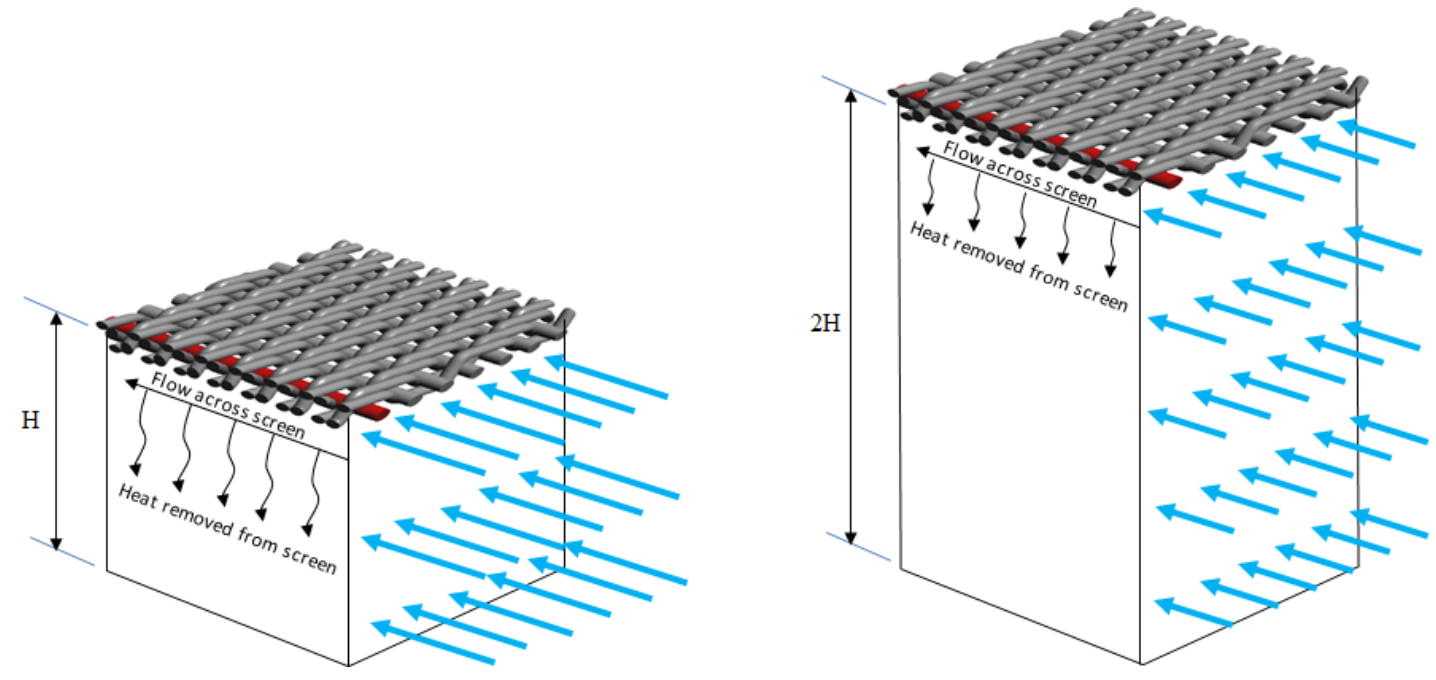

Figure 12.12 - Illustration of Convective Heat Transfer across Screen for Two Liquid Acquisition Device Channel Sections with the Same Overall Mass Flow Rate with Velocity Vectors Shown by Blue Arrows for a) LAD Channel of Height $\mathrm{H}$ and b) LAD Channel of Height 2H. Heat is transferred from the gas side to the liquid side of the screen. 
The 1-g inverted outflow simulations were repeated with the dynamic bubble point model using a value of $8.53 \times 10^{4}$ for $n_{d y n}$. With this value of $n_{d y n}$, a flow rate of $0.05 \mathrm{~kg} / \mathrm{s}$, with $\mathrm{W}=\mathrm{H}=0.0254 \mathrm{~m}$ yields $\Delta P_{B P, \text { dynamic }}=3.08 \Delta P_{B P, \text { static }}$, implying that there is over $300 \%$ gain over the static bubble point at the highest flow rate of $0.05 \mathrm{~kg} / \mathrm{s}$. This represents a substantial gain factor over the static case. Compare this gain in performance with the $165 \%$ gain in static bubble point performance between the $325 \times 2300$ at the NBP and using the 450x2750 screen, subcooling with GHe several degrees Kelvin below the NBP as reported in Section 5.5.

The differences between the updated model and data are plotted in Figure 12.13. As shown, the slope in difference between data and model is effectively accounted for, that there is a small remaining residual difference between data and model, and that the model now predicts slightly better performance than the data. There are two primary reasons for this residual difference. The first has to deal with the way the velocity profile transitions from the submerged channel portion to the exposed channel portion in the model. The velocity profile in the channel has a significant $v_{y}$ component at the location just below the L/V interface level of the tank, as shown in Figure 12.14. The model assumes that immediately after the liquid in the channel has passed the tank liquid level, the flow is fully developed, parabolic, and without any $v_{y}$ component. In reality the transition from submerged to exposed screen may promote vortices and circulation that would promote a small additional pressure drop, which would cause the model predicted breakdown point to occur earlier. This would force $L_{\text {Exposed,data }}-L_{\text {Exposed, model }}$ closer to 0 . The second possible source of error is due to the presence of the two $2.54 \mathrm{~cm}$ solid wall 
pieces in between screen sections in the physical channel, as discussed in Section 9.6.2.1. These screen "gaps" were omitted from the simulation and instead the channel screen was treated as one continuous piece of screen. With slight excess in available screen area, the simulation underpredicts the necessary overall flow rate through the porous screen and thus slightly underpredicts the total pressure drop along the LAD channel. Taking these gaps into account would thus also force $L_{\text {Exposed,data }}-L_{\text {Exposed, ,model }}$ closer to 0 . Overall, the new analytical model is shown to predict breakdown height and thus exposed screen length to within $8 \%$ of the total channel length of the data.

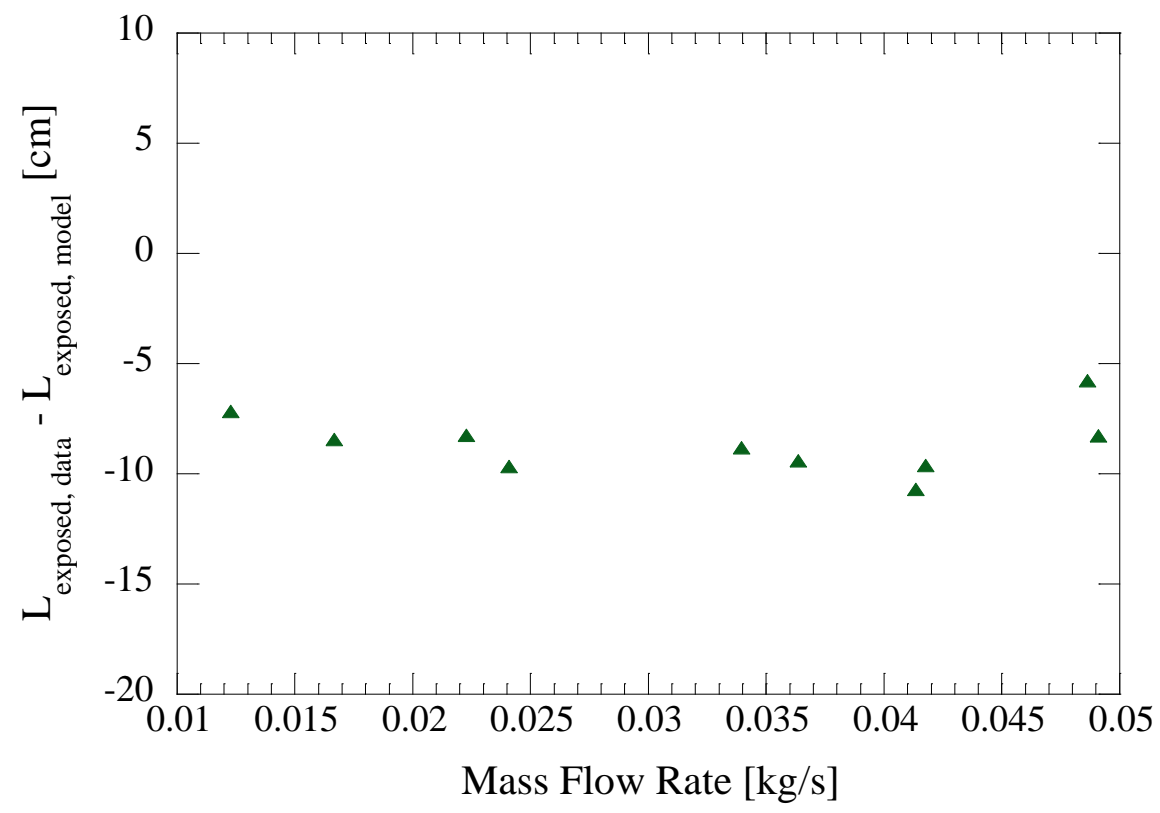

Figure 12.13 - Residual Difference between Actual and Model Predicted Exposed Screen Length using Analytical Flow Model Coupled with Dynamic Bubble Point Model 


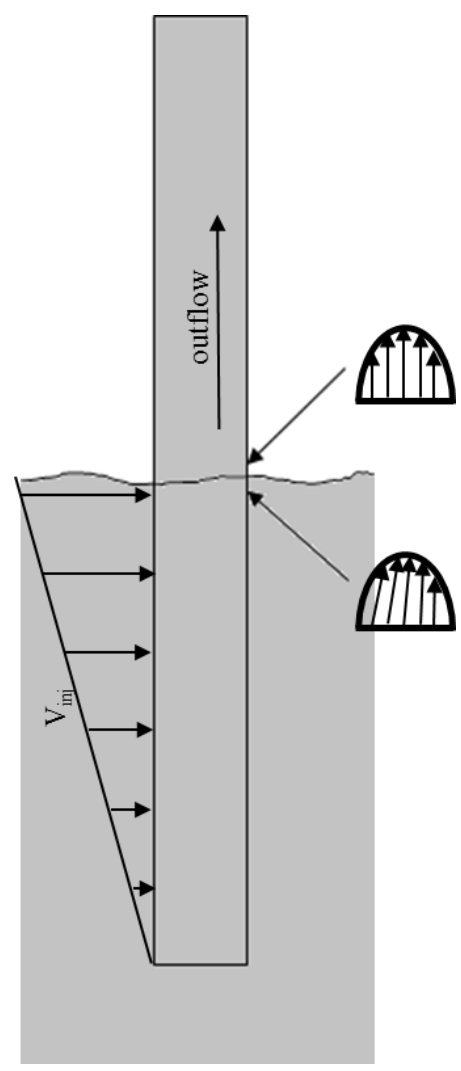

Figure 12.14 - Illustration of the Internal Channel Velocity Profile Change at the Propellant Tank Liquid/Vapor Interface. The model assumes that the velocity transitions from a skewed parabolic profile to a fully developed, parabolic profile at the tank L/V interface.

\subsection{Convective Cooling of the Liquid Acquisition Device Screen}

To quantify the effect of convective cooling of the LAD screen during dynamic outflow, the heat transfer rate across the screen is estimated for the $\mathrm{LH}_{2} \mathrm{LAD}$ outflow test runs. Due to the high Re number (based on exit) for the tests, the Nusselt $(\mathrm{Nu})$ number based on hydraulic diameter derived by Petukhov and Popov (1963) is used, which assumes fully turbulent developed flow:

$$
N u_{D_{H}}=\frac{(f / 8) \operatorname{Re}_{e} \operatorname{Pr}}{1.07+12.7(f / 8)^{1 / 2}\left(\operatorname{Pr}^{2 / 3}-1\right)}
$$


This expression is valid for $0.5<\operatorname{Pr}<200$ and $10^{4}<\mathrm{Re}_{e}<5 \times 10^{6}$. For the $\mathrm{LH}_{2}$ tests $\operatorname{Pr} \approx 1.2$ and $4.4 \times 10^{4}<\operatorname{Re}_{e}<1.6 \times 10^{5}$, therefore Equation 12.41 is valid over the range of experimental data.

The resultant heat transfer coefficient is:

$h_{\text {conv }}=\frac{k_{e f f}}{D_{H}}\left[\frac{(\mathrm{f} / 8) \operatorname{Re}_{e} \operatorname{Pr}}{1.07+12.7(\mathrm{f} / 8)^{1 / 2}\left(\operatorname{Pr}^{2 / 3}-1\right)}\right]$

where $k_{\text {eff }}$ is the thermal conductivity of the screen in the direction normal to the plane of the screen. An estimate of $k_{\text {eff }}$ is found from an equation given by Li and Peterson (2006):

$k_{\text {eff }}=\frac{k_{f}\left[k_{f}+k_{s}-(1-\varepsilon)\left(k_{f}-k_{s}\right)\right]}{\left[k_{f}+k_{s}+(1-\varepsilon)\left(k_{f}-k_{s}\right)\right]}$

where $k_{f}$ is the fluid thermal conductivity and $k_{s}$ is the thermal conductivity of the solid screen. $k_{f}$ was taken as the average of the liquid and gas thermal conductivities. $k_{s}$ is found from the conductivity temperature equation for 304SS from Marquardt et al. (2000):

$$
\begin{aligned}
\log \left(k_{s}\right) & =-1.409+1.398 \log T_{s}+0.254\left(\log T_{s}\right)^{2}-0.626\left(\log T_{s}\right)^{3}+0.233\left(\log T_{s}\right)^{4} \\
& +0.426\left(\log T_{s}\right)^{5}-0.466\left(\log T_{s}\right)^{6}+0.165\left(\log T_{s}\right)^{7}+0.020\left(\log T_{s}\right)^{8}
\end{aligned}
$$

where $T_{s}$ is the measured temperature on the liquid side of the screen. With Equations $12.42-12.44$, and the exposed screen length at breakdown, the heat transfer rate across the screen per unit length at breakdown is calculated from Newton's law of cooling: 
$\frac{\dot{Q}_{\text {conv }}}{L}=h_{\text {conv }} W\left(T_{s}-T_{\infty}\right)$

where $T_{\infty}$ is the free stream temperature in the channel, located at the bottom of the submerged portion of the LAD channel where the liquid temperature on either side of the screen is the same. Measurements of $T_{s}$ and $T_{\infty}$ were taken during each test so that Equations 12.44 and 12.45 could be evaluated. Results are plotted in Figure 12.15.

As shown in Figure 12.15, higher mass flow rates produce higher heat transfer coefficients and thus higher convective heat transfer rates across the screen. The heat transfer rates follow the same general trends as the heat transfer coefficients, except that there is more scatter due to the variation of $\left(T_{s}-T_{\infty}\right)$. As shown, even for relatively small outflow rates, for small exposed screen lengths, low-kW heat transfer per unit length is achievable for the $\mathrm{LAD}$ screen and channel in $\mathrm{LH}_{2}$.

\subsection{Concluding Remarks}

Based on first principles, an analytical model has been derived and validated to model the exact solution of cryogenic liquid flow through a porous LAD channel with one porous wall. The analytical model is solved from the Navier-Stokes equation using a modified form of the Kármán-Pohlhausen technique. Based on comparison to the literature, the new model improves upon previous solutions by 1) satisfying the no slip boundary condition at the channel dead end, 2) adding a turbulent term to the porous wall boundary condition governing the injection velocity, 3) allowing for arbitrary sized and variable suction or injection, and 4) extension of the model to predict cryogenic liquid flow through a LAD channel. 

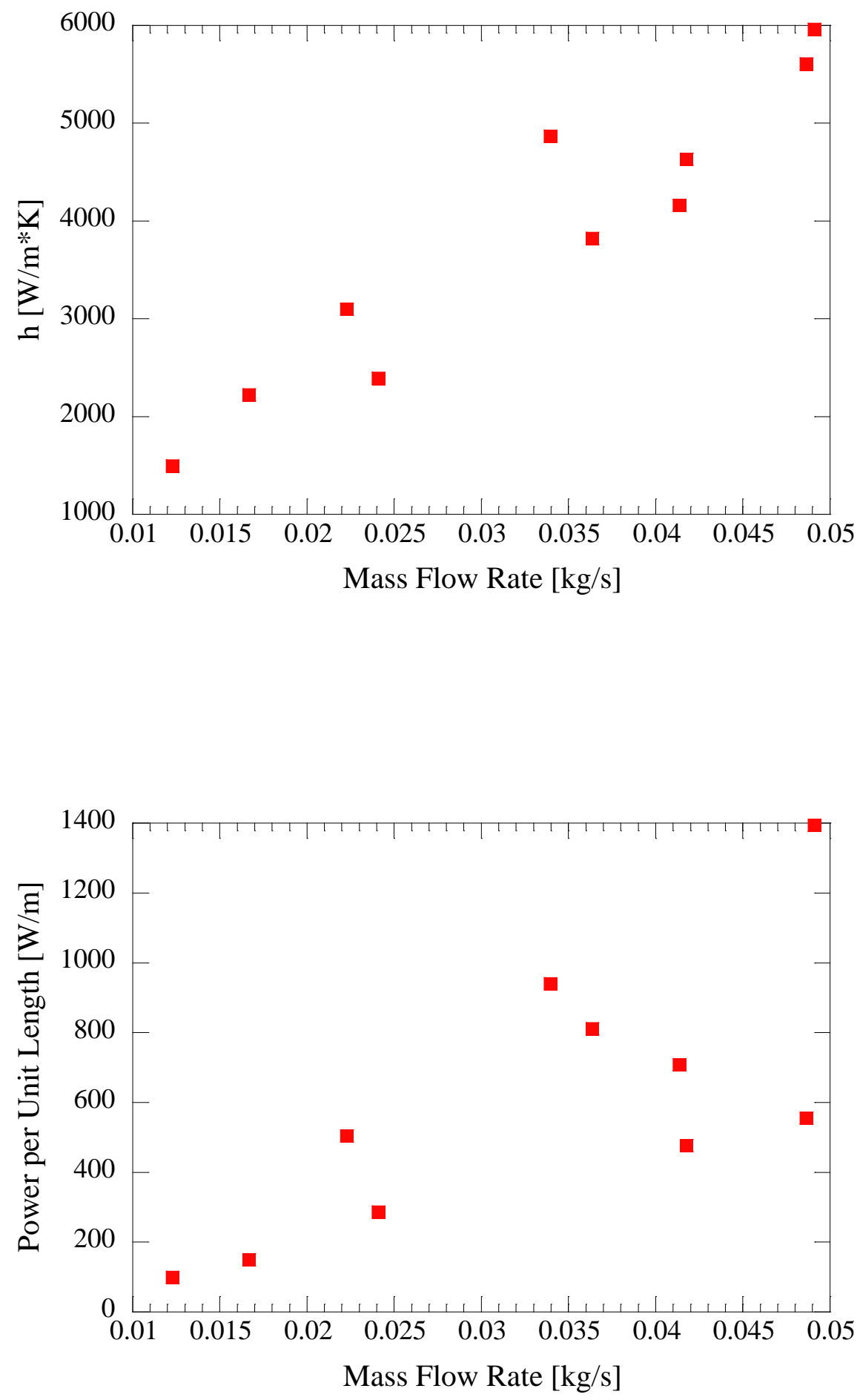

Figure 12.15 - a) Heat Transfer Coefficient and b) Power Per Unit Length across the Exposed Portion of the Screen as a Function of Mass Flow Rate through the Channel 
The solution shows that the velocity inside the channel becomes more concentrated near the outlet as the demand flow rate increases. At low Reynolds numbers, the resultant pressure drop across the screen is fairly linear, and at high Re numbers, the pressure field inside the channel becomes highly nonlinear. The implication is that, as the demand flow rate increases, the channel draws mass closest to the outlet, causing higher pressure drop across the screen near the outlet, with almost no mass entering closest to the channel dead end. Thus as demand flow rate increases, the nonlinear pressure distribution causes a higher susceptibility to breakdown near the channel outlet. Increasing both channel width and height act to prolong the breakdown point.

The raw analytical model, which is an exact solution to the flow through a finite channel with one porous wall, was used to determine velocity and pressure fields inside the LAD channel to simulate cryogenic propellant tank drain through the LAD. The code was validated through horizontal liquid oxygen LADs data as well as through $1 \mathrm{~g}$ inverted vertical outflow data in liquid hydrogen. Model validation against experimental data shows that the new model significantly improves over the simplified 1D model by accounting for non-uniform injection velocity and non-uniform frictional losses down the channel through solution of the general velocity and pressure fields as a function of the distance along the channel.

When compared to the liquid hydrogen outflow data, the difference between data and model is shown to be proportional to outflow rate. When the static bubble point model is updated with a new dynamic term, which takes into account the effect of enhanced convective cooling at the screen, the 1-g outflow model agrees well with the 
data. Heat transfer coefficient and convection heat transfer rates were also estimated for typical outflow rates for $\mathrm{LADs}$ in $\mathrm{LH}_{2}$. Combined with the cryogenic bubble point model, the new analytical model can easily be implemented into design and analysis tools for all future cryogenic liquid acquisition devices operating in microgravity conditions. 


\section{Chapter 13}

\section{Optimal Liquid Acquisition Device Screen Weave for a Liquid Hydrogen Fuel Depot}

Chapter 13 represents the first of two chapters of applying the analytical models of PMDs to real cryogenic propulsion systems. The purpose of this chapter is to apply the new cryogenic LAD modeling tools and present rationale for choosing the optimal LAD screen type and weave, and to present basic analysis and design for a LAD to be implemented into a large scale liquid hydrogen fuel depot. First the background is presented along with mission requirements. All LAD screens are initially considered for comparison. Then, from the set of influential factors from Chapter 3, bubble point pressure and FTS pressure drop are combined to define a critical mass flux used to make general comparisons between screens. Based off the minimum bubble point pressure to maintain adequate phase separation against adverse depot acceleration levels, the five finest Dutch Twill screens are then down selected for the depot. Next, the minimum screen area is defined and used to compare the remaining five screens. Then, secondary parameters such as wicking rate, screen compliance, and manufacturability are discussed to further compare performance between screens. Finally, the optimal LAD mesh is down selected and LAD channels are sized for the depot. 


\subsection{Background and Mission Requirements}

Due to very high performance relative to other propellant combinations, $\mathrm{LOX} / \mathrm{LH}_{2}$ fueled systems are most popular for implementation into future in-space cryogenic engines. A large payload like a space exploration vehicle can be transported to LEO by a sufficiently large launch vehicle, or sections of the payload can be launched separately and assembled in LEO. To continue travel out of LEO with cryogenic propellant as the fuel source, a large percentage of the vehicle mass at initial launch must be the propellant. For example, to travel from LEO to the surface of the moon would require the space vehicle mass while in $\mathrm{LEO}$ to be $75 \% \mathrm{LH}_{2} / \mathrm{LOX}(\mathrm{McLean}$ et al. 2011). In the case that the space vehicle is launched simultaneously with its propellant, the amount of propellant available to the space vehicle would be limited by the amount of mass that can be launched from the surface of the Earth at once. Therefore, the Earth departure speed that the space vehicle can achieve, dependent on the amount of propellant available in LEO, has an upper limit for this type of launch sequence. This speed barrier can easily be removed with the use of a cryogenic fuel depot. With higher Earth departure speeds, destinations like Mars and beyond can be reached faster.

The importance and role of cryogenic depots were outlined in Section 1.6.2. With a depot in orbit, a space vehicle can be launched without any propellant of its own into LEO, dock with the fuel depot, and then fill its own tank with the propellant stored in the depot. Large fuel depots can therefore contain a large percentage of the required propellant for a deep space mission, thus enabling longer duration missions. 
Because cryogenic fuel depots will be stored in the low Bond number conditions of LEO, LADs will be required to extract vapor free liquid from the depot. In high Bo number environments, gravity can be used to divide phases and even drive liquid out of the tank; in the low Bond number conditions of LEO, surface tension can be used to control the L/V interface. This is because the surface tension force of a liquid in the low Bond number environment of LEO is on the order of, or in some cases greater than, the hydrostatic force of the liquid. For example, for proposed liquid hydrogen fuel depots, the acceleration of gravity in LEO is on the order of $10^{-6} \mathrm{~m} / \mathrm{s}^{2}$. With a propellant tank hydrostatic length scale of $13.03 \mathrm{~m}$ from Table 13.1, an $\mathrm{LH}_{2}$ density and surface tension of $70.9 \mathrm{~kg} / \mathrm{m}^{3}$ and $1.952 \mathrm{mN} / \mathrm{m}$, respectively, the Bond number in LEO is 62, indicating that the surface tension force is comparable to the hydrostatic force. Therefore the same gravity-dominant method of L/V interface control on the ground is not possible in LEO.

\begin{tabular}{|c|c|c|}
\cline { 2 - 3 } \multicolumn{1}{c|}{} & Aerocapture & Propulsive Capture \\
\hline Depot Diameter & $7.8 \mathrm{~m}$ & $7.8 \mathrm{~m}$ \\
\hline Depot Length & $17.59 \mathrm{~m}$ & $36.22 \mathrm{~m}$ \\
\hline OF Ratio & 5.6 & 5.8 \\
\hline Length Ratio (LH2-to-LOX) & 2.86 & 2.76 \\
\hline Length of LH2 portion & $13.03 \mathrm{~m}$ & $26.58 \mathrm{~m}$ \\
\hline
\end{tabular}

Table 13.1 - Depot Sizing for a Mars Exploration Mission for Two Types of Architectures

A consequence of the large size of fuel depots is that relatively large accelerations (in excess of $0.1 \mathrm{~m} / \mathrm{s}^{2}$ ) will be expected during operation. This is due to the trend that larger satellites require larger accelerations for orbit and station keeping maneuvering. The importance of large accelerations will become important in the following sections when determining the optimum screen for the LAD. In addition, because the storage duration may last several years, and vehicle tanks will be large, propellant transfer will 
require relatively high flow rates, and the depot will be subject to large accelerations and vibrations in any direction. Thus vanes and sponge type PMDs are not recommended for large scale cryogenic fuel depots that experience high vehicle accelerations. The screen channel configuration can handle higher flow rates than vanes and sponges while also resisting vapor ingestion due to these random accelerations.

The effectiveness of a screen channel LAD will depend, among other things, on the thermodynamic state of the propellant. Analysis in this chapter will therefore consider a range of thermodynamic states of $\mathrm{LH}_{2}$ that eclipse the anticipated operating range of the fuel depot. The range will be $\mathrm{LH}_{2}$ at a saturation pressure of $100 \mathrm{kPa}(14.5 \mathrm{psia})$ to $\mathrm{LH}_{2}$ at a saturation pressure of $350 \mathrm{kPa}(50.8 \mathrm{psia})$. The corresponding ranges in temperature, density, viscosity, and surface tension are shown in Table 13.2. The upper limit on the mass flow rate range appropriate for $\mathrm{LH}_{2}$ fuel depots that will be considered in this analysis is $2.75 \mathrm{~kg} / \mathrm{s}$, consistent with scaling analysis performed and reported in Table 9.1 (Kutter 2011).

LADs are much more difficult to design for long term cryogenic liquid service than with storable propellants. As shown in all cryogenic experimental data in this dissertation, evaporation and condensation in cryogenic systems can alter the expected performance of LADs drastically from room temperature predictions. Significant amounts of phase change are due to the large temperature gradients that can occur in a cryogenic storage tank. Temperature gradients have such an impact on the overall performance of cryogenic storage and transfer that researchers have looked at the design of forced mixing (Ho and Rahman 2008 and 2012). Secondly, the surface tension of a cryogenic liquid is much lower compared to a storable propellant. NTO and MMH have 
surface tensions of $28.7 \mathrm{mN} / \mathrm{m}$ and $34.6 \mathrm{mN} / \mathrm{m}$, at $278 \mathrm{~K}$, respectively. $\mathrm{LOX}$ and $\mathrm{LH}_{2}$ have surface tensions of $13.1 \mathrm{mN} / \mathrm{m}$ at $90.19 \mathrm{~K}$ and $1.95 \mathrm{mN} / \mathrm{m}$ at $20.37 \mathrm{~K}$, respectively. Smaller values of surface tension diminish the performance of a given LAD by decreasing the bubble point and thus margin in LAD system design. As shown in cryogenic bubble point testing, the type of pressurant gas will also affect the performance of the LAD and therefore affect the design of the pressurization system of a fuel depot.

A strong candidate for the proposed $\mathrm{LOX} / \mathrm{LH}_{2}$ depot size for a manned Mars exploration mission is given by Tanner et al. (2006). Two different depot sizes were proposed, depending on the type of Mars approach - one for aerocapture and one for propulsive capture. The diameter, length, and $\mathrm{LOX}$-to- $\mathrm{LH}_{2}$ mass ratio, or oxidizer to fuel (OF) ratio, for both depot architectures are given in Table 13.1. With the ratio of LOX density to $\mathrm{LH}_{2}$ density at $P_{\text {sat }}=100 \mathrm{kPa}$ (approximately 16 ) and the $\mathrm{OF}$ ratio, the ratio of the $\mathrm{LH}_{2}$ tank to the length of the LOX tank can be determined (assuming both portions have the same diameter). With this ratio, the length of the depot dedicated to $\mathrm{LH}_{2}$ storage can be approximated, which is also included in Table 13.1.

\begin{tabular}{|c|c|c|}
\cline { 2 - 3 } \multicolumn{1}{c|}{} & Psat = 100kPa & Psat = 350kPa \\
\hline Temperature [K] & 20.32 & 25.42 \\
\hline Density [kg/m3] & 70.9 & 64.05 \\
\hline Viscosity [uPa·s] & 13.37 & 9.182 \\
\hline Surface Tension [mN/m] & 1.952 & 1.139 \\
\hline
\end{tabular}

Table 13.2 - Thermodynamic Properties of Liquid Hydrogen Which Bound Depot Operation

\subsection{Bubble Point Pressure and Flow-through-Screen Pressure Drop}

The two primary parameters that are influential to LAD screen selection for a particular mission as outlined in Chapter 3 are the bubble point pressure, $\Delta P_{B P}$ and the 
FTS pressure loss, $\Delta P_{F T S}$. The room temperature bubble point pressure and FTS pressure drop were modified for cryogenic fluids, and can be computed using Equations 10.18 and 3.33, respectively, using the appropriate model parameters and coefficients. The FTS pressure drop can be rewritten in the following form:

$\Delta P_{F T S}=\alpha X\left(\frac{\dot{m}}{A_{C}}\right)+\beta Y\left(\frac{\dot{m}}{A_{C}}\right)^{2}$

where $\mathrm{X}$ and $\mathrm{Y}$ are defined as:

$$
\begin{aligned}
& X=\frac{Q B \mu a^{2}}{\rho \varepsilon} \\
& Y=\frac{Q B}{\rho \varepsilon^{2} D_{P}}
\end{aligned}
$$

Temperature dependent values for $\alpha, \beta$ are in Chapter 9. The screen parameters can be evaluated from tables in Chapter 3.

The FTS pressure drop is also considered a primary performance parameter for screen channel LAD design due to the following rationale: From the steady state pressure drop analysis, in a 1-g environment, the largest pressure drop contribution is the hydrostatic head of the liquid inside the channel. In a microgravity environment, the FTS pressure loss is typically on the order of or much greater than the hydrostatic head. From Equation 13.1, as a propellant tank is drained, the area of the screen in contact with liquid decreases, and the FTS pressure drop increases proportionally. If the FTS pressure drop exceeds the bubble point value, the LAD will fail. This parameter is critical to LAD 
design, since finer meshes may produce higher bubble point pressures at the cost of higher FTS pressure drop.

Examination of Equations 10.18 and 13.1 indicates that a LAD designer will want a high bubble point value and a low FTS pressure loss, regardless of the mission. The application of interest in the current work considers low surface tension liquid acquisition and control in low Bond number conditions of LEO where these two parameters are essential for LAD design. These two primary parameters can be used to make general comparisons between all available LAD screen meshes in order to down select an appropriate subset of screens for the $\mathrm{LH}_{2}$ fuel depot.

Figure 13.1 plots the model predicted $\mathrm{LH}_{2}$ bubble point pressure values against the anticipated operating fuel depot liquid temperature range for several screen types using the new cryogenic bubble point model from Chapter 10. Figure 13.1a shows the bubble point predictions for the five finest Dutch Twill screens while Figures $13.1 \mathrm{~b}$ and 13.1c show the $\mathrm{LH}_{2}$ bubble point for much coarser Dutch Twill and Plain Dutch, and Twilled Square and Plain Square screens, respectively. Equation 10.18 was used to calculate the bubble point for all screens assuming a pressurant gas temperature equal to the liquid temperature and using GHe to pressurize the liquid held at the NBP. Comparing figures, the $450 \times 2750$ has the largest $\Delta P_{B P}$ of all screens over the range of operation. The $510 \times 3600$ has the next largest $\Delta P_{B P}$ and the $325 \times 2300$ has the third largest $\Delta P_{B P}$. The bubble points for the coarser meshes in Figures $13.1 \mathrm{~b}$ and $13.1 \mathrm{c}$ are significantly smaller than those for the fine Dutch Twill meshes. 

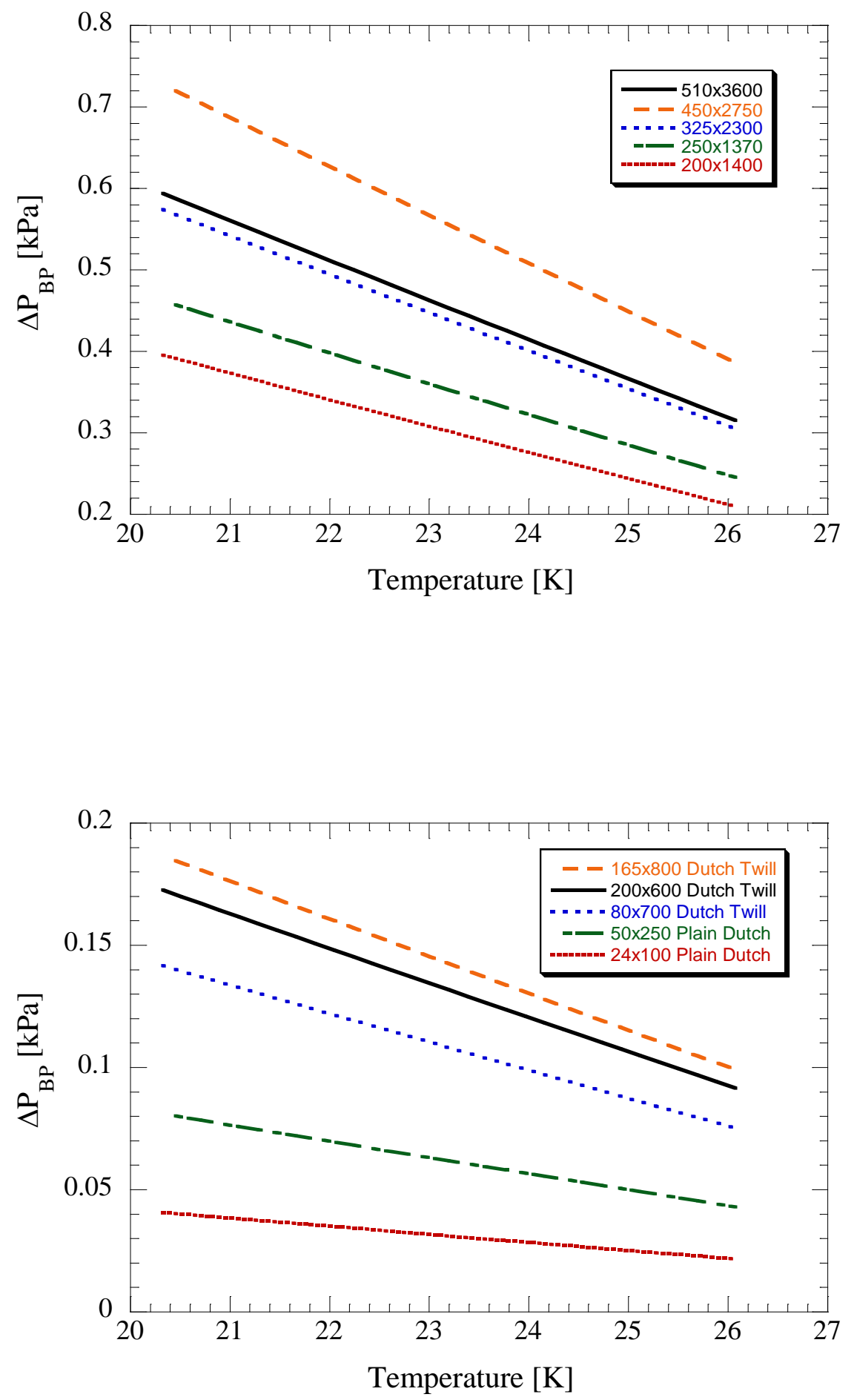


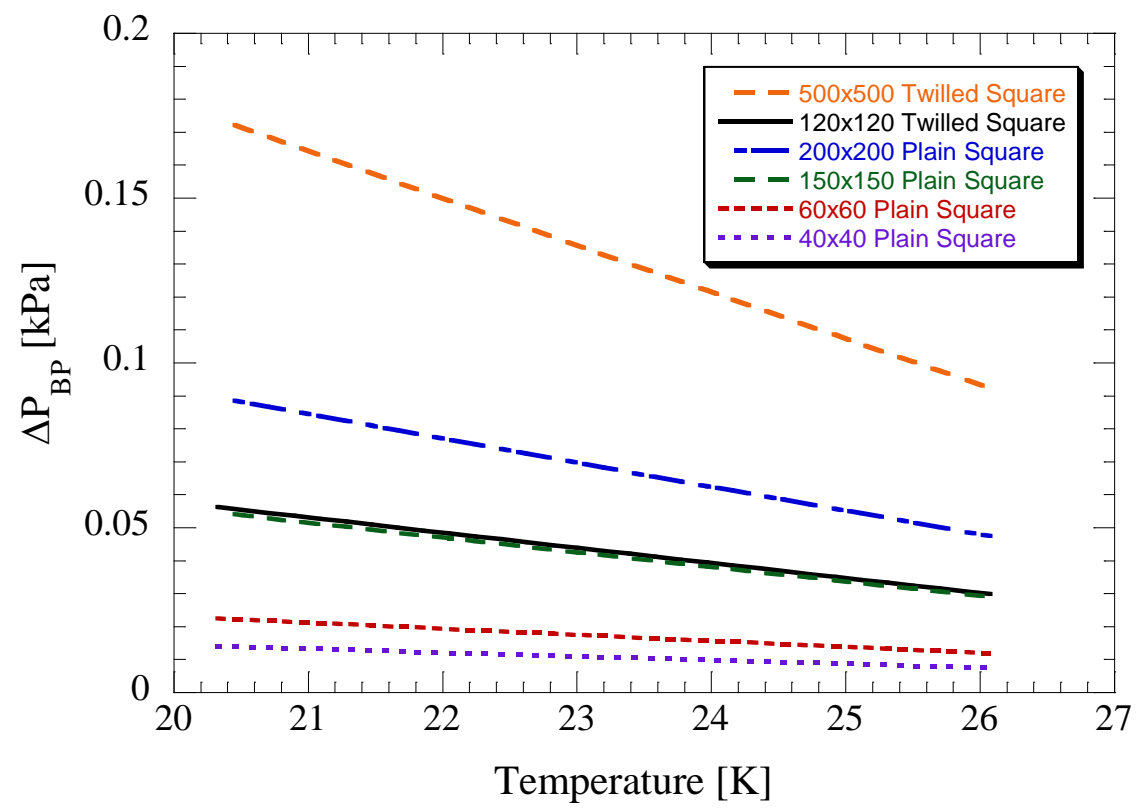

Figure 13.1 - Model Predicted Liquid Hydrogen Bubble Point Pressure Over the Anticipated Operating Range of a Cryogenic Fuel Depot for a) Five Finest Dutch Twill, b) Coarse Dutch Twill and Plain Dutch, and c) Twilled and Plain Square Mesh Screens. All curves assume GHe pressurant, with no subcooling, and $\mathrm{T}_{\mathrm{GAS}}=\mathrm{T}_{\text {LIQUID }}$.

Figure 13.2 shows the FTS pressure loss (evaluated at $\mathrm{LH}_{2}$ temperatures) versus $\mathrm{LH}_{2}$ mass flux for the same screens used in Figures 13.1. The mass flux is the mass flow rate of $\mathrm{LH}_{2}$ through the screen per unit cross sectional area of screen. A reference state of $\mathrm{LH}_{2}$ saturated at $100 \mathrm{kPa}$ is defined to evaluate density and viscosity. The FTS pressure loss predictions were plotted over a wide enough range in mass flux so that the plots included the critical mass flux, which will be discussed in Section 13.3. 

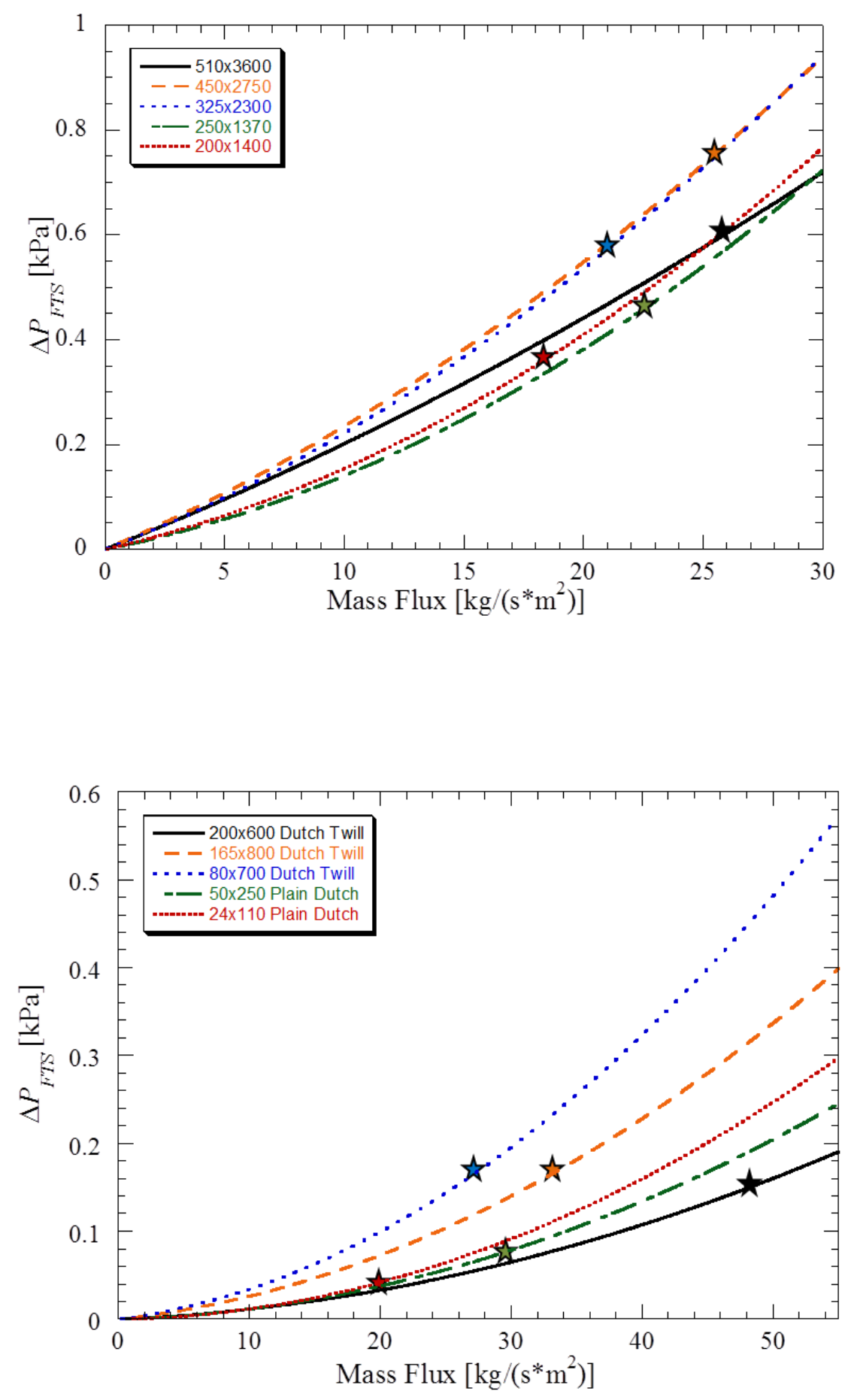


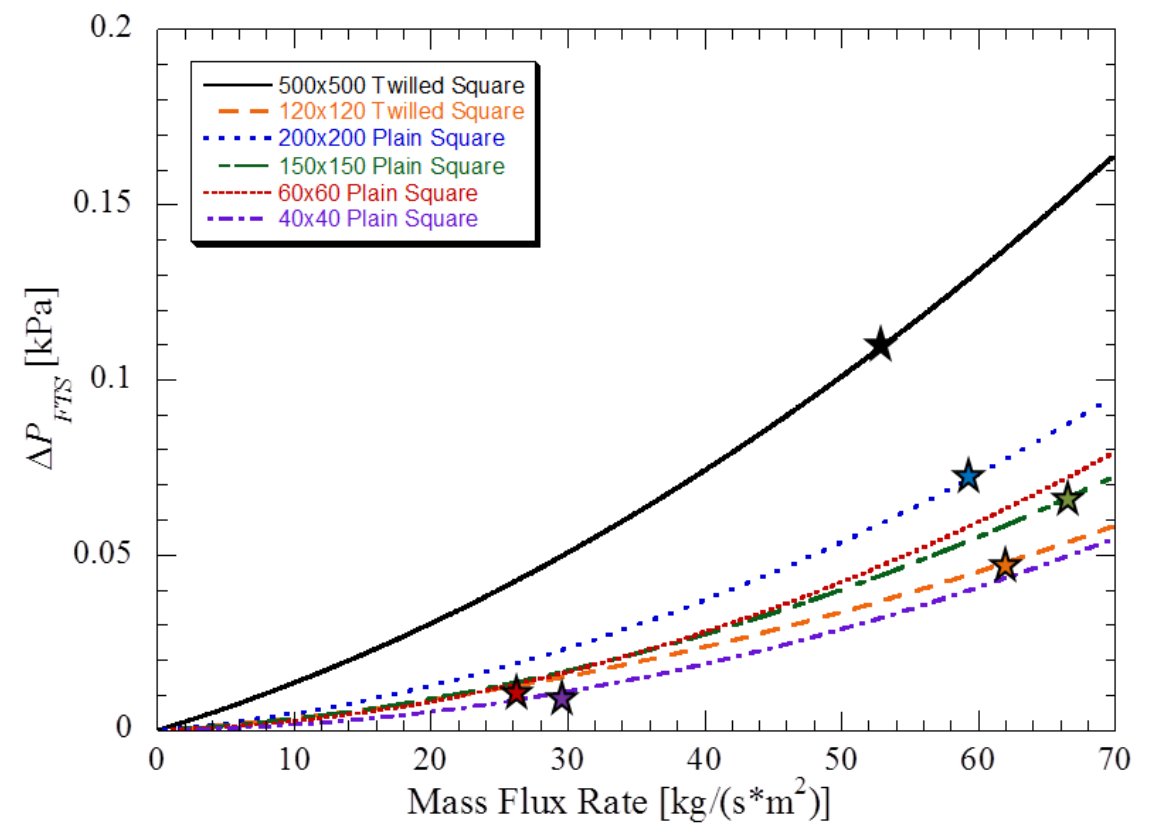

Figure 13.2 - Model Predicted Liquid Hydrogen Flow-through-Screen Pressure Drop Over the Anticipated Operating Range of a Cryogenic Fuel Depot for a) Five Finest Dutch Twill, b) Coarse Dutch Twill and Plain Dutch, and c) Twilled and Plain Square Mesh Screens. All curves evaluate $\alpha, \beta$ at liquid hydrogen temperatures.

\subsection{Critical Mass Flux}

For each screen in Figure 13.2, there is a star symbol placed at a pressure corresponding to the bubble point pressure of that screen for $\mathrm{LH}_{2}$ saturated at $100 \mathrm{kPa}$. This visual indicator ties together the FTS pressure drop and bubble point pressure by showing the amount of mass flux through a given screen that creates a FTS pressure drop equal to the bubble point of that screen. In other words, it indicates the lowest mass flux that will create vapor ingestion for a given screen and given operating conditions. Therefore this maximum allowable flux is denoted as the critical mass flux, which is valid for steady state outflow conditions, in which there are no other types of pressure loss besides the FTS pressure loss. Even though this is an idealized situation, it does 
serve as a useful parameter to compare between LAD screens. As seen from Figure 13.2a, the 510x3600 Dutch Twill has the highest critical mass flux among Dutch Twills. But, examination of Figures $13.2 \mathrm{~b}$ and $\mathrm{c}$ indicate that some of the Plain Dutch, Twilled Square, and Plain Square weaves can achieve much higher critical mass fluxes than the finer Dutch Twill meshes. A ranking of these screens based off of the critical mass flux is shown in Table 13.3. Before selecting the optimal screen based on this parameter, the minimum required bubble point of a LAD screen for the fuel depot must be examined.

\begin{tabular}{|c|c|c|c|}
\hline Ranking & Screen Type & Weave Type & Critical Mass Flux $\left[\mathrm{kg} /\left(\mathrm{m}^{\wedge} 2^{*} \mathrm{~s}\right)\right]$ \\
\hline 1 & $150 \times 150$ & Plain Square & 68.5 \\
\hline 2 & $120 \times 120$ & Twilled Square & 63.7 \\
\hline 3 & $200 \times 200$ & Plain Square & 61.8 \\
\hline 4 & $500 \times 500$ & Twilled Square & 54.4 \\
\hline 5 & $200 \times 600$ & Dutch Twill & 49 \\
\hline 6 & $165 \times 800$ & Dutch Twill & 34.5 \\
\hline 7 & $50 \times 250$ & Plain Dutch & 32.9 \\
\hline 8 & $40 \times 40$ & Plain Square & 31.2 \\
\hline 9 & $80 \times 700$ & Dutch Twill & 27.8 \\
\hline 10 & $60 \times 60$ & Plain Square & 27.7 \\
\hline 12 & $510 \times 3600$ & Dutch Twill & 25.7 \\
\hline 11 & $450 \times 2750$ & Dutch Twill & 25.2 \\
\hline 13 & $250 \times 1370$ & Dutch Twill & 22.7 \\
\hline 14 & $325 \times 2300$ & Dutch Twill & 20.9 \\
\hline 15 & $24 \times 110$ & Plain Dutch & 20.7 \\
\hline 16 & $200 \times 1400$ & Dutch Twill & 18 \\
\hline
\end{tabular}

Table 13.3 - List of Screen Meshes Ranked by Critical Mass Flux

\subsection{Minimum Bubble Point}

A propellant depot will be required to make station keeping maneuvers and adjustments to maintain its orbit. Therefore a screen must maintain phase separation during potential random accelerations of the fuel depot during maneuvering, station keeping, or settling. For storable propellants, the surface tension is large enough to provide an adequate barrier to vapor ingestion against adverse accelerations such that 
relatively coarse screens can be implemented to meet mission demands. For $\mathrm{LH}_{2}$, however, the surface tension is so low that the bubble points of coarse screens are likely too small, and bubble breakthrough is almost guaranteed during typical fuel depot maneuvering. Table 13.4 shows the bubble point for Dutch Twill, Plain Dutch, Twilled Square, and Plain Square weaves for $\mathrm{LH}_{2}$ saturated at $100 \mathrm{kPa}$.

\begin{tabular}{|c|c|c|}
\hline Screen Type & Weave Type & LH2 NBP Bubble Point, kPa \\
\hline $510 \times 3600$ & Dutch Twill & 0.5939 \\
\hline $450 \times 2750$ & Dutch Twill & 0.7279 \\
\hline $325 \times 2300$ & Dutch Twill & 0.5743 \\
\hline $250 \times 1370$ & Dutch Twill & 0.4621 \\
\hline $200 \times 1400$ & Dutch Twill & 0.3954 \\
\hline $200 \times 600$ & Dutch Twill & 0.1726 \\
\hline $165 \times 800$ & Dutch Twill & 0.1867 \\
\hline $80 \times 700$ & Dutch Twill & 0.1417 \\
\hline $50 \times 250$ & Plain Dutch & 0.0810 \\
\hline $24 \times 110$ & Plain Dutch & 0.0407 \\
\hline $500 \times 500$ & Twilled Square & 0.1741 \\
\hline $120 \times 120$ & Twilled Square & 0.0563 \\
\hline $200 \times 200$ & Plain Square & 0.0895 \\
\hline $150 \times 150$ & Plain Square & 0.0546 \\
\hline $60 \times 60$ & Plain Square & 0.0225 \\
\hline $40 \times 50$ & Plain Square & 0.0141 \\
\hline
\end{tabular}

Table 13.4 - Liquid Hydrogen Bubble Point Pressure Computed at the Normal Boiling Point for Candidate Screens for the Fuel Depot. All values assume GHe pressurant, no subcooling, and

$$
\mathrm{T}_{\mathrm{GAS}}=\mathrm{T}_{\mathrm{LIQUID}}
$$

A calculation to show the expected pressure difference due to random accelerations is given here: Consider a random acceleration of $0.1 \mathrm{~m} / \mathrm{s}^{2}$. For $\mathrm{LH}_{2}$ saturated at $100 \mathrm{kPa}$, the density is approximately $70.9 \mathrm{~kg} / \mathrm{m}^{3}$. Assuming the length of the LAD is the approximately the length of the tank in Table $13.1,26.58 \mathrm{~m}$, the hydrostatic head produced by this random acceleration for a completely liquid filled channel is equal to $0.187 \mathrm{kPa}$. From Table 13.4 it can be seen that, except for the five finest Dutch Twill 
screens - 510x3600, 450x2750, 325x2300, 250x1370, and 200x1400 - all other screens have bubble points that are less than $0.187 \mathrm{kPa}$ and are thus susceptible to vapor ingestion simply due to a random acceleration of this magnitude. Therefore only the five finest Dutch Twill screens can be considered as potential candidates for an optimal LAD screen for a liquid hydrogen fuel depot for accelerations of this magnitude. Coarser screens could be considered if vehicle maneuvering were less.

Another factor that supports the use of a finer screen in an $\mathrm{LH}_{2}$ fuel depot is the effect of heat and mass transfer across the screen. As shown experimentally from the 200x1400 and 325x2300 LOX bubble point tests in Chapter 6, as well as heated pressurant gas $\mathrm{LH}_{2}$ and $\mathrm{LN}_{2}$ bubble point tests in Chapter 8 , it can confidently be concluded that finer screens are able to provide more of a thermal barrier between the liquid and gas zones. Therefore finer screens are able to resist heat and mass transfer across the screen, minimizing the degradation of performance due to unwanted heat leak. While coarser screens are generally easier to integrate into full LAD channel designs, a mission designer should not hastily or blindly apply storable LAD design logic to cryogenic LAD systems where coarser screens experience higher degradation in performance due to heat transfer effects, which is a negligible issue in storable propulsion systems.

\subsection{Minimum Screen Area}

The required minimum screen area performance parameter is now introduced to determine which of the five finest Dutch Twill meshes is the most robust option for the cryogenic fuel depot. To illustrate the minimum area, consider the situation where a 
screen channel LAD completely surrounded by liquid is in steady state outflow under zero-gravity conditions, as shown in Figure 13.3. Neglecting any frictional losses down the channel, at steady state, the only pressure loss is due to the FTS pressure drop. This is a simplified situation, but it will help compare the coupled bubble point and FTS pressure loss performance between different Dutch Twill screen types.

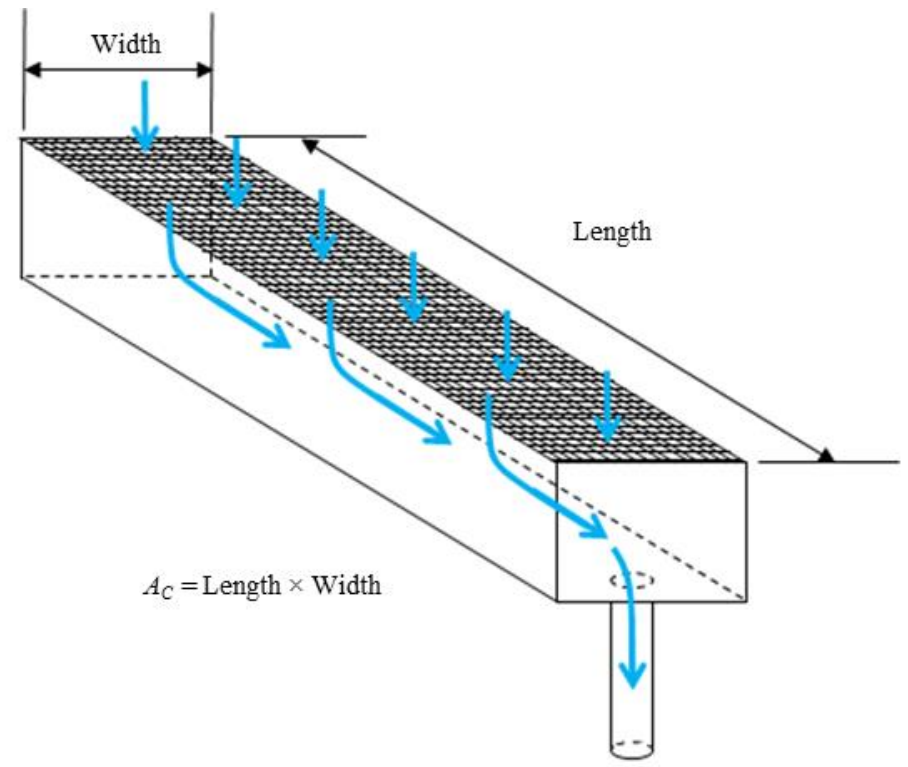

Figure 13.3 - A Screen Channel Liquid Acquisition Device under Steady Flow Conditions with Negligible Gravitational Force. The cross sectional area of the screen channel LAD is the length multiplied by the width.

In order to resist vapor ingestion, $\Delta P_{F T S}$ must be less than $\Delta P_{B P}$. All terms in Equation 13.1 are constant for a given screen type and fluid except for $\dot{m}$ and $A_{C}$. This leads to the following design criteria: for a given flow rate $\dot{m}, A_{C}$ must be large enough so that $\Delta P_{F T S}<\Delta P_{B P}$. This minimum required flow-area, $A_{C, \text { min }}$, can be solved for in terms of the flow rate, screen properties, and fluid properties by setting the bubble point equal to the maximum allowed FTS pressure loss $\Delta P_{F T S-M A X}$ : 


$$
\Delta P_{B P}=\Delta P_{F T S-M A X}=\alpha X\left(\frac{\dot{m}}{A_{C, \text { min }}}\right)+\beta Y\left(\frac{\dot{m}}{A_{C, \text { min }}}\right)^{2}
$$

A more realistic design criterion would account for other sources of pressure loss in addition to $\Delta P_{F T S}$, such as transient and hydrostatic pressure losses. The variation of $\Delta P_{F T S}$ with distance from the inlet should also be considered in a rigorous screen LAD design, using the code from Chapter 12. This analysis is useful to the designer, however, for two main reasons. First, in most cases for microgravity outflow (except for startup and accelerated maneuvering), $\Delta P_{F T S}$ is the leading order pressure loss term and therefore equating the bubble point pressure to $\Delta P_{F T S}$ is an acceptable first estimate. The term $A_{C, \text { min }}$ therefore serves as a good first estimate of the minimum required cross sectional area of the screen required to maintain $\Delta P_{F T S}$ equal to or below the bubble point.

Secondly, a quantitative performance metric, $A_{C, \min }$, can be determined for each screen type that combines the bubble point and FTS pressure loss parameters. Solving for $A_{C \text {,min }}$ from Equation 13.4:

$$
A_{C, \min }=\left[\frac{\sqrt{\alpha^{2} X^{2}+4 \beta \Delta P_{B P} Y}+\alpha X}{2 \Delta P_{B P}}\right] \dot{m}=Z \dot{m}
$$

This is a complicated equation in terms of screen parameters and fluid properties (represented by $Z$ ), but it is a simple linear relationship with mass flow rate. For a given fluid at known thermodynamic conditions and a desired mass flow rate, $A_{C, \min }$ can be compared between screen types by plugging in the screen parameter values for each screen. A low value of $Z$ is desirable from a design standpoint because a low $Z$ value 
implies that for a given flow rate there is less required screen area to keep the FTS pressure loss below the bubble point. Less required area means less system mass and therefore less launch costs and material costs.

An example is carried out for a screen channel flowing saturated $\mathrm{LH}_{2}$ at $100 \mathrm{kPa}$. Using the values of density, viscosity, and surface tension at this thermodynamic state along with screen parameter values from Chapter 3, Table 13.5 was constructed in order to compare $Z$ values between the five finest Dutch Twill screen types. Figure 13.4

illustrates the same trends by plotting $A_{C, \min }$ from zero mass flow rate up to $2.75 \mathrm{~kg} / \mathrm{s}$ for the five finest Dutch Twill screens.

\begin{tabular}{|c|c|c|}
\hline Screen Type & Weave Type $\mathbf{Z}$ [m²s/kg] \\
\hline $510 \times 3600$ & Dutch Twill & 0.023 \\
\hline $450 \times 2750$ & Dutch Twill & 0.0269 \\
\hline $325 \times 2300$ & Dutch Twill & 0.0486 \\
\hline $250 \times 2750$ & Dutch Twill & 0.0357 \\
\hline $200 \times 1400$ & Dutch Twill & 0.0392 \\
\hline
\end{tabular}

Table 13.5 - Computed Z Values for the Five Finest Dutch Twill Screens in Liquid Hydrogen

Of the five finest Dutch Twill screens the finest screen type, the 510x3600, has the lowest $Z$ value. The $450 \times 2750$ is a close second. At $2.75 \mathrm{~kg} / \mathrm{s}, A_{C \text {, min }}$ for the $510 \times 3600$ is about $15 \%$ lower than for the $450 \times 2750$. The relatively low FTS pressure loss of the 510x3600, due to its high void fraction, outweighs its lower bubble point relative to the $450 \times 2750$. This does not indicate that the 510x3600 will have a lower minimum required area for every liquid compared to the other screens. $Z$ is a function that depends, among other things, on fluid properties and it is theoretically possible that there exists a fluid that will give the 450x2750 a lower $Z$ value than the 510x3600. 


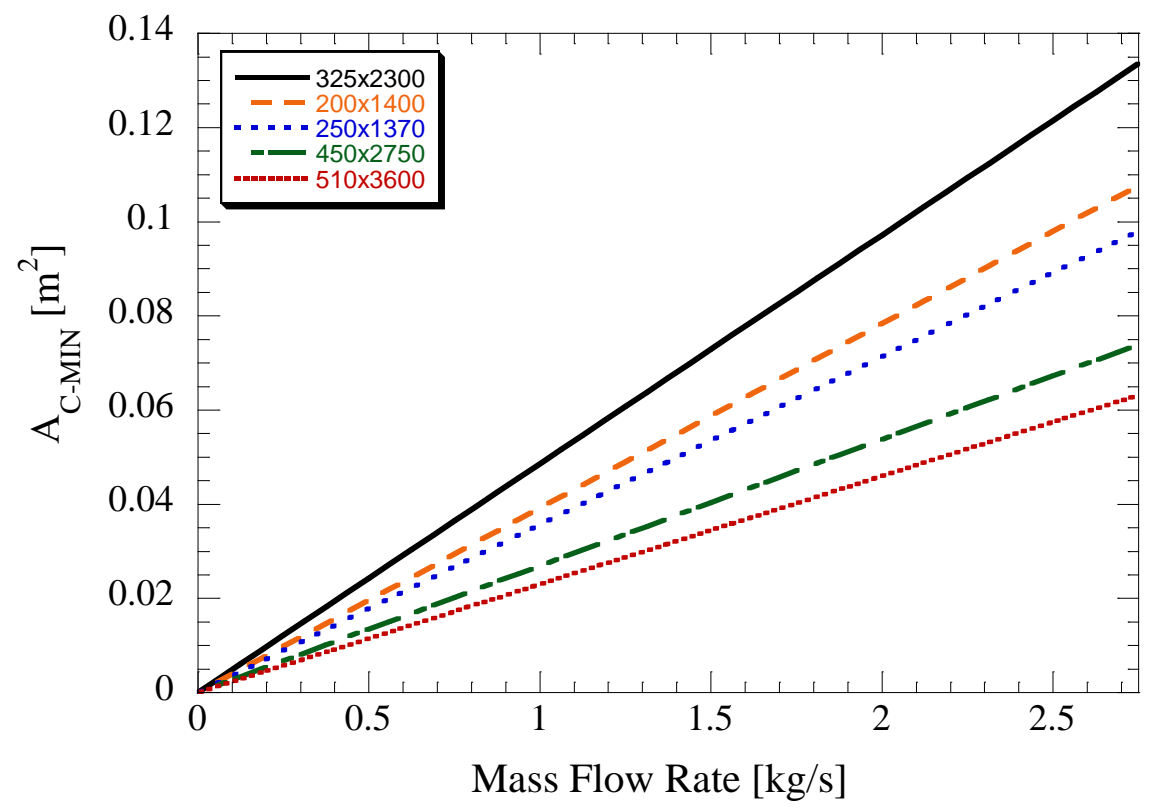

Figure 13.4 - Minimum Cross Sectional Area versus Demand Mass Flow Rate for the Five Finest Dutch Twill Screens in Liquid Hydrogen

\subsection{Other Considerations}

The $510 \times 3600$ and the $450 \times 2750$ are the outstanding choices for the optimal screen type due to the low $Z$ values compared to the other fine mesh Dutch Twill screens. There are compelling reasons to declare the $450 \times 2750$ as more optimal than the $510 \times 3600$, and vice versa. On the one hand, the $450 \times 2750$ has a higher bubble point than the 510x3600. Thus it will be able to resist vapor ingestion due to higher random accelerations. On the other hand, the $510 \times 3600$ has a slightly lower $Z$ value than the $450 \times 2750$. For a target flow rate of a $\mathrm{LH}_{2}$ fuel depot, $2.75 \mathrm{~kg} / \mathrm{s}$, this equates to a $15 \%$ smaller $A_{C \text {, min }}$ than the $450 \times 2750$. From this, one could argue that the 510x3600 screen channels will require up to $15 \%$ less flow area for a given flow rate than a LAD using $450 \times 2750$ screen channels. For a total screen channel LAD assembly, the mass savings 
from using $510 \times 3600$ over the $450 \times 2750$ screen could be significant as the depot grows in size. However, this mass savings is minimal, and secondary influential design parameters from Chapter 3 must be considered to down select the optimal screen.

First, the wicking rate is compared between each screen. Recall that another advantage of screen channel LADs is the ability to wick liquid to areas of the screen that dry out due to evaporation during tank pressurization and outflow. This property of a screen is essential for cryogenic systems because of the propensity to experience dry out due to unwanted heat leak. A higher wicking rate is desired so that the LAD can counteract higher evaporation rates. The $450 \times 2750$ has a $130 \%$ higher wicking rate perpendicular to the warp wires and a $16 \%$ higher wicking rate parallel to the warp wires over the $510 \times 3600$. Therefore the $450 \times 2750$ is much more desirable from a wicking rate perspective.

Second, the effect of screen compliance is compared between the two screens. Recall that screen compliance is the amount of deflection of the screen that can occur at the transient start of flow from the propellant tank to the transfer line through a LAD screen. A large deflection can distort the screen pores to such a degree that vapor is ingested at a portion of the screen in contact with pressurant gas. Paynter (1973a) found that when a pressure difference was applied across $325 \times 2300$ and 200x 1400 screens of the same material, the $325 \times 2300$ deformed more than the $200 \times 1400$. This supports the idea that finer meshes experience larger deflections than coarser meshes for the same pressure drop. This was also noticed visually during $\mathrm{LH}_{2}$ bubble point tests in Chapter 5 .

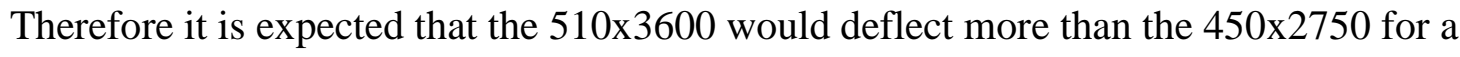
given pressure drop. This is a drawback to the $510 \times 3600$ when compared to the 
450x2750 since, as pointed out earlier, a larger screen deflection can increase the likelihood of vapor ingestion and potentially inflict more damage to the screen.

Lastly, manufacturability of the screen is compared between the two screens. It has been shown that for screens finer than the 250x1370, which include the 450x2750 and the $510 \times 3600$, the only available material for screen fabrication is SS. Al and Ti versions of the 450x2750 and 510x3600 are not possible as limited by current manufacturing methods. Therefore there is no difference between the $450 \times 2750$ and the $510 \times 3600$ in terms of the manufacturability.

The amount of drawbacks to the $510 \times 3600$ outweighs the fact that the $510 \times 3600$ will require slightly less material than the $450 \times 2750$ for a given flow rate for the $\mathrm{LH}_{2}$ fuel depot LAD. These include:

1. The $450 \times 2750$ has a much higher bubble point than the $510 \times 3600$ and thus will be able to resist vapor ingestion against higher accelerations

2. The $450 \times 2750$ is able to counteract larger evaporation rates than the $510 \times 3600$ due to its higher wicking rate

3. The deflection of a $450 \times 2750$ screen is smaller for a given pressure drop than the 510x3600, indicating that it will be less susceptible to vapor ingestion at start up.

Therefore, the $450 \times 2750$ is chosen as the optimal screen for the large scale $\mathrm{LH}_{2}$ fuel depot.

\subsection{Channel Number and Size}


As a final exercise, a 450x2750 LAD system is sized for a hypothetical $\mathrm{LH}_{2}$ fuel depot for a manned mission to Mars. With the length and diameter of the $\mathrm{LH}_{2}$ tank given by Table 13.1, channels can be sized for different screens. A worst case scenario, where only $10 \%$ of one channel has liquid contact on both sides of the screen and thus only $10 \%$ cross sectional flow area, will be examined. This scenario may be encountered when the liquid amount in the tank is very low and the liquid is mostly located at one side of the tank. The bubble point of the screen is set equal to the FTS pressure loss. Hence, this analysis signifies that screen breakdown is imminent when an $\mathrm{LH}_{2}$ depot has reached $10 \%$ liquid coverage of a single LAD channel. This is for demonstrative purposes and does not suggest that this is the best method of sizing fuel depot. Furthermore, assume that one side of the channel is porous screen while the remaining three are solid. Lastly, it will be assumed that the length of the channel is equal to the length of the $\mathrm{LH}_{2}$ tank. Since $\Delta P_{B P}$ is set equal to $\Delta P_{F T S}$, Equation 13.5 can be used to solve for the crosssectional area of the screen that represents $10 \%$ of the total screen channel LAD. The required area of the entire screen channel is then found by multiplying this by 10 . The required area of the entire LAD channel for each screen is shown in Table 13.6. The max flow rate was assumed to be $2.75 \mathrm{~kg} / \mathrm{s}$.

Since the length of the LAD channel is fixed $(13.03 \mathrm{~m}$ for aerocapture, $26.58 \mathrm{~m}$ for propulsive capture) the width of the LAD channel can be determined by simply dividing the area of the screen channel LAD by the length. The total amount of required area is the same as $A_{C, \min }$. The results are included in Table 13.6. As expected, the channel width is the smallest for the 510x3600 followed by the 450x2750, regardless of the Mars approach type. This shows that a screen channel LAD built with either of these two screen types 
would require less material than a screen channel LAD built with any of the other fine mesh Dutch Twill screens. Therefore, the lightest possible liquid transfer system for a $\mathrm{LH}_{2}$ fuel depot would consist of 510x3600 screens, and the next lightest possible system would consist of $450 \times 2750$ screens. Note that this analysis does not take into account issues that may arise due to channel fabrication with these fine screens.

\begin{tabular}{|c|c|c|c|c|}
\hline & & & Channel & Width [cm] \\
\hline Screen Type & Weave Type & Total Required Screen Area [m^^] & Aero Capture & Propulsive Capture \\
\hline $510 \times 3600$ & Dutch Twill & 1.379 & 10.59 & 5.189 \\
\hline $450 \times 2750$ & Dutch Twill & 1.614 & 12.38 & 6.071 \\
\hline $325 \times 2300$ & Dutch Twill & 2.915 & 22.37 & 10.97 \\
\hline $250 \times 1370$ & Dutch Twill & 2.14 & 16.42 & 8.051 \\
\hline $200 \times 1400$ & Dutch Twill & 2.35 & 18.03 & 8.841 \\
\hline
\end{tabular}

Table 13.6 - Required Minimum Screen Area and Channel Width for Two Different Liquid Hydrogen Depot Concepts at a Demand Flow Rate of $2.75 \mathrm{~kg} / \mathrm{s}$

\subsection{Concluding Remarks}

Analysis based on the new cryogenic analytical tools have been used to determine the optimal screen weave and channel size and number for a large scale liquid hydrogen fuel depot in Low Earth Orbit. The 450x2750 Dutch Twill screen is shown to be the optimal screen type for a large scale liquid hydrogen fuel depot that experiences large accelerations due to vehicle maneuvering in LEO. Coarser screens like the 200x600 Dutch Twill have a higher critical mass flux, but are disregarded because the low $\mathrm{LH}_{2}$ bubble point values will not maintain phase separation against the expected large adverse accelerations during depot maneuvering. In addition coarser screens are more susceptible to heat and mass transfer across the screens. Of the five screens with large enough bubble point values to be considered, the 510x3600 Dutch Twill has the lowest $Z$ value (the 
$450 \times 2750$ has the second lowest $Z$ value) and therefore is the optimal screen in terms of minimum required screen area. This is outweighed, however, by the fact that the 450x 2750 outperforms the 510x3600 in bubble point, wicking rate, and screen compliance. Therefore, the $450 \times 2750$ is the optimal LAD screen type for a large scale $\mathrm{LH}_{2}$ fuel depot experiencing large random acceleration levels. The analysis conducted in this chapter provides the rationale and logic for choosing an appropriate LAD mesh style and screen for any mission. 


\section{Chapter 14}

\section{Optimal Propellant Management Device for a}

\section{Small Scale Liquid Hydrogen Propellant Tank}

Chapter 14 represents the second chapter of applying the analytical models of PMDs to real cryogenic propulsion systems. The purpose of this chapter is to present the results of a trade study to compare performance of two different cryogenic propellant management devices inside a small scale liquid hydrogen storage tank in the microgravity of space. The purpose of the trade study is to determine operating conditions under which a vane type and a screen channel LAD type are the optimal PMD.

First the background is presented. Then, analytical models are then developed from first principles for both PMDs. The 1-g analytical flow model for screen channel LADs is modified to predict velocity and pressure fields inside a propellant tank in microgravity. Several different ullage bubble growth rates and initial locations within the tank are modeled. When coupled with the cryogenic bubble point model and ullage bubble models, the analytical flow code is used to simulate propellant tank drain in microgravity. A vane analytical model is also developed for microgravity fluid flow. Next, trade study variables are presented. Then, the analytical models are used to compare PMD size, mass, and expulsion efficiency across a wide range of demand flow 
rates out of the tank. Finally, a critical flow rate range is defined below which vanes are the optimal PMD, above which screen channel LADs are the optimal choice for a small scale liquid hydrogen propellant tank. Throughout the text, the term gallery arm is treated synonymously with a screen channel LAD.

\subsection{Background and Mission Requirements}

Full scale in-space cryogenic engines and cryogenic depots represent the highest possible level of technology maturation of level 9 on the TRL scale for CFM technology. TRL-9 implies that the technology has been implemented into an actual flight mission. Analysis performed in Chapters 3, 9, and 10, and component level and full scale ground experiments conducted in Chapters $4-9$ raise the TRL of LADs in cryogenic liquids from TRL-1 to TRL-5. TRL-6 represents a full integrated system test in 1-g while TRL-7 represents a technology demonstration mission in a relevant environment, in microgravity.

Before full scale depots can be fully realized, cryogenic propellant storage and transfer must first be demonstrated using a smaller scale propellant tank to understand and mitigate all the issues associated with storage and transfer of $\mathrm{LH}_{2}$. Any flight TDM would inevitably raise the TRL of LADs from TRL-5 to TRL-7, because the mission represents the final hurdle before enabling full scale depots. Analysis was presented for sizing a screen channel LAD for a full scale depot in Chapter 13 where the mission requirements dictated the use of the screen channel LAD over the vane or sponge type PMD. Therefore this chapter focuses on modeling propellant flow out of a small scale (relative to a full scale fuel depot) $\mathrm{LH}_{2}$ tank and considers using either a screen channel 
or vane type PMD. Steady state governing equations for screen channel LADs and vanes are presented, a trade study is conducted to compare performance, and the operating conditions under which vanes and screen channels are the optimal choice are presented for this smaller scale system.

The design concept, basic flow physics, principle of operation, and advantages and disadvantages of vanes, sponges, and screen channel LADs were outlined previously in Chapter 2. Sponges are not considered in this trade study because it was not desired to control the location of the ullage bubble. In summary, the four advantages of implementing vanes over galleries are simplicity, low mass, low manufacturing cost, and high reliability. Disadvantages of a vane PMD are that it cannot supply medium to large demand flow rates and cannot sustain vapor free liquid flow under medium to high adverse acceleration levels. Meanwhile the advantages of using screen channel galleries over vanes include higher flexibility in design, robustness, and the ability to maintain and acquire any flow rate over any adverse acceleration level. Disadvantages of the screen channel type PMD include added cost, mass, complexity, and less overall reliability relative to vanes.

The performance of any PMD is characterized by 3 primary parameters: expulsion efficiency, demand flow rate, and PMD system mass. The expulsion efficiency is the percentage of propellant that a given PMD system can remove from the tank before vapor is admitted into the transfer line, which from Chapter 2 is defined as the ratio of the tank volume minus liquid volume left in the propellant tank at the time of PMD breakdown versus the tank volume. The demand flow rate is a specified mass flow rate that determines how quickly the tank needs to be drained. The mass of the PMD system 
is a result of the desired demand flow rate or expulsion efficiency, and generally changes with the size and number of arms for the PMD. In general, the higher the desired demand flow rate, the lower the expulsion efficiency, unless the size of the PMD is increased. Therefore, for a given desired demand flow rate, an optimal PMD design is one that maximizes the expulsion efficiency while minimizing the mass of the PMD.

The performance of the two PMDs, screen channels and vanes, is analyzed by comparing ability and efficiency in draining a small scale cryogenic propellant tank in microgravity. The $\mathrm{LH}_{2}$ propellant tank is defined as a cylindrical tank with a volume of $4.05 \mathrm{~m}^{3}$, a length of $2.29 \mathrm{~m}$ (90 inches), and a radius of $0.762 \mathrm{~m}$ (30 inches). Compare these dimensions to the dimensions of the full scale depot tank defined in Table 13.1. An illustration of the screen channel and vane type PMDs mounted inside the cylindrical tank is shown in Figures 14.1a and b, respectively.

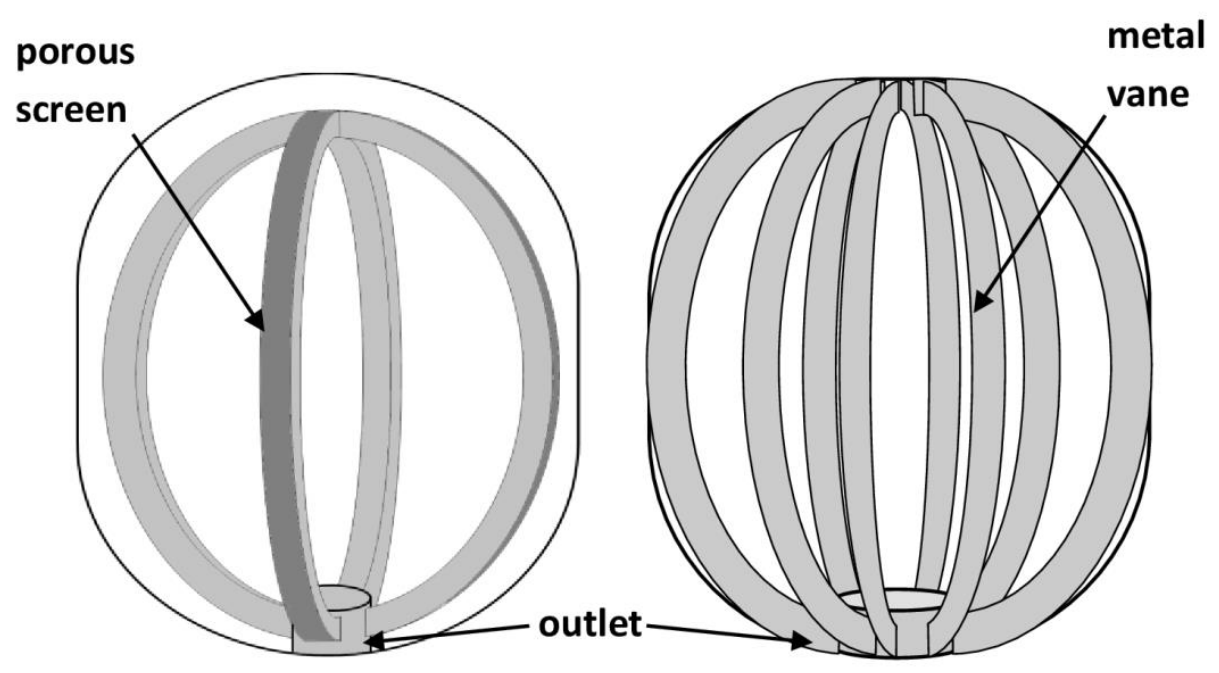

Figure 14.1 - a) Screen Channel and b) Vane Propellant Management Devices Mounted Inside the Cylindrical Liquid Hydrogen Propellant Tank 


\subsection{Analytical Screen Channel Flow Model in Microgravity}

\subsubsection{Extension of 1-g Model to Microgravity}

The analytical flow model from Chapter 12 that was used to predict pressure and velocity fields within the LAD channel during propellant tank drain in 1-g can be extended to microgravity. The primary difference between the two gravity levels is the location of the tank $\mathrm{L} / \mathrm{V}$ interface and growth rate of the ullage bubble within the tank as the tank drains, as illustrated in Figure 14.2. In 1-g, the location of the L/V interface is well known. Gravity forces the denser liquid to the bottom of the tank separating it from the vapor, creating a distinct L/V interface. As shown in Figure 14.2a, there are two regions to model for the LAD channel, a submerged portion and a portion exposed to vapor. In Region 1, the LAD is submerged in liquid, and the channel is filled with liquid, creating a liquid-liquid region at the screen. In Region 2, the LAD screen is exposed to vapor, and the channel is filled with liquid, creating a liquid-vapor region. In Region 1, the FTS pressure drop and frictional pressure drop are coupled together through the analytical flow model. For Region 2, the pressure drop is the sum of the hydrostatic, frictional, and dynamic pressure drop terms as defined in the steady state 1D pressure drop model. A depiction of the flow profile as the liquid transitions from Region 1 to Region 2 is shown to the right of the tank and LAD in Figure 14.2a.

Modeling the flow profile in LEO, however, becomes slightly more complex because in microgravity, it is generally difficult to determine the location of the $\mathrm{L} / \mathrm{V}$ interface. Surface tension forces dominate and the liquid tends to gather near the tank walls. The vapor tends to collect centrally into a bubble, which could initially be located 
anywhere in the tank. In an idealized case, after sufficiently long times in microgravity, in an unsettled configuration, the ullage bubble will tend toward the center of the tank. As the ullage bubble grows, it exposes the LAD from the middle and creates a region in which there is zero injection velocity, which forms the boundary between Regions 1 and 3 shown in Figure 12.2b. This introduces a new unknown velocity, which is deemed the interface velocity, $u_{\text {interface }}$. Because there is no mass injection in the channel in Region 2, the average velocity in Region 2 will decrease only slightly due to frictional effects.

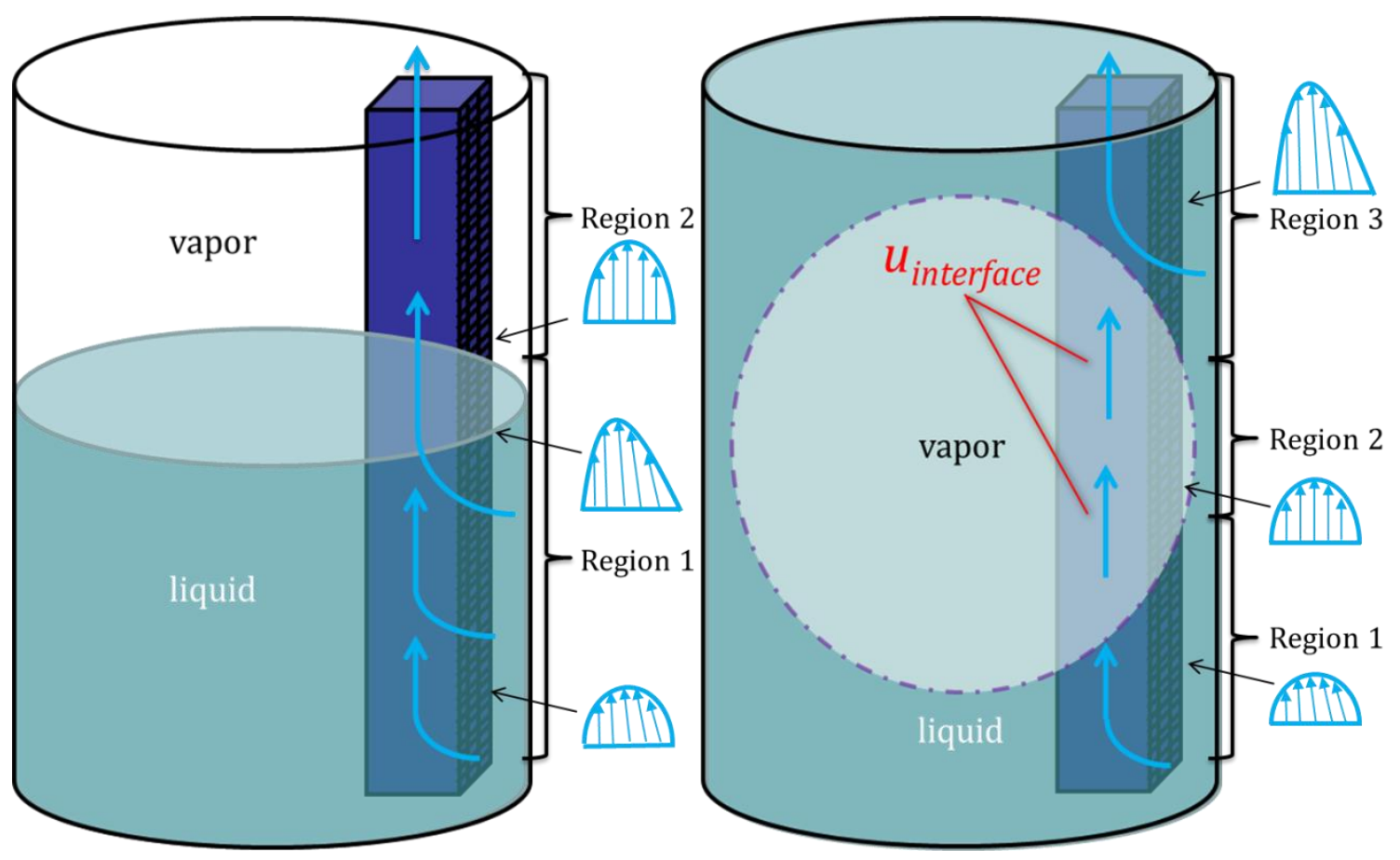

Figure 14.2 - Comparison of Liquid/Vapor Interface in a) 1-g Environment and b) Microgravity Environment

In the microgravity case, similar to the 1-g case, liquid in the channel flows through a liquid-liquid region and then a liquid-vapor region. However, there is now a second liquid-liquid region after the liquid-vapor region, as shown in Figure 12.2b. The 
interface velocity links the flow profile between these two liquid-liquid regions and is thus a vital part of solving porous channel flow in microgravity.

\subsubsection{Flow Model Derivation}

Figure 12.3 portrays the flow pattern through a rectangular screen channel LAD in microgravity. The LAD has dimensions of length $L$, width $W$, and height $H$, and it is oriented vertically with the $\mathrm{x}$-axis running along the length of the channel. Cryogenic fluid flows through the porous screen in the negative y-direction and down the channel in the positive $\mathrm{x}$-direction. Due to the nature of the $\mathrm{L} / \mathrm{V}$ interface in microgravity, the LAD is analyzed in three regions, consistent with Figure 14.2b: Region 1, the lower zone through which liquid flows, Region 2, the vapor-exposed zone, and Region 3, the upper zone through which liquid flows. The length of the regions are defined by the bottom and top $\mathrm{L} / \mathrm{V}$ interface boundaries, $L_{B}$ and $L_{T}$. Moving in the positive x-direction, $L_{B}$ defines the coordinate where the screen is first exposed to vapor, while $L_{T}$ defines the coordinate where the screen is again immersed in liquid. These values change as the bubble grows, and the screen is further exposed to vapor. The injection velocity increases from zero at the channel dead end to a maximum value at $L_{B}$ for Region 1 . There is no liquid injection between $L_{B}$ and $L_{T}$ in Region 2 . Then from $L_{T}$ to $L$, the injection velocity increases from zero to its maximum value for Region 3. 


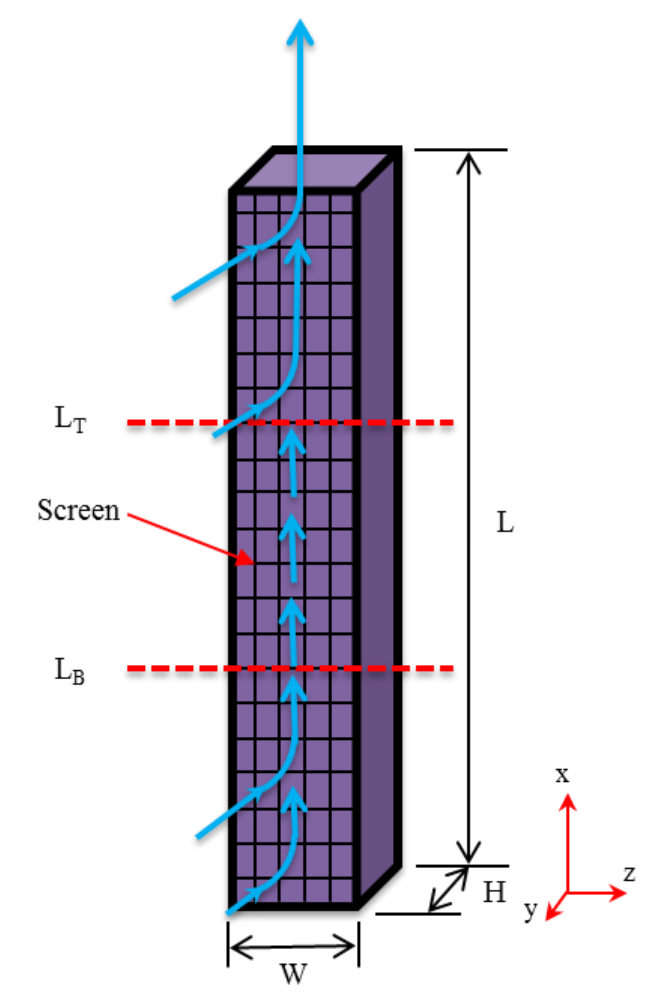

Figure 14.3 - Liquid Flow through a Screen Channel Liquid Acquisition Device in Microgravity

\section{$\underline{\text { Solution for Region } 1 \text { and Region } 3}$}

The same set of assumptions and resultant implications for the 1-g model are applied to the 0 -g model. The resultant simplified governing equations are also the same:

$$
\begin{aligned}
& \frac{\partial v_{x}}{\partial x}+\frac{\partial v_{y}}{\partial y}=0 \\
& \rho\left(v_{x} \frac{\partial v_{x}}{\partial x}+v_{y} \frac{\partial v_{x}}{\partial y}\right)=-\frac{\partial P}{\partial x}+\mu\left(\frac{\partial^{2} v_{x}}{\partial x^{2}}+\frac{\partial^{2} v_{x}}{\partial y^{2}}\right) \\
& \frac{\partial P}{\partial y}=0
\end{aligned}
$$


Six boundary conditions are required for each liquid-liquid section to solve the set of equations for Region 1 and Region 3. To enforce zero injection velocity at $x=0$ and at $x=L_{T}$, an additional seventh boundary condition is required. The seven BCs are:

\section{$\underline{\text { Boundary Conditions }}$}

\section{$\underline{\text { Region } 1}$}

1. $v_{x}(0, y)=0 \quad$ (no penetration)

2. $v_{x}\left(\mathrm{~L}_{\mathrm{B}}, y\right)=\bar{u}_{\text {interface }} g(y) \quad$ (flow demand)

3. $v_{x}(\mathrm{x}, 0)=0 \quad$ (no slip)

4. $v_{x}(\mathrm{x}, \mathrm{H})=0 \quad$ (no slip)

5. $v_{y}(\mathrm{x}, 0)=0 \quad$ (no penetration)

6. $v_{y}(\mathrm{x}, \mathrm{H})=v_{i n j, 1}(x) \quad$ (mass injection)

7. $v_{y}(0, y)=0 \quad$ (no slip)
?

\section{$\underline{\text { Region } 3}$}

1. $v_{x}\left(\mathrm{~L}_{\mathrm{T}}, y\right)=\bar{u}_{\text {interface }} g(y) \quad$ (no penetration)

2. $v_{x}(\mathrm{~L}, y)=\bar{u}_{e} g(y) \quad$ (flow demand)

3. $v_{x}(\mathrm{x}, 0)=0 \quad$ (no slip)

4. $v_{x}(\mathrm{x}, \mathrm{H})=0 \quad$ (no slip)

5. $v_{y}(\mathrm{x}, 0)=0 \quad$ (no penetration) 

6. $v_{y}(\mathrm{x}, \mathrm{H})=v_{i n j, 3}(x)$
(mass injection)
7. $v_{y}\left(\mathrm{~L}_{\mathrm{T}}, y\right)=0$
(no slip)

where $\bar{u}_{\text {interface }}$ is the average interface velocity in Region $2, \bar{u}_{e}$ is the magnitude of the demand velocity at the channel outlet, and $g(y)$ defines the shape of the $v_{x}$ profile. $v_{i n j, 1}(x)$ and $v_{i n j, 3}(x)$ are the injection velocity profiles across the screen for Region 1 and Region 3, respectively. Governing equations and boundary conditions are scaled again, using the following non-dimensional variables:

$x^{*}=\frac{x}{H} \quad y^{*}=\frac{y}{H} \quad L^{*}=\frac{L}{H} \quad L_{B}^{*}=\frac{L_{B}}{H} \quad L_{T}^{*}=\frac{L_{T}}{H}$

Y-momentum again implies that pressure inside the channel is a function of $\mathrm{x}$ only. The simplified, scaled continuity and x-momentum equations become:

$\frac{\partial v_{x}}{\partial x^{*}}+\frac{\partial v_{y}}{\partial y^{*}}=0$

$\rho\left(v_{x} \frac{\partial v_{x}}{\partial x^{*}}+v_{y} \frac{\partial v_{x}}{\partial y^{*}}\right)=-\frac{d P}{d x^{*}}+\frac{\mu}{H}\left(\frac{\partial^{2} v_{x}}{\partial x^{* 2}}+\frac{\partial^{2} v_{x}}{\partial y^{* 2}}\right)$

\section{$\underline{\text { Scaled Boundary Conditions }}$}

\section{$\underline{\text { Region } 1}$}

1. $v_{x}\left(0, y^{*}\right)=0 \quad$ (no penetration)

2. $v_{x}\left(\mathrm{~L}_{\mathrm{B}}^{*}, y^{*}\right)=\bar{u}_{\text {interface }} g\left(y^{*}\right) \quad$ (flow demand) 

3. $v_{x}\left(\mathrm{x}^{*}, 0\right)=0$
(no slip)
4. $v_{x}\left(0, y^{*}\right)=0$
(no slip)
5. $v_{y}\left(\mathrm{x}^{*}, 0\right)=0$
(no penetration)
6. $\quad v_{y}\left(\mathrm{x}^{*}, 1\right)=v_{i n j, 1}\left(x^{*}\right)$
(mass injection)
7. $v_{y}\left(0, y^{*}\right)=0$
(no slip)

\section{$\underline{\text { Region } 3}$}

1. $v_{x}\left(\mathrm{~L}_{\mathrm{T}}^{*}, y^{*}\right)=\bar{u}_{\text {interface }} g\left(y^{*}\right)$ (no penetration)

2. $v_{x}\left(\mathrm{~L}^{*}, y^{*}\right)=\bar{u}_{e} g\left(y^{*}\right) \quad$ (flow demand)

3. $v_{x}\left(\mathrm{x}^{*}, 0\right)=0 \quad$ (no slip)

4. $v_{x}\left(\mathrm{x}^{*}, 1\right)=0 \quad$ (no slip)

5. $v_{y}\left(\mathrm{x}^{*}, 0\right)=0 \quad$ (no penetration)

6. $v_{y}\left(\mathrm{x}^{*}, 1\right)=v_{i n j, 3}\left(x^{*}\right) \quad$ (mass injection)
7. $v_{y}\left(\mathrm{~L}_{\mathrm{T}}^{*}, y^{*}\right)=0$
(no slip)

The method of solution for the $0-\mathrm{g}$ case is the same as the method of solution for the $1-\mathrm{g}$ case; $\mathrm{x}$-component and $\mathrm{y}$-component velocity and pressure inside the channel are determined from: 


$$
\begin{aligned}
& \mathrm{v}_{x}\left(x^{*}, y^{*}\right)=\bar{u}_{e}\left[1+f\left(x^{*}\right)\right]\left[6 y^{*}\left(1-y^{*}\right)\right] \\
& v_{y}\left(x^{*}, y^{*}\right)=-\bar{u}_{e} \frac{d f}{d x^{*}}\left(3 y^{* 2}-2 y^{* 3}\right) \\
& P\left(x^{*}\right)=P_{0}-C_{\text {lam }} \mu \bar{u}_{e} \frac{d f}{d x^{*}}-C_{\text {turb }} \rho \bar{u}_{e}^{2}\left(\frac{d f}{d x^{*}}\right)^{2}
\end{aligned}
$$

where each is expressed in terms of the unknown variable $f$. The Kármán-Pohlhausen momentum integral technique is again applied to Regions 1 and 3 to obtain the following non-dimensional ODEs:

$$
\begin{aligned}
& \left(\frac{1}{\operatorname{Re}_{e}}+\frac{N_{\text {lam }}}{\operatorname{Re}_{e}}+2 C_{\text {turb }} f_{1}{ }^{\prime}\right) f_{1}{ }^{\prime \prime}+2 C_{\text {turb }}\left(f_{1}^{\prime \prime}\right)^{2}-\frac{12}{\operatorname{Re}_{e}} f_{1}^{\prime}-\frac{12}{5}\left(f_{1}^{\prime \prime}\left(1+f_{1}\right)+\left(f_{1}^{\prime}\right)^{2}\right)=0 \\
& \begin{array}{lll}
\text { 1. } f_{1}(0)=-1 & \text { 2. } f_{1}\left(\mathrm{~L}_{\mathrm{B}}^{*}\right)=\frac{\bar{u}_{\text {interface }}}{\bar{u}_{e}}-1 & \text { 7. } f_{1}{ }^{\prime}(0)=0
\end{array} \\
& \left(\frac{1}{\operatorname{Re}_{e}}+\frac{N_{\text {lam }}}{\operatorname{Re}_{e}}+2 C_{\text {turb }} f_{3}^{\prime}\right) f_{3}^{\prime \prime \prime}+2 C_{\text {turb }}\left(f_{3}^{\prime \prime}\right)^{2}-\frac{12}{\operatorname{Re}_{e}} f_{3}^{\prime}-\frac{12}{5}\left(f_{1}^{\prime \prime}\left(1+f_{3}\right)+\left(f_{3}^{\prime}\right)^{2}\right)=0 \\
& \begin{array}{lll}
\text { 1. } f_{3}\left(\mathrm{~L}_{\mathrm{T}}^{*}\right)=\frac{\bar{u}_{\text {interface }}}{\bar{u}_{e}}-1 & \text { 2. } f_{3}\left(\mathrm{~L}^{*}\right)=0 & \text { 7. } f_{3}^{\prime}\left(L_{\mathrm{T}}^{*}\right)=0
\end{array}
\end{aligned}
$$

where $\mathrm{Re}_{e}, N_{\text {lam }}$, and $C_{\text {turb }}$ are all defined in Chapter 12. The solution for steady, incompressible flow through a channel with a single porous wall in microgravity is comprised of the two nonlinear ODEs presented in Equations 14.9 and 14.10, along with the respective transformed boundary conditions, which can be used to determine velocity and pressure fields inside Regions 1 and 3. Coupled with an appropriate ullage bubble 
model and the cryogenic bubble point model, the new microgravity analytical flow model can be used to simulate tank drain in microgravity. However, a new unknown is introduced in the interface velocity, which will be addressed in the next section.

\section{$\underline{\text { Solution for Region } 2}$}

As illustrated in Figure 14.2b, the velocity profile for Region 2 in microgravity is assumed to be a fully-developed parabolic profile, similar to the profile for Region 2 in the 1-g case. However, in the microgravity model, the average velocity value is the interface velocity, and not the exit velocity:

$v_{x}=\bar{u}_{\text {interface }}\left(6 y^{*}\left(1-y^{*}\right)\right)$

Meanwhile, the pressure drop for Region 2 is the sum of the hydrostatic, frictional, and dynamic pressure drop terms as defined in the steady state 1D pressure drop model. Since there is no injection velocity in this region, there is no FTS pressure loss.

\subsubsection{Ullage Bubble Growth Model and Interface Velocity}

To determine the effect of pressurant gas diffuser location on performance, three ullage bubble locations and growth rates were modelled. In the idealized case (Case A), the LAD is oriented parallel with the long axis of the propellant tank. The vapor bubble and pressurant diffuser are initially located in the center of the tank, and the bubble grows spherically outwards from the center of the tank as shown in Figure 14.2b. In Case B, the vapor bubble and pressurant diffuser were located at the aft end of tank farthest away from the outlet, as shown in Figure 14.4a. The bubble grows uniformly towards the outlet, exposing the aft end of the LAD first. In Case C, the vapor bubble and pressurant 
diffuser were initially located in the center of the tank in line with the short axis of the tank, as depicted in Figure 14.4b. The LAD screen would be exposed to vapor from the center, but the amount of time elapsed before the LAD is exposed would depend on the position of the LAD with respect to the bubble. The LAD positioned directly opposite the diffuser was modeled, since it would be the last LAD that would be exposed to vapor. Assuming minimal heat transfer between ullage bubble and liquid propellant, the bubble growth rate is directly proportional to the liquid outflow rate. Combining the vapor bubble growth model with the microgravity analytical flow model allows the volume of remaining liquid, and subsequently the expulsion efficiency, to be determined at the exact moment when the LAD breaks down.

To demonstrate general model output, Case $\mathrm{A}$ is used in conjunction with the analytical model to simulate tank drain through a LAD inside a $4.05 \mathrm{~m}^{3}$ cylindrical tank with length of $2.286 \mathrm{~m}$ (90 inches) and a radius of $0.762 \mathrm{~m}$ (30 inches) filled with $\mathrm{LH}_{2}$. The length of the straight section of the screen channel LAD is approximately $2.232 \mathrm{~m}$ (87.8625 inches). Although not shown here, the LAD curves down toward the top dead center of the tank located above the outlet. Before the simulation, a vapor bubble growth code had to be developed. The code tracked the growth of the ullage bubble as well as the coordinates $L_{B}$ and $L_{T}$, which determine the length of the LAD that is exposed to vapor. In the idealized Case A that was modeled, the tank was oriented with the long axis of the tank in line with the LAD arm as shown in Figure 14.2b. The vapor bubble is initially in the center of the tank and the bubble grows proportional to the liquid outflow rate. The model assumes there is no acceleration due to thrust and that the acceleration due to gravity is $10^{-6} \mathrm{~m} / \mathrm{s}^{2}$. The initial volume of the vapor bubble is $5 \%$ of the tank volume. 

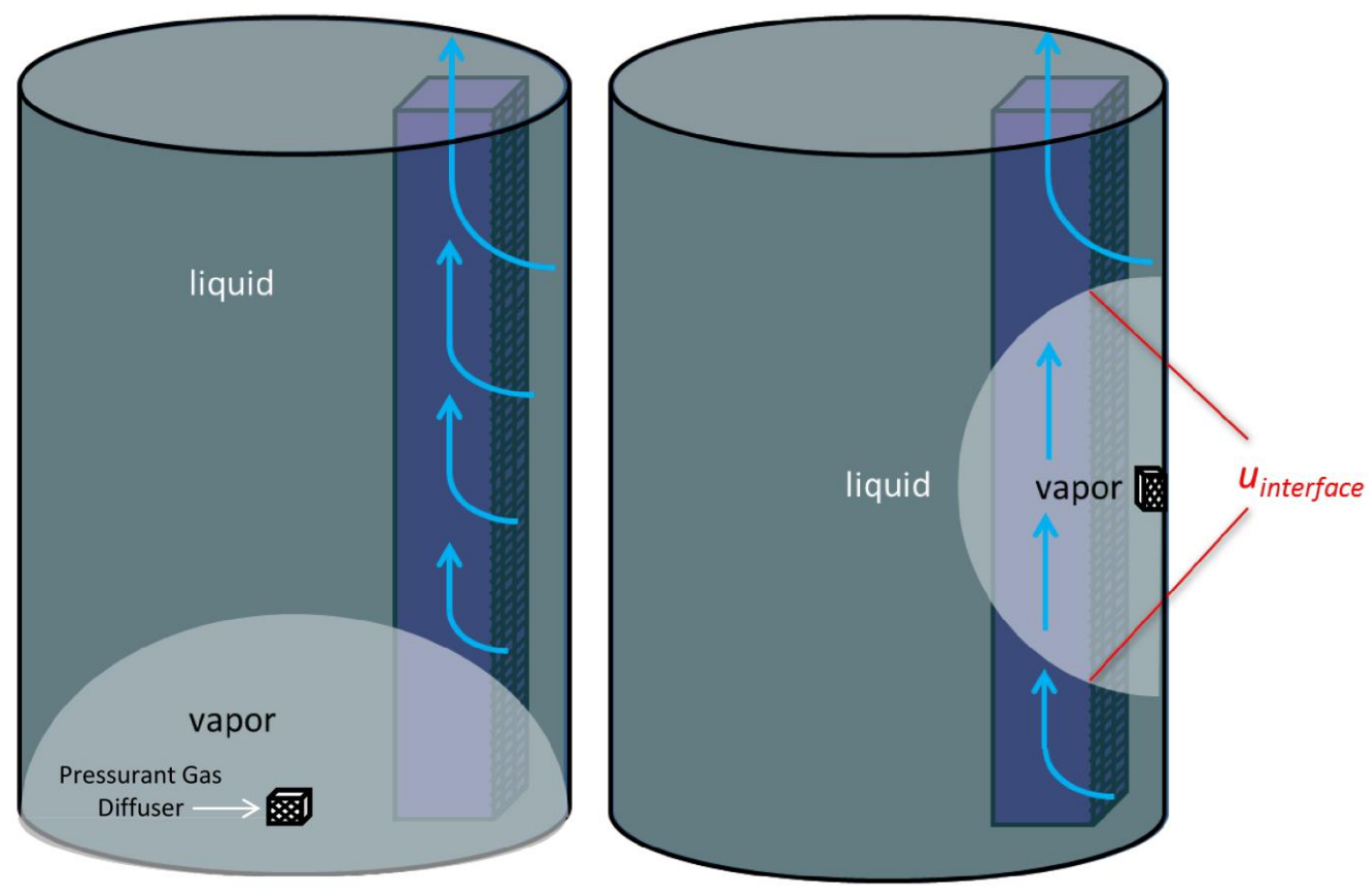

Figure 14.4 - Comparison of Microgravity Liquid/Vapor Interface in a) Case B and b) Case C

The unknown interface velocity is modeled as an exponentially decaying function of the vapor-exposed screen length as shown in Figure 14.5, based on analysis of velocity profiles for the 1-g LAD modeling and on outflow data from Chapter 9. Simulations indicate that the interface velocity is actually fairly insensitive to the assumed profile. For comparison, several interfacial velocity functional profiles (e.g. linear, exponential) were assumed, and results indicated that the screen exposure percentage at breakdown differed by less than $1 \%$ between these assumed profiles. Estimations of the interface velocity from 1-g inverted outflow data agreed quite well with this assumed profile as well.

As shown in Figure 14.5, the normalized interface velocity rapidly decays as the LAD is exposed to vapor. With an interface velocity profile, ullage bubble growth model, cryogenic bubble point model, and analytical flow model, the velocity and pressure fields 
inside the LAD can be determined in microgravity. The velocity can also be visualized as a function of time as the tank is drained.

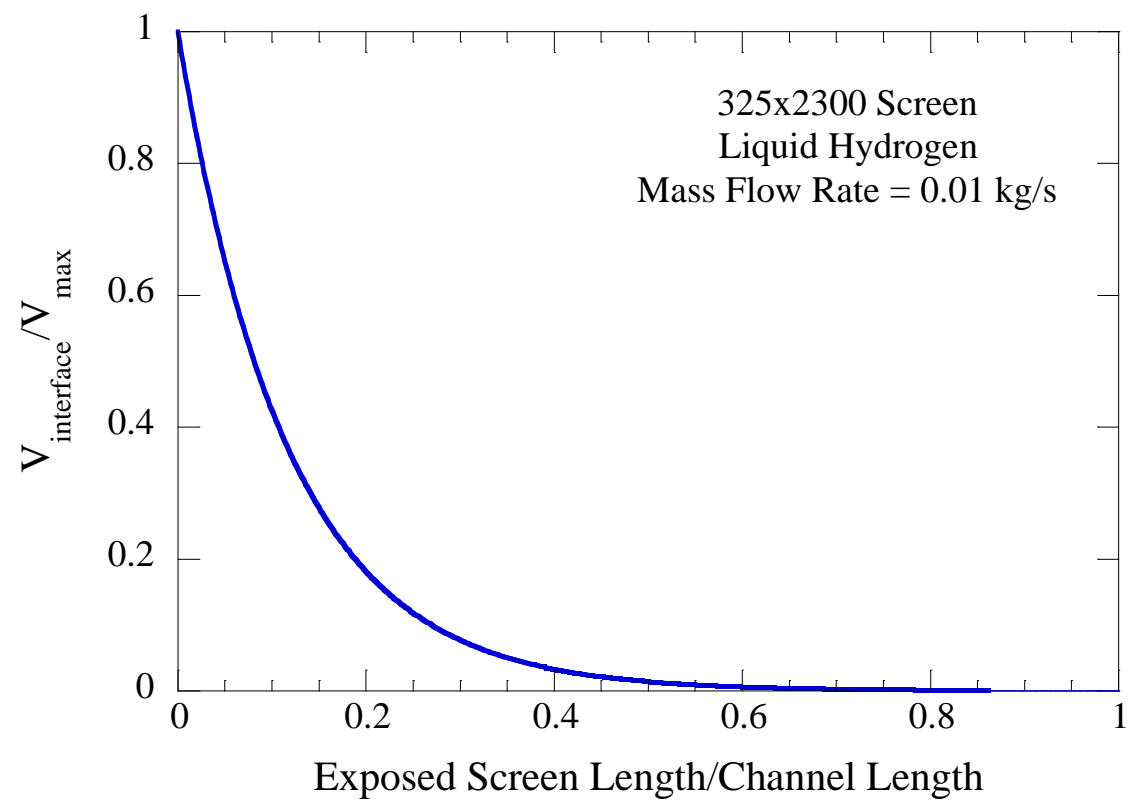

Figure 14.5 - Normalized Interface Velocity as a Function of Normalized Exposed Screen Length

\subsubsection{General Model Trends}

First, the results of the microgravity model were compared to those of the original 1-g model. Table 14.1 displays the vapor exposure percentages at breakdown for both models. As shown, the LAD clearly performs better in microgravity than in 1-g. Percent of exposure to vapor is a convenient way to compare performance between 1-g and microgravity. In microgravity, relative to the 1 -g case, a higher percentage of the screen is exposed to vapor before the LAD breaks down and vapor enters the channel. This is because the hydrostatic pressure loss term, which is the dominant pressure drop leading to breakdown in 1-g, is very small for the microgravity case. The LAD continues to drain 
the tank until the FTS pressure drop exceeds the bubble point. Thus in microgravity, the FTS pressure drop plays an exceedingly more important role in breakdown than in 1-g.

\begin{tabular}{|c|c|c|c|}
\cline { 2 - 4 } & & \multicolumn{2}{|c|}{ Percent of Screen Exposed to Vapor } \\
\hline Mass Flow Rate [kg/s] & Reynolds Number & 1-g Model & $\boldsymbol{\mu g}$ Model \\
\hline 0.005 & $1.43 \mathrm{E}+04$ & 70.9 & 91.5 \\
\hline 0.01 & $2.87 \mathrm{E}+04$ & 62 & 82.3 \\
\hline 0.02 & $5.74 \mathrm{E}+04$ & 44.1 & 59.6 \\
\hline 0.025 & $7.17 \mathrm{E}+04$ & 34.1 & 43.5 \\
\hline
\end{tabular}

Table 14.1 - Comparison of Model Predicted Percent of Screen Exposed to Pressurant Gas at Breakdown in a) 1-g and b) Microgravity for a 325x2300 Screen and Channel in Liquid Hydrogen at a Demand Flow Rate of $0.01 \mathrm{~kg} / \mathrm{s}$

Figure 14.6 shows the velocity plots inside the channel as a function of time, in terms of increasing screen exposure percentages, for a constant demand flow rate of 0.01 $\mathrm{kg} / \mathrm{s}$ for a $325 \times 2300 \mathrm{LAD}$ screen and channel in $\mathrm{LH}_{2}$. The screen exposure rate is analogous to tank fill level, therefore higher screen exposure percentages correspond to lower liquid fill levels, and that the LAD is able to drain more of the tank. As shown, the effect of exposure at the center of the channel on the velocity field is significant. Early on, the value of the interface velocity is high enough to pull mass in from the lower end of the channel (Region 1). However, as the center part of the screen becomes more exposed to vapor, the interface velocity rapidly decays, and the mass injection localizes towards the demand end of the LAD. By the time $40 \%$ of the screen is exposed, the interface velocity is very small and the majority of the mass flow is drawn through the screen through the upper portion of the LAD (Region 3). Ultimately, the LAD breaks down when most of the velocity injection occurs in the upper $10 \%$ of the LAD, in this 
case at the $80 \%$ screen exposure. At the time of breakdown, nearly $90 \%$ of the channel is unused, as mass injection is concentrated close to the demand end of the channel.

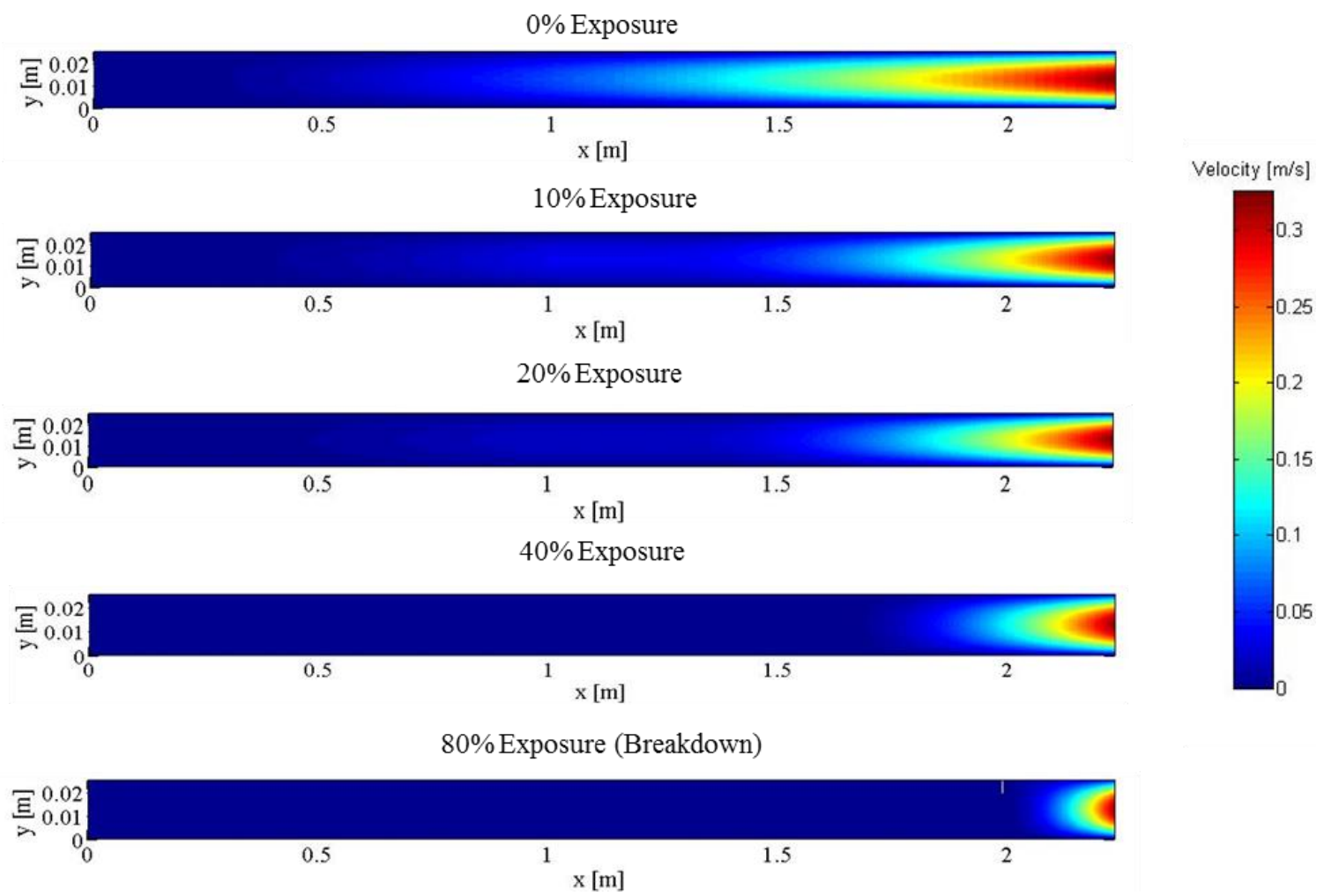

Figure 14.6 - Velocity Vector Plots at Increasing Screen Exposure Percentages (Lower Tank Fill Levels) for a 325x2300 Liquid Acquisition Device Screen and Channel in Liquid Hydrogen at a Demand Flow Rate of $0.01 \mathrm{~kg} / \mathrm{s}$. Color represents magnitude of velocity.

Figure 14.7 shows the normalized FTS pressure drop for a $325 \times 2300$ screen and channel in $\mathrm{LH}_{2}$ at several different mass flow rates. At lower mass flow rates, the pressure drop increases nearly linearly, in the laminar dominant regime. At higher mass flow rates, the curves become nonlinear and skewed to the right in the turbulent dominant regime. As the demand flow rate increases, not only does the overall pressure drop across the screen increase, but it also becomes more highly concentrated closer to the channel exit, as anticipated. Therefore, higher flow rates lead to more non-uniform FTS pressure 
drop, which would lead to earlier breakdown. For the same demand flow rate, the nonlinearity in pressure drop is slightly more pronounced in microgravity than in 1-g.

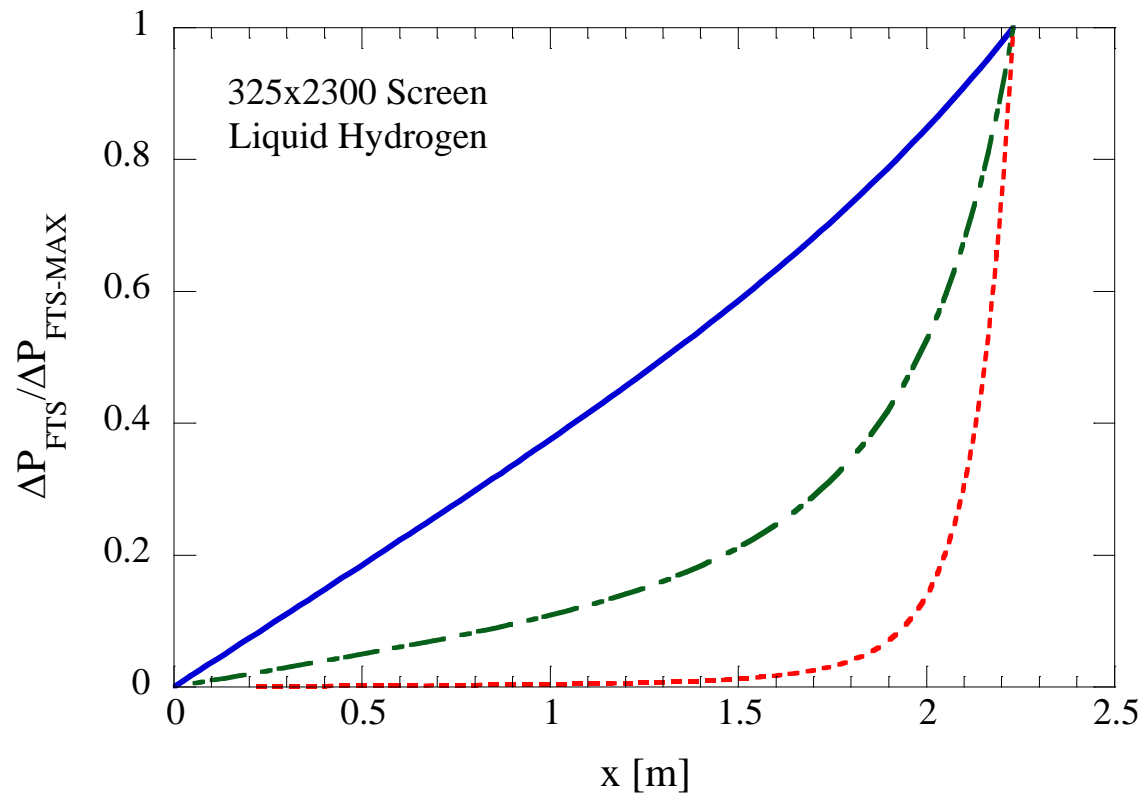

Figure 14.7 - Flow-through-Screen Pressure Drop as a Function of Distance along the Liquid Acquisition Device Channel for Varying Demand Flow Rates. Channel length is 2.23 meters.

Figure 14.8 plots the total pressure drop at breakdown as a function of the distance along the channel for different mass flow rates for a $325 \times 2300$ screen in $\mathrm{LH}_{2}$ at $20 \mathrm{~K}$ and $100 \mathrm{kPa}$ with equal gas and liquid temperatures. Note that the pressure drop is negative because it shows the difference between the inside of the channel $P(x)$ and the outside of the channel $P_{0}$, and the pressure outside the channel is always greater. 

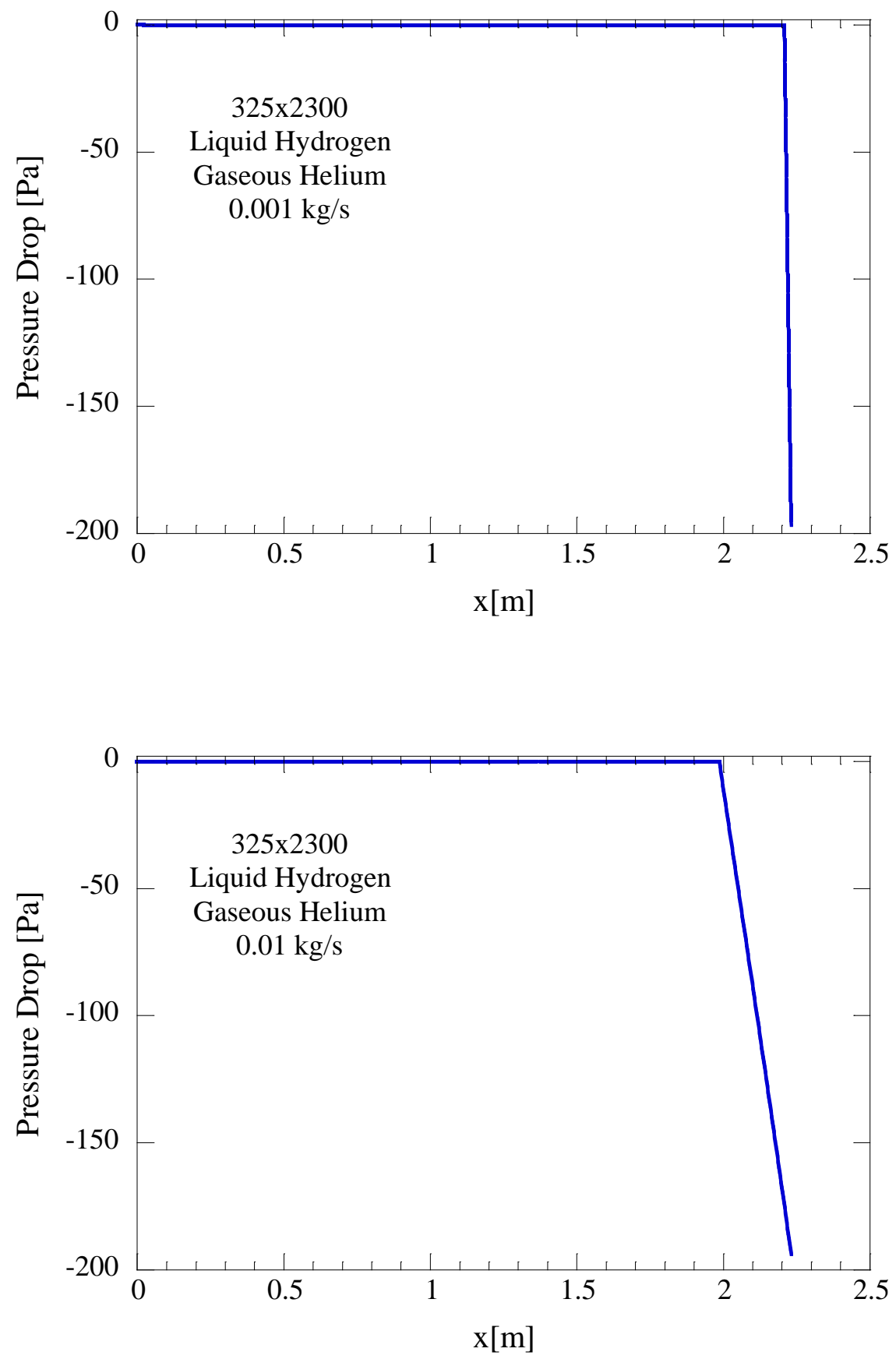

Figure 14.8 - Total Pressure Drop as a Function of Distance along Liquid Acquisition Device Channel for Demand Flow Rate of a) $0.001 \mathrm{~kg} / \mathrm{s}$ and $0.01 \mathrm{~kg} / \mathrm{s}$ at Breakdown. A safety factor of three was used in the bubble point calculation. 
In both cases, the LAD breaks down near the channel outlet where the demand for flow is highest, which is where the maximum pressure drop occurs. This indicates that breakdown occurs due to FTS pressure drop. In conjunction with the velocity plots in Figure 14.6, the concentrated flow near the outlet is the cause for breakdown. As in the 1$\mathrm{g}$ case, the slope is steeper for the lower flow rate case because a greater amount of the screen is exposed to vapor and so the region through which liquid can flow is smaller.

Finally, to examine the effect of channel dimensions and demand flow rate on channel performance in microgravity, Figure 14.9 plots the velocity profile as a function of various channel geometries and flow rates. Channel dimensions and corresponding exit Re numbers are shown on the right. It is noticeable that at a smaller height and width, the velocity is much greater near the outlet. As the LAD dimensions are increased, the flow is more uniformly distributed in the channel. Larger channels clearly support higher flow rates when compared to smaller LADs. At low flow rates, increasing the height and width have nearly the same impact on the profile. At higher mass flow rates, the magnitude of the velocity is slightly lower near the outlet when increasing the width as opposed to increasing the height. Increasing both the width and height leads to an increase in LAD mass, which is obviously undesirable from a systems level standpoint. To reduce system mass, it is far better to increase width over height, since the two solid metal sidewalls of the channel grow with the height while only the backside of the solid metal channel increases with width. The screen area also increases with width as well, but this mass is negligible because the screen thickness is so small. For each LAD design, there obviously exists an optimal design point that maximizes channel performance, but minimizes 
channel mass. The microgravity LAD model can now be used to compare performance against the vane type PMD.

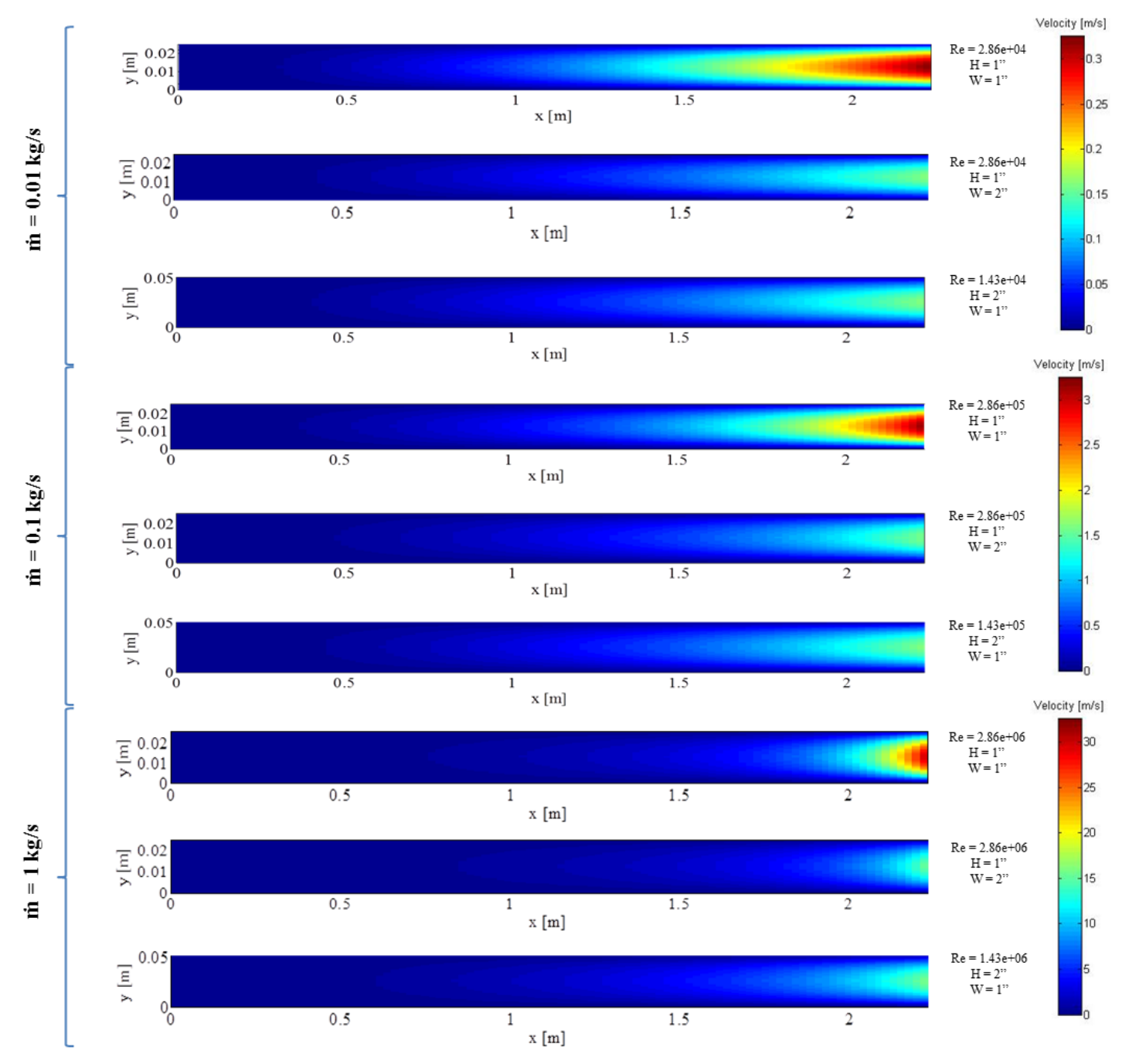

Figure 14.9 - Velocity Vector Plots inside a 325x2300 Liquid Acquisition Device Channel for Various Mass Flow Rates and Channel Dimensions. Color represents magnitude of velocity. Note color axes are different for each flow rate. 


\subsection{Analytical Vane Model in Microgravity}

Consider a vane attached to a tank wall with height $H_{V}$ and length $L_{V}$, as depicted in purple in Figure 14.10, in an end-of life (EOL) configuration, where the majority of the cryogenic propellant has been drained from the tank. Distance between successive vanes is $D_{V}$. In this case, the remaining fluid fills the corners where the vane and tank wall intersect, creating fillets with varying radii of curvature along the length of the vane as shown in blue in Figure 14.10. Liquid travels down the fillets from the aft end of the tank to the tank outlet due to the weak capillary pumping force provided by the vane. Since vanes are rarely implemented in a stand-alone configuration, the vanes feed a sump with a porous screen which covers the outlet, allowing the liquid fillets attached to the vane to flow to the sump, while the sump prevents vapor from entering the transfer line as illustrated in Figure 14.11a. Figure 14.11b shows the top down look in the propellant tank at EOL where remaining residual liquid is attached to the vanes.

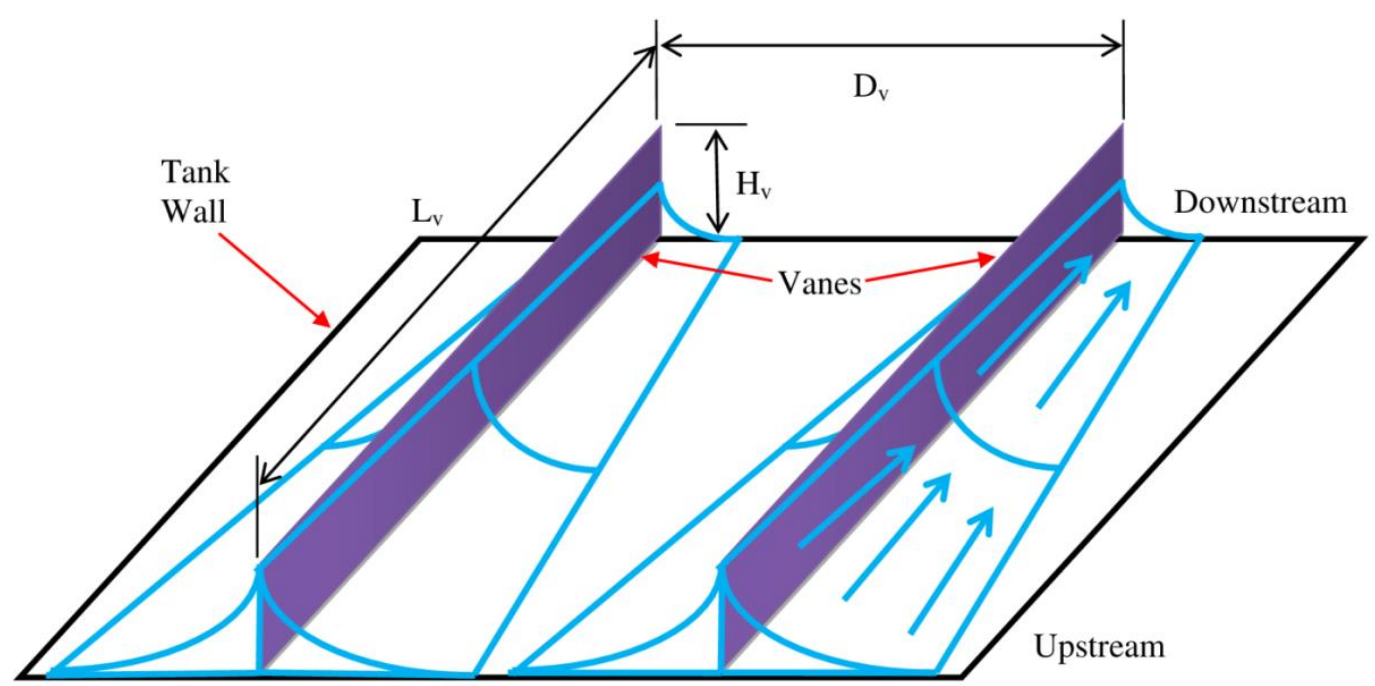

Figure 14.10 - Liquid Flow Path along Wall Mounted Vanes 

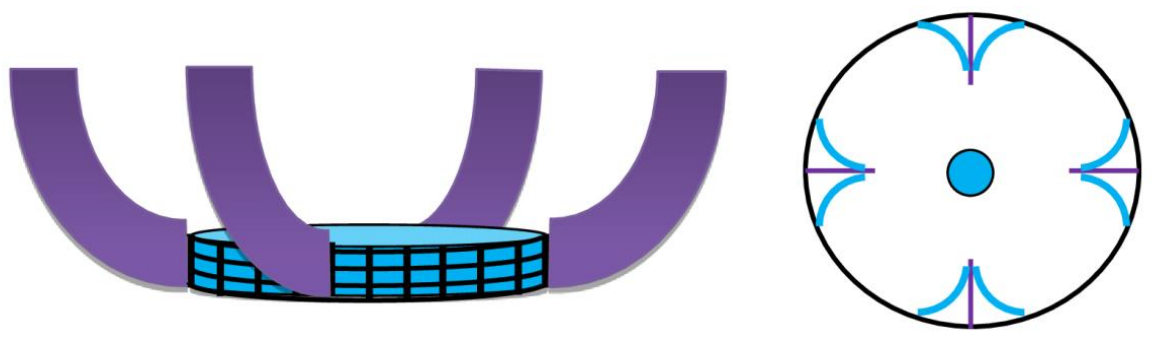

Figure 14.11 - a) Side and b) Top-Down View of Vanes and Sump

The flow of liquids down grooved geometries is well documented in the literature, see Mann et al. (1995) for example. The relationship between the driving pressure due to surface tension forces and the radii of curvature along the vane is expressed as:

$$
\Delta P_{\text {drive }}=\gamma_{L V}\left(\frac{1}{R_{D}}-\frac{1}{R_{U}}\right)
$$

where $\Delta P_{\text {drive }}$ is the driving pressure and $R_{D}$ and $R_{U}$ are the downstream and upstream radii of curvature. So long as the upstream radius of curvature is greater than the downstream radius of curvature, liquid flows downstream along the vane to the outlet. In Figure 14.10 , the radius of curvature diminishes down the vane primarily due to frictional losses.

The vane system is analyzed assuming steady state, incompressible, isothermal liquid flow along wall-mounted vanes with no center post. The governing equations for the system are continuity and momentum:

$$
d u=-\frac{\dot{V}}{A^{2}} d A
$$


$-A d p-2 \rho v \dot{V}\left(\frac{s}{A}\right)^{2} d x=\rho A u d u$

where $u$ is the velocity of the liquid down the vane, $A$ is the cross sectional area of

fillet, $\dot{V}$ is the volumetric flow rate of the fillet down the vane, $x$ is the distance along the vane, and $s$ is the wetted circumference. After substituting the surface tension equation of state, simplifying, and rearranging, mass and momentum are combined and reduced to the following differential equation:

$$
\frac{d R}{d x}=\frac{(F M U) \frac{2 v}{\dot{V}} s^{2}}{\frac{d A}{d R}-(S F) \frac{\gamma_{L V}}{\rho} \frac{1}{\dot{V}^{2}} \frac{A^{3}}{R^{2}}}
$$

where $F M U$ is the friction multiplier (set to 3), and $S F$ is set to 2. In general there are three unknowns in Equation 14.15, $R_{D}, R_{U}, \dot{m}$. Requirements on the total wetted area over the propellant tank outlet and desired expulsion efficiency generally constrain $R_{D}$ and $R_{U}$, respectively. Given one of the two constraints and a desired demand flow rate, this equation can be solved for $R$ as a function of $x$ by implementing a $4^{\text {th }}$-order Runge-Kutta method with an appropriate initial guess (constraint) for $R$. The solution method can march in either direction, but knowledge of one of the two radii of curvature is generally required.

Alternatively, a simple force balance can also be used to approximate $R$ as a function of $x$; however, there are problems associated with this method (Jaekle 1991). For example, at a given flow rate, there appear to be two downstream radii of curvature 
for every upstream radius of curvature. Figure 14.12 shows points on the right half of this curve, which is the valid solution, for a fixed flow rate of $1 \times 10^{-4} \mathrm{~kg} / \mathrm{s}$. The left half of the curve is generally not possible due to choked flow, which occurs when the denominator of Equation 14.15 goes to zero when the fluid velocity approaches the area wave propagation speed. Detailed explanation of this phenomenon is reserved in Jaekle (1991). Essentially, unsteady flow can occur depending on the nature of the singularity, but if both numerator and denominator go to zero, the flow is truly choked.

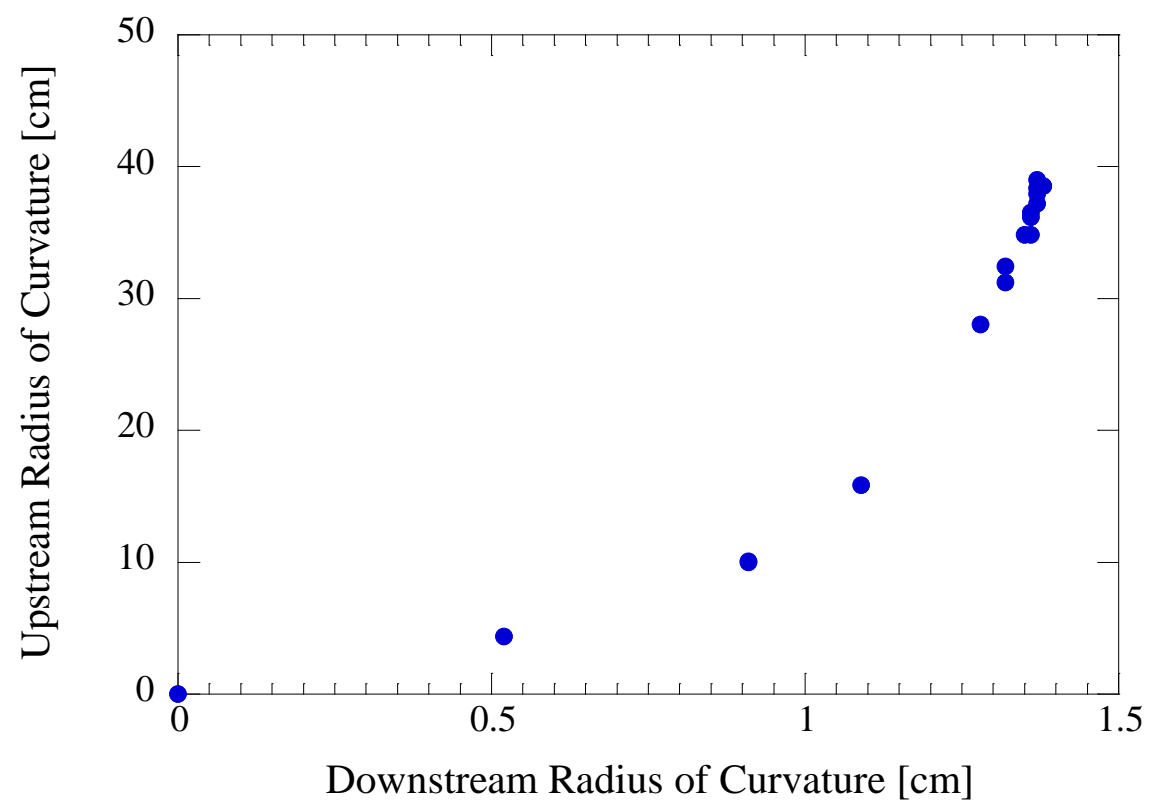

Figure 14.12 - Upstream Radius of Curvature as a Function of Downstream Radius of Curvature for a Liquid Hydrogen Demand Flow Rate of $1 \times 10^{-4} \mathrm{~kg} / \mathrm{s}$

For the current work, the downstream constraint for $R$ mandates that $R_{D}$ has to be large enough so that fillets overlap above the height of the sump, therefore always keeping the sump submerged in liquid as the tank is drained. The sump is assumed to be cylindrical with a porous metal screen around it with a solid metal top. To minimize flow 
losses, the screen for the sump is chosen to be a 200x600 Dutch Twill mesh. In microgravity, hydrostatics are minimized and the FTS pressure drop becomes the highestorder pressure drop term for the porous LAD element. Therefore, the sump screen area is sized such that $\Delta P_{F T S, \max }<\Delta P_{B P}$ for as long as the sump is submerged in liquid during tank drain. Setting $\Delta P_{B P}$ equal to $\Delta P_{F T S}$ as in Chapter 13, the minimum required sump screen area is solved for:

$A_{C, \min }=\left[\frac{\sqrt{\alpha^{2} X^{2}+4 \beta \Delta P_{B P} Y}+\alpha X}{2 \Delta P_{B P}}\right] \dot{m}=Z \dot{m}$

where $X$ and $Y$ are defined in Equations 13.2 and 13.3. Consistent with the screen channel LAD analysis, a safety factor of 3 is used for $\Delta P_{B P}$. As flow rate demand increases, and thus the size of the sump increases, the sump growth rate is assumed to have a radius to height ratio of 12 . Given the minimum screen area, the sump can be sized by solving for the sump height and radius, respectively:

$$
\begin{aligned}
& H_{S}=\sqrt{\frac{A_{C, \min }}{24 \pi}} \\
& R_{S}=12 H_{S}
\end{aligned}
$$

For a given propellant, liquid temperature and pressure, and demand flow rate, the height of the sump constrains the minimum allowable downstream radius of curvature.

The length of the vane is determined by assuming that the vane runs the length of the tank and then curves sharply towards the sump. Therefore, the approximate length of the vane is taken as 
$L_{V} \approx L_{\text {tank }}+\left(R_{\text {tank }}-R_{S}\right)$

where $L_{\text {tank }}$ is the length of the tank and $R_{\text {tank }}$ is the radius of the tank. The sump dimensions and required flow rate also constrain the total number of vanes. The distance between the vanes can be determined for a given number of vanes:

$D_{V}=\frac{2 \pi R_{S}}{N_{V}}$

where $N_{V}$ is the number of vanes. For a given vane height and number of vanes, the constraint on $R_{D}$ is enforced by iterating through increasing $R_{D}$ values. For each $R_{D}$ value, the chord length based on the height of the vane $C_{D}$, and the chord length based on the height of the sump $C_{S}$, is calculated as

$$
\begin{aligned}
& C_{D}=\sqrt{8 H_{V}\left(R_{D}-\left(H_{V} / 2\right)\right)} \\
& C_{S}=\sqrt{8 H_{S}\left(R_{D}-\left(H_{S} / 2\right)\right)}
\end{aligned}
$$

Geometrically, when the value $\left(C_{D}-C_{S}\right)$ reaches the value $D_{V}$, the $R_{D}$ value at that iteration is sufficient to keep the sump submerged in liquid. This value for $R_{D}$ provides an initial value for solving Equation 14.15. $A$ and $s$ must first be determined as a function of $R$, since both values change along the length of the vane as shown in Figure 14.10. For $\left(R>H_{V}\right)$, the functional forms for $A$ and $s$ are: 


$$
\begin{aligned}
& A=C_{V} H_{V}-\frac{R^{2}}{2}(\theta-\sin \theta) \\
& s=C_{V}+2 H_{V}
\end{aligned}
$$

where

$$
\begin{aligned}
& C_{V}=\sqrt{8 H_{V}\left(R-\left(H_{V} / 2\right)\right)} \\
& \theta=2 \sin ^{-1}\left(\frac{C_{V}}{2 R}\right)
\end{aligned}
$$

If $\left(R<H_{V}\right)$, then

$$
\begin{aligned}
& A=2 R^{2}-\frac{\pi R^{2}}{2} \\
& s=4 R
\end{aligned}
$$

Equation 14.15 is then numerically integrated to determine $R(x)$ and subsequently $R_{U}$, which is the final value of $R(x)$. To calculate the expulsion efficiency at $\operatorname{EOL}, A(x)$ is used to calculate the residual fillet volume:

$V=N_{V} \int_{0}^{L_{V}} A(x) d x$

where $V$ is the total volume of the residual liquid attached to the vanes. In summary, a demand flow rate specifies the total number and size of the vanes to keep the screened 
sump completely submerged in liquid, and the combined mass/momentum equation is used to calculate expulsion efficiency at EOL.

\subsection{Trade Study Variables}

The performance of the two PMDs, screen channels and vanes, is analyzed by comparing ability and efficiency in draining a small scale cryogenic propellant tank in microgravity. The $\mathrm{LH}_{2}$ propellant tank and LAD dimensions were defined previously. The length of the vane is dependent on the size of the sump, and can be calculated using Equation 14.19. Figure 14.13 summarizes the inputs and outputs for the trade study. In sizing the PMD system, expulsion efficiency is considered the primary performance parameter. For a given mass flow rate, the PMD is sized to maximize the expulsion efficiency and minimize the resultant PMD mass.

To size the screen channel LADs, the dimensions and number of the screen channel LADs were varied, tank drain was simulated to determine the residual liquid volume at bubble breakthrough, and resultant PMD mass and expulsion efficiency were determined. Based on general model outputs, larger channels are more efficient at sustaining vapor free liquid flow at higher mass flow rates; however, increasing the dimensions of the LAD leads to an unfavorable increase in PMD mass. Initial simulations from Section 14.2.4 indicated that it is more advantageous to increase width over height of the channel to minimize overall LAD mass. The final channel dimensions were chosen to maximize expulsion efficiency. When multiple LAD systems could achieve the same expulsion efficiency, the system with the lowest mass was selected as the optimal PMD. For a single channel, a limit on channel width was set to be $0.2794 \mathrm{~m}$ (11 inches) so that 
the total screen width around the tank would not exceed half of the tank circumference. It should be noted that a $S F$ of 3 for all bubble point pressures was used in all subsequent tank drain simulations, regardless of the ullage bubble case.

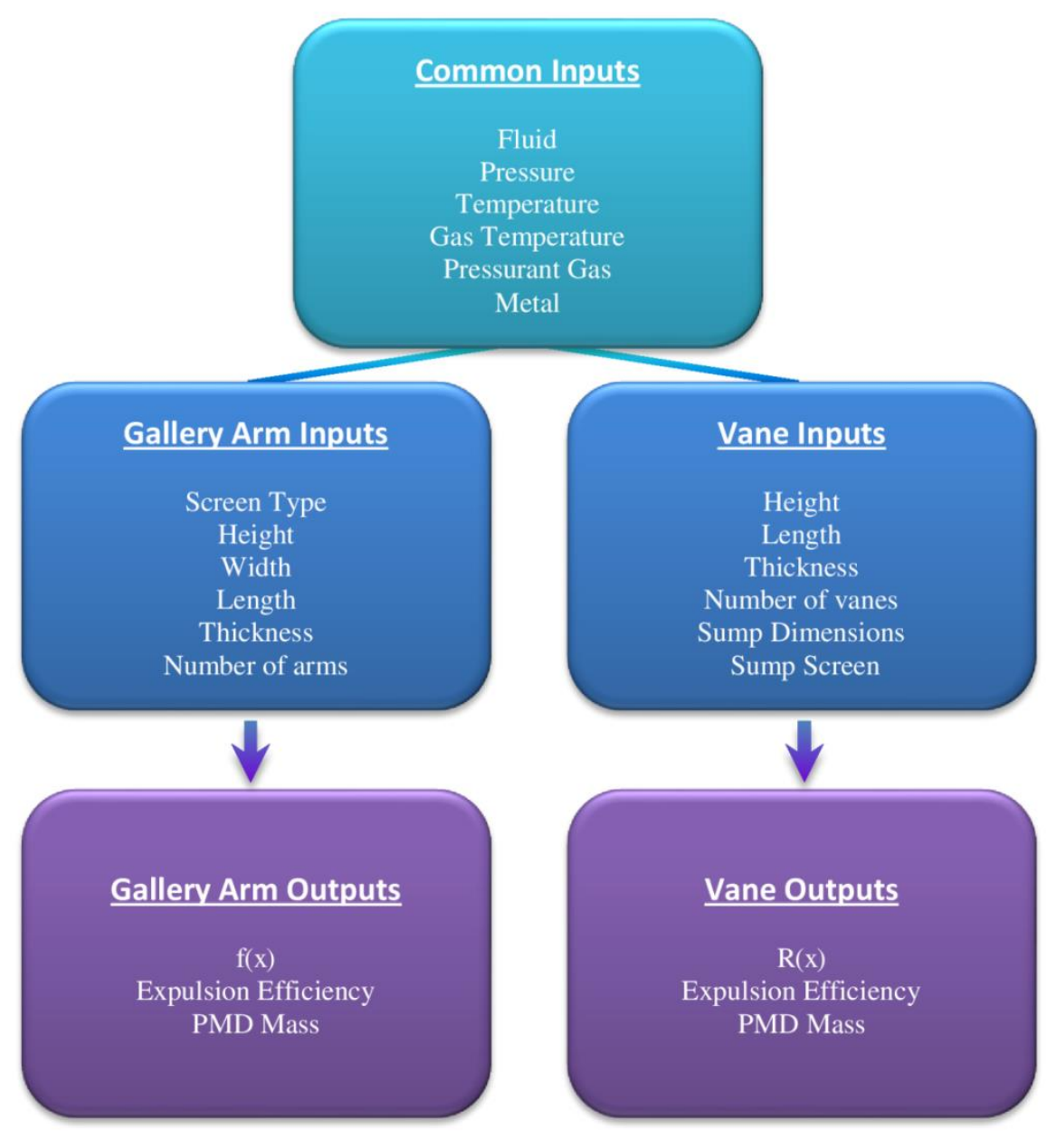

Figure 14.13 - Flow Chart Outlining Propellant Management Device Trade Study Model Inputs and Outputs

For the vanes, tank drain simulation was not required since the model already considers an EOL configuration. To size the vanes, the height and number of vanes were varied to fully submerge the sump, the liquid fillet volume was calculated (following the 
method outlined in Section 14.3), and expulsion efficiency and PMD mass were obtained. Vanes were also sized to maximize expulsion efficiency at a given demand flow rate. If multiple vane systems could achieve the same expulsion efficiency, the system with the lowest mass was chosen.

To simplify the analysis, several assumptions were applied to both PMD models. There are no adverse accelerations or acceleration due to vehicle thrust; therefore, the cryogenic propellant is in an unsettled, quiescent environment. The acceleration due to gravity is $10^{-6} \mathrm{~m} / \mathrm{s}^{2}$. Too high of adverse acceleration levels would immediately discredit the vane from the trade study. The initial volume of the vapor bubble is $5 \%$ of the tank volume. There is minimal heat transfer between the ullage bubble and the liquid propellant. The heat leak into the cryogenic propellant is negligible over the time scales of tank drain. Additionally, the temperature of the liquid is uniform throughout the tank, as is the temperature of the vapor, during expulsion. Every case assumes GHe as the pressurant gas.

A range of mass flow rates are considered in the trade study and both PMDs are sized for each flow rate. The ultimate goal of the trade study is to determine the critical mass flow rate range for vanes and galleries for a small scale cryogenic propellant tank. The critical mass flow rate range is the overlap range of flow rates where both galleries and vanes can be considered the optimal PMD. At flow rates lower than this range, vanes are the optimal choice while galleries would be the recommended PMD option beyond the critical mass flow rate range. 


\subsection{Trade Study Results}

As shown in Figure 14.13, many input variables impact the output of the PMD models. For screen channel LADs, Tables 14.2 and 14.3 display the general trends when varying several of these inputs including screen mesh, tank pressure, liquid temperature, pressurant gas temperature, number of arms, and LAD width. All of these initial simulations were run using the ideal centrally located spherical bubble growth Case A, for simplicity.

To examine basic trends in the model, tank drains were simulated for a screen channel LAD with a fixed height and width of $2.54 \mathrm{~cm}$ each at a demand flow rate of $0.01 \mathrm{~kg} / \mathrm{s}$ for a $325 \mathrm{x} 2300$ screen in $\mathrm{LH}_{2}$, using $\mathrm{GHe}$ to pressurize, varying the three main thermodynamic properties of liquid temperature, pressure, and pressurant gas temperature. All screen parameters are taken from Chapter 3.

\begin{tabular}{|c|c|c|c|c|c|}
\hline Screen/Fluid & T [K] & Tgas[K] & P [kPa] & Mass Flow Rate [kg/s] & Expulsion Efficiency \\
\hline $325 \times 2300$, LH2 & 20.3 & 20.3 & 103.4 & 0.01 & 92 \\
\hline $325 \times 2300$, LH2 & 20.3 & 20.3 & 206.8 & 0.01 & 92.2 \\
\hline $325 \times 2300$, LH2 & 20.3 & 20.3 & 344.7 & 0.01 & 92.4 \\
\hline $325 \times 2300$, LH2 & 20.3 & 20.3 & 103.4 & 0.01 & 92 \\
\hline $325 \times 2300$, LH2 & 22 & 22 & 162 & 0.01 & 91.7 \\
\hline $325 \times 2300$, LH2 & 24 & 24 & 262 & 0.01 & 91 \\
\hline $325 \times 2300$, LH2 & 20.3 & 20.3 & 103.4 & 0.01 & 92 \\
\hline $325 \times 2300$, LH2 & 20.3 & 22 & 103.4 & 0.01 & 91.9 \\
\hline $325 \times 2300$, LH2 & 20.3 & 24 & 103.4 & 0.01 & 96.7 \\
\hline $200 \times 600$, LH2 & 20.3 & 20.3 & 103.4 & 0.01 & 95.8 \\
\hline $165 \times 800$, LH2 & 20.3 & 20.3 & 103.4 & 0.01 & 92.9 \\
\hline $325 \times 2300$, LH2 & 20.3 & 20.3 & 103.4 & 0.01 & 0.01 \\
\hline $450 \times 2750$, LH2 & 20.3 & 20.3 & 103.4 & & 0.01 \\
\hline
\end{tabular}

Table 14.2 - Screen Channel Liquid Acquisition Device Expulsion Efficiencies for Variable Thermodynamic Conditions, Demand Flow Rates, and Screen Types 
Results show that expulsion efficiency is not as sensitive to changes in some of these variables. For example, increasing the tank pressure causes a small increase $(<$ $0.5 \%$ ) in the expulsion efficiency as shown in the first three rows in Table 14.2. This trend can be attributed to the gain in bubble point due to subcooling the liquid at higher pressures, which would allow the LAD to sustain vapor free liquid flow for a longer period of time and achieve higher expulsion efficiencies. Meanwhile, an increase in liquid temperature causes a drop in expulsion efficiency as shown in the next three rows in Table 14.2. This is attributed to a decrease in the bubble point pressure due to decreased surface tension when liquid temperature is increased, therefore shortening the amount of time for which the LAD can sustain vapor free liquid flow. Finally, the negligible decrease in expulsion efficiency due to increasing gas temperature shown in rows 7 - 9 in Table 14.2 can be linked to the small degradation in bubble point due to heated gas, as modeled in the cryogenic bubble point equation. If the gas temperature were raised significantly higher than the liquid temperature, then the degradation in expulsion efficiency would be much higher.

Next, simulations were run in $\mathrm{LH}_{2}$ at fixed thermodynamic properties and a fixed demand flow rate of $0.01 \mathrm{~kg} / \mathrm{s}$ using four different LAD mesh screen types. The channel had a height and width of $2.54 \mathrm{~cm}$ each. Examination of the last four rows in Table 14.2 shows that the 200x600 Dutch Twill mesh screen achieves the highest expulsion efficiency and that there is $\sim 5 \%$ difference between the $200 \times 600$ and $325 \times 2300$ meshes for the identical outflow conditions. In general coarser screens have lower FTS pressure losses, but lower bubble point pressures. Meanwhile, the finer screens have higher bubble points and slightly higher flow losses, so Table 14.2 echoes the fact that there is an 
optimum balance between the bubble point pressure and FTS loss. While the coarser meshes may have slightly lower FTS losses, a lower bubble point implies lower resistance to breakthrough against adverse accelerations. If mission requirements dictate, such as in the case of the large scale cryogenic fuel depot, finer mesh screens would be required to counter those higher adverse accelerations.

\begin{tabular}{|c|c|c|c|c|c|c|}
\hline Screen/Fluid & Mass Flow Rate [kg/s] & $\mathbf{H}$ [cm] & $\mathbf{W}$ [cm] & Number of Arms & Expulsion Efficiency & Mass [kg] \\
\hline $325 \times 2300$, LH2 & 0.01 & 2.54 & 2.54 & 4 & 92 & 8.491 \\
\hline $325 \times 2300$, LH2 & 0.01 & 2.54 & 5.08 & 4 & 94.8 & 11.43 \\
\hline $325 \times 2300$, LH2 & 0.01 & 2.54 & 2.54 & 8 & 94.8 & 16.98 \\
\hline $325 \times 2300$, LH2 & 0.01 & 2.54 & 5.08 & 8 & 96.2 & 22.86 \\
\hline
\end{tabular}

Table 14.3 - Expulsion Efficiencies for Variable Sized Screen Channel Liquid Acquisition

Devices in Terms of Height, Width, and Number of Arms

A larger sized LAD can sustain both higher flow rates as well as obtain higher expulsion efficiencies. To determine how best to size the LAD with increased flow demand, simulations were run to determine the effect of varying the width of the screen channel versus varying the number of channels for a $325 \times 2300$ screen in $\mathrm{LH}_{2}$ at a fixed liquid and pressurant gas temperature of $20.3 \mathrm{~K}$ each and a tank pressure of $101 \mathrm{kPa}$. As shown in Table 14.3, doubling the width has the same impact on expulsion efficiency as increasing the number of arms by a factor of 2 . There is a significantly greater mass increase when increasing the number of arms $(8.49 \mathrm{~kg})$ as opposed to increasing the width $(2.94 \mathrm{~kg})$.

To determine how the LADs scale with flow rates, in order to compare performance with the vane PMD, simulations were also run at multiple flow rates between $1 \times 10^{-4} \mathrm{~kg} / \mathrm{s}-2.76 \times 10^{-2} \mathrm{~kg} / \mathrm{s}$ for a $325 \times 2300$ screen in $\mathrm{LH}_{2}$ at a fixed liquid and 
pressurant gas temperature of $20.3 \mathrm{~K}$ each and a tank pressure of $101 \mathrm{kPa}$. Table 14.4 presents the resultant expulsion efficiencies and PMD mass for the screen channel LAD cases at different demand flow rates. The results show that up to a mass flow rate of $0.0049 \mathrm{~kg} / \mathrm{s}$, the screen channel LAD can achieve a maximum expulsion efficiency of $98.1 \%$ of the small scale $\mathrm{LH}_{2}$ tank. This upper limit is due to the fact that the gallery arm cannot access the small residual propellant in between consecutive arms at very low fill levels due to the closed flow path. As the flow rate increases beyond that point, the total screen width reaches the defined maximum limit; if the LAD width or number of gallery arms is increased, the total screen width exceeds half the tank circumference. At even higher flow rates, the maximum achievable expulsion efficiency begins to decrease, due to the higher FTS pressure drop at higher flow rates. As expected, the mass of the screen channel LAD system increases with increasing demand mass flow rate.

\begin{tabular}{|c|c|c|c|c|c|}
\hline Mass Flow Rate [kg/s] & Height [cm] & Width [cm] & Arms & Mass [kg] & Expulsion Efficiency \\
\hline 0.0001 & 2.54 & 2.54 & 4 & 8.491 & 98.1 \\
\hline 0.00025 & 2.54 & 2.54 & 4 & 8.491 & 98.1 \\
\hline 0.0005 & 2.54 & 5.08 & 4 & 11.43 & 98.1 \\
\hline 0.001 & 2.54 & 7.62 & 4 & 14.37 & 98.1 \\
\hline 0.0018 & 2.54 & 15.24 & 4 & 23.2 & 98.1 \\
\hline 0.003 & 2.54 & 25.4 & 4 & 34.96 & 98.1 \\
\hline 0.0049 & 2.54 & 17.78 & 8 & 52.28 & 98.1 \\
\hline 0.0075 & 2.54 & 27.94 & 8 & 75.81 & 98 \\
\hline 0.01 & 2.54 & 27.94 & 8 & 75.81 & 97.9 \\
\hline 0.0125 & 2.54 & 27.94 & 8 & 75.81 & 97.8 \\
\hline 0.015 & 2.54 & 27.94 & 8 & 75.81 & 97.7 \\
\hline 0.0175 & 2.54 & 27.94 & 8 & 75.81 & 97.7 \\
\hline 0.02 & 2.54 & 27.94 & 8 & 75.81 & 97.6 \\
\hline 0.0225 & 2.54 & 27.94 & 8 & 75.81 & 97.5 \\
\hline 0.025 & 2.54 & 27.94 & 8 & 75.81 & 97.5 \\
\hline 0.0276 & 2.54 & 27.94 & 8 & 75.81 & 97.4 \\
\hline
\end{tabular}

Table 14.4 - Screen Channel Liquid Acquisition Device Sizing as a Function of Demand Flow Rate for Case A Ullage Bubble Growth 
To examine the effect of pressurant gas diffuser location on LAD performance, Table 14.5 shows the results for gallery arm LADs for Case B and Case C ullage bubble growth, where the liquid and pressurant gas temperature was $20.3 \mathrm{~K}$ and the tank pressure was $101 \mathrm{kPa}$. At smaller flow rates, Case B bubble growth allows the LAD to achieve the maximum allowable $99.9 \%$ expulsion efficiency, with a lower mass per arm requirement than Case C. At higher flow rates, Case B bubble growth allows the LAD to achieve higher expulsion efficiency compared to Case C bubble growth. From a systems level standpoint, this is highly beneficial.

\begin{tabular}{|c|c|c|c|c|c|c|c|c|}
\hline Bubble Growth & Screen & Mass Flow Rate [kg/s] & Height [cm] & Width [cm] & Arms & Mass [kg] & Mass per arm [kg] & Expulsion Efficiency \\
\hline \multirow[t]{10}{*}{ Case B } & $325 \times 2300$ & 0.0001 & 2.54 & 2.54 & 4 & 8.697 & 2.174 & 99.9 \\
\hline & & 0.001 & 2.54 & 5.08 & 4 & 11.71 & 2.928 & 99.9 \\
\hline & & 0.01 & 2.54 & 17.78 & 8 & 53.55 & 6.693 & 99.9 \\
\hline & & 0.1 & 2.54 & 27.94 & 8 & 77.65 & 9.706 & 98.7 \\
\hline & & 1 & 2.54 & 27.94 & 8 & 77.65 & 9.706 & 87.1 \\
\hline & $200 \times 600$ & 0.0001 & 2.54 & 2.54 & 4 & 8.814 & 2.204 & 99.9 \\
\hline & & 0.001 & 2.54 & 2.54 & 4 & 8.814 & 2.204 & 99.9 \\
\hline & & 0.01 & 2.54 & 7.62 & 4 & 15.07 & 3.768 & 99.9 \\
\hline & & 0.1 & 2.54 & 27.94 & 8 & 80.22 & 10.03 & 99.8 \\
\hline & & 1 & 2.54 & 27.94 & 8 & 80.22 & 10.03 & 97.8 \\
\hline \multirow[t]{10}{*}{ Case C } & $325 \times 2300$ & 0.0001 & 2.54 & 10.16 & 1 & 4.434 & 4.434 & 99.9 \\
\hline & & 0.001 & 2.54 & 27.94 & 1 & 9.706 & 9.706 & 99.4 \\
\hline & & 0.01 & 2.54 & 27.94 & 1 & 9.706 & 9.706 & 95 \\
\hline & & 0.1 & 2.54 & 27.94 & 1 & 9.706 & 9.706 & 95 \\
\hline & & 1 & 2.54 & 27.94 & 1 & 9.706 & 9.706 & 95 \\
\hline & $200 \times 600$ & 0.0001 & 2.54 & 2.54 & 1 & 2.204 & 2.204 & 99.9 \\
\hline & & 0.001 & 2.54 & 15.24 & 1 & 6.115 & 6.115 & 99.9 \\
\hline & & 0.01 & 2.54 & 27.94 & 1 & 10.03 & 10.03 & 99 \\
\hline & & 0.1 & 2.54 & 27.94 & 1 & 10.03 & 10.03 & 95 \\
\hline & & 1 & 2.54 & 27.94 & 1 & 10.03 & 10.03 & 95 \\
\hline
\end{tabular}

Table 14.5 - Screen Channel Liquid Acquisition Device Sizing as a Function of Demand Flow Rate for Cases B and C Ullage Bubble Growth Rates

Based on these results, it is favorable to have the pressurant gas diffuser at the aft end of the tank far away from the outlet (Case B) because the LAD tends to draw more mass into the channel closer to the outlet. When the LAD is exposed from the middle, as it is in Case C, there is a greater FTS pressure drop near the demand end of the LAD, 
which eventually leads to earlier breakdown. When the LAD is exposed to vapor farther away from the outlet, as it is in Case B, there is a lower FTS pressure drop, and the LAD can sustain vapor free liquid flow for a longer period of time.

Finally, simulations for Case B and C bubble growth were run for two different mesh screens. As expected, based on the preliminary study in Table 12.2, the 200x600 screen achieved higher expulsion efficiencies than the $325 \times 2300$ screen, due to its lower FTS pressure drop. Therefore, in low adverse acceleration environments, the 200x600 would be the optimal choice for $\mathrm{LH}_{2}$ flows; in higher adverse acceleration environments such as for a fuel depot, a finer mesh like the $450 \times 2750$ would be required. If there were no compatibility issues between tank wall and PMD material and liquid, then the $165 \times 800$ would be the optimal choice because it can be constructed out of Ti.

All these arguments are predicated on storable LAD design logic. They exclude additional heat and mass transfer concerns that arise in cryogenic liquids due to enhanced evaporation and condensation at the LAD screen. As mentioned in Chapter 13, higher thermal gradients are expected at breakdown for coarser mesh screens, which would further degrade an already low bubble point pressure for the 200x600 screen.

To compare vane performance against screen channel LADs, vane PMDs were sized across the same range of mass flow rates as the gallery arms from Table 14.4. Table 14.6 presents the results for vane cases at a liquid and pressurant gas temperature of 20.3K and a tank pressure of $101 \mathrm{kPa}$. Several distinct trends are noticeable. First, vanes can achieve a higher maximum expulsion efficiency than screen channel LADs, overall. Second, to achieve higher flow rates, the size and number of required vanes grows 
significantly, as expected. At a demand flow rate of $0.005 \mathrm{~kg} / \mathrm{s}$, already 44 vanes are required, which is rather hard to conceive physically due to complexity in manufacturing. Third, as the mass flow rates increases, the expulsion efficiency of the vane PMD decreases, as expected. Fourth, the flow rate beyond which vanes are not the optimal choice for this small scale $\mathrm{LH}_{2}$ tank is approximately $4 \times 10^{-3} \mathrm{~kg} / \mathrm{s}$.

\begin{tabular}{|c|c|c|c|c|c|c|c|}
\hline $\begin{array}{c}\text { Mass Flow Rate } \\
{[\mathbf{k g} / \mathbf{s}]}\end{array}$ & $\begin{array}{c}\text { Height } \\
{[\mathbf{c m}]}\end{array}$ & $\begin{array}{c}\text { \#ans Flow Rate per Vane } \\
{[\mathbf{k g} / \mathbf{s}]}\end{array}$ & $\begin{array}{c}\text { Rup } \\
{[\mathbf{c m}]}\end{array}$ & $\begin{array}{c}\text { Rdown } \\
{[\mathbf{c m}]}\end{array}$ & $\begin{array}{c}\text { Mass } \\
{[\mathbf{k g}]}\end{array}$ & Expulsion Efficiency \\
\hline 0.0001 & 1.7 & 4 & 0.000025 & 5.3 & 0.5 & 2.112 & 99.92 \\
\hline 0.00025 & 1.9 & 4 & $6.25 \mathrm{E}-05$ & 11.8 & 0.9 & 2.365 & 99.85 \\
\hline 0.0005 & 1.9 & 8 & $6.25 \mathrm{E}-05$ & 11.8 & 0.9 & 4.728 & 99.7 \\
\hline 0.001 & 2 & 12 & $8.33 \mathrm{E}-05$ & 15.8 & 1.1 & 7.439 & 99.45 \\
\hline 0.0018 & 2 & 16 & 0.000113 & 32.5 & 1.3 & 9.909 & 99.06 \\
\hline 0.003 & 2 & 28 & 0.000107 & 28 & 1.3 & 17.32 & 98.43 \\
\hline 0.0049 & 2 & 44 & 0.000111 & 31.2 & 1.3 & 27.18 & 97.54 \\
\hline 0.0075 & 2 & 64 & 0.000117 & 36.5 & 1.4 & 39.48 & 96.65 \\
\hline 0.01 & 2 & 84 & 0.000119 & 38.4 & 1.4 & 51.75 & 96.08 \\
\hline 0.0125 & 2 & 108 & 0.000116 & 34.8 & 1.4 & 66.47 & 95.68 \\
\hline 0.015 & 2 & 128 & 0.000117 & 36.2 & 1.4 & 78.7 & 95.39 \\
\hline 0.0175 & 2 & 148 & 0.000118 & 37.2 & 1.4 & 90.92 & 95.17 \\
\hline 0.02 & 2 & 168 & 0.000119 & 37.9 & 1.4 & 103.1 & 95 \\
\hline 0.0225 & 2 & 188 & 0.00012 & 38.5 & 1.4 & 115.3 & 94.86 \\
\hline 0.025 & 2 & 212 & 0.000118 & 36.6 & 1.4 & 127.5 & 94.75 \\
\hline 0.0276 & 2 & 240 & 0.000115 & 33.6 & 1.4 & 146.9 & 94.65 \\
\hline
\end{tabular}

Table 14.6 - Vane Propellant Management Device Sizing as a Function of Demand Flow Rate

Figure 14.14 compares the expulsion efficiency of the vanes and gallery arms across the flow rate range. At lower flow rates, the vanes can deliver higher expulsion efficiencies at a significantly lower PMD mass. However, the drop off in vane expulsion efficiency at higher flow rates is greater than the drop off in gallery arm expulsion efficiency. Up to a flow rate of $3 \times 10^{-3} \mathrm{~kg} / \mathrm{s}$, the expulsion efficiency of the vane system remains higher than that of gallery arms. For flow rates beyond $\sim 4 \times 10^{-3} \mathrm{~kg} / \mathrm{s}$, the expulsion efficiency of vanes decreases dramatically below that of the gallery arms, making gallery arms the appropriate choice. 


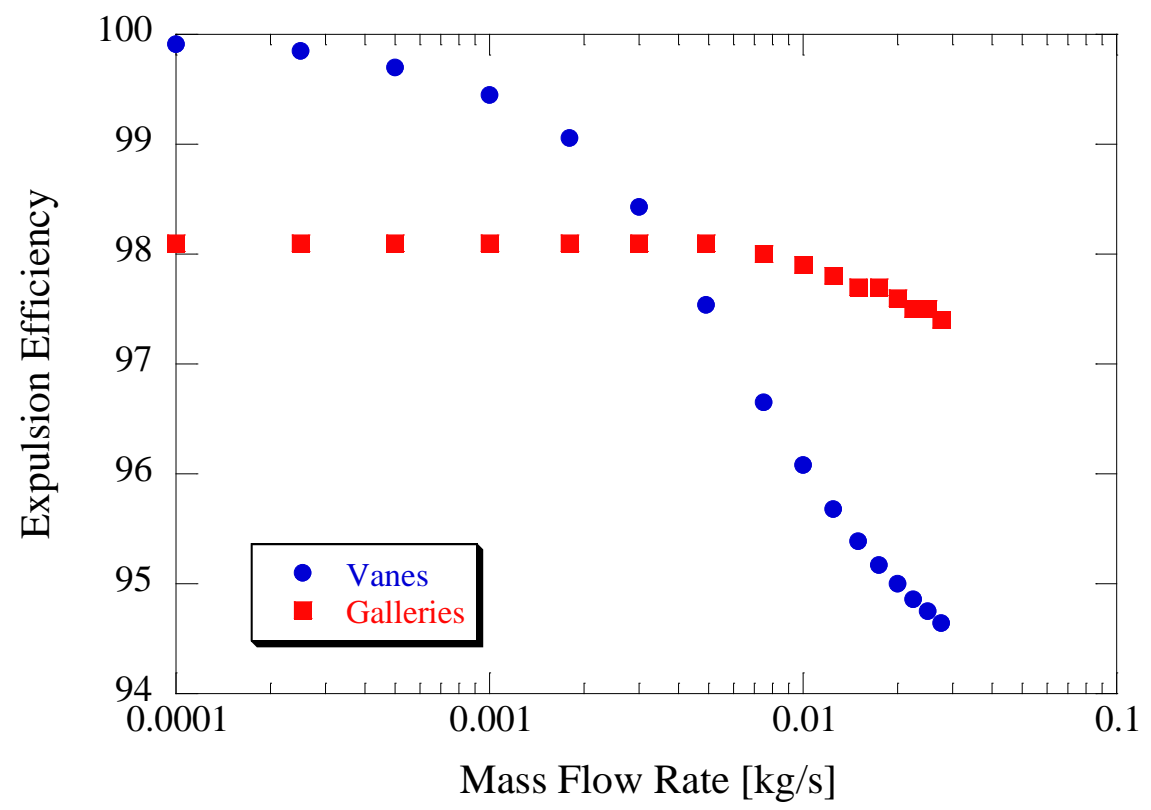

Figure 14.14 - Expulsion Efficiency as a Function of Demand Flow Rate for Vanes and Screen Channel Liquid Acquisition Devices

Fifth, comparing Tables 14.4 and 14.6, in general, for a given desired flow rate below $4 \times 10^{-3} \mathrm{~kg} / \mathrm{s}$, vanes can deliver higher expulsion efficiencies at a much lower PMD mass. Figure 14.15 plots PMD mass for vanes and screen channels as a function of demand flow rate. Clearly, vanes are the optimal choice below a demand flow rate of $1.4 \times 10^{-2} \mathrm{~kg} / \mathrm{s}$ due to a lower PMD mass; above this flow rate, the size and mass of the vane required to deliver this flow rate becomes impractically large. The mass of the vanes increases nearly linearly with demand mass flow rate. The mass of the gallery arms also initially increases linearly, but when the gallery arms system reaches the maximum allowable size, the PMD mass reaches a maximum value and remains constant for greater flow rates. Up to a mass flow rate of $1.25 \times 10^{-2} \mathrm{~kg} / \mathrm{s}$, the mass of the gallery arms is greater than the mass of the vanes. By $1.5 \times 10^{-2} \mathrm{~kg} / \mathrm{s}$, the crossover point has passed and 
vanes are more massive than gallery arms. Using interpolation, this crossover point falls near $1.4 \times 10^{-2} \mathrm{~kg} / \mathrm{s}$.

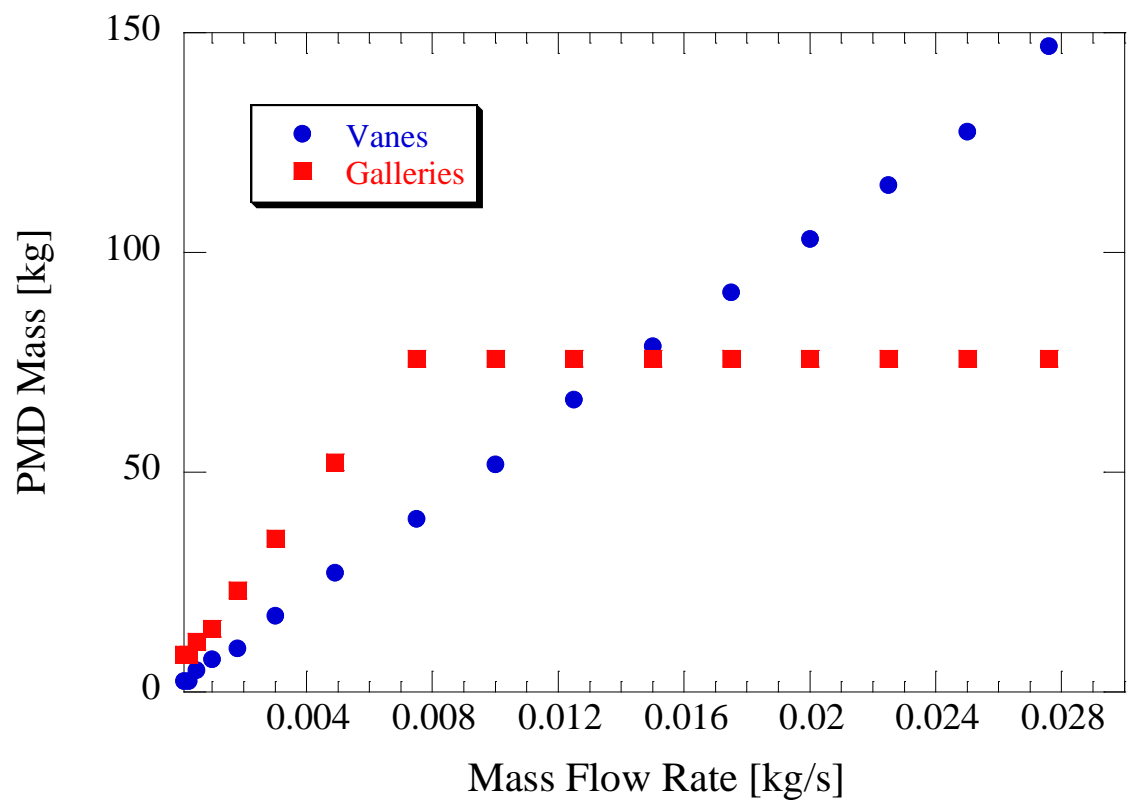

Figure 14.15 - Resultant Propellant Management Device System Mass as a Function of Demand Flow Rate for Vanes and Screen Channel Liquid Acquisition Devices

To further understand why vanes can only support lower flow rates, Figure 14.16 plots the mass flow rate per vane arm as a function of the total demand flow rate. As shown in Figure 14.16, as predicted by Equation 14.15, there is a limit to the available flow rate that a single vane can deliver, regardless of the thermodynamic conditions within the propellant tank. For this small scale $\mathrm{LH}_{2}$ tank the limiting flow rate per vane arm is approximately $1.2 \times 10^{-4} \mathrm{~kg} / \mathrm{s}$. This limiting mass flow rate is the result of the choking phenomenon exhibited by vanes, as outlined earlier. 


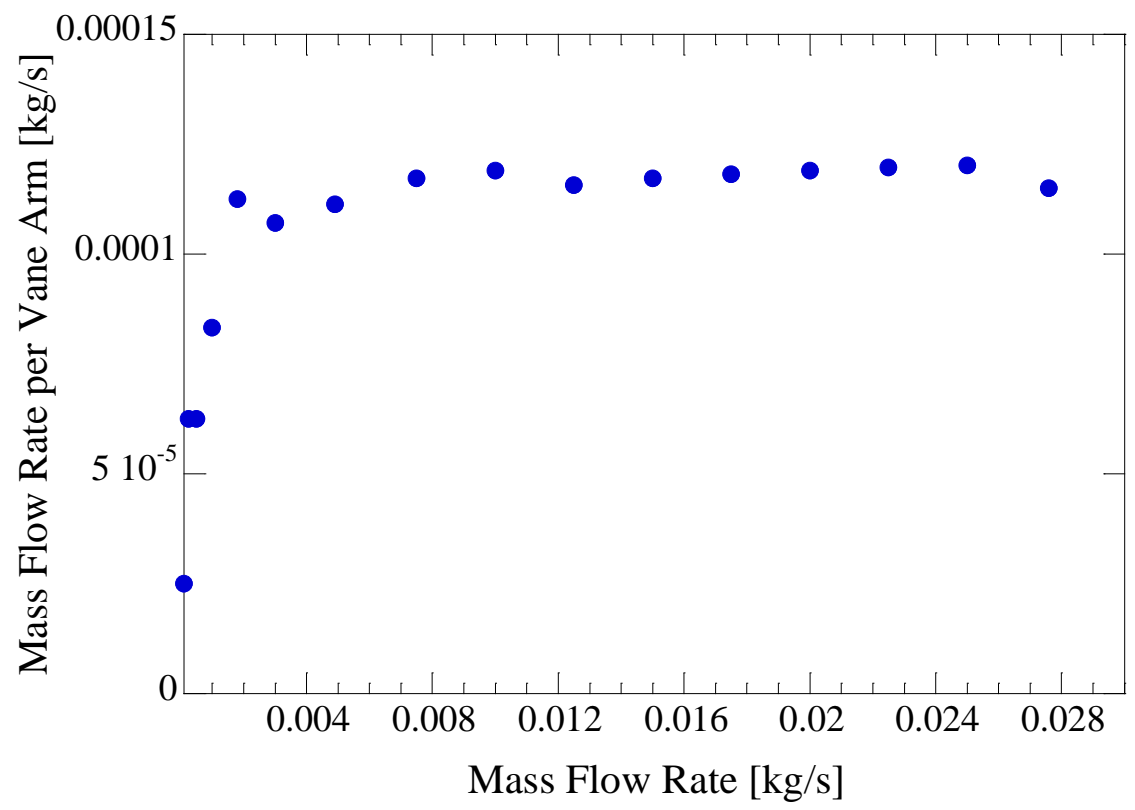

Figure 14.16 - Mass Flow Rate Delivered Per Vane Arm as a Function of Total Demand Mass Flow Rate

Figures 14.14 and 14.15 can be used to determine the range of flow rates for which vanes are the optimal choice and the range over which screen channel LADs are the more appropriate choice. The optimal PMD recommendation for a small scale $\mathrm{LH}_{2}$ storage tank in low gravity is summarized in Figure 14.17. Based on the expulsion efficiency analysis, at flow rates less than $4 \times 10^{-3} \mathrm{~kg} / \mathrm{s}$, vanes are the recommended choice, since they deliver higher expulsion efficiencies with lower PMD mass. Based on the PMD mass analysis, galleries are the recommended option at flow rates greater than $1.4 \times 10^{-2} \mathrm{~kg} / \mathrm{s}$. At higher flow rates, galleries deliver higher expulsion efficiencies and with lower PMD mass. Therefore an overlap zone exists between these two flow rates, which bound the critical mass flow rate range. In the overlap zone, gallery arms deliver higher expulsion efficiencies, but vanes have lower PMD mass. Since the primary 
performance parameter is expulsion efficiency, the recommended PMD in the overlap zone would normally be gallery arms. However, if PMD mass were more critical, vanes, though less efficient, are still an option in this zone. As in all system design, performance and system mass are generally traded against one another. By following simple rationale in the current work, Figure 14.17 can be generated for any sized propellant tank to determine the optimal PMD for that particular system.

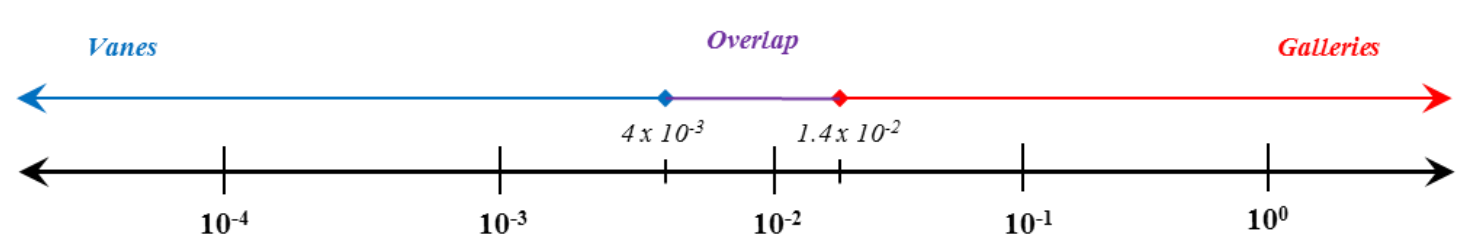

Figure 14.17 - Recommended Propellant Management Device for a Given Demand Flow Rate Range for a Small Scale Liquid Hydrogen Storage Tank in Microgravity

\subsection{Concluding Remarks}

An analytical solution for flow through a porous channel with a single porous wall was derived and used to predict the velocity and pressure fields inside a screen channel LAD mounted inside a cryogenic propellant tank in a microgravity environment. This model extends the existing 1-g model to microgravity by introducing the unknown interface velocity, which links the flow between the two liquid-liquid regions. Given specific tank and LAD dimensions, coupled with an ullage bubble growth rate and the updated cryogenic bubble point model, the new analytical flow model can be used to simulate propellant tank drain in microgravity. A comparison between the microgravity and 1-g models shows that LADs can prevent vapor ingestion for longer periods of time in microgravity. It can be concluded that since FTS pressure drop is the first order 
pressure drop term in microgravity, the screens with better resistance to FTS pressure drop should be chosen.

Results show that the LAD tends to draw more liquid from the section of the LAD closest to the demand outlet, as opposed to the back side of the channel close to the aft end of the tank. This is due to the screen being exposed at the center of the channel, which causes no injection into the channel and eventually causes the flow from the aft end of the channel to cease. Expulsion efficiencies are insensitive to the assumed profile for the interface velocity. The results also show that increasing LAD dimensions improves performance. Specifically, increasing the width leads to a lower increase in PMD mass as opposed to increasing the height. The microgravity analytical model represents the base model for designing cryogenic screen channel liquid acquisition devices for microgravity environments, which can be implemented for full propellant tank drains using either storable or cryogenic propellants. An analytical model was also developed for vanes using conservation of mass and momentum.

A preliminary trade study was developed to determine the optimal propellant management device for a small scale liquid hydrogen tank in the microgravity of Low Earth Orbit. Vanes and screen channel LADs were compared based on the performance parameters of expulsion efficiency, demand flow rate, and PMD system mass. An analytical model for screen channel LADs was used to simulate tank drain in microgravity, while vanes were modeled in an end of life configuration. Several ullage bubble growth cases were modeled and compared to determine the effect of pressurant gas diffuser location on LAD performance. The highest expulsion efficiencies are 
obtained when the diffuser is located at the aft end of the propellant tank. This is because the gallery arm pulls mass into the channel closest to the location of demand.

To compare performance between vanes and screen channels, expulsion efficiency and resultant PMD mass were determined for both PMDs across a wide range of demand flow rates. Based on the maximum achievable expulsion efficiency and resultant PMD mass, the critical mass flow rate range for the small scale $\mathrm{LH}_{2}$ tank was determined to be $4 \times 10^{-3} \mathrm{~kg} / \mathrm{s}-1.4 \times 10^{-2} \mathrm{~kg} / \mathrm{s}$, below which vanes are the optimal PMD, above which screen channels are the optimal choice. Finally, comparison of PMD mass shows that, depending on the desire to minimize PMD mass or maximize expulsion efficiency, vanes may be used to supply flow rates as high as $1.4 \times 10^{-2} \mathrm{~kg} / \mathrm{s}$ for a small scale $\mathrm{LH}_{2}$ tank in a continuous outflow. The analysis also showed that there exists a limit on the mass flow rate that a single vane can deliver, due to choking. The analytical models and rationale developed here can be used to size and trade PMD performance for any sized propellant tank in any gravitational or thermal environment. 


\section{Chapter 15}

\section{Conclusions}

This chapter presents a synopsis of the experimental and analytical results from this dissertation. Comments are given on the remaining steps required to qualify LADs for cryogenic flight. Additionally, several concepts are given to advance future cryogenic LAD technology development.

\subsection{Summary}

All future manned and robotic space exploration missions are dependent on enabling in-space cryogenic engines as well as cryogenic fuel depots, which is completely dependent on developing advanced cryogenic fluid management technology. This dissertation advances the state and technology readiness level of propellant management devices, specifically screen channel liquid acquisition devices, in low surface tension cryogenic propulsion systems operating in low gravity, by presenting the results from a battery of ground level component and full scale cryogenic tests. As a result, both component and full scale hardware has been designed, analyzed, and verified in a cryogenic environment. New and rare performance data was collected in four different cryogenic liquids, methane, oxygen, nitrogen, and hydrogen. Experimental data obtained in cryogenic liquids revealed fundamental heat and mass transfer issues which cannot be modeled or accounted for using storable propellant LAD design logic. 
The entire set of primary and secondary factors which influence LAD design were rigorously formulated and an entire suite of physics based models for the influential factors were developed and validated both in storable and cryogenic propellants. While the physics based models agreed well with historical room temperature data, all LAD models validated by cryogenic data, including bubble point pressure, reseal pressure, FTS pressure drop, TVS cooling efficiency, and full scale LAD channel pressure drop show strong temperature dependence and deviation from the room temperature behavior. The analytical models derived from first principles and validated both by the small set of historical data and large data set from this dissertation can be used to analyze, design, and size any screen channel LAD for any future in-space cryogenic or storable propulsion system in microgravity. In addition, the rationale and logic were systematically outlined for how to design and trade performance for different PMDs, as models were applied to sizing PMDs for both small scale and large scale in-space cryogenic propulsion systems.

Towards enabling NASA's desire for future long duration manned space exploration, screen channel LADs in cryogenic propellants were completely characterized experimentally and analytically and have been taken from the basic conceptual stage of TRL-1 all the way up to full scale technology demonstration in a relevant thermal environment (TRL-5/6) in just 4 years. Cryogenic LAD technology maturation requires flight demonstration in a small scale system in low gravity followed by the final full scale depot. Regardless of the space flight community and NASA's future space exploration desires, experimental data, hardware, and analytical models compiled in this work are vital to all future cryogenic LAD designs, and will be especially critical for predicting performance and eventually designing PMDs for both 
flight technology demonstration missions as well as full scale systems for in-space cryogenic engines and cryogenic depots where the highest level of LAD performance will be required to transfer such low surface tension cryogenic propellant.

In this dissertation, the general purpose, role, and challenges associated with advanced cryogenic propulsion system technology development, storage, and transfer in low gravity were outlined as they relate to cryogenic engines and cryogenic fuel depots. A detailed historical review was presented for every type of PMD. Specifically, design concept, basic flow physics, and principle of operation were given for the three primary capillary driven PMDs, vanes, sponges, and screen channel LADs. Numerous examples were given on the rich flight heritage of these three types, which include applications for basic ISS space experiments, full scale NASA missions, and military and commercial satellites. After identifying NASA's long term needs, as they relate to the development of technology for storage and transfer of cryogenic propellants over a wide range of operational and thermal conditions, screen channel LADs were selected as the PMD of choice to pursue advanced work. Models were then developed from first principles for the primary (bubble point pressure and flow-through-screen pressure drop) and secondary (wicking rate, screen compliance, material compatibility, and pressurant gas type) factors which govern LAD performance. Each model was validated with available room temperature historical data. The methodology for how to conduct tests for each of these parameters was also outlined to give mission designers insight into how to performance test the LAD.

Then a rigorous series of ground tests were performed, both in room temperature and cryogenic liquids. To validate bubble point, reseal pressure, and wicking rate models, 
the finest available LAD mesh screens were investigated in room temperature liquids. The room temperature tests showed that effective pore diameters must be calculated based on reference fluid tests, and not SEM analysis; relationships do exist between nonphysical effective pore diameter and physical dimensions of the screen (number and diameter of warp and shute wires), however. The ideal test procedure for bubble point testing was also outlined. The long standing assumption that bubble point pressure scales with the fineness of the screen was disproved, because the 450x2750 mesh yielded the maximum bubble point pressure of the four screens.

Cryogenic bubble point tests in $\mathrm{LH}_{2}$ and $\mathrm{LN}_{2}$ revealed the functional dependencies of the cryogenic bubble point pressure, namely screen type, liquid type, contact angle, liquid temperature and pressure, and pressurant gas type and temperature. A $165 \%$ increase in performance is achievable over the baseline $\mathrm{LH}_{2} 325 \times 2300$ historical value by using the finer $450 \times 2750$ mesh and pressurizing and subcooling the interface with GHe. Experiments showed that the effective pore diameter is itself temperature dependent, and that the pore shrinks at different rates depending on the specific screen mesh. Bubble point is a strong function of liquid temperature and a weak function of liquid subcooling. Warm pressurant gas in contact with the screen always acts a degradation in performance, with more degradation occurring using coarser screens and condensable gas to pressurize.

High pressure cryogenic bubble points collected in $\mathrm{LOX}$ and $\mathrm{LCH}_{4}$ show strong dependence of bubble point on the type of pressurant gas in contact with screen. Higher performance is obtained whenever subcooling the liquid below the saturation temperature (ex. 140\% gain above NBP value at a pressure $1.79 \mathrm{MPa}$ for GHe/LOX), but the 
performance gain depends on the type of gas in contact with the screen. The difference in performance is attributed to added heating and cooling of the $\mathrm{L} / \mathrm{V}$ interface due to added condensation and evaporation, verified both by experimental data and flow visualization. Pressurization with the vapor increases the interfacial temperature while pressurization with a non-condensable gas such as helium acts to reduce the interfacial temperature. For all four cryogenic liquids, higher performance is always obtained using non-condensable gases to pressure because heat and mass transfer effects are minimized. For $\mathrm{LCH}_{4}$, two gases can be considered non-condensable pressurants. Heat and mass transfer effects between three different pressurants in $\mathrm{LCH}_{4}$ were compared through quantification of heat transfer at breakdown, effective interfacial temperatures, condensation and evaporation mass fluxes, screen Re numbers, and heat conduction into the liquid. Translating bubble point pressures into allowable flow rates out of the propellant tank, experimental results in $\mathrm{LH}_{2}, \mathrm{LOX}$, and $\mathrm{LCH}_{4}$ show that fine mesh screen channel LADs can easily meet mission requirements for low pressure, low flow rate hydrogen systems and higher pressure-fed ascent engines using $\mathrm{LOX}_{\mathrm{LCH}}$.

Two full scale screen channel LADs were designed and performance tested in a thermally representative environment across a wide range of operating conditions typical of a proposed fuel depot to simulate fluid transfer, determine the functional form of each pressure drop, and assess the reliability of LADs at cryogenic temperatures. One of the LADs was thermally and structurally flight representative due to the presence of a custom built internal TVS HEX and a perforated plate. The breakdown point of the channel is dominated by liquid temperature with a secondary dependence on the demand flow rate through the LAD. Performance for the TVS cooled channel is higher than the standard 
channel due to TVS cooling of the liquid inside the channel and enhanced wicking from the perforated plate. Subcooling the liquid improved performance in the dynamic outflow performance of LADs as it did in the static case.

A simplified 1D model proposed by Jaekle (1997) only qualitatively tracks experimental results for system pressure drop calculations in cryogenic liquids. To fully account for system effects in cryogenic liquids, higher fidelity tools are required. Experimental results in cryogenic liquids were used to update analytical predictive models for bubble point pressure, reseal pressure, FTS pressure drop and full scale pressure drop through the porous LAD channel. The new cryogenic bubble point and reseal pressure models are accurate to within $3 \%$ and $4 \%$ of the data, respectively. The FTS pressure is shown to be $50 \%$ higher at $\mathrm{LH}_{2}$ temperatures, with the new model accounting for temperature effects at cryogenic temperatures through the temperature dependent fitting parameters. The controlling parameter for determining the maximum bubble point and reseal pressures is the warp to shute diameter ratio. Convective cooling of the screen and channel in the dynamic outflow environment enhanced performance by $300 \%$ over the static bubble point value. When coupled with the new cryogenic bubble point model, FTS model, and a dynamic bubble point term which accounts for enhanced convective cooling of the screen in the dynamic flow case, the new analytical pressure drop model matches the $\mathrm{LH}_{2}$ outflow data well. With an appropriate ullage bubble location and growth rate model, the analytical pressure drop model is easily integrated into a microgravity flow code.

Finally, as an added bonus, the suite of analytical tools were applied to the design, analysis, and sizing of LADs for a large scale fuel depot as well as a small scale $\mathrm{LH}_{2}$ 
propellant tank. The logic for how to size the LAD was detailed and the methodology for conducting a PMD mass versus expulsion efficiency trade study was given to compare performance of vanes against screen channel LADs. Anchoring models to the new cryogenic data collected here facilitates predictions in both storable and cryogenic propulsion systems.

\subsection{Future Work}

While screen channel LADs are at a general technology readiness level of TRL-5, there always remains more research and technology development to advance the state of LADs for cryogenic propulsion systems. On a systems level, the remaining three steps to obtain the highest possible readiness level are:

1. Integrate LADs and all other CFM components into a full scale integrated ground experiment to examine interactions between each component and to demonstrate full scale outflow.

2. Technology demonstration mission in microgravity.

3. Full scale depot and demonstration of tank to tank transfer.

Experiments conducted in microgravity can provide much needed, final performance data necessary to validate analytical flow models. Some other ideas for future work are given as follows:

First, future smaller scale ground experiments should include component level testing of coarser LAD screen meshes. Driven by the desire to acquire and maintain low surface tension cryogenic propellant liquid flow from a propellant tank against high 
adverse acceleration levels over a wide range of demand flow rates, the bulk of the work conducted in this dissertation centered on technology development of fine mesh LAD screens. However, coarser screens are easier to integrate into channels, tend to have lower flow losses, and can be constructed out of lighter materials. Specifically, testing should be conducted in $\mathrm{LH}_{2}$, where the highest deviation from room temperature performance is exhibited, where it is expected to have high degradation in performance due to heat and mass transfer effects. Coarser mesh data can be used to add to the existing cryogenic database and also anchor analytical models.

Second, due to the unavailability of historical data, full scale outflow tests should be repeated in the same thermally representative environment as in Chapter 9, but repeated using warm pressurant vapor and helium. The purpose of the test series would be to quantify the rate of degradation of LAD performance in a dynamic outflow environment to compare with degradation in the static case as well as determine the competition between degradation due to warm pressurant and gain due to convective cooling. Additionally, the optimal method and location of the pressurant gas diffuser could be determined through these experiments.

Third, performance data is needed in LHe and SFHe, which has implications for satellites and space telescope cooling. Fourth, full scale outflow tests are needed with the 450x2750 mesh. Fifth, component level bubble point tests should be conducted in higher pressures all the way up to the critical pressure. Sixth, wicking rate tests should be performed in more cryogenic fluids. Seventh, using the experimental apparatus outlined in Chapter 3, screen compliance tests need to be performed on coarse and fine mesh screens. 
Eighth, the analytical porous flow model applied to LAD channels can be updated to account for a slip velocity and/or permeability (Brown and Lai 2006) in the boundary condition at the LAD screen as suggested in previous simplified porous flow solutions analytically or experimentally using techniques such as those proposed in Appendix F. Ninth, the energy equation can be used to update the analytical porous flow solution to modify the proposed $\mathrm{x}$-velocity profile in the following manner:

$v_{x}(x, y)=h(x, y)$

where $\mathrm{x}$-momentum and energy are both used to specify the shape and magnitude of the velocity as the profile evolves down the channel. Tenth and finally, multiple different trade studies should be performed for vanes, sponges, and screen channel LADs for multiple different cryogenic systems. 


\section{Appendix A}

\section{Historical Depot Demonstration Missions}

This appendix is presented to provide historical reference into the previously attempted small scale "depot demonstration" missions. Along with the recently proposed CPST TDM, all of these missions provide a point with which to anchor test conditions for the various LADs experiments conducted in this dissertation. For each previous attempt at a depot demonstration, a brief historical description is given and relevant test parameters are outlined, including information about the PMD, where data was available in the literature. A list of many historical full scale depot designs is available in the literature (Glover 1991 and Chato 2008). Recent depot design concepts are outlined in Chapter 1.

A brief scan through the literature indicates that NASA has maintained interest in cryogenic fuel depot concepts and related depot demonstrations for over 50 years. After successful launch of the Apollo Saturn series rockets in the late 1960s, NASA soon examined options to send man to destinations beyond LEO. But in order to reduce risk and cost associated with the full development of depot concepts, smaller scale technology demonstrations (both on the ground and in LEO) were first required to ensure successful storage and transfer of a $20 \mathrm{~K}$ fluid under varying thermal and gravitational conditions. 
Over the past 50 years, NASA has successfully completed numerous ground CFM technology development programs, including both component and system level tests, across several NASA centers. Preliminary vehicle architecture design, system trade studies, and experimental requirements for CFM flight demonstrations have also been attempted, both in-house and through contracting organizations. But despite the rich history of CFM ground testing and obvious need for a low gravity cryogenic storage and transfer test, achieving a depot demonstration still remains as the elusive next step toward enabling the full scale cryogenic fuel depot.

\section{A.1 Project THERMO}

The first of several attempts to obtain low-g cryogenic fluid storage and transfer data came in 1967 under the project titled THERMO. Building off the success of the Apollo Program, the purpose of the Thermo and Hydrodynamic Experiment Research Module in Orbit was to identify and resolve CFM technology issues required to enable a manned mission to Mars by 1971 (Frederickson and Schweikle 1967a). Preliminary trade studies were conducted and a design review was held in 1967 (Frederickson and Schweikle 1967b). Originally, the in-flight experiment would be carried on a manned mission to the Moon using existing Apollo hardware, but was soon downgraded as a stand-alone experiment carried on an unmanned rocket. No mention of the specific propellant management device is available in the literature for Project THERMO. Due to budget constraints, the project was quickly terminated in 1969. 


\section{A.2 Shuttle Derived Experiments}

Initially, it was conceived that the STS cargo bay could be used as a test bed for various low-g demonstrations in LEO. Although STS-1 did not launch until April 1981, plans to derive a Shuttle compatible CFM flight experiment with $\mathrm{LH}_{2}$ were under way as early as 1976 as part of the on-orbit reusable Spacelab experimental test laboratory, beginning with conceptual designs from contractors McDonnell Douglas (Cady 1976). By 1978, these preliminary designs were fed into further investigations and an eventual preliminary data review (PDR) for a Cryogenic Fluid Management Experiment (CFME) by Martin Marietta aboard Shuttle (Eberhardt et al. 1981 and Smolak 1987). Consistent with interior Shuttle cargo bay dimensions, the proposed storage and transfer tank dimensions are listed in Table A.1 (Jetley and Scarlotti 1987).

\begin{tabular}{|c|c|c|c|c|c|c|}
\hline & & & & Depot & & \\
\hline & & Main Tank & Mass & (Cylindrical Receiver) & & \\
\hline & & Volume [m³] & LH2 [kg] & Volume [ft^3] & MAWP [kPa] & MEOP [kPa] \\
\hline 1 & CFME & 11.33 & 804 & $1.28 *$ & 345 & 138 \\
\hline 2 & CFMF & 71.6 & 5085 & 0.425 & 345 & 138 \\
\hline
\end{tabular}

Table A.1 - Shuttle Cargo Bay Derived Cryogenic Fluid Management Experiment Design Parameters

By the mid-1980s, safety and integration issues were addressed as the project was renamed into the Cryogenic Fluid and Management Facility (CFMF), despite apparent apprehension to mount an $\mathrm{LH}_{2}$ tank (Eberhardt et al. 1984 and DeFelice 1987) or LOX tank (Tunc et al. 2001) inside the Shuttle cargo bay. While design of CFME was centered on the supply aspects of an in-space fuel depot, CFMF design and analysis was tailored to address receiver tank storage and transfer aspects for an OTV. CFMF design parameters 
are also listed in Table A.1. Note that full communication capillary LADs were proposed for all fluid transfer tests on both CFME and CFMF in both supply and receiver tanks. However, no specific details about the screen mesh weave are available in the literature.

In January 1987, a year after the Challenger accident, all chances of using Shuttle cargo bay to transport an $\mathrm{LH}_{2} \mathrm{CFM}$ demonstration experiment were hopeless. There were attempts to replace the hazardous test fluid with an inert cryogen (Eberhardt and Fester 1981, Bicknell et al. 1988, and Lak et al. 1998), but many scientists within NASA felt that extrapolating results from a $77 \mathrm{~K}$ fluid like $\mathrm{LN}_{2}$ to a $20 \mathrm{~K}$ fluid would not faithfully represent the underlying physics of $\mathrm{LH}_{2}$ storage and transfer. Even still, cost to build a Shuttle based CFM $\mathrm{LN}_{2}$ demo was far too high to justify building a full flight demonstration. Therefore, the end of Challenger nearly marked the end of design and analysis of CFME, CFMF, and all other Shuttle based CFM demonstrations.

\section{A.3 COLD-SAT}

Despite the reduced morale in the NASA Space Flight Program after the Challenger accident, NASA still maintained a strong desire to obtain low-g cryogenic storage and transfer data, primarily due to the desire to continue to explore options for a manned mission to Mars. Due to safety concerns however, all subsequent designs of flight demonstrations would be delivered into LEO using EELVs carried by either an Atlas I or Delta II rocket. Since the entire payload volume on these EELVs was available for a CFM experiment, this radically changed the design and objectives of the demo. While CFME and CFMF individually targeted supply and receiver aspects of an in-space depot respectively, engineers were designing the new experiment to simultaneously 
address both aspects in a single mission in what would be called Cryogenic Orbiting Liquid Depot - Storage, Acquisition, and Transfer (COLD-SAT). While previously proposed Shuttle designs were attached to the cargo bay, COLD-SAT would be a freely flying satellite launched on an EELV and deployed in LEO (COLD-SAT 1989).

In the mid to late 1980s, due to the uncertainty of the future of Shuttle, NASA began to shift funding from these manned missions into research into LEO experiments and demos using EELVs. From 1988 - 1990, NASA funded three independent Phase A studies to General Dynamics, Ball Aerospace, and Martin Marietta to conduct trade studies, examine feasibility, propose flight architecture, and determine general experimental requirements and test procedures for COLD-SAT (Rybak et al. 1990, Schuster et al. 1990, and Bailey et al. 1990). During this time, NASA also conducted parallel analysis in-house (Arif and Kroeger 1989 and Arif 1991). Eventually, results from all four studies were compiled into a single comprehensive review (Kramer 1998).

For the transfer tests and liquid acquisition systems, COLD-SAT parameters from all four studies are compiled in Table A.2. Wherever parameters were not readily available, assumptions were made to derive those parameters. For example, MEOPs were determined from the anticipated maximum ranges of tank pressures during pressurization/outflow tests. Flow rates were determined from LAD or No Vent Fill (NVF) subsystem requirements. Re numbers in the transfer line were determined based on total flow delivery from the LAD subsystem and the internal diameter of the pipe. Line velocities were determined using conservation of mass. 


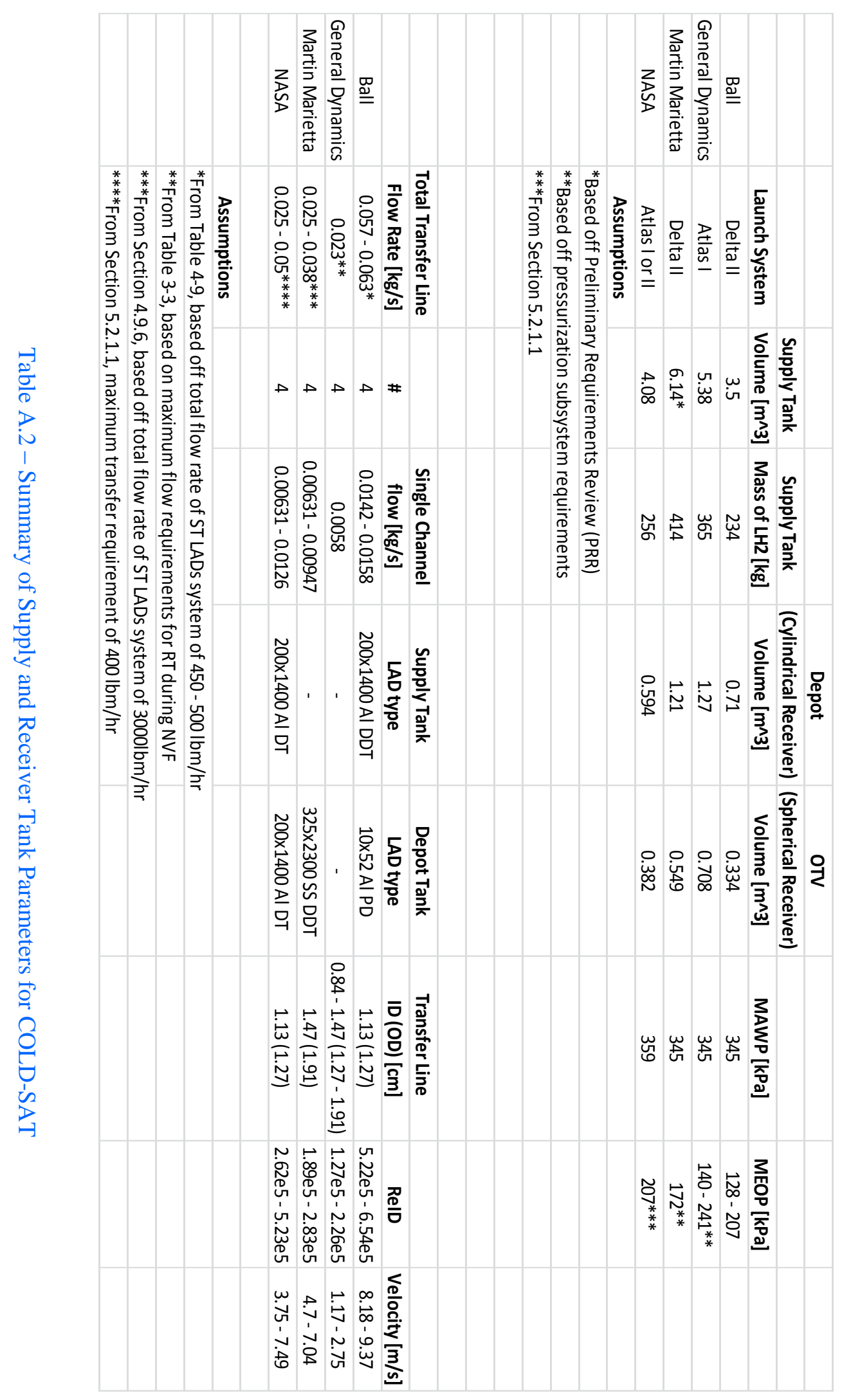


While there are numerous similarities between each system in terms of the supply tank MEOP during transfer and proposed LAD systems and flow rates, it is interesting to note that NASA's supply and receiver tanks are sized significantly smaller than the contractor's counterpart. It is also interesting to note that, wherever noted in the literature, the $200 \times 1400$ screen channel LAD was taken as the popular candidate despite its lower bubble point relative to the standard $325 \times 2300$ screen, possibly due to the lack of cryogenic data. Years later, experimentalists reported difficulty in simply achieving screen sealing and resealing at normally saturated fluid conditions in a ground test (Chato and Kudlac 2002).

\section{A.4 CONE}

Unfortunately, by the beginning of 1990, despite the swelling momentum within the NASA community to finally obtain low-g $\mathrm{LH}_{2}$ data, plans to fly COLD-SAT soon halted due to a shift in resources back to the newly invigorated Shuttle program. As an alternate to COLD-SAT which could be flown more quickly, a parallel study, called CONE (Cryogenic Orbital Nitrogen Experiment) which began and ended in 1990, was undertaken to again examine the feasibility of an $\mathrm{LN}_{2}$ flight experiment. The argument for funding an $\mathrm{LN}_{2}$ test was that quickly obtainable $\mathrm{LN}_{2}$ data could be used to modify the design of the $\mathrm{LH}_{2}$ COLD-SAT experiment. NASA once again funded two independent feasibility studies from Ball Aerospace and Martin Marietta (Bell et al. 1991 and Bailey et al. 1991). Because these studies involved the storage and transfer of nitrogen and not hydrogen, tank sizes, flow rates, and LAD parameters are excluded from this appendix. Thus the perpetual cycle of design, analysis, and fall out of funding of cryogenic 
technology demonstration missions had repeated a fifth time as the desire to obtain low-g cryogenic storage and transfer data once again eluded NASA engineers.

\section{A.5 CRYOTE}

Another recently proposed small scale depot demonstration of $\mathrm{LH}_{2}$ storage and transfer in LEO was the proposed Cryogenic Orbital Test Bed (CRYOYE-LITE) mission from the United Launch Alliance (ULA), which considered the transfer of propellant from a Centaur upper stage to the CRYOTE receiver tank. The CRYOTE tank would be launched empty as a secondary payload on Centaur; once the primary payload has been delivered, Centaur and CRYOYE would detach and Centaur would supply liquid to an empty CRYOTE tank. Once in orbit, CRYOTE could then conduct short term storage tests. Details and proposed experimental requirements for this test (Fikes et al. 2006, Gravlee et al. 2010, and McLean et al. 2011), as well as older Centaur-based experiments (Schuster et al. 1991), are available in the literature.

For the transfer portion of the test, Centaur would use a $2.54 \mathrm{~cm}(1 \mathrm{in})$ OD line to supply fluid to CRYOTE at a nominal flow rate of $0.182 \mathrm{~kg} / \mathrm{s}(0.4 \mathrm{lbm} / \mathrm{s})$, which is a full order of magnitude higher than the CPST TDM requirements. During tank drain, $\mathrm{LH}_{2}$ would be maintained at $207-276 \mathrm{kPa}(30-40$ psia) in the Centaur supply tank. For propellant outflow from Centaur, ULA considered both vane and screen channel PMD systems, although the number of vanes required to deliver that high of a flow rate would become impractical. Due to funding cuts, further work on CRYOTE was put on hold in 2012. 


\section{A.6 CPST TDM}

Recently, NASA has renewed its interest in depots through a proposed battery of $\mathrm{LH}_{2}$ storage and transfer ground tests, as well as conducting conceptual analysis for an in-space depot demonstration (Mulqueen 2010 and 2011). Before the full sized depot is implemented, it is highly desirable to characterize a much smaller system by demonstrating successful long term storage and transfer of $\mathrm{LH}_{2}$ in LEO. The CPST TDM represented the most recent attempt to obtain the coveted low-g cryogenic storage and transfer data. Based on cost, size, and payload, there were two vehicle concepts in consideration, as listed in Table 9.1, using an EELV in a similar architecture to those previously proposed in the 1980 s and 1990s. The storage tank would house up to $250 \mathrm{~kg}$ of $\mathrm{LH}_{2}$ for testing. Only gallery arms were being considered for this mission, due to robust mission requirements. Tank pressure, transfer line size, resultant transfer line velocity, and total outflow rate is listed in Table 9.1 to compare against Tables A.1 and A.2. The rest of the specific design details are proprietary at this time. Due to funding cuts, this sixth attempt at obtaining low-g cryogenic transfer data passed in 2014. 


\section{Appendix B}

\section{Summary of Previously Reported Bubble Point}

\section{Data}

This appendix presents a synopsis of the historical bubble point data collected to validate and anchor the cryogenic bubble point model in Chapter 10. A thorough and exhaustive scan of the literature reveals numerous bubble point tests conducted over a wide range of LAD screens, liquids, and thermodynamic conditions over the past 45 years. Previous bubble point experiments are summarized in Tables B.1 - B.5 in terms of the meshes, liquids, and pressurant gases tested. Tables are organized first by mesh type. As shown, there is data characterizing 40 different LAD screens and 5 different mesh types, including Plain Square, Twilled Square, Plain Dutch, Reverse Dutch, and Dutch Twill. The majority of historical references are associated with storable propulsion technology development programs and thus present data for high surface tension propellants with coarser mesh screens. Meanwhile, with the latest push towards advancing cryogenic propulsion technology, more recent references report data for finer mesh screens and lower surface tension cryogenic liquids.

Several authors also reported manufacture specified information, but not effective pore diameters, for certain screens (e.g. 325x1900). This is denoted by a blank row in 
Tables B.1 - B.5. Although no discrimination is made in the tables, there is data available for certain meshes using different metals. For example, data is available for a $165 \times 800$ mesh constructed from $\mathrm{Ti}, \mathrm{Al}$, and SS. Next, the tables are organized by liquid type and then finally by pressurant gas type.

\begin{tabular}{|c|c|c|c|c|}
\hline & Mesh & Liquid & Pressurant Gas & References \\
\hline \multirow[t]{6}{*}{1} & $510 \times 3600$ & Acetone & $\mathrm{GHe}$ & Current Work \\
\hline & & IPA & $\mathrm{GHe}$ & Current Work \\
\hline & & Methanol & $\mathrm{GHe}$ & Current Work \\
\hline & & Water & $\mathrm{GHe}$ & Current Work \\
\hline & & LN2 & $\mathrm{GHe}, \mathrm{GN} 2$ & Current Work \\
\hline & & $\mathrm{LH} 2$ & $\mathrm{GHe}, \mathrm{GH} 2$ & Current Work \\
\hline \multirow[t]{6}{*}{2} & $450 \times 2750$ & Acetone & $\mathrm{GHe}$ & Current Work \\
\hline & & IPA & $\mathrm{GHe}$ & Current Work \\
\hline & & Methanol & $\mathrm{GHe}$ & Current Work \\
\hline & & Water & $\mathrm{GHe}$ & Current Work \\
\hline & & LN2 & $\mathrm{GHe}, \mathrm{GN} 2$ & Current Work \\
\hline & & $\mathrm{LH} 2$ & $\mathrm{GHe}, \mathrm{GH} 2$ & Current Work \\
\hline \multirow[t]{18}{*}{3} & $325 \times 2300$ & $\mathrm{~N} 2 \mathrm{O} 4$ & GN2 & Paynter 1973b \\
\hline & & $\mathrm{MMH}$ & GN2 & Paynter 1973b \\
\hline & & $\mathrm{N} 2 \mathrm{H} 4$ & GN2 & Paynter 1973b \\
\hline & & Acetone & $\mathrm{GHe}$ & Current Work \\
\hline & & IPA & $\mathrm{GHe}, \mathrm{GN} 2, \mathrm{GH} 2$ & Current work, Alexander et al. 1970, Castle 1972, \\
\hline & & ' & ' & Cady 1973,1975 , and 1977, Burge and Blackmon 1973b, \\
\hline & & ' & ' & Bingham and Tegart 1977, Simon 1979, Wilson and Meserole 1986 \\
\hline & & Methanol & $\mathrm{GHe}, \mathrm{GN} 2$ & Current Work, Paynter 1973b \\
\hline & & Water & $\mathrm{GHe}$ & Current Work \\
\hline & & Ethanol & GN2 & Bingham and Tegart 1977 \\
\hline & & Freon-113 & GN2 & Bingham and Tegart 1977, Meserole and Jones 1993 \\
\hline & & Freon-114 & $\mathrm{GHe}$ & Cady 1977 \\
\hline & & $\mathrm{LCH} 4$ & $\mathrm{GHe}, \mathrm{GN} 2, \mathrm{GCH} 4$ & Current work, Jurns et al. 2007 \\
\hline & & LOX & $\mathrm{GHe}, \mathrm{GOX}$ & Current work, Paynter 1973a, Kudlac and Jurns 2005, Jurns and McQuillen 2008 \\
\hline & & LN2 & $\mathrm{GHe}, \mathrm{GN} 2$ & Current work, Castle 1972, Paynter 1973a, Chato and Kudlac 2002, Kudlac and Jurns 2005 \\
\hline & & $\mathrm{LH} 2$ & $\mathrm{GHe}, \mathrm{GH} 2$ & Current work, Cady 1973, 1975, 1977, Burge et al. 1973, \\
\hline & & ' & ' & Paynter 1973b, Stark et al. 1974, Meserole and Jones 1993, Chato and Kudlac 2002 \\
\hline & & SFHe & $\mathrm{GHe}$ & Anderson 1997 \\
\hline 4 & $325 \times 1900$ & & & Cady 1973 \\
\hline \multirow[t]{2}{*}{5} & $250 \times 1400$ & IPA & GN2 & Castle 1972 \\
\hline & & LN2 & GN2 & Castle 1972 \\
\hline \multirow[t]{15}{*}{6} & $200 \times 1400$ & IPA & $\mathrm{GHe}, \mathrm{GN} 2, \mathrm{GH} 2$ & Castle 1972, Cady 1973, 1975, 1977, Burge and Blackmon 1973b, \\
\hline & & ' & ' & Chato and Kudlac 2002, Kudlac and Jurns 2005 \\
\hline & & Methanol & GN2 & Paynter 1973a \\
\hline & & Ethanol & GN2 & Bingham and Tegart 1977 \\
\hline & & $\mathrm{N} 2 \mathrm{O} 4$ & GN2 & Paynter 1973b \\
\hline & & $\mathrm{MMH}$ & GN2 & Paynter 1973b \\
\hline & & $\mathrm{N} 2 \mathrm{H} 4$ & GN2 & Paynter 1973b \\
\hline & & $\mathrm{B} 2 \mathrm{H} 6$ & GN2 & Paynter 1970 \\
\hline & & OF2 & GN2 & Paynter 1970 \\
\hline & & Silicon Oil & Air & Conrath and Dreyer 2009 \\
\hline & & Freon-114 & $\mathrm{GHe}$ & Cady 1977 \\
\hline & & $\mathrm{LCH} 4$ & $\mathrm{GHe}$ & Jurns et al. 2007 \\
\hline & & LOX & GHe, GOX & Current work, Paynter 1973a, Kudlac and Jurns 2005, Jurns and McQuillen 2008 \\
\hline & & LN2 & $\mathrm{GHe}, \mathrm{GN} 2$ & Castle 1972, Paynter 1973a, Chato and Kudlac 2002, Kudlac and Jurns 2005 \\
\hline & & $\mathrm{LH} 2$ & $\mathrm{GHe}, \mathrm{GH} 2$ & Cady 1973, 1975, 1977, Burge et al. 1973 \\
\hline
\end{tabular}

Table B.1 - Fine Mesh Dutch Twill Meshes 


\begin{tabular}{|c|c|c|c|c|}
\hline & Mesh & Liquid & Pressurant Gas & References \\
\hline 7 & $165 \times 1400$ & & & Blatt 1970 \\
\hline \multirow[t]{5}{*}{8} & $250 \times 1370$ & IPA & $\mathrm{GHe}$ & Burge and Blackmon 1973b \\
\hline & & Methanol & $\mathrm{GHe}, \mathrm{GN} 2$ & Balzer et al. 1969, Paynter 1973b \\
\hline & & LOX & GOX & Paynter 1973a \\
\hline & & LN2 & GN2 & Paynter 1973a \\
\hline & & $\mathrm{LH} 2$ & $\mathrm{GH} 2$ & Cady 1973 \\
\hline \multirow[t]{10}{*}{9} & $165 \times 800$ & IPA & $\mathrm{GHe}, \mathrm{GN} 2, \mathrm{GH} 2$ & Castle 1972, Cady 1973, 1975, 1977 \\
\hline & & Methanol & GN2 & Paynter 1973b \\
\hline & & NTO & GN2 & Paynter 1973b \\
\hline & & $\mathrm{MMH}$ & GN2 & Paynter 1973b \\
\hline & & $\mathrm{N} 2 \mathrm{H} 4$ & GN2 & Paynter $1973 b$ \\
\hline & & Freon-114 & $\mathrm{GHe}$ & Cady 1977 \\
\hline & & Freon-113 & GN2 & Bingham and Tegart 1977 \\
\hline & & LN2 & GN2 & Castle 1972 \\
\hline & & $\mathrm{LH} 2$ & $\mathrm{GHe}, \mathrm{GH} 2$ & Burge et al. 1973, Cady 1973, 1975, 1977 \\
\hline & & & & Blatt 1970, Alexander et al. 1970 \\
\hline 10 & $150 \times 700$ & & & Blatt 1970 \\
\hline \multirow[t]{4}{*}{11} & $80 \times 700$ & IPA & GHe, GN2 & Heckman 1971, Burge and Blackmon 1973b \\
\hline & & Methanol & GN2 & Paynter $1973 b$ \\
\hline & & $\mathrm{LH} 2$ & $\mathrm{GH} 2$ & Cady 1973 \\
\hline & & & & Blatt 1970 \\
\hline \multirow[t]{4}{*}{12} & $200 \times 600$ & IPA & GHe, GN2 & Heckman 1971, Castle 1972, Burge and Blackmon 1973b \\
\hline & & LN2 & GN2 & Castle 1972 \\
\hline & & $\mathrm{LH} 2$ & $\mathrm{GH} 2$ & Cady 1973 \\
\hline & & & & Blatt 1970 \\
\hline \multirow[t]{2}{*}{13} & $50 \times 250$ & Freon-113 & GN2 & Bingham and Tegart 1977 \\
\hline & & & & Blatt 1970 \\
\hline \multirow[t]{2}{*}{14} & $30 \times 250$ & Methanol & $\mathrm{GHe}$ & Balzer et al. 1969 \\
\hline & & & & Blatt 1970, Alexander et al. 1970 \\
\hline 15 & $20 \times 250$ & & & Blatt 1970 \\
\hline 16 & $30 \times 160$ & LH2 & $\mathrm{GH} 2$ & Cady 1973 \\
\hline 17 & $24 \times 110$ & & & Alexander et al. 1970 \\
\hline
\end{tabular}

Table B.2 - Coarser Dutch Twill Meshes

\begin{tabular}{|c|c|c|c|c|}
\hline & Mesh & Liquid & Pressurant Gas & References \\
\hline \multirow[t]{3}{*}{18} & $50 \times 250$ & IPA & $\mathrm{GN} 2, \mathrm{GH} 2$ & Heckman 1971, Cady 1973 \\
\hline & & $\mathrm{C} 5 \mathrm{H} 12$ & Air & Dodge and Bowles 1984 \\
\hline & & LH2 & $\mathrm{GH} 2$ & Cady 1973 \\
\hline \multirow[t]{2}{*}{19} & $30 \times 250$ & IPA & GN2 & Heckman 1971 \\
\hline & & Methanol & GN2 & Paynter 1973b \\
\hline \multirow[t]{4}{*}{20} & $24 \times 110$ & IPA & GN2, GH2 & Heckman 1971, Cady 1973 \\
\hline & & Methanol & GN2 & Paynter 1973b \\
\hline & & $\mathrm{C} 5 \mathrm{H} 12$ & Air & Dodge and Bowles 1984 \\
\hline & & LH2 & $\mathrm{GH} 2$ & Cady 1973 \\
\hline
\end{tabular}

Table B.3 - Plain Dutch Meshes 


\begin{tabular}{|c|c|c|c|c|}
\hline & Mesh & Liquid & Pressurant Gas & References \\
\hline \multirow[t]{3}{*}{21} & $720 \times 140$ & IPA & $\mathrm{GHe}, \mathrm{GH} 2$ & Cady $1973,1975,1977$ \\
\hline & & Freon-114 & $\mathrm{GHe}$ & Cady 1977 \\
\hline & & LH2 & $\mathrm{GHe}, \mathrm{GH} 2$ & Cady 1973, 1975, 1977 \\
\hline
\end{tabular}

Table B.4 - Reverse Dutch Mesh

\begin{tabular}{|c|c|c|c|c|}
\hline & Mesh & Liquid & Pressurant Gas & References \\
\hline \multirow[t]{3}{*}{22} & $500 \times 500$ & IPA & $\mathrm{GN} 2, \mathrm{GH} 2$ & Cady 1973, 1977 \\
\hline & & Freon-114 & $\mathrm{GHe}$ & Cady 1977 \\
\hline & & $\mathrm{LH} 2$ & $\mathrm{GHe}, \mathrm{GH} 2$ & Cady 1973,1977 \\
\hline \multirow[t]{3}{*}{23} & $325 \times 325$ & IPA & GN2 & Heckman 1971 \\
\hline & & LH2 & $\mathrm{GH} 2$ & Cady 1973 \\
\hline & & & & Blatt 1970 \\
\hline \multirow[t]{3}{*}{24} & $180 \times 180$ & $\mathrm{MMH}$ & GN2 & Paynter 1970 \\
\hline & & $\mathrm{N} 2 \mathrm{O} 4$ & GN2 & Paynter 1970 \\
\hline & & $\mathrm{NH}$ & GN2 & Paynter 1970 \\
\hline \multirow[t]{2}{*}{25} & $120 \times 120$ & IPA & $\mathrm{GHe}$ & Cady 1975 \\
\hline & & $\mathrm{LH} 2$ & $\mathrm{GH} 2$ & Cady 1975 \\
\hline 26 & $100 \times 100$ & $\mathrm{NH}$ & GN2 & Paynter 1970 \\
\hline
\end{tabular}

Table B.5 - Twilled Square Meshes 


\begin{tabular}{|c|c|c|c|c|}
\hline & Mesh & Liquid & Pressurant Gas & References \\
\hline 27 & $400 \times 400$ & & & Blatt 1970 \\
\hline 28 & $230 \times 230$ & Isopropyl Alcohol & GN2 & Heckman 1971 \\
\hline \multirow[t]{6}{*}{29} & $200 \times 200$ & Methanol & GN2 & Paynter $1973 b$ \\
\hline & & Nitrogen Tetroxide & GN2 & Paynter 1973b \\
\hline & & Monomethylhydrazine & GN2 & Paynter 1973b \\
\hline & & Hydrazine & GN2 & Paynter 1973b \\
\hline & & LH2 & $\mathrm{GH} 2$ & Cady 1973 \\
\hline & & & & Alexander et al. 1970, Blatt 1970 \\
\hline 30 & $180 \times 180$ & Methanol & GN2 & Paynter 1973b \\
\hline \multirow[t]{4}{*}{31} & $150 \times 150$ & Isopropyl Alcohol & $\mathrm{GH} 2$ & Cady 1973 \\
\hline & & Methanol & GN2 & Paynter $1973 b$ \\
\hline & & LH2 & $\mathrm{GH} 2$ & Cady 1973 \\
\hline & & & & Blatt 1970 \\
\hline \multirow[t]{2}{*}{32} & $120 \times 120$ & Isopropyl Alcohol & GN2 & Heckman 1971 \\
\hline & & Methanol & GN2 & Paynter 1973b \\
\hline \multirow[t]{2}{*}{33} & $100 \times 100$ & Methanol & GN2 & Paynter 1973b \\
\hline & & & & Alexander et al. 1970, Blatt 1970 \\
\hline \multirow[t]{2}{*}{34} & $80 \times 80$ & Methanol & GN2 & Paynter $1973 b$ \\
\hline & & & & Blatt 1970 \\
\hline \multirow[t]{2}{*}{35} & $60 \times 60$ & Isopropyl Alcohol & $\mathrm{GH} 2$ & Cady 1973 \\
\hline & & LH2 & $\mathrm{GH} 2$ & Cady 1973 \\
\hline \multirow[t]{2}{*}{36} & $50 \times 50$ & Methanol & GN2 & Paynter 1973b \\
\hline & & & & Alexander et al. 1970, Blatt 1970 \\
\hline \multirow[t]{3}{*}{37} & $40 \times 40$ & Isopropyl Alcohol & $\mathrm{GH} 2$ & Cady 1973 \\
\hline & & LH2 & $\mathrm{GH} 2$ & Cady 1973 \\
\hline & & & & Blatt 1970 \\
\hline \multirow[t]{2}{*}{38} & $30 \times 30$ & Methanol & GN2 & Paynter 1973b \\
\hline & & & & Alexander et al. 1970 \\
\hline 39 & $20 \times 20$ & & & Blatt 1970 \\
\hline 40 & $12 \times 12$ & & & Alexander et al. 1970 \\
\hline
\end{tabular}

Table B.6 - Plain Square Meshes 


\section{Appendix C}

\section{Langmuir Isotherm for the Liquid/Vapor Case}

This appendix presents the Langmuir isotherm fit to the methanol/water binary mixture data from Chapter 4 . The isotherm is systematically derived from the combined $1^{\text {st }} / 2^{\text {nd }}$ Law of Thermodynamics for a binary component system. Fitting parameters are then used to predict liquid/vapor surface tension at any methanol mass fraction.

Starting with the combined form of the 1 st and 2 nd law of thermodynamics for the multi-component system, which takes into account the energy at the L/V interface:

$d U=T d S-P d V+\sum_{i=1}^{c} \mu_{i} d n_{i}+\gamma_{L V} d A$

where $U$ is the internal energy, $S$ is the entropy, $\mu_{i}$ is the chemical potential of the $i$ th component, $n_{i}$ is the number of moles of component $i$, and $d A$ is the differential area at the $\mathrm{L} / \mathrm{V}$ interface. C. 1 is written for a system that consists of two phases, $\alpha$ and $\beta$. Integrating, the Euler equation is obtained:

$U=T S-P V+\sum_{i=1}^{c} \mu_{i} n_{i}+\gamma_{L V} A$

for the entire system phase $\alpha$, the interface, and phase $\beta$. From Equations C.1 and C.2, the Gibbs-Duhem equation is obtained: 
$0=S d T-V d P+\sum_{i=1}^{c} n_{i} d \mu_{i}+A d \gamma_{L V}$

where $S, V, n_{i}$ are extensive variables that include all of phases $\alpha, \beta$ and the interface of area $A$. Note that Equation C.3 without constraints does not obey the Gibbs phase rule, which counts the number of independent intensive variables. Any two of the set of intensive variables $T, P,\left\{\mu_{i}\right\}_{i=1}^{c}$ must be dependent variables along with the L/V surface tension $\gamma_{L V}$. A number of algorithms exist for eliminating two of the dependent variables. The method of Hansen (1962) and Turkevich and Mann (1990a) is used, and the method of undetermined multipliers is employed asserting two conditions, one for each phase. The first condition is that of thermal, mechanical, and chemical equilibrium so that each of the variables, $T, P,\left\{\mu_{i}\right\}_{i=1}^{c}$ are uniform throughout phases $\alpha, \beta$ and the interface. Then two conditions are satisfied:

$$
\begin{aligned}
& 0=S^{v} d T-d P+\sum_{i=1}^{c} c^{\alpha}{ }_{i} d \mu_{i}, \\
& 0=S^{v^{\beta}} d T-d P+\sum_{i=1}^{c} c^{\beta}{ }_{i} d \mu_{i} .
\end{aligned}
$$

Let the undetermined multipliers be $\lambda^{\alpha}$ and $\lambda^{\beta}$ so that adding Equation C.4 and C.5 to C.3 gives:

$0=\left(S-\lambda^{\alpha} S^{\nu}-\lambda^{\beta} S^{v}\right) d T-\left(V-\lambda^{\alpha}-\lambda^{\beta}\right) d P+\sum_{i=1}^{c}\left(n_{i}-\lambda^{\alpha} c_{i}^{\alpha}-\lambda^{\beta} c_{i}^{\beta}\right) d \mu_{i}+A d \gamma$

where the area of the interface and the volumes of the phases are arbitrary. Define the excess quantities $S^{e}, V^{e},\left\{n_{i}^{e}\right\}_{i=1}^{c}$ by: 


$$
\begin{aligned}
& S^{e}=S-\lambda^{\alpha} S^{\alpha}-\lambda^{\beta} S^{\nu} \\
& V^{e}=V-\lambda^{\alpha}-\lambda^{\beta} \\
& n_{i}^{e}=n_{i}-\lambda^{\alpha} c_{i}^{\alpha}-\lambda^{\beta} c_{i}^{\beta}
\end{aligned}
$$

The two side conditions are taken into account and therefore the phase rule is satisfied by setting any two functions $S^{e}, V^{e}, n_{i}^{e}$ equal to zero and solving for $\lambda^{\alpha}, \lambda^{\beta}$. The selection is made for convenience; different conventions will be used for the $\mathrm{L} / \mathrm{V}$ interfacial system and for the $\mathrm{S} / \mathrm{V}$ or $\mathrm{S} / \mathrm{L}$ interfacial systems. Dividing by the area $A$, results in a formula for the differential of $\gamma_{L V}$ :

$-d \gamma=\bar{S} d T-\tau d p+\sum_{i=1}^{c} \Gamma_{i} d \mu_{i}$

where $\bar{S}$ is the excess entropy per area, $\tau$ is the excess volume per unit area, and $\Gamma_{i}$ are the excess of compositions per unit area at the interface, subject to setting to zero any two of the excess coefficients $\left(\bar{S}, \tau,\left\{\Gamma_{i}\right\}_{1}^{c}\right)$. This formula is valid regardless of whether the phases are solids, liquids or gases. It is common practice to use tension units of milliNewtons per meter for $\gamma_{L V}$ when the interface is between liquid phases or liquid-fluid phases, and energy units milli-Joules per square meter when one phase is a solid. The units of $\gamma_{L V}[=] m N / m$ are used for all interfacial tensions, including $\gamma_{S V}, \gamma_{S L}$.

Consider first the L/V interface (Figure 3.2) and in this case, the classical convention of Gibbs is used to set $V^{e}=0, n_{1}^{e}=0$ in which case, Equation C.11 is obtained after dividing through by the area of the interface, and rearranging: 
$-d \gamma_{L V}=\bar{S} d T+\sum_{i=2}^{c} \Gamma_{i} d \mu_{i}$

The majority component is labeled as $i=1$. Therefore, one can define these excesses in terms of partial derivatives:

$$
\begin{aligned}
& \bar{S}=-\left(\frac{\partial \gamma_{L V}}{\partial T}\right)_{\left\{\mu_{i} c_{2}^{c}\right.} \\
& \Gamma_{i}=-\left(\frac{\partial \gamma_{L V}}{\partial \mu_{i}}\right)_{T,\left\{\left\{\mu_{i}\right\}_{2}^{c}-\mu_{1}\right\}}
\end{aligned}
$$

In the Gibbs convention, for a two component system of solvent, $i=1$ (water), and solute, $i=2$ (methanol), Equation C.11 becomes:

$$
-d \gamma_{L V}=\bar{S} d T+\Gamma_{2} d \mu_{2}
$$

Evaluating subcooled liquid states for bubble point tests would involve an added complexity, since one would have to take into account the pressure dependence of the surface tension (Turkevich and Mann 1990a). Therefore, to permit facile interpretation of the data, reference fluid bubble point tests are conducted in equilibrium saturated liquid states. One can then use Equations C.12 - C.14 to determine the excess of composition of methanol:

$\Gamma_{\text {meth }}=-\left.\frac{\partial \gamma_{L V}}{\partial \mu_{\text {meth }}}\right|_{T, P}$

The chemical potential can be written as: 
$\mu_{\text {meth }}=\mu_{\text {meth }}^{0}+k_{B} T \log \left(a_{C, 2}\right)$

where $k_{B}$ is the Boltzmann constant, $a_{C, 2}$ is the activity of component 2, methanol in this case, which can be written as the product of the activity coefficient $\alpha_{2}$ and the concentration, $Y_{2}$ is the mass fraction of methanol:

$\mu_{\text {meth }}=\mu_{\text {meth }}^{0}+k_{B} T \log \left(\alpha_{2} Y_{2}\right)$

The assumption is made that $\alpha_{2}=1$ even though $Y_{1} \in[0,1]$. The goodness of fit to the Langmuir isotherm supports this assumption. Substituting Equation C.17 into Equation C.15:

$\Gamma_{2}=-\left.\frac{\partial \gamma_{L V}}{\partial\left(k_{B} T \log Y_{2}\right)}\right|_{T, P}$

Simplifying,

$\Gamma_{2}=-\left.\frac{1}{k_{B} T} \frac{\partial \gamma_{L V}}{\partial\left(\log Y_{2}\right)}\right|_{T, P}$

To determine $\Gamma_{2} v s Y_{2}$, an equation of state is required; the Langmuir isotherm is used herein to fit the various data sets:

$\Gamma_{2}=\Gamma_{2, \max } \frac{a Y_{2}}{1+a Y_{2}}$

where $a$ and $\Gamma_{2, \max }$ are fitting parameters. Inserting Equation C.20 into Equation C.19 and rearranging: 
$-d \gamma_{L V}=k_{B} T \Gamma_{2, \max } d \log \left(1+a Y_{2}\right)$

The "spreading pressure" can now be defined:

$\pi=\gamma_{L V}^{0}-\gamma_{L V}$

where $\gamma_{L V}^{0}$ for the liquid-vapor system is the surface tension of water without methanol present, $Y_{2}=0$. Computing the differential of both sides of Equation C.22:

$d \pi=-d \gamma_{L V}$

so that:

$d \pi=k_{B} T \Gamma_{2, \max } d \log \left(1+a Y_{2}\right)$

Integrating Equation C.24 from $Y_{2}=0$ to $Y_{2}$ :

$\pi=k_{B} T \Gamma_{2, \max } \log \left(1+a Y_{2}\right)$

The fitting equation is then:

$$
\gamma_{L V}=\gamma_{L V}^{0}-k_{B} T \Gamma_{2 \max } \log \left(1+a Y_{2}\right)
$$

Since $\gamma_{L V}, T, Y_{2}$ are known in discrete intervals, Equation C.26 can be used to determine the three fitting parameters $\gamma_{L V}^{0}, a$, and $\Gamma_{2, \max }$ by a non-linear fitting algorithm, which was applied to the $\mathrm{T}=20^{\circ} \mathrm{C}$ methanol/water data from Vazquez et al. (1995). Fitting the Langmuir isotherm through the data yields: 
$\Gamma_{2, \max }=\frac{4.44 \text { molecules }}{n m^{2}}$

$a=16.4$

After inserting these values into Equation C.26 and rearranging, a Langmuir isotherm is generated, which allows prediction of mixture fluid bubble points at any methanol mass fraction. The Langmuir isotherm is plotted with the data in Figure 4.7.

Note that $A_{2, \min }=\frac{1}{\Gamma_{2, \text { max }}}=0.23 \frac{\mathrm{nm}^{2}}{\text { molecule }}$, which suggests that the helium/water

interface is covered by a methanol monolayer that is packed. Compare this number to the co-area of a close-packed monolayer of a long-chain alcohol of $0.20 \frac{\mathrm{nm}^{2}}{\text { molecule }}$ at the solution/water interface ( $\mathrm{Li}$ et al. 1992). This result suggests the possibility of a considerable adsorption of methanol at the SS304/solution interface. Fan et al. (2011) provides experimental data from neutron diffraction studies along with molecular dynamic simulation that show that, even though water and methanol mix in all proportions, there are extended structures that transform into "bi-percolating liquid mixtures." The surface structure may be quite complex. The fact that the Langmuir isotherm does so well in fitting the experimental data is an interesting result. 


\section{Appendix D}

\section{Langmuir Isotherms for the Solid/Liquid and}

\section{Solid/Vapor Case}

This appendix presents computation of resultant solid/liquid and solid/vapor interfacial tensions from the methanol/water binary mixture bubble point data from Chapter 4. Governing equations are presented for deriving the Langmuir isotherms for the S/L and S/V data. The goodness of fits are also discussed for both cases.

The Langmuir isotherm, $\gamma_{L V}$ vs $Y_{2}$ for the methanol/water solution against its vapor can be measured directly, and the Langmuir parameters determined as described in Appendix C. However, the dependent variables, $\gamma_{S V}, \gamma_{S L}$ are not directly determined by experiment, but can be computed in the following manner: Direct, macroscopic measurement of the contact angle of methanol/water mixtures can be performed on the SS304 porous screens. It is then possible to compute the work of adhesion invoking the YLE. Once the work of adhesion is determined, an equation of state is invoked to estimate $\gamma_{S V}$. Given $\left(\gamma_{L V}, \gamma_{S V}\right)$ vs. $Y_{2}, \gamma_{S L}$ can be estimated as a function of $Y_{2}$ by invoking the YLE. Since the structure of the SS304 surface is unknown with respect to the adsorption of methanol or water components, a molecular level interpretation of the isotherms will not be attempted. EDAX data was collected on the screens used in this 
study, which showed that the distribution of chemical elements (Carbon, Nickel, Iron, etc.) on the surfaces of the screens was consistent at any location on the screen, and that the composition breakdown as expected for a SS304 piece of material.

From an engineering viewpoint it is asserted that a lumped parameter is sufficient to represent this complex system in Equation C.11 such that:

$-d \gamma=\bar{S} d T-\tau d p+\Gamma_{m e t h} d \mu_{m e t h}$

where $\Gamma_{S S 304}=0$ and $\Gamma_{\text {water }}=0$, which satisfies the phase rule, even though the analytical chemistry of component distribution in the SS304 phase and of SS304 components in the other phases is unknown. The pressure term is negligible, since $\tau=\left.\frac{\partial \gamma}{\partial p}\right|_{T, \mu_{\text {meth }}}$ is small for the liquid and solid phases (Turkevich and Mann 1990a). The experiments were done at constant temperature so that the working differential form is

$$
-d \gamma=\Gamma_{m e t h} d \mu_{m e t h}
$$

and therefore the fitting equation is:

$$
\gamma_{S X}=\gamma_{S X}^{0}-k T \Gamma_{2 \max }^{S X} \log \left(1+a^{S X} Y_{2}\right)
$$

where $S X$ represents either the solid-liquid interface $S L$, or the solid-vapor interface, $S V$. Once the parameters $\gamma_{S X}^{0}, \Gamma_{2 \max }^{S X}, a^{S X}$ are determined by fitting Equation C.26, the two excesses can thus be estimated by the Langmuir isotherm, Equation C.20, written as

$$
\Gamma_{2}^{S X}=\Gamma_{2 \max }^{S X} \frac{a^{S X} Y_{2}}{1+a^{S X} Y_{2}}
$$


The parameters obtained from the fitting equation will differ depending on the interface, liquid-vapor, SS304-vapor, or SS304-solution.

The correlation of the contact angle data for binary fluids with the "surface energy" of solids is not clear experimentally. For example Good (1977), in his Kendall award lecture cautioned against the use of liquid mixtures to span the L/V surface tension range required to estimate solid surface free energies. More recently, Li et al. (1992) reported inconsistent results of advancing Sessile drop contact angles and the estimation of $\gamma_{S V}$ using binary liquid mixtures.

Assume that $\mu_{2}^{\text {solution }}=\mu_{2}^{\text {Vapor }}$ and that the activity coefficients, $\alpha_{i}=1$. While the activity coefficient of methanol deviates from unity at small mole fractions, the data analyzed is in a concentration range in which $\alpha_{2} \approx 1$ is a close enough approximation to see the effects of adsorption. Since $\bar{W}_{a} v s \gamma_{L V}$ was established experimentally, Equation 4.11 was solved for $\gamma_{S V} v s Y_{2}$ by a direct search algorithm. Tables of $\pi_{S V} v s A_{S V}$ and $\pi_{S L} v S A_{S L}$ were constructed, where $A_{S X}=1 / \Gamma_{2}^{S X}$. Note that $\Gamma_{2}^{S V}$ or $\Gamma_{2}^{S L}$ are determined from the Langmuir isotherm using the coefficients given in Table D.1.

\begin{tabular}{|l|c|c|c|}
\hline \multicolumn{1}{|c|}{ System } & $\boldsymbol{v}^{\mathbf{0}}$ & $\boldsymbol{A}_{\text {MIN }}$ & $\boldsymbol{a}$ \\
\hline & {$[\mathrm{mN} / \mathrm{m}]$} & {$\left[\mathrm{nm}^{2} /\right.$ molecule $]$} & \\
\hline Solid - Vapor & $55.4 \pm 3.1$ & $0.315 \pm 0.030$ & $11.3 \pm 4.4$ \\
\hline Solid - Liquid & $6.12 \pm 3.0$ & $1.73 \pm 0.20$ & $33.5 \pm 5.0$ \\
Liquid - Vapor & $73.2 \pm 3.4$ & $0.22 \pm 0.012$ & $15.0 \pm 8.0$ \\
\hline
\end{tabular}

Table D.1 - Langmuir Isotherm Fitting Parameters 
The uncertainties quoted here are derived from the least squares algorithm. The residuals are small enough to suggest good fits but in each case the numbers were rather large and the uncertainties reflect that property. The fit of the S/L isotherm required special care to obtain convergence; the methanol-water cluster structure of the composition range above 0.4 mass fraction is of interest.

To obtain $\gamma_{S V}$ as a function of $\gamma_{L V}$, Equation 4.11 was solved numerically, given $\bar{W}_{a}$ and $\gamma_{L V} \cdot \beta$ is taken as 0.0001247 from Li et al. (1992). Figure D.1 shows results from this study, along with data from Addesso and Lund (1997). It is clear from both data sets that $\gamma_{S V}$ depends on $\gamma_{L V}$ and that reasonable agreement exists between both sets despite two different 304SS compositions (i.e. solid steel plane vs. porous LAD screen).

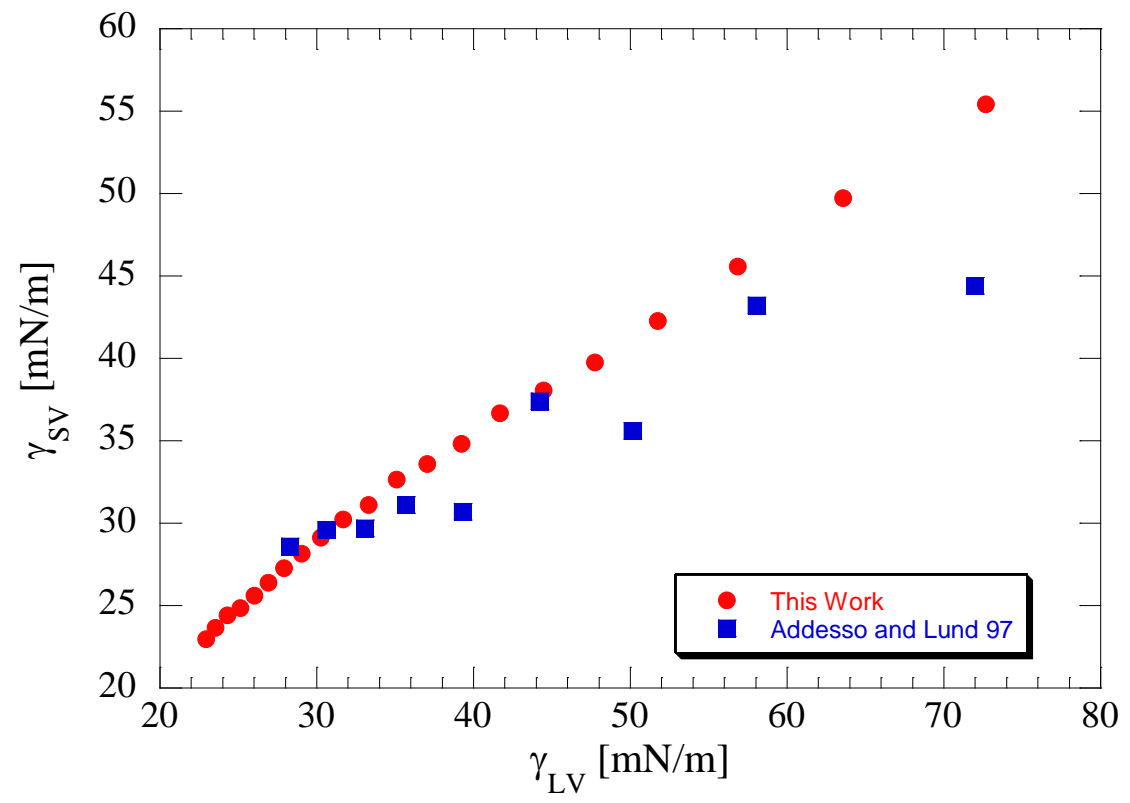

Figure D.1 - Solid/Vapor Interfacial Tension as a Function of Liquid/Vapor Interfacial Tension for Binary Methanol/Water and Stainless Steel 304 System 
Figure D.2 provides a contrasting picture of the variation of $\gamma_{S V}$ and $\gamma_{S L}$ with $Y_{2}$. The Langmuir isotherm fits the $\gamma_{S V}$ data very well throughout the range of concentrations studied. Moreover, $\gamma_{S V} \in[23,55.4) \mathrm{mN} / \mathrm{m}$ is substantially larger than the range $\gamma_{S L} \in[0,6.12) m N / m$. The fit to the $\mathrm{S} / \mathrm{V}$ data is excellent, but the fit to the $\mathrm{S} / \mathrm{L}$ data is modest up to $Y_{2}=0.4$; the data between $(0.4,1]$ did not follow the fit. The non-linear least squares procedure could not be made to converge when the $\gamma_{S L}$ data for $Y_{2}>0.4$ was included in the array. Apparently, the structure of the solution/SS304 interface does not satisfy the conditions required for the Langmuir isotherm to be valid.

Figure D.3 uses the result of computing $A^{S X}=1 / \Gamma_{2}^{S X}$ from Equation C.20. The co-area for the S/V interface is reasonable when compared to close packing of long-chain alcohols, which are in the range of $(0.2,0.3) \mathrm{nm}^{2} /$ molecule. The co-area found for the $\mathrm{S} / \mathrm{L}$ interface is sufficiently large that the $\mathrm{S} / \mathrm{L}$ interface may be mostly water-clusters with only a relatively few methanol molecules at the surface; the co-area ratio is 5.5.

A question is whether the completely soluble methanol/water solution has local structures analogous to microemulsions. Dougan et al. (2004) built a case for the possibility that methanol/water mixtures are not homogenous but tend to form clusters of various sizes. Their data suggest that at higher concentrations of methanol "the system segregates into what is effectively a molecular-scale microemulsion." They provide evidence that in the concentration range $Y_{2} \in(\sim 0.27,0.54)$ "both water and alcohol clusters percolate simultaneously." This is analogous to the bi-continuous structures that manifest in microemulsions in certain regions of their phase diagrams. 

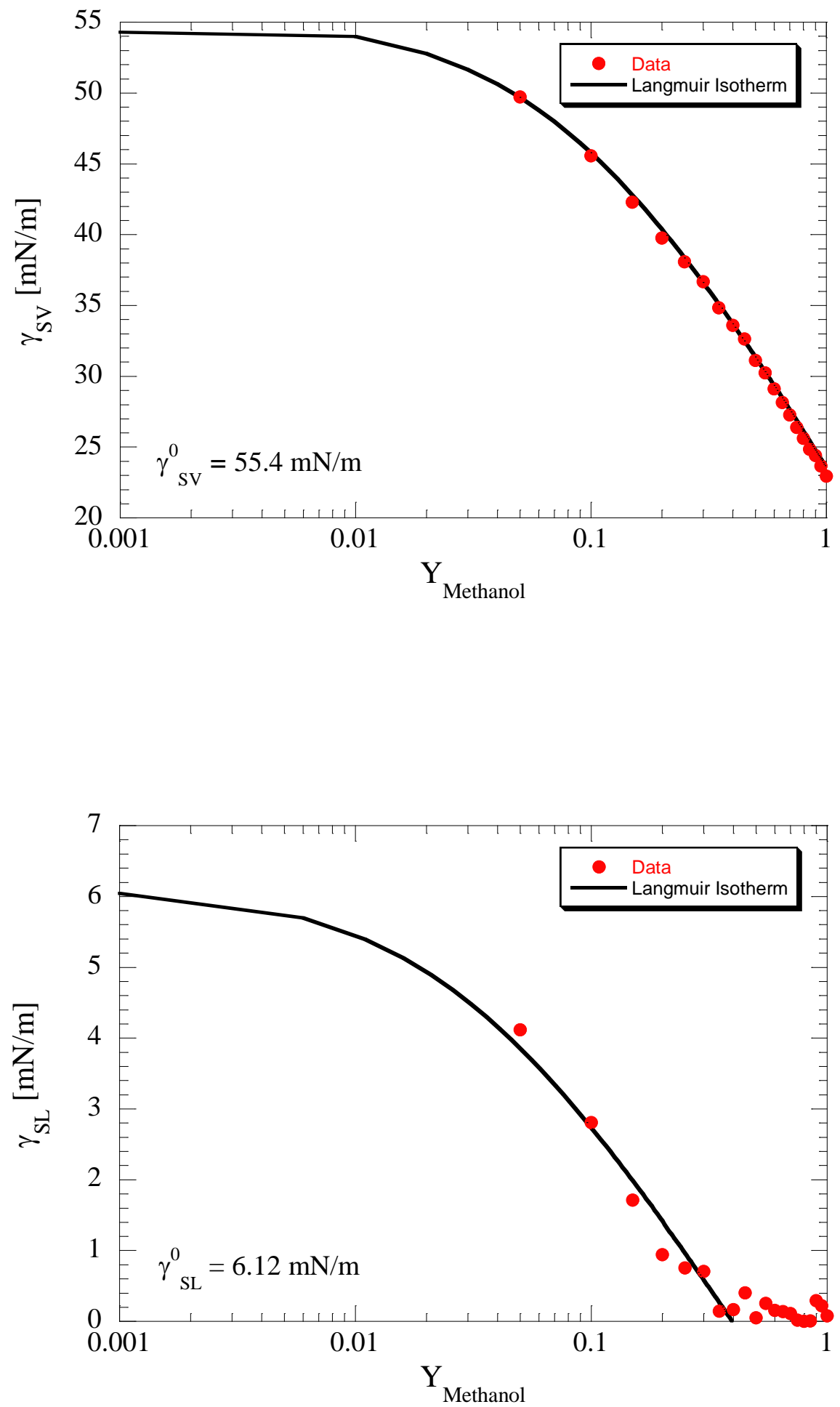

Figure D.2 - a) Solid/Vapor and b) Solid/Liquid Interfacial Tension as a Function of Methanol Mass Fraction 

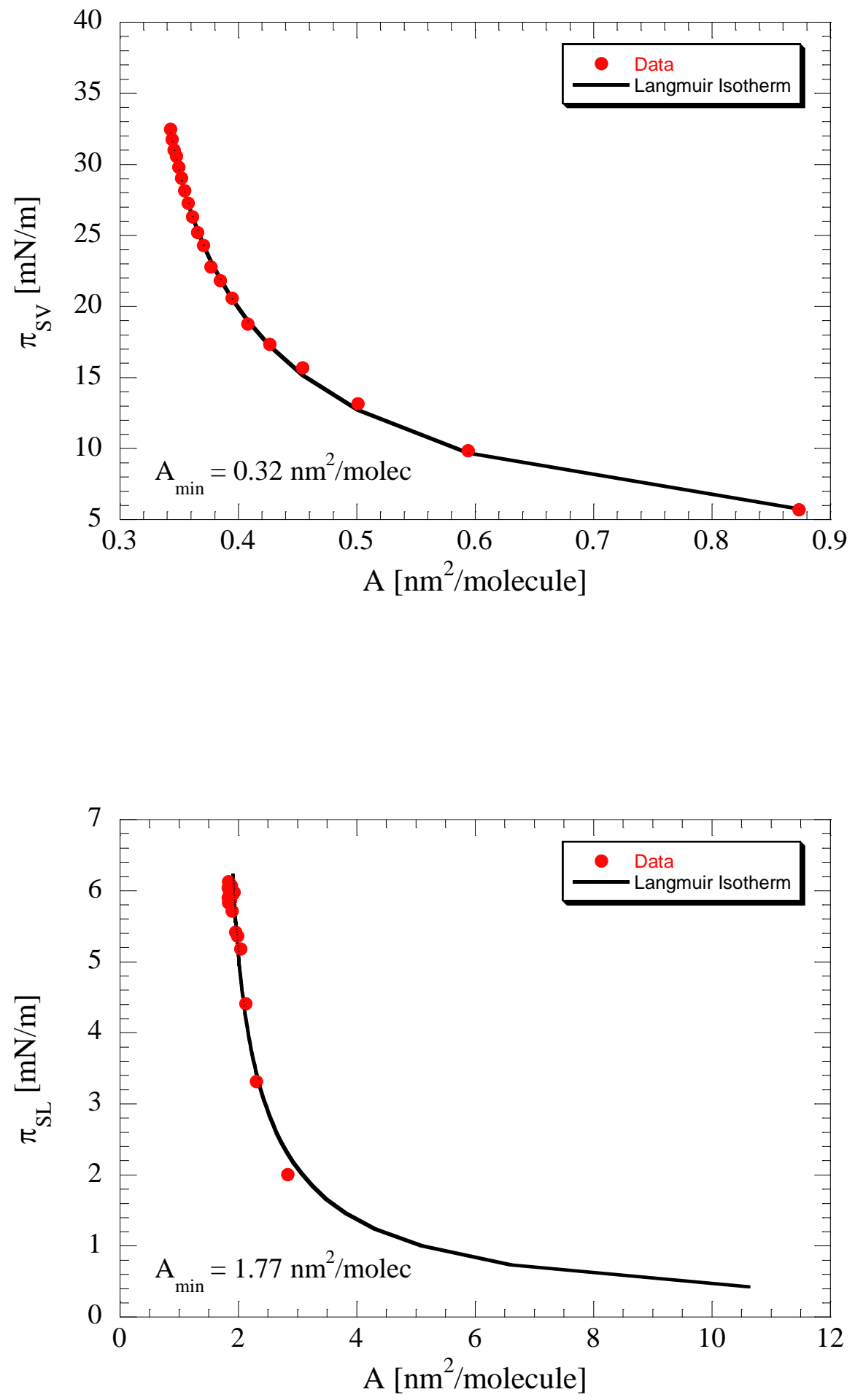

Figure D.3 - a) Solid/Vapor and b) Solid/Liquid Spreading Pressure as a Function of "A" 
The bubble point tests conducted in methanol/water mixtures were worked up to show properties of the three-phase interfaces along the complex contact line in SS304 LAD screens. In particular the variation with $Y_{2}$ of the solid/vapor interfacial tension $\gamma_{S V}$ differed from that of the solid/liquid interface $\gamma_{S L}$. The data are consistent with the Langmuir isotherm description of the thermodynamics of adsorption. The result of the analysis is that the co-areas $A_{\min }$ are $0.32 \mathrm{~nm}^{2} /$ molecule for the SS304 - vapor interface and $1.77 \mathrm{~nm}^{2} /$ molecule for the SS304 - solution interface. This implies that that methanol molecules form a dense, liquid-like monolayer at the interface of SS304 with the vapor phase, while the methanol molecules are very dilute in the interface between SS304 and the solution of methanol/water. 


\section{Appendix E}

\section{Historical Heated Pressurant Gas Liquid}

\section{Acquisition Device Tests}

This appendix presents historical warm pressurant gas LAD performance test results. A review of the literature revealed a total of 10 relevant studies of warm pressurant gas effects on LAD performance. LADs are primarily affected by the surface tension, and, thus temperature. Therefore to make meaningful comparisons, historical results are organized by propellant type (function of saturation temperature), and then by screen type. A brief overview of historical results is presented chronologically. Results are shown in Figures E.1 - E.6. To compare between all the historical results across multiple LAD testing schemes, data is cast in a non-dimensional form using Equation 8.1.

Castle (1972) first attempted to quantify the effect of heated pressurant on screen channel LAD performance. Using a standard NIBP configuration, they reported static bubble point tests conducted in $\mathrm{LN}_{2}$ using $\mathrm{GN}_{2}$ for six different screen samples. $\mathrm{GN}_{2}$ was heated electrically and forced downward on the LAD screen using a fan in an attempt to eliminate natural convection in favor of forced convection. As shown in Figure E.5, for a $\Delta \mathrm{T}=50 \mathrm{~K}$ across the screen, there was no degradation in performance for any screen 
mesh. Only the $250 \times 600$ mesh screen showed a $31 \%$ degradation in performance at a $\Delta \mathrm{T}$ $=250 \mathrm{~K}$.

Burge and Blackmon (1973b) reported heated gas $\mathrm{LH}_{2}$ bubble points using a similar NIBP configuration as Castle for three different meshes using $\mathrm{GH}_{2}$ as a pressurant, as shown in Figure E.2. A fan forced hot $\mathrm{GH}_{2}$ down on the screen, but no attempt was made to eliminate natural convection. Results show performance degradation for all of the screens tested, with the finer 250x1370 mesh performing much worse than the coarser $200 \times 600$ mesh. The 250 mesh degraded to $30 \%$ of the cold gas breakdown point. Burge and Blackmon (1973b) also reported premature breakdown for a 200x 1400 mesh with $\mathrm{GH}_{2} / \mathrm{LH}_{2}$ of $50 \%$ but did not report the gas temperature at breakdown. Due to the existence of both natural and forced convection, the screen likely broke down prematurely for all these tests. In addition, results are complicated by the fact that the liquid may have been subcooled relative to the pressurant gas temperature.

Paynter et al. (1970) then conducted the first set of IO tests in $\mathrm{LH}_{2}$ using both $\mathrm{GHe}$ and $\mathrm{GH}_{2}$. Test conditions were not reported, but Paynter reported no premature breakdown with warm gas under steady continuous outflow conditions, but premature breakdown for stepped or ramped expulsions. Blackmon (1974) later conducted IO tests for a 250x1370 mesh in $\mathrm{LH}_{2}$ using warm $\mathrm{GHe}$ and $\mathrm{GH}_{2}$. Results from Figure E.2 indicate a degradation in $\mathrm{LAD}$ performance as much as $75 \%$ at a $\Delta \mathrm{T}=35 \mathrm{~K}$. Building on Paynter's tests, Warren (1975) and Warren et al. (1975) report IO data using two layers of $325 \times 2300$ screen mesh in $\mathrm{LH}_{2}$ using both warm $\mathrm{GHe}$ and $\mathrm{GH}_{2}$. Neither saw premature LAD breakdown for continuous or stepped expulsion for a $\Delta \mathrm{T}=19-291 \mathrm{~K}$ as shown in Figure E.2. 
Cady (1974) reported NIBP test data for a standard 325x2300 screen, a pleated 325x2300, a SS and Al 200x1400 screen, a 720x140, 165x800, and a SS and Al 120x120 screen in $\mathrm{LH}_{2}$ using warm GHe. Results are plotted in Figures E.1 and E.2. As shown, there is degradation in heated bubble point values for all meshes tested, with the largest reduction in performance for the 200x 1400 screen. On average there was greater than $10 \%$ reduction in bubble point for temperature differences between gas and liquid as high as $\Delta \mathrm{T}=55 \mathrm{~K}$. When compared to other heated gas $325 \times 2300 \mathrm{LAD}$ data in Figure E.1, the data follows the general trend. Cady saw no difference between pleated and unpleated screen performance and saw no difference in performance between SS and Al screens. Examination of the test apparatus however showed that there was a direct view factor between heating source and screen. Therefore results here are complicated by the fact that heat was being conducted into the pressurant gas and also into the screen itself, which may have caused early breakdown. In addition, it is difficult to compare results from this NIBP configuration where only natural convection was present with those from Castle (1972) where forced convection is dominate with Burge and Blackmon (1973b) where forced and natural convection were present.

Wilson and Meserole (1986) report the first known heated pressurant gas LAD performance data using the IBP test configuration for a $325 \times 2300$ screen using $\mathrm{GN}_{2}$ in IPA. As shown in Figure E.6, they reported a reduction in performance of $69 \%$ of the cold gas bubble point at a $\Delta \mathrm{T}=56 \mathrm{~K}$. But this data may also be corrupted due to the presence of a direct view factor between heat source and screen; the screen may have broken down early due to additional radiation heat transfer. 
Bennett (1987) tested the liquid retention capability of a $34 \mathrm{~cm}$ tall 250x1400 start basket with a 200x 1400 window in $\mathrm{LH}_{2}$ using both non-condensable and autogenous pressurization schemes. Here the basket was submerged in liquid into a dewar, the liquid level lowered below the basket, and warm pressurant gas was introduced until the basket ingested vapor. With GHe pressurant, no degradation in performance was noted, even when the LAD was subjected to $\mathrm{GHe} 70 \mathrm{~K}$ above the liquid temperature as shown in Figure E.4. Using $\mathrm{GH}_{2}$ pressurant however, results were not repeatable as the start basket broke down at a height of 0,70 , and $90 \%$ of the cold gas height.

The most recent investigation was conducted by Meserole and Jones (1993) for a $325 \times 2300$ screen in $\mathrm{LH}_{2}$ using both $\mathrm{GHe}$ and $\mathrm{GH}_{2}$ using an IO test configuration and using $\mathrm{GN}_{2}$ in Freon in an IBP configuration. In the IBP case, $\mathrm{GN}_{2}$ was heated inside a cup with a heating element that was blocked from the screen to prevent stray radiation between heat source and screen. Gas was heated en route to the screen, which was submerged in Freon. A 325x2300 double-Dutch perforated plate, double plate backed $325 \times 2300$, and a pleated screen sandwich $(32 \times x 2300,25 \times 25,325 \times 2300)$ were tested. As shown in Figure E.6, the double-Dutch and double plated samples did not improve screen retention, as bubble point pressure degraded to $57 \%$ and $67 \%$ of the cold gas values, respectively, for $\Delta \mathrm{T}=83 \mathrm{~K}$ and $100 \mathrm{~K}$, respectively. The onset of degradation did not occur until the gas temperature had risen $40 \mathrm{~K}$ above that of the liquid. The pleated sandwich also did not improve screen retention over a single $325 \times 2300$ screen, as warm gas bubble point degraded to $75 \%$ at a $\Delta \mathrm{T}=99 \mathrm{~K}$.

Meserole and Jones (1993) also tested a 325x2300 perforated plate sample in $\mathrm{LH}_{2}$ in a modified version of the test apparatus used in the Freon tests, in the IO configuration, 
and also in hold tests where liquid outflow was stopped for a period of time to allow sufficient residence time of the warm gas inside the dewar that held the LAD channel. IO test results using GHe, para- $\mathrm{GH}_{2}$, and normal $\mathrm{GH}_{2}$ are plotted in Figure E.1 and hold tests are plotted in Figure E.3. The trends are obvious; for GHe, for a $\Delta \mathrm{T}$ as high as $62 \mathrm{~K}$, there was insignificant degradation (less than $5 \%$ reduction in performance). For hold tests with warm $\mathrm{GHe}$, there was no change in performance. For normal $\mathrm{GH}_{2}$ pressurization, only a $6 \%$ reduction in performance was reported for a $\Delta \mathrm{T}=54 \mathrm{~K}$ during continuous expulsion tests; for stepped expulsion tests, the LAD broke down at a height of $19 \%$ of the cold gas height when the $\mathrm{GH}_{2}$ was only $5 \mathrm{~K}$ above the liquid temperature. For para$\mathrm{GH}_{2}$ outflow tests, the LAD broke down at a height of $40 \%$ of the cold gas value at a $\Delta \mathrm{T}$ $=26 \mathrm{~K}$; for hold tests, the LAD broke down at a height of $19 \%$ of the cold gas height for a $\Delta \mathrm{T}=6 \mathrm{~K}$.

Although trends from Meserole and Jones (1993) are fairly obvious, in that GHe performed much better than $\mathrm{GH}_{2}$ for both IBP and IO test configurations, the results are not repeatable and are complicated by the following reasons. First, the LAD itself was not sized properly. The hydrostatic head pressure inside the LAD when the LAD was completely exposed to gas was never enough to overcome the static bubble point pressure. As a result of the poorly sized LAD, a flow restriction orifice was used on the $\mathrm{LH}_{2}$ inflow to help build up additional pressure differential. LAD breakdown was actually induced through the use of this flow restriction at the bottom of the tank by ramping the ullage pressure while still flowing through the flow restriction. The liquid level always dropped below the bottom of the LAD screen and breakdown only occurred when ramping the ullage pressure. It is unclear what the flow rate was during this 
pressurization event, and this is an indirect (and not direct) way to break the channel down. Second, warm gas was always injected at a temperature much greater than the actual temperature of the gas at the screen at breakthrough. Without direct measure of the temperature at the screen, it is unclear what the actual gas temperature was at the screen at breakdown. Third, for IO tests, a curved LAD was used, which only serves to complicate interpretation of results due to nonuniform FTS pressure losses. Fourth, while $\mathrm{GHe}$ and para-H2 tests were fairly repeatable, there is quite a large discrepancy between para- $\mathrm{GH}_{2}$ and normal $\mathrm{GH}_{2}$ results, despite the fact that there is only a $1-2 \%$ increase in surface tension for para over normal hydrogen gas. Heated pressurant gas tests conducted in Chapter 8 are the most accurate and most reliable data reported.

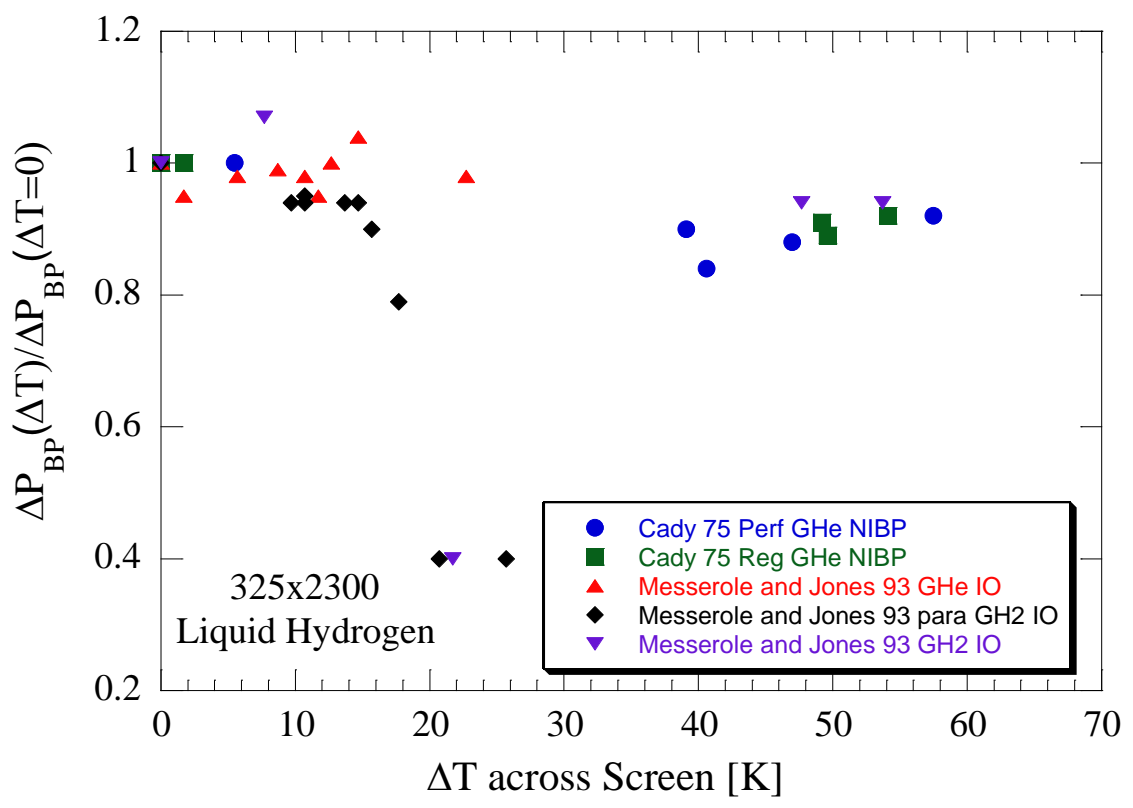

Figure E.1 - Heated Pressurant Gas Liquid Acquisition Device Performance Data for a 325x2300 Screen in Liquid Hydrogen 


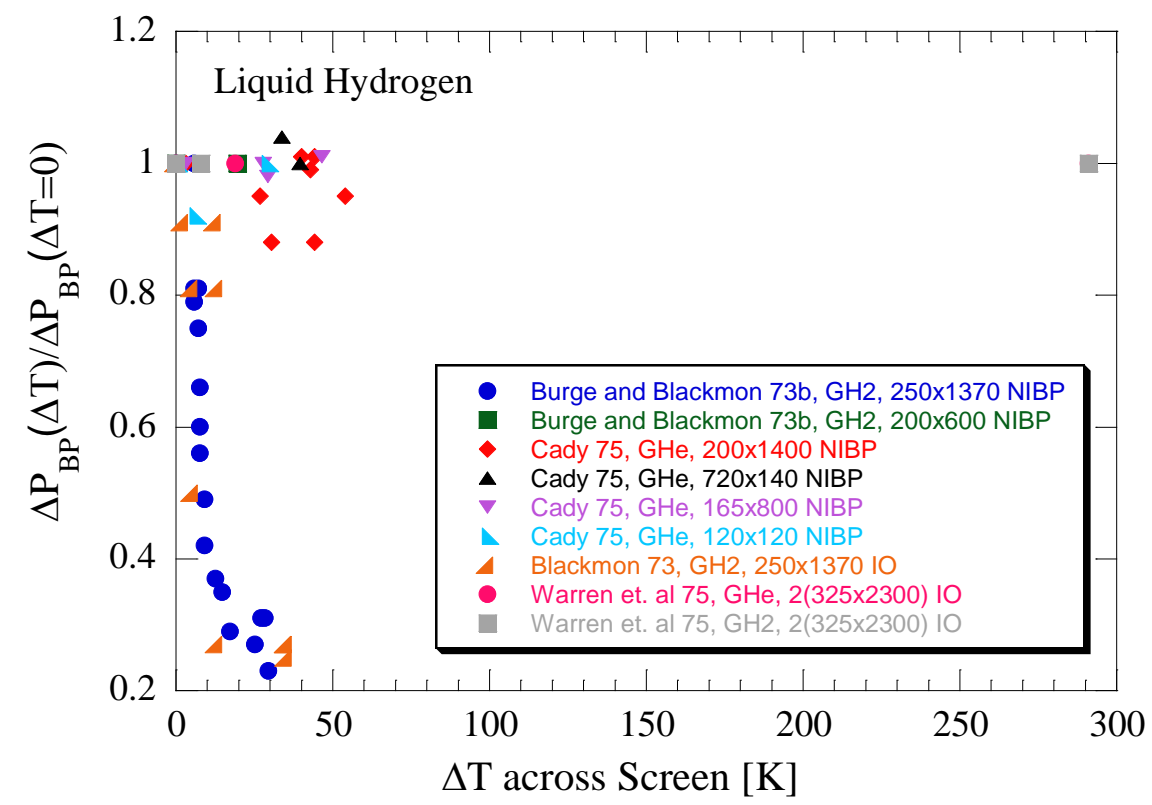

Figure E.2 - Remaining Heated Pressurant Gas Liquid Acquisition Device Data in Liquid Hydrogen for Various Screens

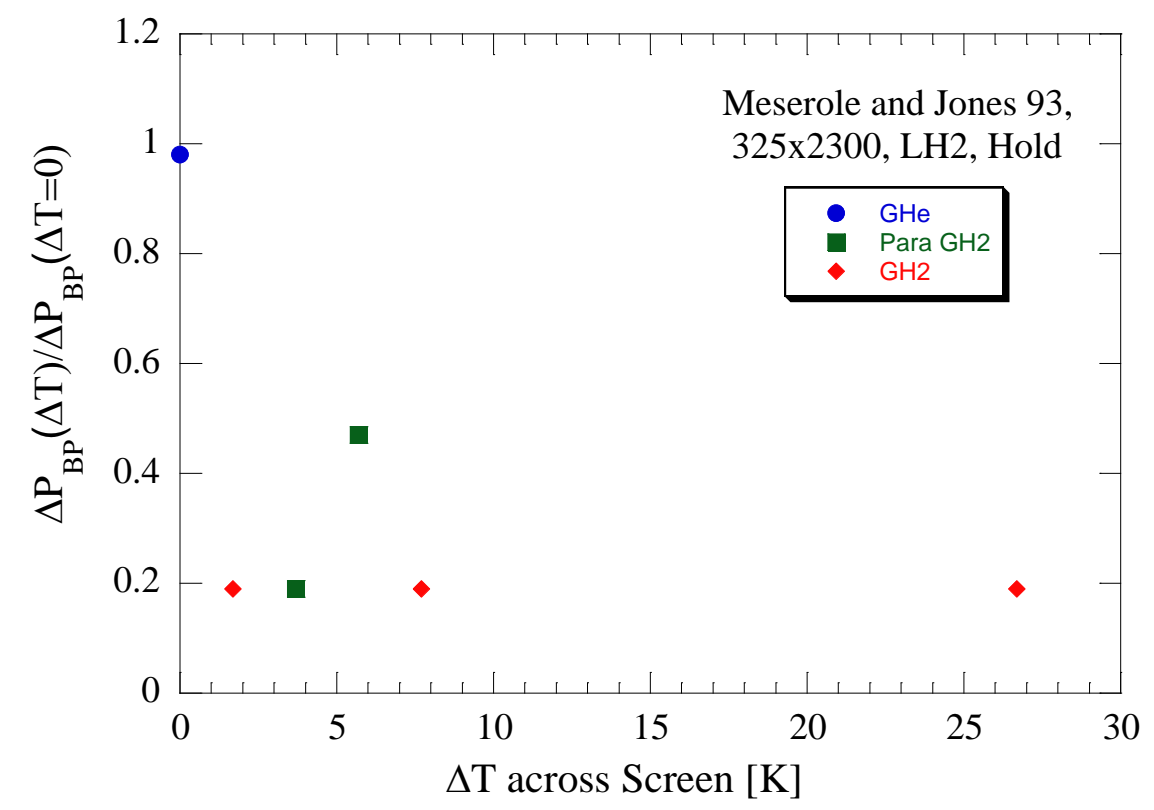

Figure E.3 - Inverted Liquid Hydrogen Outflow Hold Tests for a 325x2300 Screen from Meserole and Jones (1993) using Heated Pressure Gas 


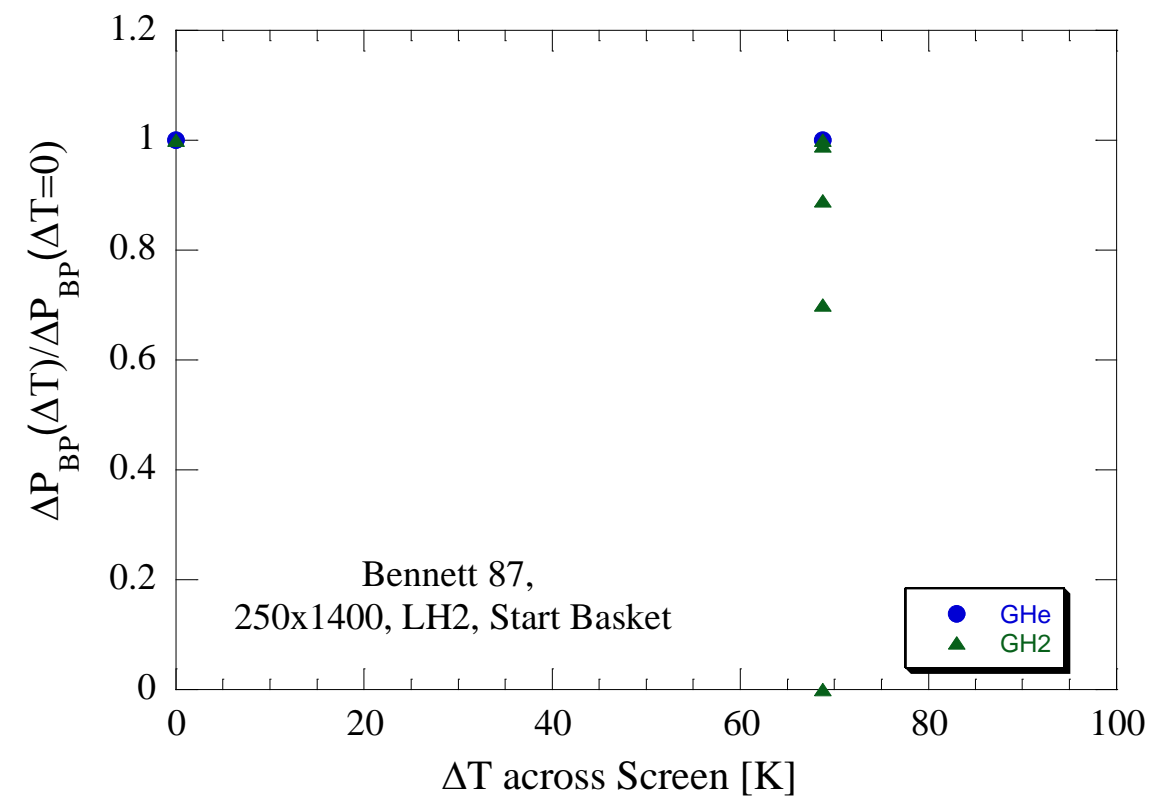

Figure E.4 - Warm Pressurant Start Basket Liquid Acquisition Device Performance Tests in Liquid Hydrogen from Bennett (1987)

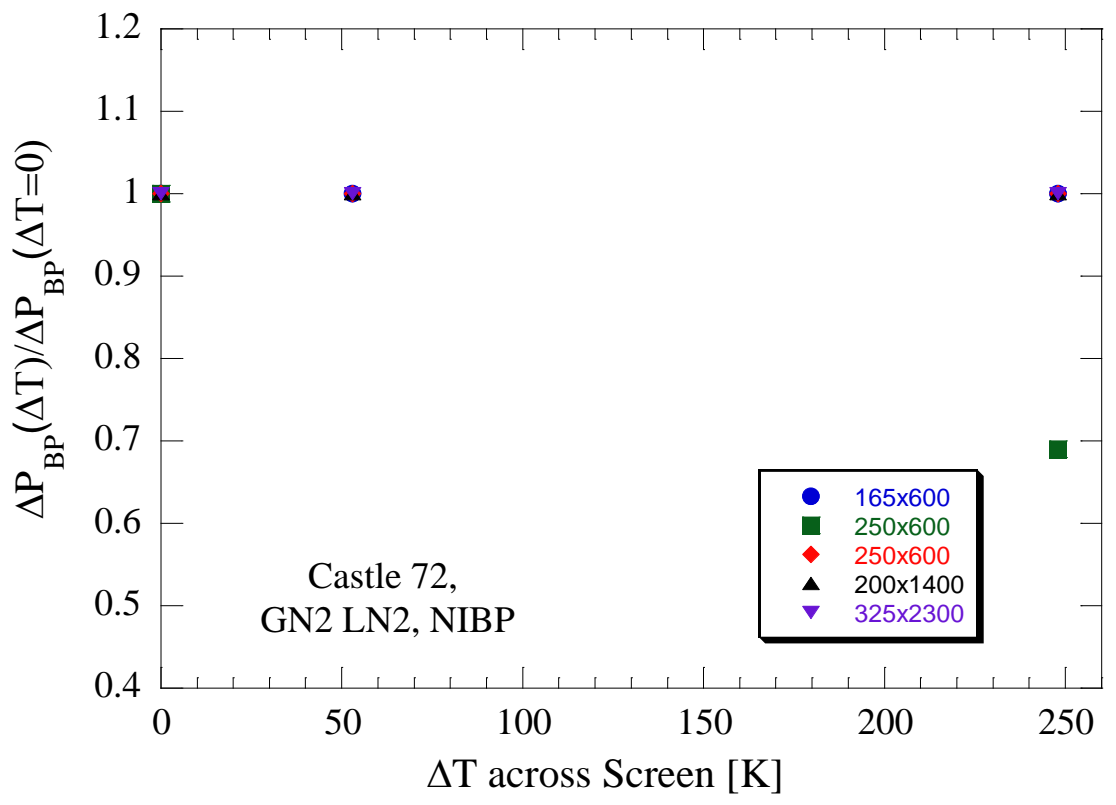

Figure E.5 - Heated Non-inverted Bubble Point Data in Liquid Nitrogen from Castle (1972) 


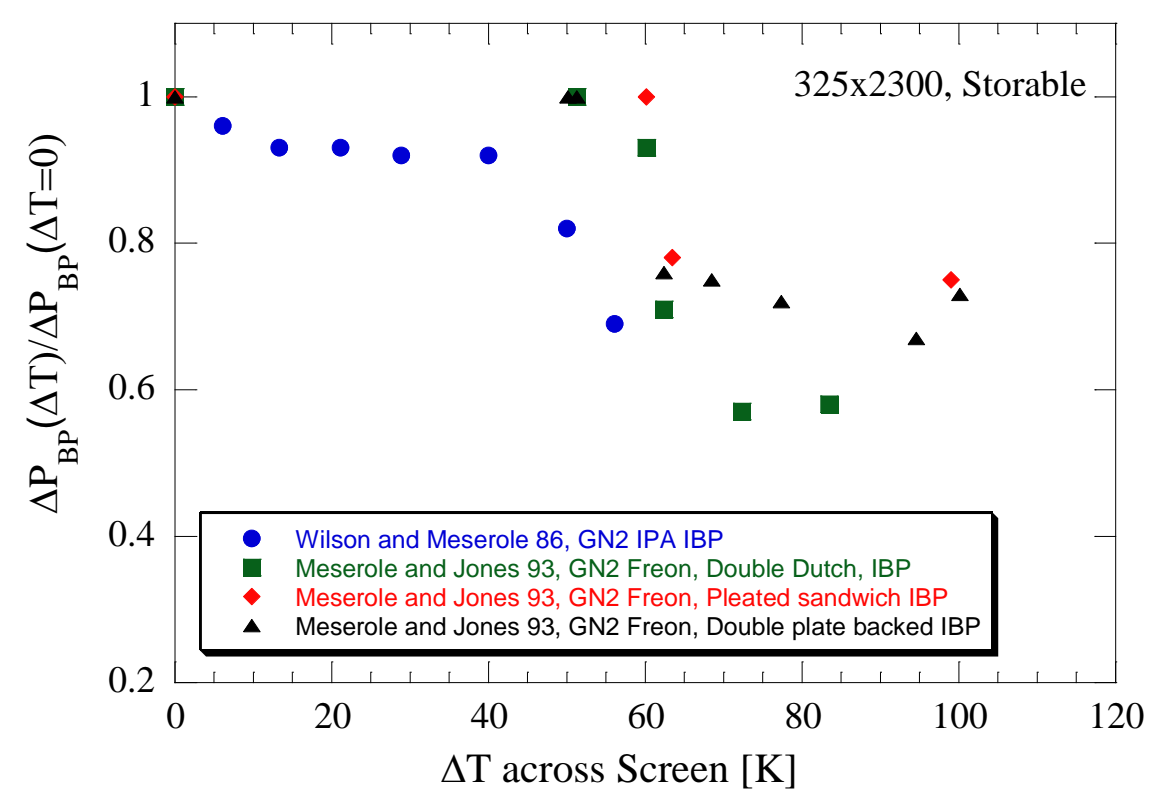

Figure E.6 - Warm Pressurant Gas Liquid Acquisition Device Performance Data for a 325x2300 Screen in Isopropyl Alcohol and Freon

In summary, past inverted outflow tests are not ideal for assessing the fundamental effect of warm pressurant gas on LAD performance due to complexities in reducing the data, in controlling pressurant gas flow, and in controlling uniformity of the gas temperature. Dynamic IO tests are more flight representative than the simple static screen sample tests because the IO tests simulate 1-g outflow through a LAD channel from a larger propellant tank. However, for IO outflow tests, it is extremely difficult to control the location and direction of pressurization and also the uniformity of the temperature of the warm gas inside the tank relative to the LAD; even small changes in the direction or temperature of the gas can cause noticeable differences in the breakdown height of the channel as is shown in the historical data. The NIBP configuration is also not preferred due to complexities in controlling pressurant gas temperature and 
controlling gas flow down on the screen (i.e. natural vs. forced convection). Meanwhile, the IBP configuration is well suited to address this effect provided that careful attempts are made to heat only the gas, and not the screen. Care must also be taken to assure uniformity of temperature rise and to precisely measure the temperature of the pressurant gas before incidence on the screen. IBP configurations are preferred over IO configurations because it is better to heat the gas and then have the gas incident on the screen, rather than warm the ullage space inside a large tank and then allow it to cool as it comes in contact with the LAD. Most discrepancies in previously reported experiments are due to these aforementioned concerns which are mitigated through the new IBP experimental design presented in Chapter 8. 


\section{Appendix F}

\section{Previously Reported Porous Channel Solutions}

The solution derived in Chapter 12 is for laminar channel flow with one porous wall with arbitrary, variable suction or injection, with no heat transfer, electric or magnetic field. This appendix presents a comprehensive review of historical work on porous element flow solutions, analytically, numerically, and experimentally. Results are organized and classified by the type of porous element and number of porous walls (for channels), the nature of flow within the porous element (laminar or turbulent), the size (small, large, arbitrary) and nature (uniform or variable) of injection/suction Re number into or out of the porous element, the type of transverse and axial boundary conditions at the porous surface (suction or injection), whether or not the flow has an electric or magnetic component or any other special feature, such as moving boundaries, nonNewtonian flow, and whether or not there is heat transfer. Heat transfer solutions are organized by boundary condition type, either isothermal or isoflux wall.

Analytical and numerical solutions exist in the literature for porous tubes/pipes for laminar channel flow with no heat transfer (Taylor 1956, Sparrow et al. 1964, Tharakan and Chau 1986, Salmon et al. 1988, Wang 1989, Heath et al. 1990, and Vassilieff 1992) with small uniform suction (Prager 1964, Agrawal 1969, Singh and Laurence 1979, and Brady 1984), with large uniform suction (Macey 1963 and 1965), 
with arbitrary uniform suction (Quaile and Levy 1975, Schnitzlein 1993, and Zaturska and Banks 1995), with small variable suction (Drake et al. 1969, Apelblat et al. 1974, Galowin et al. 1974, Ilias and Govind 1988, Karode 2001, and Munson-McGee 2002), with arbitrary variable suction (Bruining 1989), with small uniform injection (Friedman and Gillis 1967, Bundy and Weissburg 1970), with large uniform injection (Davis and Ethier 1993, Lu 1992 and 2002), with small uniform suction or injection (Yuan and Finkelstein 1956, Hornbeck et al. 1963, Kozinski et al. 1970), with large uniform suction or injection (Weissberg 1959), with arbitrary uniform suction or injection (Berman 1956, White 1962, Terrill and Thomas 1969, Erdogan and Imrak 2005 and 2008), with small variable suction or injection (Galowin and Desantis 1971 and White 1991), and with arbitrary variable suction or injection (Terrill 1982 and 1983, Mellis et al. 1993).

Special solutions exist for porous tubes/pipes for laminar channel flow with no heat transfer for non-Newtonian flow with small uniform suction or injection (Narasimhan 1961), for swirling flow with accelerating walls with small uniform suction (Banks and Zaturska 1996), for pulsating flow (Chang et al. 1989), and for expanding walls with arbitrary uniform suction (Si et al. 2011a and b) or arbitrary uniform injection (Goto and Uchida 1980).

Solutions exist for porous tubes/pipes for laminar channel flow with heat transfer (Vafai and Thiyagaraja 1987, Schnitzlein and Lowe 1990) with small uniform injection (Davis 1978) and with symmetric isothermal boundaries with small uniform suction or injection (Kinney 1968). 
Solutions exist for porous tubes/pipes for turbulent channel flow with no heat transfer (Yuan and Brogen 1961) with large uniform suction (Doshi and Gill 1974, Schildknecht et al. 1979), with arbitrary uniform suction (Kinney and Sparrow 1970, Merkine et al. 1971, Brosh and Winograd 1974, and Eroshenko 1982), with large uniform injection (Eroshenko 1981b), with large uniform suction or injection (Yeroshenko et al. 1984), with arbitrary uniform suction or injection (Eroshenko et al. 1981c), and with arbitrary uniform suction or injection with internal heat generation (Eroshenko et al. 1980).

Analytical and numerical solutions exist in the literature for a porous annulus for laminar channel flow with no heat transfer with small uniform suction or injection (Das 1966, Morel and Bernstein 1976, Moussy and Snider 2009), with large uniform suction or injection (Moussy and Snider 2011), with arbitrary uniform suction or injection (Berman 1958, Huang and Yu 1973, Terrill and Thomas 1973, Morel et al. 1977, Chatterjee and Belfort 1986, Belfort et al. 1993 and b, and Dolecek et al. 1995), and for non-Newtonian flow with arbitrary variable suction (Shrestha 1969a and Huang 1974).

Special solutions exist for a porous annulus for laminar channel flow with no heat transfer for unsteady uniform wall suction or injection (Tsangaris et al. 2007), for nonNewtonian flow with arbitrary uniform suction or injection (Huang 1977), for swirling fluid motion (Verma and Gaur 1974), for magneto-hydrodynamic (MHD) laminar channel flow with arbitrary uniform suction or injection (Nandi 1973), variable suction or injection (Nandi 1971), for unsteady MHD flow (Nandi 1970a), and for unsteady MHD flow with a moving inner boundary (Nandi 1970b). 
Solutions exist for a porous annulus for laminar channel flow with heat transfer with asymmetric isothermal boundaries (Havstad and Burns 1982 and Prasad and Kulacki 1984a) with arbitrary uniform suction or injection (Sharma and Singh 1987 and Mitrovic and Maletic 2006), with arbitrary uniform suction or injection and viscous dissipation included (Kuznetsov et al. 2003), with asymmetric isoflux boundaries with arbitrary uniform suction or injection (Prasad 1986, Chikh et al. 1995, and Srivastava and Singh 2011), with natural convection heat transfer (Prasad and Kulacki 1985 and Prasad et al. 1985), with natural convection with isoflux boundary (Prasad et al. 1986), and with natural convection, non-Newtonian flow, and entropy generation (Chauhan and Olkha 2011).

Analytical and numerical solutions exist in the literature for a porous channel with two porous walls for laminar channel flow with no heat transfer (Wight et al. 1958, Wageman and Guevara 1960, Sutton 1970, Altena and Belfort 1984, Jocelyne et al. 1989, and Breugem 2007) with small uniform suction (Berman 1953, Gill et al. 1965, Singh and Laurence 1979a), with large uniform suction (Sellars 1955, Doughty 1975, Taylor et al. 1991, MacGillivray and Lu 1994, Lu 1997b, and Jankowski and Majdalani 2002), with arbitrary uniform suction (Raithby and Knudsen 1974, Robinson 1976, Cox 1991a, Ferro and Gnavi 2000, and Haldenwang et al. 2010), with small variable suction (Doshi et al. 1971 and Haldenwang 2007), with small uniform injection (Terrill 1964, Horton and Yuan 1964, Terrill and Shrestha 1965, and Sheppard 1972), with large uniform injection (Terrill 1965a, Shrestha and Terrill 1968, Eroshenko et al. 1972, Shih 1987, Zaturska et al. 1988, Avalon et al. 1998, Casalis et al. 1998, and Griffond, Casalis 2000 and 2001, and Ashraf et al. 2009), with arbitrary uniform injection (Morduchow 1957, 
Varapaev 1969, Varapaev and Yagodkin 1969, Feraille and Casalis 2003, and Franson and Alfredsson 2003), with small uniform suction or injection (Terrill and Shrestha 1966, Shrestha 1969b, Chaudhary and Dutta 1972, Khodadadi and Kroll 1992, Lu et al. 1992, Nicoud and Angilella 1997, Erdogan 2003, Oxarango et al. 2004, and Avramenko et al. 2005), with large uniform suction or injection (Yuan 1956, Proudman 1960, Eroshenko et al. 1981a, Schmitz et al. 1992, and Hill and Koch 2002), and with arbitrary uniform suction or injection (White et al. 1958, Gorton 1959, Terrill 1966, Doughty and Perkins 1970, Aggarwala and Gangal 1977, Skalank and Wang 1978, Belfort and Nagata 1985, Wang 1991, Ariel 2002, Nield 2003, Zaturska and Banks 2003, Hewitt et al. 2003, and Hafeez and Ndikilar 2014).

Special solutions exist for a porous channel with two porous walls for laminar channel flow with no heat transfer for periodic suction or injection (Ramana et al. 2007), for sinusoidal suction or injection (Singh 1999), for an oscillatory pressure driven steady flow with large uniform injection (Majdalani and Roh 2000), and with arbitrary uniform injection (Majdalani 2001and Graham and Higdon 2002a), for an oscillatory pressure driven unsteady flow with arbitrary uniform injection (Wang 1971, Graham and Higdon 2002b, and Majdalani and Flandro 2002), for non-Newtonian flow (Hayat et al. 2011), and for non-Newtonian flow with small uniform suction or injection (Jain and Goel 1964).

Special solutions exist for a channel with two porous walls for laminar channel flow with no heat transfer with pulsating walls (Secomb 1973) with arbitrary uniform suction or injection (Skalank and Wang 1977), for a channel with non-uniform gap thickness (Makinde 1995) with small uniform suction or injection (Shivakumar et al. 
1986 and Pozrikidis 1987), for a converging or diverging channel with large uniform suction or injection (Terrill 1965c, Roy and Nayak 1982), for a channel with rotational flow (Vidyanidhi and Nigam 1967 and Vidyanidhi et al. 1975) with arbitrary uniform injection (Saad and Majdalani 2009 and Majdalani 2009), for a channel with expanding, contracting, or accelerating walls (Brady and Acrivos 1981, Watson et al. 1991) with small uniform suction or injection (Majdalani et al. 2002), with large uniform suction or injection (Majdalani and Zhou 2003 and Zhang et al. 2014), and with arbitrary uniform suction or injection (Cox 1991b, Lu 1997a, Hamza 1999, Dauenhauer and Majdalani 2003, Kamisli 2006, Dinarvand et al. 2009, and Xu et al. 2010).

Special solutions exist for a porous channel with two porous walls for laminar channel flow with no heat transfer with MHD flow (Ganesh and Krishnambal 2006) with small uniform suction (Metha and Jain 1962, Suryaprakasarao 1962, and Jain 1967), with small uniform suction or injection (Makinde and Osalusi 2006), with large uniform suction or injection (Shrestha 1967), with arbitrary uniform suction or injection (Bathaiah 1980, Abbas et al. 2006, Sai and Rao 2000, Khan et al. 2008, and Barik et al. 2014), for unsteady, time dependent suction (Govindarajulu 1978), for periodic suction and injection (Singh and Sharma 2001 and Singh 2004), for unsteady flow (Srivastava and Agarwal 1978), with arbitrary uniform suction or injection (Srivastava 1976 and Ramakrishnan and Shailendhra 2011 and 2013), for pulsatile flow (Ogulu and Amos 2007), for unsteady non-Newtonian flow (Taklifi and Aliabadi 2012), for a porous channel with one uniformly moving wall and one wall with small uniform suction (Verma and Mathub 1969), and with arbitrary uniform suction (Verma and Bansal 1966 and Ismail et al. 2014), for a converging porous channel (Bararnia et al. 2010), for a 
porous channel with expanding or contracting walls (Si et al. 2010), for a porous channel with an oscillating wall (Sahoo 2013), and a porous channel with a periodic applied magnetic field (Sengupta and Ghosh 1977).

Solutions exist in the literature for a porous channel with two porous walls for turbulent channel flow with no heat transfer with small uniform suction (Weissberg and Berman 1955), with large uniform injection (Volkov 2013a), with arbitrary uniform suction or injection (Nikitin and Pavel'ev 1998, Zhapbasbaev and Isakhanova 1998, Vigdorovich and Oberlack 2008, Zhang and Prosperetti 2009, and Liu and Prosperetti 2011), and for standard turbulent channel flow (Moin and Kim 2002).

Solutions exist for a porous channel with two porous walls for laminar channel flow with heat transfer (Doughty and Perkins 1971 Tsou and Chang 1975, Nield 1999, and Cui et al. 2000) with arbitrary uniform suction (Chow et al. 1980), with arbitrary variable suction (Singh et al. 2000), with small uniform injection (Walker and Terrill 1974), with small uniform suction or injection (Gupta and Gupta 1977), and for symmetric isothermal walls (Kaviany 1985, Nield and Kuznetsov 1999 and 2000, Narasimhan and Lage 2001, and Nield et al. 2002 and 2004a).

Solutions exist for a porous channel with two porous walls for laminar channel flow with heat transfer for asymmetric isothermal boundaries (Haji-Sheikh et al. 2004a and b, Haji-Sheikh et al. 2005, Minkowycz and Haji-Sheikh 2006, Mitrovic and Maletic 2007, and Minkowycz and Haji-Sheikh 2009), with small uniform injection (Mohais et al. 2011a and b and Tian et al. 2012), with small uniform suction or injection (Terrill 1965b, Doughty 1971, Pederson and Kinney 1971, and Hinvi et al. 2013a and b), with 
large uniform suction or injection (Terrill and Walker 1967 and Ferro and Gnavi 2002), with arbitrary uniform suction or injection (Yeroshenko et al. 1981 and Fournier et al. 2007), for periodic suction or injection (Jain and Gupta 2006), for a pressure driven unsteady flow (Fang 2004a and b) with small suction or injection (Tilton and Cortelezzi 2008), for an oscillatory pressure driven unsteady flow (Roy et al. 1981), for viscous dissipation included (Al-Hadhrami et al. 2003, Nield et al. 2003a, and Hooman and Gorji-Bandpy 2005), for viscous dissipation and entropy generation included (Mahmud and Fraser 2005), for viscous dissipation and flow work included (Nield et al. 2004b), for unsteady flow with viscous dissipation included (Attia 2009), for a channel with moving walls with arbitrary uniform suction or injection with viscous dissipation included (Si et al. 2013), for a channel with stretching walls (Chen and Char 1988) with internal heat generation included (Subhas and Veena 1998), and for a channel with oscillating wall temperature with small uniform suction or injection (Chaudhary and Sharma 2003) with radiation included (Sharma et al. 2007).

Solutions exist for a porous channel with two porous walls for laminar channel flow with heat transfer for asymmetric isoflux boundaries (Hadim 1994, Nield et al. 1996, Nield et al. 2003b, Kuznetsov and Nield 2009, and Chen and Tso 2012), with small uniform suction or injection (Doughty 1971, Raithby 1971, Doughty and Perkins 1972), with arbitrary uniform suction or injection (Lan and Khodadadi 1993), for nonNewtonian fluid flow (Mahmud and Fraser 2003), and for internal heat sources included (Vidhya and Kesavan 2010) with arbitrary uniform suction or injection (Elbashbeshy and Bazid 2004 and Cortell 2005). 
Solutions exist for a porous channel with two porous walls for laminar channel flow with heat transfer for asymmetric isothermal or isoflux boundaries (Prasad and Kulacki 1984b and Haji-Sheikh et al. 2006), with arbitrary uniform injection (Doughty and Perkins 1973), arbitrary uniform suction or injection (Rao and Prasad 1977, and Nield et al. 1996), and for oscillating walls with small uniform suction or injection (Sharma and Yadav 2005).

Special solutions exist for a porous channel with two porous walls for laminar channel flow with heat transfer and MHD flow (Attia and Kotb 1996 and Eegunjobi 2013) with asymmetric isothermal boundaries for periodic suction or injection (Ojjela and Kumar 2013), for oscillatory flow (Singh and Gupta 2005 and Singh 2012), for pulsatile flow (Radhakrishnamacharya and Maiti 1977 and Bestman 1982), for asymmetric isothermal or isoflux boundaries with small suction or injection (Shrestha 1968), for heat generation included (Gulab 1977), for flow of a non-Newtonian fluid with arbitrary uniform suction or injection (Sharma et al. 2013), for unsteady flow with temperature dependent properties (Chamkha 2001), and for laminar channel flow with heat transfer and MHD flow with natural convection heat transfer (Mishra and Muduli 1976, Raptis 1983, and Manglesh et al. 2014) with small uniform suction or injection (Rao 1963), with small variable suction or injection (Singh and Sharma 2002), with time dependent suction with radiation and viscous dissipation included (Israel-Cookey et al. 2003), for non-Newtonian fluid flow (Kumar and Gupta 2012), with radiation included (Das et al. 2012), with radiation and chemical reaction included (Sandeep et al. 2012), for a rotating channel including radiation (Singh 2013), for a channel with a moving wall (Reddy and Bathaiah 1982) with small suction or injection (Das et al. 2008a and Das 
2009a), with unsteady flow (Das et al. 2008b), for a channel with shrinking wall with arbitrary uniform suction (Muhaimin et al. 2008), for a channel with oscillating wall with internal heat source included (Das et al. 2011 and Sharma et al. 2012), with oscillating wall with oscillatory heat flux boundary (Manna et al. 2012), for a channel with oscillatory suction with internal heat source included (Das et al. 2009b), for a channel with oscillatory suction and internal heat source included (Das et al. 2010), and for a channel with radiation, diffusion, and Hall currents included (Manglesh and Gorla 2013).

Analytical and numerical solutions exist in the literature for a porous channel with one porous wall for laminar channel flow with no heat transfer (Vignes-Adler et al. 1987 and Vafai and Kim 1990) with small uniform suction (Terrill 1960, Green 1979, Verma and Bhatt 1973, Kohler 1973, Kleinstreuer and Paller 1983, Kleinstreuer and Belfort 1984, Belfort 1989, Chellam et al. 1992, Deng and Martinez 2005b, and Mkenda 2012), with large uniform suction (Novikov and Lyubin 1985 and Deng and Martinez 2005a), with arbitrary uniform suction (Cox and King 1997 and Geraldes et al. 2000), with small variable suction (Granger et al. 1986), with small uniform injection (Breugem et al. 2005), with arbitrary uniform injection (Chellam et al. 1995), with small uniform suction or injection (Sparrow et al. 1974, Gupta and Levy 1974 and 1976, and Jorne 1982), with large uniform suction or injection (Lessner and Newman 1984 and Bujurke et al. 2010), and with arbitrary uniform suction or injection (Sparrow et al. 1971 and 1972, Eroshenko et al. 1984, and King and Cox 2001).

Special solutions exist for a porous channel with one porous wall for laminar channel flow with no heat transfer with a moving wall and time dependent injection (Volkov 2013b) and for non-Newtonian flow (Baris 2001). 
Special solutions exist for a porous channel with one porous wall for laminar channel flow with no heat transfer with MHD flow with small uniform injection (Meena 2001 and Motsa et al. 2012), and with arbitrary uniform suction or injection (Manyonge et al. 2012).

Solutions exist in the literature for a porous channel with one wall for turbulent channel flow with no heat transfer with small uniform injection (Breugem and Boersma 2005), with large uniform injection (Beddini 1986, Balakrishnan et al. 1991 and Silva and Lemos 2003), with arbitrary uniform injection (Eroshenko et al. 1986, Chaouat 2001, Chaouat 2002, Apte and Yang 2003, Wasistho et al. 2004, Volkov and Emelyanov 2004, Breugem et al. 2006, Dupuy et al. 2011, and Volkov 2012), with large variable injection (Liou and Lien 1995), with arbitrary uniform injection and chemical reaction (Liou et al. 1994), with arbitrary variable injection (Tsai and Liou 1991), with large uniform suction or injection (Piomelli et al. 1989), and with arbitrary uniform suction or injection (Kim et al. 1987, Sumitani and Kasagi 1995, and Hahn et al. 2002).

Solutions exist for a porous channel with one porous wall for laminar channel flow with heat transfer for asymmetric isothermal boundaries with arbitrary uniform injection (Debruge and Han 1972 and Massey and Sunderland 1972), with small uniform suction or injection (Guria et al. 2009), and for small periodic injection with radiation and chemical reactions included (Rath et al. 2012).

Solutions exist for a porous channel with one porous wall for laminar channel flow with heat transfer for asymmetric isoflux boundaries with small variable injection 
(Sorour 1987), with small uniform suction or injection (Hwang et al. 1993a), and with arbitrary uniform suction or injection (Rhee and Edwards 1981 and Cheng et al. 1994).

Solutions exist for a porous channel with one porous wall for laminar channel flow with heat transfer for asymmetric isothermal or isoflux boundaries with arbitrary uniform suction (Rhee and Edwards 1981).

Special solutions exist for a porous channel with one porous wall for laminar channel flow with heat transfer and flow of viscoelastic fluid with arbitrary uniform suction or injection (Kurtcebe and Erim 2005) and for a channel with one porous wall with heat transfer and expanding walls with arbitrary uniform suction or injection (Tsai et al. 2009).

Special solutions exist for a porous channel with one porous wall for laminar channel flow with heat transfer and MHD flow with heat transfer with asymmetric isothermal boundaries with small uniform suction and one moving wall (Chauhan and Agrawal 2011 and 2013), with small periodic injection with radiation and chemical reactions included (Rath et al. 2013), with small uniform injection with radiation and chemical reactions included (Kar et al. 2013), and for a channel with a moving boundary and radiation included (Ahmed and Kalita 2013).

Solutions exist for a porous channel with one porous wall for turbulent channel flow with heat transfer for asymmetric isothermal boundaries with arbitrary uniform injection (Doche and Tardu 2012).

Previously reported experimental work for porous pipes and channels with one or two porous walls include measurements of the slip coefficients and permeability 
(Rajasekhara 1974 and Brown and Lai 2006), friction factors (Beavers 1970 and 1974, Aggarwal et al. 1972, and Hwang et al. 1993b) and laminar (Hwang et al. 1993b, Cheng and Hwang 1995, and Hwang et al. 1997) and turbulent (Olsen and Eckert 1966 and Lombardi et al. 1974) heat transfer coefficients. Velocity measurements inside porous media have been attempted in previous work using various techniques such as laser anemometry (Johnstone et al. 1975), laser Doppler anemometry (Gupte and Advani 1997), laser Doppler velocimetry (Dybbs and Edwards 1984 and Yarlagadda and Yoganathan 1989), particle image displacement velocimetry (Saleh et al. 1992), Particle Image Velocimetry (PIV) (Hassan et al. 1992) for laminar (Jackson and James 1986, Saleh et al. 1993, Aziz and Wong 2003, Tachie et al. 2003, Agelinchaab et al. 2006, Huang et al. 2008, and Arthur et al. 2009) and turbulent flows (Deng et al. 2001, Horton and Pokrajac 2009, Suga et al. 2010, and Bejatovic et al. 2010), and fluorescent PIV (Northrup et al. 1991 and 1993).

The effect of non-zero slip velocity at the porous wall was also addressed previously in Beavers and Joseph 1967, Taylor 1971, Richardson 1971, Sparrow et al. 1973, Larson and Higdon 1986 and 1987, Blake 1990, Sahraoui and Kaviany 1992, Chellam and Wiesner 1993a and b, Jeong 2001, James and Davis 2001, Davis and James 2003, Lauga and Cossu 2005, Chellam and Liu 2006, Makinde and Osalusi 2006, and Chandesris and Jamet 2006). 


\section{Appendix G}

\section{Summary of Cryogenic Screen Channel LAD}

\section{Design Tools}

This appendix summarizes the updated suite of analytical models that were developed in this dissertation. Models can be used to design, analyze, and predict performance of screen channel LADs operating in either storable or cryogenic propulsion systems. The methodology is also given for how to apply and run the models.

\section{G.1 Bubble Point Pressure Model}

The bubble point model has been derived in a way to make it facile for a system designer to predict performance for any screen in any fluid at any thermodynamic state. The updated bubble point equation is expressed as:

$$
\Delta P_{B P}=\frac{4 \gamma_{L V} \cos \theta_{C}}{D_{P}(T)}\left(1+n_{\text {Sub }}\left(\frac{P-P_{\text {Sat }}}{P_{C}}+\frac{T_{\text {Sat }}-T}{T_{C}}\right)\right)\left(1-n_{H o t}\left(T_{G a s}-T\right)\right)
$$

where surface tension is:

$\gamma_{L V}=\gamma_{0}\left(1-\frac{T}{T_{C}}\right)^{a_{0}+a_{1} \frac{T}{T_{C}}+a_{2} \frac{T^{2}}{T_{C}}}$ 
and the temperature dependent pore diameter, which takes into account both screen pore shrinkage at cryogenic temperatures as well as effect due to type of pressurization scheme is:

$D_{P}(T)=D_{P, 295 K}\left(1+b_{0} \xi(T)\right)$

where

$\xi(T)=3 \frac{L_{T}-L_{295 K}}{L_{295 K}}=3\left(c_{0}+c_{1} T+c_{2} T^{2}+c_{3} T^{3}+c_{4} T^{4}\right) * 10^{-5}$

Specifying the metal type (SS, Al, Ti) specifies $c_{0}, c_{1}, c_{2}, c_{3}, c_{4}$. Specifying the liquid specifies $\gamma_{0}, a_{0}, a_{1}, a_{2}, P_{\text {Sat }}, P_{C}, T_{\text {Sat }}, T_{C}$. Surface tension fitting parameters for 20 different fluids (including all major storable and cryogenic propellants) are available in Table 3.1. Fitting parameters for other fluids can easily be generated using the model in Equation G.2. Room temperature pore diameters are available in Table 10.2 for 26 different screens, and temperature dependent fitting parameters are available in Table 10.5 for 18 different screens. Where room temperature pore diameters are unavailable, one can use the relationship derived in Chapter 10, which relates effective pore diameter to the number and diameter of the warp and shute diameters:

$D_{P, 295 K}=\frac{9633}{x_{s}}$

where

$x_{s}=n_{s}^{r_{d}}$ 
Where temperature dependent pore diameters are unavailable, one can use the room temperature pore diameter from either Table 10.5 or the derived value from Equation G.5. Specifying the type of pressurant gas specifies $b_{0}$. Since subcooled parameters do not depend on the type of screen, specifying the pressurant gas and liquid type specifies

$n_{\text {Sub }}$. Specifying the screen/gas/liquid triplet specifies $n_{H o t}$. For screens where $n_{H o t}$ is not available, one can extrapolate a value for coarser screens based off work in Chapter 8. Alternatively, where warm pressurant gas is expected in a mission, safety factors can be employed. Equation G.1 can be used to predict the bubble point for any fluid, at any thermodynamic state of the liquid, using either pressurization scheme, in either saturated or subcooled states.

\section{G.2 Reseal Pressure Model}

The reseal pressure can be determined using Equation G.7:

$$
\Delta P_{R S}=\frac{4 \gamma_{L V} \cos \theta_{C}}{D_{R}(T)}\left(1+n_{S u b}\left(\frac{P-P_{S a t}}{P_{C}}+\frac{T_{S a t}-T}{T_{C}}\right)\right)\left(1-n_{H o t}\left(T_{G a s}-T\right)\right)
$$

where

$$
D_{R}(T)=D_{R, 288 K} \exp \left(\frac{b_{1}}{T^{b_{2}}}\right)
$$

Room temperature reseal diameters can be determined using Table 11.1 for 13 different screens and temperature dependent pore diameter parameters using Table 11.3 for four screens. For screens where reseal data is not available, a simple relation like Equation 
G.5 can be derived for the reseal case. Subcooled and heated pressurant gas model parameters are available from Tables 11.4 and 10.8, respectively.

\section{G.3 Flow-through-Screen Pressure Drop}

The functional form of the FTS pressure drop model derived in Chapter 3 is unchanged:

$$
\Delta P_{F T S}=\alpha\left(\frac{Q B \mu a^{2}}{\rho \varepsilon^{2}}\right) \frac{\dot{m}}{A_{C}}+\beta\left(\frac{Q B}{\rho \varepsilon^{2} D_{P}}\right)\left(\frac{\dot{m}}{A_{C}}\right)^{2}
$$

where screen parameters are available for 31 different LAD meshes in Table 10.2. Specifying the fluid specifies the thermodynamic properties. To predict FTS pressure drop at cryogenic $\mathrm{LOX}$ or $\mathrm{LH}_{2}$ temperatures, one can use temperature dependent coefficients $\alpha, \beta$ from Tables 3.4 and 9.4. Interpolation can be used to determine $\alpha, \beta$ at other temperatures, or a safety factor can be used with the room temperature coefficients. Equation G.9 can be used to determine flow losses for any sized LAD system.

\section{G.4 Wicking Rate}

For advanced LADs analysis and design, one can integrate the wicking rate equation into analytical tools to predict the effect of wicking rate:

$$
L=\sqrt{D_{E} \frac{\gamma_{L V} \cos \theta_{C}}{4 \mu} t}
$$

In cryogenic systems, wicking rate is especially important because it offsets degradation in performance due to screen dry out when the system is in contact with warm pressurant gas. Specifying the fluid specifies the thermodynamic properties. Table 4.3 can be used to 
input $D_{E}$ for 14 different screens. For screens where wicking parameters are unavailable, simple interpolation can be used to determine $D_{E}$.

\section{G.5 Total System Pressure Drop in 1-g or Microgravity}

The analytical flow model developed for 1-g in Chapter 12 and microgravity in Chapter 14 can be used to predict full scale screen channel LAD performance in any g-level for any propellant and any screen:

$\left(\frac{1}{\operatorname{Re}_{e}}+\frac{N_{\text {lam }}}{\operatorname{Re}_{e}}+2 C_{\text {turb }} f^{\prime}\right) f^{\prime \prime \prime}+2 C_{\text {turb }}\left(f^{\prime \prime}\right)^{2}-\frac{12}{\operatorname{Re}_{e}} f^{\prime}-\frac{12}{5}\left(f^{\prime \prime}(1+f)+\left(f^{\prime}\right)^{2}\right)=0$

Specifying the propellant, screen, demand mass flow rate and operating conditions specifies $N_{\text {lam }}, C_{\text {turb }}$, and $\mathrm{Re}_{e}$. Specifying a pressurant gas diffuser location within a propellant tank as well as an appropriate ullage bubble growth model allows extension of Equation G.11 into microgravity fluid flow modeling.

An optimal screen can be chosen by trading the primary influential factors such as bubble point and FTS pressure drop, as well as secondary influential factors, such as wicking rate, screen compliance, material compatibility, and pressurant gas type. Once the optimal screen is chosen which meets all mission requirements, the LAD channel dimensions can be sized based on logic presented in Chapter 14. An optimal screen channel LAD is one that delivers the desired demand flow rate, against the specified adverse acceleration level, to the desired expulsion efficiency, in the least amount of LAD mass. For transient analysis, basic equations of state from Jaekle (1997) can be used 
to further quantify LAD performance under adverse acceleration levels. Screen compliance data is however required. 


\section{Bibliography}

Abbas, Z., Sajid, M., and Hayat, T. "MHD Boundary-Layer Flow of an Upper-Convected Maxwell Fluid in a Porous Channel" Theoretical Computational Fluid Dynamics 20, $229-238.2006$.

Adamson, A.W. and Gast, A.P. Physical Chemistry of Surfaces New York: John Wiley and Sons, Inc. 1997.

Addesso, A. and Lund, D.B. "Influence of Solid Surface Energy on Protein Adsorption" Journal of Food Processing and Preservation 21, 319 - 333. 1997.

Afzal, N., Seena, A., and Bushra, A. "Power Law Velocity Profile in Fully Developed Turbulent Pipe and Channel Flows" Journal of Hydraulic Engineering 133, 1080 $-1086.2007$.

Agelinchaab, M., Tachie, M.F., and Ruth, D.W. "Velocity Measurement of Flow through a Model Three-Dimensional Porous Medium" Physics of Fluids 18, 017105. 2006.

Aggarwal, J.K., Hollingsworth, M.A., and Mayhew, Y.R. “Experimental Friction Factors for Turbulent Flow with Suction in a Porous Tube" International Journal of Heat and Mass Transfer 15, 1585 - 1602. 1972. 
Aggarwala, B.D. and Gangal, M.K. "Fully Developed Laminar Flow in Porous Ducts" Journal of Mathematical and Physical Sciences 11, 37 - 46. 1977.

Agrawal, H.L. "Laminar Flow through a Uniform Circular Pipe with Small Outward Normal Suction” Proceedings of the National Institute of Sciences of India: Physical Sciences 35A, 518 - 527. 1969.

Ahmed, S. and Kalita, K. "Magnetohydrodynamic Transient Flow through a Porous Medium Bounded by a Hot Vertical Plate in the Presence of Radiation: A Theoretical Analysis" Journal of Engineering Physics and Thermophysics 86, 30 $-39.2013$.

Al-Hadhrami, A.K., Elliot, L., and Ingham, D.B. “A New Model for Viscous Dissipation in Porous Media across a Range of Permeability Values" Transport in Porous Media 53, $117-122.2003$.

Alexander, G.E., Barksdale, T.R., Hise, R.E., Lunden, K.C., and Paynter, H.L. “Experimental Investigation of Capillary Propellant Control Devices for LowGravity Environments" NASA-CR-110755, June, 1970.

Altena, F.W. and Belfort, G. "Lateral Migration of Spherical Particles in Porous Flow Channels: Application to Membrane Filtration" Chemical Engineering Science $39,343-355.1984$.

Ambrose, D., Sprake, C.H.S., and Townsend, R. "Thermodynamic Properties of Organic Oxygen Compounds XXXIII: The Vapor Pressure of Acetone” Journal of Chemical Thermodynamics 6, 693-700. 1974. 
Ambrose, D., Sprake, C.H.S., and Townsend, R. "Thermodynamic Properties of Organic Oxygen Compounds XXXVII. Vapour Pressures of Methanol, Ethanol, Pentan-1ol, and Octan-1-ol from the Normal Boiling Temperature to the Critical Temperature" Journal of Chemical Thermodynamics 7, 185 - 190. 1975.

Amneus, J.S. “A New Pulp Drainage Model, Its Application and Verification” TAPPI Journal 48, 641 - 647. 1965.

Anderson, J.E. and DiPirro, M.J. “Acquisition System Testing with Superfluid Helium” Advances in Cryogenic Engineering 37, 909 - 916. Plenum Press, New York, 1988.

Anderson, J.E. "Superfluid Helium Acquisition System Development” Cryogenics 29, $513-516.1989$.

Anglim, D.D. "Low-g Testing of the Space Shuttle OMS Propellant Tank" AIAA-79-1258 $15^{\text {th }}$ Joint Propulsion Conference, Las Vegas, NV, June 18 - 20, 1979.

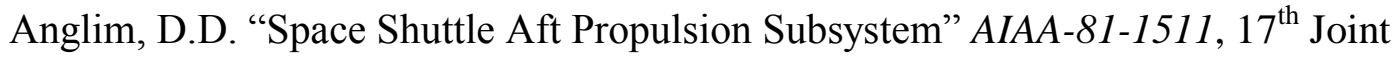
Propulsion Conference, Colorado Springs, CO, July 27 - 29, 1981.

Anonymous "Exploration Systems Architecture Study, Final Report" NASA-TM-2005214062, 2005.

Apelblat, A., Katzir-Katchalsky, A., and Silberberg, A. "A Mathematical Analysis of Capillary Tissue Fluid Exchange” Biorheology 11, 1 - 49. 1974. 
Apfel, S.L. "Optimization of the Boeing 702 for the DIRECTV Mission" AIAA-2006$5300,24^{\text {th }}$ International Communications Satellite Systems Conference, San Diego, CA, June $11-14,2006$.

Apte, S.V. and Yang, V. “A Large-Eddy Simulation Study of Transition and Flow Instability in a Porous-Walled Chamber with Mass Injection” Journal of Fluid Mechanics 477, 215 - 225. 2003.

Ariel, P.D. "On Exact Solutions of Flow Problems of a Second Grade Fluid through Two Parallel Porous Walls" International Journal of Engineering Science 40, 913 941. 2002.

Arif, H. and Kroeger, E.W. "COLD-SAT: A Technology Satellite for Cryogenic Experimentation" NASA-TM-102286. 1989.

Arif, H. "Preliminary Thermal Design of the COLD-SAT Spacecraft" NASA-TM-104440. 1991.

Arifvianto, B., Suyitno, M., Mahardika, M., Dewo, P., Iswanto, P.T., and Salim, U.A. "Effect of Surface Mechanical Attrition Treatments (SMAT) on Microhardness, Surface Roughness, and Wettability of AISI 316L" Materials Chemistry and Physics 125, $418-426.2011$.

Armour, J.C. and Cannon, J.N. "Fluid Flow through Woven Screens" AIChE Journal 14, $415-420.1968$. 
Arnold, S.L. "Physical and Thermodynamic Properties of Hypergolic Propellants: A Review and Update" JANNAF Inter-Agency Propulsion Committee PD\&CS and S\&EPS Joint Meeting, San Diego, CA, 1999.

Arthur, J.K., Ruth, D.W., and Tachie, M.F. "PIV Measurements of Flow through a Model Porous Medium with Varying Boundary Conditions" Journal of Fluid Mechanics $629,343-374.2009$.

Ashraf, M., Kamal, M.A., and Syed, K.S. "Numerical Study of Asymmetric Laminar Flow of Micropolar Fluids in a Porous Channel" Computers and Fluids 38, 1895 $-1902.2009$.

Attia, H.A. and Kotb, N.A. "MHD Flow between Two Parallel Plates with Heat Transfer" Acta Mechanica 117, 215 - 220. 1996.

Attia, H.A. "Effect of Porosity on Unsteady Couette Flow with Heat Transfer in the Presence of Uniform Suction and Injection” Kragujevac Journal of Science 31, 11 $-16.2009$.

Augustine, N. Letter to Representative Frank R. Wolf. March 23, 2010.

Austad, K.L. "The Common Centaur Upper Stage" AIAA-2001-3842, 37 ${ }^{\text {th }}$ Joint Propulsion Conference, Salt Lake City, Utah, July 8 - 11, 2001.

Avalon, G., Casalis, G., and Griffond, J. "Flow Instabilities and Acoustic Resonance of Channels with Wall Injection" AIAA-98-3218, 34 ${ }^{\text {th }}$ Joint Propulsion Conference, Cleveland, OH, July $13-15,1998$. 
Avramenko, A.A., Kuznetsov, A.V., Basok, B.I., and Blinov, D.G. "Investigation of Stability of a Laminar Flow in a Parallel-Plate Channel Filled with a Fluid Saturated Porous Medium" Physics of Fluids 17, 094102. 2005.

Aziz, A.R.A. and Wong, K.F.V. "Velocity Measurements across Fluid-Porous Medium Interface using Particle Image Velocimetry" International Journal of Modeling and Simulation 23, $1-8.2003$.

Bailey, R.V., Murphy, J.M., and Villars, A.J. “An All Aluminum Propellant Tank” AIAA86-1714, 22 $2^{\text {nd }}$ Joint Propulsion Conference, Huntsville, AL, June 16 - 18, 1986.

Bailey, W.J., Weiner, S.P., Beekman, D.H., Dennis, M.F., and Martin, T.A. "Cryogenic On-Orbit Liquid Depot Storage, Acquisition, and Transfer Satellite (COLD-SAT) Feasibility Studies" NASA-CR-185247. 1990.

Bailey, W.J., Weiner, S.P., and Beekman, D.H. "Cryogenic Orbital Nitrogen Experiment (CONE) - Phase A/B Design Study" NASA-CR-187230. 1991.

Balakrishnan, G., Linan, A., and Williams, F.A. "Compressibility Effects in Thin Channels with Injection" AIAA Journal 29, 2149 - 2154. 1991.

Balat, M. "Potential Importance of Hydrogen as a Future Solution to Environmental and Transportation Problems" International Journal of Hydrogen Energy 33, 4013 4029. 2008.

Ballinger, I.A., Lay, W.D., and Tam, W.H. "Review and History of PSI Elastomeric Diaphragm Tanks." AlAA-95-2534, 31 ${ }^{\text {st }}$ Joint Propulsion Conference and Exhibit, San Diego, CA, July 10-12, 1995. 
Ballweg, R. and Wallrapp, F. "EDRS Operations at GSOC- Relevant Heritage and New Developments" 2012.

Balzer, D.L., Barksdale, T.R., Bowman, T.E., Gilmore, D.E., Gorman, D.N., Hise, R.E. “Advanced Propellant Management System for Spacecraft Propulsion Systems, Phase 2 - Detail Design” MCR-69-436, NASA-CR-101913, September, 1969.

Balzer, D.L., Brill, Y.C., and Scott, W.R. "The Monopropellant Hydrazine Reaction Control System for the RCA SATCOM Satellite" AIAA-76-631, $12^{\text {th }}$ Joint Propulsion Conference, Palo Alto, CA, July 26 - 29, 1976.

Bamberger, H.H., Robinson, R.C., Jurns, J.J., and Grasl, S.J. “Liquid Methane Conditioning Capabilities Developed at the NASA Glenn Research Center's Small Multi-purpose Research Facility (SMiRF) for Accelerated Lunar Surface Storage Thermal Testing” NASA-CR-2011-216745. 2011.

Banks, W.H.H. and Zaturska, M.B. "Swirling Flow in a Porous Pipe with an Accelerating Wall” Acta Mechanica 119, 1 - 12. 1996.

Bararnia, H., Ganji, Z.Z., Ganji, D.D., and Moghimi, S.M. "Numerical and Analytical Approaches to MHD Jeffery-Hamel Flow in a Porous Channel” International Journal of Numerical Methods for Heat and Fluid Flow 22, 491 - 502. 2010.

Barik, R.N., Dash, G.C., and Rath, P.K. "Homotopy Perturbation Method (HPM) Solution for Flow of a Conducting Visco-Elastic Fluid through a Porous Medium" Proceedings of the National Academy of Sciences of India 84, 55 - 61. 2014. 
Baris, S. "Injection of a Non-Newtonian Fluid through One Side of a Long Vertical Channel" Acta Mechanica 151, 163 - 170. 2001.

Bathaiah, D. "MHD Flow through a Porous Straight Channel” Acta Mechanica 35, 223 229. 1980.

Beavers, G.S. and Joseph, D.D. "Boundary Conditions at a Naturally Permeable Wall" Journal of Fluid Mechanics 30, 197 - 207. 1967.

Beavers, G.S., Sparrow, E.M., and Magnuson, R.A. "Experiments on Coupled Parallel Flows in a Channel and a Bounding Porous Medium" ASME Journal of Basic Engineering 92, 843 - 848. 1970.

Beavers, G.S., Sparrow, E.M., and Masha, B.A. "Boundary Condition at a Porous Surface Which Bounds a Fluid Flow” AIChE Journal 20, 596 - 597. 1974.

Beddini, R.A. "Injection-Induced Flows in Porous-Walled Ducts" AIAA Journal 24, 1766 $-1773.1986$.

Behruzi, P. and Netter, G. "Concept Analysis of PMD Designs for Future Upper Stages” IAC-03-S.1.07, $54^{\text {th }}$ International Astronautical Congress of the International Astronautical Federation, the International Academy of Astronautics, and the International Institute of Space Law, Bremen, Germany, September 29 - October 3, 2003.

Behruzi, K.P. and Michaelis, M. "Development of a Propellant Management Device for Restartable Future Cryogenic Upper Stages" AIAA-2006-5053, 42 ${ }^{\text {nd }}$ Joint Propulsion Conference, Sacramento, CA, July 9 - 12, 2006. 
Behruzi, P., Dodd, C., and Netter, G. "Future Propellant Management Device Concepts for Restartable Cryogenic Upper Stages" AIAA-2007-5498, $43^{\text {rd }}$ Joint Propulsion Conference, Cincinnati, OH, July 8 - 11, 2007.

Bejatovic, S., Tachie, M.F., Agelinchaab, M., and Paul, S.S. "PIV Study of Turbulent Flow in Porous Media" Turbulence and Interactions: Notes on Numerical Fluid Mechanics and Multidisciplinary Design 110, 71 - 78. 2010.

Belfort, G. and Nagata, N. "Fluid Mechanics and Cross-Flow Filtration: Some Thoughts" Desalination 53, $57-79.1985$.

Belfort, G. "Fluid Mechanics in Membrane Filtration: Recent Developments" Journal of Membrane Science 40, 123 - 147. 1989.

Belfort, G., Pimbley, J.M., Greiner, A., and Chung, K.Y. "Diagnosis of Membrane Fouling using a Rotating Annular Filter. 1. Cell Culture Media” Journal of Membrane Science 77, 1 - 22. 1993a.

Belfort, G., Mikulasek, P., Pimbley, J.M., and Chung, K.Y. "Diagnosis of Membrane Fouling Using a Rotating Annular Filter. 2. Dilute Particle Suspensions of Known Particle Size” Journal of Membrane Science 77, 23 - 39. $1993 \mathrm{~b}$.

Bell, R.S., Crouch, M.A., Hanna, G.J., Cady, E.C., and Meserole, J.S. "Feasibility Study for the Cryogenic Orbital Nitrogen Experiment (CONE)" NASA-CR-187231. 1991. 
Bennett, F.O. "Design and Demonstrate the Performance of Cryogenic Components Representative of Space Vehicles, Start Basket Liquid Acquisition Device Performance Analysis” NASA-CR-179138, February, 1987.

Benton, J., Ballinger, I.A., Jaekle, D.E., and Osborn, M.F. "Design and Manufacture of a Propellant Tank Assembly” AIAA-2007 5559, 2007.

Berg, J.C. "An Introduction to Interfaces and Colloids: The Bridge to Nanoscience" Hackensack: World Scientific. 2010.

Berman, A. S. "Laminar Flow in Channels with Porous Walls" Journal of Applied Physics 24, 1232 - 1235. 1953.

Berman, A.S. "Concerning Laminar Flow in Channels with Porous Walls" Journal of Applied Physics 27, 1557 - 1558. 1956.

Berman, A.S. "Laminar Flow in an Annulus with Porous Walls" Journal of Applied Physics 29, $71-75.1958$.

Bestman, A.R. "Pulsatile Flow in Heated Porous Channel" International Journal of Heat and Mass Transfer 25, 675 - 682. 1982.

Bicknell, B.A., Fester, D.A., and Wilson, S.C. "Integration of Space Station Propulsion and Fluid Systems" AIAA-88-3289, 24 ${ }^{\text {th }}$ Joint Propulsion Conference, Boston, MA, July $11-13,1988$.

Bingham, P.E. and Tegart, J.R. "Wicking in Fine Mesh Screens" AIAA-P-77-849, $13^{\text {th }}$ Propulsion Conference, Orlando, FL, July 11 - 13, 1977. 
Biron, J. “An Aluminum Collapsible Bladder Tank for Space Systems” AIAA-90-2058, $26^{\text {th }}$ Joint Propulsion Conference, Orlando, FL, July 16-18, 1990.

Blackmon, J.B. "Design, Fabrication, Assembly, and Test of a Liquid Hydrogen Acquisition Subsystem" NASA-CR-120447. 1974.

Blake, T.D. "Slip between a Liquid and a Solid: D.M. Tolstoi's (1952) Theory Reconsidered" Colloids and Surfaces 47, 135 - 145. 1990.

Blatt, M.H. "Liquid Expulsion with Cryogenic Capillary Devices" Report \#78218, Convair Division of General Dynamics, San Diego, CA, 1970a.

Blatt, M.H. "Low Gravity Propellant Control using Capillary Devices in Large Scale Cryogenic Vehicles, Design Handbook" GDC-DDB70-006, NASA-CR-102901, Convair Division of General Dynamics, San Diego, CA, August, 1970b.

Blatt, M.H. "Low Gravity Propellant Control using Capillary Devices in Large Scale Cryogenic Tanks. Related IRAD Studies" GDC-DDB70-009, NASA-CR-102902, Convair Division of General Dynamics, San Diego CA, August, 1970c.

Blatt, M.H. "Low Gravity Propellant Control using Capillary Devices in Large Scale Cryogenic Tanks. Phase I Final Report" GDC-DDB70-008, NASA-CR-114104, Convair Division of General Dynamics, San Diego, CA, August 1970d.

Blatt, M.H. "Low Gravity Propellant Control using Capillary Devices in Large Scale Cryogenic Tanks. Phase II Final Report" GDC-DDB70-008, NASA-CR-114104, Convair Division of General Dynamics, San Diego, CA, August 1970e.

Blatt, M.H. "Orbital Cryogenic Acquisition and Transfer" N71-29611, 1971. 
Blatt, M.H. and Walter, M.D. "Centaur Propellant Acquisition System Study" CASDNAS-75-023, NASA-CR-134811, 1975.

Blatt, M.H., Pleasant, R.L., and Erickson, R.C. "Centaur Propellant Thermal Conditioning Study" CASD-NAS-76-026, NASA-CR-135032, July, 1976.

Blatt, M.H. and Aydelott, J.C. “Centaur Propellant Acquisition System” Journal of Spacecraft 13, $515-521,1976$.

Blatt, M.H. and Aydelott, J.C. “Capillary Device Passive Thermal Conditioning” Journal of Spacecraft 15, $236-241,1978$.

Blatt, M.H., Bradshaw, R.D., and Risberg, J.A. "Capillary Acquisition Devices for HighPerformance Vehicles - Executive Summary" GDC-CRAD-80-003, NASA-CR$159658,1980$.

"Boeing 601 Fleet." Boeing.com. Boeing Company, n.d. Web. August 8, 2013.

"Boeing 702HP Fleet." Boeing.com. Boeing Company, n.d. Web. August 6, 2013.

Bolshinskiy, L.G., Hastings, L.J., Statham, G., and Turpin, J.B. “Capillary Liquid Acquisition Device Heat Entrapment” NASA-TM-2007-215074, 2007.

Bolshinskiy, L.G., Hastings, L.J., and Statham, G. “Cryogenic Capillary Screen Heat Entrapment" Cryogenics 48, 232 - 237. 2008.

Boraas, S. and LaBruna, A.J. “In-Space Propellant Acquisition with Pleated Screen Tubes" Journal of Spacecraft 13, 377 - 384. 1976. 
Boruvka, L. and Neumann, A.W. "Generalization of the Classical Theory of Capillarity" Journal of Chemical Physics 66, 5464 - 5476. 1977.

Brady, J.F. and Acrivos, A. "Steady Flow in a Channel or Tube with an Accelerating Surface Velocity. An Exact Solution to the Navier-Stokes Equations with Reverse Flow" Journal of Fluid Mechanics 112, 127 - 150. 1981.

Brady, J.F. "Flow Development in a Porous Channel and Tube" Physics of Fluids 27, 1061 - 1067. 1984.

Bretherton, F.P. "The Motion of Long Bubbles in Tubes" Journal of Fluid Mechanics 10, $166-188.1961$.

Breugem, W.P. and Boersma, B.J. "Direct Numerical Simulations of Turbulent Flow Over a Permeable Wall Using a Direct and Continuum Approach" Physics of Fluids 17, 025103. 2005.

Breugem, W.P., Boersma, B.J., and Uittenbogaard, R.E. "The Laminar Boundary Layer over a Permeable Wall” Transport in Porous Media 59, 267 - 300. 2005.

Breugem, W.P., Boersma, B.J., and Uittenbogaard, R.E. "The Influence of Wall Permeability on Turbulent Channel Flow" Journal of Fluid Mechanics 562, 35 72. 2006.

Breugem, W.P. "The Effective Viscosity of a Channel-Type Porous Medium" Physics of Fluids 19, 103104. 2007. 
Brooks, J.S. and Donnelly, R.J. "The Calculated Thermodynamic Properties of Superfluid Helium-4" Journal of Physical Chemistry Reference Data 6, 51 - 104. 1977.

Brosh, A. and Winograd, Y. "Experimental Study of Turbulent Flow in a Tube with Wall Suction" ASME Journal of Heat Transfer 96, 338 - 342. 1974.

Brown, N.M. and Lai, F.C. "Measurement of Permeability and Slip Coefficient of Porous Tubes” Journal of Fluids Engineering 128, 987 - 992. 2006.

Bruining, W.J. "A General Description of Flows and Pressures in Hollow Fiber Membrane Modules" Chemical Engineering Science 44, 1441 - 1447. 1989.

Brundrett, E. "Incompressible Flow through Screens" Journal of Fluids Engineering 115, $239-242.1993$.

Buffington, B., Strange, N., and Smith, J. "Overview of the Cassini Extended Mission Trajectory" AIAA-2008-6752, Astrodynamics Specialist Conference, Honolulu, HI, August $18-21,2008$.

Bujurke, N.M., Katagi, N.N., and Awati, V.B. "Analysis of Laminar Flow in a Channel with One Porous Bounding Wall" International Journal of Fluid Mechanics Research 37, $267-281.2010$.

Bundy, R.D. and Weissburg, H.L. "Experimental Study of Fully Developed Laminar Flow in a Porous Pipe with Wall Injection" Physics of Fluids 13, 2613 - 2615. 1970. 
Burge, G.W., Blackmon, J.B., and Klevatt, P.L. "Study and Design of a Cryogenic Propellant Acquisition System, $5^{\text {th }}$ Quarterly Report” MDC-G4271, NASA-CR120387, McDonnell Douglas Company, Huntington Beach, CA, September, 1972.

Burge, G.W. and Blackmon, J.B. "Study and Design of Cryogenic Propellant Acquisition Systems. Volume I: Design Studies” NASA-CR-120300, McDonnell Douglas Company, Huntington Beach, CA, December, 1973a.

Burge, G.W. and Blackmon, J.B. "Study and Design of Cryogenic Propellant Acquisition Systems. Volume II: Supporting Experimental Program” MDC-G5038, NASACR-120301, McDonnell Douglas Company, Huntington Beach, CA, December, $1973 b$.

Burge, G.W., Blackmon, J.B., and Castle, J.N. "Design of Propellant Acquisition Systems for Advanced Cryogenic Space Propulsion Systems" AIAA-73-1287, $9^{\text {th }}$ Propulsion Conference, Las Vegas, NV, November 5 - 7, 1973.

Burk, T. and Bates, D. “Cassini Attitude Control Operations: Flight Rules and How They are Enforced” AIAA-2008-6808, Guidance, Navigation, and Control Conference, Honolulu, HI, August 18 - 21, 2008.

Cady, E.C. "Study of Thermodynamic Vent and Screen Baffle Integration for Orbital Storage and Transfer of Liquid Hydrogen - Final Report” NASA-CR-134482. 1973. 
Cady, E.C. and Blackmon, J.B. "Developments in Low-Gravity Cryogenic Propellant Acquisition and Thermal Control Systems" AIAA-74-1155, $10^{\text {th }}$ Propulsion Conference, San Diego, CA, October 21 - 23, 1974.

Cady, E.C. Design and Evaluation of Thermodynamic Vent/Screen Baffle Cryogenic Storage System. NASA-CR-134810. 1975.

Cady, E.C. "Spacelab Cryogenic Fluid Management Experiment” NAS3-19719 NASA CR-135143, November, 1976.

Cady, E.C. "Effect of Transient Liquid Flow on Retention Characteristics of Screen Acquisition Systems - Final Report” NASA-CR-135218. 1977.

Cady, E.C. "Filling of Orbital Fluid Management Systems" NASA-CR-159405, August, 1978.

Cahn, J.W. "Thermodynamics of Solid and Fluid Surfaces" Segregation to Interfaces, ASM Seminar Series 3 -23. 1978.

Casalis, G., Avalon, G., and Pineau, J.P. "Spatial Instability of Planar Channel Flow with Fluid Injection through Porous Walls" Physics of Fluids 10, 2558 - 2568. 1998.

Castellano, T.P., Raymond, E.A., Shapiro, J.C., Robinson, F.A., and Rosenthal, D.A. "Knowledge Based and Interactive Control for the Superfluid Helium On-Orbit Transfer Project" The 1989 Goddard Conference on Space Applications of Artificial Intelligence, 3 - 11, 1989.

Castle, J.N. "Heat Transfer Effects on Bubble Point Tests in Liquid Nitrogen" MDCG2653 McDonnell Douglas Company, Huntington Beach, CA, January, 1972. 
Chamkha, A.J. "Unsteady Laminar Hydromagnetic Flow and Heat Transfer in Porous Channels with Temperature-Dependent Properties" International Journal of Numerical Methods for Heat and Fluid Flow 11, 430 - 448. 2001.

Chandesris, M. and Jamet, D. "Boundary Conditions at a Planar Fluid-Porous Interface for a Poiseuille Flow" International Journal of Heat and Mass Transfer 49, 2137 $-2150.2006$.

Chandler, F., Bienhoff, D., Cronick, J., and Grayson, G. "Propellant Depots for Earth Orbit and Lunar Exploration" AIAA-2007-6081, SPACE Conference, Long Beach, CA, September 18 - 20, 2007.

Chang, H.N., Ha., J.S., Park, J.K., Kim, I.H., and Shin, H.D. "Velocity Field of a Pulsatile Flow in a Porous Tube" Journal of Biomechanics 22, 1257 - 1262. 1989.

Chaouat, B. "Simulations of Channel Flows with Effects of Spanwise Rotation or Wall Injection Using a Reynolds Stress Model" ASME Journal of Fluids Engineering 123, 2 - 10. 2001.

Chaouat, B. "Numerical Predictions of Channel Flows with Fluid Injection Using Reynolds-Stress Model” Journal of Propulsion and Power 18, 295 - 303. 2002.

Chato, D.J. and Kudlac, M.T. "Screen Channel Liquid Acquisition Devices for Cryogenic Propellants" AIAA-2002-3983, $38^{\text {th }}$ Joint Propulsion Conference, Indianapolis, IN, July $7-10,2002$.

Chato, D.J., and Martin, T.A. "Vented Tank Resupply Experiment: Flight Test Results" Journal of Spacecraft and Rockets 43, 1124 - 1130. 2006. 
Chato, D.J. "The Role of Flight Experiments in the Development of Cryogenic Fluid Management Technologies" Cryogenics 46, 82 - 88. 2006.

Chato, D.J. "Experimentation for the Maturation of Deep Space Cryogenic Refueling Technologies" NASA-TP-2008-214929. 2008.

Chatterjee, S.G. and Belfort, G. "Fluid Flow in an Idealized Spiral Wound Membrane Module" Journal of Membrane Science 28, 191 - 208. 1986.

Chaudhary, R.C. and Sharma, B.K. "Injection and Suction Effects on Three Dimensional Unsteady Flow and Heat Transfer between Two Parallel Porous Plates" Matemáticas: Enseñanza Universitaria 11, 45 - 55. 2003.

Chaudhary, T.K. and Dutta, J. "Steady Couette Flow of a Simple Fluid between Porous Plates" Pure and Applied Geophysics 97, 92 - 99. 1972.

Chauhan, D.S. and Agrawal, R. "MHD Flow and Heat Transfer in a Channel Bounded by a Shrinking Sheet and a Plate with a Porous Substrate" Journal of Engineering Physics and Thermophysics 84, 1034 - 1046. 2011.

Chauhan, D.S. and Olkha, A. "Entropy Generation and Heat Transfer Effects on NonNewtonian Fluid Flow in Annular Pipe with Naturally Permeable Boundaries" International Journal of Energy and Technology 3, 30, 1 - 9. 2011.

Chauhan, D.S. and Agrawal, R. "MHD Flow and Heat Transfer in a Channel Bounded by a Shrinking Sheet and a Porous Medium Bed: Homotopy Analysis Method" ISRN Thermodynamics Article Number 291270, 2013. 
Chellam, S., Wiesner, M.R., and Dawson, C. "Slip at a Uniformly Porous Boundary: Effect on Fluid Flow and Mass Transfer" Journal of Engineering Mathematics 26, $481-492.1992$.

Chellam, S. and Wiesner, M.R. "Laminar Flow with Slip in Channels with Uniformly Porous Walls" Journal of Hydraulic Engineering 119, 126 - 132. 1993 a.

Chellam, S. and Wiesner, M.R. "Slip Flow through Porous Media with Permeable Boundaries: Implications for the Dimensional Scaling of Packed Beds" Water Environment Research 65, 744 - 749. 1993b.

Chellam, S., Wiesner, M.R., and Dawson, C. "Laminar Flow in Porous Ducts" Reviews in Chemical Engineering 11, 54 - 99. 1995.

Chellam, S. and Liu, M. "Effect of Slip on Existence, Uniqueness, and Behavior of Similarity Solutions for Steady, Incompressible Laminar Flow in Porous Tubes and Channels" Physics of Fluids 18, 083601. 2006.

Chen, C.K. and Char, M.I. "Heat Transfer of a Continuous, Stretching Surface with Suction or Blowing” Journal of Mathematical Analysis and Applications 135, 568 $-580.1988$.

Chen, G.M. and Tso, C.P. "Field Synergy Principle Analysis on Convective Heat Transfer in Porous Medium with Uniform Heat Generation for Thermally Developing Flow" International Journal of Heat and Mass Transfer 55, 4139 4147. 2012. 
Chen, J.C. "Correlation for Boiling Heat Transfer to Saturated Fluids in Convective Flow." Industrial \& Engineering Chemistry Process Design and Development 5, $322-329.1966$.

Cheng, P., Li, D., Boruvka, D., Rotenberg, Y., Neumann, A.W. “Automation of Axisymmetric Drop Shape Analysis for Measurements of Interfacial Tensions and Contact Angles" Colloids and Surfaces 43, 151 - 167. 1990.

Cheng, Y.C., Hwang, G.J., and Ng, M.L. "Developing Laminar Flow and Heat Transfer in a Rectangular Duct with One-Walled Injection and Suction" International Journal of Heat and Mass Transfer 37, 2601 - 2613. 1994.

Cheng, Y.C. and Hwang, G.J. "Experimental Studies of Laminar Flow and Heat Transfer in a One-Porous-Wall Square Duct with Injection Flow" International Journal of Heat and Mass Transfer 38, 3475 - 3484. 1995.

Chiang, R.Y., Breckenridge, W.G., and Wong, E.C. "Self-Tuning Thruster Control for Cassini Spacecraft" AIAA-96-3822, Guidance, Navigation, and Control Conference, San Diego, CA, July 29 - 31, 1996.

Chikh, S., Boumedien, A., Bouhadef, K., and Lauriat, G. "Analytical Solution of NonDarcian Forced Convection in an Annular Duct Partially Filled with a Porous Medium" International Journal of Heat and Mass Transfer 38, 1543 - 1551. 1995. 
Chow, L.C., Campo, A., and Tien, C.L. "Heat Transfer Characteristics for Laminar Flow between Parallel Plates with Suction" International Journal of Heat and Mass Transfer 23, $740-743.1980$.

Chu, H.N. and Unterberg, W. "Improvement of Efficiency and Life of Expulsion Bladders"NAS7-506, R-6762-3, Rocketdyne, 1967.

Class, G., Raff, S., and Meyder, R. "The Mechanism of Violent Condensation Shocks" International Journal of Multiphase Flow 13, 33 - 46. 1987.

"COLD-SAT - Cryogenic On-orbit Liquid Depot - Storage, Acquisition, Transfer" NASA-TM-102308, 1989.

Collins, J.A., Melcher, J.C., and Hurlbert, E.A., "Sea-Level Flight Demonstration and Altitude Characterization of a LO2/LCH4 Based Ascent Propulsion Lander" $57^{\text {th }}$ JANNAF Propulsion Meeting, Colorado Springs, CO, May, 2010.

Conrath, M. and Dreyer, M. "Gas Separation and Bubble Behavior at a Woven Screen" 2009 Proceedings of Interdisciplinary Transport Phenomena VI, Volterra, Italy, October 4-9, 2009.

Conrath, M. and Dreyer, M. "Gas Breakthrough at a Porous Screen" International Journal of Multiphase Flow 42, 29 - 41. 2012.

Corruccini, R.J. and Gniewek, J.J. "Thermal Expansion of Technical Solids at Low Temperatures. A Compilation from the Literature" U.S. Department of Commerce, National Bureau of Standards. May, 1961. 
Cortell, R. "Flow and Heat Transfer of a Fluid through a Porous Medium Over a Stretching Surface with Internal Heat Generation/Absorption and Suction/Blowing” Fluid Dynamics Research 37, 231 - 245. 2005.

Coulbert, C.D., Cuddihy, E.F., and Fedors, R.F. "Long-Time Dynamic Compatibility of Elastomeric Materials with Hydrazine" NASA-TM-33-650. 1973.

Cox, S.M. "Two-Dimensional Flow of a Viscous Fluid in a Channel with Porous Walls" Journal of Fluid Mechanics 227, 1 - 33. 1991a.

Cox, S.M. "Analysis of Steady Flow in a Channel with One Porous Wall, or with Accelerating Walls" SIAM Journal of Applied Mathematics 51, 429 - 438. $1991 \mathrm{~b}$.

Cox, S.M. and King, A.C. "On the Asymptotic Solution of a High-Order Nonlinear Ordinary Differential Equation" Proceedings of the Royal Society of London 453, $711-728.1997$.

Crawley, E.F. and Mindell, D.A. "U.S. Human Spaceflight: The FY11 Budget and the Flexible Path: A Space Policy White Paper” 2010.

Cryogenic Propellant Storage and Transfer (CPST) Technology Demonstration Pre-Phase A Government Point-of-Departure Concept Study - Final Review, October 6, 2011.

Cui, C., Huang, X.Y., Liu, C.Y. "Forced Convection in a Porous Channel with Discrete Heat Sources" ASME Journal of Heat Transfer 123, 404 - 407. 2000. 
Das, S.P. "Slow Steady Flow of a Viscous Liquid in an Annulus with Uniform Arbitrary Injection and Suction Velocities along the Walls" ASME Journal of Applied Mechanics 33, $668-673.1966$.

Das, S.S., Tripathy, U.K., Das, J.K., Sahoo, S.K., and Mishra, S. "Magnetohydrodynamic Unsteady Flow of a Viscous Stratified Fluid through a Porous Medium Past a Porous Flat Moving Plate in the Slip Flow Regime with Heat Source" Far East Journal of Mathematics and Science 29, 71 - 88. 2008.

Das, S.S. "Effect of Suction and Injection on MHD Three Dimensional Couette Flow and Heat Transfer through a Porous Medium" Journal of Naval Architecture and Marine Engineering 41 - 51, June, 2009a.

Das, S.S., Satapathy, A., Das, J.K., and Panda, J.P. "Mass Transfer Effects on MHD Flow and Heat Transfer Past a Vertical Porous Plate through a Porous Medium under Oscillatory Suction and Heat Source" International Journal of Heat and Mass Transfer 52, $5962-5969.2009 b$.

Das, S.S., Tripathy, U.K., and Das, J.K. "Hydromagnetic Convective Flow Past a Vertical Porous Plate through a Porous Medium with Suction and Heat Source" International Journal of Energy Environment 1, 467 - 478. 2010.

Das, S.S., Mishra, L.K., and Mishra, P.K. "Effect of Heat Source on MHD Free Convection Flow Past an Oscillating Porous Plate in the Slip Flow Regime" International Journal of Energy and Environment 2, 945 - 952. 2011. 
Das, S.S., Mohanty, M., Panigrahi, S.K., Padhy, R.K., and Sahu, M. "Radiative Heat and Mass Transfer Effects on Natural Convection Couette Flow through a Porous Medium in the Slip Flow Regime” International Journal of Renewable Energy Technology Research 1, 1 - 14. 2012.

Dauenhauer, E.C. and Majdalani, J. "Exact Self-Similarity Solution of the Navier-Stokes Equations for a Porous Channel with Orthogonally Moving Walls" Physics of Fluids 15, 1485 - 1495. 2003.

Davis, A.M.J. and Ethier, C.R. "Transport through Materials Bounded by Porous Surfaces" Chemical Engineering Science 48, 1655 - 1663. 1993.

Davis, A.M.J. and James, D.F. "The Slip Velocity at the Edge of a Porous Medium: Effects of Interior Resistance and Interface Curvature” Transport in Porous Media 53, 175 - 196. 2003.

Davis, H.R. "Laminar Mass Transfer from Porous Tubes and Flat Plates with Wall Resistance” Applied Scientific Research 34, 127 - 143. 1978.

Debreceni, M.J., Lay, W.D., Kuo, T.K., Bond, D.L., McClellan, R.E., and Yeh, T.P. "Design and Development of the Intelsat VIIA and N-Star Propellant Tanks" AIAA-95-2527, $31^{\text {st }}$ Joint Propulsion Conference, San Diego, CA, July $10-12$, 1995a.

Debreceni, M.J., Lay, W.D., Newell, J.M., Jaekle, D.J., and Benard, I.J. "Design and Development of a Communications Satellite Propellant Tank” AIAA-95-2529, $31^{\text {st }}$ Joint Propulsion Conference, San Diego, CA, July 10 - 12, 1995 b. 
Debreceni, M.J., Lay, W.D., Jaekle, D.E., and Graffer, A.C. "Design and Development of the AXAF-IPS PMD and PMD Integration", AIAA-97-2812, 33 ${ }^{\text {rd }}$ Joint Propulsion Conference, Seattle, WA, July 6-9, 1997.

Debreceni, M.J., Lay, W.D., and Jaekle, D.J. “Design and Development of a PMD-Type Bipropellant Tank" AIAA-98-3200, 34 ${ }^{\text {th }}$ Joint Propulsion Conference, Cleveland, OH, July $13-15,1998$.

Debreceni, M.J., Lay, W.D., Kuo, T.K., Jaekle, D.E., and Seki, T. "Propellant Tank for an Advanced Communications Satellite", AIAA-2001-3826, 37 $7^{\text {th }}$ Joint Propulsion Conference and Exhibit, Salt Lake City, UT, July 8 - 11, 2001.

Debreceni, M.J., Kuo, T.K., and Jaekle, D.E. “Development of a Titanium Propellant Tank", AIAA-2003-4604, 2003.

Debreceni, M.J., Kuo, T.K., and Jaekle, D.E. “Development of a Composite Wrapped Propellant Tank", AIAA-2004-3505, 40 ${ }^{\text {th }}$ Joint Propulsion Conference, Ft. Lauderdale, FL, July 11 - 14, 2004.

DeBrock, S.C. “Surface Tension Devices for Management of Space Propulsion System Propellants" SAE Aerospace Systems Conference, Los Angeles, CA, June 27 30, 1967.

DeBrock, S.C. "Spacecraft Capillary Propellant Retention and Control for Long-Life Missions" AIAA-68-465, $2^{\text {nd }}$ Communications Satellite Systems Conference, San Francisco, CA, April 8 - 10, 1968. 
DeBrock, S.C., Grove, R.K., Sloma, R.O., Balzer, D.L., Brill, Y., and Yankura, G.A. “A Survey of Current Developments in Surface Tension Devices for Propellant Acquisition” Journal of Spacecraft and Rockets 8, 83 - 98. 1971.

DeBrock, S.C. and Grove, R.K. “Capillary Propellant Management for Integrated Primary and Secondary Propulsion Systems" AIAA-74-1153, $10^{\text {th }}$ Propulsion Conference, San Diego, CA, October 21 - 23, 1974.

DeBrock, S.C. and Grove, R.K. “Capillary Propellant Management for Integrated Primary and Secondary Propulsion Systems” Journal of Spacecraft 12, 261 - 270. 1975.

Debruge, L.L. and Han, L.S. "Heat Transfer in a Channel with Porous Wall for Turbine Cooling Application” ASME Journal of Heat Transfer 94, 385 - 390. 1972.

Decket, M. “ORBCOMM - A Description and Status of the LEO Satellite Mobile Data Communication System” AIAA-94-1135-CP, 1994.

DeFelice, D.M. “Cryogenic Fluid Management Flight Experiment” NASA-N87-21150. 1987.

Dempsey, P.J. and Fabik, R.H. "Using Silicon Diodes for Detecting the Liquid-Vapor Interface in Hydrogen" NASA-TM-105541, $38^{\text {th }}$ International Instrumentation Symposium, Las Vegas, NV, April 26 - 30, 1992.

Deng, C. and Martinez, D.M. "Linear Stability of a Berman Flow in a Channel Partially Filled with a Porous Medium” Physics of Fluids 17, 024102, 2005a. 
Deng, C. and Martinez, D.M. "Viscous Flow in a Channel Partially Filled with a Porous Medium and with Wall Suction" Chemical Engineering Science 60, 329 - 336. $2005 b$.

Deng, Z., Adrian, R.J., and Tomkins, C.D. "Structure of Turbulence in Channel Flow with a Fully Transpired Wall” AIAA-2001-1019, 39 ${ }^{\text {th }}$ Aerospace Sciences Meeting, Reno, NV, January 8 - 11, 2001.

DeWitt, R.L. and McIntire, T.O. "Pressurant Requirements for Discharge of Liquid Methane from a 1.52-meter- (5-ft-) Diameter Spherical Tank Under Both Static and Slosh Conditions" NASA-TN-D-7638. 1974.

Dickens, K. Personal Correspondence, January 27, 2010.

DiFrancesco, A., and Boorady, F. "The Agena Rocket Engine Story" AIAA 89-2390, $25^{\text {th }}$ Joint Propulsion Conference, Monterey, CA, July 10 - 12, 1989.

Dinarvand, S., Rashidi, M.M., and Doosthoseini, A. "Analytical Approximate Solutions for Two-Dimensional Viscous Flow through Expanding or Contracting Gaps with Pearmeable Walls" Central European Journal of Physics 7, 791 - 799. 2009.

DiPirro, M.J. "Fluid Acquisition System for Superfluid Helium” Cryogenics 29, 517 $522,1989$.

DiPirro, M.J. "Liquid Acquisition Devices for Superfluid Helium Transfer" Cryogenics 30, $193-199.1990$.

DiPirro, M.J., Schein, M.E., Boyle, R.F., Figueroa, O., and Lindauer, D.A. “The SHOOT Cryogenic Components: Testing and Applicability to Other Flight Programs" 
SPIE Volume 1340 - Cryogenic Optical Systems and Instruments IV, $291-302$.

1990.

DiPirro, M.J., Shirron, P.J., Volz, S.M., and Schein, M.E. "SHOOT Performance Testing” Advances in Cryogenic Engineering 37B, 1229 - 1236. Plenum Press: New York, 1992.

Dipprey, N.F. and Rotenberger, S.J. "Orbital Express Propellant Resupply Servicing” AIAA-2003-4898, $39^{\text {th }}$ Joint Propulsion Conference, Huntsville, AL, July 20 - 23, 2003.

DiStefano, E., Cady, E.C., and Rangel, R.H. "Method of Filling Screen Liquid Acquisition Devices in Low Gravity" Journal of Spacecraft and Rockets 31, 1099 - 1106. 1994.

Doche, O. and Tardu, S. "Mechanism of Wall Transfer under Steady Localized Blowing" International Journal of Heat and Mass Transfer 55, 1574 - 1581. 2012.

Dodge, F.T. and Bowles, E.B. "Study of Vapor Flow into a Capillary PropellantAcquisition Device" NASA-CR-167883, Southwest Research Institute, San Antonio, TX, May-June, 1984.

Dodge, F.T. "The New Dynamic Behavior of Liquids in Moving Containers" Southwest Research Institute, San Antonio, TX, 2000.

Dolecek, P., Mikulasek, P., and Belfort, G. "The Performance of a Rotating Filter 1. Theoretical Analysis of the Flow in an Annulus with a Rotating Inner Porous Wall” Journal of Membrane Science 99, 241 - 248. 1995. 
Dominick, S.M. and Tegart, J.R. "Low-G Propellant Transfer Using Capillary Devices" AIAA-81-1507, $17^{\text {th }}$ Joint Propulsion Conference, Colorado Springs, CO, July 27 $-29,1981$.

Dominick, S. and Driscoll, S., "Fluid Acquisition and Resupply Experiment (FARE I) Flight Results" AIAA-93-2424, 29 ${ }^{\text {th }}$ Joint Propulsion Conference, Monterey, CA, June $28-30,1993$.

Dominick, S.M. and Tegart, J.R. “Orbital Test Results of a Vaned Liquid Acquisition Device” AIAA-1994-3027, 30 ${ }^{\text {th }}$ Joint Propulsion Conference, Indianapolis, IN, June $27-29,1994$.

Dominick, S.M. "Design, Development, and Flight Performance of the Mars Global Surveyor Propulsion System", AIAA-99-2176, 35 ${ }^{\text {th }}$ Joint Propulsion Conference, Los Angeles, CA, June 20 - 24, 1999.

Dominick, S.M., Tegart, J.R., Driscoll, S.L., Sledd, J.D., Hastings, L.J. “Fluid Acquisition and Resupply Experiments on Space Shuttle Flights STS-53 and STS57” NASA-TP-2011-216465, 2011.

Dortmund Data Bank Software Package (DDBSP), accessed 05-01-2011.

Doshi, M.R., Dewan, A.K., and Gill, W.N. "The Effect of Concentration Dependent Viscosity and Diffusivity on Concentration Polarization in Reverse Osmosis Flow Systems” AIChE Symposium Series, Water 68, 323 - 339. 1971.

Doshi, M.R. and Gill, W.N. "Turbulent Flow in a Tube with Wall Suction" ASME Journal of Heat Transfer 96, 251 - 252. 1974. 
Dougan, L., Bates, S.P., Hargreaves, R., Fox, J.P., Crain, J., Finney, J.L., Reat, V., and Soper, A.K. "Methanol-Water Solutions: A Bi-Percolating Liquid Mixture" Journal of Chemical Physics 121, 6456 - 6462. 2004.

Doughty, J.R. and Perkins, H.C. "Hydrodynamic Entry Length for Laminar Flow between Parallel Porous Plates" ASME Journal of Applied Mechanics 37, 548 550. 1970.

Doughty, J.R. "Heat and Momentum Transfer between Parallel Porous Plates" PhD Thesis, University of Arizona. 1971.

Doughty, J.R. and Perkins, H.C. "The Thermal Entry Problem for Laminar Flow between Parallel Porous Plates" ASME Journal of Heat Transfer 93, 476 - 478. 1971.

Doughty, J.R. and Perkins, H.C. "Thermal and Combined Entry Problems for Laminar Flow between Parallel Porous Plates" ASME Journal of Heat Transfer 94, 233 234. 1972.

Doughty, J.R. and Perkins, H.C. "Variable Properties Laminar Gas Flow Heat Transfer in the Entry Region of Parallel Porous Plates" International Journal of Heat and Mass Transfer 16, 663 - 668. 1973.

Doughty, J.R. "Parallel Porous Plate Channel Flow Characteristics Resulting from Nonuniform Entry Velocity Profiles" ASME Journal of Fluids Engineering 97, 78 $-81.1975$. 
Dowdy, M.W. and De Brock, S.C. "Selection of a Surface-Tension Propellant Management System for the Viking 75 Orbiter" Journal of Spacecraft 10, 549 558. 1973.

Dowdy, M.W., Hise, R.E., and Peterson, R.G. "Development and Qualification of the Propellant Management System for the Viking 75 Orbiter" Journal of Spacecraft 14, 133 - 140. 1977.

Drake, R.L., Molz, F.J., Remson, I., and Fungaroll, A.A. "Similarity Approximation for the Radial Subsurface Flow Problem" Water Resources Research 5, 673 - 684. 1969.

Drelich, J., Miller, J.D., and Good, R.J. “The Effect of Drop (Bubble) Size on Advancing and Receding Contact Angles for Heterogeneous and Rough Solid Surfaces as Observed with Sessile-Drop and Captive-Bubble Techniques" Journal of Colloid and Interface Science 179, 37 - 50. 1996.

Ducret, E., Arnaud, R., and Rigollet, R. "Design and Development of the EUROSTAR 2000+ Propellant Tank" AIAA-96-3289, $32^{\text {nd }}$ Joint Propulsion Conference, Lake Buena Vista, FL, July 1 - 3, 1996.

Dupuy, M., Radenac, E., Fabignon, Y., and Plourde, F. "Large Eddy Simulations of Channel Flow with Fluid Injection through a Porous Wall" AIAA-2011-0288, $49^{\text {th }}$ Aerospace Sciences Meeting, Orlando, FL, January 4 - 7, 2011.

Dybbs, A. and Edwards, R.V. "An Index Matched Flow System for Measurements of Flow in Complex Geometries" Laser Anemometry in Fluid Mechanics, 2, 1984. 
Eberhardt, R.N. and Fester, D.A. "Shuttle Compatible Cryogenic Liquid Storage and Supply Systems" AIAA-81-1509, 17 $7^{\text {th }}$ Joint Propulsion Conference, Colorado Springs, CO, July $27-29,1981$.

Eberhardt, R.N., Bailey, W.J., and Fester, D.A. “Cryogenics Fluid Management Experiment" NASA CR-165495, October, 1981.

Eberhardt, R.N., Gille, J.P., Bailey, W.J., and Berry, R.L. “Cryogenic Fluid Management Facility Concept Definition Study” NASA-CR-174630. 1984.

Eegunjobi, A.S. “Analysis of Laminar Flow, Thermal Stability, and Entropy Generation in Porous Channel” Thesis, Cape Peninsula University of Technology, South Africa, 2013.

Elbashbeshy, E.M.A. and Bazid, M.A.A. "Heat Transfer in a Porous Medium over a Stretching Surface with Internal Heat Generation and Suction or Injection” Applied Mathematics and Computation 158, 799 - 807. 2004.

Enright, P.J., and Wong, E.C. "Propellant Slosh Models for the Cassini Spacecraft", AIAA-94-3730-CP, 1994.

Erdogan, M.E. "The Effects of Side Walls on Axial Flow in Rectangular Ducts with Suction and Injection” Acta Mechanica 162, 157 - 166. 2003.

Erdogan, M.E. and Imrak, C.E. "On the Axial Flow of an Incompressible Viscous Fluid in a Pipe with a Porous Boundary” Acta Mechanica 178, 187 - 197. 2005.

Erdogan, M.E. and Imrak, C.E. "On the Flow in a Uniformly Porous Pipe” International Journal of Non-Linear Mechanics 43, 292 - 301. 2008. 
Ergun, S. "Fluid Flow through Packed Columns" Chemical Engineering Progress 48, 89 -94. 1952.

Eroshenko, V.M., Ermakov, A.L., Klimov, A.A., Motulevich, V.P., and Terent'ev, Y.N. "Influence of Large Injection on Flow Stability and the Transition to Turbulent Flow" Thermophysical Properties and Gas Dynamics of High-Temperature Media, 56 - 64, Moscow, Russia, 1972.

Eroshenko, V.M., Zaichik, L.I., and Rabovskii, V.B. "Heat Exchange in Tubes with Permeable Walls in the Presence of Internal Heat Sources" Journal of Engineering Physics 38, 222 - 227. 1980.

Eroshenko, V.M., Zaichik, L.I., and Rabovski, V.B. "Stability of Fluid Flow in a Plane Channel with Uniform Injection or Suction through Porous Walls" Journal of Engineering Physics 41, 957 - 960. 1981a.

Eroshenko, V.M., Ershov, A.V., and Zaichik, L.I. “Turbulent Fluid Flow in a Circular Pipe with Uniform Blowing through Porous Walls" Journal of Engineering Physics 41, 1175 - 1178. 1981b.

Eroshenko, V.M., Ershov, A.V., and Zaichik, L.I. “Calculation of Fully Developed Turbulent Flow in a Tube with Injection and Suction" High Temperature Science $19,80-85.1981 \mathrm{c}$

Eroshenko, V.M., Ershov, A.V., and Zaichik, L.I. "Calculation of Turbulent Flow of an Incompressible Fluid in a Circular Tube with Suction through Porous Walls" Fluid Dynamics 17, 559 - 564. 1982. 
Eroshenko, V.M., Zaichik, L.I., and Rabovskii, V.B. "Flow Stability in a Plane-Parallel Channel with One Permeable Wall” Journal of Engineering Physics 46, 642 644. 1984.

Eroshenko, V.M., Ershov, A.V., and Zaichik, L.I. "Effect of Variability of Physical Properties of a Gas on Turbulent Flow and Heat Transfer in a Pipe with Permeable Walls” Journal of Engineering Physics 50, 135 - 139. 1986.

Fainerman, V.B., Miller, R., and Joos, P. “The Measurement of Dynamic Surface Tension by the Maximum Bubble Pressure Method" Colloid and Polymer Science 272, $731-739.1994$.

Fan, L., Yuan, X., Zhou, C., Zeng, A., Yu, K., Kalbassi, M., and Porter, K. “Contact Angle of Ethanol and n-Propanol Aqueous Solutions on Metal Surfaces” Chemical Engineering Technology 34, 1535 - 1542. 2011.

Fang, T. "A Note on the Incompressible Couette Flow with Porous Walls" International Communications in Heat and Mass Transfer 31, 31 - 41. 2004a.

Fang, T. "Further Discussion on the Incompressible Pressure in a Channel with Porous Walls" International Communications in Heat and Mass Transfer 31, 487 - 500. 2004b.

Feraille, T. and Casalis, G. "Channel Flow Induced by Wall Injection of Fluid and Particles" Physics of Fluids 15, 348 - 360. 2003.

Ferguson, A. and Kennedy, S.J. "Free and Total Surface Energies and Related Quantities” Transactions of the Faraday Society 32, 1474 - 1481. 1936. 
Ferro, S. and Gnavi, G. "Spatial Stability of Similarity Solutions for Viscous Flows in Channels with Porous Walls" Physics of Fluids 12, 797 - 802. 2000.

Ferro, S. and Gnavi, G. "Effects of Temperature-Dependent Viscosity in Channels with Porous Walls" Physics of Fluids 14, 839 - 849. 2002.

Fester, D.A., Eberhardt, R.N., and Tegart, J.R. "Space Shuttle Reaction Control Subsystem Propellant Acquisition" AIAA-74-1106, 10 ${ }^{\text {th }}$ Joint Propulsion Conference, San Diego, CA, October 21 - 23, 1974.

Fester, D.A., Villars, A.J., and Uney, P.E. "Surface Tension Propellant Acquisition System Technology for Space Shuttle Reaction Control Tanks" AIAA-75-1196, $11^{\text {th }}$ Propulsion Conference, Anaheim, CA, September 29 - October 1, 1975.

Fikes, J., Howell, J.T., and Henley, M. “In-Space Cryogenic Propellant Depot (ISCPD) Architecture Definitions and Systems Studies" AIAA-2006-178, IAC-06-D3.3.08. 2006.

Forchheimer, P. “Wasserbewegung Durch Boden” Forschtlft ver. D. Ing. 45, 1782 1788. 1901.

Fournier, C., Bataille, F., and Michard, M. "Heat Transfer in a Laminar Channel Flow Generated by Injection through Porous Walls" ASME Journal of Fluids Engineering 129, 1048 - 1057. 2007.

Frank, D. "Dynamics of Superfluid Helium in Low-Gravity” NASA-CR-204755, June, 1997. 
Franson, J.H.M. and Alfredsson, P.H. “On the Hydrodynamic Stability of Channel Flow with Cross Flow" Physics of Fluids 15, 436 - 441. 2003.

Fredrickson, G.O. and Schweikle, J.D. "Thermo and Hydrodynamic Experiment Research Module in Orbit” NASA-CR-85271. 1967a.

Fredrickson, G.O. and Schweikle, J.D. "Project Thermo - Phase B Prime" NASA-CR88712. $1967 b$.

Friedman, M. and Gillis, J. "Viscous Flow in a Pipe with Absorbing Walls" ASME Journal of Applied Mechanics 34, 819 - 822. 1967.

Fries, N., Odic, K., and Dreyer, M. "Wicking of Perfectly Wetting Liquids into a Metallic Mesh" Proceedings of the $2^{\text {nd }}$ International Conference on Porous Media and its Applications in Science and Engineering, Kauai, HI, June 17 - 21, 2007.

Fritz, W. "Berechnung des Maximalvolumens von Dampfblasen” Physikalische Zeitschrift 36, 379-384. 1936.

Fuks, S. and Bellemans, A. "The Surface Tension of Krypton, Methane, and Their Mixtures" Physica 32, $594-602.1966$.

Gaines, R.D. and Orton, G.F. "Recent Developments in Propellant Acquisition Technology" AIAA-84-1477, 20 ${ }^{\text {th }}$ Joint Propulsion Conference, Cincinnati, OH, June 11-13, 1984.

Galowin, L.S. and Desantis, M.J. "Theoretical Analysis of Laminar Pipe Flow in a Porous Wall Cylinder" Journal of Dynamic Systems, Measurement, and Control 93, $102-108.1971$. 
Galowin, L.S., Fletcher, L.S., and DeSantis, M.J. "Investigation of Laminar Flow in a Porous Pipe with Variable Wall Suction” AIAA Journal 12, 1585 - 1589. 1974.

Ganesh, S. and Krishnambal, S. "Magnetohydrodynamic Flow of Viscous Fluid between Two Parallel Porous Plates" Journal of Applied Sciences, Asian Network for Scientific Information 6. 2006.

Gardner, J.P., Mather, J.C., Clampin, M., Doyon, R., Greenhouse, M.A., Hammel, H.B., Hutchings, J.B., Jakobsen, P., Lilly, S.J., Long, K.S., Lunine, J.I., McCaughrean, M.J., Mountain, M., Nella, J., Rieke, G.H., Rieke, M.J., Rix, H-W., Smith, E.P., Sonneborn, G., Stiavelli, M., Stockman, H.S., Windhorst, R.A., and Wright, G.S. “The James Webb Space Telescope” Space Science Reviews 123, Issue 4, 485 606. 2006.

Garrison, T.P., Ince, M., Pizzicaroli, J., and Swan, P.A. “System Engineering Trades for the IRIDIUM Constellation” Journal of Spacecraft and Rockets 34, 675 - 680, 1997.

Gauglitz, P.A. and Radke, C.J. "The Dynamics of Liquid Film Breakup in Constricted Cylindrical Capillaries” Journal of Colloid and Interface Science 134, 14 - 40. 1990.

Geraldes, V., Semiao, V., and Pinho, M.N. "Numerical Modelling of Mass Transfer in Slits with Semi-Permeable Membrane Walls” Engineering Computations 17, 192 $-217.2000$. 
Giacalone, P.L. "Detail Design of the Surface Tension Propellant Management Device for the Intelsat VII Communication Satellite" AIAA-93-1802, $29^{\text {th }}$ Joint Propulsion Conference, Monterey, CA, June 28 - 30, 1993.

Gill, W.N., Tien, C., and Zeh, D.W. "Concentration Polarization Effects in a Reverse Osmosis System" Industrial and Engineering Chemistry Fundamentals 4, 433 439. 1965.

Gille, J.P., Martin, T.A., and McIntosh, G.E. "Fluid System Design for a Superfluid Helium Space Tanker" AIAA-89-0586, Aerospace Sciences Meeting, Reno, NV, January $9-12,1989$.

Gilmore, W. "Supplying Cryogenic Propellants for Space Based OTV” AIAA-85-1225, $21^{\text {st }}$ Joint Propulsion Conference, Monterey, CA, July 8 - 10, 1985.

Glover, D. "NASA Cryogenic Fluid Management Space Experiment Efforts" NASA-TM103752. 1991.

Goebel, D.M., Martinez-Lavin, M., Bond, T.A., and King, A.M. "Performance of XIPS Electric Propulsion in On-orbit Station Keeping of the Boeing 702 Spacecraft" AIAA-2002-4348, 38 $8^{\text {th }}$ Joint Propulsion Conference and Exhibit, Indianapolis, IN, July $7-10,2002$.

Goff, J., Kutter, B.F., Zegler, F., Bienhoff, D., Chandler, F., and Marchetta, J. "Realistic Near-Term Propellant Depots: Implementation of a Critical Spacefaring Capability" AIAA-2009-6757, 2009. 
Good, R.J. "Surface Free Energy of Solids and Liquids: Thermodynamics, Molecular Forces, and Structure" Journal of Colloid and Interface Science 59, 398 - 419. 1977.

Gorton, C.W. "Laminar Flow in a Uniformly Porous Channel" ASME Journal of Applied Mechanics 26, $469-470.1959$.

Goto, M. and Uchida, S. "Unsteady Flow in a Semi-Infinite Expanding Pipe with Injection through Wall" Journal of the Japanese Society for Aeronautical and Space Science 38, 131 - 138. 1980.

Govindarajulu, T. "Couette Flow in Hydromagnetics with Time-Dependent Suction" Indian Journal of Pure Applied Mathematics 9, 1359 - 1364. 1978.

Graham, D.R. and Higdon, J.J.L. "Oscillatory Forcing of Flow through Porous Media. Part 1. Steady Flow" Journal of Fluid Mechanics 465, 213 - 235. 2002a.

Graham, D.R. and Higdon, J.J.L. "Oscillatory Forcing of Flow through Porous Media. Part 2. Unsteady Flow" Journal of Fluid Mechanics 465, 237 - 260. $2002 \mathrm{~b}$.

Granger, J., Dodds, J., Leclerc, D., and Midoux, N. "Flow and Diffusion of Particles in a Channel with One Porous Wall: Polarization Chromatography" Chemical Engineering Science 41, 3119 -3128. 1986.

Gravlee, M, Vera, C., Wollen, M., McLean, C., and Walls, L. "Micro-gravity Cryogenic Experiment Opportunity” AIAA-2010-8838, 2010 Space Conference, Anaheim, CA, August 30 - September 2, 2010. 
Green, G. "Laminar Flow through a Channel with One Porous Wall” Course Project in Advanced Fluid Mechanics, Department of Chemical and Environmental Engineering, RPI, Troy, NY, 1979.

Griffin, P.S., Ballinger, I.A., Jaekle, D.E., and Jackson, A.C. "Design and Manufacture of a Lightweight Fuel Tank Assembly" AIAA-2003-4606, 39 ${ }^{\text {th }}$ AIAA Propulsion Conference, Huntsville, AL, July 21, 2003.

Griffond, J. and Casalis, G. "On the Dependence on the Formulation of Some Nonparallel Stability Approaches Applied to the Taylor Flow" Physics of Fluids $12,466-468.2000$.

Griffond, J. and Casalis, G. "On the Nonparallel Stability of the Injection Induced TwoDimensional Taylor Flow" Physics of Fluids 13, 1635 - 1644. 2001.

Gude, M. and Teja, A.S. "Vapor-Liquid Critical Properties of Elements and Compounds. 4. Aliphatic Alkanols" Journal of Chemical Engineering Data 40, 1025 - 1036. 1995.

Guernsey, C.S., Baker, R.S., Plachta, D., and Kittel, P. “Cryogenic Propulsion with Zero Boil-Off Storage Applied to Outer Planetary Exploration" AIAA-2005-3559, 41 ${ }^{\text {st }}$ Joint Propulsion Conference, Tucson, AZ, July 10 - 13, 2005.

Gulab, R. and Mishra, R. "Unsteady Flow through Magnetohydrodynamic Porous Media" Indian Journal of Pure Applied Mathematics 8, 637 - 642. 1977. 
Gupta, B.K. and Levy, E.K. "Laminar Flow in Annuli and Flat Plate Channels with Mass Transfer at One Wall" Thermophysics and Heat Transfer Conference Boston, MA, July $15-17.1974$.

Gupta, B.K. and Levy, E.K. "Symmetrical Laminar Channel Flow with Wall Suction" ASME Journal of Fluids Engineering 98, 469 - 474. 1976.

Gupta, P.S. and Gupta, A.S. "Heat and Mass Transfer on a Stretching Sheet with Suction or Blowing” The Canadian Journal of Chemical Engineering 55, 744 - 746. 1977.

Gupte, S.K. and Advani, S.G. "Flow Near the Permeable Boundary of a Porous Medium" An Experimental Investigation Using LDA” Experiments in Fluids 22, 408 - 422. 1997.

Guria, M., Ghosh, S.K., and Pop, I. "Three-Dimensional Free Convection Flow in a Vertical Channel Filled with a Porous Medium” Journal of Porous Media 12, 985 $-995.2009$.

Hadim, A. "Forced Convection in a Porous Channel with Localized Heat Sources" ASME Journal of Heat Transfer 116, 465 - 472. 1994.

Hafeez, H.Y. and Ndikilar, C.E. "Flow of Viscous Fluid between Two Parallel Porous Plates with Bottom Injection and Top Suction” Progress in Physics 10, 49 - 51. 2014.

Hahn, S., Jongdoo, J., and Choi, H. "Direct Numerical Simulation of Turbulent Channel Flow with Permeable Walls" Journal of Fluid Mechanics 450, 259 - 285. 2002. 
Haji-Sheikh, A., Minkowycz, W.J., and Sparrow, E.M. “Green's Function Solution of Temperature Field for Flow in Porous Passages" International Journal of Heat and Mass Transfer 47, 4685 - 4695. 2004a.

Haji-Sheikh, A., Minkowycz, W.J., and Sparrow, E.M. “A Numerical Study of the Heat Transfer to Fluid Flow through Circular Porous Passages" Numerical Heat Transfer, Part A: Applications 46, 929 - 955. 2004b.

Haji-Sheikh, A., Sparrow, E.M., Minkowycz, W.J. “Heat Transfer to Flow through Porous Passages using Extended Weighted Residuals Method - A Green's Function Solution” International Journal of Heat and Mass Transfer 48, 1330 1349. 2005.

Haji-Sheikh, A., Nield, D.A., and Hooman, K. "Heat Transfer in the Entrance Region for Flow through Rectangular Porous Passages" International Journal of Heat and Mass Transfer 49, 3004 - 3015. 2006.

Haldenwang, P. “Laminar Flow in a Two-Dimensional Plane Channel with Local Pressure-Dependent Crossflow" Journal of Fluid Mechanics 593, 463 - 473. 2007.

Haldenwang, P., Guichardon, P., Chiavassa, G., and Ibaseta, N. "Exact Solution to Mass Transfer in Berman Flow: Application to Concentration Polarization Combined with Osmosis in Crossflow Membrane Filtration” International Journal of Heat and Mass Transfer 53, 3898 - 3904. 2010. 
Hamza, E.A. "Suction and Injection Effects on a Similar Flow between Parallel Plates" Journal of Applied Physics D: Applied Physics 32, 656 - 663. 1999.

Hansen, R.S. "Thermodynamics of Interfaces between Condensed Phases" Journal of Physical Chemistry 66, 410 - 415. 1962.

Hassan, Y.A., Blanchat, T.K., and Seeley, C.H. "PIV Flow Visualization using Particle Tracking Techniques" Measurements, Science, and Technology 3, 633-642. 1992.

Hastings, L.J., Bolshinskiy, L.G., Schunk, R.G., Martin, A.K., Eskridge, R.H., Hamill, B.D., Gomez, C.F., Frenkel, A., Grayson, G., and Pendleton, M.L. "Thermal Integration of a Liquid Acquisition Device into a Cryogenic Feed System" NASATP-2011-216474, 2011.

Havstad, M.A. and Burns, P.J. "Convective Heat Transfer in Vertical Cylindrical Annuli Filled with a Porous Medium" International Journal of Heat and Mass Transfer $25,1755-1766.1982$.

Hayat, T., Naz, R., and Abbasbandy, S. "Poiseuille Flow of a Third Grade Fluid in a Porous Medium" Transport in Porous Media 87, 355 - 366. 2011.

Heald, D.A. and Merino, F. "OTV Orbital Tanking Systems" AIAA-79-1262, $15^{\text {th }}$ Joint Propulsion Conference, Las Vegas, NV, June 18 - 20, 1979.

Heath, C.A., Belfort, G., Hammer, B.E., Mirer, S.D., and Pimbley, J.M. "Magnetic Resonance Imaging and Modeling of Flow in Hollow-Fiber Bioreactors" AIChE Journal 36, $547-558.1990$. 
Heckman, B.R. "Bubble Point Characteristics of Multi-Layer Screen Elements" $M D C$ 02656, McDonnell Douglas Astronautics Company, Huntington Beach, CA, December 1971.

Heffner, K. and Davidson, G. "Performance as Promised: How the Chandra X-ray Observatory Accomplished One of NASA's Most Challenging Missions for Billions of Dollars Less than Originally Planned" AIAA-2004-5935, Space 2004 Conference, San Diego, CA, September 28 - 30, 2004.

Hendricks, J.B., Nilles, M.J., and Dingus, M.L. “A Helium-3/Helium-4 Dilution Cryocooler for Operation in Zero Gravity" NASA-CR-183632, 1988.

Hernandez, A., Calvo, J.I., Pradanos, P., and Tejerina, F. "Pore Size Distributions in Microporous Membranes. A Critical Analysis of the Bubble Point Extended Method" Journal of Membrane Science 112, 1 - 12. 1996.

Hess, D.A. and Regnier, W.W. "Design and Performance Verification of a Passive Propellant Management System" AIAA-78-1029, $14^{\text {th }}$ Joint Propulsion Conference, Las Vegas, NV, July 25 - 27, 1978.

Heubush, H. and Pugmire, T.K “Acceptability of Stainless Steel for Nitrogen Tetroxide Propellant Management Devices and Flight Tankage" AIAA-88-3024, $24^{\text {th }}$ Joint Propulsion Conference, Boston, MA, July $11-13,1988$.

Hewitt, R.E., Duck, P.W., and Al-Azhari, M. "Extensions to Three-Dimensional Flow in a Porous Channel” Fluid Dynamics Research 33, 17 - 39. 2003. 
Hibbard, R.R. and Evans, A. "On the Solubilities and Rates of Solution of Gases in Liquid Methane" NASA-TN-D-4701. August, 1968.

Hill, R.J. and Koch, D.L. "Moderate-Reynolds-Number Flow in a Wall-Bounded Porous Medium” Journal of Fluid Mechanics 453, 315 - 344. 2002.

Hines, W.J., Duncan, L.F., Lewin, J.E., and Fettel, B.E. “Apollo SPS Propellant Position Control in Low- and Zero G Environments" SD-67-655 July, 1967.

Hines, W.J. "Capillary Acquisition and Transfer, Tests of Capillary Barrier Materials" North American Rockwell Internal Letter Number SII-193-603-71-093, September 24, 1971.

Hinvi, L.A., Monwanou, A.V., and Orou, J.B.C. "Linear Stability Analysis of Hydromagnetic Couette Flow with Small Injection/Suction through the Modified Orr-Sommerfeld Equation” arXiv:1308.5530 [physics.flu-dyn], 2013a.

Hinvi, L.A., Monwanou, A.V., and Orou, J.B. "Linear Stability Analysis of Fluid Flow between Two Parallel Porous Stationary Plates with Small Suction and Injection" arXiv:1304.7210 [physics.flu-dyn], 2013b.

Ho. S. and Rahman, M.M. "Nozzle Injection Displacement Mixing in a Zero Boil-off Hydrogen Storage Tank” International Journal of Hydrogen Energy 33, 878 888. 2008.

Ho, S. and Rahman, M.M. "Forced Convective Mixing in a Zero Boil-off Cryogenic Storage Tank" International Journal of Hydrogen Energy 37, 10196 - 10209. 2012. 
Holdich, R., Starov, V.M., Prokopovich, P., Njobuenwu, D.O., Rubio, R.G., Zhdanov, S., and Velarde, M.G. "Spreading of Liquid Drops from a Liquid Source” Colloids and Surfaces A: Physicochemical Engineering Aspects 282-283, 247 - 255. 2006.

Hollingsworth, T.P., van Ommering, G., and Kim, D.J. "Evolutionary Enhancement of SS/L's 1300 Bus for Broadband Payloads" AIAA-2002-1929, 20 ${ }^{\text {th }}$ International Communication Satellite Systems Conference, Montreal, Quebec, May 12 - 15, 2002.

Hooman, K. and Gorji-Bandpy, M. "Laminar Dissipative Flow in a Porous Channel Bounded by Isothermal Parallel Plates" Applied Mathematics and Mechanics 26, $587-593.2005$.

Hopkins, R.A. and Mord, A.J. “A Design and Critical Technology Issues for On-Orbit Resupply of Superfluid Helium” Advances in Cryogenic Engineering 35, 321 333. Plenum Press: New York, 1990.

Hornbeck, R.W., Rouleau, W.T., and Osterle, F. "Laminar Entry Problem in Porous Tubes" Physics of Fluids 6, 1649 - 1654. 1963.

Horton, N.A. and Pokrajac, D. "Onset of Turbulence in a Regular Porous Medium: An Experimental Study" Physics of Fluids 21, 045104. 2009.

Horton, T.E. and Yuan, S.W. "Laminar Flow in the Entrance Region of a Porous-Wall Channel” Journal of Applied Scientific Research 14, 233 - 249. 1964. 
Huang, A.T.L., Huang, M.Y.F., Capart, H., and Chen, R.H. "Optical Measurements of Pore Geometry and Fluid Velocity in a Bed of Irregularly Packed Spheres" Experiments in Fluids 45, 309 - 321. 2008.

Huang, C.L. "Applying Quasi-Linearization to the Problem of Flow through an Annulus with Porous Walls of Different Permeability" Applied Scientific Research 29, 145 $-158.1974$.

Huang, C.L. "Laminar Non-Newtonian Fluid Flow in a Porous Annulus" Journal of Mathematical Analysis and Applications 59, 130 - 144. 1977.

Huang, J.C.P. and Yu, H.S. "Pressure Distributions in Porous Ducts of Arbitrary Cross Section" ASME Journal of Fluids Engineering 95, 342 - 348. 1973.

Hunter, D.G., Stockman, H.S., and Long, K.S. “The Role of Science and Operations in the James Webb Space Telescope Mission Development” Space OPS Conference, 2004.

Hwang, G.J., Cheng, Y.C., and Ng, M.L. "Developing Laminar Flow and Heat Transfer in a Square Duct with One-Walled Injection and Suction" International Journal of Heat and Mass Transfer 36, 2429 - 2440. 1993a.

Hwang, G.J., Cheng, Y.C., and Ng, M.L. "Friction Factors and Heat Transfer Correlations for Gaseous Reactant Flow in Fuel Cell Power Modules" Proceedings of the $6^{\text {th }}$ International Symposium on Transport Phenomena in Thermal Engineering 1, 685 - 690. $1993 \mathrm{~b}$. 
Hwang, G.J., Cheng, Y.C., and Chiang, Y.W. “An Experimental Study of Laminar Heat Transfer in a One-Porous-Wall Square Duct with Suction Flow" International Journal of Heat and Mass Transfer 40, 481 - 485. 1997.

Ilias, S. and Govind, R. "Fluid Dynamics of Dilute Suspensions and Fouling of Tubular Membrane Modules” Journal of Membrane Science 39, 125 - 141. 1988.

Incropera, F.P. and DeWitt, D.P. Fundamentals of Heat and Mass Transfer John Wiley and Sons. New York, NY. 1996.

Ingmanson, W.L., Han, S.T., Wilder, H.D., and Myers Jr., W.T. "Resistance of Wire Screens to Flow of Water" TAPPI Journal 44, 47 - 54. 1961.

Ismail, A.M., Ganesh, S., and Kirubhashankar, C.K. "Unsteady MHD Flow between Two Parallel Plates through Porous Medium with One Plate Moving Uniformly and the Other Plate at Rest with Uniform Suction” International Journal of Science, Engineering, and Technology Research 3, 6-10. 2014.

Israel-Cookey, C., Ogulu, A., and Omubo-Pepple, V.B. "Influence of Viscous Dissipation and Radiation on Unsteady MHD Free-Convection Flow Past an Infinite Heated Vertical Plate in a Porous Medium with Time-Dependent Suction” International Journal of Heat and Mass Transfer 46, 2305 - 2311. 2003.

Jackson, G.W. and James, D.F. "The Permeability of Fibrous Porous Media” Canadian Journal of Chemical Engineering 64, 364 - 374. 1986. 
Jaekle, D.E. "Propellant Management Device Conceptual Design and Analysis: Vanes" AIAA-91-2172, $27^{\text {th }}$ Joint Propulsion Conference, Sacramento, CA, June $24-26$, 1991.

Jaekle, D.E. "Propellant Management Device Conceptual Design and Analysis: Sponges" AIAA-93-1970, 29 ${ }^{\text {th }}$ Joint Propulsion Conference, Monterey, CA, June $28-30$, 1993.

Jaekle, D. E. "Propellant Management Device Conceptual Design and Analysis: Traps and Troughs" AIAA-95-2531, $31^{\text {st }}$ Joint Propulsion Conference, San Diego, CA, July $10-12,1995$.

Jaekle, D.E. "Propellant Management Device Conceptual Design and Analysis: Galleries" AIAA 97-2811, 33 ${ }^{\text {rd }}$ Joint Propulsion Conference and Exhibit, Seattle, WA, July $6-9,1997$.

Jaekle, D.J. Personal correspondence in February, 2011.

Jaekle, D.J. Personal correspondence in May, 2013.

Jain, N.C. and Gupta, P. “Three Dimensional Free Convection Couette Flow with Transpiration Cooling” Journal of Zhejiang University SCIENCE A 7, 340 - 346. 2006.

Jain, P.C. and Goel, S. "Flow of a Certain Non-Newtonian Fluid between Two Parallel Plates with Suction and Injection” ZAMM Journal of Applied Mathematics and Mechanics 44, 135 - 136. 1964. 
Jain, R.K. "Stability of Hydromagnetic Flow between Porous Parallel Plates under Transverse Magnetic Field" Proceedings of the National Institute of Sciences of India 35A, $37-43.1967$.

James, D.F. and Davis, A.M.J. "Flow at the Interface of a Model Fibrous Porous Medium" Journal of Fluid Mechanics 426, 47 - 72. 2001.

Jankowski, T.A. and Majdalani, J. "Laminar Flow in a Porous Channel with Large Wall Suction and a Weakly Oscillatory Pressure" Physics of Fluids 14, $1101-1110$. 2002.

Jasper, J.J. "The Surface Tension of Pure Liquid Compounds" Journal of Physical Chemical Reference Data 4, 841 - 1009. 1972.

Jensen, J.E., Stewart, R.B., Tuttle, W.A., Brechna, H., and Prodell, A.G. "Brookhaven National Laboratory Selected Cryogenic Data Notebook” Associated Universities, Inc., August, 1980.

Jeong, J.T. "Slip Boundary Condition on an Idealized Porous Wall” Physics of Fluids 13, $1884-1890.2001$.

Jetley, R.L. and Scarlotti, R.D. “Space Station Experiment Definition: Long-Term Cryogenic Fluid Storage” NASA-CR-1987-4072. 1987.

Jocelyne, G., John, D., and Noel, M. "Laminar Flow in Channel with Porous Walls" The Chemical Engineering Journal 42, 193 - 204. 1989.

Johnson, R.E. Dettre, R.H. “Contact Angle Hysteresis. III. Study of an Idealized Heterogeneous Surface” Journal of Physical Chemistry 68, 1744 - 1750. 1964. 
Johnson, R.E. and Dettre, R.H. "Wettability and Contact Angles" Surface and Colloid Science, 2, 85 - 153. New York: Wiley-Interscience, 1969.

Johnson, W.L., Jurns, J.M., Bamberger, H.H., and Plachta, D.W., "Launch Ascent Testing of a Representative Altair Ascent Stage Methane Tank” Cryogenics, 52, $278-282,2012$.

Johnstone, W., Dybbs, A., and Edwards, R. "Measurement of Fluid Velocity inside Porous Media with a Laser Anemometer” Physics of Fluids 18, 913 - 914. 1975.

Jones, D.P. and Krier, H. “Gas Flow Resistance Measurements through Packed Beds at High Reynolds Numbers” Journal of Fluid Engineering 105, 168 - 172. 1982.

Jorne, J. "Mass Transfer in Laminar Flow Channel with Porous Wall” Journal of the Electrochemistry Society 129, 1727 - 1733. 1982.

Joselyn, J.A., and Grubb, R.N. "The Space Environment Monitors Onboard GOES" AIAA-85-0238, $23^{\text {rd }}$ AIAA Aerospace Sciences Meeting, Reno, NV, January 14 17, 1985.

Jurns, J.M. and Kudlac, M.T. "NASA Glenn Research Center Creek Road Complex Cryogenic Testing Facilities" Cryogenics 46, 98 - 104. 2006.

Jurns, J.M., McQuillen, J.B., Gaby, J.D., and Sinacore, S.A. "Bubble Point Measurements with Liquid Methane of a Screen Channel Capillary Liquid Acquisition Device" NASA-TM-2009-215494, 54 ${ }^{\text {th }}$ JANNAF Propulsion Meeting, Denver, CO, May 14 - 17, 2007. 
Jurns, J.M. and McQuillen, J.B. "Liquid Acquisition Device Testing with Sub-cooled Liquid Oxygen" AIAA-2008-4943, 44 ${ }^{\text {th }}$ Joint Propulsion Conference and Exhibit, Hartford, CT, July $21-23,2008$.

Kale, P.P., Nickelson, R.L., and Sarles, F.W. “A Design for INSAT” AIAA-72-576, $4^{\text {th }}$ Communications Satellite Systems Conference, Washington, D.C., April 24 - 26, 1972.

Kamisli, F. "Laminar Flow of a Non-Newtonian Fluid in Channels with Wall Suction or Injection” International Journal of Engineering Science 44, 650 - 661. 2006.

Kar, M., Dash, G.C., Sahoo, S.N., and Rath, P.K. "Three-Dimensional Free Convection MHD Flow in a Vertical Channel through a Porous Medium with Heat Source and Chemical Reaction” Journal of Engineering Thermophysics 22, 203 - 215. 2013.

Karode, S.K. "Laminar Flow in Channels with Porous Walls, Revisited" Journal of Membrane Science 191, 237 - 241. 2001.

Kashani, A., Wilcox, R.A., Spivak, A.L., Daney, D.E., and Woodhouse, C.E. "SHOOT Flowmeter and Pressure Transducers” Cryogenics 30, 286 - 291. 1990.

Kaviany, M. "Laminar Flow through a Porous Channel Bounded by Isothermal Parallel Plates" International Journal of Heat and Mass Transfer 28, 851 - 858. 1985.

Khan, M., Hayat, T., and Wang, Y. "Slip Effects on Shearing Flows in a Porous Medium” Acta Mechanica Sinica 24, 51 - 59. 2008. 
Khodadadi, J.M. and Kroll, J.T. "Fluid Flow through a Porous Medium Channel with Permeable Walls" ASME Journal of Fluids Engineering 114, 124 - 126. 1992.

Kim, D.J. and Wilson, J.C. "SS/L-1300 Satellite Optimized for Land Launch" AIAA2006-5302, $24^{\text {th }}$ International Communications Satellite Systems Conference, San Diego, CA, June 11 - 14, 2006.

Kim, D.J., Wilson, J., Chiang, J., and Hom, S. "SS/L Historical Trends on Satellite Mass Growth" AIAA-2007-3220, 25 ${ }^{\text {th }}$ International Communications Satellite Systems Conference, Seoul, South Korea, 2007.

Kim, J., Moin, P., and Moser, R. "Turbulent Statistics in Fully Developed Channel Flow at Low Reynolds Number" Journal of Fluid Mechanics 177, 133 - 166. 1987.

King, J.R. and Cox, S.M. “Asymptotic Analysis of the Steady-State and Time-Dependent Berman Problem" Journal of Engineering Mathematics 39, 87 - 130. 2001.

Kinney, R.B. "Fully Developed Frictional and Heat-Transfer Characteristics of Laminar Flow in Porous Tubes" International Journal of Heat and Mass Transfer 11, 1393 $-1401.1968$.

Kinney, R.B. and Sparrow, E.M. “Turbulent Flow, Heat Transfer, and Mass Transfer in a Tube with Surface Suction” ASME Journal of Heat Transfer 92, 117 - 124. 1970.

Kleinstreuer, C. and Paller, M.S. "Laminar Dilute Suspension Flows Plate-and-Frame Ultrafiltration Units" AIChE Journal 29, 529 - 533. 1983.

Kleinstreuer, C. and Belfort, G. "Mathematical Modeling of Fluid Flow and Solute Distribution in Pressure-Driven Membrane Modules" Chapter 5, Synthetic 
Membrane Processes, Fundamentals and Water Applications Orlando, FL: Academic Press, 131 - 190. 1984.

Kohler, J.P. “An Investigation of Laminar Flow through a Porous-Walled Channel” $P h D$ Dissertation, University of Massachusetts, Amherst, 1973.

Korsmeyer, D.J., Landis, R.R., Merrill, R.G., Mazanek, D.D., Falck, R.D., and Adams, R.B. "A Flexible Path for Human and Robotic Space Exploration" 2010 SpaceOps Conference, Huntsville, AL, April 26 - 30, 2010.

Kozinski, A.A., Schmidt, F.P., and Lightfoot, E.N. "Velocity Profiles in Porous-Walled Ducts" Industrial and Engineering Chemistry Fundamentals 9, 503 - 505. 1970.

Kramer, E. "Cryogenic On-Orbit Liquid Depot-Storage Acquisition and Transfer (COLD-SAT) Experiment Conceptual Design and Feasibility Study" NASA-TP3523. 1998.

Kreis, A., Kurz, A., Klein, M., and Deloo, P. "Static and Dynamic Modelling of Diaphragm Tanks" Proceedings of International Conference on Spacecraft Structures, Materials and Mechanical Testing 2, 845-852. 1996.

Kressilk Products "Metal Filter Cloth Technical and Performance Data" Monterey Park, CA, June 23, 1969.

Krummann, W. "The Power Subsystem for the Next Generation GOES Satellite" AIAA2000-2834, $35^{\text {th }}$ Intersociety Energy Conversion Engineering Conference and Exhibit, 2000. 
Kudlac, M.T. and Jurns, J.M. "Screen Channel Liquid Acquisition Devices for Liquid Oxygen" AIAA-2006-5054, 42 ${ }^{\text {nd }}$ Joint Propulsion Conference, Sacramento, CA, July $9-12,2006$.

Kumar, N. and Gupta, S. "MHD Free-Convective Flow of Micropolar and Newtonian Fluids through Porous Medium in a Vertical Channel” Meccanica 47, 277 - 291. 2012.

Kumpel, A., Barros, P., Burg, C., Villeneuve, F., and Mavris, D. “A Conceptual Design for the Space Launch Capability of the Peacekeeper ICBM" AIAA-2002-5854, Aircraft Technology, Integration, and Operations 2002 Technical Forum, Los Angeles, CA, October 1-3, 2002.

Kurtcebe, C. and Erim, M.Z. "Heat Transfer of a Viscoelastic Fluid in a Porous Channel" International Journal of Heat and Mass Transfer 48, 5072 - 5077. 2005.

Kutter, B., Zegler, F., Lucas, S., Hines, L., Ragab, M., Spradley, I., and Hopkins, J. “Atlas Centaur Extensibility to Long-Duration In-Space Applications" AIAA2005-6738. 2005.

Kutter, B., Zegler, F., Sakla, S., Wall, J., Saks, G., Duffey, J., Hopkins, J., and Chato, D.J. “Settled Cryogenic Propellant Transfer” AIAA-2006-4436, 2006.

Kutter, B., Zegler, F., O’Neil, G., and Pitchford, B. “A Practical, Affordable Cryogenic Propellant Depot Based on ULA's Flight Experience" AIAA-2008-7644, 2008 SPACE Conference, San Diego, CA, September 9 - 11, 2008.

Kutter, B. "Propellant Depots Made Simple" FISO Presentation November 10, 2010. 
Kutter, B. Personal correspondence, September 7, 2011.

Kuznetsov, A.V., Xiong, M., and Nield, D.A. “Thermally Developing Forced Convection in a Porous Medium" Circular Duct with Walls at Constant Temperature, with Longitudinal Conduction and Viscous Dissipation Effects" Transport in Porous Media 53, $331-345.2003$.

Kuznetsov, A.V. and Nield, D.A. "Thermally Developing Forced Convection in a Porous Medium Occupied by a Rarefied Gas: Parallel Plate Channel or Circular Tube with Walls at Constant Heat Flux" Transport in Porous Media 76, 345 - 362. 2009.

Kwiatkowski, L.F., Daugherty, M.J., Cornell, C.O., King, M.A., and Riley, P.B. “The MILSTAR System" AIAA-94-1013-CP, 1994.

Lak, T., Rodriguez, H., Chandler, F.O., and Jenkins, D. "Non-toxic Cryogenic Storage for OMS/RCS Shuttle Upgrade" AIAA-98-3818, 34 ${ }^{\text {th }}$ Joint Propulsion Conference, Cleveland, OH, July $12-15,1998$.

Lan, X.K. and Khodadadi, J.M. "Fluid Flow and Heat Transfer through a Porous Medium Channel with Permeable Walls" International Journal of Heat and Mass Transfer 36, $2242-2245.1993$.

Lander, L.M., Siewierski, L.M., Brittain, W.J., and Vogler, E.A. “A Systematic Comparison of Contact Angle Methods" Langmuir 9, 2237 - 2239. 1993.

Langmuir, I. "The Constitution and Fundamental Properties of Solids and Liquids. Part I. Solids" Journal of American Chemical Society 38, 2221 - 2295. 1916. 
Lark, R.F. “Cryogenic Positive Expulsion Bladders” NASA-TM-X-1555. 1968.

Larson, R.E. and Higdon, J.J.L. "Microscopic Flow near the Surface of Two-Dimensional Porous Media. Part 1. Axial Flow" Journal of Fluid Mechanics 166, 449 - 472. 1986.

Larson, R.E. and Higdon, J.J.L. "Microscopic Flow near the Surface of Two-Dimensional Porous Media. Part 2. Transverse Flow" Journal of Fluid Mechanics 178, 119 136. 1987.

Laubengayer, A.W., Ferguson, R.P., Newkirk, A.E. Journal of American Chemical Society 63, $559-561.1941$.

Lauga, E. and Cossu, C. "A Note on the Stability of Slip Channel Flows" Physics of Fluids 17, 088106. 2005.

Laws, E.M. and Livesey, J.L. "Flow through Screens" Annual Review of Fluid Mechanics 10, 247-266. 1978.

Leach, A.E. and Szpakowski, R.J. "Low Cost Titanium Propellant Tankage” AIAA-821228, $18^{\text {th }}$ Joint Propulsion Conference, Cleveland, OH, June 21 - 23, 1982.

Lee, A.Y. and Hanover, G. “Cassini Spacecraft Attitude Control System Flight Performance" AIAA-2005-6269, Guidance, Navigation, and Control Conference, San Francisco, CA, August $15-18,2005$.

Lee, J.H., Ng, Y.S., and Brooks, W.F. “Analytical Study of He II Flow Characteristics in the SHOOT Transfer Line" Cryogenics 28, 81 - 85. 1988. 
Lee, S.I. and No, H.C. "Gravity-Driven Injection Experiments and Direct-Contact Condensation Regime Map for Passive High-Pressure Injection System" Nuclear Engineering and Design 183, 213 - 234. 1998.

Lenahen, B., Desai, M., and Gangadharan, S. “A Computational and Experimental Analysis of Spacecraft Propellant Tanks Implemented with Flexible Diaphragms" AIAA-2013-1886, $54^{\text {th }}$ Structures, Structural Dynamics, and Materials Conference, Boston, MA, April 8 - 11, 2013.

Lessnar, P. and Newman, J. "Hydrodynamics and Mass Transfer in a Porous-Wall Channel" Journal of the Electrochemistry Society 131, 1828 - 1831. 1984.

Li, C. and Peterson, G.P. "The Effective Thermal Conductivity of Wire Screen" International Journal of Heat and Mass Transfer 49, 4095 - 4105. 2006.

Li, D., Ng, C., and Neumann, A.W. "Contact Angles of Binary Liquids and Their Interpretation" Journal of Adhesion Science and Technology 6, 601 - 610. 1992.

Liou, T.M., Lien, W.Y., and Hwang, P.W. "Large-Eddy Simulations of Turbulent Reacting Flows in a Chamber with Gaseous Ethylene Injecting through the Porous Wall” Combustion and Flame 99, 591 - 600. 1994.

Liou, T.M. and Lien, W.Y. "Numerical Simulations of Injection-Driven Flows in a TwoDimensional Nozzleless Solid-Rocket Motor" Journal of Propulsion and Power 11, $600-606.1995$.

Liu, Q. and Prosperetti, A. "Pressure-Driven Flow in a Channel with Porous Walls" Journal of Fluid Mechanics 679, 77 - 100. 2011. 
Lombardi, G., Sparrow, E.M., and Eckert, E.R.G. "Experiments on Heat Transfer to Transpired Turbulent Pipe Flows" International Journal of Heat and Mass Transfer 17, $429-437.1974$.

Lord, D.R. "Spacelab: An International Success Story” NASA-SP-487, 1987.

Lu, C., MacGillivray, A.D., and Hastings, S.P. “Asymptotic Behavior of Solutions of a Similarity Equation for Laminar Flows in Channels with Porous Walls" IMA Journal of Applied Mathematics 49, 139 - 162. 1992.

Lu, C. "On the Existence of Steady Flow in a Channel with One Porous Wall or Two Accelerating Walls" Differential Equations and Computational Simulations III $159-170.1997 \mathrm{a}$.

Lu, C. “On the Asymptotic Solution of Laminar Channel Flow with Large Suction” SIAM Journal of Mathematical Analysis 28, 1113 - 1134. 1997b.

Lu, C. “Asymptotic Solutions of a Nonlinear Equation” Proceedings of the Fourth International Conference on Dynamical Systems and Differential Equations, 590 - 595, Wilmington, NC, May 24 - 27, 2002.

Ludewig, M., Omori, S. and Rao, G.L. "Pressure Drop across Woven Screens Under Uniform and Nonuniform Flow Conditions” NASA-CR-120559, October, 1974.

Macey, R.I. "Pressure Flow Patterns in a Cylinder with Reabsorbing Walls" The Bulletin of Mathematical Biophysics 25, 1 - 9. 1963.

Macey, R.I. "Hydrodynamics in a Renal Tubule" The Bulletin of Mathematical Biophysics 27, 117 - 124. 1965. 
MacGillivray, A.D. and Lu, C. “Asymptotic Solution of a Laminar Flow in a Porous Channel with Large Suction: A Nonlinear Turning Point Problem” Methods and Applications of Analysis 1, 229 - 248. 1994.

Mack, G.L. and Lee, D.A. “The Determination of Contact Angles from Measurements of the Dimensions of Small Bubbles and Drops. II. The Sessile Drop Method for Obtuse Angles” Journal of Physical Chemistry 40, 169 - 176. 1936.

Maddocks, J.R. and Van Sciver, S.W. "Pressure Drop and Helium II Flow through Fine Mesh Screens" Cryogenics 29, 503 - 508. 1989.

Mahmud, S. and Fraser, R.A. "Thermodynamic Analysis of Flow and Heat Transfer Inside a Channel with Two Parallel Plates” Exergy 2, 140 - 146. 2002.

Mahmud, S. and Fraser, R.A. "Flow, Thermal, and Entropy Generation Characteristics Inside a Porous Channel with Viscous Dissipation” International Journal of Thermal Sciences 44, 21 - 32. 2005.

Majdalani, J. and Roh, T.S. “The Oscillatory Channel Flow with Large Wall Injection” Proceedings of the Royal Society of London A: Mathematical, Physical, and Engineering Sciences 456, 1625 - 1657. 2000.

Majdalani, J. "The Oscillatory Channel Flow with Arbitrary Wall Injection” ZAMP Journal of Applied Mathematics and Physics 52, 33 - 61. 2001.

Majdalani, J. and Flandro, G.A. "The Oscillatory Pipe Flow with Arbitrary Wall Injection" Proceedings of the Royal Society of London A: Mathematical, Physical, and Engineering Sciences 458, 1621 - 1651. 2002. 
Majdalani, J., Zhou, C., and Dawson, C.A. “Two-Dimensional Viscous Flow between Slowly Expanding or Contracting Walls with Weak Permeability" Journal of Biomechanics 35, 1399 - 1403. 2002.

Majdalani, J. and Zhou, C. "Moderate-to-Large Injection and Suction Driven Channel Flows with Expanding or Contracting Walls" ZAMM - Journal of Applied Mathematics and Mechanics 83, 181 - 196. 2003.

Majdalani, J. "Multiple Asymptotic Solutions for Axially Travelling Waves in Porous Channels" Journal of Fluid Mechanics 636, 59 - 89. 2009.

Makinde, O.D. "Laminar Flow in a Channel of Varying Width with Permeable Boundaries" Romanian Journal of Physics 40, 403 - 417. 1995.

Makinde, O.D. and Osalusi, E. "MHD Steady Flow in a Channel with Slip at the Permeable Boundaries" Romanian Journal of Physics 51, 319 - 328. 2006.

Malyshenko, S.P. and Dunikov, D.O. "On the Surface Tension Corrections in Nonuniform and Nonequilibrium Liquid-Gas Systems" International Journal of Heat and Mass Transfer 45, 5201 - 5208. 2002.

Manglesh, A. and Gorla, M.G. "MHD Free Convective Flow through Porous Medium in the Presence of Hall Current, Radiation, and Thermal Diffusion" Indian Journal of Pure and Applied Mathematics 44, 743 - 756. 2013.

Manglesh, A., Gorla, M.G., and Chand, K. "Soret and Hall Effect on Heat and Mass Transfer in MHD Free Convective Flow through a Porous Medium in a Vertical 
Porous Channel" Proceedings of the National Academy of Sciences India A: Physical Sciences 84, 63 - 69. 2014.

Mann, J.A., Romero, L., Rye, R.R., and Yost, F.G. "Flow of Simple Liquids down Narrow V Grooves" Physical Review E 52, 3967 - 3972. 1995.

Manna, S.S., Das, S., and Jana, R.N. "Effects of Radiation on Unsteady MHD Free Convective Flow Past an Oscillating Vertical Porous Plate Embedded in a Porous Medium with Oscillatory Heat Flux" Advances in Applied Science Research 3, $3722-3736.2012$

Marquardt, E. D., Le, J. P., and Radebaugh, R. "Cryogenic Material Properties Database” 11th International Cryocooler Conference, Keystone, CO, June 20 - 22, 2000.

Mason, G. and Morrow, N.R. "Coexistence of Menisci and the Influence of Neighboring Pores on Capillary Displacement Curvatures in Sphere Packings" Journal of Colloid and Interface Science 100, 519 - 535. 1984.

Mason, G. and Morrow, N.R. "Meniscus Displacement Curvatures of a Perfectly Wetting Liquid in Capillary Pore Throats Formed by Spheres" Journal of Colloid and Interface Science 109, 46 - 56. 1986.

Mason, G. and Morrow, N.R. "Capillary Behavior of a Perfectly Wetting Liquid in Irregular Triangular Tubes" Journal of Colloid and Interface Science 141, 262 274. 1991. 
Mason, P. and Starin, S.R. "The Effects of Propellant Slosh Dynamics on the Solar Dynamics Observatory" AIAA-2011-6731, Guidance, Navigation, and Control Conference, Portland, OR, August 8 - 11, 2011.

Massey, W.M. and Sunderland, J.E. "Heat and Mass Transfer in Semi-Porous Channels with Application to Freeze-Drying” International Journal of Heat and Mass Transfer 15, 493 - 502. 1972.

Mayonge, W.A., Kiema, D.W., and Iyaya, C.C.W. "Steady MHD Poiseuille Flow between Two Infinite Parallel Porous Plates in an Inclined Magnetic Field” International Journal of Pure and Applied Mathematics 76, 661 - 668. 2012.

McCarty, R.D. “NBS Technical Note 1097” 1986.

McLean, C., Mustafi, S., Walls, L., Pitchford, B., Wollen, M., Schmidt, J. “Simple, Robust Cryogenic Propellant Depot for Near Term Applications” IEEE-20111044, 2011.

McQuillen, J. B., Chato, D. J., Motil, B. J., Doherty, M. P., Chao, D. F., and Zhang, N. "Porous Screen Applied in Liquid Acquisition Device Channel and CFD Simulation of Flow in the Channel” Journal of Porous Media 15, 429 - 437. 2012.

Meena, S. "Fluid Flow through a Channel with Porous Wall under a Transverse Magnetic Field" $14^{\text {th }}$ Australian Fluid Mechanics Conference, Adelaide University, Adelaide, Australia, December 10 - 14, 2001. 
Mellis, R., Gill, W.N., and Belfort, G. "Fluid Dynamics in a Tubular Membrane: Theory and Experiment” Chemical Engineering Communications 122, 103 - 125. 1993.

Menon, M.G.K. "INSAT in Perspective" AIAA-72-583, $4{ }^{\text {th }}$ Communications Satellite Systems Conference, Washington, D.C., April 24 - 26, 1972.

Merkine, L., Solan, A., and Winograd, Y. "Turbulent Flow in a Tube with Wall Suction" ASME Journal of Heat Transfer 93, 242 - 244. 1971.

Meserole, J.S. and Jones, O.S. "Pressurant Effects on Cryogenic Liquid Acquisition Devices" Journal of Spacecraft and Rockets 30, 236 - 243. 1993.

Metha, N. and Jain, R.K. "Laminar Hydromagnetic Flow in a Rectangular Channel with Porous Walls" Proceedings of the National Institute of Sciences of India A28, 846 $-856.1962$.

Minkowycz, W.J. and Haji-Sheikh, A. "Heat Transfer in Parallel Plates and Circular Porous Passages with Axial Conduction” International Journal of Heat and Mass Transfer 49, 2381 - 2390. 2006.

Minkowycz, W.J. and Haji-Sheikh, A. "Asymptotic Behaviors of Heat Transfer in Porous Passages with Axial Conduction” International Journal of Heat and Mass Transfer 52, $3101-3108.2009$.

Mishra, S.P. and Muduli, J.C. "Combined Free and Forced Convection Effects on the Magnetohydrodynamic Flow through a Porous Channel" Proceedings of the Indian Academy of Sciences 84A, 257 - 272. 1976. 
Mitchell, R.T. "Cassini/Huygens at Saturn and Titan” 56th International Astronautical Congress of the International Astronautical Federation, the International Academy of Astronautics, and the International Institute of Space Law, 2005.

Mitrovic, J. and Maletic, B. "Effect of Thermal Asymmetry on Laminar Forced Convection Heat Transfer in a Porous Annular Channel” Chemical Engineering Technology 29, $750-760.2006$.

Mitrovic, J. and Maletic, B. "Heat Transfer with Laminar Forced Convection in a Porous Channel Exposed to a Thermal Asymmetry" International Journal of Heat and Mass Transfer 50, 1106 - 1121. 2007.

Miyamoto, S. "A Theory of the Rate of Solution of Gas into Liquid. II. The Rate of Solution of Gas into Liquid Containing a Reacting Substance, and a Kinetic Derivation of the Distribution Law" Chemical Society Japan Bulletin 7, 388 398. 1932.

Mkenda, T.B. "Modeling of Fluid Flow in a Channel with Porous Wall and Navier Slip" Master's Thesis, University of Dar es Salaam, October, 2012.

Moder, J.P. "Equations for Multi-node or Multi-zone Analysis of Cryogenic Storage Tanks" (Unpublished results) 2011.

Mohais, R., Xu, C., and Dowd, P.A. "Fluid Flow and Heat Transfer within a Single Horizontal Fracture in an Enhanced Geothermal System" ASME Journal of Heat Transfer 133, 112603. 2011a. 
Mohais, R., Xu, C., and Dowd, P.A. “An Analytical Model of Coupled Fluid Flow and Heat Transfer through a Fracture with Permeable Walls in an EGS” 2011 Australian Geothermal Energy Conference, 175 - 179. 2011 b.

Moin, P. and Kim, J. "Numerical Investigation of Turbulent Channel Flow” Journal of Fluid Mechanics 118, 341 - 377. 1982.

Momirlan, M. and Veziroglu, T.N. "The Properties of Hydrogen as Fuel Tomorrow in Sustainable Energy System for a Cleaner Planet” International Journal of Hydrogen Energy 30, 795 - 802. 2005.

Moore, R.D., Ruggeri, R.S., Gelder, T.F. "Effects of Wall Pressure Distribution and Liquid Temperature on Incipient Cavitation of Freon-114 and Water in Venturi Flow" NASA-TN-D-4340, 1968.

Morduchow, M. “On Laminar Flow through a Channel or Tube with Injection: Application of Method of Averages" Quarterly of Applied Mathematics 14, 361 368. 1957.

Morel, J. and Bernstein, B. “A Method of Parameter Differentiation Applied to Flow in Porous Annuli” ZAMP Journal of Applied Mathematics and Physics 27, 289 302. 1976.

Morel, J., Lavan, Z., and Bernstein, B. "Flow through Rotating Porous Annuli" Physics of Fluids 20, 726 - 733. 1977.

Morrisey, D.C. "Historical Perspective: Viking Mars Lander Propulsion” Journal of Propulsion and Power, 8, 320 - 331. 1992. 
Motil, S.M., Meyer, M.L., and Tucker, S.P. “Cryogenic Fluid Management Technologies for Advanced Green Propulsion Systems" NASA-TM-2007-214810, 2007.

Motsa, S.S., Shateyi, S., Marewo, G.T., and Sibanda, P. “An Improved Spectral Homotopy Analysis Method for MHD Flow in a Semi-Porous Channel” Numerical Algorithms 60, 463 - 481. 2012.

Moussey, Y. and Snider, A.D. "Laminar Flow Over Pipes with Injection and Suction through the Porous Wall at Low Reynolds Number" Journal of Membrane Science 327, $104-107.2009$.

Moussy, Y. and Snider, A.D. "Flow within a Pipe Annulus with Injection and Suction through a Porous Wall with High Wall Reynolds Numbers" ASME Journal of Fluids Engineering 133, 014501. 2011.

Mudawar, I. "Assessment of High-Heat-Flux Thermal Management Schemes” IEEE Transactions on Components and Packaging Technologies 24, 122 - 141. 2001.

Muhaimin, Kandasamy, R., and Khamis, A.B. "Effects of Heat and Mass Transfer on Nonlinear MHD Boundary Layer Flow Over a Shrinking Sheet in the Presence of Suction" Applied Mathematics and Mechanics 29, 1309 - 1317. 2008.

Mulqueen, J. "CRYOSTAT Cryogenic Storage and Transfer Flagship Technology Demonstration" Pre-Phase A Mission Architecture Study, Study Close Out Plan, December 1, 2010. 
Mulqueen, J. “CRYOSTAT Cryogenic Storage and Transfer Flagship Technology Demonstration" Pre-Phase A Mission Architecture Study, Study Close-Out Summary, January 11, 2011.

Munson, B.R. "Very Low Reynolds Number Flow through Screens" Journal of Fluids Engineering 110, 462-463. 1988.

Munson-McGee, S.H. "An Approximate Analytical Solution for the Fluid Dynamics of Laminar Flow in a Porous Tube" Journal of Membrane Science 197, 223 - 230. 2002.

Murase, K., Doi, M., Fukuda, K., Okuyama, A., and Hososno, N. “Superbird-C Communications Satellite System" AIAA-98-1251, $17^{\text {th }}$ International Communications Satellite Systems Conference and Exhibit, 1998.

Myers, F.R., Jackson, W.C., Shropshire, D.P., and Viens, P.R. “Chandra X-Ray Observatory Long Term Flight Software Maintenance: Minimizing Overall Program Risk on an Evolving Spacecraft" AIAA-2008-3290, SpaceOps Conference, Heidelberg, Germany, May 12 - 16, 2008.

Nandi, S. "Unsteady Hydromagnetic Flow in a Porous Annulus with Time-Dependent Pressure Gradient" Pure and Applied Geophysics 79, 33 - 40. 1970a.

Nandi, S. "Unsteady Hydromagnetic Flow in an Annulus with Porous Walls When the Inner Boundary Starts Moving from Rest” Pure and Applied Geophysics 78, 86 95. $1970 \mathrm{~b}$. 
Nandi, S. "Laminar Hydromagnetic Flow in an Annulus with Uniformly Porous Walls of Different Permeability" Pure and Applied Geophysics 89, 139 - 150. 1971.

Nandi, S. "Steady Hydromagnetic Viscous Flow in an Annulus with Porous Walls" Pure and Applied Geophysics 105, 825 - 835. 1973.

Narasimhan, M.N.L. "Laminar Non-Newtonian Flow in a Porous Pipe" Applied Scientific Research A 10, 393 - 409. 1961.

Narasimhan, A. and Lage, J.L. "Forced Convection of a Fluid with TemperatureDependent Viscosity through a Porous Medium Channel" Numerical Heat Transfer Part A: Applications 40, 801 - 820. 2001.

Narita, T. and Yendler, B. “Thermal Propellant Gauging System for BSS 601” AIAA2007-3149, 25 ${ }^{\text {th }}$ International Communications Satellite Systems Conference, 2007.

“NASA Space Technology Roadmaps and Priorities - Restoring NASA's Technological Edge and Paving the Way for a New Era in Space" National Research Council, Washington, DC: The National Academies Press, 2012.

"Near Field InfraRed Experiment (NFIRE)" Mda.mil. Missile Defense Agency, 15 Jan. 2013. Web. 7 Aug. 2013. <http://www.mda.mil/global/documents/pdf/nfire.pdf>.

Netter, G. and Prasad, C.S. "Use of the Surface Tension Propellant Tanks in the Indian Satellite INSAT" IAF Paper 88-237, $39^{\text {th }}$ IAF International Astronautical Congress, Bangalore, India, October 8 - 15, 1988. 
Netter, G., Renner, U., and Dreyer, M. "Design and Verification of a Standard Surface Tension Propellant Tank" AIAA-99-2178, 35 ${ }^{\text {th }}$ Joint Propulsion Conference, Los Angeles, CA, June 20 - 24, 1999.

Neumann, A.W. and Good, R.J. "Techniques of Measuring Contact Angles" Surface and Colloid Science 11, 31 - 91. 1979.

Neumann, A.W. and Spelt, J.K. Applied Surface Thermodynamics. New York: Marcel Dekker, 1996.

Nicoud, F. and Angilella, J.R. "Effects of Uniform Injection at the Wall on the Stability of Couette-like Flows" Physical Review E 56, 3000 - 3009. 1997.

Nield, D.A., Junqueira, S.L.M., and Lage, J.L. "Forced Convection in a Fluid-Saturated Porous-Medium Channel with Isothermal or Isoflux Boundaries" Journal of Fluid Mechanics 322, 201 -214. 1996.

Nield, D.A. and Kuznetsov, A.V. "Local Thermal Nonequilibrium Effects in Forced Convection in a Porous Medium Channel: A Conjugate Problem" International Journal of Heat and Mass Transfer 42, 3245 - 3252. 1999.

Nield, D.A., Porneala, D.C., and Lage, J.L. “A Theoretical Study, with Experimental Verification, of the Temperature-Dependent Viscosity Effect on the Forced Convection through a Porous Medium Channel" ASME Journal of Heat Transfer 121, $500-503.1999$. 
Nield, D.A. and Kuznetsov, A.V. "Effect of Heterogeneity in Forced Convection in a Porous Medium: Parallel Plate Channel or Circular Duct” International Journal of Heat and Mass Transfer 43, 4119 - 4134. 2000.

Nield, D.A., Kuznetsov, A.V., and Xiong, M. "Effect of Local Thermal Non-equilibrium on Thermally Developed Forced Convection in a Porous Medium” International Journal of Heat and Mass Transfer 45, 4949 - 4955. 2002.

Nield, D.A. "The Stability of Flow in a Channel or Duct Occupied by a Porous Medium" International Journal of Heat and Mass Transfer 46, 4351 - 4354. 2003.

Nield, D.A., Kuznetsov, A.V., and Xiong, M. "Thermally Developing Forced Convection in a Porous Medium: Parallel Plate Channel with Walls at Uniform Temperature, with Axial Conduction and Viscous Dissipation Effects" International Journal of Heat and Mass Transfer 46, 643 - 651. 2003a.

Nield, D.A., Kuznetsov, A.V., and Xiong, M. “Thermally Developing Forced Convection in a Porous Medium: Parallel-Plate Channel or Circular Tube with Walls at Constant Heat Flux” Journal of Porous Media 6, 203 - 212. 2003 b.

Nield, D.A., Kuznetsov, A.V., and Xiong, M. "Thermally Developing Forced Convection in a Porous Medium: Parallel Plate Channel or Circular Tube with Isothermal Walls" Journal of Porous Media 7, 19 - 27. 2004a.

Nield, D.A., Kuznetsov, A.V., and Xiong, M. "Effects of Viscous Dissipation and Flow Work on Forced Convection in a Channel Filled by a Saturated Porous Medium" Transport in Porous Media 56, 351 - 367. 2004b. 
Nield, D.A., and Kuznetsov, A.V. "Forced Convection with Laminar Pulsating Flow in a Channel or Tube” International Journal of Thermal Sciences 46, 551 - 560. 2007.

Niemi, R., Mahiout, A., Siivinen, Mahlberg, R., Likonen, J., Nikkola, J., Mannila, J., Vuorio, T., Johansson, L.-S., Soderberg, O., and Hannula, S.-P. “Surface Pretreatment of Austenitic Stainless Steel and Copper by Chemical, Plasma, Electrolytic, or $\mathrm{CO}_{2}$ Cryoblasting Techniques for Sol-Gel Coating” Surface and Coatings Technology 204, 2424 - 2431. 2010.

Nikitin, N.V. and Pavel'ev, A.A. "Turbulent Flow in a Channel with Permeable Walls. Direct Numerical Simulation and Results of Three-Parameter Model” Fluid Dynamics 33, 826 - 832. 1998.

Nissen, J.A., Maytal, B., and Van Sciver, S.W. "Pressure Drop in the SHOOT Superfluid Helium Acquisition System" Cryogenics 30, 211 - 215. 1990.

Nissen, J.A. and Van Sciver, S.W. "Thermal Behavior of the SHOOT Gallery Arm" Advances in Cryogenic Engineering 37A, 115 - 121. Plenum Press, New York, 1991.

Northrup, M.A., Kulp, T.J., and Angel, S.M. "Fluorescent Particle Image Velocimetry: Application to Flow Measurement in Refractive Index-Matched Porous Media” Applied Optics 30, 3034 - 3040. 1991.

Northrup, M.A., Kulp, T.J., Angel, S.M., and Pinder, G.F. “Direct Measurement of Interstitial Velocity Field Variations in a Porous Medium Using FluorescentParticle Image Velocimetry" Chemical Engineering Science 48, 13 - 21. 1993. 
Novikov, P.A. and Lyubin, L.Y. "Analysis of Self-Similar Laminar Flows in Slot Channels with One Permeable Wall” Journal of Engineering Physics 49, 1066 1070. 1985.

Ogulu, A. and Amos, E. "Modeling Pulsatile Blood Flow within a Homogenous Porous Bed in the Presence of a Uniform Magnetic Field and Time-Dependent Suction" International Communications in Heat and Mass Transfer 34, 989 - 995. 2007.

Ojjela, O. and Kumar, N.N. "Numerical Study of MHD Flow and Heat Transfer through Porous Medium between Two Parallel Plates with Hall and Ion Slip Effects" Proceedings of the 2013 International Conference on Mechanics, Fluids, Heat, Elasticity, and Electromagnetic Fields, 161 - 167. 2013.

Olbricht, W.L. "Pore-Scale Prototypes of Multiphase Flow in Porous Media" Annual Review of Fluid Mechanics 28, 187-213. 1996.

Olsen, R.M. and Eckert, E.R.G. "Experimental Studies of Turbulent Flow in a Porous Circular Tube with Uniform Fluid Injection through the Tube Wall” ASME Journal of Applied Mechanics 33, 7 - 17. 1966.

Osborn, M., Clauss, C., Gorin, B., and Netwall, C. “Micro-Satellite Technology Experiment (MiTEx) Upper Stage Propulsion System Development” AIAA-20075434, 43 ${ }^{\text {rd }}$ Joint Propulsion Conference, Cincinnati, OH, July 8 - 11, 2007.

Oxarango, L., Schmitz, P., and Quintard, M. "Laminar Flow in Channels with Wall Suction or Injection: A New Model to Study Multi-Channel Filtration Systems" Chemical Engineering Science 59, 1039 - 1051. 2004. 
Panzarella, C. and Kassemi, M. "One-Dimensional Model of Evaporation and Condensation in the Presence of a Noncondensable Gas with Applications to Cryogenic Fluid Storage" International Journal of Heat and Mass Transfer 52, 3767 - 3777. 2009.

Paynter, H.L. "Investigation of Space Storable Propellant Acquisition Devices Final Report, Volume II - Design and Analysis" MCR-70-171, NASA-CR-111343, Denver, CO, December, 1970.

Paynter, H.L. “Acquisition/Expulsion System for Earth Orbital Propulsion System, Volume III, Cryogenic Test", MCR-73-97, NASA-CR-134155, Martin Marietta Corporation, Denver, CO. October, 1973a.

Paynter, H.L. “Acquisition/Expulsion System for Earth Orbital Propulsion System, Volume V - Final Report" MCR-73-97, NASA-CR-134157, Martin Marietta Corporation, Denver, CO, October, $1973 b$.

Pederson, R.J. and Kinney, R.B. “Entrance-Region Heat Transfer for Laminar Flow in Porous Tubes" International Journal of Heat and Mass Transfer 14, 159 - 161. 1971.

Petukhov, B. S., \& Popov, V. N. "Theoretical Calculations of Heat Exchange and Frictional Resistance in Turbulen Flow in Tubes of an Incompressible Fluid with Variable Physical Properties" High Temperature, 1, 69-83. 1963.

Pilinski, E.B. and Lee, A.Y. "Pointing-Stability Performance of the Cassini Spacecraft" Journal of Spacecraft and Rockets 46, 1007 - 1015. 2009. 
Piomelli, U., Moin, P., and Ferziger, J. "Large Eddy Simulation of the Flow in a Transpired Channel” AIAA-89-0375, $27^{\text {th }}$ Aerospace Sciences Meeting, Reno, NV, January $9-12,1989$.

Plachta, D., Sutherlin, S.G., Johnson, W.L., and Feller, J., and Jurns, J.M. "Methane Lunar Surface Thermal Control Test” NASA-TM-2012-217427, 2012.

Polzin, K.A., Markusic, T.E., and Stanojev, B.J. "Liquid Bismuth Propellant Management System for the Very High Specific Impulse Thruster with Anode Layer” NASA-TM-2007-214958. 2007.

Pong, L. and Moses, G.A., "Vapor Condensation in the Presence of a Noncondensable Gas" Physics of Fluids 29, 1796 - 1804, 1986.

Pozrikidis, C. "Creeping Flow in Two-Dimensional Channels" Journal of Fluid Mechanics 180, 495 - 514. 1987.

Prager, S. "Spiral Flow in a Stationary Porous Pipe" Physics of Fluids 7, 907 - 908. 1964.

Prasad, V. and Kulacki, F.A. "Natural Convection in a Vertical Porous Annulus" International Journal of Heat and Mass Transfer 27, 207 - 219. 1984a.

Prasad, V. and Kulacki, F.A. "Natural Convection in a Rectangular Porous Cavity with Constant Heat Flux on One Vertical Wall” ASME Journal of Heat Transfer 106, $152-157.1984 b$. 
Prasad, V. and Kulacki, F.A. "Natural Convection in Porous Media Bounded by Short Concentric Vertical Cylinders" ASME Journal of Heat Transfer 107, 147 - 154. 1985.

Prasad, V., Kulacki, F.A., and Keyhani, M. "Natural Convection in Porous Media" Journal of Fluid Mechanics 150, 89 - 119. 1985.

Prasad, V. "Numerical Study of Natural Convection in a Vertical, Porous Annulus with Constant Heat Flux on the Inner Wall" International Journal of Heat and Mass Transfer 29, $841-853.1986$.

Prasad, V., Kulacki, F.A., and Kulkarni, A.V. "Free Convection in a Vertical, Porous Annulus with Constant Heat Flux on the Inner Wall - Experimental Results" International Journal of Heat and Mass Transfer 29, 713 - 723. 1986.

Prodanovic, M. and Bryant, S.L. “A Level Set Method for Determining Critical Curvatures for Drainage and Imbibition" Journal of Colloid and Interface Science 304, $442-458.2006$.

Proudman, I. “An Example of Steady Laminar Flow at Large Reynolds Number” Journal of Fluid Mechanics 9, 593 - 602. 1960.

Purohit, G.P. and Prickett, R.P. "Modeling of the Intelsat VI Bipropellant Propulsion System" AIAA-93-2518, $29^{\text {th }}$ Joint Propulsion Conference, Monterey, CA, June 28 $-30,1993$. 
Purohit, G.P., Ellison, J.R., and Jaekle, D.E. "Propellant Management Device Analysis for Some Off-Design Operational Scecnarios" AIAA-99-2974, $35^{\text {th }}$ Joint Propulsion Conference, Los Angeles, CA, June 20 - 23, 1999.

Quaile, J.P. and Levy, E.K. "Laminar Flow in a Porous Tube with Suction" ASME Journal of Heat Transfer 97, 66 - 71. 1975.

Radcliffe, W.F. and Transue, J.R. "Problems Associated with Multiple Engine Starts in Spacecraft” American Rocket Society Journal 31, 1408 - 1412. 1961.

Radhakrishnamacharya, G. and Maiti, M.K. "Heat Transfer to Pulsatile Flow in a Porous Channel" International Journal of Heat and Mass Transfer 20, 171 - 173. 1977.

Rahimi, P. and Ward, C.A. "Kinetics of Evaporation: Statistical Rate Theory Approach" International Journal of Thermodynamics 8, 1-14. 2005.

Raithby, G. "Laminar Heat Transfer in the Thermal Entrance Region of Circular Tubes and Two-Dimensional Rectangular Ducts with Wall Suction and Injection" International Journal of Heat and Mass Transfer 14, 223 - 243. 1971.

Raithby, G.D. and Knudsen, D.C. "Hydrodynamic Development in a Duct with Suction and Blowing” ASME Journal of Applied Mechanics 41, 896 - 902. 1974.

Rajasekhara, B.M. "Experimental and Theoretical Study of Flow Past a Porous Medium" Thesis, Bangalore University, India. 1974.

Ramakrishnan, K. and Shailendhra, K. "Hydromagnetic Flow through Uniform Channel Bounded by Porous Media" Applied Mathematics and Mechanics 32, 837 - 846. 2011. 
Ramakrishnan, K. and Shailendhra, K. "Hydromagnetic Blood Flow through a Uniform Channel with Permeable Walls Covered by Porous Media of Finite Thickness" Journal of Applied Fluid Mechanics 6, 39 - 47. 2013.

Ramana, M.J.V., Srinivasa, N.C., Ojjela, O. "Viscous Fluid Flow between Two Parallel Plates with Periodic Suction and Injection" ASME Advances in Modeling B: Signal Processing and Pattern Recognition 50, 29 - 37. 2007.

Rame, E., Hartwig, J.W., and McQuillen, J.B. "Flow Visualization of Liquid Hydrogen Line Chill Down Tests" AIAA-2014-1074, SciTech Conference, National Harbor, MD, January 13 - 17, 2014.

Rao, A.K. "Laminar Natural Convection Flow with Suction or Injection" Applied Scientific Research A 11, 1-9. 1963.

Rao, V.V.R. and Prasad, V.B. "Heat Transfer in a Rotating Channel with Porous Walls" Acta Physica Academiae Scientiarum Hungaricae, Tomus 42, 143 - 149. 1977.

Raptis, A. "MHD Natural Convection and Mass Transfer through a Horizontal Porous Channel" Acta Physica Hungaria 54, 213 - 215. 1983.

Rath, P.V., Parida, T., and Dash, G.C. “Three-Dimensional Free Convective Flow through Porous Medium in a Vertical Channel with Heat Source and Chemical Reaction" Proceedings of the National Academy of Sciences A: Physical Sciences (India) 82, $225-232.2012$.

Rath, P.V., Dash, G.C., and Parida, A.K. "Three-Dimensional MHD Free Convective Flow with Heat and Mass Transfer through a Porous Medium with Periodic 
Permeability and Chemical Reaction" Proceedings of the National Academy of Sciences A: Physical Sciences (India) 83, 105 - 114. 2013.

Rattenni, L. "Design and Performance of the Orbital Star-2 Propulsion Subsystem" AIAA-2001-3394, $37^{\text {th }}$ Joint Propulsion Conference, Salt Lake City, UT, July 8 $11,2001$.

Reddy, N.B. and Bathaiah, D. "MHD Combined Free and Forced Convection Flow through Two Parallel Porous Walls" Acta Mechanica 42, 239 - 251. 1982.

Regnier, W.W. and Hess, D.A. "Design and Development of a Passive Propellant Management System" Journal of Spacecraft 15, 299 - 304. 1978a.

Rhee, S.J. and Edwards, D.K. "Laminar Entrance Flow in a Flat Plate Duct with Asymmetric Suction and Heating” Numerical Heat Transfer 4, 85-100. 1981.

Richardson, S. "A Model for the Boundary Condition of a Porous Material” Journal of Fluid Mechanics 49, 327 - 336. 1971.

Rio, O.I. and Neumann, A.W. "Axisymmetric Drop Shape Analysis" Computational Methods for the Measurement of Interfacial Properties from the Shape and Dimensions of Pendant and Sessile Drop" Journal of Colloid and Interface Science 196, 136 - 147. 1997.

Robbins, W.H. and Finger, H.B. "An Historical Perspective of the NERVA Nuclear Rocket Engine Technology Program" NASA-CR-187154. 1991. 
Robinson, W.A. "The Existence of Multiple Solutions for the Laminar Flow in a Uniformly Porous Channel with Suction at Both Walls" Journal of Engineering Mathematics 10, 23 - 40. 1976.

Roder, H.M. and Weber, L.A. “ASRDI Oxygen Technology Survey Volume I: Thermophysical Properties” NASA-SP-3071, January, 1972.

Rollins, J.R., Grove, R.K., Schatz, W.J., and Valliappan, K.L. "Design and Operational Performance of INSAT-I Propellant Tank" AIAA-83-1273, $19^{\text {th }}$ Joint Propulsion Conference, Seattle, WA, June 27 - 29, 1983.

Rollins, J.R., Grove, R.K., Lewis, A.H. "Design and Qualification of the Arabsat Propellant Tank" AIAA-84-1480, 20 ${ }^{\text {th }}$ Joint Propulsion Conference, Cincinnati, OH, June $11-13,1984$.

Rollins, J.R., Grove, R.K., and Jaekle, D.J. “Twenty-Three Years of Surface Tension Propellant Management System Design, Development, Manufacture, Test, and Operation" AIAA-85-1199, $21^{\text {st }}$ Joint Propulsion Conference, Monterey, CA, July $8-10,1985$.

Rollins, J.R., Grove, R.K., and Hobbs, L.W. "Design and Qualification of the EUROSTAR Propellant Tank" AIAA-86-1659, 22 ${ }^{\text {nd }}$ Joint Propulsion Conference, Huntsville, AL, June 16 - 18, 1986.

Rollins, J.R., Grove, R.K., and Walling, D.R. "Design and Qualification of a Surface Tension Propellant Tank for an Advanced Spacecraft" AIAA-88-2848, $24^{\text {th }}$ Joint Propulsion Conference, Boston, MA, July 11 - 13, 1988. 
Rollins, J.R., Grove, R.K., Miller, J.A., Price, J.D., and Lawrie, A. “Design and Qualification of the EUROSTAR 2000 Propellant Tank” AIAA-92-3606, $28^{\text {th }}$ Joint Propulsion Conference, Nashville, TN, July 6 - 8, 1992.

Roy, J.S., Nayak, P., and Dash, J. "Heat Transfer in Pulsatile Visco-Elastic Flow in a Porous Channel” Acta Mechanica 40, 33 - 48. 1981.

Roy, J.S. and Nayak, P. "Steady Two Dimensional Incompressible Laminar Visco-Elastic Flow in a Converging or Diverging Channel with Suction and Injection" Acta Mechanica 43, 129 - 136. 1982.

Rybak, S.C., Willen, G.S., Follett, W.H., Hanna, G.J., Cady, E.C., DiStefano, E., and Meserole, J.S. "Feasibility Study for a Cryogenic On-orbit Liquid Depot-Storage, Acquisition, and Transfer (COLD-SAT) Satellite" NASA-CR-185248. 1990.

Saad, T. and Majdalani, J. "Rotational Flowfields in Porous Channels with Arbitrary Headwall Injection” Journal of Propulsion and Power 25, 921 - 929. 2009.

Saffman, P.G. "On the Boundary Condition at the Interface of a Porous Medium" Studies in Applied Mathematics 1, 93 - 101. 1971.

Sahoo, S.N. "Heat and Mass Transfer Effect on MHD Flow of a Viscoelastic Fluid through a Porous Medium Bounded by an Oscillating Porous Plate in Slip Flow Regime” International Journal of Chemical Engineering 380679, 2013.

Sahraoui, M. and Kaviany, M. "Slip and No-Slip Velocity Boundary Conditions at Interface of Porous, Plain Media" International Journal of Heat and Mass Transfer 35, $927-943.1992$. 
Sai, K.S. and Rao, B.N. "Magnetohydrodynamic Flow in a Rectangular Duct with Suction and Injection" Acta Mechanica 140, 57 - 64. 2000.

Sakla, S., Kutter, B., and Wall, J. "Centaur Test Bed (CTB) for Cryogenic Fluid Management" NASA-CR-2006-214410, 2006.

Saleh, S., Thovert, J.F., and Adler, P.M. "Measurement of Two-Dimensional Velocity Fields in Porous Media by Particle Image Displacement Velocimetry" Experiments in Fluids 12, 210 - 212. 1992.

Saleh, S., Thovert, J.F., and Adler, P.M. "Flow along Porous Media by Particle Image Velocimetry" AIChE Journal 39, 1765 - 1776. 1993.

Salmon, P.M., Libicki, S.B., and Channing, R. “A Theoretical Investigation of Convective Transport in the Hollow-Fiber Reactor" Chemical Engineering Communications 66, 221 - 248. 1988.

Sandeep, N., Reddy, A.V.B., and Sugunamma, V. "Effect of Radiation and Chemical Reaction on Transient MHD Free Convective Flow over a Vertical Plate through Porous Media” Chemical and Process Engineering Research 2, 1 - 9. 2012.

Sangiorgi, R. Caracciolo, G., Passerone, A. "Factors Limiting the Accuracy of Measurements of Surface Tension by the Sessile Drop Method" Journal of Materials Science 17, 2895 - 2901. 1982.

Santiago, J.R. "Evolution of the RL10 Liquid Rocket Engine for a New Upperstage Application" AIAA-2006-3013, 32 $2^{\text {nd }}$ Joint Propulsion Conference, Lake Buena Vista, FL, July 1 - 3, 1996. 
Sarani, S. "Cassini Attitude Control Configuration for Huygens Probe Release" AIAA2005-6390, Guidance, Navigation, and Control Conference, San Francisco, CA, August $15-18,2005$.

Sato, H., Watanabe, K., Sengers, J.M.H., Gallagher, J.S., Hill, P.G., Straub, J., and Wagner, W. "Sixteen Thousand Evaluated Experimental Thermodynamic Property Data for Water and Steam" Journal of Physical and Chemical Reference Data 20, 1023 - 1044. 1991.

Saulsberry, R., Ramirez, J., Julien, H., Hart, M., and Smith, W. "Mars Observer Propulsion and Pyrotechnics Corrective Actions Test Program Review - 1999” AIAA-99-2305, 35 ${ }^{\text {th }}$ Joint Propulsion Conference, Los Angeles, CA, June 20 - 23, 1997.

Schildknecht, M., Miller, J.A., and Meier, G.E.A. "The Influence of Suction on the Structure of Turbulence in Fully Developed Pipe Flow" Journal of Fluid Mechanics 90, 67 - 107. 1979.

Schmidt, G.R. "Thermocapillary Flow with Evaporation and Condensation and its Effect on Liquid Retention in Low-g Fluid Acquisition Devices" NASA-TP-3463. 1994.

Schmidt, G.R., Nadarajah, A., Chung, T.J., and Karr, G.R. "Influence of Two-Phase Thermocapillary Flow on Liquid Retention in Microscopic Pores" Journal of Thermophysics and Heat Transfer 9, 151 - 158. 1995. 
Schmit, D.D., Leeds, M., and Vote, F. "In-Flight Performance of the Viking 75 Orbiter Propulsion System" AIAA-77-894, $13^{\text {th }}$ Propulsion Conference, Orlando, FL, July $11-13,1977$.

Schmit, D.D., Anderson, J.W., and Vote, F.C. “A Long Life Bipropellant System Demonstration, Viking Orbiter Propulsion System 4 Years in Space and Operating” AIAA-80-1173, $16^{\text {th }}$ Joint Propulsion Conference, Hartford, CT, June 30 - July 2, 1980.

Schmit, D.D., Anderson, J.W., and Vote, F.C. "Long-Life Bipropellant System Demonstration, Viking Orbiter Propulsion System” Journal of Spacecraft 18, 327 $-332.1981$.

Schmitz, P., Houi, D., and Wandelt, B. "Hydrodynamic Aspects of Crossflow Microfiltration. Analysis of Particle Deposition at the Membrane Surface” Journal of Membrane Science 71, 29 - 40. 1992.

Schnitzlein, K. and Lowe, A. "Numerical Simulation of the Performance of Ceramic Fiber Coil Diesel Particulate Traps” Chemical Engineering Science 45, 2671 2678. 1990.

Schnitzlein, K. "A Note on Laminar Flow in Uniformly Porous Tubes" ASME Journal of Fluids Engineering 115, 493 - 496. 1993.

Schrage, R.W. "A Theoretical Study of Interphase Mass Transfer" Columbia University Press, New York. 1953. 
Schuster, J.R. and Brown, N.S. "Long Term Orbital Storage of Cryogenic Propellants for Advanced Space Transportation Missions" SEE-A88-33426, IEEE EASCON Conference, Washington, D.C., October 14 - 16, 1987.

Schuster, J.R., Russ, E.J., Wachter, J.P. “Cryogenic On-Orbit Liquid Depot Storage, Acquisition, and Transfer Satellite (COLD-SAT) Feasibility Study Final Report NASA-CR-185249. 1990.

Schuster, J.R., Howell, D.J., Lucas Jr., S.L., Haberbusch, M.S., Gaby, J.D., Van Dresar, N.T., and Wadel, M.F. “Cold Flow Testing of Revised Engine Chilldown Methods for the Atlas Centaur" $32^{\text {nd }} A I A A-96-3014,32^{\text {nd }}$ Joint Propulsion Conference, Lake Buena Vista, FL, July 1 - 3,1996.

Schuster, J.R., Bassett, C.E., Christensen, E.H., Honkonen, S.C., Merino, F., Munko, D.S., Pietrzyk, J.R., and Wollen, M.A. "Centaur-Based Liquid Hydrogen Fluid Management Flight Experiments" AIAA-91-3539, AIAA/NASA/OAI Conference on Advanced SEI Technologies, Cleveland, OH, September 4 - 6, 1997.

Schutz, S., Kopf, P., and Piesche, M. "Prediction of Pore Size and Pressure Drop of Porous Woven Wire Cloth Filter Media on the Basis of Calculation Models" Filtration 8, 335 - 344. 2008.

Schweickert, T.F. "Design of the Aft Propulsion Subsystem for Long Life" JANNAF Propulsion Meeting, New Orleans, May 26 -28, 1981.

Secomb, T.W. "Flow in a Channel with Pulsating Walls" Journal of Fluid Mechanics 88, $273-288.1978$. 
Sefiane, K. and Ward, C. A., "Recent Advances on Thermocapillary Flows and Interfacial Conditions during the Evaporation of Liquids," Advances in Colloid and Interface Science 134-135, 201-223. 2007.

Sellars, J.R. "Laminar Flow in Channels with Porous Walls at High Suction Reynolds Numbers" Journal of Applied Physics 26, 489 - 490. 1955.

Semenov, Yu P, Lozino-Lozinsky, et al., Mnogorazoviy orbitalniy korabl 'Buran', Mashinostroenie, Moscow, 1995.

Sengupta, P.R. and Ghosh, S.K. "Slow Hydromagnetic Flow in a Channel with Porous Walls in Presence of a Periodic Magnetic Field" Czechoslovak Journal of Physics B 27, 158 - 166. 1977.

Sharma, B.K., Agarwal, M., and Chaudhary, R.C. "Radiation Effect on Temperature Distribution in Three-Dimensional Couette Flow with Suction or Injection" Applied Mathematics and Mechanics 28, 309 - 316. 2007.

Sharma, B.K., Mishra, A., and Gupta, S. "Heat and Mass Transfer in Magneto-Biofluid Flow through a Non-Darcian Porous Medium with Joule Effect” Journal of Engineering Physics and Thermophysics 86, 766 - 774. 2013.

Sharma, H.G. and Singh, K.R. "Heat Transfer in the Laminar Flow of a Non-Newtonian Fluid in a Porous Annulus by the Method of Quasi-linearization" International Journal of Heat and Mass Transfer 30, 1227 - 1231. 1987. 
Sharma, R.P. and Yadav, G.R. "Heat Transfer through Three Dimensional Couette Flow between a Stationary Porous Plate Bounded by Porous Medium and a Moving Porous Plate" Journal of Ultra Scientist of Physical Sciences 17, 351 - 360. 2005.

Sharma, S.K., Sharma, V.K., and Varshney, N.K. "Effect of Thermal Diffusion on MHD Free Convective Flow of Dusty Olrdroud Fluid through a Porous Medium Bounded by an Oscillating Porous Plate in Slip Flow Regime with Heat Source/Sink" International Journal of Mathematical Archive 3, 4711 - 4717. 2012.

Sheppard, D.M. "Hydrodynamic Stability of the Flow between Parallel Porous Walls" Physics of Fluids 15, 241 - 243. 1972.

Shih, K.G. "On the Existence of Solutions of an Equation Arising in the Theory of Laminar Flow in a Uniformly Porous Channel with Injection" SIAM Journal of Applied Mathematics 47, 526 - 533. 1987.

Shivakumar, P.N., Nagaraj, S., Veerabhadraiah, R., and Rudraiah, N. "Fluid Movement in a Channel of Varying Gap with Permeable Walls Covered by a Porous Media" Journal of Engineering Science 24, 479 - 492. 1986.

Shrestha, G.M. "Singular Perturbation Problems of Laminar Flow in a Uniformly Porous Channel in the Presence of a Transverse Magnetic Field" The Quarterly Journal of Applied Mechanics and Applied Mathematics 20, 233 - 246. 1967. 
Shrestha, G.M. "Heat Transfer in Laminar Flow in a Uniformly Porous Channel with an Applied Transverse Magnetic Field” Applied Scientific Research 19, 352 - 369. 1968.

Shrestha, G.M. and Terrill, R.M. "Laminar Flow with Large Injection through Parallel and Uniformly Porous Walls of Different Permeability" The Quarterly Journal of Mechanics and Applied Mathematics 21, 413 - 432. 1968.

Shrestha, G.M. "Laminar Non-Newtonian Flow through a Porous Annulus" ZAMM Journal of Applied Mathematics and Mechanics 49, 53 - 62. 1969a.

Shrestha, G.M. "Laminar Elasticoviscous Flow in Channels with Porous Walls” Journal of Applied Physics 40, 907 - 909. 1969b.

Si, X., Zheng, L., Zhang, X., and Chao, Y. "Perturbation Solution to Unsteady Flow in a Porous Channel with Expanding or Contracting Walls in the Presence of a Transverse Magnetic Field” Applied Mathematics and Mechanics 31, 151 - 158. 2010.

Si, X., Zheng, L., Zhang, X., Min, L., Jianhong, Y., Ying, C. "Multiple Solutions for the Laminar Flow in a Porous Pipe with Suction at Slowly Expanding or Contracting Wall” Applied Mathematics and Computation 218, 3515 - 3521. 2011a.

Si, X., Zheng, L., Zhang, X., Chao, Y. "Existence of Multiple Solutions for the Laminar Flow in a Porous Channel with Suction at both Slowly Expanding and Contracting Walls” International Journal of Minerals, Metallurgy, and Materials $18,494-501.2011 b$. 
Si, X., Zheng, L., Lin, P., Zhang, X., Zhang, Y. "Flow and Heat Transfer of a Micropolar Fluid in a Porous Channel with Expanding or Contracting Walls" International Journal of Heat and Mass Transfer 67, 885 - 895. 2013.

Silva, R.A. and Lemos, M.J.S. "Turbulent Flow in a Channel Occupied by a Porous Layer Considering the Stress Jump at the Interface" International Journal of Heat and Mass Transfer 46, 5113 - 5121. 2003.

Simon, E.D. "Environmental Requirements for Bubble Pressure Tests on Fine-Mesh Screens" Journal of Spacecraft and Rockets 16, 218 - 222. 1979.

Singh, K.D. "Three Dimensional Couette Flow with Transpiration Cooling” ZAMP Journal of Applied Mathematics and Physics 50, 661 - 668. 1999.

Singh, K.D., Sharma, R., and Chand, K. "Three Dimensional Fluctuating Flow and Heat Transfer through a Porous Medium with Variable Permeability" ZAMM Journal of Applied Mathematics and Mechanics 80, 473 - 480. 2000.

Singh, K.D. and Sharma, R. "MHD Three-Dimensional Couette Flow with Transpiration Cooling” ZAMM Journal of Applied Mathematics and Mechanics 81, 715 - 720. 2001.

Singh, K.D. and Sharma, R. “Three Dimensional Free Convective Flow and Heat Transfer through a Porous Medium with Periodic Permeability" Indian Journal of Pure Applied Mathematics 33, 941 - 949. 2002.

Singh, K.D. "Influence of Moving Magnetic Field on Three Dimensional Couette Flow" ZAMP Journal of Applied Mathematics and Physics 55, 894 - 902. 2004. 
Singh, K.D. "Visco-Elastic Mixed Convection MHD Oscillatory Flow through a Porous Medium Filled in a Vertical Channel” International Journal of Physical and Mathematical Sciences 3, 194 - 205. 2012.

Singh, K.D. "Visco-Elastic MHD Convective Periodic Flow through Porous Medium in a Rotating Vertical Channel with Thermal Radiation" Journal of Global Research in Mathematical Archives 1, 8 - 20. 2013.

Singh, P. and Gupta, C.B. "MHD Free Convective Flow of Viscous Fluid through a Porous Medium Bounded by an Oscillating Porous Plate in Slip Flow Regime with Mass Transfer” Indian Journal of Theoretical Physics 53, 111 - 120. 2005.

Singh, R. and Laurence, R.L. "Influence of Slip Velocity at a Membrane Surface on Ultrafiltration Performance - I. Channel Flow System” International Journal of Heat and Mass Transfer 22, 721 - 729. 1979a.

Singh, R. and Laurence, R.L. "Influence of Slip Velocity at a Membrane Surface on Ultrafiltration Performance - II. Tube Flow System" International Journal of Heat and Mass Transfer 22, 731 - 737. 1979b.

Sinor, J.E. and Kurata, F. "Solubility of Helium in Liquid Argon, Oxygen, and Carbon Monoxide” Journal of Chemical and Engineering Data 11, 537 - 539. 1966.

Sinor, J.E. PhD Dissertation University of Kansas, 1965.

Skalank, F.M. Wang, C.Y. "Pulsatile Flow in Tube with Wall Injection and Suction" Applied Scientific Research 33, 269 - 307. 1977. 
Skalank, F.M. and Wang, C.Y. "On the Nonunique Solutions of Laminar Flow through a Porous Tube or Channel" SIAM Journal of Applied Mathematics 34, 535 - 544. 1978.

Smith, T.D., Klem, M.D., and Fisher, K., "Propulsion Risk Reduction Activities for NonToxic Cryogenic Propulsion” AIAA-2010-8680, AIAA Space Conference, Anaheim, CA, August 30 - September 2, 2010.

Smolak, G.R. "Thermal Analysis for the Cryogenic Fluid Management Flight Experiment" N88-15935, Cryogenic Fluid Management Technology Workshop April 28, 1987.

Smolders, C.A. and Duyvis, E.M. "Contact Angles; Wetting and De-wetting of Mercury: Part I. A Critical Examination of Surface Tension Measurement by the Sessile Drop Method" Recueil des Travaux Chimiques des Pays-Bas 80, 635 - 649. 1961.

Smutny, B. and Lange, R. "Homodyne BPSK Based Optical Inter-Satellite Communication Links" AIAA-2006-5460, $24^{\text {th }}$ International Communications Satellite Systems Conference, San Diego, CA, June 11 - 14, 2006.

Sodre, J.R. and Parise, J.A.R. "Friction Factor Determination for Flow through Finite Wire-Mesh Woven-Screen Materials" Journal of Fluid Engineering 119, 847851. 1997.

Sorour, M.M., Hassab, M.A., and Estafanous, S. "Developing Laminar Flow in a Semiporous Two-Dimensional Channel with Nonuniform Transpiration" Heat and Fluid Flow 8, 44 - 54. 1987. 
Sparrow, E.M., Lin, S., and Lundgren, T.S. "Flow Development in the Hydrodynamic Entrance Region of Tubes and Ducts" Physics of Fluids 7, 338 - 347. 1964.

Sparrow, E.M., Beavers, G.S., and Hung, L.Y. "Channel and Tube Flows with Surface Mass Transfer and Velocity Slip" Physics of Fluids 14, 1312 - 1319. 1971.

Sparrow, E.M., Beavers, G.S., and Hwang, T.I. "Effect of Velocity Slip on PorousWalled Squeeze Films" Journal of Lubrication Technology 94, 260 - 264. 1972.

Sparrow, E.M., Chen, T.S, Lloyd, J.R., and Beavers, G.S. "Breakdown of the Laminar Flow Regime in Permeable-Walled Ducts" ASME Journal of Applied Mechanics 40, $337-342.1973$.

Sparrow, E.M., Beavers, G.S., and Masha, B.A. "Laminar Flow in a Rectangular Duct Bounded by a Porous Wall" Physics of Fluids 17, 1465 - 1467. 1974.

Spencer, B., Rigollet, R., Fontaine, J.M., and Salome, R. "Qualification and Future Development of Propellant Tanks for Telecommunication Satellites" AIAA-3387, $27^{\text {th }}$ Joint Propulsion Conference, Sacramento, CA, June $24-26,1991$.

Sprow, F.B. and Prausnitz, J.M. "Surface Tensions of Simple Liquids" Transactions of the Faraday Society, 62, 1097 - 1104. 1966.

Srivastava, L.M. “An Exact Solution of the Problem of MHD Unsteady Viscous Flow through a Porous Straight Channel" Acta Physica Academiae Scientiarum Hungaricae 41, 63 - 66. 1976. 
Srivastava, L.M. and Agarwal, R.P. "On Unsteady Viscous MHD Flow through a Porous Channel with Constant Suction" Acta Physica Academiae Scientiarum Hungaricae 45, 163 - 170. 1978.

Srivastava, N. and Singh, A.K. "Free Convection between Coaxial Vertical Cylinders Partially Filled with a Porous Material Having Constant Heat Flux on the Inner Cylinder" International Journal of Energy and Technology 3, 3, 1 - 6. 2011.

Standley, S.P. "Cassini-Huygens Engineering Operations at Saturn” AIAA-2006-5516, Space Ops Conference, 2006.

Stansfield, D. "The Surface Tensions of Liquid Argon and Nitrogen" Proceedings of the Physical Society 72, $854-866.1958$.

Stark, J.A., Blatt, M.H., Bennett, F.O., and Campbell, B.J. "Fluid Management Systems Technology Summaries" NASA-CR-134748, 1974.

Stiegemeier, B. and Marshall, W. "Sea-Level Testing of a 100 lbf LOX/Methane Reaction Control Engine" $5^{\text {th }}$ JANNAF Liquid Propulsion Subcommittee Meeting, May, 2010.

Stiegemeier, B., Williams, G., Melcher, J.C., and Robinson, J.W. “Altitude Testing of an Ascent Stage LOX/Methane Main Engine" $5^{\text {th }}$ JANNAF Liquid Propulsion Subcommittee Meeting, May, 2010.

Stochl, R.J., Van Dresar, N.T., and Lacovic, R.F. “Autogenous Pressurization of Cryogenic Vessels Using Submerged Vapor Injection” NASA-TM-104516, 
Cryogenic Engineering Conference and International Cryogenic Materials Conference, Huntsville, AL, June 11 - 14, 1991.

Stoltz, P.M., Krebs, M., and Baltman, R. “ORBCOMM Attitude Determination and Control” AIAA-96-3620, Astrodynamics Conference, San Diego, CA, July 29 31, 1996.

Street, D. “A Scalable Orbital Propellant Depot Design" AE8900 MS Special Problems Report, Space Systems Design Lab, Georgia Institute of Technology, Atlanta, GA, April 28, 2006.

Stultz, J.W. "Viking Mars Orbiter 1975 Solar Energy Controller" Journal of Spacecraft 14, $294-299.1977$.

Subhas, A. and Venna, P. "Visco-Elastic Fluid Flow and Heat Transfer in a Porous Medium Over a Stretching Sheet" International Journal of Non-Linear Mechanics 33, $531-540.1998$.

Suga, K., Matsumura, Y., Ashitaka, Y., Tominaga, S., and Kaneda, M. "Effects of Wall Permeability on Turbulence" International Journal of Heat and Fluid Flow 31, $974-984.2010$.

Sumitani, Y. and Kasagi, N. "Direct Numerical Simulation of Turbulent Transport with Uniform Wall Injection and Suction” AIAA Journal 33, 1220 - 1228. 1995.

Suryaprakasarao, U. "Laminar Flow in Channels with Porous Walls in the Presence of a Transverse Magnetic Field" Applied Scientific Research B 9, 374 - 382. 1962.

Sutherlin, S. Personal correspondence, September 9, 2011. 
Sutton, F.M. "Onset of Convection in a Porous Channel with Net through Flow" Physics of Fluids 13, 1931 - 1934. 1970.

Symons, E.P. "Wicking of Liquids in Screens" NASA-TN-D-7657, 1974.

Tachie, M.F., James, D.F., and Currie, I.G. "Velocity Measurements of a Shear Flow Penetrating a Porous Medium” Journal of Fluid Mechanics 493, 319 - 343. 2003.

Taklifi, A. and Aliabadi, A. "Analytical Solution of Unsteady MHD Periodic Flow of a Non-Newtonian Fluid through a Porous Channel" Journal of Porous Media 15, $1051-1059.2012$.

Tam, W.H., Debreceni, M.J., and Lay, W.D. "Design and Development of the NEAR Oxidizer Tank" AIAA-95-2528, $31^{\text {st }}$ Joint Propulsion Conference and Exhibit, San Diego, CA, July 10-12, 1995.

Tam, W.H., Lay, W.D., Hersh, M.S., Jaekle, D.E., and Epstein, S.J. “Design, Development, Qualification, and Manufacture of the HS 601 Propellant Tank", AIAA-96-2748, 32 ${ }^{\text {nd }}$ Joint Propulsion Conference, Lake Buena Vista, FL, July 1 3, 1996.

Tam, W.H., Jaekle, D.E., and Farokhi, S.A. “Design and Manufacture of the HS 601 Block II Propellant Tank Assembly” AIAA-98-3199, 34 ${ }^{\text {th }}$ Joint Propulsion Conference and Exhibit, Cleveland, OH, July 13-15, 1998.

Tam, W.H., Kawahara, G.H., Jaekle, D.J., and Larsson, L.W. "Design and Manufacture of a Propellant Tank Assembly" AIAA-2000-3444, $36^{\text {th }}$ Joint Propulsion Conference, Huntsville, AL, July 16 - 19, 2000. 
Tam, W.H., Drey, M.D., Jaekle, D., and Larsson, L.W. "Design and Manufacture of an Oxidizer Tank Assembly" AIAA-2001-3825, 37 ${ }^{\text {th }}$ Joint Propulsion Conference, Salt Lake City, UT, July 8 - 11, 2001.

Tam, W.H., Wiley, S., Dommer, K., Mosher, L., and Persons, D. "Design and Manufacture of the Messenger Propellant Tank Assembly" AIAA-2002-4139, 38 Joint Propulsion Conference, Indianapolis, IN, July 7 - 10, 2002a.

Tam, W.H., Kuo, J., and Jaekle, D.J. “Design and Manufacture of an Ultra-Lightweight Propellant Management Device" AIAA-2002-4137, 2002b.

Tam, W.H., Hersh, M., and Ballinger, I. "Hybrid Propellant Tanks for Spacecraft and Launch Vehicles" AIAA-2003-4607, 39 ${ }^{\text {th }}$ Joint Propulsion Conference, Huntsville, AL, July $20-23,2003$.

Tam, W.H. and Jaekle, D.E. "Design and Manufacture of an Oxidizer Tank with a Surface Tension PMD” AIAA-2005-3734, 2005.

Tam, W.H., Ballinger, I., and Jaekle, D.J. “Conceptual Design of Space Efficient Tanks” AIAA-2006-5058, 42 ${ }^{\text {nd }}$ Joint Propulsion Conference, Sacramento, CA, July $9-12$, 2006.

Tam, W.H., Ballinger, I., and Jaekle, D.E. "Propellant Tank with Surface Tension PMD for Tight Center-of-Mass Propellant Control” AIAA-2008-4942, 2008a.

Tam, W., Ballinger, I., and Jaekle, D.E. "Tank Trade Studies - An Overview” AIAA2008-4940, 44 ${ }^{\text {th }}$ Joint Propulsion Conference, Hartford, CT, July $21-23,2008$ b. 
Tam, W.H., Ballinger, I., and Jaekle, D.E. "Surface Tension PMD Tank for On Orbit Fluid Transfer" AIAA-2008-5105, 44 ${ }^{\text {th }}$ Joint Propulsion Conference, Hartford CT, July $21-23,2008 c$.

Tanner, C., Young, J., Thompson, R., and Wilhite, A. “On-Orbit Propellant Resupply Options for Mars Exploration Architectures” AIAA-2006-261, IAC-06-D1.1.01. 2006.

Taylor, C.L., Banks, W.H., Zaturska, M.B., and Drazin, P.G. “Three-Dimensional Flow in a Porous Channel" Quarterly Journal of Mechanics and Applied Mathematics 44, $105-133.1991$.

Taylor, G. "Fluid Flow in Regions Bounded by Porous Surfaces" Proceedings of the Royal Society A: Mathematical, Physical, and Engineering Science 234, 456 475. 1956.

Taylor, G.I. “A Model for the Boundary Condition of a Porous Material. Part 1” Journal of Fluid Mechanics 49, 319 - 326. 1971.

Tegart, J.R. and Fester, D.A. "Space Storable Propellant Acquisition System” Journal of Spacecraft and Rockets 12, $544-551.1975$.

Tegart, J.R. "Influence of Pressure Transients on the Performance of Capillary Propellant Acquisition Systems" AIAA-76-597, $12^{\text {th }}$ Propulsion Conference, Palo Alto, CA, July $26-29,1976$. 
Tegart, J.R. and Aydelott, J.C. "Effect of Vibration on Retention Characteristics of Screen Acquisition Systems" AIAA-78-1030, $14^{\text {th }}$ Joint Propulsion Conference, Las Vegas, NV, July $25-27,1978$.

Tegart, J.R. "Performance of a Capillary Propellant Management Device with Hydrazine" AIAA-79-1259, $15^{\text {th }}$ Joint Propulsion Conference, Las Vegas, NV, June $18-20,1979$.

Tegart, J.R. "A Vane Type Propellant Management Device" AIAA-97-3028, $33^{\text {rd }}$ Joint Propulsion Conference, Seattle, WA, July 6- 9, 1997.

Terrill, R.M. "Laminar Boundary-Layer Flow near Separation With and Without Suction" Philosophical Transactions of the Royal Society of London. Series A, Mathematical and Physical Sciences 253, 55 - 100. 1960.

Terrill, R.M. "Laminar Flow in a Uniformly Porous Channel" The Aeronautical Quarterly 299-310. 1964.

Terrill, R. M. "Laminar Flow in a Uniformly Porous Channel with Large Injection" The Aeronautical Quarterly 323 -332, November, 1965a.

Terrill, R.M. "Heat Transfer in Laminar Flow between Parallel Porous Plates" International Journal of Heat and Mass Transfer 8, 1491 - 1497. $1965 \mathrm{~b}$.

Terrill, R.M. "Slow Laminar Flow in a Converging or Diverging Channel with Suction at One Wall and Blowing at the Other Wall" ZAMP Journal of Applied Mathematics and Physics 16, $306-308.1965 \mathrm{c}$. 
Terrill, R.M. and Shrestha, G.M. "Laminar Flow through Parallel and Uniformly Porous Walls of Different Permeability" ZAMP Journal of Applied Mathematics and Physics 16, $470-482.1965$.

Terrill, R.M. “A Possibility of Eigensolutions for Laminar Flow in a Porous Channel” ASME Journal of Applied Mechanics 33, 200 - 201. 1966.

Terrill, R.M. and Shrestha, G.M. "Laminar Flow through a Channel with Uniformly Porous Walls of Different Permeability" Applied Scientific Research 15, 440 468. 1966.

Terrill, R.M. "Flow through a Porous Annulus" Applied Scientific Research 17, 204 222. 1967.

Terrill, R.M. and Walker, G. "Heat and Mass Transfer in Laminar Flow between Parallel Porous Plates" Applied Scientific Research 18, 193 - 220. 1967.

Terrill, R.M., and Thomas, P.W. "On Laminar Flow through a Uniformly Porous Pipe" Applied Scientific Research 21, 37 - 67. 1969.

Terrill, R.M. and Thomas, P.W. "Spiral Flow in a Porous Pipe" Physics of Fluids 16, 356 $-359.1973$.

Terrill, R.M. “An Exact Solution for Flow in a Porous Pipe” ZAMP Journal of Applied Mathematics and Physics 33, 547 - 552. 1982.

Terrill, R.M. "Laminar Flow in a Porous Tube" Journal of Fluids Engineering 105, 303 307. 1983. 
Tharakan, J.P. and Chau, P.C. "Operation and Pressure Distribution of Immobilized Cell Hollow Fiber Bioreactors" Biotechnology and Bioengineering 28, 1064 - 1071. 1986.

Thome, J.R. “Wolverine Engineering Databook III: Chapter 10: Boiling Heat Transfer Inside Plain Tubes" available online at www.wlv.com. 2004.

Thongyai, N. "Study of Stainless Steel Surface Cleanability" Master's Thesis, Institute of Technology, North Bangkok, 2005.

Tian, Z.F., Mohais, R., Xu, C., and Zhu, X. "CFD Modeling of the Velocity Profile within a Single Horizontal Fracture in an Enhanced Geothermal System" $18^{\text {th }}$ Australian Fluid Mechanics Conference, Launceston, Tasmania, December 3-7, 2012

Tilton, N. and Cortelezzi, L. "Linear Stability Analysis of Pressure-Driven Flows in Channels with Porous Walls" Journal of Fluid Mechanics 604, 411 - 445. 2008.

Tomsik, T.M., Johnson, W.L., Smuddle, T.D., and Femminineo, M.F., “A Densified Liquid Methane Delivery System for the Altair Ascent Stage" NASA-TM-2010216246, 2010.

Tsai, G.L., Lin, Y.C., Wang, H.W., Lin, Y.F., Su, Y.C., and Yang, J.T. “Cooling Transients in a Sudden-Expansion Channel with Varied Rates of Wall Transpiration" International Journal of Heat and Mass Transfer 52, 5990 - 5999. 2009. 
Tsai, M.K. and Liou, T.M. "Study of Flow Induced by Nonuniform Lateral Injection" Journal of Propulsion 7, 668 - 677. 1991.

Tsangaris, S., Kondaxakis, D., and Vlachakis, N.W. "Exact Solution for Flow in a Porous Pipe with Unsteady Wall Suction and/or Injection" Communications in Nonlinear Science and Numerical Simulation 12, 1181 - 1189. 2007.

Tsou, R.C.H. and Chang, Y.P. "On the Linearized Analysis of Entrance Flow in Heated, Porous Conduits" International Journal of Heat and Mass Transfer 19, 445 448. 1976.

Tunc, G., Wagner, H., and Bayazitoglu, Y. "Space Shuttle Upgrade Liquid Oxygen Tank Thermal Stratification" AIAA-2001-3082, 35 ${ }^{\text {th }}$ Thermophysics Conference, Anaheim, CA, June $11-14,2001$.

Turkevich, L.A. and Mann, J.A. "Pressure Dependence of the Interfacial Tension between Fluid Phases. 1. Formalism and Application to Simple Fluids" Langmuir 6, $445-456.1990 a$.

Turkevich, L.A. and Mann, J.A. "Pressure Dependence of the Interfacial Tension between Fluid Phases. 2. Application to Liquid-Vapor Interfaces and to Interfaces of Amphiphilic Solutions" Langmuir 6, 457 - 470. 1990b.

Vafai, K. and Thiyagaraja, R. "Analysis of Flow and Heat Transfer at the Interface Region of a Porous Medium" International Journal of Heat and Mass Transfer 30, $1391-1405.1987$. 
Vafai, K. and Kim, S.J. "Fluid Mechanics of the Interface Region between a Porous Medium and a Fluid Layer - An Exact Solution" International Journal of Heat and Fluid Flow 11, 254 - 256. 1990.

Valli, A., Hyvaluoma, J., Jasberg, A., Koponen, A. and Timonen, J. "Pressure Drop for Low Reynolds-Number Flows through Regular and Random Screens" Transport in Porous Media 80, 193-208. 2009.

Van Dyke, M. “Identification of Influential Factors for Liquid Acquisition Device Designs" AIAA-98-3198, 34 ${ }^{\text {th }}$ Joint Propulsion Conference, Cleveland, OH, July $13-15,1998$.

Van Sciver, S.W. “Helium Cryogenics” Plenum Press: New York, NY. 1986.

Vandermey, N. and Paczkowski, B.G. "The Cassini-Huygens Mission Overview” AIAA2006-5502, SpaceOps Conference, 2006.

Varapaev, V.N. "Viscous Fluid Flow in Initial Segment of Two-Dimensional Channel with Porous Walls" Fluid Dynamics 4, 122 - 124. 1969.

Varapaev, V.N. and Yagodkin, V.I. "Flow Stability in a Channel with Porous Walls" Fluid Dynamics 4, 60-62. 1969.

Vassilieff, C.S. “Convective Model of Cross-Flow Microfiltration” Advances in Colloid and Interface Science 40, 1 - 36. 1992.

Vazquez, G., Alvarez, E., and Navaza, J.M. "Surface Tension of Alcohol + Water from 20 to 50 "C" Journal of Chemical Engineering Data 40, 611 - 614. 1995. 
Verma, P.D. and Bansal, J.L. "Flow of a Viscous Incompressible Fluid between Two Parallel Plates, One in Uniform Motion and the Other at Rest with Uniform Suction at the Stationary Plate" Proceedings of the Indian Academy of Sciences, A 64, $385-396.1966$.

Verma, P.D. and Mathur, A.K. "Magnetohydrodynamic Flow between Two Parallel Plates, One in Uniform Motion and the Other at Rest with Uniform Suction at the Stationary Plate" Proceedings of the National Institute of Sciences of India 35A, $507-517.1969$.

Verma, P.D. and Bhatt, B.S. "Plane Couette Flow of Two Immiscible Incompressible Fluids with Uniform Suction at the Stationary Plate" Proceedings of the Indian Academy of Sciences of India A 78, 108 - 120. 1973.

Verma, P.D. and Gaur, Y.N. "Laminar Swirling Flow in an Annulus with Porous Walls" Proceedings of the Indian Academy of Sciences of India A 80, 211 - 222. 1974.

Vidhya, M. and Kesavan, S. "Laminar Convection through Porous Medium between Two Vertical Parallel Plates with Heat Source” IEEE 197 - 200. 2010.

Vidyanidhi, V. and Nigam, S.D. "Secondary Flow in Rotating Channel” Journal of Mathematics and Physical Sciences 1, 85-97. 1967.

Vidyanidhi, V., Prasad, V.B., and Rao, V.V.R. "Secondary Flow between Two Parallel Porous Walls in a Rotating System" Journal of the Physical Society of Japan 39, 1077 - 1081. 1975. 
Vigdorovich, I. and Oberlack, M. “Analytical Study of Turbulent Poiseuille Flow with Wall Transpiration" Physics of Fluids 20, 055102. 2008.

Vignes-Adler, M.M., Adler, P.M., and Gougat, P. “Transport Processes across Fractals. The Cantor-Taylor Brush” PhysicoChemical Hydrodynamics 8, 401 - 422. 1987.

Volkov, K.N. and Emelyanov, V.N. "Mathematical Models of Three-Dimensional Turbulent Flows in Channels with Injection” Journal of Mathematical Modeling $16,41-63.2004$.

Volkov, K.V. “Application of a Two-Parametric Turbulence Model to Description of Flows in Channels with Injection" Journal of Engineering Physics and Thermophysics 85, $884-894.2012$.

Volkov, K.N. "Simulation of a Nonstationary Flow in a Channel with a Distributed Blow from the Channel Walls and Forced Pressure Oscillations" Journal of Engineering Physics and Thermophysics 86, 96 - 104. $2013 \mathrm{a}$.

Volkov, K.N. "Simulation of a Fluid Flow in a Channel with a Moving Side Surface and Injection” Journal of Engineering Physics and Thermophysics 86, 1447 - 1453. $2013 b$.

Vote, F.C. and Schatz, W.J. "Development of the Propulsion Subsystem for the Viking 75 Orbiter" AIAA-73-1208, $9^{\text {th }}$ Propulsion Conference, Las Vegas, NV, November $5-7,1973$.

Wageman, W.E. and Guevara, F.A. "Fluid Flow through a Porous Channel" Physics of Fluids 3, 878 - 881. 1960. 
Wakeland, R.S. and Keolian, R.M. "Measurements of Resistance of Individual SquareMesh Screens to Oscillating Flow at Low and Intermediate Reynolds Numbers" Journal of Fluids Engineering 125, 851 - 862. 2003.

Walker, G. and Terrill, R.M. "Mass Transfer Cooling of Laminar Flow between Parallel Porous Plates" ASME Journal of Heat Transfer 96, 343 - 347. 1974.

Walker, J.A. and Weil, J. “The X-15 Program” 1963.

Wang, C.Y. "Pulsatile Flow in a Porous Channel” ASME Journal of Applied Mechanics $38,553-555.1971$.

Wang, C.Y. "Exact Solutions of the Unsteady Navier-Stokes Equations" Applied Mechanics Reviews 42, S269 - S282. 1989.

Wang, C.Y. "Exact Solutions of the Steady-State Navier-Stokes Equations" Annual Review of Fluid Mechanics 23, 159 - 177. 1991.

Warren, R.P. “Acquisition System Environmental Effects Study” NASA-CR-120768. Martin Marietta Corp., May, 1975.

Warren, R.P., Butz, J.R., Maytum, C.D., Fester, D.A., and Young, G.M. "Measurements of Capillary System Degradation” AIAA-75-1197, $11^{\text {th }}$ Propulsion Conference, Anaheim, CA, September 29 - October 1, 1975.

Washburn, E.W. “The dynamics of capillary flow” Physical Review 17, 273 - 283. 1921. 
Wasistho, B., Balachandar, S., and Moser, R.D. “Compressible Wall-Injection Flows in Laminar, Transitional, and Turbulent Regimes: Numerical Prediction" Journal of Spacecraft and Rockets 41, 915 - 924. 2004.

Watson, P., Banks, W.H.H., Zaturska, M.B., and Drazin, P.G. "Laminar Channel Flow Driven by Accelerating Walls" European Journal of Applied Mathematics 2, 359 $-385.1991$.

Weissberg, H.L. "Laminar Flow in the Entrance Region of a Porous Pipe" Physics of Fluids 2, 510 - 516. 1959.

Weissberg, H.L. and Berman, A.S. "Velocity and Pressure Distributions in Turbulent Pipe Flow with Uniform Wall Suction” Proceedings of the Heat Transfer and Fluid Mechanics Institute, 1 - 30, University of California, Los Angeles, CA, June 14, 1955.

Weisskopf, M.C., Brinkman, B., Canizares, C., Garmire, G., Murray, S., and Van Speybroeck, L.P. “An Overview of the Performance and Scientific Results from the Chandra X-Ray Observatory" Astronomical Society of the Pacific 114, 1-24. 2002.

White, F. M., Barfield, B. F., and Goglia, M. J. "Laminar Flow in a Uniformly Porous Channel” ASME Journal of Applied Mechanics 25, 613 - 617. 1958.

White, F.M. "Laminar Flow in a Uniformly Porous Tube" Journal of Applied Mechanics 29, $201-204.1962$.

White, F.M. Viscous Fluid Flow New York: McGraw-Hill Inc. 1991. 
White, G.P. “Aft Propulsion Subsystem” Addendum II to TN-E453-244, 1980.

Wight, F.M., Barfield, B.F., and Golia, M.J. "Laminar Flow in a Uniformly Porous Channel” Journal of Applied Mechanics 25, 613 - 615. 1958.

Wiley, S. and Dommer, K. "Design and Development of the Messenger Propulsion System" AIAA-2003-5078, 39 ${ }^{\text {th }}$ Joint Propulsion Conference, Huntsville, AL, July $20-23,2003$.

Willis, W.D. "The SDO Propulsion Subsystem” AIAA-2012-4329, 48 ${ }^{\text {th }}$ Joint Propulsion Conference, Atlanta GA, July 30 - August 2, 2012.

Wilson, A.C. and Meserole, J.S. "Liquid Hydrogen Acquisition Device Component Fabrication and Testing” Boeing Company, March, 1986.

Wilson, M.N., Engelbrecht, C.S., and Trela, M.D. "Flight Performance of the MESSENGER Propulsion System from Launch to Orbit Insertion" AIAA-20124333, 48 ${ }^{\text {th }}$ Joint Propulsion Conference, Atlanta, GA, July 30 - August 1, 2012.

Wilson, M.N., Engelbrecht, C.S., and Jaekle, D.J. "MESSENGER Propulsion System: Strategies for Orbit-Phase Propellant Extraction at Low Fill Fractions" AIAA2013-3757, 49 ${ }^{\text {th }}$ Joint Propulsion Conference, San Jose, CA, July $14-17,2013$.

Wintec Corporation “Cryogenic Fuel Filter Study, NAS9-11264, March 1972.

Winters, B.A. "Analysis of the Solar Thermal Upper Stage Technology Demonstrator Liquid Acquisition Device with Integrated Thermodynamic Vent System" AIAA96-2745 32 ${ }^{\text {nd }}$ Joint Propulsion Conference, Lake Buena Vista, FL, July 1 - 3, 1996. 
Woodward, R.P. "Surface Tension Measurement Using the Drop Shape Method" Firsttenangstroms.com. First Ten Angstroms. Web. <http://www.firsttenangstroms.com/pdfdocs/STPaper.pdf〉. 2010.

Wong, E.C. and Breckenridge, W.G. "An Attitude Control Design for the Cassini Spacecraft" AIAA-95-3274-CP, 1995.

Wu, W.T., Liu, J.F., Li, W.J., and Hsieh, W.H. "Measurement and Correlation of Hydraulic Resistance of Flow through Woven Metal Screens" International Journal of Heat and Mass Transfer 48, 3008 - 3017. 2005.

Xu, H., Lin, Z.L., Liao, S.J., Wu, J.Z., and Majdalani, J. "Homotopy Based Solutions of the Navier-Stokes Equations for a Porous Channel with Orthogonally Moving Walls" Physics of Fluids 22, 053601. 2010.

Yarlagadda, A.P. and Yoganathan, A.P. "Experimental Studies of Model Porous Media Fluid Dynamics" Experiments in Fluids 8, 59 - 71. 1989.

Yendler, B. and Jew, E. “Total Fuel Management at EOL” AIAA-2008-3376, SpaceOps Conference, Heidelberg, Germany, May 12 - 16, 2008.

Yeroshenko, V.M., Zaichik, L.I., and Bakhvalov, B.Y. "Heat Transfer in Laminar Plane Channel Flow with Uniform Suction or Injection" International Journal of Heat and Mass Transfer 24, 1649 - 1655. 1981.

Yeroshenko, V.M., Yershov, A.V., and Zaichik, L.I. "Heat Transfer for Turbulent Flow in a Circular Tube with Uniform Suction or Injection" International Journal of Heat and Mass Transfer 27, 1197 - 1203. 1984. 
Young, J.J., Thompson, R.W., and Wilhite, A.W. “Architecture Options for Propellant Resupply of Lunar Exploration Elements” AIAA-2006-7237, SPACE Conference, San Jose, CA, September 19 - 21, 2006.

Yuan, S. W. "Further Investigation of Laminar Flow in Channels with Porous Walls" Journal of Applied Physics 27, 267 - 269. 1956.

Yuan, S.W. and Finkelstein, A.B. "Laminar Pipe Flow with Injection and Suction through a Porous Wall” Transactions of the American Society of Mechanical Engineers 78, 719 - 724. 1956.

Yuan, S.W. and Brogen, E.W. "Turbulent Flow in a Circular Pipe with Porous Wall" Physics of Fluids 4, 368 - 372. 1961.

Zagarola, M.V., Perry, A.E., and Smits, A.J. "Log Laws or Power Laws: The Scaling in the Overlap Region” Physics of Fluids 9, 2094 - 2100. 1997.

Zaturska, M.B., Drazin, P.G., and Banks, W.H.H. "On the Flow of a Viscous Fluid Driven Along a Channel by Suction at Porous Walls" Fluids Dyanmics Research 4, $151-178.1988$.

Zaturska, M.B. and Banks, W.H.H. "Suction-Driven Flow in a Porous Pipe" ZAMM Journal of Applied Mathematics and Mechanics 75, 21 - 30. 1995.

Zaturska, M.B. and Banks, W.H.H. "New Solutions for Flow in a Channel with Porous Walls and/or Non-Rigid Wall” Fluid Dynamics Research 33, 57 - 71. 2003.

Zegler, F., Kutter, B.F., and Barr, J. “A Commercially Based Lunar Architecture” AIAA2009-6567, SPACE Conference, Pasadena, CA, September 14 - 17, 2009. 
Zegler, F. and Kutter, B. "Evolving to a Depot-Based Space Transportation Architecture" 2010 Space Conference, Anaheim, CA, August 30 - September 2, 2010.

Zhang, N., Chato, D.J., McQuillen, J.B., Motil, B.J., and Chao, D.F. "CFD Simulation of Pressure Drops in Liquid Acquisition Device Channel with Sub-cooled Oxygen" World Academy of Science, Engineering and Technology 58, 1180 - 1185, 2009.

Zhang, Q. and Prosperetti, A. "Pressure-Driven Flow in a Two-Dimensional Channel with Porous Walls" Journal of Fluid Mechanics 631, 1-21. 2009.

Zhang, Y., Lin, P., and Si, X. "Perturbation Solutions for Asymmetric Laminar Flow in Porous Channel with Expanding and Contracting Walls" Applied Mathematics and Mechanics 35, 203 - 220. 2014.

Zhapbasbaev, U.K. and Isakhanova, G.Z. "Developed Turbulent Flow in a Plane Channel with Simultaneous Injection through One Porous Wall and Suction through the Other” Journal of Applied Mechanics and Technical Physics 39, 53 - 59. 1998.

Zimmerli, G.A., Asipauskas, M. and Van Dresar, N.T. "Empirical Correlations for the Solubility of Pressurant Gases in Cryogenic Propellants" Cryogenics 50, 556 560. 2010.

Zimmerman, R.F. "The Universe in a Mirror: The Saga of the Hubble Space Telescope and the Visionaries Who Built It" New Jersey: Princeton University Press. 2008.

Zisman, W.A. "Contact Angle, Wettability, and Adhesion. Relation of the Equilibrium Contact Angle to Liquid and Solid Constitution" Advances in Chemistry 43, 1 51. 1964. 
“3D-Models.” BOPP.com. BOPP Group, n.d. Web. August 2, 2013. 